ORP-56297

Revision 0

\title{
Final Report - High Level Waste Vitrification System Improvements, VSL-07R1010-1, Rev 0, dated 04/16/07
}

Prepared for the U.S. Department of Energy

Assistant Secretary for Environmental Management

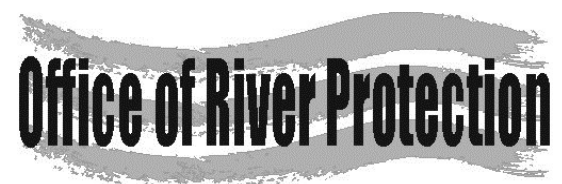

P.O. Box 450

Richland, Washington 99352 


\section{Final Report - High Level Waste Vitrification System Improvements, VSL-07R1010-1, Rev 0, dated 04/16/07}

\author{
A. A. Kruger \\ Department of Energy - Office of River Protection \\ H. Gan \\ The Catholic University of America \\ I. L. Pegg \\ The Catholic University of America \\ W. Gong \\ The Catholic University of America
}

\author{
C. C. Champman \\ The Catholic University of America \\ I. Joseph \\ The Catholic University of America \\ K. S. Matlack \\ The Catholic University of America
}

Date Published

April 2007

Prepared for the U.S. Department of Energy Assistant Secretary for Environmental Management

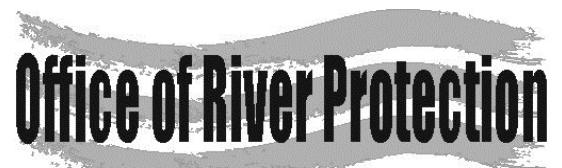

\section{P.O. Box 450}

Richland, Washington 99352

\section{APPROVED}

By Shauna Adams at 12:52 pm, Nov 13, 2013 
ORP-56297

Revision 0

TRADEMARK DISCLAIMER

Reference herein to any specific commercial product, process, or service by tradename, trademark, manufacturer, or otherwise, does not necessarily constitute or imply its endorsement, recommendation, or favoring by the United States Government or any agency thereof or its contractors or subcontractors.

This report has been reproduced from the best available copy.

Printed in the United States of America 
VSL-07R1010-1

\title{
Final Report
}

High Level Waste Vitrification System Improvements

\author{
prepared by
}

Keith S. Matlack, Hao Gan, Weiliang Gong, and Ian L. Pegg

Vitreous State Laboratory

The Catholic University of America

Washington, DC 20064

and

Christopher C. Chapman, Innocent Joseph

Duratek, Inc.

Columbia, MD 21046

for

Department of Energy

Office of River Protection

February 28, 2007

Rev. 0; 4/16/07 
Document Title: High Level Waste Vitrification System Improvements

\author{
Document Number \\ and Revision: VSL-07R1010-1, Rev. 0
}

Issue Date: $\quad 4 / 16 / 06$

Performing Organization: Vitreous State Laboratory, The Catholic University of America

Test Plan:

High Level Waste Vitrification System Improvements, VSL-

06T1010-1, Rev. 0

This report describes the results of testing specified by the above Test Plan. The work was performed in compliance with the quality assurance requirements specified in the Test Plan. Results required by the Test Plan are reported. The test results and this report have been reviewed for correctness, technical adequacy, completeness, and accuracy.

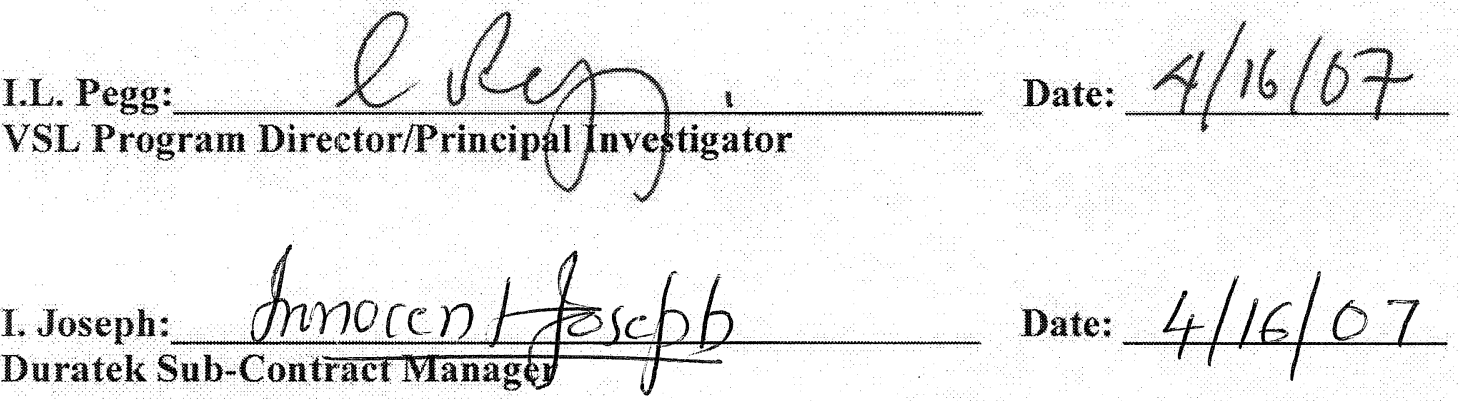


The Catholic University of America Vitreous State Laboratory
High Level Waste Vitrification System Improvements Final Report, VSL-07R1010-1, Rev. 0

\section{TABLE OF CONTENTS}

LIST OF TABLES.

LIST OF FIGURES

LIST OF ABBREVIATIONS

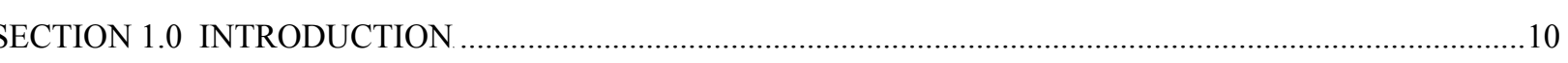

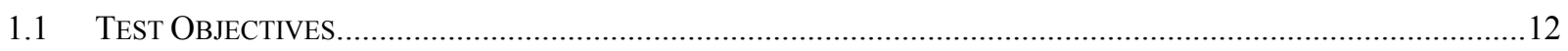

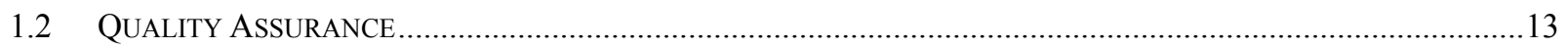

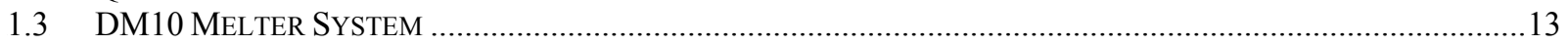

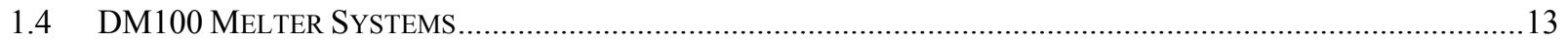

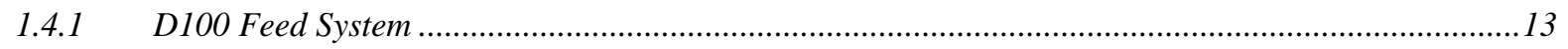

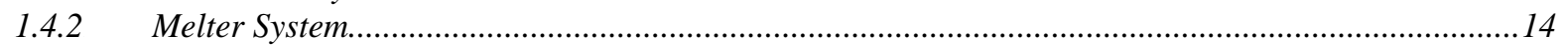

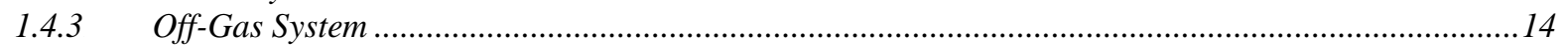

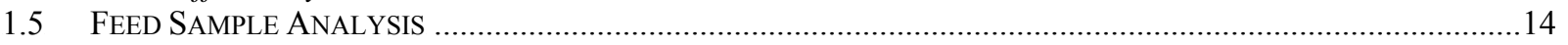

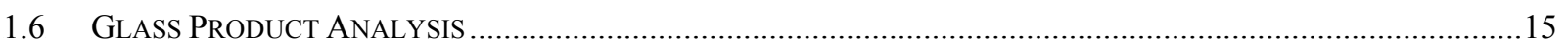

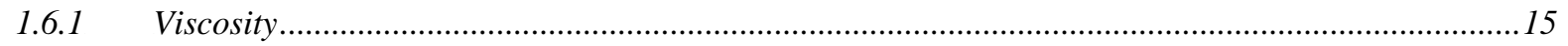

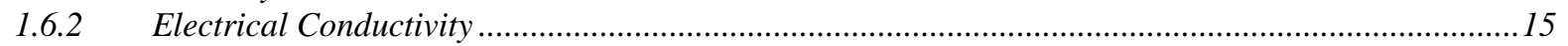

1.6.3 Product Consistency Test (PCT)....................................................................................................16

1.6.4 Toxicity Characteristic Leaching Procedure (TCLP).................................................................16

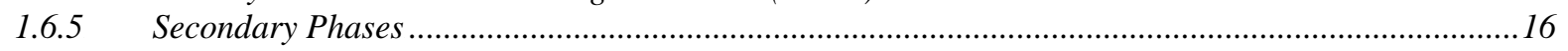

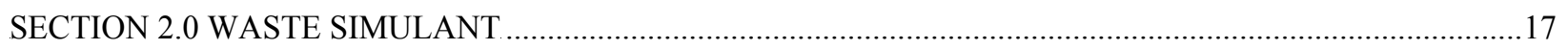

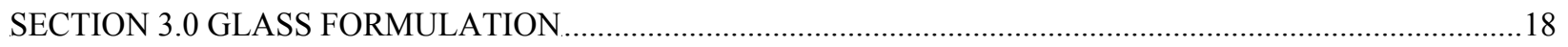

3.1 ENHANCED GLASS FormULATION FOR BI-LIMITED WASTE …..............................................................18

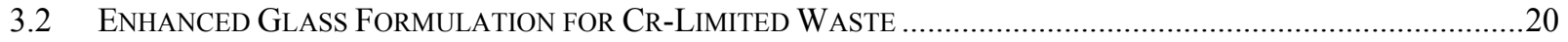

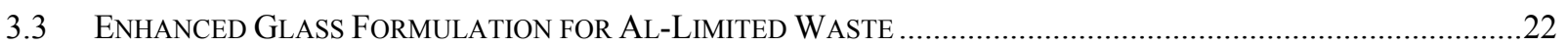

3.4 ENHANCED GLASS FORMULATION FOR AL-NA-LimitED WASTE.....................................................................24

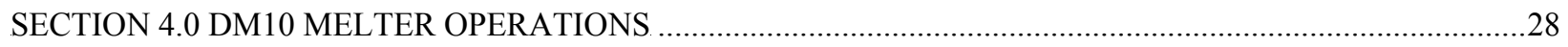

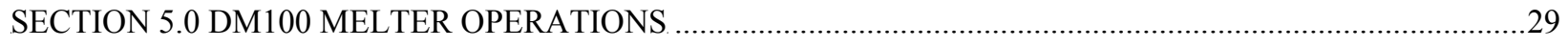

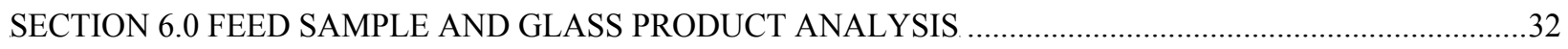

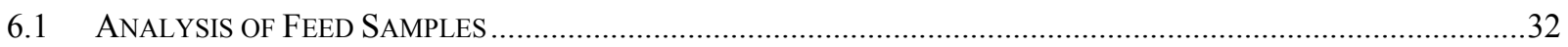

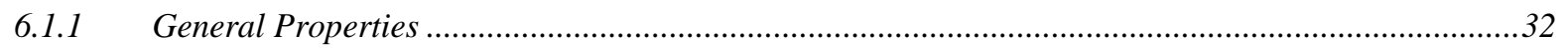

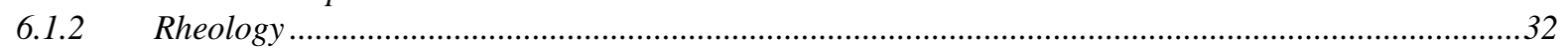

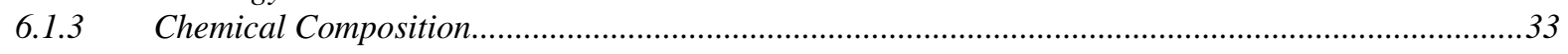

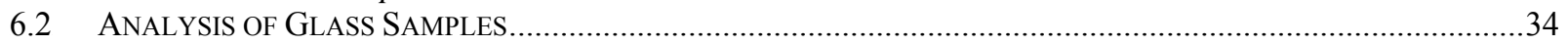

6.2.1 Compositional Analysis of Discharge Glasses ..................................................................................34

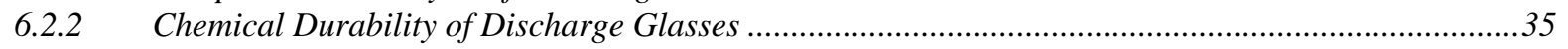

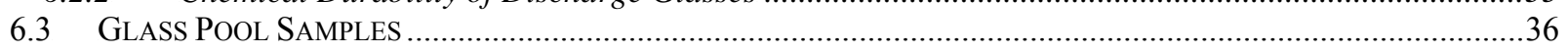

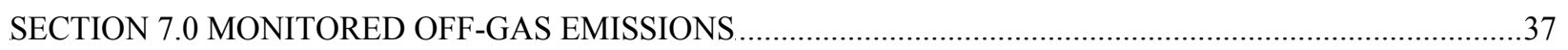

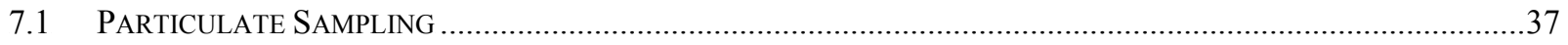

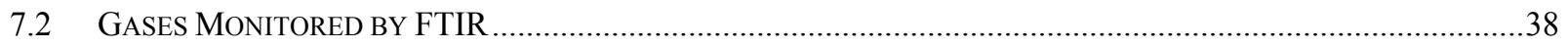

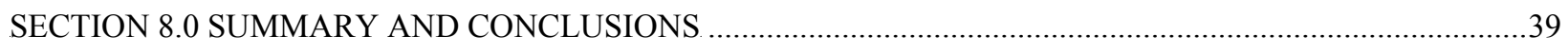

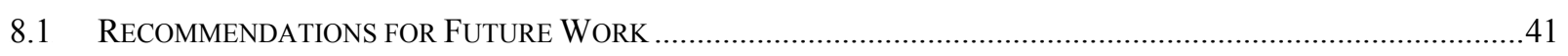

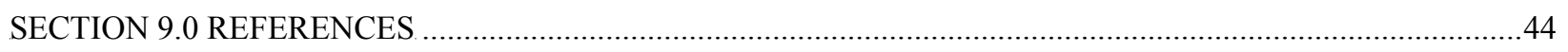

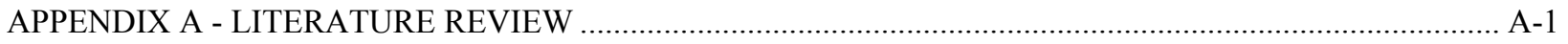




\section{List of Tables}

Table 2.1. Oxide Compositions of Limiting Waste Streams

Table 2.2. Compositions of the Bi-Limited Waste (Oxide Basis) and the HLW Waste Simulant to Produce $100 \mathrm{~kg}$ of Waste Oxides (20 wt \% suspended solids).

Table 2.3. Compositions of the Cr-Limited Waste (Oxide Basis) and the HLW Waste Simulant to Produce $100 \mathrm{~kg}$ of Waste Oxides (20 wt $\%$ suspended solids).

Table 2.4. Compositions of the Al-Limited Waste (Oxide Basis) and the HLW Waste Simulant to Produce $100 \mathrm{~kg}$ of Waste Oxides (20 wt $\%$ suspended solids).

Table 2.5. Compositions of the Al- and Na-Limited Waste (Oxide Basis) and the HLW
Waste Simulant to Produce $100 \mathrm{~kg}$ of Waste Oxides ( $20 \mathrm{wt} \%$ suspended solids).

Table 3.1. Target Composition and XRF Analysis of Glasses Formulated for Bi-Limited Waste

Table 3.2. Characterization of Glasses Formulated for Bi-Limited Waste.

Table 3.3. TCLP Results (ppm) for Glasses Selected for Melter Tests and Their Corresponding Radioactive Glasses.

Table 3.4. Composition and Properties of Bismuth Limited Waste and Glass Formulation at 50\% Waste Loading Used in Melter Tests (wt\%).

Table 3.5. Target Composition and XRF Analysis of Glasses Formulated for Cr-Limited Waste.

Table 3.6. Characterization of Glasses Formulated for Cr-Limited Waste.

$\mathrm{T}-12$

$\mathrm{T}-15$

Table 3.7. XRF Analysis of Yellow Salt Phase Collected from Glass Surface of a Crucible Melt. $\mathrm{B}_{2} \mathrm{O}_{3}$ and $\mathrm{Li}_{2} \mathrm{O}$ were not analyzed by XRF.

Table 3.8. Composition and Properties of Chromium Limited Waste and Glass Formulation at 40\% Waste Loading Initially Used in DM10 Melter Tests (wt\%).

Table 3.9. Target Composition and XRF Analysis of Glasses Formulated for Al-Limited Waste.

Table 3.10. Characterization of Glasses Formulated for Al-limited Waste.

Table 3.11. Composition and Properties of Aluminum Limited Waste and Glass Formulation at $45 \%$ Waste Loading Used in Melter Tests (wt\%).

Table 3.12. Target Composition and XRF Analysis of Glasses Formulated for Al-Na-Limited Waste.

Table 3.13. Characterization of Glasses Formulated for Al-Na-Limited Waste.

Table 3.14. Composition and Properties of Aluminum-Plus-Sodium-Limited Waste and Glass Formulation at $47 \%$ Waste Loading Used in Melter Tests (wt $\%$ ).

Table 4.1. Summary of Chromium-Limited Waste DM10 Test Conditions and Results.

Table 4.2. Composition and Properties of Chromium Limited Waste and Glass Formulation at $32.5 \%$ Waste Loading Used in DM100 Melter Tests (wt \%).

Table 5.1. Summary of Results from DM100 Bismuth Limited Waste Tests.

Table 5.6. Summary of Measured DM100 Parameters for Bismuth-Limited Waste Tests.

Table 5.7. Summary of Measured DM100 Parameters for Chromium-Limited Waste Tests.

Table 5.8. Summary of Measured DM100 Parameters for Aluminum-Limited Waste Tests.

Table 5.9. Summary of Measured DM100 Parameters for Al + Na-Limited Waste Tests.

Table 6.1. Characteristics of Melter Feed Samples from DM100 Tests.

Table 6.2. XRF Analyzed Compositions of Vitrified Melter Feed Samples (wt\%).

Table 6.3. Listing of Glass Discharged, Masses, and Analysis Performed During Bismuth-Limited DM100 Tests.

Table 6.4. List of Glass Discharged, Masses, and Analysis Performed During Chromium-Limited DM100 Tests. 
Table 6.5. List of Glass Discharged, Masses, and Analysis Performed During Aluminum-Limited DM100 Tests.

Table 6.6. List of Glass Discharged, Masses, and Analysis Performed During Aluminum-PlusSodium-Limited DM100 Tests.

Table 6.7. XRF-Analyzed Compositions of Discharged Glass Samples from the DM100 Tests with the Bismuth-Limited Composition (wt\%).

Table 6.8. XRF-Analyzed Compositions of Discharged Glass Samples from the DM100 Tests with the Chromium-Limited Composition (wt\%).

Table 6.9. XRF-Analyzed Compositions of Discharged Glass Samples from the DM100 Tests with the Aluminum-Limited Composition (wt $\%$ ).

Table 6.10. XRF-Analyzed Compositions of Discharged Glass Samples from the DM100 Tests with the Aluminum-Plus-Sodium-Limited Composition (wt $\%$ ).

Table 6.11. XRF and DCP Analysis of Selected Glass Samples (wt\%).

Table 6.12. PCT Results for Melter Glasses.

Table 6.13 TCLP Results for Melter Glasses (mg/L).

Table 6.14. Glass Pool Samples and Secondary Phase Observations. $\quad$ T-77

Table 7.1. Results from Melter Off-Gas Emission Samples.

Table 7.2. Average Concentration (ppmv) of Selected Species in Off-Gas Measured by FTIR Spectroscopy. 


\section{List of Figures}

Figure 1.1. Schematic diagram of DuraMelter 100-BL vitrification system.

$\underline{\text { Page }}$

Figure 1.2.a. Schematic diagram showing cross-section through the DM100-BL-melter. Plan view showing locations of lid ports.

Figure 1.2.b. Schematic diagram showing cross-section through the DM100-BL melter.

Figure 3.1. Volume \% of crystalline phase in the heat treated glasses vs. heat treatment temperature.

Figure 3.2. Top view of CCC sample of HLW-E-Bi6. Foaming observed is concentrated near the top of the sample.

F-6

Figure 3.3. Volume $\%$ of crystalline phase in the heat treated glasses vs. heat treatment temperature for HLW-E-Bi-6 and HLW-E-BiUThR1. Two arrows indicate the volume $\%$ of crystals in the corresponding samples after CCC treatment.

F-7

Figure 3.4. Volume $\%$ of crystalline phase in the heat treated glasses vs. heat treatment temperature for HLW-E-Cr-M and HLW-E-CrMUTh. Two arrows indicate the volume \% of crystals in the corresponding samples after CCC treatment. The result from HLW-E-Cr-10 is also plotted for comparison.

Figure 3.5. Volume\% of crystalline phase in the heat treated glasses vs. heat treatment temperature for HLW-E-Al-27 and HLW-E-Al-27iUThR2. Two arrows indicate the volume\% of crystals in the corresponding samples after CCC treatment.

Figure 3.6. Volume $\%$ of crystalline phase in the heat treated glasses vs. heat treatment temperature for HLW-E-ANa-22 and HLW-E-ANa22UTh. Two arrows indicate the volume\% of crystals In the corresponding samples after CCC treatment.

Figure 4.1. Sulfur and chromium concentrations measured by XRF in glasses from DM10 tests.

Figure 4.2. Select oxide concentrations measured by XRF in glasses from DM10 tests.

Figure 5.1.a. Glass production rates (hourly moving averages) for DM100 Tests 1A and 1B. Figure 5.1.b. Glass production rates (hourly moving averages) for DM100 Tests 2A and 2B. Figure 5.1.c. Glass production rates (hourly moving averages) for DM100 Tests 3B and 4A. Figure 5.1.d. Glass production rates (hourly moving averages) for DM100 Tests 5A and 5B. Figure 5.1.e. Glass production rates (hourly moving averages) for DM100 Tests 6A and 6B. Figure 5.1.f. Glass production rates (hourly moving averages) for DM100 Test 6C. Figure 5.1.g. Glass production rates (hourly moving averages) for DM100 Tests 7A and 7B. Figure 5.1.h. Glass production rates (hourly moving averages) for DM100 Tests 8A and 8B. Figure 5.1.i. Glass production rates (hourly moving averages) for DM100 Test 8C.

Figure 5.2. Steady-state glass production rates during DM100 tests vs. bubbling rate; feed solids content $500( \pm 50) \mathrm{g}$ glass per liter feed.

Figure 5.3. Steady-state glass production rates during DM100 tests vs. glass temperature; feed solids content $500( \pm 50) \mathrm{g}$ glass per liter feed, glass pool bubbling rate $9 \mathrm{lpm}$.

Figure 5.4. Steady-state glass production rates during DM100 tests vs. feed solids content;

glass temperature $1150^{\circ} \mathrm{C}$, glass pool bubbling rate $9 \mathrm{lpm}$.
Figure 5.5.a. Glass pool bubbling rate for DM100 Tests $1 \mathrm{~A}$ and $1 \mathrm{~B}$.

Figure 5.5.b. Glass pool bubbling rate for DM100 Tests 5A and 5B. 
Figure 5.6.i. Glass temperatures (hourly averages) during DM100 Test 8C.

Figure 5.7.c. Plenum temperatures (hourly averages) during DM100 Tests 3B and 4A.

Figure 5.8.a. Electrode temperatures and power (hourly averages) during DM100 Tests 1A and $1 \mathrm{~B}$.

Figure 5.8.b. Electrode temperatures and power (hourly averages) during DM100 Tests 2A and $2 \mathrm{~B}$.

Figure 5.8.c. Electrode temperatures and power (hourly averages) during DM100 Tests 3B and $4 \mathrm{~A}$.

Figure 5.8.d. Electrode temperatures and power (hourly averages) during DM100 Tests 5A and $5 \mathrm{~B}$.

Figure 5.8.e. Electrode temperatures and power (hourly averages) during DM100 Tests 6A and $6 \mathrm{~B}$.

Figure 5.8.f. Electrode temperatures and power (hourly averages) during DM100 Test 6C.

Figure 5.8.g. Electrode temperatures and power (hourly averages) during DM100 Tests 7A and $7 \mathrm{~B}$.

Figure 5.8.h. Electrode temperatures and power (hourly averages) during DM100 Tests 8A and $8 \mathrm{~B}$.

Figure 5.8.i. Electrode temperatures and power (hourly averages) during DM100 Test 8C.

Figure 5.9.c. Melt pool resistance and total electrode power during DM100 Tests 3B and 4A.

Figure 6.1. Measured viscosities of feed samples at target 250 and $500 \mathrm{~g} / 1$ solids content.

Figure 7.1. Percent feed solids emitted as particles during DM100 tests vs. bubbling rate; feed solids content $500( \pm 50) \mathrm{g}$ glass per liter feed.

Figure 7.2. Percent feed solids emitted as particles during DM100 tests vs. glass temperature;

Figure 7.3. Percent feed solids emitted as particles during DM100 tests vs. feed solids content; glass temperature $1150^{\circ} \mathrm{C}$, glass pool bubbling rate $9 \mathrm{lpm}$.

Figure 7.4. NO and HF FTIR monitored emissions during DM100 Tests $1 \mathrm{~A}$ and 1B. 
Figure 7.8. NO and HF FTIR monitored emissions during DM100 Tests 6A and 6B.

Figure 7.9. NO and HF FTIR monitored emissions during DM100 Test 6C.

Figure 7.10. NO and HF FTIR monitored emissions during DM100 Tests 7A and 7B.

Figure 7.11. NO and HF FTIR monitored emissions during DM100 Tests 8A and 8B.

Figure 7.12. NO and HF FTIR monitored emissions during DM100 Test 8C.

Figure 8.1. Comparison of glass production rates obtained in the present work for the high solids content feeds with comparable high-iron feeds tested previously.

Figure 8.1. Waste Oxide Processing Rates during DM100 tests; glass temperature $1150^{\circ} \mathrm{C}$, glass pool bubbling rate $9 \mathrm{lpm}$, and feed solids content $500( \pm 50) \mathrm{g}$ glass per liter feed. 


\section{List of Abbreviations}

\begin{tabular}{|c|c|}
\hline AA & Atomic Absorption Spectroscopy \\
\hline ADS & Air Displacement Slurry \\
\hline $\mathrm{CCC}$ & Canister Center Line Cooling \\
\hline DCP-AES & Direct Current Plasma Atomic Emission Spectroscopy \\
\hline DF & Decontamination Factor \\
\hline $\mathrm{DM}$ & DuraMelter ${ }^{\circledR}$ \\
\hline DOE & Department of Energy \\
\hline DRE & Destruction \& Removal Efficiency \\
\hline DWPF & Defense Waste Processing Facility \\
\hline EPA & Environmental Protection Agency \\
\hline EDS & Energy Dispersive X-Ray Spectroscopy \\
\hline FTIR & Fourier Transform Infrared Spectroscopy \\
\hline $\mathrm{GC}$ & Gas Chromatography \\
\hline HEPA & High-Efficiency Particulate Air Filter \\
\hline HLW & High Level Waste \\
\hline IHLW & Immobilized High Level Waste \\
\hline LAW & Low Activity Waste \\
\hline M & Molarity \\
\hline $\mathrm{N}$ & Normality \\
\hline NIST & National Institute of Standards and Technology \\
\hline PCT & Product Consistency Test \\
\hline QA & Quality Assurance \\
\hline QAPjP & Quality Assurance Project Plan for Testing Programs Generating Environmental Regulatory Data \\
\hline QAPP & Quality Assurance Project Plan \\
\hline QC & Quality Control \\
\hline RPP & River Protection Project \\
\hline SEM & Scanning Electron Microscopy \\
\hline SIPP & Semi Integrated Pilot Plant \\
\hline SRM & Standard Reference Material \\
\hline SRNL & Savannah River National Laboratory \\
\hline TCLP & Toxicity Characteristic Leaching Procedure \\
\hline VSL & Vitreous State Laboratory \\
\hline WTP & Hanford Tank Waste Treatment and Immobilization Plant \\
\hline WVDP & West Valley Demonstration Project \\
\hline XRD & $\mathrm{X}$-ray Diffraction \\
\hline XRF & X-Ray Fluorescence \\
\hline
\end{tabular}




\section{SECTION 1.0 INTRODUCTION}

This report describes work conducted to support the development and testing of new glass formulations that extend beyond those that have been previously investigated for the Hanford Waste Treatment and Immobilization Plant (WTP). The principal objective was to investigate maximization of the incorporation of several waste components that are expected to limit waste loading and, consequently, high level waste (HLW) processing rates and canister count. The work was performed with four waste compositions specified by the Office of River Protection (ORP); these wastes contain high concentrations of bismuth, chromium, aluminum, and aluminum plus sodium. The tests were designed to identify glass formulations that maximize waste loading while meeting all processing and product quality requirements. The work included preparation and characterization of crucible melts in support of subsequent DuraMelter 100 (DM100) tests designed to examine the effects of enhanced glass formulations, increased glass processing temperature, increased crystallinity, and feed solids content on waste processing rate and product quality. This work builds on previous work performed at VSL for DOE to increase waste loading and processing rates for high-iron HLW waste streams [1]. The scope of this study was outlined in a Test Plan [2] that was prepared in response to an ORP-supplied statement of work [3].

DOE HLW treatment programs have featured joule heated ceramic melter technology for the vitrification of high level tank waste. The melter technology used at the West Valley Demonstration Project (WVDP) in New York and at the Defense Waste Processing Facility (DWPF) at the Savannah River Site (SRS) process(ed) HLW in ceramic melters at an operating temperature of $1150^{\circ} \mathrm{C}$. Historically, HLW melters are operated at temperatures of $1150^{\circ} \mathrm{C}$ to allow for sufficient temperature control for normal as well as upset conditions in an operating melter, while still protecting the electrodes from potential damage due to unanticipated high temperature swings. Since the HLW melters deployed in the United States at West Valley and DWPF do not actively mix the glass pool, temperature variations within the glass pool can be relatively large $\left(\sim \pm 75^{\circ} \mathrm{C}\right)$ with respect to the nominal operating temperature since natural convection within the glass pool is limited in the viscous molten glass. In advancing the technology, Duratek/VSL have demonstrated on very large scale melters (Duratek M-Area facility, Duratek RPP-WTP HLW pilot melter, and the Duratek RPP-WTP LAW pilot melter) that active mixing of the glass pool using Duratek's patented bubbler technology significantly reduces the temperature gradient within the glass pool and allows the melter to be controlled in a tighter operating band. As a result, the operating temperature of the melter can be modestly increased by some 25 to $50^{\circ} \mathrm{C}$ with the current materials of construction, (and yet higher with changes of electrode and bubbler materials) while maintaining the operating integrity of the melter at the higher temperature. For HLW waste streams, increases in operating temperature have the advantage of increased processing rate as well as increased waste loading, both of which translate into significant cost savings.

The waste loading of high-level nuclear waste streams is typically limited by crystal formation because of the relatively high concentrations of elements such as $\mathrm{Fe}, \mathrm{Cr}, \mathrm{Ni}, \mathrm{Mn}, \mathrm{Zr}$, 
The Catholic University of America Vitreous State Laboratory
High Level Waste Vitrification System Improvements Final Report, VSL-07R1010-1, Rev. 0

$\mathrm{Al}, \mathrm{Th}, \mathrm{Bi}$, etc. in the waste and their tendency to form crystalline phases. While the presence of crystalline phases rarely affects the quality of the glass product, it can present a significant processing concern since such phases can settle and accumulate in the melter, ultimately reducing the life of the melter. For this reason, HLW glasses (e.g., DWPF and WVDP) have traditionally been designed to have a liquidus temperature that is lower than the nominal processing temperature by some target value. Since the basic practical issue is crystal settling and accumulation, depending upon the crystal size and density, non-zero amounts of near-liquidus phases can often be maintained in suspension and, therefore, tolerated in bubbled melters. These considerations have led Duratek/VSL to develop and implement an "operational liquidus" constraint in which a non-zero fraction of crystals is tolerated at a given temperature. Glasses designed for DuraMelter vitrification systems have employed a limit of $<1$ vol. \% crystals at a reference temperature (typically $950^{\circ} \mathrm{C}$ ) below the operating temperature. Based on this experience and recommendation, this constraint has now been formally adopted for use at the Hanford WTP in place of the traditional, much more conservative, liquidus-temperature constraint. Additional increases in the amount of crystals allowable in the glass would further increase waste loadings and therefore further reduce waste treatment costs. However, at present, although it is expected that the actively mixed DuraMelter systems should be able to tolerate a larger amount of crystals than is the case for conventional melters, the maximum concentration of crystals such melter systems can tolerate is not yet known. For the current work, glass formulations with modestly higher amounts of crystals (i.e., closer to the $1 \mathrm{vol} \%$ limit than is typical of the current WTP baseline glasses) were considered for melter testing, if it resulted in higher waste loading. The maximum amount of crystals in the glass formulations selected for melter testing was decided on a case-by-case basis, in consultation with ORP, and was based on the specific glass formulation and the composition and characteristics of the crystal phase. Previous DM10 work demonstrated successful processing of high-iron wastes with glasses formulated with up to $\sim 3 \mathrm{vol} \%$ crystals at $950^{\circ} \mathrm{C}[1]$.

Under a separate contract to support the WTP, the VSL is developing and testing glass formulations for WTP HLW waste compositions to provide data to meet the WTP contract requirements and to support system design activities [4-6]. That work is based upon small-scale batch melts ("crucible melts") using waste simulants. Selected formulations have also been tested in small-scale, continuously fed, joule-heated melters (DM100) [7-10] and, ultimately, in the HLW DM1200 Pilot Melter [9-17]. Such melter tests provide information on key process factors such as feed processing behavior, dynamic effects during processing, secondary phase formation, processing rates, off-gas amounts and compositions, foaming control, etc., that cannot be reliably obtained from crucible melts. This sequential scale-up approach in the vitrification testing program ensures that maximum benefit is obtained from the more costly melter tests and that the most effective use is made of those resources.

The glass formulation and melter testing work described in this report is aimed at identifying glass compositions that maximize waste loadings for the four waste streams specified by ORP. This information provides ORP with a basis for projection of the amount of Immobilized High Level Waste (IHLW) to be produced at Hanford, and evaluation of the likely potential for future enhancements of the WTP over and above the present well-developed baseline. It should be noted that the compositions of the four specified waste streams differ significantly from those of the feed tanks (AZ-101, AZ-102, C-16/AY-102, and C-104/AY-101) 
that have been the focus of the extensive technology development and design work performed for the WTP baseline. In this regard, the work described in this report is complementary to and necessarily of a more exploratory nature than the work in support of the current WTP baseline. It should be noted, therefore, that to the extent that the present effort is successful, considerable further work would be required to bring the level of confidence in the new glass composition regions to a similar level of maturity to that of the current WTP baseline. Additional testing in larger melters such as the DM1200 will be needed to confirm feed processing characteristics at larger scales. Additional testing at the crucible and melter scales will be needed to determine the robustness of the new compositions with respect to variations in the feed compositions that may results from process variations. Off-gas characteristics will have to be determined and data to support engineering and permitting requirements will need to be collected using a WTP prototypic off-gas system. In addition, since the high waste loading glass compositions are likely to be in a new composition space as compared to the current WTP compositions, additional effort will be required to develop and extend the current qualified glass composition region and supporting models to include these new compositions.

Literature information on glass formulations containing the components of interest in the present work ( $\mathrm{Cr}, \mathrm{Bi}, \mathrm{Al}$, and $\mathrm{Na})$ is summarized and discussed in the Appendix. The concentrations of these components in the formulations developed in the present work are also compared to expectations based on literature information.

\section{$1.1 \quad$ Test Objectives}

The principal objectives of this work were [2, 3] to test and evaluate HLW glass compositions to determine the maximum waste loading that can be achieved and still produce a simulated glass composition that has acceptable durability and processing characteristics. Glass composition development was based on four HLW waste compositions specified by ORP that have high concentrations of bismuth, chromium, aluminum, and aluminum in combination with sodium. These objectives were addressed through a combination of crucible-scale tests and confirmation tests on the DM100 melter system. The DM100-BL unit was selected for these tests. The DM100-BL was used for previous tests on HLW glass compositions [7-10] that were used to support subsequent tests on the HLW Pilot Melter [9-17]. The volatility of cesium and rhenium (as a surrogate for technetium) during the vitrification of an HLW AZ-102 composition was also studied on the DM100-BL [18]. The same melter was selected for the present tests in order to maintain comparisons between the previously collected data. The tests provided information on melter processing characteristics and off-gas data, including formation of secondary phases and partitioning. In addition, the work evaluated the effect on production rate of modest increases in melter operating temperature and changes in feed solids content. Once glass formulation development was completed for each of the four waste compositions, the data were reviewed with ORP prior to making a decision on whether to proceed directly to DM100-BL tests or to first conduct screening tests on the DM10.

Per the Statement of Work [3], a parametric "crucible scale" study of the effects of waste loading was conducted for each waste composition. Waste loadings were increased until the maximum expected waste loading was achieved, or the limits of a glass property were exceeded. 
Glass properties for evaluation included: viscosity, electrical conductivity, crystallinity, salt phase separation, and durability per the 7-day Product Consistency Test (PCT, ASTM-1285). Glass property limits were based upon the reference properties for the WTP HLW melter. However, the WTP crystallinity limit $\left(<1\right.$ vol $\%$ at $\left.950^{\circ} \mathrm{C}\right)$ was not used as a waste loading constraint for the crucible melts. Instead, in the present work, a less conservative approach was employed such that crucible melt tests evaluated glasses that exhibit up to about $5-10$ vol\% crystallization [2].

\subsection{Quality Assurance}

This work was conducted under a quality assurance program that is based on NQA-1 (1989) and NQA-2a (1990) Part 2.7 that is in place at the VSL. This program is supplemented by a Quality Assurance Project Plan for RPP-WTP work [19] that is conducted at VSL. Test and procedure requirements by which the testing activities are planned and controlled are also defined in this plan. The program is supported by VSL standard operating procedures that were used for this work [20]. In addition, the requirements of DOE/RW-0333P were applicable to the following specific aspects of this work:

- Crucible melt preparation

- Analysis of crucible melt glasses

- PCT

\subsection{DM10 Melter System}

The DM10 unit is a ceramic refractory-lined melter fitted with two Inconel 690 plate electrodes that are used for joule-heating of the glass pool and a bubbler for stirring the melt. The glass product is removed from the melter by means of an air-lift discharge system. The DM10 unit has a melt surface area of $0.021 \mathrm{~m}^{2}$ and a glass inventory of about $8 \mathrm{~kg}$, less than a tenth of that of the DM100. As with the DM100, the melter feed is introduced in batches into a stirred feed container that is mounted on a load cell for weight monitoring. The feed is constantly recirculated except for periodic, momentary interruptions during which the weight is recorded. The feed can be introduced into the melter by means of a simulated air-displacement slurry (ADS) pump system or through a peristaltic pump; the latter was used for the present tests. The recirculation loop extends to the top of the melter where feed is diverted from the recirculation loop into the melter through a Teflon-lined feed line and water-cooled, vertical feed tube. The off-gas system is similar to that for the DM100, which is described below. Samples for analysis are taken from the discharged glass and by dip-sampling of the glass pool using a threaded rod.

\subsection{DM100 Melter Systems 1.4.1 D100 Feed System}

A schematic diagram of the DM100 vitrification system is shown in Figure 1.1. The melter feed is introduced in batches into a feed container that is mounted on a load cell for 
weight monitoring. The feed is stirred with a variable speed mixer and constantly recirculated except for periodic, momentary interruptions during which the weight is recorded. Feed is normally introduced into the melter via a system designed to mimic the operation of an ADS pump, which is the present WTP baseline; however, a peristaltic pump was used in these tests to facilitate observations of any differences in processing rates and feed behavior. In this system, a recirculation loop extends to the top of the melter where feed is diverted from the recirculation loop to the peristaltic pump and subsequently into the melter through a Teflon-lined feed line and water-cooled, vertical feed tube.

\subsubsection{Melter System}

Cross-sectional diagrams of the DM100-BL melter are shown in Figures 1.2.a-c. The DM100-BL unit is a ceramic refractory-lined melter fitted with five electrodes: two pairs of opposing Inconel 690 plate electrodes and a bottom electrode. Power can be supplied in either three-phase or single-phase configurations. All of the tests in the present work were performed with the upper and lower electrodes on each side connected together and powered by a single-phase supply; the bottom electrode was not powered. Melt pool agitation is achieved by either a removable lance entering from the top of the melter or a permanent bubbler installed through the bottom electrode. In these tests the lance bubbler was used. The glass product is removed from the melter by means of an airlift discharge system. The melter has a melt surface area of $0.108 \mathrm{~m}^{2}$ and a variable glass inventory of between $110 \mathrm{~kg}$, when only the bottom pair of electrodes is used, and about $170 \mathrm{~kg}$ when both pairs of electrodes are used, which was the case in the present tests.

\subsubsection{Off-Gas System}

For operational simplicity, the DM100-BL is equipped with a dry off-gas treatment system involving gas filtration operations only. Exhaust gases leave the melter plenum through a film cooler device that minimizes the formation of solid deposits. The film-cooler air has constant flow rate and its temperature is thermostatically controlled. Consequently, under steady-state operating conditions, the exhaust gases passing through the transition line (between the melter and the first filtration device) can be sampled at constant temperature and airflow rate. The geometry of the transition line conforms to the requirements of the 40-CFR-60 air sampling techniques. Immediately downstream of the transition line are cyclonic filters followed by conventional pre-filters and HEPA filters. The temperature of the cyclonic filters is maintained above $150^{\circ} \mathrm{C}$ while the temperatures in the HEPAs are kept sufficiently high to prevent moisture condensation. The entire train of gas filtration operations is duplicated and each train is used alternately. An induced draft fan completes the system.

\subsection{Feed Sample Analysis}

Feed samples were taken directly from the feed recirculation line during each test. Feed samples were poured into a platinum/gold crucible that was placed into a programmed furnace 
for drying and fusion to form a glass. The glass produced from this fusion was ground to less than 200 mesh and sealed in 20-ml vials for subsequent analysis by $\mathrm{x}$-ray fluorescence spectroscopy (XRF), or by acid digestion followed by direct current plasma - atomic emission spectroscopy (DCP-AES) on the resulting solution. The feed samples were also characterized for their rheological properties, density, $\mathrm{pH}$, water content, and glass yield.

\subsection{Glass Product Analysis}

The glass product is discharged from the melter into 5-gallon steel pails periodically using an air-lift system. The discharged product glass was sampled at the end of each test by removing sufficient glass from the top of the cans for compositional analysis and secondary phase determinations. In addition, the Product Consistency Test ( 7 days at $90^{\circ} \mathrm{C}$ ) and Toxicity Characteristic Leaching Procedure (TCLP) were performed on samples of the glass product from the DM100 melter tests. Prior to those tests, the PCT and TCLP were also performed on the crucible melt compositions that were selected for the melter tests to ensure their compliance with the present WTP contract requirements. All of these procedures are routinely conducted at VSL and, therefore, standard operating procedures (SOPs) are in place.

Sample preparation for chemical analysis typically involves size reduction and sieving. All samples were subjected to XRF to determine the concentration of all elements except boron and lithium. A series of National Institute of Standards and Technology (NIST) reference materials were used for confirmation of the XRF data. Boron and lithium were determined by total acid dissolution of ground glass samples in $\mathrm{HF} / \mathrm{HNO}_{3}$ and subjecting the resulting solutions to DCP-AES analysis.

\subsubsection{Viscosity}

The melt viscosity, $\eta$, is measured using a Brookfield viscometer. Measurements are performed in the temperature range of $950-1250^{\circ} \mathrm{C}$ and the data are interpolated to standard temperatures using the Vogel-Fulcher equation: $\ln \eta=\left[\mathrm{A} /\left(\mathrm{T}-\mathrm{T}_{\mathrm{o}}\right)\right]+\mathrm{B}$, where $\mathrm{A}, \mathrm{B}$, and $\mathrm{T}_{\mathrm{o}}$ are fitting parameters. The equipment is calibrated at room temperature using standard oils of known viscosity and then checked at $950-1250^{\circ} \mathrm{C}$ using a NIST standard reference glass (SRM 711). Both precision and accuracy of the viscosity measurements are estimated to be within \pm 15 relative $\%$.

\subsubsection{Electrical Conductivity}

The electrical conductivity, $\sigma$, of each glass melt is determined by measuring the resistance of the glass melt as a function of frequency using a calibrated platinum/rhodium electrode probe attached to a Hewlett-Packard model 4194A impedance analyzer. Measurements are performed over similar temperature ranges to those employed for the melt viscosity measurements. The results are analyzed and modeled to obtain the DC electrical conductivity. 
The electrical conductivity data are then interpolated to standard temperatures using the Vogel-Fulcher equation: $\ln \sigma=\left[\mathrm{A} /\left(\mathrm{T}-\mathrm{T}_{\mathrm{o}}\right)\right]+\mathrm{B}$, where $\mathrm{A}, \mathrm{B}$ and $\mathrm{T}_{\mathrm{o}}$ are fitting parameters. Estimated uncertainties in the electrical conductivity measurements are \pm 20 relative $\%$.

\subsubsection{Product Consistency Test (PCT)}

The product consistency test (PCT; ASTM C 1285) is used to evaluate the relative chemical durability of glasses by measuring the concentrations of the chemical species released from 100-200 mesh crushed glass $(75-149 \mu \mathrm{m})$ to the test solution (de-ionized water in this case). PCT tests on the HLW glasses are performed at $90^{\circ} \mathrm{C}$, in accordance with the current WTP contract requirement. The ratio of the glass surface area to the solution volume for this test is about $2000 \mathrm{~m}^{-1}$ (typically, $10 \mathrm{~g}$ of 100-200 mesh glass is immersed in $100 \mathrm{ml}$ deionized water). All tests are conducted in triplicate, in 304L stainless steel vessels, and in parallel with a standard glass included in each test set. The internal standard is the ANL-LRM reference glass [21] and/or the DWPF-EA glass, both of which have undergone round-robin testing. The leachates are sampled at predetermined times, the first of which is seven days. One milliliter of sampled leachate is mixed with $20 \mathrm{ml}$ of $1 \mathrm{M} \mathrm{HNO}_{3}$ and the resulting solution is analyzed by DCP-AES; another $3 \mathrm{ml}$ of sampled leachate is used for $\mathrm{pH}$ measurement.

\subsubsection{Toxicity Characteristic Leaching Procedure (TCLP)}

The TCLP was performed at VSL using SW-846 Method 1311, which employs leaching of crushed glass $\left(<3 / 8^{\prime}\right)$ in a sodium acetate buffer solution for 18 hours at $22^{\circ} \mathrm{C}$ with constant end-over-end agitation. A mass of about 100 grams of glass is leached in 2 liters of TCLP extract, according to the extraction method for non-volatiles. The surface area to volume ratio for this test is about $20 \mathrm{~m}^{-1}$, which is about two orders of magnitude lower than that in the PCT. The leachates are analyzed by DCP-AES according to VSL standard operating procedures.

\subsubsection{Secondary Phases}

Secondary phases in the glass samples were determined by optical microscopy and scanning electron microscopy coupled with energy dispersive x-ray spectroscopy (SEM-EDS). Secondary phases due to crystallization and phase separation can be identified using these methods. Quantitative determination of the amount of crystals in glass samples were made by SEM in conjunction with image analysis. 


\section{SECTION 2.0 WASTE SIMULANT}

The waste stream compositions provided by DOE are given in Table 2.1 on an oxide basis [3] under the heading "Actual". Omission of radioactive components and renormalization of the simulated waste yields the compositions shown in Table 2.1 as "Non-Rad"; these compositions were used for the majority of the crucible formulations and all of the melter testing. However, once the final formulations were selected, additional crucible melts were performed using the appropriate radioactive components (i.e., thorium and uranium).

Actual HLW Hanford tank wastes are aqueous solutions with suspended solids and dissolved salts including hydroxides, nitrates, nitrites, halides, and carbonates. For the purpose of the present work, the concentrations of the volatile components (i.e., carbonate, nitrite, nitrate, and organic carbon) are assumed to be similar to those found for the AZ-102 HLW waste [14]. With the waste compositions defined, formulation of the HLW waste simulant proceeds in a straightforward fashion from the oxide compositions listed in Table 2.1. In general, oxides and hydroxides were used as the starting materials, including a slurry of iron (III) hydroxide (13\% by weight). Volatile inorganic components were added as the sodium salts, whereas organic carbon was added as oxalic acid. Finally, the water content was adjusted to target a glass yield of $500 \mathrm{~g}$ of glass per liter of feed. Feeds were diluted by the addition of water for the last DM100-BL test segments, targeting a glass yield of $250 \mathrm{~g}$ per liter. The compositions of the resulting four HLW waste simulants that were used for melter testing are given in Tables 2.2 - 2.5. 


\section{SECTION 3.0 GLASS FORMULATION}

\subsection{Enhanced Glass Formulation for Bi-Limited Waste}

Crucible melts spanning a range of waste loadings were prepared and tested to determine the maximum viable waste loading for the bismuth-limited waste composition supplied by ORP [3]. Table 3.1 presents the compositions of the one radioactive and 16 non-radioactive glasses investigated in this work, along with the radioactive and non-radioactive versions of the Bi-limited waste. The non-radioactive version of the waste was obtained by renormalizing the waste composition to $100 \%$ after removing radioactive components $\mathrm{U}$ and Th. Besides the high concentration of $\mathrm{Bi}_{2} \mathrm{O}_{3}$, the Bi-limited waste contains several other ingredients that often limit waste loading in borosilicate glasses, notably, $\mathrm{P}_{2} \mathrm{O}_{5}(\sim 10 \mathrm{wt} \%), \mathrm{NiO}(\sim 4 \mathrm{wt} \%), \mathrm{Cr}_{2} \mathrm{O}_{3}(\sim 1 \mathrm{wt} \%)$, $\mathrm{SO}_{3}(\sim 1 \mathrm{wt} \%)$ and $\mathrm{F}(\sim 1.6 \mathrm{wt} \%)$. Phosphates can cause crystallization and liquid-liquid phase separation in borosilicate glass melts at levels of a few percent. Nickel and chromium oxides are major constituents of spinel, which is the most common near-liquidus phase in HLW glasses. The solubility of sulfate is rather low in silicate systems and can be further reduced by addition of halides $(\mathrm{F}, \mathrm{Cl})$.

The formulation work for the Bi-limited waste started with crucible melts targeting $30 \mathrm{wt} \%$ waste loading, which is double the contract-specified minimum of $15 \mathrm{wt} \%$. HLW-E-Bi11 (Table 3.1), one of the glasses with $30 \mathrm{wt} \%$ waste loading, is a typical high-sodium borosilicate waste glass, with around $44 \mathrm{wt} \%$ silica and moderate amounts of aluminum and iron oxides. The presence of about $4 \mathrm{wt} \%$ of $\mathrm{Bi}_{2} \mathrm{O}_{3}$ and $3 \mathrm{wt} \%$ of $\mathrm{P}_{2} \mathrm{O}_{5}$ from the Bi-limited waste did not adversely affect the glass properties. As reported in Table 3.2, the as-melted glass from the crucible melt and the sample after heat treat treatment for 70 hours at $950^{\circ} \mathrm{C}$ were crystal-free, as determined by X-ray diffraction (XRD) and SEM analysis. Glasses with $40 \mathrm{wt} \%$ waste loading were formulated with lower $\mathrm{SiO}_{2}$ concentrations (see HLW-E-Bi-1, -2, -3, and -4, in Table 3.1). The $\mathrm{ZrO}_{2}$ concentration in these glasses was varied from nearly zero (HLW-E-Bi-1) to more than 2 percent (HLW-E-Bi-4) to investigate its effect on PCT response because the normalized PCT leach rates, although well within the acceptable range, were higher than those for the baseline WTP HLW glasses (Table 3.2). However, even though the 7-day PCT results from three glasses with $40 \mathrm{wt} \%$ waste loading and increasing levels of $\mathrm{ZrO}_{2}$ showed improvement, the magnitude of the improvement was not large enough to justify the introduction of another glass former.

Overall, at $40 \mathrm{wt} \%$ waste loading, as-melted glasses and samples heat treated for 70 hours at $950^{\circ} \mathrm{C}$ showed minimal amounts of crystallization (about $0.1 \mathrm{vol} \%$ of spinel), moderate viscosity and electrical conductivity, and acceptable leaching performance (see Table 3.2). Further increase of the waste loading, by $5 \mathrm{wt} \%$ to $45 \mathrm{wt} \%$, did not result in significant changes to the glass properties. Four glasses with waste loadings of $45 \%$ were prepared with varying concentrations of $\mathrm{ZrO}_{2}$ (HLW-E-Bi-5), $\mathrm{B}_{2} \mathrm{O}_{3}$ (HLW-E-Bi-8), $\mathrm{Na}_{2} \mathrm{O}$ (HLW-E-Bi-9), and $\mathrm{K}_{2} \mathrm{O}$ (HLW-E-Bi-10). All four glasses showed similar melting behavior and heat treatment results ( $\sim 0.5 \mathrm{vol} \%$ of spinel after 70 hours at $950^{\circ} \mathrm{C}$, see Table 3.2$)$. Crystallization of spinel increased 
The Catholic University of America Vitreous State Laboratory
High Level Waste Vitrification System Improvements Final Report, VSL-07R1010-1, Rev. 0

considerably when the waste loading was increased by another $5 \mathrm{wt} \%$ to $50 \mathrm{wt} \%$. HLW-E-Bi-6, with $50 \mathrm{wt} \%$ waste loading, showed good melting behavior, slightly higher viscosity (96 poise at $1150^{\circ} \mathrm{C}$ ), and acceptable leaching rate (Table 3.2 ), but a considerably higher vol\% of crystalline phase in the heat treated glass. As shown in Table 3.2, and Figure 3.1 (vol \% crystals vs. temperature for four Bi glasses), 1.8 vol\% of crystalline phases consisting largely of spinel was present in HLW-E-Bi-6 after heat treatment for 70 hours at $950^{\circ} \mathrm{C}$. The percentage of the crystalline phases increases roughly linearly with decreasing heat treatment temperature to about $4 \mathrm{vol} \%$ at $800^{\circ} \mathrm{C}$. As shown in Figure 3.1, the amount of crystals increases further with increasing waste loading. The crystal content in the glasses heat treated for 70 hours at $950^{\circ} \mathrm{C}$ increases from $1.8 \mathrm{vol} \%$ at $50 \mathrm{wt} \%$ loading to $2.7 \mathrm{vol} \%$ at $55 \mathrm{wt} \%$ loading (HLW-E-Bi-7, -13 , and -15 ), and further to $4.2 \mathrm{vol} \%$ at $60 \mathrm{wt} \%$ waste loading. Moreover, as-melted glasses with more than $50 \mathrm{wt} \%$ waste loading displayed a heterogeneous texture that is indicative of the presence of crystalline phases even at the glass melting temperature of $1200^{\circ} \mathrm{C}$. Thus, although the glasses with $55 \mathrm{wt} \%, 60 \mathrm{wt} \%$ and $65 \mathrm{wt} \%$ waste loading are reasonably leach resistant and show acceptable viscosity and electrical conductivity, the presence of crystallization at the melting temperature was considered undesirable. Therefore, HLW-E-Bi-6 with 50 wt $\%$ waste loading was selected as a reasonable high waste loading glass formulation with acceptable glass properties.

Heat treatment of this glass according to the WTP HLW canister center line cooling (CCC) profile resulted in around $5.6 \mathrm{vol} \%$ of crystallization, with roughly half spinel and half phosphate phases. It should also be noted that the glass after CCC treatment showed clear signs of foaming, especially near the top surface in contact with air (see Figure 3.2). Foaming was not observed in the glass sample after isothermal heat treatment at $950,900,850$ or $800^{\circ} \mathrm{C}$. Consequently, the foaming behavior appears to be associated with the particular CCC temperature profile. This observation is of concern in that during waste processing, the glass poured into the canister may foam sufficiently to spill out of the canister and it, therefore, deserves further study. The normalized 7-day PCT results for HLW-E-Bi-6 after CCC heat treatment are quite comparable to those of the quenched crucible melt (Table 3.2). HLW-E-Bi6UThR1, the radioactive version of HLW-E-Bi-6, showed properties that are virtually identical to those of HLW-E-Bi-6 (see Table 3.2, Table 3.3, and Figure 3.3).

HLW-E-Bi-6 has a relatively high melt viscosity at the typical melter operating temperature of $1150^{\circ} \mathrm{C}$. Substitution of $2 \mathrm{wt} \%$ of $\mathrm{K}_{2} \mathrm{O}$ for $\mathrm{Na}_{2} \mathrm{O}$ and $\mathrm{SiO}_{2}$ in HLW-E-Bi-6 produced HLW-E-Bi-16 (Tables 3.1 and 3.2), which showed a lower melt viscosity of 70 poise at $1150^{\circ} \mathrm{C}$. However, the volume $\%$ of crystals after heat treatment at $950^{\circ} \mathrm{C}$ for 75 hours increased from $1.8 \%$ for HLW-E-Bi-6 to $2.7 \%$ for HLW-E-Bi-16. Although the measured viscosity of $\mathrm{HLW}-\mathrm{E}-\mathrm{Bi}-6$ of 96 poise at $1150^{\circ} \mathrm{C}$ is within the range acceptable for melter operation, minor modifications would be advisable for the production glass in order to bring the viscosity closer to the center of the operating range.

The composition and properties of the high-Bi glass selected for melter testing are given in Table 3.4. The selected glass formulation, HLW-E-Bi-6, meets all of the processing and product quality requirements imposed for these tests and has a waste oxide loading of $50 \mathrm{wt} \%$. This exceeds both the minimum and maximum expected waste loadings provided in the scope of work [3] of $15 \mathrm{wt} \%$ and $40 \mathrm{wt} \%$, respectively. The glass contains $6.7 \mathrm{wt} \% \mathrm{Bi}_{2} \mathrm{O}_{3}$ and close to 
$5 \mathrm{wt} \% \mathrm{P}_{2} \mathrm{O}_{5}$. The $\mathrm{Bi}_{2} \mathrm{O}_{3}$ content is more than three times the WTP contract minimum for $\mathrm{Bi}_{2} \mathrm{O}_{3}$ $(2 \mathrm{wt} \%)$. The PCT leach rates are over an order of magnitude lower than those of the DWPF-EA glass and the TCLP leachate concentrations are all below the WTP Delisting Limits. The measured processing parameters are within acceptable ranges. As noted above, the viscosity is towards the high end of the acceptable range, which is appropriate in view of the fact that a portion of the testing was performed at elevated temperature. One concern that remains is the potential risk of foaming during cooling of the poured glass in the canister. The potential foaming observed after CCC heat treatment and its mitigation requires further investigation. The phenomenon is likely associated with the redox behavior of $\mathrm{Bi}$ and other glass components with variable valence states.

\subsection{Enhanced Glass Formulation for Cr-Limited Waste}

Crucible melts spanning a range of waste loadings were prepared and tested to determine the maximum viable waste loading for the chromium-limited waste composition. Table 3.5 presents the compositions of one radioactive and 19 non-radioactive glasses investigated in this work, along with the Cr-limited waste composition specified by ORP [3]. The non-radioactive version of the Cr-limited waste composition, obtained by removing the radioactive components $\mathrm{U}$ and $\mathrm{Th}$ and renormalizing, is also given in Table 3.5. Beside its relatively high $\mathrm{Cr}_{2} \mathrm{O}_{3}$ concentration, the Cr-limited waste contains considerable amount of $\mathrm{SO}_{3}$ and $\mathrm{F}(\sim 1.5 \mathrm{wt} \%$ and $2 \mathrm{wt} \%$, respectively). Chromium interacts with sulfates in borosilicate melts through the formation of chromates, which are isomorphous with sulfates. High concentrations of $\mathrm{Cr}_{2} \mathrm{O}_{3}$ and $\mathrm{SO}_{3}$ can promote the formation of a separate chromium-containing sulfate or sulfur-containing chromate phase during glass melting or subsequent heat treatment. Fluorine can also increase the tendency towards the formation of molten sulfate phases. Thus, in view of the typically low solubility of sulfate and chromium oxide in borosilicate glasses, it is possible that waste loading of this waste would be limited by salt formation rather than chromium crystallization, which would be the case if the sulfate content was lower.

Formulation work for Cr-limited waste was started with glasses at $30 \mathrm{wt} \%$ waste loading, which is $50 \%$ over the contract-specified minimum of $20 \mathrm{wt} \%$. HLW-E-Cr-14 (Table 3.6) is a typical sodium borosilicate waste glass except for its high $\mathrm{K}_{2} \mathrm{O}$ content of $6 \mathrm{wt} \%$. Potassium oxide was used as a glass former because previous work [1] indicated its potential advantage in suppressing the formation of Fe-rich spinel from iron-rich borosilicate glasses. Other work at VSL suggested that potassium and lead oxides would help enhance the solubility of chromium oxide in borosilicate glasses. The heat treated sample of HLW-E-Cr-14 showed minimal crystallization $\left(\sim 0.1 \%\right.$ spinel after 70 hours at $\left.950^{\circ} \mathrm{C}\right)$. For this borosilicate waste glass, which contains $1 \mathrm{wt} \% \mathrm{Cr}_{2} \mathrm{O}_{3}$, a few percent of $\mathrm{Fe}_{2} \mathrm{O}_{3}$, and some $\mathrm{NiO}$, this amount of crystallization is atypically low, probably due to the presence of potassium oxide. However, as the waste loading was increased further, sulfate/chromate phase separation, rather than Cr-rich spinel crystallization, became the limiting factor in identifying an acceptable glass formulation. Among eleven glasses formulated with $40 \mathrm{wt} \%$ waste loading $\left(1.33 \mathrm{wt} \% \mathrm{Cr}_{2} \mathrm{O}_{3}, 0.46 \mathrm{wt} \% \mathrm{NiO}\right.$, and $0.66 \mathrm{wt} \% \mathrm{SO}_{3}$, see Table 3.6), five developed a yellow colored salt phase (HLW-E-Cr-1, -2, -3 , $-8,-9$ in Table 3.5), and three appeared partially crystallized (HLW-E-Cr-4, -5, -6 in Table 3.6). Analysis of the collected salt phase indicated a mixture of chromate-sulfate salt that had 
The Catholic University of America Vitreous State Laboratory
High Level Waste Vitrification System Improvements Final Report, VSL-07R1010-1, Rev. 0

segregated from the glass melt during the melting process (Table 3.7). The crystalline phases were mostly spinel, with lesser amounts of escolaite and perhaps phosphate (Table 3.6). A close examination of the properties and composition of these eleven glasses at $40 \mathrm{wt} \%$ waste loading revealed a subtle balance between the segregation of sulfate salt phase and precipitation of spinel crystals. As shown in Table 3.7, potassium is a major component in the segregated chromate-sulfate salt phase. It is then logical that salt formation would decrease with decreasing $\mathrm{K}_{2} \mathrm{O}$ concentration in the glass. Indeed, as $\mathrm{K}_{2} \mathrm{O}$ was decreased from $11.16 \mathrm{wt} \%$ (HLW-E-Cr-1), $10.16 \mathrm{wt} \%$ (HLW-E-Cr-2), $7.66 \mathrm{wt} \%$ (HLW-E-Cr-3) to below 5 wt\% (HLW-E-Cr-4, -5, -6), the yellow salt phase disappeared. However, reduced $\mathrm{K}_{2} \mathrm{O}$ in the glasses also reduced the solubility of Cr-rich spinel, as manifested by the crystals in the glass samples HLW-E-Cr-4, -5 , -6. Fine-tuning of the concentrations of $\mathrm{Li}_{2} \mathrm{O}$ (HLW-E-Cr-8) and $\mathrm{B}_{2} \mathrm{O}_{3}$ (HLW-E-Cr-9) resulted in an acceptable glass, HLW-E-Cr-7, with only trace amounts of crystalline phases and no apparent separation of chromate/sulfate phase. The role of $\mathrm{PbO}$ in suppressing crystallization of $\mathrm{Cr}$ containing spinel was also investigated in this group of glasses but with inconclusive results. For glasses that showed spinel crystallization (HLW-E-Cr-4, -5 and -6), $\mathrm{PbO}$ concentrations from $1.21 \mathrm{wt} \%$ (HLW-E-Cr-4, -5) to $2.21 \mathrm{wt} \%$ (HLW-E-Cr-6) did not appear to be effective in suppressing spinel crystallization. Nevertheless, HLW-E-Cr-10, which is a variation of HLW-E-Cr-7 without PbO did show a moderate increase in the total volume of crystals after heat treatment in the temperature range of 800 to $950^{\circ} \mathrm{C}$. Another variation of HLW-E-Cr-7 with increased $\mathrm{ZrO}_{2}$ concentration and no $\mathrm{PbO}$, HLW-E-Cr-11, did not show any improvement in the PCT response, as shown in Table 3.6. All formulations with higher waste loadings $(42.5 \mathrm{wt} \%$, $45 \mathrm{wt} \%$, and $50 \mathrm{wt} \%$ ) resulted in, to various extents, formation of yellow chromate/sulfate salt during glass melting. No beneficial effect on salt formation was observed with the addition of $\mathrm{V}_{2} \mathrm{O}_{5}$ (HLW-E-Cr-15, and $\mathrm{Cr}-16$ ), $\mathrm{CaO}$ (HLW-E-Cr-18), reduced $\mathrm{SiO}_{2}$ (HLW-E-Cr-17), or simply renormalization from Cr-10 (HLW-E-Cr-12 and HLW-E-Cr-13).

In conclusion, three Cr-rich borosilicate glasses with $40 \mathrm{wt} \%$ waste loading that meet all processing and product quality requirements were identified. The three glasses differ only in that HLW-E-Cr-7 contains $3 \mathrm{wt} \%$ of PbO, HLW-E-Cr-11 contains 1 wt $\% \mathrm{ZrO}_{2}$, whereas HLW-E-Cr-10 contains neither of these additives. Considering their rather similar properties and performance, the simplest version of the three glass formulations, HLW-E-Cr-10, was chosen as the candidate for melter testing. However, in view of the importance of salt phase formation, screening tests on the DM10 melter were recommended, and approved by ORP, prior to performing the DM100 tests.

The composition and properties of HLW-E-Cr-10 are given in Table 3.8. The selected glass formulation meets all of the processing and product quality requirements imposed for these tests and has a waste oxide loading of $40 \mathrm{wt} \%$. This exceeds the minimum and equals the maximum expected waste loadings provided in the scope of work [3] of $20 \mathrm{wt} \%$ and $40 \mathrm{wt} \%$, respectively. The target glass composition contains $1.33 \mathrm{wt} \% \mathrm{Cr}_{2} \mathrm{O}_{3}, 0.66 \mathrm{wt} \% \mathrm{SO}_{3}$, and over $3 \mathrm{wt} \% \mathrm{Bi}_{2} \mathrm{O}_{3}$. The $\mathrm{Cr}_{2} \mathrm{O}_{3}, \mathrm{SO}_{3}, \mathrm{Bi}_{2} \mathrm{O}_{3}$, and $\mathrm{Al}_{2} \mathrm{O}_{3}$ contents all equal or exceed their WTP contract minimum values of $0.5,0.5,2$, and $11 \mathrm{wt} \%$, respectively. This glass formulation has the highest waste loading tested that did not result in a separate salt layer on the crucible melt surface. The PCT leach rates are about five times lower than those of the DWPF-EA glass and the TCLP leachate concentrations are all below the WTP Delisting Limits. The measured processing parameters are within acceptable ranges. 
The screening tests performed on the DM10 melter tests with HLW-E-Cr-10 at $40 \mathrm{wt} \%$ waste loading resulted in separate salt phase formation on the melt surface. This is not inconsistent with the batch crucible melt results because salt phase formation is influenced by both thermodynamic and kinetic factors, and due to kinetic factors, in a continuously fed melter, salt phase formation can occur even before the solubility limits for the respective components are reached in the melt. Indeed, this was exactly why the screening tests were performed. To address salt phase formation, the waste loading in the melter glass formulation was progressively reduced to determine the maximum loading at which no salt phase was seen (See Section 4.0). Based on the results of the DM10 melter tests, the glass formulation with $32.5 \mathrm{wt} \%$ waste loading was selected for further tests on the larger DM100 melter. Both radioactive (HLW-E-CrMUTh) and non-radioactive (HLW-E-Cr-M) versions of the glass at $32.5 \mathrm{wt} \%$ were prepared at the crucible scale and characterized. The results are discussed below.

The compositions and the characterization results for HLW-E-Cr-M and HLW-E-CrMUTh are listed together with results for HLW-E-Cr-10 in Tables 3.3, 3.5, and 3.6. The radioactive and non-radioactive versions of the selected Cr-limited glass yield similar PCT and TCLP results. The volume percent of crystalline phases after isothermal and CCC heat treatments are generally lower in HLW-E-CrMUTh (see Figure 3.4). Nevertheless, the same crystalline phases were observed in both glasses, including the submicron sized bubble-like feature in glass samples subjected to CCC heat treatment. Both HLW-E-Cr-M and HLW-E-CrMUTh meet all product quality (PCT and TCLP) and processing requirements (crystallinity, viscosity, and electrical conductivity). The waste loading achieved (32.5 wt $\%$ ) significantly exceeded the minimum $(20 \mathrm{wt} \%)$, specified in the contract [3].

In summary, although this waste stream is designated a "chromium-limited" waste, there are several components, including sulfur, phosphorus, and bismuth, that are present at high enough levels to potentially challenge borosilicate glass formulations. Indeed, the results of the present tests showed that the factor that limited waste loading was not the Cr content alone, but a combination of $\mathrm{Cr}$ and $\mathrm{S}$ concentrations and the interaction between these two components. As a result, the limiting factor was not Cr-rich spinel crystallization, rather the formation of a separate chromate-sulfate salt phase.

\subsection{Enhanced Glass Formulation for Al-Limited Waste}

Crucible melts spanning a range of waste loadings were prepared and tested to determine the maximum viable waste loading for the aluminum-limited waste composition [3]. Table 3.9 presents the compositions of one radioactive and 29 non-radioactive glasses investigated in this work, along with the Al-limited waste composition supplied by OPR [3] and the non-radioactive version of the Al-limited waste composition obtained by removing the radioactive components $U$ and $\mathrm{Th}$ and renormalizing. Beside its high $\mathrm{Al}_{2} \mathrm{O}_{3}$ concentration $(\sim 50 \mathrm{wt} \%)$, the Al-limited waste contains considerable amounts of $\mathrm{Fe}_{2} \mathrm{O}_{3}$ and $\mathrm{Cr}_{2} \mathrm{O}_{3}(\sim 13 \mathrm{wt} \%$ and $1.2 \mathrm{wt} \%$, respectively). All three oxides are major constituents of a typical spinel phase. More importantly, high concentrations of $\mathrm{Al}_{2} \mathrm{O}_{3}$, with $\mathrm{SiO}_{2}$ and alkali oxides in the glass matrix promote the formation of alkali-aluminosilicates; such phases can often form in very large amounts. Amongst these 
The Catholic University of America Vitreous State Laboratory

phases, nepheline $\left(\mathrm{NaAlSiO}_{4}\right)$ forms fairly readily and can significantly degrade PCT performance. A "nepheline index" has been proposed as a guideline for glass formulation in order to prevent nepheline formation [22,23]. From the perspective of a simple chemical reaction, formation of nepheline should be influenced most significantly by the concentrations of its major constituents, $\mathrm{Na}_{2} \mathrm{O}, \mathrm{Al}_{2} \mathrm{O}_{3}$, and $\mathrm{SiO}_{2}$. Since $\mathrm{Al}_{2} \mathrm{O}_{3}$ is the most abundant component from the Al-limited waste, it is thus prudent to avoid addition of $\mathrm{Na}_{2} \mathrm{O}$ and to limit the $\mathrm{SiO}_{2}$ concentration to the minimum level necessary to meet other glass property requirements. A principal aspect of the strategy employed in the formulation of Al-limited waste glasses was, therefore, the evaluation of flux chemicals other than $\mathrm{Na}_{2} \mathrm{O}$.

The formulation work for the Al-limited waste was started at $35 \mathrm{wt} \%$ waste loading, which is $40 \%$ over the contract-specified minimum of $25 \mathrm{wt} \%$ waste loading. HLW-E-Al-24 (Table 3.9) has an $\mathrm{Al}_{2} \mathrm{O}_{3}$ content of more than $18 \mathrm{wt} \%$. Moderate amounts of $\mathrm{Na}_{2} \mathrm{O}$ and $\mathrm{Li}_{2} \mathrm{O}$ were added along with $\mathrm{B}_{2} \mathrm{O}_{3}$ as fluxes while $\mathrm{SiO}_{2}$ was maintained at a relatively low $\sim 35 \mathrm{wt} \%$. Heat treatment of this glass at $800^{\circ} \mathrm{C}$ and under $\mathrm{CCC}$ conditions showed around $0.1-0.2 \mathrm{vol} \%$ of spinel crystallization (Table 3.10), which is well within acceptable processing limits.

Seven glasses with $45 \mathrm{wt} \%$ waste loading were tested with various combinations of alkaline and alkaline earth oxide $\left(\mathrm{Na}_{2} \mathrm{O}, \mathrm{Li}_{2} \mathrm{O}\right.$, and $\left.\mathrm{CaO}\right)$ contents and at different concentrations of $\mathrm{SiO}_{2}$. However, at $45 \%$ waste loading, a considerable amount of spinel was observed in two of the as-melted glasses (HLW-E-Al-23 and Al-26) at similar $\mathrm{Na}_{2} \mathrm{O}$ and $\mathrm{Li}_{2} \mathrm{O}$ concentrations as in HLW-E-Al-24. Two other glasses with higher $\mathrm{CaO}$ contents resulted in melt viscosities that were lower than desirable (HLW-E-Al-12 and Al-18, Table 3.10). Addition of $\mathrm{P}_{2} \mathrm{O}_{5}$ also resulted in considerable crystallization in the as-melted glass (HLW-E-Al-16). Glasses HLW-E-Al-25 and Al-27 with moderate $\mathrm{CaO}, \mathrm{Li}_{2} \mathrm{O}$, and $\mathrm{Na}_{2} \mathrm{O}$ were largely crystal free on melting, with acceptable viscosity and crystallization on heat treatment (isothermal and CCC, see Table 3.10). Addition of one more percent $\mathrm{B}_{2} \mathrm{O}_{3}$ in HLW-E-Al-27 as compared to HLW-E-Al-25 was effective in reducing the melt viscosity from 60 poise to 46 poise at $1150^{\circ} \mathrm{C}$ while suppressing crystallization of calcium phosphate in samples subjected to CCC treatment.

Fourteen glasses with waste loading equal to or greater than $50 \mathrm{wt} \%$, the maximum value specified in the contract [3], were prepared and characterized. All glasses with moderate to high contents of $\mathrm{CaO}$ displayed significant crystallization either in the as-melted glass (HLW-E-Al-2, $3,-5$ and -10 with $\mathrm{CaO}$ from $14.2 \mathrm{wt} \%$ to $11.2 \mathrm{wt} \%$ ) or after heat treatment at $950^{\circ} \mathrm{C}$ (HLW-E-Al-7, -8, -9, -11 with $\mathrm{CaO}$ at $20.2 \mathrm{wt} \%, 18.2 \mathrm{wt} \%, 16.2 \mathrm{wt} \%$ and $14.2 \mathrm{wt} \%$, respectively). Most remarkably, about $30 \mathrm{vol} \%$ of alkali aluminosilicate, apatite, and spinel was observed in HLW-E-Al-4 and Al-6 after 70 hours at $950^{\circ} \mathrm{C}$. Substitution of $\mathrm{MgO}$ for $\mathrm{CaO}$ in HLW-E-Al-1 also resulted in heavy crystallization in the as-melted glass in addition to its unacceptable viscosity. The higher $\mathrm{Li}_{2} \mathrm{O}$ concentration in HLW-E-Al-14 at $50 \mathrm{wt} \%$ waste loading and HLW-E-Al-17 at $55 \mathrm{wt} \%$ waste loading were not effective in suppressing crystallization in the as-melted glass. Addition of $\mathrm{Na}_{2} \mathrm{O}$ with $\mathrm{Li}_{2} \mathrm{O}$ in HLW-E-Al-19 also was not effective in suppressing crystallization. Since none of the $50 \mathrm{wt} \%$ waste loading glasses had acceptable properties, a slightly lower waste loading of $47.5 \%$ was chosen for further tests.

Seven glasses at $47.5 \mathrm{wt} \%$ waste loading were tested. All seven glasses showed signs of crystallization in the as-melted samples. The majority of them also displayed rather low 
viscosity, as judged visually on pouring. Among them are two glasses with moderate $\mathrm{Na}_{2} \mathrm{O}$ and $\mathrm{CaO}$ concentrations (HLW-E-Al-28 and Al-29), one with moderate $\mathrm{Na}_{2} \mathrm{O}$ and added $\mathrm{K}_{2} \mathrm{O}$ (HLW-E-Al-22), two with high $\mathrm{Na}_{2} \mathrm{O}$ concentrations (HLW-E-Al-20 and Al-21) and two with low $\mathrm{Na}_{2} \mathrm{O}$ and higher $\mathrm{Li}_{2} \mathrm{O}$ and $\mathrm{CaO}$ concentrations (HLW-E-Al-15 and Al-13).

Based on these results, $45 \mathrm{wt} \%$ was selected as the optimum waste loading for this waste stream and the glass HLW-E-Al-27 was selected for melter testing. This waste loading is slightly lower than the maximum expected waste loading of $50 \mathrm{wt} \%$ [3]. After heat treatment at $950^{\circ} \mathrm{C}$ HLW-E-Al-27 shows about 1 vol\% crystalline phases, which is the current operational crystallization limit for WTP. The amount of crystallization increased with decreasing temperature up to about $7 \mathrm{vol} \%$ at $800^{\circ} \mathrm{C}$. The glass sample after CCC treatment showed less than 2 vol\% spinel crystals.

The compositions and the characterization results of the non-radioactive and radioactive versions of the selected glass (HLW-E-Al-27 and HLW-E-Al-27UTh) are given in Tables 3.3, 3.9, and 3.10. Both of the glasses show similar PCT and TCLP responses. The volume percent of the crystalline phases after isothermal and $\mathrm{CCC}$ heat treatments are generally lower in HLW-E-Al-27UTh than in HLW-E-Al-27 (Figure 3.5). Both glass samples, however, showed the same types of crystals. Table 3.11 presents the composition of the glass selected for processing on the DM100 and the measured properties of the crucible glass. The selected glass formulation, HLW-E-Al-27, meets all of the processing and product quality requirements imposed for these tests and has a waste oxide loading of $45 \mathrm{wt} \%$. This exceeds both the minimum waste loading of $25 \mathrm{wt} \%$ and approaches the maximum expected waste loading of 50 $\mathrm{wt} \%$ provided in the Scope of Work [3]. The glass contains $23.97 \mathrm{wt} \% \mathrm{Al}_{2} \mathrm{O}_{3}$, which is more than two times the WTP contract minimum for $\mathrm{Al}_{2} \mathrm{O}_{3}(11 \mathrm{wt} \%)$. All of the measured processing parameters are within acceptable ranges. The PCT leach rates are over an order of magnitude lower than those of the DWPF-EA glass and the TCLP leachate concentrations are all below the WTP Delisting Limits. Sodium aluminosilicate formation (e.g., nepheline) on heat treatment (especially canister centerline cooling (CCC) heat treatment) is a known concern with high-aluminum formulations and was the waste-loading-limiting factor in the present work. The selected glass produced very little crystallization $(\sim 1.9 \mathrm{vol} \%)$ after CCC heat treatment and the heat treated glass also meet the PCT requirements by a wide margin. It is worth noting that the frequently-employed "nepheline discriminator" [22, 23] would have erroneously rejected this glass in favor of lower-waste-loading alternatives.

\subsection{Enhanced Glass Formulation for Al-Na-Limited Waste}

A total of one radioactive and 26 non-radioactive glasses were tested to identify a suitable glass formulation for the Al-Na-limited waste stream. As shown in Table 3.12, in addition to $43 \mathrm{wt} \%$ of $\mathrm{Al}_{2} \mathrm{O}_{3}$, which is close to that in the Al-limited waste, the Al-Na limited waste also contains $26 \mathrm{wt} \% \mathrm{Na}_{2} \mathrm{O}$ and more than $4 \mathrm{wt} \% \mathrm{P}_{2} \mathrm{O}_{5}$. The Al-Na-rich waste was the most challenging among the four waste streams because a melt rich in $\mathrm{Al}_{2} \mathrm{O}_{3}, \mathrm{Na}_{2} \mathrm{O}$, and $\mathrm{SiO}_{2}$ tends to crystallize aluminosilicate phases such as nepheline, sodalite, and other silicates. To further complicate the formulation, the relatively high content of $\mathrm{P}_{2} \mathrm{O}_{5}$ in the Al-Na limited waste (almost double that of the Al-limited waste) tends to increase the propensity for precipitation of 
alkaline or alkaline earth phosphates (e.g., lithium phosphate, calcium phosphate, etc.). Consequently, the formulation selected for the Al-limited waste may not be suitable for this waste stream. In addition, the high $\mathrm{Na}_{2} \mathrm{O}$ concentration in the waste stream allows even less flexibility in glass formulation. Similar to the Al-limited formulation, the formation of crystalline silicate or phosphate phases could be detrimental because of the potential for extensive crystallization of silicates or the formation of phases that degrade PCT response such as nepheline and lithium phosphate. The formulation work on Al-Na limited wastes was, therefore, focused on optimizing the balance between maximizing waste loading and the inhibition of the aforementioned crystallization.

Crucible melts spanning a range of waste loadings were prepared and tested to determine the maximum viable waste loading for the aluminum-sodium limited waste composition. Table 3.12 presents the compositions of the 26 glasses investigated in this work, along with the Al-Na-limited waste and non-radioactive version of the Al-Na-limited waste obtained by removing the radioactive components $U$ and $T h$ and renormalizing. The strategy employed in the formulation of Al-Na-limited waste was focused on identifying the appropriate amounts of flux chemicals other than $\mathrm{Na}_{2} \mathrm{O}$ (in particular, $\mathrm{CaO}$ and $\mathrm{Li}_{2} \mathrm{O}$, the two components used successfully for the Al-limited waste formulation).

The formulation work for Al-Na-limited waste was started at $42 \mathrm{wt} \%$ waste loading, which is more than double the specified minimum of $20 \mathrm{wt} \%$ [3]. HLW-E-ANa-1 (Table 3.12) is similar to the glass HLW-E-Al-24 (Table 3.9, $35 \mathrm{wt} \%$ loading), with similar $\mathrm{Al}_{2} \mathrm{O}_{3}$, higher $\mathrm{Na}_{2} \mathrm{O}$ (from the waste), and higher $\mathrm{P}_{2} \mathrm{O}_{5}$. Although the as-melted glass appeared normal and only $0.3 \mathrm{vol} \%$ of $\mathrm{Cr}-\mathrm{Fe}$ oxide crystallized after 70 hours at $950^{\circ} \mathrm{C}$, the melt viscosity judged visually by glass pouring seemed rather high. No further characterization was conducted on this glass in order to concentrate on the development of Al-Na-limited glasses with higher waste loading.

The formulation using $\mathrm{Li}$ and $\mathrm{Ca}$ as the major fluxes (in addition to $\mathrm{Na}$ from the waste and added B) resulted in extensive crystallization, in particular, of calcium phosphate and spinel. As evident from Table 3.13, at waste loadings of $43 \mathrm{wt} \%, 45 \mathrm{wt} \%$, and $47 \mathrm{wt} \%$, as-melted glass samples with both $\mathrm{Li}$ and $\mathrm{Ca}$ as fluxes showed minor or trace amounts of crystals. However, considerable amounts of calcium phosphate formed on CCC heat treatment in glasses HLW-E-ANa-19 (43 wt\% waste loading with 5.7\% CaO), HLW-E-ANa-17, -18 (45 wt\% waste loading with 5.69\% CaO), and HLW-E-ANa-13, -20, and -21 (47 wt \% waste loading with 5.72\% $\mathrm{CaO}$ for $\mathrm{ANa}-13$ and $3.72 \% \mathrm{CaO}$ for the other two). The amounts of phosphate and sodium aluminosilicate crystallization became massive at higher waste loadings for glasses with both $\mathrm{Li}$ and $\mathrm{Ca}$, as demonstrated by HLW-E-ANa-14, -15 and -16 at $52 \mathrm{wt} \%$ loading. The volume percent of crystalline phases after CCC heat treatment ranged from 20 to $60 \mathrm{vol} \%$. These results suggest that $\mathrm{CaO}$ is not a suitable fluxing additive for the Al-Na-limited waste due to its high $\mathrm{P}_{2} \mathrm{O}_{5}$ content. Therefore lithium oxide was preferred, particularly considering that $\mathrm{Li}^{+}$is too small to be incorporated into the nepheline structure.

The formulation using $\mathrm{Li}$ as the main flux (in addition to $\mathrm{Na}$ from waste and added $\mathrm{B}$ ) started with HLW-E-ANa-2 at $47 \mathrm{wt} \%$ waste loading (Tables 3.12 and 3.13). The as-melted glass was crystal free with acceptable PCT responses. Heat treatment at $950^{\circ} \mathrm{C}$ resulted in only $0.3 \mathrm{vol} \%$ spinel crystallization. However, the glass melt seemed rather viscous, as judged 
The Catholic University of America Vitreous State Laboratory

visually on pouring. The viscosity was still relatively high for HLW-E-ANa-4, which had 1 wt\% $\mathrm{Li}_{2} \mathrm{O}$ added (89 poise, Table 3.13). Although this glass with additional Li had very similar PCT response and isothermal heat treatment result as HLW-E-ANa-2, up to 5 vol\% of spinel and nepheline-like phases were identified in the sample after CCC heat treatment. Addition of another $4 \mathrm{wt} \% \mathrm{~B}_{2} \mathrm{O}_{3}$ (HLW-E-ANa-22 and HLW-E-ANa-23) significantly suppressed the crystallization and, in particular, that of the nepheline-like crystalline phases. HLW-E-ANa-23 with an additional $3 \% \mathrm{ZrO}_{2}$ replacing $\mathrm{SiO}_{2}$ resulted in about $1.3 \mathrm{vol} \%$ of spinel and $\mathrm{Fe}-\mathrm{Cr}$ oxide after CCC heat treatment; HLW-E-ANa-22 with increased $\mathrm{B}_{2} \mathrm{O}_{3}$ showed only $0.5 \%$ of spinel and $\mathrm{Fe}-\mathrm{Cr}$ oxide after $\mathrm{CCC}$ treatment. Two glasses of similar composition but with higher waste loadings $(50 \%)$ resulted in more crystallization after CCC treatment: $2-3 \mathrm{vol} \%$ of spinel and nepheline crystals were present in HLW-E-ANa-24 and 1.5-2 vol\% crystals were present in HLW-E-ANa-25 (which contains $1 \mathrm{wt} \%$ additional $\mathrm{B}_{2} \mathrm{O}_{3}$ ). The majority of the glasses made using $\mathrm{Li}$ as the flux with waste loadings greater than $50 \mathrm{wt} \%$ encountered problems associated with crystallization of either nepheline-like phases after CCC heat treatment (HLW-E-ANa-5, ANa-26, ANa-9, ANa-11) or in as-melted glass samples (HLW-E-ANa-8, ANa-10, ANa-7 and ANa-12). Two glasses (HLW-E-ANa-3 and ANa-6) displayed melt viscosities that appeared too high for processing and, therefore, were not further characterized.

The test results suggest that an optimum waste loading for the Al-Na-limited waste is around 47 to $50 \mathrm{wt} \%$, as exemplified by HLW-E-ANa-22 and $\mathrm{ANa}-25$. With the $\mathrm{Al}_{2} \mathrm{O}_{3}$ content around $22 \mathrm{wt} \%$ and the $\mathrm{Na}_{2} \mathrm{O}$ content around $13 \mathrm{wt} \%$, the addition of $\mathrm{B}_{2} \mathrm{O}_{3}$ seemed most effective at suppressing crystallization of nepheline-like crystalline phases. From HLW-E-ANa-22 to ANa-25, the $3 \mathrm{wt} \%$ gain in waste loading comes with an increased level of crystallization under CCC conditions and, in particular, crystallization of nepheline. The other test data suggest that the amount of nepheline can rise quickly above about $50 \mathrm{wt} \%$ waste loading, potentially leaving little room for process variations. Therefore, although the two glasses have very similar PCT responses, viscosity, and electrical conductivity, the slightly lower waste loading HLW-E-ANa-22, which showed no sodium aluminosilicate crystallization, was selected as the best candidate for the DM100 tests.

The compositions and properties of the non-radioactive and radioactive versions of the selected glass (HLW-E-ANa-22 and HLW-E-ANa22UTh) are listed together in Tables 3.3, 3.12, and 3.13. Both glasses show similar PCT and TCLP responses. The volume percent of the crystalline phases after isothermal and $\mathrm{CCC}$ heat treatments are generally lower in HLW-E-CrMUTh (Figure 3.6). The same crystal phases were, however, observed in both glasses. Table 3.14 presents the composition of the glass selected for testing on the DM100 and the measured properties of the crucible glass. The selected glass formulation, HLW-E-ANa-22, meets all of the processing and product quality requirements imposed for these tests and has a waste oxide loading of $47 \mathrm{wt} \%$. This is more than twice the minimum waste loading of $20 \mathrm{wt} \%$ and approaches the maximum expected waste loading of $60 \mathrm{wt} \%$ provided in the Scope of Work [3]. The glass contains $21.34 \mathrm{wt} \% \mathrm{Al}_{2} \mathrm{O}_{3}$, which is nearly twice the WTP contract minimum for $\mathrm{Al}_{2} \mathrm{O}_{3}(11 \mathrm{wt} \%)$. The glass also contains $12.71 \mathrm{wt} \% \mathrm{Na}_{2} \mathrm{O}$; this is below the WTP contract minimum for $\mathrm{Na}_{2} \mathrm{O}+\mathrm{K}_{2} \mathrm{O}(15 \mathrm{wt} \%)$ because of the high aluminum concentration. The measured processing parameters are within acceptable ranges. The PCT leach rates are more than a factor of four lower than those of the DWPF-EA glass and the TCLP leachate concentrations are all below the WTP Delisting Limits. Sodium aluminosilicate formation (e.g., nepheline) on heat 
treatment (especially canister centerline cooling heat treatment) is a known concern with highaluminum formulations and was the waste-loading-limiting factor in the present work. The selected glass produced very little crystallization $(\sim 0.5 \mathrm{vol} \%)$ after $\mathrm{CCC}$ heat treatment and the heat treated glass also meets the PCT requirements by a wide margin. It is worth noting that the frequently-employed "nepheline discriminator" [22, 23] would have erroneously rejected this glass in favor of lower-waste-loading alternatives. 


\section{SECTION 4.0 DM10 MELTER OPERATIONS}

Results from crucible scale testing with chromium limited waste showed that in spite of the high chromium content, the waste loading was not limited by crystallization but by sulfatechromate salt formation due to the relatively high sulfate concentration in this waste, compounded by the chemical similarity and known interactions between sulfate and chromate. For this reason, screening tests were performed on the DM10 melter system to determine the maximum waste loading possible without forming secondary sulfate phases on the melt surface. A summary of the twelve tests conducted on the DM10, including feed composition, melter operating conditions, and sulfate layer identification, is provided in Table 4.1. The variation in the concentration of several oxides in the glass discharges over the course of the tests is shown in Figures 4.1 and 4.2.

Based on results from the crucible tests, the first test employed the $40 \%$ waste loading glass. A secondary sulfate layer was observed at the end of this test and, therefore, tests were conducted at successively lower waste loadings until a separated sulfate phase was not observed on the melt pool surface. No separated sulfate phase was observed at $30 \%$ waste loading and initial tests at $32.5 \%$ waste loading were not definitive due to the incompleteness of turnover in Test 5 and the minute trace of a sulfate phase on a single dip sample from Test 6 . At the end of a repeated test at $32.5 \%$ waste loading conducted at the lower melt temperature of $1150^{\circ} \mathrm{C}$, no secondary sulfate phase was observed on dip samples and therefore the glass composition with this waste loading was advanced for testing on the DM100. The composition of this glass and select measured properties are provided in Table 4.2.

Tests conducted using vanadium and sugar as additives were unsuccessful in increasing the waste loading attainable without the formation of a secondary sulfate phase or had other undesirable effects. In Test 2, $10 \mathrm{~g}$ sugar per liter was added to the feed, which reduced some of the sulfur thereby increasing sulfur emissions while decreasing the amount of sulfur in the glass product. As a result, no separate sulfate layer was observed at $40 \%$ waste loading. However this level of sugar addition resulted in the reduction of $28 \%$ of the iron to the divalent state and caused the measured concentration of bismuth in the product glass to drop by more than a factor of two, presumably due to reduction of bismuth to the metallic state causing it to settle out of the glass melt. The redox sensitivity of bismuth is an issue that deserves further investigation for bismuth-containing wastes since metal deposition could seriously affect melter lifetime and operations. The decreases in sulfur and bismuth concentrations are readily observed at about $40 \mathrm{~kg}$ glass production in Figures 4.1 and 4.2. Based on results from high-sulfur LAW melter tests, vanadium was added to the feed to achieve one percent vanadium oxide in the glass product in several tests to determine if it decreased the tendency to form a separate salt phase. However, the results of these tests are indistinguishable from comparable tests without vanadium, even when small amounts of sugar were added to the feed. Therefore, since no increase in waste loading was observed with vanadium, the $1 \mathrm{wt} \% \mathrm{~V}_{2} \mathrm{O}_{5}$ addition was not tested on the DM100. Instead, the $32.5 \mathrm{wt} \%$ waste loading formulation was selected as the best option. 


\section{SECTION 5.0 DM100 MELTER OPERATIONS}

Melter tests were conducted on the DM100-BL between 7/17/06 and 11/20/06. These tests produced over three metric tons of glass from almost nine and a half metric tons of feed. Prior to feeding a new glass composition, the glass inventory was reduced from about $180 \mathrm{~kg}$ to about $100 \mathrm{~kg}$ in order to decrease the feeding time required to change over the composition of the glass pool. The series of sixteen nominally 50-hour tests were divided as follows:

- Bismuth-Limited Waste - $794 \mathrm{~kg}$ of Glass Produced

o $1175^{\circ} \mathrm{C}$ glass temperature, optimized bubbling, $500 \mathrm{~g}$ glass per liter feed.

o $1175^{\circ} \mathrm{C}$ glass temperature, $9 \mathrm{lpm}$ bubbling, $500 \mathrm{~g}$ glass per liter feed.

o $1150^{\circ} \mathrm{C}$ glass temperature, $9 \mathrm{lpm}$ bubbling, $500 \mathrm{~g}$ glass per liter feed.

o $1150^{\circ} \mathrm{C}$ glass temperature, $9 \mathrm{lpm}$ bubbling, $250 \mathrm{~g}$ glass per liter feed.

- Chromium-Limited Waste - $540 \mathrm{~kg}$ of Glass Produced

o $1175^{\circ} \mathrm{C}$ glass temperature, $9 \mathrm{lpm}$ bubbling, $500 \mathrm{~g}$ glass per liter feed.

o $1150^{\circ} \mathrm{C}$ glass temperature, $9 \mathrm{lpm}$ bubbling, $500 \mathrm{~g}$ glass per liter feed.

- Aluminum-Limited Waste - $793 \mathrm{~kg}$ of Glass Produced

o $1175^{\circ} \mathrm{C}$ glass temperature, optimized bubbling, $500 \mathrm{~g}$ glass per liter feed.

o $1175^{\circ} \mathrm{C}$ glass temperature, $9 \mathrm{lpm}$ bubbling, $500 \mathrm{~g}$ glass per liter feed.

o $1150^{\circ} \mathrm{C}$ glass temperature, $9 \mathrm{lpm}$ bubbling, $500 \mathrm{~g}$ glass per liter feed.

o $1150^{\circ} \mathrm{C}$ glass temperature, 9 lpm bubbling, $250 \mathrm{~g}$ glass per liter feed.

$0 \quad 1150^{\circ} \mathrm{C}$ glass temperature, optimized bubbling, $500 \mathrm{~g}$ glass per liter feed.

- Aluminum-Plus-Sodium-Limited Waste - $934 \mathrm{~kg}$ of Glass Produced

o $1175^{\circ} \mathrm{C}$ glass temperature, optimized bubbling, $500 \mathrm{~g}$ glass per liter feed.

o $1175^{\circ} \mathrm{C}$ glass temperature, $9 \mathrm{lpm}$ bubbling, $500 \mathrm{~g}$ glass per liter feed.

o $1150^{\circ} \mathrm{C}$ glass temperature, $9 \mathrm{lpm}$ bubbling, $500 \mathrm{~g}$ glass per liter feed.

- $1150^{\circ} \mathrm{C}$ glass temperature, $9 \mathrm{lpm}$ bubbling, $250 \mathrm{~g}$ glass per liter feed.

o $1150^{\circ} \mathrm{C}$ glass temperature, optimized bubbling, $500 \mathrm{~g}$ glass per liter feed.

Fewer tests were performed on the DM100 with the chromium-limited waste because of the need to perform the screening tests on the DM10 for this waste stream.

Summaries of the tests are provided in Tables 5.1-5.4. Attempts were made to replicate the melter configuration and operating conditions used for previous tests with HLW simulants $[7-10,17,18,24]$. These conditions include a near-complete cold cap, which is between $80-95 \%$ melt surface coverage for the DM100 since a 100\% cold cap tends to lead to "bridging" in smaller melters. The bubbling rate was either fixed at $9 \mathrm{lpm}$ and the feed rate was adjusted to maintain a complete cold cap or the bubbling rate was optimized to achieve the maximum production rate. This use of bubbling is in contrast to some previous tests where the production 
The Catholic University of America Vitreous State Laboratory

rate was fixed between 1000 to $1050 \mathrm{~kg} / \mathrm{m}^{2} /$ day and the bubbling rate was adjusted to maintain the complete cold cap [7-10,18]. The approach used in the present tests permits the evaluation of the effects of waste composition, glass temperature, glass pool bubbling, and feed solids content on production rate. Figures 5.1.a - 5.1.i illustrate the glass production rates as moving hourly averages throughout the tests. Steady-state production rates for current and previous tests [7, 17, 24] are tabulated in Table 5.5 and depicted in Figures 5.2 - 5.4. All parameters tested, including waste/feed composition, glass temperature, glass pool bubbling, and feed solids content, had an effect on glass production rate. As expected, glass production rates increased with increased bubbling rate, glass pool temperature, and feed solids content; however, the effect of waste composition was greater than any other tested variable. Notice in Figure 5.2 at constant bubbling rate and feed solids content, as well as in Figure 5.3 at constant glass temperature and feed solids content, that glass production rate ranged between 400 and $1400 \mathrm{~kg} / \mathrm{m}^{2} /$ day over the waste compositions tested. WTP waste compositions previously tested, the vast majority of which were high in iron (as also has been the case at WVDP and DWPF), processed at the highest rates, whereas waste compositions high in aluminum processed at the slowest rates. The higher processing rates are not only attributable to the differences in waste composition but also the lower waste loadings (24-28 vs. $45-47$ wt. \% oxide) used in many of the previous tests [7, 17]. It is also noteworthy that of the four glass compositions tested, the highest production rates at constant bubbling were obtained for the chromium-limited waste, which had the lowest waste loading $(32.5 \mathrm{wt} \%)$. The observed increases in production rate in response to increases in melt pool bubbling, glass temperature, and feed solids content also showed large variations with waste composition. Glass production rates increased 40-300\% with optimized glass pool bubbling, $0-125 \%$ with a $25^{\circ} \mathrm{C}$ increase in glass pool temperature, and $40-60 \%$ with a doubling of the feed solids content.

Overall, there were no significant difficulties in processing these feed and glass compositions during these tests. Cold cap conditions were similar to the range of conditions observed in previous tests with HLW feeds [7-10, 17, 18, 24]. The chromium-limited waste formed a more fluid cold cap, which spread more evenly across the melt surface and was less likely to adhere to melter walls to form "shelves" and "bridges". In contrast, the aluminum-limited waste streams showed a greater tendency to form shelves and bridges that limited the steady-state production rates. On occasions during Tests $1 \mathrm{a}, 5 \mathrm{~b}, 6 \mathrm{~b}, 7 \mathrm{~b}$ and $8 \mathrm{c}$, feeding was intentionally paused to allow deposits in the plenum space to be assimilated into the glass in order to better determine the actual steady-state, cold-cap-limited feed rate. Other, shorter interruptions were required during testing in order to energize the top pair of electrodes, transfer feed to the feed tank, adjust the feed line in the peristaltic pump as a result of wear from the pump rollers, and to perform other maintenance activities. Spikes in feed rate often occurred immediately after feed transfers due to adjustments in tank mixer speeds and pump settings. During steady-state feeding periods, production rates typically vary by about ten percent from the mean rate. No foamy glass was observed in the glass discharge and no foam was observed on the melt pool surface or cold cap.

The results of various operational measurements that were made during these tests are given in Tables 5.5 - 5.9. Glass bubbling rates are shown in Figures 5.5.a - 5.5.e, glass temperatures are shown in Figures 5.6.a - 5.6.i, plenum temperatures in Figures 5.7.a - 5.7.i, electrode temperatures in Figures 5.8.a - 5.8.i, and glass resistance in Figures 5.9.a - 5.9.i; 
electrode power is included in the figures with electrode temperatures and glass resistance. The target bubbling rate of $9 \mathrm{lpm}$ was maintained throughout the designated tests. Conversely, in other tests, the cold cap was constantly monitored and the bubbling rate was frequently adjusted to achieve the maximum cold-cap-limited feed rate. The test-average optimized bubbling rates were one-and-a-half to two times higher than the nominal bubbling rate of $9 \mathrm{lpm}$. Bulk glass temperatures (measured at 5 and 10 inches from the bottom of the melt pool) were largely within $10^{\circ} \mathrm{C}$ of the target glass temperatures of $1175^{\circ} \mathrm{C}$ and $1150^{\circ} \mathrm{C}$ throughout the vast majority of the tests. The test-segment-average bulk glass temperatures were $1166-1178^{\circ} \mathrm{C}$ and $1148-1161^{\circ} \mathrm{C}$ for tests targeting glass temperatures of $1175^{\circ} \mathrm{C}$ and $1150^{\circ} \mathrm{C}$, respectively. Glass temperatures closer to the top of the melt pool (measured at 16 and 27 inches from the bottom) are not reliable indicators of bulk glass temperatures as a result of their sensitivity to variations in the level of glass in the melter and gradients near the melt surface. Glass temperatures measured at these locations were even lower at the beginning of tests with each new waste composition, prior to the glass level in the melter being increased to above the upper pair of electrodes. Plenum temperatures typically ranged from 250 to $450^{\circ} \mathrm{C}$, which is lower than the 550 to $650^{\circ} \mathrm{C}$ target. These lower temperatures were the consequence of maintaining a more complete cold cap. The lower pair of electrodes was hotter than the upper pair of electrodes at the beginning of tests with each composition due to the lower glass levels at the beginning and end of the tests with each composition. The top pair of electrodes was also colder than the bottom pair in tests with production rates below $500 \mathrm{~kg} / \mathrm{m}^{2} /$ day. Once the melter was filled with glass above the top electrodes, the two electrode pairs typically averaged 50 to 100 degrees lower than the glass pool. The bottom electrode, which was not powered, ranged between 630 and $830^{\circ} \mathrm{C}$. Power supplied to the electrodes typically varied between 9 and $26 \mathrm{~kW}$. As expected, more power was required as the bubbling rate and, therefore, production rate increased. The opposite trend is observed when power usage is normalized to glass production due to the amount of energy required to maintain the glass pool at the target melting temperature. The calculated glass pool resistance decreased dramatically as the melter was filled with glass, as would be expected. Relative increases in resistance were observed with glass pool temperature decreases, feed water content increases, and with the aluminum-limited waste composition.

The gas temperature at the film cooler averaged between $254-282^{\circ} \mathrm{C}$ and depended on the plenum temperature, the amount of added film cooler air, and the temperature of the added film cooler air. Drops of less than twelve degrees in gas temperature were observed across the (insulated) transition line; the high temperature is maintained in order to prevent condensation in the downstream filtration units. 


\section{SECTION 6.0 \\ FEED SAMPLE AND GLASS PRODUCT ANALYSIS}

\subsection{Analysis of Feed Samples \\ 6.1.1 General Properties}

Feed samples from each test were analyzed to confirm physical properties and chemical composition. Samples were taken during melter testing from an inline sampling port. Sample names, sampling dates, and measured properties are given in Table 6.1. All samples were measured for both density and $\mathrm{pH}$; at least one sample per unique waste composition and water content was analyzed for water content, glass conversion ratio, rheological properties, and oxide composition by XRF. The measured glass conversion ratio for all feed samples from tests targeting $500 \mathrm{~g}$ glass per liter were all within five percent of the target (see Tables 5.1-5.4 for target values) on a weight per weight basis, validating the use of the target conversion ratio for calculating glass production rates. Samples from tests targeting $250 \mathrm{~g}$ glass per liter had measured glass conversion ratios of 2 to $15 \%$ below the target values due to the difficulty in obtaining representative sub-samples for analysis. The low bias in measured glass conversion ratios can partially be attributed to water added to flush feed lines during feed transfers. The water content, density, glass yield, and $\mathrm{pH}$ varied within a narrow range for each feed type and water content. As intended, values for these parameters are lower for comparable samples at the lower target solids content of $250 \mathrm{~g}$ glass per liter.

\subsubsection{Rheology}

Samples of the melter feeds that were used for these tests were also subjected to rheological characterization. The results from rheological characterization of a variety of other melter feeds and waste simulants, as well as the effects of a range of test variables, are described in detail in a separate report [25]. Melter feeds were characterized using a Haake RS75 rheometer, which was equipped with either a Z40DIN or a FL22-SZ40 sensor. A typical set of measurements consists of identifying the flow characteristics of the slurry by measuring the shear stress on the slurry at controlled shear rates and temperatures. In these measurements, the shear rate values are preset and are increased stepwise from $0.01 \mathrm{~s}^{-1}$ to $200 \mathrm{~s}^{-1}\left(70 \mathrm{~s}^{-1}\right.$ for FL22-SZ40) with a sufficient delay (typically 15 to 30 seconds) between steps to ensure that the shear stress is allowed to fully relax and therefore is measured at equilibrium. This approach is somewhat different from the "flow curve" approach in which the shear rate is ramped up to some maximum value and then ramped back down to produce a hysteresis curve that is dependent on the selected ramp rate. The viscosity of the sample as a function of the shear rate is then calculated as the ratio of the shear stress to the shear rate. The yield stress data for the melter feeds were measured using a controlled-stress mode in which the torque on the rotor was slowly increased while the resulting deformation of the fluid was monitored. The discontinuity in the measured deformation-torque curve was identified as the yield stress. It should be noted that this direct measurement of the yield stress can be quite different from the value that is often reported as the yield stress, which is obtained by extrapolation of the shear stress-shear rate curve to zero 
shear rate. All of the measurements in this work were made at $25^{\circ} \mathrm{C}$; previous work [25], which examined a range of temperatures, showed a relatively weak effect of temperature.

Rheograms for the melter feeds, which show the feed viscosity versus shear rate, are presented in Figure 6.1; measured values for viscosity at selected shear rates and the yield stress are shown in Table 6.1. The aluminum-limited waste stream resulted in the most viscous feed, even when diluted to $250 \mathrm{~g}$ glass per liter. The least viscous feeds were those for the bismuthand aluminum-plus-sodium-limited waste streams. As expected, each feed became significantly less viscous when diluted to $250 \mathrm{~g}$ glass per liter. The yield stress values exhibited the same trends as those described for the viscosity at low shear rates. All feeds were processed by the feed system without significant difficulties.

\subsubsection{Chemical Composition}

The methods used for analysis of feed sample chemical compositions are described in Section 1.5. The boron and lithium oxide target values were used for normalizing the XRF data since their concentrations were not determined by XRF. These results, compared to the target composition in Table 6.2, generally corroborate the consistency of the feed compositions and show good agreement with the target compositions for the major elements. All oxides with target concentrations greater than one percent deviated less than $10 \%$ from target for feed samples with bismuth- and aluminum-limited waste. The feed samples for the chromium-limited waste were within $10 \%$ of the target except for chromium and phosphorus oxides, which averaged 0.12 and 0.16 wt $\%$ absolute deviation above the target values. Deviations from target in the analysis of the feed sample for the aluminum-plus-sodium-limited waste are partially attributable to segregation during sub-sampling. This is discerned from the low glass recovery (Table 6.1) and the oxide deviations for sample BLN-F-107A. Samples with low solids content are difficult to sub-sample due to rapid settling of particulate matter. Once a biased sub-sample is taken, the remaining parent sample is also biased with respect to solids and, therefore, is no longer representative of the original feed, thus preventing further sub-sampling and analysis. Measurements from other feed samples with the aluminum-plus-sodium-limited waste are much closer to target values, supporting this contention. The composition of this feed is further corroborated by comparison to the product glasses (see Section 6.2.1), which shows all oxides with concentrations greater than $1 \mathrm{wt} \%$ in the target composition to be within $10 \%$ of the target except for iron, which had a absolute deviation of only $0.32 \mathrm{wt} \%$ in the non-segregated feed samples (BLN-F-9A and BLN-F-152A).

Low concentrations of manganese in all feeds, as well as titanium, neodymium, and chlorine in some feed compositions, were measured, even though they are not included in the target composition. Also, common elements such as magnesium, titanium, zirconium, potassium, barium, and calcium, when targeted at low concentrations, were typically above these targets. These positive deviations are often observed in melter feeds due to their ubiquity in the raw materials used to make up the simulants and in the glass forming additives. Cadmium was not detected in feed samples by XRF even though it is included at very low concentrations in two of the simulated waste streams. Analysis of the product glass using a method other than XRF (see Section 6.2.1) indicates the cadmium is present at the low target concentrations; however, these 
concentrations are below the sensitivity of the XRF for these glass matrices. Analyzed sulfur concentrations are below target concentrations due to volatilization during sample preparation.

\subsection{Analysis of Glass Samples}

Over three metric tons of glass was produced in these tests. The glass was discharged from the melter periodically into 5-gallon carbon steel pails using an airlift system. The discharged product glass was sampled at the end of each test by removing sufficient glass from the top of the cans for total inorganic analysis. Product glass masses, discharge date, and analysis performed are given in Tables 6.3-6.6. Glass samples were also obtained by dipping a rod into the glass pool at the beginning and end of each test. These "dip samples" underwent visual examinations to detect the presence of separate sulfate or crystalline phases on the glass surface as well as examination by SEM-EDS to determine the extent of crystallization in the melt pool.

\subsubsection{Compositional Analysis of Discharge Glasses}

All discharge glass samples were crushed and analyzed directly by XRF. The target values for boron and lithium oxides, which are not determined by XRF, were used for normalizing the XRF data to $100 \mathrm{wt} \%$. The XRF analyzed compositions of discharged glass samples are provided in Tables 6.7-6.10. The majority of the XRF analysis results compared very favorably to their corresponding target values and feed sample analyses (see Section 6.1.3). Oxides with a target concentration greater than 1 weight percent showed less than $10 \%$ deviation from the target values. Exceptions are a deficit of nickel in the glasses from the bismuth-limited composition; surpluses of bismuth and chromium in the glasses from the chromium-limited composition due to insufficient glass production during the test to reduce the high concentrations of these elements present from the previous test; and a surplus of iron in the glasses from the aluminum-plus-sodium-limited composition, also observed in the feed samples. The origin of the nickel deficit in the bismuth-limited glasses is unclear since it was not observed in the feed samples. Also, nickel-bearing crystalline phases, although detected in dip samples, were lower in concentration in the bismuth-limited dip sample glasses than the in the dip sample glasses from the aluminum-plus-sodium composition, which did not have an analyzed nickel deficit. Similar to feed sample analysis, manganese in all glasses, as well as chlorine, neodymium, and titanium in some glasses (which are not in the target compositions), were measured at low concentrations in the product glasses. Also similar to feed sample analysis, common elements such as magnesium, titanium, zirconium, potassium, barium, and calcium, when targeted at low concentrations, were typically above their respective targets. Sulfur is below target for almost all glasses due to volatilization from the glass pool and cold cap.

Corroborative analysis using DCP on solutions of acid-dissolved glass was performed on select glasses produced from each test; the results are compared to the XRF analysis in Table 6.11. Values for all the major oxides compare favorably with the XRF analysis and target composition except for sodium, which often exhibits a low bias using this procedure [13] and chromium. The closeness of the DCP boron and lithium analysis to the target (deviations less 
than $10 \%$ ) validates the use of the target boron and lithium concentrations for normalizing the XRF data. Relative deviations for lithium are larger for glass generated from the bismuth-limited waste due to the low lithium concentrations (lithium was not used as an additive in this test); however, the absolute deviations are less than $0.14 \mathrm{wt} \%$. Cadmium concentrations measured by DCP are very close to target values, whereas the XRF method was not able to detect these low levels of cadmium.

Compositional trends for selected oxides shown in Figures 6.2-6.5 illustrate the closeness to targets at the end of tests with each composition. Exceptions include volatile species such as sulfur and fluorine, which remain significantly below target concentrations as a result of significant release to the melter exhaust. During tests with bismuth-limited wastes, bismuth, nickel, phosphorus, potassium, sulfur, sodium, fluorine, and chromium increase in concentration at the expense of iron, manganese, zinc, and silicon as the steady-state composition is approached. Subsequently, silicon, potassium, sulfur, fluorine, and chromium increase in concentration at the expense of sodium, bismuth, nickel, phosphorus, and iron as the glass pool transitioned from the bismuth- to the chromium-limited composition. The subsequent transition to the aluminum-limited waste composition resulted in increases in the concentrations of aluminum, calcium, sodium, fluorine, and lead at the expense of bismuth, potassium, and silicon. Testing ended with sodium, phosphorus, silicon, and sulfur increasing in concentration in the product glass at the expense of aluminum, calcium, iron, and lead as the glass pool transitioned to the aluminum-plus-sodium-limited composition. Common elements that are present in the target composition at low concentrations, such as potassium and magnesium, are observed at above target concentrations in the discharged glass, presumably due to contaminants in the glass forming chemicals. Chromium and nickel are observed at above target concentrations in some of the discharged glasses, presumably due to corrosion of the high-chromium melter bricks and Inconel components.

\subsubsection{Chemical Durability of Discharge Glasses}

Glass discharge samples from the end of at least two test segments for each waste composition were evaluated for chemical durability using the PCT and TCLP methods. The PCT results are compared to those for the benchmark DWPF-EA glass in Table 6.12 and the TCLP results are compared to the WTP delisting limits [29, 30] and Universal Treatment Standard (UTS) limits in Table 6.13. The chemical durability determined by both of these methods is excellent for the melter glasses. All measured PCT concentrations and normalized leach rates on discharge glass samples are at least ninety times lower than the corresponding values for the DWPF-EA glass. All regulated TCLP leachate concentrations are less than $1 \mathrm{mg} / \mathrm{l}$ and more than an order of magnitude less than WTP delisting limits. All measured concentrations are also well below the UTS limits. The highest TCLP leachate concentrations were for bismuth, which were less than or equal to $1 \mathrm{mg} / \mathrm{l}$. The chemical durability of these glasses produced from wastes limited by bismuth, chromium, aluminum, and aluminum plus sodium is similar to that for glasses formulated with iron-limited waste [1]. The measured durability is also similar to durability for corresponding radioactive glasses produced in crucible melts (see Table 3.3). 
These results confirm that glasses can be formulated from a variety of waste loading limiting constituents without compromising the quality of the vitrified product.

\subsection{Glass Pool Samples}

Glass pool samples were obtained to provide a conservative estimate of the extent of secondary phases in the glass melt in each test. However, even though the small dip samples cool much more quickly that the discharge samples, there is still the potential for crystallization during cooling of the dip sample. As a result, the crystal contents found in the dip samples will always overestimate the amount actually present in the melt pool at the time of sampling. A list of all dip samples including sample names, sampling dates, target glass pool temperature, visual observations of secondary phases, and SEM analysis are given in Table 6.14. These samples were also useful in detecting secondary phases on the melt pool surface, particularly sulfate in the tests with the chromium-limited waste. There was no visual evidence of secondary phases in the samples at the time of sampling as either material adhering to the sampling rod indicative of a surface layer or macroscopic features indicative of crystalline phases in the glass. However, SEM observations indicated that the glasses contained between 0.2 to 1.1 volume percent crystals. These crystal phases in glass samples were primarily spinels rich in iron, chromium, nickel and aluminum in all tests except tests conducted with chromium-limited waste. The amount of aluminum in the crystal phases observed varied with the concentration of aluminum in the product glass. No bismuth was observed in any of the secondary phases. The secondary phases observed in glasses produced from the chromium-limited waste were fine grained chromium oxide aggregates accounting for only 0.3 volume percent of the glass. The amount of crystalline phases observed in these glasses is consistent with observations on the same glass compositions from crucible scale testing (see Section 3.0). The ease of discharging these glasses during testing coupled with the low crystal content of these glass samples indicate that secondary phase formation should not be a concern in processing these compositions. The lack of secondary sulfate adhering to sampling rods used during the tests with the chromium-limited waste confirms the results obtained in the DM10 screening tests. 


\section{SECTION 7.0 \\ MONITORED OFF-GAS EMISSIONS}

\subsection{Particulate Sampling}

The melter exhaust was sampled for metals/particles according to 40-CFR-60 Methods 3, 5, and 29 at steady-state operating conditions during each test segment. The concentrations of off-gas species that are present as particulates and gaseous species that are collected in impinger solutions were derived from laboratory data on solutions extracted from air samples (filters and various solutions) together with measurements of the volume of air sampled. Particulate collection required isokinetic sampling, which entails removing gas from the exhaust at the same velocity that the air is flowing in the duct (40-CFR-60, Methods 1-5). Typically, a sample size of 30 dscf was taken at a rate of between 0.5 and $0.75 \mathrm{dscfm}$. Total particulate loading was determined by combining gravimetric analysis of the standard particle filter and chemical analysis of probe rinse solutions. An additional impinger containing $2 \mathrm{~N} \mathrm{NaOH}$ was added to the sampling train to ensure complete scrubbing of all acid gases and, particularly, iodine. The collected materials were analyzed using direct current plasma atomic emission spectroscopy for the majority of the constituents and ion chromatography (IC) for anions. Melter emission fluxes are compared to feed fluxes in Table 7.1. Notice the distinction that is made between constituents sampled as particles and as "gas". The "gaseous" constituents are operationally defined as those species that are scrubbed in the impinger solutions after the air stream has passed through a 0.3 $\mu \mathrm{m}$ heated filter. All samples are well within the $90-110 \%$ limits for isokinetic sampling.

Particulate emissions from the melter constituted 0.06 to 0.57 percent of feed solids. This level of carry-over is less than that measured for HLW AZ-102 (0.57 - 1.47 percent) and HLW C-106/AY-102 SIPP (0.61 to 0.81 percent) simulants processed on the same melter $[8,18]$. The higher carry-over in many of the previous tests is due to higher proportions of volatile species in the feed such as rhenium, cesium, and halogens. The carry-over is comparable to previous tests conducted with HLW AZ-101 simulants while bubbling the melt pool [7]. The effects of melt pool bubbling, glass pool temperature, and feed solids content on solids carry-over from the melter is depicted in Figures 7.1 - 7.3. Notice that no clear emissions trends can be seen as a function of the tested variables. Increases in particulate carry-over with melt pool bubbling have been extensively documented on the DM1200 with HLW waste streams [11-13, 16, 17]. The lack of an increase in emissions with bubbling in these DM100 tests maybe due to the proximity of the bubbling outlet to exhaust outlet of the melter as well as the height of the plenum. Tests with the DM1200 have demonstrated that the bubbler configuration as well as the bubbling rate has an effect on melter emissions $[16,17]$. The results from previous tests have also shown that particulate melter emissions tend to increase with increasing glass processing temperature [13, 31-34]; however, the effect of only a $25^{\circ} \mathrm{C}$ increase is thought to be within the variability of the measurements [1, 34]. Tests on the DM10 and DM1200 with HLW wastes have also demonstrated that carry-over increases with increasing water content due presumably to the entrainment of solids with volatilized water $[1,11]$. This same trend was not observed in these 
tests due perhaps to the differences in plenum geometry and blockage by plenum deposits formed during these tests.

As expected, the feed elements emitted at the lowest melter DF were clearly fluorine and sulfur. Other elements exhibiting volatile behavior in some of the tests include chromium, lead, boron, and alkali metals. The relative volatility of barium, cadmium, and titanium is difficult to evaluate due to the low target concentrations in the feed. Bismuth emissions range from $<0.03$ to 0.87 percent of bismuth fed to the melter. Emissions of chlorine were measured during all tests even though they were not included in simulant recipes or in analyzed compositions. Chlorine has frequently been observed in melter emissions when not present in feed recipe or at levels exceeding the amount in the feed recipe due to its ubiquity in raw materials, presence in tap water, and high volatility at glass melting temperatures. Boron, sulfur, and the halides were the only elements detected in the impinger solutions collected downstream of the heated particle filter in the sampling train, which constitutes the "gas" fraction of the melter emissions. The proportion of these elements present in the gas phase increases while processing feeds with higher water content due to the volatilization of acid species.

\subsection{Gases Monitored by FTIR}

Melter emissions were monitored in each test for a variety of gaseous components, most notably CO and nitrogen species, by Fourier Transform Infra-Red Spectroscopy (FTIR). The off-gas system temperature is maintained well above $100^{\circ} \mathrm{C}$ beyond the sampling port downstream of the HEPA filter to prevent analyte loss due to condensation prior to monitoring. A summary of average concentrations monitored during each test is provided in Table 7.2. The concentrations of two of the monitored species are plotted in Figures 7.4 - 7.12. The analytes listed in Table 7.2 are those that were thought likely to be observed during the test based on previous work; no other species were detected in the off-gas stream by FTIR. Generally, emissions were low as a result of the low concentrations of nitrogen, organic carbon, ammonia, and chlorine in the feed. The most abundant nitrogen species monitored was NO, which is in keeping with previous melter tests with both HLW and LAW feeds. Little or no nitrogen was detected as other species, except as $\mathrm{NO}_{2}$, which was 10 to 20 times lower in concentration than $\mathrm{NO}$ in all but the test with chromium-limited wastes where no $\mathrm{NO}_{2}$ was detected. The variability in the NO concentrations shown in Figures 7.4-7.12 is attributable to the dynamic conditions in the cold cap and in keeping with previous melter tests. The concentration of water in the exhaust increased with increasing feed rate for each waste type. Water emissions were elevated with respect to feed rate when processing feed with low solids content. Consistent with the gaseous chlorine and fluorine concentrations observed using the Method 5-type sampling discussed earlier, $\mathrm{HF}$ or $\mathrm{HCl}$ were observed by FTIR. The variations in emissions over the course of each test segment are due in part to changes in the melt pool cold cap. Hydrogen fluoride concentrations were lower at the beginning of testing due to the lack of fluorine in the glass pool and the processing time required for the glass to reach steady-state concentration with respect to fluorine. Sulfur dioxide emissions were higher in tests with higher feed sulfur concentrations (chromium- and bismuth-limited wastes) and increase with increasing feed rate for each waste composition. However, gaseous sulfur emissions can also be present in forms other than sulfur dioxide that are not monitored by the FTIR, such as sulfuric acid. 


\section{SECTION 8.0 SUMMARY AND CONCLUSIONS}

A series of tests was conducted on the DM100-BL vitrification system installed at VSL to evaluate enhanced HLW glass formulations for WTP waste streams that are compositionally very different from those extensively studied to date, which are predominantly limited by iron. The waste streams employed for this work were provided by ORP; their loadings in glass are expected to be limited by bismuth, chromium, aluminum, and aluminum plus sodium. The melter tests evaluated the effects of modest increases in glass processing temperature, optimized glass pool bubbling, and feed solids content on glass productions rates. New glass formulations were developed with the objectives of achieving high waste loadings and determining the waste loading-limiting factors for each waste type. The formulations used for melter testing were selected based on the test results from a series of crucible melt glasses that were prepared and characterized for each waste type. Each of the selected formulations meets all of the product quality, processability, and waste loading requirements. The results of this work provide new information on waste loadings and glass production rates that are needed to underpin overall WTP waste treatment rate projections. The work also identifies new risks associated with these previously unstudied waste types. This information provides ORP with a basis for projection of the amount of Immobilized High Level Waste (IHLW) to be produced at Hanford, potential waste treatment rates, and evaluation of the prospects for future enhancements of the WTP over and above the present well-developed baseline.

Glass formulations that meet all processing and product quality requirements for each of the four waste streams were developed with waste loadings ranging from 40 to $50 \mathrm{wt} \%$ on a waste oxide basis. The $40 \mathrm{wt} \%$ loading selected for the chromium-limited waste was decreased to $32.5 \mathrm{wt} \%$ based on DM10 screening tests in order to mitigate sulfate/chromate salt formation. However, all of the final waste loadings far exceed the minimum and approach or meet the maximum levels specified by ORP.

Melter testing on the DM100 was performed with each of the four selected glass formulations in 50-hour test segments. These test segments employed glass pool temperatures of $1150^{\circ} \mathrm{C}$ and $1175^{\circ} \mathrm{C}$, bubbling rates of $9 \mathrm{lpm}$ and optimized flow, and 250 and $500 \mathrm{~g}$ glass per liter feed solids concentration. Almost nine and a half metric tons of feed was processed to produce over three metric tons of glass. Analysis was performed on discharge and glass pool samples throughout the tests for total composition and secondary phases. All of the melter tests were successfully completed with no evidence of processing issues. Glass production rates above the WTP requirement of $800 \mathrm{~kg} / \mathrm{m}^{2} /$ day were obtained for each waste composition. However, only the bismuth- and chromium-limited wastes achieved this rate without increased bubbling and/or increased melt temperature. All compositions with low solids content processed at production rates well below $800 \mathrm{~kg} / \mathrm{m}^{2} /$ day, in keeping with previous HLW tests with iron-limited waste streams. 
Figure 8.1 shows the variation in glass production rates obtained in the present work as compared to results obtained previously on the DM100 melter with high-iron feeds. If the rates for the high-iron feeds are used as the baseline, these results show that there is a significant potential for production rate short-falls as the waste type is varied. In view of the large variations in glass production rates and waste loadings across the waste types studied in the present work and for the WTP baseline, it is instructive to consider the waste oxide processing rate, which is the product of these two factors. The waste oxide processing rate ties most directly to the overall question of the rate at which waste can be treated. Accordingly, Figure 8.2 shows the waste oxide processing rates for the results from the present work and other results obtained on the DM100 for WTP high-iron wastes. A strong dependence on waste type is evident. The chromium and bismuth limited wastes processed more rapidly than three of the four iron-limited wastes, whereas the two aluminum-limited wastes processed at considerably slower rates.

During each test, melter exhaust was sampled for particulate and gaseous species to determine the effect of the variations in feed composition, bubbling rate, and glass temperature on emissions. Total particulate carry-over from the melter into the off-gas stream was only 0.06 to 0.57 percent, which is below the range measured previously on the DM10 and DM100 when processing HLW simulants. Melter DFs were determined for most elements in the feed for all eight test segments performed. The most volatile species were sulfur and fluorine, which is typical. Particulate emissions from the melter were not significantly affected by glass pool temperature, bubbling rate, or water content but did change with waste type. Gaseous emissions of nitrogen oxides and byproducts of incomplete combustion, such as carbon monoxide and ammonia, were very low due to the lack of nitrates and organic carbon in the feed.

Glass samples from the crucible and melter tests were subjected to leach testing using the PCT and TCLP methods in order to evaluate product quality. The crucible melt results, with and without the addition of radioactive components ( $U$ and $T h$ ), were confirmed by tests performed on glass samples from the melter runs. Despite the higher waste loadings and range of waste loading limiting components, the glass products significantly out-performed the DWPF-EA benchmark glass on the PCT leaching procedure by factors of at least 90 and exhibited TCLP leachate concentrations that were well below the WTP delisting limits. Overall, the results from the melter tests and the associated processing and product quality data support the viability of the proposed HLW enhancement approaches.

Waste loadings, waste-loading-limiting factors, and key observations are summarized for each waste type below:

- Bismuth-Limited Waste

o $50 \mathrm{wt} \%$ waste loading

o Limited by crystallization (spinel + phosphate)

o $\mathrm{Bi}_{2} \mathrm{O}_{3}$ content $=6.71 \mathrm{wt} \%$ vs. WTP contract minimum of $2 \mathrm{wt} \%$, giving a $70 \%$ reduction in the volume of glass produced from this waste

o Melt foaming on CCC heat treatment

o Bismuth redox sensitivity (facile reduction to metal)

o Low intrinsic melt rate 
- Chromium-Limited Waste

o $32.5 \mathrm{wt} \%$ waste loading

o Limited by sulfate/chromate salt formation (not crystallization)

o $\mathrm{Cr}_{2} \mathrm{O}_{3}$ content $=1.08 \mathrm{wt} \%$ vs. WTP contract minimum of $0.5 \mathrm{wt} \%$, giving a $54 \%$ reduction in the volume of glass produced from this waste

o Importance of sulfate-chromate interaction

o Importance of salt formation mitigation for HLW (previously treated as a uniquely LAW issue)

o High bismuth content

- Aluminum-Limited Waste

o $45 \mathrm{wt} \%$ waste loading

0 Limited by crystallization on CCC and impact on PCT

o $\quad \mathrm{Al}_{2} \mathrm{O}_{3}$ content $=23.97 \mathrm{wt} \%$ vs. WTP contract minimum of $11 \mathrm{wt} \%$, giving a $54 \%$ reduction in the volume of glass produced from this waste

o Low intrinsic melt rate

- Aluminum-Plus-Sodium-Limited Waste

o $47 \mathrm{wt} \%$ waste loading

o Limited by crystallization on CCC and impact on PCT

o $\mathrm{Al}_{2} \mathrm{O}_{3}$ content $=21.34 \mathrm{wt} \%$ vs. WTP contract minimum of $11 \mathrm{wt} \%$, giving a $48 \%$ reduction in the volume of glass produced from this waste

o Low intrinsic melt rate

As indicated above, compared to the current WTP HLW contract minima, the glass formulations developed in the present work achieve significantly higher waste loadings, which translate into reductions in the volume of glass produced from these streams of between 48 and $70 \%$. Clearly, the extent of the increase in waste loading, and decrease in glass volume, will vary from tank to tank as a result of the differences in tank waste compositions. However, if even a fraction of this benefit can be realized across the entire Hanford HLW inventory, the potential cost savings are enormous. Taking an overall glass volume reduction of $30 \%$ for illustration, which appears conservative based on the present results, and further assuming a baseline canister count of about 15,000, this would reduce that number by about 4500 canisters to about 10500 . Assuming a disposal cost of $\sim \$ 1 \mathrm{M}$ per canister and a production cost of $\sim \$ 1 \mathrm{M}$ per canister, this amounts to a cost saving of about $\$ 9 \mathrm{~B}$. Furthermore, there would likely be additional cost savings through the potential for schedule acceleration. Consequently, the potential return on the investment made in the development of these enhancements is extremely favorable.

\subsection{Recommendations for Future Work}

The results of the testing presented herein clearly demonstrate the viability of the enhancement and optimization strategies for the four HLW waste streams tested. Furthermore, simple estimates of the impacts of these enhancements show the potential for enormous savings in cost and schedule. As a result, it is recommended that testing and evaluation of these strategies 
be continued in order to provide a solid basis for their broad implementation in order to maximize the cost and schedule benefits while minimizing technical risk. Some of the elements of such a program are summarized below. While the discussion is centered around Hanford HLW streams, many of these enhancement strategies would also be applicable to other HLW streams, such as those being treated at the DWPF, with the potential for similarly large benefits.

- Other WTP HLW Waste Types: The present testing was based on four HLW compositions from the Hanford tanks. While these results are also relevant to waste from several other tanks, the diversity of the Hanford tank wastes means that there are several such waste-loading-limiting constituents, each of which needs to be addressed in order to maximize the benefit from these enhancements. As a starting point, a similar test program to the one performed in the present work should be performed for each of the principal waste-loading-limiting constituents. Particular attention should be paid to waste streams containing sulfur due to the potential of forming secondary phases on the melt pool surface. Key issues identified in the present work include:

- Interaction between chromium and sulfate can lead to molten chromate-sulfate salt formation during HLW vitrification.

- Redox sensitivity of bismuth and the potential for molten metal formation in the HLW melter. Appropriate redox operating ranges and process controls need to be developed and tested.

- Potential for foaming of high-bismuth glasses in the HLW canister during cooling. The underlying cause and appropriate mitigation strategies need to be developed and tested.

- Throughput: A key risk area identified in the present work relates to the strong dependence of glass production rates on waste composition. The extent of this variation across the full spectrum of HLW waste types needs to be quantified in order to accurately project waste treatment rates. The vast majority of the previous testing for the WTP has been performed on iron-limited wastes and those results have formed the basis for vitrification system capacity projections. The results of the present work suggest that this basis may not be appropriate for other waste types. Testing also needs to be performed to develop approaches to mitigate the potential short-fall in glass production rates.

- Formulating Glasses with higher crystal contents: Previous tests with HLW iron-limited wastes showed that allowing a higher crystal content product can allow significantly increased waste loadings. Evaluation of this enhanced "operational liquidus temperature" approach for other waste streams would result in further waste loading increases.

- Scale-Up Testing: Testing should be extended to larger-scale melter systems in order to address potential risks associated with scale-up, particularly with respect to processing rates. Testing should be conducted at the DM1200 WTP HLW Pilot Melter scale $\left(1.2 \mathrm{~m}^{2}\right)$. Optimization of bubbling rate was critical in achieving the required production 
rate in the DM100 tests and therefore testing with bubblers in the prototypical orientation at larger scale is required to confirm these findings.

- Integrated System Testing: Testing on the DM1200 WTP HLW Pilot Melter system would provide data from a one-third scale system with a prototypical feed delivery system and off-gas treatment train. Such testing is necessary to evaluate potential interactive effects on system operation arising from implementation of the enhancement strategies and to provide data on the performance of each unit operation, input for flow-sheet models and regulatory requirements, and information of recycle streams.

- Longer-Duration Testing: Once the results of this work are validated at larger scale, the duration of testing should be extended in order to address and quantify any chronic issues, such as the slow accumulation of crystals in the melter cavity, any degradation in the ability to discharge glass, and effects on off-gas line plugging. 


\section{SECTION 9.0 \\ REFERENCES}

[1] "HLW Enhancement Tests on the DuraMelter" 10 with Hanford AZ-102 Tank Waste Simulants," Final Report, K.S. Matlack, W.K. Kot, H. Gan, W. Gong and I.L. Pegg, VSL-06R6260-1, Rev. 0, Vitreous State Laboratory, The Catholic University of America, Washington, DC, 2/28/06.

[2] "High Level Waste Vitrification System Improvements," Test Plan, K.S. Matlack, W.K. Kot, and I.L. Pegg, VSL-06T1010-1, Rev. 0, Vitreous State Laboratory, The Catholic University of America, Washington, DC, 5/8/06.

[3] "Test and Evaluate High Level Waste (HLW) Vitrification System Improvements," Contract Number DE-AC27-06RV14790, US Department of Energy, Office of River Protection, Richland, WA, April, 2006.

[4] "Glass Formulation and Testing with RPP-WTP HLW Simulants," Final Report, W.K. Kot, and I.L. Pegg, VSL-01R2540-2, Rev. 0, Vitreous State Laboratory, The Catholic University of America, Washington, DC, 2/16/01.

[5] "Glass Formulation to Support Melter Runs with HLW Simulants," Final Report, W.K. Kot, K. Klatt, and I.L. Pegg, VSL-03R3760-2, Rev. 0, Vitreous State Laboratory, The Catholic University of America, Washington, DC, 8/8/03.

[6] "HLW Glass Formulation to Support C-106/AY-102 Actual Waste Testing," Final Report, W.K. Kot and I.L. Pegg, VSL-04R4770-1, Rev. 0, Vitreous State Laboratory, The Catholic University of America, Washington, DC, 8/12/04.

[7] "Melter Tests with AZ-101 HLW Simulant Using a DuraMelter 100 Vitrification System," Final Report, K.S. Matlack, W.K. Kot, and I.L. Pegg, VSL-01R10N0-1, Rev. 1, Vitreous State Laboratory, The Catholic University of America, Washington, DC, $2 / 25 / 01$.

[8] "DuraMelter 100 HLW Simulant Validation Tests with C-106/AY-102 Feeds," Final Report, K.S. Matlack, W. Gong and I.L. Pegg, VSL-05R5710-1, Rev. 0, Vitreous State Laboratory, The Catholic University of America, Washington, DC, 6/2/05.

[9] "Integrated DM1200 Melter Testing of HLW C-106/AY-102 Composition Using Bubblers," Final Report, K.S. Matlack, W. Gong, T. Bardakci, N. D’Angelo, W. Kot and I.L. Pegg, VSL-03R3800-1, Rev. 0, Vitreous State Laboratory, The Catholic University of America, Washington, DC, 9/15/03. 
[10] "Integrated DM1200 Melter Testing of HLW C-104/AY-101 Compositions Using Bubblers," Final Report, K.S. Matlack, W. Gong, T. Bardakci, N. D’Angelo, W. Kot and I.L. Pegg, VSL-03R3800-3, Rev. 0, Vitreous State Laboratory, The Catholic University of America, Washington, DC, 11/24/03.

[11] "DM1200 Tests with AZ-101 HLW Simulants," Final Report, K.S. Matlack, W. Gong, T. Bardakci, N. D'Angelo, W.K. Kot, and I.L. Pegg, VSL-03R3800-4, Rev. 0, Vitreous State Laboratory, The Catholic University of America, Washington, DC, 2/17/04.

[12] "Start-Up and Commissioning Tests on the DM1200 HLW Pilot Melter System Using AZ-101 Waste Simulants," Final Report, K.S. Matlack, M. Brandys, and I.L. Pegg, VSL01R0100-2, Rev. 1, Vitreous State Laboratory, The Catholic University of America, Washington, DC, 10/31/01.

[13] "Tests on the DuraMelter 1200 HLW Pilot Melter System Using AZ-101 HLW Simulants," Final Report, K.S. Matlack, W.K. Kot, T. Bardakci, T.R. Schatz, W. Gong, and I.L. Pegg, VSL-02R0100-2, Rev. 0, Vitreous State Laboratory, The Catholic University of America, Washington, DC, 6/11/02.

[14] "Integrated DM1200 Melter Testing of HLW AZ-102 Compositions Using Bubblers," Final Report, K.S. Matlack, W. Gong, T. Bardakci, N. D’Angelo, W. Kot and I.L. Pegg, VSL-03R3800-2, Rev. 0, Vitreous State Laboratory, The Catholic University of America, Washington, DC, 9/24/03.

[15] "Integrated DM1200 Melter Testing of Redox Effects Using HLW AZ-101 and C-106/AY-102 Simulants," Final Report, K.S. Matlack, W. Gong, T. Bardakci, N. D’Angelo, W. Lutze, P. M. Bizot, R. A. Callow, M. Brandys, W.K. Kot, and I.L. Pegg, VSL-04R4800-1, Rev. 0, Vitreous State Laboratory, The Catholic University of America, Washington, DC, 5/6/04.

[16] "Integrated DM1200 Melter Testing of Bubbler Configurations Using HLW AZ-101 Simulants," Final Report, K.S. Matlack, W. Gong, T. Bardakci, N. D’Angelo, W. Lutze, R. A. Callow, M. Brandys, W.K. Kot, and I.L. Pegg, VSL-04R4800-4, Rev. 0, Vitreous State Laboratory, The Catholic University of America, Washington, DC, 10/5/04.

[17] "Integrated DM1200 Melter Testing Using AZ-102 and C-106/AY-102 HLW Simulants: HLW Simulant Verification," Final Report, K.S. Matlack, W. Gong, T. Bardakci, N. D’Angelo, M. Brandys, W.K. Kot, and I.L. Pegg, VSL-05T5800-1, Rev. 0, Vitreous State Laboratory, The Catholic University of America, Washington, DC, 6/27/05.

[18] "Technetium/Cesium Volatility in DM100 Tests Using HLW AZ-102 and LAW SubEnvelope A1 Simulants," Final Report, K.S. Matlack, W.K. Kot, and I.L. Pegg, VSL04R4710-1, Rev. 0, Vitreous State Laboratory, The Catholic University of America, Washington, DC, 9/28/04. 
[19] "Quality Assurance Project Plan for RPP-WTP Support Activities Conducted by VSL," Vitreous State Laboratory, QAPP Rev. 7, Vitreous State Laboratory, The Catholic University of America, Washington, DC, 8/13/04.

[20] "Master List of Controlled VSL Manuals and Standard Operating Procedures in Use," QA-MLCP, Rev. 14, Vitreous State Laboratory, The Catholic University of America, Washington, DC, 2/8/05.

[21] "Round Robin Testing of a Reference Glass for Low-Activity Waste Forms," W.L. Ebert and S.F. Wolf, Department of Energy report ANL-99/22, Argonne National Laboratory, Argonne, IL, 1999.

[22] "Effects of $\mathrm{Al}_{2} \mathrm{O}_{3}, \mathrm{~B}_{2} \mathrm{O}_{3}, \mathrm{Na}_{2} \mathrm{O}$, and $\mathrm{SiO}_{2}$ on Nepheline Formation in Borosilicate Glasses: Chemical and Physical Correlations", H. Li, P. Hrma, J.D. Vienna, M. Qian, Y. Su, and D.E. Smith, J. Non-Crystalline Solids, 331, 202 (2003).

[23] "Frit Development Efforts for Sludge Batch 4", D.K. Peeler and T.E. Edwards, WSRCRP-2006-00002, SRNL, Aiken, SC.

[24] "DuraMelter 100 HLW Simulant Validation Tests with C-106/AY-102 Feeds," Final Report, K.S. Matlack, W. Gong, and I.L. Pegg, Final Report, VSL-05R5710-1, Rev 0, Vitreous State Laboratory, The Catholic University of America, Washington, DC, 6/2/05.

[25] "Physical and Rheological Properties of Waste Simulants and Melter Feeds for RPPWTP HLW Vitrification,” Final Report, K. Kot, H. Gan, and I.L. Pegg, VSL-00R2520-1, Rev. 0, Vitreous State Laboratory, The Catholic University of America, Washington, DC, 10/31/01.

[29] "New Delisting Limits for Arsenic and Chrome," D. Blumenkranz, e-mail message to J. Westsik, CCN 069211, River Protection Project, Waste Treatment Plant, Richland, WA. 9/15/03.

[30] "Data Quality Objectives Process in Support of LDR/Delisting at the WTP," J. Cook and D. Blumenkranz, 24590-WTP-RPT-ENV-01-012, Rev. 2, River Protection Project, Waste Treatment Plant, Richland, WA, 3/26/03.

[31] "High-Temperature Melter Tests for Vitrification of BNFL Nuclear Wastes," K.S. Matlack, I.S. Muller, C. Ahearn, R.K. Mohr, and I.L. Pegg, Final Report to BNFL, plc, Vitreous State Laboratory, The Catholic University of America, Washington, DC, May 1998.

[32] "Vitrification," I.L. Pegg and I. Joseph, Chapter 4.2 in "Hazardous and Radioactive Waste Treatment Technologies Handbook,” Ed. C.H. Oh, CRC Press, LLC, 2001. 
[33] "Vitrification Testing for Fernald CRU4 Silo Wastes," S.S. Fu, K.S. Matlack, R.K. Mohr, C. Paul, I.L. Pegg, and P.B. Macedo, Final Report, Vitreous State Laboratory, The Catholic University of America, Washington, DC, 9/27/96.

[34] "Small Scale Melter Testing to Assess Impact of Higher Temperature Melter Operations," K.S. Matlack, W. Gong, and I.L. Pegg, Final Report to Dept. of Energy, Office of River Protection, VSL-04R4980-1, Rev. 0, Vitreous State Laboratory, The Catholic University of America, Washington, DC, 2/13/04. 
Table 2.1. Oxide Compositions of Limiting Waste Streams.

\begin{tabular}{|c|c|c|c|c|c|c|c|c|}
\hline \multirow{2}{*}{$\begin{array}{c}\text { Waste } \\
\text { Oxides }\end{array}$} & \multicolumn{2}{|c|}{ Bi Limited } & \multicolumn{2}{|c|}{ Cr Limited } & \multicolumn{2}{|c|}{ Al Limited } & \multicolumn{2}{|c|}{$\mathrm{Al}$ and Na Limited } \\
\hline & Actual & Non-Rad. & Actual & Non-Rad. & Actual & Non-Rad. & Actual & Non-Rad \\
\hline $\mathrm{Al}_{2} \mathrm{O}_{3}$ & $22.45 \%$ & $23.32 \%$ & $25.53 \%$ & $27.64 \%$ & $49.21 \%$ & $53.27 \%$ & $43.30 \%$ & $45.40 \%$ \\
\hline $\mathrm{B}_{2} \mathrm{O}_{3}$ & $0.58 \%$ & $0.60 \%$ & $0.53 \%$ & $0.57 \%$ & $0.39 \%$ & $0.42 \%$ & $0.74 \%$ & $0.78 \%$ \\
\hline $\mathrm{CaO}$ & $1.61 \%$ & $1.67 \%$ & $2.47 \%$ & $2.67 \%$ & $2.21 \%$ & $2.39 \%$ & $1.47 \%$ & $1.54 \%$ \\
\hline $\mathrm{Fe}_{2} \mathrm{O}_{3}$ & $13.40 \%$ & $13.92 \%$ & $13.13 \%$ & $14.21 \%$ & $12.11 \%$ & $13.11 \%$ & $5.71 \%$ & $5.99 \%$ \\
\hline $\mathrm{Li}_{2} \mathrm{O}$ & $0.31 \%$ & $0.32 \%$ & $0.36 \%$ & $0.39 \%$ & $0.35 \%$ & $0.38 \%$ & $0.15 \%$ & $0.16 \%$ \\
\hline $\mathrm{MgO}$ & $0.82 \%$ & $0.85 \%$ & $0.16 \%$ & $0.17 \%$ & $0.24 \%$ & $0.26 \%$ & $0.44 \%$ & $0.46 \%$ \\
\hline $\mathrm{Na}_{2} \mathrm{O}$ & $12.97 \%$ & $13.47 \%$ & $20.09 \%$ & $21.75 \%$ & $7.35 \%$ & $7.96 \%$ & $25.79 \%$ & $27.04 \%$ \\
\hline $\mathrm{SiO}_{2}$ & $12.04 \%$ & $12.51 \%$ & $10.56 \%$ & $11.43 \%$ & $10.05 \%$ & $10.88 \%$ & $6.22 \%$ & $6.52 \%$ \\
\hline $\mathrm{TiO}_{2}$ & $0.30 \%$ & $0.31 \%$ & $0.01 \%$ & $0.01 \%$ & $0.02 \%$ & $0.02 \%$ & $0.35 \%$ & $0.37 \%$ \\
\hline $\mathrm{ZnO}$ & $0.31 \%$ & $0.32 \%$ & $0.25 \%$ & $0.27 \%$ & $0.17 \%$ & $0.18 \%$ & $0.36 \%$ & $0.38 \%$ \\
\hline $\mathrm{ZrO}_{2}$ & $0.40 \%$ & $0.42 \%$ & $0.11 \%$ & $0.12 \%$ & $0.81 \%$ & $0.88 \%$ & $0.25 \%$ & $0.26 \%$ \\
\hline $\mathrm{SO}_{3}$ & $0.91 \%$ & $0.95 \%$ & $1.52 \%$ & $1.65 \%$ & $0.41 \%$ & $0.44 \%$ & $0.44 \%$ & $0.46 \%$ \\
\hline $\mathrm{Bi}_{2} \mathrm{O}_{3}$ & $12.91 \%$ & $13.41 \%$ & $7.29 \%$ & $7.89 \%$ & $2.35 \%$ & $2.54 \%$ & $2.35 \%$ & $2.46 \%$ \\
\hline $\mathrm{ThO}_{2}$ & $0.25 \%$ & Omitted & $0.04 \%$ & Omitted & $0.37 \%$ & Omitted & $0.04 \%$ & Omitted \\
\hline $\mathrm{Cr}_{2} \mathrm{O}_{3}$ & $1.00 \%$ & $1.04 \%$ & $3.07 \%$ & $3.32 \%$ & $1.07 \%$ & $1.16 \%$ & $1.44 \%$ & $1.51 \%$ \\
\hline $\mathrm{K}_{2} \mathrm{O}$ & $0.89 \%$ & $0.92 \%$ & $0.37 \%$ & $0.40 \%$ & $0.29 \%$ & $0.31 \%$ & $1.34 \%$ & $1.40 \%$ \\
\hline $\mathrm{U}_{3} \mathrm{O}_{8}$ & $3.48 \%$ & Omitted & $7.59 \%$ & Omitted & $7.25 \%$ & Omitted & $4.58 \%$ & Omitted \\
\hline $\mathrm{BaO}$ & $0.02 \%$ & $0.02 \%$ & $0.03 \%$ & $0.03 \%$ & $0.11 \%$ & $0.12 \%$ & $0.06 \%$ & $0.06 \%$ \\
\hline $\mathrm{CdO}$ & $0.00 \%$ & $0.00 \%$ & $0.01 \%$ & $0.01 \%$ & $0.05 \%$ & $0.05 \%$ & $0.02 \%$ & $0.02 \%$ \\
\hline $\mathrm{NiO}$ & $3.71 \%$ & $3.85 \%$ & $1.06 \%$ & $1.15 \%$ & $0.82 \%$ & $0.89 \%$ & $0.20 \%$ & $0.21 \%$ \\
\hline $\mathrm{PbO}$ & $0.48 \%$ & $0.50 \%$ & $0.48 \%$ & $0.52 \%$ & $0.84 \%$ & $0.91 \%$ & $0.18 \%$ & $0.19 \%$ \\
\hline $\mathrm{P}_{2} \mathrm{O}_{5}$ & $9.60 \%$ & $9.97 \%$ & $3.34 \%$ & $3.62 \%$ & $2.16 \%$ & $2.34 \%$ & $4.10 \%$ & $4.30 \%$ \\
\hline F- & $1.58 \%$ & $1.64 \%$ & $2.00 \%$ & $2.17 \%$ & $1.37 \%$ & $1.48 \%$ & $0.46 \%$ & $0.48 \%$ \\
\hline Total & $100.00 \%$ & $100.0 \%$ & $100.00 \%$ & $100.0 \%$ & $100.00 \%$ & $100.0 \%$ & $100.00 \%$ & $100.0 \%$ \\
\hline
\end{tabular}


The Catholic University of America

Table 2.2. Compositions of the Bi-Limited Waste (Oxide Basis) and the HLW Waste Simulant to Produce $100 \mathrm{~kg}$ of Waste Oxides (20 wt\% suspended solids).

\begin{tabular}{|c|c|c|c|}
\hline \multicolumn{2}{|c|}{ Bi-Limited Waste Composition } & \multicolumn{2}{|c|}{ Bi-Limited HLW Waste Simulant } \\
\hline Waste Oxide & Wt\% & Starting Materials & Target Weight (kg) $^{1}$ \\
\hline $\mathrm{Al}_{2} \mathrm{O}_{3}$ & $22.45 \%$ & $\mathrm{Al}_{2} \mathrm{O}_{3}$ & 22.677 \\
\hline $\mathrm{B}_{2} \mathrm{O}_{3}$ & $0.58 \%$ & $\mathrm{H}_{3} \mathrm{BO}_{3}$ & 1.041 \\
\hline $\mathrm{CaO}$ & $1.61 \%$ & $\mathrm{CaO}$ & 1.643 \\
\hline $\mathrm{Fe}_{2} \mathrm{O}_{3}$ & $13.40 \%$ & $\mathrm{Fe}(\mathrm{OH})_{3}(13 \%$ Slurry $)$ & 26.752 \\
\hline $\mathrm{Li}_{2} \mathrm{O}$ & $0.31 \%$ & $\mathrm{Li}_{2} \mathrm{CO}_{3}$ & 0.786 \\
\hline $\mathrm{MgO}$ & $0.82 \%$ & $\mathrm{MgO}$ & 0.863 \\
\hline $\mathrm{Na}_{2} \mathrm{O}$ & $12.97 \%$ & $\mathrm{NaOH}$ & 11.108 \\
\hline $\mathrm{SiO}_{2}$ & $12.04 \%$ & $\mathrm{SiO}_{2}$ & 12.162 \\
\hline $\mathrm{TiO}_{2}$ & $0.30 \%$ & $\mathrm{TiO}_{2}$ & 0.303 \\
\hline $\mathrm{ZnO}$ & $0.31 \%$ & $\mathrm{ZnO}$ & 0.313 \\
\hline $\mathrm{ZrO}_{2}$ & $0.40 \%$ & $\mathrm{Zr}(\mathrm{OH})_{4} \cdot x \mathrm{H}_{2} \mathrm{O}$ & 1.034 \\
\hline $\mathrm{SO}_{3}$ & $0.91 \%$ & $\mathrm{Na}_{2} \mathrm{SO}_{4}$ & 1.632 \\
\hline $\mathrm{Bi}_{2} \mathrm{O}_{3}$ & $12.91 \%$ & $\mathrm{Bi}_{2} \mathrm{O}_{3}$ & 13.040 \\
\hline $\mathrm{ThO}_{2}$ & $0.25 \%$ & \multicolumn{2}{|c|}{ Omitted } \\
\hline $\mathrm{Cr}_{2} \mathrm{O}_{3}$ & $1.00 \%$ & $\mathrm{Cr}_{2} \mathrm{O}_{3} \cdot 1.5 \mathrm{H}_{2} \mathrm{O}$ & 1.190 \\
\hline $\mathrm{K}_{2} \mathrm{O}$ & $0.89 \%$ & $\mathrm{KNO}_{3}$ & 1.940 \\
\hline $\mathrm{U}_{3} \mathrm{O}_{8}$ & $3.48 \%$ & \multicolumn{2}{|c|}{ Omitted } \\
\hline $\mathrm{BaO}$ & $0.02 \%$ & $\mathrm{BaCO}_{3}$ & 0.026 \\
\hline $\mathrm{CdO}$ & $0.00 \%$ & $\mathrm{CdO}$ & 0.000 \\
\hline $\mathrm{NiO}$ & $3.71 \%$ & $\mathrm{Ni}(\mathrm{OH})_{2}$ & 4.771 \\
\hline $\mathrm{PbO}$ & $0.48 \%$ & $\mathrm{PbO}$ & 0.485 \\
\hline $\mathrm{P}_{2} \mathrm{O}_{5}$ & $9.60 \%$ & $\mathrm{FePO}_{4} \cdot x \mathrm{H}_{2} \mathrm{O}$ & 25.501 \\
\hline $\mathrm{F}$ & $1.58 \%$ & $\mathrm{NaF}$ & 3.510 \\
\hline Carbonate & $1.20^{2}$ & $\mathrm{Na}_{2} \mathrm{CO}_{3}$ & 1.011 \\
\hline Nitrite & 0.50 & $\mathrm{NaNO}_{2}$ & 0.769 \\
\hline Nitrate & 2.00 & $\mathrm{NaNO}_{3}$ & 1.141 \\
\hline Organic Carbon & 0.05 & $\mathrm{H}_{2} \mathrm{C}_{2} \mathrm{O}_{4} \cdot 2 \mathrm{H}_{2} \mathrm{O}$ & 0.264 \\
\hline- & - & Water & 353.500 \\
\hline TOTAL & $100.0 \%$ & TOTAL & 487.463 \\
\hline
\end{tabular}

${ }^{1}$ Target weights adjusted for assay information of starting materials

${ }^{2}$-Unit for volatile components is $\mathrm{g} / 100 \mathrm{~g}$ of waste oxide

— Empty data field 
Table 2.3. Compositions of the Cr-Limited Waste (Oxide Basis) and the HLW Waste Simulant to Produce $100 \mathrm{~kg}$ of Waste Oxides (20 wt\% suspended solids).

\begin{tabular}{|c|c|c|c|}
\hline \multicolumn{2}{|c|}{ Cr-Limited Waste Composition } & \multicolumn{2}{|c|}{ Cr-Limited HLW Waste Simulant } \\
\hline Waste Oxide & $\mathrm{Wt} \%$ & Starting Materials & Target Weight (kg) \\
\hline $\mathrm{Al}_{2} \mathrm{O}_{3}$ & $25.53 \%$ & $\mathrm{Al}_{2} \mathrm{O}_{3}$ & 25.788 \\
\hline $\mathrm{B}_{2} \mathrm{O}_{3}$ & $0.53 \%$ & $\mathrm{H}_{3} \mathrm{BO}_{3}$ & 0.951 \\
\hline $\mathrm{CaO}$ & $2.47 \%$ & $\mathrm{CaO}$ & 2.520 \\
\hline $\mathrm{Fe}_{2} \mathrm{O}_{3}$ & $13.13 \%$ & $\mathrm{Fe}(\mathrm{OH})_{3}(13 \%$ Slurry $)$ & 96.476 \\
\hline $\mathrm{Li}_{2} \mathrm{O}$ & $0.36 \%$ & $\mathrm{Li}_{2} \mathrm{CO}_{3}$ & 0.913 \\
\hline $\mathrm{MgO}$ & $0.16 \%$ & $\mathrm{MgO}$ & 0.168 \\
\hline $\mathrm{Na}_{2} \mathrm{O}$ & $20.09 \%$ & $\mathrm{NaOH}$ & 18.730 \\
\hline $\mathrm{SiO}_{2}$ & $10.56 \%$ & $\mathrm{SiO}_{2}$ & 10.667 \\
\hline $\mathrm{TiO}_{2}$ & $0.01 \%$ & $\mathrm{TiO}_{2}$ & 0.010 \\
\hline $\mathrm{ZnO}$ & $0.25 \%$ & $\mathrm{ZnO}$ & 0.253 \\
\hline $\mathrm{ZrO}_{2}$ & $0.11 \%$ & $\mathrm{Zr}(\mathrm{OH})_{4} \cdot x \mathrm{H}_{2} \mathrm{O}$ & 0.284 \\
\hline $\mathrm{SO}_{3}$ & $1.52 \%$ & $\mathrm{Na}_{2} \mathrm{SO}_{4}$ & 2.726 \\
\hline $\mathrm{Bi}_{2} \mathrm{O}_{3}$ & $7.29 \%$ & $\mathrm{Bi}_{2} \mathrm{O}_{3}$ & 7.364 \\
\hline $\mathrm{ThO}_{2}$ & $0.04 \%$ & \multicolumn{2}{|c|}{ Omitted } \\
\hline $\mathrm{Cr}_{2} \mathrm{O}_{3}$ & $3.07 \%$ & $\mathrm{Cr}_{2} \mathrm{O}_{3} \cdot 1.5 \mathrm{H}_{2} \mathrm{O}$ & 3.652 \\
\hline $\mathrm{K}_{2} \mathrm{O}$ & $0.37 \%$ & $\mathrm{KNO}_{3}$ & 0.806 \\
\hline $\mathrm{U}_{3} \mathrm{O}_{8}$ & $7.59 \%$ & \multicolumn{2}{|c|}{ Omitted } \\
\hline $\mathrm{BaO}$ & $0.03 \%$ & $\mathrm{BaCO}_{3}$ & 0.039 \\
\hline $\mathrm{CdO}$ & $0.01 \%$ & $\mathrm{CdO}$ & 0.010 \\
\hline $\mathrm{NiO}$ & $1.06 \%$ & $\mathrm{Ni}(\mathrm{OH})_{2}$ & 1.363 \\
\hline $\mathrm{PbO}$ & $0.48 \%$ & $\mathrm{PbO}$ & 0.485 \\
\hline $\mathrm{P}_{2} \mathrm{O}_{5}$ & $3.34 \%$ & $\mathrm{FePO}_{4} \cdot x \mathrm{H}_{2} \mathrm{O}$ & 8.872 \\
\hline $\mathrm{F}$ & $2.00 \%$ & $\mathrm{NaF}$ & 4.443 \\
\hline Carbonate & $1.20^{2}$ & $\mathrm{Na}_{2} \mathrm{CO}_{3}$ & 0.826 \\
\hline Nitrite & 0.50 & $\mathrm{NaNO}_{2}$ & 0.769 \\
\hline Nitrate & 2.00 & $\mathrm{NaNO}_{3}$ & 2.085 \\
\hline Organic Carbon & 0.05 & $\mathrm{H}_{2} \mathrm{C}_{2} \mathrm{O}_{4} \cdot 2 \mathrm{H}_{2} \mathrm{O}$ & 0.264 \\
\hline- & - & Water & 293.300 \\
\hline- & - & - & - \\
\hline TOTAL & $100.0 \%$ & TOTAL & 483.765 \\
\hline
\end{tabular}

${ }^{1}$ Target weights adjusted for assay information of starting materials

${ }^{2}$-Unit for volatile components is $\mathrm{g} / 100 \mathrm{~g}$ of waste oxide

— Empty data field 
Table 2.4. Compositions of the Al-Limited Waste (Oxide Basis) and the HLW Waste Simulant to Produce $100 \mathrm{~kg}$ of Waste Oxides (20 wt\% suspended solids).

\begin{tabular}{|c|c|c|c|}
\hline \multicolumn{2}{|c|}{ Al-Limited Waste Composition } & \multicolumn{2}{|c|}{ Al-Limited HLW Waste Simulant } \\
\hline Waste Oxide & Wt\% & Starting Materials & Target Weight (kg) $^{1}$ \\
\hline $\mathrm{Al}_{2} \mathrm{O}_{3}$ & $49.21 \%$ & $\mathrm{Al}_{2} \mathrm{O}_{3}$ & 499.707 \\
\hline $\mathrm{B}_{2} \mathrm{O}_{3}$ & $0.39 \%$ & $\mathrm{H}_{3} \mathrm{BO}_{3}$ & 0.700 \\
\hline $\mathrm{CaO}$ & $2.21 \%$ & $\mathrm{CaO}$ & 2.255 \\
\hline $\mathrm{Fe}_{2} \mathrm{O}_{3}$ & $12.11 \%$ & $\mathrm{Fe}(\mathrm{OH})_{3}(13 \%$ Slurry $)$ & 99.643 \\
\hline $\mathrm{Li}_{2} \mathrm{O}$ & $0.35 \%$ & $\mathrm{Li}_{2} \mathrm{CO}_{3}$ & 0.888 \\
\hline $\mathrm{MgO}$ & $0.24 \%$ & $\mathrm{MgO}$ & 0.253 \\
\hline $\mathrm{Na}_{2} \mathrm{O}$ & $7.35 \%$ & $\mathrm{NaOH}$ & 4.235 \\
\hline $\mathrm{SiO}_{2}$ & $10.05 \%$ & $\mathrm{SiO}_{2}$ & 10.152 \\
\hline $\mathrm{TiO}_{2}$ & $0.02 \%$ & $\mathrm{TiO}_{2}$ & 0.020 \\
\hline $\mathrm{ZnO}$ & $0.17 \%$ & $\mathrm{ZnO}$ & 0.172 \\
\hline $\mathrm{ZrO}_{2}$ & $0.81 \%$ & $\mathrm{Zr}(\mathrm{OH})_{4} \cdot x \mathrm{H}_{2} \mathrm{O}$ & 2.093 \\
\hline $\mathrm{SO}_{3}$ & $0.41 \%$ & $\mathrm{Na}_{2} \mathrm{SO}_{4}$ & 0.735 \\
\hline $\mathrm{Bi}_{2} \mathrm{O}_{3}$ & $2.35 \%$ & $\mathrm{Bi}_{2} \mathrm{O}_{3}$ & 2.374 \\
\hline $\mathrm{ThO}_{2}$ & $0.37 \%$ & \multicolumn{2}{|c|}{ Omitted } \\
\hline $\mathrm{Cr}_{2} \mathrm{O}_{3}$ & $1.07 \%$ & $\mathrm{Cr}_{2} \mathrm{O}_{3} \cdot 1.5 \mathrm{H}_{2} \mathrm{O}$ & 1.273 \\
\hline $\mathrm{K}_{2} \mathrm{O}$ & $0.29 \%$ & $\mathrm{KNO}_{3}$ & 0.632 \\
\hline $\mathrm{U}_{3} \mathrm{O}_{8}$ & $7.25 \%$ & \multicolumn{2}{|c|}{ Omitted } \\
\hline $\mathrm{BaO}$ & $0.11 \%$ & $\mathrm{BaCO}_{3}$ & 0.143 \\
\hline $\mathrm{CdO}$ & $0.05 \%$ & $\mathrm{CdO}$ & 0.051 \\
\hline $\mathrm{NiO}$ & $0.82 \%$ & $\mathrm{Ni}(\mathrm{OH})_{2}$ & 1.055 \\
\hline $\mathrm{PbO}$ & $0.84 \%$ & $\mathrm{PbO}$ & 0.848 \\
\hline $\mathrm{P}_{2} \mathrm{O}_{5}$ & $2.16 \%$ & $\mathrm{FePO}_{4} \cdot x \mathrm{H}_{2} \mathrm{O}$ & 5.738 \\
\hline $\mathrm{F}$ & $1.37 \%$ & $\mathrm{NaF}$ & 3.044 \\
\hline Carbonate & $1.20^{2}$ & $\mathrm{Na}_{2} \mathrm{CO}_{3}$ & 0.806 \\
\hline Nitrite & 0.50 & $\mathrm{NaNO}_{2}$ & 0.769 \\
\hline Nitrate & 2.00 & $\mathrm{NaNO}_{3}$ & 2.230 \\
\hline Organic Carbon & 0.05 & $\mathrm{H}_{2} \mathrm{C}_{2} \mathrm{O}_{4} \cdot 2 \mathrm{H}_{2} \mathrm{O}$ & 0.264 \\
\hline- & - & Water & 279.400 \\
\hline TOTAL & $100.0 \%$ & TOTAL & 469.478 \\
\hline
\end{tabular}

${ }^{1}$ Target weights adjusted for assay information of starting materials

${ }^{2}$-Unit for volatile components is $\mathrm{g} / 100 \mathrm{~g}$ of waste oxide

— Empty data field 
Table 2.5. Compositions of the Al- and Na-Limited Waste (Oxide Basis) and the HLW Waste Simulant to Produce $100 \mathrm{~kg}$ of Waste Oxides (20 wt\% suspended solids).

\begin{tabular}{|c|c|c|c|}
\hline \multicolumn{2}{|c|}{ Al- and Na-Limited Waste Composition } & \multicolumn{2}{|c|}{ Al- and Na-Limited HLW Waste Simulant } \\
\hline Waste Oxide & Wt\% & Starting Materials & 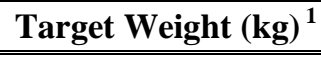 \\
\hline $\mathrm{Al}_{2} \mathrm{O}_{3}$ & $43.30 \%$ & $\mathrm{Al}_{2} \mathrm{O}_{3}$ & 43.737 \\
\hline $\mathrm{B}_{2} \mathrm{O}_{3}$ & $0.74 \%$ & $\mathrm{H}_{3} \mathrm{BO}_{3}$ & 1.328 \\
\hline $\mathrm{CaO}$ & $1.47 \%$ & $\mathrm{CaO}$ & 1.500 \\
\hline $\mathrm{Fe}_{2} \mathrm{O}_{3}$ & $5.71 \%$ & $\mathrm{Fe}(\mathrm{OH})_{3}(13 \%$ Slurry $)$ & 11.292 \\
\hline $\mathrm{Li}_{2} \mathrm{O}$ & $0.15 \%$ & $\mathrm{Li}_{2} \mathrm{CO}_{3}$ & 0.380 \\
\hline $\mathrm{MgO}$ & $0.44 \%$ & $\mathrm{MgO}$ & 0.463 \\
\hline $\mathrm{Na}_{2} \mathrm{O}$ & $25.79 \%$ & $\mathrm{NaOH}$ & 31.057 \\
\hline $\mathrm{SiO}_{2}$ & $6.22 \%$ & $\mathrm{SiO}_{2}$ & 6.283 \\
\hline $\mathrm{TiO}_{2}$ & $0.35 \%$ & $\mathrm{TiO}_{2}$ & 0.354 \\
\hline $\mathrm{ZnO}$ & $0.36 \%$ & $\mathrm{ZnO}$ & 0.364 \\
\hline $\mathrm{ZrO}_{2}$ & $0.25 \%$ & $\mathrm{Zr}(\mathrm{OH})_{4} \cdot x \mathrm{H}_{2} \mathrm{O}$ & 0.646 \\
\hline $\mathrm{SO}_{3}$ & $0.44 \%$ & $\mathrm{Na}_{2} \mathrm{SO}_{4}$ & 0.789 \\
\hline $\mathrm{Bi}_{2} \mathrm{O}_{3}$ & $2.35 \%$ & $\mathrm{Bi}_{2} \mathrm{O}_{3}$ & 2.374 \\
\hline $\mathrm{ThO}_{2}$ & $0.04 \%$ & \multicolumn{2}{|c|}{ Omitted } \\
\hline $\mathrm{Cr}_{2} \mathrm{O}_{3}$ & $1.44 \%$ & $\mathrm{Cr}_{2} \mathrm{O}_{3} \cdot 1.5 \mathrm{H}_{2} \mathrm{O}$ & 1.713 \\
\hline $\mathrm{K}_{2} \mathrm{O}$ & $1.34 \%$ & $\mathrm{KNO}_{3}$ & 2.921 \\
\hline $\mathrm{U}_{3} \mathrm{O}_{8}$ & $4.58 \%$ & \multicolumn{2}{|c|}{ Omitted } \\
\hline $\mathrm{BaO}$ & $0.06 \%$ & $\mathrm{BaCO}_{3}$ & 0.078 \\
\hline $\mathrm{CdO}$ & $0.02 \%$ & $\mathrm{CdO}$ & 0.020 \\
\hline $\mathrm{NiO}$ & $0.20 \%$ & $\mathrm{Ni}(\mathrm{OH})_{2}$ & 0.257 \\
\hline $\mathrm{PbO}$ & $0.18 \%$ & $\mathrm{PbO}$ & 0.182 \\
\hline $\mathrm{P}_{2} \mathrm{O}_{5}$ & $4.10 \%$ & $\mathrm{FePO}_{4} \cdot x \mathrm{H}_{2} \mathrm{O}$ & 10.891 \\
\hline $\mathrm{F}$ & $0.46 \%$ & $\mathrm{NaF}$ & 1.022 \\
\hline Carbonate & $1.20^{2}$ & $\mathrm{Na}_{2} \mathrm{CO}_{3}$ & 1.554 \\
\hline Nitrite & 0.50 & $\mathrm{NaNO}_{2}$ & 0.769 \\
\hline Nitrate & 2.00 & $\mathrm{NaNO}_{3}$ & 0.325 \\
\hline Organic Carbon & 0.05 & $\mathrm{H}_{2} \mathrm{C}_{2} \mathrm{O}_{4} \cdot 2 \mathrm{H}_{2} \mathrm{O}$ & 0.264 \\
\hline- & - & Water & 381.400 \\
\hline TOTAL & $100.0 \%$ & TOTAL & 501.964 \\
\hline
\end{tabular}

${ }^{1}$ Target weights adjusted for assay information of starting materials

${ }^{2}$-Unit for volatile components is $\mathrm{g} / 100 \mathrm{~g}$ of waste oxide

— Empty data field 
Table 3.1. Target Composition and XRF Analysis of Glasses Formulated for Bi-Limited Waste.

\begin{tabular}{|c|c|c|c|c|c|c|c|c|c|c|c|c|c|c|c|c|c|c|}
\hline \multirow{2}{*}{ - } & \multirow{2}{*}{$\begin{array}{l}\text { Bi Limited } \\
\text { Waste }\end{array}$} & \multirow{2}{*}{$\begin{array}{l}\text { Bi Limited Waste } \\
\text { (Non Rad)\# }\end{array}$} & \multicolumn{2}{|c|}{ HLW-E-Bi-1 } & \multicolumn{2}{|c|}{ HLW-E-Bi-2 } & \multicolumn{2}{|c|}{ HLW-E-Bi-3 } & \multicolumn{2}{|c|}{ HLW-E-Bi-4 } & \multicolumn{2}{|c|}{ HLW-E-Bi-5 } & \multicolumn{2}{|c|}{ HLW-E-Bi-6 } & \multicolumn{2}{|c|}{ HLW-E-Bi-7 } & \multicolumn{2}{|c|}{ HLW-E-Bi-8 } \\
\hline & & & Target & XRF & Target & XRF & Target & XRF & Target & $\mathrm{XRF}$ & Target & $\mathrm{XRF}$ & Target & $\mathrm{XRF}$ & Target & XRF & Target & $\mathrm{XRF}$ \\
\hline $\mathrm{Al}_{2} \mathrm{O}_{3}$ & 22.45 & 23.31 & 9.32 & 9.60 & 9.32 & 9.14 & 9.32 & 9.68 & 9.32 & 9.49 & 10.49 & 10.54 & 11.66 & 11.85 & 12.82 & 12.89 & 10.49 & 10.50 \\
\hline $\mathrm{B}_{2} \mathrm{O}_{3}$ & 0.58 & 0.60 & 13.74 & 13.74 & 12.74 & 12.74 & 13.74 & 13.74 & 12.74 & 12.74 & 12.27 & 12.27 & 11.30 & 11.30 & 10.33 & 10.33 & 14.27 & 14.27 \\
\hline $\mathrm{BaO}$ & 0.02 & 0.02 & 0.01 & 0.00 & 0.01 & 0.00 & 0.01 & 0.00 & 0.01 & 0.01 & 0.01 & 0.01 & 0.01 & 0.02 & 0.01 & 0.02 & 0.01 & 0.01 \\
\hline $\mathrm{Bi}_{2} \mathrm{O}_{3} *$ & 12.91 & 13.41 & 5.36 & 5.60 & 5.36 & 5.78 & 5.36 & 5.41 & 5.36 & 5.63 & 6.03 & 6.12 & 6.71 & 6.83 & 7.38 & 7.61 & 6.03 & 6.39 \\
\hline $\mathrm{CaO}$ & 1.61 & 1.67 & 0.67 & 0.70 & 0.67 & 0.72 & 0.67 & 0.71 & 0.67 & 0.72 & 0.75 & 0.80 & 0.84 & 0.57 & 0.92 & 0.94 & 0.75 & 0.80 \\
\hline $\mathrm{Cr}_{2} \mathrm{O}_{3}$ & 1.00 & 1.04 & 0.42 & 0.51 & 0.42 & 0.53 & 0.42 & 0.51 & 0.42 & 0.52 & 0.47 & 0.59 & 0.52 & 0.61 & 0.57 & 0.68 & 0.47 & 0.58 \\
\hline $\mathrm{F}^{*}$ & 1.58 & 1.64 & 0.66 & - & 0.66 & - & 0.66 & - & 0.66 & - & 0.74 & - & 0.82 & - & 0.90 & - & 0.74 & - \\
\hline $\mathrm{Fe}_{2} \mathrm{O}_{3}$ & 13.40 & 13.92 & 5.57 & 5.50 & 5.57 & 5.57 & 5.57 & 5.30 & 5.57 & 5.50 & 6.26 & 6.02 & 6.96 & 6.69 & 7.66 & 7.40 & 6.26 & 6.20 \\
\hline $\mathrm{K}_{2} \mathrm{O}$ & 0.89 & 0.92 & 0.37 & 0.45 & 0.37 & 0.43 & 0.37 & 0.43 & 0.37 & 0.44 & 0.41 & 0.48 & 0.46 & 0.50 & 0.51 & 0.56 & 0.41 & 0.47 \\
\hline $\mathrm{Li}_{2} \mathrm{O}^{*}$ & 0.31 & 0.32 & 0.13 & 0.13 & 0.13 & 0.13 & 0.13 & 0.13 & 0.13 & 0.13 & 0.14 & 0.14 & 0.16 & 0.16 & 0.18 & 0.18 & 0.14 & 0.14 \\
\hline $\mathrm{MgO}$ & 0.82 & 0.85 & 0.34 & 0.28 & 0.34 & 0.28 & 0.34 & 0.34 & 0.34 & 0.32 & 0.38 & 0.34 & 0.43 & 0.38 & 0.47 & 0.44 & 0.38 & 0.37 \\
\hline $\mathrm{Na}_{2} \mathrm{O}$ & 12.97 & 13.47 & 17.39 & 17.79 & 16.39 & 15.78 & 17.39 & 17.86 & 16.39 & 16.78 & 16.06 & 16.63 & 15.74 & 16.44 & 15.41 & 15.72 & 16.06 & 15.90 \\
\hline $\mathrm{NiO}$ & 3.71 & 3.85 & 1.54 & 1.51 & 1.54 & 1.57 & 1.54 & 1.47 & 1.54 & 1.51 & 1.73 & 1.65 & 1.93 & 1.83 & 2.12 & 1.96 & 1.73 & 1.70 \\
\hline $\mathrm{P}_{2} \mathrm{O}_{5}$ & 9.60 & 9.97 & 3.99 & 4.26 & 3.99 & 4.86 & 3.99 & 4.67 & 3.99 & 4.34 & 4.49 & 4.95 & 4.99 & 5.48 & 5.48 & 6.10 & 4.49 & 5.08 \\
\hline $\mathrm{PbO}$ & 0.48 & 0.50 & 0.20 & 0.19 & 0.20 & 0.20 & 0.20 & 0.18 & 0.20 & 0.19 & 0.23 & 0.20 & 0.25 & 0.24 & 0.28 & 0.26 & 0.23 & 0.22 \\
\hline $\mathrm{SiO}_{2}$ & 12.04 & 12.51 & 39.50 & 38.87 & 39.50 & 39.40 & 38.00 & 37.37 & 39.00 & 38.46 & 37.63 & 37.42 & 36.26 & 35.84 & 33.88 & 33.75 & 36.63 & 36.46 \\
\hline $\mathrm{ThO}_{2}$ & 0.25 & 0.00 & 0.00 & 0.00 & 0.00 & 0.00 & 0.00 & 0.00 & 0.00 & 0.00 & 0.00 & 0.00 & 0.00 & 0.00 & 0.00 & 0.00 & 0.00 & 0.00 \\
\hline $\mathrm{TiO}_{2}$ & 0.30 & 0.31 & 0.12 & 0.16 & 0.12 & 0.15 & 0.12 & 0.15 & 0.12 & 0.16 & 0.14 & 0.17 & 0.16 & 0.18 & 0.17 & 0.21 & 0.14 & 0.17 \\
\hline $\mathrm{SO}_{3}$ & 0.91 & 0.95 & 0.38 & 0.35 & 0.38 & 0.30 & 0.38 & 0.30 & 0.38 & 0.29 & 0.43 & 0.33 & 0.48 & 0.32 & 0.52 & 0.43 & 0.43 & 0.30 \\
\hline $\mathrm{ZnO}$ & 0.31 & 0.32 & 0.13 & 0.15 & 1.13 & 1.18 & 1.13 & 1.11 & 0.63 & 0.65 & 0.14 & 0.16 & 0.16 & 0.18 & 0.18 & 0.20 & 0.14 & 0.17 \\
\hline $\mathrm{ZrO}_{2}$ & 0.40 & 0.42 & 0.17 & 0.15 & 1.17 & 1.14 & 0.67 & 0.60 & 2.17 & 2.02 & 1.19 & 1.08 & 0.21 & 0.20 & 0.23 & 0.21 & 0.19 & 0.19 \\
\hline $\mathrm{U}_{3} \mathrm{O}_{8}$ & 3.48 & 0.00 & 0.00 & 0.00 & 0.00 & 0.00 & 0.00 & 0.00 & 0.00 & 0.00 & 0.00 & 0.00 & 0.00 & 0.00 & 0.00 & 0.00 & 0.00 & 0.00 \\
\hline
\end{tabular}

*In XRF analysis target concentrations are used for $\mathrm{B}_{2} \mathrm{O}_{3}$ and $\mathrm{Li}_{2} \mathrm{O}$; F was not analyzed.

\#The non-radioactive version of the waste is calculated by renormalization of the waste constituents after removing $\mathrm{U}_{3} \mathrm{O}_{8}$ and $\mathrm{ThO}_{2}$.

- Empty data field 
Table 3.1. Target Composition and XRF Analysis of Glasses Formulated for Bi-Limited Waste (continued).

\begin{tabular}{|c|c|c|c|c|c|c|c|c|c|c|c|c|c|c|c|c|c|c|}
\hline \multirow{2}{*}{ - } & \multicolumn{2}{|c|}{ HLW-E-Bi-9 } & \multicolumn{2}{|c|}{ HLW-E-Bi-10 } & \multicolumn{2}{|c|}{ HLW-E-Bi-11 } & \multicolumn{2}{|c|}{ HLW-E-Bi-12 } & \multicolumn{2}{|c|}{ HLW-E-Bi-13 } & \multicolumn{2}{|c|}{ HLW-E-Bi-14 } & \multicolumn{2}{|c|}{ HLW-E-Bi-15 } & \multicolumn{2}{|c|}{ HLW-E-Bi-16 } & \multicolumn{2}{|c|}{ HLW-E-Bi6UTh } \\
\hline & Comp & XRF & Comp & XRF & Comp & XRF & Comp & XRF & Comp & XRF & Comp & XRF & Comp & XRF & Comp & XRF & Comp & XRF \\
\hline $\mathrm{Al}_{2} \mathrm{O}_{3}$ & 10.49 & 10.70 & 10.49 & 10.62 & 6.99 & 7.68 & 13.99 & 13.83 & 12.82 & 12.80 & 15.15 & 15.31 & 12.82 & 12.87 & 11.66 & 11.81 & 11.22 & 11.35 \\
\hline $\mathrm{B}_{2} \mathrm{O}_{3}{ }^{*}$ & 12.27 & 12.27 & 12.27 & 12.27 & 12.68 & 12.68 & 11.36 & 11.36 & 10.33 & 10.33 & 10.39 & 10.39 & 10.33 & 0.00 & 11.30 & 11.30 & 11.29 & 11.29 \\
\hline $\mathrm{BaO}$ & 0.01 & 0.01 & 0.01 & 0.01 & 0.01 & 0.03 & 0.01 & 0.01 & 0.01 & 0.02 & 0.01 & 0.01 & 0.01 & 0.02 & 0.01 & 0.00 & 0.01 & 0.02 \\
\hline $\mathrm{Bi}_{2} \mathrm{O}_{3}$ & 6.03 & 6.01 & 6.03 & 6.17 & 4.02 & 4.34 & 8.05 & 8.17 & 7.38 & 7.41 & 8.72 & 9.18 & 7.38 & 7.92 & 6.71 & 7.35 & 6.45 & 7.03 \\
\hline $\mathrm{CaO}$ & 0.75 & 0.78 & 0.75 & 0.80 & 0.50 & 0.66 & 1.00 & 1.05 & 0.92 & 0.95 & 1.09 & 1.12 & 0.92 & 0.97 & 0.84 & 0.89 & 0.80 & 0.85 \\
\hline $\mathrm{Cr}_{2} \mathrm{O}_{3}$ & 0.47 & 0.56 & 0.47 & 0.57 & 0.31 & 0.40 & 0.62 & 0.71 & 0.57 & 0.67 & 0.68 & 0.57 & 0.57 & 0.75 & 0.52 & 0.67 & 0.50 & 0.63 \\
\hline $\mathrm{F}^{*}$ & 0.74 & - & 0.74 & - & 0.49 & - & 0.98 & - & 0.90 & - & 1.07 & - & 0.90 & - & 0.82 & - & 0.79 & - \\
\hline $\mathrm{Fe}_{2} \mathrm{O}_{3}$ & 6.26 & 5.93 & 6.26 & 6.03 & 4.18 & 4.21 & 8.35 & 7.89 & 7.66 & 7.27 & 9.05 & 7.90 & 7.66 & 7.81 & 6.96 & 7.09 & 6.70 & 6.78 \\
\hline $\mathrm{K}_{2} \mathrm{O}$ & 0.41 & 0.46 & 2.41 & 2.45 & 0.28 & 0.35 & 0.55 & 0.64 & 2.51 & 2.54 & 0.60 & 0.64 & 2.51 & 2.50 & 2.46 & 2.50 & 0.44 & 0.50 \\
\hline $\mathrm{Li}_{2} \mathrm{O}^{*}$ & 0.14 & 0.14 & 0.14 & 0.00 & 0.10 & 0.10 & 0.19 & 0.19 & 0.18 & 0.18 & 0.21 & 0.21 & 2.18 & 2.18 & 0.16 & 0.16 & 0.15 & 0.15 \\
\hline $\mathrm{MgO}$ & 0.38 & 0.38 & 0.38 & 0.34 & 0.26 & 0.24 & 0.51 & 0.46 & 0.47 & 0.43 & 0.55 & 0.52 & 0.47 & 0.42 & 0.43 & 0.36 & 0.41 & 0.38 \\
\hline $\mathrm{Na}_{2} \mathrm{O}$ & 18.06 & 18.66 & 16.06 & 16.00 & 18.04 & 18.15 & 15.08 & 15.38 & 14.91 & 14.49 & 15.76 & 16.73 & 12.91 & 13.18 & 14.74 & 14.87 & 15.48 & 15.13 \\
\hline $\mathrm{NiO}$ & 1.73 & 1.62 & 1.73 & 1.66 & 1.16 & 1.16 & 2.31 & 2.05 & 2.12 & 1.94 & 2.50 & 1.78 & 2.12 & 2.04 & 1.93 & 1.94 & 1.85 & 1.86 \\
\hline $\mathrm{P}_{2} \mathrm{O}_{5}$ & 4.49 & 5.05 & 4.49 & 5.61 & 2.99 & 3.28 & 5.98 & 7.45 & 5.48 & 6.89 & 6.48 & 7.38 & 5.48 & 5.98 & 4.99 & 5.41 & 4.80 & 5.28 \\
\hline $\mathrm{PbO}$ & 0.23 & 0.21 & 0.23 & 0.21 & 0.15 & 0.14 & 0.30 & 0.29 & 0.28 & 0.24 & 0.33 & 0.32 & 0.28 & 0.26 & 0.25 & 0.25 & 0.24 & 0.23 \\
\hline $\mathrm{SiO}_{2}$ & 36.63 & 36.26 & 36.63 & 36.15 & 44.25 & 43.10 & 29.51 & 29.32 & 32.38 & 32.65 & 26.13 & 26.57 & 32.38 & 31.59 & 35.26 & 34.27 & 36.02 & 35.40 \\
\hline $\mathrm{ThO}_{2}$ & 0.00 & 0.00 & 0.00 & 0.00 & 0.00 & 0.00 & 0.00 & 0.00 & 0.00 & 0.00 & 0.00 & 0.00 & 0.00 & 0.00 & 0.00 & 0.00 & 0.12 & 0.13 \\
\hline $\mathrm{TiO}_{2}$ & 0.14 & 0.17 & 0.14 & 0.17 & 0.09 & 0.12 & 0.19 & 0.21 & 0.17 & 0.21 & 0.20 & 0.24 & 0.17 & 0.22 & 0.16 & 0.20 & 0.15 & 0.18 \\
\hline $\mathrm{SO}_{3}$ & 0.43 & 0.37 & 0.43 & 0.38 & 0.29 & 0.24 & 0.57 & 0.46 & 0.52 & 0.49 & 0.62 & 0.51 & 0.52 & 0.44 & 0.48 & 0.40 & 0.45 & 0.38 \\
\hline $\mathrm{ZnO}$ & 0.14 & 0.16 & 0.14 & 0.16 & 1.10 & 1.13 & 0.19 & 0.22 & 0.18 & 0.20 & 0.21 & 0.23 & 0.18 & 0.21 & 0.16 & 0.19 & 0.15 & 0.18 \\
\hline $\mathrm{ZrO}_{2}$ & 0.19 & 0.17 & 0.19 & 0.17 & 2.13 & 1.89 & 0.25 & 0.24 & 0.23 & 0.21 & 0.27 & 0.25 & 0.23 & 0.21 & 0.21 & 0.21 & 0.20 & 0.18 \\
\hline $\mathrm{U}_{3} \mathrm{O}_{8}$ & 0.00 & 0.00 & 0.00 & 0.00 & 0.00 & 0.00 & 0.00 & 0.00 & 0.00 & 0.00 & 0.00 & 0.00 & 0.00 & 0.00 & 0.00 & 0.00 & 1.74 & 1.99 \\
\hline
\end{tabular}

$*$ In XRF analysis target concentrations are used for $\mathrm{B}_{2} \mathrm{O}_{3}$ and $\mathrm{Li}_{2} \mathrm{O} ; \mathrm{F}$ was not analyzed.

- Empty data field 
Table 3.2. Characterization of Glasses Formulated for Bi-Limited Waste.

\begin{tabular}{|c|c|c|c|c|c|c|c|c|c|}
\hline & Glass Name & HLW-E-Bi-11 & HLW-E-Bi-1 & HLW-E-Bi-2 & HLW-E-Bi-3 & HLW-E-Bi-4 & HLW-E-Bi-5 & HLW-E-Bi-9 & HLW-E-Bi-10 \\
\hline & Waste loading (wt\%) & $30 \%$ & $40 \%$ & $40 \%$ & $40 \%$ & $40 \%$ & $45 \%$ & $45 \%$ & $45 \%$ \\
\hline \multirow{7}{*}{ 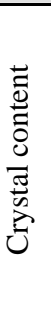 } & Glass as melted & Free & Trace & Trace & Trace & Trace & Trace & Trace & Trace \\
\hline & $\mathrm{Xl}$ at $950 \mathrm{C}$ & - & $\mathrm{Sp}$ & Sp & $\mathrm{Sp}$ & - & Sp & $\mathrm{Sp}$ & $\mathrm{Sp}$ \\
\hline & Glass after $\mathrm{HT}$ at $950^{\circ} \mathrm{C}$ (vol\%) & $<0.1$ & 0.125 & 0.1 & 0.1 & - & 0.4 & 0.5 & 0.4 \\
\hline & Glass after $\mathrm{HT}$ at $900^{\circ} \mathrm{C}(\mathrm{vol} \%)$ & - & - & 0.1 & - & - & - & - & - \\
\hline & Glass after $\mathrm{HT}$ at $850^{\circ} \mathrm{C}$ (vol\%) & - & - & 1.0 & - & - & - & - & - \\
\hline & Glass after $\mathrm{HT}$ at $800^{\circ} \mathrm{C}$ (vol\%) & - & - & 0.5 & - & - & - & - & - \\
\hline & Glass after HT of CCC (vol\%) & - & - & - & - & - & - & - & - \\
\hline \multirow{10}{*}{ 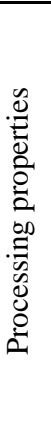 } & Highly viscous during pouring $(\mathrm{Y} / \mathrm{N})$ & $\mathrm{N}$ & $\mathrm{N}$ & $\mathrm{N}$ & $\mathrm{N}$ & $\mathrm{N}$ & $\mathrm{N}$ & $\mathrm{N}$ & $\mathrm{N}$ \\
\hline & Viscosity at $1250^{\circ} \mathrm{C}$ (poise) & - & - & 34.6 & - & - & - & - & - \\
\hline & Viscosity at $1150^{\circ} \mathrm{C}$ (poise) & - & - & 76.5 & - & - & - & - & - \\
\hline & Viscosity at $1050^{\circ} \mathrm{C}$ (poise) & - & - & 211.1 & - & - & - & - & - \\
\hline & Viscosity at $950^{\circ} \mathrm{C}$ (poise) & - & - & 813.1 & - & - & - & - & - \\
\hline & Electric conductivity at $1250^{\circ} \mathrm{C}(\mathrm{S} / \mathrm{cm})$ & - & - & 0.45 & - & - & - & - & - \\
\hline & Electric conductivity at $1150^{\circ} \mathrm{C}(\mathrm{S} / \mathrm{cm})$ & - & - & 0.34 & - & - & - & - & - \\
\hline & Electric conductivity at $1050^{\circ} \mathrm{C}(\mathrm{S} / \mathrm{cm})$ & - & - & 0.24 & - & - & - & - & - \\
\hline & Electric conductivity at $950^{\circ} \mathrm{C}(\mathrm{S} / \mathrm{cm})$ & - & - & 0.15 & - & - & - & - & - \\
\hline & Glass transition temperature $\left({ }^{\circ} \mathrm{C}\right)$ & - & - & - & - & - & - & - & - \\
\hline \multirow{19}{*}{ 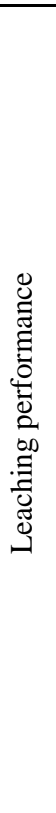 } & PCT & - & - & - & - & - & - & - & - \\
\hline & B (ppm) & - & 158.8 & 61.4 & - & 52.7 & 31.1 & - & - \\
\hline & Li (ppm) & - & 1.6 & 0.6 & - & 0.4 & 0.3 & - & - \\
\hline & $\mathrm{Na}(\mathrm{ppm})$ & - & 243.6 & 111.9 & - & 89.4 & 73.8 & - & - \\
\hline & Si (ppm) & - & 49.1 & 51.5 & - & 43.6 & 46.1 & - & - \\
\hline & B (g/L) & - & 3.72 & 1.55 & - & 1.33 & 0.82 & - & - \\
\hline & $\mathrm{Li}(\mathrm{g} / \mathrm{L})$ & - & 2.67 & 0.97 & - & 0.67 & 0.50 & - & - \\
\hline & $\mathrm{Na}(\mathrm{g} / \mathrm{L})$ & - & 1.89 & 0.92 & - & 0.74 & 0.62 & - & - \\
\hline & Si (g/L) & - & 0.27 & 0.28 & - & 0.24 & 0.26 & - & - \\
\hline & PCT (for CCC sample) & - & - & - & - & - & - & - & - \\
\hline & $\mathrm{B}(\mathrm{ppm})$ & - & - & - & - & - & - & - & - \\
\hline & $\mathrm{Li}(\mathrm{ppm})$ & - & - & - & - & - & - & - & - \\
\hline & $\mathrm{Na}(\mathrm{ppm})$ & - & - & - & - & - & - & - & - \\
\hline & $\mathrm{Si}(\mathrm{ppm})$ & - & - & - & - & - & - & - & - \\
\hline & $\mathrm{B}(\mathrm{g} / \mathrm{L})$ & - & - & - & - & - & - & - & - \\
\hline & $\mathrm{Li}(\mathrm{g} / \mathrm{L})$ & - & - & - & - & - & - & - & - \\
\hline & $\mathrm{Na}(\mathrm{g} / \mathrm{L})$ & - & - & - & - & - & - & - & - \\
\hline & Si (g/L) & - & - & - & - & - & - & - & - \\
\hline & TCLP (Pass/Fail) & - & - & Pass & - & - & - & - & - \\
\hline
\end{tabular}


Table 3.2. Characterization of Glasses Formulated for Bi-Limited Waste (continued).

\begin{tabular}{|c|c|c|c|c|c|c|c|c|c|c|}
\hline & Glass Name & HLW-E-Bi-8 & HLW-E-Bi-6 & HLW-E-Bi6UThR1 & HLW-E-Bi-16 & HLW-E-Bi-7 & HLW-E-Bi-13 & HLW-E-Bi-15 & HLW-E-Bi-12 & HLW-E-Bi-14 \\
\hline & Waste loading (wt\%) & $45 \%$ & $50 \%$ & $50 \%$ & $50 \%$ & $55 \%$ & $55 \%$ & $55 \%$ & $60 \%$ & $65 \%$ \\
\hline \multirow{7}{*}{ 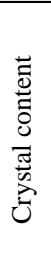 } & Glass as melted & Trace & Trace & Not analyzed & Trace & Xtals & xtals & Xtals & Xtals & Xtals \\
\hline & $\mathrm{Xl}$ at $950 \mathrm{C}$ & Sp & Sp + Pho & Sp + Pho & Sp + Pho & Sp +Pho & Sp + Pho & Sp & Sp + Pho & Sp + Pho \\
\hline & Glass after $\mathrm{HT}$ at $950^{\circ} \mathrm{C}(\mathrm{vol} \%)$ & 0.5 & 1.8 & 1.4 & 2.7 & 2.7 & 3.2 & 2.1 & 4.2 & 3.3 \\
\hline & Glass after $\mathrm{HT}$ at $900^{\circ} \mathrm{C}(\mathrm{vol} \%)$ & - & 2.3 & 1.9 & - & 3.5 & 3.4 & - & 5.0 & - \\
\hline & Glass after $\mathrm{HT}$ at $850^{\circ} \mathrm{C}$ (vol\%) & - & 3.3 & 2.8 & - & 4.9 & 4.5 & - & 5.6 & - \\
\hline & Glass after $\mathrm{HT}$ at $800^{\circ} \mathrm{C}(\mathrm{vol} \%)$ & - & 4.0 & 3.5 & - & 5.5 & 5.3 & - & 5.0 & - \\
\hline & Glass after HT of CCC (vol\%) & - & $5.6(\mathrm{Pho}+\mathrm{Sp}+\dagger)$ & $5.0(\mathrm{Pho}+\mathrm{Sp}+\dagger)$ & - & - & - & - & - & - \\
\hline \multirow{10}{*}{ 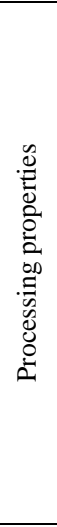 } & $\begin{array}{l}\text { Highly viscous during pouring } \\
(\mathrm{Y} / \mathrm{N})\end{array}$ & $\mathrm{N}$ & $\mathrm{N}$ & $\mathrm{N}$ & $\mathrm{N}$ & $\mathrm{N}$ & $\mathrm{N}$ & $\mathrm{N}$ & $\mathrm{N}$ & $\mathrm{N}$ \\
\hline & Viscosity at $1250^{\circ} \mathrm{C}$ (poise) & - & 42.3 & 36.1 & 34.3 & 38.2 & 32.2 & - & - & - \\
\hline & Viscosity at $1150^{\circ} \mathrm{C}$ (poise) & - & 96.0 & 81.8 & 74.8 & 96.6 & 76.4 & - & - & - \\
\hline & Viscosity at $1050^{\circ} \mathrm{C}$ (poise) & - & 266.6 & 242.8 & 193.9 & 286.8 & 210.6 & - & - & - \\
\hline & Viscosity at $950^{\circ} \mathrm{C}$ (poise) & - & 987.2 & 1114.6 & 637.9 & 1048.5 & 704.1 & - & - & - \\
\hline & $\begin{array}{l}\text { Electric conductivity at } 1250^{\circ} \mathrm{C} \\
(\mathrm{S} / \mathrm{cm})\end{array}$ & - & 0.61 & 0.47 & 0.51 & 0.57 & 0.53 & - & - & - \\
\hline & $\begin{array}{l}\text { Electric conductivity at } 1150^{\circ} \mathrm{C} \\
(\mathrm{S} / \mathrm{cm})\end{array}$ & - & 0.47 & 0.36 & 0.41 & 0.43 & 0.38 & - & - & - \\
\hline & $\begin{array}{l}\text { Electric conductivity at } 1050^{\circ} \mathrm{C} \\
(\mathrm{S} / \mathrm{cm})\end{array}$ & - & 0.35 & 0.26 & 0.30 & 0.31 & 0.24 & - & - & - \\
\hline & $\begin{array}{l}\text { Electric conductivity at } 950^{\circ} \mathrm{C} \\
(\mathrm{S} / \mathrm{cm})\end{array}$ & - & 0.24 & 0.16 & 0.19 & 0.20 & 0.11 & - & - & - \\
\hline & Glass transition temperature $\left({ }^{\circ} \mathrm{C}\right)$ & - & 484 & - & - & - & - & - & - & - \\
\hline \multirow{19}{*}{ 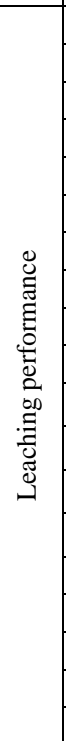 } & PCT & - & & - & - & - & - & - & - & - \\
\hline & B (ppm) & - & 17.2 & 17.2 & - & 15.8 & 21.4 & - & 27.8 & - \\
\hline & $\mathrm{Li}(\mathrm{ppm})$ & - & 0.1 & 0.4 & - & 0.1 & 0.4 & - & 0.6 & - \\
\hline & $\mathrm{Na}(\mathrm{ppm})$ & - & 61.9 & 54.8 & - & 58.6 & 72.0 & - & 83.5 & - \\
\hline & Si (ppm) & - & 45.7 & 39.5 & - & 40.3 & 39.3 & - & 39.8 & - \\
\hline & $B(g / L)$ & - & 0.49 & 0.49 & - & 0.49 & 0.67 & - & 0.79 & - \\
\hline & $\mathrm{Li}(\mathrm{g} / \mathrm{L})$ & - & 0.18 & 0.55 & - & 0.18 & 0.52 & - & 0.66 & - \\
\hline & $\mathrm{Na}(\mathrm{g} / \mathrm{L})$ & - & 0.53 & 0.48 & - & 0.51 & 0.65 & - & 0.75 & - \\
\hline & $\mathrm{Si}(\mathrm{g} / \mathrm{L})$ & - & 0.27 & 0.23 & - & 0.25 & 0.26 & - & 0.29 & - \\
\hline & PCT (for CCC sample) & - & & - & - & - & - & - & - & - \\
\hline & $\mathrm{B}(\mathrm{ppm})$ & - & 16.9 & - & - & - & - & - & - & - \\
\hline & $\mathrm{Li}(\mathrm{ppm})$ & - & 0.5 & - & - & - & - & - & - & - \\
\hline & $\mathrm{Na}(\mathrm{ppm})$ & - & 69.4 & - & - & - & - & - & - & - \\
\hline & Si (ppm) & - & 42.5 & - & - & - & - & - & - & - \\
\hline & $B(g / L)$ & - & 0.48 & - & - & - & - & - & - & - \\
\hline & $\mathrm{Li}(\mathrm{g} / \mathrm{L})$ & - & 0.67 & - & - & - & - & - & - & - \\
\hline & $\mathrm{Na}(\mathrm{g} / \mathrm{L})$ & - & 0.59 & - & - & - & - & - & - & - \\
\hline & $\mathrm{Si}(\mathrm{g} / \mathrm{L})$ & - & 0.25 & - & - & - & - & - & - & - \\
\hline & TCLP (Pass/Fail) & - & Pass & Pass & - & Pass & Pass & - & - & - \\
\hline
\end{tabular}

$\mathrm{Sp}=$ spinel; Pho $=$ phosphate; Nas = sodium aluminosilicate; †bubbles submicron size

- Empty data field 
The Catholic University of America Vitreous State Laboratory
High Level Waste Vitrification System Improvements

Final Report, VSL-07R1010-1, Rev. 0

Table 3.3. TCLP Results (ppm) for Glasses Selected for Melter Tests and Their Corresponding Radioactive Glasses.

\begin{tabular}{|l|c|c|c|c|c|c|c|}
\hline Element & $\mathrm{Ba}$ & $\mathrm{Bi}$ & $\mathrm{Cd}$ & $\mathrm{Cr}$ & $\mathrm{Ni}$ & $\mathrm{Pb}$ & $\mathrm{Zn}$ \\
\hline UTS Limits & 21 & $\mathrm{~N} / \mathrm{A}$ & 0.11 & 0.6 & 11 & 0.75 & 4.3 \\
\hline Delisting Limits & 100 & N/A & 0.48 & 4.95 & 22.6 & 5 & 225 \\
\hline HLW-E-Al27R1 & 0.12 & 0.91 & $\mathrm{NA}$ & 0.21 & 0.20 & 0.37 & 0.13 \\
\hline HLW-E-Al27UThR2 & 0.12 & 0.55 & 0.04 & 0.31 & 0.39 & 0.58 & 0.13 \\
\hline HLW-E-ANa22R1 & 0.09 & 0.46 & NA & 0.21 & $<0.04$ & $<0.1$ & 0.11 \\
\hline HLW-E-ANa22UTh & 0.05 & 0.26 & $<0.03$ & 0.19 & 0.04 & $<0.1$ & 0.07 \\
\hline HLW-E-Bi6 & 0.10 & NA & NA & 0.06 & 0.48 & $<0.1$ & 0.08 \\
\hline HLW-E-Bi6UThR1 & 0.04 & 0.80 & $<0.03$ & 0.06 & 0.48 & $<0.1$ & 0.05 \\
\hline HLW-E-Cr-MR1 & 0.08 & 0.44 & NA & 0.07 & $<0.04$ & $<0.1$ & 0.06 \\
\hline HLW-E-Cr-MUTh & 0.06 & 0.50 & $<0.03$ & 0.20 & 0.11 & $<0.1$ & 0.05 \\
\hline HLW-E-Cr-10 & 0.13 & NA & NA & 0.31 & 0.15 & $<0.1$ & 0.08 \\
\hline
\end{tabular}

N/A- Not Applicable

NA-Not analyzed 


\section{Table 3.4. Composition and Properties of Bismuth Limited Waste and Glass} Formulation at $50 \%$ Waste Loading Used in Melter Tests (wt\%).

\begin{tabular}{|c|c|c|c|c|}
\hline- & $\begin{array}{c}\text { Bi-Limited } \\
\text { Waste* }\end{array}$ & Waste in Glass & $\begin{array}{c}\text { Glass Forming } \\
\text { Additives }\end{array}$ & $\begin{array}{c}\text { Target Glass } \\
\text { HLW-E-Bi-6 }\end{array}$ \\
\hline $\mathrm{Al}_{2} \mathrm{O}_{3}$ & 23.31 & 11.66 & - & 11.66 \\
\hline $\mathrm{B}_{2} \mathrm{O}_{3}$ & 0.60 & 0.30 & 11.00 & 11.30 \\
\hline $\mathrm{BaO}$ & 0.02 & 0.01 & - & 0.01 \\
\hline $\mathrm{Bi}_{2} \mathrm{O}_{3}$ & 13.41 & 6.71 & - & 6.71 \\
\hline $\mathrm{CaO}$ & 1.67 & 0.84 & - & 0.84 \\
\hline $\mathrm{Cr}_{2} \mathrm{O}_{3}$ & 1.04 & 0.52 & - & 0.52 \\
\hline $\mathrm{F}$ & 1.64 & 0.82 & - & 0.82 \\
\hline $\mathrm{Fe}_{2} \mathrm{O}_{3}$ & 13.92 & 6.96 & - & 6.96 \\
\hline $\mathrm{K}_{2} \mathrm{O}$ & 0.92 & 0.46 & - & 0.46 \\
\hline $\mathrm{Li}_{2} \mathrm{O}$ & 0.32 & 0.16 & - & 0.16 \\
\hline $\mathrm{MgO}^{\mathrm{Na} O}$ & 0.85 & 0.43 & - & 0.43 \\
\hline $\mathrm{NiO}_{2} \mathrm{O}$ & 13.47 & 6.74 & 9.00 & 15.74 \\
\hline $\mathrm{P}_{2} \mathrm{O}_{5}$ & 3.85 & 1.93 & - & 1.93 \\
\hline $\mathrm{PbO}^{3 \mathrm{O} O}$ & 9.97 & 4.99 & - & 4.99 \\
\hline $\mathrm{SiO}_{2}$ & 0.50 & 0.25 & - & 0.25 \\
\hline $\mathrm{TiO}_{2}$ & 12.51 & 6.26 & 30.00 & 36.26 \\
\hline $\mathrm{SO}_{3}$ & 0.31 & 0.16 & - & 0.16 \\
\hline $\mathrm{ZnO}_{\mathrm{ZrO}}$ & 0.95 & 0.48 & - & 0.48 \\
\hline $\mathrm{Sum}_{2}$ & 0.42 & 0.16 & - & 0.16 \\
\hline $\mathrm{Ren}$ & 100 & 0.21 & - & 0.21 \\
\hline
\end{tabular}

* Renormalized from Ref. [3] after removal of radioactive components

\begin{tabular}{|c|c|c|c|}
\hline \multicolumn{3}{|c|}{ Viscosity @1150, P } & 96 \\
\hline \multicolumn{3}{|c|}{ Conductivity @1150ㄷ, S/cm } & 0.47 \\
\hline \multicolumn{3}{|c|}{ Crystal Content, As Melted } & Trace \\
\hline \multicolumn{3}{|c|}{ Crystal Content, $72 \mathrm{hr}$ at $950^{\circ} \mathrm{C}$} & $\sim 1.8 \mathrm{vol} \%$ \\
\hline \multicolumn{3}{|c|}{ TCLP } & Pass \\
\hline \multirow{4}{*}{ PCT, g/L } & - & DWPF-EA & HLW-E-Bi-6 \\
\hline & $\mathrm{B}$ & 16.7 & 0.49 \\
\hline & $\mathrm{Li}$ & 9.6 & 0.18 \\
\hline & $\mathrm{Na}$ & 13.3 & 0.53 \\
\hline
\end{tabular}

- Empty data field 
Table 3.5. Target Composition and XRF Analysis of Glasses Formulated for Cr-Limited Waste.

\begin{tabular}{|c|c|c|c|c|c|c|c|c|c|c|c|c|c|c|}
\hline \multirow{2}{*}{ - } & \multirow{2}{*}{$\begin{array}{l}\text { Cr-Limited } \\
\text { Waste }\end{array}$} & \multirow{2}{*}{$\begin{array}{l}\text { Cr-limited waste } \\
\text { (non-rad)\# }\end{array}$} & \multicolumn{2}{|c|}{ HLW-E-Cr-1 } & \multicolumn{2}{|c|}{ HLW-E-Cr-2 } & \multicolumn{2}{|c|}{ HLW-E-Cr-3 } & \multicolumn{2}{|c|}{ HLW-E-Cr-4 } & \multicolumn{2}{|c|}{ HLW-E-Cr-5 } & \multicolumn{2}{|c|}{ HLW-E-Cr-6 } \\
\hline & & & Target & XRF & Target & XRF & Target & XRF & Target & XRF & Target & XRF & Target & XRF \\
\hline $\mathrm{Al}_{2} \mathrm{O}_{3}$ & 25.53 & 27.64 & 11.06 & 11.18 & 11.06 & 11.42 & 11.06 & 11.68 & 11.06 & 11.64 & 11.06 & 11.37 & 11.06 & 11.47 \\
\hline $\mathrm{B}_{2} \mathrm{O}_{3} *$ & 0.53 & 0.57 & 14.23 & 14.23 & 13.73 & 13.73 & 13.73 & 13.73 & 13.73 & 13.73 & 13.73 & 13.73 & 13.73 & 13.73 \\
\hline $\mathrm{BaO}$ & 0.03 & 0.03 & 0.01 & 0.019 & 0.01 & 0.02 & 0.01 & 0.01 & 0.01 & 0.02 & 0.01 & 0.00 & 0.01 & 0.03 \\
\hline $\mathrm{Bi}_{2} \mathrm{O}_{3}$ & 7.29 & 7.89 & 3.16 & 3.34 & 3.16 & 3.25 & 3.16 & 3.24 & 3.16 & 3.29 & 3.16 & 3.33 & 3.16 & 3.36 \\
\hline $\mathrm{CaO}$ & 2.47 & 2.67 & 1.07 & 1.13 & 1.07 & 1.10 & 1.07 & 1.12 & 1.07 & 1.14 & 1.07 & 1.11 & 1.07 & 1.15 \\
\hline $\mathrm{CdO}$ & 0.01 & 0.01 & 0.00 & 0.00 & 0.00 & 0.00 & 0.00 & 0.00 & 0.00 & 0.00 & 0.00 & 0.05 & 0.00 & 0.00 \\
\hline $\mathrm{Cr}_{2} \mathrm{O}_{3}$ & 3.07 & 3.32 & 1.33 & 1.28 & 1.33 & 1.38 & 1.33 & 1.39 & 1.33 & 0.57 & 1.33 & 1.58 & 1.33 & 1.51 \\
\hline $\mathrm{F}^{*}$ & 2.00 & 2.17 & 0.87 & 0.00 & 0.87 & - & 0.87 & - & 0.87 & - & 0.87 & - & 0.87 & - \\
\hline $\mathrm{Fe}_{2} \mathrm{O}_{3}$ & 13.13 & 14.22 & 5.69 & 5.77 & 5.69 & 5.58 & 5.69 & 5.58 & 5.69 & 5.59 & 5.69 & 5.63 & 5.69 & 5.73 \\
\hline $\mathrm{K}_{2} \mathrm{O}$ & 0.37 & 0.40 & 11.16 & 11.56 & 10.16 & 10.12 & 7.66 & 7.65 & 2.66 & 2.75 & 5.16 & 5.17 & 3.66 & 3.79 \\
\hline $\mathrm{Li}_{2} \mathrm{O}^{*}$ & 0.36 & 0.39 & 0.16 & 0.16 & 1.16 & 1.16 & 2.66 & 2.66 & 3.16 & 3.16 & 2.16 & 3.16 & 3.16 & 3.16 \\
\hline $\mathrm{MgO}$ & 0.16 & 0.17 & 0.07 & 0.072 & 0.07 & 0.06 & 0.07 & 0.05 & 0.07 & 0.10 & 0.07 & 0.06 & 0.07 & 0.08 \\
\hline $\mathrm{Na}_{2} \mathrm{O}$ & 20.09 & 21.75 & 8.70 & 8.32 & 8.70 & 8.74 & 8.70 & 8.70 & 8.70 & 9.18 & 8.70 & 8.82 & 8.70 & 8.60 \\
\hline $\mathrm{NiO}$ & 1.06 & 1.15 & 0.46 & 0.44 & 0.46 & 0.45 & 0.46 & 0.45 & 0.46 & 0.44 & 0.46 & 0.45 & 0.46 & 0.45 \\
\hline $\mathrm{P}_{2} \mathrm{O}_{5}$ & 3.34 & 3.62 & 1.45 & 1.71 & 1.45 & 1.60 & 1.45 & 1.58 & 1.45 & 1.56 & 1.45 & 1.55 & 1.45 & 1.59 \\
\hline $\mathrm{PbO}$ & 0.48 & 0.52 & 0.71 & 0.654 & 1.21 & 1.12 & 2.21 & 2.04 & 1.21 & 1.12 & 1.21 & 1.16 & 2.21 & 2.09 \\
\hline $\mathrm{SiO}_{2}$ & 10.56 & 11.43 & 39.07 & 39.57 & 39.07 & 39.65 & 39.07 & 39.37 & 44.57 & 44.00 & 40.57 & 39.67 & 42.57 & 42.50 \\
\hline $\mathrm{SrO}$ & 0.00 & 0.00 & 0.00 & 0.015 & 0.00 & 0.02 & 0.00 & 0.02 & 0.00 & 0.02 & 2.50 & 2.43 & 0.00 & 0.02 \\
\hline $\mathrm{ThO}_{2}$ & 0.04 & 0.00 & 0.00 & 0.00 & 0.00 & 0.00 & 0.00 & 0.00 & 0.00 & 0.00 & 0.00 & 0.00 & 0.00 & 0.00 \\
\hline $\mathrm{TiO}_{2}$ & 0.01 & 0.01 & 0.00 & 0.05 & 0.00 & 0.03 & 0.00 & 0.05 & 0.00 & 0.06 & 0.00 & 0.06 & 0.00 & 0.04 \\
\hline $\mathrm{SO}_{3}$ & 1.52 & 1.65 & 0.66 & 0.315 & 0.66 & 0.32 & 0.66 & 0.41 & 0.66 & 0.39 & 0.66 & 0.40 & 0.66 & 0.44 \\
\hline $\mathrm{V}_{2} \mathrm{O}_{5}$ & 0.00 & 0.00 & 0.00 & 0.00 & 0.00 & 0.00 & 0.00 & 0.00 & 0.00 & 0.00 & 0.00 & 0.00 & 0.00 & 0.00 \\
\hline $\mathrm{ZnO}$ & 0.25 & 0.27 & 0.11 & 0.108 & 0.11 & 0.14 & 0.11 & 0.13 & 0.11 & 0.14 & 0.11 & 0.13 & 0.11 & 0.12 \\
\hline $\mathrm{ZrO}_{2}$ & 0.11 & 0.12 & 0.05 & 0.052 & 0.05 & 0.07 & 0.05 & 0.04 & 0.05 & 0.06 & 0.05 & 0.05 & 0.05 & 0.05 \\
\hline $\mathrm{U}_{3} \mathrm{O}_{8}$ & 7.59 & 0.00 & 0.00 & 0.00 & 0.00 & 0.00 & 0.00 & 0.00 & 0.00 & 0.00 & 0.00 & 0.00 & 0.00 & 0.00 \\
\hline
\end{tabular}

*In XRF analysis target concentrations are used for $\mathrm{B}_{2} \mathrm{O}_{3}$ and $\mathrm{Li}_{2} \mathrm{O}$; $\mathrm{F}$ was not analyzed.

\#The non-radioactive version of the waste is calculated by renormalization of the waste constituents after removing $\mathrm{U}_{3} \mathrm{O}_{8}$ and $\mathrm{ThO}_{2}$

-Empty data field 
Table 3.5. Target Composition and XRF Analysis of Glasses Formulated for Cr-Limited Waste (continued).

\begin{tabular}{|c|c|c|c|c|c|c|c|c|c|c|c|c|c|c|c|c|}
\hline & \multicolumn{2}{|c|}{ HLW-E-Cr-7 } & \multicolumn{2}{|c|}{ HLW-E-Cr-8 } & \multicolumn{2}{|c|}{ HLW-E-Cr-9 } & \multicolumn{2}{|c|}{ HLW-E-Cr-10 } & \multicolumn{2}{|c|}{ HLW-E-Cr-11 } & \multicolumn{2}{|c|}{ HLW-E-Cr-12 } & \multicolumn{2}{|c|}{ HLW-E-Cr-13 } & \multicolumn{2}{|c|}{ HLW-E-Cr-14 } \\
\hline & Target & XRF & Target & XRF & Target & XRF & Target & XRF & Target & XRF & Target & XRF & Target & XRF & Target & XRF \\
\hline $\mathrm{Al}_{2} \mathrm{O}_{3}$ & 11.06 & 11.75 & 11.06 & 11.28 & 11.06 & 11.05 & 11.06 & 11.24 & 11.06 & 11.37 & 12.44 & 12.56 & 13.82 & 14.07 & 8.29 & 8.92 \\
\hline $\mathrm{B}_{2} \mathrm{O}_{3} *$ & 13.73 & 13.73 & 13.73 & 13.73 & 15.73 & 15.73 & 14.44 & 14.44 & 14.44 & 14.44 & 14.47 & 14.27 & 14.00 & 14.27 & 14.38 & 14.38 \\
\hline $\mathrm{BaO}$ & 0.01 & 0.03 & 0.01 & 0.02 & 0.01 & 0.02 & 0.01 & 0.03 & 0.01 & 0.02 & 0.01 & 0.02 & 0.02 & 0.02 & 0.01 & 0.01 \\
\hline $\mathrm{Bi}_{2} \mathrm{O}_{3}$ & 3.16 & 3.16 & 3.16 & 3.18 & 3.16 & 3.28 & 3.16 & 3.36 & 3.16 & 3.25 & 3.55 & 3.70 & 3.95 & 4.02 & 2.37 & 2.49 \\
\hline $\mathrm{CaO}$ & 1.07 & 1.06 & 1.07 & 1.07 & 1.07 & 1.14 & 1.07 & 1.11 & 1.07 & 1.09 & 1.20 & 1.24 & 1.34 & 1.31 & 1.59 & 1.67 \\
\hline $\mathrm{CdO}$ & 0.00 & 0.00 & 0.00 & 0.07 & 0.00 & 0.00 & 0.00 & 0.00 & 0.00 & 0.00 & 0.00 & 0.00 & 0.01 & 0.00 & 0.00 & 0.00 \\
\hline $\mathrm{Cr}_{2} \mathrm{O}_{3}$ & 1.33 & 1.42 & 1.33 & 1.39 & 1.33 & 1.47 & 1.33 & 1.49 & 1.33 & 1.46 & 1.50 & 1.73 & 1.66 & 1.73 & 1.00 & 1.19 \\
\hline F* & 0.87 & - & 0.87 & - & 0.87 & - & 0.87 & - & 0.87 & - & 0.97 & - & 1.08 & - & 0.65 & - \\
\hline $\mathrm{Fe}_{2} \mathrm{O}_{3}$ & 5.69 & 5.39 & 5.69 & 5.41 & 5.69 & 5.57 & 5.69 & 5.65 & 5.69 & 5.51 & 6.40 & 6.27 & 7.11 & 6.86 & 4.26 & 4.22 \\
\hline $\mathrm{K}_{2} \mathrm{O}$ & 5.16 & 5.06 & 7.66 & 7.41 & 5.16 & 5.15 & 5.42 & 5.62 & 5.42 & 5.47 & 5.44 & 5.27 & 5.20 & 4.90 & 6.12 & 6.14 \\
\hline $\mathrm{Li}_{2} \mathrm{O}^{*}$ & 3.16 & 3.16 & 2.66 & 2.66 & 3.16 & 3.16 & 3.31 & 3.31 & 3.31 & 3.31 & 3.33 & 2.68 & 2.20 & 2.20 & 4.12 & 4.12 \\
\hline $\mathrm{MgO}$ & 0.07 & 0.07 & 0.07 & 0.00 & 0.07 & 0.06 & 0.07 & 0.05 & 0.07 & 0.00 & 0.08 & 0.07 & 0.09 & 0.06 & 0.05 & 0.08 \\
\hline $\mathrm{Na}_{2} \mathrm{O}$ & 8.70 & 9.39 & 8.70 & 9.07 & 8.70 & 9.13 & 8.70 & 8.67 & 8.70 & 8.93 & 9.79 & 10.32 & 10.87 & 11.16 & 6.52 & 6.58 \\
\hline $\mathrm{NiO}$ & 0.46 & 0.43 & 0.46 & 0.45 & 0.46 & 0.45 & 0.46 & 0.46 & 0.46 & 0.44 & 0.52 & 0.50 & 0.57 & 0.54 & 0.34 & 0.34 \\
\hline $\mathrm{P}_{2} \mathrm{O}_{5}$ & 1.45 & 1.58 & 1.45 & 1.77 & 1.45 & 1.77 & 1.45 & 1.61 & 1.45 & 1.63 & 1.63 & 1.89 & 1.81 & 1.99 & 1.08 & 1.23 \\
\hline $\mathrm{PbO}$ & 3.21 & 2.91 & 3.21 & 2.90 & 3.21 & 2.95 & 0.21 & 0.20 & 0.21 & 0.20 & 0.23 & 0.22 & 0.26 & 0.23 & 0.16 & 0.15 \\
\hline $\mathrm{SiO}_{2}$ & 40.07 & 40.15 & 38.07 & 38.79 & 38.07 & 38.26 & 41.94 & 41.99 & 40.94 & 41.22 & 37.51 & 38.51 & 35.00 & 35.83 & 45.43 & 45.04 \\
\hline $\mathrm{SrO}$ & 0.00 & 0.02 & 0.00 & 0.00 & 0.00 & 0.00 & 0.00 & 0.02 & 0.00 & 0.02 & 0.00 & 0.02 & 0.00 & 0.01 & 0.00 & 0.02 \\
\hline $\mathrm{ThO}_{2}$ & 0.00 & 0.00 & 0.00 & 0.00 & 0.00 & 0.00 & 0.00 & 0.00 & 0.00 & 0.00 & 0.00 & 0.00 & 0.00 & 0.00 & 0.00 & 0.00 \\
\hline $\mathrm{TiO}_{2}$ & 0.00 & 0.05 & 0.00 & 0.04 & 0.00 & 0.05 & 0.00 & 0.06 & 0.00 & 0.05 & 0.00 & 0.04 & 0.01 & 0.04 & 0.00 & 0.04 \\
\hline $\mathrm{SO}_{3}$ & 0.66 & 0.39 & 0.66 & 0.49 & 0.66 & 0.45 & 0.66 & 0.46 & 0.66 & 0.38 & 0.74 & 0.47 & 0.82 & 0.48 & 0.49 & 0.36 \\
\hline $\mathrm{V}_{2} \mathrm{O}_{5}$ & 0.00 & 0.00 & 0.00 & 0.00 & 0.00 & 0.00 & 0.00 & 0.00 & 0.00 & 0.00 & 0.00 & 0.00 & 0.00 & 0.00 & 0.00 & 0.00 \\
\hline $\mathrm{ZnO}$ & 0.11 & 0.13 & 0.11 & 0.13 & 0.11 & 0.13 & 0.11 & 0.13 & 0.11 & 0.13 & 0.12 & 0.14 & 0.14 & 0.15 & 0.08 & 0.10 \\
\hline $\mathrm{ZrO}_{2}$ & 0.05 & 0.03 & 0.05 & 0.04 & 0.05 & 0.05 & 0.05 & 0.04 & 1.05 & 0.98 & 0.05 & 0.05 & 0.06 & 0.06 & 3.04 & 2.80 \\
\hline $\mathrm{U}_{3} \mathrm{O}_{8}$ & 0.00 & 0.00 & 0.00 & 0.00 & 0.00 & 0.00 & 0.00 & 0.00 & 0.00 & 0.00 & 0.00 & 0.00 & 0.00 & 0.00 & 0.00 & 0.00 \\
\hline
\end{tabular}

*In XRF analysis target concentrations are used for $\mathrm{B}_{2} \mathrm{O}_{3}$ and $\mathrm{Li}_{2} \mathrm{O}$; F was not analyzed.

- Empty data field 
Table 3.5. Target Composition and XRF Analysis of Glasses Formulated for Cr-Limited Waste (continued).

\begin{tabular}{|c|c|c|c|c|c|c|c|c|c|c|c|c|}
\hline \multirow{2}{*}{-} & \multicolumn{2}{|c|}{ HLW-E-Cr-15 } & \multicolumn{2}{|c|}{ HLW-E-Cr-16 } & \multicolumn{2}{|c|}{ HLW-E-Cr-17 } & \multicolumn{2}{|c|}{ HLW-E-Cr-18 } & \multicolumn{2}{|c|}{ HLW-E-Cr-Mrl } & \multicolumn{2}{|c|}{ HLW-E-Cr-MUTh } \\
\hline & Target & XRF & Target & XRF & Target & XRF & Target & XRF & Target & XRF & Target & XRF \\
\hline $\mathrm{Al}_{2} \mathrm{O}_{3}$ & 12.44 & 12.49 & 13.82 & 13.95 & 11.75 & 12.13 & 11.75 & 12.03 & 8.98 & 9.77 & 8.30 & 8.88 \\
\hline $\mathrm{BaO}$ & 0.01 & 0.01 & 0.02 & 0.02 & 0.01 & 0.02 & 0.01 & 0.01 & 0.01 & 0.02 & 0.01 & 0.03 \\
\hline $\mathrm{Bi}_{2} \mathrm{O}_{3}$ & 3.55 & 3.67 & 3.95 & 4.15 & 3.35 & 3.52 & 3.35 & 3.45 & 2.57 & 2.76 & 2.37 & 2.31 \\
\hline $\mathrm{CaO}$ & 1.20 & 1.24 & 1.34 & 1.36 & 1.14 & 1.16 & 3.17 & 3.11 & 0.87 & 0.92 & 0.80 & 0.81 \\
\hline $\mathrm{CdO}$ & 0.00 & 0.00 & 0.01 & 0.00 & 0.00 & 0.00 & 0.00 & 0.00 & 0.00 & 0.00 & 0.00 & 0.00 \\
\hline $\mathrm{Cr}_{2} \mathrm{O}_{3}$ & 1.50 & 1.63 & 1.66 & 1.84 & 1.41 & 1.52 & 1.41 & 1.56 & 1.08 & 1.24 & 1.00 & 1.14 \\
\hline $\mathrm{F}^{*}$ & 0.97 & - & 1.08 & - & 0.92 & - & 0.92 & - & 0.70 & - & 0.65 & - \\
\hline $\mathrm{Fe}_{2} \mathrm{O}_{3}$ & 6.40 & 6.26 & 7.11 & 6.99 & 6.04 & 5.96 & 6.04 & 5.92 & 4.62 & 4.65 & 4.27 & 3.93 \\
\hline $\mathrm{K}_{2} \mathrm{O}$ & 5.44 & 5.39 & 5.20 & 5.28 & 5.43 & 5.37 & 5.43 & 5.42 & 6.05 & 5.73 & 6.04 & 5.82 \\
\hline $\mathrm{Li}_{2} \mathrm{O}^{*}$ & 3.33 & 3.33 & 2.21 & 2.21 & 3.31 & 3.31 & 3.17 & 3.17 & 3.68 & 3.68 & 3.67 & 3.67 \\
\hline $\mathrm{Na}_{2} \mathrm{O}$ & 9.79 & 10.30 & 10.87 & 11.11 & 9.24 & 9.23 & 9.24 & 9.22 & 7.07 & 6.36 & 6.53 & 6.86 \\
\hline $\mathrm{NiO}$ & 0.52 & 0.49 & 0.57 & 0.54 & 0.49 & 0.47 & 0.49 & 0.48 & 0.37 & 0.36 & 0.34 & 0.30 \\
\hline $\mathrm{P}_{2} \mathrm{O}_{5}$ & 1.63 & 2.05 & 1.81 & 2.03 & 1.54 & 1.70 & 1.54 & 1.69 & 1.18 & 1.34 & 1.09 & 1.25 \\
\hline $\mathrm{PbO}$ & 0.23 & 0.22 & 0.26 & 0.25 & 0.22 & 0.20 & 0.22 & 0.21 & 0.17 & 0.17 & 0.16 & 0.13 \\
\hline $\mathrm{SiO}_{2}$ & 36.01 & 35.97 & 33.41 & 33.81 & 39.75 & 40.14 & 37.86 & 38.42 & 45.76 & 45.92 & 45.47 & 45.58 \\
\hline $\mathrm{SrO}$ & 0.00 & 0.01 & 0.00 & 0.01 & 0.00 & 0.02 & 0.00 & 0.02 & 0.00 & 0.02 & 0.00 & 0.02 \\
\hline $\mathrm{ThO}_{2}$ & 0.00 & 0.00 & 0.00 & 0.00 & 0.00 & 0.00 & 0.00 & 0.00 & 0.00 & 0.00 & 0.01 & 0.00 \\
\hline $\mathrm{TiO}_{2}$ & 0.00 & 0.05 & 0.01 & 0.04 & 0.00 & 0.08 & 0.00 & 0.05 & 0.00 & 0.05 & 0.00 & 0.04 \\
\hline $\mathrm{SO}_{3}$ & 0.74 & 0.51 & 0.82 & 0.49 & 0.70 & 0.43 & 0.70 & 0.48 & 0.54 & 0.60 & 0.49 & 0.38 \\
\hline $\mathrm{V}_{2} \mathrm{O}_{5}$ & 1.50 & 1.57 & 1.50 & 1.58 & 0.00 & 0.00 & 0.00 & 0.00 & 0.00 & 0.00 & 0.00 & 0.00 \\
\hline $\mathrm{ZnO}$ & 0.12 & 0.15 & 0.14 & 0.15 & 0.12 & 0.14 & 0.12 & 0.13 & 0.09 & 0.12 & 0.08 & 0.13 \\
\hline $\mathrm{ZrO}_{2}$ & 0.05 & 0.05 & 0.06 & 0.05 & 0.05 & 0.05 & 0.05 & 0.07 & 0.04 & 0.04 & 0.04 & 0.03 \\
\hline $\mathrm{U}_{3} \mathrm{O}_{8}$ & 0.00 & 0.00 & 0.00 & 0.00 & 0.00 & 0.00 & 0.00 & 0.00 & 0.00 & 0.00 & 2.47 & 2.43 \\
\hline
\end{tabular}

*In XRF analysis target concentrations are used for $\mathrm{B}_{2} \mathrm{O}_{3}$ and $\mathrm{Li}_{2} \mathrm{O}$; $\mathrm{F}$ was not analyzed.

- Empty data field 
Table 3.6. Characterization of Glasses Formulated for Cr-Limited Waste.

\begin{tabular}{|c|c|c|c|c|c|c|c|c|c|c|c|}
\hline & Glass Name & HLW-E-Cr-14 & HLW-E-Cr-1 & HLW-E-Cr-2 & HLW-E-Cr-3 & HLW-E-Cr-4 & HLW-E-Cr-5 & HLW-E-Cr-6 & HLW-E-Cr-7 & HLW-E-Cr-8 & HLW-E-Cr-9 \\
\hline & Waste Loading (wt\%) & $30 \%$ & $40 \%$ & $40 \%$ & $40 \%$ & $40 \%$ & $40 \%$ & $40 \%$ & $40 \%$ & $40 \%$ & $40 \%$ \\
\hline \multirow{16}{*}{ 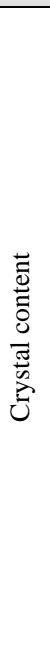 } & Glass as melted & No salt & $\mathrm{Cr} / \mathrm{S}$ salt & $\mathrm{Cr} / \mathrm{S}$ salt & $\mathrm{Cr} / \mathrm{S}$ salt & $\mathrm{Xl}+$ Trace salt & $\mathrm{Xl+Trace} \mathrm{salt}$ & Xl+Trace salt & No salt & $\mathrm{Cr} / \mathrm{S}$ salt & $\mathrm{Cr} / \mathrm{S}$ salt \\
\hline & $\mathrm{Xl}$ at $950^{\circ} \mathrm{C}$ & Sp & Sp + Pho & Sp + Cr-Fe & Sp & Sp & Sp + Pho & Sp + Cr-Fe & Sp & Sp & Sp \\
\hline & Glass after $\mathrm{HT}$ at $950^{\circ} \mathrm{C}$ & 0.1 & 0.5 & 0.3 & 0.3 & 0.8 & 0.9 & 0.9 & 0.5 & 0.2 & 0.7 \\
\hline & Glass after $\mathrm{HT}$ at $900^{\circ} \mathrm{C}$ & - & 0.9 & - & - & - & - & - & 0.6 & - & - \\
\hline & Glass after $\mathrm{HT}$ at $850^{\circ} \mathrm{C}$ & - & - & - & - & - & - & - & 0.6 & - & - \\
\hline & Glass after $\mathrm{HT}$ at $800^{\circ} \mathrm{C}$ & - & - & - & - & - & - & - & 1.2 & - & - \\
\hline & Glass after HT of CCC & - & - & - & - & - & - & - & & - & - \\
\hline & Viscosity at $1250^{\circ} \mathrm{C}$ & - & - & - & - & - & - & - & 23.0 & - & - \\
\hline & Viscosity at $1150^{\circ} \mathrm{C}$ & - & - & - & - & - & - & - & 46.8 & - & - \\
\hline & Viscosity at $1050^{\circ} \mathrm{C}$ & - & - & - & - & - & - & - & 120.3 & - & - \\
\hline & Viscosity at $950^{\circ} \mathrm{C}$ & - & - & - & - & - & - & - & 451.7 & - & - \\
\hline & Electric conductivity at $1250^{\circ} \mathrm{C}$ & - & - & - & - & - & - & - & 0.34 & - & - \\
\hline & Electric conductivity at $1150^{\circ} \mathrm{C}$ & - & - & - & - & - & - & - & 0.26 & - & - \\
\hline & Electric conductivity at $1050^{\circ} \mathrm{C}$ & - & - & - & - & - & - & - & 0.18 & - & - \\
\hline & Electric conductivity at $950^{\circ} \mathrm{C}$ & - & - & - & - & - & - & - & 0.11 & - & - \\
\hline & Glass transition temperature $\left({ }^{\circ} \mathrm{C}\right)$ & - & - & - & - & - & - & - & - & - & - \\
\hline \multirow{19}{*}{ 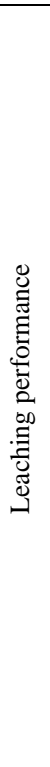 } & PCT & - & - & - & - & - & - & - & - & - & - \\
\hline & $\mathrm{B}(\mathrm{ppm})$ & - & - & - & - & - & - & - & - & - & 312.2 \\
\hline & $\mathrm{Li}(\mathrm{ppm})$ & - & - & - & - & - & - & - & - & - & 62.2 \\
\hline & $\mathrm{Na}(\mathrm{ppm})$ & - & - & - & - & - & - & - & - & - & 225.1 \\
\hline & Si (ppm) & - & - & - & - & - & - & - & - & - & 34.4 \\
\hline & $B(g / L)$ & - & - & - & - & - & - & - & - & - & 6.39 \\
\hline & Li (g/L) & - & - & - & - & - & - & - & - & - & 4.25 \\
\hline & $\mathrm{Na}(\mathrm{g} / \mathrm{L})$ & - & - & - & - & - & - & - & - & - & 3.49 \\
\hline & $\mathrm{Si}(\mathrm{g} / \mathrm{L})$ & - & - & - & - & - & - & - & - & - & 0.19 \\
\hline & PCT (for CCC sample) & - & - & - & - & - & - & - & - & - & - \\
\hline & $\mathrm{B}(\mathrm{ppm})$ & - & - & - & - & - & - & - & - & - & - \\
\hline & $\mathrm{Li}(\mathrm{ppm})$ & - & - & - & - & - & - & - & - & - & - \\
\hline & $\mathrm{Na}(\mathrm{ppm})$ & - & - & - & - & - & - & - & - & - & - \\
\hline & Si (ppm) & - & - & - & - & - & - & - & - & - & - \\
\hline & $B(g / L)$ & - & - & - & - & - & - & - & - & - & - \\
\hline & $\mathrm{Li}(\mathrm{g} / \mathrm{L})$ & - & - & - & - & - & - & - & - & - & - \\
\hline & $\mathrm{Na}(\mathrm{g} / \mathrm{L})$ & - & - & - & - & - & - & - & - & - & - \\
\hline & $\mathrm{Si}(\mathrm{g} / \mathrm{L})$ & - & - & - & - & - & - & - & - & - & - \\
\hline & TCLP (Pass/Fail) & - & - & - & - & - & - & - & Pass & - & - \\
\hline
\end{tabular}

$\mathrm{Sp}=$ spinel; Pho = phosphate; Nas = sodium aluminosilicate; $\mathrm{Cr}$-Fe = Cr-Fe-oxide

- Empty data field 
Table 3.6. Characterization of Glasses Formulated for Cr-Limited Waste (continued).

\begin{tabular}{|c|c|c|c|c|c|c|c|c|c|c|c|}
\hline & Glass Name & HLW-E-Cr-10 & HLW-E-Cr-11 & HLW-E-Cr-17 & HLW-E-Cr-18 & HLW-E-Cr-12 & HLW-E-Cr-15 & HLW-E-Cr-13 & HLW-E-Cr-16 & HLW-E-Cr-M & HLW-E-Cr-MUTh \\
\hline & Waste Loading (wt\%) & $40 \%$ & $40 \%$ & $42.5 \%$ & $42.5 \%$ & $45 \%$ & $45 \%$ & $50 \%$ & $50 \%$ & $32.50 \%$ & $32.50 \%$ \\
\hline \multirow{16}{*}{ 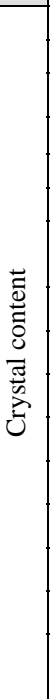 } & Glass as melted & No salt & No salt & $\mathrm{Cr} / \mathrm{S}$ salt & $\mathrm{Cr} / \mathrm{S}$ salt & Trace salt & $\mathrm{Cr} / \mathrm{S}$ salt & $\mathrm{Cr} / \mathrm{S}$ salt & $\mathrm{Cr} / \mathrm{S}$ salt & No salt & No salt \\
\hline & $\mathrm{Xl}$ at $950^{\circ} \mathrm{CSP}$ & - & Sp & Sp +Pho & $\mathrm{Sp}$ & Sp & Sp & $\mathrm{Sp}+\mathrm{Cr}-\mathrm{Fe}$ & $\mathrm{Sp}+\mathrm{Cr}-\mathrm{Fe}$ & Ni-rich oxide & $\mathrm{Sp}+\mathrm{Cr}-\mathrm{Fe}$ \\
\hline & Glass after $\mathrm{HT}$ at $950^{\circ} \mathrm{C}$ & 0.6 & - & 1.1 & 0.6 & 0.7 & 0.7 & 1.2 & 2.0 & 0.8 & 0.2 \\
\hline & Glass after $\mathrm{HT}$ at $900^{\circ} \mathrm{C}$ & 0.8 & - & - & - & - & - & - & - & 0.9 & 0.2 \\
\hline & Glass after $\mathrm{HT}$ at $850^{\circ} \mathrm{C}$ & 0.8 & - & - & - & - & - & - & - & NA & 0.4 \\
\hline & Glass after $\mathrm{HT}$ at $800^{\circ} \mathrm{C}$ & 2.0 & - & - & - & - & - & - & - & 2.9 & 0.1 \\
\hline & Glass after $\mathrm{HT}$ of CCC & $0.7(\mathrm{Sp}+\mathrm{Cr}-\mathrm{Fe})$ & - & - & - & - & - & - & - & $\begin{array}{c}1.3(\text { Sp + Pho } \\
++)\end{array}$ & $0.3(\mathrm{CrFe}+\mathrm{Sp}+\dagger)$ \\
\hline & Viscosity at $1250^{\circ} \mathrm{C}$ & 29.8 & - & - & - & - & - & - & - & 36.2 & 26.1 \\
\hline & Viscosity at $1150^{\circ} \mathrm{C}$ & 59.5 & - & - & - & - & - & - & - & 77.9 & 54.6 \\
\hline & Viscosity at $1050^{\circ} \mathrm{C}$ & 144.0 & - & - & - & - & - & - & - & 201.0 & 143.2 \\
\hline & Viscosity at $950^{\circ} \mathrm{C}$ & 466.1 & - & - & - & - & - & - & - & 664.7 & 529.5 \\
\hline & Electric conductivity at $1250^{\circ} \mathrm{C}$ & 0.49 & - & - & - & - & - & - & - & 0.28 & 0.31 \\
\hline & Electric conductivity at $1150^{\circ} \mathrm{C}$ & 0.37 & - & - & - & - & - & - & - & 0.20 & 0.24 \\
\hline & Electric conductivity at $1050^{\circ} \mathrm{C}$ & 0.25 & - & - & - & - & - & - & - & 0.14 & 0.16 \\
\hline & Electric conductivity at $950^{\circ} \mathrm{C}$ & 0.14 & - & - & - & - & - & - & - & 0.09 & 0.09 \\
\hline & $\begin{array}{c}\text { Glass transition temperature } \\
\left({ }^{\circ} \mathrm{C}\right)\end{array}$ & - & - & - & - & - & - & - & - & 455 & - \\
\hline \multirow{19}{*}{ 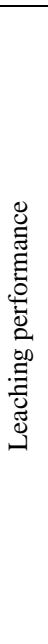 } & PCT & - & - & - & - & - & - & - & - & - & - \\
\hline & B (ppm) & 130.1 & 138.3 & 133.1 & - & 98.5 & - & 174.3 & - & 229.7 & 238.5 \\
\hline & $\mathrm{Li}(\mathrm{ppm})$ & 30.6 & 31.2 & 31.2 & - & 17.1 & - & 24.9 & - & 57.5 & 62.4 \\
\hline & $\mathrm{Na}(\mathrm{ppm})$ & 96.7 & 104.2 & 102.3 & - & 89.1 & - & 153.0 & - & 112.0 & 132.8 \\
\hline & Si (ppm) & 45.5 & 42.3 & 46.6 & - & 38.6 & - & 34.8 & - & 47.7 & 42.4 \\
\hline & $\mathrm{B}(\mathrm{g} / \mathrm{L})$ & 2.90 & 3.08 & 2.97 & - & 2.19 & - & 4.01 & - & 4.57 & 4.75 \\
\hline & $\mathrm{Li}(\mathrm{g} / \mathrm{L})$ & 1.99 & 2.02 & 2.03 & - & 1.10 & - & 2.44 & - & 3.36 & 3.66 \\
\hline & $\mathrm{Na}(\mathrm{g} / \mathrm{L})$ & 1.50 & 1.61 & 1.49 & - & 1.23 & - & 1.90 & - & 2.14 & 2.74 \\
\hline & Si $(g / L)$ & 0.23 & 0.22 & 0.25 & - & 0.22 & - & 0.21 & - & 0.22 & 0.20 \\
\hline & PCT (for CCC sample) & - & - & - & - & - & - & - & - & - & - \\
\hline & $\mathrm{B}(\mathrm{ppm})$ & 113.0 & - & - & - & - & - & - & - & - & - \\
\hline & Li (ppm) & 42.2 & - & - & - & - & - & - & - & - & - \\
\hline & $\mathrm{Na}(\mathrm{ppm})$ & 99.1 & - & - & - & - & - & - & - & - & - \\
\hline & Si (ppm) & 51.1 & - & - & - & - & - & - & - & - & - \\
\hline & $B(g / L)$ & 2.52 & - & - & - & - & - & - & - & - & - \\
\hline & $\mathrm{Li}(\mathrm{g} / \mathrm{L})$ & 2.74 & - & - & - & - & - & - & - & - & - \\
\hline & $\mathrm{Na}(\mathrm{g} / \mathrm{L})$ & 1.54 & - & - & - & - & - & - & - & - & - \\
\hline & $\mathrm{Si}(\mathrm{g} / \mathrm{L})$ & 0.26 & - & - & - & - & - & - & - & - & - \\
\hline & TCLP (Pass/Fail) & Pass & - & - & - & - & - & - & - & Pass & Pass \\
\hline
\end{tabular}

$\mathrm{Sp}=$ spinel; $\mathrm{Pho}=$ phosphate; Nas = sodium aluminosilicate; †bubbles submicron size

- Empty data field 
The Catholic University of America

Table 3.7. XRF Analysis of Yellow Salt Phase Collected from Glass Surface of a Crucible Melt. $\mathrm{B}_{2} \mathrm{O}_{3}$ and $\mathrm{Li}_{2} \mathrm{O}$ were not analyzed by XRF.

\begin{tabular}{|c|c|}
\hline Component & wt \% \\
\hline $\mathrm{Al}_{2} \mathrm{O}_{3}$ & 4.1 \\
\hline $\mathrm{Bi}_{2} \mathrm{O}_{3}$ & 1.1 \\
\hline $\mathrm{CaO}$ & 2.6 \\
\hline $\mathrm{Cr}_{2} \mathrm{O}_{3}$ & 16.1 \\
\hline $\mathrm{Fe}_{2} \mathrm{O}_{3}$ & 1.0 \\
\hline $\mathrm{K}_{2} \mathrm{O}$ & 22.8 \\
\hline $\mathrm{Na}_{2} \mathrm{O}$ & 20.1 \\
\hline $\mathrm{NiO}$ & 0.2 \\
\hline $\mathrm{P}_{2} \mathrm{O}_{5}$ & 6.2 \\
\hline $\mathrm{PbO}$ & 0.5 \\
\hline $\mathrm{SO}_{3}$ & 21.2 \\
\hline $\mathrm{SiO}_{2}$ & 4.2 \\
\hline $\mathrm{Sum}_{2}$ & 100 \\
\hline
\end{tabular}


Table 3.8. Composition and Properties of Chromium Limited Waste and Glass Formulation at $40 \%$ Waste Loading Initially Used in DM10 Melter Tests (wt\%).

\begin{tabular}{|c|c|c|c|c|}
\hline- & $\begin{array}{l}\text { Cr-Limited } \\
\text { Waste* }\end{array}$ & Waste in Glass & $\begin{array}{c}\text { Glass Forming } \\
\text { Additives }\end{array}$ & $\begin{array}{c}\text { Target Glass } \\
\text { HLW-E-Cr-10 }\end{array}$ \\
\hline $\mathrm{Al}_{2} \mathrm{O}_{3}$ & 27.64 & 11.06 & - & 11.06 \\
\hline $\mathrm{B}_{2} \mathrm{O}_{3}$ & 0.57 & 0.23 & 14.21 & 14.44 \\
\hline $\mathrm{BaO}$ & 0.03 & 0.01 & - & 0.01 \\
\hline $\mathrm{Bi}_{2} \mathrm{O}_{3}$ & 7.89 & 3.16 & - & 3.16 \\
\hline $\mathrm{CaO}$ & 2.67 & 1.07 & - & 1.07 \\
\hline $\mathrm{CdO}$ & 0.01 & 0.00 & - & $<0.01$ \\
\hline $\mathrm{Cr}_{2} \mathrm{O}_{3}$ & 3.32 & 1.33 & - & 1.33 \\
\hline $\mathrm{F}$ & 2.17 & 0.87 & - & 0.87 \\
\hline $\mathrm{Fe}_{2} \mathrm{O}_{3}$ & 14.22 & 5.69 & - & 5.69 \\
\hline $\mathrm{K}_{2} \mathrm{O}$ & 0.40 & 0.16 & 5.26 & 5.42 \\
\hline $\mathrm{Li}_{2} \mathrm{O}$ & 0.39 & 0.16 & 3.16 & 3.31 \\
\hline $\mathrm{MgO}$ & 0.17 & 0.07 & - & 0.07 \\
\hline $\mathrm{Na}_{2} \mathrm{O}$ & 21.75 & 8.70 & - & 8.70 \\
\hline $\mathrm{NiO}$ & 1.15 & 0.46 & - & 0.46 \\
\hline $\mathrm{P}_{2} \mathrm{O}_{5}$ & 3.62 & 1.45 & - & 1.45 \\
\hline $\mathrm{PbO}$ & 0.52 & 0.21 & - & 0.21 \\
\hline $\mathrm{SiO}_{2}$ & 11.43 & 4.57 & 37.37 & 41.94 \\
\hline $\mathrm{TiO}_{2}$ & 0.01 & $<0.01$ & - & $<0.01$ \\
\hline $\mathrm{SO}_{3}$ & 1.65 & 0.66 & - & 0.66 \\
\hline $\mathrm{ZnO}$ & 0.27 & 0.11 & - & 0.11 \\
\hline $\mathrm{ZrO}_{2}$ & 0.12 & 0.05 & - & 0.05 \\
\hline Sum & 100 & 40 & 60 & 100 \\
\hline
\end{tabular}

\begin{tabular}{|c|c|c|c|}
\hline \multicolumn{3}{|c|}{ Viscosity @1150 $\mathrm{C}, \mathrm{P}$} & 59 \\
\hline \multicolumn{3}{|c|}{ Conductivity @1150ㄷ, S/cm } & 0.37 \\
\hline \multicolumn{3}{|c|}{ Crystal Content, As Melted } & None \\
\hline \multicolumn{3}{|c|}{ Crystal Content, $72 \mathrm{hr}$ at $950^{\circ} \mathrm{C}$} & $\sim 0.6 \mathrm{vol} \%$ \\
\hline \multicolumn{3}{|c|}{ TCLP } & Pass \\
\hline \multirow{4}{*}{ PCT, g/L } & - & DWPF-EA & HLW-E-Cr-10 \\
\hline & B & 16.7 & 2.9 \\
\hline & $\mathrm{Li}$ & 9.6 & 2 \\
\hline & $\mathrm{Na}$ & 13.3 & 1.5 \\
\hline
\end{tabular}

* Renormalized from Ref. [3] after removal of radioactive components

- Empty data field 
Table 3.9. Target Composition and XRF Analysis of Glasses Formulated for Al-Limited Waste.

\begin{tabular}{|c|c|c|c|c|c|c|c|c|c|c|c|c|c|c|}
\hline \multirow{2}{*}{-} & \multirow{2}{*}{$\begin{array}{l}\text { Al-Limited } \\
\text { Waste }\end{array}$} & \multirow{2}{*}{$\begin{array}{l}\text { Al-Limited Waste } \\
\text { (non rad)\# }\end{array}$} & \multicolumn{2}{|c|}{ HLW-E-Al-1 } & \multicolumn{2}{|c|}{ HLW-E-Al-2 } & \multicolumn{2}{|c|}{ HLW-E-Al-3 } & \multicolumn{2}{|c|}{ HLW-E-Al-4 } & \multicolumn{2}{|c|}{ HLW-E-Al-5 } & \multicolumn{2}{|c|}{ HLW-E-Al-6 } \\
\hline & & & Target & XRF & Target & $\mathrm{XRF}$ & Target & XRF & Target & XRF & Target & XRF & Target & XRF \\
\hline $\mathrm{Al}_{2} \mathrm{O}_{3}$ & 49.21 & 53.27 & 26.63 & 24.82 & 26.63 & 25.91 & 26.63 & 26.02 & 26.63 & 25.74 & 26.63 & 25.97 & 26.63 & 25.87 \\
\hline $\mathrm{B}_{2} \mathrm{O}_{3}{ }^{*}$ & 0.39 & 0.42 & 10.21 & 10.21 & 3.21 & 3.21 & 8.21 & 8.21 & 10.21 & 10.21 & 8.21 & 8.21 & 12.21 & 12.21 \\
\hline $\mathrm{BaO}$ & 0.11 & 0.12 & 0.06 & 0.06 & 0.06 & 0.06 & 0.06 & 0.05 & 0.06 & 0.06 & 0.06 & 0.05 & 0.06 & 0.05 \\
\hline $\mathrm{Bi}_{2} \mathrm{O}_{3}$ & 2.35 & 2.54 & 1.27 & 1.31 & 1.27 & 1.37 & 1.27 & 1.30 & 1.27 & 1.37 & 1.27 & 1.41 & 1.27 & 1.33 \\
\hline $\mathrm{CaO}$ & 2.21 & 2.39 & 1.20 & 1.33 & 14.20 & 14.72 & 14.20 & 14.32 & 16.20 & 16.53 & 11.20 & 11.61 & 18.20 & 18.31 \\
\hline $\mathrm{CdO}$ & 0.05 & 0.05 & 0.03 & 0.00 & 0.03 & 0.00 & 0.03 & 0.00 & 0.03 & 0.00 & 0.03 & 0.00 & 0.03 & 0.00 \\
\hline $\mathrm{Cr}_{2} \mathrm{O}_{3}$ & 1.07 & 1.16 & 0.58 & 0.78 & 0.58 & 0.70 & 0.58 & 0.67 & 0.58 & 0.62 & 0.58 & 0.68 & 0.58 & 0.65 \\
\hline $\mathrm{F}^{*}$ & 1.37 & 1.48 & 0.74 & - & 0.74 & - & 0.74 & - & 0.74 & - & 0.74 & - & 0.74 & - \\
\hline $\mathrm{Fe}_{2} \mathrm{O}_{3}$ & 12.11 & 13.11 & 6.55 & 6.99 & 6.55 & 6.56 & 6.55 & 6.38 & 6.55 & 6.59 & 6.55 & 6.69 & 6.55 & 6.46 \\
\hline $\mathrm{K}_{2} \mathrm{O}$ & 0.29 & 0.31 & 0.16 & 0.23 & 1.56 & 1.72 & 0.16 & 0.26 & 0.16 & 0.25 & 0.16 & 0.26 & 0.16 & 0.25 \\
\hline $\mathrm{Li}_{2} \mathrm{O}^{*}$ & 0.35 & 0.38 & 0.19 & 0.19 & 0.19 & 0.19 & 0.19 & 0.19 & 0.19 & 0.19 & 0.19 & 0.19 & 0.19 & 0.19 \\
\hline $\mathrm{MgO}$ & 0.24 & 0.26 & 9.13 & 7.77 & 0.13 & 0.10 & 0.13 & 0.13 & 0.13 & 0.11 & 0.13 & 0.08 & 0.13 & 0.11 \\
\hline $\mathrm{Na}_{2} \mathrm{O}$ & 7.35 & 7.96 & 10.98 & 11.73 & 9.58 & 9.51 & 8.98 & 9.94 & 6.98 & 7.32 & 11.98 & 12.32 & 4.98 & 5.35 \\
\hline $\mathrm{NiO}$ & 0.82 & 0.89 & 0.44 & 0.44 & 0.44 & 0.41 & 0.44 & 0.40 & 0.44 & 0.40 & 0.44 & 0.41 & 0.44 & 0.41 \\
\hline $\mathrm{P}_{2} \mathrm{O}_{5}$ & 2.16 & 2.34 & 1.17 & 1.31 & 1.17 & 1.51 & 1.17 & 1.35 & 1.17 & 1.19 & 1.17 & 1.29 & 1.17 & 1.16 \\
\hline $\mathrm{PbO}$ & 0.84 & 0.91 & 0.45 & 0.42 & 0.45 & 0.43 & 0.45 & 0.40 & 0.45 & 0.44 & 0.45 & 0.46 & 0.45 & 0.42 \\
\hline $\mathrm{SiO}_{2}$ & 10.05 & 10.88 & 29.44 & 31.66 & 32.44 & 32.92 & 29.44 & 29.80 & 27.44 & 28.05 & 29.44 & 29.55 & 25.44 & 26.40 \\
\hline $\mathrm{ThO}_{2}$ & 0.37 & 0.00 & 0.00 & 0.00 & 0.00 & 0.00 & 0.00 & 0.00 & 0.00 & 0.00 & 0.00 & 0.00 & 0.00 & 0.00 \\
\hline $\mathrm{TiO}_{2}$ & 0.02 & 0.02 & 0.01 & 0.05 & 0.01 & 0.04 & 0.01 & 0.04 & 0.01 & 0.07 & 0.01 & 0.06 & 0.01 & 0.06 \\
\hline $\mathrm{SO}_{3}$ & 0.41 & 0.44 & 0.22 & 0.17 & 0.22 & 0.29 & 0.22 & 0.24 & 0.22 & 0.28 & 0.22 & 0.21 & 0.22 & 0.22 \\
\hline $\mathrm{ZnO}$ & 0.17 & 0.18 & 0.09 & 0.09 & 0.09 & 0.10 & 0.09 & 0.09 & 0.09 & 0.10 & 0.09 & 0.10 & 0.09 & 0.10 \\
\hline $\mathrm{ZrO}_{2}$ & 0.81 & 0.88 & 0.44 & 0.39 & 0.44 & 0.18 & 0.44 & 0.17 & 0.44 & 0.40 & 0.44 & 0.40 & 0.44 & 0.39 \\
\hline $\mathrm{U}_{3} \mathrm{O}_{8}$ & 7.25 & 0.00 & 0.00 & 0.00 & 0.00 & 0.00 & 0.00 & 0.00 & 0.00 & 0.00 & 0.00 & 0.00 & 0.00 & 0.00 \\
\hline
\end{tabular}

*In XRF analysis target concentrations are used for $\mathrm{B}_{2} \mathrm{O}_{3}$ and $\mathrm{Li}_{2} \mathrm{O} ; \mathrm{F}$ was not analyzed.

\#The non-radioactive version of the waste is calculated by renormalization of the waste constituents after removing $\mathrm{U}_{3} \mathrm{O}_{8}$ and $\mathrm{ThO}_{2}$.

- Empty data field 
Table 3.9. Target Composition and XRF Analysis of Glasses Formulated for Al-Limited Waste (continued).

\begin{tabular}{|c|c|c|c|c|c|c|c|c|c|c|c|c|c|c|c|c|c|c|}
\hline \multirow{2}{*}{-} & \multicolumn{2}{|c|}{ HLW-E-Al-7 } & \multicolumn{2}{|c|}{ HLW-E-Al-8 } & \multicolumn{2}{|c|}{ HLW-E-Al-9 } & \multicolumn{2}{|c|}{ HLW-E-Al-10 } & \multicolumn{2}{|c|}{ HLW-E-A1-11 } & \multicolumn{2}{|c|}{ HLW-E-Al-12 } & \multicolumn{2}{|c|}{ HLW-E-Al-13 } & \multicolumn{2}{|c|}{ HLW-E-Al-14 } & \multicolumn{2}{|c|}{ HLW-E-Al-15 } \\
\hline & Target & XRF & Target & XRF & Target & XRF & Target & XRF & Target & XRF & Target & XRF & Target & XRF & Target & XRF & Target & XRF \\
\hline $\mathrm{Al}_{2} \mathrm{O}_{3}$ & 26.63 & 26.02 & 26.63 & 26.32 & 26.63 & 26.25 & 26.63 & 26.16 & 26.63 & 25.89 & 23.97 & 23.34 & 25.30 & 24.78 & 26.63 & 26.76 & 25.30 & 25.00 \\
\hline $\mathrm{B}_{2} \mathrm{O}_{3}{ }^{*}$ & 15.21 & 15.21 & 18.21 & 18.21 & 18.21 & 18.21 & 20.21 & 20.21 & 16.21 & 16.21 & 20.19 & 20.19 & 19.29 & 19.29 & 18.21 & 18.21 & 20.20 & 20.20 \\
\hline $\mathrm{BaO}$ & 0.06 & 0.07 & 0.06 & 0.07 & 0.06 & 0.07 & 0.06 & 0.00 & 0.06 & 0.06 & 0.05 & 0.06 & 0.06 & 0.05 & 0.06 & 0.05 & 0.06 & 0.06 \\
\hline $\mathrm{Bi}_{2} \mathrm{O}_{3}$ & 1.27 & 1.33 & 1.27 & 1.26 & 1.27 & 1.33 & 1.27 & 1.34 & 1.27 & 1.35 & 1.14 & 1.20 & 1.21 & 1.27 & 1.27 & 1.37 & 1.21 & 1.36 \\
\hline $\mathrm{CaO}$ & 20.20 & 20.28 & 18.20 & 17.99 & 16.20 & 16.49 & 14.20 & 14.46 & 14.20 & 14.20 & 14.08 & 14.36 & 13.55 & 14.00 & 1.20 & 1.25 & 1.14 & 1.28 \\
\hline CdO & 0.03 & 0.00 & 0.03 & 0.00 & 0.03 & 0.00 & 0.03 & 0.00 & 0.03 & 0.00 & 0.02 & 0.00 & 0.03 & 0.00 & 0.03 & 0.00 & 0.03 & 0.00 \\
\hline $\mathrm{Cr}_{2} \mathrm{O}_{3}$ & 0.58 & 0.67 & 0.58 & 0.62 & 0.58 & 0.68 & 0.58 & 0.64 & 0.58 & 0.68 & 0.52 & 0.62 & 0.55 & 0.66 & 0.58 & 0.67 & 0.55 & 0.62 \\
\hline $\mathrm{F}^{*}$ & 0.74 & - & 0.74 & - & 0.74 & - & 0.74 & - & 0.74 & - & 0.67 & - & 0.70 & - & 0.74 & - & 0.70 & - \\
\hline $\mathrm{Fe}_{2} \mathrm{O}_{3}$ & 6.55 & 6.25 & 6.55 & 6.10 & 6.55 & 6.43 & 6.55 & 6.41 & 6.55 & 6.39 & 5.90 & 5.77 & 6.23 & 6.14 & 6.55 & 6.42 & 6.23 & 6.43 \\
\hline $\mathrm{K}_{2} \mathrm{O}$ & 0.16 & 0.20 & 0.16 & 0.20 & 0.16 & 0.21 & 0.16 & 0.24 & 0.16 & 0.21 & 0.14 & 0.20 & 0.15 & 0.24 & 0.16 & 0.25 & 0.15 & 0.23 \\
\hline $\mathrm{Li}_{2} \mathrm{O}^{*}$ & 1.19 & 1.19 & 1.19 & 1.19 & 3.19 & 3.09 & 5.19 & 5.19 & 5.19 & 5.19 & 5.17 & 5.17 & 4.95 & 4.95 & 7.19 & 7.19 & 7.68 & 7.68 \\
\hline $\mathrm{MgO}$ & 0.13 & 0.11 & 0.13 & 0.07 & 0.13 & 0.10 & 0.13 & 0.12 & 0.13 & 0.10 & 0.12 & 0.11 & 0.12 & 0.11 & 0.13 & 0.07 & 0.12 & 0.00 \\
\hline $\mathrm{Na}_{2} \mathrm{O}$ & 3.98 & 4.14 & 3.98 & 4.19 & 3.98 & 4.09 & 3.98 & 4.05 & 3.98 & 4.67 & 3.58 & 3.65 & 3.78 & 3.94 & 3.98 & 4.03 & 3.78 & 3.93 \\
\hline $\mathrm{NiO}$ & 0.44 & 0.42 & 0.44 & 0.41 & 0.44 & 0.41 & 0.44 & 0.43 & 0.44 & 0.42 & 0.40 & 0.40 & 0.42 & 0.41 & 0.44 & 0.42 & 0.42 & 0.42 \\
\hline $\mathrm{P}_{2} \mathrm{O}_{5}$ & 1.17 & 1.39 & 1.17 & 1.41 & 1.17 & 1.27 & 1.17 & 1.26 & 1.17 & 1.48 & 1.05 & 1.24 & 1.11 & 1.25 & 1.17 & 1.29 & 1.11 & 1.24 \\
\hline $\mathrm{PbO}$ & 0.45 & 0.43 & 0.45 & 0.42 & 0.45 & 0.43 & 0.45 & 0.43 & 0.45 & 0.44 & 0.41 & 0.39 & 0.43 & 0.40 & 0.45 & 0.43 & 0.43 & 0.44 \\
\hline $\mathrm{SiO}_{2}$ & 20.44 & 21.37 & 19.44 & 20.68 & 19.44 & 19.99 & 17.44 & 18.20 & 21.44 & 21.95 & 21.90 & 22.63 & 21.39 & 21.78 & 30.44 & 30.71 & 30.17 & 30.26 \\
\hline $\mathrm{ThO}_{2}$ & 0.00 & 0.00 & 0.00 & 0.00 & 0.00 & 0.00 & 0.00 & 0.00 & 0.00 & 0.00 & 0.00 & 0.00 & 0.00 & 0.00 & 0.00 & 0.00 & 0.00 & 0.00 \\
\hline $\mathrm{TiO}_{2}$ & 0.01 & 0.03 & 0.01 & 0.03 & 0.01 & 0.05 & 0.01 & 0.06 & 0.01 & 0.03 & 0.01 & 0.03 & 0.01 & 0.03 & 0.01 & 0.07 & 0.01 & 0.04 \\
\hline $\mathrm{SO}_{3}$ & 0.22 & 0.26 & 0.22 & 0.24 & 0.22 & 0.19 & 0.22 & 0.19 & 0.22 & 0.15 & 0.20 & 0.13 & 0.21 & 0.21 & 0.22 & 0.26 & 0.21 & 0.22 \\
\hline $\mathrm{ZnO}$ & 0.09 & 0.12 & 0.09 & 0.11 & 0.09 & 0.11 & 0.09 & 0.12 & 0.09 & 0.11 & 0.08 & 0.10 & 0.09 & 0.11 & 0.09 & 0.11 & 0.09 & 0.12 \\
\hline $\mathrm{ZrO}_{2}$ & 0.44 & 0.43 & 0.44 & 0.40 & 0.44 & 0.41 & 0.44 & 0.41 & 0.44 & 0.41 & 0.39 & 0.37 & 0.42 & 0.30 & 0.44 & 0.40 & 0.42 & 0.39 \\
\hline $\mathrm{U}_{3} \mathrm{O}_{8}$ & 0.00 & 0.00 & 0.00 & 0.00 & 0.00 & 0.00 & 0.00 & 0.00 & 0.00 & 0.00 & 0.00 & 0.00 & 0.00 & 0.00 & 0.00 & 0.00 & 0.00 & 0.00 \\
\hline
\end{tabular}

*In XRF analysis target concentrations are used for $\mathrm{B}_{2} \mathrm{O}_{3}$ and $\mathrm{Li}_{2} \mathrm{O}$; $\mathrm{F}$ was not analyzed.

- Empty data field 
Table 3.9. Target Composition and XRF Analysis of Glasses Formulated for Al-Limited Waste (continued).

\begin{tabular}{|c|c|c|c|c|c|c|c|c|c|c|c|c|c|c|c|c|c|c|}
\hline & \multicolumn{2}{|c|}{ HLW-E-Al-16 } & \multicolumn{2}{|c|}{ HLW-E-A1-17 } & \multicolumn{2}{|c|}{ HLW-E-A1-18 } & \multicolumn{2}{|c|}{ HLW-E-Al-19 } & \multicolumn{2}{|c|}{ HLW-E-Al-20 } & \multicolumn{2}{|c|}{ HLW-E-Al-21 } & \multicolumn{2}{|c|}{ HLW-E-Al-22 } & \multicolumn{2}{|c|}{ HLW-E-Al-23 } & \multicolumn{2}{|c|}{ HLW-E-Al-24 } \\
\hline & Target & XRF & Target & XRF & Target & XRF & Target & XRF & Target & XRF & Target & XRF & Target & XRF & Target & XRF & Target & XRF \\
\hline $\mathrm{Al}_{2} \mathrm{O}_{3}$ & 23.97 & 24.05 & 29.30 & 29.05 & 23.97 & 23.32 & 26.63 & 26.47 & 25.30 & 25.34 & 25.30 & 25.42 & 25.30 & 25.39 & 23.97 & 23.81 & 18.64 & 18.54 \\
\hline $\mathrm{B}_{2} \mathrm{O}_{3}{ }^{*}$ & 20.19 & 20.19 & 18.23 & 18.23 & 19.19 & 19.19 & 14.41 & 14.41 & 14.40 & 14.40 & 14.00 & 14.00 & 14.00 & 14.00 & 14.19 & 14.19 & 15.15 & 15.15 \\
\hline $\mathrm{BaO}$ & 0.05 & 0.05 & 0.07 & 0.08 & 0.05 & 0.08 & 0.06 & 0.07 & 0.06 & 0.06 & 0.06 & 0.08 & 0.06 & 0.06 & 0.05 & 0.05 & 0.04 & 0.05 \\
\hline $\mathrm{Bi}_{2} \mathrm{O}_{3}$ & 1.14 & 1.20 & 1.40 & 1.46 & 1.14 & 1.26 & 1.27 & 1.32 & 1.21 & 1.24 & 1.21 & 1.23 & 1.21 & 1.15 & 1.14 & 1.19 & 0.89 & 1.00 \\
\hline $\mathrm{CaO}$ & 1.08 & 1.12 & 1.32 & 1.39 & 13.08 & 13.71 & 1.20 & 1.27 & 1.14 & 1.19 & 1.14 & 1.18 & 1.14 & 1.12 & 1.08 & 1.12 & 0.84 & 0.91 \\
\hline $\mathrm{CdO}$ & 0.02 & 0.00 & 0.03 & 0.00 & 0.02 & 0.00 & 0.03 & 0.00 & 0.03 & 0.00 & 0.03 & 0.00 & 0.03 & 0.00 & 0.02 & 0.00 & 0.02 & 0.00 \\
\hline $\mathrm{Cr}_{2} \mathrm{O}_{3}$ & 0.52 & 0.60 & 0.64 & 0.74 & 0.52 & 0.64 & 0.58 & 0.67 & 0.55 & 0.64 & 0.55 & 0.66 & 0.55 & 0.52 & 0.52 & 0.64 & 0.41 & 0.51 \\
\hline $\mathrm{F}^{*}$ & 0.67 & - & 0.82 & - & 0.67 & - & 0.74 & - & 0.70 & - & 0.70 & - & 0.70 & - & 0.67 & - & 0.52 & - \\
\hline $\mathrm{Fe}_{2} \mathrm{O}_{3}$ & 5.90 & 5.78 & 7.21 & 0.70 & 5.90 & 5.99 & 6.55 & 6.30 & 6.23 & 5.93 & 6.23 & 5.94 & 6.23 & 5.61 & 5.90 & 5.66 & 4.59 & 4.69 \\
\hline $\mathrm{K}_{2} \mathrm{O}$ & 0.14 & 0.23 & 0.17 & 0.25 & 0.14 & 0.21 & 0.16 & 0.21 & 0.15 & 0.22 & 0.15 & 0.22 & 5.15 & 4.91 & 0.14 & 0.18 & 0.11 & 0.19 \\
\hline $\mathrm{Li}_{2} \mathrm{O}^{*}$ & 7.67 & 7.67 & 7.71 & 7.71 & 5.17 & 5.17 & 3.49 & 3.49 & 4.18 & 4.18 & 5.03 & 5.03 & 5.03 & 5.03 & 3.57 & 3.57 & 3.53 & 3.53 \\
\hline $\mathrm{MgO}$ & 0.12 & 0.08 & 0.14 & 0.11 & 0.12 & 0.08 & 0.13 & 0.10 & 0.12 & 0.10 & 0.12 & 0.07 & 0.12 & 0.10 & 0.12 & 0.08 & 0.09 & 0.14 \\
\hline $\mathrm{Na}_{2} \mathrm{O}$ & 3.58 & 3.66 & 4.38 & 4.62 & 3.58 & 3.62 & 13.98 & 14.82 & 13.78 & 14.32 & 14.78 & 14.90 & 9.78 & 10.68 & 14.08 & 14.78 & 14.78 & 14.61 \\
\hline $\mathrm{NiO}$ & 0.40 & 0.38 & 0.49 & 0.45 & 0.40 & 0.40 & 0.44 & 0.40 & 0.42 & 0.38 & 0.42 & 0.40 & 0.42 & 0.37 & 0.40 & 0.38 & 0.31 & 0.32 \\
\hline $\mathrm{P}_{2} \mathrm{O}_{5}$ & 3.55 & 3.82 & 1.29 & 1.42 & 1.05 & 1.14 & 1.17 & 1.30 & 1.11 & 1.25 & 1.11 & 1.26 & 1.11 & 1.28 & 1.05 & 1.23 & 0.82 & 0.94 \\
\hline $\mathrm{PbO}$ & 0.41 & 0.38 & 0.50 & 0.48 & 0.41 & 0.41 & 0.45 & 0.41 & 0.43 & 0.39 & 0.43 & 0.39 & 0.43 & 0.36 & 0.41 & 0.37 & 0.32 & 0.34 \\
\hline $\mathrm{SiO}_{2}$ & 29.90 & 29.97 & 25.48 & 26.04 & 21.90 & 22.00 & 27.94 & 27.89 & 29.47 & 29.54 & 28.02 & 28.35 & 28.02 & 28.58 & 32.00 & 31.95 & 35.81 & 35.72 \\
\hline $\mathrm{ThO}_{2}$ & 0.00 & 0.00 & 0.00 & 0.00 & 0.00 & 0.00 & 0.00 & 0.00 & 0.00 & 0.00 & 0.00 & 0.00 & 0.00 & 0.00 & 0.00 & 0.00 & 0.00 & 0.00 \\
\hline $\mathrm{TiO}_{2}$ & 0.01 & 0.05 & 0.01 & 0.05 & 0.01 & 0.04 & 0.01 & 0.04 & 0.01 & 0.04 & 0.01 & 0.05 & 0.01 & 0.04 & 0.01 & 0.07 & 0.01 & 0.05 \\
\hline $\mathrm{SO}_{3}$ & 0.20 & 0.23 & 0.24 & 0.25 & 0.20 & 0.19 & 0.22 & 0.26 & 0.21 & 0.23 & 0.21 & 0.27 & 0.21 & 0.26 & 0.20 & 0.26 & 0.16 & 0.20 \\
\hline $\mathrm{ZnO}$ & 0.08 & 0.10 & 0.10 & 0.13 & 0.08 & 0.11 & 0.09 & 0.12 & 0.09 & 0.10 & 0.09 & 0.12 & 0.09 & 0.10 & 0.08 & 0.10 & 0.06 & 0.09 \\
\hline $\mathrm{ZrO}_{2}$ & 0.39 & 0.36 & 0.48 & 0.45 & 2.39 & 2.32 & 0.44 & 0.40 & 0.42 & 0.38 & 0.42 & 0.39 & 0.42 & 0.37 & 0.39 & 0.32 & 2.91 & 2.90 \\
\hline $\mathrm{U}_{3} \mathrm{O}_{8}$ & 0.00 & 0.00 & 0.00 & 0.00 & 0.00 & 0.00 & 0.00 & 0.00 & 0.00 & 0.00 & 0.00 & 0.00 & 0.00 & 0.00 & 0.00 & 0.00 & 0.00 & 0.00 \\
\hline
\end{tabular}

*In XRF analysis target concentrations are used for $\mathrm{B}_{2} \mathrm{O}_{3}$ and $\mathrm{Li}_{2} \mathrm{O}$; $\mathrm{F}$ was not analyzed.

- Empty data field 
Table 3.9. Target Composition and XRF Analysis of Glasses Formulated for Al-Limited Waste (continued).

\begin{tabular}{|c|c|c|c|c|c|c|c|c|c|c|c|c|c|}
\hline \multirow{2}{*}{-} & \multicolumn{2}{|c|}{ HLW-E-Al-25 } & \multicolumn{2}{|c|}{ HLW-E-Al-26 } & \multicolumn{3}{|c|}{ HLW-E-Al-27 } & \multicolumn{2}{|c|}{ HLW-E-Al-28 } & \multicolumn{2}{|c|}{ HLW-E-Al-29 } & \multicolumn{2}{|c|}{ HLW-E-Al27UTh } \\
\hline & Target & $\mathrm{XRF}$ & Target & XRF & Target & XRF-27 & XRF-27R1 & Target & XRF-28 & Target & XRF-29 & Target & XRF \\
\hline $\mathrm{Al}_{2} \mathrm{O}_{3}$ & 23.97 & 23.63 & 23.97 & 22.37 & 23.97 & 23.68 & 23.58 & 25.30 & 25.03 & 25.30 & 24.86 & 22.14 & 22.03 \\
\hline $\mathrm{B}_{2} \mathrm{O}_{3}{ }^{*}$ & 14.19 & 14.19 & 16.19 & 16.19 & 15.19 & 15.19 & 15.19 & 14.40 & 14.40 & 14.40 & 14.40 & 15.18 & 15.18 \\
\hline $\mathrm{BaO}$ & 0.05 & 0.05 & 0.05 & 0.05 & 0.05 & 0.07 & 0.07 & 0.06 & 0.06 & 0.06 & 0.06 & 0.05 & 0.03 \\
\hline $\mathrm{Bi}_{2} \mathrm{O}_{3}$ & 1.14 & 1.24 & 1.14 & 1.31 & 1.14 & 1.31 & 1.26 & 1.21 & 1.35 & 1.21 & 1.34 & 1.06 & 1.14 \\
\hline $\mathrm{CaO}$ & 6.08 & 6.42 & 1.08 & 1.19 & 6.08 & 6.41 & 6.17 & 6.14 & 6.46 & 9.14 & 9.31 & 5.99 & 6.29 \\
\hline $\mathrm{CdO}$ & 0.02 & 0.00 & 0.02 & 0.00 & 0.02 & 0.00 & 0.00 & 0.03 & 0.00 & 0.03 & 0.00 & 0.02 & 0.00 \\
\hline $\mathrm{Cr}_{2} \mathrm{O}_{3}$ & 0.52 & 0.63 & 0.52 & 0.66 & 0.52 & 0.54 & 0.62 & 0.55 & 0.67 & 0.55 & 0.66 & 0.48 & 0.58 \\
\hline $\mathrm{F}^{*}$ & 0.67 & - & 0.67 & - & 0.67 & - & - & 0.70 & - & 0.70 & - & 0.62 & - \\
\hline $\mathrm{Fe}_{2} \mathrm{O}_{3}$ & 5.90 & 6.00 & 5.90 & 6.21 & 5.90 & 6.18 & 5.89 & 6.23 & 6.39 & 6.23 & 6.27 & 5.45 & 5.43 \\
\hline $\mathrm{K}_{2} \mathrm{O}$ & 0.14 & 0.19 & 0.14 & 0.24 & 0.14 & 0.19 & 0.22 & 0.15 & 0.21 & 0.15 & 0.23 & 0.13 & 0.20 \\
\hline $\mathrm{Li}_{2} \mathrm{O}^{*}$ & 3.57 & 3.57 & 3.57 & 3.57 & 3.57 & 3.57 & 3.57 & 3.68 & 3.68 & 2.68 & 2.68 & 3.56 & 3.56 \\
\hline $\mathrm{MgO}$ & 0.12 & 0.07 & 0.12 & 0.11 & 0.12 & 0.00 & 0.10 & 0.12 & 0.07 & 0.12 & 0.08 & 0.11 & 0.11 \\
\hline $\mathrm{Na}_{2} \mathrm{O}$ & 9.58 & 10.04 & 13.08 & 13.42 & 9.58 & 9.78 & 10.13 & 9.78 & 10.26 & 9.78 & 10.36 & 9.31 & 9.08 \\
\hline $\mathrm{NiO}$ & 0.40 & 0.40 & 0.40 & 0.41 & 0.40 & 0.42 & 0.38 & 0.42 & 0.42 & 0.42 & 0.41 & 0.37 & 0.37 \\
\hline $\mathrm{P}_{2} \mathrm{O}_{5}$ & 1.05 & 1.19 & 1.05 & 1.27 & 1.05 & 1.20 & 1.16 & 1.11 & 1.26 & 1.11 & 1.21 & 0.97 & 1.11 \\
\hline $\mathrm{PbO}$ & 0.41 & 0.40 & 0.41 & 0.42 & 0.41 & 0.42 & 0.39 & 0.43 & 0.44 & 0.43 & 0.43 & 0.38 & 0.37 \\
\hline $\mathrm{SiO}_{2}$ & 31.50 & 31.18 & 31.00 & 31.66 & 30.50 & 30.13 & 30.46 & 28.97 & 28.44 & 26.97 & 26.81 & 30.12 & 30.19 \\
\hline $\mathrm{ThO}_{2}$ & 0.00 & 0.00 & 0.00 & 0.00 & 0.00 & 0.00 & 0.00 & 0.00 & 0.00 & 0.00 & 0.00 & 0.17 & 0.02 \\
\hline $\mathrm{TiO}_{2}$ & 0.01 & 0.03 & 0.01 & 0.04 & 0.01 & 0.04 & 0.05 & 0.01 & 0.05 & 0.01 & 0.06 & 0.01 & 0.06 \\
\hline $\mathrm{SO}_{3}$ & 0.20 & 0.23 & 0.20 & 0.26 & 0.20 & 0.22 & 0.22 & 0.21 & 0.25 & 0.21 & 0.23 & 0.18 & 0.24 \\
\hline $\mathrm{ZnO}$ & 0.08 & 0.10 & 0.08 & 0.11 & 0.08 & 0.11 & 0.11 & 0.09 & 0.11 & 0.09 & 0.11 & 0.08 & 0.11 \\
\hline $\mathrm{ZrO}_{2}$ & 0.39 & 0.37 & 0.39 & 0.40 & 0.39 & 0.41 & 0.36 & 0.42 & 0.39 & 0.42 & 0.41 & 0.36 & 0.30 \\
\hline $\mathrm{U}_{3} \mathrm{O}_{8}$ & 0.00 & 0.00 & 0.00 & 0.00 & 0.00 & 0.00 & 0.00 & 0.00 & 0.00 & 0.00 & 0.00 & 3.26 & 3.44 \\
\hline
\end{tabular}

*In XRF analysis target concentrations are used for $\mathrm{B}_{2} \mathrm{O}_{3}$ and $\mathrm{Li}_{2} \mathrm{O}$; F was not analyzed.

- Empty data field 
Table 3.10. Characterization of Glasses Formulated for Al-Limited Waste.

\begin{tabular}{|c|c|c|c|c|c|c|c|c|}
\hline & Glass Name & HLW-E-Al-24 & HLW-E-Al-16 & HLW-E-Al-12 & HLW-E-Al-18 & HLW-E-Al-23 & HLW-E-Al-26 & HLW-E-Al-25 \\
\hline & Waste loading (wt\%) & 35 & 45 & 45 & 45 & 45 & 45 & 45 \\
\hline & Glass former type & $\mathrm{Na}+\mathrm{Li}$ & $\mathrm{Li}$ & $\mathrm{Ca}+\mathrm{Li}$ & $\mathrm{Ca}+\mathrm{Li}$ & $\mathrm{Na}+\mathrm{Li}$ & $\mathrm{Na}+\mathrm{Li}$ & $\mathrm{Na}+\mathrm{Li}+\mathrm{Ca}$ \\
\hline \multirow{7}{*}{ 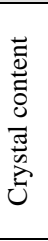 } & Glass as melted & Free & Xtls & Trace & Trace & Xtls & Xtls & Trace \\
\hline & $\mathrm{Xl}$ at $950^{\circ} \mathrm{C}$ & - & - & Sp & $\mathrm{Sp}+\mathrm{ZrO} 2$ & - & - & Sp + Pho \\
\hline & Glass after $\mathrm{HT}$ at $950^{\circ} \mathrm{C}$ (vol\%) & - & - & 1.6 & $\sim 1.8$ & - & - & 2.0 \\
\hline & Glass after $\mathrm{HT}$ at $900^{\circ} \mathrm{C}$ (vol\%) & - & - & - & - & - & - & 3.7 \\
\hline & Glass after $\mathrm{HT}$ at $850^{\circ} \mathrm{C}(\mathrm{vol} \%)$ & - & - & 3.5 & - & - & - & 5.1 \\
\hline & Glass after $\mathrm{HT}$ at $800^{\circ} \mathrm{C}(\mathrm{vol} \%)$ & 0.2 & - & 4.1 & - & 12.3 & - & 5.5 \\
\hline & Glass after HT of CCC (vol\%) & 0.1 (Spinel) & - & - & - & - & - & $1.9(\mathrm{Sp}+\mathrm{Pho})$ \\
\hline \multirow{10}{*}{ 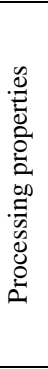 } & Highly viscous during pouring $(\mathrm{Y} / \mathrm{N})$ & $\mathrm{N}$ & $\mathrm{N}$ & $\mathrm{N}$ & $\mathrm{N}$ & $\mathrm{N}$ & $\mathrm{N}$ & $\mathrm{N}$ \\
\hline & Viscosity at $1250^{\circ} \mathrm{C}$ (poise) & - & - & 5.5 & 4.6 & - & - & 25.1 \\
\hline & Viscosity at $1150^{\circ} \mathrm{C}$ (poise) & - & - & 10.9 & 10.2 & - & - & 59.4 \\
\hline & Viscosity at $1050^{\circ} \mathrm{C}$ (poise) & - & - & 27.0 & 27.2 & - & - & 172.6 \\
\hline & Viscosity at $950^{\circ} \mathrm{C}$ (poise) & - & - & 96.7 & 92.5 & - & - & 669.1 \\
\hline & Electric conductivity at $1250^{\circ} \mathrm{C}(\mathrm{S} / \mathrm{cm})$ & - & - & 0.37 & 0.29 & - & - & 0.35 \\
\hline & Electric conductivity at $1150^{\circ} \mathrm{C}(\mathrm{S} / \mathrm{cm})$ & - & - & 0.25 & 0.20 & - & - & 0.26 \\
\hline & Electric conductivity at $1050^{\circ} \mathrm{C}(\mathrm{S} / \mathrm{cm})$ & - & - & 0.16 & 0.12 & - & - & 0.17 \\
\hline & Electric conductivity at $950^{\circ} \mathrm{C}(\mathrm{S} / \mathrm{cm})$ & - & - & 0.09 & 0.07 & - & - & 0.10 \\
\hline & Glass transition temperature $\left({ }^{\circ} \mathrm{C}\right)$ & - & - & & & - & - & - \\
\hline \multirow{19}{*}{ 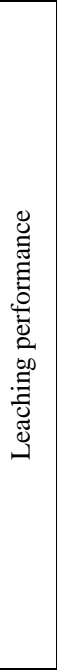 } & PCT & - & - & & & - & - & - \\
\hline & B (ppm) & - & - & 32.7 & 42.2 & - & - & - \\
\hline & Li (ppm) & - & - & 12.2 & 15.2 & - & - & - \\
\hline & $\mathrm{Na}(\mathrm{ppm})$ & - & - & 14.2 & 18.4 & - & - & - \\
\hline & Si (ppm) & - & - & 4.4 & 4.4 & - & - & - \\
\hline & $B(g / L)$ & - & - & 0.52 & 0.71 & - & - & - \\
\hline & $\mathrm{Li}(\mathrm{g} / \mathrm{L})$ & - & - & 0.51 & 0.63 & - & - & - \\
\hline & $\mathrm{Na}(\mathrm{g} / \mathrm{L})$ & - & - & 0.54 & 0.69 & - & - & - \\
\hline & $\mathrm{Si}(\mathrm{g} / \mathrm{L})$ & - & - & 0.04 & 0.04 & - & - & - \\
\hline & PCT (for CCC sample) & - & - & - & - & - & - & - \\
\hline & $\mathrm{B}(\mathrm{ppm})$ & - & - & - & - & - & - & - \\
\hline & $\mathrm{Li}(\mathrm{ppm})$ & - & - & - & - & - & - & - \\
\hline & $\mathrm{Na}(\mathrm{ppm})$ & - & - & - & - & - & - & - \\
\hline & Si (ppm) & - & - & - & - & - & - & - \\
\hline & $\mathrm{B}(\mathrm{g} / \mathrm{L})$ & - & - & - & - & - & - & - \\
\hline & $\mathrm{Li}(\mathrm{g} / \mathrm{L})$ & - & - & - & - & - & - & - \\
\hline & $\mathrm{Na}(\mathrm{g} / \mathrm{L})$ & - & - & - & - & - & - & - \\
\hline & $\mathrm{Si}(\mathrm{g} / \mathrm{L})$ & - & - & - & - & - & - & - \\
\hline & TCLP (Pass/Fail) & - & - & Pass & Pass & - & - & - \\
\hline
\end{tabular}

Sp = spinel; Pho = phosphate; Nas = sodium aluminosilicate - Empty data field 
Table 3.10. Characterization of Glasses Formulated for Al-Limited Waste (continued).

\begin{tabular}{|c|c|c|c|c|c|c|c|c|}
\hline & Glass Name & HLW-E-Al-27 & HLW-E-Al-27UThR2 & HLW-E-Al-15 & HLW-E-Al-13 & HLW-E-Al-20 & HLW-E-Al-21 & HLW-E-Al-22 \\
\hline & Waste loading (wt\%) & 45 & 45 & 47.5 & 47.5 & 47.5 & 47.5 & 47.5 \\
\hline & Glass former type & $\mathrm{Na}+\mathrm{Li}+\mathrm{Ca}$ & $\mathrm{Na}+\mathrm{Li}+\mathrm{Ca}$ & $\mathrm{Li}$ & $\mathrm{Ca}+\mathrm{Li}$ & $\mathrm{Na}+\mathrm{Li}$ & $\mathrm{Na}+\mathrm{Li}$ & $\mathrm{Na}+\mathrm{Li}$ \\
\hline \multirow{7}{*}{ 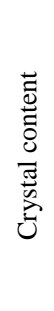 } & Glass as melted & Trace & - & Xtls & Trace & Xtls & Xtls & Xtls \\
\hline & $\mathrm{Xl}$ at $950^{\circ} \mathrm{C}$ & Sp & Sp & - & Sp + Apatite & Sp & Sp & Sp \\
\hline & Glass after $\mathrm{HT}$ at $950^{\circ} \mathrm{C}$ (vol\%) & 1.0 & 1.0 & - & 1.5 & 1.1 & 0.5 & 2.2 \\
\hline & Glass after $\mathrm{HT}$ at $900^{\circ} \mathrm{C}$ (vol\%) & 3.8 & Not analyzed & - & - & - & - & - \\
\hline & Glass after $\mathrm{HT}$ at $850^{\circ} \mathrm{C}$ (vol\%) & 3.4 & 3.0 & - & - & - & - & - \\
\hline & Glass after $\mathrm{HT}$ at $800^{\circ} \mathrm{C}$ (vol\%) & 7.2 & 3.1 & - & - & - & - & - \\
\hline & Glass after HT of CCC (vol\%) & 1.9 (spinel) & 1.7 (spinel) & - & - & - & - & - \\
\hline \multirow{10}{*}{ 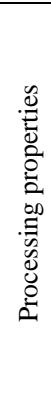 } & Highly viscous during pouring $(\mathrm{Y} / \mathrm{N})$ & $\mathrm{N}$ & $\mathrm{N}$ & $\mathrm{N}$ & $\mathrm{N}$ & $\mathrm{N}$ & $\mathrm{N}$ & $\mathrm{N}$ \\
\hline & Viscosity at $1250^{\circ} \mathrm{C}$ (poise) & 19.5 & 17.5 & - & - & - & - & - \\
\hline & Viscosity at $1150^{\circ} \mathrm{C}$ (poise) & 45.6 & 38.7 & - & - & - & - & - \\
\hline & Viscosity at $1050^{\circ} \mathrm{C}$ (poise) & 129.8 & 107.0 & - & - & - & - & - \\
\hline & Viscosity at $950^{\circ} \mathrm{C}$ (poise) & 488.8 & 407.8 & - & - & - & - & - \\
\hline & Electric conductivity at $1250^{\circ} \mathrm{C}(\mathrm{S} / \mathrm{cm})$ & 0.35 & 0.41 & - & - & - & - & - \\
\hline & Electric conductivity at $1150^{\circ} \mathrm{C}(\mathrm{S} / \mathrm{cm})$ & 0.26 & 0.29 & - & - & - & - & - \\
\hline & Electric conductivity at $1050^{\circ} \mathrm{C}(\mathrm{S} / \mathrm{cm})$ & 0.18 & 0.19 & - & - & - & - & - \\
\hline & Electric conductivity at $950^{\circ} \mathrm{C}(\mathrm{S} / \mathrm{cm})$ & 0.10 & 0.12 & - & - & - & - & - \\
\hline & Glass transition temperature $\left({ }^{\circ} \mathrm{C}\right)$ & 483 & - & - & - & - & - & - \\
\hline \multirow{19}{*}{ 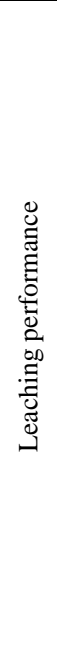 } & PCT & - & - & - & - & - & - & - \\
\hline & B (ppm) & 12.7 & 12.6 & - & - & - & - & 81.8 \\
\hline & Li (ppm) & 7.3 & 6.4 & - & - & - & - & 35.0 \\
\hline & $\mathrm{Na}(\mathrm{ppm})$ & 21.5 & 18.9 & - & - & - & - & 93.2 \\
\hline & Si (ppm) & 20.1 & 16.3 & - & - & - & - & 36.1 \\
\hline & $\mathrm{B}(\mathrm{g} / \mathrm{L})$ & 0.27 & 0.27 & - & - & - & - & 1.88 \\
\hline & $\mathrm{Li}(\mathrm{g} / \mathrm{L})$ & 0.44 & 0.38 & - & - & - & - & 1.50 \\
\hline & $\mathrm{Na}(\mathrm{g} / \mathrm{L})$ & 0.30 & 0.27 & - & - & - & - & 1.28 \\
\hline & $\mathrm{Si}(\mathrm{g} / \mathrm{L})$ & 0.14 & 0.12 & - & - & - & - & 0.28 \\
\hline & PCT (for CCC sample) & - & - & - & - & - & - & - \\
\hline & B (ppm) & 14.72 & - & - & - & - & - & - \\
\hline & $\mathrm{Li}(\mathrm{ppm})$ & 7.303 & - & - & - & - & - & - \\
\hline & Na (ppm) & 23.1 & - & - & - & - & - & - \\
\hline & Si (ppm) & 23.25 & - & - & - & - & - & - \\
\hline & $\mathrm{B}(\mathrm{g} / \mathrm{L})$ & 0.3121 & - & - & - & - & - & - \\
\hline & Li (g/L) & 0.4403 & - & - & - & - & - & - \\
\hline & $\mathrm{Na}(\mathrm{g} / \mathrm{L})$ & 0.3250 & - & - & - & - & - & - \\
\hline & Si (g/L) & 0.1631 & - & - & - & - & - & - \\
\hline & TCLP (Pass/Fail) & Pass & Pass & - & - & - & - & Pass \\
\hline
\end{tabular}

Sp = spinel; Pho = phosphate; Nas = sodium aluminosilicate

- Empty data field 
Table 3.10. Characterization of Glasses Formulated for Al-Limited Waste (continued).

\begin{tabular}{|c|c|c|c|c|c|c|c|c|c|}
\hline & Glass Name & HLW-E-Al-28 & HLW-E-Al-29 & HLW-E-Al-14 & HLW-E-Al-7 & HLW-E-Al-8 & HLW-E-Al-9 & HLW-E-Al-10 & HLW-E-Al-11 \\
\hline & Waste loading (wt\%) & 47.5 & 47.5 & 50 & 50 & 50 & 50 & 50 & 50 \\
\hline & Glass former type & $\mathrm{Na}+\mathrm{Li}+\mathrm{Ca}$ & $\mathrm{Na}+\mathrm{Li}+\mathrm{Ca}$ & $\mathrm{Li}$ & $\mathrm{Ca}+\mathrm{Li}$ & $\mathrm{Ca}+\mathrm{Li}$ & $\mathrm{Ca}+\mathrm{Li}$ & $\mathrm{Ca}+\mathrm{Li}$ & $\mathrm{Ca}+\mathrm{Li}$ \\
\hline \multirow{7}{*}{ 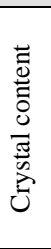 } & Glass as melted & Xtls & Xtls & Xtls & Trace & Trace & Trace & Xtls & Trace \\
\hline & $\mathrm{Xl}$ at $950^{\circ} \mathrm{C}$ & Sp + Pho & - & - & $\mathrm{Pho}+\mathrm{Nas}+\mathrm{Sp}$ & $\mathrm{Sp}+\mathrm{Nas}$ & Sp + Pho & Sp + Pho & Sp + Pho \\
\hline & Glass after $\mathrm{HT}$ at $950^{\circ} \mathrm{C}$ (vol\%) & 2.8 & - & - & 6.7 & 6.8 & 4.0 & 2.1 & 2.9 \\
\hline & Glass after $\mathrm{HT}$ at $900^{\circ} \mathrm{C}(\mathrm{vol} \%)$ & - & - & - & - & - & - & - & 4.2 \\
\hline & Glass after HT at $850^{\circ} \mathrm{C}$ (vol\%) & - & - & - & - & - & - & - & 5.3 \\
\hline & Glass after $\mathrm{HT}$ at $800^{\circ} \mathrm{C}$ (vol\%) & - & - & - & - & - & - & - & 7.8 \\
\hline & Glass after HT of CCC (vol\%) & - & - & - & - & - & - & - & \\
\hline \multirow{10}{*}{ 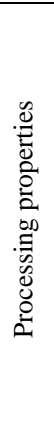 } & Highly viscous during pouring $(\mathrm{Y} / \mathrm{N})$ & $\mathrm{N}$ & $\mathrm{N}$ & $\mathrm{N}$ & $\mathrm{N}$ & $\mathrm{N}$ & $\mathrm{N}$ & $\mathrm{N}$ & $\mathrm{N}$ \\
\hline & Viscosity at $1250^{\circ} \mathrm{C}$ (poise) & - & - & - & - & 9.9 & - & - & 6.3 \\
\hline & Viscosity at $1150^{\circ} \mathrm{C}$ (poise) & - & - & - & - & 27.1 & - & - & 14.0 \\
\hline & Viscosity at $1050^{\circ} \mathrm{C}$ (poise) & - & - & - & - & 103.6 & - & - & 35.7 \\
\hline & Viscosity at $950^{\circ} \mathrm{C}$ (poise) & - & - & - & - & 677.7 & - & - & 108.3 \\
\hline & Electric conductivity at $1250^{\circ} \mathrm{C}(\mathrm{S} / \mathrm{cm})$ & - & - & - & - & 0.13 & - & - & 0.49 \\
\hline & Electric conductivity at $1150^{\circ} \mathrm{C}(\mathrm{S} / \mathrm{cm})$ & - & - & - & - & 0.07 & - & - & 0.31 \\
\hline & Electric conductivity at $1050^{\circ} \mathrm{C}(\mathrm{S} / \mathrm{cm})$ & - & - & - & - & 0.03 & - & - & 0.18 \\
\hline & Electric conductivity at $950^{\circ} \mathrm{C}(\mathrm{S} / \mathrm{cm})$ & - & - & - & - & 0.01 & - & - & 0.10 \\
\hline & Glass transition temperature $\left({ }^{\circ} \mathrm{C}\right)$ & - & - & - & - & - & - & - & - \\
\hline \multirow{19}{*}{ 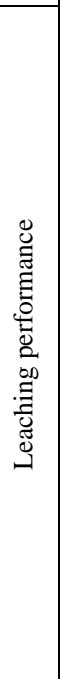 } & PCT & - & - & - & - & - & - & - & - \\
\hline & B (ppm) & - & - & - & - & - & - & 111.9 & 22.8 \\
\hline & $\mathrm{Li}(\mathrm{ppm})$ & - & - & - & - & - & - & 44.3 & 13.1 \\
\hline & $\mathrm{Na}(\mathrm{ppm})$ & - & - & - & - & - & - & 55.5 & 15.5 \\
\hline & Si (ppm) & - & - & - & - & - & - & 2.2 & 7.4 \\
\hline & $B(g / L)$ & - & - & - & - & - & - & 1.78 & 0.45 \\
\hline & $\mathrm{Li}(\mathrm{g} / \mathrm{L})$ & - & - & - & - & - & - & 1.84 & 0.55 \\
\hline & $\mathrm{Na}(\mathrm{g} / \mathrm{L})$ & - & - & - & - & - & - & 1.88 & 0.52 \\
\hline & $\mathrm{Si}(\mathrm{g} / \mathrm{L})$ & - & - & - & - & - & - & 0.03 & 0.07 \\
\hline & PCT (for CCC sample) & - & - & - & - & - & - & - & - \\
\hline & B (ppm) & - & - & - & - & - & - & - & - \\
\hline & $\mathrm{Li}(\mathrm{ppm})$ & - & - & - & - & - & - & - & - \\
\hline & $\mathrm{Na}(\mathrm{ppm})$ & - & - & - & - & - & - & - & - \\
\hline & $\mathrm{Si}(\mathrm{ppm})$ & - & - & - & - & - & - & - & - \\
\hline & $B(g / L)$ & - & - & - & - & - & - & - & - \\
\hline & $\mathrm{Li}(\mathrm{g} / \mathrm{L})$ & - & - & - & - & - & - & - & - \\
\hline & $\mathrm{Na}(\mathrm{g} / \mathrm{L})$ & - & - & - & - & - & - & - & - \\
\hline & Si (g/L) & - & - & - & - & - & - & - & - \\
\hline & TCLP (Pass/Fail) & - & - & - & - & - & - & - & Pass \\
\hline
\end{tabular}

Sp = spinel; Pho = phosphate; Nas = sodium aluminosilicate

- Empty data field 
Table 3.10. Characterization of Glasses Formulated for Al-Limited Waste (continued).

\begin{tabular}{|c|c|c|c|c|c|c|c|c|c|}
\hline & Glass Name & \begin{tabular}{|l|} 
HLW-E-Al-19 \\
\end{tabular} & HLW-E-Al-2 & HLW-E-Al-3 & HLW-E-Al-4 & HLW-E-Al-5 & HLW-E-Al-6 & \begin{tabular}{|l|} 
HLW-E-Al-1 \\
\end{tabular} & HLW-E-Al-17 \\
\hline & Waste loading (wt\%) & \begin{tabular}{|l|}
50 \\
\end{tabular} & 50 & 50 & 50 & 50 & 50 & 50 & 55 \\
\hline & Glass former type & $\mathrm{Na}+\mathrm{Li}$ & $\mathrm{Ca}+\mathrm{Na}$ & $\mathrm{Ca}+\mathrm{Na}$ & $\mathrm{Ca}+\mathrm{Na}$ & $\mathrm{Ca}+\mathrm{Na}$ & $\mathrm{Ca}+\mathrm{Na}$ & $\mathrm{Mg}+\mathrm{Na}$ & $\mathrm{Li}$ \\
\hline \multirow{7}{*}{ 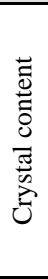 } & Glass as melted & Xtls & Xtls & Xtls & Trace & Xtls & Xtls & Xtls & Xtls \\
\hline & $\mathrm{Xl}$ at $950^{\circ} \mathrm{C}$ & $\mathrm{Sp}+\mathrm{Nas}$ & - & - & $\mathrm{Nas}+\mathrm{Pho}+\mathrm{Sp}$ & - & $\mathrm{Nas}+\mathrm{Pho}+\mathrm{Sp}$ & - & - \\
\hline & Glass after HT at $950^{\circ} \mathrm{C}$ (vol\%) & 4.1 & - & - & 34.0 & - & 31.9 & - & - \\
\hline & Glass after $\mathrm{HT}$ at $900^{\circ} \mathrm{C}$ (vol\%) & 3.2 & - & - & - & - & - & - & - \\
\hline & Glass after HT at $850^{\circ} \mathrm{C}$ (vol\%) & 5.2 & - & - & - & - & - & - & - \\
\hline & Glass after $\mathrm{HT}$ at $800^{\circ} \mathrm{C}$ (vol\%) & 2.7 & - & - & - & - & - & - & - \\
\hline & Glass after HT of CCC (vol\%) & - & - & - & - & - & - & - & - \\
\hline \multirow{10}{*}{ 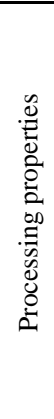 } & Highly viscous during pouring $(\mathrm{Y} / \mathrm{N})$ & $\mathrm{N}$ & $\mathrm{Y}$ & $\mathrm{N}$ & $\mathrm{N}$ & $\mathrm{N}$ & $\mathrm{N}$ & $\mathrm{Y}$ & $\mathrm{N}$ \\
\hline & Viscosity at $1250^{\circ} \mathrm{C}$ (poise) & - & - & - & - & - & 28.9 & - & - \\
\hline & Viscosity at $1150^{\circ} \mathrm{C}$ (poise) & - & - & - & - & - & 101.5 & - & - \\
\hline & Viscosity at $1050^{\circ} \mathrm{C}$ (poise) & - & - & - & - & - & 568.1 & - & - \\
\hline & Viscosity at $950^{\circ} \mathrm{C}$ (poise) & - & - & - & - & - & 6961.8 & - & - \\
\hline & Electric conductivity at $1250^{\circ} \mathrm{C}(\mathrm{S} / \mathrm{cm})$ & - & - & - & - & - & 0.06 & - & - \\
\hline & Electric conductivity at $1150^{\circ} \mathrm{C}(\mathrm{S} / \mathrm{cm})$ & - & - & - & - & - & 0.03 & - & - \\
\hline & Electric conductivity at $1050^{\circ} \mathrm{C}(\mathrm{S} / \mathrm{cm})$ & - & - & - & - & - & 0.01 & - & - \\
\hline & Electric conductivity at $950^{\circ} \mathrm{C}(\mathrm{S} / \mathrm{cm})$ & - & - & - & - & - & 0.00 & - & - \\
\hline & Glass transition temperature $\left({ }^{\circ} \mathrm{C}\right)$ & - & - & - & - & - & - & - & - \\
\hline \multirow{19}{*}{ 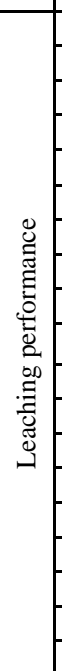 } & PCT & - & - & - & - & - & - & - & - \\
\hline & $\mathrm{B}(\mathrm{ppm})$ & - & - & - & - & - & - & - & - \\
\hline & $\mathrm{Li}(\mathrm{ppm})$ & - & - & - & - & - & - & - & - \\
\hline & $\mathrm{Na}(\mathrm{ppm})$ & - & - & - & - & - & - & - & - \\
\hline & Si (ppm) & - & - & - & - & - & - & - & - \\
\hline & $\mathrm{B}(\mathrm{g} / \mathrm{L})$ & - & - & - & - & - & - & - & - \\
\hline & $\mathrm{Li}(\mathrm{g} / \mathrm{L})$ & - & - & - & - & - & - & - & - \\
\hline & $\mathrm{Na}(\mathrm{g} / \mathrm{L})$ & - & - & - & - & - & - & - & - \\
\hline & $\mathrm{Si}(\mathrm{g} / \mathrm{L})$ & - & - & - & - & - & - & - & - \\
\hline & PCT (for CCC sample) & - & - & - & - & - & - & - & - \\
\hline & $\mathrm{B}(\mathrm{ppm})$ & - & - & - & - & - & - & - & - \\
\hline & $\mathrm{Li}(\mathrm{ppm})$ & - & - & - & - & - & - & - & - \\
\hline & $\mathrm{Na}(\mathrm{ppm})$ & - & - & - & - & - & - & - & - \\
\hline & Si (ppm) & - & - & - & - & - & - & - & - \\
\hline & $\mathrm{B}(\mathrm{g} / \mathrm{L})$ & - & - & - & - & - & - & - & - \\
\hline & $\mathrm{Li}(\mathrm{g} / \mathrm{L})$ & - & - & - & - & - & - & - & - \\
\hline & $\mathrm{Na}(\mathrm{g} / \mathrm{L})$ & - & - & - & - & - & - & - & - \\
\hline & $\mathrm{Si}(\mathrm{g} / \mathrm{L})$ & - & - & - & - & - & - & - & - \\
\hline & TCLP (Pass/Fail) & - & - & - & - & - & - & - & - \\
\hline
\end{tabular}

$\mathrm{Sp}=$ spinel; $\mathrm{Pho}=$ phosphate; Nas = sodium aluminosilicate

- Empty data field 
Table 3.11. Composition and Properties of Aluminum Limited Waste and Glass Formulation at 45\% Waste Loading Used in Melter Tests (wt\%).

\begin{tabular}{|c|c|c|c|c|}
\hline- & $\begin{array}{c}\text { Al-Limited } \\
\text { Waste* }\end{array}$ & Waste in Glass & $\begin{array}{c}\text { Glass Forming } \\
\text { Additives }\end{array}$ & $\begin{array}{c}\text { Target Glass } \\
\text { HLW-E-Al-27 }\end{array}$ \\
\hline $\mathrm{Al}_{2} \mathrm{O}_{3}$ & 53.27 & 23.97 & - & 23.97 \\
\hline $\mathrm{B}_{2} \mathrm{O}_{3}$ & 0.42 & 0.19 & 15.00 & 15.19 \\
\hline $\mathrm{BaO}$ & 0.12 & 0.05 & - & 0.05 \\
\hline $\mathrm{Bi}_{2} \mathrm{O}_{3}$ & 2.54 & 1.14 & - & 1.14 \\
\hline $\mathrm{CaO}$ & 2.39 & 1.08 & 5.00 & 6.08 \\
\hline $\mathrm{CdO}$ & 0.05 & 0.02 & - & 0.02 \\
\hline $\mathrm{Cr}_{2} \mathrm{O}_{3}$ & 1.16 & 0.52 & & 0.52 \\
\hline $\mathrm{F}$ & 1.48 & 0.67 & - & 0.67 \\
\hline $\mathrm{Fe}_{2} \mathrm{O}_{3}$ & 13.11 & 5.90 & - & 5.90 \\
\hline $\mathrm{K}_{2} \mathrm{O}$ & 0.31 & 0.14 & - & 0.14 \\
\hline $\mathrm{Li}_{2} \mathrm{O}$ & 0.38 & 0.17 & 3.40 & 3.57 \\
\hline $\mathrm{MgO}^{\mathrm{Na}} \mathrm{O}$ & 0.26 & 0.12 & - & 0.12 \\
\hline $\mathrm{NiO}^{2} \mathrm{O}$ & 7.96 & 3.58 & 6.00 & 9.58 \\
\hline $\mathrm{P}_{2} \mathrm{O}_{5}$ & 0.89 & 0.40 & - & 0.40 \\
\hline $\mathrm{PbO}_{\mathrm{SiO}}$ & 2.34 & 1.05 & - & 1.05 \\
\hline $\mathrm{TiO}_{2}$ & 0.91 & 0.41 & - & 0.41 \\
\hline $\mathrm{SO}_{3}$ & 10.88 & 4.90 & 25.60 & 30.50 \\
\hline $\mathrm{ZnO}$ & 0.02 & 0.01 & - & 0.01 \\
\hline $\mathrm{ZrO}_{2}$ & 0.44 & 0.20 & - & 0.20 \\
\hline $\mathrm{Sum}$ & 0.18 & 0.08 & - & 0.08 \\
\hline $\mathrm{Re}$ & 100.00 & 0.39 & - & 0.39 \\
\hline
\end{tabular}

* Renormalized from Ref. [3] after removal of radioactive components

\begin{tabular}{|c|c|c|c|}
\hline \multicolumn{3}{|c|}{ Viscosity @1150º, P } & 46 \\
\hline \multicolumn{3}{|c|}{ Conductivity @1150º, S/cm } & 0.26 \\
\hline \multicolumn{3}{|c|}{ Crystal Content, As Melted } & Trace \\
\hline \multicolumn{3}{|c|}{ Crystal Content, $72 \mathrm{hr}$ at $950^{\circ} \mathrm{C}$} & $\sim 1.0 \mathrm{vol} \%$ \\
\hline \multicolumn{3}{|c|}{ Crystal Content, CCC } & $\sim 1.9 \mathrm{vol} \%$ \\
\hline \multicolumn{3}{|c|}{ TCLP } & Pass \\
\hline \multirow{4}{*}{ PCT, g/L } & - & DWPF-EA & HLW-E-Al-27 \\
\hline & B & 16.7 & 0.27 \\
\hline & $\mathrm{Li}$ & 9.6 & 0.44 \\
\hline & $\mathrm{Na}$ & 13.3 & 0.30 \\
\hline
\end{tabular}

- Empty data field 
Table 3.12. Target Composition and XRF Analysis of Glasses Formulated for Al-Na-Limited Waste.

\begin{tabular}{|c|c|c|c|c|c|c|c|c|c|c|c|c|c|c|c|c|c|c|}
\hline \multirow{2}{*}{ - } & \multirow{2}{*}{$\begin{array}{l}\text { Al-Na-limited } \\
\text { waste }\end{array}$} & \multirow{2}{*}{$\begin{array}{l}\text { Al-Na-limited waste } \\
\text { (non rad)\# }\end{array}$} & \multicolumn{2}{|c|}{ HLW-E-ANa-1 } & \multicolumn{2}{|c|}{ HLW-E-ANa-2 } & \multicolumn{2}{|c|}{ HLWE-ANa-3 } & \multicolumn{2}{|c|}{ HLWE-ANa-4 } & \multicolumn{2}{|c|}{ HLWE-ANa-5 } & \multicolumn{2}{|c|}{ HLWE-ANa-6 } & \multicolumn{2}{|c|}{ HLWE-ANa-7 } & \multicolumn{2}{|c|}{ HLWE-ANa-8 } \\
\hline & & & Target & XRF & Target & XRF & Target & XRF & Target & XRF & Target & XRF & Target & XRF & Target & XRF & Target & XRF \\
\hline $\mathrm{Al}_{2} \mathrm{O}_{3}$ & 43.30 & 45.40 & 19.07 & 18.99 & 21.34 & 21.10 & 23.61 & 23.32 & 21.34 & 21.03 & 23.61 & 23.39 & 25.88 & 25.37 & 27.24 & 26.89 & 25.88 & 25.41 \\
\hline $\mathrm{B}_{2} \mathrm{O}_{3} *$ & 0.74 & 0.78 & 14.33 & 14.33 & 14.37 & 14.37 & 13.41 & 13.41 & 14.37 & 14.37 & 14.41 & 14.41 & 14.44 & 14.44 & 14.47 & 14.47 & 14.44 & 14.44 \\
\hline $\mathrm{BaO}$ & 0.06 & 0.06 & 0.03 & 0.02 & 0.03 & 0.04 & 0.03 & 0.02 & 0.03 & 0.03 & 0.03 & 0.03 & 0.03 & 0.04 & 0.04 & 0.04 & 0.03 & 0.04 \\
\hline $\mathrm{Bi}_{2} \mathrm{O}_{3}$ & 2.35 & 2.46 & 1.03 & 1.09 & 1.16 & 1.21 & 1.28 & 1.26 & 1.16 & 1.15 & 1.28 & 1.35 & 1.40 & 1.61 & 1.48 & 1.53 & 1.40 & 1.50 \\
\hline $\mathrm{CaO}$ & 1.47 & 1.54 & 0.65 & 0.67 & 0.72 & 0.78 & 0.80 & 0.88 & 0.72 & 0.76 & 0.80 & 0.85 & 0.88 & 1.00 & 0.92 & 1.00 & 0.88 & 0.95 \\
\hline $\mathrm{CdO}$ & 0.02 & 0.02 & 0.01 & 0.00 & 0.01 & 0.00 & 0.01 & 0.00 & 0.01 & 0.00 & 0.01 & 0.00 & 0.01 & 0.00 & 0.01 & 0.00 & 0.01 & 0.00 \\
\hline $\mathrm{Cr}_{2} \mathrm{O}_{3}$ & 1.44 & 1.51 & 0.63 & 0.77 & 0.71 & 0.83 & 0.79 & 0.96 & 0.71 & 0.85 & 0.79 & 1.02 & 0.86 & 1.12 & 0.91 & 1.08 & 0.86 & 1.07 \\
\hline $\mathrm{F}^{*}$ & 0.46 & 0.48 & 0.20 & - & 0.23 & - & 0.25 & - & 0.23 & - & 0.25 & - & 0.27 & - & 0.29 & - & 0.27 & - \\
\hline $\mathrm{Fe}_{2} \mathrm{O}_{3}$ & 5.71 & 5.99 & 2.52 & 2.51 & 2.82 & 2.76 & 3.11 & 3.12 & 2.82 & 2.75 & 3.11 & 3.19 & 3.41 & 3.73 & 3.59 & 3.56 & 3.41 & 3.42 \\
\hline $\mathrm{K}_{2} \mathrm{O}$ & 1.34 & 1.41 & 0.59 & 0.69 & 0.66 & 0.73 & 0.73 & 0.08 & 0.66 & 0.75 & 0.73 & 0.80 & 0.80 & 0.90 & 0.85 & 0.88 & 0.80 & 0.86 \\
\hline $\mathrm{Li}_{2} \mathrm{O}^{*}$ & 0.15 & 0.16 & 4.07 & 4.07 & 3.08 & 3.08 & 2.58 & 2.58 & 4.08 & 4.08 & 3.58 & 3.58 & 2.81 & 2.81 & 2.82 & 2.82 & 3.59 & 3.59 \\
\hline $\mathrm{MgO}$ & 0.44 & 0.46 & 0.19 & 0.17 & 0.22 & 0.20 & 0.24 & 0.19 & 0.22 & 0.19 & 0.24 & 0.20 & 0.26 & 0.22 & 0.28 & 0.23 & 0.26 & 0.24 \\
\hline $\mathrm{Na}_{2} \mathrm{O}$ & 25.79 & 27.04 & 11.36 & 11.40 & 12.71 & 12.96 & 14.06 & 14.09 & 12.71 & 12.65 & 14.06 & 14.56 & 15.41 & 15.16 & 16.22 & 15.88 & 15.41 & 15.65 \\
\hline $\mathrm{NiO}$ & 0.20 & 0.21 & 0.09 & 0.08 & 0.10 & 0.10 & 0.11 & 0.10 & 0.10 & 0.10 & 0.11 & 0.11 & 0.12 & 0.14 & 0.13 & 0.12 & 0.12 & 0.13 \\
\hline $\mathrm{P}_{2} \mathrm{O}_{5}$ & 4.10 & 4.30 & 3.81 & 4.10 & 2.02 & 2.51 & 2.24 & 2.51 & 2.02 & 2.55 & 2.24 & 2.44 & 2.45 & 2.68 & 2.58 & 2.90 & 2.45 & 2.70 \\
\hline $\mathrm{PbO}$ & 0.18 & 0.19 & 0.08 & 0.09 & 0.09 & 0.08 & 0.10 & 0.09 & 0.09 & 0.09 & 0.10 & 0.09 & 0.11 & 0.11 & 0.11 & 0.11 & 0.11 & 0.11 \\
\hline $\mathrm{SiO}_{2}$ & 6.22 & 6.52 & 39.74 & 39.39 & 39.06 & 38.47 & 35.89 & 35.83 & 38.06 & 37.87 & 33.89 & 33.10 & 30.00 & 29.67 & 27.19 & 27.53 & 29.22 & 28.96 \\
\hline $\mathrm{ThO}_{2}$ & 0.04 & 0.00 & 0.00 & 0.00 & 0.00 & 0.00 & 0.00 & 0.00 & 0.00 & 0.00 & 0.00 & 0.00 & 0.00 & 0.00 & 0.00 & 0.00 & 0.00 & 0.00 \\
\hline $\mathrm{TiO}_{2}$ & 0.35 & 0.37 & 0.16 & 0.20 & 0.17 & 0.21 & 0.19 & 0.23 & 0.17 & 0.21 & 0.19 & 0.23 & 0.21 & 0.27 & 0.22 & 0.26 & 0.21 & 0.25 \\
\hline $\mathrm{SO}_{3}$ & 0.44 & 0.46 & 0.19 & 0.18 & 0.22 & 0.22 & 0.24 & 0.23 & 0.22 & 0.24 & 0.24 & 0.23 & 0.26 & 0.26 & 0.28 & 0.28 & 0.26 & 0.27 \\
\hline $\mathrm{ZnO}$ & 0.36 & 0.38 & 0.16 & 0.17 & 0.18 & 0.19 & 0.20 & 0.21 & 0.18 & 0.19 & 0.20 & 0.21 & 0.22 & 0.26 & 0.23 & 0.24 & 0.22 & 0.23 \\
\hline $\mathrm{ZrO}_{2}$ & 0.25 & 0.26 & 1.11 & 1.01 & 0.12 & 0.12 & 0.14 & 0.10 & 0.12 & 0.11 & 0.14 & 0.14 & 0.15 & 0.16 & 0.16 & 0.15 & 0.15 & 0.15 \\
\hline $\mathrm{U}_{3} \mathrm{O}_{8}$ & 4.58 & 0.00 & 0.00 & 0.00 & 0.00 & 0.00 & 0.00 & 0.00 & 0.00 & 0.00 & 0.00 & 0.00 & 0.00 & 0.00 & 0.00 & 0.00 & 0.00 & 0.00 \\
\hline
\end{tabular}

*In XRF analysis target concentrations are used for $\mathrm{B}_{2} \mathrm{O}_{3}$ and $\mathrm{Li}_{2} \mathrm{O} ; \mathrm{F}$ was not analyzed.

\#The non-radioactive version of the waste is calculated by renormalization of the waste constituents after removing $\mathrm{U}_{3} \mathrm{O}_{8}$ and $\mathrm{ThO}_{2}$.

- Empty data field 
Table 3.12. Target Composition and XRF Analysis of Glasses Formulated for Al-Na-Limited Waste (continued).

\begin{tabular}{|c|c|c|c|c|c|c|c|c|c|c|c|c|c|c|c|c|c|c|c|c|c|c|}
\hline & \multicolumn{2}{|c|}{ HLWE-ANa-9 } & \multicolumn{2}{|c|}{ HLWE-ANa-10 } & \multicolumn{2}{|c|}{ HLWE-ANa-11 } & \multicolumn{2}{|c|}{ HLWE-ANa-12 } & \multicolumn{2}{|c|}{ HLWE-ANa-13 } & \multicolumn{2}{|c|}{ HLWE-ANa-14 } & \multicolumn{2}{|c|}{ HLWE-ANa-15 } & \multicolumn{2}{|c|}{ HLWE-ANa-16 } & \multicolumn{2}{|c|}{ HLWE-ANa-17 } & \multicolumn{2}{|c|}{ HLWE-ANa-18 } & \multicolumn{2}{|c|}{ HLWE-ANa-19 } \\
\hline & Target & XRF & Target & XRF & Target & XRF & Target & XRF & Target & XRF & Target & XRF & Target & XRF & Target & XRF & Target & XRF & Target & XRF & Target & XRF \\
\hline${ }_{2} \mathrm{O}_{3}$ & 25.88 & 25.29 & 25.88 & 25.28 & 25.88 & 25.45 & 29.51 & 28.96 & 21.34 & 20.02 & 23.61 & 23.28 & 23.61 & 23.23 & 23.61 & 23.17 & 20.43 & 20.41 & 20.43 & 20.23 & 19.52 & 19.45 \\
\hline $\mathrm{B}_{2} \mathrm{O}_{3}{ }^{*}$ & 14.44 & 14.44 & 15.94 & 15.94 & 13.44 & 13.44 & 13.51 & 13.51 & 14.37 & 14.37 & 14.41 & 14.41 & 15.41 & 15.41 & 14.41 & 14.41 & 15.35 & 15.35 & 15.35 & 15.35 & 15.34 & 15.34 \\
\hline $\mathrm{BaO}$ & 0.03 & 0.03 & 0.03 & 0.05 & 0.03 & 0.05 & 0.04 & 0.06 & 0.03 & 0.03 & 0.03 & 0.04 & 0.03 & 0.03 & 0.03 & 0.04 & 0.03 & 0.02 & 0.03 & 0.02 & 0.03 & 0.03 \\
\hline $\mathrm{Bi}_{2} \mathrm{O}_{3}$ & 1.40 & 1.66 & 1.40 & 1.53 & 1.40 & 1.49 & 1.60 & 1.65 & 1.16 & 1.29 & 1.28 & 1.35 & 1.28 & 1.38 & 1.28 & 1.45 & 1.11 & 1.02 & 1.11 & 1.11 & 1.06 & 1.04 \\
\hline $\mathrm{CaO}$ & 0.88 & 0.90 & 0.88 & 0.94 & 0.88 & 0.94 & 1.00 & 1.03 & 5.72 & 5.95 & 5.80 & 5.82 & 5.80 & 5.91 & 5.80 & 6.03 & 5.69 & 5.37 & 5.69 & 5.52 & 5.66 & 5.52 \\
\hline $\mathrm{CdO}$ & 0.01 & 0.00 & 0.01 & 0.00 & 0.01 & 0.00 & 0.01 & 0.00 & 0.01 & 0.00 & 0.01 & 0.00 & 0.01 & 0.00 & 0.01 & 0.00 & 0.01 & 0.00 & 0.01 & 0.00 & 0.01 & 0.00 \\
\hline $\mathrm{Cr}_{2} \mathrm{O}_{3}$ & 0.86 & 1.01 & 0.86 & 1.07 & 0.86 & 1.05 & 0.98 & 1.13 & 0.71 & 0.90 & 0.79 & 0.93 & 0.79 & 0.97 & 0.79 & 1.00 & 0.68 & 0.73 & 0.68 & 0.77 & 0.65 & 0.74 \\
\hline $\mathrm{F}^{*}$ & 0.27 & - & 0.27 & - & 0.27 & - & 0.31 & - & 0.23 & - & 0.25 & - & 0.25 & - & 0.25 & - & 0.22 & - & 0.22 & - & 0.21 & - \\
\hline $\mathrm{Fe}_{2} \mathrm{O}_{3}$ & 3.41 & 3.23 & 3.41 & 3.47 & 3.41 & 3.41 & 3.89 & 3.73 & 2.82 & 2.89 & 3.11 & 3.08 & 3.11 & 3.16 & 3.11 & 3.24 & 2.70 & 2.37 & 2.70 & 2.54 & 2.58 & 2.38 \\
\hline $\mathrm{K}_{2} \mathrm{O}$ & 0.80 & 0.82 & 0.80 & 0.85 & 0.80 & 0.86 & 0.92 & 0.93 & 0.66 & 0.72 & 0.73 & 0.76 & 0.73 & 0.76 & 0.73 & 0.7 & 0.63 & 0.63 & 0.63 & 0.68 & 0.61 & 0.61 \\
\hline $\mathrm{Li}_{2} \mathrm{O}^{*}$ & 5.09 & 5.09 & 3.59 & 3.59 & 5.09 & 5.09 & 2.10 & 2.10 & 3.08 & 3.08 & 2.58 & 2.58 & 2.58 & 2.58 & 3.58 & 3.58 & 3.07 & 3.07 & 3.07 & 3.07 & 3.57 & 3.57 \\
\hline MgO & 0.26 & 0.21 & 0.26 & 0.21 & 0.26 & 0.23 & 0.30 & 0.25 & 0.22 & 0.20 & 0.24 & 0.17 & 0.24 & 0.18 & 0.24 & 0.19 & 0.21 & 0.17 & 0.21 & 0.19 & 0.20 & 0.19 \\
\hline $\mathrm{Na}_{2} \mathrm{O}$ & 15.41 & 16.11 & 15.41 & 15.88 & 15.41 & 15.17 & 17.58 & 18.85 & 12.71 & 13.12 & 14.06 & 14.59 & 14.06 & 14.46 & 14.06 & 14.03 & 12.17 & 12.95 & 12.17 & 12.41 & 11.63 & 12.08 \\
\hline $\mathrm{NiO}$ & 0.12 & 0.12 & 0.12 & 0.12 & 0.12 & 0.12 & 0.14 & 0.12 & 0.10 & 0.10 & 0.11 & 0.11 & 0.11 & 0.11 & 0.11 & 0.11 & 0.09 & 0.09 & 0.09 & 0.08 & 0.09 & 0.08 \\
\hline $\mathrm{P}_{2} \mathrm{O}_{5}$ & 2.45 & 2.70 & 2.45 & 2.69 & 2.45 & 2.74 & 2.80 & 2.97 & 2.02 & 2.32 & 2.24 & 2.40 & 2.24 & 2.50 & 2.24 & 2.48 & 1.94 & 2.23 & 1.94 & 2.21 & 1.85 & 2.08 \\
\hline $\mathrm{PbO}$ & 0.11 & 0.10 & 0.1 & 0.11 & 0.1 & 0. & 0.1 & 0. & 0.0 & 0.09 & 0.10 & 0.09 & 0.10 & 0.10 & 0.10 & 0.10 & 0.09 & 0.06 & 0.09 & 0.07 & 0.08 & 0.07 \\
\hline $\mathrm{SiO}_{2}$ & 27.72 & 27.40 & 27.72 & 27.31 & 28.72 & 28.87 & 24.24 & 23.60 & 34.06 & 34.11 & 29.89 & 29.50 & 28.89 & 28.37 & 28.89 & 28.48 & 34.93 & 34.81 & 32.93 & 33.29 & 34.30 & 34.18 \\
\hline $\mathrm{ThO}_{2}$ & 0.00 & 0.00 & 0.00 & 0.00 & 0.00 & 0.00 & 0.00 & 0.00 & 0.00 & 0.00 & 0.00 & 0.00 & 0.00 & 0.00 & 0.00 & 0.00 & 0.00 & 0.00 & 0.00 & 0.00 & 0.00 & 0.00 \\
\hline $\mathrm{TiO}_{2}$ & 0.21 & 0.22 & 0.21 & 0.24 & 0.21 & 0.25 & 0.24 & 0.26 & 0.17 & 0.22 & 0.19 & 0.22 & 0.19 & 0.23 & 0.19 & 0.23 & 0.17 & 0.18 & 0.17 & 0.19 & 0.16 & 0.38 \\
\hline $\mathrm{SO}_{3}$ & 0.26 & 0.28 & 0.26 & 0.27 & 0.26 & 0.32 & 0.30 & 0.28 & 0.22 & 0.22 & 0.24 & 0.26 & 0.24 & 0.25 & 0.24 & 0.26 & 0.21 & 0.24 & 0.21 & 0.19 & 0.20 & 0.19 \\
\hline $\mathrm{ZnO}$ & 0.22 & 0.22 & 0.22 & 0.24 & 0.22 & 0.24 & 0.25 & 0.24 & 0.18 & 0.20 & 0.20 & 0.21 & 0.20 & 0.21 & 0.20 & 0.22 & 0.17 & 0.16 & 0.17 & 0.16 & 0.16 & 0.16 \\
\hline $\mathrm{ZrO}_{2}$ & 0.15 & 0.13 & 0.15 & 0.15 & 0.15 & 0.15 & 0.17 & 0.16 & 0.12 & 0.13 & 0.14 & 0.14 & 0.14 & 0.13 & 0.14 & 0.14 & 0.12 & 0.09 & 2.12 & 1.82 & 2.11 & 1.83 \\
\hline $\mathrm{U}_{3} \mathrm{O}_{8}$ & 0.00 & 0.00 & 0.00 & 0.00 & 0.00 & 0.00 & 0.00 & 0.00 & 0.00 & 0.00 & 0.00 & 0.00 & 0.00 & 0.00 & 0.00 & 0.00 & 0.00 & 0.00 & 0.00 & 0.00 & 0.00 & 0.00 \\
\hline
\end{tabular}

*In XRF analysis target concentrations are used for $\mathrm{B}_{2} \mathrm{O}_{3}$ and $\mathrm{Li}_{2} \mathrm{O} ; \mathrm{F}$ was not analyzed.

- Empty data field 
Table 3.12. Target Composition and XRF Analysis of Glasses Formulated for Al-Na-Limited Waste (continued).

\begin{tabular}{|c|c|c|c|c|c|c|c|c|c|c|c|c|c|c|c|c|c|c|}
\hline & \multicolumn{2}{|c|}{ HLWE-ANa-20 } & \multicolumn{2}{|c|}{ HLWE-ANa-21 } & \multicolumn{3}{|c|}{ HLWE-ANa-22 } & \multicolumn{2}{|c|}{ HLWE-ANa-23 } & \multicolumn{2}{|c|}{ HLWE-ANa-24 } & \multicolumn{3}{|c|}{ HLWE-ANa-25 } & \multicolumn{2}{|c|}{ HLWE-ANa-26 } & \multicolumn{2}{|c|}{ HLW-E-ANa22UTh } \\
\hline & Target & XRF & Target & XRF & Target & XRF & XRF-22R1 & Target & XRF & Target & XRF & Target & XRF & XRF-25R1 & Target & XRF & Target & XRF \\
\hline $\mathrm{Al}_{2} \mathrm{O}_{3}$ & 21.34 & 21.00 & 21.34 & 20.81 & 21.34 & 21.21 & 21.15 & 21.34 & 21.29 & 22.70 & 22.51 & 22.70 & 25.52 & 22.47 & 23.61 & 23.32 & 20.35 & 20.17 \\
\hline $\mathrm{B}_{2} \mathrm{O}_{3}{ }^{*}$ & 14.37 & 14.37 & 15.37 & 15.37 & 18.37 & 18.37 & 18.37 & 18.37 & 18.37 & 18.39 & 18.39 & 19.39 & 19.39 & 19.39 & 19.41 & 19.41 & 18.35 & 18.35 \\
\hline $\mathrm{BaO}$ & 0.03 & 0.03 & 0.03 & 0.03 & 0.03 & 0.04 & 0.05 & 0.03 & 0.03 & 0.03 & 0.03 & 0.03 & 0.03 & 0.04 & 0.03 & 0.04 & 0.03 & 0.03 \\
\hline $\mathrm{Bi}_{2} \mathrm{O}_{3}$ & 1.16 & 1.31 & 1.16 & 1.28 & 1.16 & 1.21 & 1.35 & 1.16 & 1.11 & 1.23 & 1.32 & 1.23 & 1.34 & 1.41 & 1.28 & 1.38 & 1.10 & 1.13 \\
\hline $\mathrm{CaO}$ & 3.72 & 3.97 & 3.72 & 3.78 & 0.72 & 0.77 & 0.83 & 0.72 & 0.73 & 0.77 & 0.81 & 0.77 & 0.82 & 0.87 & 0.80 & 0.85 & 0.69 & 0.70 \\
\hline $\mathrm{CdO}$ & 0.01 & 0.00 & 0.01 & 0.00 & 0.01 & 0.00 & 0.00 & 0.01 & 0.00 & 0.01 & 0.00 & 0.01 & 0.00 & 0.00 & 0.01 & 0.00 & 0.01 & 0.00 \\
\hline $\mathrm{Cr}_{2} \mathrm{O}_{3}$ & 0.71 & 0.93 & 0.71 & 0.91 & 0.71 & 0.86 & 0.95 & 0.71 & 0.79 & 0.76 & 0.92 & 0.76 & 0.94 & 0.96 & 0.79 & 0.98 & 0.68 & 0.79 \\
\hline $\mathrm{F}^{*}$ & 0.23 & - & 0.23 & - & 0.23 & - & - & 0.23 & - & 0.24 & - & 0.24 & - & - & 0.25 & - & 0.22 & - \\
\hline $\mathrm{Fe}_{2} \mathrm{O}_{3}$ & 2.82 & 2.97 & 2.82 & 2.89 & 2.82 & 2.78 & 3.03 & 2.82 & 2.57 & 3.00 & 3.01 & 3.00 & 3.02 & 3.16 & 3.11 & 3.16 & 2.68 & 2.56 \\
\hline $\mathrm{K}_{2} \mathrm{O}$ & 0.66 & 0.72 & 0.66 & 1.13 & 0.66 & 0.69 & 0.72 & 0.66 & 0.67 & 0.71 & 0.72 & 0.71 & 0.75 & 0.74 & 0.73 & 0.76 & 0.63 & 0.69 \\
\hline $\mathrm{Li}_{2} \mathrm{O}^{*}$ & 3.08 & 3.08 & 3.08 & 3.08 & 3.58 & 3.58 & 3.58 & 3.58 & 3.58 & 3.08 & 3.08 & 3.08 & 3.08 & 3.08 & 3.08 & 3.08 & 3.57 & 3.57 \\
\hline $\mathrm{MgO}$ & 0.22 & 0.19 & 0.22 & 0.17 & 0.22 & 0.20 & 0.21 & 0.22 & 0.16 & 0.23 & 0.18 & 0.23 & 0.18 & 0.17 & 0.24 & 0.21 & 0.21 & 0.21 \\
\hline $\mathrm{Na}_{2} \mathrm{O}$ & 12.71 & 12.73 & 12.71 & 12.81 & 12.71 & 12.75 & 12.34 & 12.71 & 13.45 & 13.52 & 13.70 & 13.52 & 13.24 & 13.73 & 14.06 & 14.32 & 12.12 & 12.25 \\
\hline $\mathrm{NiO}$ & 0.10 & 0.10 & 0.10 & 0.10 & 0.10 & 0.09 & 0.10 & 0.10 & 0.08 & 0.11 & 0.12 & 0.11 & 0.11 & 0.12 & 0.11 & 0.11 & 0.09 & 0.09 \\
\hline $\mathrm{P}_{2} \mathrm{O}_{5}$ & 2.02 & 2.27 & 2.02 & 2.24 & 2.02 & 2.28 & 2.29 & 2.02 & 2.36 & 2.15 & 2.38 & 2.15 & 2.41 & 2.43 & 2.24 & 2.45 & 1.93 & 2.17 \\
\hline $\mathrm{PbO}$ & 0.09 & 0.10 & 0.09 & 0.09 & 0.09 & 0.09 & 0.10 & 0.09 & 0.00 & 0.10 & 0.09 & 0.10 & 0.09 & 0.11 & 0.10 & 0.11 & 0.08 & 0.09 \\
\hline $\mathrm{SiO}_{2}$ & 32.06 & 31.55 & 30.06 & 29.43 & 34.56 & 34.25 & 34.09 & 31.56 & 31.47 & 32.26 & 31.91 & 31.26 & 31.25 & 30.49 & 29.39 & 28.93 & 34.42 & 34.29 \\
\hline $\mathrm{ThO}_{2}$ & 0.00 & 0.00 & 0.00 & 0.00 & 0.00 & 0.00 & 0.00 & 0.00 & 0.00 & 0.00 & 0.00 & 0.00 & 0.00 & 0.00 & 0.00 & 0.00 & 0.02 & 0.01 \\
\hline $\mathrm{TiO}_{2}$ & 0.17 & 0.22 & 2.17 & 2.34 & 0.17 & 0.22 & 0.23 & 0.17 & 0.19 & 0.19 & 0.22 & 0.19 & 0.23 & 0.23 & 0.19 & 0.25 & 0.16 & 0.20 \\
\hline $\mathrm{SO}_{3}$ & 0.22 & 0.21 & 0.22 & 0.23 & 0.22 & 0.24 & 0.21 & 0.22 & 0.23 & 0.23 & 0.22 & 0.23 & 0.24 & 0.22 & 0.24 & 0.23 & 0.21 & 0.20 \\
\hline $\mathrm{ZnO}$ & 0.18 & 0.21 & 0.18 & 0.19 & 0.18 & 0.20 & 0.21 & 0.18 & 0.17 & 0.19 & 0.21 & 0.19 & 0.21 & 0.21 & 0.20 & 0.22 & 0.17 & 0.18 \\
\hline $\mathrm{ZrO}_{2}$ & 4.12 & 3.90 & 3.12 & 2.99 & 0.12 & 0.13 & 0.14 & 3.12 & 2.62 & 0.13 & 0.13 & 0.13 & 0.13 & 0.14 & 0.14 & 0.14 & 0.12 & 0.09 \\
\hline $\mathrm{U}_{3} \mathrm{O}_{8}$ & 0.00 & 0.00 & 0.00 & 0.00 & 0.00 & 0.00 & 0.00 & 0.00 & 0.00 & 0.00 & 0.00 & 0.00 & 0.00 & 0.00 & 0.00 & 0.00 & 2.15 & 2.21 \\
\hline
\end{tabular}

*In XRF analysis target concentrations are used for $\mathrm{B}_{2} \mathrm{O}_{3}$ and $\mathrm{Li}_{2} \mathrm{O}$; $\mathrm{F}$ was not analyzed.

- Empty data field 
Table 3.13. Characterization of Glasses Formulated for Al-Na-Limited Waste.

\begin{tabular}{|c|c|c|c|c|c|c|c|c|c|c|}
\hline & Glass Name & HLW-E-ANa-1 & $\begin{array}{c}\text { HLW-E-ANa- } \\
19\end{array}$ & HLW-E-ANa-17 & HLW-E-ANa-18 & HLW-E-ANa-13 & HLW-E-ANa-20 & HLW-E-ANa-21 & HLW-E-ANa-23 & HLW-E-ANa-2 \\
\hline & Waste loading (wt\%) & 42 & 43 & 45 & 45 & 47 & 47 & 47 & 47 & 47 \\
\hline & Glass former type & Li, Zr, P & Li,Ca, Zr & $\mathrm{Li}, \mathrm{Ca}$ & LI,Ca, Zr & $\mathrm{Li}, \mathrm{Ca}$ & Li,Ca, Zr & Li,Ca, Zr, Ti & $\mathrm{Li}, \mathrm{Zr}$ & $\mathrm{Li}$ \\
\hline \multirow{7}{*}{ 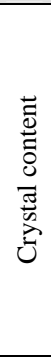 } & Glass as melted & $\begin{array}{c}\text { Trace } \\
\left(\text { Spinel/ } / \mathrm{Cr}_{2} \mathrm{O}_{3}\right) \\
\end{array}$ & $\begin{array}{c}\text { Trace (Ca } \\
\text { phosphate) }\end{array}$ & $\begin{array}{l}\text { Minor (Ca } \\
\text { phosphate) }\end{array}$ & $\begin{array}{c}\text { Trace (Ca } \\
\text { phosphate) }\end{array}$ & $\begin{array}{c}\text { Minor (Ca } \\
\text { phosphate+Nephline) }\end{array}$ & Minor (silicate) & Free & Free & Free \\
\hline & $\mathrm{Xl}$ at $950^{\circ} \mathrm{C}$ & Cr-Fe oxide & - & - & - & - & - & $\mathrm{ZrO}_{2}+\mathrm{Sp}$ & $\mathrm{ZrO}_{2}+\mathrm{Sp}$ & Sp \\
\hline & Glass after $\mathrm{HT}$ at $950^{\circ} \mathrm{C}(\mathrm{vol} \%)$ & 0.3 & - & - & - & - & 1.9 & 0.4 & 1.0 & 0.3 \\
\hline & Glass after $\mathrm{HT}$ at $900^{\circ} \mathrm{C}(\mathrm{vol} \%)$ & 0.3 & - & - & - & - & 3.3 & 1.6 & 1.0 & - \\
\hline & Glass after $\mathrm{HT}$ at $850^{\circ} \mathrm{C}(\mathrm{vol} \%)$ & 1.2 & - & - & - & - & 4.6 & 2.6 & 1.2 & - \\
\hline & Glass after $\mathrm{HT}$ at $800^{\circ} \mathrm{C}$ (vol\%) & 3.9 & $\begin{array}{c}4.1(\mathrm{Sp}+ \\
\text { Pho) }\end{array}$ & 5.4 (Sp + Pho) & $8.2(\mathrm{Pho}+\mathrm{Sp})$ & - & $\sim 12.5$ & $\sim 20$ & 1.8 & - \\
\hline & Glass after HT of CCC (vol\%) & - & $\begin{array}{c}\text { Considerate } \\
\text { amount }\end{array}$ & $\begin{array}{c}5.6(\mathrm{Pho}+\mathrm{Sp}+ \\
\text { Nas) }\end{array}$ & $\begin{array}{c}3.6 \text { (Pho }+\mathrm{Sp}+ \\
\text { Nas) }\end{array}$ & $\begin{array}{l}\text { 12 (Apatite, Na-Al- } \\
\text { Silicate, Spinel) }\end{array}$ & $\begin{array}{c}\sim 31 \text { (Nas + Pho } \\
\left.+\mathrm{ZrO}_{2}\right)\end{array}$ & $\begin{array}{c}\text { Phase } \\
\text { separated }\end{array}$ & 1.3 (spinel) & - \\
\hline \multirow{9}{*}{ 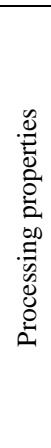 } & Highly viscous during pouring $(\mathrm{Y} / \mathrm{N})$ & $\mathrm{Y}$ & $\mathrm{N}$ & $\mathrm{N}$ & $\mathrm{N}$ & $\mathrm{N}$ & $\mathrm{N}$ & $\mathrm{N}$ & $\mathrm{N}$ & $\mathrm{Y}$ \\
\hline & Viscosity at $1250^{\circ} \mathrm{C}$ (poise) & - & - & - & - & 22.1 & - & - & - & - \\
\hline & Viscosity at $1150^{\circ} \mathrm{C}$ (poise) & - & - & - & - & 46.9 & - & - & - & - \\
\hline & Viscosity at $1050^{\circ} \mathrm{C}$ (poise) & - & - & - & - & 119.0 & - & - & - & - \\
\hline & Viscosity at $950^{\circ} \mathrm{C}$ (poise) & - & - & - & - & 385.5 & - & - & - & - \\
\hline & Electric conductivity at $1150^{\circ} \mathrm{C}(\mathrm{S} / \mathrm{cm})$ & - & - & - & - & 0.31 & - & - & - & - \\
\hline & Electric conductivity at $1050^{\circ} \mathrm{C}(\mathrm{S} / \mathrm{cm})$ & - & - & - & - & 0.22 & - & - & - & - \\
\hline & Electric conductivity at $950^{\circ} \mathrm{C}(\mathrm{S} / \mathrm{cm})$ & - & - & - & - & 0.14 & - & - & - & - \\
\hline & Glass transition temperature $\left({ }^{\circ} \mathrm{C}\right)$ & - & - & - & - & - & - & - & - & - \\
\hline \multirow{16}{*}{ 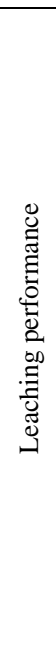 } & PCT & - & - & - & - & - & - & - & - & - \\
\hline & B (ppm) & - & - & - & - & - & - & - & - & 15.5 \\
\hline & Li (ppm) & - & - & - & - & - & - & - & - & 9.4 \\
\hline & $\mathrm{Na}(\mathrm{ppm})$ & - & - & - & - & - & - & - & - & 27.0 \\
\hline & $\mathrm{Si}(\mathrm{ppm})$ & - & - & - & - & - & - & - & - & 57.4 \\
\hline & $\mathrm{B}(\mathrm{g} / \mathrm{L})$ & - & - & - & - & - & - & - & - & 0.35 \\
\hline & $\mathrm{Li}(\mathrm{g} / \mathrm{L})$ & - & - & - & - & - & - & - & - & 0.66 \\
\hline & $\mathrm{Na}(\mathrm{g} / \mathrm{L})$ & - & - & - & - & - & - & - & - & 0.29 \\
\hline & $\mathrm{Si}(\mathrm{g} / \mathrm{L})$ & - & - & - & - & - & - & - & - & 0.31 \\
\hline & PCT (for CCC sample) & - & - & - & - & - & - & - & - & - \\
\hline & B (ppm) & - & - & - & - & - & - & - & - & - \\
\hline & $\mathrm{Li}(\mathrm{ppm})$ & - & - & - & - & - & - & - & - & - \\
\hline & $\mathrm{Na}(\mathrm{ppm})$ & - & - & - & - & - & - & - & - & - \\
\hline & Si (ppm) & - & - & - & - & - & - & - & - & - \\
\hline & $\mathrm{Si}(\mathrm{g} / \mathrm{L})$ & - & - & - & - & - & - & - & - & - \\
\hline & TCLP (Pass/Fail) & - & - & - & - & - & - & - & - & - \\
\hline
\end{tabular}

Sp = spinel; Pho = phosphate; Nas = sodium aluminosilicate

- Empty data field 
Table 3.13. Characterization of Glasses Formulated for Al-Na-Limited Waste (continued).

\begin{tabular}{|c|c|c|c|c|c|c|c|c|}
\hline & Glass Name & HLW-E-ANa-4 & HLW-E-ANa-22 & HLW-E-ANa-22UTh & HLW-E-ANa-24 & HLW-E-ANa-25 & HLW-E-ANa-14 & HLW-E-ANa-15 \\
\hline & Waste loading (wt\%) & 47 & 47 & 47 & 50 & 50 & 52 & 52 \\
\hline & Glass former type & $\mathrm{Li}$ & $\mathrm{Li}$ & $\mathrm{Li}$ & $\mathrm{Li}$ & $\mathrm{Li}$ & $\mathrm{Li}, \mathrm{Ca}$ & $\mathrm{Li}, \mathrm{Ca}$ \\
\hline \multirow{7}{*}{ 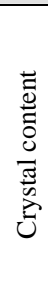 } & Glass as melted & Free & Free & NA & Free & Free & $\begin{array}{c}\text { Minor (Ca- } \\
\text { phosphate+Nephline) }\end{array}$ & $\begin{array}{c}\text { Minor (Ca- } \\
\text { phosphate+Nephline) }\end{array}$ \\
\hline & $\mathrm{Xl}$ at $950^{\circ} \mathrm{C}$ & Sp & Sp & Sp & Sp & Sp & Pho + Sp & Nas + Sp + Pho \\
\hline & Glass after $\mathrm{HT}$ at $950^{\circ} \mathrm{C}$ (vol\%) & 0.2 & 0.3 & 0.3 & 0.5 & 0.5 & 1.7 & 5.4 \\
\hline & Glass after $\mathrm{HT}$ at $900^{\circ} \mathrm{C}$ (vol\%) & 0.2 & 0.5 & 0.2 & 0.7 & 0.7 & - & - \\
\hline & Glass after $\mathrm{HT}$ at $850^{\circ} \mathrm{C}$ (vol\%) & 0.2 & 0.5 & 0.4 & 0.7 & 0.7 & - & - \\
\hline & Glass after $\mathrm{HT}$ at $800^{\circ} \mathrm{C}(\mathrm{vol} \%)$ & 2.1 & 0.5 & 0.3 & 1.0 & 0.9 & - & - \\
\hline & Glass after HT of CCC (vol\%) & $2-5(\mathrm{Sp}+\mathrm{Nas})$ & $0.5(\mathrm{Sp})$ & $0.3(\mathrm{Sp})$ & $3(\mathrm{Sp}+\mathrm{Nas})$ & $2(\mathrm{Sp}+\mathrm{Nas})$ & $\begin{aligned} & \text { 50-60 (Nas } \\
+ & \text { Apatite + Sp) }\end{aligned}$ & $\begin{array}{c}\text { 20-50 (Nas + Apatite } \\
\text { + Sp) }\end{array}$ \\
\hline \multirow{10}{*}{ 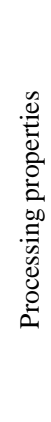 } & Highly viscous during pouring $(\mathrm{Y} / \mathrm{N})$ & $\mathrm{N}$ & $\mathrm{N}$ & $\mathrm{N}$ & $\mathrm{N}$ & $\mathrm{N}$ & $\mathrm{N}$ & $\mathrm{Y}$ \\
\hline & Viscosity at $1250^{\circ} \mathrm{C}$ (poise) & 42.5 & 28.7 & 29.3 & - & 25.6 & - & - \\
\hline & Viscosity at $1150^{\circ} \mathrm{C}$ (poise) & 89.4 & 59.8 & 63.4 & - & 55.4 & - & - \\
\hline & Viscosity at $1050^{\circ} \mathrm{C}$ (poise) & 226.4 & 144.7 & 174.2 & - & 143.8 & - & - \\
\hline & Viscosity at $950^{\circ} \mathrm{C}$ (poise) & 747.5 & 428.4 & 690.5 & - & 481.6 & - & - \\
\hline & Electric conductivity at $1250^{\circ} \mathrm{C}(\mathrm{S} / \mathrm{cm})$ & 0.17 & 0.48 & 0.57 & - & 0.48 & - & - \\
\hline & Electric conductivity at $1150^{\circ} \mathrm{C}(\mathrm{S} / \mathrm{cm})$ & 0.25 & 0.38 & 0.43 & - & 0.36 & - & - \\
\hline & Electric conductivity at $1050^{\circ} \mathrm{C}(\mathrm{S} / \mathrm{cm})$ & 0.34 & 0.28 & 0.30 & - & 0.25 & - & - \\
\hline & Electric conductivity at $950^{\circ} \mathrm{C}(\mathrm{S} / \mathrm{cm})$ & 0.44 & 0.18 & 0.19 & - & 0.16 & - & - \\
\hline & Glass transition temperature $\left({ }^{\circ} \mathrm{C}\right)$ & - & 469 & - & - & - & - & - \\
\hline \multirow{19}{*}{ 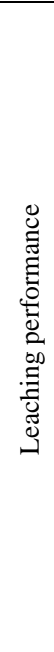 } & PCT & - & - & - & - & - & - & - \\
\hline & B (ppm) & 18.7 & 145.2 & 102.3 & - & 229.3 & - & - \\
\hline & Li (ppm) & 11.4 & 34.8 & 25.7 & - & 42.8 & - & - \\
\hline & $\mathrm{Na}(\mathrm{ppm})$ & 31.9 & 111.4 & 76.2 & - & 183.5 & - & - \\
\hline & Si (ppm) & 61.2 & 49.8 & 46.3 & - & 43.2 & - & - \\
\hline & $\mathrm{B}(\mathrm{g} / \mathrm{L})$ & 0.42 & 2.55 & 1.80 & - & 3.81 & - & - \\
\hline & $\mathrm{Li}(\mathrm{g} / \mathrm{L})$ & 0.60 & 2.09 & 1.55 & - & 2.99 & - & - \\
\hline & $\mathrm{Na}(\mathrm{g} / \mathrm{L})$ & 0.34 & 1.18 & 0.85 & - & 1.83 & - & - \\
\hline & $\mathrm{Si}(\mathrm{g} / \mathrm{L})$ & 0.34 & 0.31 & 0.29 & - & 0.30 & - & - \\
\hline & PCT (for CCC sample) & - & - & - & - & & - & - \\
\hline & B (ppm) & $32.1^{*}$ & 144.4 & - & - & 523.2 & - & - \\
\hline & Li (ppm) & $22.2^{*}$ & 34.2 & - & - & 100.5 & - & - \\
\hline & $\mathrm{Na}(\mathrm{ppm})$ & $44.1^{*}$ & 109.6 & - & - & 356.2 & - & - \\
\hline & Si (ppm) & $62.4^{*}$ & 51.0 & - & - & 36.6 & - & - \\
\hline & $\mathrm{B}(\mathrm{g} / \mathrm{L})$ & $0.72 *$ & 2.53 & - & - & 8.69 & - & - \\
\hline & Li (g/L) & $1.18^{*}$ & 2.06 & - & - & 7.02 & - & - \\
\hline & $\mathrm{Na}(\mathrm{g} / \mathrm{L})$ & $0.47^{*}$ & 1.16 & - & - & 3.55 & - & - \\
\hline & Si (g/L) & $0.35^{*}$ & 0.32 & - & - & 0.25 & - & - \\
\hline & TCLP (Pass/Fail) & PASS & PASS & PASS & - & PASS & - & - \\
\hline
\end{tabular}

*Single sample analysis

$\mathrm{Sp}=$ spinel; Pho = phosphate; Nas = sodium aluminosilicate

- Empty data field 
Table 3.13. Characterization of Glasses Formulated for Al-Na-Limited Waste (continued).

\begin{tabular}{|c|c|c|c|c|c|c|}
\hline & Glass Name & HLW-E-ANa-16 & HLW-E-ANa-3 & HLW-E-ANa-5 & HLW-E-ANa26 & HLW-E-ANa6 \\
\hline & Waste loading (wt\%) & 52 & 52 & 52 & 52 & 57 \\
\hline & Glass former type & $\mathrm{Li}, \mathrm{Ca}$ & $\mathrm{Li}$ & $\mathrm{Li}$ & $\mathrm{Li}$ & $\mathrm{Li}$ \\
\hline \multirow{7}{*}{ 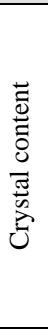 } & Glass as melted & Trace (Ca-phosphate+Nephline) & Trace (Sp) & Trace (Sp) & Free & Trace (Sp) \\
\hline & $\mathrm{Xl}$ at $950^{\circ} \mathrm{C}$ & $\mathrm{Sp}+\mathrm{Pho}+\mathrm{Nas}$ & Sp & Sp & Sp & Sp \\
\hline & Glass after $\mathrm{HT}$ at $950^{\circ} \mathrm{C}$ (vol\%) & 8.8 & 0.5 & 0.3 & 0.6 & 0.8 \\
\hline & Glass after $\mathrm{HT}$ at $900^{\circ} \mathrm{C}$ (vol\%) & - & - & 0.5 & 0.7 & 5.6 \\
\hline & Glass after $\mathrm{HT}$ at $850^{\circ} \mathrm{C}$ (vol\%) & - & - & $\sim 5.4$ & 0.9 & $\sim 16$ \\
\hline & Glass after $\mathrm{HT}$ at $800^{\circ} \mathrm{C}(\mathrm{vol} \%)$ & $\sim 36$ & - & $\sim 5.7$ & 0.6 & $\sim 11$ \\
\hline & Glass after HT of CCC (vol\%) & 30-50 (Pho+Na-Al-Silicate+Spinel) & - & $\begin{array}{c}\sim 50 \text { (Na-Al- } \\
\text { Silicate) }\end{array}$ & $\begin{array}{l}\text { 5.6 (P-rich Na-Al- } \\
\text { Silicate+spinel) }\end{array}$ & - \\
\hline \multirow{10}{*}{ 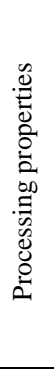 } & Highly viscous during pouring $(\mathrm{Y} / \mathrm{N})$ & $\mathrm{N}$ & $\mathrm{Y}$ & $\mathrm{N}$ & $\mathrm{Y}$ & $\mathrm{N}$ \\
\hline & Viscosity at $1250^{\circ} \mathrm{C}$ (poise) & - & - & 35.6 & - & 48.5 \\
\hline & Viscosity at $1150^{\circ} \mathrm{C}$ (poise) & - & - & 81.9 & - & 115.0 \\
\hline & Viscosity at $1050^{\circ} \mathrm{C}$ (poise) & - & - & 213.7 & - & 342.1 \\
\hline & Viscosity at $950^{\circ} \mathrm{C}$ (poise) & - & - & 652.3 & - & 1416.1 \\
\hline & Electric conductivity at $1250^{\circ} \mathrm{C}(\mathrm{S} / \mathrm{cm})$ & - & - & 0.17 & - & 0.65 \\
\hline & Electric conductivity at $1150^{\circ} \mathrm{C}(\mathrm{S} / \mathrm{cm})$ & - & - & 0.25 & - & 0.49 \\
\hline & Electric conductivity at $1050^{\circ} \mathrm{C}(\mathrm{S} / \mathrm{cm})$ & - & - & 0.35 & - & 0.35 \\
\hline & Electric conductivity at $950^{\circ} \mathrm{C}(\mathrm{S} / \mathrm{cm})$ & - & - & 0.47 & - & 0.24 \\
\hline & Glass transition temperature $\left({ }^{\circ} \mathrm{C}\right)$ & - & - & - & - & - \\
\hline \multirow{19}{*}{ 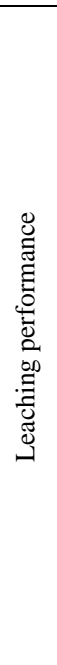 } & PCT & - & - & - & - & - \\
\hline & B (ppm) & - & 13.7 & 28.7 & - & 58.0 \\
\hline & $\mathrm{Li}(\mathrm{ppm})$ & - & 8.0 & 13.5 & - & 15.6 \\
\hline & $\mathrm{Na}(\mathrm{ppm})$ & - & 40.5 & 62.5 & - & 106.8 \\
\hline & Si (ppm) & - & 54.3 & 61.0 & - & 52.1 \\
\hline & $\mathrm{B}(\mathrm{g} / \mathrm{L})$ & - & 0.33 & 0.64 & - & 1.29 \\
\hline & $\mathrm{Li}(\mathrm{g} / \mathrm{L})$ & - & 0.67 & 0.81 & - & 1.20 \\
\hline & $\mathrm{Na}(\mathrm{g} / \mathrm{L})$ & - & 0.39 & 0.60 & - & 0.93 \\
\hline & $\mathrm{Si}(\mathrm{g} / \mathrm{L})$ & - & 0.32 & 0.39 & - & 0.37 \\
\hline & PCT (for CCC sample) & - & - & - & - & - \\
\hline & B (ppm) & - & - & - & - & - \\
\hline & $\mathrm{Li}(\mathrm{ppm})$ & - & - & - & - & - \\
\hline & $\mathrm{Na}(\mathrm{ppm})$ & - & - & - & - & - \\
\hline & Si (ppm) & - & - & - & - & - \\
\hline & $\mathrm{B}(\mathrm{g} / \mathrm{L})$ & - & - & - & - & - \\
\hline & $\mathrm{Li}(\mathrm{g} / \mathrm{L})$ & - & - & - & - & - \\
\hline & $\mathrm{Na}(\mathrm{g} / \mathrm{L})$ & - & - & - & - & - \\
\hline & Si (g/L) & - & - & - & - & - \\
\hline & TCLP (Pass/Fail) & - & - & PASS & - & PASS \\
\hline
\end{tabular}

Sp = spinel; Pho $=$ phosphate; Nas = sodium aluminosilicate

- Empty data field 
Table 3.13. Characterization of Glasses Formulated for Al-Na-Limited Waste (continued).

\begin{tabular}{|c|c|c|c|c|c|c|c|}
\hline & Glass Name & HLW-E-ANa8 & HLW-E-ANa9 & HLW-E-ANa10 & HLW-E-ANa11 & HLW-E-ANa7 & HLW-E-ANa12 \\
\hline & Waste loading (wt\%) & 57 & 57 & 57 & 57 & 60 & 65 \\
\hline & Glass former type & $\mathrm{Li}$ & $\mathrm{Li}$ & $\mathrm{Li}$ & $\mathrm{Li}$ & $\mathrm{Li}$ & $\mathrm{Li}$ \\
\hline \multirow{7}{*}{ 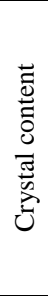 } & Glass as melted & Heterogeneous & Free & Heterogeneous & Free & Heterogeneous & Heterogeneous \\
\hline & $\mathrm{Xl}$ at $950^{\circ} \mathrm{C}$ & - & Spinel & $\mathrm{Sp}+\mathrm{Nas}$ & - & - & $\mathrm{Nas}+\mathrm{Sp}$ \\
\hline & Glass after $\mathrm{HT}$ at $950^{\circ} \mathrm{C}$ (vol\%) & - & 0.4 & 1.6 & - & - & $\sim 10$ \\
\hline & Glass after $\mathrm{HT}$ at $900^{\circ} \mathrm{C}$ (vol\%) & 5.4 & 2.5 & 5.7 & - & - & - \\
\hline & Glass after $\mathrm{HT}$ at $850^{\circ} \mathrm{C}$ (vol\%) & - & 6.6 & $\sim 10$ & - & - & - \\
\hline & Glass after $\mathrm{HT}$ at $800^{\circ} \mathrm{C}$ (vol\%) & - & $\sim 35$ & $\sim 16$ & - & - & - \\
\hline & Glass after HT of CCC (vol\%) & - & > 50 (Nephline+Sodalite) & & $>50$ (Nephline+Sodalite) & - & - \\
\hline \multirow{10}{*}{ 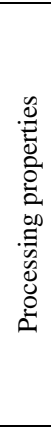 } & Highly viscous during pouring $(\mathrm{Y} / \mathrm{N})$ & $\mathrm{N}$ & $\mathrm{N}$ & $\mathrm{N}$ & $\mathrm{N}$ & $\mathrm{N}$ & $\mathrm{Y}$ \\
\hline & Viscosity at $1250^{\circ} \mathrm{C}$ (poise) & - & 22.4 & - & 21.6 & - & - \\
\hline & Viscosity at $1150^{\circ} \mathrm{C}$ (poise) & - & 38.9 & - & 43.7 & - & - \\
\hline & Viscosity at $1050^{\circ} \mathrm{C}$ (poise) & - & 83.0 & - & 102.3 & - & - \\
\hline & Viscosity at $950^{\circ} \mathrm{C}$ (poise) & - & 239.5 & - & 293.1 & - & - \\
\hline & Electric conductivity at $1250^{\circ} \mathrm{C}(\mathrm{S} / \mathrm{cm})$ & - & 0.61 & - & 0.65 & - & - \\
\hline & Electric conductivity at $1150^{\circ} \mathrm{C}(\mathrm{S} / \mathrm{cm})$ & - & 0.48 & - & 0.50 & - & - \\
\hline & Electric conductivity at $1050^{\circ} \mathrm{C}(\mathrm{S} / \mathrm{cm})$ & - & 0.37 & - & 0.37 & - & - \\
\hline & Electric conductivity at $950^{\circ} \mathrm{C}(\mathrm{S} / \mathrm{cm})$ & - & 0.27 & - & 0.26 & - & - \\
\hline & Glass transition temperature $\left({ }^{\circ} \mathrm{C}\right)$ & - & - & - & - & - & - \\
\hline \multirow{19}{*}{ 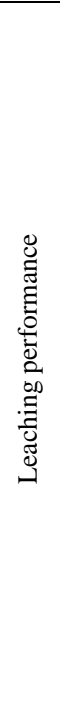 } & PCT & - & - & - & - & - & - \\
\hline & B (ppm) & 83.5 & 121.8 & 111.8 & 97.1 & - & - \\
\hline & $\mathrm{Li}(\mathrm{ppm})$ & 25.2 & 47.4 & 28.3 & 37.4 & - & - \\
\hline & $\mathrm{Na}(\mathrm{ppm})$ & 140.9 & 201.8 & 162.3 & 172.1 & - & - \\
\hline & Si (ppm) & 47.4 & 45.9 & 37.5 & 52.7 & - & - \\
\hline & $\mathrm{B}(\mathrm{g} / \mathrm{L})$ & 1.86 & 2.72 & 2.26 & 2.33 & - & - \\
\hline & $\mathrm{Li}(\mathrm{g} / \mathrm{L})$ & 1.51 & 2.00 & 1.69 & 1.58 & - & - \\
\hline & $\mathrm{Na}(\mathrm{g} / \mathrm{L})$ & 1.23 & 1.76 & 1.42 & 1.51 & - & - \\
\hline & $\mathrm{Si}(\mathrm{g} / \mathrm{L})$ & 0.35 & 0.35 & 0.29 & 0.39 & - & - \\
\hline & PCT (for CCC sample) & - & - & - & - & - & - \\
\hline & B (ppm) & - & $3630 * *$ & - & - & - & - \\
\hline & $\mathrm{Li}(\mathrm{ppm})$ & - & $1155.3^{* *}$ & - & - & - & - \\
\hline & $\mathrm{Na}(\mathrm{ppm})$ & - & $2107.5^{* *}$ & - & - & - & - \\
\hline & $\mathrm{Si}(\mathrm{ppm})$ & - & $<0.84^{* *}$ & - & - & - & - \\
\hline & $\mathrm{B}(\mathrm{g} / \mathrm{L})$ & - & $81.07 * *$ & - & - & - & - \\
\hline & $\mathrm{Li}(\mathrm{g} / \mathrm{L})$ & - & $49.33^{* *}$ & - & - & - & - \\
\hline & $\mathrm{Na}(\mathrm{g} / \mathrm{L})$ & - & $18.43^{* *}$ & - & - & - & - \\
\hline & $\mathrm{Si}(\mathrm{g} / \mathrm{L})$ & - & - & - & - & - & - \\
\hline & TCLP (Pass/Fail) & PASS & PASS & PASS & PASS & - & - \\
\hline
\end{tabular}

**Not standard test; single sample result; experiment terminated after 6 days.

$\mathrm{Sp}=$ spinel; Pho $=$ phosphate; Nas = sodium aluminosilicate

- Empty data field 
Table 3.14. Composition and Properties of Aluminum-Plus-Sodium-Limited Waste and Glass Formulation at $47 \%$ Waste Loading Used in Melter Tests (wt\%).

\begin{tabular}{|c|c|c|c|c|}
\hline- & $\begin{array}{c}\text { Al-Na Limited } \\
\text { Waste* }\end{array}$ & Waste in Glass & $\begin{array}{c}\text { Glass Forming } \\
\text { Additives }\end{array}$ & $\begin{array}{c}\text { Target Glass } \\
\text { HLW-E-ANa-22 }\end{array}$ \\
\hline $\mathrm{Al}_{2} \mathrm{O}_{3}$ & 45.40 & 21.34 & 0.00 & 21.34 \\
\hline $\mathrm{B}_{2} \mathrm{O}_{3}$ & 0.78 & 0.37 & 18.00 & 18.37 \\
\hline $\mathrm{BaO}$ & 0.06 & 0.03 & 0.00 & 0.03 \\
\hline $\mathrm{Bi}_{2} \mathrm{O}_{3}$ & 2.46 & 1.16 & 0.00 & 1.16 \\
\hline $\mathrm{CaO}$ & 1.54 & 0.72 & 0.00 & 0.72 \\
\hline $\mathrm{CdO}$ & 0.02 & 0.01 & 0.00 & 0.01 \\
\hline $\mathrm{Cr}_{2} \mathrm{O}_{3}$ & 1.51 & 0.71 & 0.00 & 0.71 \\
\hline F & 0.48 & 0.23 & 0.00 & 0.23 \\
\hline $\mathrm{Fe}_{2} \mathrm{O}_{3}$ & 5.99 & 2.82 & 0.00 & 2.82 \\
\hline $\mathrm{K}_{2} \mathrm{O}$ & 1.41 & 0.66 & 0.00 & 0.66 \\
\hline $\mathrm{Li}_{2} \mathrm{O}$ & 0.16 & 0.08 & 3.50 & 3.58 \\
\hline $\mathrm{MgO}$ & 0.46 & 0.22 & 0.00 & 0.22 \\
\hline $\mathrm{Na}_{2} \mathrm{O}$ & 27.04 & 12.71 & 0.00 & 12.71 \\
\hline $\mathrm{NiO}$ & 0.21 & 0.10 & 0.00 & 0.10 \\
\hline $\mathrm{P}_{2} \mathrm{O}_{5}$ & 4.30 & 2.02 & 0.00 & 2.02 \\
\hline $\mathrm{PbO}$ & 0.19 & 0.09 & 0.00 & 0.09 \\
\hline $\mathrm{SiO}_{2}$ & 6.52 & 3.06 & 31.50 & 34.56 \\
\hline $\mathrm{TiO}_{2}$ & 0.37 & 0.17 & 0.00 & 0.17 \\
\hline $\mathrm{SO}_{3}$ & 0.46 & 0.22 & 0.00 & 0.22 \\
\hline $\mathrm{ZnO}$ & 0.38 & 0.18 & 0.00 & 0.18 \\
\hline $\mathrm{ZrO}_{2}$ & 0.26 & 0.12 & 0.00 & 0.12 \\
\hline Sum & 100.00 & 47.00 & 53.00 & 100.00 \\
\hline
\end{tabular}

* Renormalized from Ref. [3] after removal of radioactive components

\begin{tabular}{|c|c|c|c|}
\hline \multicolumn{3}{|c|}{ Viscosity @1150º, P } & 60 \\
\hline \multicolumn{3}{|c|}{ Conductivity @1150º, S/cm } & 0.38 \\
\hline \multicolumn{3}{|c|}{ Crystal Content, As Melted } & Trace \\
\hline \multicolumn{3}{|c|}{ Crystal Content, $72 \mathrm{hr}$ at $950^{\circ} \mathrm{C}$} & $\sim 0.3 \mathrm{vol} \%$ \\
\hline \multicolumn{3}{|c|}{ Crystal Content, CCC } & $\sim 0.5$ vol \% \\
\hline \multicolumn{3}{|c|}{ TCLP } & Pass \\
\hline \multirow{4}{*}{ PCT, g/L } & - & DWPF-EA & HLW-E-ANa-22 \\
\hline & B & 16.7 & 2.55 \\
\hline & $\mathrm{Li}$ & 9.6 & 2.09 \\
\hline & $\mathrm{Na}$ & 13.3 & 1.18 \\
\hline
\end{tabular}

- Empty data field 
ORP-56297 Rev. 0

The Catholic University of America

High Level Waste Vitrification System Improvements

Vitreous State Laboratory

Final Report, VSL-07R1010-1, Rev. 0

Table 4.1. Summary of Chromium-Limited Waste DM10 Test Conditions and Results.

\begin{tabular}{|c|c|c|c|c|c|c|c|}
\hline \multicolumn{2}{|r|}{ Test } & 1 & 2 & 3 & 4 & 5 & 6 \\
\hline \multirow{5}{*}{ Time } & \multirow[t]{2}{*}{ Feed Start } & 7/31/06 & 8/1/06 & $8 / 2 / 06$ & 8/3/06 & 8/4/06 & 8/7/06 \\
\hline & & 10:00 & 11:35 & 13:11 & $14: 11$ & 14:09 & $7: 20$ \\
\hline & \multirow{2}{*}{ Feed End } & 8/1/06 & 8/2/06 & 8/3/06 & 8/4/06 & 8/5/06 & 8/7/06 \\
\hline & & 9:30 & $12: 30$ & $12: 45$ & $12: 20$ & 4:00 & $19: 45$ \\
\hline & Net Slurry Feeding (hr) & 23.5 & 24.9 & 23.6 & 22.2 & 13.9 & 12.4 \\
\hline \multicolumn{2}{|r|}{ Glass Temperature $\left({ }^{\circ} \mathrm{C}\right)$} & 1150 & 1150 & 1150 & 1150 & 1150 & 1175 \\
\hline \multirow{5}{*}{ Feed } & Waste Loading & $40 \%$ & $40 \%$ & $37.5 \%$ & $35 \%$ & $32.5 \%$ & $32.5 \%$ \\
\hline & wt $\% \mathrm{Cr}_{2} \mathrm{O}_{3}$ as glass & 1.33 & 1.33 & 1.25 & 1.16 & 1.08 & 1.08 \\
\hline & $\mathrm{wt} \% \mathrm{SO}_{3}$ as glass & 0.66 & 0.66 & 0.62 & 0.58 & 0.53 & 0.53 \\
\hline & Extra Additives & None & $10 \mathrm{~g} / \mathrm{l}$ sugar & None & None & None & None \\
\hline & Feed Used (kg) & 65.1 & 68.2 & 67.8 & 67.5 & 47.0 & 62.1 \\
\hline \multicolumn{2}{|r|}{ Average Production Rate $\left(\mathrm{kg} / \mathrm{m}^{2} /\right.$ day $)$} & 1154 & 1139 & 1192 & 1271 & 1413 & 2095 \\
\hline \multicolumn{2}{|r|}{ Average Bubbling Rate (lpm) } & 0.79 & 1.08 & 0.47 & 0.53 & 0.54 & 1.17 \\
\hline \multirow{3}{*}{ Product } & Secondary Phases on Melt Surface at Test End & Yes & No & Yes & Yes & Yes & Trace \\
\hline & Measured wt $\% \mathrm{Cr}_{2} \mathrm{O}_{3}$ & 1.23 & 1.51 & 1.17 & 1.12 & 1.05 & 1.17 \\
\hline & Measured wt $\% \mathrm{SO}_{3}$ & 0.49 & 0.31 & 0.55 & 0.53 & 0.46 & 0.43 \\
\hline
\end{tabular}


ORP-56297 Rev. 0

The Catholic University of America High Level Waste Vitrification System Improvements Vitreous State Laboratory Final Report, VSL-07R1010-1, Rev. 0

Table 4.1. Summary of Chromium-Limited-Waste DM10 Test Conditions and Results (continued).

\begin{tabular}{|c|c|c|c|c|c|c|c|}
\hline \multicolumn{2}{|r|}{ Test } & $7 \mathrm{~A}$ & $8 B$ & 9 & $10 \mathrm{~A}$ & $10 \mathrm{C}$ & 12 \\
\hline \multirow{4}{*}{ Time } & Feed Start & $\begin{array}{c}8 / 8 / 06 \\
7: 00\end{array}$ & $\begin{array}{c}8 / 9 / 06 \\
7: 00\end{array}$ & $\begin{array}{c}8 / 14 / 06 \\
8: 50\end{array}$ & $\begin{array}{c}\text { 8/15/06 } \\
3: 00\end{array}$ & $\begin{array}{c}8 / 15 / 06 \\
21: 30\end{array}$ & $\begin{array}{c}8 / 16 / 06 \\
17: 00\end{array}$ \\
\hline & \multirow{2}{*}{ Feed End } & $8 / 8 / 06$ & $8 / 9 / 06$ & $8 / 14 / 06$ & $8 / 15 / 06$ & $8 / 16 / 06$ & $8 / 17 / 06$ \\
\hline & & 19:30 & $20: 30$ & 23:04 & $19: 45$ & $16: 00$ & $11: 32$ \\
\hline & Net Slurry Feeding (hr) & 12.5 & 13.5 & 14.2 & 16.75 & 18.5 & 18.5 \\
\hline \multicolumn{2}{|r|}{ Glass Temperature $\left({ }^{\circ} \mathrm{C}\right)$} & 1175 & 1150 & 1175 & 1175 & 1150 & 1150 \\
\hline \multirow{5}{*}{ Feed } & Waste Loading & $30 \%$ & $30 \%$ & $34.7 \%$ & $34.7 \%$ & $32.2 \%$ & $32.5 \%$ \\
\hline & $\mathrm{wt} \% \mathrm{Cr}_{2} \mathrm{O}_{3}$ as glass & 1.0 & 1.0 & 1.15 & 1.15 & 1.07 & 1.08 \\
\hline & $\mathrm{wt} \% \mathrm{SO}_{3}$ as glass & 0.49 & 0.49 & 0.57 & 0.57 & 0.52 & 0.53 \\
\hline & Extra Additives & None & None & Vanadium & $\begin{array}{l}\text { Vanadium }+2 \mathrm{~g} / \mathrm{l} \\
\text { sugar }\end{array}$ & Vanadium & None \\
\hline & Feed Used (kg) & 67.0 & 65.1 & 66.1 & 67.2 & 66.7 & 66.5 \\
\hline \multicolumn{2}{|c|}{ Average Production Rate $\left(\mathrm{kg} / \mathrm{m}^{2} /\right.$ day) } & 2179 & 2023 & 1947 & 1678 & 1508 & 1504 \\
\hline \multicolumn{2}{|r|}{ Average Bubbling Rate (lpm) } & 1.31 & 1.31 & 1.32 & 1.54 & 1.55 & 1.42 \\
\hline \multirow{3}{*}{ Product } & $\begin{array}{l}\text { Secondary Phases on Melt Surface } \\
\text { at Test End }\end{array}$ & No & No & Yes & Yes & No & No \\
\hline & Measured wt $\% \mathrm{Cr}_{2} \mathrm{O}_{3}$ & 1.12 & 1.13 & 1.21 & 1.23 & 1.16 & 1.17 \\
\hline & Measured wt $\% \mathrm{SO}_{3}$ & 0.38 & 0.42 & 0.64 & 0.41 & 0.39 & 0.45 \\
\hline
\end{tabular}


Table 4.2. Composition and Properties of Chromium-Limited Waste and Glass Formulation at 32.5\% Waste Loading Used in DM100 Melter Tests (wt\%).

\begin{tabular}{|c|c|c|c|c|}
\hline- & $\begin{array}{c}\text { Cr-Limited } \\
\text { Waste* }\end{array}$ & Waste in Glass & $\begin{array}{c}\text { Glass Forming } \\
\text { Additives }\end{array}$ & $\begin{array}{c}\text { Target Glass } \\
\text { HLW-E-Cr-M }\end{array}$ \\
\hline $\mathrm{Al}_{2} \mathrm{O}_{3}$ & 27.64 & 8.98 & - & 8.98 \\
\hline $\mathrm{B}_{2} \mathrm{O}_{3}$ & 0.57 & 0.19 & 15.99 & 16.17 \\
\hline $\mathrm{BaO}$ & 0.03 & 0.01 & - & 0.01 \\
\hline $\mathrm{Bi}_{2} \mathrm{O}_{3}$ & 7.89 & 2.56 & - & 2.56 \\
\hline $\mathrm{CaO}$ & 2.67 & 0.87 & - & 0.87 \\
\hline $\mathrm{CdO}$ & 0.01 & 0.00 & - & $<0.01$ \\
\hline $\mathrm{Cr}_{2} \mathrm{O}_{3}$ & 3.32 & 1.08 & - & 1.08 \\
\hline $\mathrm{F}$ & 2.17 & 0.70 & - & 0.70 \\
\hline $\mathrm{Fe}_{2} \mathrm{O}_{3}$ & 14.22 & 4.62 & - & 4.62 \\
\hline $\mathrm{K}_{2} \mathrm{O}$ & 0.40 & 0.13 & 5.92 & 6.05 \\
\hline $\mathrm{Li}_{2} \mathrm{O}$ & 0.39 & 0.13 & 3.55 & 3.68 \\
\hline $\mathrm{MgO}$ & 0.17 & 0.06 & - & 0.06 \\
\hline $\mathrm{Na}_{2} \mathrm{O}$ & 21.75 & 7.07 & - & 7.07 \\
\hline $\mathrm{NiO}$ & 1.15 & 0.37 & - & 0.37 \\
\hline $\mathrm{P}_{2} \mathrm{O}_{5}$ & 3.62 & 1.18 & - & 1.18 \\
\hline $\mathrm{PbO}$ & 0.52 & 0.17 & - & 0.17 \\
\hline $\mathrm{SiO}_{2}$ & 11.43 & 3.72 & 42.04 & 45.76 \\
\hline $\mathrm{TiO}_{2}$ & 0.01 & 0.00 & - & $<0.01$ \\
\hline $\mathrm{SO}_{3}$ & 1.65 & 0.53 & - & 0.53 \\
\hline $\mathrm{ZnO}$ & 0.27 & 0.09 & - & 0.09 \\
\hline $\mathrm{ZrO}_{2}$ & 0.12 & 0.04 & - & 0.04 \\
\hline Sum & 100 & 32.5 & 67.5 & 100 \\
\hline
\end{tabular}

* Renormalized from Ref. [3] after removal of radioactive components

\begin{tabular}{|c|c|c|c|}
\hline \multicolumn{3}{|c|}{ Viscosity @1150ㄷ, P } & 77.9 \\
\hline \multicolumn{3}{|c|}{ Conductivity @1150º, S/cm } & 0.20 \\
\hline \multicolumn{3}{|c|}{ Crystal Content, As Melted } & None \\
\hline \multicolumn{3}{|c|}{ Crystal Content, $72 \mathrm{hr}$ at $950^{\circ} \mathrm{C}$} & 0.8 vol \% \\
\hline \multicolumn{3}{|c|}{ Crystal Content, CCC } & $1.3 \mathrm{vol} \%$ \\
\hline \multicolumn{3}{|c|}{ TCLP } & Pass \\
\hline \multirow{4}{*}{ PCT, g/L } & - & DWPF-EA & HLW-E-Cr-M \\
\hline & $\mathrm{B}$ & 16.7 & 4.57 \\
\hline & $\mathrm{Li}$ & 9.6 & 3.36 \\
\hline & $\mathrm{Na}$ & 13.3 & 2.14 \\
\hline
\end{tabular}

- Empty data field 
Table 5.1. Summary of Results from DM100 Bismuth-Limited Waste Tests.

\begin{tabular}{|c|c|c|c|c|c|}
\hline \multicolumn{2}{|r|}{ Test } & $1 \mathrm{~A}$ & $1 \mathrm{~B}$ & $2 \mathrm{~A}$ & $2 B$ \\
\hline \multirow{3}{*}{$\stackrel{\mathscr{g}}{g}$} & Feed Start & 7/17/06 8:50 & 7/19/06 15:00 & 7/24/06 8:30 & 7/26/06 12:30 \\
\hline & Feed End & 7/19/06 13:00 & 7/21/06 17:00 & 7/26/06 11:00 & 7/28/06 14:30 \\
\hline & Interval & $52.2 \mathrm{hr}$ & $50.0 \mathrm{hr}$ & $50.5 \mathrm{hr}$ & $50.0 \mathrm{hr}$ \\
\hline \multicolumn{2}{|c|}{ Water Feeding for Cold Cap } & $0.5 \mathrm{hr}$ & NA & $0.5 \mathrm{hr}$ & NA \\
\hline \multicolumn{2}{|r|}{ Slurry Feeding } & $51.7 \mathrm{hr}$ & $50.0 \mathrm{hr}$ & $50.0 \mathrm{hr}$ & $50.0 \mathrm{hr}$ \\
\hline \multicolumn{2}{|c|}{ Feeding Interruptions } & $131 \min$ & $12 \mathrm{~min}$ & $6 \mathrm{~min}$ & $25 \mathrm{~min}$ \\
\hline \multicolumn{2}{|r|}{ Cold cap burn } & NA & $4.3 \mathrm{hr}$ & NA & $5.5 \mathrm{hr}$ \\
\hline \multicolumn{2}{|c|}{ Target Glass Temperature } & $1175^{\circ} \mathrm{C}$ & $1175^{\circ} \mathrm{C}$ & $1150^{\circ} \mathrm{C}$ & $1150^{\circ} \mathrm{C}$ \\
\hline \multicolumn{2}{|c|}{ Average Bubbling Rate } & $14.1 \mathrm{lpm}$ & 9 lpm & 9 lpm & 9 lpm \\
\hline \multirow{4}{*}{ 离 } & Used & $812 \mathrm{~kg}$ & $642 \mathrm{~kg}$ & $523 \mathrm{~kg}$ & $549 \mathrm{~kg}$ \\
\hline & \multirow{2}{*}{ Target Glass yield } & $500 \mathrm{~g} / \mathrm{l}$ & $500 \mathrm{~g} / \mathrm{l}$ & $500 \mathrm{~g} / \mathrm{l}$ & $250 \mathrm{~g} / \mathrm{l}$ \\
\hline & & $0.358 \mathrm{~kg} / \mathrm{kg}$ & $0.358 \mathrm{~kg} / \mathrm{kg}$ & $0.358 \mathrm{~kg} / \mathrm{kg}$ & $0.209 \mathrm{~kg} / \mathrm{kg}$ \\
\hline & Average Feed Rate & $15.7 \mathrm{~kg} / \mathrm{hr}$ & $12.8 \mathrm{~kg} / \mathrm{hr}$ & $10.5 \mathrm{~kg} / \mathrm{hr}$ & $11.0 \mathrm{~kg} / \mathrm{hr}$ \\
\hline \multirow{5}{*}{ 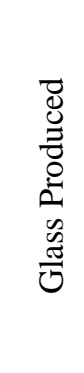 } & Poured & $193 \mathrm{~kg}$ & $233 \mathrm{~kg}$ & $168 \mathrm{~kg}$ & $200 \mathrm{~kg}$ \\
\hline & Average Rate ${ }^{\$}$ & NC & $1036 \mathrm{~kg} / \mathrm{m}^{2} /$ day & $747 \mathrm{~kg} / \mathrm{m}^{2} /$ day & NC \\
\hline & Average Rate ${ }^{*}$ & $1249 \mathrm{~kg} / \mathrm{m}^{2} /$ day & $1021 \mathrm{~kg} / \mathrm{m}^{2} /$ day & $832 \mathrm{~kg} / \mathrm{m}^{2} /$ day & $510 \mathrm{~kg} / \mathrm{m}^{2} /$ day \\
\hline & Steady State Rate $^{*}$ & $1200 \mathrm{~kg} / \mathrm{m}^{2} /$ day & $1000 \mathrm{~kg} / \mathrm{m}^{2} /$ day & $830 \mathrm{~kg} / \mathrm{m}^{2} /$ day & $510 \mathrm{~kg} / \mathrm{m}^{2} /$ day \\
\hline & Average Power Use & $4.1 \mathrm{~kW} \mathrm{hr} / \mathrm{kg}$ glass & $4.3 \mathrm{~kW} \mathrm{hr} / \mathrm{kg}$ glass & $\begin{array}{l}4.6 \mathrm{~kW} \mathrm{hr} / \mathrm{kg} \\
\text { glass }\end{array}$ & $\begin{array}{c}7.7 \mathrm{~kW} \mathrm{hr} / \mathrm{kg} \\
\text { glass }\end{array}$ \\
\hline
\end{tabular}

$\$$ - Rates calculated from glass poured.

*- Rates calculated from feed data.

NC- Not calculated during Tests $1 \mathrm{a}$ and $2 \mathrm{~b}$ due to the changing of the level of glass in the melter.

Note: Rates do not take into account the time for water feeding and cold cap burn-off. 
Table 5.2. Summary of Results from DM100 Chromium-Limited Waste Tests.

\begin{tabular}{|c|c|c|c|}
\hline & Test & 3B & $4 \mathrm{~A}$ \\
\hline & Feed Start & 8/21/06 8:33 & 8/23/06 16:06 \\
\hline$\Xi$ & Feed End & 8/23/06 15:30 & 8/25/06 19:43 \\
\hline & Interval & $55.0 \mathrm{hr}$ & $51.6 \mathrm{hr}$ \\
\hline Wate & Feeding for Cold Cap & $0.5 \mathrm{hr}$ & NA \\
\hline & Slurry Feeding & $54.5 \mathrm{hr}$ & $51.6 \mathrm{hr}$ \\
\hline & eding Interruptions & $56 \mathrm{~min}$ & $4 \mathrm{~min}$ \\
\hline & Cold cap burn & NA & $0.5 \mathrm{hr}$ \\
\hline Tary & th Glass Temperature & $1175^{\circ} \mathrm{C}$ & $1150^{\circ} \mathrm{C}$ \\
\hline & rage Bubbling Rate & $9 \mathrm{lpm}$ & $9 \mathrm{lpm}$ \\
\hline & Waste Loading & $32.5 \%$ & $32.5 \%$ \\
\hline & Used & $866.5 \mathrm{~kg}$ & $720.4 \mathrm{~kg}$ \\
\hline$\underset{\mathbb{Q}}{\mathbb{Q}}$ & & $500 \mathrm{~g} / \mathrm{l}$ & $500 \mathrm{~g} / \mathrm{l}$ \\
\hline & & $0.366 \mathrm{~kg} / \mathrm{kg}$ & $0.366 \mathrm{~kg} / \mathrm{kg}$ \\
\hline & Average Feed Rate & $15.9 \mathrm{~kg} / \mathrm{hr}$ & $14.0 \mathrm{~kg} / \mathrm{hr}$ \\
\hline $\bar{d}$ & Poured & $248.5 \mathrm{~kg}$ & $291.8 \mathrm{~kg}$ \\
\hline $\bar{z}$ & Average Rate ${ }^{*}$ & $1293 \mathrm{~kg} / \mathrm{m}^{2} /$ day & $1136 \mathrm{~kg} / \mathrm{m}^{2} /$ day \\
\hline $\begin{array}{l}A_{1} \\
\tilde{n}^{2}\end{array}$ & Steady State Rate ${ }^{*}$ & $1300 \mathrm{~kg} / \mathrm{m}^{2} /$ day & $1150 \mathrm{~kg} / \mathrm{m}^{2} /$ day \\
\hline $\bar{J}$ & Average Power Use & $4.0 \mathrm{~kW} \mathrm{hr/kg} \mathrm{glass}$ & $4.0 \mathrm{~kW} \mathrm{hr/kg} \mathrm{glass}$ \\
\hline $\begin{array}{r}\text { Sec } \\
\text { ob }\end{array}$ & $\begin{array}{l}\text { 7dary sulfates phases } \\
\text { erved on melt pool } \\
\text { samples }\end{array}$ & None & None \\
\hline
\end{tabular}

*- Rates calculated from feed data.

NA - Not applicable

Notes: Rates do not take into account the time for water feeding and cold cap burn-off.

Production rates not calculated from amounts of poured glass due to the changing of the level of glass in the melter. 
ORP-56297 Rev. 0

The Catholic University of America Vitreous State Laboratory

Table 5.3. Summary of Results from DM100 Aluminum-Limited Waste Tests.

\begin{tabular}{|c|c|c|c|c|c|c|}
\hline \multicolumn{2}{|r|}{ Test } & $5 \mathrm{~A}$ & $5 B$ & $6 \mathrm{~A}$ & $6 B$ & $6 \mathrm{C}$ \\
\hline \multirow{3}{*}{ 䍐 } & Feed Start & 9/11/06 9:00 & 9/13/06 14:00 & 9/18/06 9:00 & 9/20/06 12:30 & 9/26/06 10:00 \\
\hline & Feed End & 9/13/06 11:32 & 9/15/06 18:00 & 9/20/06 11:30 & 9/22/06 19:00 & 9/28/06 08:30 \\
\hline & Interval & $50.5 \mathrm{hr}$ & $52.0 \mathrm{hr}$ & $50.5 \mathrm{hr}$ & 54.5 & $46.5 \mathrm{hr}$ \\
\hline \multicolumn{2}{|c|}{ Water Feeding for Cold Cap } & $0.5 \mathrm{hr}$ & NA & $0.5 \mathrm{hr}$ & NA & $0.5 \mathrm{hr}$ \\
\hline \multicolumn{2}{|r|}{ Slurry Feeding } & $50.0 \mathrm{hr}$ & $52.0 \mathrm{hr}$ & $50.0 \mathrm{hr}$ & $54.5 \mathrm{hr}$ & $46.0 \mathrm{hr}$ \\
\hline \multicolumn{2}{|c|}{ Feeding Interruptions } & $32 \mathrm{~min}$ & $99 \mathrm{~min}$ & $0 \mathrm{~min}$ & $93 \mathrm{~min}$ & $20 \mathrm{~min}$ \\
\hline \multicolumn{2}{|r|}{ Cold cap burn } & NA & $6 \mathrm{hr}$ & NA & $5.5 \mathrm{hr}$ & $6 \mathrm{hr}$ \\
\hline \multicolumn{2}{|c|}{ Target Glass Temperature } & $1175^{\circ} \mathrm{C}$ & $1175^{\circ} \mathrm{C}$ & $1150^{\circ} \mathrm{C}$ & $1150{ }^{\circ} \mathrm{C}$ & $1150^{\circ} \mathrm{C}$ \\
\hline \multicolumn{2}{|c|}{ Average Bubbling Rate } & $18 \mathrm{lpm}$ & 9 lpm & 9 lpm & 9 lpm & $16.6 \mathrm{lpm}$ \\
\hline \multirow{4}{*}{ 离 } & Used & $667 \mathrm{~kg}$ & $394 \mathrm{~kg}$ & $361 \mathrm{~kg}$ & $449 \mathrm{~kg}$ & 594 kg \\
\hline & \multirow{2}{*}{ Target Glass yield } & $500 \mathrm{~g} / \mathrm{l}$ & $500 \mathrm{~g} / \mathrm{l}$ & $500 \mathrm{~g} / \mathrm{l}$ & $250 \mathrm{~g} / \mathrm{l}$ & $500 \mathrm{~g} / \mathrm{l}$ \\
\hline & & $0.365 \mathrm{~kg} / \mathrm{kg}$ & $0.365 \mathrm{~kg} / \mathrm{kg}$ & 0.365 kg/kg & $0.211 \mathrm{~kg} / \mathrm{kg}$ & $0.365 \mathrm{~kg} / \mathrm{kg}$ \\
\hline & Average Feed Rate & $13.3 \mathrm{~kg} / \mathrm{hr}$ & $7.6 \mathrm{~kg} / \mathrm{hr}$ & $7.2 \mathrm{~kg} / \mathrm{hr}$ & $8.2 \mathrm{~kg} / \mathrm{hr}$ & $12.9 \mathrm{~kg} / \mathrm{hr}$ \\
\hline \multirow{5}{*}{ 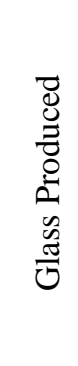 } & Poured & $171 \mathrm{~kg}$ & $134 \mathrm{~kg}$ & $137 \mathrm{~kg}$ & $93 \mathrm{~kg}$ & 259 kg \\
\hline & Average Rate $\$$ & NC & $567 \mathrm{~kg} / \mathrm{m}^{2} /$ day & $608 \mathrm{~kg} / \mathrm{m}^{2} /$ day & $379 \mathrm{~kg} / \mathrm{m}^{2} /$ day & NC \\
\hline & Average Rate ${ }^{*}$ & $1082 \mathrm{~kg} / \mathrm{m}^{2} /$ day & $615 \mathrm{~kg} / \mathrm{m}^{2} /$ day & $587 \mathrm{~kg} / \mathrm{m}^{2} /$ day & $386 \mathrm{~kg} / \mathrm{m}^{2} /$ day & 1047 kg/m²/day \\
\hline & Steady State Rate ${ }^{*}$ & $1000 \mathrm{~kg} / \mathrm{m}^{2} /$ day & $550 \mathrm{~kg} / \mathrm{m}^{2} /$ day & $550 \mathrm{~kg} / \mathrm{m}^{2} /$ day & $400 \mathrm{~kg} / \mathrm{m}^{2} /$ day & $1000 \mathrm{~kg} / \mathrm{m}^{2} /$ day \\
\hline & Average Power Use & $\begin{array}{l}4.4 \mathrm{~kW} \text { hr } / \mathrm{kg} \\
\text { glass }\end{array}$ & $\begin{array}{l}5.8 \mathrm{~kW} \mathrm{hr} / \mathrm{kg} \\
\text { glass }\end{array}$ & $\begin{array}{l}6.4 \mathrm{~kW} \mathrm{hr} / \mathrm{kg} \\
\text { glass }\end{array}$ & $\begin{array}{l}9.2 \mathrm{~kW} \mathrm{hr} / \mathrm{kg} \\
\text { glass }\end{array}$ & $\begin{array}{l}4.5 \mathrm{~kW} \mathrm{hr} / \mathrm{kg} \\
\text { glass }\end{array}$ \\
\hline
\end{tabular}

$\$$ - Rates calculated from glass poured.

*- Rates calculated from feed data.

NC- Not calculated during Tests $5 \mathrm{a}$ and $6 \mathrm{c}$ due to the changing of the level of glass in the melter.

Note: Rates do not take into account the time for water feeding and cold cap burn-off. 
ORP-56297 Rev. 0

The Catholic University of America Vitreous State Laboratory

Table 5.4. Summary of Results from DM100 Aluminum-Plus-Sodium-Limited Waste Tests.

\begin{tabular}{|c|c|c|c|c|c|c|}
\hline \multirow{2}{*}{$\underset{\Xi}{\Xi}$} & Feed Start & 10/2/06 11:45 & 10/4/06 14:15 & 10/16/06 8:30 & 10/18/06 20:40 & 11/18/06 00:00 \\
\hline & Interval & $50.0 \mathrm{hr}$ & $51.3 \mathrm{hr}$ & $55.5 \mathrm{hr}$ & 50.0 & $57.5 \mathrm{hr}$ \\
\hline \multicolumn{2}{|r|}{ Slurry Feeding } & $49.5 \mathrm{hr}$ & $51.3 \mathrm{hr}$ & $55.0 \mathrm{hr}$ & $50.0 \mathrm{hr}$ & $57.0 \mathrm{hr}$ \\
\hline \multicolumn{2}{|c|}{ Feeding Interruptions } & $22 \min$ & $98 \mathrm{~min}$ & $176 \mathrm{~min}$ & $28 \mathrm{~min}$ & $680 \mathrm{~min}$ \\
\hline \multicolumn{2}{|r|}{ Cold cap burn } & NA & $3.5 \mathrm{hr}$ & NA & $6 \mathrm{hr}$ & $3.5 \mathrm{hr}$ \\
\hline \multirow{4}{*}{ 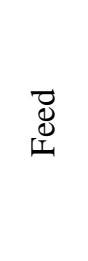 } & Used & $872 \mathrm{~kg}$ & $604 \mathrm{~kg}$ & $385 \mathrm{~kg}$ & $252 \mathrm{~kg}$ & $717 \mathrm{~kg}$ \\
\hline & \multirow{2}{*}{ Target Glass yield } & $500 \mathrm{~g} / \mathrm{l}$ & $500 \mathrm{~g} / \mathrm{l}$ & $500 \mathrm{~g} / \mathrm{l}$ & $250 \mathrm{~g} / \mathrm{l}$ & $500 \mathrm{~g} / \mathrm{l}$ \\
\hline & & $0.358 \mathrm{~kg} / \mathrm{kg}$ & $0.358 \mathrm{~kg} / \mathrm{kg}$ & $0.358 \mathrm{~kg} / \mathrm{kg}$ & $0.209 \mathrm{~kg} / \mathrm{kg}$ & $0.358 \mathrm{~kg} / \mathrm{kg}$ \\
\hline & Average Feed Rate & $17.6 \mathrm{~kg} / \mathrm{hr}$ & $11.8 \mathrm{~kg} / \mathrm{hr}$ & $7.0 \mathrm{~kg} / \mathrm{hr}$ & $5.0 \mathrm{~kg} / \mathrm{hr}$ & $12.6 \mathrm{~kg} / \mathrm{hr}$ \\
\hline \multirow{3}{*}{ 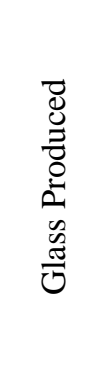 } & Poured & $259 \mathrm{~kg}$ & $217 \mathrm{~kg}$ & $154 \mathrm{~kg}$ & $47 \mathrm{~kg}$ & $256 \mathrm{~kg}$ \\
\hline & Average Rate & $\mathrm{NC}$ & $940 \mathrm{~kg} / \mathrm{m}^{2} /$ day & $621 \mathrm{~kg} / \mathrm{m}^{2} /$ day & 209 kg/m²/day & NC \\
\hline & Average Rate* & 1401 kg/m²/day & 936 kg/m²/day & $557 \mathrm{~kg} / \mathrm{m}^{2} /$ day & 234 kg/m²/day & $1002 \mathrm{~kg} / \mathrm{m}^{2} /$ day \\
\hline
\end{tabular}

$\$$ - Rates calculates from glass poured.

*- Rates calculated from feed data.

NC- Not calculated during Tests 7A and 8C due to the changing of the level of glass in the melter.

Note: Rates do not take into account the time for water feeding and cold cap burn-off but do include feeding interruptions required to stabilize the cold during, transfer feed, and perform maintenance during testing, 
ORP-56297 Rev. 0

The Catholic University of America Vitreous State Laboratory

Table 5.5. Steady-State Production Rates Achieved on the DM100 with HLW Compositions and Comparison to Previous Results with High-Iron Feeds.

\begin{tabular}{|c|c|c|c|c|}
\hline HLW Waste & $\begin{array}{l}\text { Bubbling Rate } \\
\text { (lpm) }\end{array}$ & Glass Yield (g/L) & $\begin{array}{c}\text { Glass Temperature } \\
\left({ }^{\circ} \mathrm{C}\right)\end{array}$ & $\begin{array}{c}\text { Production Rate } \\
\mathrm{kg} / \mathrm{m}^{2} / \text { day }\end{array}$ \\
\hline \multirow{4}{*}{ Bismuth Limited } & Optimized & 500 & 1175 & 1200 \\
\hline & 9 & 500 & 1175 & 1000 \\
\hline & 9 & 500 & 1150 & 830 \\
\hline & 9 & 250 & 1150 & 510 \\
\hline \multirow{2}{*}{ Chromium Limited } & 9 & 500 & 1175 & 1300 \\
\hline & 9 & 500 & 1150 & 1150 \\
\hline \multirow{5}{*}{ Aluminum Limited } & Optimized & 500 & 1175 & 1000 \\
\hline & 9 & 500 & 1175 & 550 \\
\hline & 9 & 500 & 1150 & 550 \\
\hline & 9 & 250 & 1150 & 400 \\
\hline & Optimized & 500 & 1150 & 1000 \\
\hline \multirow{5}{*}{$\begin{array}{l}\text { Aluminum and } \\
\text { Sodium Limited }\end{array}$} & Optimized & 500 & 1175 & 1400 \\
\hline & 9 & 500 & 1175 & 900 \\
\hline & 9 & 500 & 1150 & 400 \\
\hline & 9 & 250 & 1150 & 200 \\
\hline & Optimized & 500 & 1150 & 1250 \\
\hline AZ-101 [7] & 9 & 530 & 1150 & 1300 \\
\hline $\begin{array}{c}\text { AZ-102, } \\
\text { Nominal Rheology } \\
{[17]}\end{array}$ & 9 & 550 & 1150 & 1200 \\
\hline $\begin{array}{c}\text { AZ-102, } \\
\text { Adjusted Rheology } \\
{[17]}\end{array}$ & 9 & 550 & 1150 & 1400 \\
\hline $\begin{array}{l}\text { C-106/AY-102, High } \\
\text { Waste Loading [17] }\end{array}$ & Optimized & 420 & 1150 & 1350 \\
\hline $\begin{array}{c}\text { C-106/AY-102, } \\
\text { Nominal Rheology } \\
{[24]} \\
\end{array}$ & 9 & 435 & 1150 & 1100 \\
\hline $\begin{array}{c}\text { C-106/AY-102, } \\
\text { Adjusted Rheology } \\
{[24]}\end{array}$ & 9 & 435 & 1150 & 1150 \\
\hline $\begin{array}{l}\text { C-106/AY-102, } \\
\text { SIPP [24] }\end{array}$ & 9 & 470 & 1150 & 1180 \\
\hline
\end{tabular}


Table 5.6. Summary of Measured DM100 Parameters for Bismuth-Limited Waste Tests.

\begin{tabular}{|c|c|c|c|c|c|c|c|c|c|c|c|c|c|c|}
\hline \multirow{2}{*}{\multicolumn{3}{|c|}{ Test }} & \multicolumn{3}{|c|}{$1 \mathrm{~A}$} & \multicolumn{3}{|c|}{$1 \mathrm{~B}$} & \multicolumn{3}{|c|}{$2 \mathrm{~A}$} & \multicolumn{3}{|c|}{$2 B$} \\
\hline & & & I & MIN & MAX & VG & IN & MAX & $\mathrm{VG}$ & AIN & & & IIN & $\mathrm{M} t$ \\
\hline \multirow{14}{*}{$\begin{array}{c}\mathrm{T} \\
\mathrm{E} \\
\mathrm{M} \\
\mathrm{P} \\
\mathrm{E} \\
\mathrm{R} \\
\mathrm{A} \\
\mathrm{T} \\
\mathrm{U} \\
\mathrm{R} \\
\mathrm{E} \\
\text { (C) }\end{array}$} & \multirow{4}{*}{ Electrode } & East Upper & 915 & 601 & 1077 & 1053 & 1031 & 1090 & 1012 & 981 & 1038 & 982 & 704 & 1033 \\
\hline & & West Upper & 1056 & 675 & 1123 & 1097 & 1085 & 1110 & 1057 & 1031 & 1073 & 1032 & 903 & 1069 \\
\hline & & West Lower & 1093 & 1057 & 1118 & 1080 & 1069 & 1090 & 1037 & 1005 & 1049 & 1038 & 1026 & 1050 \\
\hline & & Bottom & 747 & 736 & 763 & 722 & 715 & 732 & 676 & 635 & 686 & 684 & 682 & 688 \\
\hline & \multirow{4}{*}{ Glass } & 27" from bottom & 912 & 159 & 1169 & 1126 & 1059 & 1165 & 1090 & 999 & 1143 & 974 & 431 & 1133 \\
\hline & & 16 " from bottom & 1056 & 638 & 1177 & 1157 & 1127 & 1176 & 1131 & 1078 & 1150 & 1118 & 976 & 1146 \\
\hline & & 10 " from $b$ & 1176 & 1112 & 1198 & 1178 & 1153 & 1193 & 1153 & 1124 & 1164 & 1152 & 1121 & 1166 \\
\hline & & 5" from botto & 1176 & 1145 & 1201 & 1176 & 1157 & 1190 & 1151 & 1129 & 1163 & 1152 & 1129 & 1169 \\
\hline & \multirow{2}{*}{ Plenum } & a & 467 & 147 & 669 & 465 & 358 & 542 & 408 & 308 & 584 & 372 & 293 & 467 \\
\hline & & herr & 411 & 216 & 45 & 380 & 30 & 504 & 363 & 296 & 556 & 327 & 254 & 435 \\
\hline & \multirow{2}{*}{ Discharge } & Chamber & 1030 & 973 & 1055 & 1046 & 1029 & 1060 & 1019 & 989 & 1036 & 1029 & 1010 & 1044 \\
\hline & & Air Lift & 998 & 927 & 1105 & 1016 & 998 & 1107 & 983 & 926 & 1070 & 993 & 970 & 1084 \\
\hline & \multicolumn{2}{|c|}{ Film Cooler Outlet } & 281 & 200 & 322 & 273 & 215 & 286 & 265 & 244 & 302 & 260 & 242 & 276 \\
\hline & \multicolumn{2}{|c|}{ Transition Line Outlet } & 279 & 222 & 326 & 267 & 239 & 283 & 261 & 242 & 300 & 254 & 239 & 270 \\
\hline \multicolumn{3}{|c|}{ Lance Bubbling (lpm) } & 14.1 & 2.5 & 20.5 & 9.0 & 8.8 & 9.1 & 9.0 & 8.7 & 9.6 & 9.0 & 8.8 & 9.2 \\
\hline \multicolumn{3}{|c|}{ Melter Pressure (inches water) } & -1.81 & -3.13 & 0.25 & -1.76 & -2.64 & 0.39 & -1.84 & -4.58 & 0.27 & -1.61 & -2.96 & 0.26 \\
\hline \multicolumn{3}{|c|}{ Total Electrode Voltage (V) } & 45.5 & 1.7 & 55.3 & 39.1 & 37.0 & 40.8 & 38.5 & 36.0 & 41.9 & 39.6 & 36.4 & 45.2 \\
\hline \multicolumn{3}{|c|}{ Total Power $(\mathrm{kW})$} & 22.9 & 0.3 & 28.0 & 19.7 & 17.9 & 21.5 & 17.3 & 15.4 & 20.0 & 17.6 & 15.0 & 19.5 \\
\hline \multicolumn{3}{|c|}{ Glass Resistance (ohms) } & 0.091 & 0.011 & 0.153 & 0.077 & 0.074 & 0.083 & 0.086 & 0.081 & 0.093 & 0.089 & 0.081 & 0.110 \\
\hline
\end{tabular}


The Catholic University of America Vitreous State Laboratory
High Level Waste Vitrification System Improvements Final Report, VSL-07R1010-1, Rev. 0

Table 5.7. Summary of Measured DM100 Parameters for Chromium-Limited Waste Tests.

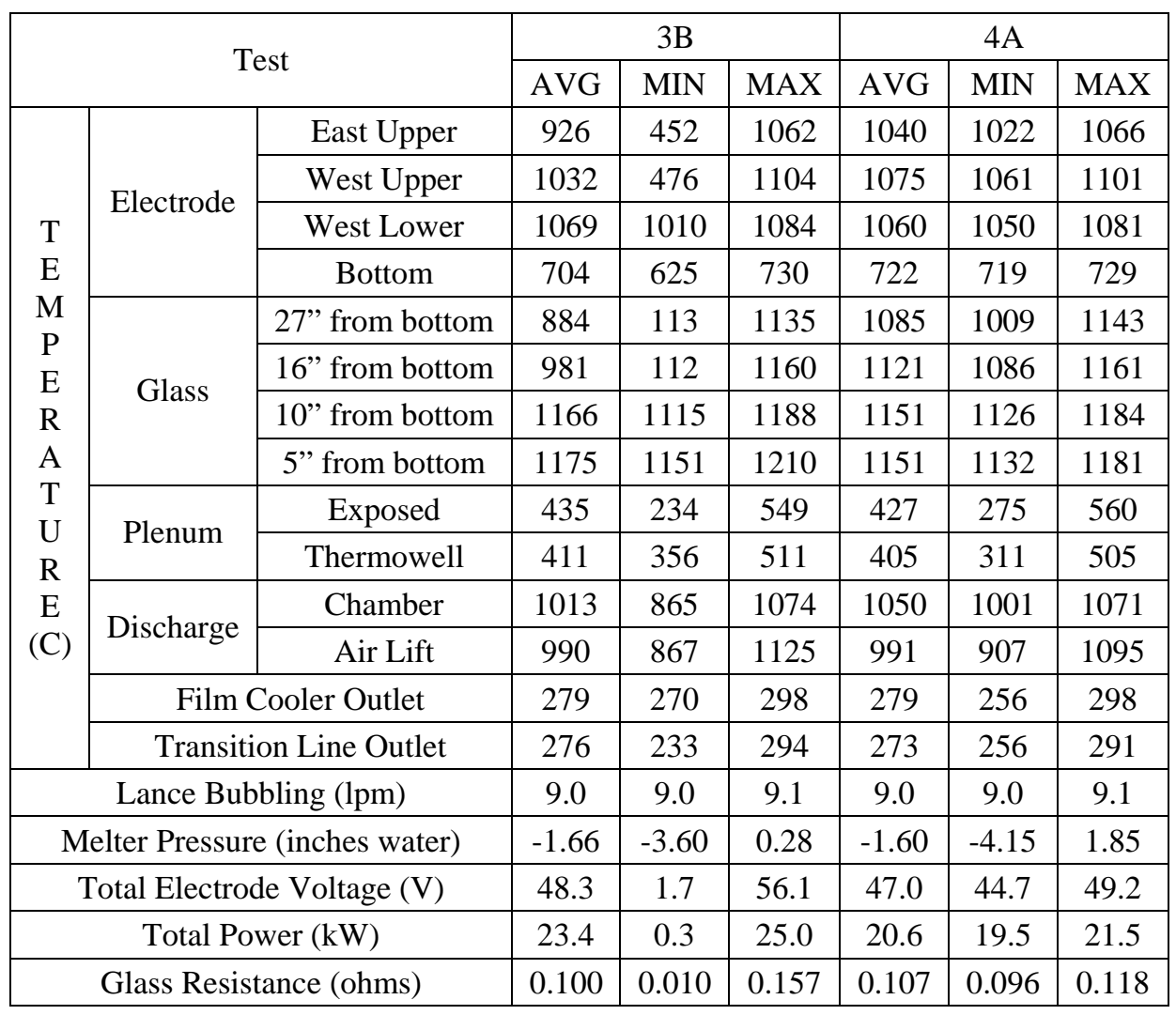


Table 5.8. Summary of Measured DM100 Parameters for Aluminum-Limited Waste Tests.

\begin{tabular}{|c|c|c|c|c|c|c|c|c|c|c|c|c|c|c|c|c|c|}
\hline \multirow{2}{*}{\multicolumn{3}{|c|}{ Test }} & \multicolumn{3}{|c|}{$5 \mathrm{~A}$} & \multicolumn{3}{|c|}{$5 B$} & \multicolumn{3}{|c|}{$6 \mathrm{~A}$} & \multicolumn{3}{|c|}{$6 \mathrm{~B}$} & \multicolumn{3}{|c|}{$6 \mathrm{C}$} \\
\hline & & & AVG & MIN & MAX & AVG & MIN & MAX & AVG & MIN & MAX & $\mathrm{AVG}$ & MIN & MAX & AVG & MIN & MAX \\
\hline \multirow{14}{*}{$\begin{array}{c}\mathrm{T} \\
\mathrm{E} \\
\mathrm{M} \\
\mathrm{P} \\
\mathrm{E} \\
\mathrm{R} \\
\mathrm{A} \\
\mathrm{T} \\
\mathrm{U} \\
\mathrm{R} \\
\mathrm{E} \\
\text { (C) }\end{array}$} & \multirow{4}{*}{ Electrode } & East Upper & 970 & 652 & 1087 & 1054 & 1013 & 1084 & 1050 & 1011 & 1070 & 980 & 869 & 1082 & 1080 & 1031 & 1112 \\
\hline & & West Upper & 1087 & 694 & 1128 & 1102 & 1083 & 1117 & 1066 & 1037 & 1092 & 1011 & 902 & 1095 & 1068 & 1002 & 1103 \\
\hline & & $\begin{array}{c}\text { West } \\
\text { Lower }\end{array}$ & 1093 & 1075 & 1107 & 1081 & 1071 & 1093 & 1055 & 1034 & 1074 & 1053 & 1021 & 1076 & 1061 & 1031 & 1085 \\
\hline & & Bottom & 788 & 782 & 801 & 770 & 762 & 785 & 751 & 721 & 830 & 719 & 706 & 729 & 716 & 701 & 724 \\
\hline & \multirow{4}{*}{ Glass } & $\begin{array}{l}27 ” \text { from } \\
\text { bottom }\end{array}$ & 932 & 217 & 1151 & 1104 & 961 & 1166 & 1121 & 1059 & 1143 & 954 & 680 & 1148 & 1130 & 1016 & 1177 \\
\hline & & $\begin{array}{l}16 " \text { from } \\
\text { bottom }\end{array}$ & 1037 & 540 & 1169 & 1152 & 1120 & 1179 & 1133 & 1104 & 1155 & 1106 & 1056 & 1155 & 1129 & 1070 & 1170 \\
\hline & & $\begin{array}{l}10 ” \text { from } \\
\text { bottom }\end{array}$ & 1172 & 1129 & 1197 & 1178 & 1153 & 1197 & 1153 & 1118 & 1179 & 1157 & 1103 & 1186 & 1154 & 1087 & 1197 \\
\hline & & $\begin{array}{l}5 " \text { from } \\
\text { bottom }\end{array}$ & 1173 & 1131 & 1196 & 1174 & 1150 & 1194 & 1148 & 1108 & 1174 & 1152 & 1093 & 1179 & 1151 & 1095 & 1190 \\
\hline & \multirow{2}{*}{ Plenum } & Exposed & 445 & 348 & 663 & 398 & 273 & 527 & 448 & 361 & 523 & 366 & 189 & 526 & 432 & 259 & 536 \\
\hline & & Thermowell & 408 & 327 & 637 & 356 & 246 & 467 & 425 & 376 & 503 & 332 & 210 & 495 & 448 & 171 & 615 \\
\hline & \multirow{2}{*}{ Discharge } & Chamber & 1024 & 924 & 1064 & 1056 & 978 & 1112 & 1058 & 1011 & 1077 & 1067 & 1045 & 1080 & 1066 & 975 & 1089 \\
\hline & & Air Lift & 946 & 870 & 1051 & 1008 & 930 & 1147 & 1015 & 936 & 1112 & 1016 & 1000 & 1117 & 1040 & 1000 & 1130 \\
\hline & \multicolumn{2}{|c|}{ Film Cooler Outlet } & 277 & 260 & 304 & 261 & 233 & 279 & 274 & 265 & 293 & 260 & 233 & 290 & 282 & 247 & 294 \\
\hline & \multicolumn{2}{|c|}{ Transition Line Outlet } & 278 & 258 & 316 & 258 & 229 & 276 & 270 & 259 & 288 & 253 & 231 & 287 & 275 & 238 & 281 \\
\hline \multicolumn{3}{|c|}{ Lance Bubbling (lpm) } & 18.0 & -2.3 & 22.9 & 9.0 & 8.7 & 17.9 & 9.1 & 9.0 & 9.3 & 9.0 & 8.9 & 9.3 & 16.6 & 9.5 & 23.6 \\
\hline \multicolumn{3}{|c|}{ Melter Pressure (inches water) } & -1.80 & -4.56 & 1.50 & -1.93 & -4.26 & 0.19 & -1.93 & -2.95 & 0.23 & -1.94 & -3.22 & 2.89 & -1.87 & -2.99 & 0.23 \\
\hline \multicolumn{3}{|c|}{ Total Electrode Voltage (V) } & 48.7 & 2.3 & 60.0 & 39.9 & 36.5 & 44.7 & 43.3 & 39.3 & 48.8 & 44.2 & 39.0 & 50.9 & 50.0 & 44.1 & 58.2 \\
\hline \multicolumn{3}{|c|}{ Total Power $(\mathrm{kW})$} & 21.3 & 0.3 & 24.4 & 16.2 & 13.9 & 19.4 & 16.9 & 13.4 & 21.5 & 16.0 & 11.4 & 21.5 & 21.0 & 14.9 & 26.4 \\
\hline \multicolumn{3}{|c|}{ Glass Resistance (ohms) } & 0.112 & 0.019 & 0.155 & 0.098 & 0.091 & 0.108 & 0.111 & 0.100 & 0.123 & 0.125 & 0.099 & 0.169 & 0.121 & 0.101 & 0.174 \\
\hline
\end{tabular}


Table 5.9. Summary of Measured DM100 Parameters for Al + Na-Limited Waste Tests.

\begin{tabular}{|c|c|c|c|c|c|c|c|c|c|c|c|c|c|c|c|c|c|}
\hline \multirow{2}{*}{\multicolumn{3}{|c|}{ Test }} & \multicolumn{3}{|c|}{$7 \mathrm{~A}$} & \multicolumn{3}{|c|}{$7 B$} & \multicolumn{3}{|c|}{$8 \mathrm{~A}$} & \multicolumn{3}{|c|}{$8 B$} & \multicolumn{3}{|c|}{$8 C$} \\
\hline & & & AVG & MIN & MAX & AVG & MIN & MAX & AVG & MIN & MAX & AVG & MIN & MAX & AVG & MIN & MAX \\
\hline \multirow{4}{*}{$\begin{array}{c}\mathrm{T} \\
\mathrm{E} \\
\mathrm{M}\end{array}$} & \multirow{4}{*}{ Electrode } & East Upper & 1021 & 722 & 1139 & 1109 & 1048 & 1134 & 1019 & 854 & 1089 & 1048 & 1009 & 1085 & 1088 & 981 & 1116 \\
\hline & & West Upper & 1094 & 738 & 1143 & 1118 & 1072 & 1140 & 994 & 805 & 1097 & 1026 & 991 & 1056 & 1073 & 1044 & 1114 \\
\hline & & West Lower & 1105 & 1067 & 1126 & 1102 & 1080 & 1118 & 1062 & 1029 & 1099 & 1045 & 1020 & 1061 & 1070 & 1045 & 1099 \\
\hline & & Bottom & 746 & 716 & 752 & 740 & 736 & 744 & 773 & 747 & 825 & 747 & 741 & 754 & 755 & 724 & 768 \\
\hline \multirow{4}{*}{$\begin{array}{l}\mathrm{P} \\
\mathrm{E} \\
\mathrm{R}\end{array}$} & \multirow{4}{*}{ Glass } & 27” from bottom & 1026 & 324 & 1180 & 1144 & 1012 & 1184 & 1012 & 430 & 1176 & 1123 & 994 & 1175 & 1148 & 1037 & 1195 \\
\hline & & 16” from bottom & 1120 & 812 & 1167 & 1148 & 1110 & 1178 & 1118 & 1027 & 1170 & 1134 & 1091 & 1170 & 1132 & 1088 & 1162 \\
\hline & & 10” from bottom & 1170 & 1126 & 1193 & 1175 & 1129 & 1200 & 1161 & 1117 & 1190 & 1160 & 1119 & 1185 & 1155 & 1124 & 1184 \\
\hline & & 5” from bottom & 1171 & 1135 & 1203 & 1174 & 1135 & 1202 & 1149 & 1118 & 1180 & 1142 & 1109 & 1160 & 1148 & 1111 & 1178 \\
\hline $\mathrm{U}$ & \multirow{2}{*}{ Plenum } & Exposed & 401 & 307 & 464 & 356 & 289 & 398 & 353 & 245 & 574 & 328 & 248 & 391 & 518 & 306 & 865 \\
\hline $\begin{array}{l}\mathrm{K} \\
\mathrm{F}\end{array}$ & & Thermowell & 439 & 341 & 516 & 402 & 324 & 470 & 373 & 219 & 649 & 346 & 225 & 488 & 414 & 109 & 666 \\
\hline \multirow[t]{4}{*}{ (C) } & \multirow{2}{*}{ Discharge } & Chamber & 1069 & 1000 & 1106 & 1081 & 1050 & 1103 & 1063 & 991 & 1091 & 1066 & 1049 & 1090 & 996 & 966 & 1028 \\
\hline & & Air Lift & 1009 & 847 & 1186 & 930 & 748 & 1100 & 857 & 756 & 1015 & 891 & 827 & 982 & 860 & 832 & 879 \\
\hline & \multicolumn{2}{|c|}{ Film Cooler Outlet } & 282 & 274 & 290 & 276 & 142 & 289 & 275 & 257 & 302 & 271 & 257 & 284 & 288 & 245 & 393 \\
\hline & \multicolumn{2}{|c|}{ Transition Line Outlet } & 279 & 271 & 285 & 272 & 159 & 280 & 268 & 246 & 309 & 263 & 251 & 270 & 276 & 241 & 367 \\
\hline \multicolumn{3}{|c|}{ Lance Bubbling (lpm) } & 15.7 & 5.0 & 30.3 & 9.1 & 9.0 & 9.2 & 9.1 & 3.5 & 9.2 & 9.1 & 8.9 & 9.2 & 15.0 & 0.6 & 23.0 \\
\hline \multicolumn{3}{|c|}{ Melter Pressure (inches water) } & -1.77 & -5.06 & 0.26 & -1.68 & -4.41 & 0.66 & -1.45 & -3.08 & 0.28 & -1.35 & -2.63 & 0.32 & -1.64 & -3.74 & 0.88 \\
\hline \multicolumn{3}{|c|}{ Total Electrode Voltage (V) } & 48.3 & 1.9 & 58.2 & 38.3 & 34.3 & 42.0 & 38.9 & 30.7 & 49.3 & 38.5 & 1.8 & 52.3 & 44.9 & 28.4 & 52.4 \\
\hline \multicolumn{3}{|c|}{ Total Power (kW) } & 26.0 & 0.3 & 28.5 & 20.2 & 16.9 & 23.0 & 15.2 & 10.5 & 23.0 & 14.0 & 0.3 & 20.8 & 21.2 & 9.5 & 28.0 \\
\hline \multicolumn{3}{|c|}{ Glass Resistance (ohms) } & 0.091 & 0.012 & 0.189 & 0.073 & 0.067 & 0.088 & 0.101 & 0.075 & 0.127 & 0.106 & 0.012 & 0.132 & 0.096 & 0.075 & 0.120 \\
\hline
\end{tabular}


Table 6.1. Characteristics of Melter Feed Samples from DM100 Tests.

\begin{tabular}{|c|c|c|c|c|c|c|c|c|c|c|c|}
\hline \multirow[b]{2}{*}{ Test } & \multirow{2}{*}{ Date } & \multirow{2}{*}{ Name } & \multirow{2}{*}{$\begin{array}{c}\% \\
\text { Water }\end{array}$} & \multirow{2}{*}{\begin{tabular}{|c|} 
Density \\
$(\mathrm{g} / \mathrm{ml})$ \\
\end{tabular}} & \multicolumn{2}{|c|}{ Glass Yield } & \multirow[b]{2}{*}{ pH } & \multirow{2}{*}{$\begin{array}{c}\text { Yield } \\
\text { Stress } \\
\text { (Pa) }\end{array}$} & \multicolumn{3}{|c|}{ Viscosity (Poise) } \\
\hline & & & & & $(\mathrm{kg} / \mathrm{kg})$ & (g/l) & & & $1 / \mathrm{s}$ & $10 / \mathrm{s}$ & $100 / \mathrm{s}$ \\
\hline \multirow{3}{*}{$1 \mathrm{~A}$} & $7 / 17 / 06$ & BLK-F-71A & 61.88 & 1.39 & 0.353 & 491 & 10.51 & 0.04 & 1.03 & 0.21 & 0.05 \\
\hline & 7/18/06 & BLK-F-84A & NA & 1.36 & NA & NA & 10.58 & NA & NA & NA & NA \\
\hline & $7 / 19 / 06$ & BLK-F-100A & NA & 1.32 & NA & NA & 10.41 & NA & NA & NA & NA \\
\hline $1 \mathrm{~B}$ & $7 / 20 / 06$ & BLK-F-112A & 61.65 & 1.38 & 0.353 & 487 & 10.72 & NA & NA & NA & NA \\
\hline \multirow{2}{*}{$2 \mathrm{~A}$} & $7 / 24 / 06$ & BLK-F-146A & NA & 1.37 & NA & NA & 10.48 & NA & NA & NA & NA \\
\hline & $7 / 25 / 06$ & BLK-F-152A & 60.94 & 1.39 & 0.354 & 492 & 10.72 & NA & NA & NA & NA \\
\hline \multirow{2}{*}{$2 \mathrm{~B}$} & 7/26/06 & BLL-F-20A & 78.48 & 1.18 & 0.192 & 227 & 10.25 & NA & NA & NA & NA \\
\hline & $7 / 27 / 06$ & BLL-F-30A & 79.62 & 1.19 & 0.188 & 223 & 10.25 & 0.03 & 0.12 & 0.03 & 0.02 \\
\hline \multirow{3}{*}{ 3B } & 8/21/06 & BLL-F-65A & NA & 1.36 & NA & NA & 9.56 & NA & NA & NA & NA \\
\hline & $8 / 22 / 06$ & BLL-F-73A & 59.52 & 1.37 & 0.355 & 487 & 9.58 & 0.1 & 2.65 & 0.65 & 0.19 \\
\hline & $8 / 23 / 06$ & BLL-F-86A & NA & 1.37 & NA & NA & 9.65 & NA & NA & NA & NA \\
\hline $4 \mathrm{~A}$ & $8 / 24 / 05$ & BLL-F-100A & 59.65 & 1.37 & 0.359 & 492 & 9.58 & 0.2 & 2.40 & 0.67 & 0.20 \\
\hline \multirow{3}{*}{$5 \mathrm{~A}$} & 9/11/06 & BLL-F-132A & 60.78 & 1.36 & 0.362 & 492 & 10.18 & 9.5 & 35.89 & 4.63 & 1.16 \\
\hline & $\begin{array}{l}9 / 11 / 06 \\
\end{array}$ & BLL-F-135A & NA & 1.34 & NA & NA & 10.21 & NA & NA & NA & NA \\
\hline & 9/12/06 & BLL-F-147A & NA & 1.37 & NA & NA & 10.24 & NA & NA & NA & NA \\
\hline $5 \mathrm{~B}$ & 9/13/06 & BLM-F-14A & 59.42 & 1.36 & 0.351 & 478 & 10.24 & NA & NA & NA & NA \\
\hline $6 \mathrm{~A}$ & $9 / 18 / 06$ & BLM-F-53A & 59.60 & 1.37 & 0.361 & 494 & 10.34 & NA & NA & NA & NA \\
\hline \multirow{2}{*}{$6 \mathrm{~B}$} & 9/20/06 & BLM-F-75A & 77.51 & 1.16 & 0.206 & 239 & 10.10 & 1.9 & 4.83 & 0.54 & 0.27 \\
\hline & 9/21/06 & BLM-F-91A & NA & 1.18 & NA & NA & 10.12 & NA & NA & NA & NA \\
\hline \multirow{2}{*}{$6 C$} & 9/26/06 & BLM-F-123A & 60.14 & 1.38 & 0.356 & 492 & 10.28 & NA & NA & NA & NA \\
\hline & 9/27/06 & BLM-F-133A & NA & 1.38 & NA & NA & 10.30 & NA & NA & NA & NA \\
\hline \multirow{4}{*}{$7 \mathrm{~A}$} & $10 / 2 / 06$ & BLN-F-9A & 61.23 & 1.37 & 0.344 & 471 & 10.90 & 0.1 & 1.39 & 0.27 & 0.06 \\
\hline & $10 / 3 / 06$ & BLN-F-21A & NA & 1.38 & NA & NA & 10.92 & NA & $\mathrm{NA}$ & NA & NA \\
\hline & $10 / 3 / 06$ & BLN-F-29A & NA & 1.38 & NA & NA & 10.95 & NA & NA & NA & NA \\
\hline & $10 / 4 / 06$ & BLN-F-35A & NA & 1.38 & NA & NA & 10.8 & NA & NA & NA & NA \\
\hline \multirow{2}{*}{$7 \mathrm{~B}$} & $10 / 5 / 06$ & BLN-F-48A & NA & 1.38 & NA & NA & 10.97 & NA & NA & NA & NA \\
\hline & $\begin{array}{l}10 / 6 / 06 \\
\end{array}$ & BLN-F-62A & NA & 1.39 & NA & NA & 10.97 & NA & NA & NA & NA \\
\hline $8 \mathrm{~A}$ & $10 / 16 / 06$ & BLN-F-88A & NA & 1.37 & NA & NA & 10.99 & NA & NA & NA & NA \\
\hline $8 \mathrm{~B}$ & $10 / 18 / 06$ & BLN-F-107A & 79.84 & 1.16 & 0.177 & 205 & 10.39 & 0.1 & 0.04 & 0.02 & 0.01 \\
\hline \multirow{3}{*}{$8 C$} & $11 / 18 / 06$ & BLN-F-146A & NA & 1.36 & NA & NA & 10.75 & NA & NA & NA & NA \\
\hline & $11 / 18 / 06$ & BLN-F-152A & 61.16 & 1.38 & 0.345 & 476 & 10.78 & NA & NA & NA & NA \\
\hline & $11 / 19 / 06$ & BLO-F-15A & NA & 1.36 & NA & NA & 10.77 & NA & NA & NA & NA \\
\hline
\end{tabular}

NA - Not analyzed. 
Table 6.2. XRF Analyzed Compositions of Vitrified Melter Feed Samples (wt\%).

\begin{tabular}{|c|c|c|c|c|c|c|c|c|c|c|c|c|c|}
\hline \multirow[b]{2}{*}{ Sample } & \multicolumn{8}{|c|}{ Bi Limited } & \multicolumn{5}{|c|}{ Cr Limited } \\
\hline & Target & $\begin{array}{l}\text { BLK- } \\
\text { F-71A }\end{array}$ & \begin{tabular}{|c} 
BLK- \\
F- \\
$112 \mathrm{~A}$
\end{tabular} & \begin{tabular}{|c|} 
BLK- \\
F- \\
$152 \mathrm{~A}$ \\
\end{tabular} & \begin{tabular}{|c|} 
BLL- \\
F-G- \\
20A \\
\end{tabular} & $\begin{array}{l}\text { BLL- } \\
\text { F-30A }\end{array}$ & Avg. & \%Dev. & Target & $\begin{array}{l}\text { BLL- } \\
\text { F-73A }\end{array}$ & \begin{tabular}{|c} 
BLL- \\
F- \\
$100 \mathrm{~A}$
\end{tabular} & Avg. & $\%$ Dev \\
\hline $\mathrm{Al}_{2} \mathrm{O}_{3}$ & 11.66 & 11.67 & 12.49 & 11.91 & 12.54 & 12.58 & 12.24 & 4.96 & 8.98 & 9.34 & 9.75 & 9.55 & 6.31 \\
\hline $\mathrm{B}_{2} \mathrm{O}_{3} *$ & 11.30 & 11.30 & 11.30 & 11.30 & 11.30 & 11.30 & 11.30 & $\mathrm{NC}$ & 16.17 & 16.17 & 16.17 & 16.17 & $\mathrm{NC}$ \\
\hline $\mathrm{BaO}$ & 0.01 & \begin{tabular}{|l|}
0.02 \\
\end{tabular} & 0.02 & 0.03 & 0.03 & 0.02 & 0.02 & $\mathrm{NC}$ & 0.01 & 0.01 & 0.02 & 0.02 & $\mathrm{NC}$ \\
\hline $\mathrm{Bi}_{2} \mathrm{O}_{3}$ & 6.71 & 6.31 & 6.64 & 6.15 & 6.67 & 6.11 & 6.38 & -4.96 & 2.56 & 2.32 & 2.78 & 2.55 & -0.59 \\
\hline $\mathrm{CaO}$ & 0.84 & 0.86 & 0.81 & 0.82 & 0.83 & 0.83 & 0.83 & $\mathrm{NC}$ & 0.87 & 0.78 & \begin{tabular}{|l|}
0.87 \\
\end{tabular} & 0.83 & $\mathrm{NC}$ \\
\hline $\mathrm{CdO}$ & $\S$ & $<0.01$ & $<0.01$ & $<0.01$ & $<0.01$ & $<0.01$ & $<0.01$ & NC & $\S$ & $<0.01$ & $<0.01$ & $<0.01$ & NC \\
\hline $\mathrm{Cl}$ & $\S$ & 0.02 & 0.03 & 0.04 & 0.03 & 0.03 & 0.03 & NC & $\S$ & $<0.01$ & $<0.01$ & $<0.01$ & $\mathrm{NC}$ \\
\hline $\mathrm{Cr}_{2} \mathrm{O}_{3}$ & 0.52 & 0.61 & 0.68 & 0.61 & 0.66 & 0.63 & 0.64 & NC & 1.08 & 1.12 & 1.27 & 1.20 & 10.80 \\
\hline $\mathrm{F}^{*}$ & 0.82 & 0.82 & 0.82 & 0.82 & 0.82 & 0.82 & 0.82 & NC & 0.70 & 0.70 & 0.70 & 0.70 & NC \\
\hline $\mathrm{Fe}_{2} \mathrm{O}_{3}$ & 6.96 & 7.15 & 7.56 & 7.05 & 7.53 & 7.11 & 7.28 & 4.61 & 4.62 & 4.28 & 4.91 & 4.60 & -0.53 \\
\hline $\mathrm{K}_{2} \mathrm{O}$ & 0.46 & 0.50 & 0.54 & 0.51 & 0.52 & 0.51 & 0.52 & $\mathrm{NC}$ & 6.05 & 5.49 & 5.68 & 5.59 & -7.60 \\
\hline $\mathrm{La}_{2} \mathrm{O}_{3}$ & $\S$ & 0.01 & 0.02 & 0.02 & 0.03 & 0.02 & 0.02 & NC & $\S$ & $<0.01$ & 0.01 & $<0.01$ & $\mathrm{NC}$ \\
\hline \begin{tabular}{|l|}
$\mathrm{Li}_{2} \mathrm{O} *$ \\
\end{tabular} & 0.16 & 0.16 & 0.16 & 0.16 & 0.16 & 0.16 & 0.16 & NC & 3.68 & 3.68 & 3.68 & 3.68 & $\mathrm{NC}$ \\
\hline MgO & 0.43 & 0.48 & 0.50 & 0.41 & 0.42 & 0.46 & \begin{tabular}{|l|}
0.45 \\
\end{tabular} & NC & 0.06 & 0.25 & 0.23 & 0.24 & $\mathrm{NC}$ \\
\hline $\mathrm{MnO}$ & $\S$ & \begin{tabular}{|l|}
0.12 \\
\end{tabular} & 0.09 & 0.10 & 0.09 & 0.11 & \begin{tabular}{|l|}
0.10 \\
\end{tabular} & NC & $\S$ & 0.01 & \begin{tabular}{|l|}
0.02 \\
\end{tabular} & 0.02 & $\mathrm{NC}$ \\
\hline $\mathrm{Na}_{2} \mathrm{O}$ & 15.74 & 15.16 & 15.80 & 16.31 & 15.78 & 14.83 & 15.57 & -1.05 & 7.07 & 7.23 & \begin{tabular}{|l|}
6.67 \\
\end{tabular} & 6.95 & -1.65 \\
\hline $\mathrm{Nd}_{2} \mathrm{O}_{3}$ & $\S$ & \begin{tabular}{|l|}
0.05 \\
\end{tabular} & 0.05 & \begin{tabular}{|l|}
0.05 \\
\end{tabular} & \begin{tabular}{|l|}
0.05 \\
\end{tabular} & 0.05 & \begin{tabular}{|l|}
0.05 \\
\end{tabular} & NC & $\S$ & $<0.01$ & \begin{tabular}{|l|}
0.01 \\
\end{tabular} & $<0.01$ & NC \\
\hline $\mathrm{NiO}$ & 1.93 & 1.78 & 1.90 & 1.76 & 1.85 & 1.72 & 1.80 & -6.69 & 0.37 & 0.34 & \begin{tabular}{|l|}
0.40 \\
\end{tabular} & 0.37 & $\mathrm{NC}$ \\
\hline $\mathrm{P}_{2} \mathrm{O}_{5}$ & 4.99 & 5.10 & 5.43 & 5.24 & 5.59 & 5.42 & 5.36 & 7.32 & 1.18 & 1.36 & \begin{tabular}{|l|}
1.33 \\
\end{tabular} & 1.34 & \begin{tabular}{|l|l|}
14.40 \\
\end{tabular} \\
\hline $\mathrm{PbO}$ & 0.25 & 0.29 & 0.30 & 0.28 & 0.28 & 0.27 & 0.28 & $\mathrm{NC}$ & \begin{tabular}{|l|}
0.17 \\
\end{tabular} & 0.14 & 0.16 & 0.15 & $\mathrm{NC}$ \\
\hline $\mathrm{SiO}_{2}$ & 36.26 & 36.53 & 33.67 & 35.30 & 33.62 & 35.86 & 35.00 & -3.49 & 45.76 & 46.31 & 44.74 & 45.52 & -0.51 \\
\hline $\mathrm{SO}_{3}$ & \begin{tabular}{|l|}
0.48 \\
\end{tabular} & \begin{tabular}{|l|}
0.32 \\
\end{tabular} & 0.44 & \begin{tabular}{|l|}
0.43 \\
\end{tabular} & \begin{tabular}{|l|}
0.40 \\
\end{tabular} & 0.43 & 0.40 & $\mathrm{NC}$ & \begin{tabular}{|l|}
0.53 \\
\end{tabular} & 0.32 & \begin{tabular}{|l|}
0.37 \\
\end{tabular} & 0.34 & NC \\
\hline $\mathrm{TiO}_{2}$ & 0.16 & \begin{tabular}{|l|}
0.19 \\
\end{tabular} & 0.19 & \begin{tabular}{|l|}
0.19 \\
\end{tabular} & 0.20 & 0.19 & 0.19 & NC & $\S$ & 0.02 & \begin{tabular}{|l|}
0.04 \\
\end{tabular} & 0.03 & $\mathrm{NC}$ \\
\hline $\mathrm{ZnO}$ & 0.16 & 0.18 & 0.19 & 0.18 & 0.20 & 0.18 & \begin{tabular}{|l|}
0.18 \\
\end{tabular} & NC & \begin{tabular}{|l|}
0.09 \\
\end{tabular} & 0.08 & \begin{tabular}{|l|}
0.09 \\
\end{tabular} & 0.09 & $\mathrm{NC}$ \\
\hline $\mathrm{ZrO}_{2}$ & 0.21 & 0.38 & 0.38 & 0.36 & 0.39 & 0.36 & 0.37 & $\mathrm{NC}$ & 0.04 & 0.05 & \begin{tabular}{|l|}
0.06 \\
\end{tabular} & 0.05 & $\mathrm{NC}$ \\
\hline Sum & 100 & 100 & 100 & 100 & 100 & 100 & 100 & 100 & 100 & 100 & 100 & 100 & $\mathrm{NC}$ \\
\hline
\end{tabular}


The Catholic University of America Vitreous State Laboratory
High Level Waste Vitrification System Improvements Final Report, VSL-07R1010-1, Rev. 0

Table 6.2. XRF Analyzed Compositions of Vitrified Melter Feed Samples (wt \%) (continued).

\begin{tabular}{|c|c|c|c|c|c|c|c|c|}
\hline \multirow[b]{2}{*}{ Sample } & \multicolumn{8}{|c|}{ Al Limited } \\
\hline & Target & $\begin{array}{c}\text { BLL-F- } \\
132 A\end{array}$ & $\begin{array}{c}\text { BLM-F- } \\
14 \mathrm{~A}\end{array}$ & $\begin{array}{c}\text { BLM-F- } \\
53 \mathrm{~A}\end{array}$ & $\begin{array}{c}\text { BLM-F- } \\
75 \mathrm{~A}\end{array}$ & $\begin{array}{c}\text { BLM-F- } \\
123 \mathrm{~A}\end{array}$ & Avg. & \%Dev. \\
\hline $\mathrm{Al}_{2} \mathrm{O}_{3}$ & 23.97 & 22.89 & 22.67 & 22.62 & 23.90 & 22.63 & 22.94 & -4.29 \\
\hline $\mathrm{B}_{2} \mathrm{O}_{3}{ }^{*}$ & 15.19 & 15.19 & 15.19 & 15.19 & 15.19 & 15.19 & 15.19 & NC \\
\hline $\mathrm{BaO}$ & 0.05 & 0.38 & 0.40 & 0.34 & 0.34 & 0.33 & 0.36 & $\mathrm{NC}$ \\
\hline $\mathrm{Bi}_{2} \mathrm{O}_{3}$ & 1.14 & 1.24 & 1.19 & 1.15 & 1.16 & 1.14 & 1.17 & 2.47 \\
\hline $\mathrm{CaO}$ & 6.08 & 5.78 & 5.73 & 5.70 & 5.67 & 5.74 & 5.73 & -5.76 \\
\hline $\mathrm{CdO}$ & 0.02 & $<0.01$ & $<0.01$ & $<0.01$ & $<0.01$ & $<0.01$ & $<0.01$ & $\mathrm{NC}$ \\
\hline $\mathrm{Cl}$ & $\S$ & $<0.01$ & $<0.01$ & $<0.01$ & $<0.010$ & $<0.01$ & $<0.01$ & $\mathrm{NC}$ \\
\hline $\mathrm{Cr}_{2} \mathrm{O}_{3}$ & 0.52 & 0.58 & 0.56 & 0.54 & 0.57 & 0.54 & 0.56 & NC \\
\hline $\mathrm{F}^{*}$ & 0.67 & 0.67 & 0.67 & 0.67 & 0.67 & 0.67 & 0.67 & $\mathrm{NC}$ \\
\hline $\mathrm{Fe}_{2} \mathrm{O}_{3}$ & 5.90 & 6.24 & 6.08 & 5.95 & 6.02 & 5.92 & 6.04 & 2.40 \\
\hline $\mathrm{K}_{2} \mathrm{O}$ & 0.14 & 0.35 & 0.34 & 0.31 & 0.34 & 0.33 & 0.34 & $\mathrm{NC}$ \\
\hline $\mathrm{La}_{2} \mathrm{O}_{3}$ & $\S$ & $<0.01$ & $<0.01$ & $<0.01$ & $<0.01$ & $<0.01$ & $<0.01$ & NC \\
\hline $\mathrm{Li}_{2} \mathrm{O}^{*}$ & 3.57 & 3.57 & 3.57 & 3.57 & 3.57 & 3.57 & 3.57 & $\mathrm{NC}$ \\
\hline $\mathrm{MgO}$ & 0.12 & 0.38 & 0.39 & 0.44 & 0.40 & 0.39 & 0.40 & $\mathrm{NC}$ \\
\hline $\mathrm{MnO}$ & $\S$ & 0.02 & 0.02 & 0.02 & 0.02 & 0.02 & 0.02 & $\mathrm{NC}$ \\
\hline $\mathrm{Na}_{2} \mathrm{O}$ & 9.58 & 8.63 & 9.39 & 9.95 & 8.18 & 9.91 & 9.21 & -3.85 \\
\hline $\mathrm{Nd}_{2} \mathrm{O}_{3}$ & $\S$ & 0.01 & 0.01 & 0.01 & $<0.01$ & $<0.01$ & 0.01 & $\mathrm{NC}$ \\
\hline $\mathrm{NiO}$ & 0.40 & 0.38 & 0.38 & 0.36 & 0.37 & 0.37 & 0.37 & NC \\
\hline $\mathrm{P}_{2} \mathrm{O}_{5}$ & 1.05 & 1.10 & 1.13 & 1.13 & 1.13 & 1.11 & 1.12 & 6.05 \\
\hline $\mathrm{PbO}$ & 0.41 & 0.37 & 0.36 & 0.35 & 0.35 & 0.34 & 0.35 & $\mathrm{NC}$ \\
\hline $\mathrm{SiO}_{2}$ & 30.50 & 31.42 & 31.15 & 30.98 & 31.38 & 31.09 & 31.21 & 2.33 \\
\hline $\mathrm{SO}_{3}$ & 0.20 & 0.16 & 0.18 & 0.16 & 0.18 & 0.16 & 0.17 & $\mathrm{NC}$ \\
\hline $\mathrm{TiO}_{2}$ & 0.01 & 0.04 & 0.04 & 0.04 & 0.04 & 0.04 & 0.04 & $\mathrm{NC}$ \\
\hline $\mathrm{ZnO}$ & 0.08 & 0.09 & 0.09 & 0.08 & 0.08 & 0.08 & 0.08 & $\mathrm{NC}$ \\
\hline $\mathrm{ZrO}_{2}$ & 0.39 & 0.50 & 0.49 & 0.45 & 0.45 & 0.45 & 0.47 & $\mathrm{NC}$ \\
\hline Sum & 100.00 & 100.00 & 100.00 & 100.00 & 100.00 & 100.00 & 100.00 & NC \\
\hline
\end{tabular}


The Catholic University of America Vitreous State Laboratory
High Level Waste Vitrification System Improvements Final Report, VSL-07R1010-1, Rev. 0

Table 6.2. XRF Analyzed Compositions of Vitrified Melter Feed Samples (wt\%) (continued).

\begin{tabular}{|c|c|c|c|c|c|c|c|}
\hline \multirow[b]{2}{*}{ Sample } & \multicolumn{7}{|c|}{ Al + Na Limited } \\
\hline & Target & $\begin{array}{c}\text { BLN-F- } \\
107 \mathrm{~A}\end{array}$ & $\%$ Dev & BLN-F-9A & $\begin{array}{c}\text { BLN-F- } \\
152 \mathrm{~A}\end{array}$ & Avg. & \%Dev. \\
\hline $\mathrm{Al}_{2} \mathrm{O}_{3}$ & 21.34 & 23.43 & 9.83 & 21.73 & 21.55 & 21.64 & 1.41 \\
\hline $\mathrm{B}_{2} \mathrm{O}_{3}{ }^{*}$ & 18.37 & 18.37 & $\mathrm{NC}$ & 18.37 & 18.37 & 18.37 & $\mathrm{NC}$ \\
\hline $\mathrm{BaO}$ & 0.03 & 0.04 & $\mathrm{NC}$ & 0.05 & 0.04 & 0.04 & $\mathrm{NC}$ \\
\hline $\mathrm{Bi}_{2} \mathrm{O}_{3}$ & 1.16 & 1.38 & 19.08 & 1.27 & 1.21 & 1.24 & 7.00 \\
\hline $\mathrm{CaO}$ & 0.72 & 1.03 & $\mathrm{NC}$ & 1.13 & 1.00 & 1.06 & NC \\
\hline $\mathrm{CdO}$ & 0.01 & $<0.01$ & NC & $<0.01$ & $<0.01$ & $<0.01$ & $\mathrm{NC}$ \\
\hline $\mathrm{Cl}$ & $\S$ & $<0.01$ & NC & 0.01 & 0.01 & 0.01 & $\mathrm{NC}$ \\
\hline $\mathrm{Cr}_{2} \mathrm{O}_{3}$ & 0.71 & 0.93 & NC & 0.85 & 0.86 & 0.85 & $\mathrm{NC}$ \\
\hline $\mathrm{F}^{*}$ & 0.23 & 0.23 & NC & 0.23 & 0.23 & 0.23 & $\mathrm{NC}$ \\
\hline $\mathrm{Fe}_{2} \mathrm{O}_{3}$ & 2.82 & 3.39 & 20.48 & 3.19 & 3.14 & 3.17 & 12.51 \\
\hline $\mathrm{K}_{2} \mathrm{O}$ & 0.66 & 0.81 & $\mathrm{NC}$ & 0.71 & 0.71 & 0.71 & $\mathrm{NC}$ \\
\hline $\mathrm{La}_{2} \mathrm{O}_{3}$ & $\S$ & $<0.01$ & NC & $<0.01$ & $<0.01$ & $<0.01$ & $\mathrm{NC}$ \\
\hline $\mathrm{Li}_{2} \mathrm{O}^{*}$ & 3.58 & 3.58 & NC & 3.58 & 3.58 & 3.58 & $\mathrm{NC}$ \\
\hline $\mathrm{MgO}$ & 0.22 & 0.25 & NC & 0.25 & 0.20 & 0.23 & $\mathrm{NC}$ \\
\hline $\mathrm{MnO}$ & $\S$ & 0.01 & NC & 0.02 & 0.02 & 0.02 & $\mathrm{NC}$ \\
\hline $\mathrm{Na}_{2} \mathrm{O}$ & 12.71 & 11.93 & -6.11 & 11.77 & 12.25 & 12.01 & -5.48 \\
\hline $\mathrm{Nd}_{2} \mathrm{O}_{3}$ & $\S$ & 0.01 & NC & 0.01 & $<0.01$ & 0.01 & NC \\
\hline $\mathrm{NiO}$ & 0.10 & 0.13 & NC & 0.13 & 0.12 & 0.12 & NC \\
\hline $\mathrm{P}_{2} \mathrm{O}_{5}$ & 2.02 & 2.39 & 18.47 & 2.07 & 2.12 & 2.10 & 3.67 \\
\hline $\mathrm{PbO}$ & 0.09 & 0.11 & $\mathrm{NC}$ & 0.11 & 0.09 & 0.10 & $\mathrm{NC}$ \\
\hline $\mathrm{SiO}_{2}$ & 34.56 & 31.25 & -9.60 & 33.80 & 33.71 & 33.76 & -2.34 \\
\hline $\mathrm{SO}_{3}$ & 0.17 & 0.15 & NC & 0.18 & 0.19 & 0.19 & $\mathrm{NC}$ \\
\hline $\mathrm{TiO}_{2}$ & 0.22 & 0.20 & NC & 0.18 & 0.20 & 0.19 & $\mathrm{NC}$ \\
\hline $\mathrm{ZnO}$ & 0.18 & 0.20 & NC & 0.18 & 0.21 & 0.20 & $\mathrm{NC}$ \\
\hline $\mathrm{ZrO}_{2}$ & 0.12 & 0.19 & $\mathrm{NC}$ & 0.19 & 0.20 & 0.20 & $\mathrm{NC}$ \\
\hline Sum & 100.00 & 100.00 & NC & 100.00 & 100.00 & 100.00 & $\mathrm{NC}$ \\
\hline
\end{tabular}

$\S$ - Not a target constituent

* Target values

NC -Not calculated 
Table 6.3. Listing of Glass Discharged, Masses, and Analysis Performed During Bismuth-Limited DM100 Tests.

\begin{tabular}{|c|c|c|c|c|c|c|}
\hline Test & $\mathbf{T}\left({ }^{\circ} \mathbf{C}\right)$ & Date & Sample Name & Analysis & Mass (kg) & \begin{tabular}{|c|} 
Cumulative Mass \\
(kg)
\end{tabular} \\
\hline \multirow{21}{*}{$1 \mathrm{~A}$} & \multirow{39}{*}{1175} & \multirow{14}{*}{ 7/18/06 } & BLK-G-78A & - & - & - \\
\hline & & & BLK-G-79A & - & - & - \\
\hline & & & BLK-G-79B & XRF, DCP, F & 13.60 & 13.60 \\
\hline & & & BLK-G-81A & - & - & - \\
\hline & & & BLK-G-81B & - & - & - \\
\hline & & & BLK-G-83A & - & - & - \\
\hline & & & BLK-G-83B & XRF, DCP & 26.50 & 40.10 \\
\hline & & & BLK-G-83C & - & - & - \\
\hline & & & BLK-G-84A & XRF, DCP & 14.46 & 54.56 \\
\hline & & & BLK-G-84B & - & - & - \\
\hline & & & BLK-G-84C & XRF, DCP, F & 19.74 & 74.30 \\
\hline & & & BLK-G-88A & - & - & - \\
\hline & & & BLK-G-89A & XRF, DCP & 33.64 & 107.94 \\
\hline & & & BLK-G-91A & - & - & - \\
\hline & & \multirow{10}{*}{ 7/19/06 } & BLK-G-95A & XRF, DCP & 22.82 & 130.76 \\
\hline & & & BLK-G-96A & - & - & - \\
\hline & & & BLK-G-96B & XRF, F & 24.24 & 155.00 \\
\hline & & & BLK-G-97A & - & - & - \\
\hline & & & BLK-G-99A & XRF, DCP & 17.60 & 172.60 \\
\hline & & & BLK-G-100A & - & - & - \\
\hline & & & BLK-G-100B & XRF & 20.24 & 192.84 \\
\hline \multirow{18}{*}{$1 \mathrm{~B}$} & & & BLK-G-103A & - & - & - \\
\hline & & & BLK-G-105A & XRF, DCP, F & 23.88 & 216.72 \\
\hline & & & BLK-G-105B & - & - & - \\
\hline & & \multirow{15}{*}{ 7/20/06 } & BLK-G-108A & XRF, DCP & 26.46 & 243.18 \\
\hline & & & BLK-G-110A & - & - & - \\
\hline & & & BLK-G-110B & XRF & 26.02 & 269.20 \\
\hline & & & BLK-G-111A & - & - & - \\
\hline & & & BLK-G-111B & XRF, F & 21.40 & 290.60 \\
\hline & & & BLK-G-112A & - & - & - \\
\hline & & & BLK-G-112B & XRF & 26.36 & 316.96 \\
\hline & & & BLK-G-117A & - & - & - \\
\hline & & & BLK-G-119A & XRF, DCP & 26.22 & 343.18 \\
\hline & & & BLK-G-119B & - & - & - \\
\hline & & & BLK-G-122A & XRF, F & 26.76 & 369.94 \\
\hline & & & BLK-G-124A & - & - & - \\
\hline & & & BLK-G-124B & XRF & 22.64 & 392.58 \\
\hline & & & BLK-G-124C & - & - & - \\
\hline & & & BLK-G-129A & $\begin{array}{l}\text { XRF, DCP, PCT, } \\
\text { TCLP }\end{array}$ & 33.46 & 426.04 \\
\hline \multirow{4}{*}{$2 \mathrm{~A}$} & \multirow{4}{*}{1150} & \multirow{4}{*}{ 7/24/06 } & BLK-G-146A & - & - & - \\
\hline & & & BLK-G-146B & XRF & 18.16 & 444.20 \\
\hline & & & BLK-G-147A & - & - & - \\
\hline & & & BLK-G-149A & XRF & 19.68 & 463.88 \\
\hline
\end{tabular}

- Empty data field 
The Catholic University of America Vitreous State Laboratory
High Level Waste Vitrification System Improvements Final Report, VSL-07R1010-1, Rev. 0

\section{Table 6.3. List of Glass Discharged, Masses, and Analysis Performed During Bismuth-Limited DM100 Tests (continued).}

\begin{tabular}{|c|c|c|c|c|c|c|}
\hline Test & $\mathrm{T}\left({ }^{\circ} \mathrm{C}\right)$ & Date & Sample Name & Analysis & Mass (kg) & $\begin{array}{c}\text { Cumulative } \\
\text { Mass (kg) }\end{array}$ \\
\hline \multirow{12}{*}{$2 \mathrm{~A}$} & \multirow{25}{*}{1150} & $7 / 24 / 05$ & BLK-G-150A & - & - & - \\
\hline & & \multirow{8}{*}{ 7/25/06 } & BLK-G-150B & XRF, DCP, F & 25.54 & 489.42 \\
\hline & & & BLK-G-151A & - & - & - \\
\hline & & & BLK-G-151B & XRF, DCP & 16.98 & 506.40 \\
\hline & & & BLL-G-9A & - & - & - \\
\hline & & & BLL-G-9B & XRF & 17.64 & 524.04 \\
\hline & & & BLL-G-9C & - & - & - \\
\hline & & & BLL-G-10A & XRF, DCP & 24.42 & 548.46 \\
\hline & & & BLL-G-11A & - & - & - \\
\hline & & \multirow{6}{*}{ 7/26/06 } & BLL-G-11B & XRF, DCP & 23.82 & 572.28 \\
\hline & & & BLL-G-15A & - & - & - \\
\hline & & & BLL-G-15B & XRF, F & 21.96 & 594.24 \\
\hline \multirow{13}{*}{ 2B } & & & BLL-G-20A & - & - & - \\
\hline & & & BLL-G-23A & XRF & 19.76 & 614.00 \\
\hline & & & BLL-G-27A & - & - & - \\
\hline & & \multirow{6}{*}{ 7/27/06 } & BLL-G-27B & XRF, DCP & 21.12 & 635.12 \\
\hline & & & BLL-G-28A & - & - & - \\
\hline & & & BLL-G-30A & XRF, F & 15.02 & 650.14 \\
\hline & & & BLL-G-31A & - & - & - \\
\hline & & & BLL-G-35A & XRF, DCP & 22.82 & 672.96 \\
\hline & & & BLL-G-38A & - & - & - \\
\hline & & \multirow{4}{*}{ 7/28/06 } & BLL-G-40A & XRF, DCP & 30.32 & 703.28 \\
\hline & & & BLL-G-41A & $\mathrm{XRF}$ & 29.80 & 733.08 \\
\hline & & & BLL-G-46A & XRF & 28.12 & 761.20 \\
\hline & & & BLL-G-46B & $\begin{array}{c}\text { XRF, DCP, PCT, } \\
\text { TCLP, F }\end{array}$ & 33.14 & 794.34 \\
\hline
\end{tabular}

-Empty data field 
Table 6.4. List of Glass Discharged, Masses, and Analysis Performed During Chromium-Limited DM100 Tests.

\begin{tabular}{|c|c|c|c|c|c|c|}
\hline Test & $\mathrm{T}\left({ }^{\circ} \mathrm{C}\right)$ & Date & Name & Analysis & Mass (kg) & \begin{tabular}{|c|}
$\begin{array}{c}\text { Cumulative Mass } \\
\text { (kg) }\end{array}$ \\
\end{tabular} \\
\hline \multirow{18}{*}{ 3B } & \multirow{18}{*}{1175} & $8 / 21 / 06$ & BLL-G-70A & - & - & - \\
\hline & & \multirow{10}{*}{$8 / 22 / 06$} & BLL-G-70B & XRF, F & 24.94 & 24.94 \\
\hline & & & BLL-G-71A & - & - & - \\
\hline & & & BLL-G-71B & XRF & 32.34 & 57.28 \\
\hline & & & BLL-G-73A & - & - & - \\
\hline & & & BLL-G-73B & XRF & 24.38 & 81.66 \\
\hline & & & BLL-G-76A & - & - & - \\
\hline & & & BLL-G-76B & XRF, F & 25.42 & 107.08 \\
\hline & & & BLL-G-77A & - & - & - \\
\hline & & & BLL-G-77B & XRF & 28.10 & 135.18 \\
\hline & & & BLL-G-79A & - & - & - \\
\hline & & \multirow{9}{*}{$8 / 23 / 06$} & BLL-G-83A & XRF & 35.60 & 170.78 \\
\hline & & & BLL-G-83B & - & - & - \\
\hline & & & BLL-G-83C & XRF, F & 32.02 & 202.80 \\
\hline & & & BLL-G-86A & - & - & - \\
\hline & & & BLL-G-86B & XRF & 17.32 & 220.12 \\
\hline & & & BLL-G-86C & - & - & - \\
\hline & & & BLL-G-88A & XRF, DCP, TCLP, PCT & 28.44 & 248.56 \\
\hline \multirow{22}{*}{$4 \mathrm{~A}$} & \multirow{22}{*}{1150} & & BLL-G-89A & - & - & - \\
\hline & & & BLL-G-91A & XRF, F & 27.56 & 276.12 \\
\hline & & \multirow{10}{*}{$8 / 24 / 06$} & BLL-G-91B & - & - & - \\
\hline & & & BLL-G-94A & XRF & 30.18 & 306.30 \\
\hline & & & BLL-G-96A & - & - & - \\
\hline & & & BLL-G-96B & $\mathrm{XRF}$ & 22.86 & 329.16 \\
\hline & & & BLL-G-100A & - & - & - \\
\hline & & & BLL-G-100B & XRF, F & 21.00 & 350.16 \\
\hline & & & BLL-G-100C & - & - & - \\
\hline & & & BLL-G-101A & XRF & 25.40 & 375.56 \\
\hline & & & BLL-G-101B & - & - & - \\
\hline & & & BLL-G-105A & XRF & 28.56 & 404.12 \\
\hline & & \multirow{10}{*}{$8 / 25 / 06$} & BLL-G-105B & - & - & - \\
\hline & & & BLL-G-107A & XRF, DCP, F & 29.88 & 434.00 \\
\hline & & & BLL-G-107B & - & - & - \\
\hline & & & BLL-G-107C & XRF & 19.34 & 453.34 \\
\hline & & & BLL-G-111A & - & - & - \\
\hline & & & BLL-G-111B & XRF, DCP & 27.76 & 481.10 \\
\hline & & & BLL-G-111C & - & - & - \\
\hline & & & BLL-G-113A & XRF, DCP, TCLP & 26.44 & 507.54 \\
\hline & & & BLL-G-113B & - & - & - \\
\hline & & & BLL-G-121A & $\begin{array}{c}\text { XRF, DCP, TCLP, } \\
\text { PCT, F }\end{array}$ & 32.86 & 540.40 \\
\hline
\end{tabular}

- Empty data field 
Table 6.5. List of Glass Discharged, Masses, and Analysis Performed During Aluminum-Limited DM100 Tests.

\begin{tabular}{|c|c|c|c|c|c|c|}
\hline Test & $\mathrm{T}\left({ }^{\circ} \mathrm{C}\right)$ & Date & Name & Analysis & Mass (kg) & $\begin{array}{c}\text { Cumulative } \\
\text { Mass (kg) }\end{array}$ \\
\hline \multirow{14}{*}{$5 \mathrm{~A}$} & \multirow{26}{*}{ 1175C } & $9 / 11 / 06$ & BLL-G-137A & - & - & - \\
\hline & & \multirow{10}{*}{$9 / 12 / 06$} & BLL-G-137B & XRF, F & 26.9 & 26.90 \\
\hline & & & BLL-G-140A & - & - & - \\
\hline & & & BLL-G-142A & XRF & 21.56 & 48.46 \\
\hline & & & BLL-G-143A & - & - & - \\
\hline & & & BLL-G-143B & XRF & 20.88 & 69.34 \\
\hline & & & BLL-G-147A & - & - & - \\
\hline & & & BLL-G-148A & XRF & 25.62 & 94.96 \\
\hline & & & BLL-G-149A & - & - & - \\
\hline & & & BLL-G-149B & XRF, F & 22.56 & 117.52 \\
\hline & & & BLL-G-153A & - & - & - \\
\hline & & \multirow{5}{*}{ 9/13/06 } & BLL-G-154A & XRF & 27.56 & 145.08 \\
\hline & & & BLL-G-155A & - & - & - \\
\hline & & & BLM-G-9A & XRF & 25.84 & 170.92 \\
\hline \multirow{12}{*}{$5 B$} & & & BLM-G-11A & - & - & - \\
\hline & & & BLM-G-13A & XRF & 23.82 & 194.74 \\
\hline & & \multirow{7}{*}{ 9/14/06 } & BLM-G-14A & - & - & - \\
\hline & & & BLM-G-17A & XRF, F & 18.57 & 213.31 \\
\hline & & & BLM-G-18A & - & - & - \\
\hline & & & BLM-G-20A & $\mathrm{XRF}$ & 15.84 & 229.15 \\
\hline & & & BLM-G-24A & - & - & - \\
\hline & & & BLM-G-24B & XRF & 21.5 & 250.65 \\
\hline & & & BLM-G-27A & - & - & - \\
\hline & & \multirow{3}{*}{ 9/15/06 } & BLM-G-31A & XRF & 29.56 & 280.21 \\
\hline & & & BLM-G-32A & - & - & - \\
\hline & & & BLM-G-36A & XRF, F, DCP, TCLP, PCT & 25 & 305.21 \\
\hline \multirow{13}{*}{$6 \mathrm{~A}$} & \multirow{17}{*}{$1150 \mathrm{C}$} & \multirow{4}{*}{ 9/18/06 } & BLM-G-53A & - & - & - \\
\hline & & & BLM-G-54A & $\mathrm{XRF}$ & 20.92 & 326.13 \\
\hline & & & BLM-G-55A & - & - & - \\
\hline & & & BLM-G-57A & XRF & 24.56 & 350.69 \\
\hline & & \multirow{6}{*}{ 9/19/06 } & BLM-G-60A & - & - & - \\
\hline & & & BLM-G-62A & XRF & 20 & 370.69 \\
\hline & & & BLM-G-62B & - & - & - \\
\hline & & & BLM-G-65A & XRF, F & 20.6 & 391.29 \\
\hline & & & BLM-G-67A & - & - & - \\
\hline & & & BLM-G-71A & $\mathrm{XRF}$ & 22.04 & 413.33 \\
\hline & & \multirow{5}{*}{ 9/20/06 } & BLM-G-72A & - & - & - \\
\hline & & & BLM-G-73A & XRF & 18.84 & 432.17 \\
\hline & & & BLM-G-73B & $\mathrm{XRF}$ & 9.86 & 442.03 \\
\hline \multirow{4}{*}{$6 B$} & & & BLM-G-79A & - & - & - \\
\hline & & & BLM-G-80A & XRF, F & 21.1 & 463.13 \\
\hline & & \multirow{2}{*}{ 9/21/06 } & BLM-G-83A & - & - & - \\
\hline & & & BLM-G-85A & $\mathrm{XRF}$ & 14.89 & 478.02 \\
\hline
\end{tabular}

- Empty data field 


\section{Table 6.5. List of Glass Discharged, Masses, and Analysis Performed During Aluminum-Limited DM100 Tests (continued).}

\begin{tabular}{|c|c|c|c|c|c|c|}
\hline Test & $\mathrm{T}\left({ }^{\circ} \mathrm{C}\right)$ & Date & Name & Analysis & Mass (kg) & $\begin{array}{c}\text { Cumulative } \\
\text { Mass (kg) }\end{array}$ \\
\hline \multirow{7}{*}{$6 \mathrm{~B}$} & \multirow{35}{*}{1150} & \multirow{2}{*}{ 9/21/06 } & BLM-G-88A & - & - & - \\
\hline & & & BLM-G-90A & XRF & 26.26 & 504.28 \\
\hline & & \multirow{2}{*}{$9 / 22 / 06$} & BLM-G-91A & - & - & - \\
\hline & & & BLM-G-100A & XRF & 11.5 & 515.78 \\
\hline & & $9 / 23 / 06$ & BLM-G-110A & XRF, F, DCP, TCLP, PCT & 12.92 & 528.70 \\
\hline & & $9 / 25 / 06$ & BLM-G-111A & XRF & 6.18 & 534.88 \\
\hline & & \multirow{7}{*}{$9 / 26 / 06$} & BLM-G-112A & - & - & - \\
\hline \multirow{28}{*}{$6 \mathrm{C}$} & & & BLM-G-123A & - & - & - \\
\hline & & & BLM-G-123B & XRF & 23.3 & 558.18 \\
\hline & & & BLM-G-123C & - & - & - \\
\hline & & & BLM-G-124A & XRF & 11.98 & 570.16 \\
\hline & & & BLM-G-125A & - & - & - \\
\hline & & & BLM-G-127A & XRF & 17.32 & 587.48 \\
\hline & & \multirow{10}{*}{$9 / 27 / 06$} & BLM-G-128A & - & - & - \\
\hline & & & BLM-G-131A & XRF, F, DCP & 28.58 & 616.06 \\
\hline & & & BLM-G-133A & - & - & - \\
\hline & & & BLM-G-133B & XRF & 18.38 & 634.44 \\
\hline & & & BLM-G-134A & - & - & - \\
\hline & & & BLM-G-134B & XRF & 19.68 & 654.12 \\
\hline & & & BLM-G-134C & - & - & \\
\hline & & & BLM-G-138A & XRF & 18.48 & 672.60 \\
\hline & & & BLM-G-138B & - & - & - \\
\hline & & & BLM-G-139A & XRF, F, DCP & 26.32 & 698.92 \\
\hline & & \multirow{12}{*}{$9 / 28 / 06$} & BLM-G-143A & - & - & - \\
\hline & & & BLM-G-144A & XRF & 19.56 & 718.48 \\
\hline & & & BLM-G-144B & - & - & \\
\hline & & & BLM-G-145A & XRF, F, DCP & 17.68 & 736.16 \\
\hline & & & BLM-G-145B & - & - & \\
\hline & & & BLM-G-146A & - & - & - \\
\hline & & & BLM-G-146B & XRF, DCP, PCT, TCLP & 19.52 & 755.68 \\
\hline & & & BLM-G-146C & - & - & - \\
\hline & & & BLM-G-152A & - & - & - \\
\hline & & & BLM-G-152B & XRF, F & 27.08 & 782.76 \\
\hline & & & BLM-G-152C & - & - & - \\
\hline & & & BLM-G-152D & XRF & 10.62 & 793.38 \\
\hline
\end{tabular}

- Empty data field 
Table 6.6. List of Glass Discharged, Masses, and Analysis Performed During Aluminum-Plus-Sodium-Limited DM100 Tests.

\begin{tabular}{|c|c|c|c|c|c|c|}
\hline Test & $\mathrm{T}\left({ }^{\circ} \mathrm{C}\right)$ & Date & Name & Analysis & Mass (kg) & $\begin{array}{c}\text { Cumulative } \\
\text { Mass (kg) }\end{array}$ \\
\hline \multirow{21}{*}{$7 \mathrm{~A}$} & \multirow{39}{*}{1175} & $10 / 2 / 06$ & BLN-G-15A & - & - & - \\
\hline & & \multirow{12}{*}{ 10/3/06 } & BLN-G-17A & XRF, F & 19.46 & 19.46 \\
\hline & & & BLN-G-18A & - & - & - \\
\hline & & & BLN-G-21A & XRF & 21.7 & 41.16 \\
\hline & & & BLN-G-22A & - & - & - \\
\hline & & & BLN-G-22B & XRF & 26.36 & 67.52 \\
\hline & & & BLN-G-22C & - & - & - \\
\hline & & & BLN-G-24A & XRF & 28 & 95.52 \\
\hline & & & BLN-G-24B & - & - & - \\
\hline & & & BLN-G-24C & XRF & 23.02 & 118.54 \\
\hline & & & BLN-G-27A & - & - & - \\
\hline & & & BLN-G-27B & XRF, F & 25.24 & 143.78 \\
\hline & & & BLN-G-29A & - & - & - \\
\hline & & \multirow{11}{*}{$10 / 4 / 06$} & BLN-G-32A & XRF & 23.1 & 166.88 \\
\hline & & & BLN-G-32B & - & - & - \\
\hline & & & BLN-G-32C & XRF & 27.86 & 194.74 \\
\hline & & & BLN-G-34A & - & - & - \\
\hline & & & BLN-G-35A & XRF & 24.42 & 219.16 \\
\hline & & & BLN-G-35B & - & - & - \\
\hline & & & BLN-G-36A & XRF, DCP & 26.46 & 245.62 \\
\hline & & & BLN-G-40A & XRF & 13.86 & 259.48 \\
\hline \multirow{18}{*}{$7 \mathrm{~B}$} & & & BLN-G-40B & - & - & - \\
\hline & & & BLN-G-41A & XRF, F & 21.88 & 281.36 \\
\hline & & & BLN-G-41B & - & - & - \\
\hline & & \multirow{8}{*}{$10 / 5 / 06$} & BLN-G-43A & XRF & 21.64 & 303.00 \\
\hline & & & BLN-G-43B & - & - & - \\
\hline & & & BLN-G-46A & XRF & 22.18 & 325.18 \\
\hline & & & BLN-G-49A & - & - & - \\
\hline & & & BLN-G-52A & XRF & 17.94 & 343.12 \\
\hline & & & BLN-G-52B & - & - & - \\
\hline & & & BLN-G-52C & XRF & 30.66 & 373.78 \\
\hline & & & BLN-G-55A & - & - & - \\
\hline & & \multirow{7}{*}{ 10/6/06 } & BLN-G-55B & XRF & 25.24 & 399.02 \\
\hline & & & BLN-G-56A & - & - & - \\
\hline & & & BLN-G-60A & XRF & 26.66 & 425.68 \\
\hline & & & BLN-G-62A & - & - & - \\
\hline & & & BLN-G-62B & XRF, F & 23.56 & 449.24 \\
\hline & & & BLN-G-65A & - & - & - \\
\hline & & & BLN-G-66A & XRF, DCP, TCLP, PCT & 27.4 & 476.64 \\
\hline \multirow{4}{*}{$8 \mathrm{~A}$} & \multirow{4}{*}{1150} & \multirow{4}{*}{$10 / 16 / 06$} & BLN-G-81A & - & - & - \\
\hline & & & BLN-G-81B & $\mathrm{XRF}$ & 19.3 & 495.94 \\
\hline & & & BLN-G-82A & - & - & - \\
\hline & & & BLN-G-82B & XRF, F & 17.18 & 513.12 \\
\hline
\end{tabular}

- Empty data field 
Table 6.6. List of Glass Discharged, Masses, and Analysis Performed During Aluminum-Plus-Sodium-Limited DM100 Tests (continued).

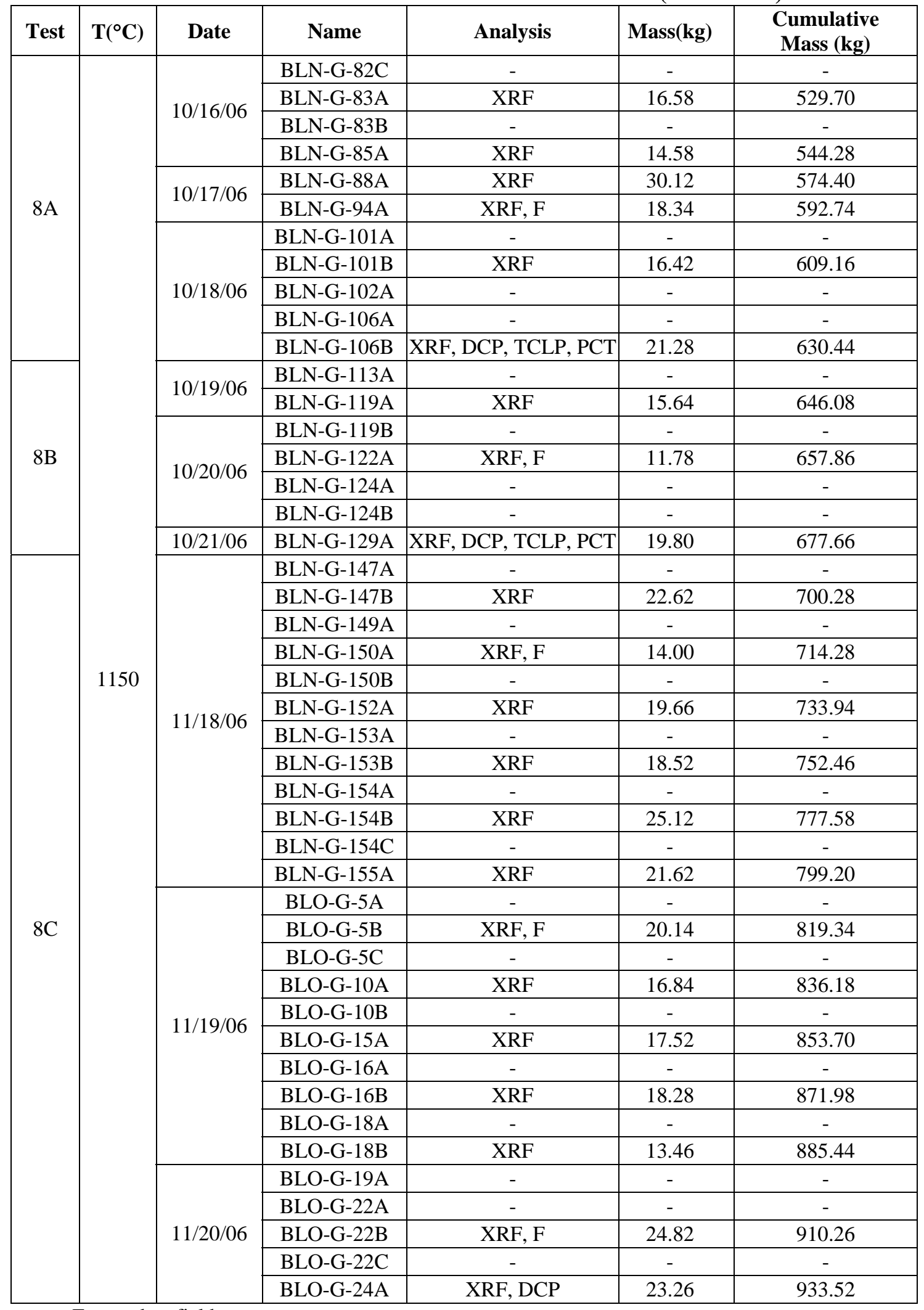

- Empty data field 
ORP-56297 Rev. 0

The Catholic University of America

Vitreous State Laboratory

High Level Waste Vitrification System Improvements

Final Report, VSL-07R1010-1, Rev. 0

Table 6.7. XRF-Analyzed Compositions of Discharged Glass Samples from the DM100 Tests with the Bismuth-Limited Composition (wt\%).

\begin{tabular}{|c|c|c|c|c|c|c|c|c|c|c|c|}
\hline \multirow{2}{*}{\multicolumn{2}{|c|}{$\frac{\text { Test }}{\text { Glass (ko) }}$}} & \multicolumn{10}{|c|}{$1 \mathrm{~A}$} \\
\hline & & 0.00 & 13.60 & 40.10 & 54.56 & 74.30 & 107.94 & 130.76 & 155.00 & 172.60 & 192.84 \\
\hline \multirow{2}{*}{ Constituent } & \multirow{2}{*}{ Target } & BLK-G & BLK-G- & -BLK-G & -BLK-G & BLK-G & BLK-G- & BLK-G- & BLK-G & BLK-G- & BLK-G- \\
\hline & & $58 \mathrm{~A}$ & $79 B$ & 83B & $84 \mathrm{~A}$ & $84 \mathrm{C}$ & $89 \mathrm{~A}$ & $95 \mathrm{~A}$ & $96 \mathrm{~B}$ & $99 \mathrm{~A}$ & 100B \\
\hline $\mathrm{Ag}_{2} \mathrm{O}$ & $\S$ & 0.08 & 0.06 & 0.03 & 0.03 & 0.03 & 0.03 & 0.03 & 0.02 & 0.02 & 0.02 \\
\hline $\mathrm{Al}_{2} \mathrm{O}_{3}$ & 11.66 & 7.06 & 8.59 & 9.19 & 9.27 & 9.80 & 10.34 & 10.10 & 10.32 & 10.26 & 10.74 \\
\hline $\mathrm{B}_{2} \mathrm{O}_{3} *$ & 11.30 & 9.97 & 10.42 & 10.54 & 10.60 & 10.67 & 10.78 & 10.84 & 10.90 & 10.94 & 10.97 \\
\hline $\mathrm{BaO}$ & 0.01 & 0.07 & 0.07 & 0.05 & 0.04 & 0.06 & 0.05 & 0.05 & 0.04 & 0.03 & 0.03 \\
\hline $\mathrm{Bi}_{2} \mathrm{O}_{3}$ & 6.71 & $<0.01$ & 2.28 & 3.18 & 3.42 & 3.41 & 3.79 & 4.21 & 4.57 & 4.71 & 4.85 \\
\hline $\mathrm{CaO}$ & 0.84 & 0.60 & 0.66 & 0.70 & 0.71 & 0.73 & 0.74 & 0.78 & 0.77 & 0.78 & 0.78 \\
\hline $\mathrm{CeO}_{2}$ & $\S$ & 0.10 & 0.07 & 0.04 & 0.04 & 0.04 & 0.03 & 0.03 & 0.01 & 0.02 & 0.01 \\
\hline $\mathrm{Cl}$ & $\S$ & 0.01 & 0.02 & 0.02 & 0.03 & 0.03 & 0.03 & 0.03 & 0.04 & 0.03 & 0.03 \\
\hline $\mathrm{Cr}_{2} \mathrm{O}_{3}$ & 0.52 & 0.21 & 0.36 & 0.41 & 0.43 & 0.41 & 0.43 & 0.45 & 0.48 & 0.49 & 0.48 \\
\hline$F$ & 0.82 & $<0.1^{\#}$ & 0.17 & 0.23 & 0.26 & $0.30^{\#}$ & 0.32 & 0.33 & $0.35^{\#}$ & 0.36 & 0.38 \\
\hline $\mathrm{Fe}_{2} \mathrm{O}_{3}$ & 6.96 & 12.79 & 10.63 & 9.29 & 9.40 & 8.86 & 8.60 & 8.90 & 8.29 & 8.32 & 7.97 \\
\hline $\mathrm{K}_{2} \mathrm{O}$ & 0.46 & 0.26 & 0.36 & 0.40 & 0.40 & 0.40 & 0.43 & 0.44 & 0.45 & 0.47 & 0.46 \\
\hline $\mathrm{Li}_{2} \mathrm{O} *$ & 0.16 & 2.62 & 1.95 & 1.70 & 1.59 & 1.44 & 1.22 & 1.09 & 0.98 & 0.90 & 0.82 \\
\hline $\mathrm{MgO}$ & 0.43 & 0.30 & 0.37 & 0.40 & 0.41 & 0.40 & 0.47 & 0.45 & 0.44 & 0.48 & 0.41 \\
\hline $\mathrm{MnO}$ & $\S$ & 2.39 & 1.57 & 1.12 & 1.11 & 1.01 & 0.89 & 0.86 & 0.69 & 0.66 & 0.57 \\
\hline $\mathrm{Na}_{2} \mathrm{O}$ & 15.74 & 12.21 & 13.50 & 14.47 & 14.66 & 14.69 & 14.33 & 14.98 & 15.16 & 14.90 & 15.51 \\
\hline $\mathrm{Nd}_{2} \mathrm{O}_{3}$ & $\S$ & 0.15 & 0.12 & 0.09 & 0.09 & 0.09 & 0.08 & 0.07 & 0.07 & 0.07 & 0.06 \\
\hline $\mathrm{NiO}$ & 1.93 & 0.34 & 0.79 & 1.02 & 1.04 & 1.02 & 1.09 & 1.23 & 1.30 & 1.38 & 1.34 \\
\hline $\mathrm{P}_{2} \mathrm{O}_{5}$ & 4.99 & 0.43 & 2.15 & 3.05 & 3.10 & 3.24 & 3.52 & 3.55 & 3.98 & 4.15 & 4.22 \\
\hline $\mathrm{PbO}$ & 0.25 & 0.42 & 0.37 & 0.32 & 0.34 & 0.31 & 0.31 & 0.33 & 0.30 & 0.31 & 0.29 \\
\hline $\mathrm{SiO}_{2}$ & 36.26 & 47.90 & 43.75 & 42.19 & 41.49 & 41.57 & 41.07 & 39.73 & 39.46 & 39.34 & 38.70 \\
\hline $\mathrm{SnO}_{2}$ & $\S$ & 0.04 & 0.03 & 0.02 & 0.01 & 0.02 & 0.01 & 0.01 & 0.01 & $<0.01$ & 0.01 \\
\hline $\mathrm{SO}_{3}$ & 0.48 & 0.07 & 0.21 & 0.31 & 0.30 & 0.30 & 0.32 & 0.32 & 0.35 & 0.37 & 0.36 \\
\hline $\mathrm{SrO}$ & $\S$ & 0.14 & 0.09 & 0.07 & 0.07 & 0.06 & 0.06 & 0.06 & 0.05 & 0.05 & 0.04 \\
\hline $\mathrm{TiO}_{2}$ & 0.16 & 0.05 & 0.10 & 0.12 & 0.11 & 0.13 & 0.14 & 0.15 & 0.14 & 0.15 & 0.16 \\
\hline $\mathrm{ZnO}$ & 0.16 & 0.93 & 0.65 & 0.49 & 0.49 & 0.46 & 0.41 & 0.42 & 0.36 & 0.35 & 0.32 \\
\hline $\mathrm{ZrO}_{2}$ & 0.21 & 0.87 & 0.68 & 0.55 & 0.56 & 0.52 & 0.50 & 0.52 & 0.47 & 0.47 & 0.45 \\
\hline Sum & 100.00 & 100.00 & 100.00 & 100.00 & 100.00 & 100.00 & 100.00 & 100.00 & 100.00 & 100.00 & 100.00 \\
\hline
\end{tabular}


Table 6.7. XRF-Analyzed Compositions of Discharged Glass Samples from the DM100 Tests with the Bismuth-Limited Composition (wt\%) (continued).

\begin{tabular}{|c|c|c|c|c|c|c|c|c|c|c|c|}
\hline \multirow{2}{*}{\multicolumn{2}{|c|}{$\frac{\text { Test }}{\text { lass (kg) }}$}} & \multicolumn{9}{|c|}{$1 \mathrm{~B}$} & \multirow{2}{*}{\begin{tabular}{|c|}
$2 \mathrm{~A}$ \\
444.20 \\
\end{tabular}} \\
\hline & & \multirow{2}{*}{\begin{tabular}{|c|}
216.72 \\
BLK-G-
\end{tabular}} & \multirow{2}{*}{$\begin{array}{r}243.18 \\
\text { BLK-G }\end{array}$} & \multirow{2}{*}{\begin{tabular}{r|}
269.20 \\
BLK-G-
\end{tabular}} & \multirow{2}{*}{\begin{tabular}{|l|}
290.60 \\
BLK-G-
\end{tabular}} & \multirow{2}{*}{$\frac{316.96}{\text { BLK-G }}$} & \multirow{2}{*}{$\begin{array}{r}343.18 \\
\text { BLK-G- }\end{array}$} & \multirow{2}{*}{\begin{tabular}{|l|}
369.94 \\
BLK-G-
\end{tabular}} & \multirow{2}{*}{$\begin{array}{r}392.58 \\
\text { BLK-G }\end{array}$} & \multirow{2}{*}{\begin{tabular}{|l}
426.04 \\
BLK-G
\end{tabular}} & \\
\hline & & & & & & & & & & & BLK-G \\
\hline |Constituent & Target & $105 \mathrm{~A}$ & $108 \mathrm{~A}$ & 110B & 111B & $112 \mathrm{~B}$ & 119A & $122 \mathrm{~A}$ & $124 \mathrm{~B}$ & $129 \mathrm{~A}$ & 146B \\
\hline $\mathrm{Ag}_{2} \mathrm{O}$ & $\S$ & 0.01 & 0.01 & 0.01 & $<0.01$ & $<0.01$ & $<0.01$ & 0.00 & 0.01 & 0.01 & $<0.01$ \\
\hline $\mathrm{Al}_{2} \mathrm{O}_{3}$ & 11.66 & 10.56 & 10.82 & 10.95 & 11.31 & 11.30 & 11.34 & 11.43 & 11.33 & 11.26 & 11.67 \\
\hline $\mathrm{B}_{2} \mathrm{O}_{3} *$ & 11.30 & 11.02 & 11.05 & 11.09 & \begin{tabular}{|l|}
11.11 \\
\end{tabular} & \begin{tabular}{|l|l|}
11.14 \\
\end{tabular} & 11.16 & \begin{tabular}{|l|}
11.18 \\
\end{tabular} & \begin{tabular}{|l|}
11.19 \\
\end{tabular} & 11.21 & 11.22 \\
\hline $\mathrm{BaO}$ & 0.01 & 0.03 & 0.02 & 0.04 & 0.02 & 0.02 & 0.02 & 0.03 & 0.03 & 0.03 & 0.03 \\
\hline $\mathrm{Bi}_{2} \mathrm{O}_{3}$ & 6.71 & 4.94 & 5.06 & 5.19 & 5.57 & 5.42 & 5.56 & 5.19 & 6.32 & 5.75 & 5.70 \\
\hline $\mathrm{CaO}$ & 0.84 & 0.78 & 0.81 & 0.81 & 0.84 & 0.80 & 0.80 & 0.77 & 0.88 & 0.82 & 0.82 \\
\hline $\mathrm{CeO}_{2}$ & $\S$ & 0.01 & 0.02 & $<0.01$ & $<0.01$ & 0.01 & $<0.01$ & $<0.01$ & $<0.01$ & $<0.01$ & $<0.01$ \\
\hline $\mathrm{Cl}$ & $\S$ & 0.04 & 0.04 & 0.03 & 0.04 & 0.04 & 0.05 & 0.04 & 0.04 & 0.04 & 0.04 \\
\hline $\mathrm{Cr}_{2} \mathrm{O}_{3}$ & 0.52 & 0.48 & 0.47 & 0.48 & 0.50 & 0.47 & 0.48 & 0.45 & 0.52 & 0.49 & 0.45 \\
\hline $\mathrm{F}$ & 0.82 & $0.40^{\#}$ & 0.40 & 0.40 & $0.40^{\#}$ & 0.42 & 0.43 & $0.45^{\#}$ & 0.45 & 0.45 & 0.44 \\
\hline $\mathrm{Fe}_{2} \mathrm{O}_{3}$ & 6.96 & 7.87 & 7.60 & 7.47 & 7.73 & 7.41 & 7.23 & 6.80 & 7.79 & 7.13 & 6.88 \\
\hline $\mathrm{K}_{2} \mathrm{O}$ & 0.46 & 0.45 & 0.48 & 0.48 & 0.51 & 0.49 & 0.48 & 0.49 & 0.52 & 0.51 & 0.51 \\
\hline $\mathrm{Li}_{2} \mathrm{O}^{*}$ & 0.16 & 0.74 & 0.66 & 0.59 & 0.54 & 0.49 & 0.45 & 0.41 & 0.38 & 0.34 & 0.32 \\
\hline $\mathrm{MgO}$ & 0.43 & 0.49 & 0.52 & 0.52 & 0.48 & 0.50 & 0.52 & 0.53 & 0.50 & 0.51 & 0.53 \\
\hline $\mathrm{MnO}$ & $\S$ & 0.53 & 0.45 & 0.40 & 0.39 & 0.37 & 0.31 & 0.27 & 0.28 & 0.26 & 0.22 \\
\hline $\mathrm{Na}_{2} \mathrm{O}$ & 15.74 & 15.90 & 15.60 & 15.74 & 14.39 & 15.42 & 15.81 & 16.04 & 14.40 & 16.00 & 16.38 \\
\hline $\mathrm{Nd}_{2} \mathrm{O}_{3}$ & $\S$ & 0.07 & 0.06 & 0.05 & 0.06 & 0.06 & 0.05 & 0.06 & 0.06 & 0.06 & 0.05 \\
\hline $\mathrm{NiO}$ & 1.93 & 1.31 & 1.39 & 1.43 & 1.48 & 1.42 & 1.44 & 1.34 & 1.64 & 1.49 & 1.42 \\
\hline $\mathrm{P}_{2} \mathrm{O}_{5}$ & 4.99 & 4.30 & 4.58 & 4.61 & 4.74 & 4.71 & 4.76 & 4.96 & 4.92 & 4.92 & 4.97 \\
\hline $\mathrm{PbO}$ & 0.25 & 0.29 & 0.29 & 0.28 & 0.29 & 0.29 & 0.28 & 0.26 & 0.31 & 0.28 & 0.27 \\
\hline $\mathrm{SiO}_{2}$ & 36.26 & 38.47 & 38.41 & 38.15 & 38.27 & 37.96 & \begin{tabular}{|l|}
37.57 \\
\end{tabular} & 38.11 & 37.08 & 37.24 & 36.84 \\
\hline $\mathrm{SnO}_{2}$ & $\S$ & $<0.01$ & 0.01 & $<0.01$ & 0.01 & 0.01 & $<0.01$ & $<0.01$ & 0.01 & 0.01 & 0.01 \\
\hline $\mathrm{SO}_{3}$ & 0.48 & 0.37 & 0.39 & 0.39 & 0.41 & 0.40 & 0.41 & 0.42 & 0.43 & 0.41 & 0.44 \\
\hline $\mathrm{SrO}$ & $\S$ & 0.04 & 0.03 & 0.04 & 0.03 & 0.03 & 0.03 & 0.03 & 0.03 & 0.03 & 0.03 \\
\hline $\mathrm{TiO}_{2}$ & 0.16 & 0.16 & 0.16 & 0.16 & 0.18 & 0.17 & 0.18 & 0.16 & 0.19 & 0.18 & 0.18 \\
\hline $\mathrm{ZnO}$ & 0.16 & 0.31 & 0.28 & 0.26 & 0.27 & 0.25 & 0.24 & 0.22 & 0.25 & 0.22 & 0.21 \\
\hline $\mathrm{ZrO}_{2}$ & 0.21 & 0.44 & 0.40 & 0.41 & 0.42 & 0.41 & 0.39 & 0.36 & 0.44 & 0.39 & 0.37 \\
\hline Sum & 100.00 & 100.00 & 100.00 & 100.00 & 100.00 & 100.00 & 100.00 & 100.00 & 100.00 & 100.00 & 100.00 \\
\hline
\end{tabular}


Table 6.7. XRF-Analyzed Compositions of Discharged Glass Samples from the DM100 Tests with the Bismuth-Limited Composition (wt\%) (continued).

\begin{tabular}{|c|c|c|c|c|c|c|c|c|c|c|c|}
\hline \multirow{2}{*}{\multicolumn{2}{|c|}{$\begin{array}{l}\text { Test } \\
\text { lass }(\mathrm{kg})\end{array}$}} & \multicolumn{7}{|c|}{$2 \mathrm{~A}$} & \multicolumn{3}{|c|}{$2 B$} \\
\hline & & 463.88 & 489.42 & 506.40 & 524.04 & 548.46 & 572.28 & 594.24 & 614.00 & 635.12 & 650.14 \\
\hline renctituont & Tarrat & BLK-G- & BLK-G- & BLK-G- & BLL-G- & BLL-G- & BLL-G- & BLL-G- & BLL-G & BLL-G- & BLL-G- \\
\hline Conistituentit & Idiget & $149 \mathrm{~A}$ & 150B & 151B & 9B & $10 \mathrm{~A}$ & $11 \mathrm{~B}$ & 15B & $23 \mathrm{~A}$ & $27 \mathrm{~B}$ & $30 \mathrm{~A}$ \\
\hline $\mathrm{Ag}_{2} \mathrm{O}$ & $\S$ & $<0.01$ & $<0.01$ & $<0.01$ & $<0.01$ & $<0.01$ & $<0.01$ & $<0.01$ & $<0.01$ & $<0.01$ & $<0.01$ \\
\hline $\mathrm{Al}_{2} \mathrm{O}_{3}$ & 11.66 & \begin{tabular}{|l}
11.52 \\
\end{tabular} & \begin{tabular}{|l}
11.53 \\
\end{tabular} & 11.47 & \begin{tabular}{|l|}
11.81 \\
\end{tabular} & 11.76 & 11.63 & 11.59 & 11.96 & 11.69 & 11.75 \\
\hline $\mathrm{B}_{2} \mathrm{O}_{3} *$ & \begin{tabular}{|l|}
11.30 \\
\end{tabular} & \begin{tabular}{|l|}
11.23 \\
\end{tabular} & \begin{tabular}{|l}
11.24 \\
\end{tabular} & 11.24 & 11.25 & 11.25 & 11.26 & 11.27 & 11.27 & 11.27 & 11.27 \\
\hline $\mathrm{BaO}$ & 0.01 & 0.03 & 0.03 & 0.02 & 0.02 & 0.03 & 0.03 & 0.03 & 0.03 & 0.03 & 0.02 \\
\hline $\mathrm{Bi}_{2} \mathrm{O}_{3}$ & 6.71 & 6.12 & 6.08 & 6.15 & 5.97 & 6.06 & 6.20 & 6.43 & 5.81 & 6.15 & 6.15 \\
\hline $\mathrm{CaO}$ & 0.84 & 0.84 & 0.82 & 0.84 & 0.83 & 0.82 & 0.84 & 0.86 & 0.82 & 0.85 & 0.83 \\
\hline $\mathrm{CeO}_{2}$ & $\S$ & $<0.01$ & $<0.01$ & $<0.01$ & $<0.01$ & $<0.01$ & $<0.01$ & $<0.01$ & $<0.01$ & $<0.01$ & $<0.01$ \\
\hline $\mathrm{Cl}$ & $\S$ & 0.04 & 0.04 & 0.05 & 0.04 & 0.05 & 0.05 & 0.05 & 0.04 & 0.04 & 0.05 \\
\hline $\mathrm{Cr}_{2} \mathrm{O}_{3}$ & 0.52 & 0.47 & 0.46 & 0.47 & 0.47 & 0.47 & 0.46 & 0.48 & 0.44 & 0.47 & 0.45 \\
\hline $\mathrm{F}$ & 0.82 & 0.44 & $0.44^{\#}$ & 0.44 & 0.43 & 0.43 & 0.42 & $0.42^{\#}$ & 0.43 & 0.45 & $0.46^{\#}$ \\
\hline $\mathrm{Fe}_{2} \mathrm{O}_{3}$ & 6.96 & 7.18 & 7.09 & 7.19 & 6.92 & 6.90 & 7.04 & 7.22 & 6.58 & 6.86 & 6.91 \\
\hline $\mathrm{K}_{2} \mathrm{O}$ & 0.46 & 0.52 & 0.51 & 0.51 & 0.52 & 0.50 & 0.51 & 0.52 & 0.50 & 0.52 & 0.52 \\
\hline $\mathrm{Li}_{2} \mathrm{O}^{*}$ & 0.16 & 0.31 & 0.29 & 0.28 & 0.27 & 0.25 & 0.24 & 0.23 & 0.22 & 0.22 & 0.21 \\
\hline $\mathrm{MgO}$ & 0.43 & 0.49 & 0.50 & 0.46 & 0.51 & 0.46 & 0.45 & 0.47 & 0.46 & 0.46 & 0.41 \\
\hline $\mathrm{MnO}$ & $\S$ & 0.22 & 0.21 & 0.21 & 0.18 & 0.17 & 0.17 & 0.16 & 0.15 & 0.15 & 0.15 \\
\hline $\mathrm{Na}_{2} \mathrm{O}$ & 15.74 & 15.40 & 16.10 & 16.10 & 16.02 & 16.38 & 16.21 & 15.35 & 16.52 & 16.38 & 16.42 \\
\hline $\mathrm{Nd}_{2} \mathrm{O}_{3}$ & $\S$ & 0.06 & 0.05 & 0.05 & 0.05 & 0.05 & 0.05 & 0.05 & 0.05 & 0.05 & 0.05 \\
\hline $\mathrm{NiO}$ & 1.93 & 1.50 & 1.45 & 1.51 & 1.47 & 1.47 & 1.54 & 1.59 & 1.43 & 1.51 & 1.53 \\
\hline $\mathrm{P}_{2} \mathrm{O}_{5}$ & 4.99 & 5.04 & 4.96 & 4.94 & 5.00 & 4.99 & 5.04 & 5.14 & 5.11 & 5.14 & 5.03 \\
\hline $\mathrm{PbO}$ & 0.25 & 0.29 & 0.28 & 0.30 & 0.27 & 0.28 & 0.29 & 0.30 & 0.27 & 0.29 & 0.28 \\
\hline $\mathrm{SiO}_{2}$ & 36.26 & 37.01 & 36.60 & 36.40 & 36.76 & 36.45 & 36.32 & 36.57 & 36.71 & 36.27 & 36.33 \\
\hline $\mathrm{SnO}_{2}$ & $\S$ & $<0.01$ & 0.01 & $<0.01$ & $<0.01$ & $<0.01$ & $<0.01$ & $<0.01$ & $<0.01$ & $<0.01$ & $<0.01$ \\
\hline $\mathrm{SO}_{3}$ & 0.48 & 0.45 & 0.49 & 0.53 & 0.42 & 0.44 & 0.45 & 0.45 & 0.45 & 0.44 & 0.42 \\
\hline $\mathrm{SrO}$ & $\S$ & 0.03 & 0.03 & 0.03 & 0.02 & 0.03 & 0.03 & 0.03 & 0.03 & 0.03 & 0.02 \\
\hline $\mathrm{TiO}_{2}$ & 0.16 & 0.19 & 0.18 & 0.19 & 0.19 & 0.19 & 0.18 & 0.19 & 0.18 & 0.18 & 0.18 \\
\hline $\mathrm{ZnO}$ & 0.16 & 0.21 & 0.21 & 0.21 & 0.20 & 0.19 & 0.20 & 0.20 & 0.18 & 0.18 & 0.19 \\
\hline $\mathrm{ZrO}_{2}$ & 0.21 & 0.40 & 0.40 & 0.41 & 0.38 & 0.38 & 0.39 & 0.41 & 0.36 & 0.38 & 0.38 \\
\hline Sum & 100.00 & 100.00 & 100.00 & 100.00 & 100.00 & 100.00 & 100.00 & 100.00 & 100.00 & 100.00 & 100.00 \\
\hline
\end{tabular}


Table 6.7. XRF-Analyzed Compositions of Discharged Glass Samples from the DM100 Tests with the Bismuth-Limited Composition (wt\%) (continued).

\begin{tabular}{|c|c|c|c|c|c|c|c|c|}
\hline \multirow{2}{*}{\multicolumn{2}{|c|}{$\begin{array}{c}\text { Test } \\
\text { Glass }(\mathrm{kg}) \\
\end{array}$}} & \multicolumn{5}{|c|}{$2 B$} & \multirow{2}{*}{\multicolumn{2}{|c|}{$\begin{array}{c}\text { Post Turnover } \\
572-795\end{array}$}} \\
\hline & & \multirow{2}{*}{\begin{tabular}{|c|}
672.96 \\
BLL-G- \\
$35 A$
\end{tabular}} & \multirow{2}{*}{$\begin{array}{c}703.28 \\
\text { BLL-G- } \\
40 \mathrm{~A}\end{array}$} & \multirow{2}{*}{$\begin{array}{c}733.08 \\
\text { BLL-G- } \\
41 \mathrm{~A}\end{array}$} & \multirow{2}{*}{$\begin{array}{c}761.20 \\
\text { BLL-G- } \\
46 \mathrm{~A}\end{array}$} & \multirow{2}{*}{$\begin{array}{c}794.34 \\
\text { BLL-G- } \\
46 B\end{array}$} & & \\
\hline Constituent & Target & & & & & & Average & \%Dev. \\
\hline $\mathrm{Ag}_{2} \mathrm{O}$ & $\S$ & $<0.01$ & $<0.01$ & $<0.01$ & $<0.01$ & $<0.01$ & $<0.01$ & NC \\
\hline $\mathrm{Al}_{2} \mathrm{O}_{3}$ & 11.66 & 11.63 & 11.69 & 11.73 & 11.83 & 11.48 & 11.70 & 0.33 \\
\hline $\mathrm{B}_{2} \mathrm{O}_{3} *$ & 11.30 & 11.28 & 11.28 & 11.28 & 11.29 & \begin{tabular}{|l|}
11.29 \\
\end{tabular} & 11.28 & NC \\
\hline $\mathrm{BaO}$ & 0.01 & 0.02 & 0.02 & 0.03 & 0.02 & 0.03 & 0.03 & $\mathrm{NC}$ \\
\hline $\mathrm{Bi}_{2} \mathrm{O}_{3}$ & 6.71 & 6.31 & 6.25 & 6.30 & 5.93 & 6.67 & 6.22 & -7.31 \\
\hline $\mathrm{CaO}$ & 0.84 & 0.87 & 0.86 & 0.86 & 0.83 & 0.87 & 0.85 & 0.93 \\
\hline $\mathrm{CeO}_{2}$ & $\S$ & $<0.01$ & $<0.01$ & $<0.01$ & $<0.01$ & $<0.01$ & $<0.01$ & $\mathrm{NC}$ \\
\hline $\mathrm{Cl}$ & $\S$ & 0.04 & 0.04 & 0.04 & 0.05 & 0.05 & 0.04 & NC \\
\hline $\mathrm{Cr}_{2} \mathrm{O}_{3}$ & 0.52 & 0.46 & 0.46 & 0.47 & 0.45 & 0.51 & 0.47 & $\mathrm{NC}$ \\
\hline $\mathrm{F}$ & 0.82 & 0.46 & 0.47 & 0.48 & 0.48 & $0.49^{\#}$ & 0.46 & $\mathrm{NC}$ \\
\hline $\mathrm{Fe}_{2} \mathrm{O}_{3}$ & 6.96 & 7.05 & 6.90 & 7.04 & 6.65 & 7.38 & 6.96 & 0.04 \\
\hline $\mathrm{K}_{2} \mathrm{O}$ & 0.46 & 0.52 & 0.52 & 0.53 & 0.49 & 0.54 & 0.52 & $\mathrm{NC}$ \\
\hline $\mathrm{Li}_{2} \mathrm{O}^{*}$ & 0.16 & 0.21 & 0.20 & 0.19 & 0.19 & 0.18 & 0.21 & $\mathrm{NC}$ \\
\hline $\mathrm{MgO}$ & 0.43 & 0.46 & 0.44 & 0.41 & 0.47 & 0.50 & 0.45 & $\mathrm{NC}$ \\
\hline $\mathrm{MnO}$ & $\S$ & 0.15 & 0.14 & 0.14 & 0.14 & 0.15 & 0.15 & $\mathrm{NC}$ \\
\hline $\mathrm{Na}_{2} \mathrm{O}$ & 15.74 & 15.21 & 15.89 & 15.62 & 16.15 & 15.23 & 15.90 & 0.99 \\
\hline $\mathrm{Nd}_{2} \mathrm{O}_{3}$ & $\S$ & 0.05 & 0.06 & 0.06 & 0.05 & 0.05 & 0.05 & NC \\
\hline $\mathrm{NiO}$ & 1.93 & 1.54 & 1.55 & 1.56 & 1.46 & 1.67 & 1.54 & -20.45 \\
\hline $\mathrm{P}_{2} \mathrm{O}_{5}$ & 4.99 & 5.19 & 5.12 & 5.14 & 5.23 & 5.06 & 5.12 & 2.63 \\
\hline $\mathrm{PbO}$ & 0.25 & 0.29 & 0.28 & 0.29 & 0.27 & 0.30 & 0.29 & $\mathrm{NC}$ \\
\hline $\mathrm{SiO}_{2}$ & 36.26 & 37.04 & 36.65 & 36.62 & 36.85 & 36.27 & 36.56 & 0.83 \\
\hline $\mathrm{SnO}_{2}$ & $\S$ & $<0.01$ & $<0.01$ & $<0.01$ & $<0.01$ & $<0.01$ & $<0.01$ & $\mathrm{NC}$ \\
\hline $\mathrm{SO}_{3}$ & 0.48 & 0.44 & 0.41 & 0.41 & 0.44 & 0.43 & 0.43 & $\mathrm{NC}$ \\
\hline $\mathrm{SrO}$ & $\S$ & 0.02 & 0.02 & 0.02 & 0.02 & 0.03 & 0.03 & $\mathrm{NC}$ \\
\hline $\mathrm{TiO}_{2}$ & 0.16 & 0.19 & 0.19 & 0.19 & 0.17 & 0.19 & 0.19 & $\mathrm{NC}$ \\
\hline $\mathrm{ZnO}$ & 0.16 & 0.19 & 0.18 & 0.19 & 0.18 & 0.20 & 0.19 & $\mathrm{NC}$ \\
\hline $\mathrm{ZrO}_{2}$ & 0.21 & 0.39 & 0.38 & 0.39 & 0.36 & 0.42 & 0.39 & NC \\
\hline Sum & 100.00 & 100.00 & 100.00 & 100.00 & 100.00 & 100.00 & \begin{tabular}{|l|}
100.00 \\
\end{tabular} & NC \\
\hline
\end{tabular}


Table 6.8. XRF-Analyzed Compositions of Discharged Glass Samples from the DM100 Tests with the Chromium-Limited Composition (wt\%).

\begin{tabular}{|c|c|c|c|c|c|c|c|c|c|c|c|c|c|}
\hline \multirow{2}{*}{\multicolumn{2}{|c|}{$\frac{\text { Test }}{\text { Glass (kg) }}$}} & \multicolumn{9}{|c|}{ 3B } & \multicolumn{3}{|c|}{ 4A } \\
\hline & & 24.94 & 57.28 & 81.66 & 107.08 & 135.18 & 170.78 & 202.8 & 220.12 & 248.56 & 276.12 & 306.30 & 329.16 \\
\hline Constituent & Target & $\begin{array}{c}\text { BLL-G- } \\
70 \mathrm{~B}\end{array}$ & $\begin{array}{c}\text { BLL-G- } \\
71 B\end{array}$ & $\begin{array}{c}\text { BLL-G- } \\
73 B\end{array}$ & $\begin{array}{c}\text { BLL-G- } \\
76 B\end{array}$ & $\begin{array}{c}\text { BLL-G- } \\
77 \mathrm{~B}\end{array}$ & $\begin{array}{c}\text { BLL-G- } \\
\text { 83A }\end{array}$ & $\begin{array}{c}\text { BLL-G- } \\
83 \mathrm{C}\end{array}$ & $\begin{array}{c}\text { BLL-G- } \\
86 B\end{array}$ & $\begin{array}{c}\text { BLL-G- } \\
88 \mathrm{~A}\end{array}$ & $\begin{array}{c}\text { BLL-G- } \\
\text { 91A }\end{array}$ & $\begin{array}{c}\text { BLL-G- } \\
94 \mathrm{~A}\end{array}$ & $\begin{array}{c}\text { BLL-G- } \\
96 B\end{array}$ \\
\hline $\mathrm{Al}_{2} \mathrm{O}_{3}$ & 8.98 & 11.18 & 10.32 & 10.37 & 10.50 & 10.30 & 9.95 & 9.81 & 9.75 & 9.43 & 9.81 & 9.89 & 9.66 \\
\hline $\mathrm{B}_{2} \mathrm{O}_{3} *$ & 16.17 & 11.92 & 12.62 & 13.07 & 13.48 & 13.87 & 14.28 & 14.59 & 14.73 & 14.95 & 15.12 & 15.28 & 15.39 \\
\hline $\mathrm{BaO}$ & 0.01 & 0.03 & 0.03 & 0.02 & 0.01 & 0.02 & 0.02 & 0.02 & 0.01 & 0.02 & 0.02 & 0.02 & 0.02 \\
\hline $\mathrm{Bi}_{2} \mathrm{O}_{3}$ & 2.56 & 4.42 & 4.64 & 4.05 & 3.48 & 3.51 & 3.52 & 3.32 & 3.23 & 3.36 & 3.00 & 2.87 & 2.88 \\
\hline $\mathrm{CaO}$ & 0.87 & 0.84 & 0.88 & 0.83 & 0.80 & 0.82 & 0.85 & 0.82 & 0.81 & 0.84 & 0.81 & 0.80 & 0.81 \\
\hline $\mathrm{Cr}_{2} \mathrm{O}_{3}$ & 1.08 & 0.81 & 0.94 & 0.90 & 0.92 & 1.00 & 1.07 & 1.07 & 1.11 & 1.16 & 1.12 & 1.12 & 1.15 \\
\hline $\mathrm{F}$ & 0.70 & $0.35 \#$ & 0.39 & 0.41 & $0.44 \#$ & 0.43 & 0.43 & 0.42\# & 0.42 & 0.43 & $0.44 \#$ & 0.45 & 0.45 \\
\hline $\mathrm{Fe}_{2} \mathrm{O}_{3}$ & 4.62 & 5.80 & 6.13 & 5.57 & 5.14 & 5.27 & 5.43 & 5.29 & 5.21 & 5.43 & 5.02 & 4.90 & 4.98 \\
\hline $\mathrm{K}_{2} \mathrm{O}$ & 6.05 & 2.91 & 3.48 & 3.62 & 3.86 & 4.18 & 4.51 & 4.64 & 4.72 & 4.94 & 4.89 & 4.93 & 4.99 \\
\hline $\mathrm{Li}_{2} \mathrm{O}^{*}$ & 3.68 & 0.64 & 1.14 & 1.46 & 1.75 & 2.03 & 2.33 & 2.55 & 2.65 & 2.80 & 2.93 & 3.04 & 3.12 \\
\hline $\mathrm{MgO}$ & 0.06 & 0.36 & 0.32 & 0.33 & 0.33 & 0.30 & 0.28 & 0.29 & 0.27 & 0.28 & 0.24 & 0.29 & 0.31 \\
\hline $\mathrm{MnO}$ & $\S$ & 0.09 & 0.08 & 0.07 & 0.05 & 0.05 & 0.06 & 0.05 & 0.04 & 0.05 & 0.04 & 0.04 & 0.04 \\
\hline $\mathrm{Na}_{2} \mathrm{O}$ & 7.07 & 12.94 & 12.17 & 11.64 & 10.91 & 10.02 & 9.13 & 9.31 & 9.47 & 8.90 & 8.73 & 8.65 & 8.67 \\
\hline $\mathrm{Nd}_{2} \mathrm{O}_{3}$ & $\S$ & 0.03 & 0.03 & 0.03 & 0.02 & 0.02 & 0.02 & $<0.01$ & 0.01 & 0.01 & 0.01 & 0.01 & 0.01 \\
\hline $\mathrm{NiO}$ & 0.37 & 1.00 & 1.02 & 0.89 & 0.79 & 0.79 & 0.78 & 0.76 & 0.72 & 0.74 & 0.66 & 0.63 & 0.61 \\
\hline $\mathrm{P}_{2} \mathrm{O}_{5}$ & 1.18 & 3.56 & 3.13 & 2.97 & 2.67 & 2.49 & 2.27 & 2.13 & 1.98 & 1.91 & 1.82 & 1.75 & 1.71 \\
\hline $\mathrm{PbO}$ & 0.17 & 0.22 & 0.23 & 0.21 & 0.18 & 0.18 & 0.19 & 0.18 & 0.18 & 0.19 & 0.17 & 0.16 & 0.18 \\
\hline $\mathrm{SiO}_{2}$ & 45.76 & 42.08 & 41.63 & 42.78 & 43.93 & 44.02 & 44.18 & 44.10 & 44.03 & 43.93 & 44.51 & 44.54 & 44.39 \\
\hline $\mathrm{SO}_{3}$ & 0.53 & 0.33 & 0.33 & 0.35 & 0.36 & 0.35 & 0.36 & 0.36 & 0.36 & 0.37 & 0.37 & 0.37 & 0.39 \\
\hline $\mathrm{SrO}$ & $\S$ & 0.01 & 0.01 & 0.01 & 0.01 & 0.01 & 0.01 & 0.01 & $<0.01$ & 0.01 & $<0.01$ & $<0.01$ & $<0.01$ \\
\hline $\mathrm{TiO}_{2}$ & $\S$ & 0.12 & 0.10 & 0.10 & 0.09 & 0.08 & 0.07 & 0.06 & 0.06 & 0.05 & 0.05 & 0.05 & 0.05 \\
\hline $\mathrm{ZnO}$ & 0.09 & 0.14 & 0.15 & 0.13 & 0.11 & 0.11 & 0.12 & 0.12 & 0.11 & 0.12 & 0.11 & 0.10 & 0.10 \\
\hline $\mathrm{ZrO}_{2}$ & 0.04 & 0.23 & 0.23 & 0.19 & 0.15 & 0.15 & 0.14 & 0.13 & 0.11 & 0.12 & 0.10 & 0.09 & 0.09 \\
\hline Sum & 100.00 & 100.00 & 100.00 & 100.00 & 100.00 & 100.00 & 100.00 & 100.00 & 100.00 & 100.00 & 100.00 & 100.00 & 100.00 \\
\hline
\end{tabular}

$\S$ - Not a target constituent

* - Target values calculated using simple well-stirred tank model

\# - Fluorine was measured by XRF on polished samples, values for other samples calculated by interpolation 
The Catholic University of America Vitreous State Laboratory
High Level Waste Vitrification System Improvements Final Report, VSL-07R1010-1, Rev. 0

\section{Table 6.8. XRF-Analyzed Compositions of Discharged Glass Samples from the DM100 Tests with the Chromium-Limited Composition (wt\%) (continued).}

\begin{tabular}{|c|c|c|c|c|c|c|c|c|c|c|}
\hline \multirow{2}{*}{\multicolumn{2}{|c|}{$\begin{array}{c}\text { Test } \\
\text { Glass (kg) }\end{array}$}} & \multicolumn{8}{|c|}{ 4A } & \multirow{3}{*}{\begin{tabular}{|c} 
\%Dev. \\
from \\
target for \\
last \\
sample
\end{tabular}} \\
\hline & & \multirow{2}{*}{$\begin{array}{c}350.16 \\
\text { BLL-G- } \\
100 B\end{array}$} & \multirow{2}{*}{$\begin{array}{c}375.56 \\
\text { BLL-G- } \\
101 \mathrm{~A} \\
\end{array}$} & \multirow{2}{*}{$\begin{array}{c}404.12 \\
\text { BLL-G- } \\
105 A \\
\end{array}$} & \multirow{2}{*}{$\begin{array}{c}434.00 \\
\text { BLL-G- } \\
107 A \\
\end{array}$} & \multirow{2}{*}{$\begin{array}{c}453.34 \\
\text { BLL-G- } \\
107 C\end{array}$} & \multirow{2}{*}{$\begin{array}{c}481.10 \\
\text { BLL-G- } \\
111 \mathrm{~B} \\
\end{array}$} & \multirow{2}{*}{$\begin{array}{c}507.54 \\
\text { BLL-G- } \\
113 \mathrm{~A}\end{array}$} & \multirow{2}{*}{$\begin{array}{c}540.40 \\
\text { BLL-G- } \\
121 \mathrm{~A} \\
\end{array}$} & \\
\hline Constituent & Target & & & & & & & & & \\
\hline $\mathrm{Al}_{2} \mathrm{O}_{3}$ & 8.98 & 9.76 & 9.66 & 9.74 & 9.36 & 9.36 & 9.72 & 9.07 & 9.37 & 4.35 \\
\hline $\mathrm{B}_{2} \mathrm{O}_{3} *$ & 16.17 & 15.47 & 15.57 & 15.66 & 15.73 & 15.78 & 15.84 & $\begin{array}{l}15.88 \\
\end{array}$ & 15.93 & NC \\
\hline $\mathrm{BaO}$ & 0.01 & 0.01 & 0.02 & 0.02 & 0.01 & 0.02 & 0.01 & 0.02 & 0.01 & $\mathrm{NC}$ \\
\hline $\mathrm{Bi}_{2} \mathrm{O}_{3}$ & 2.56 & 2.82 & 2.88 & 2.58 & 2.93 & 2.87 & 2.69 & 2.90 & 2.88 & 12.42 \\
\hline $\mathrm{CaO}$ & 0.87 & 0.81 & 0.82 & 0.76 & 0.84 & 0.82 & 0.80 & 0.83 & 0.81 & NC \\
\hline $\mathrm{Cr}_{2} \mathrm{O}_{3}$ & 1.08 & 1.15 & 1.14 & 1.06 & 1.19 & 1.19 & 1.14 & 1.19 & 1.19 & 9.74 \\
\hline $\mathrm{F}$ & 0.70 & $0.46 \#$ & 0.46 & 0.46 & $0.46 \#$ & 0.47 & 0.48 & 0.49 & $0.51 \#$ & $\mathrm{NC}$ \\
\hline $\mathrm{Fe}_{2} \mathrm{O}_{3}$ & 4.62 & 4.89 & 5.02 & 4.56 & 5.13 & 5.02 & 4.77 & 5.06 & 5.06 & 9.60 \\
\hline $\mathrm{K}_{2} \mathrm{O}$ & 6.05 & 5.02 & 5.18 & 4.98 & 5.41 & 5.43 & 5.36 & 5.53 & 5.51 & -8.97 \\
\hline $\mathrm{Li}_{2} \mathrm{O}^{*}$ & 3.68 & 3.18 & 3.25 & 3.31 & 3.37 & 3.40 & 3.44 & 3.47 & 3.51 & $\mathrm{NC}$ \\
\hline $\mathrm{MgO}$ & 0.06 & 0.28 & 0.28 & 0.28 & 0.25 & 0.24 & 0.25 & 0.22 & 0.23 & $\mathrm{NC}$ \\
\hline $\mathrm{MnO}$ & $\S$ & 0.04 & 0.04 & 0.03 & 0.04 & 0.03 & 0.03 & 0.03 & 0.04 & NC \\
\hline $\mathrm{Na}_{2} \mathrm{O}$ & 7.07 & 8.46 & 8.31 & 8.84 & 7.75 & 7.75 & 7.44 & 7.68 & 7.62 & 7.74 \\
\hline $\mathrm{Nd}_{2} \mathrm{O}_{3}$ & 0.00 & 0.01 & 0.01 & 0.01 & 0.01 & $<0.01$ & 0.01 & $<0.01$ & 0.01 & $\mathrm{NC}$ \\
\hline $\mathrm{NiO}$ & 0.37 & 0.59 & 0.60 & 0.52 & 0.58 & 0.55 & 0.50 & 0.53 & 0.54 & $\mathrm{NC}$ \\
\hline $\mathrm{P}_{2} \mathrm{O}_{5}$ & 1.18 & 1.66 & 1.62 & 1.57 & 1.57 & 1.52 & 1.51 & 1.48 & 1.46 & 23.96 \\
\hline $\mathrm{PbO}$ & 0.17 & 0.16 & 0.16 & 0.15 & 0.17 & 0.17 & 0.15 & 0.17 & 0.16 & $\mathrm{NC}$ \\
\hline $\mathrm{SiO}_{2}$ & 45.76 & 44.58 & 44.36 & 44.87 & 44.58 & 44.74 & 45.22 & 44.82 & 44.51 & -2.74 \\
\hline $\mathrm{SO}_{3}$ & 0.53 & 0.39 & 0.39 & 0.39 & 0.40 & 0.40 & 0.41 & 0.41 & 0.41 & NC \\
\hline $\mathrm{SrO}$ & $\S$ & $<0.01$ & $<0.01$ & $<0.01$ & $<0.01$ & $<0.01$ & $<0.01$ & $<0.01$ & $<0.01$ & $\mathrm{NC}$ \\
\hline $\mathrm{TiO}_{2}$ & $\S$ & 0.05 & 0.04 & 0.04 & 0.04 & 0.04 & 0.05 & 0.03 & 0.04 & $\mathrm{NC}$ \\
\hline $\mathrm{ZnO}$ & 0.09 & 0.10 & 0.11 & 0.09 & 0.10 & 0.10 & 0.10 & 0.11 & 0.11 & $\mathrm{NC}$ \\
\hline $\mathrm{ZrO}_{2}$ & 0.04 & 0.09 & 0.09 & 0.07 & 0.08 & 0.08 & 0.07 & 0.07 & 0.08 & $\mathrm{NC}$ \\
\hline Sum & 100.00 & 100.00 & 100.00 & 100.00 & 100.00 & 100.00 & 100.00 & 100.00 & 100.00 & $\mathrm{NC}$ \\
\hline
\end{tabular}

$\S$ - Not a target constituent

* - Target values calculated using simple well-stirred tank model

\# - Fluorine was measured by XRF on polished samples, values for other samples calculated by interpolation 


\section{Table 6.9. XRF-Analyzed Compositions of Discharged Glass Samples from the DM100 Tests with the Aluminum-Limited} Composition (wt\%).

\begin{tabular}{|c|c|c|c|c|c|c|c|c|c|c|c|c|c|c|c|c|}
\hline \multirow{2}{*}{\multicolumn{2}{|c|}{$\frac{\text { Test }}{\text { Glass (kg) }}$}} & \multicolumn{7}{|c|}{$5 A$} & \multicolumn{6}{|c|}{$5 B$} & \multicolumn{2}{|c|}{ 6A } \\
\hline & & 26.9 & 48.46 & 69.34 & 94.96 & 117.52 & 145.08 & 170.92 & 194.74 & 213.31 & 229.15 & 250.65 & 280.21 & 305.21 & 326.13 & 350.69 \\
\hline Constituent & Target & $\begin{array}{c}\text { BLL-G- } \\
\text { 137B }\end{array}$ & $\begin{array}{c}\text { BLL-G- } \\
142 \mathrm{~A}\end{array}$ & $\begin{array}{c}\text { BLL-G- } \\
143 B\end{array}$ & $\begin{array}{c}\text { BLL-G- } \\
148 \mathrm{~A}\end{array}$ & $\begin{array}{c}\text { BLL-G- } \\
149 B\end{array}$ & $\begin{array}{c}\text { BLL-G- } \\
154 \mathrm{~A}\end{array}$ & $\begin{array}{l}\text { BLM- } \\
\text { G-9A }\end{array}$ & $\begin{array}{l}\text { BLM- } \\
\text { G-13A }\end{array}$ & \begin{tabular}{|l} 
BLM- \\
G-17A
\end{tabular} & $\begin{array}{l}\text { BLM- } \\
\text { G-20A }\end{array}$ & $\begin{array}{l}\text { BLM- } \\
\text { G-24B }\end{array}$ & $\begin{array}{l}\text { BLM- } \\
\text { G-31A }\end{array}$ & $\begin{array}{l}\text { BLM- } \\
\text { G-36A }\end{array}$ & $\begin{array}{c}\text { BLM-G- } \\
54 \mathrm{~A}\end{array}$ & $\begin{array}{c}\text { BLM-G } \\
57 \mathrm{~A}\end{array}$ \\
\hline $\mathrm{Al}_{2} \mathrm{O}_{3}$ & 23.97 & 14.43 & 15.58 & 16.38 & 17.44 & 18.11 & 18.70 & 18.98 & 19.90 & 20.20 & 20.42 & 20.79 & 21.13 & 20.95 & 21.34 & 21.50 \\
\hline $\mathrm{B}_{2} \mathrm{O}_{3} *$ & 15.19 & 15.83 & 15.76 & 15.69 & 15.63 & 15.58 & 15.52 & 15.48 & 15.44 & 15.42 & 15.40 & 15.37 & 15.35 & 15.33 & 15.31 & 15.30 \\
\hline $\mathrm{BaO}$ & 0.05 & 0.12 & 0.19 & 0.22 & 0.24 & 0.28 & 0.27 & 0.24 & 0.30 & 0.30 & 0.31 & 0.30 & 0.30 & 0.32 & 0.36 & 0.36 \\
\hline $\mathrm{Bi}_{2} \mathrm{O}_{3}$ & 4 & 2.19 & 1.97 & 1.92 & 1.80 & 1.76 & 1.65 & 1.66 & 1.52 & 1.46 & 1.41 & 1.37 & 1.37 & 1.41 & 1.40 & 1.38 \\
\hline $\mathrm{CaO}$ & .08 & 2.71 & 2.98 & 3.35 & 3.75 & 4.03 & 4.24 & 4.39 & 4.65 & 4.64 & 4.67 & 4.79 & 5.02 & 5.25 & 5.30 & 5.32 \\
\hline $\mathrm{CdO}$ & 0.02 & $<0.01$ & $<0.01$ & $<0.01$ & $<0.01$ & $<0.01$ & $<0.01$ & $<0.01$ & $<0.01$ & $<0.01$ & $<0.01$ & $<0.01$ & $<0.01$ & $<0.01$ & $<0.01$ & $<0.01$ \\
\hline $\mathrm{Cr}_{2} \mathrm{O}_{3}$ & 0.52 & 0.90 & 0.83 & 0.79 & 0.74 & 0.72 & 0.68 & 0.66 & 0.63 & 0.59 & 0.58 & 0.56 & 0.57 & 0.56 & 0.55 & 0.55 \\
\hline$F$ & 0.67 & $0.30 \#$ & 0.32 & 0.33 & 0.35 & $0.37 \#$ & 0.39 & 0.41 & 0.43 & $0.44 \#$ & 0.43 & 0.43 & 0.42 & $0.41 \#$ & 0.42 & 0.43 \\
\hline $\mathrm{Fe}_{2} \mathrm{O}_{3}$ & 5.90 & 5.78 & 5.62 & 5.71 & 5.73 & 5.88 & 5.82 & 5.94 & 5.81 & 5.67 & 5.60 & 5.54 & 5.77 & 5.97 & 5.97 & 5.91 \\
\hline $\mathrm{K}_{2} \mathrm{O}$ & 0.14 & 3.36 & 2.94 & 2.67 & 2.25 & 2.03 & 1.79 & 1.69 & 1.41 & 1.29 & 1.28 & 1.12 & 0.99 & 0.96 & 0.91 & 0.82 \\
\hline $\mathrm{Li}_{2} \mathrm{O}^{*}$ & 3.57 & 3.52 & 3.52 & 3.53 & 3.53 & 3.54 & 3.54 & 3.55 & 3.55 & 3.55 & 3.55 & 3.55 & 3.56 & 3.56 & 3.56 & 3.56 \\
\hline $\mathrm{MgO}$ & 0.12 & 0.30 & 0.31 & 0.30 & 0.33 & 0.33 & 0.33 & 0.33 & 0.35 & 0.35 & 0.35 & 0.36 & 0.38 & 0.35 & 0.34 & 0.38 \\
\hline $\mathrm{MnO}$ & $\S$ & 0.12 & 0.11 & 0.10 & 0.09 & 0.08 & 0.07 & 0.08 & 0.06 & 0.06 & 0.06 & 0.05 & 0.05 & 0.05 & 0.06 & 0.06 \\
\hline $\mathrm{Na}_{2} \mathrm{O}$ & 9.58 & 8.53 & 8.59 & 8.51 & 9.20 & 8.81 & 9.27 & 9.36 & 9.18 & 9.64 & 9.22 & 9.76 & 9.32 & 9.64 & 9.23 & 9.44 \\
\hline $\mathrm{Nd}_{2} \mathrm{O}_{3}$ & $\S$ & 0.01 & 0.01 & 0.01 & 0.01 & 0.01 & 0.01 & $<0.01$ & 0.01 & 0.01 & 0.01 & 0.01 & 0.01 & $<0.01$ & 0.01 & 0.01 \\
\hline $\mathrm{NiO}$ & 40 & 0.55 & 0.51 & 0.48 & 0.47 & 0.47 & 0.43 & 0.42 & 0.41 & 0.38 & 0.37 & 0.36 & 0.35 & 0.37 & 0.35 & 0.33 \\
\hline $\mathrm{P}_{2} \mathrm{O}_{5}$ & 1.05 & 1.32 & 1.31 & 1.30 & 1.25 & 1.23 & 1.24 & 1.21 & 1.18 & 1.17 & 1.16 & 1.16 & 1.17 & 1.17 & 1.19 & 1.15 \\
\hline $\mathrm{PbO}$ & 0.41 & 0.24 & 0.25 & 0.28 & 0.29 & 0.30 & 0.31 & 0.32 & 0.33 & 0.32 & 0.31 & 0.31 & 0.33 & 0.35 & 0.36 & 0.36 \\
\hline $\mathrm{SiO}_{2}$ & 30.50 & 39.15 & 38.57 & 37.74 & 36.23 & 35.76 & 35.06 & 34.60 & 34.15 & 33.83 & 34.17 & 33.47 & 33.19 & 32.62 & 32.62 & 32.39 \\
\hline $\mathrm{SO}_{3}$ & 0.20 & 0.22 & 0.20 & 0.20 & 0.18 & 0.20 & 0.16 & 0.16 & 0.16 & 0.15 & 0.15 & 0.16 & 0.16 & 0.15 & 0.16 & 0.15 \\
\hline $\mathrm{TiO}_{2}$ & 0.01 & 0.04 & 0.05 & 0.04 & 0.04 & 0.05 & 0.04 & 0.04 & 0.04 & 0.04 & 0.05 & 0.04 & 0.05 & 0.03 & 0.04 & 0.04 \\
\hline $\mathrm{ZnO}$ & 0.08 & 0.12 & 0.12 & 0.12 & 0.11 & 0.11 & 0.10 & 0.10 & 0.10 & 0.09 & 0.09 & 0.08 & 0.09 & 0.09 & 0.09 & 0.09 \\
\hline $\mathrm{ZrO}_{2}$ & 0.39 & 0.26 & 0.27 & 0.31 & 0.33 & 0.36 & 0.37 & 0.38 & 0.40 & 0.39 & 0.39 & 0.39 & 0.42 & 0.44 & 0.46 & 0.46 \\
\hline Sum & 100.00 & 100.00 & 100.00 & 100.00 & 100.00 & 100.00 & 100.00 & 100.00 & 100.00 & 100.00 & 100.00 & 100.00 & 100.00 & 100.00 & 100.00 & 100.00 \\
\hline
\end{tabular}

$\S$ - Not a target constituent

* - Target values calculated using simple well-stirred tank model

\# - Fluorine was measured by XRF on polished samples, values for other samples calculated by interpolation 
Table 6.9. XRF-Analyzed Compositions of Discharged Glass Samples from the DM100 Tests with the Aluminum-Limited Composition (wt\%) (continued).

\begin{tabular}{|c|c|c|c|c|c|c|c|c|c|c|c|c|c|c|}
\hline \multirow{2}{*}{\multicolumn{2}{|c|}{$\frac{\text { Test }}{\text { Glass (kg) }}$}} & \multicolumn{5}{|c|}{ 6A } & \multicolumn{6}{|c|}{ 6B } & \multicolumn{2}{|c|}{$6 C$} \\
\hline & & 370.69 & 391.29 & 413.33 & 432.17 & 442.03 & 463.13 & 478.02 & 504.28 & 515.78 & 528.70 & 534.88 & 558.18 & 570.16 \\
\hline Constituent & Target & $\begin{array}{c}\text { BLM-G- } \\
62 \mathrm{~A}\end{array}$ & $\begin{array}{c}\text { BLM-G- } \\
65 \mathrm{~A}\end{array}$ & $\begin{array}{c}\text { BLM-G- } \\
71 \mathrm{~A}\end{array}$ & $\begin{array}{c}\text { BLM-G- } \\
73 \mathrm{~A}\end{array}$ & $\begin{array}{c}\text { BLM-G- } \\
73 B\end{array}$ & $\begin{array}{c}\text { BLM-G- } \\
80 \mathrm{~A}\end{array}$ & $\begin{array}{c}\text { BLM-G- } \\
85 \mathrm{~A}\end{array}$ & $\begin{array}{c}\text { BLM-G- } \\
90 \mathrm{~A}\end{array}$ & $\begin{array}{c}\text { BLM-G- } \\
100 \mathrm{~A}\end{array}$ & $\begin{array}{c}\text { BLM-G- } \\
110 A-2\end{array}$ & $\begin{array}{c}\text { BLM-G- } \\
111 \mathrm{~A}\end{array}$ & $\begin{array}{c}\text { BLM-G- } \\
123 B\end{array}$ & $\begin{array}{c}\text { BLM-G- } \\
124 \mathrm{~A}\end{array}$ \\
\hline $\mathrm{Al}_{2} \mathrm{O}_{3}$ & 23.97 & 21.46 & 21.95 & 21.71 & 21.92 & 21.98 & 21.96 & 21.93 & 22.16 & 22.27 & 22.54 & 22.46 & 22.56 & 22.37 \\
\hline $\mathrm{B}_{2} \mathrm{O}_{3} *$ & 15.19 & 15.28 & 15.27 & 15.26 & 15.26 & 15.25 & 15.25 & 15.24 & 15.23 & 15.23 & 15.23 & 15.23 & 15.22 & 15.22 \\
\hline $\mathrm{BaO}$ & 0.05 & 0.35 & 0.35 & 0.35 & 0.32 & 0.29 & 0.41 & 0.33 & 0.39 & 0.33 & 0.29 & 0.32 & 0.30 & 0.30 \\
\hline $\mathrm{Bi}_{2} \mathrm{O}_{3}$ & 1.14 & 1.43 & 1.25 & 1.38 & 1.31 & 1.30 & 1.35 & 1.26 & 1.25 & 1.24 & 1.19 & 1.25 & 1.18 & 1.24 \\
\hline $\mathrm{CaO}$ & 6.08 & 5.54 & 5.31 & 5.54 & 5.53 & 5.59 & 5.71 & 5.52 & 5.46 & 5.48 & 5.44 & 5.62 & 5.31 & 5.57 \\
\hline $\mathrm{CdO}$ & 0.02 & $<0.01$ & $<0.01$ & $<0.01$ & $<0.01$ & $<0.01$ & $<0.01$ & $<0.01$ & $<0.01$ & $<0.01$ & $<0.01$ & $<0.01$ & $<0.01$ & $<0.01$ \\
\hline $\mathrm{Cr}_{2} \mathrm{O}_{3}$ & 0.52 & 0.58 & 0.53 & 0.53 & 0.54 & 0.52 & 0.54 & 0.53 & 0.48 & 0.50 & 0.49 & 0.48 & 0.48 & 0.49 \\
\hline $\mathrm{F}$ & 0.67 & 0.43 & $0.44 \#$ & 0.44 & 0.43 & 0.43 & $0.43 \#$ & 0.42 & 0.40 & 0.40 & $0.39 \#$ & 0.39 & 0.39 & 0.39 \\
\hline $\mathrm{Fe}_{2} \mathrm{O}_{3}$ & 5.90 & 6.26 & 5.78 & 6.09 & 6.05 & 5.97 & 6.17 & 5.92 & 5.75 & 5.75 & 5.58 & 5.80 & 5.49 & 5.80 \\
\hline $\mathrm{K}_{2} \mathrm{O}$ & 0.14 & 0.83 & 0.72 & 0.72 & 0.67 & 0.63 & 0.62 & 0.58 & 0.59 & 0.56 & 0.54 & 0.54 & 0.52 & 0.53 \\
\hline $\mathrm{Li}_{2} \mathrm{O}^{*}$ & 3.57 & 3.56 & 3.56 & 3.56 & 3.56 & 3.57 & 3.57 & 3.57 & 3.57 & 3.57 & 3.57 & 3.57 & 3.57 & 3.57 \\
\hline $\mathrm{MgO}$ & 0.12 & 0.36 & 0.38 & 0.38 & 0.40 & 0.36 & 0.37 & 0.42 & 0.40 & 0.36 & 0.43 & 0.43 & 0.44 & 0.40 \\
\hline $\mathrm{MnO}$ & $\S$ & 0.05 & 0.05 & 0.06 & 0.05 & 0.05 & 0.04 & 0.04 & 0.04 & 0.04 & 0.04 & 0.04 & 0.05 & 0.05 \\
\hline $\mathrm{Na}_{2} \mathrm{O}$ & 9.58 & 8.69 & 9.34 & 9.37 & 9.42 & 9.58 & 9.00 & 10.15 & 9.60 & 9.20 & 9.29 & 9.21 & 10.21 & 9.68 \\
\hline $\mathrm{Nd}_{2} \mathrm{O}_{3}$ & $\S$ & 0.01 & 0.01 & $<0.01$ & $<0.01$ & $<0.01$ & 0.01 & $<0.01$ & 0.01 & 0.01 & 0.01 & 0.01 & 0.01 & $<0.01$ \\
\hline $\mathrm{NiO}$ & 0.40 & 0.36 & 0.34 & 0.33 & 0.35 & 0.34 & 0.35 & 0.33 & 0.32 & 0.31 & 0.30 & 0.31 & 0.30 & 0.31 \\
\hline $\mathrm{P}_{2} \mathrm{O}_{5}$ & 1.05 & 1.18 & 1.16 & 1.16 & 1.15 & 1.15 & 1.15 & 1.13 & 1.16 & 1.15 & 1.15 & 1.14 & 1.13 & 1.15 \\
\hline $\mathrm{PbO}$ & 0.41 & 0.38 & 0.34 & 0.37 & 0.35 & 0.35 & 0.38 & 0.35 & 0.35 & 0.35 & 0.35 & 0.36 & 0.34 & 0.34 \\
\hline $\mathrm{SiO}_{2}$ & 30.50 & 32.46 & 32.50 & 31.99 & 31.95 & 31.93 & 31.91 & 31.54 & 32.11 & 32.53 & 32.48 & 32.09 & 31.83 & 31.88 \\
\hline $\mathrm{SO}_{3}$ & 0.20 & 0.15 & 0.15 & 0.13 & 0.16 & 0.16 & 0.14 & 0.15 & 0.15 & 0.15 & 0.15 & 0.13 & 0.12 & 0.12 \\
\hline $\mathrm{TiO}_{2}$ & 0.01 & 0.04 & 0.05 & 0.04 & 0.04 & 0.04 & 0.05 & 0.03 & 0.04 & 0.05 & 0.05 & 0.05 & 0.04 & 0.04 \\
\hline $\mathrm{ZnO}$ & 0.08 & 0.10 & 0.09 & 0.10 & 0.09 & 0.09 & 0.09 & 0.09 & 0.08 & 0.09 & 0.08 & 0.10 & 0.09 & 0.09 \\
\hline $\mathrm{ZrO}_{2}$ & 0.39 & 0.49 & 0.44 & 0.50 & 0.46 & 0.45 & 0.50 & 0.47 & 0.47 & 0.45 & 0.44 & 0.47 & 0.44 & 0.45 \\
\hline Sum & 100.00 & 100.00 & 100.00 & 100.00 & 100.00 & 100.00 & 100.00 & 100.00 & 100.00 & 100.00 & 100.00 & 100.00 & 100.00 & 100.00 \\
\hline
\end{tabular}

* - Target values calculated using simple well-stirred tank model

\# - Fluorine was measured by XRF on polished samples, values for other samples calculated by interpolation 
Table 6.9. XRF-Analyzed Compositions of Discharged Glass Samples from the DM100 Tests with the Aluminum-Limited Composition (wt\%) (continued).

\begin{tabular}{|c|c|c|c|c|c|c|c|c|c|c|c|c|c|c|}
\hline \multirow{2}{*}{\multicolumn{2}{|c|}{$\begin{array}{c}\text { Test } \\
\text { Glass (kg) }\end{array}$}} & \multicolumn{11}{|c|}{ 6C } & \multirow{2}{*}{\multicolumn{2}{|c|}{$\begin{array}{c}\text { Steady State } \\
558-794\end{array}$}} \\
\hline & & 587.48 & 616.06 & 634.44 & 654.12 & 672.6 & 698.92 & 718.48 & 736.16 & 755.68 & 782.76 & 793.38 & & \\
\hline Constituent & Target & $\begin{array}{c}\text { BLM-G- } \\
127 \mathrm{~A}\end{array}$ & $\begin{array}{c}\text { BLM-G- } \\
131 \mathrm{~A}\end{array}$ & $\begin{array}{c}\text { BLM-G- } \\
133 B\end{array}$ & \begin{tabular}{|c|} 
BLM-G- \\
134B
\end{tabular} & $\begin{array}{c}\text { BLM-G- } \\
138 \mathrm{~A}\end{array}$ & $\begin{array}{c}\text { BLM-G- } \\
139 A\end{array}$ & $\begin{array}{c}\text { BLM-G- } \\
144 \mathrm{~A}\end{array}$ & $\begin{array}{c}\text { BLM-G- } \\
145 \mathrm{~A}\end{array}$ & $\begin{array}{c}\text { BLM-G- } \\
146 B\end{array}$ & $\begin{array}{c}\text { BLM-G- } \\
152 B\end{array}$ & $\begin{array}{c}\text { BLM-G- } \\
\text { 152D }\end{array}$ & Avg. & \%Dev. \\
\hline $\mathrm{Al}_{2} \mathrm{O}_{3}$ & 23.97 & 22.23 & 22.28 & 22.08 & 22.68 & 22.53 & 22.40 & 22.37 & 22.29 & 22.43 & 22.44 & 22.14 & 22.37 & -6.68 \\
\hline $\mathrm{B}_{2} \mathrm{O}_{3} *$ & 15.19 & 15.22 & 15.21 & 15.21 & 15.21 & 15.21 & 15.21 & 15.20 & 15.20 & 15.20 & 15.20 & 15.20 & 15.21 & $\mathrm{NC}$ \\
\hline $\mathrm{BaO}$ & 0.05 & 0.28 & 0.31 & 0.32 & 0.25 & 0.30 & 0.36 & 0.36 & 0.32 & 0.27 & 0.33 & 0.35 & 0.31 & $\mathrm{NC}$ \\
\hline $\mathrm{Bi}_{2} \mathrm{O}_{3}$ & 1.14 & 1.32 & 1.30 & 1.32 & 1.17 & 1.25 & 1.22 & 1.23 & 1.30 & 1.21 & 1.21 & 1.18 & 1.24 & 8.18 \\
\hline $\mathrm{CaO}$ & 6.08 & 5.82 & 5.80 & 5.90 & 5.62 & 5.76 & 5.74 & 5.80 & 5.87 & 5.86 & 5.61 & 5.43 & 5.70 & -6.21 \\
\hline $\mathrm{CdO}$ & 0.02 & $<0.01$ & $<0.01$ & $<0.01$ & $<0.01$ & $<0.01$ & $<0.01$ & $<0.01$ & $<0.01$ & $<0.01$ & $<0.01$ & $<0.01$ & $<0.01$ & $\mathrm{NC}$ \\
\hline $\mathrm{Cr}_{2} \mathrm{O}_{3}$ & 0.52 & 0.53 & 0.52 & 0.53 & 0.49 & 0.51 & 0.51 & 0.52 & 0.52 & 0.52 & 0.49 & 0.45 & 0.50 & $\mathrm{NC}$ \\
\hline$F$ & 0.67 & 0.40 & $0.40 \#$ & 0.40 & 0.40 & 0.40 & $0.40 \#$ & 0.41 & 0.41 & 0.42 & $0.43 \#$ & 0.43 & 0.41 & NC \\
\hline $\mathrm{Fe}_{2} \mathrm{O}_{3}$ & 5.90 & 6.19 & 6.13 & 6.22 & 5.72 & 5.95 & 5.94 & 5.97 & 6.16 & 6.00 & 5.79 & 5.54 & 5.92 & 0.28 \\
\hline $\mathrm{K}_{2} \mathrm{O}$ & 0.14 & 0.51 & 0.47 & 0.48 & 0.46 & 0.44 & 0.43 & 0.42 & 0.42 & 0.42 & 0.45 & 0.49 & 0.46 & NC \\
\hline $\mathrm{Li}_{2} \mathrm{O}^{*}$ & 3.57 & 3.57 & 3.57 & 3.57 & 3.57 & 3.57 & 3.57 & 3.57 & 3.57 & 3.57 & 3.57 & 3.57 & 3.57 & NC \\
\hline $\mathrm{MgO}$ & 0.12 & 0.38 & 0.38 & 0.42 & 0.40 & 0.41 & 0.36 & 0.35 & 0.43 & 0.38 & 0.39 & 0.41 & 0.40 & $\mathrm{NC}$ \\
\hline $\mathrm{MnO}$ & $\S$ & 0.05 & 0.04 & 0.04 & 0.04 & 0.04 & 0.03 & 0.03 & 0.03 & 0.04 & 0.04 & 0.05 & 0.04 & $\mathrm{NC}$ \\
\hline $\mathrm{Na}_{2} \mathrm{O}$ & 9.58 & 9.24 & 9.29 & 9.44 & 9.73 & 9.47 & 9.69 & 9.85 & 9.80 & 9.83 & 9.98 & 10.19 & 9.72 & 1.48 \\
\hline $\mathrm{Nd}_{2} \mathrm{O}_{3}$ & $\S$ & 0.01 & 0.01 & 0.01 & 0.01 & 0.01 & 0.01 & 0.01 & 0.01 & 0.01 & 0.01 & 0.01 & 0.01 & $\mathrm{NC}$ \\
\hline $\mathrm{NiO}$ & 0.40 & 0.35 & 0.34 & 0.34 & 0.31 & 0.33 & 0.32 & 0.32 & 0.33 & 0.32 & 0.30 & 0.28 & 0.32 & $\mathrm{NC}$ \\
\hline $\mathrm{P}_{2} \mathrm{O}_{5}$ & 1.05 & 1.16 & 1.14 & 1.12 & 1.14 & 1.14 & 1.12 & 1.10 & 1.09 & 1.12 & 1.13 & 1.16 & 1.13 & 7.34 \\
\hline $\mathrm{PbO}$ & 0.41 & 0.38 & 0.37 & 0.37 & 0.33 & 0.36 & 0.37 & 0.36 & 0.37 & 0.35 & 0.35 & 0.33 & 0.35 & NC \\
\hline $\mathrm{SiO}_{2}$ & 30.50 & 31.64 & 31.60 & 31.48 & 31.81 & 31.59 & 31.56 & 31.36 & 31.09 & 31.34 & 31.54 & 32.05 & 31.60 & 3.62 \\
\hline $\mathrm{SO}_{3}$ & 0.20 & 0.13 & 0.13 & 0.12 & 0.13 & 0.14 & 0.15 & 0.15 & 0.15 & 0.15 & 0.16 & 0.17 & 0.14 & NC \\
\hline $\mathrm{TiO}_{2}$ & 0.01 & 0.04 & 0.13 & 0.04 & 0.05 & 0.05 & 0.05 & 0.05 & 0.05 & 0.04 & 0.05 & 0.03 & 0.05 & $\mathrm{NC}$ \\
\hline $\mathrm{ZnO}$ & 0.08 & 0.09 & 0.09 & 0.09 & 0.08 & 0.08 & 0.09 & 0.08 & 0.09 & 0.08 & 0.09 & 0.09 & 0.09 & NC \\
\hline $\mathrm{ZrO}_{2}$ & 0.39 & 0.47 & 0.48 & 0.49 & 0.43 & 0.47 & 0.48 & 0.48 & 0.49 & 0.45 & 0.47 & 0.48 & 0.47 & $\mathrm{NC}$ \\
\hline Sum & 100.00 & 100.00 & 100.00 & 100.00 & 100.00 & 100.00 & 100.00 & 100.00 & 100.00 & 100.00 & 100.00 & 100.00 & 100.00 & NC \\
\hline
\end{tabular}

$\S$ - Not a target constituent

* - Target values calculated using simple well-stirred tank model

\# - Fluorine was measured by XRF on polished samples, values for other samples calculated by interpolation 
Table 6.10. XRF-Analyzed Compositions of Discharged Glass Samples from the DM100 Tests with the Aluminum-Plus-Sodium-Limited Composition (wt\%).

\begin{tabular}{|c|c|c|c|c|c|c|c|c|c|c|c|c|c|c|c|c|}
\hline \multirow{2}{*}{\multicolumn{2}{|c|}{$\frac{\text { Test }}{\text { Slass (kg) }}$}} & \multicolumn{11}{|c|}{ 7A } & \multicolumn{4}{|c|}{ 7B } \\
\hline & & 19.46 & 41.16 & 67.52 & 95.52 & 118.54 & 143.78 & 166.88 & 194.74 & 219.16 & 245.62 & 259.48 & 281.36 & 303.00 & 325.18 & 343.12 \\
\hline Constituent & Target & $\begin{array}{c}\text { BLN-G- } \\
17 \mathrm{~A}\end{array}$ & $\begin{array}{c}\text { BLN-G- } \\
21 \mathrm{~A}\end{array}$ & $\begin{array}{c}\text { BLN-G- } \\
22 \mathrm{~B}\end{array}$ & $\begin{array}{c}\text { BLN-G- } \\
24 \mathrm{~A}\end{array}$ & $\begin{array}{c}\text { BLN-G- } \\
24 \mathrm{C}\end{array}$ & $\begin{array}{c}\text { BLN-G- } \\
27 \mathrm{~B}\end{array}$ & $\begin{array}{c}\text { BLN-G- } \\
\text { 32A }\end{array}$ & $\begin{array}{c}\text { BLN-G- } \\
32 \mathrm{C}\end{array}$ & $\begin{array}{c}\text { BLN-G- } \\
35 \mathrm{~A}\end{array}$ & $\begin{array}{c}\text { BLN-G- } \\
36 \mathrm{~A}\end{array}$ & $\begin{array}{c}\text { BLN-G- } \\
40 \mathrm{~A}\end{array}$ & $\begin{array}{c}\text { BLN-G- } \\
41 \mathrm{~A}\end{array}$ & $\begin{array}{c}\text { BLN-G- } \\
43 \mathrm{~A}\end{array}$ & $\begin{array}{c}\text { BLN-G- } \\
46 \mathrm{~A}\end{array}$ & $\begin{array}{c}\text { BLN-G- } \\
52 \mathrm{~A}\end{array}$ \\
\hline $\mathrm{Al}_{2} \mathrm{O}_{3}$ & 21.34 & 22.37 & 21.98 & 21.87 & 21.71 & 21.66 & 21.43 & 21.42 & 21.19 & 21.24 & 21.13 & 21.34 & 21.27 & 21.11 & 21.05 & 21.11 \\
\hline $\mathrm{B}_{2} \mathrm{O}_{3} *$ & 18.37 & 15.52 & 15.85 & 16.19 & 16.50 & 16.73 & 16.94 & 17.11 & 17.29 & 17.43 & 17.56 & 17.62 & 17.70 & 17.78 & 17.85 & 17.90 \\
\hline $\mathrm{BaO}$ & 0.03 & 0.22 & 0.22 & 0.17 & 0.14 & 0.16 & 0.11 & 0.12 & 0.11 & 0.12 & 0.11 & 0.09 & 0.10 & 0.09 & 0.08 & 0.08 \\
\hline $\mathrm{Bi}_{2} \mathrm{O}_{3}$ & 1.16 & 1.14 & 1.23 & 1.15 & 1.19 & 1.16 & 1.15 & 1.15 & 1.20 & 1.17 & 1.21 & 1.17 & 1.20 & 1.15 & 1.25 & 1.14 \\
\hline $\mathrm{CaO}$ & 0.72 & 3.95 & 3.61 & 3.25 & 2.81 & 2.61 & 2.31 & 2.07 & 2.00 & 1.90 & 1.70 & 1.64 & 1.59 & 1.49 & 1.46 & 1.34 \\
\hline $\mathrm{CdO}$ & 0.01 & $<0.01$ & $<0.01$ & $<0.01$ & $<0.01$ & $<0.01$ & $<0.01$ & $<0.01$ & $<0.01$ & $<0.01$ & $<0.01$ & $<0.01$ & $<0.01$ & $<0.01$ & $<0.01$ & $<0.01$ \\
\hline $\mathrm{Cr}_{2} \mathrm{O}_{3}$ & 0.71 & 0.57 & 0.64 & 0.64 & 0.70 & 0.70 & 0.74 & 0.74 & 0.78 & 0.76 & 0.78 & 0.78 & 0.80 & 0.77 & 0.83 & 0.79 \\
\hline $\mathrm{F}$ & 0.23 & $0.28 \#$ & 0.27 & 0.25 & 0.24 & 0.22 & $0.21 \#$ & 0.21 & 0.21 & 0.21 & 0.21 & 0.21 & $0.21 \#$ & 0.21 & 0.21 & 0.21 \\
\hline $\mathrm{Fe}_{2} \mathrm{O}_{3}$ & 2.82 & 4.67 & 4.64 & 4.28 & 4.13 & 4.03 & 3.79 & 3.66 & 3.74 & 3.59 & 3.52 & 3.41 & 3.46 & 3.30 & 3.48 & 3.24 \\
\hline $\mathrm{K}_{2} \mathrm{O}$ & 0.66 & 0.51 & 0.55 & 0.55 & 0.58 & 0.60 & 0.60 & 0.62 & 0.64 & 0.63 & 0.66 & 0.64 & 0.66 & 0.65 & 0.68 & 0.67 \\
\hline $\mathrm{Li}_{2} \mathrm{O}^{*}$ & 3.58 & 3.57 & 3.57 & 3.57 & 3.57 & 3.57 & 3.57 & 3.57 & 3.57 & 3.57 & 3.57 & 3.57 & 3.57 & 3.57 & 3.57 & 3.57 \\
\hline $\mathrm{MgO}$ & 0.22 & 0.37 & 0.39 & 0.34 & 0.35 & 0.33 & 0.36 & 0.32 & 0.30 & 0.29 & 0.32 & 0.31 & 0.27 & 0.28 & 0.27 & 0.32 \\
\hline $\mathrm{MnO}$ & $\S$ & 0.04 & 0.04 & 0.04 & 0.04 & 0.04 & 0.03 & 0.03 & 0.04 & 0.03 & 0.03 & 0.04 & 0.03 & 0.03 & 0.03 & 0.04 \\
\hline $\mathrm{Na}_{2} \mathrm{O}$ & 12.71 & 11.10 & 10.86 & 11.84 & 11.51 & 11.67 & 12.07 & 12.31 & 12.36 & 12.34 & 12.05 & 12.22 & 11.98 & 12.66 & 12.11 & 12.26 \\
\hline $\mathrm{Nd}_{2} \mathrm{O}_{3}$ & $\S$ & 0.01 & 0.01 & 0.01 & 0.01 & $<0.01$ & 0.01 & 0.01 & 0.01 & 0.01 & 0.01 & 0.01 & 0.01 & 0.01 & 0.01 & 0.01 \\
\hline $\mathrm{NiO}$ & 0.10 & 0.26 & 0.24 & 0.22 & 0.23 & 0.22 & 0.21 & 0.20 & 0.20 & 0.20 & 0.19 & 0.18 & 0.19 & 0.18 & 0.18 & 0.17 \\
\hline $\mathrm{P}_{2} \mathrm{O}_{5}$ & 2.02 & 1.51 & 1.63 & 1.59 & 1.76 & 1.76 & 1.86 & 1.85 & 1.89 & 1.87 & 1.92 & 1.93 & 1.96 & 1.97 & 1.98 & 2.00 \\
\hline $\mathrm{PbO}$ & 0.09 & 0.26 & 0.24 & 0.22 & 0.19 & 0.18 & 0.17 & 0.15 & 0.16 & 0.15 & 0.14 & 0.13 & 0.13 & 0.13 & 0.13 & 0.11 \\
\hline $\mathrm{SiO}_{2}$ & 34.56 & 32.99 & 33.33 & 33.13 & 33.65 & 33.69 & 33.76 & 33.76 & 33.61 & 33.77 & 34.18 & 34.02 & 34.17 & 33.93 & 34.10 & 34.34 \\
\hline $\mathrm{SO}_{3}$ & 0.22 & 0.11 & 0.13 & 0.13 & 0.14 & 0.14 & 0.15 & 0.15 & 0.15 & 0.14 & 0.16 & 0.16 & 0.15 & 0.16 & 0.17 & 0.18 \\
\hline $\mathrm{TiO}_{2}$ & 0.17 & 0.09 & 0.11 & 0.12 & 0.13 & 0.13 & 0.14 & 0.14 & 0.15 & 0.15 & 0.16 & 0.16 & 0.17 & 0.16 & 0.17 & 0.17 \\
\hline $\mathrm{ZnO}$ & 0.18 & 0.11 & 0.13 & 0.12 & 0.14 & 0.14 & 0.14 & 0.15 & 0.16 & 0.15 & 0.16 & 0.15 & 0.16 & 0.16 & 0.17 & 0.16 \\
\hline $\mathrm{ZrO}_{2}$ & 0.12 & 0.35 & 0.35 & 0.31 & 0.28 & 0.27 & 0.25 & 0.24 & 0.24 & 0.24 & 0.23 & 0.21 & 0.22 & 0.21 & 0.22 & 0.19 \\
\hline Sum & 100.00 & 100.00 & 100.00 & 100.00 & 100.00 & 100.00 & 100.00 & 100.00 & 100.00 & 100.00 & 100.00 & 100.00 & 100.00 & 100.00 & 100.00 & 100.00 \\
\hline
\end{tabular}

$\S$ - Not a target constituent

* - Target values calculated using simple well-stirred tank model

\# - Fluorine was measured by XRF on polished samples, values for other samples calculated by interpolation 


\section{Table 6.10. XRF-Analyzed Compositions of Discharged Glass Samples from the DM100 Tests with the Aluminum-Plus-Sodium-Limited Composition (wt\%) (continued).}

\begin{tabular}{|c|c|c|c|c|c|c|c|c|c|c|c|c|c|c|c|c|}
\hline \multirow{2}{*}{\multicolumn{2}{|c|}{$\frac{\text { Test }}{\text { lass (kg) }}$}} & \multicolumn{5}{|c|}{ 7B } & \multicolumn{8}{|c|}{ 8A } & \multicolumn{2}{|c|}{ 8B } \\
\hline & & 373.78 & 399.02 & 425.68 & 449.24 & 476.64 & 495.94 & 513.12 & 529.70 & 544.28 & 574.40 & 592.74 & 609.16 & 630.44 & 646.08 & 657.86 \\
\hline Constituent & Target & $\begin{array}{c}\text { BLN-G- } \\
52 \mathrm{C}\end{array}$ & $\begin{array}{c}\text { BLN-G- } \\
55 \mathrm{~B}\end{array}$ & \begin{tabular}{|c|} 
BLN-G- \\
$60 \mathrm{~A}$
\end{tabular} & $\begin{array}{c}\text { BLN-G- } \\
62 \mathrm{~B}\end{array}$ & $\begin{array}{c}\text { BLN-G- } \\
66 \mathrm{~A}\end{array}$ & $\begin{array}{c}\text { BLN-G- } \\
81 \mathrm{~B}\end{array}$ & \begin{tabular}{|c|} 
BLN-G- \\
$82 \mathrm{~B}$
\end{tabular} & $\begin{array}{c}\text { BLN-G- } \\
83 \mathrm{~A}\end{array}$ & $\begin{array}{c}\text { BLN-G- } \\
85 \mathrm{~A}\end{array}$ & \begin{tabular}{|c|} 
BLN-G- \\
$88 \mathrm{~A}$
\end{tabular} & \begin{tabular}{|c|} 
BLN-G- \\
$94 \mathrm{~A}$
\end{tabular} & $\begin{array}{c}\text { BLN-G- } \\
101 \mathrm{~B}\end{array}$ & $\begin{array}{c}\text { BLN-G- } \\
106 \mathrm{~B}\end{array}$ & \begin{tabular}{|c|} 
BLN-G- \\
$119 A$
\end{tabular} & $\begin{array}{c}\text { BLN-G- } \\
122 \mathrm{~A}\end{array}$ \\
\hline $\mathrm{Al}_{2} \mathrm{O}_{3}$ & 21.34 & 21.20 & 21.02 & 20.94 & 21.29 & 20.92 & 20.84 & 20.92 & 20.88 & 20.81 & 20.76 & 21.05 & 20.61 & 20.81 & 20.69 & 20.74 \\
\hline $\mathrm{B}_{2} \mathrm{O}_{3} *$ & 18.37 & 17.97 & 18.02 & 18.07 & 18.11 & 18.14 & 18.17 & 18.18 & 18.20 & 18.21 & 18.24 & 18.25 & 18.26 & 18.27 & 18.28 & 18.28 \\
\hline $\mathrm{BaO}$ & 0.03 & 0.06 & 0.06 & 0.07 & 0.05 & 0.06 & 0.07 & 0.06 & 0.07 & 0.07 & 0.04 & 0.07 & 0.06 & 0.07 & 0.06 & 0.05 \\
\hline $\mathrm{Bi}_{2} \mathrm{O}_{3}$ & 16 & 1.17 & 1.23 & 1.18 & 1.05 & 1.18 & 1.17 & 1.15 & 1.17 & 1.19 & 1.18 & 1.15 & 1.22 & 1.20 & 1.18 & 1.19 \\
\hline $\mathrm{CaO}$ & 72 & 1.30 & 1.27 & 1.22 & 1.10 & 1.14 & 1.35 & 1.32 & 1.33 & 1.32 & 1.30 & 1.19 & 1.20 & 1.16 & 1.12 & 1.12 \\
\hline $\mathrm{CdO}$ & 0.01 & $<0.01$ & $<0.01$ & $<0.01$ & $<0.01$ & $<0.01$ & $<0.01$ & $<0.01$ & $<0.01$ & $<0.01$ & $<0.01$ & $<0.01$ & $<0.01$ & $<0.01$ & $<0.01$ & $<0.01$ \\
\hline $\mathrm{Cr}_{2} \mathrm{O}_{3}$ & 0.71 & 0.79 & 0.83 & 0.79 & 0.74 & 0.79 & 0.78 & 0.77 & 0.79 & 0.79 & 0.80 & 0.76 & 0.80 & 0.77 & 0.79 & 0.79 \\
\hline$F$ & 0.23 & 0.21 & 0.21 & 0.21 & $0.21 \#$ & 0.20 & 0.19 & $0.18 \#$ & 0.18 & 0.19 & 0.20 & $0.20 \#$ & 0.21 & 0.22 & 0.22 & $0.23 \#$ \\
\hline $\mathrm{Fe}_{2} \mathrm{O}_{3}$ & 2.82 & 3.27 & 3.30 & 3.14 & 2.92 & 3.14 & 3.38 & 3.30 & 3.38 & 3.38 & 3.37 & 3.16 & 3.33 & 3.20 & 3.14 & 3.16 \\
\hline $\mathrm{K}_{2} \mathrm{O}$ & 66 & 0.68 & 0.69 & 0.68 & 0.64 & 0.66 & 0.69 & 0.67 & 0.69 & 0.69 & 0.71 & 0.68 & 0.73 & 0.69 & 0.69 & 0.68 \\
\hline $\mathrm{Li}_{2} \mathrm{O}^{*}$ & 58 & 3.57 & 3.57 & 3.57 & 3.57 & 3.57 & 3.57 & 3.57 & 3.57 & 3.57 & 3.57 & 3.57 & 3.58 & 3.58 & 3.58 & 3.58 \\
\hline $\mathrm{MgO}$ & 0.22 & 0.31 & 0.27 & 0.32 & 0.29 & 0.28 & 0.30 & 0.28 & 0.29 & 0.27 & 0.33 & 0.30 & 0.31 & 0.26 & 0.28 & 0.31 \\
\hline $\mathrm{MnO}$ & $\S$ & 0.03 & 0.04 & 0.03 & 0.03 & 0.03 & 0.07 & 0.06 & 0.07 & 0.07 & 0.06 & 0.05 & 0.05 & 0.05 & 0.05 & 0.05 \\
\hline $\mathrm{Na}_{2} \mathrm{O}$ & .71 & 12.07 & 12.31 & 12.40 & 12.52 & 12.79 & 11.94 & 12.47 & 12.27 & 12.19 & 12.16 & 12.42 & 12.24 & 12.74 & 12.64 & 12.71 \\
\hline $\mathrm{Nd}_{2} \mathrm{O}_{3}$ & $\S$ & 0.01 & 0.01 & 0.01 & 0.01 & 0.01 & 0.01 & $<0.01$ & 0.01 & 0.01 & 0.01 & 0.01 & 0.01 & 0.01 & 0.01 & 0.01 \\
\hline $\mathrm{NiO}$ & 10 & 0.17 & 0.18 & 0.17 & 0.15 & 0.16 & 0.21 & 0.21 & 0.21 & 0.21 & 0.20 & 0.18 & 0.19 & 0.17 & 0.16 & 0.17 \\
\hline $\mathrm{P}_{2} \mathrm{O}_{5}$ & 2.02 & 2.02 & 2.05 & 2.08 & 2.03 & 2.05 & 1.98 & 1.96 & 1.98 & 1.98 & 2.00 & 1.99 & 2.02 & 2.03 & 2.00 & 2.00 \\
\hline $\mathrm{PbO}$ & 0.09 & 0.11 & 0.11 & 0.11 & 0.09 & 0.11 & 0.12 & 0.11 & 0.12 & 0.11 & 0.11 & 0.11 & 0.11 & 0.11 & 0.10 & 0.11 \\
\hline $\mathrm{SiO}_{2}$ & 34.56 & 34.38 & 34.12 & 34.35 & 34.54 & 34.07 & 34.43 & 34.08 & 34.09 & 34.22 & 34.24 & 34.13 & 34.35 & 33.95 & 34.28 & 34.11 \\
\hline $\mathrm{SO}_{3}$ & 0.22 & 0.16 & 0.17 & 0.16 & 0.15 & 0.17 & 0.18 & 0.18 & 0.18 & 0.18 & 0.18 & 0.19 & 0.18 & 0.17 & 0.17 & 0.17 \\
\hline $\mathrm{TiO}_{2}$ & 0.17 & 0.17 & 0.18 & 0.16 & 0.17 & 0.16 & 0.17 & 0.17 & 0.16 & 0.16 & 0.17 & 0.17 & 0.17 & 0.16 & 0.17 & 0.17 \\
\hline $\mathrm{ZnO}$ & 0.18 & 0.16 & 0.17 & 0.17 & 0.15 & 0.17 & 0.17 & 0.16 & 0.16 & 0.17 & 0.17 & 0.16 & 0.17 & 0.16 & 0.17 & 0.17 \\
\hline $\mathrm{ZrO}_{2}$ & 0.12 & 0.19 & 0.20 & 0.19 & 0.16 & 0.19 & 0.21 & 0.20 & 0.20 & 0.20 & 0.19 & 0.20 & 0.20 & 0.20 & 0.20 & 0.20 \\
\hline Sum & 100.00 & 100.00 & 100.00 & 100.00 & 100.00 & 100.00 & 100.00 & 100.00 & 100.00 & 100.00 & 100.00 & 100.00 & 100.00 & 100.00 & 100.00 & 100.00 \\
\hline
\end{tabular}

$\S$ - Not a target constituent

* - Target values calculated using simple well-stirred tank model

\# - Fluorine was measured by XRF on polished samples, values for other samples calculated by interpolation 
Table 6.10. XRF-Analyzed Compositions of Discharged Glass Samples from the DM100 Tests with the AluminumPlus-Sodium-Limited Composition (wt\%) (continued).

\begin{tabular}{|c|c|c|c|c|c|c|c|c|c|c|c|c|c|c|c|c|c|}
\hline \multirow{2}{*}{\multicolumn{2}{|c|}{$\frac{\text { Test }}{\text { lass (kg) }}$}} & \multirow{2}{*}{\begin{tabular}{|c|}
$\mathbf{8 B}$ \\
677.66 \\
\end{tabular}} & \multicolumn{13}{|c|}{$8 C$} & \multirow{2}{*}{\multicolumn{2}{|c|}{$\begin{array}{c}\text { Steady State } \\
544-934\end{array}$}} \\
\hline & & & 700.28 & 714.28 & 733.94 & 752.46 & \begin{tabular}{|l|}
777.58 \\
\end{tabular} & \begin{tabular}{|l|}
799.20 \\
\end{tabular} & 819.34 & 836.18 & 853.70 & 871.98 & 885.44 & 910.26 & 933.52 & & \\
\hline \multirow{2}{*}{ Constituent } & \multirow[b]{2}{*}{ Target } & BLN-G- & BLN-G- & BLN-G- & BLN-G- & BLN-G- & BLN-G- & BLN-G- & BLO-G- & BLO-G- & BLO-G- & BLO-G- & BLO-G- & BLO-G- & BLO-G- & \multirow[b]{2}{*}{ Avg. } & \multirow{2}{*}{ \%Dev. } \\
\hline & & $129 \mathrm{~A}$ & 147B & $150 \mathrm{~A}$ & $152 \mathrm{~A}$ & 153B & 154B & $155 \mathrm{~A}$ & $5 B$ & $10 \mathrm{~A}$ & $15 \mathrm{~A}$ & $16 \mathrm{~B}$ & $18 \mathrm{~B}$ & $22 B$ & $24 \mathrm{~A}$ & & \\
\hline $\mathrm{Al}_{2} \mathrm{O}_{3}$ & 21.34 & 20.74 & 20.53 & 20.62 & 20.59 & 20.50 & 20.54 & 20.73 & 20.72 & 20.74 & 20.81 & 20.86 & 20.94 & 21.06 & 20.98 & 20.75 & -2.74 \\
\hline $\mathrm{B}_{2} \mathrm{O}_{3} *$ & 18.37 & 18.29 & 18.30 & 18.31 & 18.31 & 18.32 & 18.32 & 18.33 & 18.33 & 18.34 & 18.34 & 18.34 & 18.34 & 18.35 & 18.35 & 18.30 & $\mathrm{NC}$ \\
\hline $\mathrm{BaO}$ & 0.03 & 0.07 & 0.08 & 0.06 & 0.06 & 0.07 & 0.06 & 0.06 & 0.08 & 0.07 & 0.05 & 0.06 & 0.06 & 0.06 & 0.06 & 0.06 & $\mathrm{NC}$ \\
\hline $\mathrm{Bi}_{2} \mathrm{O}_{3}$ & 1.16 & 1.17 & 1.22 & 1.23 & 1.17 & 1.22 & 1.22 & 1.18 & 1.21 & 1.22 & 1.22 & 1.19 & 1.18 & 1.19 & 1.20 & 1.20 & 3.47 \\
\hline $\mathrm{CaO}$ & 0.72 & 1.10 & 1.17 & 1.18 & 1.11 & 1.17 & 1.11 & 1.06 & 1.08 & 1.08 & 1.07 & 1.04 & 1.04 & 1.03 & 1.03 & 1.13 & $\mathrm{NC}$ \\
\hline $\mathrm{CdO}$ & 0.01 & $<0.01$ & $<0.01$ & $<0.01$ & $<0.01$ & $<0.01$ & $<0.01$ & $<0.01$ & $<0.01$ & $<0.01$ & $<0.01$ & $<0.01$ & $<0.01$ & $<0.01$ & $<0.01$ & $<0.01$ & $\mathrm{NC}$ \\
\hline $\mathrm{Cr}_{2} \mathrm{O}_{3}$ & 0.71 & 0.78 & 0.70 & 0.75 & 0.74 & 0.75 & 0.78 & 0.77 & 0.78 & 0.79 & 0.79 & 0.78 & 0.79 & 0.77 & 0.78 & 0.77 & NC \\
\hline $\mathrm{F}$ & 0.23 & 0.18 & 0.13 & $0.10 \#$ & 0.11 & 0.13 & 0.14 & 0.16 & $0.17 \#$ & 0.17 & 0.17 & 0.17 & 0.17 & $0.17 \#$ & 0.17 & 0.17 & $\mathrm{NC}$ \\
\hline $\mathrm{Fe}_{2} \mathrm{O}_{3}$ & 2.82 & 3.12 & 3.35 & 3.43 & 3.22 & 3.36 & 3.30 & 3.18 & 3.23 & 3.23 & 3.26 & 3.17 & 3.15 & 3.14 & 3.15 & 3.24 & 14.97 \\
\hline $\mathrm{K}_{2} \mathrm{O}$ & 0.66 & 0.70 & 0.69 & 0.68 & 0.67 & 0.70 & 0.69 & 0.67 & 0.69 & 0.69 & 0.68 & 0.67 & 0.68 & 0.69 & 0.68 & 0.69 & $\mathrm{NC}$ \\
\hline $\mathrm{Li}_{2} \mathrm{O}^{*}$ & 3.58 & 3.58 & 3.58 & 3.58 & 3.58 & 3.58 & 3.58 & 3.58 & 3.58 & 3.58 & 3.58 & 3.58 & 3.58 & 3.58 & 3.58 & 3.58 & $\mathrm{NC}$ \\
\hline $\mathrm{MgO}$ & 0.22 & 0.26 & 0.28 & 0.31 & 0.26 & 0.26 & 0.27 & 0.28 & 0.21 & 0.24 & 0.25 & 0.22 & 0.20 & 0.25 & 0.22 & 0.26 & $\mathrm{NC}$ \\
\hline $\mathrm{MnO}$ & $\S$ & 0.05 & 0.10 & 0.10 & 0.08 & 0.09 & 0.07 & 0.07 & 0.06 & 0.06 & 0.06 & 0.06 & 0.05 & 0.05 & 0.04 & 0.06 & $\mathrm{NC}$ \\
\hline $\mathrm{Na}_{2} \mathrm{O}$ & 12.71 & 12.82 & 12.78 & 12.27 & 13.27 & 12.39 & 12.49 & 13.03 & 12.42 & 12.40 & 12.57 & 12.70 & 12.59 & 12.35 & 12.48 & 12.56 & -1.20 \\
\hline $\mathrm{Nd}_{2} \mathrm{O}_{3}$ & $\S$ & 0.01 & 0.01 & 0.01 & 0.01 & 0.01 & 0.01 & 0.01 & 0.01 & 0.01 & 0.01 & 0.01 & 0.01 & 0.01 & 0.01 & 0.01 & $\mathrm{NC}$ \\
\hline $\mathrm{NiO}$ & 0.10 & 0.16 & 0.21 & 0.21 & 0.19 & 0.21 & 0.19 & 0.18 & 0.18 & 0.17 & 0.17 & 0.17 & 0.16 & 0.15 & 0.15 & 0.18 & $\mathrm{NC}$ \\
\hline $\mathrm{P}_{2} \mathrm{O}_{5}$ & 2.02 & 1.99 & 2.05 & 2.08 & 2.01 & 2.04 & 2.07 & 2.00 & 2.07 & 2.08 & 2.03 & 2.05 & 2.06 & 2.05 & 2.09 & 2.03 & 0.55 \\
\hline $\mathrm{PbO}$ & 0.09 & 0.10 & 0.11 & 0.11 & 0.11 & 0.11 & 0.11 & 0.10 & 0.10 & 0.10 & 0.11 & 0.10 & 0.10 & 0.10 & 0.10 & 0.11 & $\mathrm{NC}$ \\
\hline $\mathrm{SiO}_{2}$ & 34.56 & 34.17 & 33.98 & 34.24 & 33.75 & 34.32 & 34.28 & 33.89 & 34.32 & 34.27 & 34.07 & 34.11 & 34.15 & 34.26 & 34.17 & 34.16 & -1.18 \\
\hline $\mathrm{SO}_{3}$ & 0.22 & 0.16 & 0.14 & 0.15 & 0.18 & 0.16 & 0.16 & 0.15 & 0.16 & 0.17 & 0.15 & 0.15 & 0.16 & 0.17 & 0.17 & 0.17 & $\mathrm{NC}$ \\
\hline $\mathrm{TiO}_{2}$ & 0.17 & 0.16 & 0.16 & 0.17 & 0.16 & 0.17 & 0.18 & 0.17 & 0.18 & 0.18 & 0.18 & 0.18 & 0.18 & 0.18 & 0.18 & 0.17 & $\mathrm{NC}$ \\
\hline $\mathrm{ZnO}$ & 0.18 & 0.17 & 0.17 & 0.18 & 0.18 & 0.19 & 0.19 & 0.18 & 0.19 & 0.19 & 0.19 & 0.19 & 0.19 & 0.19 & 0.19 & 0.18 & NC \\
\hline $\mathrm{ZrO}_{2}$ & 0.12 & 0.20 & 0.24 & 0.24 & 0.24 & 0.26 & 0.24 & 0.23 & 0.23 & 0.24 & 0.24 & 0.23 & 0.23 & 0.22 & 0.22 & 0.22 & $\mathrm{NC}$ \\
\hline Sum & 100.00 & 100.00 & 100.00 & 100.00 & 100.00 & 100.00 & 100.00 & 100.00 & 100.00 & 100.00 & 100.00 & 100.00 & 100.00 & 100.00 & 100.00 & 100.00 & $\mathrm{NC}$ \\
\hline
\end{tabular}

$\S$ - Not a target constituent

* - Target values calculated using simple well-stirred tank model

\# - Fluorine was measured by XRF on polished samples, values for other samples calculated by interpolation 
Table 6.11. XRF and DCP Analysis of Selected Glass Samples (wt\%).

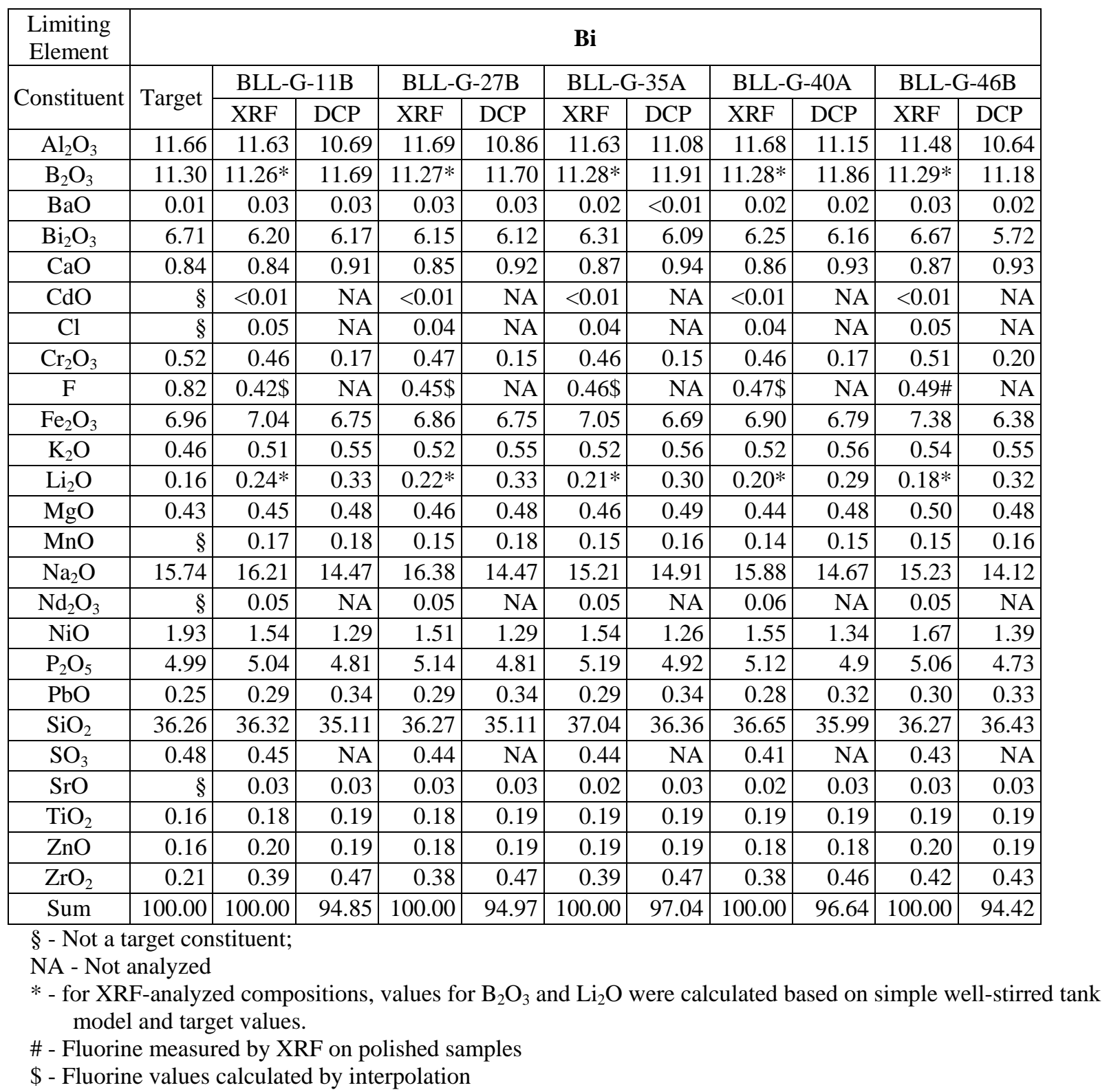


Table 6.11. XRF and DCP Analysis of Selected Glass Samples (wt\%) (continued).

\begin{tabular}{|c|c|c|c|c|c|c|c|c|c|c|c|c|}
\hline $\begin{array}{l}\text { Limiting } \\
\text { Element }\end{array}$ & \multicolumn{9}{|c|}{$\mathrm{Cr}$} & \multicolumn{3}{|c|}{ Al } \\
\hline \multirow{2}{*}{ Constituent } & \multirow{2}{*}{ Target } & \multicolumn{2}{|c|}{ BLL-G-88A } & \multicolumn{2}{|c|}{ BLL-G-107A } & \multicolumn{2}{|c|}{ BLL-G-111B } & \multicolumn{2}{|c|}{ BLL-G-121A } & \multirow{2}{*}{ Target } & \multicolumn{2}{|c|}{ BLM-G-36A } \\
\hline & & XRF & DCP & XRF & DCP & XRF & DCP & XRF & DCP & & XRF & DCP \\
\hline $\mathrm{Al}_{2} \mathrm{O}_{3}$ & 8.98 & 9.43 & 8.38 & 9.36 & 8.22 & 9.72 & 8.33 & 9.37 & 8.06 & 23.97 & 20.95 & 19.94 \\
\hline $\mathrm{B}_{2} \mathrm{O}_{3}$ & 16.17 & $14.95 *$ & 15.51 & 15.73* & 15.92 & 15.84* & 16.15 & 15.93* & 16.43 & 15.19 & 15.33* & 15.74 \\
\hline $\mathrm{BaO}$ & 0.01 & 0.02 & 0.01 & 0.01 & 0.02 & 0.01 & 0.01 & 0.01 & 0.02 & 0.05 & 0.32 & 0.28 \\
\hline $\mathrm{Bi}_{2} \mathrm{O}_{3}$ & .56 & 3.36 & 91 & 2.93 & 55 & 2.69 & .72 & 2.88 & 2.71 & .14 & 1.41 & 1.31 \\
\hline $\mathrm{CaO}$ & 0.87 & 0.84 & 94 & 0.84 & 93 & 0.80 & 0.94 & 0.81 & 0.94 & 3.08 & 5.25 & 4.69 \\
\hline $\mathrm{CdO}$ & $\S$ & $<0.01$ & 0.01 & $<0.01$ & 0.01 & $<0.01$ & 0.01 & $<0.01$ & 0.01 & 0.02 & $<0.01$ & 0.02 \\
\hline $\mathrm{Cl}$ & $\S$ & $<0.01$ & NA & $<0.01$ & NA & $<0.01$ & NA & $<0.01$ & NA & $\S$ & $<0.01$ & NA \\
\hline $\mathrm{Cr}_{2} \mathrm{O}_{3}$ & 1.08 & 1.16 & 0.61 & 1.19 & 0.51 & 1.14 & 0.58 & 1.19 & 0.56 & 0.52 & 0.56 & 0.21 \\
\hline $\mathrm{F}$ & 0.70 & $0.43 \$$ & NA & $0.46 \#$ & NA & $0.48 \$$ & NA & $0.51 \#$ & NA & 0.67 & $0.41 \#$ & NA \\
\hline $\mathrm{Fe}_{2} \mathrm{O}_{3}$ & 4.62 & 5.43 & 5.41 & 5.13 & 5.11 & 4.77 & 5.19 & 5.06 & 5.01 & 5.90 & 5.97 & 5.25 \\
\hline $\mathrm{K} 2 \mathrm{O}$ & 6.05 & 4.94 & 4.20 & 5.41 & 4.54 & 5.36 & 4.71 & 5.51 & 4.69 & 0.14 & 0.96 & 1.03 \\
\hline $\mathrm{Li}_{2} \mathrm{O}$ & 3.68 & $2.80 *$ & 3.09 & 3.37* & 3.33 & $3.44^{*}$ & 3.43 & $3.51^{*}$ & 3.47 & 3.57 & 3.56* & 3.53 \\
\hline $\mathrm{MgO}$ & 0.06 & & & & & & 0.29 & & 0.29 & 0.12 & 0.35 & 0.42 \\
\hline $\mathrm{MnO}$ & 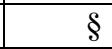 & & & 4 & & & 0.05 & + & .04 & $\S$ & 0.05 & 0.05 \\
\hline $\mathrm{Na}_{2} \mathrm{O}$ & 7.07 & 8.90 & 7.63 & 7.75 & 6.95 & 7.44 & 6.85 & 7.62 & 6.82 & 9.58 & 9.64 & 8.70 \\
\hline $\mathrm{Nd}_{2} \mathrm{O}_{3}$ & $\S$ & 0.01 & NA & 0.01 & NA & 0.01 & NA & 0.01 & NA & $\S$ & $<0.01$ & NA \\
\hline $\mathrm{NiO}$ & 0.37 & 0.74 & 0.72 & 0.58 & 0.57 & 0.50 & 0.53 & 0.54 & 0.52 & 0.40 & 0.37 & 0.27 \\
\hline $\mathrm{P}_{2} \mathrm{O}_{5}$ & 1.18 & 1.9 & 1.8 & 1.57 & 1.52 & 1.51 & 1.46 & 1.46 & 1.46 & 1.05 & 1.17 & 1.01 \\
\hline $\mathrm{PbO}$ & 0.17 & 0.19 & 0.20 & 0.17 & 0.19 & & 0.19 & 0.16 & 0.19 & 0.41 & 0.35 & 0.38 \\
\hline $\mathrm{SiO}_{2}$ & 45.76 & 43.93 & 42.59 & 44.58 & 44.200 & 45.22 & 45.51 & 44.51 & 44.83 & 30.50 & 32.62 & 32.75 \\
\hline $\mathrm{SO}_{3}$ & 0.53 & 0.37 & NA & 0.40 & NA & 0.41 & NA & 0.41 & NA & 0.20 & 0.15 & NA \\
\hline $\mathrm{SrO}$ & $\S$ & 0.01 & 0.01 & $<0.01$ & $<0.01$ & $<0.01$ & 0.01 & $<0.01$ & $<0.01$ & $\S$ & $<0.01$ & $<0.01$ \\
\hline $\mathrm{TiO}_{2}$ & $\S$ & 0.05 & 0.05 & 0.04 & 0.04 & 0.05 & 0.04 & 0.04 & 0.03 & 0.01 & 0.03 & 0.04 \\
\hline $\mathrm{ZnO}$ & 0.09 & 0.12 & 0.11 & 0.10 & 0.10 & 0.10 & 0.11 & 0.11 & 0.11 & 0.08 & 0.09 & 0.08 \\
\hline $\mathrm{ZrO}_{2}$ & 0.04 & 0.12 & 0.13 & 0.08 & 0.10 & 0.07 & 0.10 & 0.08 & 0.09 & 0.39 & 0.44 & 0.49 \\
\hline Sum & 100.00 & 100.00 & 94.74 & 100.00 & 95.14 & 100.00 & 97.21 & 100.00 & 96.28 & 100.00 & 100.00 & 96.19 \\
\hline
\end{tabular}

$\S$ - Not a target constituent;

NA - Not analyzed

* - for XRF-analyzed compositions, values for $\mathrm{B}_{2} \mathrm{O}_{3}$ and $\mathrm{Li}_{2} \mathrm{O}$ were calculated based on simple well-stirred tank model and target values.

\# - Fluorine measured by XRF on polished samples

$\$$ - Fluorine values calculated by interpolation 
Table 6.11. XRF and DCP Analysis of Selected Glass Samples (wt \%) (continued).

\begin{tabular}{|c|c|c|c|c|c|c|c|c|c|c|c|}
\hline $\begin{array}{l}\text { Limiting } \\
\text { Element }\end{array}$ & \multicolumn{11}{|c|}{ Al } \\
\hline \multirow{2}{*}{ Constituent } & \multirow{2}{*}{ Target } & \multicolumn{2}{|c|}{ BLM-G-110A } & \multicolumn{2}{|c|}{ BLM-G-131A } & \multicolumn{2}{|c|}{ BLM-G-139A } & \multicolumn{2}{|c|}{ BLM-G-145A } & \multicolumn{2}{|c|}{ BLM-G-146B } \\
\hline & & XRF & DCP & XRF & DCP & XRF & $\overline{D C P}$ & XRF & DCP & XRF & DCP \\
\hline $\mathrm{Al}_{2} \mathrm{O}_{3}$ & 23.97 & 22.54 & 20.29 & 22.28 & 21.23 & 22.40 & 21.43 & 22.29 & 21.51 & 22.43 & 21.74 \\
\hline $\mathrm{B}_{2} \mathrm{O}_{3}$ & 15.19 & 15.23* & 15.50 & 15.21* & 16.07 & 15.21* & 15.97 & $15.20 *$ & 16.27 & $15.20 *$ & 16.49 \\
\hline $\mathrm{BaO}$ & 0.05 & \begin{tabular}{l|l}
0.29 \\
\end{tabular} & 0.30 & 0.31 & 0.31 & 0.36 & 0.32 & 0.32 & 0.31 & \begin{tabular}{l|l|}
0.27 \\
\end{tabular} & 0.32 \\
\hline $\mathrm{Bi}_{2} \mathrm{O}_{3}$ & 1.14 & 1.19 & 1.19 & 1.30 & 1.25 & 1.22 & 1.20 & 1.30 & 1.16 & 1.21 & 1.24 \\
\hline $\mathrm{CaO}$ & 6.08 & 5.44 & 5.32 & 5.80 & 5.14 & 5.74 & 5.22 & 5.87 & 5.28 & 5.86 & 5.28 \\
\hline $\mathrm{CdO}$ & 0.02 & $<0.01$ & 0.03 & $<0.01$ & 0.02 & $<0.01$ & 0.03 & $<0.01$ & 0.02 & $<0.01$ & 0.02 \\
\hline $\mathrm{Cr}_{2} \mathrm{O}_{3}$ & 0.52 & 0.49 & 0.24 & 0.52 & 0.23 & 0.51 & 0.20 & 0.52 & 0.21 & 0.52 & 0.21 \\
\hline $\mathrm{F}$ & 0.67 & $0.39 \#$ & NA & $0.40 \#$ & NA & $0.40 \#$ & NA & $0.41 \$$ & NA & $0.42 \$$ & NA \\
\hline $\mathrm{Fe}_{2} \mathrm{O}_{3}$ & 5.90 & 5.58 & 5.62 & 6.13 & 5.37 & 5.94 & 5.31 & 6.16 & 5.36 & 6.00 & 5.38 \\
\hline $\mathrm{K}_{2} \mathrm{O}$ & 0.14 & 0.54 & 0.58 & 0.47 & 0.48 & 0.43 & \begin{tabular}{l|l|}
0.43 \\
\end{tabular} & 0.42 & 0.42 & \begin{tabular}{l|l|}
0.42 \\
\end{tabular} & 0.42 \\
\hline $\mathrm{Li}_{2} \mathrm{O}$ & \begin{tabular}{|l|}
3.57 \\
\end{tabular} & 3.57* & 3.63 & 3.57* & 3.52 & $3.57 \#$ & \begin{tabular}{|l|}
3.53 \\
\end{tabular} & 3.57* & 3.53 & 3.57* & 3.52 \\
\hline $\mathrm{MgO}$ & 0.12 & 0.43 & 0.44 & 0.38 & 0.45 & 0.36 & 0.44 & 0.43 & 0.44 & 0.38 & 0.44 \\
\hline $\mathrm{MnO}$ & $\S$ & 0.04 & 0.04 & 0.04 & 0.05 & 0.03 & 0.04 & 0.03 & 0.04 & 0.04 & 0.04 \\
\hline $\mathrm{Na}_{2} \mathrm{O}$ & \begin{tabular}{|l|}
9.58 \\
\end{tabular} & \begin{tabular}{|l|l|}
9.29 \\
\end{tabular} & 8.64 & \begin{tabular}{|l|}
9.29 \\
\end{tabular} & 9.03 & 9.69 & \begin{tabular}{|l|l|}
8.98 \\
\end{tabular} & 9.80 & 9.02 & \begin{tabular}{|l|}
9.83 \\
\end{tabular} & 9.08 \\
\hline $\mathrm{Nd}_{2} \mathrm{O}_{3}$ & $\S$ & 0.01 & NA & 0.01 & NA & 0.01 & NA & 0.01 & NA & 0.01 & NA \\
\hline $\mathrm{NiO}$ & 0.40 & \begin{tabular}{l|l|}
0.30 \\
\end{tabular} & 0.24 & 0.34 & 0.29 & 0.32 & 0.26 & 0.33 & 0.26 & \begin{tabular}{l|l|}
0.32 \\
\end{tabular} & 0.24 \\
\hline $\mathrm{P}_{2} \mathrm{O}_{5}$ & 1.05 & 1.15 & 1.04 & 1.14 & 0.97 & 1.12 & 0.98 & 1.09 & 0.83 & 1.12 & 1.05 \\
\hline $\mathrm{PbO}$ & \begin{tabular}{l|l|}
0.41 \\
\end{tabular} & \begin{tabular}{l|l|}
0.35 \\
\end{tabular} & 0.41 & \begin{tabular}{l|l|}
0.37 \\
\end{tabular} & 0.41 & 0.37 & \begin{tabular}{l|l|}
0.41 \\
\end{tabular} & 0.37 & 0.41 & \begin{tabular}{ll|}
0.35 \\
\end{tabular} & 0.41 \\
\hline $\mathrm{SiO}_{2}$ & 30.50 & 32.48 & 31.03 & 31.60 & 31.49 & 31.56 & 30.97 & 31.09 & 30.98 & 31.34 & 31.59 \\
\hline $\mathrm{SO}_{3}$ & \begin{tabular}{l|l}
0.20 \\
\end{tabular} & \begin{tabular}{l|l|}
0.15 \\
\end{tabular} & NA & 0.13 & NA & 0.15 & NA & 0.15 & NA & $\begin{array}{ll}0.15 \\
\end{array}$ & NA \\
\hline $\mathrm{SrO}$ & $\S$ & $<0.01$ & NA & $<0.01$ & NA & $<0.01$ & \begin{tabular}{l|l|}
0.01 \\
\end{tabular} & $<0.01$ & 0.01 & $<0.01$ & 0.01 \\
\hline $\mathrm{TiO}_{2}$ & 0.01 & 0.05 & 0.05 & 0.13 & 0.04 & 0.05 & 0.04 & 0.05 & 0.05 & 0.04 & 0.04 \\
\hline $\mathrm{ZnO}$ & \begin{tabular}{l|l}
0.08 \\
\end{tabular} & \begin{tabular}{l|l|}
0.08 \\
\end{tabular} & 0.08 & 0.09 & 0.08 & 0.09 & \begin{tabular}{l|l}
0.08 \\
\end{tabular} & 0.09 & 0.08 & \begin{tabular}{l|l}
0.08 \\
\end{tabular} & 0.08 \\
\hline $\mathrm{ZrO}_{2}$ & 0.39 & 0.44 & 0.54 & 0.48 & 0.55 & 0.48 & 0.55 & 0.49 & 0.54 & 0.45 & 0.55 \\
\hline Sum & 100.00 & \begin{tabular}{|c|}
100.00 \\
\end{tabular} & 95.21 & 100.00 & 96.98 & 100.00 & 96.40 & 100.00 & 96.73 & 100.00 & 98.15 \\
\hline $\begin{array}{l}\S \text { - Not a ta } \\
\text { NA - Not a } \\
* \text { - for XRF } \\
\text { model } \\
\text { \# - Fluorin } \\
\$ \text { - Fluorine }\end{array}$ & $\begin{array}{l}\text { arget con } \\
\text { analyzed }\end{array}$ & stituent; & on 1 & shed & les & . & 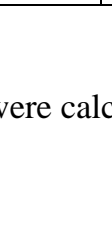 & 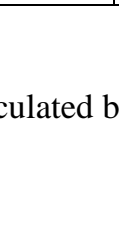 & 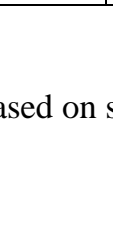 & . & 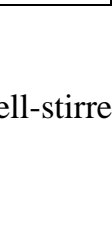 \\
\hline
\end{tabular}


Table 6.11. XRF and DCP Analysis of Selected Glass Samples (wt\%) (continued).

\begin{tabular}{|c|c|c|c|c|c|c|c|c|c|c|c|}
\hline \multirow[t]{3}{*}{$\begin{array}{l}\text { Limiting } \\
\text { Elements }\end{array}$} & \multicolumn{11}{|c|}{$\mathbf{A l}+\mathbf{N a}$} \\
\hline & \multirow{2}{*}{ Target } & \multicolumn{2}{|c|}{ BLN-G-36A } & \multicolumn{2}{|c|}{ BLN-G-66A } & \multicolumn{2}{|c|}{ BLN-G-106B } & \multicolumn{2}{|c|}{ BLN-G-129A } & \multicolumn{2}{|c|}{ BLO-G-24A } \\
\hline & & XRF & DCP & XRF & DCP & XRF & DCP & XRF & DCP & XRF & DCP \\
\hline $\mathrm{Al}_{2} \mathrm{O}_{3}$ & 21.34 & 21.13 & 19.84 & 20.92 & 20.06 & 20.81 & 20.12 & 20.74 & 19.83 & 20.98 & 19.95 \\
\hline $\mathrm{B}_{2} \mathrm{O}_{3}$ & 18.37 & 17.56* & 17.24 & 18.14* & 17.58 & 18.27* & 17.68 & 18.29* & 17.80 & 18.35* & 17.26 \\
\hline $\mathrm{BaO}$ & 0.03 & 0.11 & 0.08 & 0.06 & 0.05 & 0.07 & 0.05 & 0.07 & 0.05 & 0.06 & 0.04 \\
\hline $\mathrm{Bi}_{2} \mathrm{O}_{3}$ & 1.16 & 1.21 & 1.2 & 1.18 & 1.18 & 1.20 & 1.18 & 1.17 & 1.16 & 1.20 & 1.22 \\
\hline $\mathrm{CaO}$ & 0.72 & 1.70 & 1.71 & 1.14 & 1.22 & 1.16 & 1.24 & 1.10 & 1.16 & 1.03 & 1.10 \\
\hline $\mathrm{CdO}$ & 0.01 & $<0.01$ & 0.01 & $<0.01$ & 0.01 & $<0.01$ & 0.01 & $<0.01$ & 0.01 & $<0.01$ & 0.01 \\
\hline $\mathrm{Cr}_{2} \mathrm{O}_{3}$ & 0.71 & 0.78 & 0.39 & 0.79 & \begin{tabular}{l|l}
0.47 \\
\end{tabular} & 0.77 & 0.43 & 0.78 & 0.45 & 0.78 & 0.45 \\
\hline $\mathrm{F}$ & 0.23 & $0.21 \$$ & NA & $0.20 \$$ & NA & $0.22 \$$ & NA & $0.18 \$$ & NA & $0.17 \$$ & NA \\
\hline $\mathrm{Fe}_{2} \mathrm{O}_{3}$ & 2.82 & 3.52 & 3.01 & 3.14 & 3.05 & 3.20 & 2.96 & 3.12 & 2.82 & 3.15 & 2.84 \\
\hline $\mathrm{K}_{2} \mathrm{O}$ & 0.66 & 0.66 & 0.69 & 0.66 & 0.72 & 0.69 & 0.75 & 0.70 & 0.77 & 0.68 & 0.76 \\
\hline $\mathrm{Li}_{2} \mathrm{O}$ & 3.58 & $3.57 *$ & 3.58 & 3.57* & 3.61 & 3.58* & 3.62 & $3.58^{*}$ & 3.64 & 3.58* & 3.47 \\
\hline $\mathrm{MgO}$ & 0.22 & 0.32 & 0.34 & 0.28 & \begin{tabular}{l|l|}
0.32 \\
\end{tabular} & 0.26 & 0.30 & 0.26 & 0.30 & 0.22 & 0.27 \\
\hline $\mathrm{MnO}$ & $\S$ & 0.03 & 0.04 & 0.03 & \begin{tabular}{l|l|}
0.04 \\
\end{tabular} & 0.05 & 0.05 & 0.05 & 0.05 & 0.04 & 0.05 \\
\hline $\mathrm{Na} 2 \mathrm{O}$ & 12.71 & 12.05 & 11.19 & 12.79 & 11.65 & 12.74 & 11.63 & 12.82 & 11.67 & 12.48 & 11.66 \\
\hline $\mathrm{Nd}_{2} \mathrm{O}_{3}$ & $\S$ & 0.01 & NA & 0.01 & NA & 0.01 & NA & 0.01 & NA & 0.01 & NA \\
\hline $\mathrm{NiO}$ & 0.10 & 0.19 & 0.16 & 0.16 & 0.15 & 0.17 & 0.15 & 0.16 & 0.14 & 0.15 & 0.13 \\
\hline $\mathrm{P}_{2} \mathrm{O}_{5}$ & 2.02 & 1.92 & 1.72 & 2.05 & 1.71 & 2.03 & 1.85 & 1.99 & 1.87 & 2.09 & 2.02 \\
\hline $\mathrm{PbO}$ & 0.09 & 0.14 & 0.15 & 0.11 & 0.12 & 0.11 & 0.12 & 0.10 & 0.12 & 0.10 & 0.11 \\
\hline $\mathrm{SiO}_{2}$ & 34.56 & 34.18 & 33.92 & 34.07 & 33.74 & 33.95 & 33.90 & 34.17 & 33.41 & 34.17 & 34.6 \\
\hline $\mathrm{SO}_{3}$ & 0.22 & 0.16 & NA & 0.17 & NA & 0.17 & NA & 0.16 & NA & 0.17 & NA \\
\hline $\mathrm{TiO}_{2}$ & 0.17 & 0.16 & 0.17 & 0.16 & 0.18 & 0.16 & 0.18 & 0.16 & 0.18 & 0.18 & 0.20 \\
\hline $\mathrm{ZnO}$ & 0.18 & 0.16 & 0.14 & 0.17 & \begin{tabular}{l|l|}
0.16 \\
\end{tabular} & 0.16 & 0.14 & 0.17 & 0.14 & 0.19 & 0.19 \\
\hline $\mathrm{ZrO}_{2}$ & 0.12 & 0.23 & \begin{tabular}{l|l|}
0.22 \\
\end{tabular} & 0.19 & \begin{tabular}{|l|}
0.17 \\
\end{tabular} & 0.20 & 0.18 & 0.20 & 0.18 & 0.22 & 0.25 \\
\hline Sum & 100.00 & 100.00 & 95.80 & 100.00 & \begin{tabular}{|c|}
96.19 \\
\end{tabular} & 100.00 & 96.54 & 100.00 & 95.75 & 100.00 & 96.58 \\
\hline
\end{tabular}


Table 6.12. PCT Results for Melter Glasses.

\begin{tabular}{|c|c|c|c|c|c|c|c|c|c|c|c|}
\hline \multirow{2}{*}{\multicolumn{2}{|c|}{$\begin{array}{l}\text { Limiting Element } \\
\text { Glass Samples }\end{array}$}} & \multicolumn{2}{|c|}{ Chromium } & \multicolumn{2}{|c|}{ Bismuth } & \multicolumn{2}{|c|}{ Aluminum } & \multicolumn{3}{|c|}{ Aluminum + Sodium } & \multirow[b]{2}{*}{$\begin{array}{c}\text { DWPF- } \\
\text { EA }\end{array}$} \\
\hline & & $\begin{array}{l}\text { BLK- } \\
\text { G- }\end{array}$ & $\begin{array}{c}\text { BLL- } \\
\text { G- }\end{array}$ & $\begin{array}{c}\text { BLL- } \\
\text { G- }\end{array}$ & $\begin{array}{c}\text { BLL- } \\
\text { G- }\end{array}$ & $\begin{array}{l}\text { BLM- } \\
\text { G- }\end{array}$ & $\begin{array}{l}\text { BLM- } \\
\text { G- }\end{array}$ & $\begin{array}{l}\text { BLN- } \\
\text { G- }\end{array}$ & $\begin{array}{c}\text { BLN- } \\
\text { G- }\end{array}$ & $\begin{array}{c}\text { BLN- } \\
\text { G- }\end{array}$ & \\
\hline \multirow{4}{*}{$\begin{array}{l}\text { 7-Day PCT } \\
\text { Concentration } \\
\text { in } \mathrm{mg} / \mathrm{L}\end{array}$} & B & 9.71 & 8.04 & 183.10 & 164.80 & 1.57 & 10.72 & 88.32 & 83.69 & 90.61 & - \\
\hline & $\mathrm{Li}$ & 0.91 & 0.74 & 43.40 & 40.46 & 5.99 & 5.84 & 21.76 & 21.27 & 22.57 & - \\
\hline & $\mathrm{Na}$ & 56.27 & 53.82 & 135.00 & 118.50 & 16.95 & 17.22 & 74.54 & 72.21 & 75.49 & - \\
\hline & $\mathrm{Si}$ & 39.92 & 35.32 & 50.25 & 49.03 & 21.76 & 16.22 & 44.21 & 46.26 & 47.03 & 3920 \\
\hline \multirow{5}{*}{$\begin{array}{c}\text { 7-Day PCT } \\
\text { Normalized } \\
\text { Concentrations, } \\
\text { g/L }\end{array}$} & $\mathrm{B}$ & 0.28 & 0.23 & 3.71 & 3.33 & 0.03 & 0.23 & 1.57 & 1.48 & 1.60 & 16.7 \\
\hline & $\mathrm{Li}$ & 0.57 & 0.87 & 2.69 & 2.48 & 0.36 & 0.35 & 1.31 & 1.28 & 1.36 & 9.6 \\
\hline & $\mathrm{Na}$ & 0.47 & 0.48 & 2.37 & 2.10 & 0.24 & 0.25 & 0.78 & 0.76 & 0.79 & 13.3 \\
\hline & $\mathrm{Si}$ & 0.23 & 0.21 & 0.24 & 0.24 & 0.14 & 0.11 & 0.28 & 0.29 & 0.29 & - \\
\hline & $\mathrm{pH}$ & 9.70 & 9.65 & 9.72 & 9.71 & 9.53 & 9.75 & 9.53 & 9.53 & 9.54 & - \\
\hline \multirow{4}{*}{$\begin{array}{c}\text { 7-Day PCT } \\
\text { Normalized } \\
\text { Mass Loss } \\
\left(\mathrm{g} / \mathrm{m}^{2}\right) \\
\end{array}$} & B & 0.14 & 0.11 & 1.86 & 1.67 & 0.02 & 0.11 & 0.78 & 0.74 & 0.80 & - \\
\hline & $\mathrm{Li}$ & 0.29 & 0.43 & 1.35 & 1.24 & 0.18 & 0.18 & 0.66 & 0.64 & 0.68 & - \\
\hline & $\mathrm{Na}$ & 0.24 & 0.24 & 1.18 & 1.05 & 0.12 & 0.12 & 0.39 & 0.38 & 0.40 & - \\
\hline & $\mathrm{Si}$ & 0.11 & 0.10 & 0.12 & 0.12 & 0.07 & 0.05 & 0.14 & 0.15 & 0.15 & - \\
\hline \multirow{4}{*}{$\begin{array}{l}\text { 7-Day PCT } \\
\text { Normalized } \\
\text { Loss Rate, } \\
\mathrm{g} / \mathrm{d} / \mathrm{m}^{2}\end{array}$} & B & 0.02 & 0.02 & 0.27 & 0.24 & $<0.01$ & 0.02 & 0.11 & 0.11 & 0.11 & - \\
\hline & $\mathrm{Li}$ & 0.04 & 0.06 & 0.19 & 0.18 & 0.03 & 0.03 & 0.09 & 0.09 & 0.10 & - \\
\hline & $\mathrm{Na}$ & 0.03 & 0.03 & 0.17 & 0.15 & 0.02 & 0.02 & 0.06 & 0.05 & 0.06 & - \\
\hline & $\mathrm{Si}$ & 0.02 & 0.01 & 0.02 & 0.02 & 0.01 & 0.01 & 0.02 & 0.02 & 0.02 & - \\
\hline
\end{tabular}

- Empty data field 
The Catholic University of America Vitreous State Laboratory
High Level Waste Vitrification System Improvements Final Report, VSL-07R1010-1, Rev.0

Table 6.13. TCLP Results for Melter Glasses (mg/L).

\begin{tabular}{|c|c|c|c|c|c|c|c|c|}
\hline \multirow{3}{*}{$\begin{array}{c}\text { Limiting } \\
\text { Element }\end{array}$} & Sample I.D. & $\mathrm{Ba}$ & $\mathrm{Bi}$ & $\mathrm{Cd}$ & $\mathrm{Cr}$ & $\mathrm{Ni}$ & $\mathrm{Pb}$ & $\mathrm{Zn}$ \\
\cline { 2 - 9 } & UTS Limits & 21 & N/A & 0.11 & 0.60 & 11.00 & 0.75 & 4.3 \\
\cline { 2 - 9 } & Delisting Limits & 100 & N/A & 0.48 & 4.95 & 22.6 & 5.00 & 225 \\
\hline \multirow{3}{*}{$\mathrm{Bi}$} & BLK-G-129A & 0.22 & 0.56 & NA & $<0.01$ & 0.41 & $<0.1$ & 0.13 \\
\cline { 2 - 9 } & BLK-G-46B & 0.13 & 0.72 & NA & $<0.01$ & 0.68 & $<0.1$ & 0.10 \\
\hline \multirow{3}{*}{$\mathrm{Cr}$} & BLL-G-88A & 0.09 & 0.51 & NA & 0.05 & 0.12 & $<0.1$ & 0.05 \\
\cline { 2 - 9 } & BLL-G-113A & 0.14 & 0.58 & NA & 0.06 & 0.10 & $<0.1$ & 0.08 \\
\cline { 2 - 9 } & BLL-G-121A & 0.13 & 0.53 & NA & 0.07 & 0.10 & $<0.1$ & 0.09 \\
\hline \multirow{3}{*}{$\mathrm{Al}$} & BLM-G-36A & 0.35 & 1.00 & 0.04 & 0.05 & 0.10 & 0.35 & 0.10 \\
\cline { 2 - 9 } & BLM-G-110A & 0.42 & 0.74 & 0.04 & 0.06 & 0.13 & 0.46 & 0.09 \\
\hline \multirow{3}{*}{$\mathrm{Al}+\mathrm{Na}$} & BLN-G-66A & 0.11 & 0.47 & $<0.03$ & 0.11 & 0.07 & 0.11 & 0.09 \\
\cline { 2 - 9 } & BLN-G-106B & 0.11 & 0.46 & $<0.03$ & 0.11 & 0.07 & 0.11 & 0.08 \\
\cline { 2 - 9 } & BLN-G-129A & 0.11 & 0.62 & $<0.03$ & 0.11 & 0.06 & 0.13 & 0.07 \\
\hline
\end{tabular}

N/A - Not available

NA - Not analyzed, Cd is a target constituent only in aluminum- and aluminum-plus-sodium-limited formulations. 
Table 6.14. Glass Pool Samples and Secondary Phase Observations.

\begin{tabular}{|c|c|c|c|c|c|c|c|}
\hline & Test & $\mathrm{T}\left({ }^{\circ} \mathrm{C}\right)$ & Date & Sample I.D. & $\begin{array}{l}\text { Visual Observations } \\
\text { During Sampling }\end{array}$ & SEM Observations & $\begin{array}{c}\text { Volume } \\
\text { Percent } \\
\text { Crystals } \\
\text { Determined } \\
\text { by SEM }\end{array}$ \\
\hline \multirow{6}{*}{$\mathrm{Bi}$} & End of Test $1 \mathrm{~A}$ & \multirow{3}{*}{1175} & $7 / 19 / 06$ & BLK-D-100A & No secondary phases & Fe-Cr-Ni Spinel & 0.3 \\
\hline & \multirow{2}{*}{ End of Test 1B } & & \multirow{2}{*}{$7 / 20 / 06$} & BLK-D-125A & No secondary phases & Fe-Cr-Ni Spinel, some with Cr oxide cores & 0.3 \\
\hline & & & & BLK-D-129A & No secondary phases & NA & NA \\
\hline & End of Test $2 \mathrm{~A}$ & \multirow{3}{*}{1150} & $7 / 26 / 06$ & BLL-D-16A & No secondary phases & Fe-Cr-Ni Spinel & 0.5 \\
\hline & \multirow{2}{*}{ End of Test 2B } & & \multirow{2}{*}{ 7/28/06 } & BLL-D-45A & No secondary phases & NA & NA \\
\hline & & & & BLL-D-46A & No secondary phases & Fe-Cr-Ni Spinel, some with Cr oxide cores & 0.4 \\
\hline \multirow{8}{*}{$\mathrm{Cr}$} & \multirow{4}{*}{ End of Test 3B } & \multirow{4}{*}{1175} & \multirow{4}{*}{ 8/23/06 } & BLL-D-88A & No secondary phases & Finely grained chromium oxide aggregate & 0.3 \\
\hline & & & & BLL-D-88B & No secondary phases & NA & NA \\
\hline & & & & BLL-D-88C & No secondary phases & NA & NA \\
\hline & & & & BLL-D-88D & No secondary phases & NA & NA \\
\hline & \multirow{4}{*}{ End of Test 4A } & \multirow{4}{*}{1150} & \multirow{4}{*}{$8 / 25 / 06$} & BLL-D-113A & No secondary phases & Finely grained chromium oxide aggregate & 0.3 \\
\hline & & & & BLL-D-113B & No secondary phases & NA & NA \\
\hline & & & & BLL-D-113C & No secondary phases & NA & NA \\
\hline & & & & BLL-D-113D & No secondary phases & NA & NA \\
\hline \multirow{5}{*}{$\mathrm{Al}$} & End of Test $5 A$ & \multirow{2}{*}{1175} & 9/13/06 & BLM-D-9A & No secondary phases & Fe-Ni-Cr spinel with trace of $\mathrm{Zn}, \mathrm{Mg}, \mathrm{Al}$ & 0.9 \\
\hline & End of Test 5B & & 9/15/06 & BLM-D-37A & No secondary phases & Fe-Ni-Cr spinel with trace of $\mathrm{Al}, \mathrm{Mg}, \mathrm{Zn}$ & 0.7 \\
\hline & End of Test $6 \mathrm{~A}$ & \multirow{3}{*}{1150} & $9 / 20 / 06$ & BLM-D-73A & No secondary phases & Fe-Ni-Cr-Al spinel with trace of Mg, Zn & 0.4 \\
\hline & End of Test $6 \mathrm{~B}$ & & 9/23/06 & BLM-D-110A & No secondary phases & Fe-Ni-Cr-Al spinel with trace of Mg, Zn & 0.6 \\
\hline & End of Test 6C & & $9 / 28 / 06$ & BLM-D-146A & No secondary phases & Fe-Ni-Cr-Al spinel with trace of Mg & 0.6 \\
\hline \multirow{5}{*}{$\mathrm{Al}+\mathrm{Na}$} & End of Test 7A & \multirow{2}{*}{1175} & $10 / 04 / 06$ & BLN-D-40A & No secondary phases & $\begin{array}{l}\text { Fe-Al-Cr spinel with trace of } \mathrm{Ni} \mathrm{Zn}, \mathrm{Mg} \text {; } \\
\text { Fe-Cr-Ni spinel with trace of } \mathrm{Zn}\end{array}$ & 1.1 \\
\hline & End of Test 7B & & $10 / 06 / 06$ & BLN-D-66A & No secondary phases & $\begin{array}{l}\text { Fe-Al-Cr spinel with trace of } \mathrm{Zn}, \mathrm{Mg} \text {; } \\
\mathrm{Cr} \text { oxide platelets with } \mathrm{Fe} \text { and } \mathrm{Al}\end{array}$ & 0.2 \\
\hline & End of Test 8A & \multirow{3}{*}{1150} & $10 / 18 / 06$ & BLN-D-107B & No secondary phases & Fe-Cr spinel with trace of $\mathrm{Ni}, \mathrm{Zn}, \mathrm{Al}, \mathrm{Mg}$ & 0.2 \\
\hline & End of Test 8B & & $10 / 21 / 06$ & BLN-D-129A & No secondary phases & Fe-Cr spinel with trace of $\mathrm{Zn}, \mathrm{Ni}, \mathrm{Al}, \mathrm{Mg}$ & 0.2 \\
\hline & End of Test 8C & & $11 / 20 / 06$ & BLO-D-24A & No secondary phases & Fe-Cr spinel with trace of $\mathrm{Mg}$ & 0.2 \\
\hline
\end{tabular}

NA - Not Analyzed 
Table 7.1. Results from Melter Off-Gas Emission Samples.

\begin{tabular}{|c|c|c|c|c|c|c|c|c|c|}
\hline & \multicolumn{4}{|l|}{ Test 1A } & \multicolumn{4}{|l|}{ Test 1B } \\
\hline & & \multicolumn{4}{|c|}{$\begin{array}{r}07 / 19 / 06 \text { 11:26 - 12:26 } \\
\text { 98.6\% Isokinetic, 9.8\% Moisture }\end{array}$} & \multicolumn{4}{|c|}{$\begin{array}{r}07 / 21 / 0614: 25-15: 25 \\
90.47 \% \text { Isokinetic, } 12.3 \% \text { Moisture } \\
\end{array}$} \\
\hline & & $\begin{array}{l}\text { Feed Rate } \\
\text { (mg/min) }\end{array}$ & $\begin{array}{l}\text { Emissions } \\
\text { Rate } \\
\text { (mg/min) }\end{array}$ & \% Feed & DF & $\begin{array}{l}\text { Feed Rate } \\
\text { (mg/min) }\end{array}$ & $\begin{array}{l}\text { Emissions } \\
\text { Rate } \\
\text { (mg/min) }\end{array}$ & $\%$ Feed & DF \\
\hline \multirow{23}{*}{ 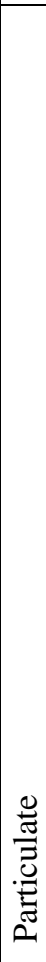 } & Total $^{\$}$ & 97710 & 183 & 0.19 & 535 & 81420 & 392 & 0.48 & 208 \\
\hline & $\mathrm{Al}$ & 5603 & 0.66 & 0.01 & 8479 & 4669 & 3.66 & 0.08 & 1277 \\
\hline & $\mathrm{B}$ & 3185 & 0.57 & 0.02 & 5598 & 2655 & 18.50 & 0.70 & 144 \\
\hline & $\mathrm{Ba}$ & 8 & $<0.10$ & $<1.3$ & $>80$ & 7 & $<0.10$ & $<1.43$ & $>70$ \\
\hline & $\mathrm{Bi}$ & 5467 & 4.49 & 0.08 & 1219 & 4556 & 24.70 & 0.54 & 185 \\
\hline & $\mathrm{Ca}$ & 545 & 0.13 & 0.02 & 4357 & 454 & 0.49 & 0.11 & 922 \\
\hline & $\mathrm{Cd}$ & 0 & $<0.10$ & NC & NC & 0 & $<0.10$ & NC & NC \\
\hline & $\mathrm{Cl}^{*}$ & 0 & 1.59 & NC & NC & 0 & 8.52 & NC & NC \\
\hline & $\mathrm{Cr}$ & 323 & 1.31 & 0.40 & 248 & 269 & 3.43 & 1.27 & 78.5 \\
\hline & $\mathrm{F}^{*}$ & 745 & 3.85 & 0.52 & 194 & 621 & 30.2 & 4.86 & 20.6 \\
\hline & $\mathrm{Fe}$ & 4421 & 1.31 & 0.03 & 3374 & 3684 & 9.47 & 0.26 & 389 \\
\hline & $\mathrm{K}$ & 347 & 3.43 & 0.99 & 101 & 289 & 6.83 & 2.36 & 42.3 \\
\hline & $\mathrm{Li}$ & 68 & 0.79 & 1.16 & 86.0 & 56 & 0.66 & 1.17 & 85.1 \\
\hline & $\mathrm{Mg}$ & 236 & $<0.10$ & $<0.10$ & $>2360$ & 196 & 0.52 & 0.27 & 376 \\
\hline & $\mathrm{Na}$ & 10608 & 41.32 & 0.39 & 257 & 8840 & 75.21 & 0.85 & 118 \\
\hline & $\mathrm{Ni}$ & 1378 & 0.34 & 0.02 & 4103 & 1148 & 2.71 & 0.24 & 424 \\
\hline & $\mathrm{P}$ & 1979 & 0.56 & 0.03 & 3507 & 1649 & 5.40 & 0.33 & 305 \\
\hline & $\mathrm{Pb}$ & 211 & 1.00 & 0.47 & 211 & 176 & 1.54 & 0.88 & 114 \\
\hline & $\mathrm{S}^{*}$ & 175 & 34.2 & 19.54 & 5.1 & 146 & 27.8 & 19.0 & 5.25 \\
\hline & $\mathrm{Si}$ & 15397 & 1.25 & 0.01 & 12287 & 12830 & 11.06 & 0.09 & 1160 \\
\hline & $\mathrm{Ti}$ & 87 & $<0.10$ & $<0.11$ & $>870$ & 73 & 0.35 & 0.48 & 207 \\
\hline & $\mathrm{Zn}$ & 117 & $<0.10$ & $<0.10$ & $>1170$ & 97 & 0.28 & 0.29 & 342 \\
\hline & $\mathrm{Zr}$ & 141 & $<0.10$ & $<0.10$ & $>1410$ & 118 & 0.19 & 0.16 & 612 \\
\hline \multirow{4}{*}{, } & B & 3185 & 16.64 & 0.52 & 192 & 2655 & 46.29 & 1.74 & 57.3 \\
\hline & $\mathrm{Cl}$ & 0 & 24.46 & NC & NC & 0 & 20.17 & NC & $\mathrm{NC}$ \\
\hline & $\mathrm{F}$ & 745 & 125.82 & 16.89 & 5.9 & 621 & 156.36 & 25.19 & 4.0 \\
\hline & $\mathrm{S}$ & 175 & 39.07 & 22.36 & 4.5 & 146 & 49.89 & 34.26 & 2.9 \\
\hline
\end{tabular}

$\$$ - From gravimetric analysis of filters and particulate nitric acid rinses.

* Based on analysis of front-half rinse and water dissolution of filter particulate.

\# - Feed rate calculated from target composition and production rate.

NC - Not Calculated 
Table 7.1. Results from Melter Off-Gas Emission Samples (continued).

\begin{tabular}{|c|c|c|c|c|c|c|c|c|c|}
\hline & \multicolumn{4}{|c|}{ Test 2A } & \multicolumn{4}{|c|}{ Test 2B } \\
\hline & & \multicolumn{4}{|c|}{$\begin{array}{c}07 / 25 / 06 \text { 19:42 - 20:45 } \\
\text { 97.8\% Isokinetic, 8.3\% Moisture }\end{array}$} & \multicolumn{4}{|c|}{$\begin{array}{c}\text { 07/28/06 9:45 - 10:45 } \\
\text { 102\% Isokinetic, } 12.1 \% \text { Moisture }\end{array}$} \\
\hline & & $\begin{array}{c}\text { Feed Rate } \\
(\mathrm{mg} / \mathrm{min})\end{array}$ & $\begin{array}{c}\text { Emissions } \\
\text { Rate } \\
\text { (mg/min) }\end{array}$ & $\%$ Feed & DF & $\begin{array}{l}\text { Feed Rate } \\
\text { (mg/min) }\end{array}$ & $\begin{array}{c}\text { Emissions } \\
\text { Rate } \\
\text { (mg/min) }\end{array}$ & $\%$ Feed & $\mathrm{DF}$ \\
\hline \multirow{23}{*}{ 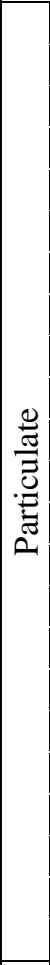 } & Total $^{\$}$ & 67580 & 215 & 0.32 & 314 & 37640 & 118 & 0.31 & 319 \\
\hline & $\mathrm{Al}$ & 3875 & 2.53 & 0.07 & 1534 & 2381 & 0.72 & 0.03 & 3295 \\
\hline & B & 2203 & 7.95 & 0.36 & 277.3 & 1354 & 3.41 & 0.25 & 398 \\
\hline & $\mathrm{Ba}$ & 6 & $<0.10$ & $<1.67$ & $>60$ & 3 & $<0.10$ & $<3.33$ & $>30$ \\
\hline & $\mathrm{Bi}$ & 3781 & 10.81 & 0.29 & \begin{tabular}{|l}
349.8 \\
\end{tabular} & 2324 & 5.26 & 0.23 & 442 \\
\hline & $\mathrm{Ca}$ & 377 & 0.29 & 0.08 & 1319 & 232 & 0.18 & 0.08 & 1303 \\
\hline & $\mathrm{Cd}$ & 0 & $<0.10$ & NC & NC & 0 & $<0.10$ & NC & $\mathrm{NC}$ \\
\hline & $\mathrm{Cl}^{*}$ & 0 & 3.61 & $\mathrm{NC}$ & NC & 0 & 1.97 & NC & $\mathrm{NC}$ \\
\hline & $\mathrm{Cr}$ & 224 & 1.40 & 0.63 & 160 & 137 & 0.94 & 0.69 & 145 \\
\hline & $\mathrm{F}^{*}$ & 515 & 10.56 & 2.05 & 48.77 & 317 & 12.4 & 3.91 & 25.6 \\
\hline & $\mathrm{Fe}$ & 3058 & 5.30 & 0.17 & 577 & 1879 & 2.14 & 0.11 & 878 \\
\hline & $\mathrm{K}$ & 240 & 3.21 & 1.34 & 74.8 & 147 & 2.33 & 1.58 & 63.4 \\
\hline & $\mathrm{Li}$ & 47 & 0.31 & 0.66 & 152 & 29 & 0.16 & 0.54 & 185 \\
\hline & $\mathrm{Mg}$ & 163 & 0.28 & 0.17 & 584 & 100 & 0.12 & 0.12 & 835 \\
\hline & $\mathrm{Na}$ & 7337 & 39.90 & 0.54 & 184 & 4508 & 24.43 & 0.54 & 185 \\
\hline & $\mathrm{Ni}$ & 953 & 1.55 & 0.16 & 616 & 585 & 0.59 & 0.10 & 988 \\
\hline & $\mathrm{P}$ & 1369 & 1.67 & 0.12 & 819 & 841 & 0.74 & 0.09 & 1139 \\
\hline & $\mathrm{Pb}$ & 146 & 0.70 & 0.48 & 209 & 90 & 0.56 & 0.62 & 161 \\
\hline & S* & 121 & 22.06 & 18.23 & 5.5 & 74 & 14.4 & 19.5 & 5.1 \\
\hline & Si & 10649 & 6.57 & 0.06 & 1622 & 6544 & 2.71 & 0.04 & 2417 \\
\hline & $\mathrm{Ti}$ & 60 & 0.11 & 0.17 & 572 & 37 & $<0.10$ & $<0.27$ & $>370$ \\
\hline & $\mathrm{Zn}$ & 81 & 0.11 & 0.14 & 710 & 50 & $<0.10$ & $<0.20$ & $>500$ \\
\hline & $\mathrm{Zr}$ & 98 & $<0.10$ & $<0.10$ & $>980$ & 60 & $<0.10$ & $<0.17$ & $>600$ \\
\hline \multirow{4}{*}{ 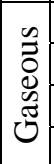 } & $B$ & 2203 & 25.74 & 1.17 & 85.6 & 1354 & 25.63 & 1.89 & 52.8 \\
\hline & $\mathrm{Cl}$ & 0 & 13.81 & NC & NC & 0 & 17.30 & NC & $\mathrm{NC}$ \\
\hline & $\mathrm{F}$ & 515 & 132.71 & 25.76 & 3.9 & 317 & 148.60 & 46.94 & 2.1 \\
\hline & $\bar{S}$ & 121 & 24.36 & 20.16 & 5.0 & 74 & 25.41 & 34.21 & 2.9 \\
\hline
\end{tabular}

$\$$ - From gravimetric analysis of filters and particulate nitric acid rinses.

* Based on analysis of front-half rinse and water dissolution of filter particulate.

\# - Feed rate calculated from target composition and production rate.

NC - Not Calculated 
Table 7.1. Results from Melter Off-Gas Emission Samples (continued).

\begin{tabular}{|c|c|c|c|c|c|c|c|c|c|}
\hline & \multicolumn{4}{|c|}{ Test 3B } & \multicolumn{4}{|c|}{ Test 4A } \\
\hline & & \multicolumn{4}{|c|}{$\begin{array}{c}\text { 08/23/06 13:42-14:42 } \\
\text { 108.5\% Isokinetic, } 12.2 \% \text { Moisture }\end{array}$} & \multicolumn{4}{|c|}{$\begin{array}{c}\text { 08/25/06 14:20 - 15:20 } \\
\text { 103.0\% Isokinetic, } 11.3 \% \text { Moisture }\end{array}$} \\
\hline & & $\begin{array}{c}\text { Feed Rate } \\
\text { (mg/min) }\end{array}$ & $\begin{array}{c}\text { Emissions } \\
\text { Rate } \\
\text { (mg/min) }\end{array}$ & $\%$ Feed & $\mathrm{DF}$ & $\begin{array}{l}\text { Feed Rate }{ }^{\#} \\
\text { (mg/min) }\end{array}$ & $\begin{array}{c}\text { Emissions } \\
\text { Rate } \\
\text { (mg/min) }\end{array}$ & $\%$ Feed & DF \\
\hline \multirow{23}{*}{ 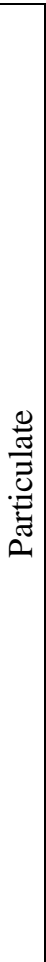 } & Total $^{\$}$ & 108660 & 441 & 0.41 & 246 & 96120 & 264 & 0.27 & 364 \\
\hline & $\mathrm{Al}$ & 5758 & 2.13 & 0.04 & 2701 & 5093 & 0.96 & 0.02 & 5317 \\
\hline & B & 4410 & 27.24 & 0.62 & 162 & 3901 & 17.10 & 0.44 & 228 \\
\hline & $\mathrm{Ba}$ & 9 & 0.16 & 1.81 & 55.3 & 8 & $<0.10$ & $<1.25$ & $>80$ \\
\hline & $\mathrm{Bi}$ & 2789 & 11.17 & 0.40 & 250 & 2467 & 5.80 & 0.24 & 425 \\
\hline & $\mathrm{Ca}$ & 753 & 1.23 & 0.16 & 610 & 666 & 0.56 & 0.08 & 1186 \\
\hline & $\mathrm{Cd}$ & 0 & $<0.10$ & NC & NC & 0 & $<0.10$ & NC & NC \\
\hline & $\mathrm{Cl}$ & 0 & 1.83 & NC & NC & 0 & 1.11 & NC & NC \\
\hline & $\mathrm{Cr}$ & 895 & 9.00 & 1.00 & 99.5 & 792 & 7.40 & 0.93 & 107 \\
\hline & $\mathrm{F}$ & 856 & 79.27 & 9.26 & 10.8 & 757 & 50.48 & 6.67 & 15.0 \\
\hline & $\mathrm{Fe}$ & 3915 & 7.41 & 0.19 & 528 & 3463 & 4.00 & 0.12 & 865 \\
\hline & $\mathrm{K}$ & 4428 & 48.42 & 1.09 & 91.4 & 3917 & 35.62 & 0.91 & 110 \\
\hline & $\mathrm{Li}$ & 1513 & 5.59 & 0.37 & 271 & 1338 & 4.32 & 0.32 & 310 \\
\hline & $\mathrm{Mg}$ & 42 & 0.37 & 0.90 & 112 & 37 & 0.20 & 0.55 & 181 \\
\hline & $\mathrm{Na}$ & 6352 & 31.96 & 0.50 & 199 & 5619 & 18.73 & 0.33 & 300 \\
\hline & $\mathrm{Ni}$ & 356 & 0.52 & 0.15 & 689 & 315 & 0.31 & 0.10 & 103 \\
\hline & $\mathrm{P}$ & 623 & 0.85 & 0.14 & 736 & 551 & 0.42 & 0.08 & 1306 \\
\hline & $\mathrm{Pb}$ & 192 & 0.88 & 0.46 & 217 & 170 & 0.51 & 0.30 & 333 \\
\hline & $\mathrm{S}$ & 260 & 30.3 & 11.65 & 8.58 & 230 & 20.78 & 9.03 & 11.1 \\
\hline & $\mathrm{Si}$ & 19292 & 11.31 & 0.06 & 1705 & 17066 & 4.67 & 0.03 & 3657 \\
\hline & $\mathrm{Ti}$ & 0 & $<0.10$ & NC & NC & 0 & $<0.10$ & NC & NC \\
\hline & $\mathrm{Zn}$ & 87 & 0.18 & 0.20 & 489 & 77 & $<0.10$ & $<0.13$ & $>770$ \\
\hline & $\mathrm{Zr}$ & 36 & $<0.10$ & $<0.28$ & $>360$ & 32 & $<0.10$ & $<0.31$ & $>320$ \\
\hline \multirow{4}{*}{ 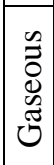 } & B & 4410 & 59.71 & 1.35 & 73.9 & 3901 & 44.87 & 1.15 & 86.9 \\
\hline & $\mathrm{Cl}$ & 0 & 7.96 & NC & NC & 0 & 6.91 & NC & NC \\
\hline & $\mathrm{F}$ & 856 & 141.62 & 16.54 & 6.0 & 757 & 140.66 & 18.57 & 5.4 \\
\hline & $S$ & 260 & $\begin{array}{l}69.18 \\
\end{array}$ & 26.58 & 3.8 & 230 & 56.32 & 24.46 & 4.1 \\
\hline
\end{tabular}

$\$$ - From gravimetric analysis of filters and particulate nitric acid rinses.

* Based on analysis of front-half rinse and water dissolution of filter particulate.

\# - Feed rate calculated from target composition and production rate.

NC - Not Calculated 
Table 7.1. Results from Melter Off-Gas Emission Samples (continued).

\begin{tabular}{|c|c|c|c|c|c|c|c|c|c|}
\hline & & Test 5 & & & \multicolumn{4}{|c|}{ Test 5B } \\
\hline & & \multicolumn{4}{|c|}{$\begin{array}{c}\text { 09/13/06 10:04 - 11:04 } \\
\text { 100.8\% Isokinetic, 10.2\% Moisture }\end{array}$} & \multicolumn{4}{|c|}{$\begin{array}{c}\text { 09/15/06 13:58 - 14:58 } \\
\text { 98.4\% Isokinetic, } 6.3 \% \text { Moisture } \\
\end{array}$} \\
\hline & & $\begin{array}{c}\text { Feed Rate }^{\#} \\
\text { (mg/min) }\end{array}$ & $\begin{array}{c}\text { Emissions } \\
\text { Rate } \\
\text { (mg/min) }\end{array}$ & \% Feed & DF & $\begin{array}{c}\text { Feed Rate } \\
\text { (mg/min) }\end{array}$ & $\begin{array}{c}\text { Emissions } \\
\text { Rate } \\
\text { (mg/min) }\end{array}$ & $\%$ Feed & DF \\
\hline \multirow{23}{*}{ 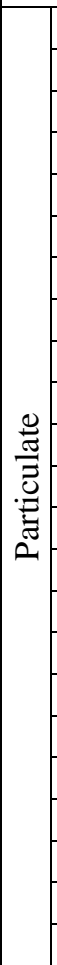 } & Total $^{\$}$ & 82980 & 105 & 0.13 & 787 & 45640 & 41.0 & 0.09 & 1112 \\
\hline & $\mathrm{Al}$ & 9599 & 2.18 & 0.02 & 4394 & 5279 & 0.64 & 0.01 & 8239 \\
\hline & B & 3568 & 4.31 & 0.12 & 827 & 1963 & 0.94 & 0.05 & 2084 \\
\hline & $\mathrm{Ba}$ & 34 & 0.24 & 0.70 & 142 & 19 & 0.13 & 0.71 & 141 \\
\hline & $\mathrm{Bi}$ & 774 & 1.30 & 0.17 & 597 & 426 & 0.58 & 0.14 & 731 \\
\hline & $\mathrm{Ca}$ & 3290 & 1.45 & 0.04 & 2266 & 1809 & 0.60 & 0.03 & 3017 \\
\hline & $\mathrm{Cd}$ & 14 & $<0.10$ & $<0.71$ & $>140$ & 7 & $<0.10$ & $<1.43$ & $>70$ \\
\hline & $\mathrm{Cl}$ & 0 & $0.40 *$ & $\mathrm{NC}$ & NC & 0 & $<0.10^{\&}$ & NC & NC \\
\hline & $\mathrm{Cr}$ & 269 & 2.50 & 0.93 & 108 & 148 & 1.01 & 0.68 & 147 \\
\hline & $\mathrm{F}$ & 507 & $10.69 *$ & 2.11 & 47.4 & 279 & $5.09^{8}$ & 1.82 & 54.8 \\
\hline & $\mathrm{Fe}$ & 3123 & 3.12 & 0.10 & 1000 & 1718 & 1.13 & 0.07 & 1515 \\
\hline & $\mathrm{K}$ & 88 & 5.44 & 6.18 & 16.2 & 48 & 1.49 & 3.08 & 32.5 \\
\hline & $\mathrm{Li}$ & 1255 & 2.46 & 0.20 & 511 & 690 & 0.98 & 0.14 & 701 \\
\hline & $\mathrm{Mg}$ & 55 & 0.16 & 0.29 & 341 & 30 & $<0.10$ & $<0.33$ & $>300$ \\
\hline & $\mathrm{Na}$ & 5380 & 12.85 & 0.24 & 419 & 2959 & 5.99 & 0.20 & 494 \\
\hline & $\mathrm{Ni}$ & 238 & $<0.10$ & $<0.04$ & $>2380$ & 131 & $<0.10$ & $<0.08$ & $>1310$ \\
\hline & $\mathrm{P}$ & 347 & $<0.10$ & $<0.03$ & $>3470$ & 191 & $<0.10$ & $<0.05$ & $>1910$ \\
\hline & $\mathrm{Pb}$ & 288 & 0.74 & 0.26 & 390 & 158 & 0.36 & 0.23 & 439 \\
\hline & $\mathrm{S}$ & 61 & $9.96 *$ & 16.33 & 6.12 & 33 & $1.89^{8}$ & $<5.73$ & $>17.5$ \\
\hline & $\mathrm{Si}$ & 10792 & 3.55 & 0.03 & 3042 & 5936 & 1.88 & 0.03 & 3151 \\
\hline & $\mathrm{Ti}$ & 5 & $<0.10$ & $<2.00$ & $>50$ & 2 & $<0.10$ & $<5.00$ & $>20$ \\
\hline & $\mathrm{Zn}$ & 49 & $<0.10$ & $<0.82$ & $>123$ & 27 & $<0.10$ & $<0.37$ & $>270$ \\
\hline & $\mathrm{Zr}$ & 219 & $<0.10$ & $<0.05$ & $>2190$ & 120 & $<0.10$ & $<0.08$ & $>1200$ \\
\hline \multirow{4}{*}{ 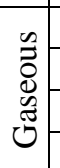 } & $\mathrm{B}$ & 3568 & 36.54 & 1.02 & 97.7 & 1963 & 15.34 & 0.78 & 128 \\
\hline & $\mathrm{Cl}$ & 0 & 5.89 & NC & $\mathrm{NC}$ & 0 & 3.54 & NC & $\mathrm{NC}$ \\
\hline & $F$ & 507 & 139.59 & 27.52 & 3.6 & 279 & 96.88 & 34.73 & 2.9 \\
\hline & $S$ & 61 & 28.42 & 46.84 & 2.1 & 33 & 17.13 & 51.34 & 1.9 \\
\hline
\end{tabular}

$\$$ - From gravimetric analysis of filters and particulate nitric acid rinses.

* Based on analysis of front-half rinse and water dissolution of filter particulate.

$\&$ Based on direct analysis of front-half rinse.

\# - Feed rate calculated from target composition and production rate.

NC - Not Calculated 
Table 7.1. Results from Melter Off-Gas Emission Samples (continued).

\begin{tabular}{|c|c|c|c|c|c|c|c|c|c|}
\hline & \multicolumn{4}{|c|}{ Test 6A } & \multicolumn{4}{|c|}{ Test 6B } \\
\hline & & \multicolumn{4}{|c|}{$\begin{array}{c}\text { 09/20/06 08:59 - 09:59 } \\
\text { 97.1\% Isokinetic, 6.4\% Moisture }\end{array}$} & \multicolumn{4}{|c|}{$\begin{array}{c}\text { 09/22/06 11:55 - 12:55 } \\
\text { 99.1\% Isokinetic, 8.3\% Moisture }\end{array}$} \\
\hline & & $\begin{array}{c}\text { Feed Rate } \\
\text { (mg/min) }\end{array}$ & $\begin{array}{c}\text { Emissions } \\
\text { Rate } \\
\text { (mg/min) }\end{array}$ & $\%$ Feed & DF & $\begin{array}{c}\text { Feed Rate }^{\#} \\
\text { (mg/min) }\end{array}$ & $\begin{array}{c}\text { Emissions } \\
\text { Rate } \\
\text { (mg/min) }\end{array}$ & $\%$ Feed & DF \\
\hline \multirow{23}{*}{ 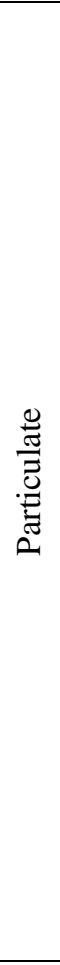 } & Total $^{\$}$ & 45640 & 259 & 0.57 & 176 & 32270 & 46.5 & 0.14 & 694 \\
\hline & $\mathrm{Al}$ & 5279 & 5.56 & 0.11 & 950 & 3839 & 0.67 & 0.02 & 5757 \\
\hline & B & 1963 & 16.34 & 0.83 & 120 & 1427 & 1.87 & 0.13 & 762 \\
\hline & $\mathrm{Ba}$ & 19 & 1.05 & 5.63 & 17.8 & 14 & 0.13 & 0.99 & 101 \\
\hline & $\mathrm{Bi}$ & 426 & 3.72 & 0.87 & 114 & 310 & 0.58 & 0.19 & 538 \\
\hline & $\mathrm{Ca}$ & 1809 & 7.23 & 0.40 & 250 & 1316 & 0.73 & 0.06 & 1813 \\
\hline & $\mathrm{Cd}$ & 7 & 0.11 & 1.45 & 69.1 & 5 & $<0.10$ & $<2.00$ & $>50$ \\
\hline & $\mathrm{Cl}$ & 0 & $0.41^{*}$ & $\mathrm{NC}$ & $\mathrm{NC}$ & 0 & $<0.10^{\&}$ & $\mathrm{NC}$ & $\mathrm{NC}$ \\
\hline & $\mathrm{Cr}$ & 148 & 1.93 & 1.30 & 76.9 & 108 & 0.86 & 0.80 & 125 \\
\hline & $\mathrm{F}$ & 279 & 15.23* & 5.46 & 18.3 & 203 & $5.71^{8}$ & 2.81 & 35.6 \\
\hline & $\mathrm{Fe}$ & 1718 & 14.32 & 0.83 & 120 & 1249 & 1.43 & 0.11 & 872 \\
\hline & $\mathrm{K}$ & 48 & 2.76 & 5.71 & 17.5 & 35 & 0.95 & 2.69 & 37.1 \\
\hline & $\mathrm{Li}$ & 690 & 4.95 & 0.72 & 140 & 502 & 1.35 & 0.27 & 372 \\
\hline & Mg & 30 & 0.82 & 2.74 & 36.6 & 22 & $<0.10$ & $<0.45$ & $>220$ \\
\hline & $\mathrm{Na}$ & 2959 & 26.74 & 0.90 & 111 & 2152 & 7.08 & 0.33 & 304 \\
\hline & $\mathrm{Ni}$ & 131 & 0.92 & 0.70 & 142 & 95 & $<0.10$ & $<0.11$ & $>950$ \\
\hline & $\mathrm{P}$ & 191 & 0.78 & 0.41 & 246 & 139 & $<0.10$ & $<0.07$ & $>1390$ \\
\hline & $\mathrm{Pb}$ & 158 & 1.66 & 1.05 & 95.6 & 115 & 0.41 & 0.35 & 282 \\
\hline & $\mathrm{S}$ & 33 & $2.05 *$ & 6.21 & 16.1 & 24 & $1.52^{\&}$ & 6.33 & $\begin{array}{l}15.8 \\
\end{array}$ \\
\hline & $\mathrm{Si}$ & 5936 & 16.65 & 0.28 & 357 & 4317 & 1.39 & 0.03 & 3110 \\
\hline & $\mathrm{Ti}$ & 2 & $<0.10$ & $<5.00$ & $>20$ & 2 & $<0.10$ & $<5.00$ & $>20$ \\
\hline & $\mathrm{Zn}$ & 27 & 0.25 & 0.94 & 107 & 19 & 0.10 & 0.54 & 186 \\
\hline & $\mathrm{Zr}$ & 120 & 0.64 & 0.54 & 187 & 87 & $<0.10$ & $<0.11$ & $>870$ \\
\hline \multirow{4}{*}{ 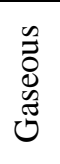 } & B & 1963 & 22.39 & 1.14 & 87.6 & 1427 & 21.16 & 1.48 & 67.5 \\
\hline & $\mathrm{Cl}$ & 0 & 3.14 & NC & NC & 0 & 2.79 & NC & NC \\
\hline & $\mathrm{F}$ & 279 & 82.79 & 29.68 & 3.4 & 203 & 81.42 & 40.14 & 2.5 \\
\hline & $\mathrm{S}$ & 33 & 11.78 & 35.31 & 2.8 & 24 & 14.80 & 61.00 & 1.6 \\
\hline
\end{tabular}

$\$$ - From gravimetric analysis of filters and particulate nitric acid rinses.

* Based on analysis of front-half rinse and water dissolution of filter particulate.

$\&$ Based on direct analysis of front-half rinse.

\# - Feed rate calculated from target composition and production rate.

NC - Not Calculated 
Table 7.1. Results from Melter Off-Gas Emission Samples (continued).

\begin{tabular}{|c|c|c|c|c|c|c|c|c|c|}
\hline & \multicolumn{4}{|c|}{ Test 6C } & \multicolumn{4}{|c|}{ Test 7A } \\
\hline & & \multicolumn{4}{|c|}{$\begin{array}{c}\text { 09/27/06 21:45 - 22:45 } \\
\text { 100.7\% Isokinetic, 9.3\% Moisture }\end{array}$} & \multicolumn{4}{|c|}{$\begin{array}{c}\text { 10/04/06 10:04 - 10:44 } \\
\text { 102.9\% Isokinetic, 13.5\% Moisture }\end{array}$} \\
\hline & & $\begin{array}{c}\text { Feed Rate } \\
(\mathrm{mg} / \mathrm{min})\end{array}$ & $\begin{array}{c}\text { Emissions } \\
\text { Rate } \\
\text { (mg/min) }\end{array}$ & $\%$ Feed & DF & $\begin{array}{c}\text { Feed Rate } \\
\text { (mg/min) }\end{array}$ & $\begin{array}{c}\text { Emissions } \\
\text { Rate } \\
\text { (mg/min) }\end{array}$ & $\%$ Feed & DF \\
\hline \multirow{23}{*}{ 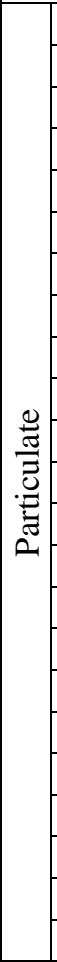 } & Total $^{\$}$ & 82980 & 438 & 0.53 & 189 & 114970 & 480 & 0.42 & 240 \\
\hline & $\mathrm{Al}$ & 9599 & 14.36 & 0.15 & 668 & 11963 & 12.66 & 0.11 & 945 \\
\hline & B & 3568 & 24.49 & 0.69 & 146 & 6042 & 39.63 & 0.66 & 152 \\
\hline & $\mathrm{Ba}$ & 34 & 1.75 & 5.16 & 19.4 & 28 & 0.42 & 1.49 & 67.0 \\
\hline & $\mathrm{Bi}$ & 774 & 5.94 & 0.77 & 130 & 1103 & 5.84 & 0.53 & 189 \\
\hline & $\mathrm{Ca}$ & 3290 & 12.84 & 0.39 & 256 & 545 & 2.98 & 0.55 & 183 \\
\hline & $\mathrm{Cd}$ & 14 & 0.18 & 1.33 & 75.1 & 9 & 0.17 & 1.77 & 56.4 \\
\hline & $\mathrm{Cl}^{*}$ & 0 & 0.52 & NC & NC & 0 & 0.59 & NC & $\mathrm{NC}$ \\
\hline & $\mathrm{Cr}$ & 269 & 2.63 & 0.98 & 102 & 515 & 7.48 & 1.45 & 68.8 \\
\hline & $\mathrm{F}^{*}$ & 507 & 30.97 & 6.11 & 16.37 & 244 & 19.34 & 7.93 & 12.6 \\
\hline & $\mathrm{Fe}$ & 3123 & 23.41 & 0.75 & 133 & 2090 & 7.75 & 0.37 & 270 \\
\hline & $\mathrm{K}$ & 88 & 3.68 & 4.18 & 23.9 & 581 & 8.29 & 1.43 & 70.0 \\
\hline & $\mathrm{Li}$ & 1255 & 7.72 & 0.61 & 163 & 1762 & 9.17 & 0.52 & 192 \\
\hline & $\mathrm{Mg}$ & 55 & 1.36 & 2.49 & 40.2 & 141 & 0.59 & 0.42 & 239 \\
\hline & $\mathrm{Na}$ & 5380 & 42.29 & 0.79 & 127 & 9993 & 72.03 & 0.72 & 139 \\
\hline & $\mathrm{Ni}$ & 238 & 1.58 & 0.66 & 151 & 83 & 0.40 & 0.48 & 208 \\
\hline & $\mathrm{P}$ & 347 & 1.36 & 0.39 & 256 & 935 & 4.89 & 0.52 & 191 \\
\hline & $\mathrm{Pb}$ & 288 & 2.80 & 0.97 & 103 & 89 & 0.88 & 1.00 & 100 \\
\hline & $\mathrm{S}^{*}$ & 61 & 5.62 & 9.21 & 10.9 & 93 & 14.83 & 15.95 & 6.3 \\
\hline & $\mathrm{Si}$ & 10792 & 30.94 & 0.29 & 349 & 17120 & 23.90 & 0.14 & 717 \\
\hline & $\mathrm{Ti}$ & 5 & $<0.10$ & $<2.00$ & $>50$ & 108 & 0.54 & 0.50 & 198 \\
\hline & $\mathrm{Zn}$ & 49 & 0.46 & 0.95 & 105 & 153 & 0.82 & 0.53 & 188 \\
\hline & $\mathrm{Zr}$ & 219 & 1.30 & 0.59 & 168 & 94 & 0.32 & 0.34 & 292 \\
\hline \multirow{4}{*}{$\left|\begin{array}{c}0 \\
0 \\
0 \\
心 \\
心 \\
心\end{array}\right|$} & B & 3568 & 37.87 & 1.06 & 94.2 & 6042 & 49.29 & 0.82 & 123 \\
\hline & $\mathrm{Cl}$ & 0 & 4.33 & NC & $\mathrm{NC}$ & 0 & 3.17 & NC & $\mathrm{NC}$ \\
\hline & $\mathrm{F}$ & 507 & 118.72 & 23.41 & 4.3 & 244 & 62.01 & 25.44 & 3.9 \\
\hline & $S$ & 61 & 15.52 & 25.58 & 3.9 & 93 & 22.65 & 24.24 & 4.1 \\
\hline
\end{tabular}

$\$$ - From gravimetric analysis of filters and particulate nitric acid rinses.

* Based on analysis of front-half rinse and water dissolution of filter particulate.

\# - Feed rate calculated from target composition and production rate.

NC - Not Calculated 
The Catholic University of America Vitreous State Laboratory
High Level Waste Vitrification System Improvements Final Report, VSL-07R1010-1, Rev. 0

Table 7.1. Results from Melter Off-Gas Emission Samples (continued).

\begin{tabular}{|c|c|c|c|c|c|c|c|c|c|}
\hline & \multicolumn{4}{|c|}{ Test 7B } & \multicolumn{4}{|c|}{ Test 8A } \\
\hline & & \multicolumn{4}{|c|}{$\begin{array}{c}\text { 10/06/06 12:32 - 13:32 } \\
\text { 101.9\% Isokinetic, } 10.0 \% \text { Moisture }\end{array}$} & \multicolumn{4}{|c|}{$\begin{array}{c}\text { 10/18/06 11:59 - 12:59 } \\
\text { 100.3\% Isokinetic, 6.1\% Moisture }\end{array}$} \\
\hline & & $\begin{array}{l}\text { Feed Rate } \\
\text { (mg/min) }\end{array}$ & $\begin{array}{c}\text { Emissions } \\
\text { Rate } \\
\text { (mg/min) }\end{array}$ & $\%$ Feed & DF & $\begin{array}{c}\text { Feed Rate } \\
(\mathrm{mg} / \mathrm{min})\end{array}$ & $\begin{array}{c}\text { Emissions } \\
\text { Rate (mg/min) }\end{array}$ & $\%$ Feed & DF \\
\hline \multirow{23}{*}{ 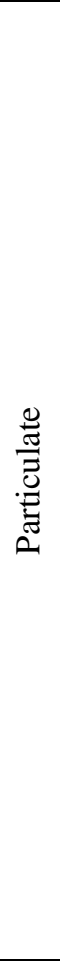 } & Total $^{\$}$ & 73910 & 69.4 & 0.09 & 1066 & 32850 & 19.7 & 0.06 & 1670 \\
\hline & $\mathrm{Al}$ & 7691 & 0.59 & 0.01 & 13075 & 3418 & 0.27 & 0.01 & 12731 \\
\hline & B & 3884 & 1.11 & 0.03 & 3484 & 1726 & 0.33 & 0.02 & 5242 \\
\hline & $\mathrm{Ba}$ & 18 & $<0.10$ & $<0.56$ & $>180$ & 8 & $<0.10$ & $<1.25$ & $>80$ \\
\hline & $\mathrm{Bi}$ & 709 & 0.71 & 0.10 & 993 & 315 & $<0.10$ & $<0.03$ & $>3150$ \\
\hline & $\mathrm{Ca}$ & 351 & 0.24 & 0.07 & 1463 & 156 & 0.63 & 0.40 & 248 \\
\hline & $\mathrm{Cd}$ & 6 & $<0.10$ & $<1.67$ & $>60$ & 3 & $<0.10$ & $<3.33$ & $>30$ \\
\hline & $\mathrm{Cl}^{\&}$ & 0 & $<0.10$ & NC & NC & 0 & $<0.10$ & NC & NC \\
\hline & $\mathrm{Cr}$ & 331 & 3.00 & 0.91 & 110 & 147 & 0.82 & 0.56 & 180 \\
\hline & $\mathrm{F}^{\&}$ & 157 & 8.50 & 5.41 & 18.5 & 70 & 0.63 & 0.90 & 111 \\
\hline & $\mathrm{Fe}$ & 1343 & 0.26 & 0.02 & 5189 & 597 & 0.23 & 0.04 & 2542 \\
\hline & $\mathrm{K}$ & 373 & 2.15 & 5.76 & 173 & 166 & 0.65 & 0.39 & 255 \\
\hline & $\mathrm{Li}$ & 1133 & 1.89 & 0.17 & 600 & 504 & 0.35 & 0.07 & 1429 \\
\hline & Mg & 90 & $<0.10$ & $<0.11$ & $>900$ & 40 & $<0.10$ & $<0.25$ & $>400$ \\
\hline & $\mathrm{Na}$ & 6424 & 12.82 & 0.20 & 501 & 2855 & 3.66 & 0.13 & 779 \\
\hline & $\mathrm{Ni}$ & 54 & $<0.10$ & $<0.19$ & $>540$ & 24 & $<0.10$ & $<0.42$ & $>240$ \\
\hline & $\mathrm{P}$ & 601 & $<0.10$ & $<0.02$ & $>6010$ & 267 & $<0.10$ & $<0.04$ & $>2670$ \\
\hline & $\mathrm{Pb}$ & 57 & 0.21 & 0.37 & 274 & 25 & $<0.10$ & $<0.40$ & $>250$ \\
\hline & $S^{\&}$ & 60 & 7.83 & 13.05 & 7.66 & 27 & 0.79 & 2.93 & 34.2 \\
\hline & $\mathrm{Si}$ & 11006 & 1.33 & 0.01 & 8252 & 4892 & 1.11 & 0.02 & 4424 \\
\hline & $\mathrm{Ti}$ & 69 & $<0.10$ & $<0.14$ & $>690$ & 31 & $<0.10$ & $<0.32$ & $>310$ \\
\hline & $\mathrm{Zn}$ & 99 & $<0.10$ & $<0.10$ & $>990$ & 44 & $<0.10$ & $<0.23$ & $>440$ \\
\hline & $\mathrm{Zr}$ & 61 & $<0.10$ & $<0.16$ & $>610$ & 27 & $<0.10$ & $<0.37$ & $>270$ \\
\hline \multirow{4}{*}{ 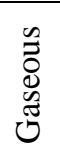 } & B & 3884 & 24.25 & 0.62 & 160 & 1726 & 4.77 & 0.28 & 362 \\
\hline & $\mathrm{Cl}$ & 0 & 3.02 & NC & $\mathrm{NC}$ & 0 & 0.41 & NC & NC \\
\hline & $\mathrm{F}$ & 157 & 65.46 & 41.78 & 2.4 & 70 & 4.39 & 6.31 & 15.9 \\
\hline & $\mathrm{S}$ & 60 & 19.64 & 32.70 & 3.1 & 27 & 8.30 & 31.09 & 3.2 \\
\hline
\end{tabular}

$\$$ - From gravimetric analysis of filters and particulate nitric acid rinses.

\& Based on direct analysis of front-half rinse.

\# - Feed rate calculated from target composition and production rate.

NC - Not Calculated 
Table 7.1. Results from Melter Off-Gas Emission Samples (continued).

\begin{tabular}{|c|c|c|c|c|c|c|c|c|c|}
\hline & \multicolumn{4}{|c|}{ Test 8B } & \multicolumn{4}{|c|}{ Test 8C } \\
\hline & & \multicolumn{4}{|c|}{$\begin{array}{c}\text { 10/20/06 14:55 - 15:55 } \\
\text { 101.2\% Isokinetic, 6.3\% Moisture }\end{array}$} & \multicolumn{4}{|c|}{$\begin{array}{c}\text { 11/19/06 22:15 - 23:15 } \\
\text { 98.9\% Isokinetic, 8.6\% Moisture }\end{array}$} \\
\hline & & $\begin{array}{c}\text { Feed Rate }^{\#} \\
(\mathrm{mg} / \mathrm{min})\end{array}$ & $\begin{array}{c}\text { Emissions } \\
\text { Rate } \\
\text { (mg/min) }\end{array}$ & $\%$ Feed & DF & $\begin{array}{l}\text { Feed Rate }^{\#} \\
\text { (mg/min) }\end{array}$ & $\begin{array}{c}\text { Emissions } \\
\text { Rate } \\
\text { (mg/min) }\end{array}$ & \% Feed & DF \\
\hline \multirow{23}{*}{ 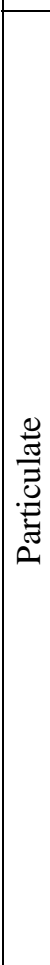 } & Total $^{\$}$ & 14600 & 24.6 & 0.17 & 594 & 102653 & 122 & 0.12 & 840 \\
\hline & $\mathrm{Al}$ & 1709 & 0.40 & 0.02 & 4310 & 10682 & 2.09 & 0.02 & 5104 \\
\hline & B & 863 & 1.22 & 0.14 & 709 & 5394 & 8.30 & 0.15 & 650 \\
\hline & $\mathrm{Ba}$ & 4 & $<0.10$ & $<2.50$ & $>40$ & 25 & 0.11 & 0.42 & 238 \\
\hline & $\mathrm{Bi}$ & 158 & $<0.10$ & $<0.06$ & $>1580$ & 985 & 1.04 & 0.11 & 948 \\
\hline & Ca & 78 & 0.59 & 0.76 & 132 & 487 & 0.67 & 0.14 & 732 \\
\hline & $\mathrm{Cd}$ & 1 & $<0.10$ & $<10.00$ & $>10$ & 8 & $<0.10$ & $<1.25$ & $>80$ \\
\hline & $\mathrm{Cl}$ & 0 & $<0.10^{\&}$ & NC & NC & 0 & $0.59 *$ & NC & NC \\
\hline & $\mathrm{Cr}$ & 74 & 0.94 & 1.28 & 78.2 & 460 & 3.73 & 0.81 & 123 \\
\hline & $F$ & 35 & $0.55^{8}$ & 1.57 & 63.6 & 218 & 4.66* & 2.14 & 46.8 \\
\hline & $\mathrm{Fe}$ & 299 & 0.51 & 0.17 & 580 & 1866 & 1.66 & 0.09 & 1121 \\
\hline & $\bar{K}$ & 83 & 0.68 & 0.82 & 122 & 518 & 3.08 & 0.59 & 168 \\
\hline & $\mathrm{Li}$ & 252 & 0.39 & 0.15 & 648 & 1573 & 2.33 & 0.15 & 676 \\
\hline & $\mathrm{Mg}$ & 20 & $<0.10$ & $<0.50$ & $>200$ & 126 & $<0.10$ & $<0.08$ & $>1260$ \\
\hline & $\mathrm{Na}$ & 1428 & 4.45 & 0.31 & 321 & 8922 & 19.56 & 0.22 & 456 \\
\hline & $\mathrm{Ni}$ & 12 & $<0.10$ & $<0.83$ & $>120$ & 74 & $<0.10$ & $<0.14$ & $>740$ \\
\hline & $\bar{P}$ & 134 & $<0.10$ & $<0.07$ & $>1340$ & 835 & 0.63 & 0.08 & 1333 \\
\hline & $\mathrm{Pb}$ & 13 & $<0.10$ & $<0.77$ & $>130$ & 79 & 0.25 & 0.31 & 322 \\
\hline & $\mathrm{S}$ & 13 & $0.57^{\&}$ & 4.38 & 22.8 & 83 & $6.87^{*}$ & 8.28 & 12.1 \\
\hline & $\mathrm{Si}$ & 2446 & 1.00 & 0.04 & 2445 & 15286 & 5.37 & 0.04 & 2849 \\
\hline & $\mathrm{Ti}$ & 15 & $<0.10$ & $<0.67$ & $>150$ & 96 & $<0.10$ & $<0.01$ & $>960$ \\
\hline & Zn & 22 & $<0.10$ & $<0.45$ & $>220$ & 137 & 0.26 & 0.19 & 535 \\
\hline & $\mathrm{Zr}$ & 13 & $<0.10$ & $<0.77$ & $>130$ & 84 & $<0.10$ & $<0.12$ & $>840$ \\
\hline \multirow{4}{*}{$\begin{array}{l}0 \\
0 \\
0 \\
\tilde{d} \\
0 \\
0\end{array}$} & B & 863 & 7.15 & 0.83 & 121 & 5394 & 20.21 & 0.37 & 267 \\
\hline & $\mathrm{Cl}$ & 0 & 0.42 & NC & $\mathrm{NC}$ & 0 & 0.53 & NC & NC \\
\hline & $\bar{F}$ & 35 & 8.79 & 25.25 & 4.0 & 218 & 20.16 & 9.27 & 10.8 \\
\hline & $S$ & 13 & 8.20 & 61.43 & 1.6 & 83 & 13.74 & 16.47 & 6.1 \\
\hline
\end{tabular}

$\$$ - From gravimetric analysis of filters and particulate nitric acid rinses.

* Based on direct analysis of front-half rinse and water dissolution of filter particulate.

$\&$ Based on direct analysis of front-half rinse.

\# - Feed rate calculated from target composition and production rate.

NC - Not Calculated 
Table 7.2. Average Concentration (ppmv) of Selected Species in Off-Gas Measured by FTIR Spectroscopy.

\begin{tabular}{|c|c|c|c|c|c|c|c|c|c|c|c|c|}
\hline \multirow{3}{*}{ Test } & \multicolumn{8}{|c|}{ Bi-Limited } & \multicolumn{4}{|c|}{ Cr-Limited } \\
\hline & \multicolumn{2}{|r|}{$1 \mathrm{~A}$} & \multicolumn{2}{|r|}{$1 \mathrm{~B}$} & \multicolumn{2}{|r|}{$2 \mathrm{~A}$} & \multicolumn{2}{|r|}{$2 B$} & \multicolumn{2}{|r|}{$3 B$} & \multicolumn{2}{|r|}{$4 \mathrm{~A}$} \\
\hline & Avg. & Range & Avg. & Range & Avg. & Range & Avg. & Range & Avg. & Range & Avg. & Range \\
\hline $\mathrm{N}_{2} \mathrm{O}$ & 1.6 & $<1.0-14.2$ & 1.2 & $<1.0-8.3$ & 1.2 & $<1.0-12.4$ & $<1.0$ & $<1.0-4.0$ & $<1.0$ & $<1.0-3.1$ & $<1.0$ & $<1.0-2.1$ \\
\hline $\mathrm{NO}$ & 193 & $<1.0-1361$ & 152 & $31.6-486$ & 117 & $21.5-774$ & 72.9 & $<1.0-396$ & 59.2 & $8.8-193$ & 51.2 & $9.4-136$ \\
\hline $\mathrm{NO}_{2}$ & 20.4 & $<1.0-221$ & 13.8 & $<1.0-79.7$ & 11.2 & $<1.0-114$ & 5.0 & $<1.0-53.2$ & $<1.0$ & $<1.0-3.6$ & $<1.0$ & $<1.0-4.5$ \\
\hline $\mathrm{NH}_{3}$ & $<1.0$ & $<1.0-4.5$ & $<1.0$ & $<1.0-1.4$ & $<1.0$ & NA & $<1.0$ & NA & $<1.0$ & NA & $<1.0$ & NA \\
\hline $\mathrm{H}_{2} \mathrm{O}[\%]$ & 7.6 & $<1.0-13.1$ & 6.1 & $3.7-13.6$ & 5.3 & $2.3-23.1$ & 6.9 & $1.7-20.4$ & 7.5 & $2.8-19.2$ & 6.9 & $2.4-19.2$ \\
\hline $\mathrm{CO}_{2}$ & 1204 & $8-4975$ & 1022 & $361-2843$ & 906 & $302-3924$ & 898 & $494-2452$ & 1878 & $934-5794$ & 1709 & $924-3770$ \\
\hline Nitrous Acid & $<1.0$ & $<1.0-2.8$ & $<1.0$ & NA & $<1.0$ & $<1.0-2.2$ & $<1.0$ & NA & $<1.0$ & NA & $<1.0$ & NA \\
\hline Nitric Acid & $<1.0$ & NA & $<1.0$ & NA & $<1.0$ & $<1.0-5.5$ & $<1.0$ & NA & $<1.0$ & NA & $<1.0$ & NA \\
\hline HCN & $<1.0$ & NA & $<1.0$ & NA & $<1.0$ & NA & $<1.0$ & NA & $<1.0$ & NA & $<1.0$ & NA \\
\hline $\mathrm{SO}_{2}$ & 3.7 & $<1.0-8.8$ & 4.5 & $<1.0-9.0$ & 3.2 & $<1.0-7.8$ & 4.1 & $<1.0-9.2$ & 8.0 & $<1.0-12.7$ & 5.5 & $<1.0-9.0$ \\
\hline $\mathrm{CO}$ & 1.6 & $<1.0-12.6$ & 1.1 & $<1.0-6.3$ & 1.0 & $<1.0-7.7$ & $<1.0$ & $<1.0-6.1$ & 1.1 & $<1.0-3.0$ & $<1.0$ & $<1.0-2.0$ \\
\hline $\mathrm{HCl}$ & $<1.0$ & $<1.0-1.6$ & 1.7 & $<1.0-7.1$ & 3.1 & $<1.0-8.3$ & 1.8 & $<1.0-4.4$ & 2.3 & $<1.0-5.4$ & 1.7 & $<1.0-3.1$ \\
\hline $\mathrm{HF}$ & 1.3 & $<1.0-4.9$ & 5.7 & $1.2-20.3$ & 15.2 & $4.0-29.4$ & 19.4 & $10.7-33.1$ & 17.6 & $1.1-35.9$ & 22.4 & $13.8-27.1$ \\
\hline
\end{tabular}

NA - Not applicable. 
Table 7.2. Average Concentration (ppmv) of Selected Species in Off-Gas Measured by FTIR Spectroscopy (continued).

\begin{tabular}{|c|c|c|c|c|c|c|c|c|c|c|}
\hline \multirow{3}{*}{ Test } & \multicolumn{10}{|c|}{ Al-Limited } \\
\hline & \multicolumn{2}{|r|}{$5 \mathrm{~A}$} & \multicolumn{2}{|r|}{$5 B$} & \multicolumn{2}{|c|}{$6 \mathrm{~A}$} & \multicolumn{2}{|r|}{$6 \mathrm{~B}$} & \multicolumn{2}{|r|}{$6 \mathrm{C}$} \\
\hline & Avg. & Range & Avg. & Range & Avg. & Range & Avg. & Range & Avg. & Range \\
\hline $\mathrm{N}_{2} \mathrm{O}$ & $<1.0$ & $<1.0-1.2$ & $<1.0$ & $<1.0-1.0$ & $<1.0$ & $<1.0-1.3$ & $<1.0$ & NA & $<1.0$ & $<1.0-1.3$ \\
\hline $\mathrm{NO}$ & 74.0 & $<1.0-460$ & 40.3 & $0-273$ & 34.9 & $<1.0-368$ & 19.6 & $<1.0-152$ & 64.8 & $<1.0-449$ \\
\hline $\mathrm{NO}_{2}$ & 4.0 & $<1.0-71.3$ & 3.4 & $<1.0-61.8$ & 3.4 & $<1.0-70.9$ & 2.2 & $<1.0-32.4$ & 5.8 & $<1.0-86.5$ \\
\hline $\mathrm{NH}_{3}$ & $<1.0$ & NA & $<1.0$ & NA & $<1.0$ & NA & $<1.0$ & $<1.0-4.9$ & $<1.0$ & NA \\
\hline $\mathrm{H}_{2} \mathrm{O}[\%]$ & 6.4 & $3.1-19.6$ & 4.6 & $1.7-16.3$ & 4.1 & $2.0-19.2$ & 4.9 & $1.3-18.5$ & 5.8 & $2.7-22.5$ \\
\hline $\mathrm{CO}_{2}$ & 1263 & $380-5233$ & 978 & $498-3971$ & 935 & $476-5359$ & 862 & $468-2958$ & 1087 & $245-5319$ \\
\hline Nitrous Acid & $<1.0$ & NA & $<1.0$ & NA & $<1.0$ & NA & $<1.0$ & NA & $<1.0$ & $<1.0-1.4$ \\
\hline Nitric Acid & $<1.0$ & NA & $<1.0$ & NA & $<1.0$ & NA & $<1.0$ & NA & $<1.0$ & NA \\
\hline $\mathrm{HCN}$ & $<1.0$ & NA & $<1.0$ & NA & $<1.0$ & NA & $<1.0$ & NA & $<1.0$ & NA \\
\hline $\mathrm{SO}_{2}$ & 4.1 & $<1.0-15.1$ & $<1.0$ & $<1.0-3.7$ & 1.2 & $<1.0-4.2$ & $<1.0$ & $<1.0-13.9$ & $<1.0$ & $<1.0-16.4$ \\
\hline $\mathrm{CO}$ & $<1.0$ & $<1.0-1.7$ & $<1.0$ & $<1.0-1.0$ & $<1.0$ & NA & $<1.0$ & $<1.0-1.9$ & $<1.0$ & $<1.0-29.1$ \\
\hline $\mathrm{HCl}$ & 1.7 & $<1.0-3.4$ & $<1.0$ & $<1.0-1.8$ & $<1.0$ & $<1.0-1.5$ & $<1.0$ & $<1.0-2.8$ & 1.4 & $<1.0-2.9$ \\
\hline $\mathrm{HF}$ & 21.9 & $12.4-37.1$ & 15.4 & $7.3-25.3$ & 16.1 & $9.2-25.7$ & 11.2 & $3.9-24.6$ & 25.0 & $10.5-38.6$ \\
\hline
\end{tabular}

NA - Not applicable. 
Table 7.2. Average Concentration (ppmv) of Selected Species in Off-Gas Measured by FTIR Spectroscopy (continued).

\begin{tabular}{|c|c|c|c|c|c|c|c|c|c|c|}
\hline \multirow{3}{*}{ Test } & \multicolumn{10}{|c|}{$\mathrm{Al}+\mathrm{Na}$-Limited } \\
\hline & \multicolumn{2}{|r|}{ 7A } & \multicolumn{2}{|r|}{$7 \mathrm{~B}$} & \multicolumn{2}{|r|}{$8 \mathrm{~A}$} & \multicolumn{2}{|r|}{$8 B$} & \multicolumn{2}{|r|}{$8 C$} \\
\hline & Avg. & Range & Avg. & Range & Avg. & Range & Avg. & Range & Avg. & Range \\
\hline $\mathrm{N}_{2} \mathrm{O}$ & $<1.0$ & NA & $<1.0$ & NA & $<1.0$ & NA & $<1.0$ & NA & 2.7 & $<1.0-2.7$ \\
\hline $\mathrm{NO}$ & 99.4 & $<1.0-566$ & 72.0 & $11.7-304$ & 35.9 & $<1.0-249$ & 13.5 & $<1.0-166$ & 93.4 & $<1.0-636$ \\
\hline $\mathrm{NO}_{2}$ & 6.1 & $<1.0-79.4$ & 4.3 & $<1.0-47.9$ & 8.0 & $<1.0-53.2$ & $<1.0$ & $<1.0-24.4$ & 3.4 & $<1.0-78.1$ \\
\hline $\mathrm{NH}_{3}$ & $<1.0$ & NA & $<1.0$ & NA & $<1.0$ & NA & $<1.0$ & NA & $<1.0$ & $<1.0-6.7$ \\
\hline $\mathrm{H}_{2} \mathrm{O}[\%]$ & 7.7 & $2.9-22.4$ & 6.2 & $1.6-16.2$ & 4.3 & $1.1-16.7$ & 4.2 & $2.4-15.1$ & 7.5 & $0.6-30.0$ \\
\hline $\mathrm{CO}_{2}$ & 1432 & $478-7255$ & 1159 & $545-3968$ & 941 & $425-4626$ & 754 & $296-3211$ & 1333 & $421-7823$ \\
\hline Nitrous Acid & $<1.0$ & $<1.0-1.0$ & $<1.0$ & NA & $<1.0$ & NA & $<1.0$ & NA & $<1.0$ & $<1.0-1.6$ \\
\hline Nitric Acid & $<1.0$ & NA & $<1.0$ & NA & $<1.0$ & NA & $<1.0$ & NA & $<1.0$ & $<1.0-1.5$ \\
\hline $\mathrm{HCN}$ & $<1.0$ & NA & $<1.0$ & NA & $<1.0$ & NA & $<1.0$ & NA & $<1.0$ & NA \\
\hline $\mathrm{SO}_{2}$ & 1.7 & $<1.0-5.2$ & 1.9 & $<1.0-5.8$ & $<1.0$ & $<1.0-3.4$ & $<1.0$ & $<1.0-2.2$ & 1.6 & $<1.0-5.4$ \\
\hline $\mathrm{CO}$ & $<1.0$ & $<1.0-1.4$ & $<1.0$ & NA & $<1.0$ & NA & $<1.0$ & NA & $<1.0$ & $<1.0-22.8$ \\
\hline $\mathrm{HCl}$ & 1.1 & $<1.0-4.2$ & $<1.0$ & NA & $<1.0$ & $<1.0-2.5$ & $<1.0$ & NA & 1.4 & $<1.0-2.9$ \\
\hline $\mathrm{HF}$ & 20.5 & $12.1-25.7$ & 10.5 & $2.9-14.5$ & 3.2 & $<1.0-8.4$ & 2.2 & $1.0-3.7$ & 3.8 & $1.0-6.9$ \\
\hline
\end{tabular}

NA - Not applicable. 
The Catholic University of America Vitreous State Laboratory
High Level Waste Vitrification System Improvements Final Report, VSL-07R1010-1, Rev. 0

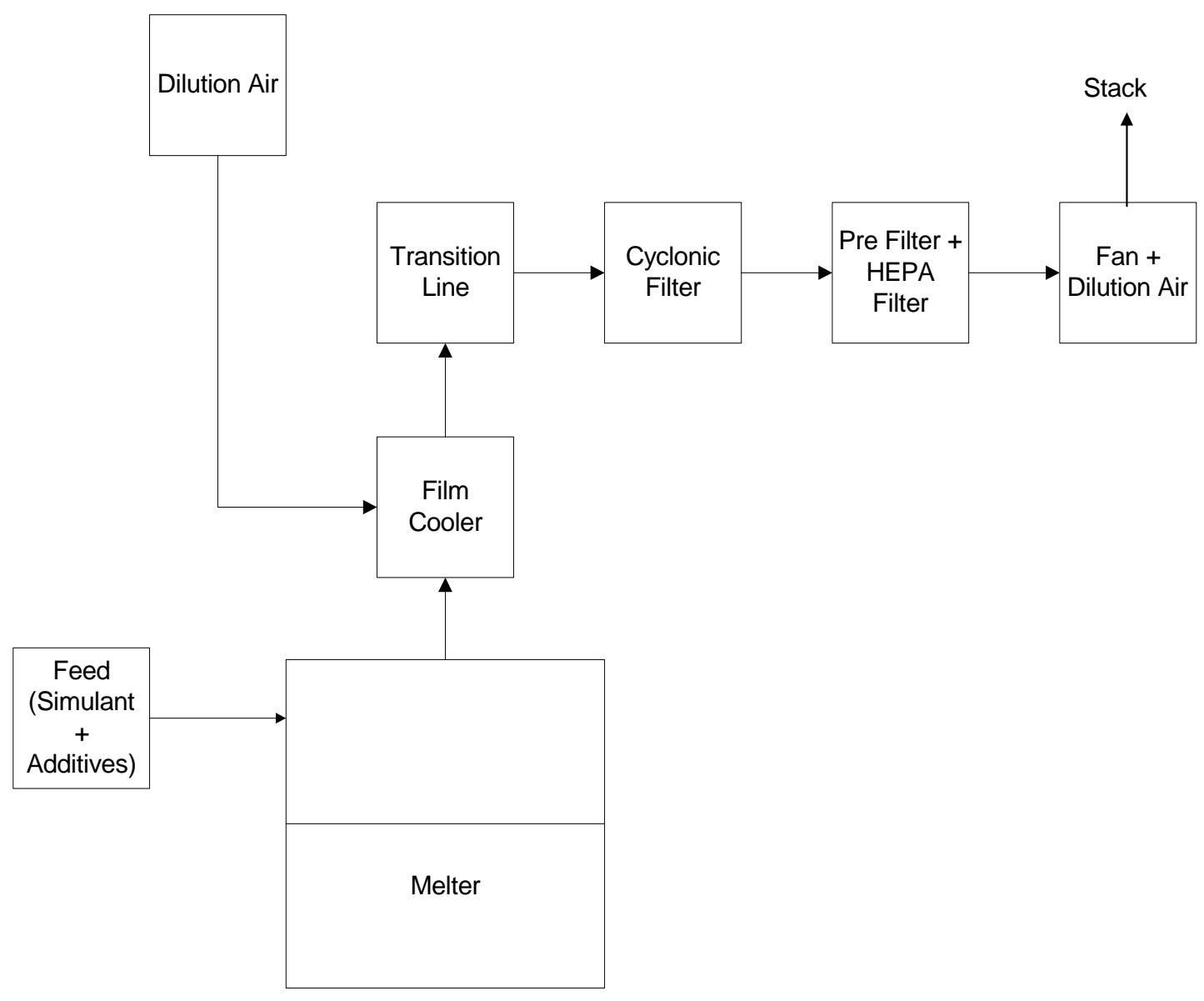

Figure 1.1. Schematic diagram of DuraMelter 100-BL vitrification system. 
The Catholic University of America Vitreous State Laboratory
High Level Waste Vitrification System Improvements Final Report, VSL-07R1010-1, Rev. 0

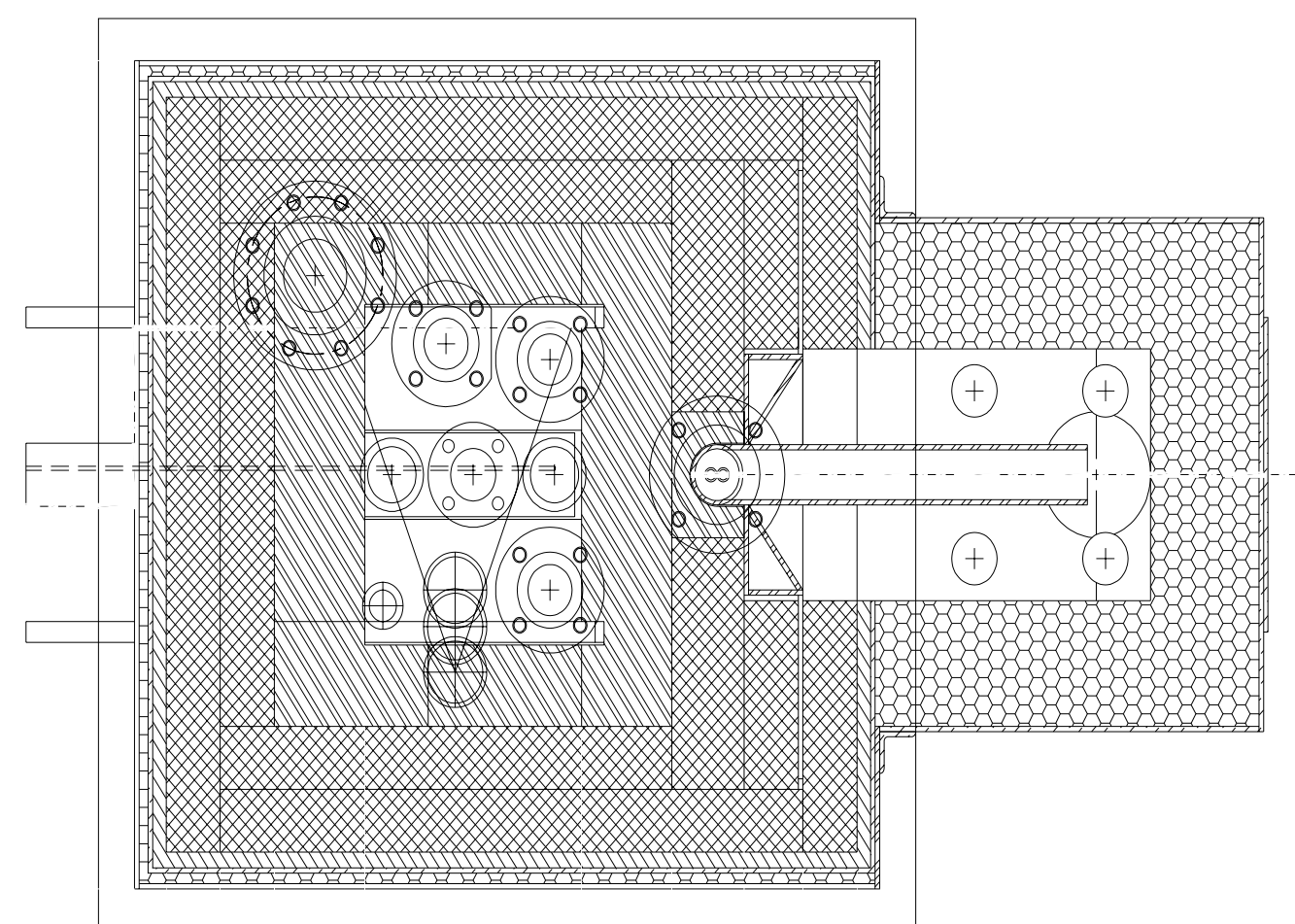

Figure 1.2.a. Schematic diagram showing cross-section through the DM100-BL-melter. Plan view showing locations of lid ports. 
The Catholic University of America Vitreous State Laboratory
High Level Waste Vitrification System Improvements Final Report, VSL-07R1010-1, Rev. 0

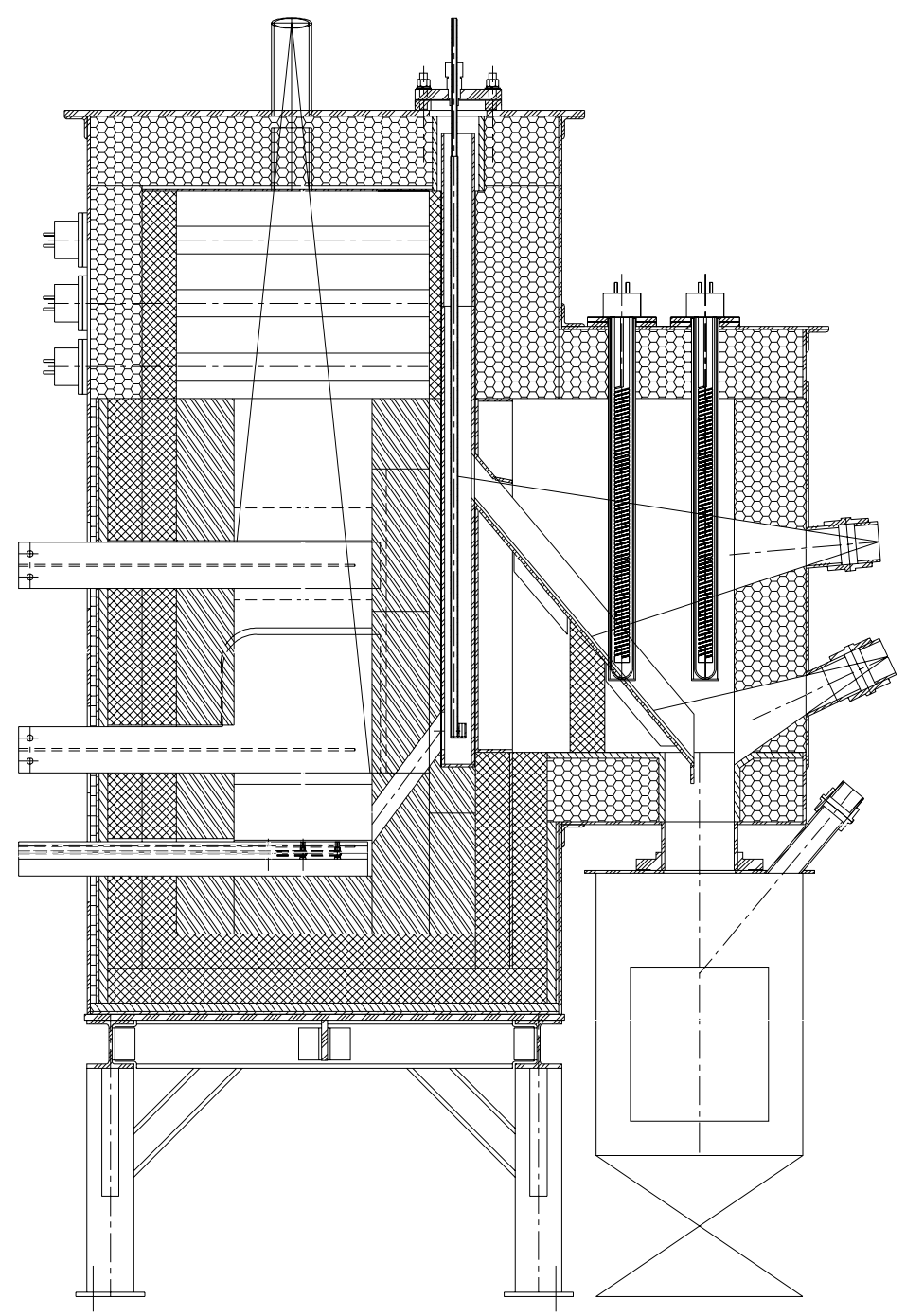

Figure 1.2.b. Schematic diagram showing cross-section through the DM100-BL melter. 
The Catholic University of America Vitreous State Laboratory
High Level Waste Vitrification System Improvements Final Report, VSL-07R1010-1, Rev. 0

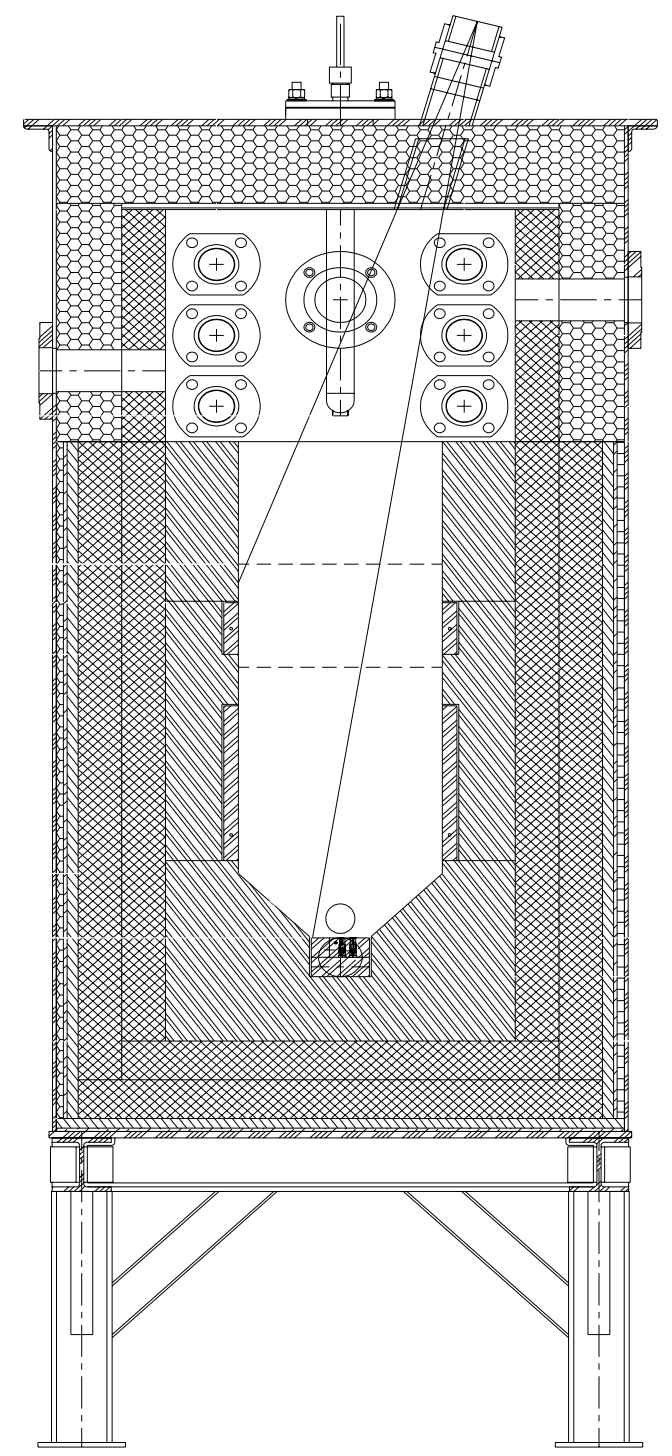

Figure 1.2.c. Schematic diagram showing cross-section through the DM100-BL melter. 


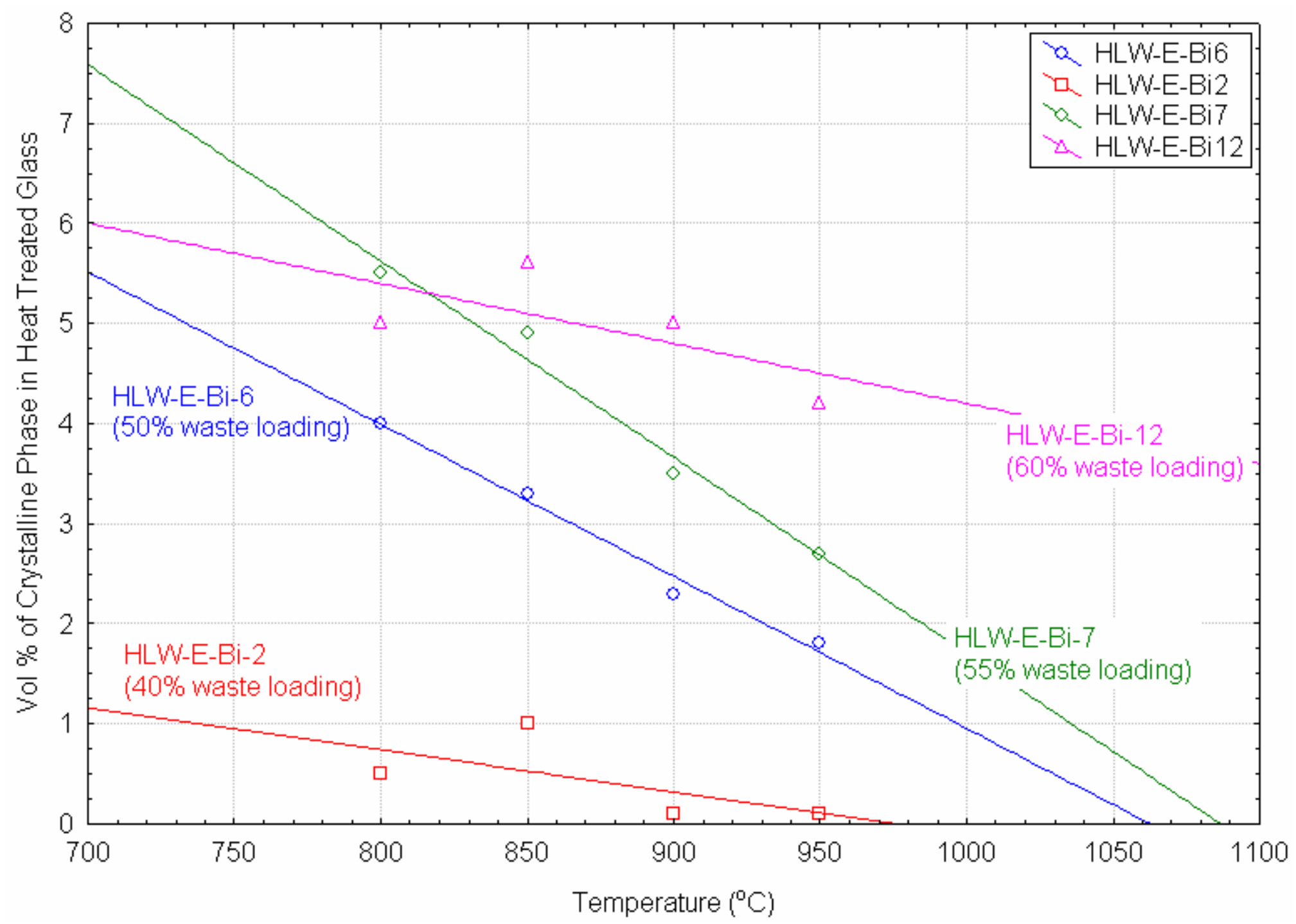

Figure 3.1. Volume \% of crystalline phase in the heat treated glasses vs. heat treatment temperature. Lines are from linear regression of the available data points of each glass. 


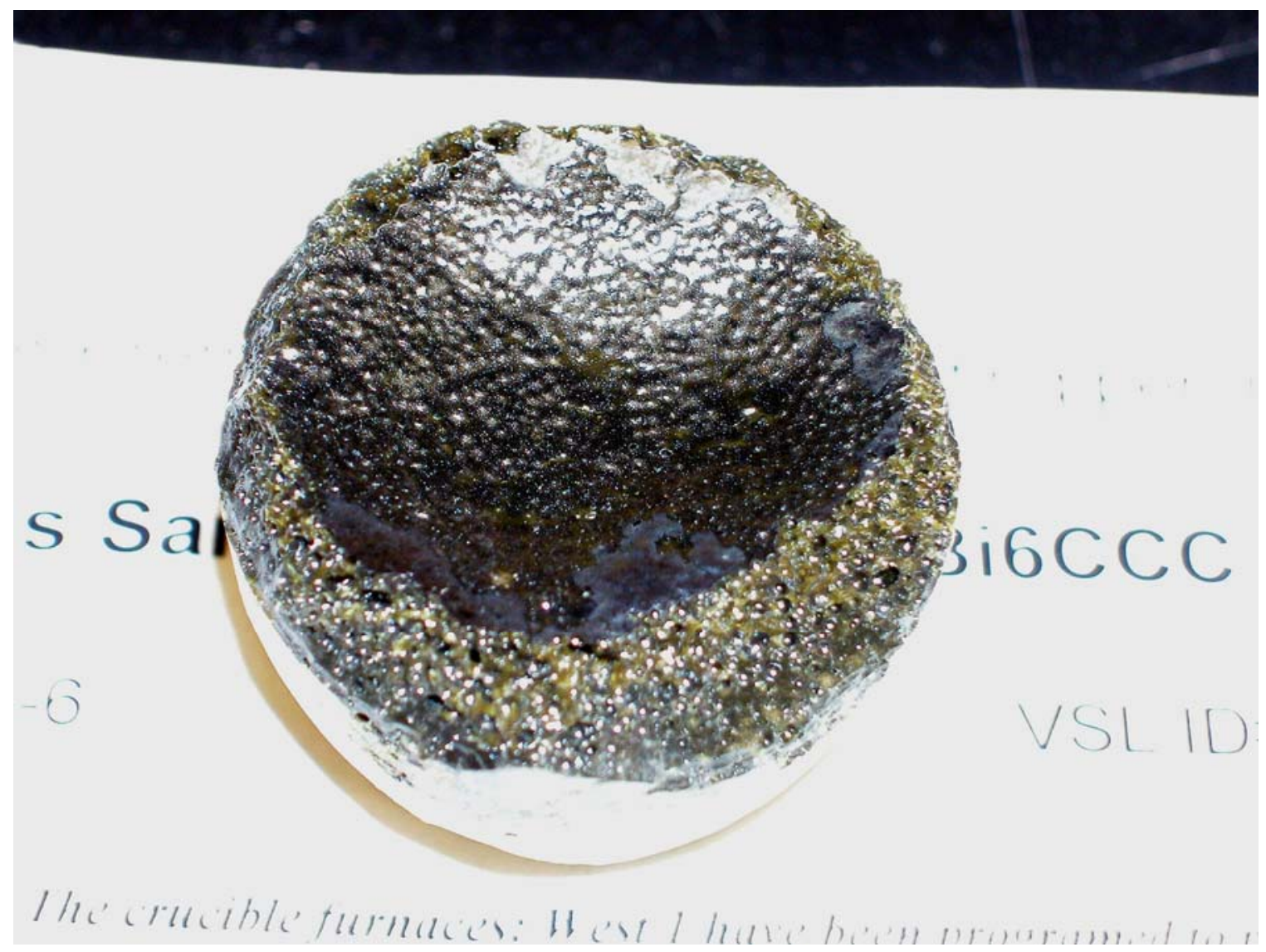

Figure 3.2. Top view of CCC sample of HLW-E-Bi6. Foaming observed is concentrated near the top of the sample. 


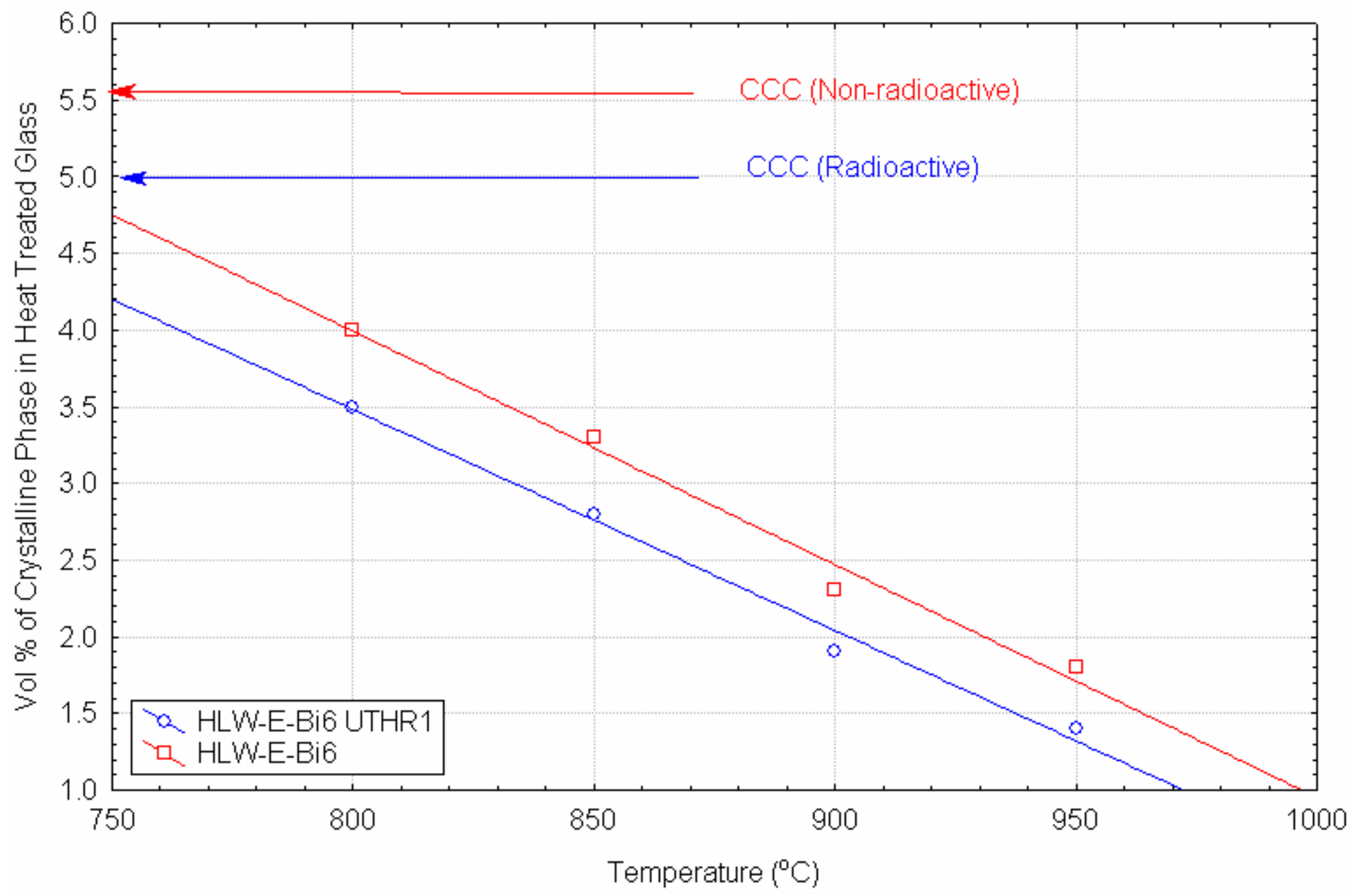

Figure 3.3. Volume $\%$ of crystalline phase in the heat treated glasses vs. heat treatment temperature for HLW-E-Bi-6 and HLW-E-BiUThR1. Two arrows indicate the volume\% of crystals in the corresponding samples after CCC treatment. 


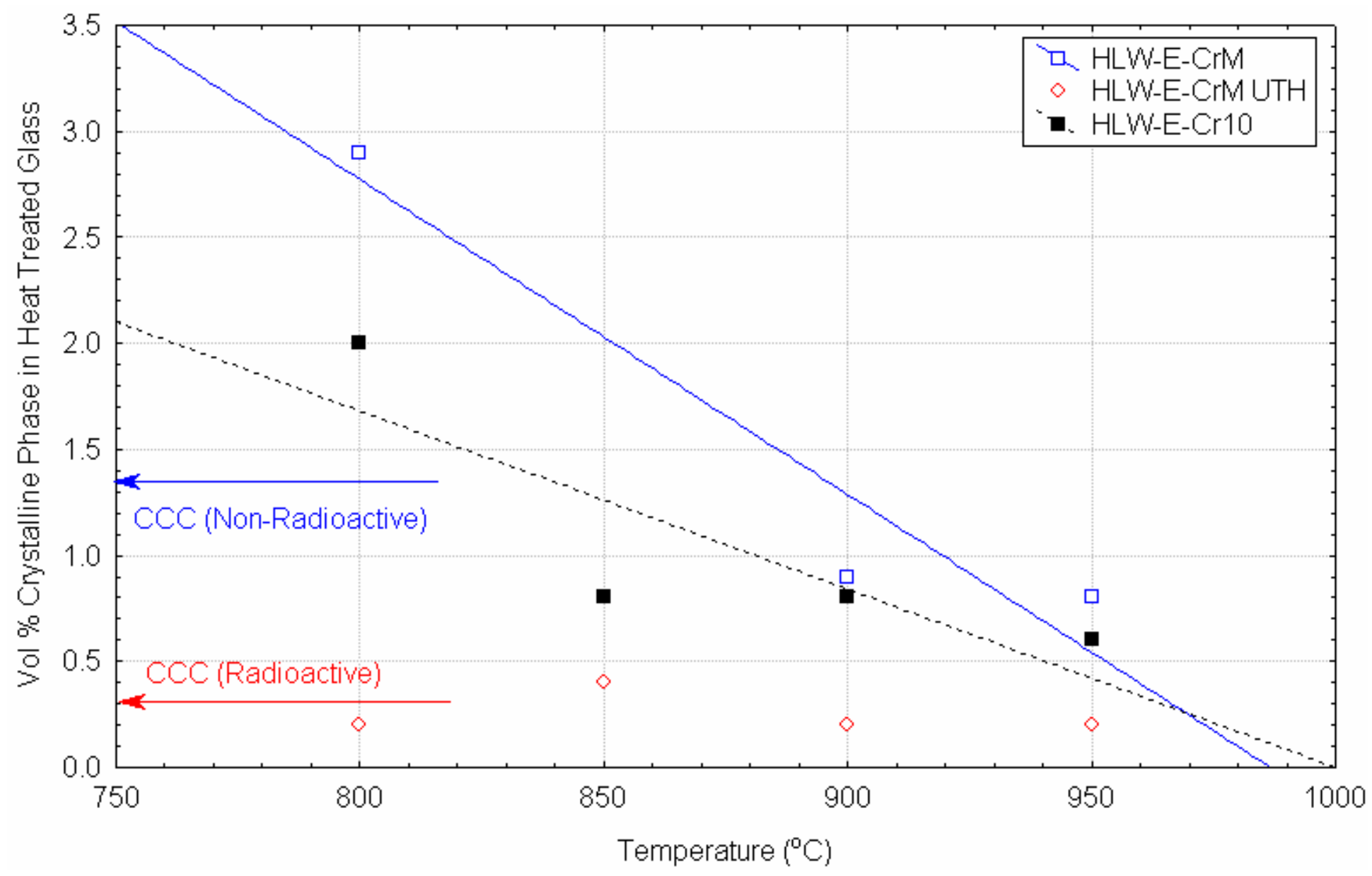

Figure 3.4. Volume\% of crystalline phase in the heat treated glasses vs. heat treatment temperature for HLW-E-Cr-M and HLW-E-CrMUTh. Two arrows indicate the volume\% of crystals in the corresponding samples after CCC treatment. The result from HLW-E-Cr-10 is also plotted for comparison. 


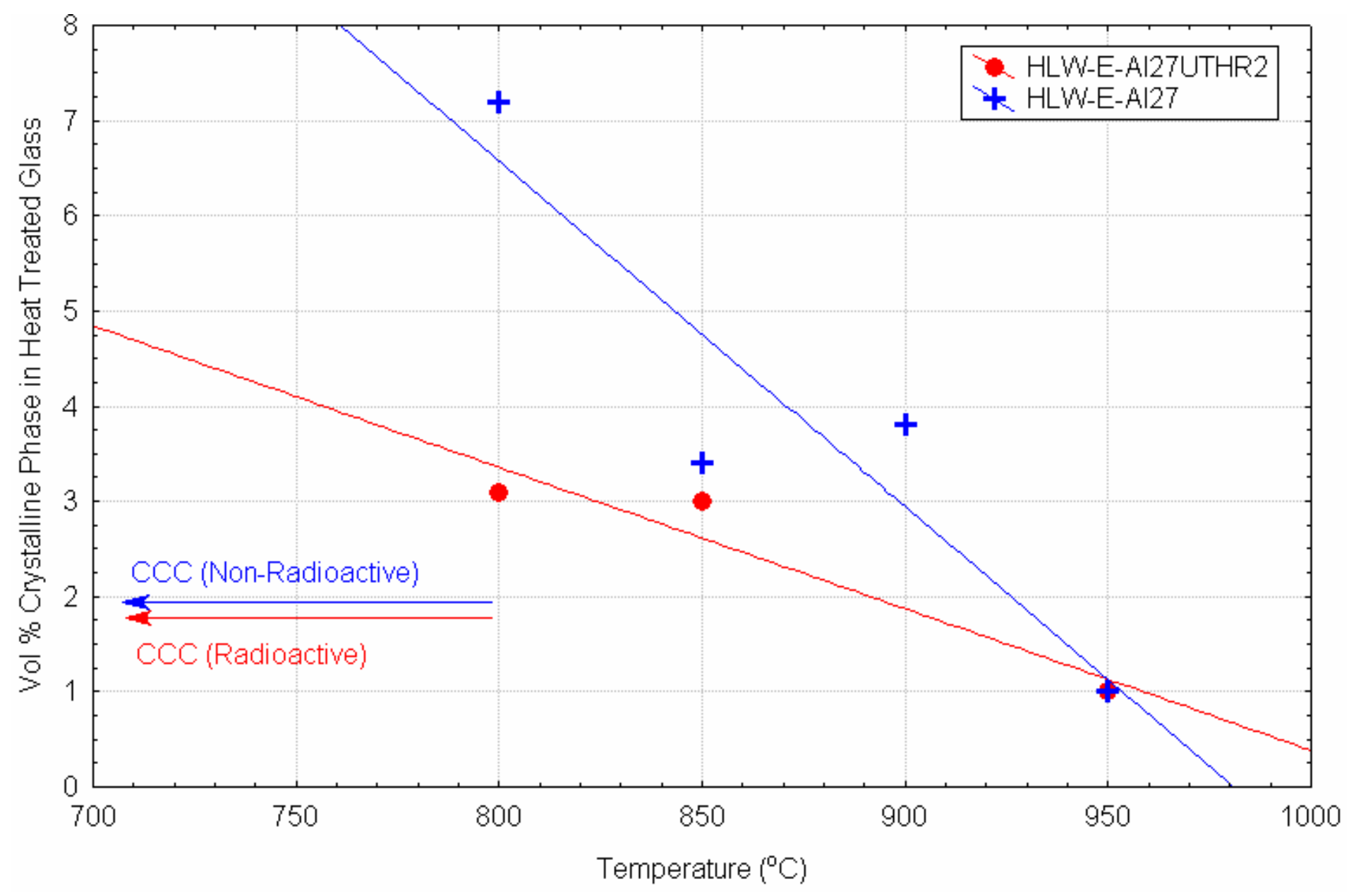

Figure 3.5. Volume\% of crystalline phase in the heat treated glasses vs. heat treatment temperature for HLW-E-Al-27 and HLW-E-Al-27iUThR2. Two arrows indicate the volume \% of crystals in the corresponding samples after CCC treatment. 


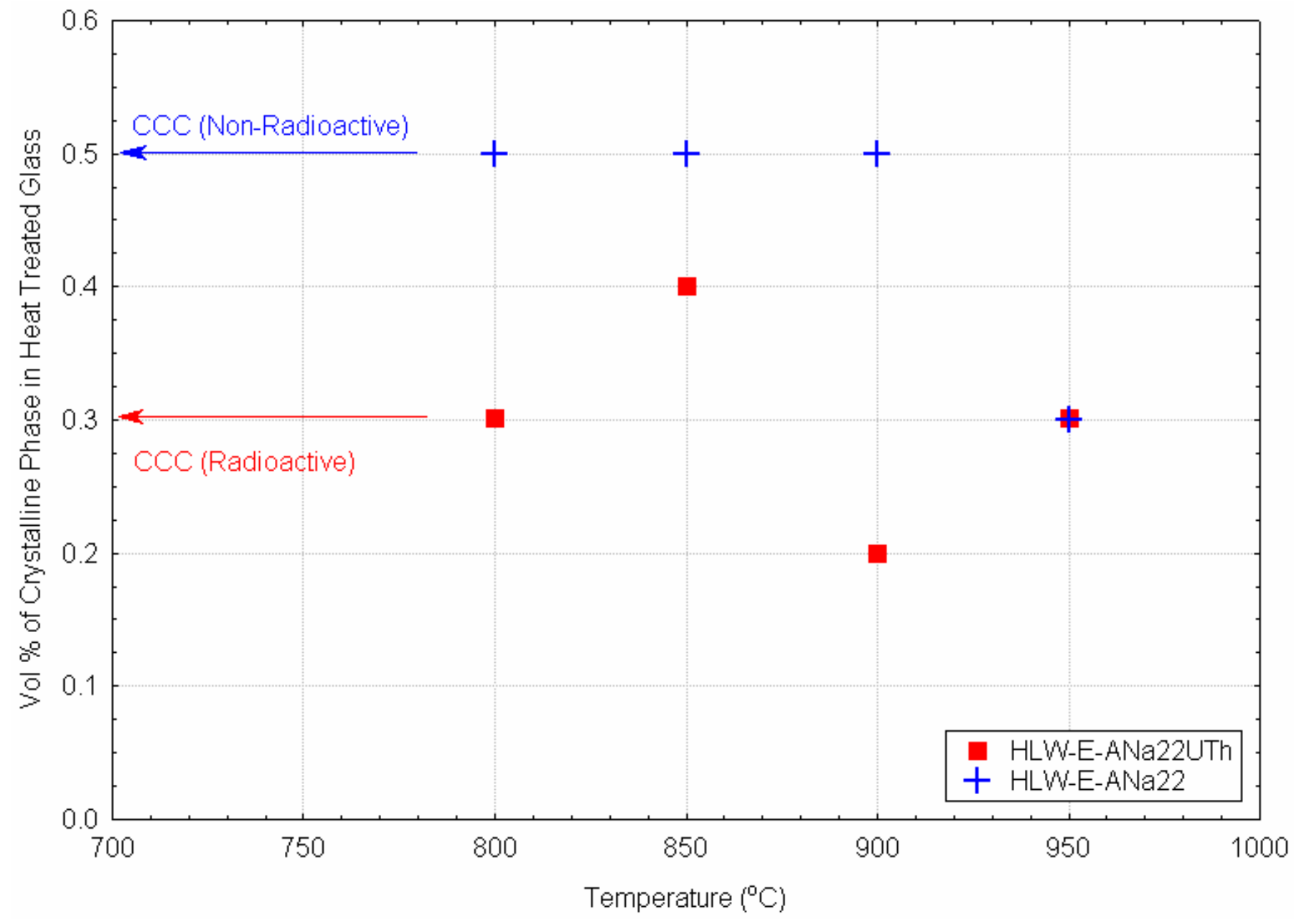

Figure 3.6. Volume\% of crystalline phase in the heat treated glasses vs. heat treatment temperature for HLW-E-ANa-22 and HLW-E-ANa22UTh. Two arrows indicate the volume\% of crystals in the corresponding samples after CCC treatment. 
The Catholic University of America Vitreous State Laboratory
High Level Waste Vitrification System Improvements Final Report, VSL-07R1010-1, Rev. 0
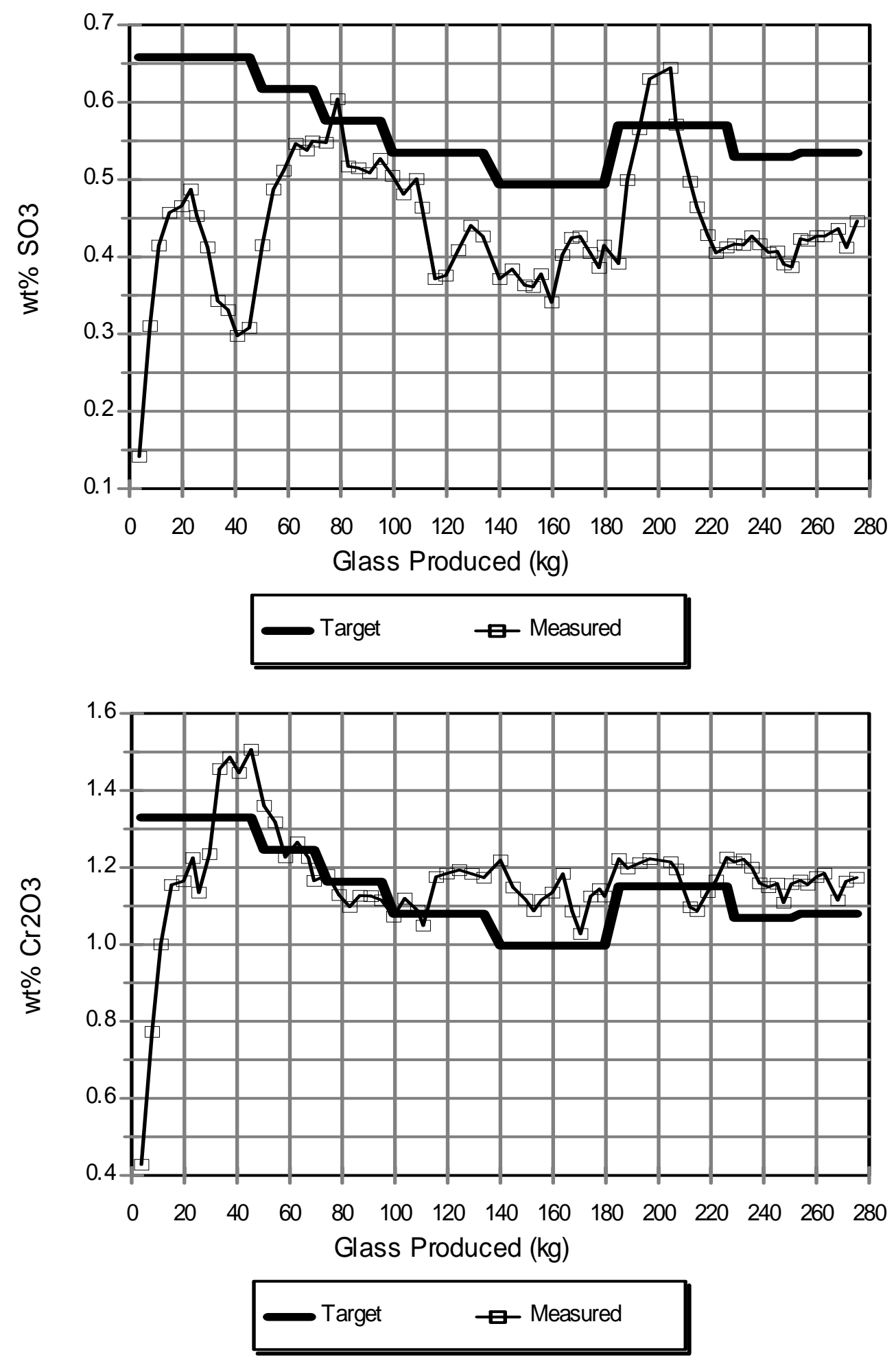

Figure 4.1. Sulfur and chromium concentrations measured by XRF in glasses from DM10 tests. 
The Catholic University of America Vitreous State Laboratory
High Level Waste Vitrification System Improvements Final Report, VSL-07R1010-1, Rev. 0
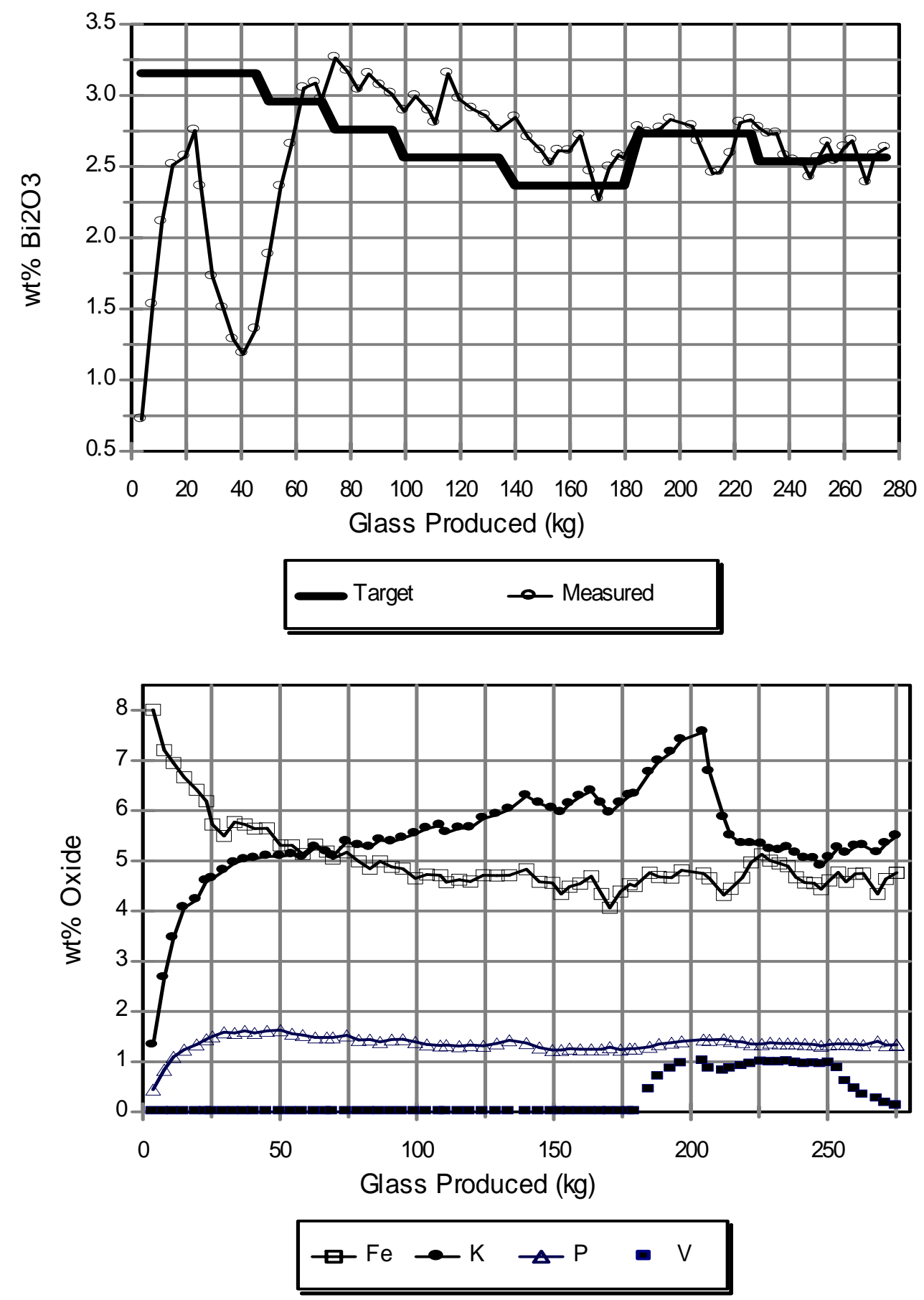

Figure 4.2. Select oxide concentrations measured by XRF in glasses from DM10 tests. 


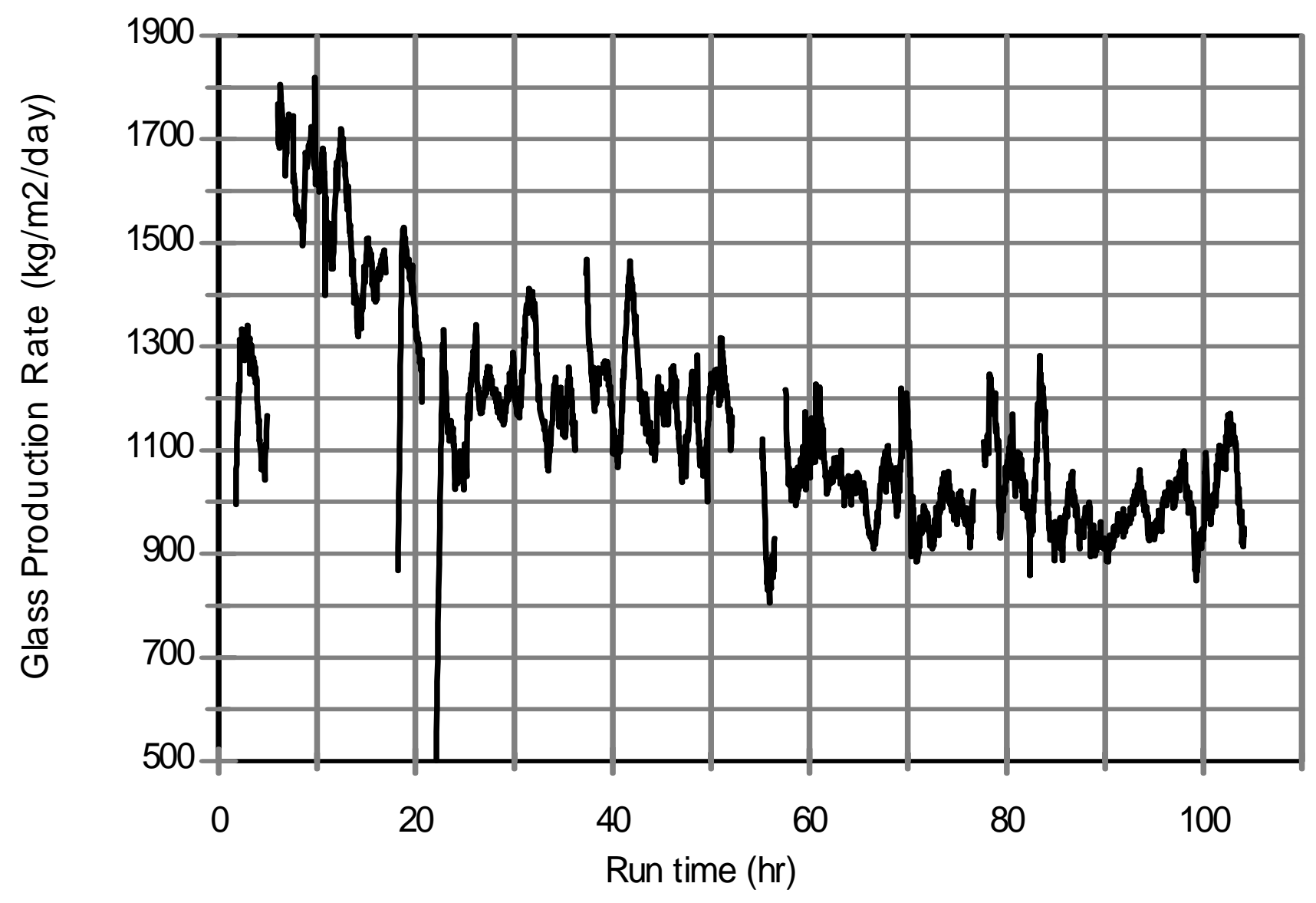

Figure 5.1.a. Glass production rates (hourly moving averages) for DM100 Tests 1A and 1B. 


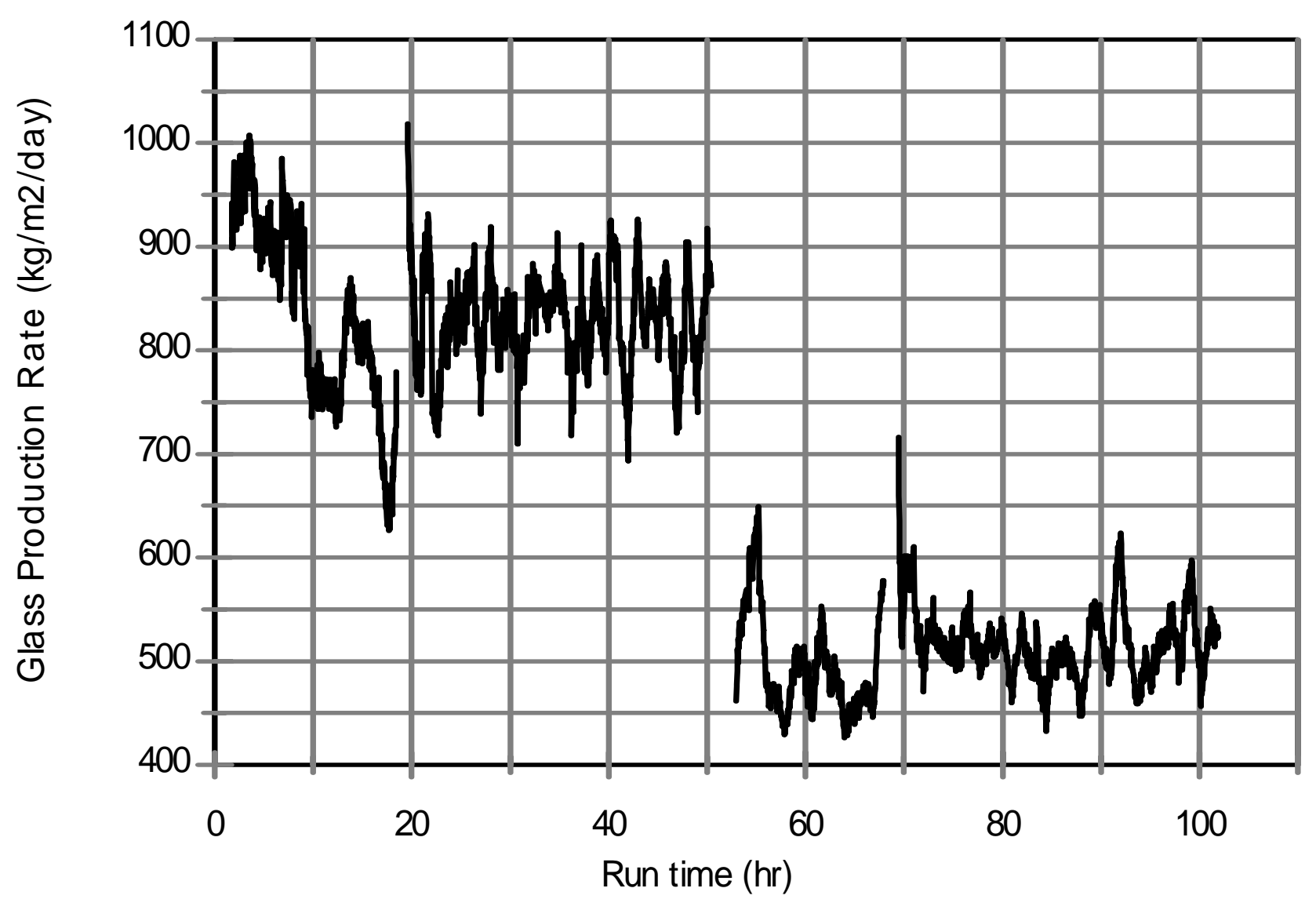

Figure 5.1.b. Glass production rates (hourly moving averages) for DM100 Tests 2A and $2 \mathrm{~B}$. 
The Catholic University of America Vitreous State Laboratory
High Level Waste Vitrification System Improvements Final Report, VSL-07R1010-1, Rev. 0

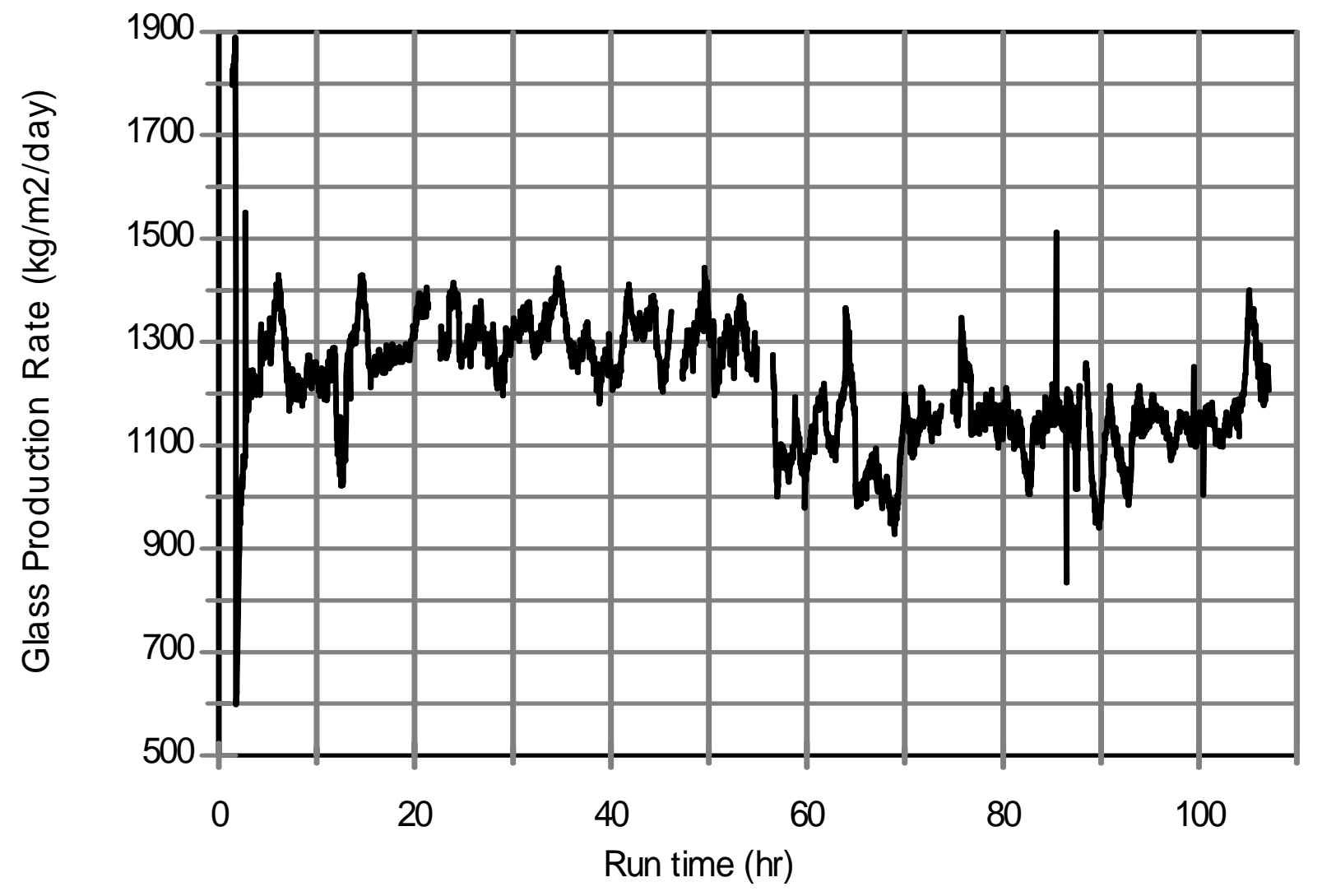

Figure 5.1.c. Glass production rates (hourly moving averages) for DM100 Tests 3B and 4A. 
The Catholic University of America Vitreous State Laboratory
High Level Waste Vitrification System Improvements

Final Report, VSL-07R1010-1, Rev. 0

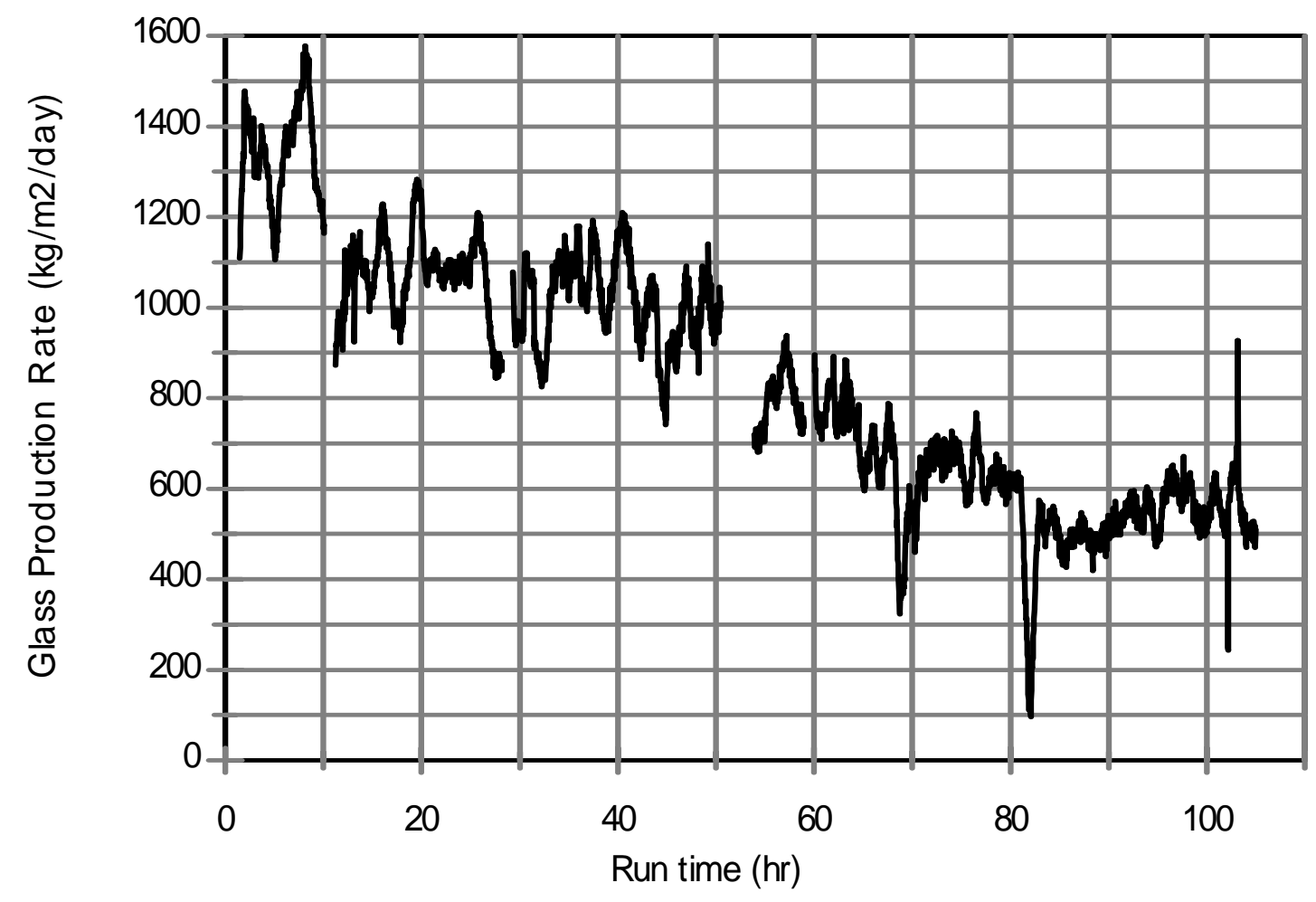

Figure 5.1.d. Glass production rates (hourly moving averages) for DM100 Tests 5A and 5B. 


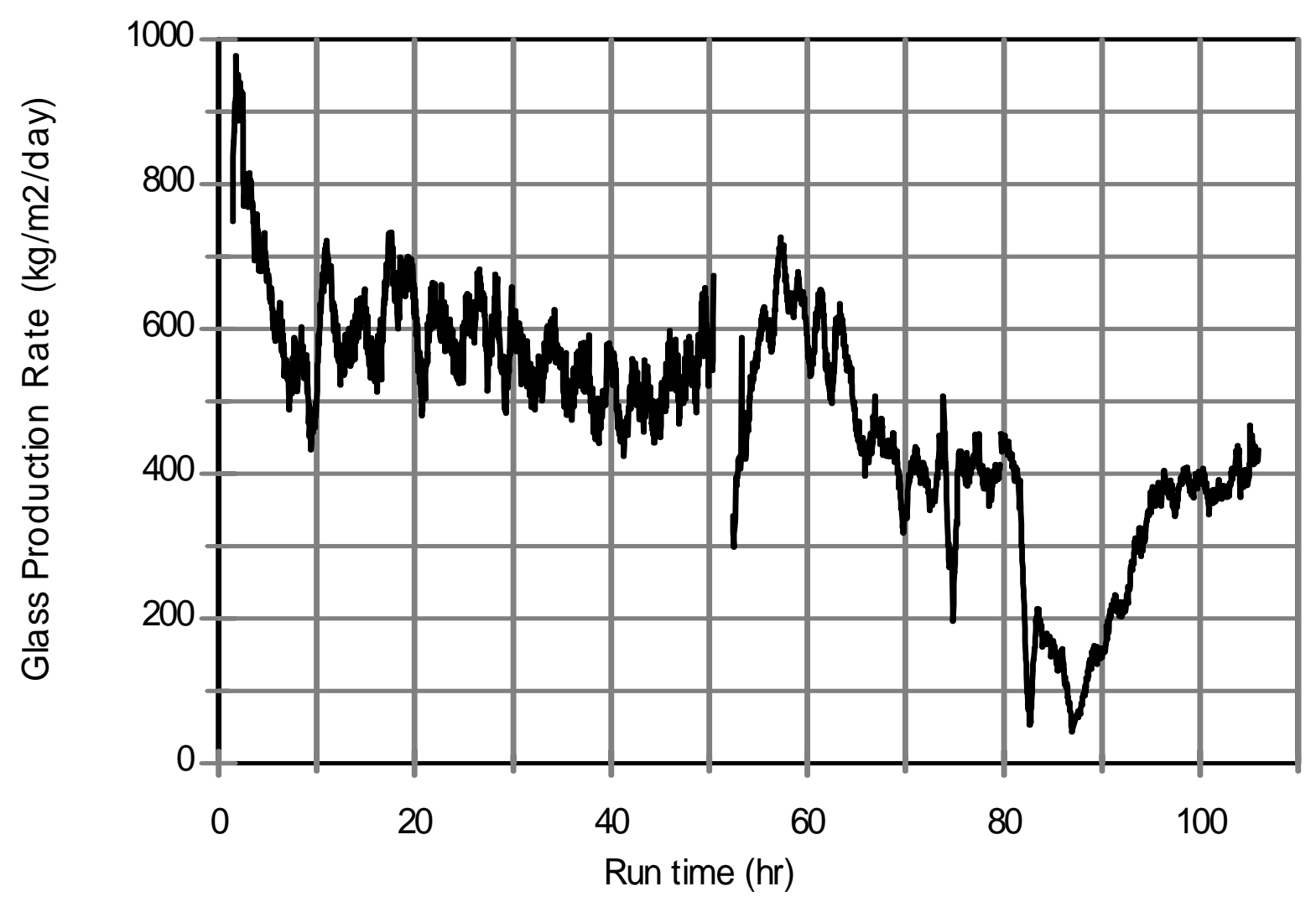

Figure 5.1.e. Glass production rates (hourly moving averages) for DM100 Tests 6A and 6B. 
The Catholic University of America Vitreous State Laboratory
High Level Waste Vitrification System Improvements

Final Report, VSL-07R1010-1, Rev. 0

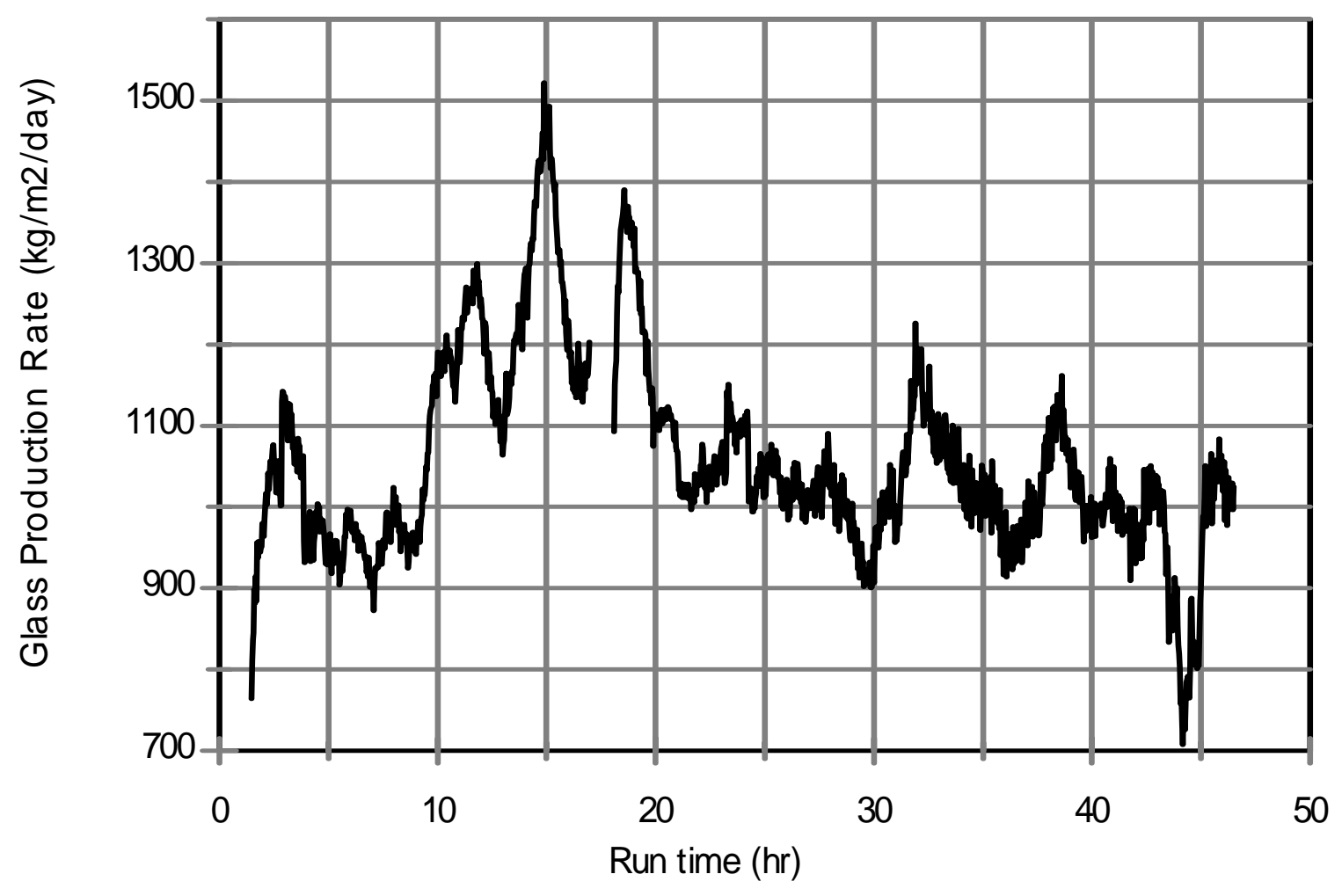

Figure 5.1.f. Glass production rates (hourly moving averages) for DM100 Test 6C. 
The Catholic University of America Vitreous State Laboratory
High Level Waste Vitrification System Improvements

Final Report, VSL-07R1010-1, Rev. 0

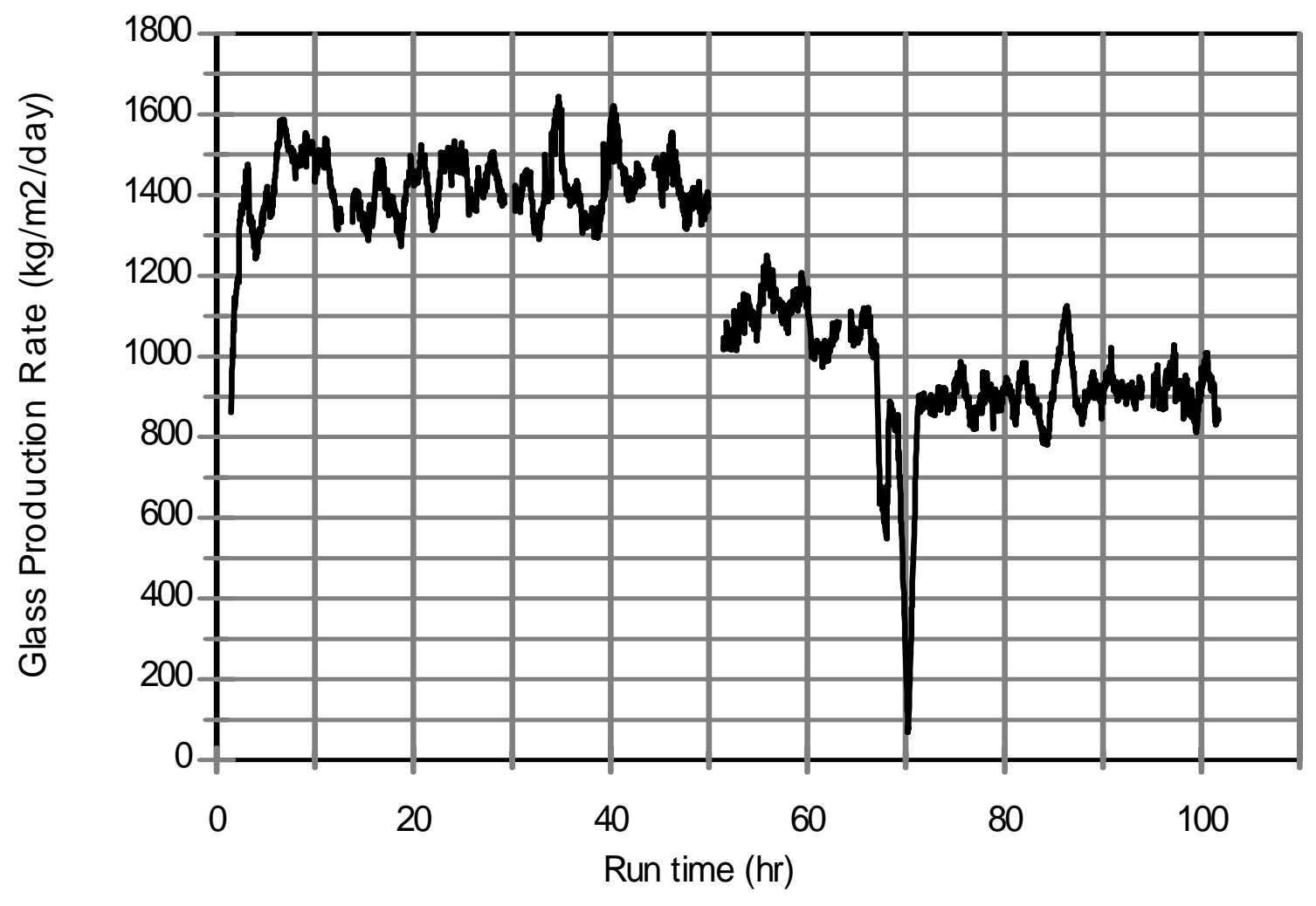

Figure 5.1.g. Glass production rates (hourly moving averages) for DM100 Tests 7A and 7B. 


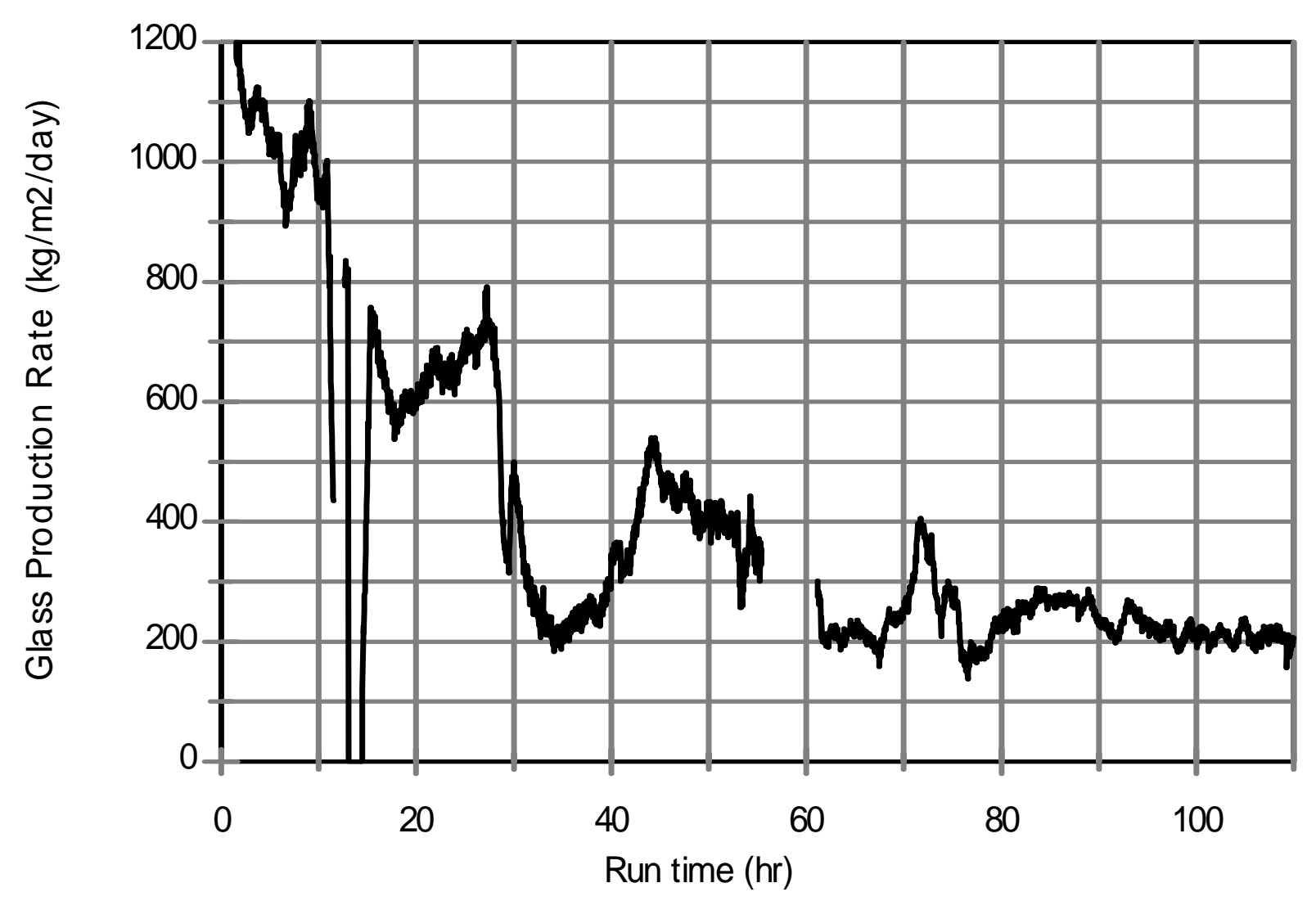

Figure 5.1.h. Glass production rates (hourly moving averages) for DM100 Tests 8A and 8B. 
The Catholic University of America Vitreous State Laboratory
High Level Waste Vitrification System Improvements

Final Report, VSL-07R1010-1, Rev. 0

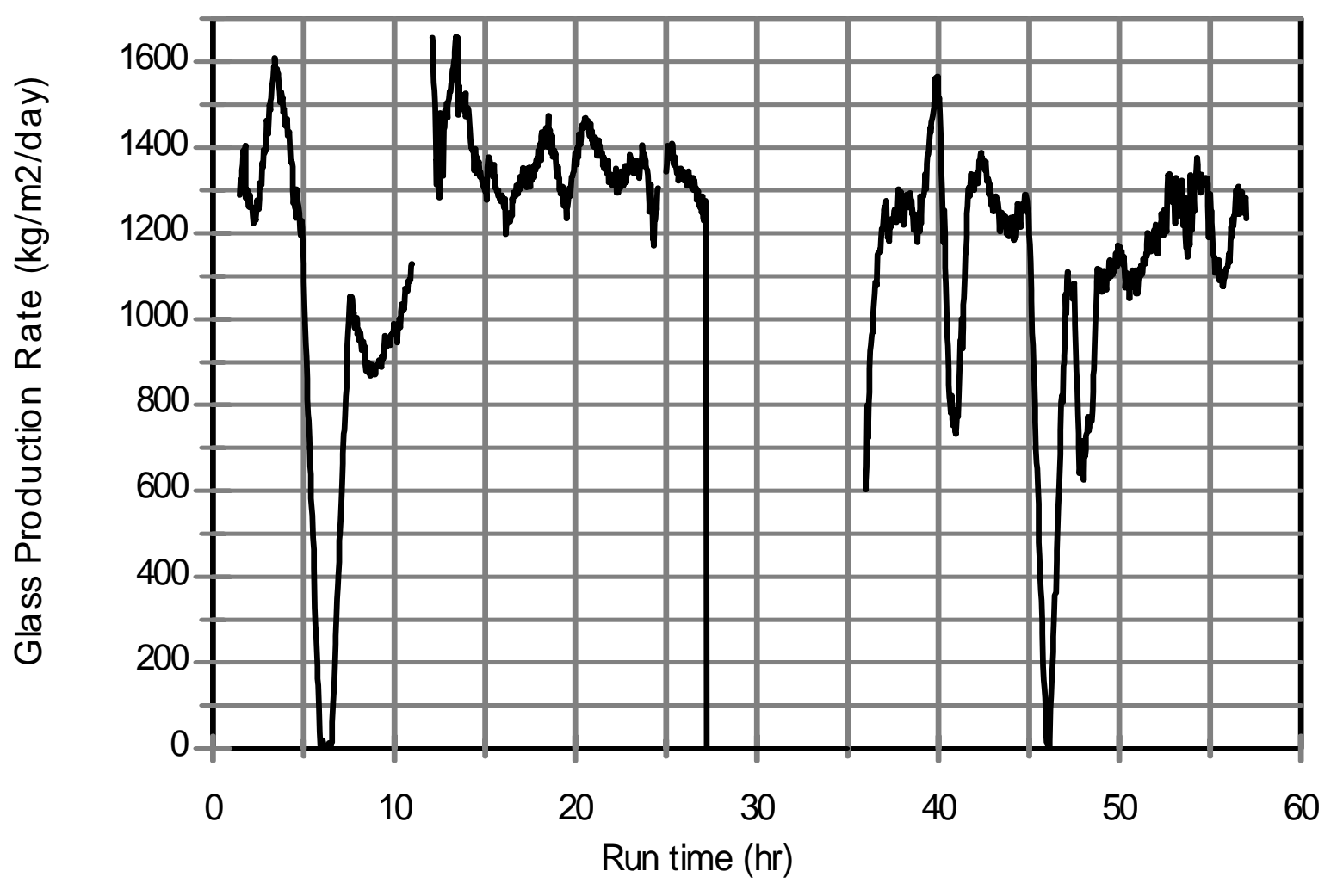

Figure 5.1.i. Glass production rates (hourly moving averages) for DM100 Test 8C. 


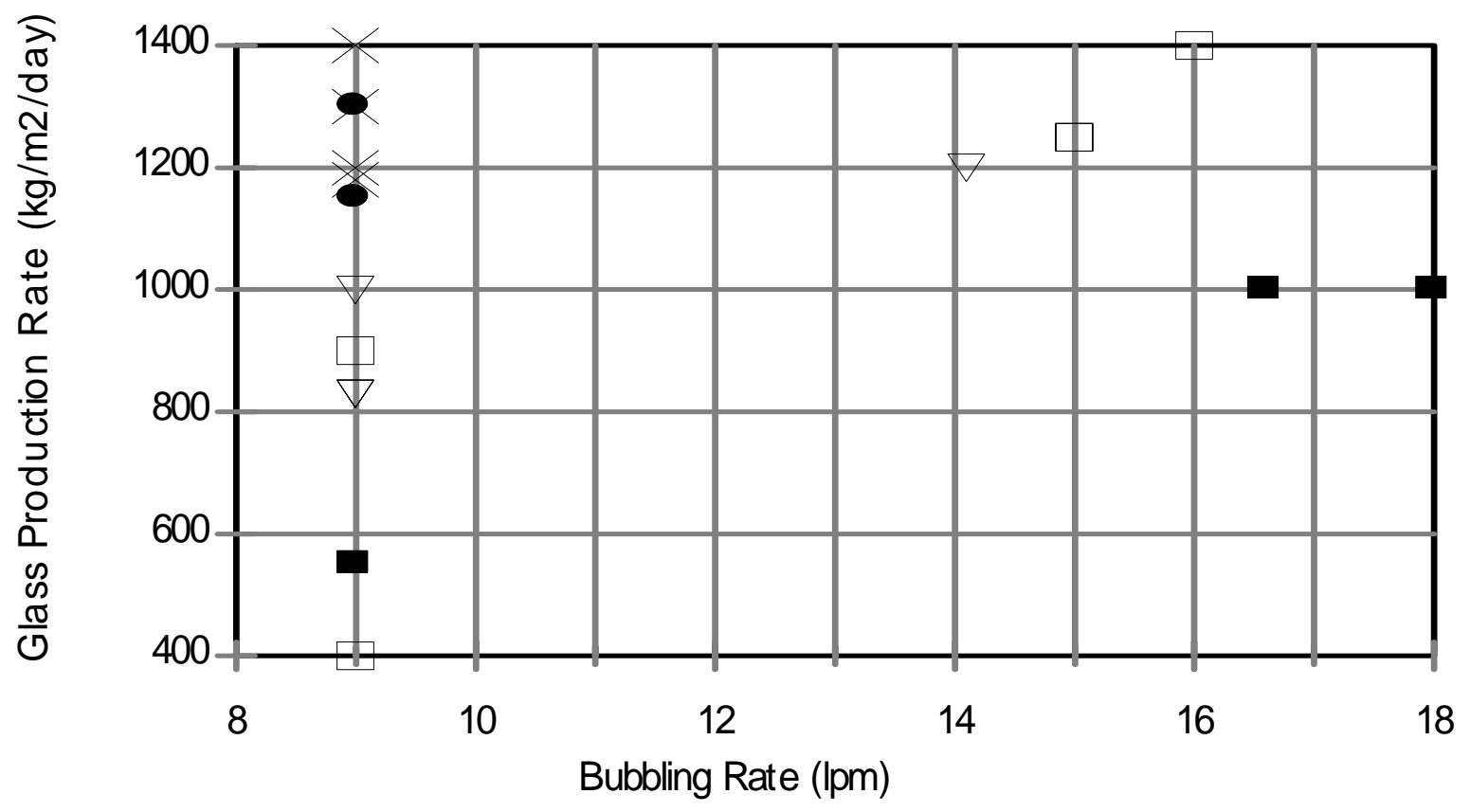
$\nabla$ Bi Limited
- Cr Limited
- Al Limited
$\square \mathrm{Al}+\mathrm{Na}$ Limited
$\times$ Others

Figure 5.2. Steady-state glass production rates during DM100 tests vs. bubbling rate; feed solids content $500( \pm 50)$ g glass per liter feed. 


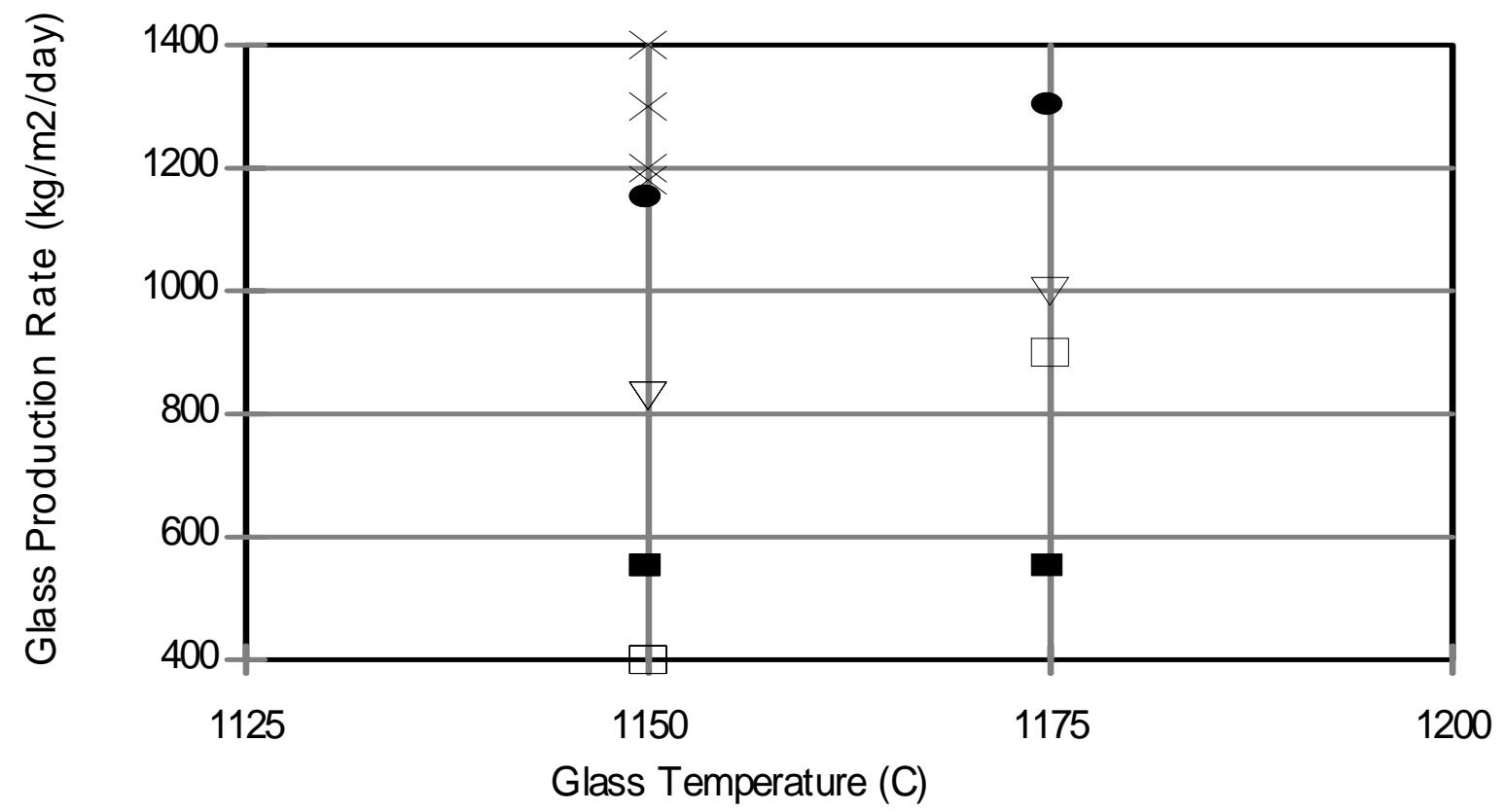
$\nabla$ Bi Limited
- Cr Limited
Al Limited
$\square$ Al + Na Limited
$\times$ Others

Figure 5.3. Steady-state glass production rates during DM100 tests vs. glass temperature; feed solids content $500( \pm 50)$ g glass per liter feed, glass pool bubbling rate 9 lpm. 


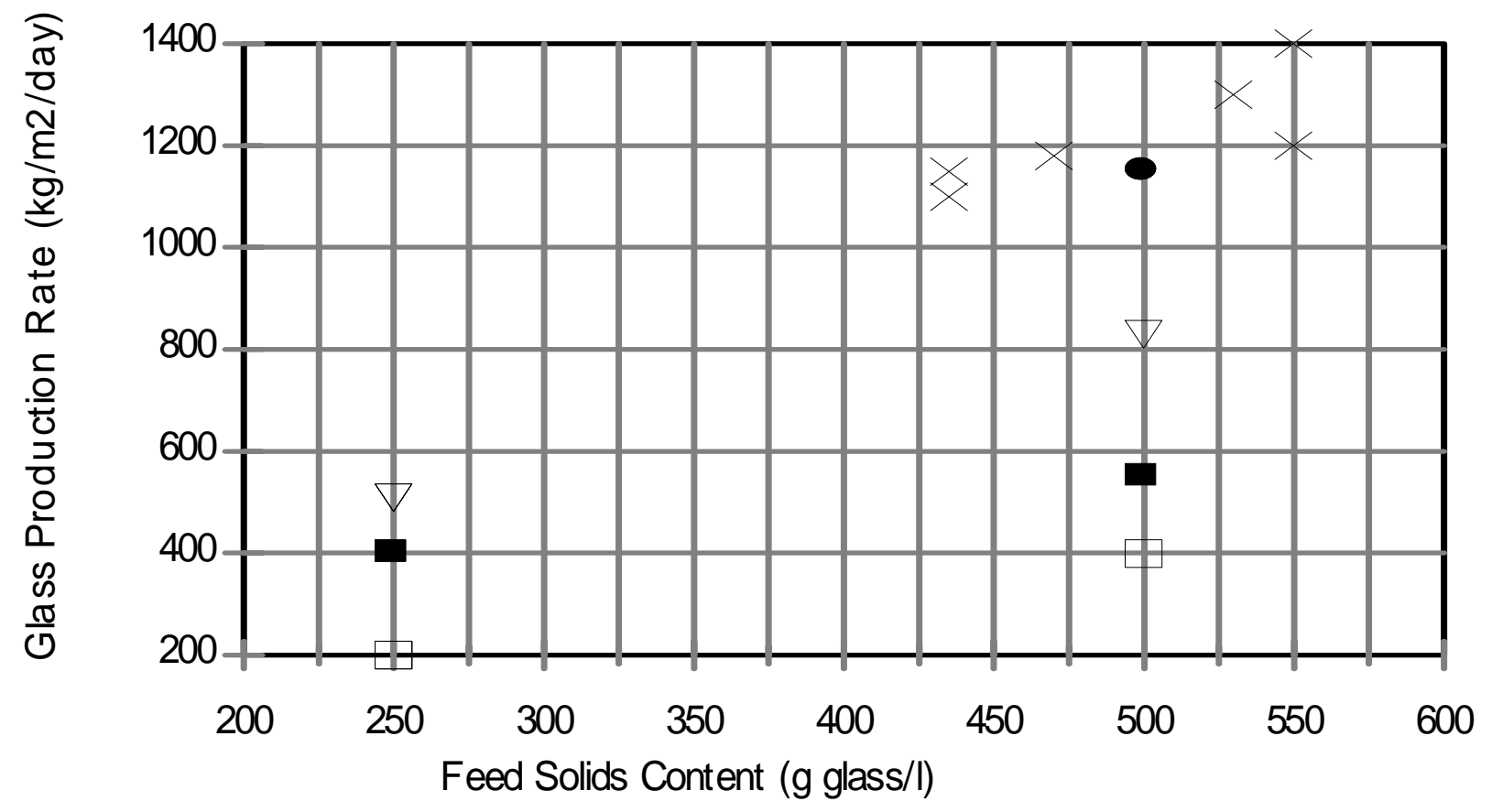

$\boldsymbol{\nabla}$ Bi Limited

- Cr Limited

- Al Limited

$\square \mathrm{Al}+\mathrm{Na}$ Limited

$\times$ Others

Figure 5.4. Steady-state glass production rates during DM100 tests vs. feed solids content; glass temperature $1150^{\circ} \mathrm{C}$, glass pool bubbling rate $9 \mathrm{lpm}$. 


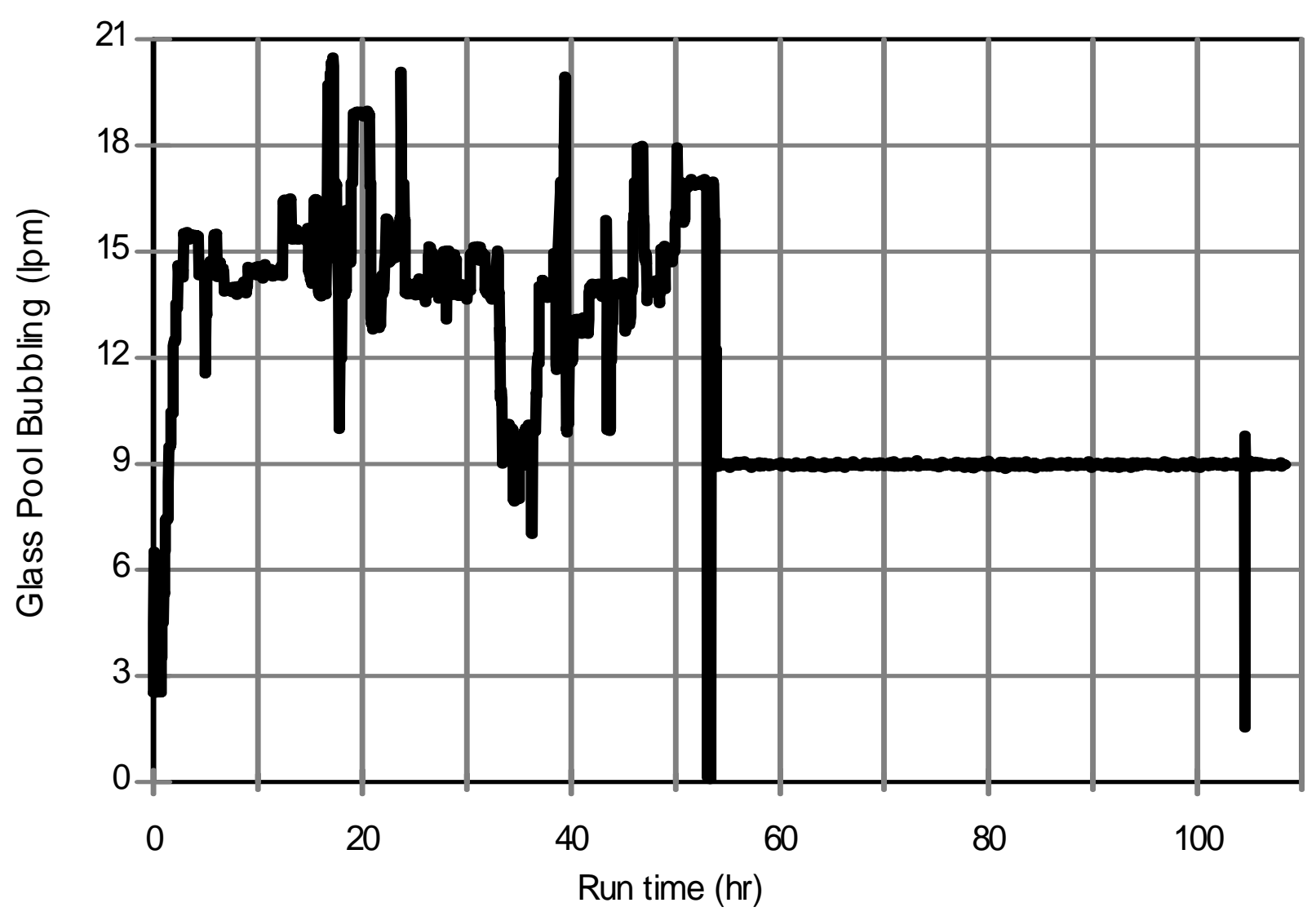

Figure 5.5.a. Glass pool bubbling rate for DM100 Tests 1A and 1B. 


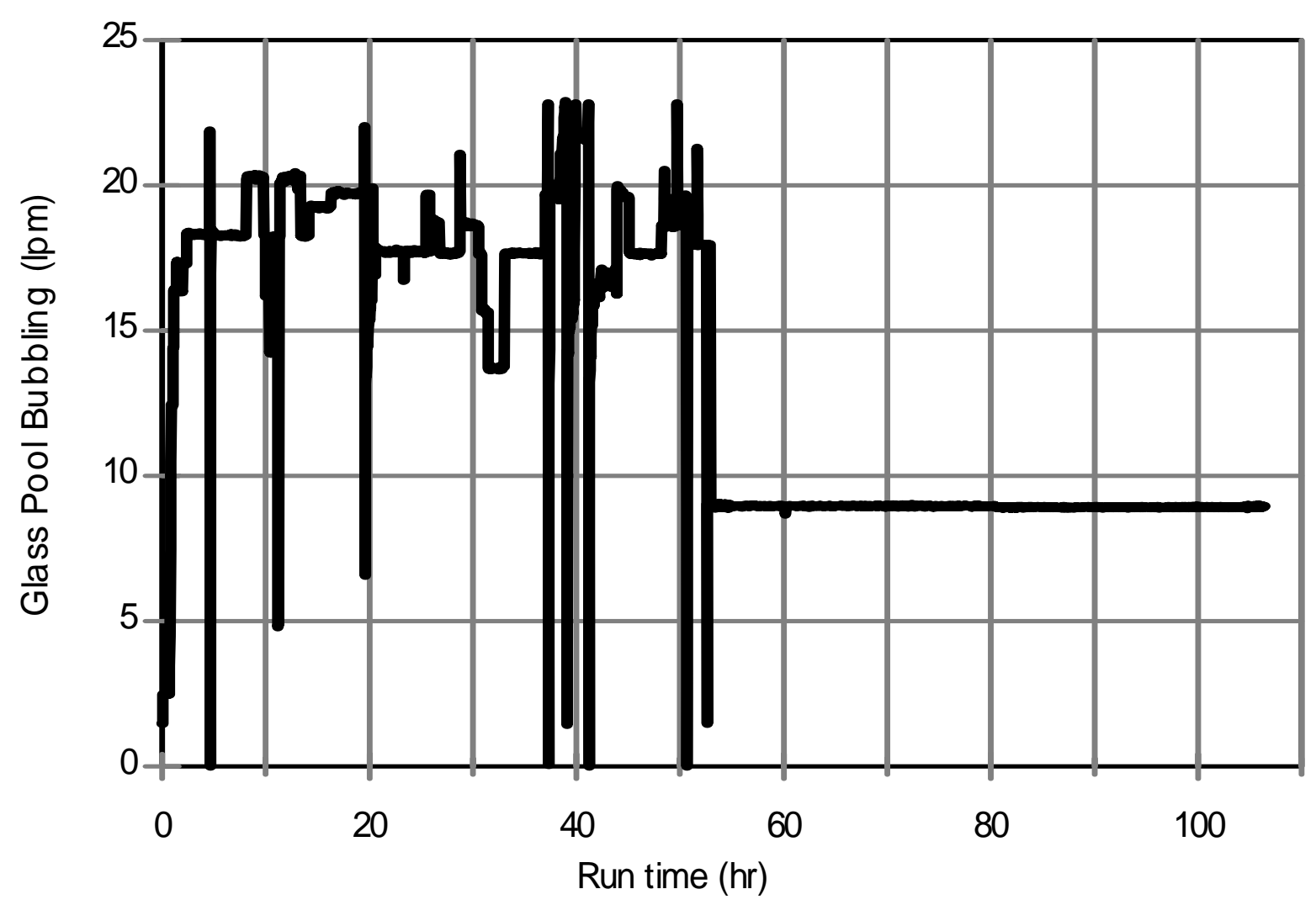

Figure 5.5.b. Glass pool bubbling rate for DM100 Tests 5A and 5B. 
The Catholic University of America Vitreous State Laboratory
High Level Waste Vitrification System Improvements

Final Report, VSL-07R1010-1, Rev. 0

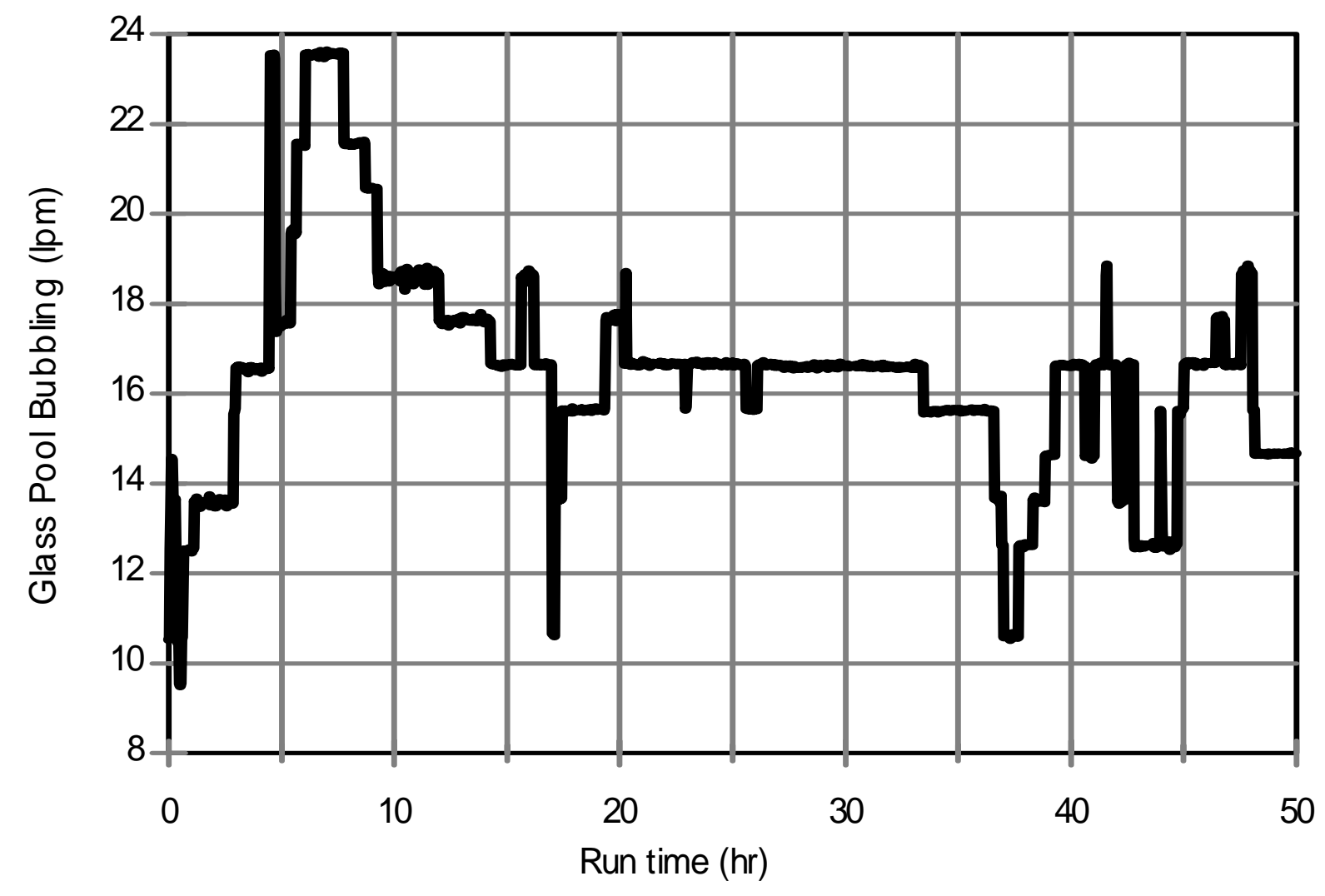

Figure 5.5.c. Glass pool bubbling rate for DM100 Test 6C. 
The Catholic University of America Vitreous State Laboratory
High Level Waste Vitrification System Improvements Final Report, VSL-07R1010-1, Rev. 0

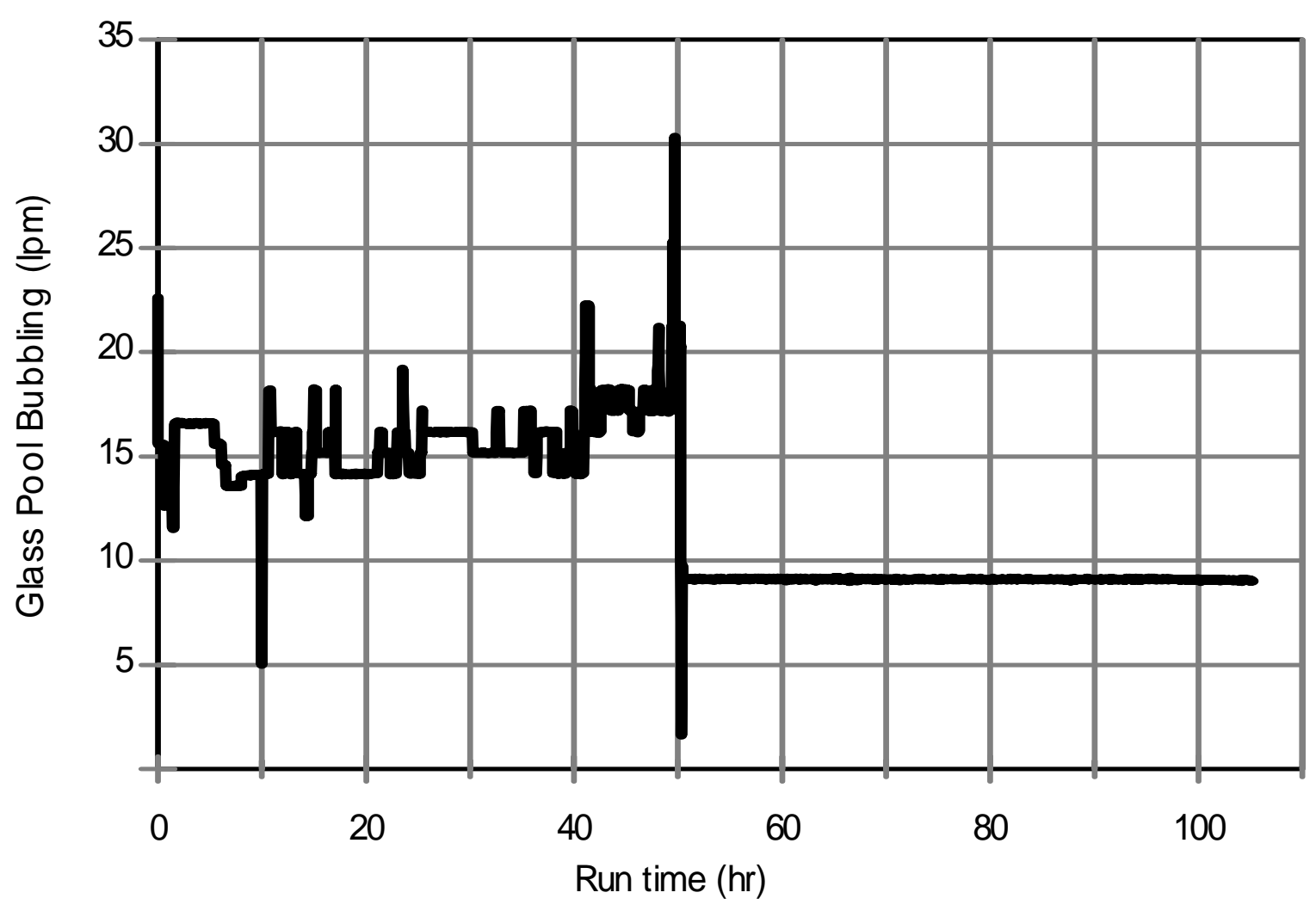

Figure 5.5.d. Glass pool bubbling rates for DM100 Tests 7A and 7B. 


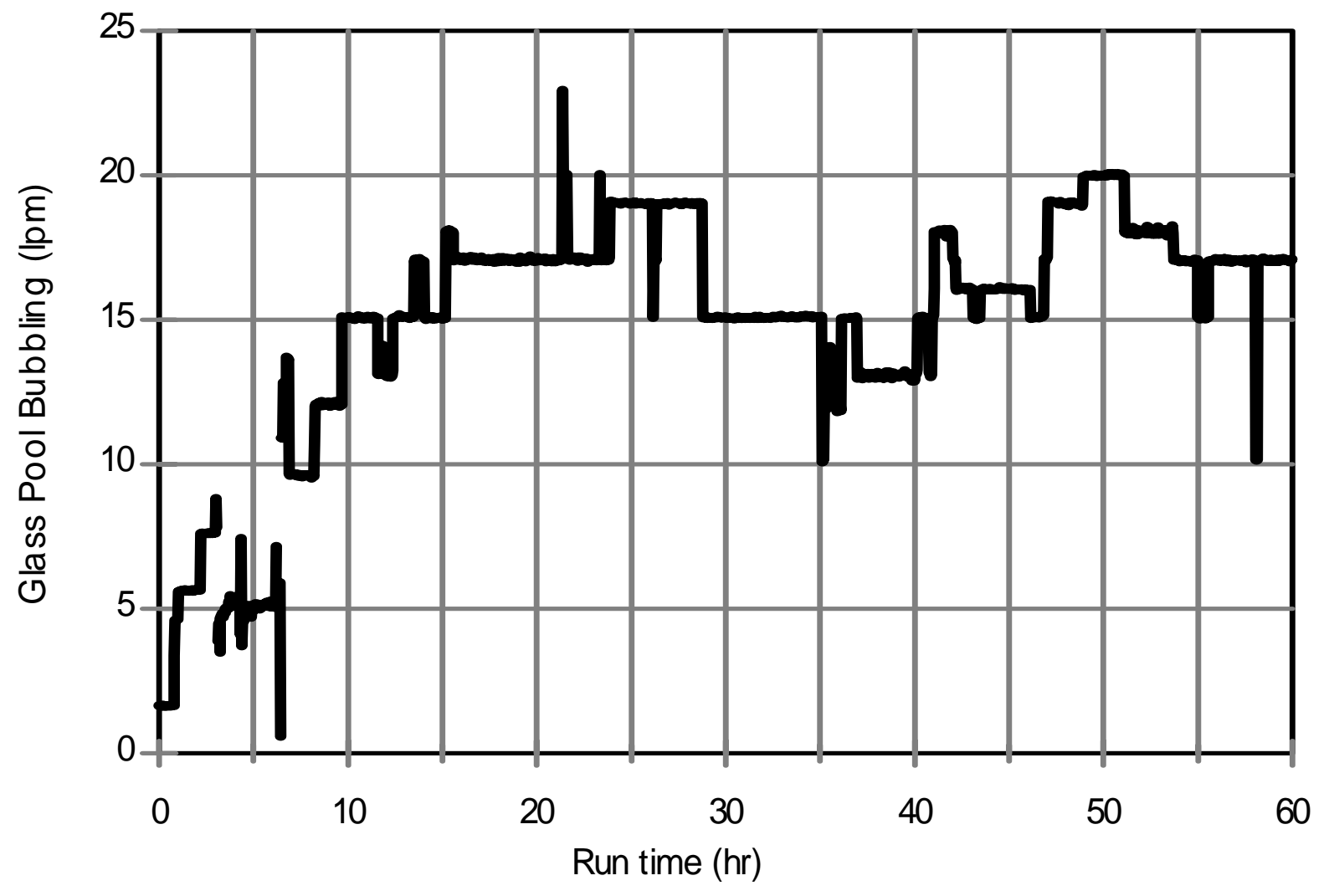

Figure 5.5.e. Glass pool bubbling rates for DM100 Test 8C. 
The Catholic University of America Vitreous State Laboratory
High Level Waste Vitrification System Improvements Final Report, VSL-07R1010-1, Rev. 0

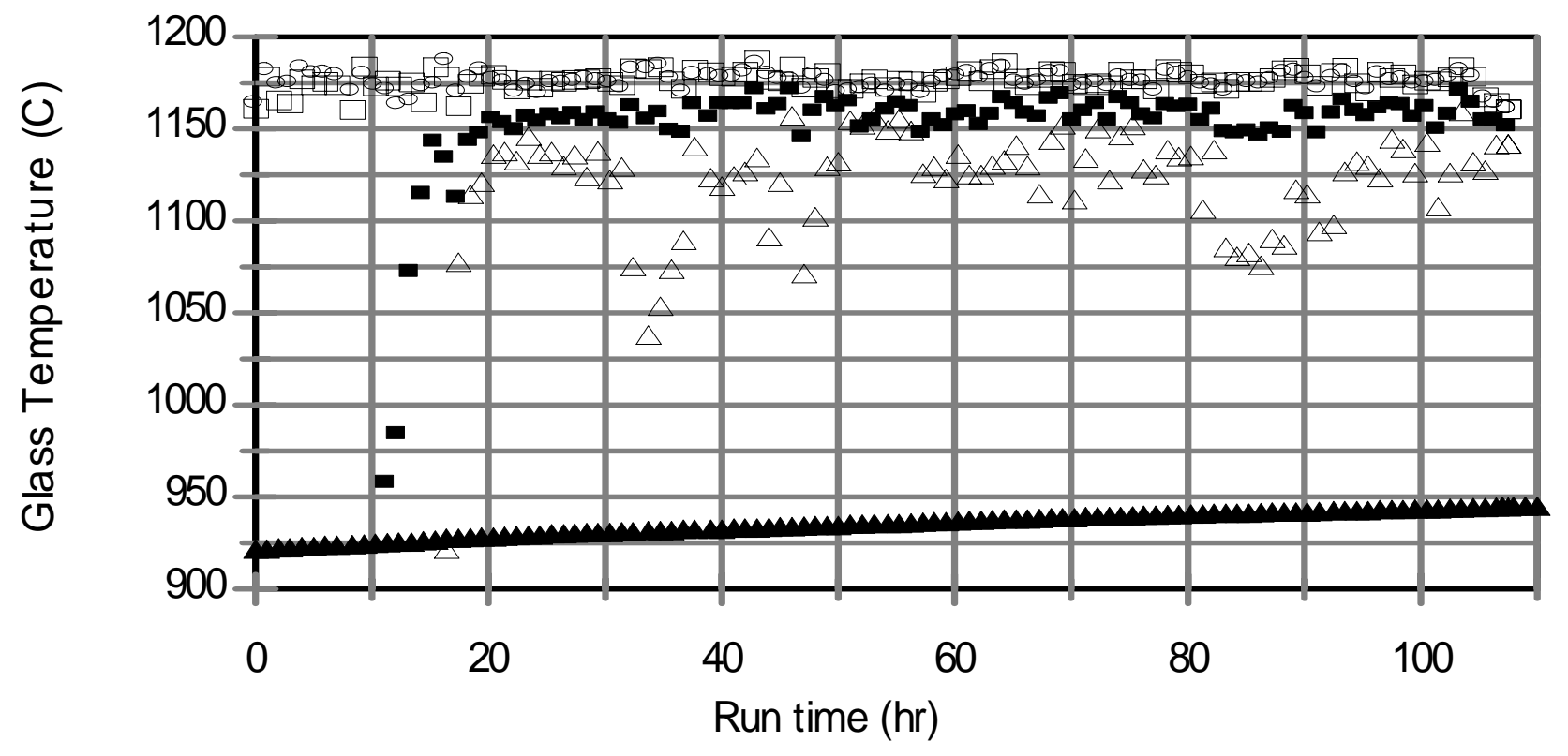

Figure 5.6.a. Glass temperatures (hourly averages) during DM100 Tests 1A and $1 B$. 


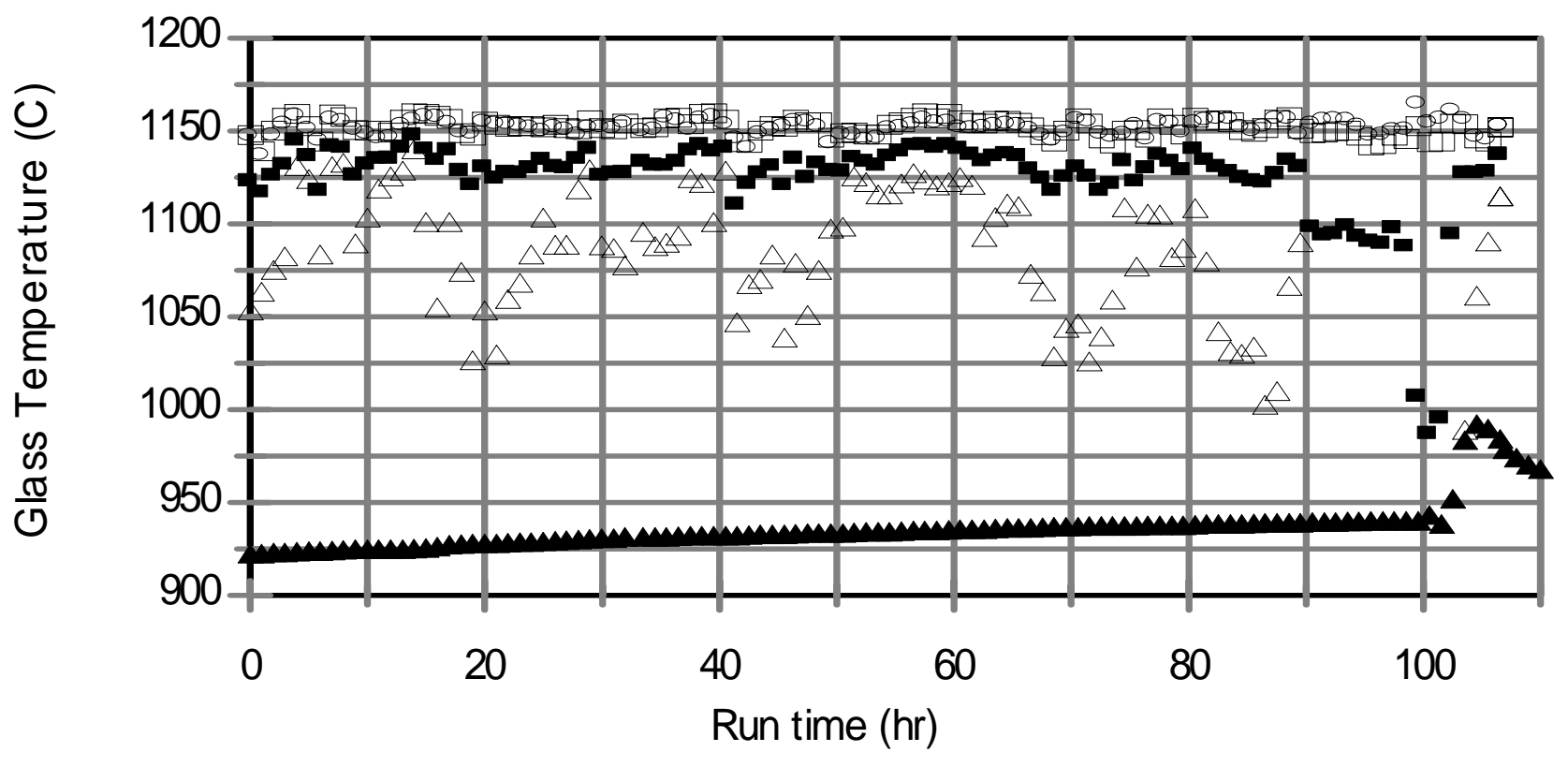

$\Delta \quad 27^{\prime \prime}$ from bottom

- 16 " from bottom

ㅁ $10^{\prime \prime}$ from bottom

- $5^{\prime \prime}$ from bottom

- Air Lift

Figure 5.6.b. Glass temperatures (hourly averages) during DM100 Tests 2A and 2B. 
The Catholic University of America Vitreous State Laboratory
High Level Waste Vitrification System Improvements Final Report, VSL-07R1010-1, Rev. 0

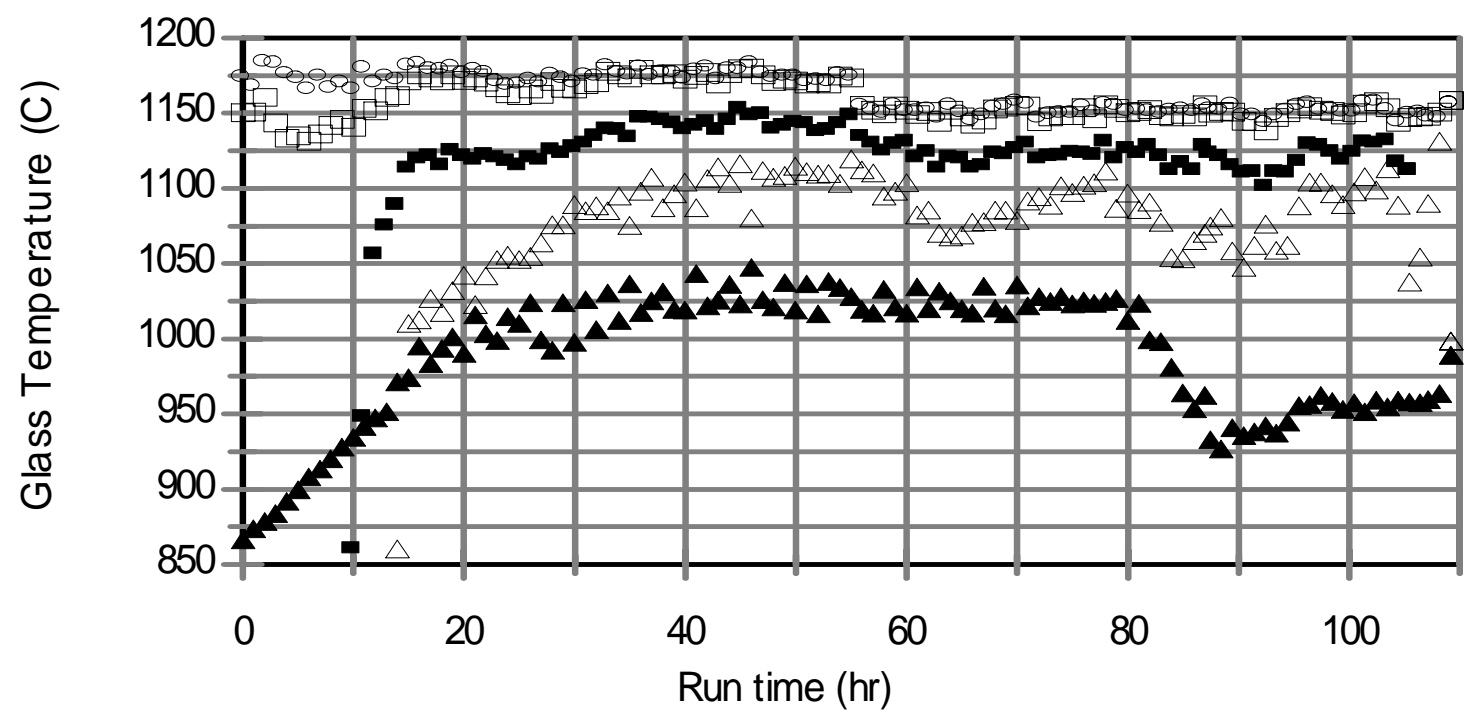
$\Delta \quad 27$ " from bottom
- 16 " from bottom
ㅁ 10 " from bottom
- 5" from bottom
- Airlift

Figure 5.6.c. Glass temperatures (hourly averages) during DM100 Tests 3B and 4A. 
The Catholic University of America Vitreous State Laboratory
High Level Waste Vitrification System Improvements Final Report, VSL-07R1010-1, Rev. 0

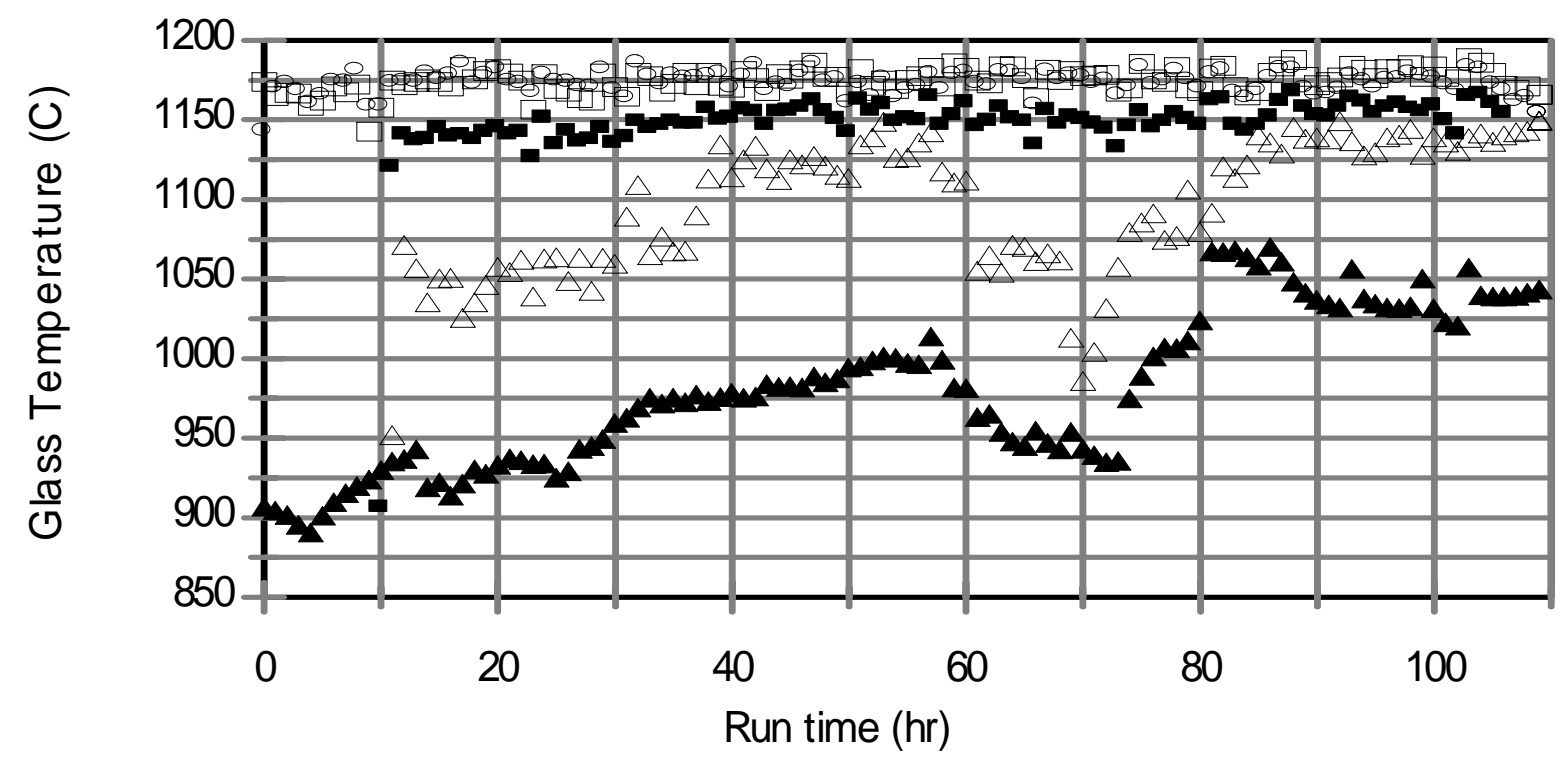

Figure 5.6.d. Glass temperatures (hourly averages) during DM100 Tests 5A and 5B. 


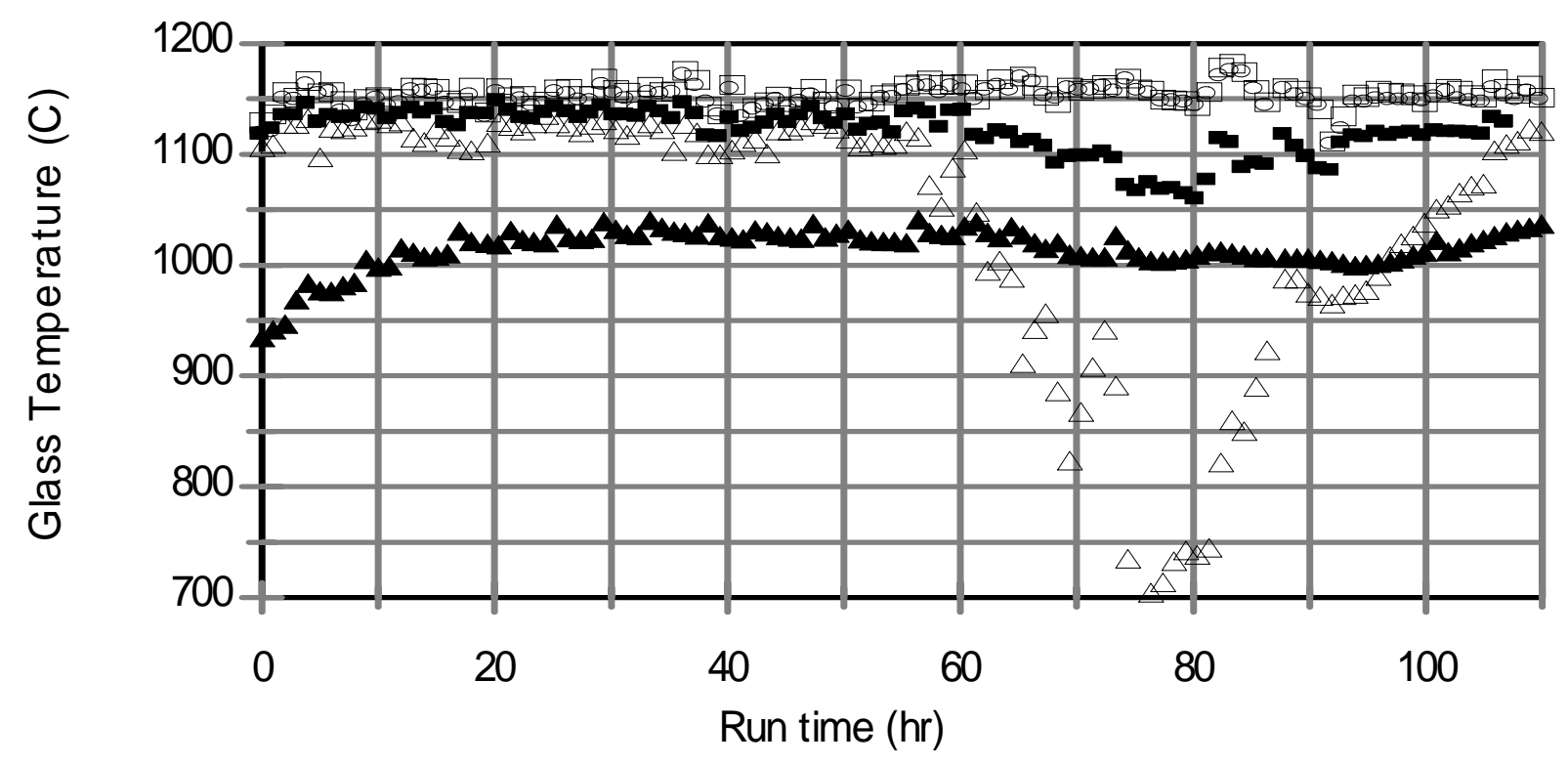

Figure 5.6.e. Glass temperatures (hourly averages) during DM100 Tests 6A and 6B. 
The Catholic University of America Vitreous State Laboratory
High Level Waste Vitrification System Improvements Final Report, VSL-07R1010-1, Rev. 0

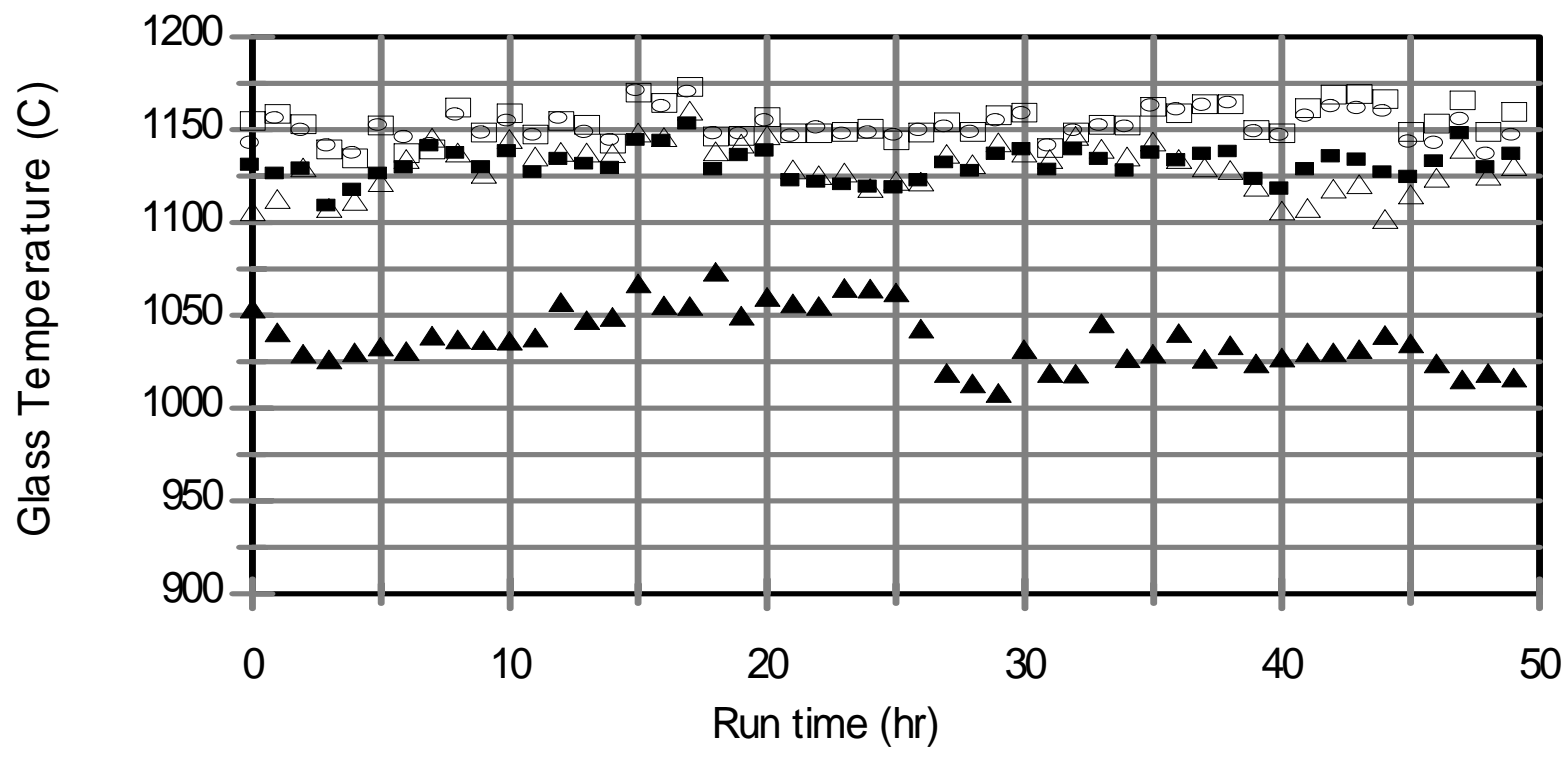
$\Delta \quad 27$ " from bottom
- $16^{\prime \prime}$ from bottom
ㅁ 10 " from bottom
- $5^{\prime \prime}$ from bottom

$\triangle$ Airlift

Figure 5.6.f. Glass temperatures (hourly averages) during DM100 Test 6C. 


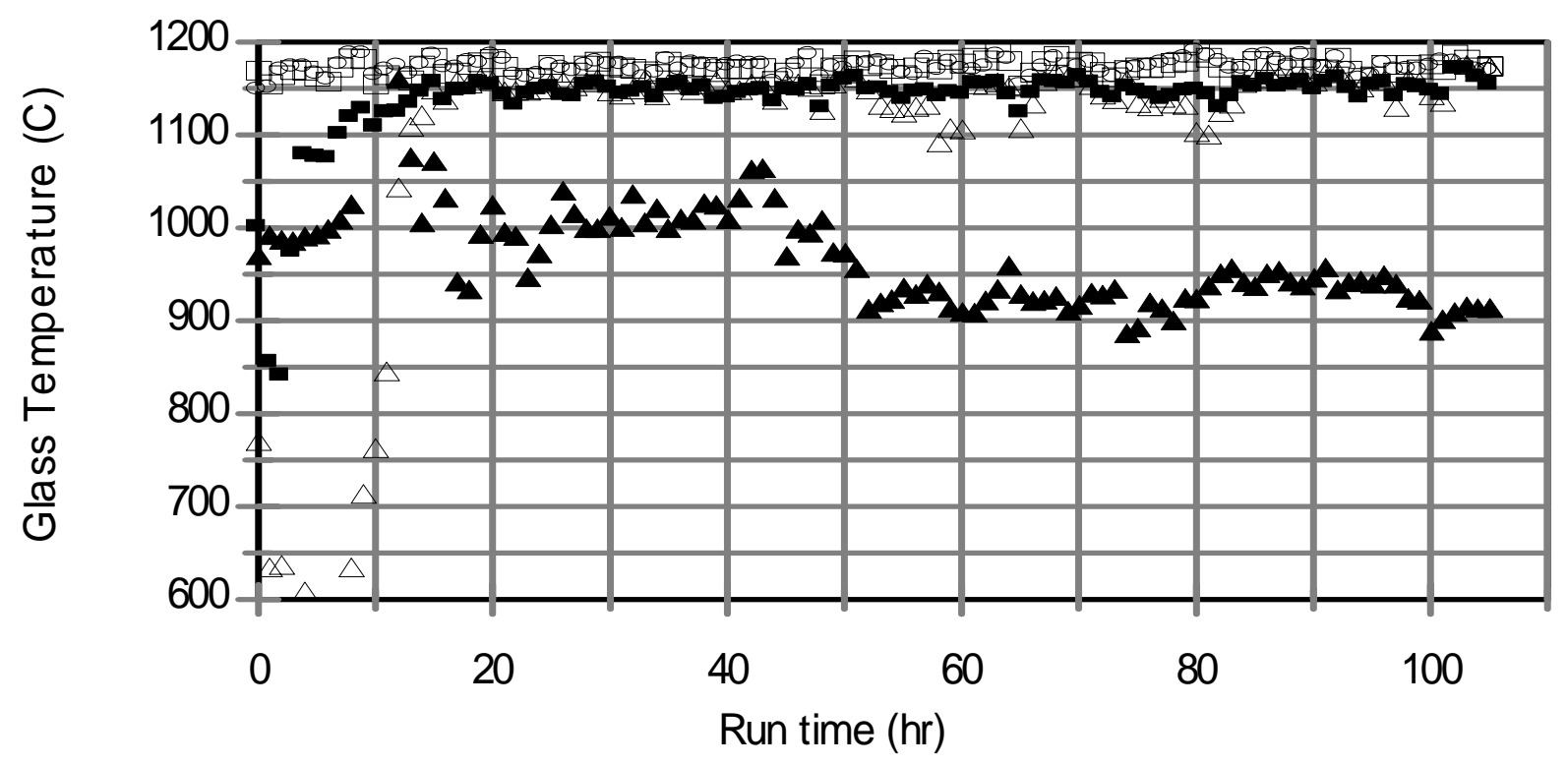

Figure 5.6.g. Glass temperatures (hourly averages) during DM100 Tests 7A and 7B. 
The Catholic University of America Vitreous State Laboratory
High Level Waste Vitrification System Improvements Final Report, VSL-07R1010-1, Rev. 0

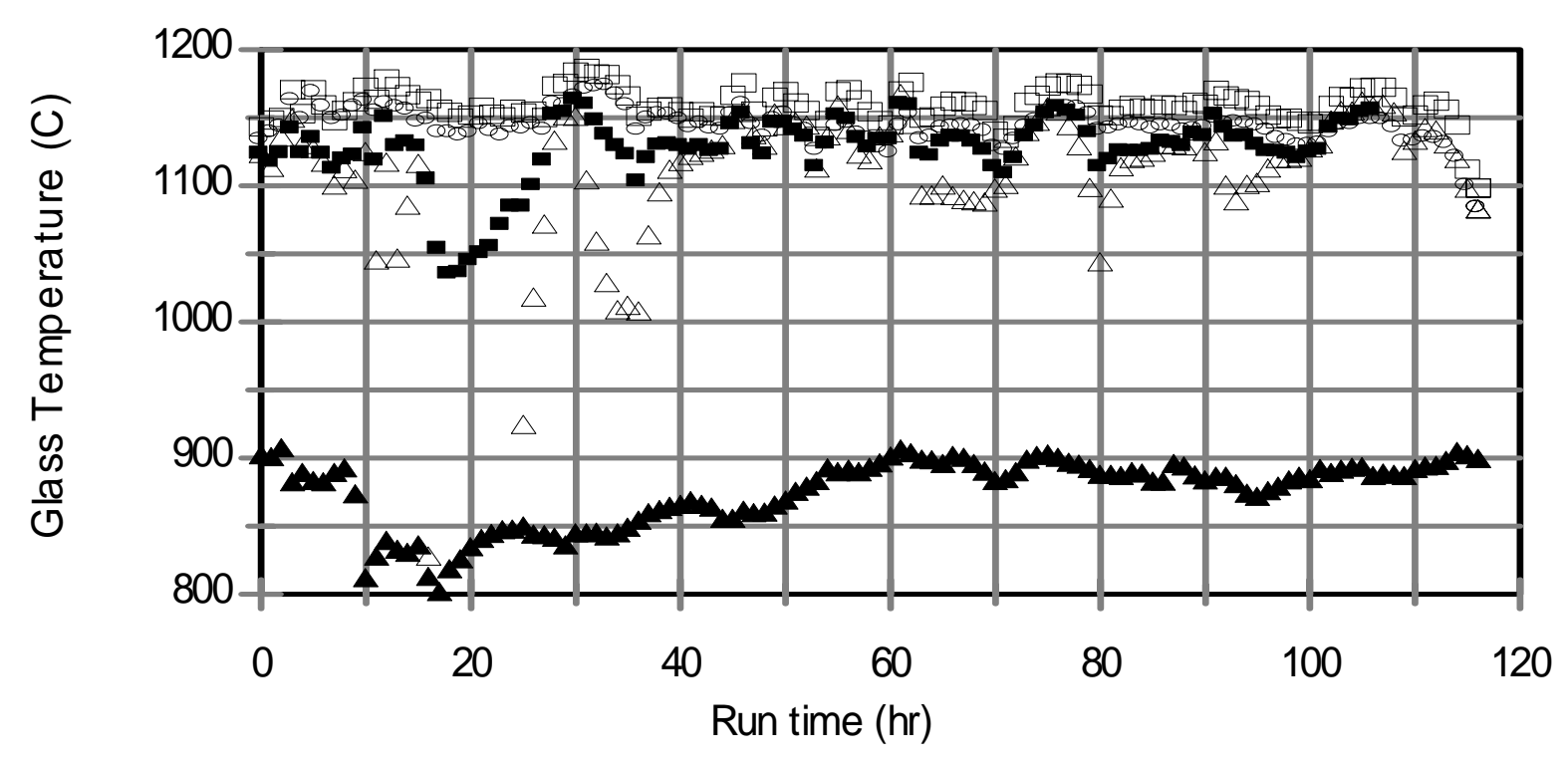

Figure 5.6.h. Glass temperatures (hourly averages) during DM100 Tests 8A and 8B. 


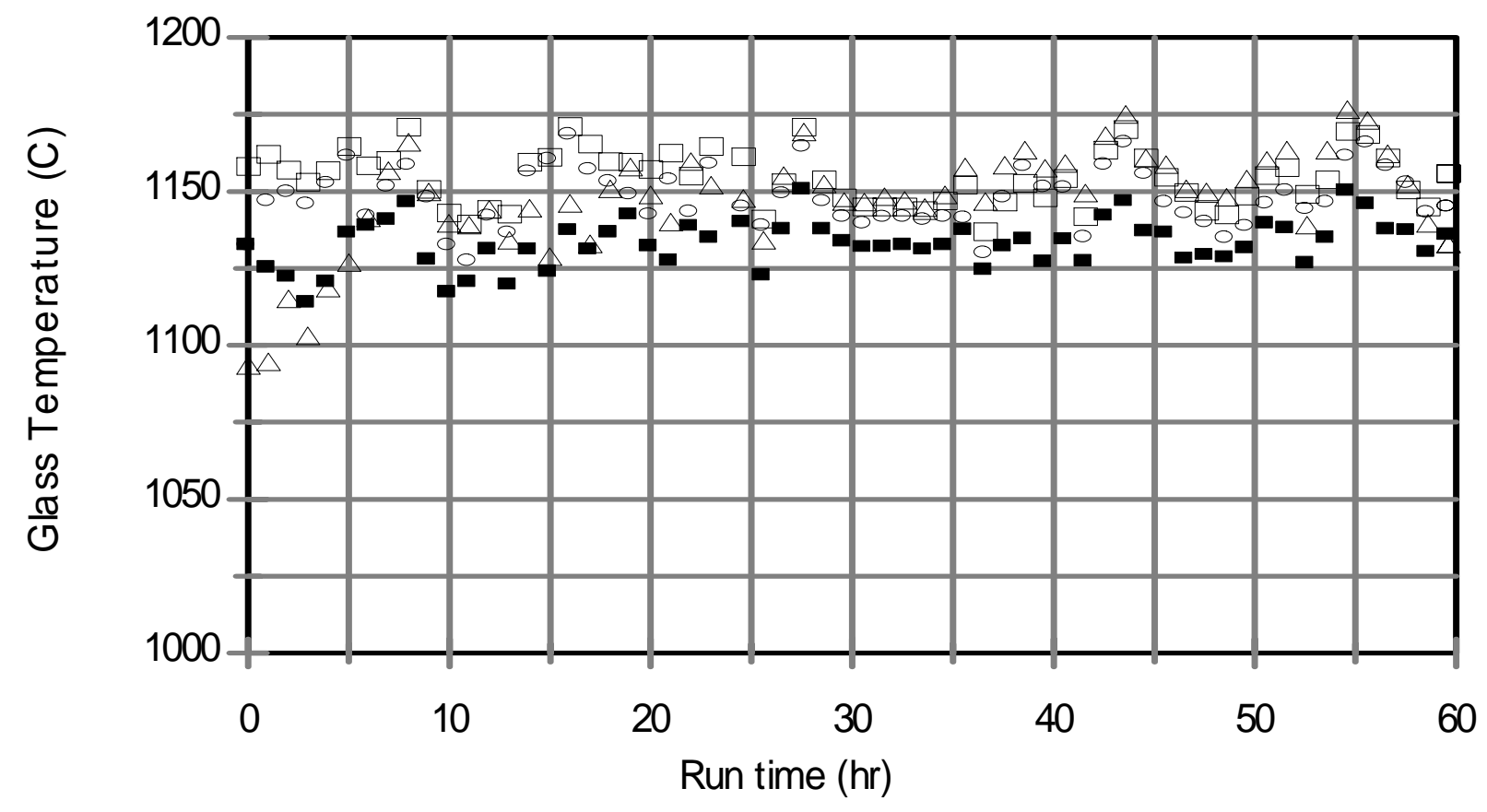

$\Delta \quad 27^{\prime \prime}$ from bottom

- $16^{\prime \prime}$ from bottom

ㅁ $10^{\prime \prime}$ from bottom

- $5^{\prime \prime}$ from bottom

Figure 5.6.i. Glass temperatures (hourly averages) during DM100 Test 8C. 
The Catholic University of America Vitreous State Laboratory
High Level Waste Vitrification System Improvements Final Report, VSL-07R1010-1, Rev. 0

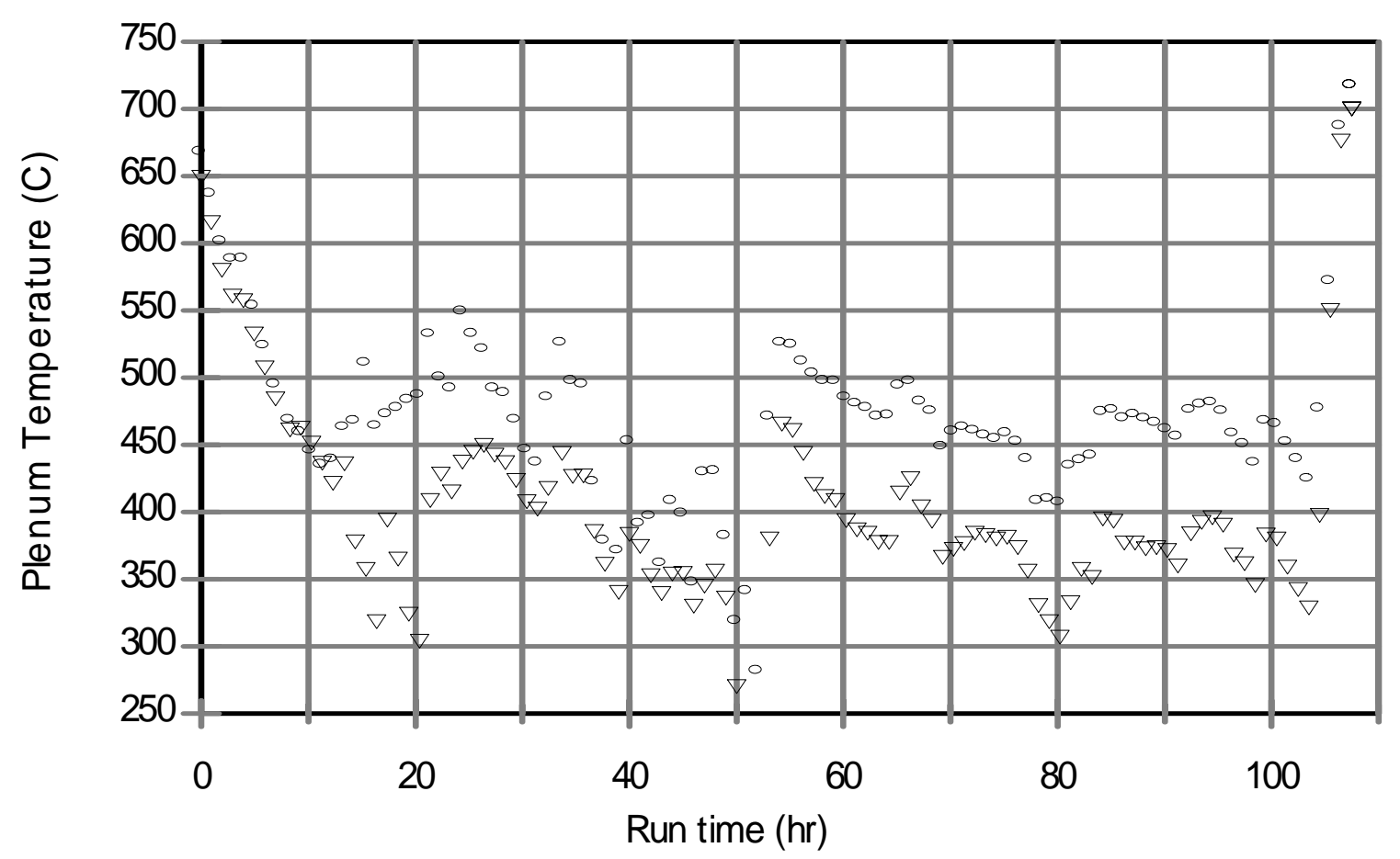

$\nabla \quad 17^{\prime \prime}$ from top, Thermowell

17" from top, Exposed

Figure 5.7.a. Plenum temperatures (hourly averages) during DM100 Tests 1A and $1 B$. 
The Catholic University of America Vitreous State Laboratory
High Level Waste Vitrification System Improvements Final Report, VSL-07R1010-1, Rev. 0

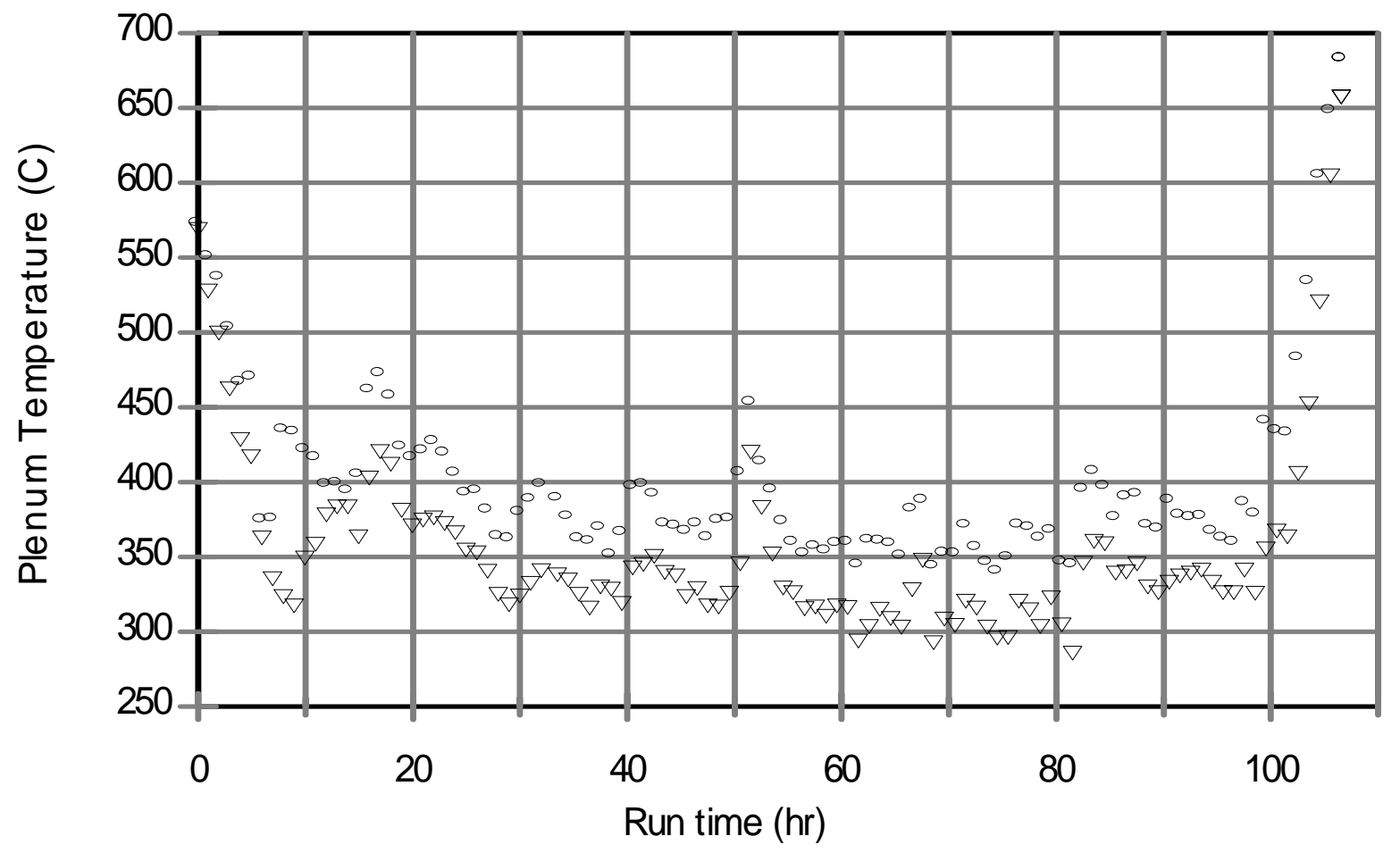

$\nabla \quad 17$ " from top, Thermowell

17" from top, Exposed

Figure 5.7.b. Plenum temperatures (hourly averages) during DM100 Tests 2A and $2 \mathrm{~B}$. 
The Catholic University of America Vitreous State Laboratory
High Level Waste Vitrification System Improvements Final Report, VSL-07R1010-1, Rev. 0

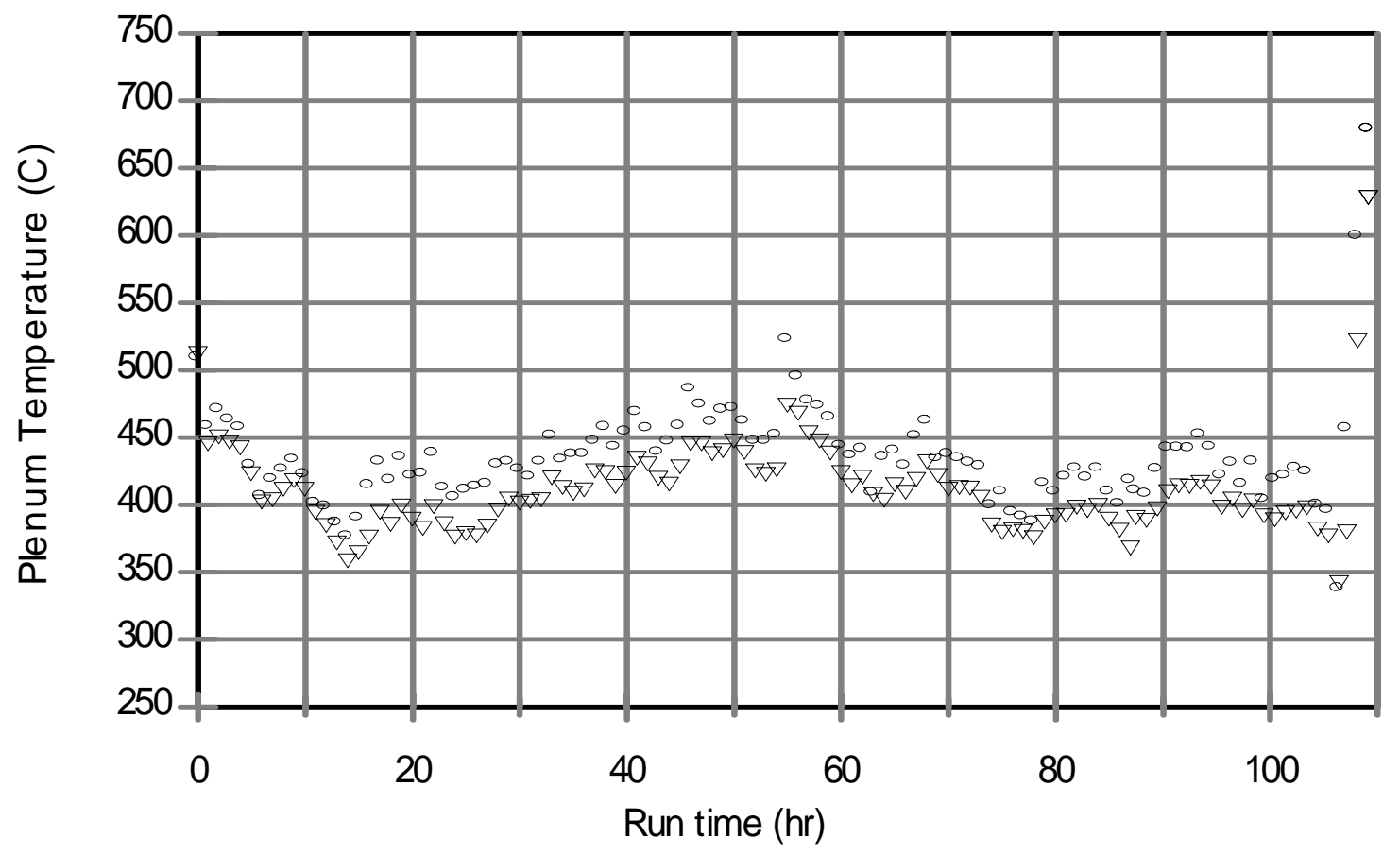

$\boldsymbol{\nabla} 17$ " from top, Thermowell

- 17 from top, Exposed

Figure 5.7.c. Plenum temperatures (hourly averages) during DM100 Tests 3B and 4A. 
The Catholic University of America Vitreous State Laboratory
High Level Waste Vitrification System Improvements Final Report, VSL-07R1010-1, Rev. 0

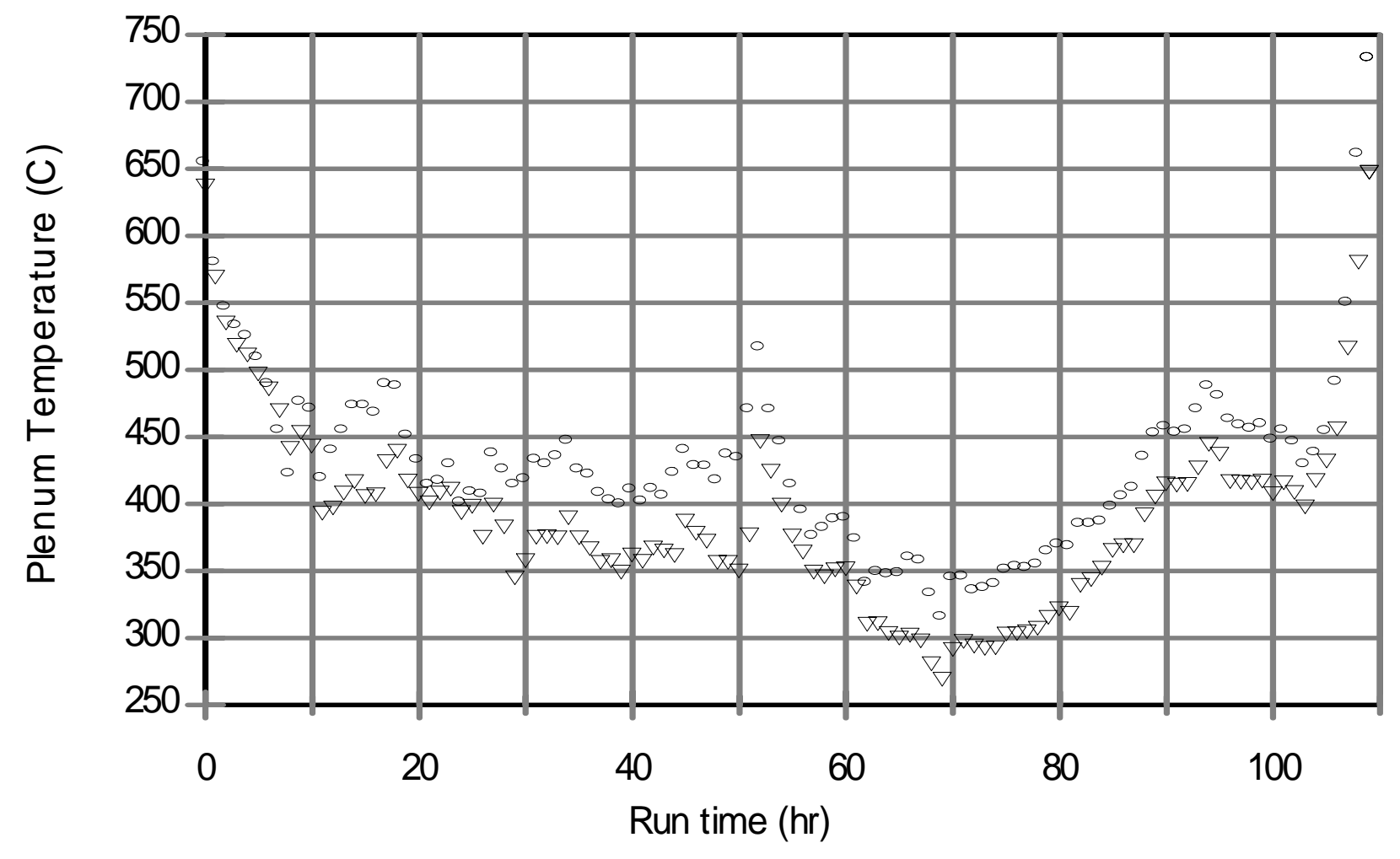

17" from top, Thermowell

- $17^{\prime \prime}$ from top, Exposed

Figure 5.7.d. Plenum temperatures (hourly averages) during DM100 Tests 5A and 5B. 
The Catholic University of America Vitreous State Laboratory
High Level Waste Vitrification System Improvements Final Report, VSL-07R1010-1, Rev. 0

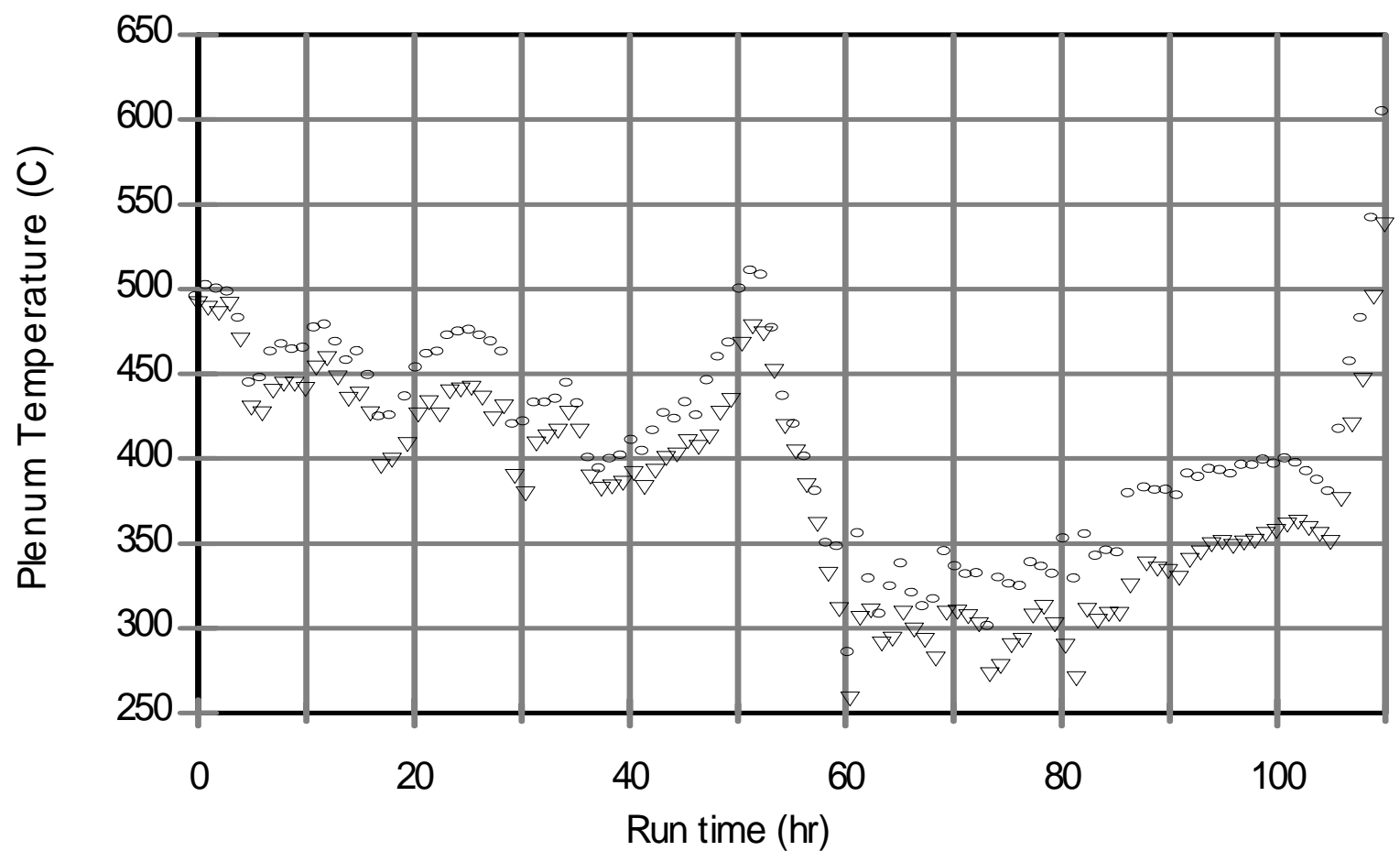

$\boldsymbol{\nabla} 17^{\prime \prime}$ from top, Thermowell

- $17^{\prime \prime}$ from top, Exposed

Figure 5.7.e. Plenum temperatures (hourly averages) during DM100 Tests 6A and 6B. 
The Catholic University of America Vitreous State Laboratory
High Level Waste Vitrification System Improvements Final Report, VSL-07R1010-1, Rev. 0

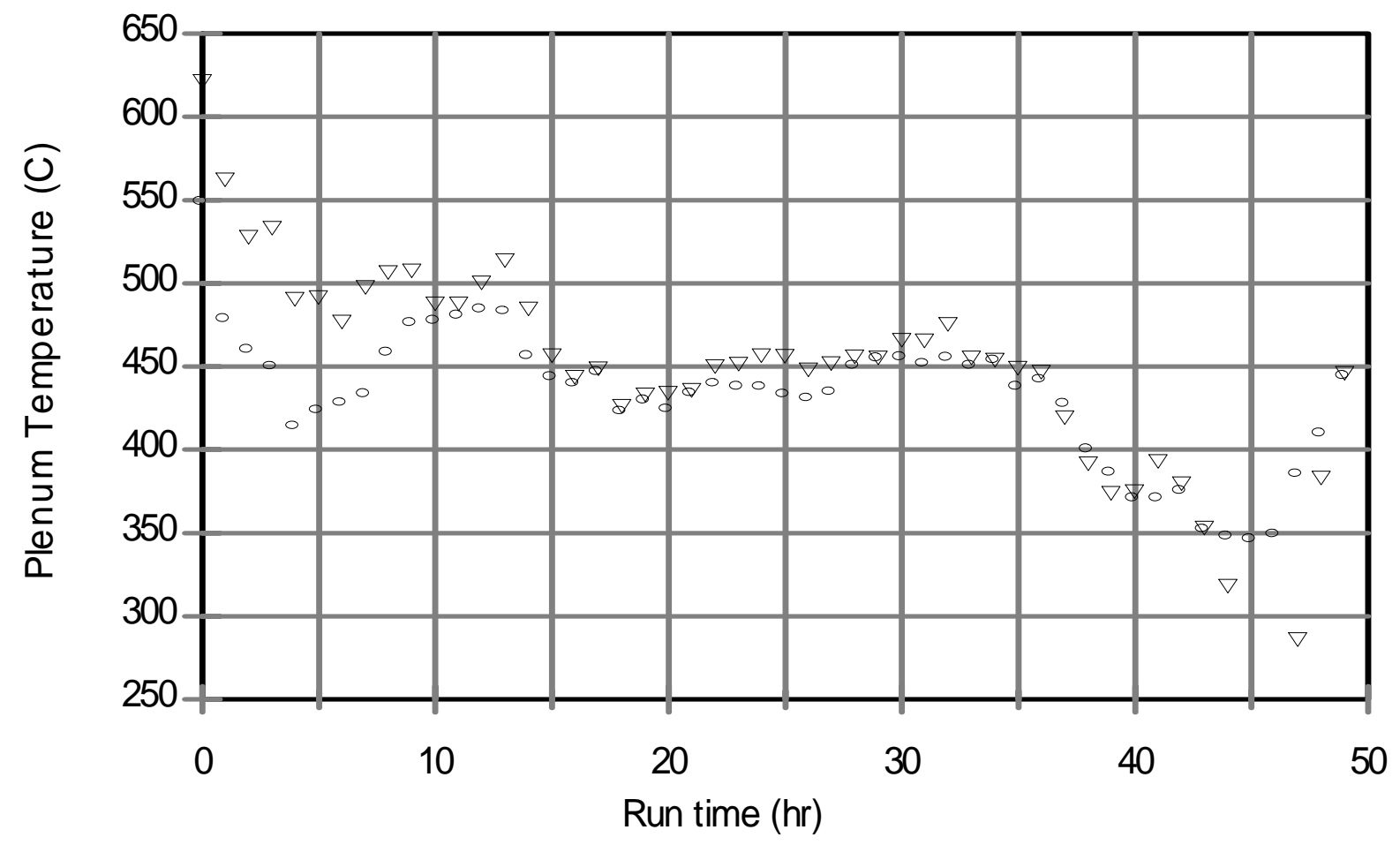

$\begin{array}{lll}\nabla & 17 \text { " from top, Thermowell } & -\end{array}$

Figure 5.7.f. Plenum temperatures (hourly averages) during DM100 Test 6C. 
The Catholic University of America Vitreous State Laboratory
High Level Waste Vitrification System Improvements Final Report, VSL-07R1010-1, Rev. 0

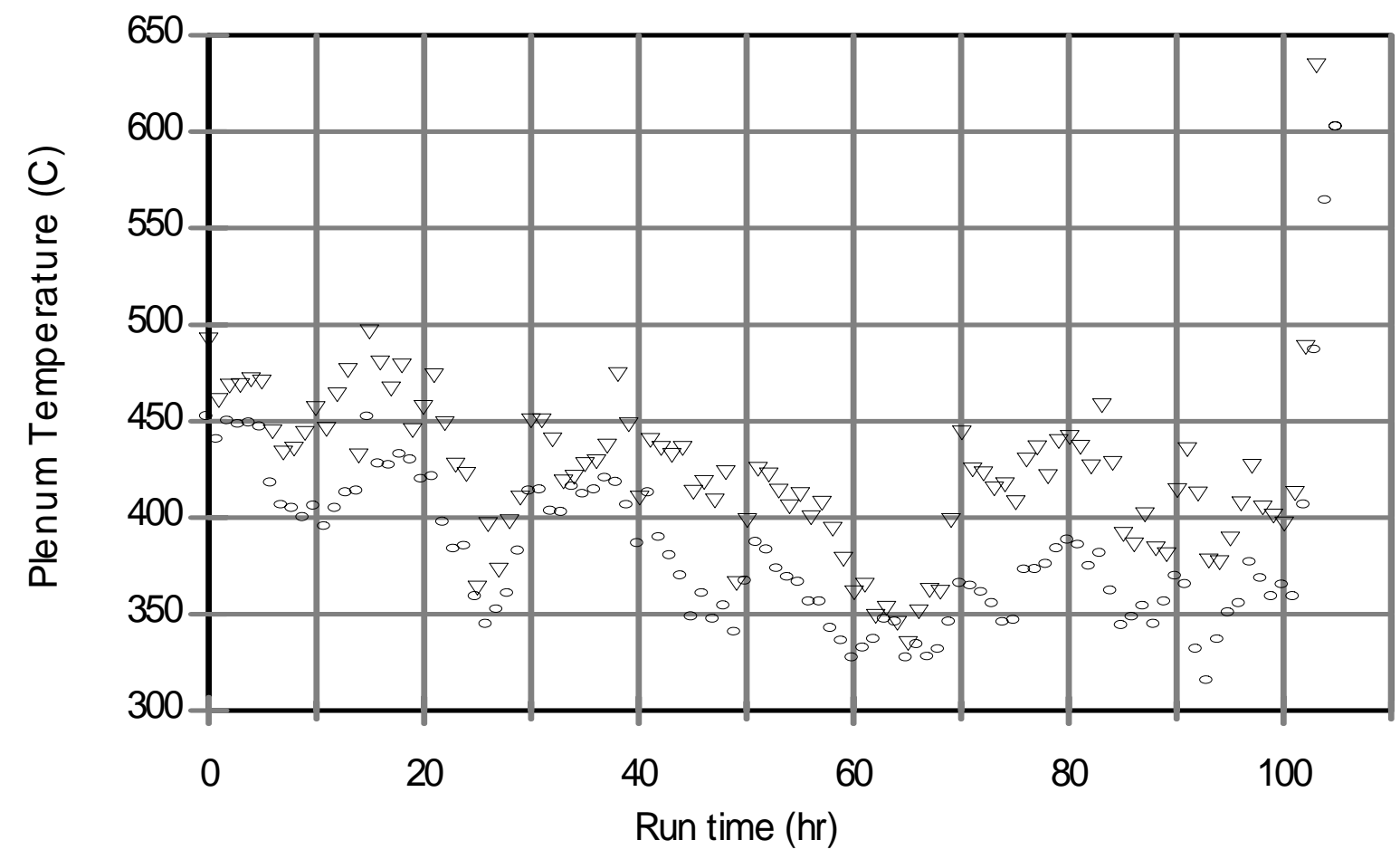

$\boldsymbol{\nabla}$ 17" from top, Thermowell

- 17 " from top, Exposed

Figure 5.7.g. Plenum temperatures (hourly averages) during DM100 Tests 7A and 7B. 
The Catholic University of America Vitreous State Laboratory
High Level Waste Vitrification System Improvements Final Report, VSL-07R1010-1, Rev. 0

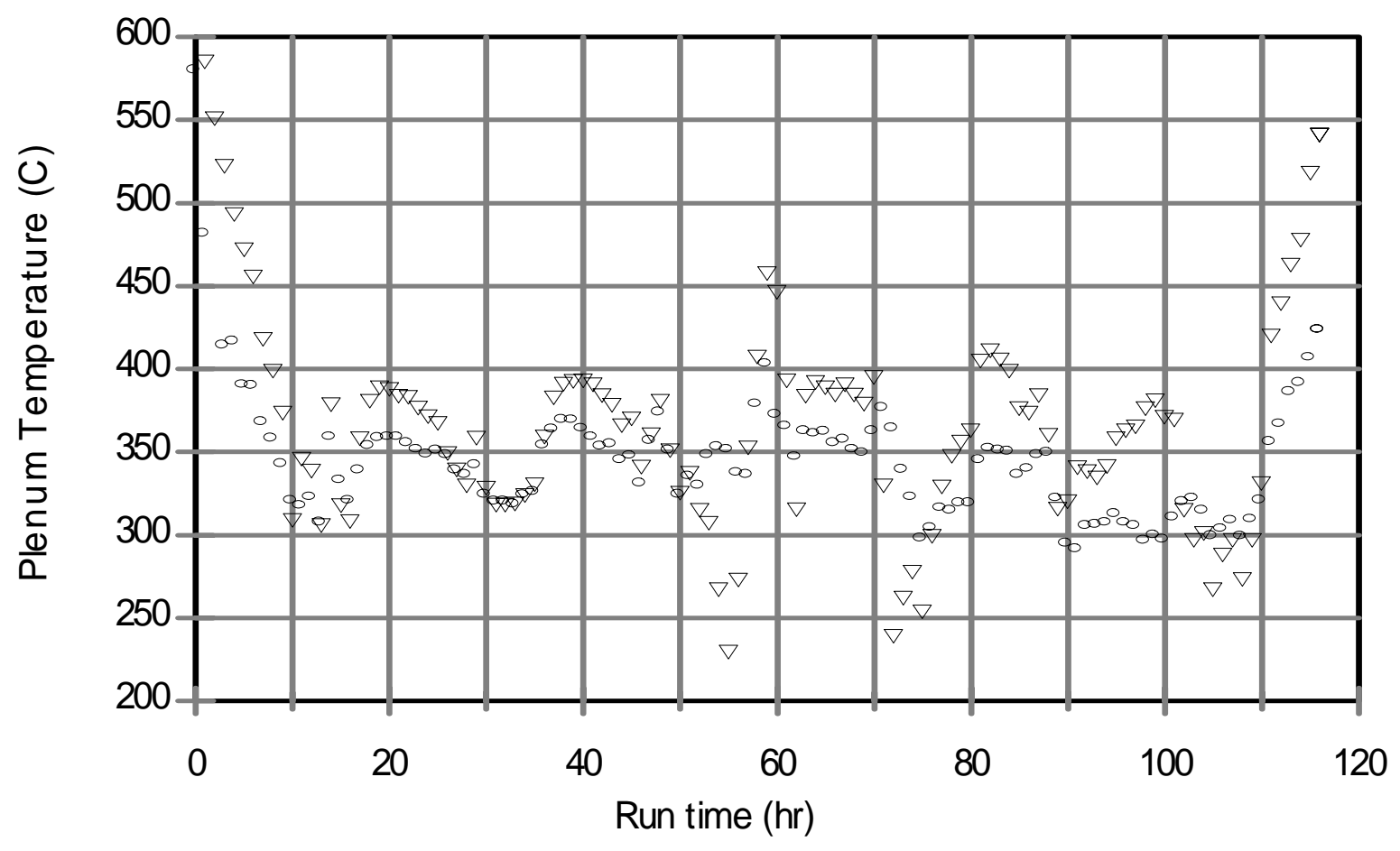

$\boldsymbol{\nabla} 17$ " from top, Thermowell

- $\quad 17$ " from top, Exposed

Figure 5.7.h. Plenum temperatures (hourly averages) during DM100 Tests 8A and 8B. 
The Catholic University of America Vitreous State Laboratory
High Level Waste Vitrification System Improvements Final Report, VSL-07R1010-1, Rev. 0

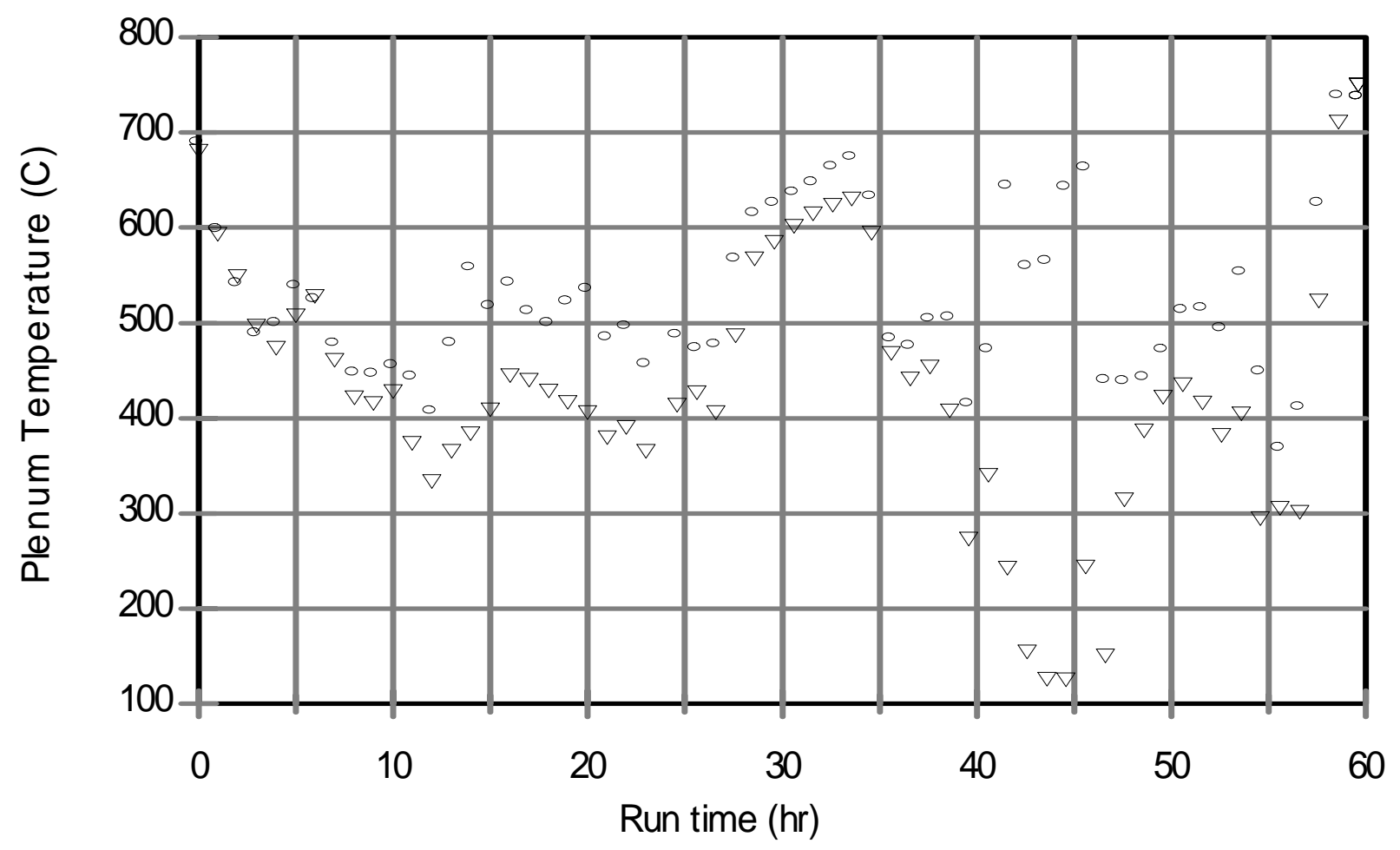

$\boldsymbol{\nabla} \quad 17^{\prime \prime}$ from top, Thermowell

- $17^{\prime \prime}$ from top, Exposed

Figure 5.7.i. Plenum temperatures (hourly averages) during DM100 Test 8C. 
The Catholic University of America Vitreous State Laboratory
High Level Waste Vitrification System Improvements

Final Report, VSL-07R1010-1, Rev. 0

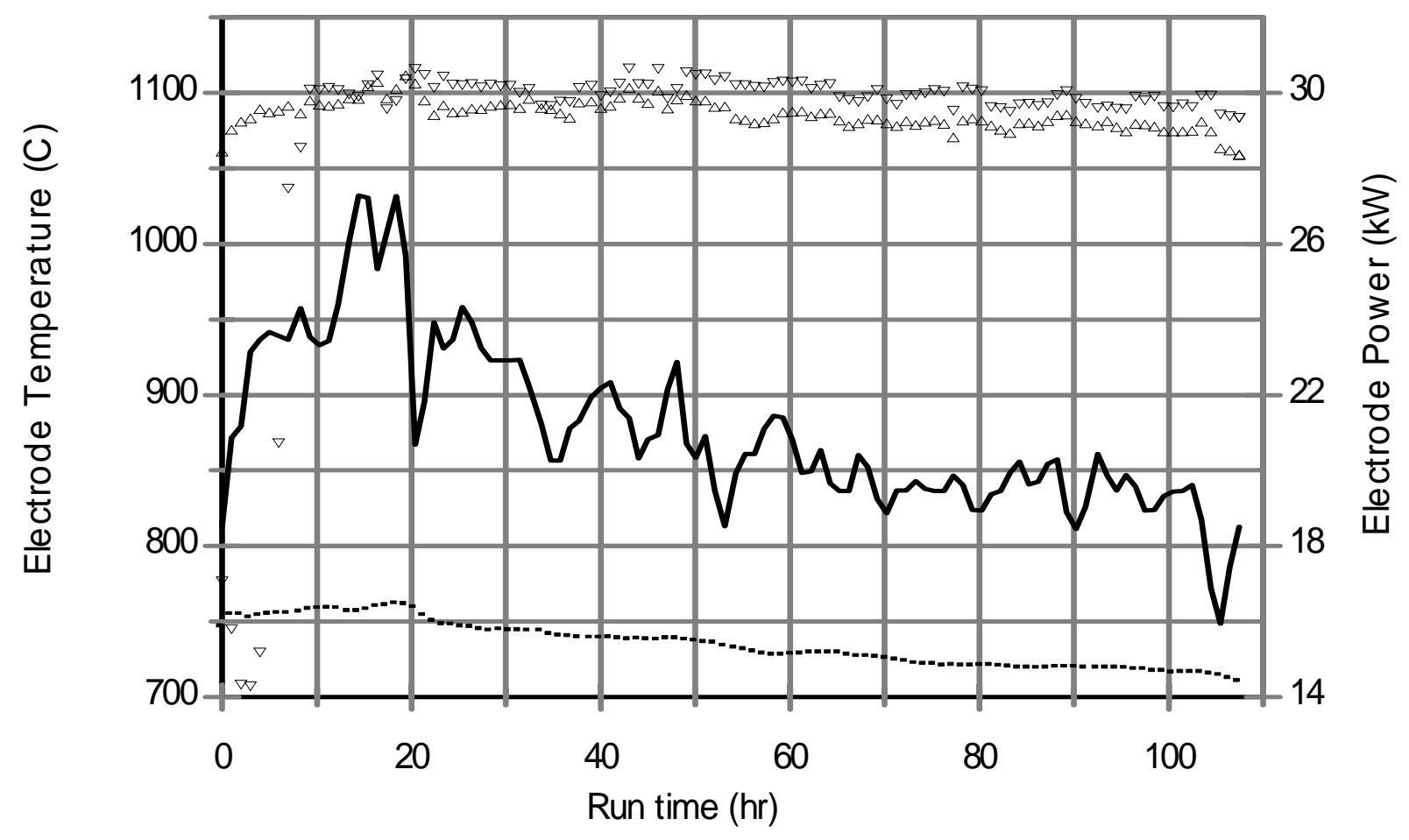

Figure 5.8.a. Electrode temperatures and power (hourly averages) during DM100 Tests 1A and 1B. 
The Catholic University of America Vitreous State Laboratory
High Level Waste Vitrification System Improvements Final Report, VSL-07R1010-1, Rev. 0

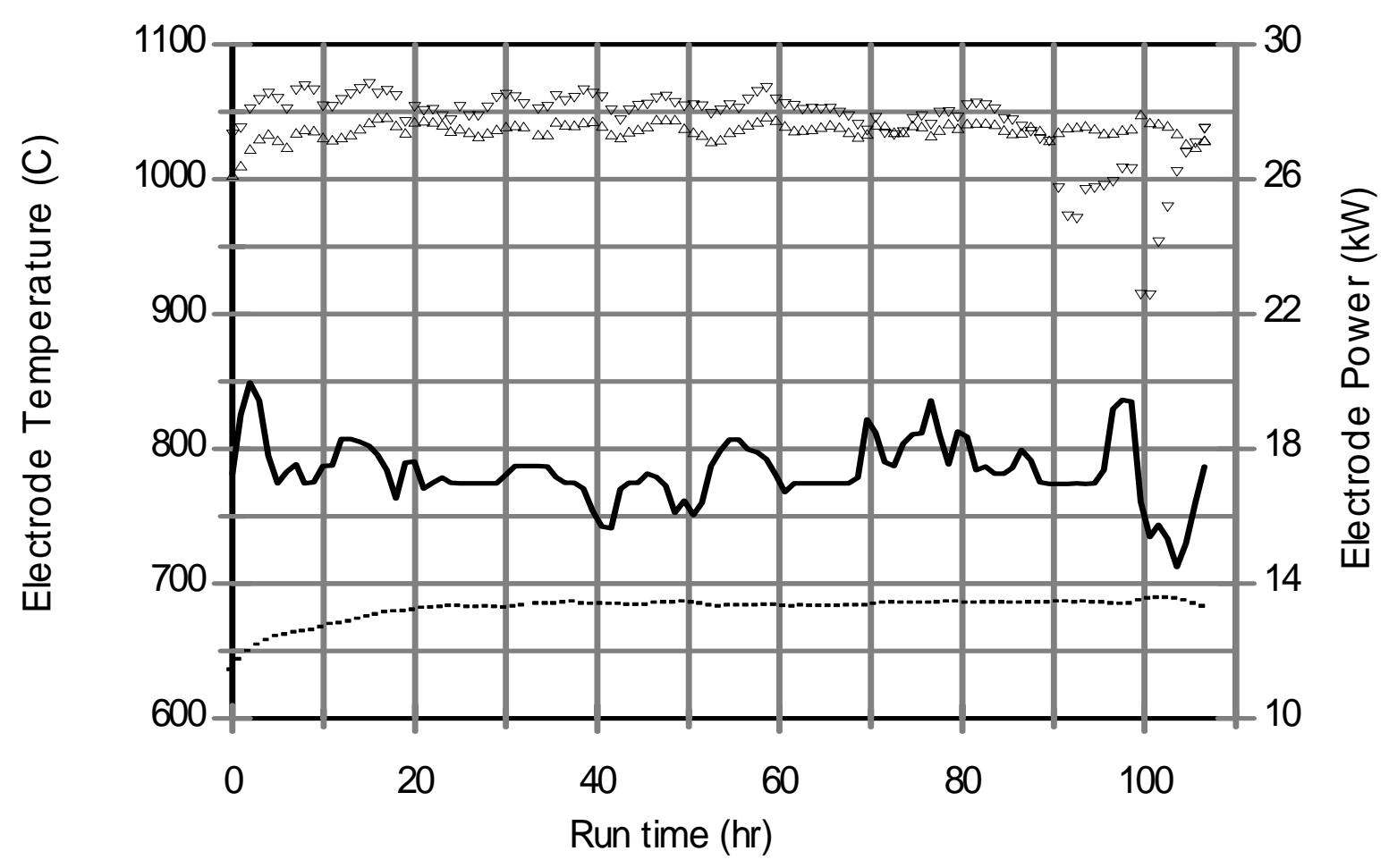

Figure 5.8.b. Electrode temperatures and power (hourly averages) during DM100 Tests 2A and 2B. 
The Catholic University of America Vitreous State Laboratory
High Level Waste Vitrification System Improvements Final Report, VSL-07R1010-1, Rev. 0

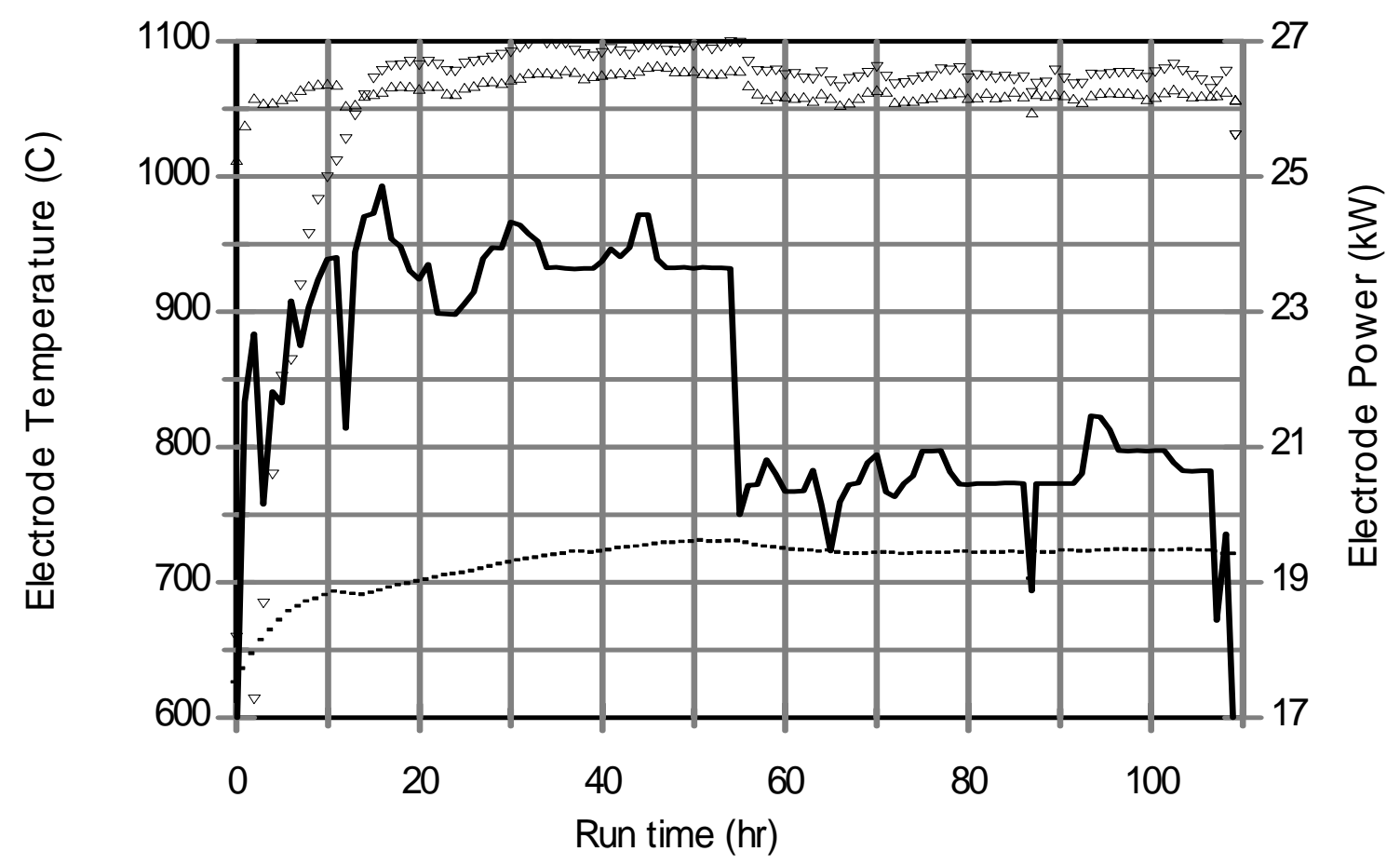

- Upper

- Lower

- Bottom

Power

Figure 5.8.c. Electrode temperatures and power (hourly averages) during DM100 Tests 3B and 4A. 
The Catholic University of America Vitreous State Laboratory
High Level Waste Vitrification System Improvements Final Report, VSL-07R1010-1, Rev. 0

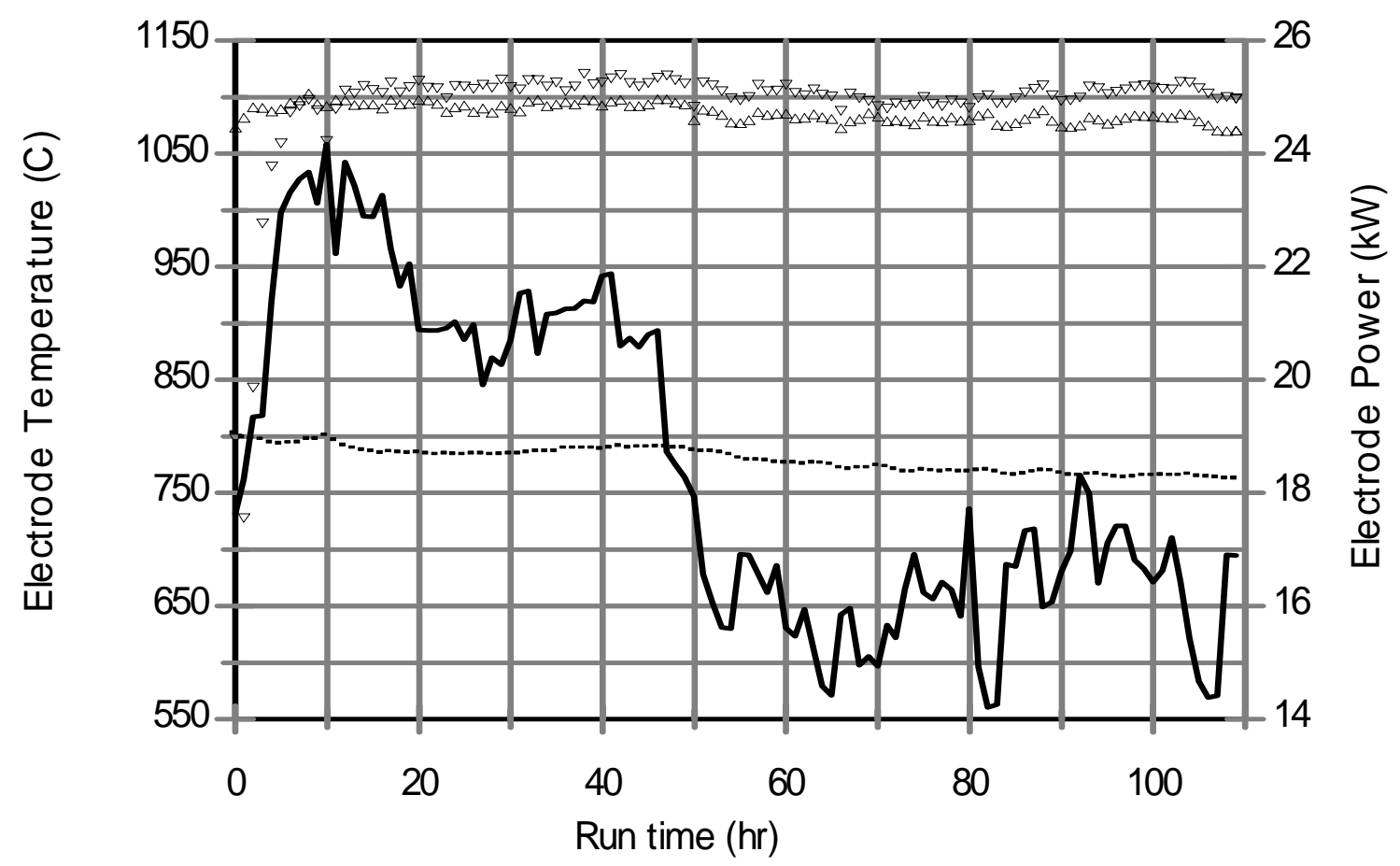

- Upper

- Lower

- Bottom

Power

Figure 5.8.d. Electrode temperatures and power (hourly averages) during DM100 Tests 5A and $5 B$. 


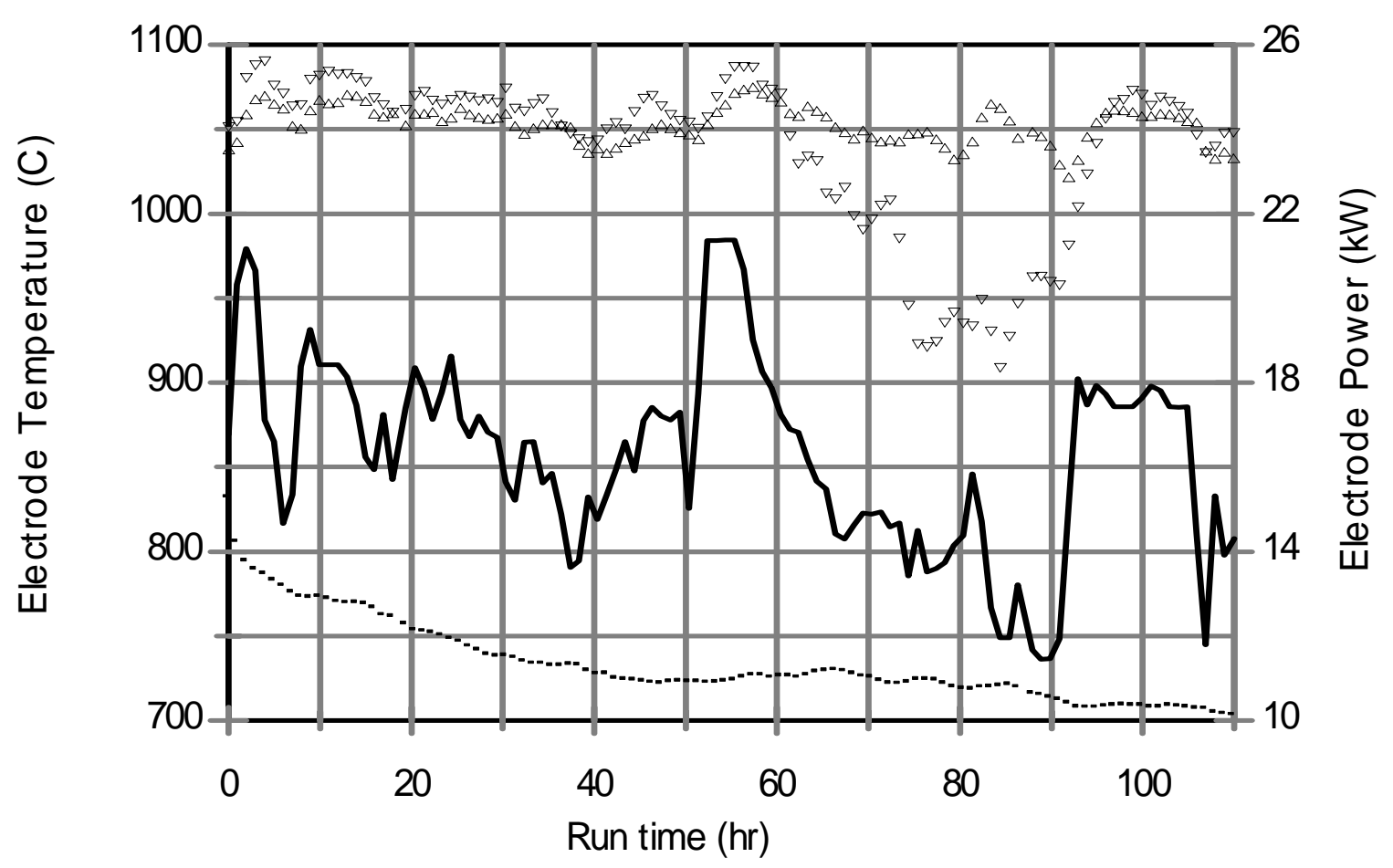

Figure 5.8.e. Electrode temperatures and power (hourly averages) during DM100 Tests 6A and 6B. 
The Catholic University of America Vitreous State Laboratory
High Level Waste Vitrification System Improvements Final Report, VSL-07R1010-1, Rev. 0

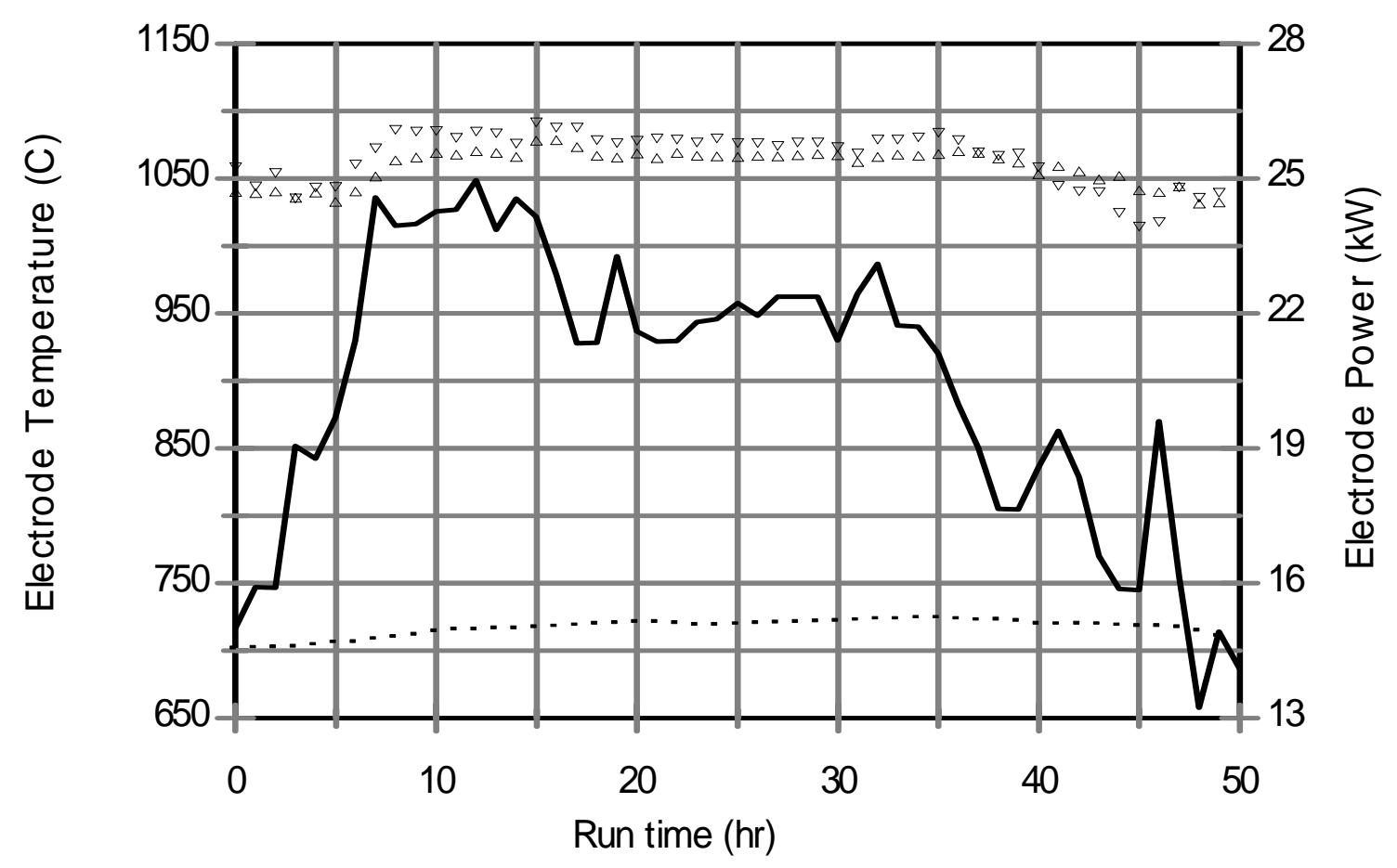

- Upper

- Lower

- Bottom

Power

Figure 5.8.f. Electrode temperatures and power (hourly averages) during DM100 Test 6C. 
The Catholic University of America Vitreous State Laboratory
High Level Waste Vitrification System Improvements Final Report, VSL-07R1010-1, Rev. 0
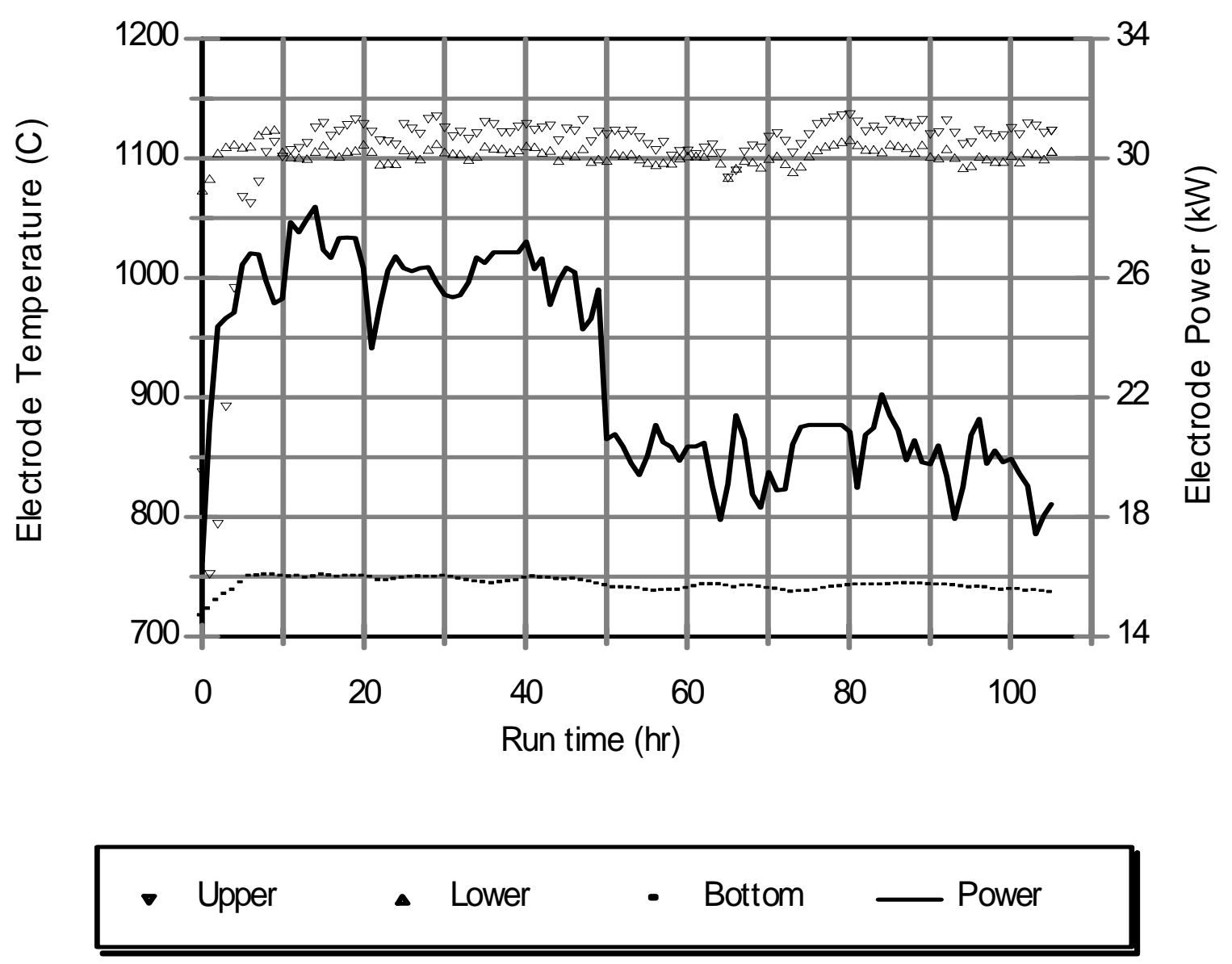

Figure 5.8.g. Electrode temperatures and power (hourly averages) during DM100 Tests 7A and 7B. 
The Catholic University of America Vitreous State Laboratory
High Level Waste Vitrification System Improvements Final Report, VSL-07R1010-1, Rev. 0

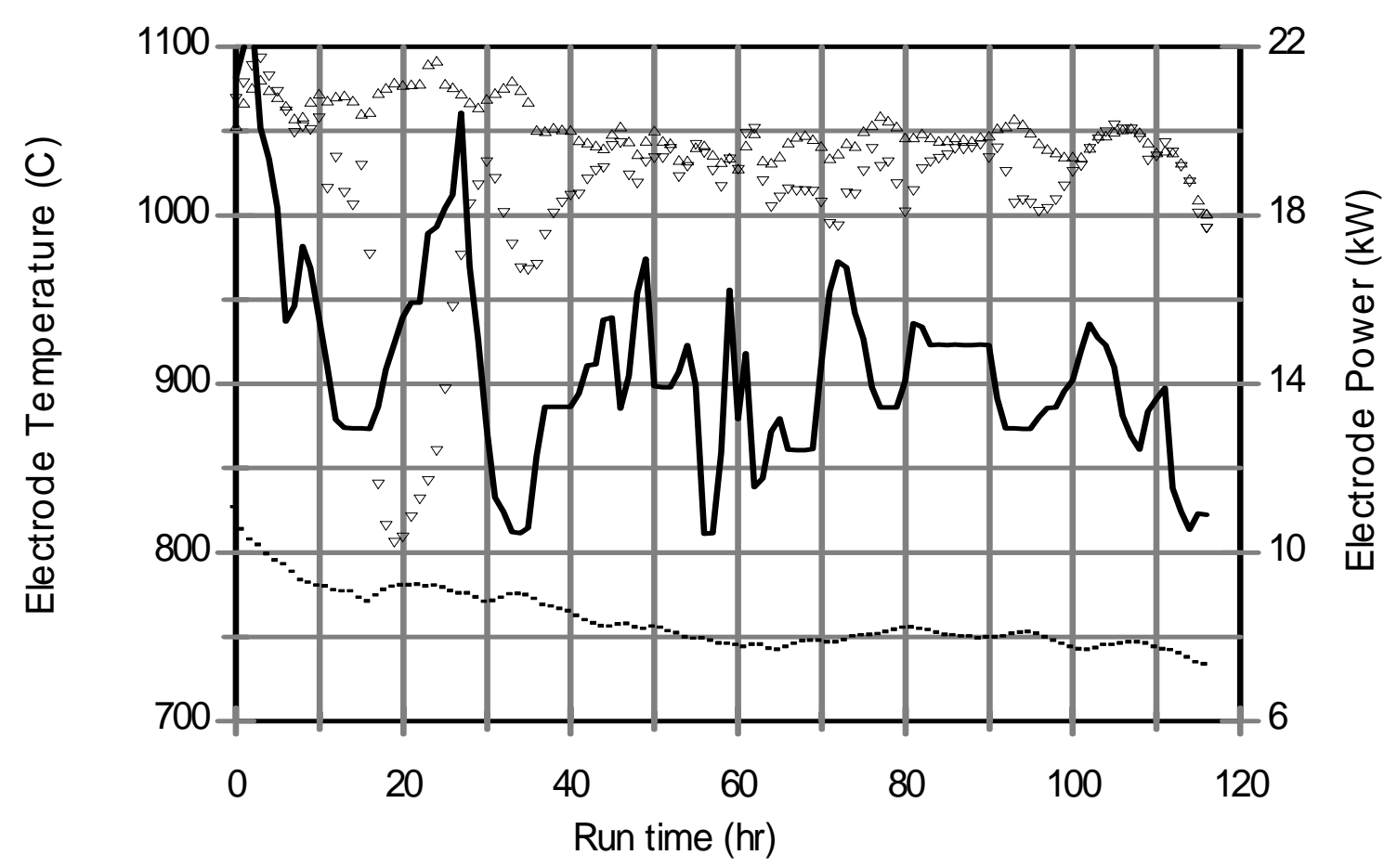

- Upper

- Lower

- Bottom

Power

Figure 5.8.h. Electrode temperatures and power (hourly averages) during DM100 Tests 8A and 8B. 
The Catholic University of America Vitreous State Laboratory
High Level Waste Vitrification System Improvements Final Report, VSL-07R1010-1, Rev. 0

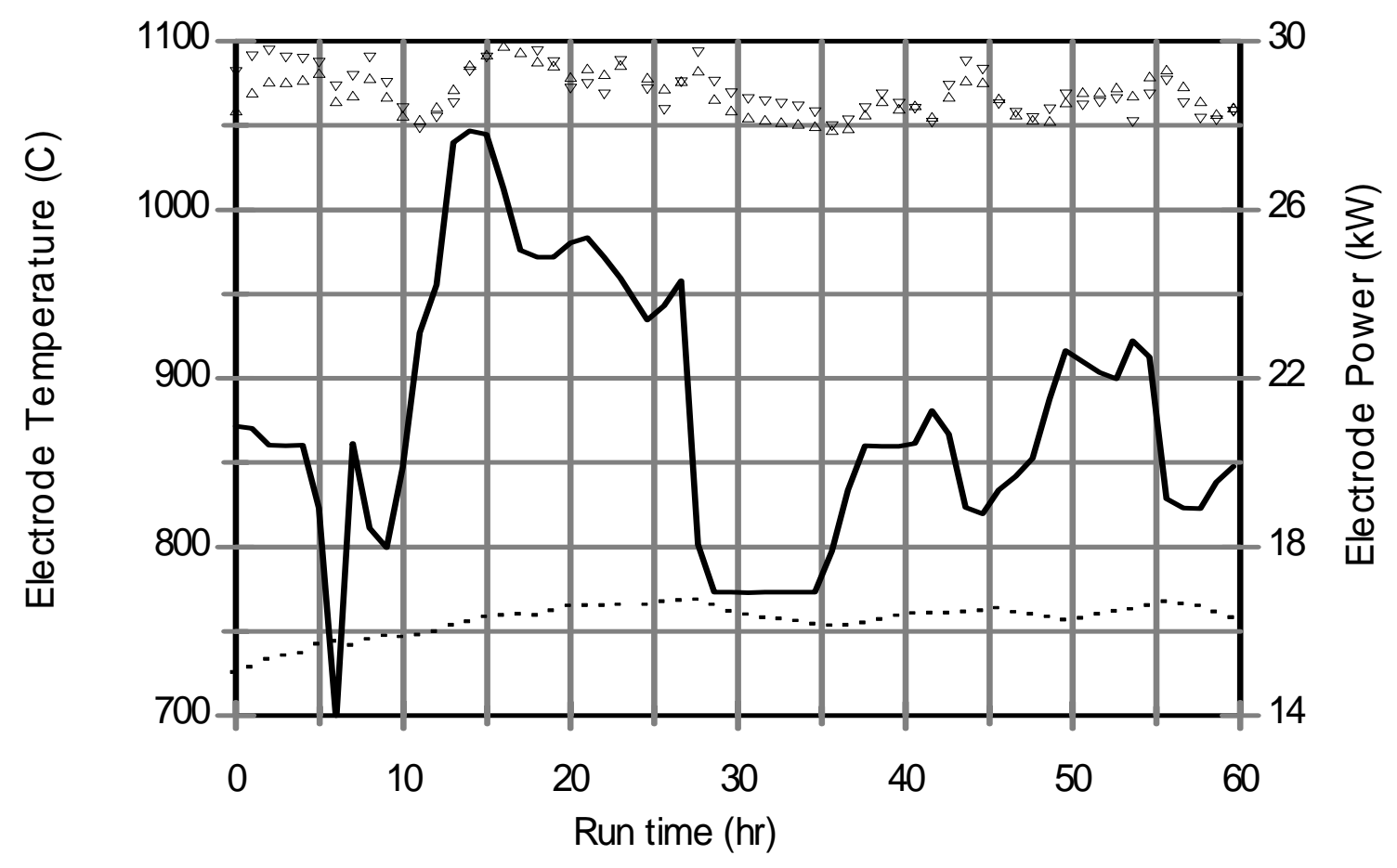

Figure 5.8.i. Electrode temperatures and power (hourly averages) during DM100 Test 8C. 
The Catholic University of America Vitreous State Laboratory
High Level Waste Vitrification System Improvements Final Report, VSL-07R1010-1, Rev. 0

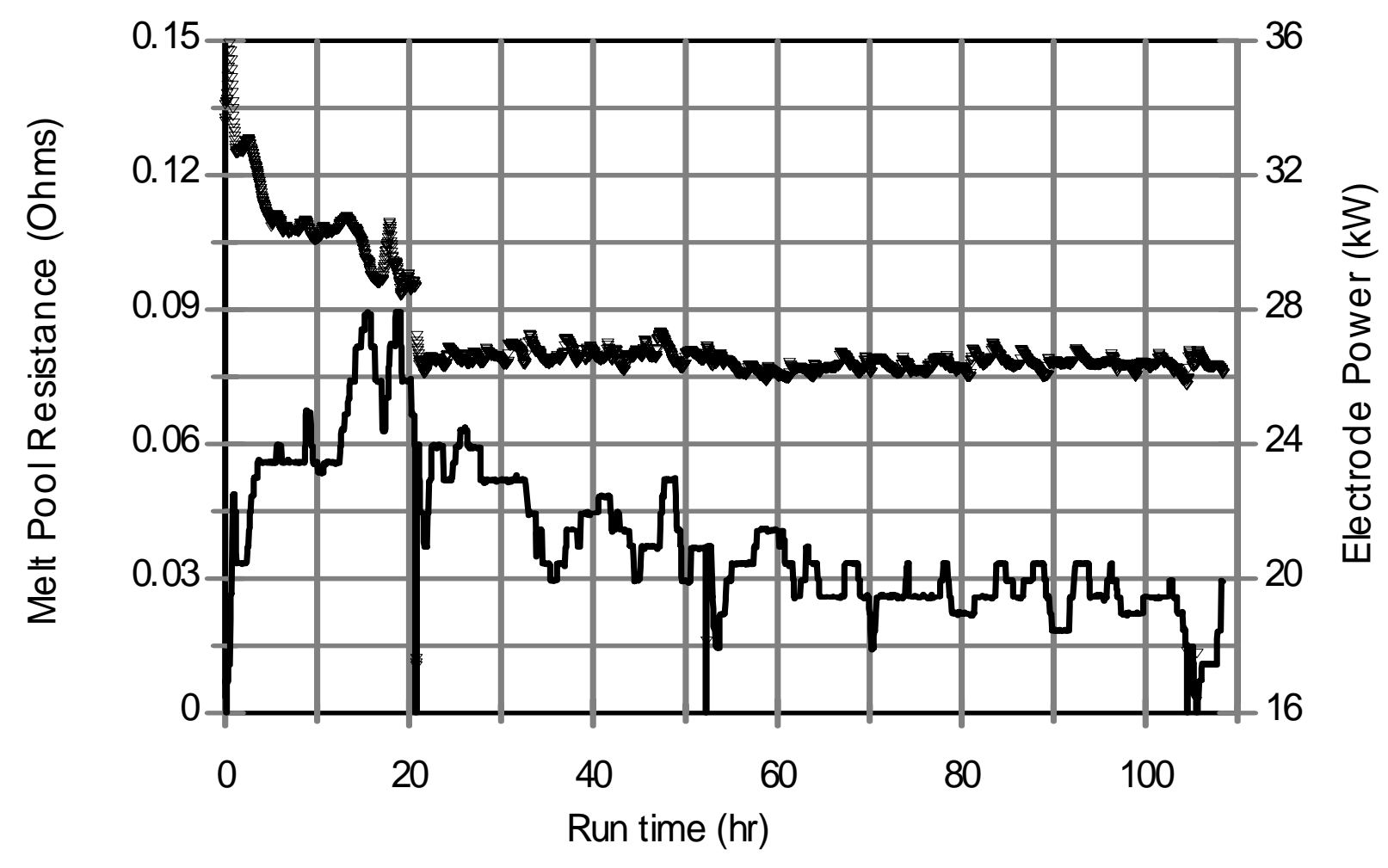

Figure 5.9.a. Melt pool resistance and total electrode power during DM100 Tests $1 \mathrm{~A}$ and $1 B$. 


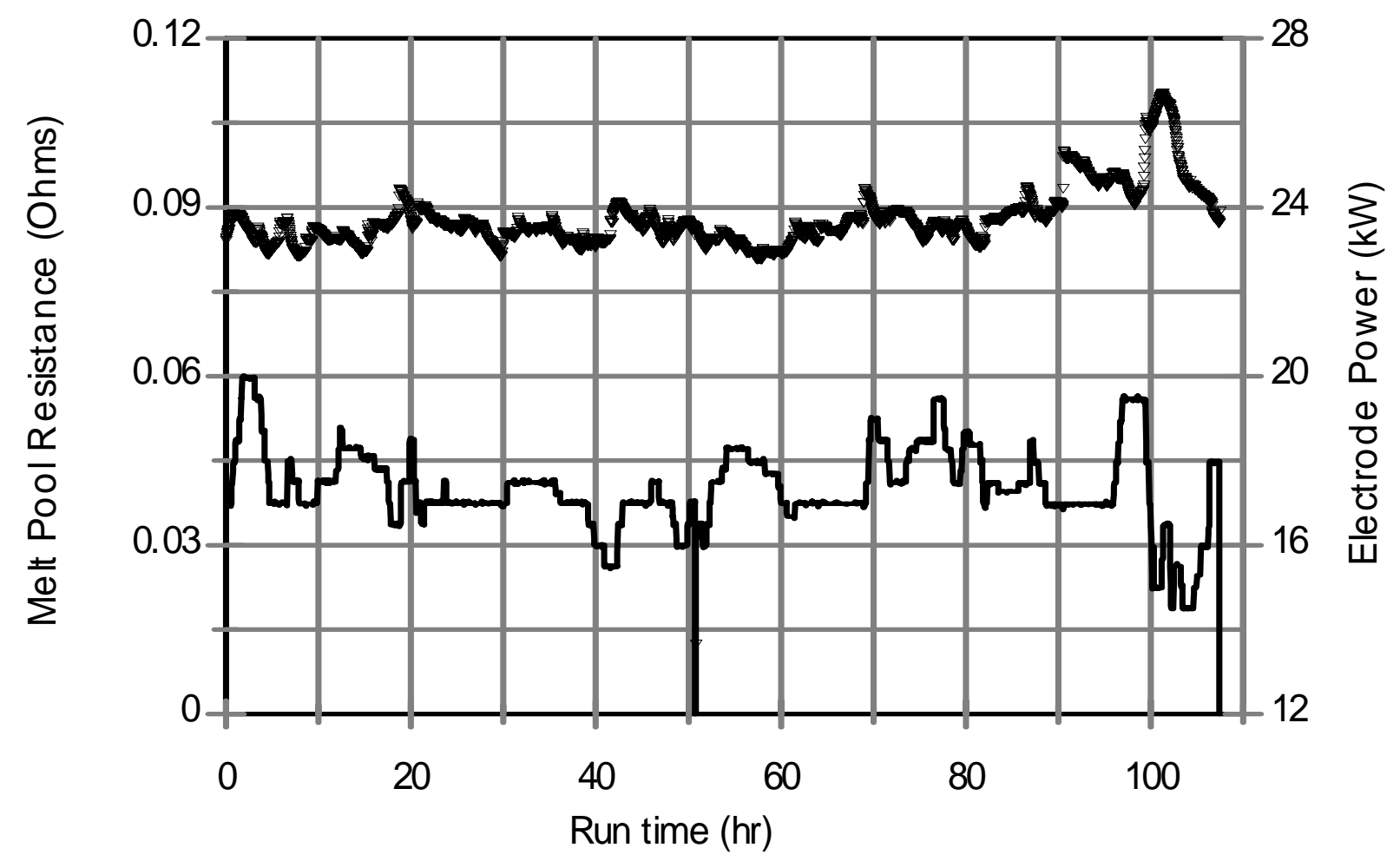

- Resistance Power

Figure 5.9.b. Melt pool resistance and total electrode power during DM100 Tests 2A and 2B. 
The Catholic University of America Vitreous State Laboratory
High Level Waste Vitrification System Improvements Final Report, VSL-07R1010-1, Rev. 0

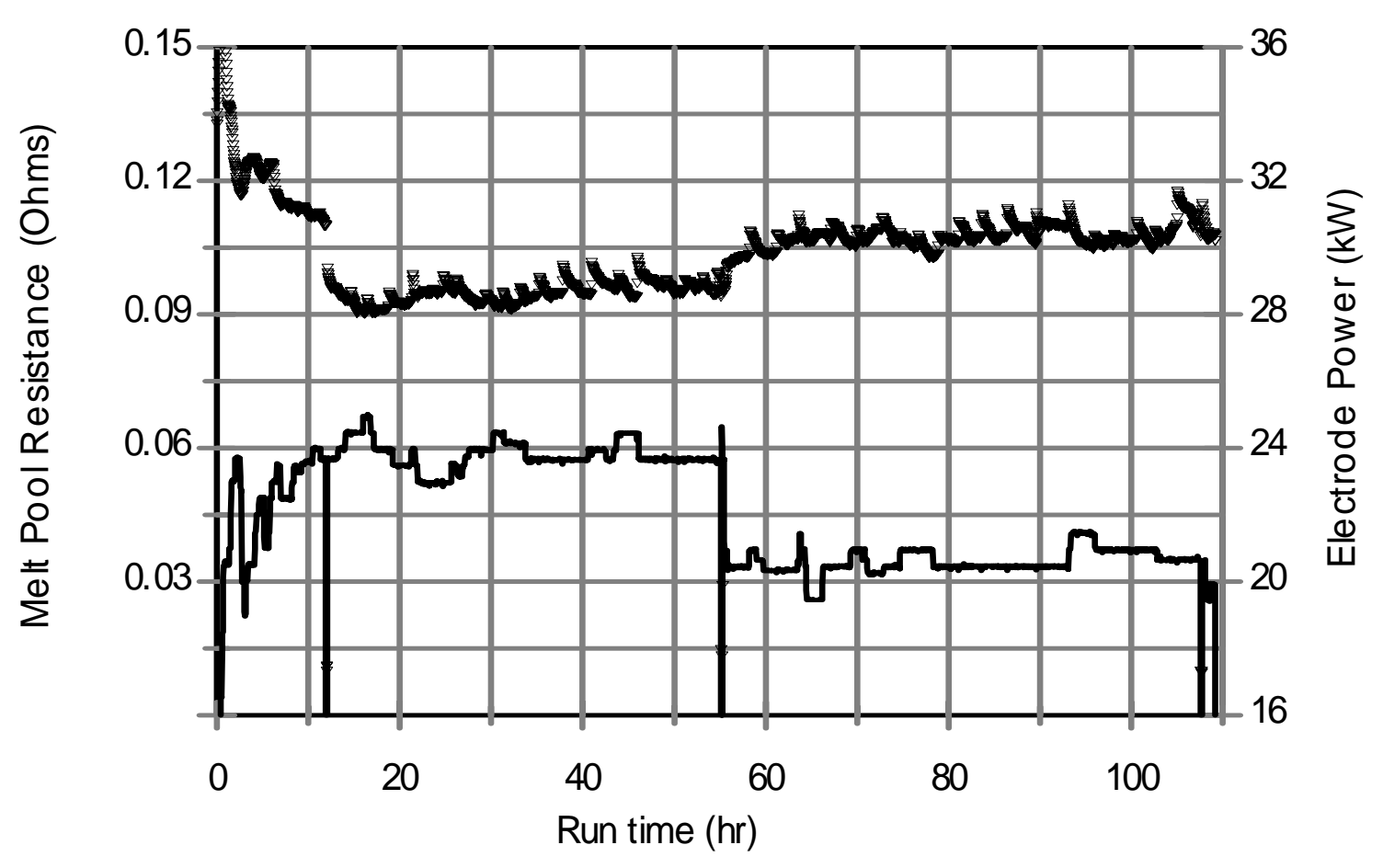

Figure 5.9.c. Melt pool resistance and total electrode power during DM100 Tests 3B and 4A. 
The Catholic University of America Vitreous State Laboratory
High Level Waste Vitrification System Improvements Final Report, VSL-07R1010-1, Rev. 0

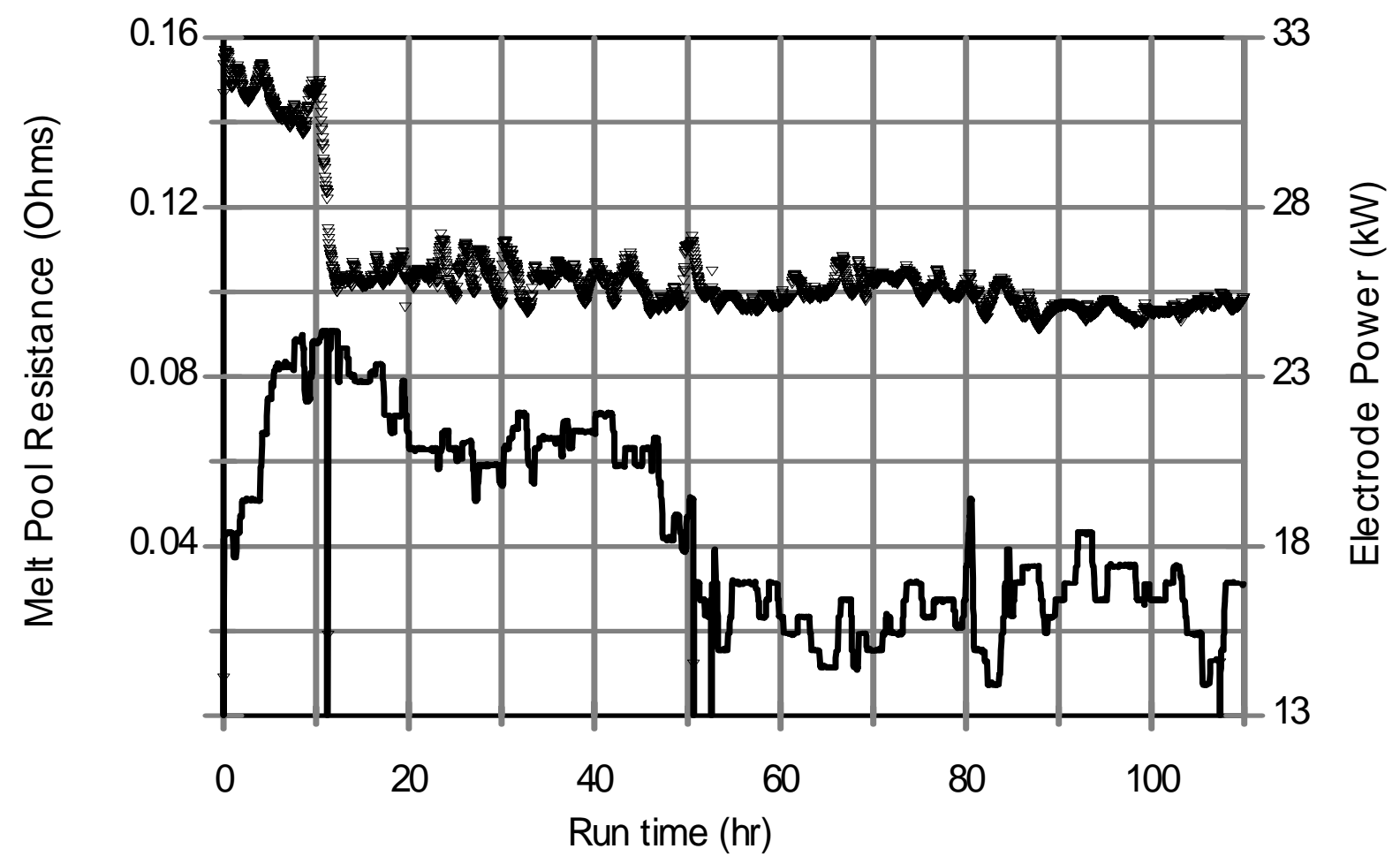

- Resistance Power

Figure 5.9.d. Melt pool resistance and total electrode power during DM100 Tests 5A and 5B. 
The Catholic University of America Vitreous State Laboratory
High Level Waste Vitrification System Improvements Final Report, VSL-07R1010-1, Rev. 0

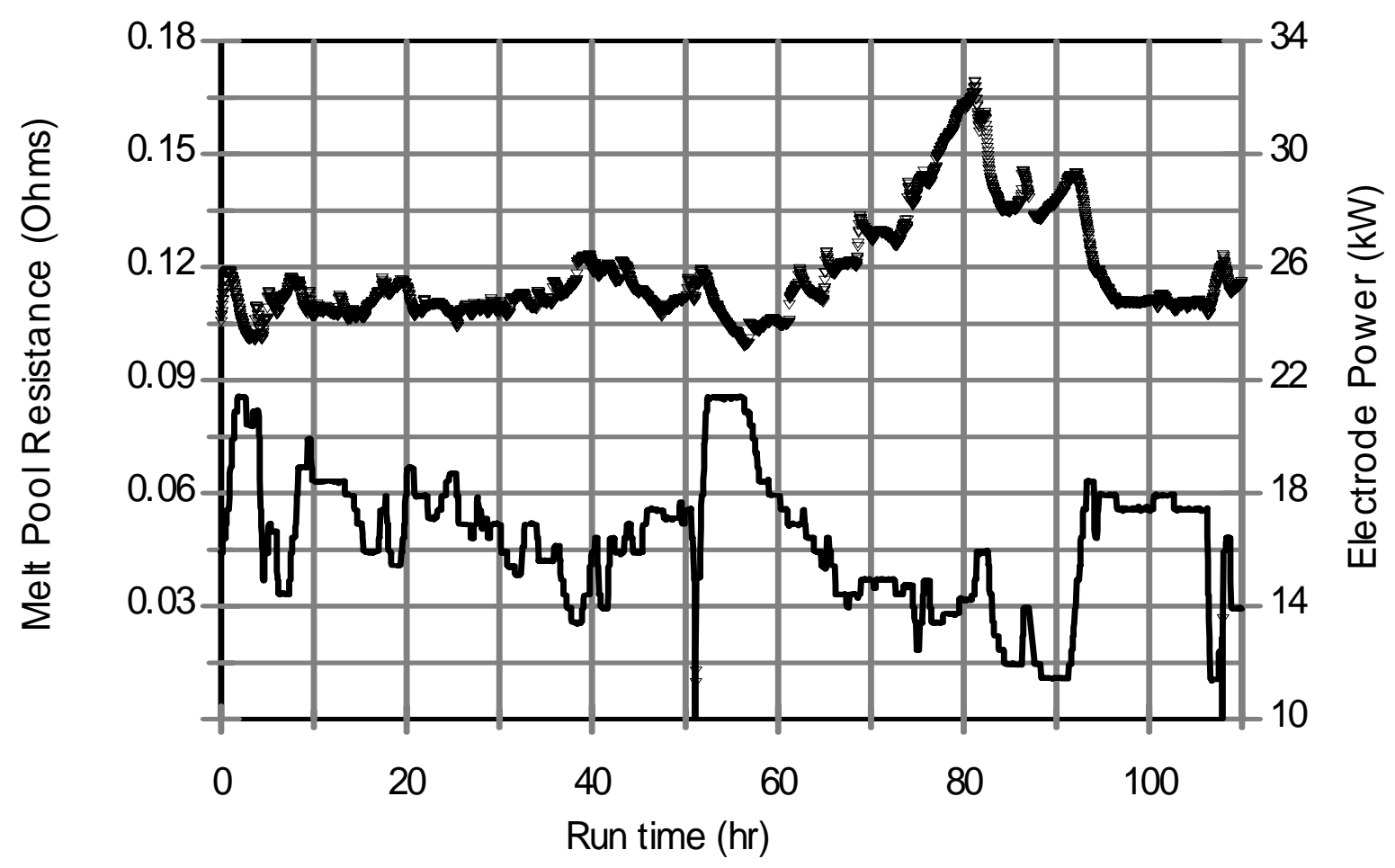

- Resistance Power

Figure 5.9.e. Melt pool resistance and total electrode power during DM100 Tests 6A and 6B. 


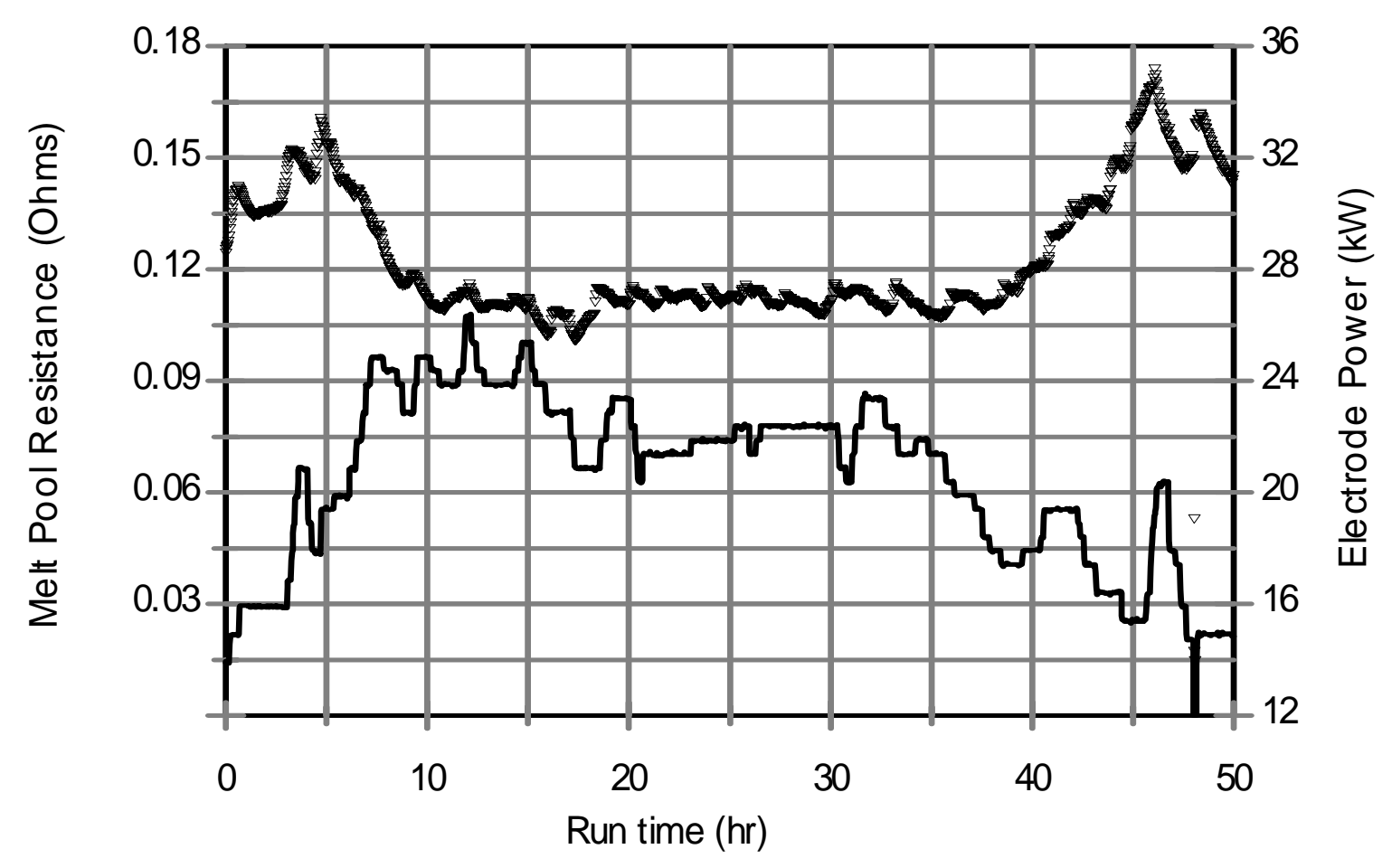

Figure 5.9.f. Melt pool resistance and total electrode power during DM100 Test 6C. 
The Catholic University of America Vitreous State Laboratory
High Level Waste Vitrification System Improvements Final Report, VSL-07R1010-1, Rev. 0

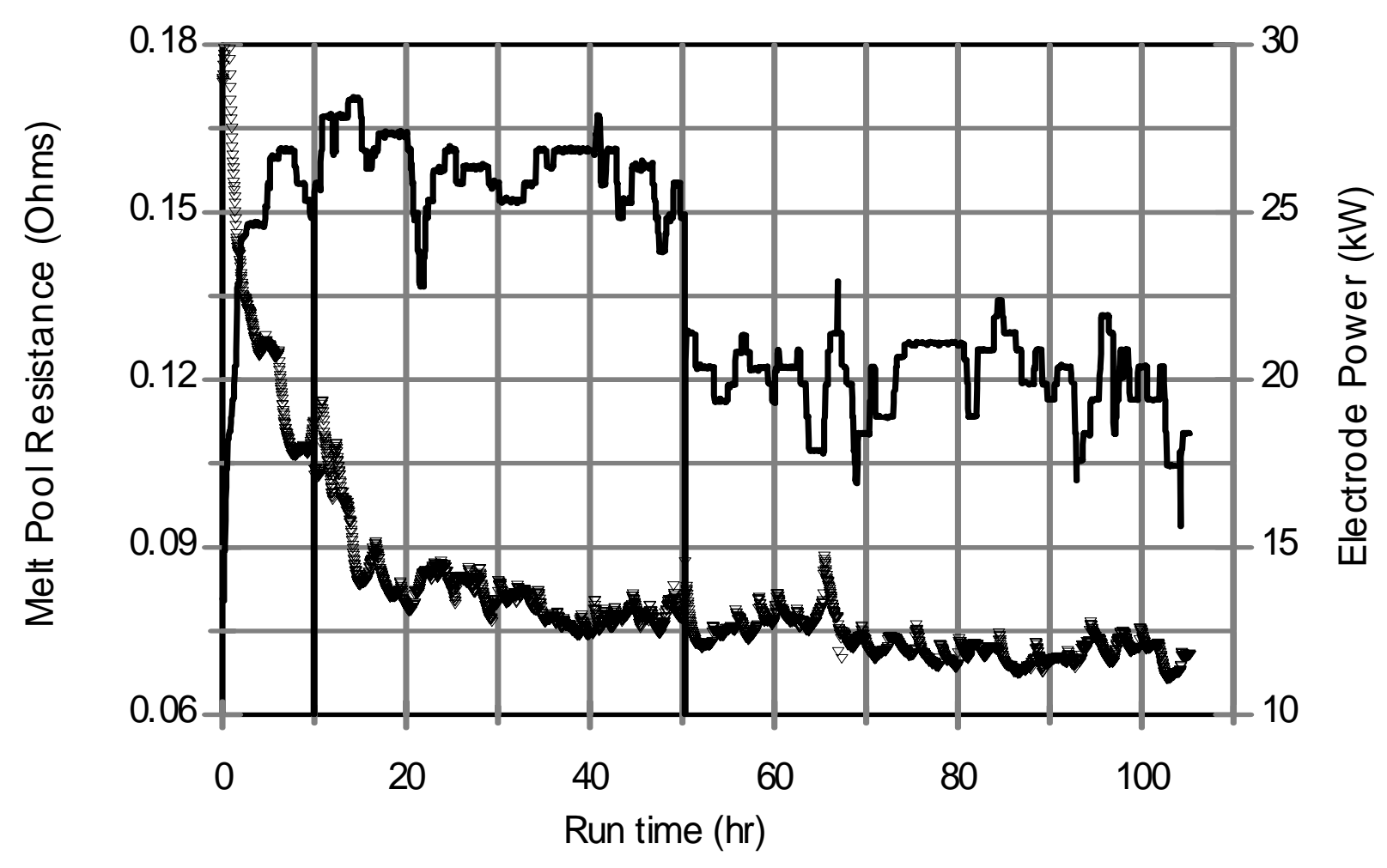

Figure 5.9.g. Melt pool resistance and total electrode power during DM100 Tests 7A and 7B. 
The Catholic University of America Vitreous State Laboratory
High Level Waste Vitrification System Improvements Final Report, VSL-07R1010-1, Rev. 0

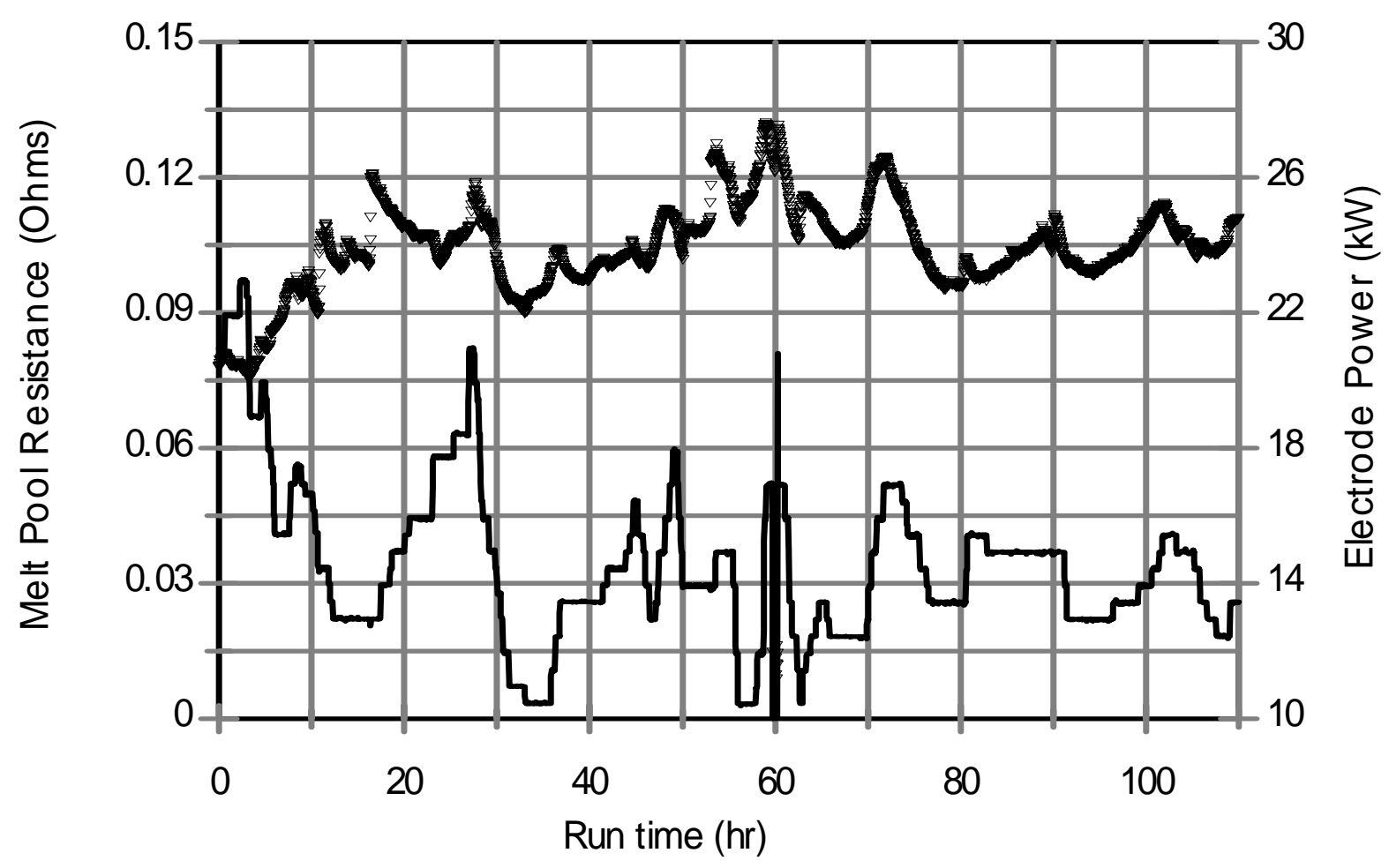

- Resistance - Power

Figure 5.9.h. Melt pool resistance and total electrode power during DM100 Tests 8A and 8B. 
The Catholic University of America Vitreous State Laboratory
High Level Waste Vitrification System Improvements Final Report, VSL-07R1010-1, Rev. 0

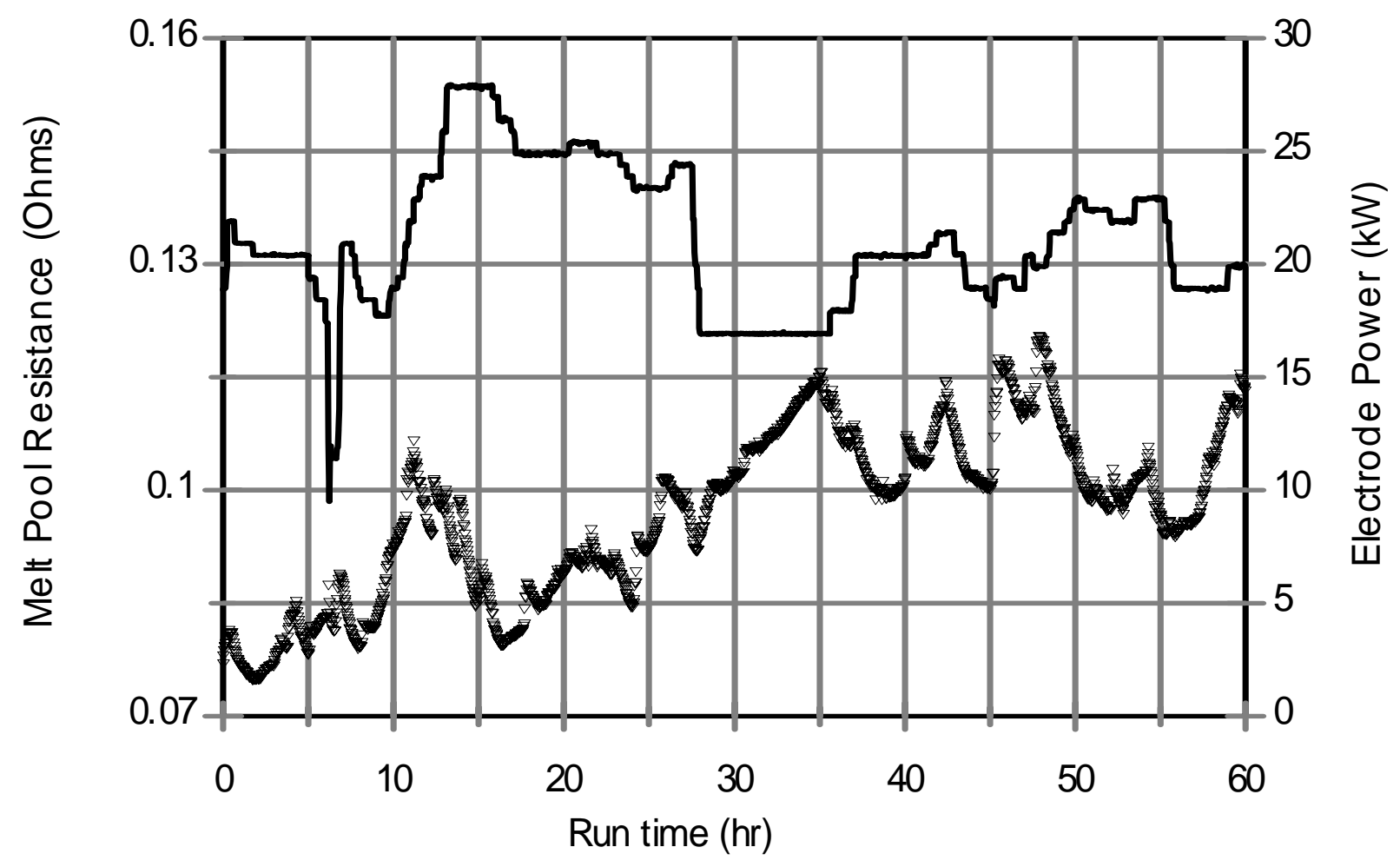

Figure 5.9.i. Melt pool resistance and total electrode power during DM100 Test 8C. 
The Catholic University of America Vitreous State Laboratory
High Level Waste Vitrification System Improvements Final Report, VSL-07R1010-1, Rev. 0

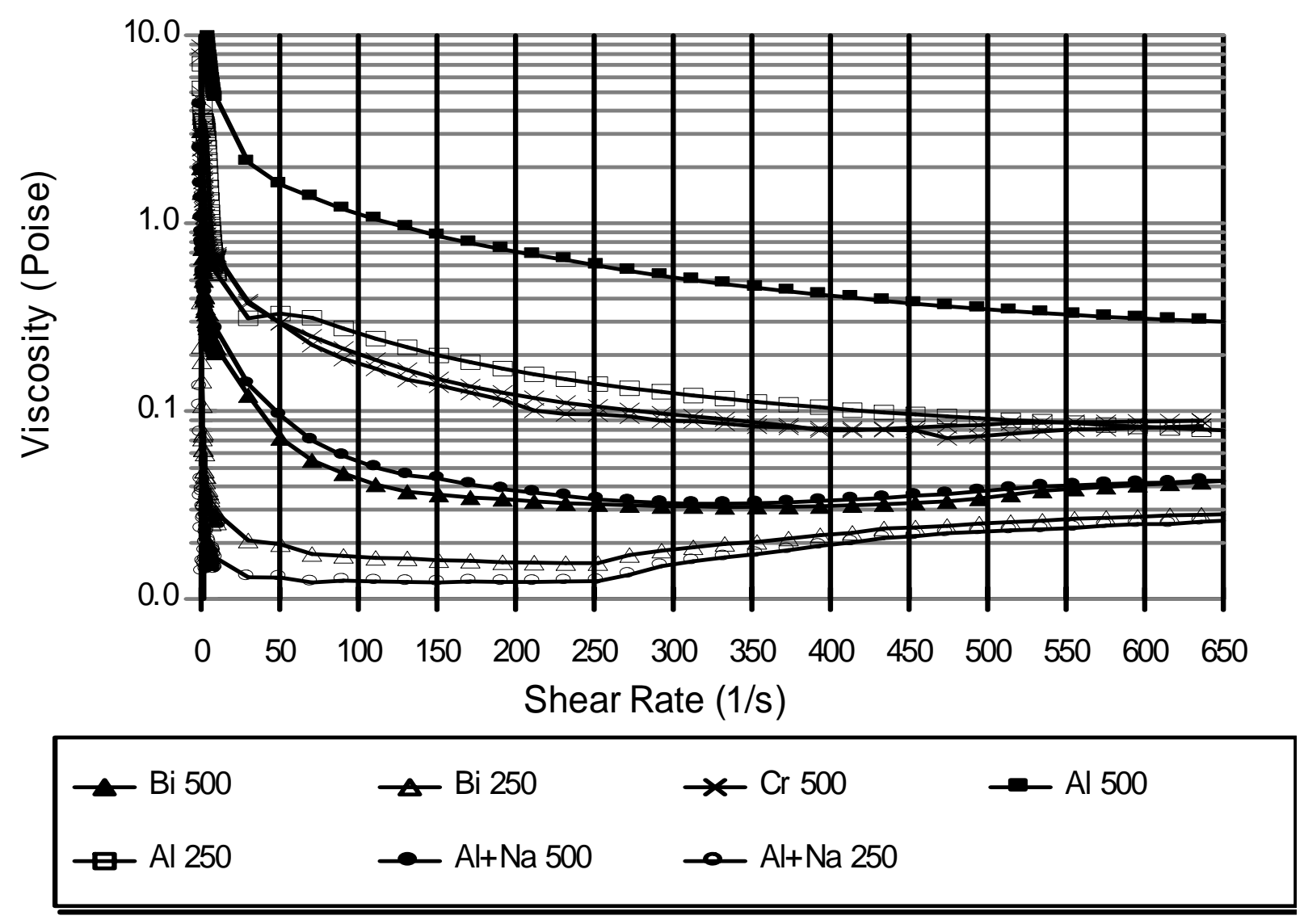

Figure 6.1. Measured viscosities of feed samples at target 250 and $500 \mathrm{~g} / \mathrm{l}$ solids content. 
The Catholic University of America Vitreous State Laboratory
High Level Waste Vitrification System Improvements Final Report, VSL-07R1010-1, Rev. 0

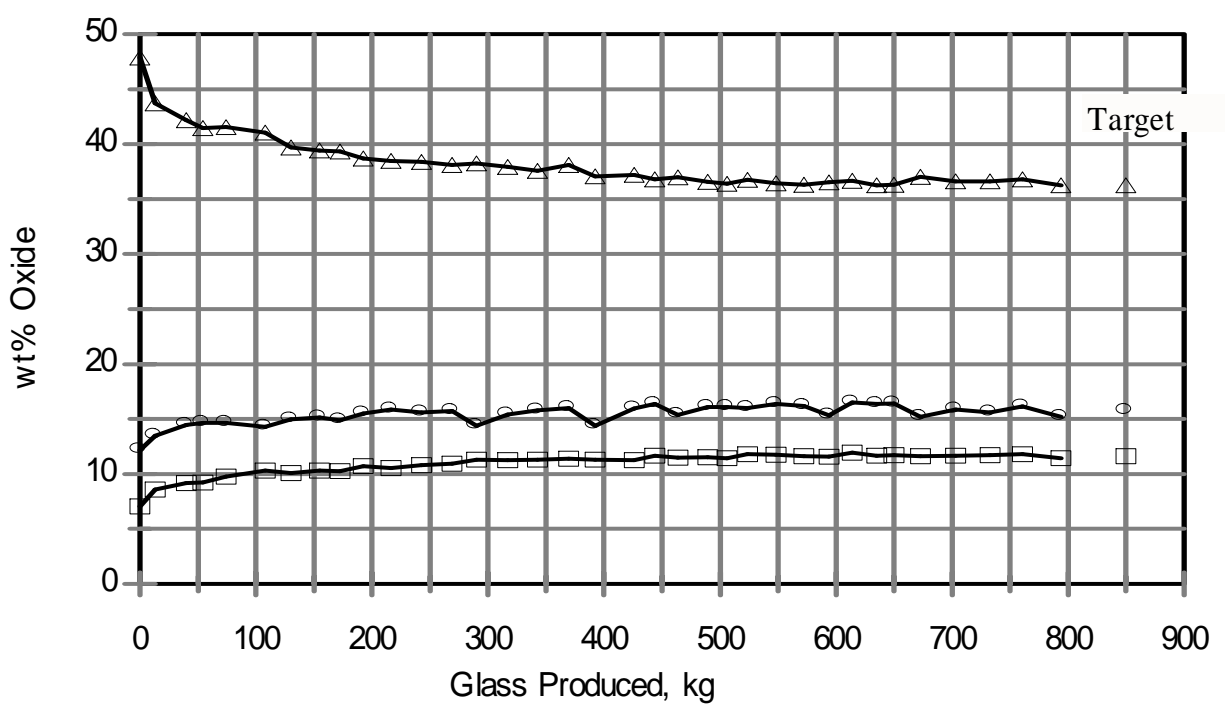

$\because \mathrm{Al} \quad-\mathrm{O}-\mathrm{Na} \rightarrow \mathrm{Si}$
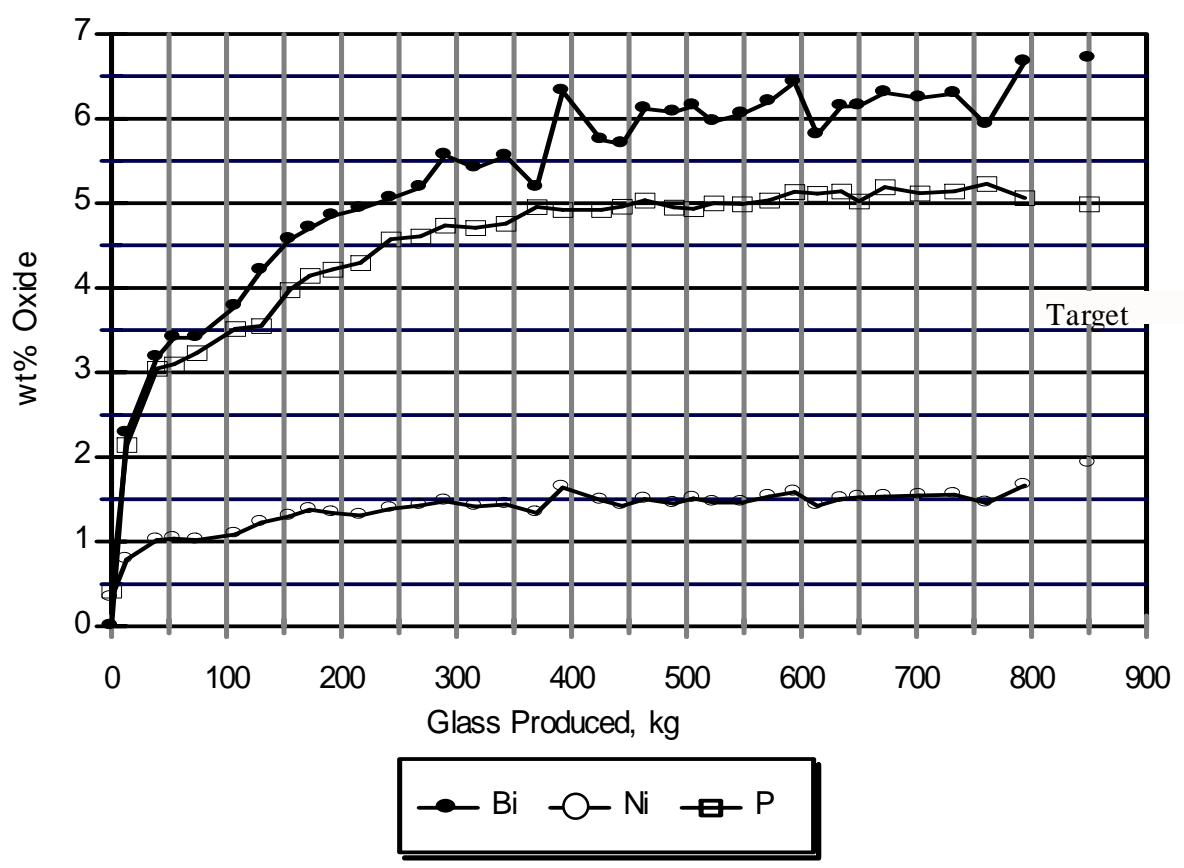

Figure 6.2.a. DM100 bismuth-limited product glass composition determined by XRF. 
The Catholic University of America Vitreous State Laboratory
High Level Waste Vitrification System Improvements Final Report, VSL-07R1010-1, Rev. 0
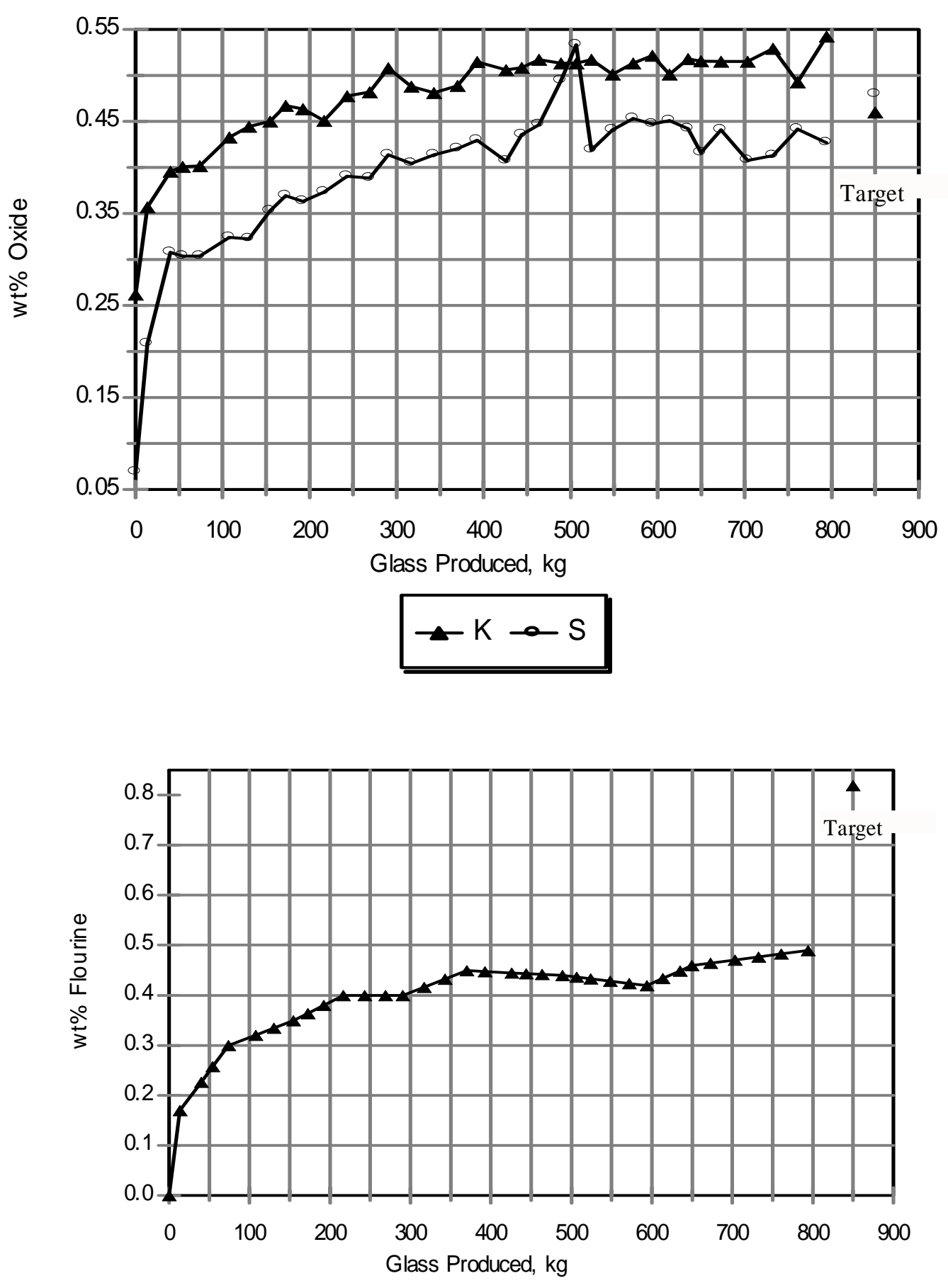

Figure 6.2.b. DM100 bismuth-limited product glass composition determined by XRF. 
The Catholic University of America Vitreous State Laboratory
High Level Waste Vitrification System Improvements Final Report, VSL-07R1010-1, Rev. 0

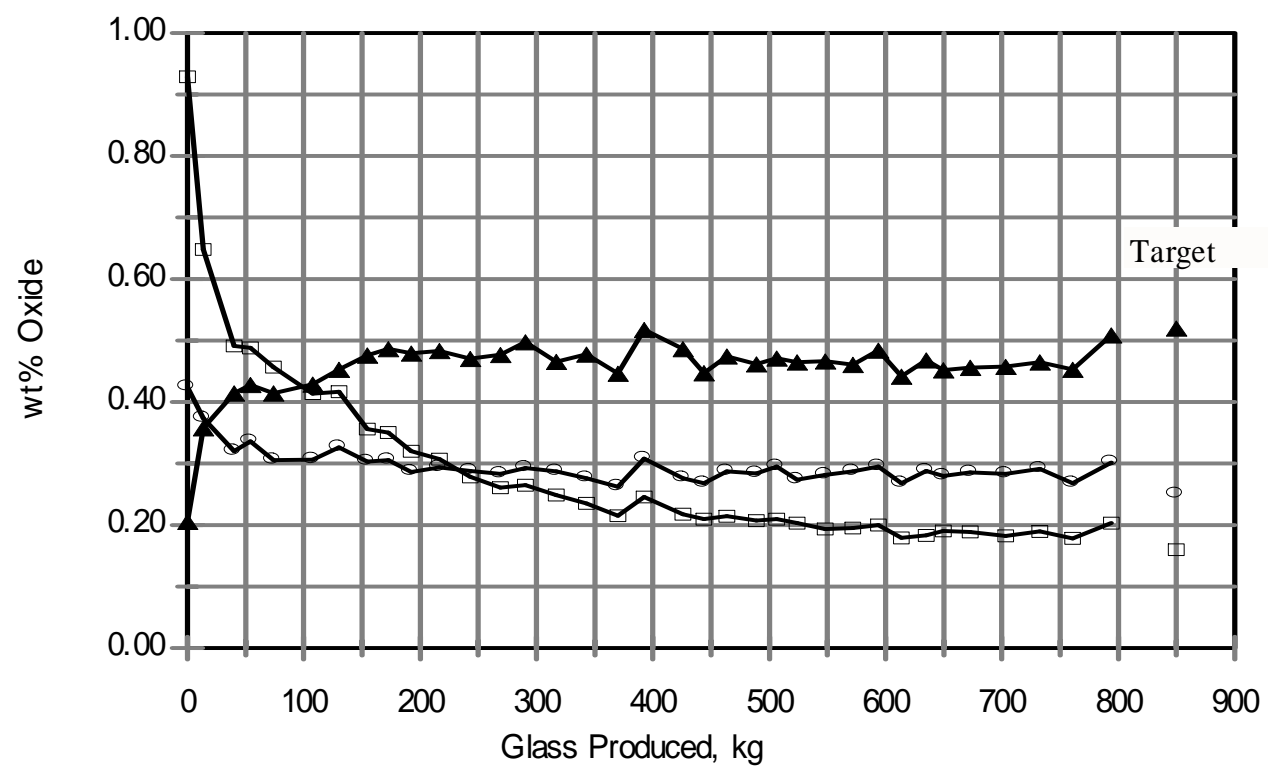

$\rightarrow \mathrm{Cr} \quad-\mathrm{O} \mathrm{Pb} \quad-\square-\mathrm{Zn}$

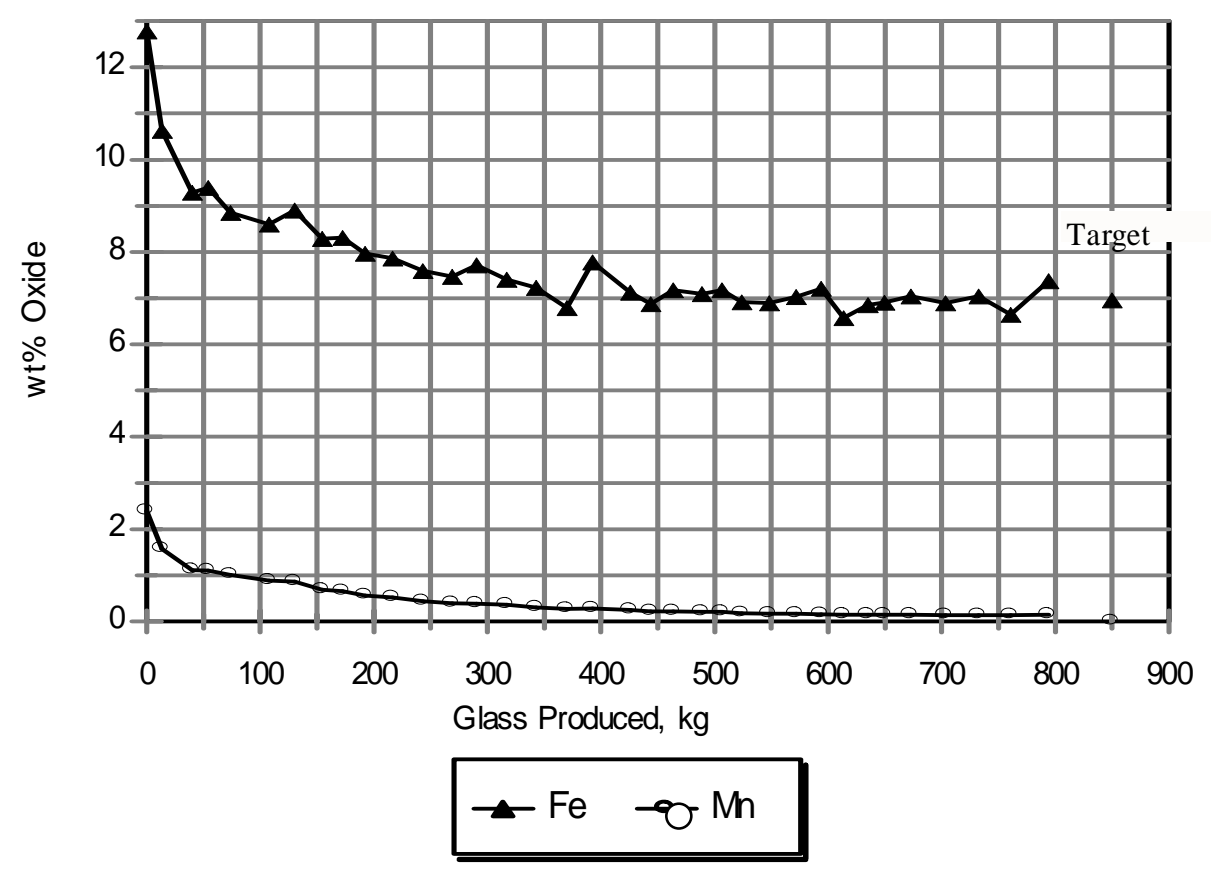

Figure 6.2.c. DM100 bismuth-limited product glass composition determined by XRF. 
The Catholic University of America Vitreous State Laboratory
High Level Waste Vitrification System Improvements Final Report, VSL-07R1010-1, Rev. 0
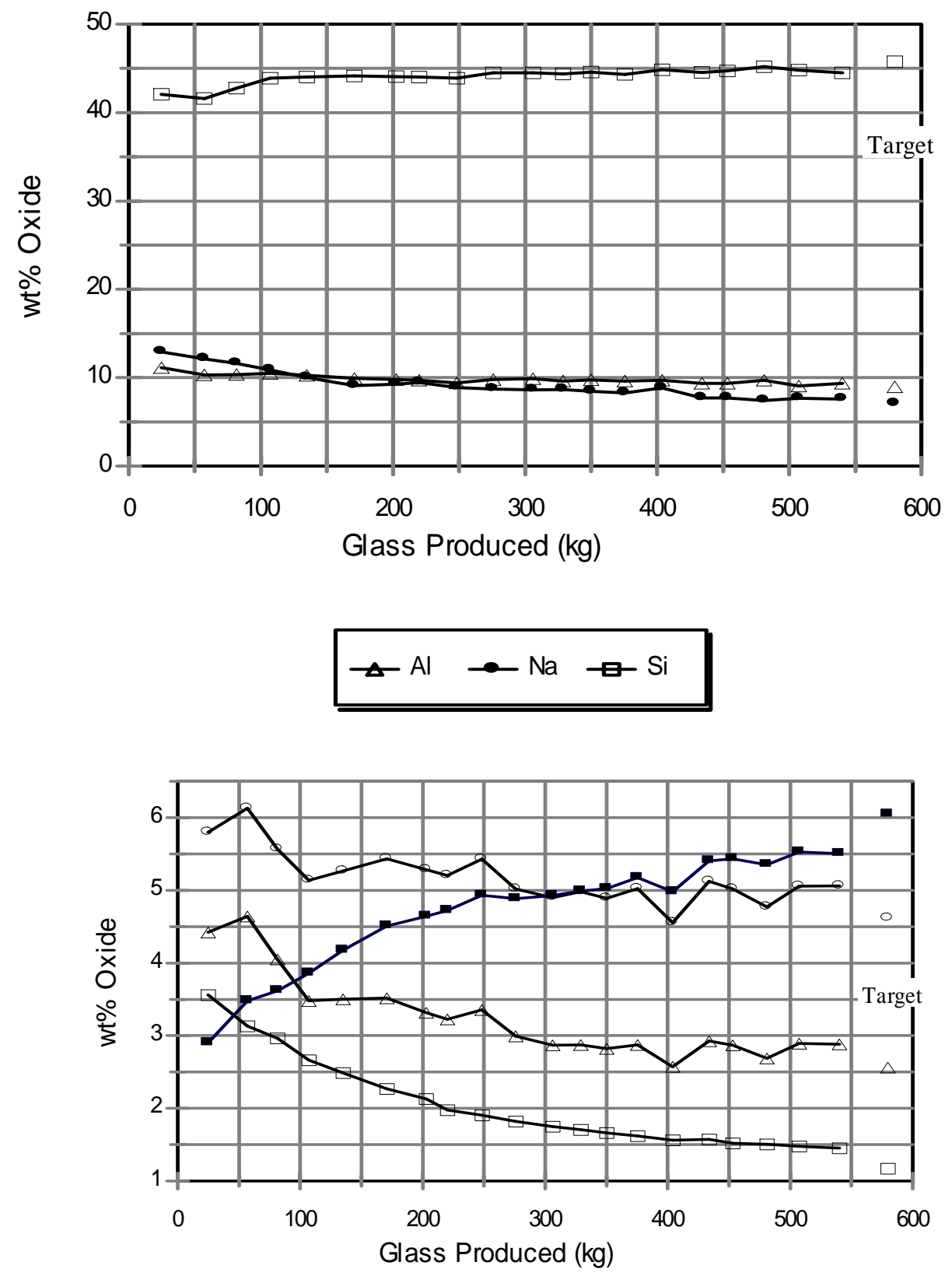

$$
\triangle \mathrm{Bi} \rightarrow \mathrm{Fe} \rightarrow \mathrm{K} \quad \boldsymbol{\mathrm { B }}
$$

Figure 6.3.a. DM100 chromium-limited product glass composition determined by XRF. 
The Catholic University of America Vitreous State Laboratory
High Level Waste Vitrification System Improvements Final Report, VSL-07R1010-1, Rev. 0
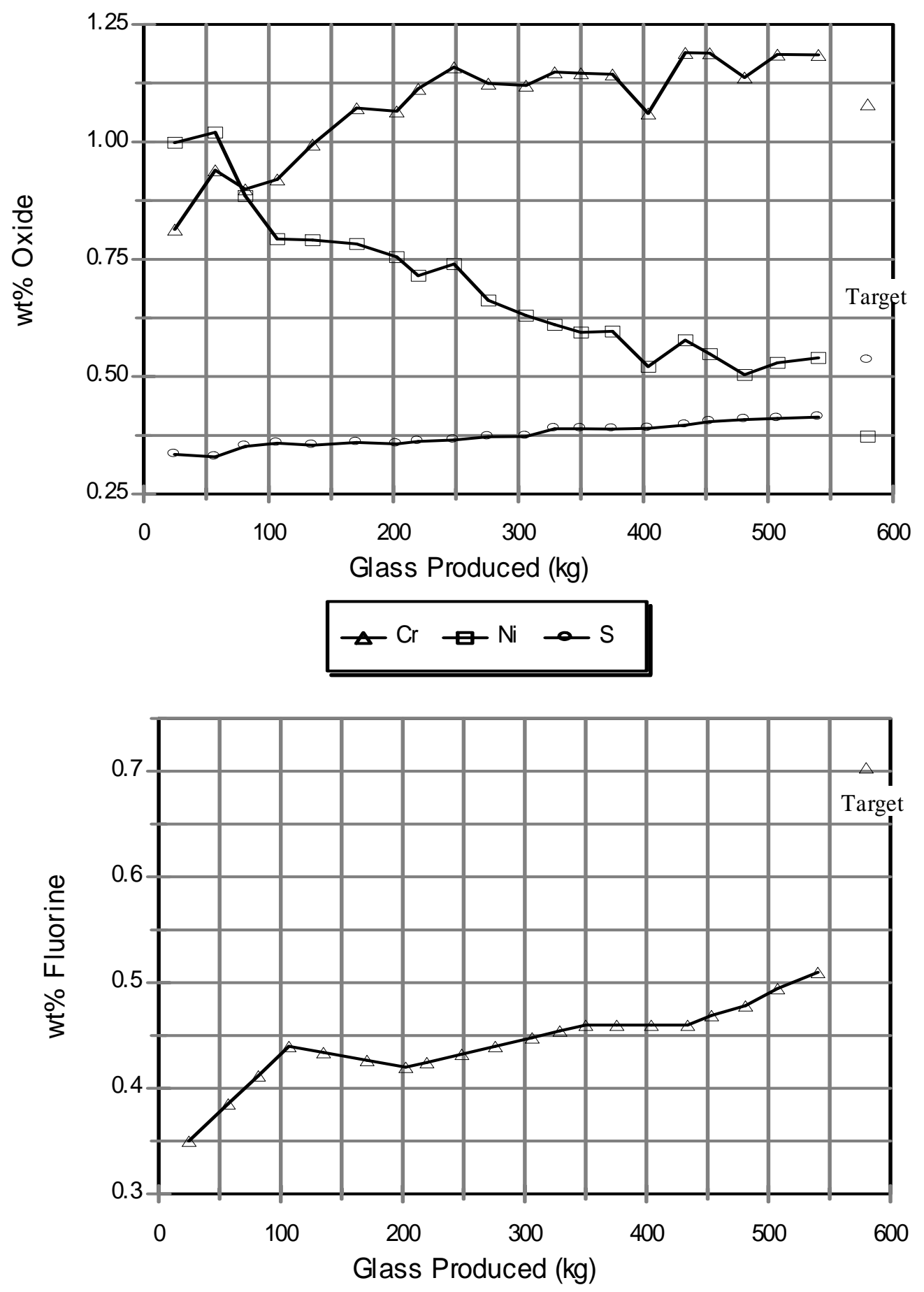

Figure 6.3.b. DM100 chromium-limited product glass composition determined by XRF. 
The Catholic University of America Vitreous State Laboratory
High Level Waste Vitrification System Improvements Final Report, VSL-07R1010-1, Rev. 0

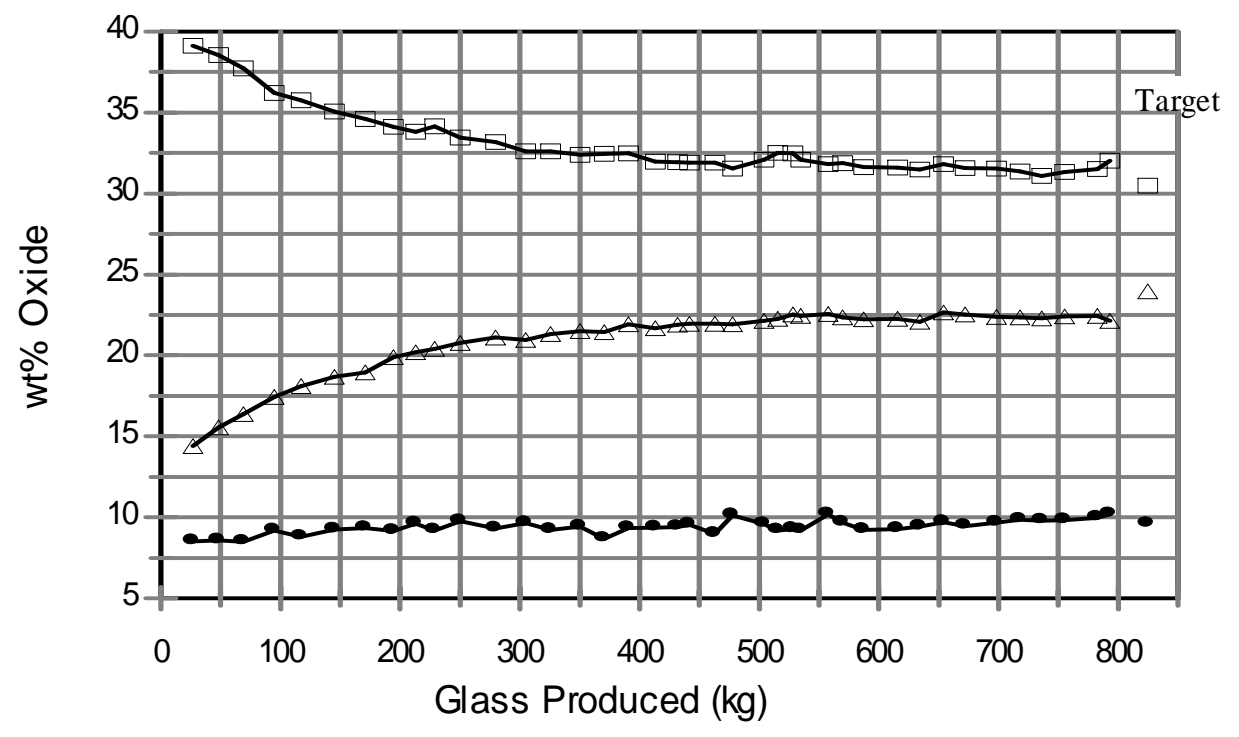

$\triangle \mathrm{Al} \longrightarrow \mathrm{Na} \longrightarrow \mathrm{Si}$
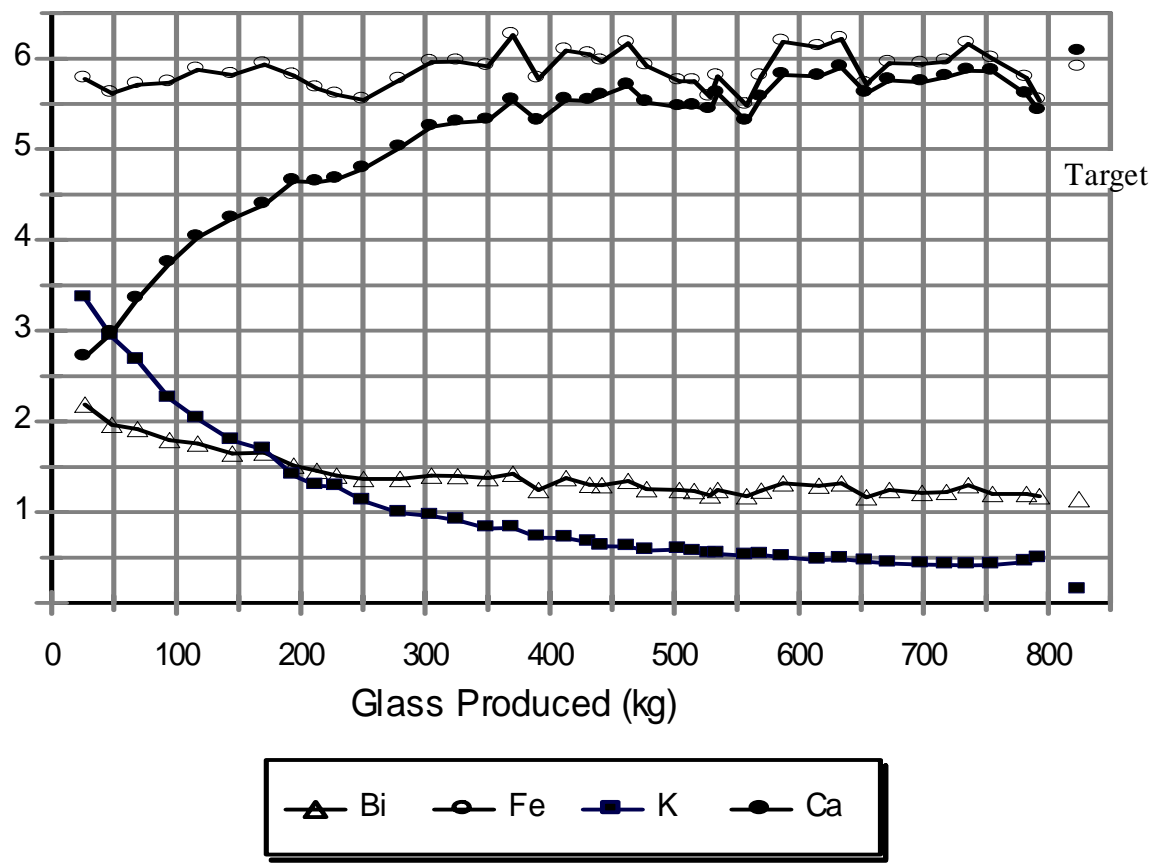

Figure 6.4.a. DM100 aluminum-limited product glass composition determined by XRF. 
The Catholic University of America Vitreous State Laboratory
High Level Waste Vitrification System Improvements Final Report, VSL-07R1010-1, Rev. 0

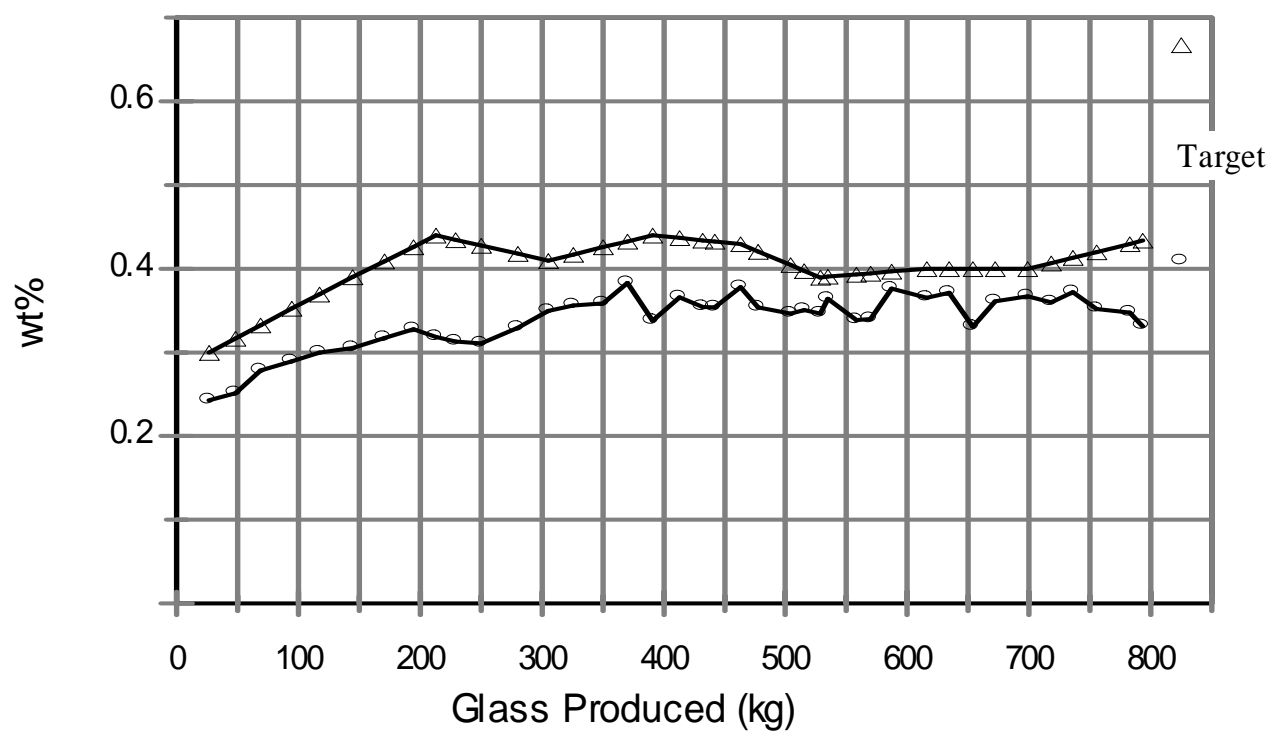

$\triangle F \quad-\mathrm{PbO}$
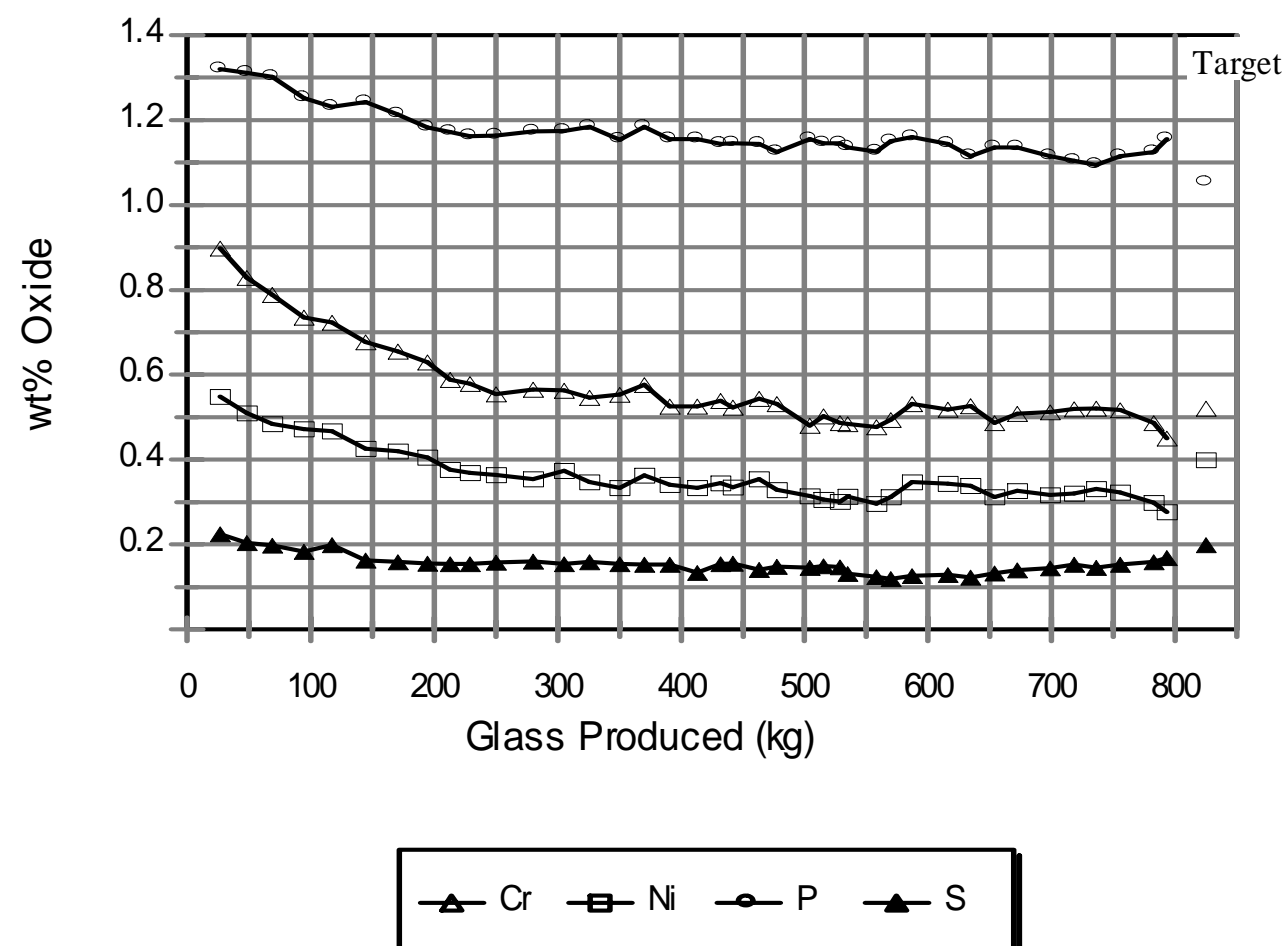

Figure 6.4.b. DM100 aluminum-limited product glass composition determined by XRF. 
The Catholic University of America Vitreous State Laboratory
High Level Waste Vitrification System Improvements Final Report, VSL-07R1010-1, Rev. 0
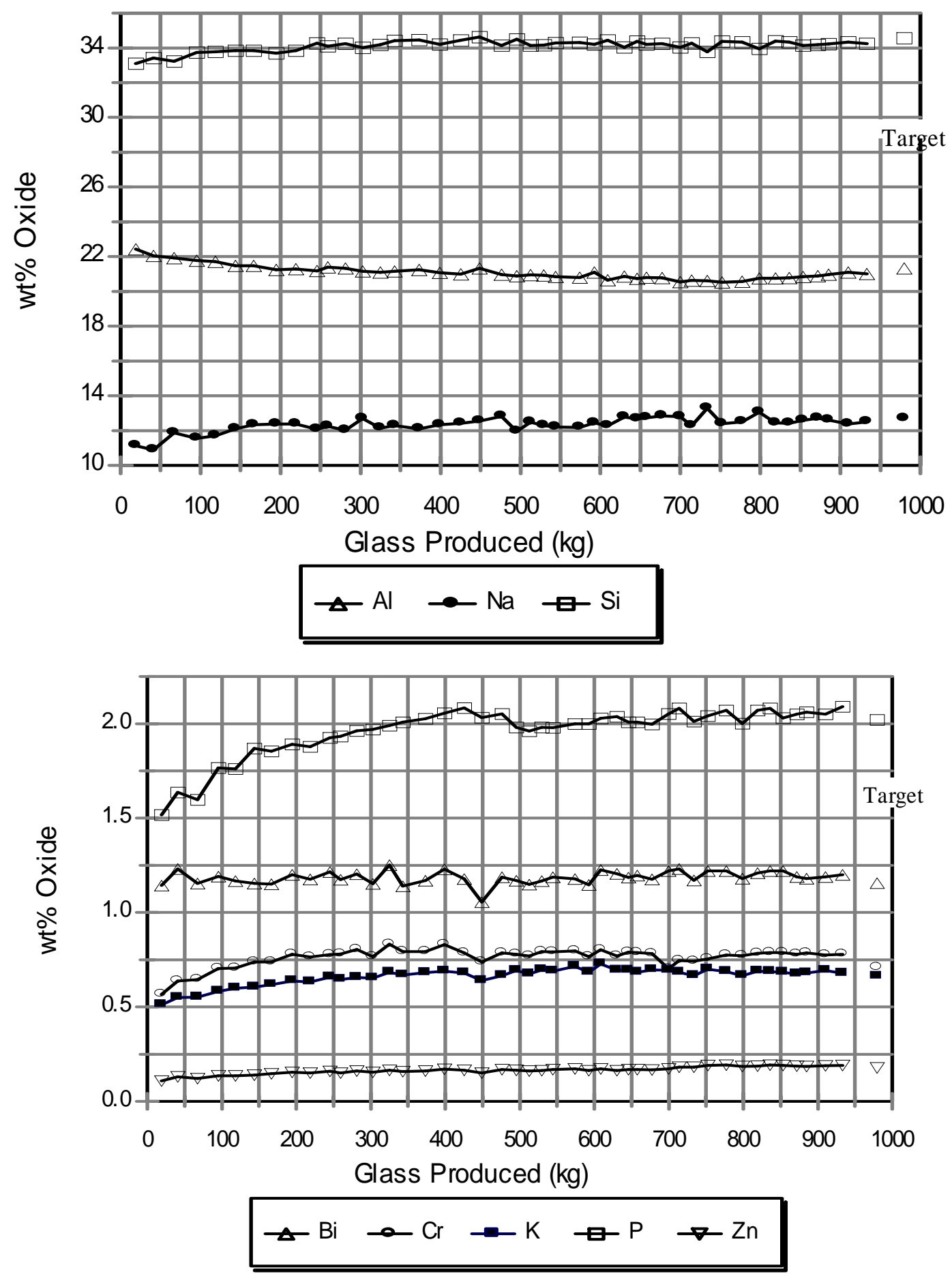

Figure 6.5.a. DM100 aluminum-plus-sodium-limited product glass composition determined by XRF. 
The Catholic University of America Vitreous State Laboratory
High Level Waste Vitrification System Improvements Final Report, VSL-07R1010-1, Rev. 0
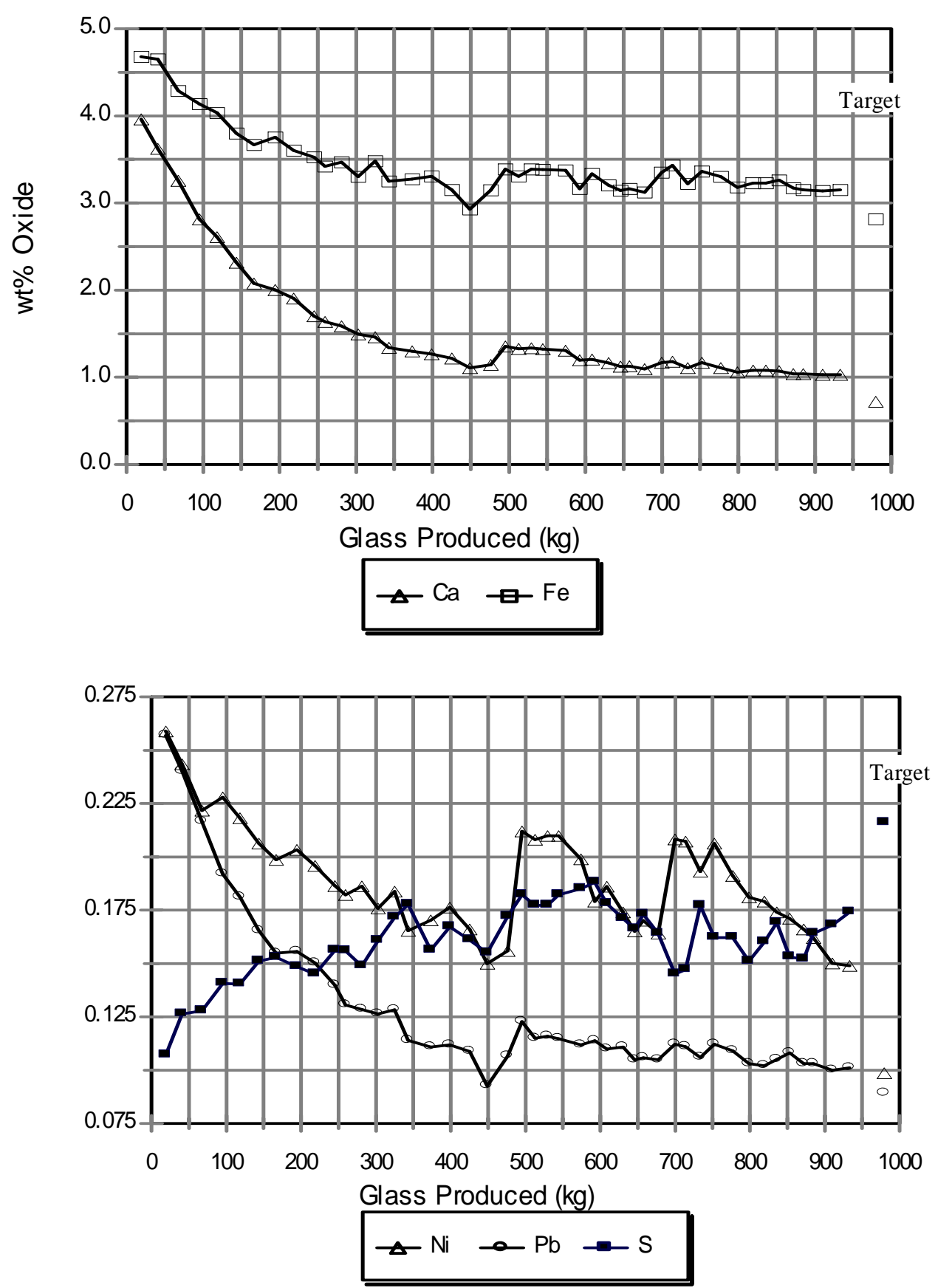

Figure 6.5.b. DM100 aluminum-plus-sodium-limited product glass composition determined by XRF. 
The Catholic University of America Vitreous State Laboratory
High Level Waste Vitrification System Improvements Final Report, VSL-07R1010-1, Rev. 0

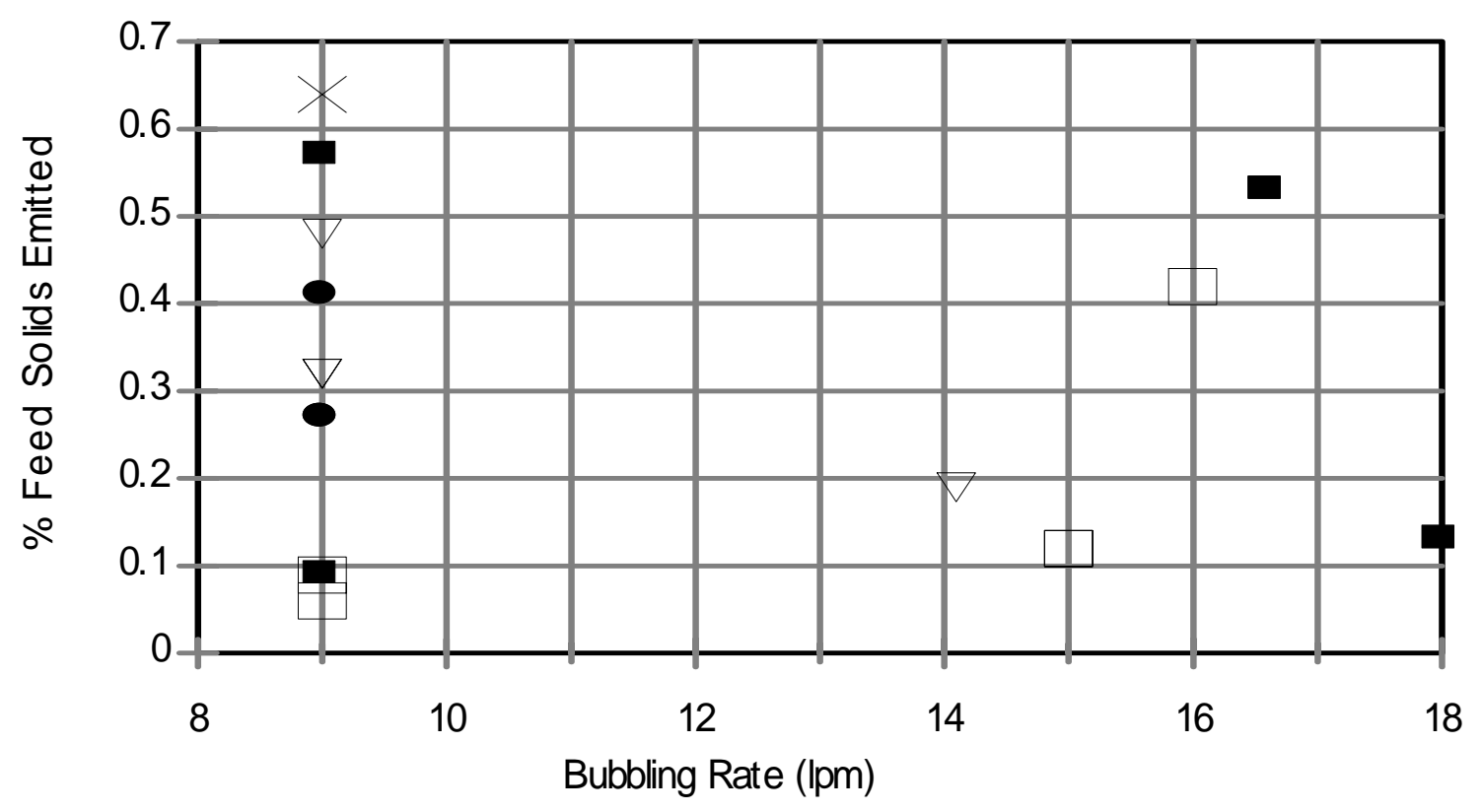

Bi Limited

- Cr Limited

Al Limited

Al + Na Limited

$\times$ SIPP

Figure 7.1. Percent feed solids emitted as particles during DM100 tests vs. bubbling rate; feed solids content $500( \pm 50)$ g glass per liter feed. 
The Catholic University of America Vitreous State Laboratory
High Level Waste Vitrification System Improvements Final Report, VSL-07R1010-1, Rev. 0

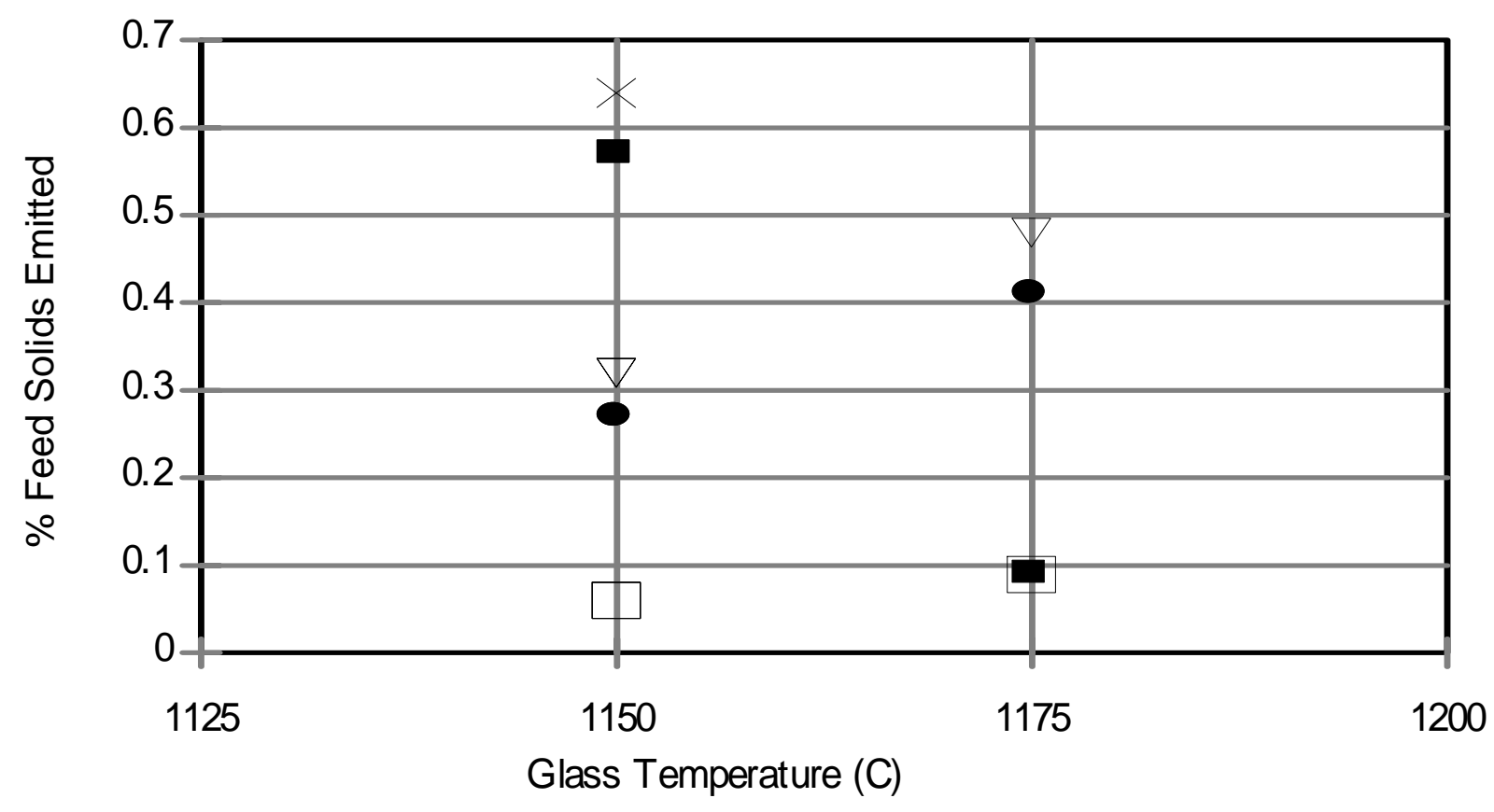

\begin{tabular}{|ll|}
\hline$\nabla$ Bi Limited & - Cr Limited \\
$\square$ Al + Na Limited & $\times$ SIPP \\
\hline \hline
\end{tabular}

Figure 7.2. Percent feed solids emitted as particles during DM100 tests vs. glass temperature; feed solids content $500( \pm 50)$ g glass per liter feed, glass pool bubbling rate 9 lpm. 
The Catholic University of America Vitreous State Laboratory
High Level Waste Vitrification System Improvements Final Report, VSL-07R1010-1, Rev. 0

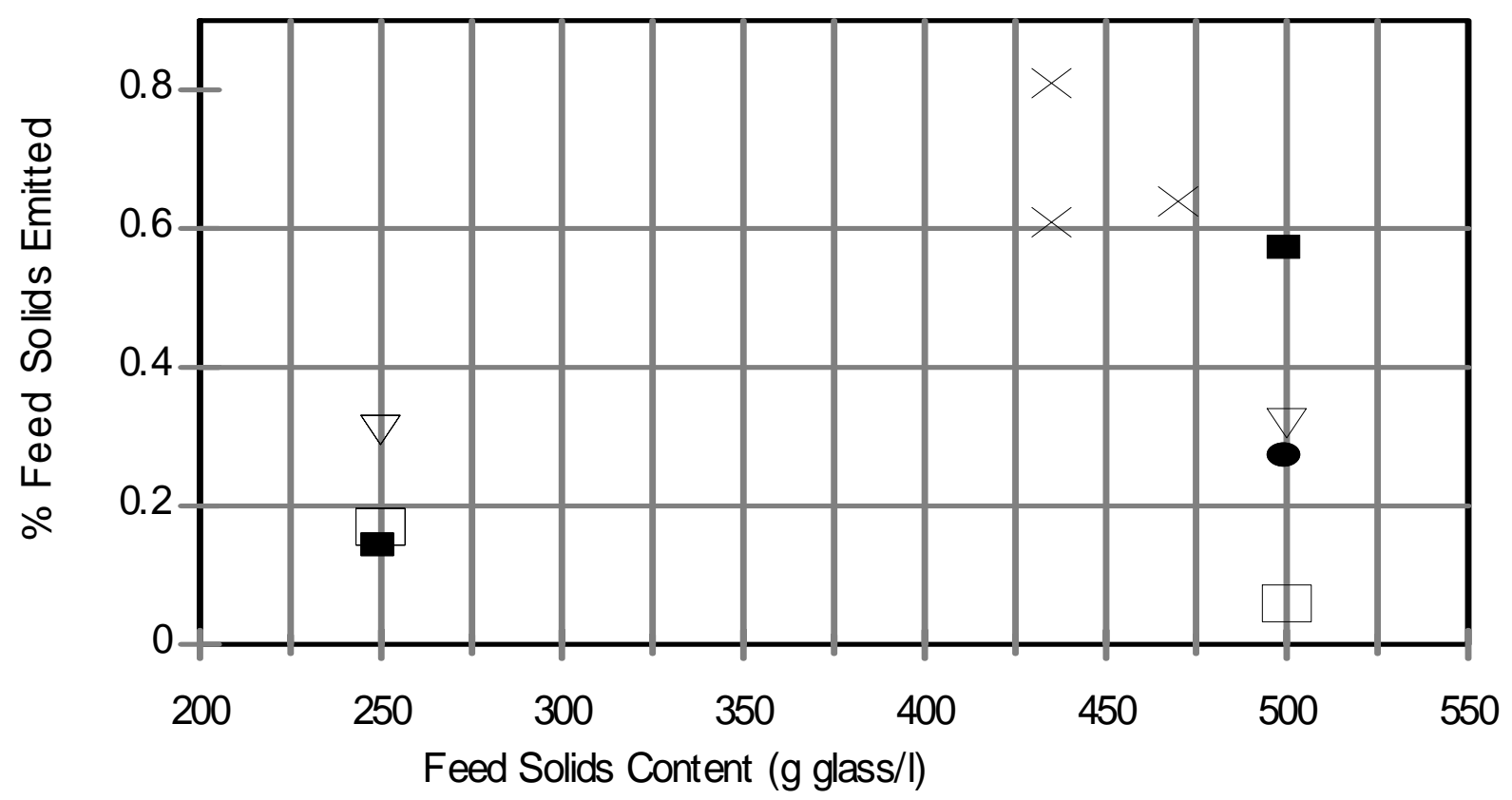

$\nabla$ Bi Limited

- Cr Limited

Al Limited

$\square \mathrm{Al}+\mathrm{Na}$ Limited

$\times$ Others

Figure 7.3. Percent feed solids emitted as particles during DM100 tests vs. feed solids content; glass temperature $1150^{\circ} \mathrm{C}$, glass pool bubbling rate $9 \mathrm{lpm}$. 
The Catholic University of America Vitreous State Laboratory
High Level Waste Vitrification System Improvements Final Report, VSL-07R1010-1, Rev. 0
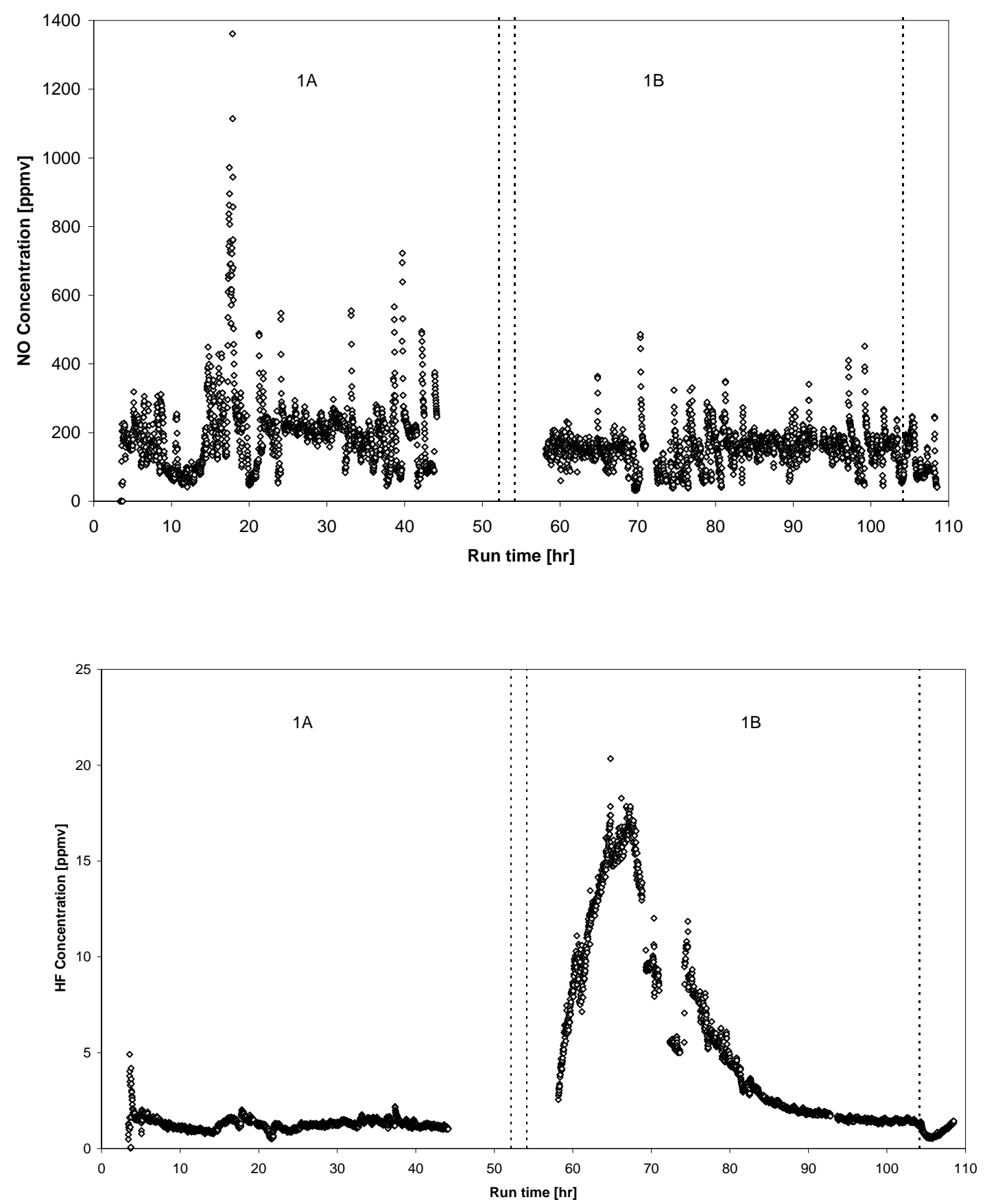

Figure 7.4. NO and HF FTIR monitored emissions during DM100 Tests 1A and $1 B$. 
The Catholic University of America Vitreous State Laboratory
High Level Waste Vitrification System Improvements Final Report, VSL-07R1010-1, Rev. 0
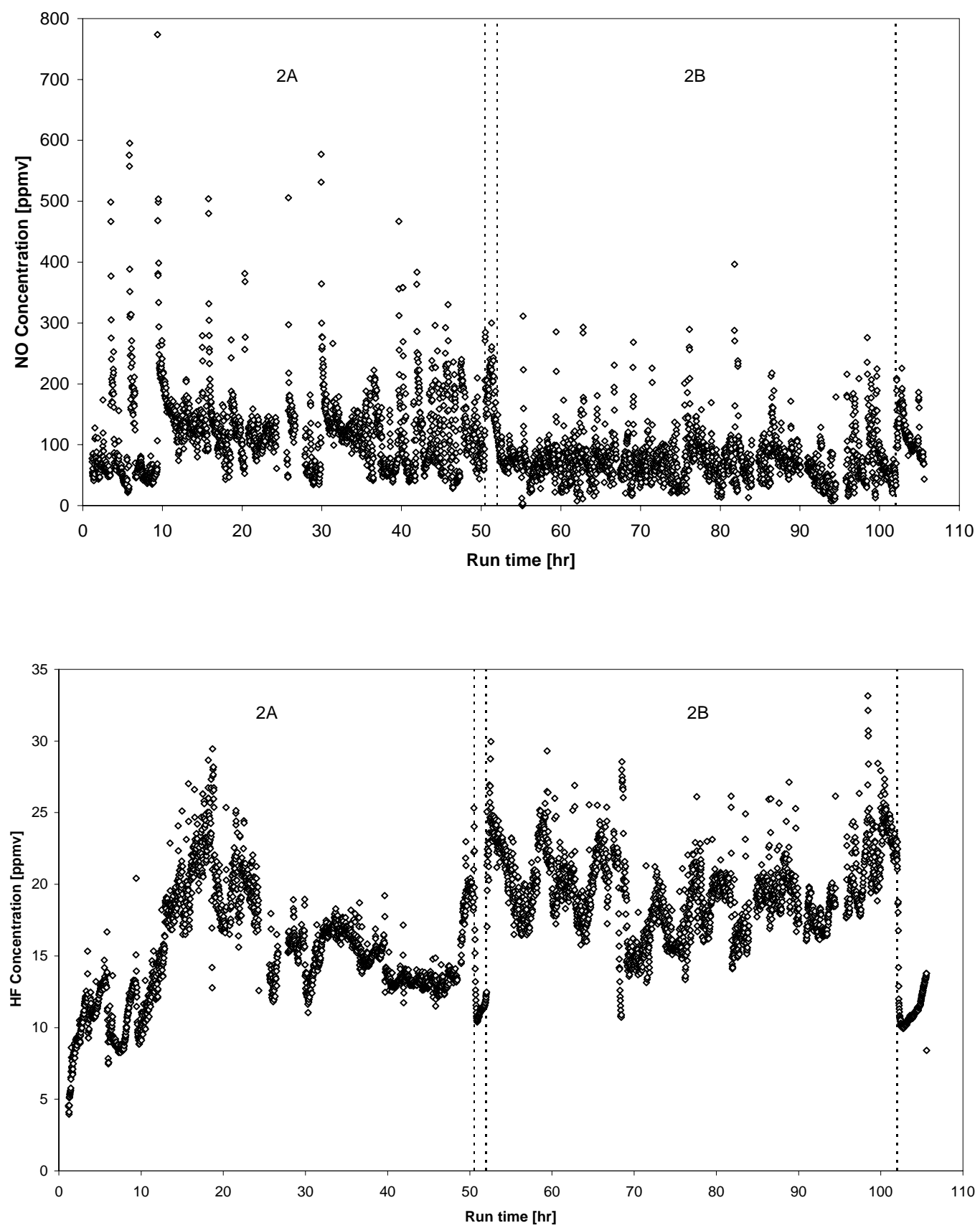

Figure 7.5. NO and HF FTIR monitored emissions during DM100 Tests 2A and 2B. 
The Catholic University of America Vitreous State Laboratory
High Level Waste Vitrification System Improvements Final Report, VSL-07R1010-1, Rev. 0
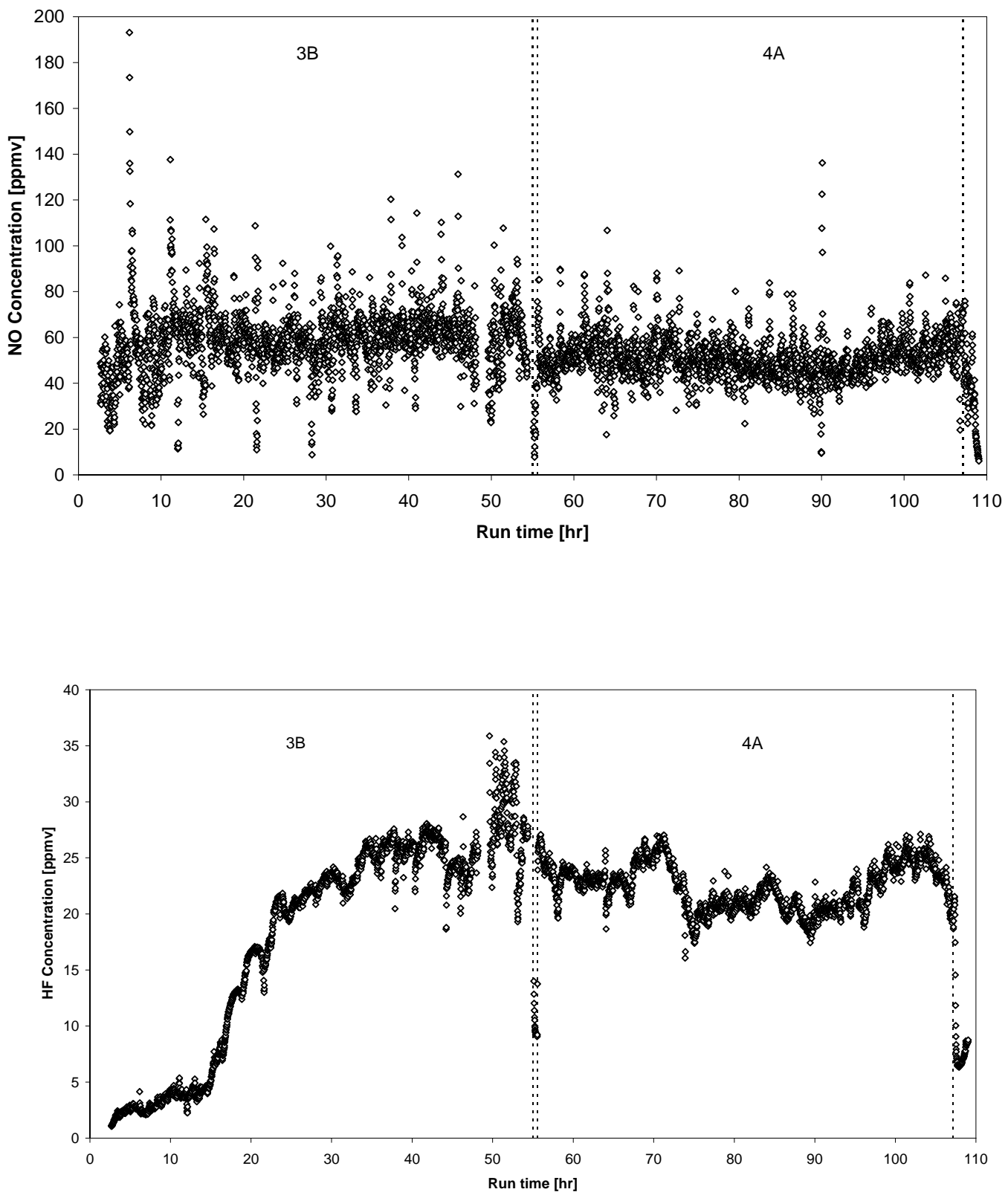

Figure 7.6. NO and HF FTIR monitored emissions during DM100 Tests 3B and 4A. 
The Catholic University of America Vitreous State Laboratory
High Level Waste Vitrification System Improvements Final Report, VSL-07R1010-1, Rev. 0
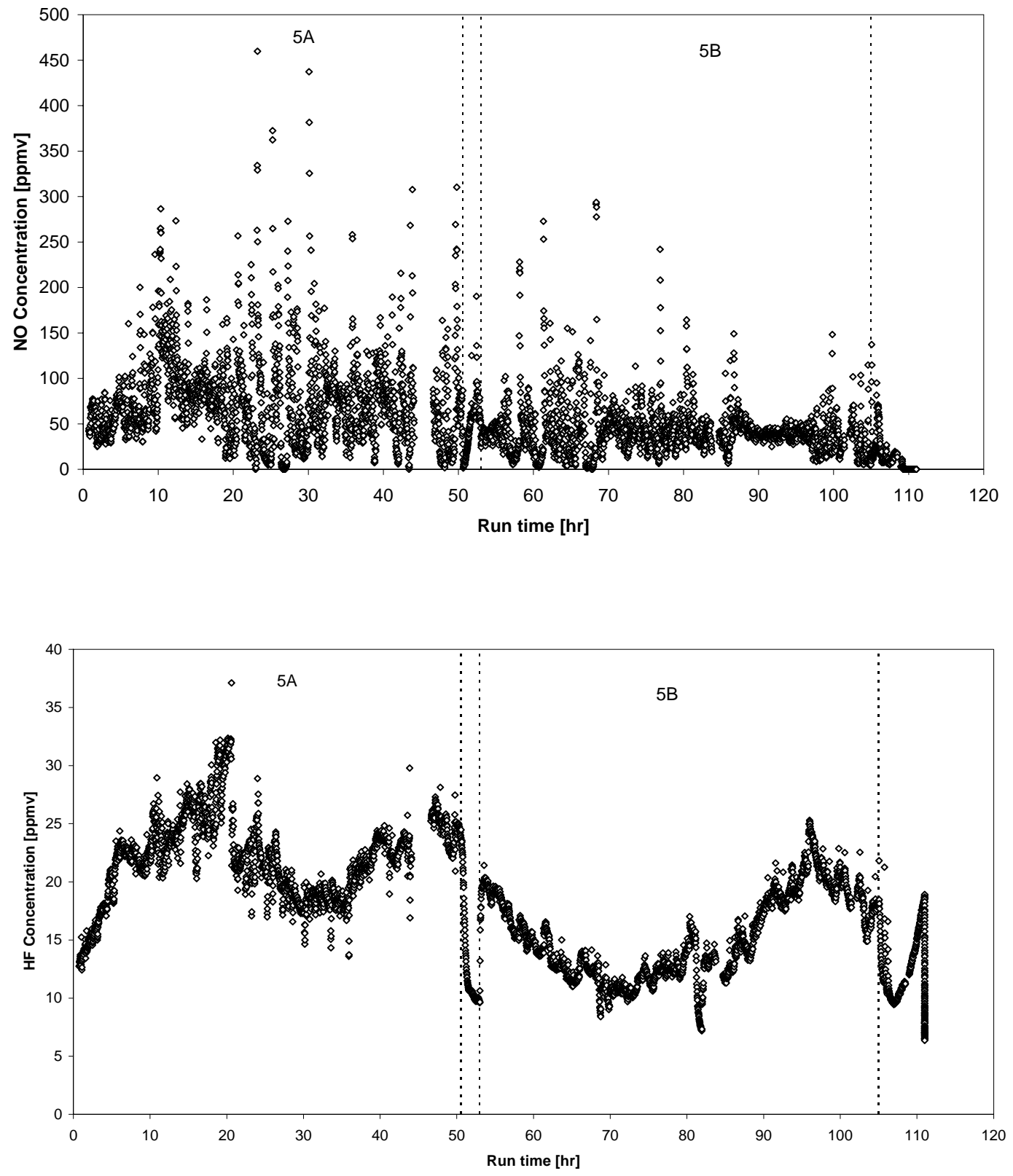

Figure 7.7. NO and HF FTIR monitored emissions during DM100 Tests 5A and 5B. 
The Catholic University of America Vitreous State Laboratory
High Level Waste Vitrification System Improvements Final Report, VSL-07R1010-1, Rev. 0
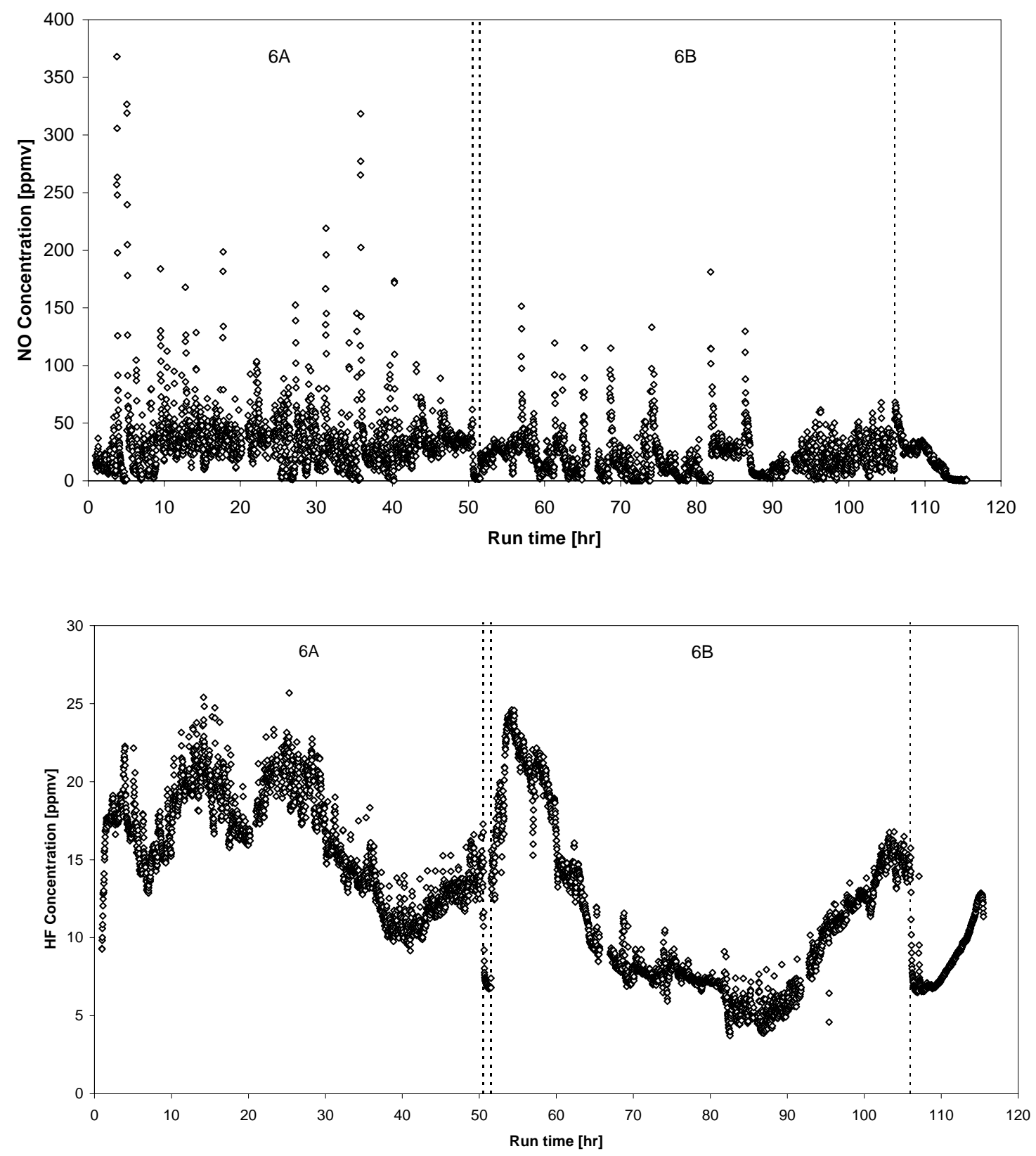

Figure 7.8. NO and HF FTIR monitored emissions during DM100 Tests 6A and 6B. 
The Catholic University of America Vitreous State Laboratory
High Level Waste Vitrification System Improvements Final Report, VSL-07R1010-1, Rev. 0
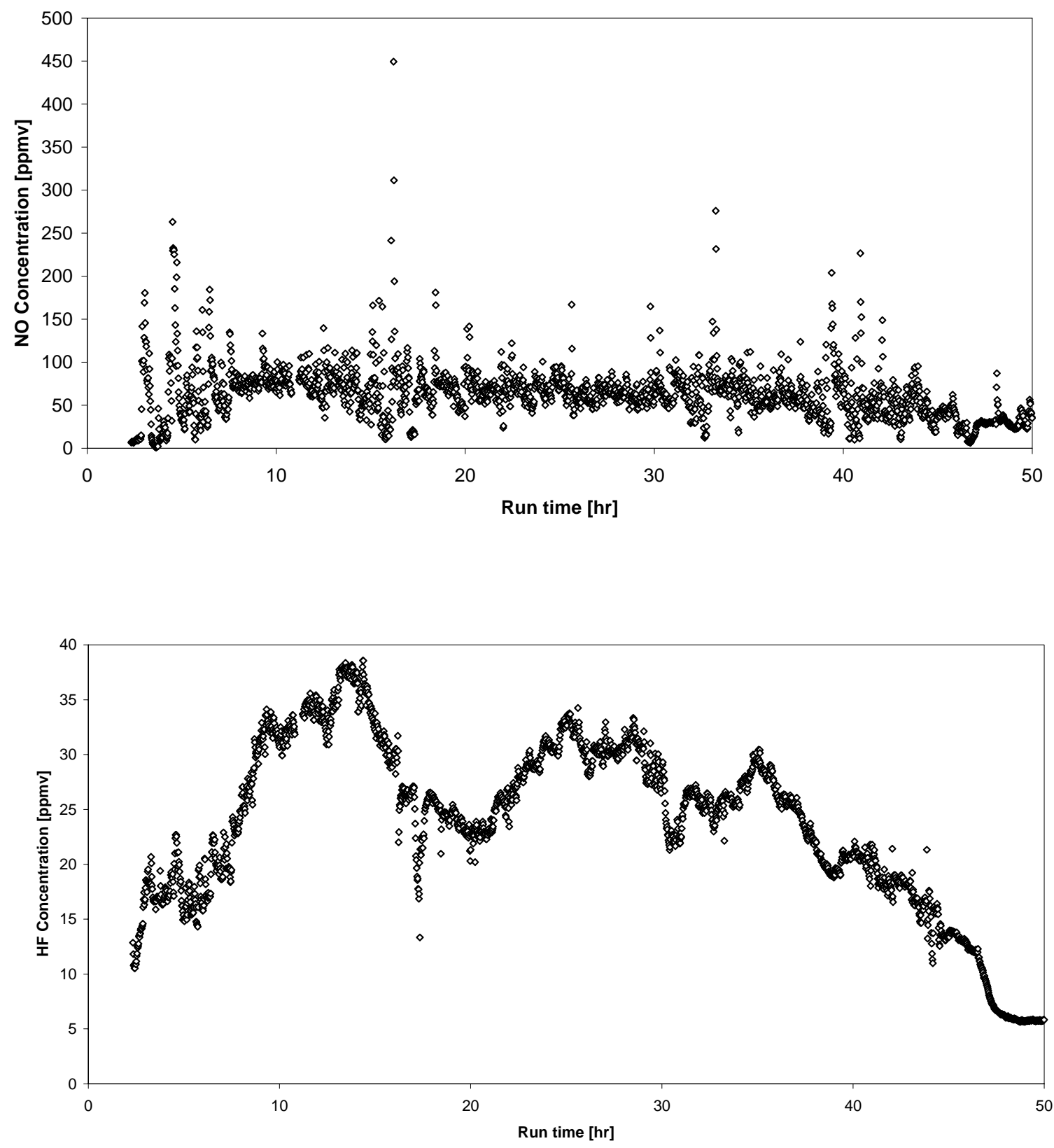

Figure 7.9. NO and HF FTIR monitored emissions during DM100 Test 6C. 
The Catholic University of America Vitreous State Laboratory
High Level Waste Vitrification System Improvements Final Report, VSL-07R1010-1, Rev. 0
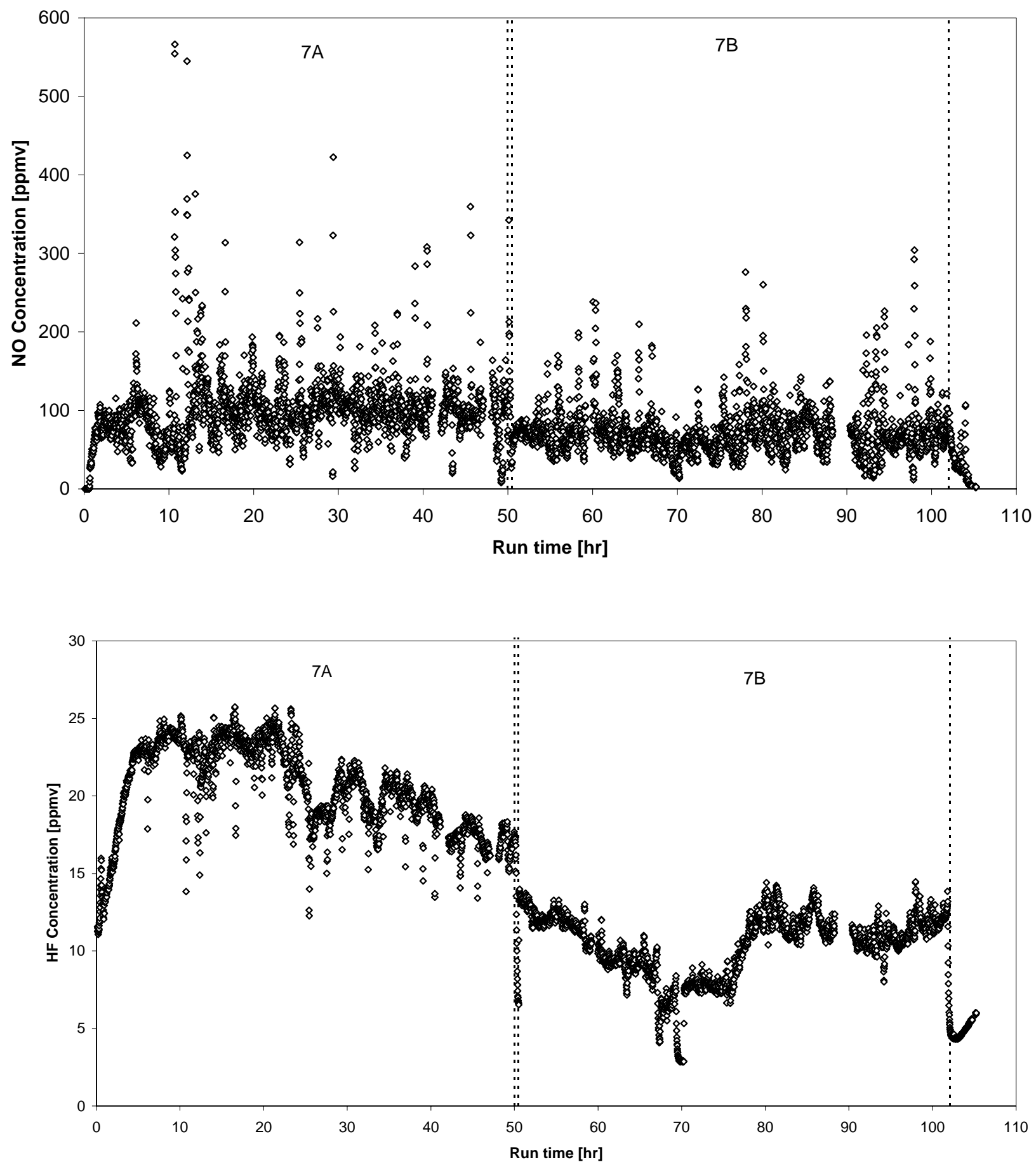

Figure 7.10. NO and HF FTIR monitored emissions during DM100 Tests 7A and 7B. 
The Catholic University of America Vitreous State Laboratory
High Level Waste Vitrification System Improvements Final Report, VSL-07R1010-1, Rev. 0
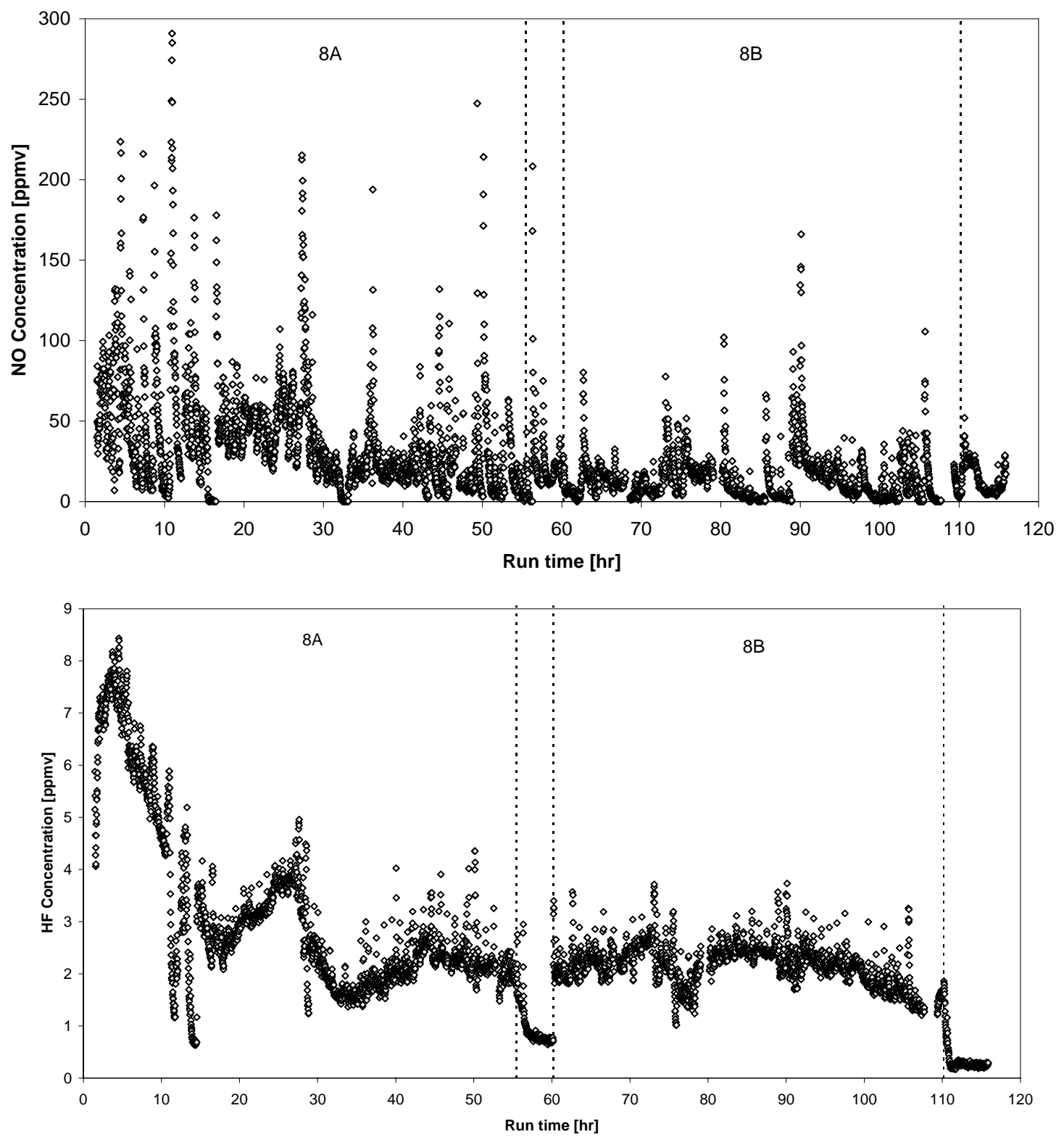

Figure 7.11. NO and HF FTIR monitored emissions during DM100 Tests 8A and 8B. 
The Catholic University of America Vitreous State Laboratory
High Level Waste Vitrification System Improvements Final Report, VSL-07R1010-1, Rev. 0
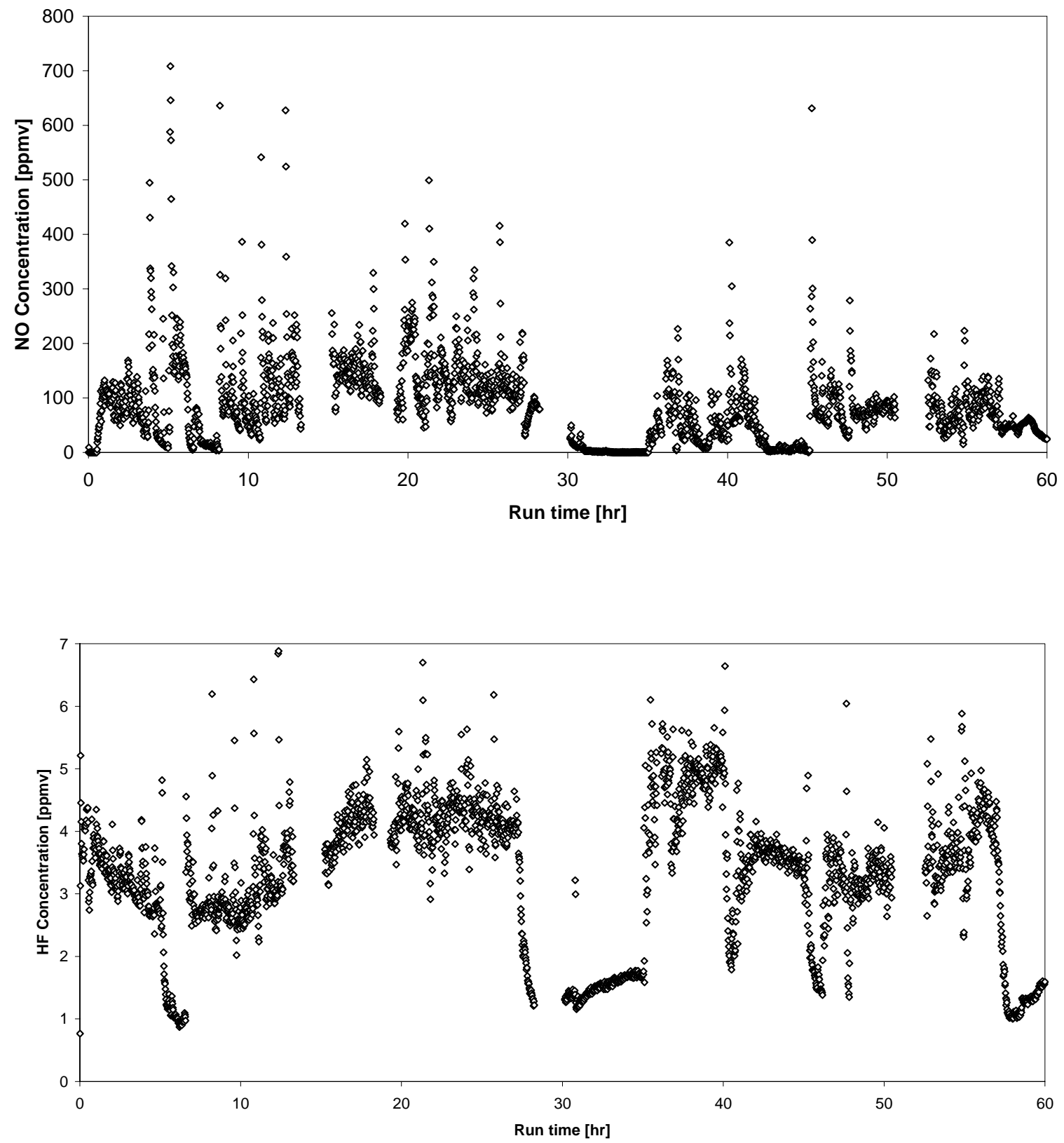

Figure 7.12. NO and HF FTIR monitored emissions during DM100 Test 8C. 


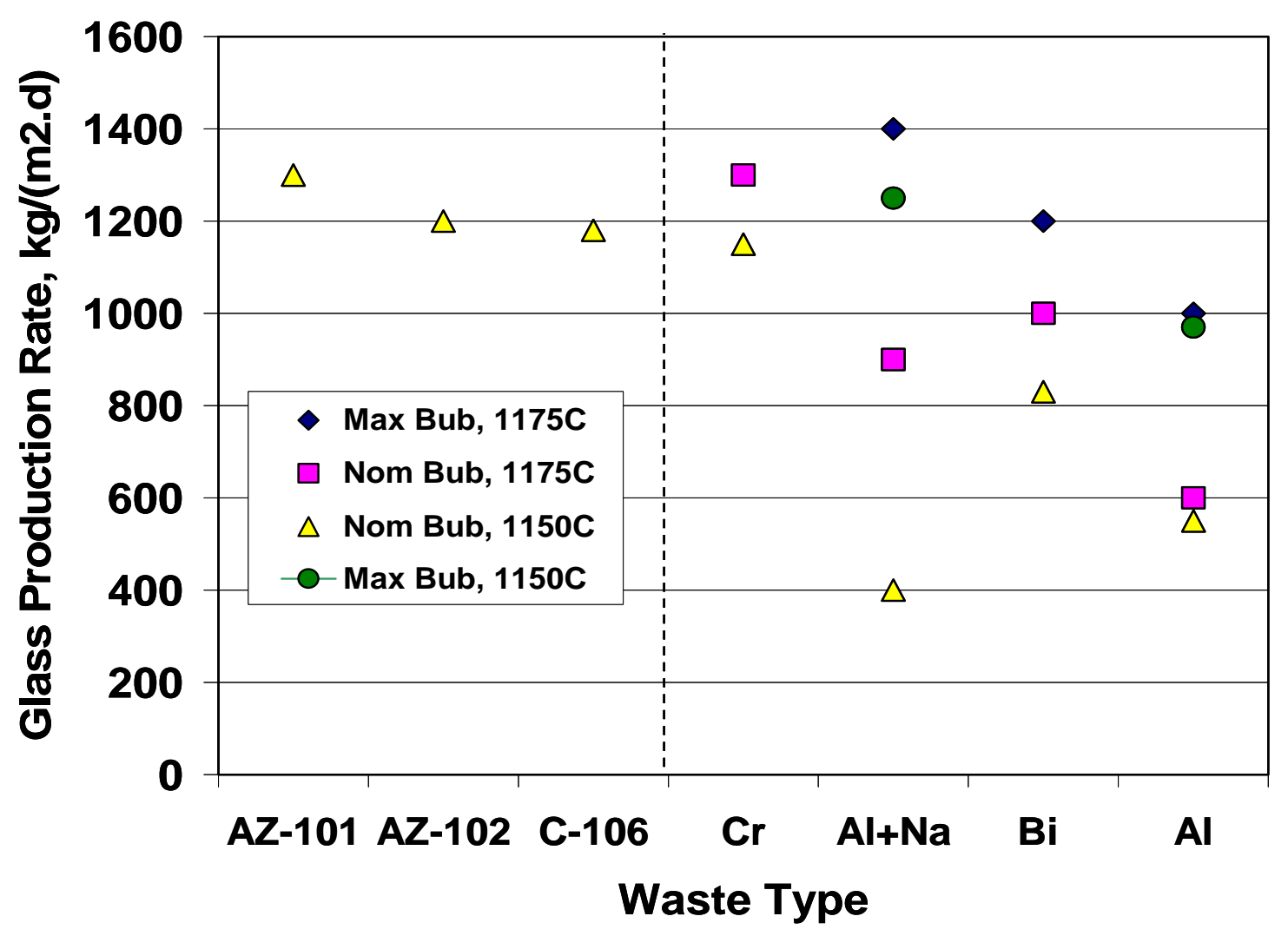

Figure 8.1. Comparison of glass production rates obtained in the present work for the high solids content feeds with comparable high-iron feeds tested previously. 


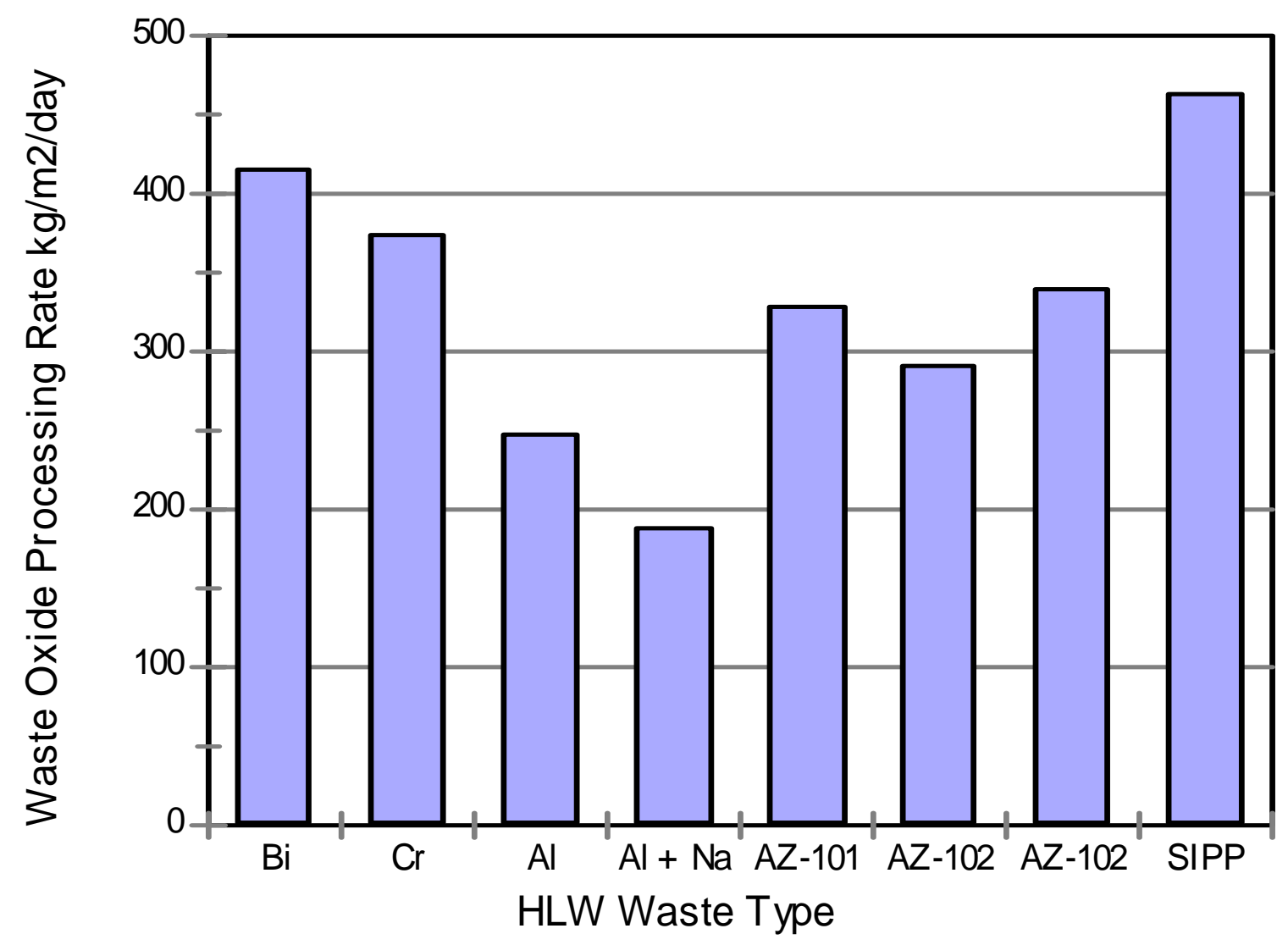

Figure 8.2. Waste oxide processing rates during DM100 tests; glass temperature $1150^{\circ} \mathrm{C}$, glass pool bubbling rate $9 \mathrm{lpm}$, and feed solids content $500( \pm 50)$ g glass per liter feed. 


\section{APPENDIX A LITERATURE REVIEW}

In parallel with the glass formulation development work to identify high waste loading HLW glasses, a literature review was conducted to identify glass formulations that have been tested with high concentrations of the components of interest, namely chromium, bismuth, aluminum, and aluminum with sodium. Since the VSL glass databases already contain most of the waste glasses, the scope of the literature review was expanded to cover a larger universe of glass compositions including commercial glasses, glass frits, glazes, basalts, volcanic glasses, etc. Journal articles, project reports, literature review reports, patents, etc. were searched for relevant glass composition data. Most of these glass compositions from other sources could not be used directly in the present work because of unacceptable properties such as high melting temperatures, high viscosities, or excessive crystallization. However, these glass compositions were helpful in identifying viable upper concentration limits of the components of interest and other glass components that may be advantageous in increasing the acceptable concentration limits of the components of interest. A compilation of the glass compositions from the literature review along with the references is provided at the end of this appendix in Table A1, where a total of 1805 glass compositions are tabulated. Where available, the melting temperature, liquidus temperature, temperature at which the glass melt viscosity is 100 poise, and PCT normalized boron release are also given in Table A1. In addition to these glass compositions, information from the VSL database, which contains several thousand glass compositions, was also used to guide the glass formulation work. While a number of the glass compositions given in this appendix have properties that are outside of the acceptable range for HLW processing at Hanford, large numbers of the glass compositions in the VSL database comply with processing and product quality requirements for Hanford.

In the following sub-sections, the chromium, bismuth, aluminum, and sodium concentrations in the glasses from the literature review are discussed and compared to their concentrations in the glasses developed during the present work.

\section{A.1 Chromium}

Seven hundred and fifty four compositions containing $\mathrm{Cr}_{2} \mathrm{O}_{3}$ are tabulated in this appendix. The highest $\mathrm{Cr}_{2} \mathrm{O}_{3}$ concentration in any of these compositions is $25 \mathrm{wt} \%$, with the majority containing less than $1 \mathrm{wt} \% \mathrm{Cr}_{2} \mathrm{O}_{3}$. The compositions with greater than $5 \mathrm{wt} \% \mathrm{Cr}_{2} \mathrm{O}_{3}$ are mostly glazes and will not be suitable for $\mathrm{HLW}$ processing. A limited number of the compositions contain more than $1 \mathrm{wt} \% \mathrm{Cr}_{2} \mathrm{O}_{3}$ and were melted at around $1150^{\circ} \mathrm{C}$, but they contain comparatively lesser amounts of other transition metal oxides (e.g. $\mathrm{Fe}_{2} \mathrm{O}_{3}$ ) than the ORP Chromium-Limited waste stream used in this study. As noted previously, as the $\mathrm{Cr}_{2} \mathrm{O}_{3}$ concentration in the glass composition is increased, the common waste-loading limiting phenomenon is crystallization of Cr-containing spinels or $\mathrm{Cr}_{2} \mathrm{O}_{3}$ crystals. Spinel crystal formation is enhanced as the concentration of other transition metal oxides in the composition is increased. Higher concentrations of alkali oxides and $\mathrm{B}_{2} \mathrm{O}_{3}$ tend to increase $\mathrm{Cr}_{2} \mathrm{O}_{3}$ solubility in 
the glass. Information from the VSL database suggested that addition of $\mathrm{K}_{2} \mathrm{O}$ and $\mathrm{PbO}$ are also beneficial in improving $\mathrm{Cr}_{2} \mathrm{O}_{3}$ solubility in the glass. The high $\mathrm{Cr}_{2} \mathrm{O}_{3}$ solubilities $(\sim 1.4 \mathrm{wt} \%)$ were generally seen in glasses with high alkali and low transition metal oxide concentrations. Based on available literature data, a $\mathrm{Cr}_{2} \mathrm{O}_{3}$ concentration of about $1 \mathrm{wt} \%$ was judged to be an achievable target for the ORP Chromium-Limited waste stream, except for the complications caused by the relatively high sulfur content in this waste stream. Information in the literature suggests that the interaction of $\mathrm{SO}_{3}$ with $\mathrm{Cr}_{2} \mathrm{O}_{3}$ can increase the tendency for secondary phase formation, thus reducing the achievable $\mathrm{Cr}_{2} \mathrm{O}_{3}$ loading in the glass, as was confirmed in the present work. The initially selected VSL formulation for this waste stream, LAW-E-Cr-10, given in Table 3.8, has a $\mathrm{Cr}_{2} \mathrm{O}_{3}$ concentration of $1.33 \mathrm{wt} \%$, which is higher than any of the $\mathrm{Cr}_{2} \mathrm{O}_{3}$ concentrations reported in the literature for similar glass compositions and property constraints. However, the $\mathrm{Cr}_{2} \mathrm{O}_{3}$ loading was indeed limited by secondary phase formation as a result of its interaction with $\mathrm{SO}_{3}$ (sulfate-chromate salts) rather than by chromium-based crystallization. The final VSL glass composition selected after the DM10 melter test, HLW-E-Cr-M (see Table 4.2), has a $\mathrm{Cr}_{2} \mathrm{O}_{3}$ concentration of $1.08 \mathrm{wt} \%$, despite the high sulfate content. Again, based on the information in the literature, the $\mathrm{Cr}_{2} \mathrm{O}_{3}$ concentration in the final glass composition is higher than what would have been expected for a fully compliant glass to be processed in a melter at a nominal operating temperature of $1150^{\circ} \mathrm{C}$.

\section{A.2 Bismuth}

One hundred and eighty nine compositions containing $\mathrm{Bi}_{2} \mathrm{O}_{3}$ are tabulated in this appendix with the highest concentration being $70.8 \mathrm{wt} \%$. All of the compositions with high $\mathrm{Bi}_{2} \mathrm{O}_{3}$ concentrations $(>10 \mathrm{wt} \%)$ have high melting temperatures $\left(1300-1400^{\circ} \mathrm{C}\right)$ with applications as enamels or glazes. The compositions in the literature do not contain most of the other potentially waste loading limiting constituents in the ORP Bismuth-Limited waste stream such as $\mathrm{Fe}_{2} \mathrm{O}_{3}, \mathrm{Cr}_{2} \mathrm{O}_{3}, \mathrm{NiO}, \mathrm{P}_{2} \mathrm{O}_{5}$, and $\mathrm{SO}_{3}$. The literature information was, therefore, not very useful in guiding the development of suitable glass formulations to process this waste stream. However, the literature review did provide some general information about the effect of $\mathrm{Bi}_{2} \mathrm{O}_{3}$ addition on the properties of glass melts. The VSL selected glass composition for this waste stream in given in Table 3.4. The glass has a waste loading of $50 \mathrm{wt} \%$. The waste loading limiting factor was crystallization of spinel and phosphate phases and not the concentration of $\mathrm{Bi}_{2} \mathrm{O}_{3}$ in the glass. As is evident from Table 3.4, the only additives are $\mathrm{B}_{2} \mathrm{O}_{3}, \mathrm{Na}_{2} \mathrm{O}$, and $\mathrm{SiO}_{2}$, all of which are essential components of an alkali-borosilicate glass. For waste vitrification, the levels of these components are also impractically low in the highest bismuth glasses found in the literature.

\section{A.3 Aluminum}

Sixteen hundred and eighty eight glasses containing $\mathrm{Al}_{2} \mathrm{O}_{3}$ are tabulated in this appendix, with the highest concentration being $74 \mathrm{wt} \%$. The majority of the glasses with high $\mathrm{Al}_{2} \mathrm{O}_{3}$ concentration $(>20 \mathrm{wt} \%)$ are calcium-aluminosilicates with very high melting points $\left(>1350^{\circ} \mathrm{C}\right)$ 
that have commercial applications. The literature review did reveal a number of waste glasses with medium to high $\mathrm{Al}_{2} \mathrm{O}_{3}$ concentrations $(10-20 \mathrm{wt} \%)$, and nominal a processing temperature of $1150^{\circ} \mathrm{C}$. The formation of nepheline $\left(\mathrm{NaAlSiO}_{4}\right)$ was reported as a major issue for waste glass compositions with high $\mathrm{Al}_{2} \mathrm{O}_{3}$ concentrations. To avoid the formation of nepheline, some authors (Li, et al. 1997), (Peeler, et al. 2005) use a nepheline discriminator based on the mass fractions of $\mathrm{Al}_{2} \mathrm{O}_{3}, \mathrm{Na}_{2} \mathrm{O}$, and $\mathrm{SiO}_{2}$ in the glass with the requirement that $\mathrm{SiO}_{2} /\left(\mathrm{Al}_{2} \mathrm{O}_{3}+\mathrm{Na}_{2} \mathrm{O}+\mathrm{SiO}_{2}\right)$ be greater than 0.62. In the VSL glass formulation development work, since increasing the $\mathrm{Al}_{2} \mathrm{O}_{3}$ loading was the primary objective, the concentrations of the other two nepheline constituents, $\mathrm{SiO}_{2}$ and $\mathrm{Na}_{2} \mathrm{O}$ were decreased and concentrations of $\mathrm{B}_{2} \mathrm{O}_{3}$ and $\mathrm{Li}_{2} \mathrm{O}$ were increased. However, based on prior VSL experience, the nepheline discriminator was judged to be misleading and likely to exclude compliant high aluminum loading glasses. Accordingly, this constraint was not used in the present glass formulation development work. In agreement with the literature information, nepheline formation, especially during canister center-line cooling (CCC) heat treatment, was found to be the waste loading limiting factor in the present work. However, the compliant glass formulation for the ORP Aluminum-Limited waste stream given in Table 3.11 has a $\mathrm{SiO}_{2} /\left(\mathrm{Al}_{2} \mathrm{O}_{3}+\mathrm{Na}_{2} \mathrm{O}+\mathrm{SiO}_{2}\right)$ ratio of 0.48 , validating the decision to disregard nepheline discriminator as an overly conservative constraint in the glass formulation development for this waste stream. The $\mathrm{Al}_{2} \mathrm{O}_{3}$ loading of $23.97 \mathrm{wt} \%$ in the selected glass is higher than what would have been expected for a fully compliant glass from the literature data.

\section{A.4 Aluminum + Sodium}

The same literature data that was used to support the development of glass formulation for ORP Aluminum-Limited waste stream was used for the Aluminum+Sodium-Limited waste stream. The only difference was that, since this waste stream contributed more $\mathrm{Na}_{2} \mathrm{O}$ to the final glass composition than the Aluminum-Limited waste stream, the aluminum loading in the final glass composition had to be lowered slightly from $23.97 \mathrm{wt} \%$ to $21.34 \mathrm{wt} \%$ in order to obtain a compliant glass formulation. The composition of the selected glass is given in Table 3.14. Again, the nepheline discriminator was not used in glass formulation development work for the reasons discussed above. The final glass composition has a $\mathrm{SiO}_{2} /\left(\mathrm{Al}_{2} \mathrm{O}_{3}+\mathrm{Na}_{2} \mathrm{O}+\mathrm{SiO}_{2}\right)$ ratio of 0.50 . This glass composition would have been unnecessarily excluded if the nepheline discriminator was used. While the information from the literature is valuable in supporting and guiding glass formulation development for Hanford waste steams, caution should be used in using empirical relationships from the literature to constrain composition regions to be studied because empirical relationships that may be valid in certain composition regions may not be valid in other regions. Indeed, some of the greatest gains in waste loading can be achieved by challenging such constraints. 


\section{A.5 References}

Ackerman, K R, (2006). "Chromium Bearing Forehearth Color Concentrate". US Patent $6,984,597 \mathrm{~B} 2$.

Barantseva, S E, et al., (2004). "Capacity of Granitoid-Based Glasses for Glass Ceramics Formation". Glass and Ceramics Vol. 61 Nos. 7 - 8.

Barbieri, L C, et al., (1994). "Solubility Reactivity and Nucleation Effect of Cr2O3 in the $\mathrm{CaO}-\mathrm{MgO}-\mathrm{A} 12 \mathrm{O} 3-\mathrm{SiO} 2$ Glassy System". Journal of Materials Science 29 (1994) 6273-6280.

Battigelli, J, et al., (1997). "Method for the Production of Mineral Wool". US Patent 5,601,628.

Belyusenko, N I and Kareev, Y P, (1979). "Effect of P2O5 on the Properties of Glazes". Translated from Steklo i Keramika No. 4 p. 18.

Bernard, J L, et al., (2005). "Mineral Wool Composition". United States Patent 6,897,173 B2.

Bloomer, P E, et al., (1999). "Effect of Crystallization, Redox and Waste Loading on the Properties of Several Glassy Waste Forms". J. Am. Ceram. Soc., 82 [11] 2999-3011 (1999).

Bouhifd, M A, et al., (2004). "Redox State, Microstructure and Viscosity of a Partially Crystallized Basalt Melt". Earth and Planetary Science Letters 218 (2004) 31-44.

Bras, E L, (1977). "Vitroceramic Materials and Process of a Making the Same". US Patent $4,043,821$.

Brouns, R A, et al., (1988). "LFCM Vitrification Technology Quarterly Progress Report, January - March 1987". .

Brouns, R A, et al., (1988a). "LFCM Vitrification Technology Quarterly Progress Report, April June 1987". .

Brown, K G and Jantzen, C M, (2001). "Relating Liquidus Temperature to Composition for Defense Waste Processing Facility (DWPF) Process Control (U)". WSRC-TR-200140520, Rev. 0 .

Bulkley, S A, and Vienna, J D, (1997). "Composition Effects on Viscosity and Chemical Durability of Simulated Plutonium Residue Glasses". Mat. Res. Soc. Symp. Proc., 465:1243-50.

Cantale, C, et al., (1991). "A Borosilicate Glass for the Italian High Level Waste: Characterization and Behaviour". Radioactive Waste Management and the Nuclear Fuel Cycle, vol. 16 (1), pp. 25-47. 
Carter, J G, et al., (1988). "Process Performance of the Pilot Scale ISV of a Simulated Waste Disposal Site at ORNL". PNL-6530.

Certa, P J, et al., (2005). "Sensitivity of Hanford Immobilized High-Level Waste Glass Mass to Chromium and Aluminum Partitioning Assumptions". RPP-20003.

Chernushkin, I T, (1960). "Low Melting Glaze Based on Volcanic Ash". Glass and Ceramics, volume 15, Number 9, pages 495-496.

Chick, L A, et al., (1984). "West Valley High-Level Nuclear Waste Glass Development: a Statistically Designed Mixture Study". PNL-4992.

Clifford, J F, (1994). "Glass Compositions". US Patent 5,308,803.

Crawford, C L, et al., (1998). "Production of a High-Level Waste Glass from Hanford Waste Samples". Proceedings of the International Conference on Decommissioning and Decontamination and on Nuclear Hazardous Waste Management.

Danyushevsky, L V, (2001). "The Effect of Small Amounts of H2O on Crystallization of Mid-Ocean Ridge and Backarc Basin Magmas". Journal of Volcanology and Geothermal Research 110 (2001) 265-280.

Dingwell, DB, et al., (2004). "Viscosity of Peridotite Liquid". Earth and Planetary Science Letters 226 (2004) 127-138.

Dvorkin, L I and Galushko, I K, (1969). "Glazes Based on Basalts". Translated from Steklo i Keramika, No. 11, pp. 36-38.

Dvorkin, L I and Galushko, I K, (1971). "Fritted Basalt Glazes". Translated from Steklo i Keramika, No.4, pp. 38-41.

Dzhigiris, D D, et al., (1983). "Continuous Basalt Fiber". Translated from Steklo i Keramika, No. 9, pp. 14-16.

Edwards, T B, et al., (1999). "Composition and Property Measurements for Phase 3 Glasses (U)". WSRC-TR-99-00292.

Edwards, T B, et al., (1999a). "Composition and Property Measurements for Phase 4 Glasses". WSRC-TR-99-00294, Rev. 0.

Ewest, E and Wiese, H. "High Level Liquid Waste Vitrification with the Pamela Plant in Belgium". IAEA-CN-48/177, pp. 269-280.

Ferrara, D M, et al., (1998). "Vitrification of Three Low-Activity Radioactive Waste Streams from Hanford". Proceedings of the International Conference on Decommissioning and Decontamination and Nuclear Hazardous Waste Management. 
Fisher, J G, et al., (2005). "Soda Lime Zirconia Silicate Glasses as Prospective Hosts for Zirconia-Containing Radioactive Wastes". Journal of Non-Crystalline Solids 351 (2005) 623631.

Flinn, J E, et al., (1981). "Annual Report on the TRU Waste Form Studies with Special Reference to Iron-Enriched Basalt". EGG-FM-5366.

Francis, A A, (2005). "Non-Isothermal Crystallization Kinetics of a Blast Furnace Slag Glass". Central Metallurgical Research and Development Institute (CMRDI), Helwan, Cairo, Egypt, J. Am. Ceram. Soc., 88 [7] 1859-1863.

Fu, S S, and Pegg, I L, (1998). "Glass Formulation and Testing With TWRS HLW Simulants, VSL Final Report". Vitreous State Laboratory, The Catholic University of America, Washington D. C.

Fu, S S, et al., (1997). "Optimization of Savannah River M-Area Mixed Waste for Vitrification". MRS Symposium Proceedings, Vol. 465:139-146.

Fyles, K M, et al., (1999). "Compositions for High Temperature Fiberisation". US Patent $5,962,354$.

Gal'Perina, M K, et al., (1981). "Colored Glazes Based on Andesite". Translated from Steklo i Keramika, No. 10, pp. 19-20.

Galushko, I K, (1974). "Crystallization Kinetics in Glaze Coatings". Translated from Steklo i Keramika, Vol. 31, No. 4, pp. 23-25.

Galushko, I K and Dvorkin, L I, (1971). "Glass-Crystalline Glazes for Chemically Stable Ceramics". Translated from Steklo i Keramika, No. 9, pp. 36-39.

Gerasimov, V V and Spirina, O V, (2004). "Modern Low-Melting Borosilicate Glasses and Glazes for Majolica and Pottery a Review". Glass and Ceramics Vol. 61, Nos. 5 - 6.

Gerasimov, V V and Spirina, O V, (2004a). "Low Melting Borosilicate Glazes for Special-Purpose and Construction Ceramics a Review". Glass and Ceramics Vol. 61, Nos. 1112.

Giordano, D, et al., (2002). "Experimental Determinations and Modeling of the Viscosity of Multicomponent Natural Silicate Melts: Volcanological Implications". Inaugural Dissertation Zur Erlangung Des Doktorgrades Der Fakultät Für Geowissenschaften Der Ludwig-Maximilians-Universität München Vorgelegt Von Daniele Giordano, München.

Giordano, D, and Dingwell, D B, (2003). "Viscosity of Hydrous Etna Basalt: Implications for Plinian-Style Basaltic Eruptions". Bull Volcanol (2003) 65:8-14. 
Giordano, D, and Dingwell, D B, (2003a). "Non-Arrhenian Multicomponent Melt Viscosity: A Model". Earth and Planetary Science Letters 208 (2003) 337-349.

Giordano, D, (2006). "An Expanded Non-Arrhenian Model for Silicate Melt Viscosity: A Treatment for Metaluminous Peraluminous and Peralkaline Liquids". Chemical Geology 229 (2006) $42-56$.

Grandy, J D, et al., (1993). "Property and Process Correlations for Iron-Enriched Basalt Waste Forms". EGG-MS-10657.

Grum-Grzhimailo, O S, et al., (1978). "Effect of Alkaline-Earth Oxides on Crystallization of Zirconium Compounds In Low-Melting Glazes". Translated from Steklo i Keramika, No. 1, pp. 27-29.

Grum-Grzhimailo, O S, et al., (1991). "Fluorides in Zirconia Glazes". Translated from Steklo Keramika, No. 5, pp. 20-21.

Hrma, P, et al., (2001). "Increasing High-Level Waste Loading in Glass without Changing the Baseline Melter Technology". WM'01 Conference, February 25-March 1, 2001, Tucson, AZ.

Hrma, P, et al., (1994). "Property/Composition Relationships for Hanford High-Level Waste Glasses Melting at $1150^{\circ} \mathrm{C}^{\prime \prime}$. PNL-10359.

Hrma, P, (1999). "Liquidus Temperature Data for DWPF Glass". PNNL-11790, Pacific Northwest National Laboratory, Richland, WA.

Hrma, P, et al., (2006). "Chromium Phase Behavior in a Multi-Component Borosilicate Glass Melt". Journal of Non-Crystalline Solids 352 (2006) 2114-2122.

Huang, D, et al., (2004). "Incorporation of Chromium(III) and Chromium(VI) Oxides In a Simulated Basaltic Industrial Waste Glass-Ceramic". J. Am. Ceram. Soc., 87 [11] 2047-2052.

Ioffe, et al., (1987). "Glass Enamel Coatings for Electrical Domestic Machines and Appliances". Translated from Steklo i Keramika, No. 3, pp. 18-19.

Jain, V J, (1990). "Viscosity Data on the Compositions Processed During FACTS Campaigns Between December 1984 and December 1989".

Jantzen, C M, et al., (1995). "Process/Product Models for the Defense Waste Processing Facility (DWPF): Part I. Predicting Glass Durability from Composition Using a Thermodynamic Hydration Energy Reaction". WSRC-TR-93-672, Rev. 1.

Jensen, C, (1999). "Method of Making Fibers From Mineral Melts which Have a Viscosity of not More than 18 Poise at $1400^{\circ} \mathrm{C} "$. US Patent 5,954,852. 
Jantzen, C. M., et al., (2000). "Crystalline Phase Separation in Phosphate Containing Waste Glasses: Relevancy to Vitrification of Idaho National Engineering and Environmental Laboratory (INEEL) High Activity Waste (U)". WSRC-TR-2000-00339, Westinghouse Savannah River Company, Aiken, SC..

Jiricka, A, and Helebrant, A, (2000). "Dissolution of Soda-Lime Silica and High-Level Waste Glass by Static and Single-Pass Flow-Through Tests". Environmental Issues and Waste Management Technologies in the Ceramic and Nuclear Industries V (Ceramic Transactions, Vol 107), The American Ceramic Society, Westerville, OH, USA, pp. 309-316.

Jiricka, M, et al., (2002). "Structural and Mechanical Response to a Thermo-Rheologic History of Spinel Sludge in High-Level Waste Glass". Ceramics - Silikáty 46 (1) 1-7 2002.

Jiřička, M, and Hrma, P, (2003). "The Effect of Composition on Spinel Crystals Equilibrium in Low-Silica High-Level Waste Glasses". Journal of Non-Crystalline Solids 319 (2003) 280-288.

Johnston, J W, et al., (1990). "Evaluation of Empirical Models for Glass Durability". Letter Report Prepared for West Valley Nuclear Services, Pacific Northwest National Laboratory, Richland, WA.

Karamanov, A, et al., (2000). "Influence of $\mathrm{Fe}+3 / \mathrm{Fe}+2$ Ratio on the Crystallization of Iron-Rich Glasses Made With Industrial Wastes". J. Am. Ceram. Soc., 83 [12] 3153-57 (2000).

Karell, R, et al., (2006). "Properties of Selected Zirconia Containing Silicate Glasses". Ceramics - Silikáty 50 (2) 78-82 (2006).

Khizanishvili, I G and Gaprindashvili, G G, (1968). "A Trachyte-Based Opaque Glaze". Translated from Steklo i Keramika, No. 9, pp. 34-35.

Khizanishvili, I G, et al., (1970). "Some Physical and Chemical Properties of Glazes Based on Volcanic Rock". Translated from Steklo i Keramika, No. 11, pp.38-40.

Khizanishvili, I G and Gaprindashvili, G G, (1973). "Perlite Glazes". Translated from Steklo i Keramika, No. 8, pp. 25-26.

Kim, Cheon-Woo, et al., (1996). "PNL Vitrification Technology Development Project High-Waste Loaded High-Level Waste Glasses for High-Temperature Melter: Letter Report". PNL-10984.

Kim, D, and Hrma, P, (2001). "Enthalpies of Chromium Oxide Solution in Soda Lime Borosilicate Glass Systems". J. Am. Ceram. Soc., 84 [12] 2987-90.

Kondratiev, A, and Jak, E, (2005). "A Quasi Chemical Viscosity Model for Fully Liquid Slags in the A12O3-CaO-FeO-SiO2 System". Metallurgical and Materials Transactions; 36B, 5; ProQuest Science Journals pg. 623. 
Kukolev, G V and Shtefan, G E, (1969). "Obtaining Glazes with Predetermined Properties". Translated from Steklo i Keramika, No. 9, pp. 32-337.

Langowski, M H, (1996). "The Incorporation of Phosphorus, Sulfur, Chromium, Fluorine, Chlorine, Iodine, Manganese, Titanium, Uranium, and Bismuth into Simulated Nuclear Waste Glasses: Literature Study". PNNL-10980.

Li, H, et al., (1996). "Minor Components Study for a Simulated High-Level Nuclear Waste (Draft)". PNNL-10996.

Li, H, Langowski, et al., (1997). "Nepheline Precipitation in High-Level Waste Glasses: Compositional Effects and Impact on the Waste Form Acceptability". Scientific Basis for Nuclear Waste Management XX, pp. 261-269.

Mahoney, L A and Vienna, J D, (2005). "Feed Variability and Bulk Vitrification Glass Performance Assessment, Letter Report". PNNL-14985 Rev. 0.

Mangat, H K, (1997). "Glass Compositions". US Patent 5,605,869.

Matlack, K S, et al., (1997). "Results of Melter Tests Using TWRS Law Envelope C Simulants". Vitreous State Laboratory, The Catholic University of America, Washington D. C.

Mcgetchin, T R, and Smyth, J R, (1978). "The Mantle of Mars: Some Possible Geological Implications of Its High Density". ICARUS 34, 512-536 .

Merrill, R A and Janke, D S, (1993). "Results of Vitrifying Fernald OU-4 Wastes". PNL-SA-21856.

Merrill, R, et al., (1995). "Vitrification of NAC Process Residue". PNL-SA-26015, ASME 5th International Conference on Radioactive Waste Management and Environmental Remediation, September 3-9, 1995, Berlin, Germany.

Mika, M, et al., (1997). "Liquidus Temperature of Spinel Precipitating High-Level Waste Glasses". Mat. Res. Soc. Symp. Proc., 465:71-8.

Mohammad, J, et al., (2003). "Operating Range for High Temperature Borosilicate Waste Glasses: Simulated Hanford Envelope". WM'03 Conference, February 23-27, 2003, Tucson, AZ.

Mukhamedzhanova, M T, et al., (2001). "Tinted Glaze Containing Chromium-Bearing Waste". Glass and Ceramics Vol. 58, Nos. 5 - 6, 2001, Translated from Steklo i Keramika, No. 5, pp. 2122.

Muller, I S, and Pegg, I L, (1998). "Glass Formulation and Testing With TWRS LAW Simulants". Final Report for GTS Durateck Inc. and BNFL Inc., Vitreous State Laboratory, The Catholic University of America, Washington D.C.. 
Musick, C A, et al., (2000). "Technical Status Report: Vitrification Technology Development Using INEEL Run 78 Pilot". INEEL/EXT-2000-00110.

Oksoy, D L, et al., (1994). "Canonical Correlation of Waste Glass Compositions and Durability, Including Ph". Ceram. Trans. 39:365-380.

Olson, K M, (1993). "Fabrication and Leaching of West Valley Demonstration Project Glasses: Ten Quarter 2 and Ten Quarter 3 Glasses".

Olson, K M, (1994). "Viscosity Testing of 30 WVDP Glasses". WVSP 94 -16.

Olson, K M, et al., (1994). "Product Consistency Testing of West Valley Compositional Variation Glasses". PNL-10191.

Paloschi, F, (2001). "Vitrifiable Mixture for Quality Glass". US Patent 6,235,667.

Peeler, D K, and Hrma, P, (2005). "Low-Li2O Frits: Selecting Glasses That Support the Melt Rate Studies and Challenge the Current Durability Model". WSRC-TR-2005-00306 Rev. 0.

Peeler, D, et al., (1998). "Predicting Liquid Immiscibility in Multicomponent Nuclear Waste Glasses". Ceram. Trans. 45:219-229.

Peeler, D K and Edwards, T B, (1999). "Composition/Property Relationships for the Phase 2 Am-Cm Glass Variability Study". WSRC-TR-99-O0393 Rev. 0.

Peeler, D K, et al., (2005). "Nepheline Formation Study for Sludge Batch 4 (SB4): Phase 1 Experimental Results". WSRC-TR-2005-00371 Rev. 0.

Peters, R D, et al., (1993). "Database for Waste Glass Composition and Properties". 1993 International Conf on Nuclear Waste Management \& Environmental Remediation, September 1993.

Peters, R D, et al., (1995). "Vitrification Development for Mixed Wastes". PNL-SA-25764, Presented at the Waste Management 1995 Conference February 26 - March 1, 1995 Tucson, Arizona.

Pittman, D J, et al., (2001). "Property-Composition Relationships for the DP Glasses: Effect of Crystallization on Durability (U)". WSRC-TR-2001-00166.

Plodinec, M J, (1978). "Viscosity of Glasses Containing Simulated Savannah River Plant Waste". DP-1507.

Pretorius, E B and Muan, A, (1992). "Activity-Composition Relations of Chromium Oxide In Silicate Melts at $1500^{\circ} \mathrm{C}$ Under Strongly Reducing Conditions". J. Am. Ceram. Soc., 75 [6] 1364-77. 
Prunchak, R, (1997). "High Bismuth Oxide Based Flux and Paint Composition for Glass Substrates". US Patent 5,629,247.

Pye, L D, (1985). "The Physical and Thermal Properties of Simulated Nuclear Waste Glasses and Their Melts". DPST-85-397.

Quang, R D, et al., (2003). "Vitrification of HLW Produced by Uranium/Molybdenum Fuel Reprocessing in Cogema's Cold Crucible Melter". WM'03 Conference, February 23-27, 2003, Tucson.

Radchenko, Yu S and Levitskii, I A, (2001). "Processes Occurring in Synthesis of Iron-Bearing Glaze Frits". Glass and Ceramics Vol. 58, Nos. 7 - 8, 2001 Translated from Steklo i Keramika, No. 8, pp. $12-16$.

Rapp, C F, et al., (1985). "Basalt Compositions and Their Fibers". US Patent Number: $4,560,606$.

Reimus, M A H, et al., (1988). "West Valley Glass Product Qualification Durability Studies, FY 1987-1988: Effects of Composition, Redox State, Thermal History, and Groundwater". PNL-6723.

Reinherz, B P, (1990). "Lead-Free Glass Frit Compositions". US Patent 4892847.

Resce, J L, et al., (1995). "The Effect of Chemical Composition on the PCT Durability of Mixed Waste Glass from Wastewater Treatment Sludges". Waste Management '95, Tucson, AZ, February 26-March 2, 1995.

Reynolds, J G, and Hrma, P, (1997). "The Kinetics of Spinel Crystallization from a High-Level Waste Glass". MRS Symposium Proceedings, Vol. 465:65-69.

Riley, B J, et al., (2001). "Impact of HLW Glass Crystallinity on the PCT Response". PNNL-13491.

Roa, Q, et al., (1997). "Liquidus Temperatures of HLW Glasses with Zirconium-Containing Primary Crystalline Phases". Journal of Non-Crystalline Solids 220(1997) 17-29.

Roberts, G J, (1994). "Bismuth Containing Lead-Free Glass Enamels and Glazes of Low Silica Content". US pat 5,326,591.

Rocabois, P, et al., (2001). "Crystallization Kinetics of A12O3-CaO-SiO2 Based Oxide Inclusions". Journal of Non-Crystalline Solids 282 (2001) 98-109.

Rudkovskaya, N V and Mikhailenko, N Y, (2001). "Decorative Zinc-Containing Crystalline Glazes for Ornamental Ceramics (A Review)". Glass and Ceramics Vol. 58, Nos. $11-12$. 
Russell, J K and Giordano, D, (2005). "A Model for Silicate Melt Viscosity in the System CaMgSi2O6 CaAl2Si2O8 NaAlSi3O8". Geochimica et Cosmochimica Acta, Vol. 69, No. 22, pp. 5333-5349.

Sakoske, G E, (1998). "Partially Crystallizing Lead-Free Enamel Compositions for Automobile Glass". US Patent 5,783,507.

Sato, H, (2005). "Viscosity Measurement of Subliquidus Magmas: 1707 Basalt of Fuji Volcano". Journal of Mineralogical and Petrological Sciences, Volume 100, page 133-142.

Scarfe, C M, (1983). "Viscosity Temperature Relationships at 1 Atm in the System Diopside-Anorthite". American Mineralogist, Volume 68, pages 1083-1088.

Scarfe, C M, and Cronin, D J, (1986). "Viscosity-Temperature Relationships of Melts at 1 Atm in the System Diopside-Albite". American Mineralogist, Volume 71, pages 767-771.

Scholes, B A, et al., (2000). "The Preparation and Characterization of INTEC Phase 3 Composition Variation Study Glasses". INEEL/EXT-2000-01566.

Scholes, BA, et al., (2002). "The Preparation and Characterization of INTEC Sodium Bearing Waste Phase 1 Composition Variation Study Glasses". INEEL/EXT-02-00386.

Seetharaman, S, et al., (2000). "Estimation of Liquidus Temperatures for Multicomponent Silicates from Activation Energies for Viscous Flow". Metallurgical and Materials Transactions; 31B, 1; ProQuest Science Journals pg. 111.

Sheng, J, (2001). "Vitrification of Borate Waste from Nuclear Power Plant Using Coal Fly Ash. (I) Glass Formulation Development". Fuel 80 (2001) 1365-1369.

Sipp, A, and Richet, P, (2002). "Equivalence of Volume Enthalpy and Viscosity Relaxation Kinetics in Glass-Forming Silicate Liquids". Journal of Non-Crystalline Solids 298 (2002) 202212.

Solvang, M, (2002). "Rheological Response To Compositional Variation in High Aluminosilicate Melts". Ph. D. Thesis, Department of Production, Aalborg University, Denmark.

Soper, P D, et al., (1982). "Optimization of Glass Composition from the Vitrification of Nuclear Waste at the Savannah River Plant". DP-MS-81-108.

Sridharam, S, (2005). "Durable Glass Enamel Composition". US Patent 6,936,556 B2.

Staples, B A, et al., (1999). "The Preparation and Characterization of Intec HAW Phase 1 Composition Variation Study Glasses". INEEL/EXT-98-00970.

Staples, B A, et al., (2000). "The Preparation and Characterization of Intec Phase 2b Composition Variation Study Glasses". INNEL/EXT-99-01322. 
Stempin, J L, and Wexell, D R, (1985). "Spontaneously Formed Machinable Glass Ceramics". US Patent 4,536,452.

Taniguchi, H, (1993). "On the Volume Dependence of Viscosity of Some Magmatic Silicate Melts". Mineralogy and Petrology (1993) 49:13-25.

Toplis, M J, et al., (1994). "The Effect of Phosphorus on the Iron Redox Ratio, Viscosity and Density of an Evolved Ferro Basalt". Mineral Petrol (1994) 117:293-304.

Vienna J, et al., (1996). "Effect of Composition and Temperature on the Properties of High-Level Waste Glass Melting Above $1200^{\circ} \mathrm{C}^{\prime}$. PNNL-10987.

Vienna, J D, et al., (1999). "Glass Formulation Development for INEEL Sodium-Bearing Waste". PNNL-12234.

Vienna, J D, et al., (1999a). "Glass Formulation for Idaho National Engineering and Environmental Laboratory Zirconia Calcine High-Activity Waste". PNNL-12202.

Vienna, J D, et al., (2000). "Glass Formulation for Idaho Engineering Environmental Laboratory Zirconia High-Activity Waste," Ceram. Trans. 107:451-459.

Vienna, J D, et al., (2002). "Database and Interim Glass Property Models for Hanford HLW and LAW Glasses". PNNL-14060.

Vojtech, O, et al., (1996). "The Effect of Chromium Oxide on the Properties of Simulated Nuclear Waste Glasses". PNNL-10986.

Volf, M B, (1984). Glass Science and Technology, 7: Chemical Approach to Glass. Elsevier Scientific Publishers, New York.

West Valley Nuclear Services, (2000). "Viscosity of a CaO MgO A12O3 SiO2 Melt Containing Spinel Particles at 1646 K". Metallurgical I old Materials Transactions; 31B. 1; ProQuest Science Journals pg. 97.

Wright, K S, et al., (1995). "An Evaluation of Glass-Crystal Composites for the Disposal of Nuclear and Hazardous Waste Materials". Waste Management '95 Tucson, Arizona February 26March 2, 1995.

Wroniciewicz, D J, et al., (1995). "West Valley Demonstration Project Waste Form Qualification Report". WVDP-186, Section 1.3, Rev. 1.

Yamagishi, T, (1985). "Thallium Containing Optical Glass". US Patent 4,495,295.

Yan, Q Y, et al., (2001). "Physical Properties of Basalt and Numerical Simulation of the Melting Process in Basalt Particle Beds". International Journal of Thermophysics, Vol. 22, No. 3. 
Yee, Tin Boo, et al., (1955). "Relation of Composition to Viscosity of Enamel Glasses". Journal of The American Ceramic Society Vol. 38, No. 10, page 378.

Yoshida, S, (1993). "Overglaze Colors for Pottery". US Patent 5,262,363.

Zubekhin, A P, et al., (1993). "Glass Formation and Crystallization in the $\mathrm{SiO} 2-\mathrm{A} 12 \mathrm{O} 3-\mathrm{CaO}-\mathrm{MgO}-\mathrm{Fe} 2 \mathrm{O} 3-\mathrm{MnO} 2-\mathrm{K} 2 \mathrm{O}-\mathrm{Na} 2 \mathrm{O}$ for Synthesizing Heat Resistant Coatings". Translated from Steklo i Keramika, No. 5, pp 26-28. 


\begin{tabular}{|c|c|c|c|c|c|c|c|c|c|c|c|c|c|c|c|c|c|c|c|c|c|c|c|c|c|c|c|c|c|c|c|c|c|c|c|}
\hline Ref & 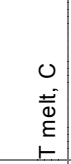 & $\begin{array}{l}0 \\
0 \\
\stackrel{0}{0} \\
\stackrel{0}{0}\end{array}$ & 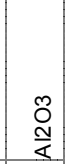 & 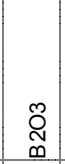 & D & 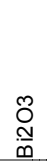 & $\begin{array}{l}0 \\
\mathbb{0} \\
0\end{array}$ & $\begin{array}{l}\text { סे } \\
0 \\
0 \\
\tilde{ల} \\
0 \\
0 \\
0\end{array}$ & $\begin{array}{l}0 \\
0 \\
0 \\
0 \\
0 \\
0 \\
0\end{array}$ & ֻొ & $\begin{array}{l}O \\
3 \\
0\end{array}$ & $\leftarrow$ & 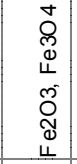 & ¿̊ & 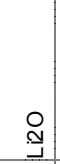 & $\begin{array}{l}\circ \\
\sum \\
\Sigma\end{array}$ & $\begin{array}{l}\tilde{O} \\
\tilde{\Sigma}\end{array}$ & $\begin{array}{l}00 \\
\text { Oొ } \\
\Sigma\end{array}$ & $\begin{array}{l}\underset{\pi}{ } \\
\text { Z }\end{array}$ & $\frac{\circ}{z}$ & \begin{tabular}{l}
$\mathcal{N}$ \\
\multirow{0}{0}{}
\end{tabular} & $\bar{\Xi}$ & $\begin{array}{l}\text { مి } \\
\text { a }\end{array}$ & $\frac{8}{2}$ & 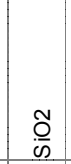 & $\begin{array}{l}0 \\
\text { O) } \\
\text { in }\end{array}$ & 온 & 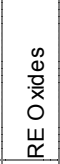 & $\begin{array}{l}\tilde{\tilde{c}} \\
\stackrel{\varepsilon}{⺊}\end{array}$ & 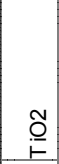 & 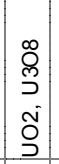 & $\begin{array}{c}Q \\
N \\
N\end{array}$ & ญั & $\underset{\hat{O}}{\underline{O}}$ & $\begin{array}{l}\vec{\Xi} \\
\underline{0} \\
\underline{\underline{D}} \\
\vec{z}\end{array}$ \\
\hline Ackerman, 2006 & 1250 & & & 11.0 & & & 2.0 & & 0.5 & 25.0 & & & & 5.0 & 1.0 & & & & 15.0 & & & & & & 38.5 & & & & & 2.0 & & & & & \\
\hline Barantseva, 2004 & 1425 & & 13.6 & & & & 6.1 & 1.0 & & & & & 6.4 & & & 4.0 & & & 11.5 & & & 0.8 & & & 57.2 & & & & & & & & & & \\
\hline Barbieri, 1994 & & & 9.2 & & & & 24.5 & & & & & & & & & 13.9 & & & & & & & & & 52.5 & & & & & & & & & & \\
\hline Barbieri, 1994 & & & 9.1 & & & & 24.2 & & & 0.5 & & & & & & 13.8 & & & & & & & & & 52.3 & & & & & & & & & & \\
\hline Barbieri, 1994 & & & 9.1 & & & & 23.8 & & & 1.3 & & & & & & 13.8 & & & & & & & & & 52.1 & & & & & & & & & & \\
\hline Barbieri, 1994 & & & 9.0 & & & & 23.5 & & & 1.8 & & & & & & 13.7 & & & & & & & & & 51.9 & & & & & & & & & & \\
\hline Barbieri, 1994 & & & 8.4 & & & & 18.4 & & & 12.2 & & & & & & 12.7 & & & & & & & & & 48.2 & & & & & & & & & & \\
\hline Barbieri, 1994 & & & 8.9 & & & & 22.5 & & & 3.9 & & & & & & 13.5 & & & & & & & & & 51.2 & & & & & & & & & & \\
\hline Barbieri, 1994 & & & 18.3 & & & & 23.1 & & & & & & & & & 9.2 & & & & & & & & & 49.4 & & & & & & & & & & \\
\hline Barbieri, 1994 & & & 18.2 & & & & 22.8 & & & 0.5 & & & & & & 9.2 & & & & & & & & & 49.2 & & & & & & & & & & \\
\hline Barbieri, 1994 & & & 18.2 & & & & 22.4 & & & 1.2 & & & & & & 9.2 & & & & & & & & & 49.0 & & & & & & & & & & \\
\hline Barbieri, 1994 & & & 18.1 & & & & 22.2 & & & 1.7 & & & & & & 9.1 & & & & & & & & & 48.9 & & & & & & & & & & \\
\hline Barbieri, 1994 & & & 17.9 & & & & 21.2 & & & 3.7 & & & & & & 9.0 & & & & & & & & & 48.3 & & & & & & & & & & \\
\hline Barbieri, 1994 & & & 16.9 & & & & 17.5 & & & 11.5 & & & & & & 8.5 & & & & & & & & & 45.6 & & & & & & & & & & \\
\hline Battigelli, 1997 & & 1423 & 18.0 & & & & 8.9 & & & & & & 10.1 & 0.6 & & 6.4 & 0.2 & & 3.5 & & & & 0.1 & & 51.5 & & & & & 0.7 & & & & & \\
\hline Belyusenko, 1979 & 1350 & & 3.5 & 23.0 & 4.5 & & 4.3 & & & & & & & 0.3 & & 0.5 & & & 6.5 & & & & 3.0 & & 35.0 & & 4.5 & & & & & 5.7 & 7.0 & & \\
\hline Belyusenko, 1979 & 1350 & & 6.0 & 22.6 & 3.6 & & 7.7 & & & & & 4.7 & & 3.0 & & & & & 7.1 & & & & 6.0 & & 33.0 & & 2.4 & & & & & 3.9 & & & \\
\hline Bernard, 2005 & & 1349 & 18.6 & & & & 6.2 & & & & & & 7.2 & 5.2 & & 7.1 & & & 8.0 & & & & & & 47.7 & & & & & & & & & 1260 & \\
\hline Bernard, 2005 & & 1284 & 18.1 & & & & 22.7 & & & & & & 2.5 & 7.4 & & 0.2 & & & 6.3 & & & & & & 42.6 & & & & & & & & & 1200 & \\
\hline Bernard, 2005 & & & 17.3 & & & & 21.7 & & & & & & 3.0 & 7.1 & & 0.4 & & & 6.0 & & & & & & 44.4 & & & & & & & & & 1190 & \\
\hline Bernard, 2005 & & 1297 & 17.2 & & & & 15.3 & & & & & & 6.6 & 7.8 & & 0.5 & & & 6.2 & & & & & & 45.2 & & & & & & & & & 1160 & \\
\hline Bernard, 2005 & & 1333 & 18.1 & & & & 13.5 & & & & & & 7.3 & 8.1 & & 0.5 & & & 6.5 & & & & & & 45.4 & & & & & & & & & 1160 & \\
\hline Bernard, 2005 & & 1324 & 17.6 & & & & 15.0 & & & & & & 8.4 & 7.6 & & 0.5 & & & 6.4 & & & & & & 43.9 & & & & & & & & & 1120 & \\
\hline Bernard, 2005 & & & 17.6 & & & & 13.3 & & & & & & 9.8 & 7.9 & & 0.5 & & & 6.3 & & & & & & 44.2 & & & & & & & & & 1100 & \\
\hline Bernard, 2005 & & & 17.6 & & & & 14.2 & & & & & & 9.2 & 7.9 & & 0.5 & & & 6.4 & & & & & & 43.8 & & & & & & & & & 1110 & \\
\hline Bernard, 2005 & & & 17.4 & & & & 13.2 & & & & & & 8.3 & 7.8 & & 0.5 & & & 6.3 & & & & & & 46.1 & & & & & & & & & 1140 & \\
\hline Bernard, 2005 & & 1353 & 17.6 & & & & 11.9 & & & & & & 11.3 & 8.0 & & 0.5 & & & 6.4 & & & & & & 43.8 & & & & & & & & & 1160 & \\
\hline Bernard, 2005 & & 1364 & 15.7 & & & & 9.8 & & & & & & 12.1 & 8.0 & & 0.4 & & & 6.4 & & & & & & 47.1 & & & & & & & & & 1200 & \\
\hline Bernard, 2005 & & 1355 & 20.9 & & & & 14.5 & & & & & & 8.7 & 7.4 & & 0.5 & & & 6.1 & & & & & & 41.9 & & & & & & & & & 1140 & \\
\hline Bernard, 2005 & & 1442 & 19.8 & & & & 14.0 & & & & & & 4.2 & 7.2 & & 0.5 & & & 6.0 & & & & & & 48.2 & & & & & & & & & 1160 & \\
\hline Bernard, 2005 & & 1403 & 22.5 & & & & 14.3 & & & & & & 6.3 & 7.1 & & 0.5 & & & 6.0 & & & & & & 43.2 & & & & & & & & & 1140 & \\
\hline Bernard, 2005 & & 1393 & 19.3 & & & & 13.9 & & & & & & 6.8 & 7.1 & & 0.5 & & & 6.0 & & & & & & 46.3 & & & & & & & & & 1110 & \\
\hline Bernard, 2005 & & 1372 & 18.8 & & & & 13.9 & & & & & & 8.3 & 7.2 & & 0.5 & & & 5.9 & & & & & & 45.4 & & & & & & & & & 1110 & \\
\hline Bernard, 2005 & & 1365 & 19.7 & & & & 14.1 & & & & & & 9.5 & 7.2 & & 0.5 & & & 6.0 & & & & & & 43.0 & & & & & & & & & 1110 & \\
\hline Bernard, 2005 & & & 19.8 & & & & 13.4 & & & & & & 9.3 & 3.7 & & 0.7 & & & 8.3 & & & & & & 44.3 & & & & & & & & & 1170 & \\
\hline Bernard, 2005 & & 1382 & 21.5 & & & & 14.1 & & & & & & 7.5 & 7.3 & & 0.5 & & & 6.0 & & & & & & 43.0 & & & & & & & & & 1140 & \\
\hline Bernard, 2005 & & 1404 & 18.4 & & & & 13.8 & & & & & & 6.2 & 7.3 & & 0.5 & & & 6.0 & & & & & & 47.7 & & & & & & & & & 1150 & \\
\hline Bernard, 2005 & & 1430 & 22.4 & & & & 13.9 & & & & & & 4.2 & 7.3 & & 0.5 & & & 6.0 & & & & & & 45.6 & & & & & & & & & 1150 & \\
\hline Bernard, 2005 & & 1382 & 21.2 & & & & 14.1 & & & & & & 7.4 & 7.2 & & 0.5 & & & 6.0 & & & & & & 43.5 & & & & & & & & & 1120 & \\
\hline Bernard, 2005 & & 1392 & 22.2 & & & & 14.0 & & & & & & 6.9 & 7.2 & & 0.5 & & & 6.0 & & & & & & 43.1 & & & & & & & & & 1160 & \\
\hline Bernard, 2005 & & 1386 & 25.1 & & & & 13.9 & & & & & & 6.9 & 7.2 & & 0.5 & & & 6.0 & & & & & & 40.3 & & & & & & & & & 1170 & \\
\hline Bernard, 2005 & & & 21.7 & & & & 13.1 & & & & & & 8.7 & 7.7 & & 0.6 & & & 5.9 & & & & & & 42.3 & & & & & & & & & 1160 & \\
\hline Bernard, 2005 & & & 24.6 & & & & 13.2 & & & & & & 4.0 & 7.6 & & 0.6 & & & 5.9 & & & & & & 43.9 & & & & & & & & & & \\
\hline Bernard, 2005 & & & 24.7 & & & & 13.4 & & & & & & 6.0 & 7.6 & & 0.6 & & & 6.2 & & & & & & 41.5 & & & & & & & & & 1180 & \\
\hline Bernard, 2005 & & & 24.9 & & & & 13.3 & & & & & & 8.1 & 7.6 & & 0.5 & & & 6.3 & & & & & & 39.3 & & & & & & & & & 1200 & \\
\hline Bernard, 2005 & & & 18.2 & & & & 13.9 & & & & & & 7.5 & 3.9 & & 0.6 & & & 8.1 & & & & & & 47.3 & & & & & & & & & 1160 & \\
\hline Bernard, 2005 & & 1323 & 19.2 & & & & 12.9 & & & & & & 7.5 & 5.7 & & 0.8 & & & 7.9 & & & & & & 45.3 & & & & & & & & & 1150 & \\
\hline Bernard, 2005 & & & 20.5 & & & & 12.9 & & & & & & 7.4 & 3.8 & & 0.8 & & & 8.3 & & & & & & 45.3 & & & & & & & & & 1180 & \\
\hline Bernard, 2005 & & & 22.5 & & & & 12.7 & & & & & & 7.5 & 3.7 & & 0.8 & & & 7.9 & & & & & & 44.0 & & & & & & & & & 1200 & \\
\hline Bernard, 2005 & & 1335 & 19.2 & & & & 12.4 & & & & & & 7.4 & 3.9 & & 0.8 & & & 8.8 & & & & & & 46.5 & & & & & & & & & 1150 & \\
\hline
\end{tabular}


ORP-56297 Rev. 0

The Catholic University of America

High Level Waste Vitrification System Improvements Vitreous State Laboratory

Table A1. Glass Compositions from Literature Review (continued)

\begin{tabular}{|c|c|c|c|c|c|c|c|c|c|c|c|c|c|c|c|c|c|c|c|c|c|c|c|c|c|c|c|c|c|c|c|c|c|c|c|}
\hline Ref & 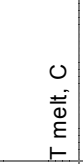 & $\begin{array}{l}0 \\
\stackrel{0}{2} \\
\stackrel{0}{\circ} \\
上\end{array}$ & $\begin{array}{l}\text { Oొ } \\
\stackrel{N}{<}\end{array}$ & బ్లి & $\begin{array}{l}\text { D } \\
\infty \\
\infty\end{array}$ & $\begin{array}{l}\text { Oొ } \\
\stackrel{N}{0}\end{array}$ & $\begin{array}{l}0 \\
0 \\
0\end{array}$ & $\begin{array}{l}\widetilde{̃} \\
0 \\
0 \\
\tilde{ల} \\
0 \\
0 \\
0\end{array}$ & $\begin{array}{l}0 \\
0 \\
0 \\
0 \\
0 \\
0 \\
0\end{array}$ & $\begin{array}{l}0 \\
\text { N } \\
\text { U }\end{array}$ & \begin{tabular}{l}
0 \\
\multirow{3}{3}{}
\end{tabular} & 4 & 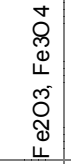 & ָָ & $\stackrel{O}{\beth}$ & $\begin{array}{l}0 \\
\dot{O} \\
\Sigma\end{array}$ & $\begin{array}{l}\tilde{O} \\
\Sigma \\
\Sigma\end{array}$ & $\begin{array}{l}0 \\
0 \\
\Sigma \\
\Sigma\end{array}$ & $\begin{array}{l}\text { D } \\
\stackrel{\pi}{Z}\end{array}$ & $\stackrel{\circ}{\bar{z}}$ & $\begin{array}{l}\text { N } \\
\text { t }\end{array}$ & 吾 & 号 & $\frac{8}{a}$ & $\frac{\tilde{O}}{\omega}$ & $\begin{array}{l}0 \\
0 \\
\infty\end{array}$ & $\begin{array}{l}\text { o } \\
\text { is }\end{array}$ & 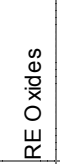 & 尽 & 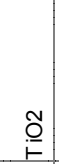 & 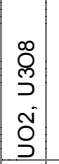 & $\frac{Q}{N}$ & $\begin{array}{l}\text { Õ } \\
\text { N }\end{array}$ & $\begin{array}{l}\overline{0} \\
\underline{1}\end{array}$ & $\begin{array}{l}\bar{\Xi} \\
\text { 음 } \\
\underline{\underline{m}}\end{array}$ \\
\hline Bloomer, 1999 & 1375 & & 15.4 & & & & 3.0 & 0.3 & & 0.1 & & & 20.0 & 0.2 & & 0.4 & 0.1 & & 15.0 & 0.1 & & 0.3 & & 0.3 & 44.2 & 0.1 & & & & & & & & & \\
\hline Bloomer, 1999 & 1450 & & 18.6 & & & & 3.6 & 0.3 & & 0.1 & & & 24.2 & 0.2 & & 0.5 & 0.1 & & 2.6 & 0.1 & & 0.6 & & 0.3 & 48.2 & 0.1 & & & & & & & & & \\
\hline Bloomer, 1999 & 1280 & & 6.0 & 5.9 & & & 2.3 & & & 0.0 & & & 20.0 & & & 0.4 & 0.0 & & 15.0 & & & 0.1 & 0.1 & & 50.0 & & & & & 0.1 & & & & & \\
\hline Bloomer, 1999 & 1450 & & 5.2 & & & & 6.7 & & & 0.0 & & & 58.8 & & & 1.1 & 0.0 & & 1.2 & 0.0 & & 0.2 & 0.1 & & 25.7 & & & & & 0.3 & & & 0.0 & & \\
\hline Bloomer, 1999 & 1300 & & 7.8 & & 0.1 & & 19.3 & 0.4 & & 1.1 & 0.3 & & 18.1 & 1.7 & & 1.6 & 0.1 & & 6.0 & 0.4 & & & 0.1 & 0.1 & 40.0 & 0.1 & & & & 0.4 & & & 0.4 & & \\
\hline Bloomer, 1999 & 1425 & & 8.7 & & 0.1 & & 21.6 & 0.4 & & 1.2 & 0.3 & & 20.2 & 1.9 & & 1.8 & 0.1 & & 1.5 & 0.5 & & & 0.1 & 0.1 & 38.1 & 0.1 & & & & 0.4 & & & 0.5 & & \\
\hline Bouhifd, 2004 & & 1198 & 13.3 & & & & 10.0 & & & & & & 11.2 & 1.4 & & 9.2 & & & 5.6 & & & & & & 46.6 & & & & & 2.5 & & & & & \\
\hline Bouhifd, 2004 & & 1325 & 12.8 & & & & 9.6 & & & & & & 9.9 & 1.5 & & 8.1 & & & 6.1 & & & & & & 49.3 & & & & & 2.1 & & & & & \\
\hline Bouhifd, 2004 & & 1355 & 12.1 & & & & 9.2 & & & & & & 8.9 & 1.8 & & 7.2 & & & 6.5 & & & & & & 51.6 & & & & & 1.5 & & & & & \\
\hline Bouhifd, 2004 & & 1506 & 9.9 & & & & 5.3 & & & & & & 7.2 & 2.3 & & 4.6 & & & 9.0 & & & & & & 59.0 & & & & & 1.6 & & & & & \\
\hline Bouhifd, 2004 & & 1312 & 13.1 & & & & 10.0 & & & & & & 10.2 & 1.5 & & 8.9 & & & 5.6 & & & & & & 48.2 & & & & & 2.3 & & & & & \\
\hline Bouhifd, 2004 & & 1696 & 4.2 & & & & 2.0 & & & & & & 1.2 & 3.4 & & 2.0 & & & 11.3 & & & & & & 74.8 & & & & & 0.5 & & & & & \\
\hline Bras, 1977 & $<1450$ & & 4.3 & 4.0 & & & 39.0 & & & 0.8 & & & 22.0 & & & & & & & & & & & & 30.0 & & & & & & & & & & \\
\hline Bras, 1977 & $<1450$ & & 4.3 & 4.0 & & & 37.0 & & & 0.8 & & & 22.0 & & & & & & & & & & & & 32.0 & & & & & & & & & & \\
\hline Bras, 1977 & $<1450$ & & 4.3 & 4.0 & & & 35.0 & & & 0.8 & & & 22.0 & & & & & & & & & & & & 34.0 & & & & & & & & & & \\
\hline Bras, 1977 & $<1450$ & & 4.3 & 4.0 & & & 29.0 & & & 0.8 & & & 22.0 & & & & & & & & & & & & 40.0 & & & & & & & & & & \\
\hline Bras, 1977 & $<1450$ & & 4.3 & 4.0 & & & 27.0 & & & 0.8 & & & 22.0 & & & & & & & & & & & & 42.0 & & & & & & & & & & \\
\hline Bras, 1977 & $<1450$ & & 4.3 & 4.0 & & & 25.0 & & & 0.8 & & & 22.0 & & & & & & & & & & & & 44.0 & & & & & & & & & & \\
\hline Bras, 1977 & $<1450$ & & 4.3 & 4.0 & & & 39.0 & & & 0.8 & & & 15.0 & & & & & & & & & & & & 37.0 & & & & & & & & & & \\
\hline Bras, 1977 & $<1450$ & & 4.3 & 4.0 & & & 36.0 & & & 0.8 & & & 18.0 & & & & & & & & & & & & 37.0 & & & & & & & & & & \\
\hline \begin{tabular}{|l|} 
Bras, 1977 \\
\end{tabular} & $<1450$ & & 4.3 & 4.0 & & & 34.0 & & & 0.8 & & & 20.0 & & & & & & & & & & & & 37.0 & & & & & & & & & & \\
\hline Bras, 1977 & $<1450$ & & 4.3 & 4.0 & & & 30.0 & & & 0.8 & & & 24.0 & & & & & & & & & & & & 37.0 & & & & & & & & & & \\
\hline Bras, 1977 & $<1450$ & & 4.3 & 4.0 & & & 28.0 & & & 0.8 & & & 26.0 & & & & & & & & & & & & 37.0 & & & & & & & & & & \\
\hline Bras, 1977 & $<1450$ & & 4.3 & 4.0 & & & 26.0 & & & 0.8 & & & 28.0 & & & & & & & & & & & & 37.0 & & & & & & & & & & \\
\hline Bras, 1977 & $<1450$ & & 4.3 & 4.0 & & & 24.0 & & & 0.8 & & & 30.0 & & & & & & & & & & & & 37.0 & & & & & & & & & & \\
\hline Bras, 1977 & $<1450$ & & 4.3 & 4.0 & & & 32.0 & & & 0.8 & & & 15.0 & & & & & & & & & & & & 44.0 & & & & & & & & & & \\
\hline Bras, 1977 & $<1450$ & & 4.3 & 4.0 & & & 32.0 & & & 0.8 & & & 18.0 & & & & & & & & & & & & 41.0 & & & & & & & & & & \\
\hline Bras, 1977 & $<1450$ & & 4.3 & 4.0 & & & 32.0 & & & 0.8 & & & 20.0 & & & & & & & & & & & & 39.0 & & & & & & & & & & \\
\hline Bras, 1977 & $<1450$ & & 4.3 & 4.0 & & & 32.0 & & & 0.8 & & & 24.0 & & & & & & & & & & & & 35.0 & & & & & & & & & & \\
\hline Bras, 1977 & $<1450$ & & 4.3 & 4.0 & & & 32.0 & & & 0.8 & & & 26.0 & & & & & & & & & & & & 33.0 & & & & & & & & & & \\
\hline Bras, 1977 & $<1450$ & & 4.3 & 4.0 & & & 32.0 & & & 0.8 & & & 29.0 & & & & & & & & & & & & 30.0 & & & & & & & & & & \\
\hline Bras, 1977 & $<1450$ & & 4.3 & 4.0 & & & 23.0 & & & 0.8 & & & 28.0 & & & & & & & & & & & & 40.0 & & & & & & & & & & \\
\hline Bras, 1977 & $<1450$ & & 4.3 & 4.0 & & & 26.0 & & & 0.8 & & & 31.0 & & & & & & & & & & & & 34.0 & & & & & & & & & & \\
\hline Bras, 1977 & $<1450$ & & 4.3 & 4.0 & & & 42.0 & & & 0.8 & & & 15.0 & & & & & & & & & & & & 34.0 & & & & & & & & & & \\
\hline Bras, 1977 & $<1450$ & & 4.3 & 4.0 & & & 38.0 & & & 0.8 & & & 13.0 & & & & & & & & & & & & 40.0 & & & & & & & & & & \\
\hline Bras, 1977 & $<1450$ & & 4.3 & 4.0 & & & 30.0 & & & 0.8 & & & 22.0 & & & 2.0 & & & & & & & & & 37.0 & & & & & & & & & & \\
\hline Bras, 1977 & $<1450$ & & 4.3 & 4.0 & & & 26.0 & & & 0.8 & & & 22.0 & & & 6.0 & & & & & & & & & 37.0 & & & & & & & & & & \\
\hline Bras, 1977 & $<1450$ & & 4.3 & 4.0 & & & 22.0 & & & 0.8 & & & 22.0 & & & 10.0 & & & & & & & & & 37.0 & & & & & & & & & & \\
\hline Bras, 1977 & $<1450$ & & 4.3 & 4.0 & & & 31.0 & & & 0.8 & & & 21.0 & & & & & & 2.0 & & & & & & 37.0 & & & & & & & & & & \\
\hline Bras, 1977 & $<1450$ & & 4.3 & 4.0 & & & 29.0 & & & 0.8 & & & 20.0 & & & & & & 5.0 & & & & & & 37.0 & & & & & & & & & & \\
\hline Bras, 1977 & $<1450$ & & 4.3 & 4.0 & & & 32.0 & & & 0.5 & & & 22.0 & & & & & & & & & & & & 36.8 & & & & & & & & & & \\
\hline Bras, 1977 & $<1450$ & & 4.3 & 4.0 & & & 31.5 & & & 2.0 & & & 21.5 & & & & & & & & & & & & 36.3 & & & & & & & & & & \\
\hline Brouns, 1988 & & & 8.1 & 12.1 & 0.1 & & 0.6 & 0.1 & & 0.2 & & & 12.2 & 1.3 & 2.0 & 1.3 & 1.3 & 0.0 & 9.3 & 0.3 & & 0.2 & 2.5 & & 42.6 & 0.3 & 0.0 & 0.2 & 3.6 & 1.0 & 0.6 & & 0.3 & & \\
\hline Brouns, 1988 & & & 8.0 & 12.1 & 0.1 & & 0.6 & 0.1 & & 0.2 & & & 12.2 & 1.3 & 2.0 & 1.3 & 1.3 & 0.0 & 9.3 & 0.3 & & 0.3 & 2.5 & & 42.6 & 0.3 & 0.0 & 0.2 & 3.6 & 1.0 & 0.6 & & 0.3 & & \\
\hline Brouns, 1988 & & & 7.8 & 11.7 & 0.1 & & 0.6 & 0.1 & & 0.2 & & & 11.8 & 1.3 & 2.0 & 1.3 & 1.3 & 0.0 & 12.2 & 0.3 & & 0.2 & 2.4 & & 41.2 & 0.3 & 0.0 & 0.2 & 3.5 & 1.0 & 0.5 & & 0.3 & & \\
\hline Brouns, 1988 & & & 8.0 & 12.0 & 0.1 & & 0.6 & 0.1 & & 0.2 & & & 12.1 & 1.3 & 2.0 & 1.3 & 1.3 & 0.0 & 9.2 & 0.3 & & 0.2 & 3.5 & & 42.2 & 0.3 & 0.0 & 0.2 & 3.6 & 1.0 & 0.6 & & 0.3 & & \\
\hline Brouns, 1988 & & & 7.9 & 11.9 & 0.1 & & 0.6 & 0.1 & & 0.2 & & & 12.0 & 1.3 & 2.0 & 1.3 & 1.3 & 0.0 & 9.1 & 0.3 & & 0.2 & 2.5 & & 41.8 & 0.3 & 0.0 & 0.2 & 5.4 & 1.0 & 0.6 & & 0.3 & & \\
\hline Brouns, 1988 & & & 8.5 & 7.3 & 0.1 & & 0.6 & 0.1 & & 0.2 & & & 12.9 & 1.4 & 2.1 & 1.4 & 1.4 & 0.0 & 9.8 & 0.3 & & 0.2 & 2.7 & & 44.9 & 0.3 & 0.0 & 0.2 & 3.8 & 1.1 & 0.6 & & 0.3 & & \\
\hline Brouns, 1988a & & & 9.0 & 13.0 & 0.3 & & 1.4 & 1.2 & & 0.2 & 0.0 & & 9.0 & 1.7 & 2.4 & 1.3 & 2.5 & 0.1 & 10.5 & 0.5 & 0.3 & 0.3 & 1.5 & & 39.0 & \begin{tabular}{|l|l|} 
& 0.4 \\
\end{tabular} & 0.0 & 0.3 & 2.0 & 1.4 & 1.0 & 0.0 & 0.7 & & \\
\hline Brouns, 1988a & & & 9.0 & 13.0 & 0.3 & & 1.4 & 0.4 & & 0.1 & 0.1 & & 15.0 & 1.4 & 1.9 & 1.3 & 2.2 & 0.0 & 8.4 & 0.2 & & 0.1 & 3.5 & & 39.0 & 0.1 & 0.0 & 0.1 & 2.0 & 0.4 & 0.2 & 0.0 & 0.2 & & \\
\hline
\end{tabular}


ORP-56297 Rev. 0

The Catholic University of America

Table A1. Glass Compositions from Literature Review (continued)

\begin{tabular}{|c|c|c|c|c|c|c|c|c|c|c|c|c|c|c|c|c|c|c|c|c|c|c|c|c|c|c|c|c|c|c|c|c|c|c|c|}
\hline Ref & 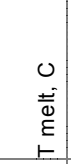 & $\begin{array}{l}0 \\
0 \\
\stackrel{0}{0} \\
\stackrel{0}{1} \\
上\end{array}$ & $\begin{array}{c}\substack{N \\
\mathbb{N}} \\
\end{array}$ & 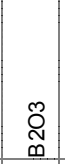 & $\begin{array}{l}\text { రి } \\
\infty\end{array}$ & $\begin{array}{l}\text { Õ } \\
\stackrel{N}{0}\end{array}$ & $\begin{array}{l}0 \\
\mathbb{\pi} \\
0\end{array}$ & $\begin{array}{l}\text { Õ } \\
0 \\
0 \\
\tilde{N} \\
0 \\
0 \\
0\end{array}$ & $\begin{array}{l}\text { O } \\
\text { N } \\
0 \\
0 \\
0 \\
0 \\
0\end{array}$ & 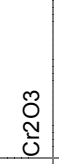 & $\stackrel{\circ}{3}$ & \llcorner & 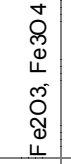 & $\begin{array}{l}\text { Zิ } \\
\underline{x}\end{array}$ & 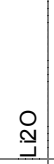 & $\begin{array}{l}\circ \\
\stackrel{O}{\Sigma}\end{array}$ & $\begin{array}{l}\tilde{O} \\
\stackrel{\Sigma}{\Sigma}\end{array}$ & $\begin{array}{l}0 \\
0 \\
\Sigma\end{array}$ & $\begin{array}{l}\text { న } \\
\text { Z }\end{array}$ & $\stackrel{\circ}{z}$ & $\begin{array}{l}\text { N } \\
\text { t̃ }\end{array}$ & $\overline{\bar{E}}$ & 足 & $\frac{8}{2}$ & $\stackrel{\widetilde{N}}{\mathrm{~s}}$ & $\begin{array}{l}\text { o } \\
\text { ¿n }\end{array}$ & $\begin{array}{l}\text { 임 } \\
\text { con }\end{array}$ & 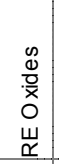 & $\begin{array}{c}\tilde{Q} \\
\stackrel{\varepsilon}{F}\end{array}$ & 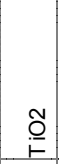 & 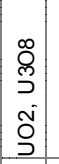 & $\frac{Q}{N}$ & ญ & 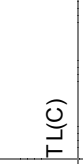 & $\begin{array}{l}\overrightarrow{\vec{J}} \\
\stackrel{0}{\underline{m}} \\
\overrightarrow{\underline{z}}\end{array}$ \\
\hline Brouns, 1988a & & & 9.0 & 10.2 & 0.3 & & 1.4 & 1.3 & & 0.3 & 0.0 & & 9.0 & 1.7 & 2.4 & 1.3 & 2.5 & 0.1 & 10.5 & 0.6 & & 0.3 & 1.5 & & 39.0 & 0.4 & 0.0 & 0.3 & 5.1 & 1.6 & 0.2 & 0.1 & 0.8 & & \\
\hline Brouns, 1988a & & & 9.0 & 11.0 & 0.1 & & 0.5 & 0.4 & & 0.1 & 0.0 & & 15.0 & 1.8 & 2.5 & 0.4 & 0.5 & 0.0 & 11.2 & 0.2 & & 0.1 & 1.5 & & 42.5 & 0.1 & 0.0 & 0.1 & 2.4 & 0.5 & 0.2 & & 0.2 & & \\
\hline Brouns, 1988a & & & 12.0 & 10.4 & 0.3 & & 1.4 & 0.4 & & 0.1 & 0.1 & & 15.0 & 1.6 & 2.2 & 1.3 & 0.5 & 0.0 & 9.5 & 0.2 & & 0.1 & 1.5 & & 36.7 & 0.1 & 0.0 & 0.1 & 5.3 & 0.4 & 2.0 & & 0.2 & & \\
\hline Brouns, 1988a & & & 12.0 & 11.1 & 0.3 & & 1.4 & 1.3 & & 0.3 & 0.0 & & 9.0 & 1.5 & 2.0 & 1.3 & 0.5 & 0.1 & 9.0 & 0.6 & & 0.3 & 3.5 & & 39.4 & 0.4 & 0.0 & 0.3 & 2.0 & 1.6 & 2.0 & 0.0 & 0.8 & & \\
\hline Brouns, 1988a & & & 12.0 & 10.3 & 0.1 & & 0.5 & 0.4 & & 0.1 & 0.1 & & 15.0 & 1.7 & 2.4 & 0.4 & 2.5 & 0.0 & 10.6 & 0.2 & & 0.1 & 3.5 & & 36.5 & 0.1 & 0.0 & 0.1 & 2.0 & 0.5 & 2.0 & 0.1 & 0.2 & & \\
\hline Brouns, 1988a & & & 12.0 & 11.8 & 0.1 & & 0.5 & 1.3 & & 0.3 & 0.0 & & 15.0 & 1.4 & 1.9 & 0.4 & 2.5 & 0.1 & 8.5 & 0.6 & & 0.3 & 1.5 & & 36.3 & 0.4 & 0.0 & 0.3 & 2.0 & 1.5 & 0.2 & 0.0 & 0.7 & & \\
\hline Brouns, 1988a & & & 9.4 & 12.5 & 0.1 & & 0.5 & 0.4 & & 0.1 & & & 14.2 & 1.9 & 1.8 & 0.5 & 0.4 & 0.0 & 8.2 & 0.2 & & 0.1 & 3.3 & & 39.3 & 0.1 & 0.0 & 0.1 & 5.0 & 0.5 & 1.0 & 0.0 & 0.2 & & \\
\hline Brouns, 1988a & & & 12.3 & 13.2 & 0.1 & & 0.5 & 0.4 & & 0.1 & 0.0 & & 8.7 & 2.5 & 2.1 & 0.6 & 2.0 & 0.0 & 9.6 & 0.2 & & 0.1 & 1.4 & & 42.1 & 0.1 & 0.0 & 0.1 & 1.9 & 0.5 & 1.0 & 0.0 & 0.2 & & \\
\hline Brouns, 1988a & & & 9.3 & 10.9 & 0.1 & & 0.5 & 0.4 & & 0.1 & 0.0 & & 8.7 & 2.0 & 2.0 & 0.5 & 2.0 & 0.0 & 9.1 & 0.2 & & 0.1 & 3.1 & & 45.0 & 0.1 & 0.0 & 0.1 & 4.4 & 0.5 & 0.1 & & 0.2 & & \\
\hline Brouns, 1988a & & & 12.3 & 13.4 & 0.3 & & 1.3 & 0.4 & & 0.1 & 0.0 & & 8.7 & 2.6 & 2.0 & 1.4 & 0.5 & 0.0 & 9.1 & 0.2 & & 0.1 & 1.6 & & 41.0 & 0.1 & 0.0 & 0.1 & 3.8 & 0.5 & 0.1 & 0.0 & 0.3 & & \\
\hline Brouns, 1988a & & & 12.2 & 12.6 & 0.1 & & 0.5 & 1.4 & & 0.3 & 0.1 & & 8.7 & 2.5 & 2.3 & 0.6 & 0.4 & 0.1 & 10.1 & 0.6 & & 0.3 & 3.5 & & 36.7 & 0.5 & 0.0 & 0.4 & 3.5 & 1.7 & 0.1 & 0.1 & 0.8 & & \\
\hline Brouns, 1988a & & & 9.7 & 10.7 & 0.1 & & 0.5 & 1.3 & & 0.3 & 0.1 & & 12.1 & 1.4 & 2.1 & 0.6 & 0.4 & 0.1 & 8.9 & 0.6 & & 0.3 & 1.5 & & 43.4 & 0.4 & 0.0 & 0.4 & 1.7 & 1.6 & 1.0 & 0.1 & 0.8 & & \\
\hline Brouns, 1988a & & & 9.3 & 11.5 & 0.3 & & 1.3 & 0.4 & & 0.1 & 0.0 & & 8.7 & 1.8 & 2.6 & 1.4 & 0.4 & 0.0 & 10.7 & 0.2 & & 0.1 & 3.3 & & 44.7 & 0.1 & 0.0 & 0.1 & 1.9 & 0.5 & 0.1 & 0.0 & 0.2 & & \\
\hline Brouns, 1988a & & & 10.1 & 11.9 & 0.2 & & 0.8 & 0.8 & & 0.2 & 0.0 & & 11.5 & 1.8 & 2.1 & 1.0 & 1.0 & 0.0 & 9.6 & 0.4 & & 0.2 & 2.4 & & 40.1 & 0.3 & 0.0 & 0.2 & 3.3 & 0.9 & 0.6 & 0.0 & 0.5 & & \\
\hline Brown, 2001 & 1150 & & 6.5 & 6.4 & & & 1.3 & & & 0.0 & & & 13.6 & 3.1 & 3.3 & 0.5 & 3.3 & & 8.8 & 1.1 & & & & & 49.0 & & & & & 1.3 & & & 0.1 & 1096 & \\
\hline Brown, 2001 & 1150 & & 14.1 & 11.4 & & & 0.4 & & & 0.0 & & & 4.1 & 0.1 & 4.2 & 1.4 & 2.7 & & 14.9 & 0.6 & & & & & 44.6 & & & & & 0.8 & & & 0.8 & 863 & \\
\hline Brown, 2001 & 1150 & & 14.1 & 11.4 & & & 0.4 & & & 0.0 & & & 4.1 & 0.1 & 4.2 & 1.4 & 2.7 & & 14.9 & 0.6 & & & & & 44.6 & & & & & 0.8 & & & 0.8 & 835 & \\
\hline Brown, 2001 & 1150 & & 13.5 & 10.9 & & & 0.4 & & & & & & 4.7 & & 4.1 & 1.4 & 2.5 & & 14.1 & 0.6 & & & & & 46.4 & & & & & 0.7 & & & 0.7 & 990 & \\
\hline Brown, 2001 & 1150 & & 4.3 & 7.7 & & & 0.8 & & & 0.0 & & & 11.5 & 0.1 & 4.4 & 0.7 & 2.8 & & 10.4 & 1.0 & & & & & 54.7 & & & & & 0.1 & & & 1.3 & 995 & \\
\hline Brown, 2001 & 1150 & & 2.3 & 7.3 & & & 1.0 & & & & & & 17.0 & & 4.1 & 0.7 & 0.9 & & 10.9 & 2.6 & & & & & 51.4 & & & & & 0.1 & & & 0.9 & 1035 & \\
\hline Brown, 2001 & 1150 & & 4.4 & 7.6 & & & 0.8 & & & & & & 11.9 & & 4.3 & 0.7 & 2.6 & & 9.9 & 1.0 & & & & & 55.0 & & & & & 0.1 & & & 0.9 & 996 & \\
\hline Brown, 2001 & 1150 & & 1.0 & 7.5 & & & 1.1 & & & 0.0 & & & 17.9 & 0.1 & 4.2 & 0.7 & 1.1 & & 11.1 & 2.9 & & & & & 51.1 & & & & & 0.1 & & & 1.2 & 1075 & \\
\hline Brown, 2001 & 1150 & & 2.3 & 7.3 & & & 1.0 & & & & & & 17.0 & & 4.1 & 0.7 & 0.9 & & 10.9 & 2.6 & & & & & 51.4 & & & & & 0.1 & & & 0.9 & 1035 & \\
\hline Brown, 2001 & 1150 & & 2.3 & 7.3 & & & 1.0 & & & & & & 17.0 & & 4.1 & 0.7 & 0.9 & & 10.9 & 2.6 & & & & & 51.4 & & & & & 0.1 & & & 0.9 & 1109 & \\
\hline Brown, 2001 & 1150 & & 2.3 & 7.3 & & & 1.0 & & & & & & 17.0 & & 4.1 & 0.7 & 0.9 & & 10.9 & 2.6 & & & & & 51.4 & & & & & 0.1 & & & 0.9 & 1107 & \\
\hline Brown, 2001 & 1150 & & 6.4 & 7.2 & & & 1.3 & & & 0.1 & & & 13.4 & 3.1 & 4.1 & 1.0 & 3.2 & & 6.5 & 1.1 & & & & & 50.2 & & & & & 1.3 & & & 0.1 & 1111 & \\
\hline Brown, 2001 & 1150 & & 13.3 & 7.6 & & & 0.5 & & & & & & 4.2 & 0.1 & 4.3 & 0.7 & 2.8 & & 11.1 & 0.6 & & & & & 52.7 & & & & & 0.1 & & & 1.3 & 863 & \\
\hline Brown, 2001 & 1150 & & 13.3 & 7.6 & & & 0.5 & & & & & & 4.2 & 0.1 & 4.3 & 0.7 & 2.8 & & 11.1 & 0.6 & & & & & 52.7 & & & & & 0.1 & & & 1.3 & 946 & \\
\hline Brown, 2001 & 1150 & & 13.4 & 7.3 & & & 0.5 & & & & & & 4.8 & & 4.2 & 0.7 & 2.6 & & 10.6 & 0.7 & & & & & 53.6 & & & & & & & & 0.8 & 840 & \\
\hline Brown, 2001 & 1150 & & 5.3 & 7.3 & & & 0.7 & & & 0.1 & & & 12.1 & 0.1 & 5.1 & 0.7 & 2.8 & & 10.3 & 1.1 & & & & & 53.1 & & & & & 0.2 & & & 0.7 & 917 & \\
\hline Brown, 2001 & 1150 & & 5.2 & 6.6 & & & 1.0 & & & & & & 11.6 & & 5.0 & 0.7 & 2.6 & & 10.0 & 1.0 & & & & & 55.3 & & & & & & & & 0.8 & 1000 & \\
\hline Brown, 2001 & 1150 & & 5.1 & 7.3 & & & 0.9 & & & 0.0 & & & 12.0 & 0.1 & 5.1 & 0.7 & 2.8 & & 10.2 & 1.0 & & & & & 53.3 & & & & & 0.1 & & & 0.8 & 1006 & \\
\hline Brown, 2001 & 1150 & & 1.4 & 7.3 & & & 1.4 & & & & & & 16.4 & & 4.1 & 0.7 & 1.1 & & 10.7 & 3.0 & & & & & 52.0 & & & & & & & & 0.9 & 1015 & \\
\hline Brown, 2001 & 1150 & & 1.5 & \begin{tabular}{|l|l|}
7.4 & \\
\end{tabular} & & & 1.4 & & & & & & $\begin{array}{l}16.5 \\
\end{array}$ & 0.0 & 4.3 & 0.6 & 1.0 & & 11.2 & 2.6 & & & & & 49.6 & & & & & & & & 0.9 & 1135 & \\
\hline Brown, 2001 & 1150 & & 1.3 & 7.5 & & & 1.5 & & & 0.0 & & & 16.4 & 0.0 & 4.2 & 0.7 & 1.1 & & 11.2 & 3.1 & & & & & 51.7 & & & & & 0.1 & & & 1.3 & 1102 & \\
\hline Brown, 2001 & 1150 & & 1.3 & 7.5 & & & 1.5 & & & 0.0 & & & 16.4 & 0.0 & 4.2 & 0.7 & 1.1 & & 11.2 & 3.1 & & & & & 51.7 & & & & & 0.1 & & & 1.3 & 920 & \\
\hline Brown, 2001 & 1150 & & 1.4 & 7.3 & & & 1.4 & & & & & & 16.4 & & 4.1 & 0.7 & 1.1 & & 10.7 & 3.0 & & & & & 52.0 & & & & & & & & 0.9 & 1085 & \\
\hline Brown, 2001 & 1150 & & 1.4 & 7.3 & & & 1.4 & & & & & & 16.4 & & 4.1 & 0.7 & 1.1 & & 10.7 & 3.0 & & & & & 52.0 & & & & & & & & 0.9 & 1086 & \\
\hline Brown, 2001 & 1150 & & 1.4 & 7.3 & & & 1.4 & & & & & & 16.4 & & 4.1 & 0.7 & 1.1 & & 10.7 & 3.0 & & & & & 52.0 & & & & & & & & 0.9 & 1113 & \\
\hline Brown, 2001 & 1150 & & 14.2 & 12.1 & & & 0.4 & & & 0.0 & & & 3.5 & 0.2 & 4.2 & 0.7 & 2.7 & & 10.4 & 0.5 & & & & & 47.7 & & & & & 0.1 & & & 0.7 & 846 & \\
\hline Brown, 2001 & 1150 & & 6.7 & 13.7 & & & 0.1 & & & & & & 2.5 & & 5.3 & 0.9 & 1.1 & & 11.8 & 0.3 & & & & & 56.4 & & & & & & & & 0.9 & 720 & \\
\hline Brown, 2001 & 1150 & & 5.3 & 12.7 & & & 0.7 & & & 0.0 & & & 11.5 & 0.1 & 4.3 & 0.7 & 2.7 & & 10.3 & 1.0 & & & & & 50.4 & & & & & 0.1 & & & 0.8 & 1014 & \\
\hline Brown, 2001 & 1150 & & 5.3 & 12.7 & & & 0.7 & & & 0.0 & & & 11.5 & 0.1 & 4.3 & 0.7 & 2.7 & & 10.3 & 1.0 & & & & & 50.4 & & & & & 0.1 & & & 0.8 & 925 & \\
\hline Brown, 2001 & 1150 & & 5.6 & 10.6 & & & 0.7 & & & & & & 11.1 & & 4.2 & 0.7 & 2.6 & & 10.1 & 1.0 & & & & & 51.6 & & & & & & & & 0.7 & 990 & \\
\hline Brown, 2001 & 1150 & & 5.6 & 10.6 & & & 0.7 & & & & & & 11.1 & & 4.2 & 0.7 & 2.6 & & 10.1 & 1.0 & & & & & 51.6 & & & & & & & & 0.7 & 980 & \\
\hline Brown, 2001 & 1150 & & 5.3 & 12.7 & & & 0.7 & & & 0.0 & & & 11.5 & 0.1 & 4.3 & 0.7 & 2.7 & & 10.3 & 1.0 & & & & & 50.4 & & & & & 0.1 & & & 0.8 & 967 & \\
\hline Brown, 2001 & 1150 & & 5.3 & 12.7 & & & 0.7 & & & 0.0 & & & 11.5 & 0.1 & 4.3 & 0.7 & 2.7 & & 10.3 & 1.0 & & & & & 50.4 & & & & & 0.1 & & & 0.8 & 971 & \\
\hline Brown, 2001 & 1150 & & 3.3 & 12.0 & & & 1.3 & & & & & & 17.4 & 0.0 & 4.0 & 0.7 & 1.0 & & 13.8 & 2.7 & & & & & 42.5 & & & & & & & & 0.7 & 1130 & \\
\hline Brown, 2001 & 1150 & & 1.4 & 11.7 & & & 1.1 & & & 0.0 & & & 13.1 & 0.1 & 4.2 & 0.7 & 0.7 & & 11.2 & 2.8 & & & & & 53.1 & & & & & 0.0 & & & 0.7 & 1022 & \\
\hline Brown, 2001 & 1150 & & 2.5 & 11.4 & & & 1.4 & & & & & & 15.6 & & 4.1 & 0.7 & 1.0 & & 10.8 & 2.8 & & & & & 48.3 & & & & & & & & 0.7 & 1085 & \\
\hline
\end{tabular}


ORP-56297 Rev. 0

The Catholic University of America

High Level Waste Vitrification System Improvements Vitreous State Laboratory

Table A1. Glass Compositions from Literature Review (continued)

\begin{tabular}{|c|c|c|c|c|c|c|c|c|c|c|c|c|c|c|c|c|c|c|c|c|c|c|c|c|c|c|c|c|c|c|c|c|c|c|c|}
\hline Ref & 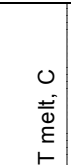 & $\begin{array}{l}0 \\
\stackrel{0}{a} \\
\stackrel{0}{0} \\
上\end{array}$ & 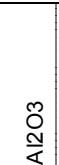 & ్ֻలె & 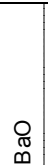 & Oొָ & $\begin{array}{l}0 \\
\mathbb{J} \\
0\end{array}$ & 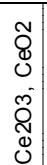 & $\begin{array}{l}\text { Oొ } \\
\text { Oे } \\
0 \\
0 \\
0 \\
0\end{array}$ & $\begin{array}{l}\widetilde{D} \\
\text { N }\end{array}$ & $\stackrel{\circ}{3}$ & 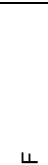 & 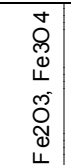 & \& & $\stackrel{ }{N}$ & $\stackrel{\circ}{\circ}$ & $\begin{array}{l}\tilde{O} \\
\text { L } \\
\Sigma\end{array}$ & $\begin{array}{l}\text { Oొ } \\
\text { ¿ }\end{array}$ & $\begin{array}{l}\stackrel{\mathbb{N}}{\pi} \\
\underset{Z}{Z}\end{array}$ & $\frac{O}{z}$ & $\begin{array}{c}\mathcal{N} \\
\tilde{0}\end{array}$ & $\overline{\overline{\bar{c}}}$ & $\begin{array}{l}\text { مి } \\
\text { م) }\end{array}$ & 8 & $\frac{\tilde{O}}{\omega}$ & Oొ & 온 & 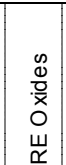 & 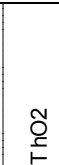 & $\stackrel{\widetilde{O}}{\underline{\underline{O}}}$ & $\begin{array}{l}\text { o̊ } \\
\text { ગ } \\
\text { دิ } \\
\text { రิ }\end{array}$ & $\stackrel{q}{N}$ & ญั & 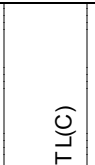 & 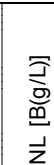 \\
\hline Brown, 2001 & 1150 & & 2.5 & 11.4 & & & 1.4 & & & & & & 15.6 & & $\overrightarrow{4.1}$ & 0.7 & 1.0 & & 10.8 & 2.8 & & & & & 48.3 & & & & & & & & 0.7 & 900 & \\
\hline Brown, 2001 & 1150 & & 13.9 & 10.3 & & & 0.6 & & & 0.0 & & & 4.0 & 3.3 & 2.6 & 1.3 & 2.6 & & 10.9 & 0.6 & & & & & 47.7 & & & & & 1.8 & & & 0.0 & 929 & \\
\hline Brown, 2001 & 1150 & & 13.4 & 10.2 & & & 0.5 & & & & & & 4.5 & 3.1 & 2.7 & 1.3 & 2.5 & & 10.6 & 0.6 & & & & & 48.4 & & & & & 1.7 & & & 0.0 & 845 & \\
\hline Brown, 2001 & 1150 & & 5.2 & 10.2 & & & 0.9 & & & 0.0 & & & 11.3 & 3.2 & 2.7 & 1.3 & 2.8 & & 10.1 & 1.0 & & & & & 49.2 & & & & & 1.6 & & & 0.1 & 997 & \\
\hline Brown, 2001 & 1150 & & 5.1 & 10.3 & & & 0.6 & & & & & & 11.9 & 3.2 & 2.7 & 1.2 & 2.6 & & 9.8 & 1.0 & & & & & 49.5 & & & & & 1.4 & & & 0.0 & 985 & \\
\hline Brown, 2001 & 1150 & & 5.9 & 10.1 & & & 0.7 & & & 0.0 & & & 11.6 & 3.1 & 3.2 & 1.2 & 2.7 & & 9.8 & 1.0 & & & & & 49.0 & & & & & 1.3 & & & 0.1 & 996 & \\
\hline Brown, 2001 & 1150 & & 2.1 & 10.1 & & & 0.9 & & & & & & 15.7 & 3.2 & 2.6 & 1.2 & 1.0 & & 10.6 & 2.6 & & & & & 47.4 & & & & & 1.8 & & & 0.0 & 1070 & \\
\hline Brown, 2001 & 1150 & & 1.4 & 9.9 & & & 1.5 & & & & & & 16.6 & 3.2 & 2.6 & 1.3 & 1.0 & & 11.0 & 2.8 & & & & & 46.5 & & & & & 1.7 & & & 0.0 & 895 & \\
\hline Brown, 2001 & 1150 & & 1.4 & 10.4 & & & 1.0 & & & 0.0 & & & 15.9 & 3.3 & 2.5 & 1.3 & 1.0 & & 11.0 & 2.7 & & & & & 47.4 & & & & & 1.9 & & & 0.1 & 1126 & \\
\hline Brown, 2001 & 1150 & & 2.1 & 10.1 & & & 0.9 & & & & & & 15.7 & 3.2 & 2.6 & 1.2 & 1.0 & & 10.6 & 2.6 & & & & & 47.4 & & & & & 1.8 & & & 0.0 & 1065 & \\
\hline Brown, 2001 & 1150 & & 2.1 & 10.1 & & & 0.9 & & & & & & 15.7 & 3.2 & 2.6 & 1.2 & 1.0 & & 10.6 & 2.6 & & & & & 47.4 & & & & & 1.8 & & & 0.0 & 1113 & \\
\hline Brown, 2001 & 1150 & & 2.1 & 10.1 & & & 0.9 & & & & & & 15.7 & 3.2 & 2.6 & 1.2 & 1.0 & & 10.6 & 2.6 & & & & & 47.4 & & & & & 1.8 & & & 0.0 & 1062 & \\
\hline Brown, 2001 & 1150 & & 13.7 & 7.5 & & & 0.4 & & & 0.0 & & & 3.9 & 3.5 & 4.3 & 1.3 & 2.6 & & 7.6 & 0.6 & & & & & 52.2 & & & & & 1.8 & & & 0.1 & 959 & \\
\hline Brown, 2001 & 1150 & & 13.9 & 7.4 & & & 0.4 & & & & & & 4.4 & 3.3 & 4.2 & 1.3 & 2.5 & & 7.3 & 0.6 & & & & & 52.4 & & & & & 1.7 & & & 0.0 & 965 & \\
\hline Brown, 2001 & 1150 & & 5.1 & 7.6 & & & 0.7 & & & 0.0 & & & 11.3 & 3.1 & 4.4 & 1.1 & 2.7 & & 6.8 & 1.0 & & & & & 54.2 & & & & & 1.3 & & & 0.1 & 965 & \\
\hline Brown, 2001 & 1150 & & 5.0 & 7.6 & & & 0.7 & & & 0.0 & & & 11.7 & 3.5 & 4.4 & 1.3 & 2.7 & & 6.8 & 1.0 & & & & & 53.3 & & & & & 1.4 & & & 0.1 & 967 & \\
\hline Brown, 2001 & 1150 & & 5.0 & 7.4 & & & 0.7 & & & & & & 11.9 & 3.3 & 4.3 & 1.3 & 2.6 & & 6.6 & 1.0 & & & & & 54.1 & & & & & 1.4 & & & 0.0 & 1010 & \\
\hline Brown, 2001 & 1150 & & 1.4 & 7.1 & & & 1.0 & & & & & & 15.8 & 3.3 & 4.3 & 1.3 & 1.0 & & 7.6 & 2.7 & & & & & 52.5 & & & & & 1.7 & & & 0.0 & 1160 & \\
\hline Brown, 2001 & 1150 & & 1.0 & 7.3 & & & 1.4 & & & & & & 15.9 & 3.3 & 4.4 & 1.3 & 1.0 & & 7.8 & 2.7 & & & & & 50.1 & & & & & 1.8 & & & 0.0 & 1100 & \\
\hline Brown, 2001 & 1150 & & 1.4 & 7.3 & & & 1.0 & & & 0.0 & & & 16.7 & 3.5 & 4.2 & 1.3 & 1.0 & & 7.7 & 2.9 & & & & & 51.0 & & & & & 1.8 & & & 0.1 & 1123 & \\
\hline Brown, 2001 & 1150 & & 1.4 & 7.1 & & & 1.0 & & & & & & 15.8 & 3.3 & 4.3 & 1.3 & 1.0 & & 7.6 & 2.7 & & & & & 52.5 & & & & & 1.7 & & & 0.0 & 1110 & \\
\hline Brown, 2001 & 1150 & & 1.4 & 7.1 & & & 1.0 & & & & & & 15.8 & 3.3 & 4.3 & 1.3 & 1.0 & & 7.6 & 2.7 & & & & & 52.5 & & & & & 1.7 & & & 0.0 & 1118 & \\
\hline Brown, 2001 & 1150 & & 1.4 & 7.1 & & & 1.0 & & & & & & 15.8 & 3.3 & 4.3 & 1.3 & 1.0 & & 7.6 & 2.7 & & & & & 52.5 & & & & & 1.7 & & & 0.0 & 1127 & \\
\hline Brown, 2001 & 1150 & & 5.5 & 7.0 & & & 0.7 & & & 0.0 & & & 11.4 & 3.2 & 3.8 & 0.6 & 2.6 & & 9.2 & 1.0 & & & & & 53.1 & & & & & 1.3 & & & 0.0 & 991 & \\
\hline Brown, 2001 & 1150 & & 6.0 & 8.8 & & & 0.7 & & & 0.0 & & & 11.6 & 3.1 & 3.5 & 0.6 & 2.6 & & 9.2 & 1.0 & & & & & 50.9 & & & & & 1.3 & & & 0.1 & 1000 & \\
\hline Brown, 2001 & 1150 & & 6.0 & 8.8 & & & 0.7 & & & 0.0 & & & 11.6 & 3.1 & 3.5 & 0.6 & 2.6 & & 9.2 & 1.0 & & & & & 50.9 & & & & & 1.3 & & & 0.1 & 778 & \\
\hline Brown, 2001 & 1150 & & 4.6 & 6.8 & & & 1.6 & & & & & & 11.6 & 0.1 & 4.9 & 0.8 & 2.0 & & 11.2 & 0.8 & & & & & 54.4 & & & & & 0.2 & & & 0.8 & 909 & \\
\hline Brown, 2001 & 1150 & & 4.6 & 8.5 & & & 1.5 & & & 0.1 & & & 14.1 & 2.7 & 3.2 & 0.9 & 1.9 & & 11.5 & 1.1 & & & & & 48.1 & & & & & 1.2 & & & 0.3 & 1066 & \\
\hline Brown, 2001 & 1150 & & 4.5 & 8.4 & & & 1.5 & & & 0.1 & & & 14.3 & 2.7 & 3.2 & 0.9 & 2.0 & & 11.5 & 1.1 & & & & & 48.0 & & & & & 1.2 & & & 0.2 & 997 & \\
\hline Brown, 2001 & 1150 & & 4.7 & 8.7 & & & 1.4 & & & 0.1 & & & 14.2 & 2.7 & 3.3 & 0.8 & 1.9 & & 11.6 & 1.1 & & & & & 47.6 & & & & & 1.2 & & & 0.2 & 1062 & \\
\hline Brown, 2001 & 1150 & & 4.5 & 8.4 & & & 1.5 & & & 0.1 & & & 14.3 & 2.7 & 3.2 & 0.9 & 2.0 & & 11.5 & 1.1 & & & & & 48.0 & & & & & 1.2 & & & 0.2 & 1012 & \\
\hline Brown, 2001 & 1150 & & & 8.5 & & & & & & & & & & & 6.9 & 1.0 & & & 12.8 & & & & & & 70.1 & & & & & & & & 0.8 & 732 & \\
\hline Brown, 2001 & 1107 & & 2.5 & 10.2 & & & 2.0 & & & 0.1 & & & 14.1 & 3.8 & 5.9 & 0.5 & 1.0 & & 6.2 & 2.1 & & & & & 42.7 & & & & & 0.7 & 4.5 & & & 1124 & \\
\hline Brown, 2001 & 1250 & & 2.6 & 5.1 & & & 1.9 & & & 0.0 & & & 6.0 & \begin{tabular}{|l|}
3.8 \\
\end{tabular} & 5.9 & 2.4 & 3.0 & & 11.0 & 0.1 & & & & & 57.8 & & & & & 0.2 & & & & 775 & \\
\hline Brown, 2001 & 1250 & & 4.0 & 9.4 & & & 1.5 & & & 0.2 & & & 11.8 & 2.1 & 3.4 & 1.9 & 2.4 & & 9.9 & 1.6 & & & & & 46.6 & & & & & 0.3 & 3.5 & & & 1164 & \\
\hline Brown, 2001 & 1300 & & 8.3 & 4.9 & & & 0.3 & & & 0.1 & & & 14.7 & 1.5 & 6.0 & 2.6 & 1.0 & & 6.2 & 2.1 & & & & & 51.8 & & & & & 0.2 & 0.3 & & & 1261 & \\
\hline Brown, 2001 & 1300 & & 5.6 & 7.7 & & & 1.2 & & & 0.2 & & & 10.6 & 2.7 & 4.4 & 1.6 & 2.0 & & 8.5 & 1.1 & & & & & 52.3 & & & & & 0.4 & 2.5 & & & 1084 & \\
\hline Brown, 2001 & 1284 & & 5.6 & 7.8 & & & 1.1 & & & 0.2 & & & 10.4 & 2.5 & 4.0 & 1.4 & 2.0 & & 8.6 & 1.1 & & & & & 51.1 & & & & & 0.4 & 2.4 & & & 1082 & \\
\hline Brown, 2001 & 1322 & & 7.9 & 5.0 & & & 2.0 & & & 0.1 & & & 14.3 & 3.8 & 3.0 & 0.5 & 1.0 & & 11.0 & 0.1 & & & & & 47.9 & & & & & 0.7 & 0.3 & & & 911 & \\
\hline Brown, 2001 & 1294 & & 8.1 & 10.6 & & & 0.3 & & & 0.3 & & & 5.8 & 3.6 & 5.4 & 2.3 & 2.9 & & 6.0 & 0.1 & & & & & 53.3 & & & & & 0.2 & 0.3 & & & 950 & \\
\hline Brown, 2001 & 1330 & & 4.1 & 6.5 & & & 1.6 & & & 0.1 & & & 12.5 & 3.2 & 3.4 & 2.1 & 2.4 & & 7.5 & 0.6 & & & & & 54.3 & & & & & 0.3 & 1.5 & & & 1114 & \\
\hline Brown, 2001 & 1200 & & 8.2 & 10.1 & & & 2.0 & & & 0.3 & & & 14.8 & 1.5 & 5.8 & 0.5 & 1.0 & & 6.3 & 0.1 & & & & & 44.0 & & & & & 0.2 & 4.8 & & & 1173 & \\
\hline Brown, 2001 & 1275 & & 4.0 & 6.7 & & & 0.8 & & & 0.3 & & & 8.2 & 3.2 & 5.3 & 2.0 & 2.5 & & 7.5 & 1.6 & & & & & 54.2 & & & & & 0.3 & 3.6 & & & 1098 & \\
\hline Brown, 2001 & 1264 & & 3.9 & 9.5 & & & 0.8 & & & 0.1 & & & 8.1 & 2.1 & 5.1 & 1.9 & 1.5 & & 9.7 & 0.6 & & & & & 53.5 & & & & & 0.3 & 1.5 & & & 895 & \\
\hline Brown, 2001 & 1384 & & 2.6 & 5.0 & & & 0.3 & & & 0.3 & & & 14.7 & 1.5 & 3.0 & 2.5 & 1.0 & & 11.1 & 0.0 & & & & & 56.4 & & & & & 0.2 & 0.3 & & & 1030 & \\
\hline Brown, 2001 & 1400 & & 2.6 & 9.8 & & & 0.3 & & & 0.3 & & & 8.4 & 1.5 & 5.9 & 0.5 & 2.9 & & 6.0 & 2.1 & & & & & 56.7 & & & & & 0.2 & 0.3 & & & 1063 & \\
\hline Brown, 2001 & 1160 & & 2.7 & 11.0 & & & 0.3 & & & 0.1 & & & 15.1 & 3.7 & 2.7 & 2.6 & 2.9 & & 11.3 & 0.1 & & & & & 43.3 & & & & & 0.2 & 5.1 & & & 951 & \\
\hline Brown, 2001 & 1285 & & 2.5 & 10.1 & & & 1.9 & & & 0.1 & & & 5.9 & 1.5 & 6.0 & 2.5 & 0.9 & & 6.1 & 2.1 & & & & & 55.9 & & & & & 0.6 & 0.7 & & & 935 & \\
\hline Brown, 2001 & 1250 & & 6.9 & 6.3 & & & 1.6 & & & 0.1 & & & 8.4 & 2.1 & 5.2 & 2.0 & 2.4 & & 9.9 & 0.6 & & & & & 50.1 & & & & & 0.5 & 3.7 & & & 995 & \\
\hline Brown, 2001 & 1160 & & 4.0 & 7.9 & & & 1.6 & & & 0.1 & & & 12.4 & 3.2 & 5.3 & 1.0 & 1.5 & & 10.0 & 1.6 & & & & & 45.7 & & & & & 0.5 & 3.6 & & & 1075 & \\
\hline
\end{tabular}


ORP-56297 Rev. 0

The Catholic University of America

High Level Waste Vitrification System Improvements Vitreous State Laboratory

Table A1. Glass Compositions from Literature Review (continued)

\begin{tabular}{|c|c|c|c|c|c|c|c|c|c|c|c|c|c|c|c|c|c|c|c|c|c|c|c|c|c|c|c|c|c|c|c|c|c|c|c|}
\hline Ref & 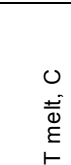 & $\begin{array}{l}0 \\
0 \\
\stackrel{0}{0} \\
\stackrel{0}{0} \\
\vdash\end{array}$ & 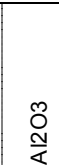 & 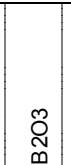 & $\begin{array}{l}\mathscr{D} \\
\infty \\
\infty\end{array}$ & Oొָ & $\begin{array}{l}0 \\
\widetilde{\pi} \\
0\end{array}$ & 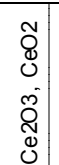 & 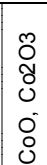 & $\begin{array}{l}\text { Oొ } \\
\text { Un }\end{array}$ & $\stackrel{\circ}{3}$ & ч & 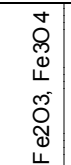 & \& & $\stackrel{?}{\cong}$ & $\begin{array}{l}O \\
\text { D } \\
\Sigma\end{array}$ & $\begin{array}{l}\text { Õ } \\
\text { ¿ }\end{array}$ & $\begin{array}{l}0 \\
0 \\
\Sigma\end{array}$ & $\begin{array}{l}\text { R } \\
\underset{Z}{Z}\end{array}$ & $\frac{O}{z}$ & $\begin{array}{l}\mathcal{N} \\
\text { Oे }\end{array}$ & 空 & 足 & $\frac{8}{0}$ & $\stackrel{\tilde{O}}{\infty}$ & Oొ & $\begin{array}{l}\frac{9}{i n} \\
\text { in }\end{array}$ & 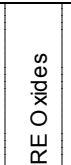 & $\begin{array}{l}\text { ญิ } \\
\text { \&ิ }\end{array}$ & $\underline{\tilde{N}}$ & 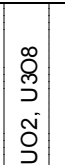 & $\frac{q}{N}$ & 饮 & 产 & $\begin{array}{l}\bar{\Xi} \\
\text { o } \\
\underline{\underline{U}} \\
z\end{array}$ \\
\hline Brown, 2001 & 1142 & & 2.5 & 10.4 & & & 0.3 & & & 0.3 & & & 14.4 & 1.5 & 5.9 & 0.5 & 2.8 & & 10.9 & 0.0 & & & & & 46.8 & & & & & 0.6 & 0.3 & & & 859 & \\
\hline Brown, 2001 & 1142 & & 2.7 & 10.3 & & & 0.3 & & & 0.3 & & & 14.7 & 1.5 & 5.9 & 0.5 & 2.9 & & 10.9 & 0.0 & & & & & 47.8 & & & & & 0.6 & 0.3 & & & 869 & \\
\hline Brown, 2001 & 1140 & & 6.6 & 10.3 & & & 0.3 & & & 0.3 & & & 5.8 & 3.7 & 5.9 & 0.5 & 1.0 & & 10.9 & 2.2 & & & & & 44.4 & & & & & 0.2 & 4.7 & & & 929 & \\
\hline Brown, 2001 & 1240 & & 8.3 & 5.0 & & & 2.0 & & & 0.1 & & & 6.1 & 1.5 & 5.9 & 2.6 & 1.0 & & 11.1 & 0.1 & & & & & 51.5 & & & & & 0.6 & 5.0 & & & 799 & \\
\hline Brown, 2001 & 1284 & & 4.0 & 8.9 & & & 1.6 & & & 0.2 & & & 7.9 & 2.0 & 5.2 & 1.0 & 2.3 & & 7.2 & 1.6 & & & & & 53.4 & & & & & 0.5 & 1.6 & & & 987 & \\
\hline Brown, 2001 & 1246 & & 6.9 & 6.5 & & & 1.5 & & & 0.3 & & & 12.7 & 2.1 & 5.2 & 1.0 & 1.5 & & 9.8 & 1.6 & & & & & 50.0 & & & & & 0.3 & 1.6 & & & 1145 & \\
\hline Brown, 2001 & 1304 & & 4.3 & 6.5 & & & 1.6 & & & 0.3 & & & 8.0 & 3.1 & 3.4 & 1.9 & 1.5 & & 9.9 & 1.6 & & & & & 53.5 & & & & & 0.5 & 1.5 & & & 1069 & \\
\hline Brown, 2001 & 1345 & & 2.6 & 5.2 & & & 0.3 & & & 0.1 & & & 11.8 & 1.5 & 5.8 & 0.5 & 1.0 & & 6.1 & 0.1 & & & & & 58.5 & & & & & 0.7 & 4.7 & & & 995 & \\
\hline Brown, 2001 & 1333 & & 7.9 & 11.5 & & & 0.4 & & & 0.1 & & & 14.4 & 3.7 & 2.7 & 2.4 & 1.0 & & 6.6 & 2.1 & & & & & 47.1 & & & & & 0.2 & 0.3 & & & 1310 & \\
\hline Brown, 2001 & 1304 & & 4.1 & 6.7 & & & 0.8 & & & 0.2 & & & 12.5 & 2.1 & 3.8 & 1.0 & 1.5 & & 10.1 & 0.6 & & & & & 52.3 & & & & & 0.5 & 3.7 & & & 1071 & \\
\hline Brown, 2001 & 1218 & & 7.0 & 9.4 & & & 1.5 & & & 0.3 & & & 11.1 & 3.3 & 5.1 & 2.0 & 1.5 & & 7.4 & 0.6 & & & & & 47.2 & & & & & 0.3 & 3.6 & & & 1086 & \\
\hline Brown, 2001 & 1150 & & 2.6 & 10.5 & & & 2.0 & & & 0.1 & & & 14.7 & 1.5 & 6.0 & 0.5 & 1.0 & & 11.2 & 0.1 & & & & & 49.5 & & & & & 0.2 & 0.3 & & & 833 & \\
\hline Brown, 2001 & 1280 & & 8.1 & 5.2 & & & 0.3 & & & 0.1 & & & 5.8 & 1.5 & 6.2 & 0.5 & 2.9 & & 11.2 & 0.1 & & & & & 51.4 & & & & & 0.7 & 4.7 & & & 811 & \\
\hline Brown, 2001 & 1157 & & 8.0 & 5.1 & & & 1.9 & & & 0.1 & & & 5.9 & 3.7 & 5.4 & 2.4 & 2.9 & & 10.9 & 2.1 & & & & & 44.1 & & & & & 0.2 & 4.5 & & & 1030 & \\
\hline Brown, 2001 & 1149 & & 8.4 & 11.1 & & & 2.0 & & & 0.1 & & & 15.2 & 3.7 & 5.3 & 2.7 & 2.9 & & 6.2 & 0.1 & & & & & 43.1 & & & & & 0.7 & 0.3 & & & 1081 & \\
\hline Brown, 2001 & 1250 & & 8.2 & 10.6 & & & 0.3 & & & 0.1 & & & 14.9 & 1.5 & 6.0 & 0.5 & 1.0 & & 10.9 & 2.1 & & & & & 43.0 & & & & & 0.6 & 0.3 & & & 1132 & \\
\hline Brown, 2001 & 1145 & & 8.4 & 10.4 & & & 2.0 & & & 0.3 & & & 6.2 & 3.8 & 5.9 & 0.5 & 2.9 & & 10.6 & 2.1 & & & & & 47.6 & & & & & 0.6 & 0.3 & & & 943 & \\
\hline Brown, 2001 & 1320 & & 8.3 & 9.6 & & & 2.0 & & & 0.3 & & & 14.6 & 1.5 & 3.0 & 2.5 & 2.9 & & 6.4 & 0.1 & & & & & 42.1 & & & & & 0.6 & 4.8 & & & 1282 & \\
\hline Brown, 2001 & 1200 & & 8.1 & 5.3 & & & 0.3 & & & 0.3 & & & 13.9 & 3.7 & 6.1 & 2.4 & 2.9 & & 11.0 & 2.1 & & & & & 41.8 & & & & & 0.7 & 0.3 & & & 1231 & \\
\hline Brown, 2001 & 1265 & & 2.5 & \begin{tabular}{|l|l|}
10.4 \\
\end{tabular} & & & 2.0 & & & 0.3 & & & 5.7 & 3.7 & 3.0 & 0.5 & 1.0 & & 11.0 & 0.1 & & & & & 51.4 & & & & & 0.2 & 4.4 & & & 813 & \\
\hline Brown, 2001 & 1268 & & 2.6 & 10.3 & & & 2.0 & & & 0.3 & & & 5.8 & 3.8 & 5.9 & 2.4 & 1.0 & & 6.0 & 0.3 & & & & & 58.2 & & & & & 0.7 & 0.3 & & & 944 & \\
\hline Brown, 2001 & 1151 & & 2.7 & 11.1 & & & 0.3 & & & 0.1 & & & 14.7 & 3.7 & 2.7 & 2.6 & 3.0 & & 11.3 & 0.1 & & & & & 43.3 & & & & & 0.7 & 5.1 & & & 897 & \\
\hline Brown, 2001 & 1322 & & 2.6 & 5.4 & & & 2.0 & & & 0.3 & & & 14.4 & 1.5 & 3.0 & 0.5 & 2.9 & & 11.2 & 2.1 & & & & & 52.2 & & & & & 0.6 & 0.3 & & & 1164 & \\
\hline Brown, 2001 & 1300 & & 8.2 & 10.8 & & & 0.3 & & & 0.3 & & & 5.9 & 1.4 & 2.7 & 2.4 & 1.0 & & 11.0 & 2.1 & & & & & 46.9 & & & & & 0.7 & 4.7 & & & 1173 & \\
\hline Brown, 2001 & 1300 & & 8.1 & 11.1 & & & 2.0 & & & 0.1 & & & 14.5 & 1.6 & 2.8 & 0.5 & 2.9 & & 6.5 & 2.0 & & & & & 42.4 & & & & & 0.2 & 4.9 & & & 1304 & \\
\hline Brown, 2001 & 1160 & & 4.6 & 9.2 & & & 0.7 & & & 0.2 & & & 12.2 & 3.2 & 5.1 & 1.9 & 2.4 & & 9.8 & 0.6 & & & & & 46.0 & & & & & 0.5 & 1.6 & & & 990 & \\
\hline Brown, 2001 & 1317 & & 6.8 & 8.8 & & & 0.7 & & & 0.2 & & & 8.1 & 3.2 & $\begin{array}{ll}3.8 \\
\end{array}$ & 1.0 & 2.5 & & 9.7 & 0.6 & & & & & 51.5 & & & & & 0.3 & 1.6 & & & 924 & \\
\hline Brown, 2001 & 1330 & & 7.0 & 9.2 & & & 0.7 & & & 0.2 & & & 12.8 & 2.1 & 3.7 & 2.0 & 1.5 & & 7.6 & 1.6 & & & & & 51.0 & & & & & 0.5 & 1.7 & & & 1244 & \\
\hline Brown, 2001 & 1317 & & 2.6 & 10.6 & & & 2.0 & & & 0.1 & & & 5.8 & 1.5 & 3.0 & 2.4 & 2.9 & & 10.8 & 2.1 & & & & & 55.9 & & & & & 0.2 & 0.3 & & & 936 & \\
\hline Brown, 2001 & 1250 & & 2.7 & 5.2 & & & 0.3 & & & 0.3 & & & 14.8 & 3.9 & 5.9 & 2.5 & 1.0 & & 6.5 & 2.1 & & & & & 49.2 & & & & & 0.6 & 4.9 & & & 1247 & \\
\hline Brown, 2001 & 1193 & & 2.7 & 5.0 & & & 2.0 & & & 0.3 & & & 14.9 & 1.5 & 5.8 & 2.5 & 1.0 & & 11.1 & 2.1 & & & & & 45.6 & & & & & 0.2 & 5.0 & & & 1144 & \\
\hline Brown, 2001 & 1356 & & 2.7 & 9.8 & & & 0.3 & & & 0.1 & & & 5.7 & 3.7 & 3.0 & 0.5 & 1.0 & & 10.7 & 2.1 & & & & & 56.7 & & & & & 0.7 & 0.3 & & & 862 & \\
\hline Brown, 2001 & 1315 & & 2.9 & 5.1 & & & 0.3 & & & 0.1 & & & 8.0 & 3.7 & 5.3 & 0.5 & 2.9 & & 6.2 & 0.1 & & & & & 59.5 & & & & & 0.2 & 4.7 & & & 877 & \\
\hline Brown, 2001 & 1320 & & 2.7 & 5.4 & & & 2.0 & & & 0.3 & & & 14.9 & 3.7 & 3.0 & 0.5 & 2.9 & & 6.3 & 2.1 & & & & & 49.3 & & & & & 0.6 & 4.8 & & & 1285 & \\
\hline Brown, 2001 & 1326 & & 8.0 & 5.2 & & & 2.0 & & & 0.3 & & & 14.8 & 3.7 & 3.0 & 0.5 & 1.0 & & 10.9 & 0.1 & & & & & 48.9 & & & & & 0.2 & 0.3 & & & 1033 & \\
\hline Bulkley, 1997 & 1242 & 1126 & 10.0 & 5.7 & 0.2 & & 4.6 & 4.3 & & 0.4 & 0.0 & 0.1 & 1.5 & 2.8 & 3.8 & 1.9 & & 0.6 & 9.5 & 0.5 & & 5.2 & 0.3 & 1.0 & 42.6 & & & 0.8 & & 1.0 & & & 2.8 & & \\
\hline Cantale, 1991 & & 1060 & 13.8 & 9.7 & 0.1 & & 3.0 & & & & & & 4.4 & & 4.7 & 0.3 & & 0.1 & 16.1 & & & 0.4 & & & 46.8 & & 0.3 & & & 0.0 & & & 0.1 & & \\
\hline Cantale, 1991 & & & 13.5 & 10.3 & 0.1 & & 2.7 & & & 0.1 & & & 0.2 & 0.4 & 4.9 & 0.5 & & & 17.3 & 0.0 & 4.2 & & & & 45.7 & 0.1 & 0.1 & & & 0.2 & & & & & \\
\hline \begin{tabular}{|l|} 
Carter, 1988 \\
\end{tabular} & & 1325 & 10.3 & & 0.1 & & 3.9 & & & & & & 5.6 & 0.9 & 0.0 & 1.8 & 0.1 & & 17.8 & 0.0 & 0.0 & 2.2 & & & 52.2 & & 3.7 & & & 1.0 & & & 0.0 & & \\
\hline Carter, 1988 & & 1325 & 10.2 & 0.1 & 0.1 & & 18.1 & & & 0.1 & & & 5.5 & 1.5 & 0.1 & 1.9 & 0.1 & & 3.0 & 0.3 & 0.0 & 2.0 & & & 51.1 & & 3.6 & & & 0.9 & & & 0.0 & & \\
\hline Carter, 1988 & & 1665 & 12.2 & 0.1 & 0.1 & & 4.5 & & & & & & 6.6 & 1.9 & & 2.2 & 0.1 & & 3.1 & 0.1 & 0.0 & 2.2 & & & 61.6 & & 3.7 & & & 1.1 & & & 0.0 & & \\
\hline Carter, 1988 & & 1324 & 13.6 & 0.2 & 0.1 & & 13.9 & & & & & & 3.8 & 3.2 & 0.1 & 10.1 & 0.1 & & 1.1 & 0.0 & & 2.0 & & & 48.5 & & 4.2 & & & 0.6 & & & 0.0 & & \\
\hline Chernushkin, $196 \mathrm{C}$ & 1150 & & 10.7 & & & & 6.7 & & & & & & 3.2 & & & 0.3 & 2.8 & & 13.4 & & & & & & 54.4 & & & & & & & 8.5 & & & \\
\hline Chick, 1984 & & 1122 & 8.0 & 7.4 & & & 5.8 & & & & & & 8.5 & & & & & & 18.8 & & 8.0 & & & & 43.7 & & & & & & & & & & \\
\hline Chick, 1984 & & 1031 & 12.0 & & & & 15.0 & & & & & & 8.0 & & & & & & 30.0 & & & & & & 35.0 & & & & & & & & & & \\
\hline Chick, 1984 & & 1586 & 8.0 & & & & & & & & & & 20.0 & & & & & & 12.0 & & & & & & 60.0 & & & & & & & & & & \\
\hline Chick, 1984 & & 1388 & 10.9 & 18.2 & & & & & & & & & & & & & & & 10.0 & & 20.0 & & & & 40.9 & & & & & & & & & & \\
\hline Chick, 1984 & & 1285 & 12.0 & 20.0 & & & & & & & & & 24.0 & & & & & & 9.0 & & & & & & 35.0 & & & & & & & & & & \\
\hline Chick, 1984 & & 1231 & 12.0 & & & & & 0.3 & & 1.6 & & & & 0.1 & & & 1.0 & 0.4 & 30.0 & 0.8 & & 0.3 & 6.0 & & 36.0 & & 0.3 & 0.4 & 7.8 & & 2.5 & & 0.4 & & \\
\hline Chick, 1984 & & 1217 & 12.0 & 9.0 & & & 15.0 & 0.3 & & 1.6 & & & & 0.1 & & & 1.0 & 0.4 & 7.0 & 0.8 & & 0.3 & 6.0 & & 35.0 & & 0.3 & 0.4 & 7.8 & & 2.5 & & 0.4 & & \\
\hline
\end{tabular}


Table A1. Glass Compositions from Literature Review (continued)

\begin{tabular}{|c|c|c|c|c|c|c|c|c|c|c|c|c|c|c|c|c|c|c|c|c|c|c|c|c|c|c|c|c|c|c|c|c|c|c|c|}
\hline Ref & 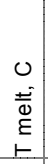 & $\begin{array}{l}0 \\
0 \\
0 \\
\stackrel{0}{0} \\
1\end{array}$ & $\begin{array}{l}\stackrel{0}{N} \\
\stackrel{N}{\leftarrow}\end{array}$ & 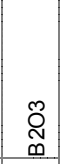 & $\begin{array}{l}\text { ષ } \\
\infty\end{array}$ & 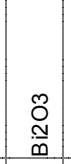 & $\begin{array}{l}0 \\
\pi \\
0\end{array}$ & 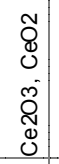 & $\begin{array}{l}\text { Oొ } \\
\text { Nర } \\
0 \\
0 \\
0 \\
0\end{array}$ & ญ̃ & $\stackrel{0}{3}$ & 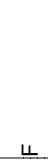 & 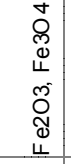 & 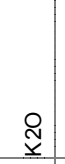 & 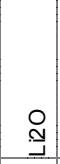 & $\stackrel{0}{\circ}$ & $\begin{array}{l}\text { O } \\
\text { L } \\
\Sigma\end{array}$ & $\begin{array}{l}0 \\
0 \\
\Sigma\end{array}$ & $\begin{array}{l}\text { N } \\
\underset{z}{z}\end{array}$ & $\frac{0}{z}$ & \begin{tabular}{l} 
N \\
\multirow{0}{0}{}
\end{tabular} & $\begin{array}{l}\bar{c} \\
\overline{0}\end{array}$ & 足 & $\frac{8}{a}$ & $\frac{\widetilde{O}}{\infty}$ & $\begin{array}{l}\text { Oొ } \\
\text { in }\end{array}$ & $\begin{array}{l}\text { Q } \\
\text { in }\end{array}$ & 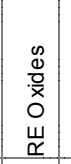 & 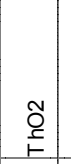 & 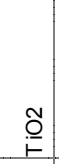 & 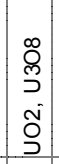 & $\frac{Q}{N}$ & $\begin{array}{c}\tilde{N} \\
\text { N }\end{array}$ & $\begin{array}{l}\underline{0} \\
\underline{1} \\
1\end{array}$ & $\begin{array}{l}\bar{\Xi} \\
\text { o్ } \\
\underline{\underline{Z}} \\
\bar{z}\end{array}$ \\
\hline Chick, 1984 & & 810 & 8.0 & 20.0 & & & 7.0 & & & & & & & & & & & & 30.0 & & & & & & 35.0 & & & & & & & & & & \\
\hline Chick, 1984 & & 1380 & 12.0 & 12.0 & & & & & & & & & & & & & & & 16.0 & & & & & & 60.0 & & & & & & & & & & \\
\hline Chick, 1984 & & 1240 & 8.0 & 2.0 & & & & & & & & & & & & & & & 30.0 & & & & & & 60.0 & & & & & & & & & & \\
\hline Chick, 1984 & & 1220 & 8.0 & & & & 15.0 & 0.3 & & 1.6 & & & & 0.1 & & & 1.0 & 0.4 & 12.0 & 0.8 & & 0.3 & 6.0 & & 43.0 & & 0.3 & 0.4 & 7.8 & & 2.5 & & 0.4 & & \\
\hline Chick, 1984 & & 1294 & 8.0 & & & & 15.0 & & & & & & & & & & & & 17.0 & & & & & & 60.0 & & & & & & & & & & \\
\hline Chick, 1984 & & 1233 & 12.0 & 20.0 & & & & 0.3 & & 1.6 & & & & 0.1 & & & 1.0 & 0.4 & 11.0 & 0.8 & & 0.3 & 6.0 & & 35.0 & & 0.3 & 0.4 & 7.8 & & 2.5 & & 0.4 & & \\
\hline Chick, 1984 & & 1327 & 8.0 & 20.0 & & & & & & & & & & & & & & & 12.0 & & & & & & 60.0 & & & & & & & & & & \\
\hline Chick, 1984 & & 1189 & 8.0 & 20.0 & & & & 0.3 & & 1.6 & & & & 0.1 & & & 1.0 & 0.4 & 12.0 & 0.8 & & 0.3 & 6.0 & & 38.0 & & 0.3 & 0.4 & 7.8 & & 2.5 & & 0.4 & & \\
\hline Chick, 1984 & & 1112 & 8.0 & & & & & & & & & & 24.0 & & & & & & 30.0 & & & & & & 38.0 & & & & & & & & & & \\
\hline Chick, 1984 & & 1481 & 12.0 & & & & 13.0 & & & & & & 6.0 & & & & & & 7.0 & & 6.0 & & & & 56.0 & & & & & & & & & & \\
\hline Chick, 1984 & & 1366 & 12.0 & 10.5 & & & 1.0 & & & & & & 7.0 & & & & & & 16.5 & & & & & & 53.0 & & & & & & & & & & \\
\hline Chick, 1984 & & 1191 & 8.0 & & & & & 0.3 & & 1.6 & & & & 0.1 & & & 1.0 & 0.4 & 30.0 & 0.8 & & 0.3 & 6.0 & & 40.0 & & 0.3 & 0.4 & 7.8 & & 2.5 & & 0.4 & & \\
\hline Chick, 1984 & & 1202 & 8.0 & & & & 7.5 & & & & & & 24.0 & & & & & & 12.0 & & 13.5 & & & & 35.0 & & & & & & & & & & \\
\hline Chick, 1984 & & 1324 & 8.0 & & & & & 0.3 & & 1.6 & & & 23.0 & 0.1 & & & 1.0 & \begin{tabular}{|l|l|}
0.4 \\
\end{tabular} & 12.0 & 0.8 & & 0.3 & 6.0 & & 35.0 & & 0.3 & 0.4 & 7.8 & & 2.5 & & 0.4 & & \\
\hline Chick, 1984 & & 962 & 12.0 & 20.0 & & & 15.0 & & & & & & & & & & & & 18.0 & & & & & & 35.0 & & & & & & & & & & \\
\hline Chick, 1984 & & 807 & 8.0 & 20.0 & & & 7.0 & & & & & & & & & & & & 30.0 & & & & & & 35.0 & & & & & & & & & & \\
\hline Chick, 1984 & & 1402 & 8.0 & 20.0 & & & & & & & & & & & & & & & 12.0 & & & & & & 60.0 & & & & & & & & & & \\
\hline Chick, 1984 & & & 8.0 & 20.0 & & & & & & & & & 24.0 & & & & & & 12.0 & & & & & & 36.0 & & & & & & & & & & \\
\hline Chick, 1984 & & 1191 & 12.0 & & & & & & & & & & 23.5 & & & & & & 29.5 & & & & & & 35.0 & & & & & & & & & & \\
\hline Chick, 1984 & & 1007 & 8.0 & & & & 15.0 & & & & & & 6.0 & & & & & & 30.0 & & 6.0 & & & & 35.0 & & & & & & & & & & \\
\hline Chick, 1984 & & 1187 & 8.0 & & & & 15.0 & & & & & & 24.0 & & & & & & 12.0 & & & & & & 41.0 & & & & & & & & & & \\
\hline Chick, 1984 & & 1168 & 8.0 & 2.0 & & & & & & & & & & & & & & & 30.0 & & & & & & 60.0 & & & & & & & & & & \\
\hline Chick, 1984 & & 1307 & 8.0 & & & & 15.0 & & & & & & & & & & & & 17.0 & & & & & & 60.0 & & & & & & & & & & \\
\hline Chick, 1984 & & & 8.0 & 8.0 & & & 15.0 & 0.3 & & 1.6 & & & & 0.1 & & & 1.0 & 0.4 & 12.0 & 0.8 & & 0.3 & 6.0 & & 35.0 & & 0.3 & 0.4 & 7.8 & & 2.5 & & 0.4 & & \\
\hline Chick, 1984 & & & 8.0 & 20.0 & & & 15.0 & & & & & & 10.0 & & & & & & 12.0 & & & & & & 35.0 & & & & & & & & & & \\
\hline Chick, 1984 & & 1166 & 8.0 & & & & 15.0 & & & & & & 24.0 & & & & & & 12.0 & & & & & & 41.0 & & & & & & & & & & \\
\hline Chick, 1984 & & 1348 & 12.0 & & & & & & & & & & & & & & & & 28.0 & & & & & & 60.0 & & & & & & & & & & \\
\hline Chick, 1984 & & 1078 & 7.3 & & & & 13.6 & & & & & & 5.4 & & & & & & 27.2 & & 5.4 & & & & 41.1 & & & & & & & & & & \\
\hline Chick, 1984 & & 1231 & 7.1 & 17.7 & & & & & & & & & 21.2 & & & & & & 12.0 & & & & & & 42.0 & & & & & & & & & & \\
\hline Chick, 1984 & & 1327 & 12.0 & 10.0 & & & & 0.1 & & 0.8 & & & 12.0 & 0.0 & & & 0.5 & 0.2 & 15.0 & 0.4 & & 0.2 & 2.9 & & 40.2 & & 0.2 & 0.2 & 3.8 & & 1.2 & & 0.2 & & \\
\hline Chick, 1984 & & 1275 & 12.0 & & & & 10.0 & 0.1 & & 0.8 & & & 12.0 & 0.0 & & & 0.5 & 0.2 & 15.0 & 0.4 & & 0.2 & 2.9 & & 40.2 & & 0.2 & 0.2 & 3.8 & & 1.2 & & 0.2 & & \\
\hline Clifford, 1994 & & & 3.7 & 11.7 & & 43.7 & & & & & & & & & 1.0 & & & & 7.1 & & & & & & 28.3 & & & & & 2.0 & & & 2.5 & & \\
\hline Clifford, 1994 & & & 2.9 & 11.9 & & 19.7 & & & & & & & & 2.0 & 3.0 & & & & 6.9 & & & & & & 49.4 & & & & & 2.0 & & & 2.2 & & \\
\hline Clifford, 1994 & & & 2.0 & 25.3 & & 5.0 & 1.0 & & & & & & & 2.0 & 4.0 & & & & 8.1 & & & & & & 47.5 & & & & & 2.0 & & & 3.0 & & \\
\hline Clifford, 1994 & & & 3.6 & 11.4 & & 42.5 & & & & & & & & 2.4 & 6.4 & & & & 2.3 & & & & & & 26.9 & & & & & & & & 1.7 & & \\
\hline Clifford, 1994 & & & 2.9 & 6.3 & & 19.3 & 1.1 & & & & & & & 10.0 & 2.4 & & & & 1.7 & & & & & & 50.9 & & & & & & & & 1.9 & & \\
\hline Clifford, 1994 & & & 2.8 & 10.1 & & 19.1 & & 2.2 & & & & & & 1.9 & 2.9 & & & & 6.7 & & & & & & 46.9 & & 1.3 & & & & & & 2.1 & & \\
\hline Clifford, 1994 & & & 3.9 & 12.3 & & 45.5 & & & & & & & & & & & & & 6.6 & & & & & & 28.8 & & & & & 1.6 & & & 1.3 & & \\
\hline Clifford, 1994 & & & 3.7 & 11.8 & & 45.9 & & & & & & & & & & & & & 6.4 & & & & & & 28.2 & & & & & 1.6 & & & 2.4 & & \\
\hline Clifford, 1994 & & & 3.0 & 11.3 & & 15.0 & 1.2 & & & & & & & 5.2 & 2.5 & & & & 6.9 & & & & & & 52.8 & & & & & & & & 2.0 & & \\
\hline Clifford, 1994 & & & 3.0 & 16.4 & & 9.9 & 1.2 & & & & & & & 0.2 & 2.5 & & & & 11.9 & & & & & & 52.9 & & & & & & & & 2.0 & & \\
\hline Clifford, 1994 & & & 3.0 & 4.4 & & 20.6 & 1.2 & & & & & & & 10.5 & 2.6 & & & & 1.8 & & & & & & 53.8 & & & & & & & & 2.1 & & \\
\hline Clifford, 1994 & & & 3.1 & 15.4 & & 15.3 & & & & & & & & 2.6 & 2.6 & & & & 7.2 & & & & & & 46.2 & & & & & & & & 7.6 & & \\
\hline Clifford, 1994 & & & 3.4 & 22.8 & & 11.1 & 0.6 & & & & & & & 2.3 & 3.4 & & & & 9.1 & & & & & & 45.7 & & & & & & & & 1.7 & & \\
\hline Clifford, 1994 & & & 3.1 & 25.9 & & 10.1 & 0.5 & & & & & & & 2.0 & 4.2 & & & & 10.4 & & & & & & 41.5 & & & & & & & & 2.3 & & \\
\hline Clifford, 1994 & & & 2.0 & 25.3 & & 5.0 & 1.0 & & & & & & & 3.0 & 6.1 & & & & 10.1 & & & & & & 41.6 & & & & & 2.0 & & & 3.7 & & \\
\hline Clifford, 1994 & & & 1.0 & 25.3 & & 9.9 & & & & & & & & 3.0 & 5.1 & & & & 10.1 & & & & & & 39.5 & & & & & 3.0 & & & 3.0 & & \\
\hline Clifford, 1994 & & & 2.0 & 25.4 & & 5.0 & 1.0 & & & & & & & 3.0 & 6.1 & & & & 10.1 & & & & & & 42.1 & & & & & & & & 5.2 & & \\
\hline Clifford, 1994 & & & 2.9 & 11.7 & & 19.5 & & & & & & & & 7.0 & 2.0 & & & & 2.8 & & & & & & 48.8 & & & & & 1.9 & & & 3.3 & & \\
\hline Clifford, 1994 & & & 2.9 & 11.6 & & 19.3 & & & & & & & & 6.9 & 1.9 & & & & 2.8 & & & & & & 48.3 & & & & & 1.9 & & & 4.3 & & \\
\hline
\end{tabular}




\begin{tabular}{|c|c|c|c|c|c|c|c|c|c|c|c|c|c|c|c|c|c|c|c|c|c|c|c|c|c|c|c|c|c|c|c|c|c|c|c|}
\hline Ref & 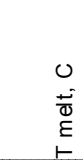 & $\begin{array}{l}0 \\
\frac{0}{2} \\
8 \\
0 \\
1\end{array}$ & $\begin{array}{l}\stackrel{0}{N} \\
\stackrel{N}{<}\end{array}$ & $\underset{\infty}{\widetilde{D}}$ & $\begin{array}{l}\mathcal{O} \\
\mathbb{\infty} \\
\infty\end{array}$ & $\begin{array}{l}\stackrel{0}{0} \\
\stackrel{N}{0}\end{array}$ & $\begin{array}{l}0 \\
\widetilde{J}\end{array}$ & $\begin{array}{l}\tilde{~} \\
0 \\
\text { ల్ } \\
0 \\
0 \\
0\end{array}$ & $\begin{array}{l}0 \\
\text { N } \\
8 \\
0 \\
0 \\
0\end{array}$ & $\begin{array}{l}\text { Õ } \\
\text { บั }\end{array}$ & $\stackrel{0}{3}$ & 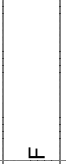 & 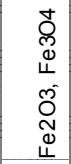 & 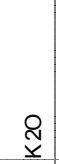 & 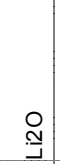 & $\frac{8}{2}$ & $\begin{array}{l}\tilde{O} \\
\frac{\varepsilon}{\Sigma}\end{array}$ & $\frac{0}{8}$ & $\begin{array}{l}\text { N } \\
\text { Z } \\
Z\end{array}$ & $\stackrel{\circ}{\bar{z}}$ & $\begin{array}{l}\mathcal{N} \\
\text { ָे }\end{array}$ & $\overline{\bar{\Xi}}$ & $\begin{array}{l}\text { ¿̊ } \\
\Sigma \\
\Sigma\end{array}$ & $\frac{O}{\alpha}$ & $\frac{\tilde{O}}{\infty}$ & $\begin{array}{l}\text { OO } \\
\text { in }\end{array}$ & 잉 & 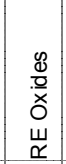 & $\begin{array}{l}\tilde{O} \\
\stackrel{F}{F}\end{array}$ & $\stackrel{\text { Oิ }}{i=}$ & $\begin{array}{l}\infty \\
0 \\
\mathcal{S} \\
\text { ก̃ } \\
\text { ป }\end{array}$ & $\begin{array}{l}0 \\
\stackrel{N}{N}\end{array}$ & $\begin{array}{l}\text { Oे } \\
\text { กิ }\end{array}$ & $\stackrel{\widehat{O}}{\vec{H}}$ & $\begin{array}{l}\vec{\Xi} \\
\text { O్ } \\
\underline{\underline{E}} \\
\dot{z}\end{array}$ \\
\hline Clifford, 1994 & & & 2.9 & 11.7 & & 19.4 & & & & & & & & 0.9 & 8.4 & & & & 1.8 & & & & & & 48.6 & & & & & 1.9 & & & 4.3 & & \\
\hline Clifford, 1994 & & & 2.4 & 14.1 & & 3.5 & & & & & & & & 1.7 & 4.3 & & & & 2.5 & & & & & & 67.9 & & & & & 2.0 & & & 1.5 & & \\
\hline Clifford, 1994 & & & 2.3 & 13.4 & & 3.4 & & & & & & & & 8.0 & 2.2 & & & & 2.4 & & & & & & 64.9 & & & & & 1.9 & & & 1.4 & & \\
\hline Clifford, 1994 & & & 2.4 & 14.1 & & 3.5 & & & & & & & & 1.7 & 4.3 & & & & 2.5 & & & & & & 67.9 & & & & & 2.0 & & & 1.5 & & \\
\hline Clifford, 1994 & & & 4.2 & 23.7 & & 7.1 & 1.7 & & & & & & & & 3.4 & & & & 15.4 & & & & & & 33.4 & & & & & & & & 11.0 & & \\
\hline Clifford, 1994 & & & 1.7 & 18.2 & & 4.9 & 1.0 & & & & & & & 3.1 & 6.0 & & & & \begin{tabular}{|c|}
9.7 \\
\end{tabular} & & & & & & 43.7 & & & & & 2.1 & & & 9.6 & & \\
\hline Clifford, 1994 & & & 2.8 & 10.4 & & 19.3 & & & & & & & & 1.9 & 2.9 & & & & 6.7 & & 3.0 & 3.5 & & & 45.8 & & & 1.7 & & & & & 2.1 & & \\
\hline Clifford, 1994 & & & 2.7 & 1.6 & & 30.4 & & & & 1.8 & & & & 9.3 & 2.3 & 0.8 & & 1.7 & 1.6 & & & & & & 47.2 & & & & & & & & 0.8 & & \\
\hline Clifford, 1994 & & & 0.4 & 25.4 & & 5.8 & 6.2 & & & & & & & 3.0 & 5.2 & 0.6 & & & 10.1 & & & & & & 38.3 & & & & & & & 2.6 & \begin{tabular}{|l|l|}
5 & 2.4 \\
\end{tabular} & & \\
\hline Clifford, 1994 & & & 4.9 & 6.6 & & 56.3 & 0.3 & & & & & & & 0.1 & 0.6 & & & & 3.0 & & & & & & 27.7 & & & & & & & & 0.5 & & \\
\hline Crawford, 1998 & & & 10.1 & 7.0 & 0.0 & & 4.0 & & & 0.0 & & & 23.0 & 0.9 & 3.0 & 0.1 & 0.4 & & 15.7 & 0.1 & & & 0.7 & 0.2 & 30.2 & & 4.2 & & & 0.2 & & 0.0 & & & \\
\hline Dingwell, 2004 & & 1122 & 5.0 & & & & 6.5 & & & 0.4 & & & 8.8 & & & 32.3 & & & 0.3 & & & & & & 46.9 & & & & & 0.2 & & & & & \\
\hline Dvorkin, 1969 & & & 13.8 & 4.6 & & & 8.6 & & & & & & 9.2 & & & 3.7 & & & 4.6 & & & & & & 55.0 & & & & & 1.6 & & & & & \\
\hline Dvorkin, 1969 & & & 11.9 & & & & 8.4 & & & & & & 9.3 & & & 4.9 & & & 6.2 & & & & & & 57.8 & & & & & 1.6 & & & & & \\
\hline Dvorkin, 1969 & & & 10.5 & & & & 7.9 & & & & & & 7.1 & & & 4.6 & & & 7.7 & & & & & & 61.2 & & & & & 1.2 & & & & & \\
\hline Dvorkin, 1969 & & & 15.6 & 6.5 & & & 11.4 & & & & & & 13.4 & & & 4.6 & & & 2.8 & & & & & & 43.6 & & & & & 2.0 & & & & & \\
\hline Dvorkin, 1971 & $\sim 1200$ & & 14.3 & & & & 10.3 & & & & & & 14.9 & & & 5.5 & & & 3.0 & & & & & & 49.7 & & & & & 2.3 & & & & & \\
\hline Dvorkin, 1971 & $\sim 1200$ & & 10.2 & 13.2 & & & 7.4 & & & & & & 10.7 & & & 4.0 & & & 2.2 & & & & & & 47.4 & & & & & 1.7 & & & & & \\
\hline Dvorkin, 1971 & $\sim 1200$ & & 8.8 & 7.5 & & & 6.4 & & & & & & 9.2 & & & 3.4 & & & 9.2 & & & & & & 54.2 & & & & & 1.4 & & & & & \\
\hline Dvorkin, 1971 & $\sim 1200$ & & 9.5 & 7.6 & & & 6.8 & & & & & & 9.9 & & & 3.6 & & & 6.9 & & & & & & 54.0 & & & & & 1.5 & & & & & \\
\hline Dvorkin, 1971 & $\sim 1200$ & & 12.1 & 9.3 & & & 8.7 & & & & & & 12.7 & & & 4.7 & & & \begin{tabular}{|l|l|}
7.4 \\
\end{tabular} & & & & & & 42.0 & & & & & 2.0 & & & & & \\
\hline Dvorkin, 1971 & $\sim 1200$ & & 8.4 & & & & 6.1 & & & & & & 8.8 & & & 3.2 & & & 13.4 & & & & & & 60.0 & & & & & 1.4 & & & & & \\
\hline Dvorkin, 1971 & $\sim 1200$ & & 9.8 & & & & 7.1 & & & & & & 10.2 & & & 3.8 & & & 12.4 & & & & & & 55.0 & & & & & 1.6 & & & & & \\
\hline Dvorkin, 1971 & $\sim 1200$ & & 7.9 & & & & 5.6 & & & & & & 8.4 & & & 3.1 & & & 10.0 & & & & & & 64.0 & & & & & 1.3 & & & & & \\
\hline Dvorkin, 1971 & $\sim 1200$ & & 10.6 & 8.0 & & & 7.6 & & & & & & 11.0 & & & 4.3 & & & 2.2 & & & & & & 54.5 & & & & & 1.7 & & & & & \\
\hline Dzhigiris, 1983 & 1375 & & 16.3 & & & & 5.0 & & & & & & 14.6 & & & 9.4 & & & 2.8 & & & & & & 50.0 & & & & & 0.7 & & & & & \\
\hline Dzhigiris, 1983 & 1375 & & 13.9 & & & & 7.4 & & & & & & 12.4 & & & 9.8 & & & 3.0 & & & & & & 49.2 & & & & & 1.2 & & & & & \\
\hline Dzhigiris, 1983 & 1440 & & 15.1 & & & & 10.4 & & & & & & 10.5 & & & 6.6 & & & 3.1 & & & & & & 52.1 & & & & & 0.9 & & & & & \\
\hline Edwards, 1999 & & & 2.5 & 8.0 & 0.1 & & 0.9 & & & 0.1 & 0.6 & 0.0 & 9.9 & 3.4 & 4.8 & 1.4 & 1.7 & & 7.9 & 0.9 & & & 0.0 & 0.1 & 53.7 & 0.2 & & & & 1.1 & 2.0 & 0.1 & 0.1 & & 1.5 \\
\hline Edwards, 1999 & & & 2.5 & 8.0 & 0.1 & & 0.9 & & & 0.1 & 0.6 & 0.0 & 9.9 & 3.4 & \begin{tabular}{ll|}
4.8 \\
\end{tabular} & 1.4 & 1.7 & & \begin{tabular}{|l|}
7.9 \\
\end{tabular} & 0.9 & & & 0.0 & 0.1 & 53.7 & 0.2 & & & & 1.1 & 2.0 & 0.1 & 0.1 & & 1.3 \\
\hline Edwards, 1999 & & & 2.5 & 9.6 & 0.1 & & 0.9 & & & 0.1 & 1.0 & 0.0 & 9.9 & 6.1 & 4.4 & 1.3 & 1.7 & & 8.2 & 0.9 & & & 0.0 & 0.1 & 49.1 & 0.2 & & & & 1.1 & 2.0 & 0.1 & 0.1 & & 1.8 \\
\hline Edwards, 1999 & & & 2.5 & 7.9 & 0.1 & & 0.9 & & & 0.1 & 0.6 & 0.0 & 9.9 & 3.4 & 4.7 & 1.4 & 1.7 & & 7.9 & 0.9 & & & 0.0 & 0.1 & 52.7 & 0.2 & & & & 2.2 & 2.0 & 0.1 & 0.1 & & 1.5 \\
\hline Edwards, 1999 & & & 2.5 & 8.7 & 0.1 & & 0.9 & & & 0.1 & 0.8 & 0.0 & 9.9 & 4.7 & 4.5 & 1.4 & 1.7 & & 8.1 & 0.9 & & & 0.0 & 0.1 & 50.5 & 0.2 & & & & 2.2 & 2.0 & 0.1 & 0.1 & & 1.3 \\
\hline Edwards, 1999 & & & 2.5 & 9.5 & 0.1 & & 0.9 & & & 0.1 & 1.0 & 0.0 & 9.9 & 6.1 & 4.3 & 1.3 & 1.7 & & 8.2 & 0.9 & & & 0.0 & 0.1 & 48.2 & 0.2 & & & & 2.2 & 2.0 & 0.1 & 0.1 & & 1.6 \\
\hline Edwards, 1999 & & & 2.9 & 7.7 & 0.1 & & 1.1 & & & 0.1 & 0.6 & 0.0 & 11.7 & 3.4 & 4.5 & 1.4 & 2.0 & & \begin{tabular}{|l|}
8.1 \\
\end{tabular} & 1.1 & & & 0.0 & 0.1 & 50.8 & 0.2 & & & & 1.1 & 2.4 & 0.1 & 0.1 & & \\
\hline Edwards, 1999 & & & 2.9 & 8.5 & 0.1 & & 1.1 & & & 0.1 & 0.8 & 0.0 & 11.7 & 4.7 & 4.3 & 1.3 & 2.0 & & 8.3 & 1.1 & & & 0.0 & 0.1 & 48.5 & 0.2 & & & & 1.1 & 2.4 & 0.1 & 0.1 & & \\
\hline Edwards, 1999 & & & 2.9 & 9.3 & 0.1 & & 1.1 & & & 0.1 & 1.0 & 0.0 & 11.7 & 6.1 & 4.1 & 1.3 & 2.0 & & \begin{tabular}{|l|l|}
8.4 \\
\end{tabular} & 1.1 & & & 0.0 & 0.1 & 46.2 & 0.2 & & & & 1.1 & 2.4 & 0.1 & 0.1 & & \\
\hline Edwards, 1999 & & & 2.9 & 7.6 & 0.1 & & 1.1 & & & 0.1 & 0.6 & 0.0 & 11.7 & 3.4 & 4.4 & 1.4 & 2.0 & & 8.2 & 1.1 & & & 0.0 & 0.1 & 49.8 & 0.2 & & & & 2.2 & 2.4 & 0.1 & 0.1 & & \\
\hline Edwards, 1999 & & & 2.9 & 8.4 & 0.1 & & 1.1 & & & 0.1 & 0.8 & 0.0 & 11.7 & 4.7 & 4.2 & 1.3 & 2.0 & & 8.3 & 1.1 & & & 0.0 & 0.1 & 47.5 & 0.2 & & & & 2.2 & 2.4 & 0.1 & 0.1 & & \\
\hline Edwards, 1999 & & & 2.9 & 9.2 & 0.1 & & 1.1 & & & 0.1 & 1.0 & 0.0 & 11.7 & 6.1 & 4.0 & 1.2 & 2.0 & & 8.5 & 1.1 & & & 0.0 & 0.1 & 45.3 & 0.2 & & & & 2.2 & 2.4 & 0.1 & 0.1 & & \\
\hline Edwards, 1999 & & & 3.3 & 7.3 & 0.1 & & 1.2 & & & 0.1 & 0.6 & 0.0 & 13.5 & 3.4 & 4.2 & 1.3 & 2.4 & & 8.4 & 1.3 & & & 0.0 & 0.1 & 47.8 & 0.2 & & & & 1.1 & 2.7 & 0.1 & 0.1 & & \\
\hline Edwards, 1999 & & & 3.2 & 8.2 & 0.1 & & 1.2 & & & 0.1 & 0.8 & 0.0 & 13.5 & 4.8 & 4.0 & 1.3 & 2.4 & & 8.5 & 1.3 & & & 0.0 & 0.1 & 45.6 & 0.2 & & & & 1.1 & 2.7 & 0.1 & 0.1 & & \\
\hline Edwards, 1999 & & & 3.2 & 9.0 & 0.1 & & 1.2 & & & 0.1 & 1.0 & 0.0 & 13.5 & 6.1 & 3.8 & 1.2 & 2.4 & & 8.7 & 1.3 & & & 0.0 & 0.1 & 43.3 & 0.2 & & & & 1.1 & 2.7 & 0.1 & 0.1 & & \\
\hline Edwards, 1999 & & & 3.3 & 7.2 & 0.1 & & 1.2 & & & 0.1 & 0.6 & 0.0 & 13.5 & 3.4 & 4.2 & 1.3 & 2.4 & & \begin{tabular}{|l|l|}
8.4 & \\
\end{tabular} & 1.3 & & & 0.0 & 0.1 & 46.9 & 0.2 & & & & 2.2 & 2.7 & 0.1 & 0.1 & & \\
\hline Edwards, 1999 & & & 3.2 & 8.1 & 0.1 & & 1.2 & & & 0.1 & 0.8 & 0.0 & 13.5 & 4.8 & 3.9 & 1.2 & 2.4 & & 8.6 & 1.3 & & & 0.0 & 0.1 & 44.6 & 0.2 & & & & 2.2 & 2.7 & 0.1 & 0.1 & & \\
\hline Edwards, 1999 & & & 3.2 & 8.9 & 0.1 & & 1.2 & & & 0.1 & 1.0 & 0.0 & 13.5 & 6.1 & 3.7 & 1.2 & 2.4 & & 8.7 & 1.3 & & & 0.0 & 0.1 & 42.3 & 0.2 & & & & 2.2 & 2.7 & 0.1 & 0.1 & & \\
\hline Edwards, $1999 a$ & & & 6.0 & 8.8 & 0.0 & & 0.5 & & & 0.1 & 0.8 & 0.0 & 6.4 & 4.7 & 4.6 & 1.5 & 2.0 & & 7.8 & 0.3 & & & 0.0 & 0.1 & 54.1 & 0.1 & & & & 1.1 & 0.7 & 0.0 & 0.1 & 916 & 0.5 \\
\hline Edwards, 1999a & & & 7.0 & 8.5 & 0.1 & & 0.6 & & & 0.1 & 0.8 & 0.0 & 7.5 & 4.7 & 4.3 & 1.4 & 2.3 & & 8.0 & 0.4 & & & 0.0 & 0.1 & 51.7 & 0.2 & & & & 1.1 & 0.8 & 0.0 & 0.1 & 928 & 0.5 \\
\hline Edwards, 1999a & & & 8.1 & 8.2 & 0.1 & & 0.6 & & & 0.1 & \begin{tabular}{|l|}
0.8 \\
\end{tabular} & 0.1 & 8.7 & 4.7 & 4.0 & \begin{tabular}{|l|}
1.4 \\
\end{tabular} & 2.7 & & \begin{tabular}{|l|}
8.2 \\
\end{tabular} & 0.5 & & & 0.0 & 0.1 & 49.2 & 0.2 & & & & 1.1 & 0.9 & 0.0 & 0.2 & 959 & 0.5 \\
\hline
\end{tabular}


Table A1. Glass Compositions from Literature Review (continued)

\begin{tabular}{|c|c|c|c|c|c|c|c|c|c|c|c|c|c|c|c|c|c|c|c|c|c|c|c|c|c|c|c|c|c|c|c|c|c|c|c|}
\hline Ref & 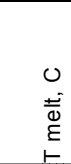 & 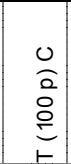 & 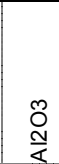 & ల్లై & $\begin{array}{l}\mathbb{Z} \\
\infty\end{array}$ & @ָ & $\begin{array}{l}0 \\
\mathbb{\pi} \\
0\end{array}$ & 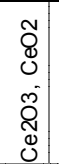 & $\begin{array}{l}0 \\
0 \\
0 \\
0 \\
0 \\
0 \\
0\end{array}$ & $\begin{array}{l}\text { Õ } \\
\text { Ũ }\end{array}$ & $\stackrel{O}{3}$ & 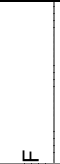 & 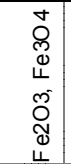 & $\underset{\tilde{\tau}}{\underline{x}}$ & 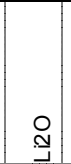 & $\begin{array}{l}0 \\
\sum \\
\Sigma\end{array}$ & $\begin{array}{l}\tilde{O} \\
\sum \\
\Sigma\end{array}$ & $\begin{array}{l}00 \\
0 \\
\Sigma\end{array}$ & $\begin{array}{l}\stackrel{O}{N} \\
\underset{Z}{Z}\end{array}$ & $\stackrel{\circ}{z}$ & 疋 & $\overline{\bar{F}}$ & مి & $\frac{8}{0}$ & $\frac{\tilde{O}}{\infty}$ & Oొ & $\begin{array}{l}\text { O } \\
\text { in }\end{array}$ & 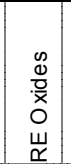 & $\begin{array}{l}\text { Q } \\
\text { \& }\end{array}$ & $\stackrel{\tilde{O}}{\llcorner}$ & $\begin{array}{l}\text { Oొ } \\
\text { ગ } \\
\text { ปิ } \\
\text { రิ }\end{array}$ & $\stackrel{q}{N}$ & ญิ & $\underset{\substack{0 \\
1}}{ }$ & 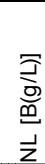 \\
\hline Ferrara, 1998 & & & 9.7 & 4.3 & & & 4.5 & & & 0.0 & & & 7.4 & 2.3 & 2.1 & 2.1 & & & 20.0 & & & & & & 41.0 & & & & & & & 3.3 & 3.1 & & \\
\hline Ferrara, 1998 & & & 8.1 & 8.1 & & & 7.0 & & & 0.1 & & & 8.0 & 0.4 & 4.1 & 3.0 & & & 10.0 & & & & & & 44.0 & & & & & & & 4.0 & 3.0 & & \\
\hline Ferrara, 1998 & & & 9.9 & 3.0 & & & 4.8 & & & 0.0 & & & 6.4 & 0.4 & 4.0 & 3.0 & & & 20.0 & & & & & & 42.0 & & & & & & & 3.3 & 3.0 & & \\
\hline Fisher, 2005 & 1450 & & & & & & 3.8 & & & & & & & & & & & & 22.0 & & & & & & 74.2 & & & & & & & & & & \\
\hline Fisher, 2005 & 1450 & & & & & & 3.5 & & & & & & & & & & & & 18.6 & & & & & & 71.1 & & & & & & & & 6.8 & & \\
\hline Fisher, 2005 & 1450 & & & & & & 3.3 & & & & & & & & & & & & 19.0 & & & & & & 68.4 & & & & & & & & 9.2 & & \\
\hline Fisher, 2005 & 1450 & & & & & & 2.8 & & & & & & & & & & & & 15.2 & & & & & & 70.8 & & & & & & & & 11.2 & & \\
\hline Fisher, 2005 & 1630 & & & & & & 3.2 & & & & & & & & & & & & 17.1 & & & & & & 63.7 & & & & & & & & 15.9 & & \\
\hline Fisher, 2005 & 1630 & & & & & & 3.0 & & & & & & & & & & & & 16.7 & & & & & & 62.8 & & & & & & & & 17.5 & & \\
\hline Fisher, 2005 & 1630 & & & & & & 2.9 & & & & & & & & & & & & 16.6 & & & & & & 59.8 & & & & & & & & 20.7 & & \\
\hline Fisher, 2005 & 1630 & & & & & & 3.0 & & & & & & & & & & & & 16.7 & & & & & & 58.9 & & & & & & & & 21.4 & & \\
\hline Flinn, 1981 & & 1294 & 7.4 & & & & 8.3 & & & & & & 34.5 & 2.4 & & 4.6 & & & 4.8 & & & & & & 38.0 & & & & & & & & & & \\
\hline Flinn, 1981 & & 1481 & 9.0 & & & & 9.1 & & & & & & 26.4 & 2.5 & & 4.0 & & & 3.9 & & & & & & 45.1 & & & & & & & & & & \\
\hline Flinn, 1981 & & & 9.6 & & & & 9.0 & & & & & & 18.2 & 5.6 & & 3.2 & & & 7.1 & & & & & & 47.3 & & & & & & & & & & \\
\hline Flinn, 1981 & & 1395 & 9.1 & & & & 8.6 & & & & & & 17.4 & 7.2 & & 3.1 & & & 9.3 & & & & & & 45.2 & & & & & & & & & & \\
\hline Flinn, 1981 & & 1541 & 10.3 & & & & 9.7 & & & & & & 19.6 & 2.6 & & 3.5 & & & 3.2 & & & & & & 51.0 & & & & & & & & & & \\
\hline Flinn, 1981 & & & 11.4 & & & & 10.3 & & & & & & 13.9 & 2.7 & & 3.1 & & & 2.6 & & & & & & 56.0 & & & & & & & & & & \\
\hline Flinn, 1981 & & & 12.1 & & & & 10.0 & & & & & & 9.5 & 2.8 & & 2.8 & & & 2.2 & & & & & & 60.6 & & & & & & & & & & \\
\hline Francis, 2005 & 1350 & & 11.8 & & & & 43.5 & & & & & & 0.3 & 0.5 & & 6.2 & 0.7 & & 0.1 & & & & 0.1 & & 35.8 & 0.8 & & & & 0.8 & & & & & \\
\hline Francis, 2005 & 1350 & & 14.8 & & 6.2 & & 26.6 & & & & & & 0.3 & 0.7 & & 6.7 & 3.3 & & 1.4 & & & & & & 37.0 & 1.1 & & & & & & & & & \\
\hline Francis, 2005 & 1350 & & 27.9 & & & & 29.4 & & & & & & 1.8 & 1.5 & & 8.0 & & & 0.4 & & & & & & 34.4 & & & & & 1.0 & & & & & \\
\hline Francis, 2005 & 1350 & & 6.8 & & & & 38.8 & & & & & & 0.6 & 0.7 & & 9.7 & & & 0.8 & & & & & & 39.7 & 0.6 & & & & 0.6 & & & & & \\
\hline Fu, 1997 & & & 9.0 & 13.0 & & & 0.5 & & & & & & 0.3 & 2.0 & 11.0 & & & & 17.0 & 0.2 & 0.3 & & 2.0 & & 44.0 & & & & & & 0.1 & & 0.1 & & \\
\hline Fu, 1997 & & & 9.0 & 13.0 & & & 0.6 & & & & & & 0.2 & 2.0 & 4.0 & & & & 22.0 & 0.2 & 0.4 & & 2.0 & & 46.0 & & & & & & 0.1 & & 0.1 & & \\
\hline Fu, 1997 & & & 9.0 & 13.0 & & & 0.6 & & & & & & 0.2 & 2.0 & 0.1 & & & & 27.0 & 0.2 & 0.4 & & 2.0 & & 46.0 & & & & & & 0.1 & & & & \\
\hline Fu, 1997 & & & 9.0 & 17.0 & & & 0.6 & & & & & & 0.2 & 2.0 & 0.1 & & & & 23.0 & 0.2 & 0.4 & & 2.0 & & 46.0 & & & & & & 0.1 & & & & \\
\hline Fu, 1997 & & & 9.0 & 21.0 & & & 0.6 & & & & & & 0.2 & 2.0 & 0.1 & & & & 19.0 & 0.2 & 0.4 & & 2.0 & & 46.0 & & & & & & 0.1 & & & & \\
\hline Fu, 1997 & & & 9.0 & 16.0 & & & 0.6 & & & & & & 0.2 & 2.0 & 11.0 & & & & \begin{tabular}{|l|}
13.0 \\
\end{tabular} & 0.2 & 0.4 & & 2.0 & & 45.0 & & & & & & 0.1 & & 0.1 & & \\
\hline Fu, 1998 & 1150 & 963 & 9.0 & 6.9 & 0.0 & & 5.6 & & & 0.0 & & & 22.6 & 0.1 & 2.9 & 0.1 & 0.4 & & 15.6 & 0.1 & & 0.1 & 0.7 & 0.2 & \begin{tabular}{|l|l|}
2 & 31.4
\end{tabular} & & 4.1 & & & 0.2 & & 0.0 & & & \\
\hline Fu, 1998 & 1150 & 1184 & 7.6 & 8.4 & 1.5 & & 2.9 & 0.3 & 0.2 & 0.3 & 0.2 & 0.8 & 11.9 & 0.5 & 0.1 & 1.0 & 3.0 & 0.2 & \begin{tabular}{|l|}
27.9 \\
\end{tabular} & 0.9 & 0.9 & 1.7 & 1.2 & 0.3 & $\begin{array}{ll}336.3 \\
\end{array}$ & 0.5 & 0.2 & 0.6 & & 0.6 & 4.5 & & 5.8 & & \\
\hline Fu, 1998 & 1150 & 1058 & 7.5 & 8.4 & 1.5 & 0.9 & 2.9 & 0.3 & 0.2 & 0.3 & 0.2 & 0.8 & 11.8 & 0.5 & 0.1 & 1.0 & 3.0 & 0.2 & 212.9 & 0.9 & \begin{tabular}{|l|}
0.9 \\
\end{tabular} & 1.7 & 1.2 & 0.3 & 330.9 & 0.5 & 0.2 & 0.6 & & 0.6 & 4.4 & & 5.8 & & \\
\hline Fu, 1998 & 1150 & 1070 & 7.5 & 13.5 & 1.5 & 0.9 & 2.9 & 0.3 & 0.2 & 0.3 & 0.2 & 0.8 & 11.8 & 0.5 & 0.1 & 1.0 & 3.0 & 0.2 & \begin{tabular}{|l|}
27.8 \\
\end{tabular} & 0.9 & 0.9 & 1.7 & 1.2 & 0.3 & $\begin{array}{l}330.9 \\
\end{array}$ & 0.5 & 0.2 & 0.6 & & 0.6 & 4.4 & & 5.8 & & \\
\hline Fu, 1998 & 1150 & 1076 & 7.5 & 8.4 & 1.5 & 0.9 & 8.0 & 0.3 & 0.2 & 0.3 & 0.2 & 0.8 & 11.8 & 0.5 & 0.1 & 1.0 & 3.0 & 0.2 & 27.8 & 0.9 & 0.9 & 1.7 & 1.2 & 0.3 & 330.9 & 0.5 & 0.2 & 0.6 & & 0.6 & 4.4 & & 5.8 & & \\
\hline Fu, 1998 & 1150 & 1015 & 7.5 & 8.4 & 1.5 & 0.9 & 2.9 & 0.3 & 0.2 & 0.3 & 0.2 & 0.8 & 11.8 & 0.5 & 3.2 & 1.0 & 3.0 & 0.2 & \begin{tabular}{|l|}
27.8 \\
\end{tabular} & 0.9 & 0.9 & 1.7 & 1.2 & 0.3 & $\begin{array}{ll}3 & 32.9 \\
\end{array}$ & 0.5 & 0.2 & 0.6 & & 0.6 & 4.4 & & 5.8 & & \\
\hline Fu, 1998 & 1150 & 1033 & 11.8 & 6.1 & 0.1 & 0.1 & 1.6 & 0.0 & & 0.1 & 0.0 & 0.4 & 26.3 & 1.1 & 3.0 & 0.3 & 0.6 & 0.0 & 14.6 & 0.3 & 0.0 & 0.0 & 1.1 & 0.4 & 428.5 & 0.1 & 2.6 & 0.0 & & 0.3 & 0.3 & & 0.2 & & \\
\hline $\mathrm{Fu}, 1998$ & 1150 & & 13.3 & 6.7 & 0.1 & & 0.5 & & & 0.2 & 0.1 & 0.1 & 21.9 & 0.5 & 4.0 & 0.1 & 0.7 & & 11.1 & 0.8 & 0.6 & 0.9 & 0.0 & 0.1 & 127.3 & & 2.9 & & & 0.1 & 0.9 & & 6.6 & & \\
\hline Fu, 1998 & 1150 & & 16.1 & 6.6 & & & 1.9 & & & & 0.5 & 2.8 & 5.7 & 1.3 & 3.1 & & & 0.6 & \begin{tabular}{|l|l|}
5 & 9.5 \\
\end{tabular} & 0.2 & & 2.1 & & 0.9 & $\begin{array}{ll}929.3 \\
\end{array}$ & 1.3 & 2.3 & & & 1.7 & & 0.4 & 12.3 & & \\
\hline Fu, 1998 & 1150 & 1117 & 16.0 & 15.1 & & & & & 0.4 & 0.7 & & & 4.7 & 1.1 & 3.1 & 2.3 & 6.7 & & 9.4 & 2.1 & 0.1 & 0.1 & & & 23.6 & & 1.8 & & & & & & 12.3 & & \\
\hline Fu, 1998 & 1150 & 920 & 10.0 & 15.3 & 0.1 & 0.1 & 0.6 & 0.0 & & 0.1 & 0.0 & & 22.1 & 0.0 & 4.1 & 0.2 & 0.5 & 0.0 & 11.3 & 0.2 & $\begin{array}{l} \\
\end{array}$ & 0.1 & 0.8 & 0.4 & 430.7 & 0.0 & 2.1 & 0.0 & & 0.2 & 0.2 & 0.0 & 0.2 & & \\
\hline Fu, 1998 & 1150 & & 7.5 & 11.2 & 0.6 & 0.1 & 1.6 & 0.2 & 0.2 & 0.4 & 0.1 & 0.1 & 17.8 & 0.6 & 2.0 & 0.6 & 0.6 & & \begin{tabular}{|l|}
16.9 \\
\end{tabular} & 0.6 & 0.2 & 1.8 & 1.1 & 0.3 & $\begin{array}{ll}326.5 \\
\end{array}$ & 0.6 & 3.4 & & 0.1 & 0.2 & 3.3 & 0.1 & 0.1 & & \\
\hline Fu, 1998 & 1150 & & 9.5 & 10.4 & 0.1 & & 6.1 & & & 0.1 & 0.1 & 0.1 & 15.6 & 0.4 & 3.1 & 0.1 & 0.5 & & 8.0 & 0.6 & \begin{tabular}{|l|} 
\\
\end{tabular} & 1.4 & 0.0 & 0.1 & 135.1 & & 2.4 & & & 0.1 & 0.6 & 0.1 & 4.7 & & \\
\hline Fu, 1998 & 1150 & 1109 & 8.5 & 6.7 & 1.2 & 0.7 & 2.3 & 0.2 & 0.1 & 0.5 & 0.1 & 0.8 & 15.9 & 0.4 & 2.9 & 0.8 & 2.4 & 0.2 & \begin{tabular}{|l|}
27.1 \\
\end{tabular} & 1.0 & 0.7 & 1.3 & 0.9 & 0.3 & $\begin{array}{ll}331.9 \\
\end{array}$ & 0.5 & 2.9 & 0.5 & & 0.5 & 3.6 & & 4.7 & & \\
\hline Fu, 1998 & 1150 & 1050 & 16.1 & 6.6 & & & 7.6 & 0.6 & & & & & \begin{tabular}{|l|}
4.7 \\
\end{tabular} & & 3.1 & & & & 18.9 & 0.2 & 1.8 & 0.4 & & & 27.4 & & 1.8 & 1.2 & 0.4 & & 9.1 & & & & \\
\hline $\mathrm{Fu}, 1998$ & 1150 & 876 & 8.5 & 6.6 & 3.9 & 2.4 & & & & & & & 23.6 & & 3.1 & & & & 18.9 & 0.2 & & 4.1 & 3.1 & & 23.6 & & 1.8 & & & & & & & & \\
\hline Fu, 1998 & 1150 & 970 & 9.2 & 12.0 & 0.2 & 0.0 & 5.6 & 0.1 & 0.1 & 0.6 & 0.0 & 0.0 & 17.0 & 0.2 & 3.0 & 0.2 & 0.2 & & 11.4 & 1.2 & 0.1 & 0.8 & 0.4 & 0.1 & \begin{tabular}{|l|l|} 
& 33.8 \\
\end{tabular} & 0.6 & 1.4 & & 0.1 & 0.1 & 1.3 & 0.0 & 0.1 & & \\
\hline Fu, 1998 & 1150 & 1018 & 8.3 & $\begin{array}{l}7.1 \\
\end{array}$ & 1.0 & & 2.0 & 0.3 & 0.1 & 0.2 & 0.1 & 0.7 & 9.7 & 2.1 & 0.1 & 0.7 & 2.1 & 0.2 & 219.5 & 0.6 & 0.6 & 1.2 & 1.9 & 0.2 & 230.2 & 0.4 & 2.5 & 0.4 & 0.2 & 0.4 & 3.1 & & 4.0 & & \\
\hline Fu, 1998 & 1150 & & 16.2 & 7.6 & 3.9 & 2.4 & 7.6 & & & & & & 10.5 & & 3.2 & & & & 9.5 & 0.2 & & 4.1 & 3.1 & & 29.6 & & 1.8 & & & & & & & & \\
\hline Fu, 1998 & 1150 & 900 & 16.1 & 16.1 & & & 7.6 & & & & 0.5 & 2.8 & 8.5 & 1.3 & 3.1 & & & 0.6 & \begin{tabular}{|l|l|}
5 & 9.5 \\
\end{tabular} & 0.2 & & 2.1 & & 0.9 & $\begin{array}{l}923.6 \\
9\end{array}$ & 1.3 & 2.3 & & & 1.7 & & 0.4 & & & \\
\hline Fu, 1998 & 1150 & & 16.1 & 6.6 & 3.6 & 2.2 & & & & & & & \begin{tabular}{|l|}
4.7 \\
\end{tabular} & & 3.1 & & & & 18.9 & 0.2 & & 3.8 & 2.8 & & 23.6 & & 1.8 & & & & & & \begin{tabular}{|l|}
12.3 \\
\end{tabular} & & \\
\hline $\mathrm{Fu}, 1998$ & 1150 & 950 & 16.1 & 11.3 & & & & & & & & & 18.9 & & 3.1 & & & & 18.9 & 0.2 & & 0.1 & & & 29.3 & & 1.8 & & & & & & & & \\
\hline
\end{tabular}


Table A1. Glass Compositions from Literature Review (continued)

\begin{tabular}{|c|c|c|c|c|c|c|c|c|c|c|c|c|c|c|c|c|c|c|c|c|c|c|c|c|c|c|c|c|c|c|c|c|c|c|c|}
\hline Ref & 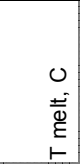 & $\begin{array}{l}0 \\
\text { a } \\
\stackrel{0}{0} \\
\stackrel{0}{=} \\
\vdash\end{array}$ & $\begin{array}{l}\text { Oొ } \\
\frac{\mathbb{1}}{\alpha}\end{array}$ & 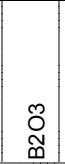 & $\begin{array}{c}\stackrel{\bigcirc}{\mathbb{D}} \\
\stackrel{\infty}{2}\end{array}$ & 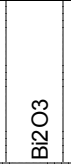 & $\begin{array}{l}0 \\
\mathbb{3}\end{array}$ & $\begin{array}{l}\text { N } \\
\mathcal{D} \\
0 \\
\tilde{N} \\
\mathcal{N} \\
\mathcal{S}\end{array}$ & 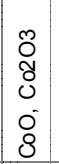 & 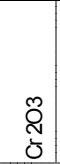 & $\stackrel{\circ}{3}$ & 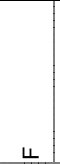 & 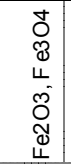 & 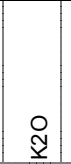 & 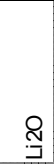 & $\begin{array}{l}\circ \\
\sum \\
\Sigma\end{array}$ & $\begin{array}{l}\tilde{O} \\
\sum \\
\Sigma\end{array}$ & $\begin{array}{l}0 \\
\text { Oे } \\
\stackrel{0}{2}\end{array}$ & $\begin{array}{l}\stackrel{ }{\mathbb{N}} \\
\mathbb{Z}\end{array}$ & $\stackrel{\circ}{z}$ & ָิ & 吾 & $\underset{N}{\stackrel{D}{\alpha}}$ & $\begin{array}{l}\circ \\
\frac{0}{2}\end{array}$ & ก๊ & ర్ల & 임 & 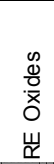 & $\begin{array}{l}\tilde{N} \\
\text { \& }\end{array}$ & 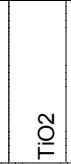 & 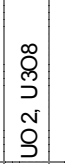 & ్N & ֻั & 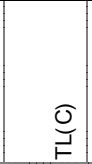 & $\begin{array}{l}\bar{\partial} \\
\frac{0}{0} \\
\frac{1}{z}\end{array}$ \\
\hline $\mathrm{Fu}, 1998$ & 1150 & & 17.1 & 8.9 & 0.3 & 0.0 & 1.5 & 0.1 & 0.1 & 0.8 & 0.0 & 0.1 & 10.6 & 0.9 & & 0.3 & 0.7 & & 22.0 & 0.3 & 0.1 & 1.1 & 0.8 & 0.2 & 28.6 & 0.3 & 2.3 & & 0.1 & 0.1 & 1.9 & 0.1 & 0.2 & & \\
\hline Fyles, 1999 & & & 14.1 & & & & 15.4 & & & & & & 5.2 & 0.6 & & 12.6 & 0.2 & & 2.5 & & & & & & 47.6 & & & & & 1.7 & & & & & \\
\hline Fyles, 1999 & & & 1.6 & & & 3.5 & 8.0 & & & & & & 0.3 & 1.0 & & 3.8 & & & 15.5 & & & & & & 66.2 & 0.2 & & & & & & & & & \\
\hline Fyles, 1999 & & 1412 & 1.6 & & & & 8.7 & & & & & & 0.3 & 0.9 & & 4.0 & & & 15.0 & & & & & & 69.3 & 0.2 & & & & & & & & & \\
\hline Fyles, 1999 & & 1424 & 1.7 & & & & 9.2 & & & & & & 0.3 & 0.8 & & 4.2 & & & 13.0 & & & & & & 70.6 & 0.2 & & & & & & & & & \\
\hline Fyles, 1999 & & 1480 & 1.7 & & & & 9.2 & & & & & & 0.3 & 0.8 & & 4.2 & & & 11.0 & & & & & & 72.6 & 0.2 & & & & & & & & & \\
\hline Fyles, 1999 & & 1509 & 1.7 & & & & 9.2 & & & & & & 0.3 & 0.8 & & 4.2 & & & 10.0 & & & & & & 73.6 & 0.2 & & & & & & & & & \\
\hline Fyles, 1999 & & 1537 & 1.7 & & & & 9.2 & & & & & & 0.3 & 0.8 & & 4.2 & & & 9.0 & & & & & & 74.6 & 0.2 & & & & & & & & & \\
\hline Fyles, 1999 & & & 1.7 & & & & 9.2 & & & & & & 0.3 & 0.8 & & 4.2 & & & 7.0 & & & & & & 76.6 & 0.2 & & & & & & & & & \\
\hline Fyles, 1999 & & 1397 & 16.0 & & & & 19.0 & & & & & & 4.0 & 1.0 & & 4.0 & & & 2.0 & & & & & & 53.8 & 0.2 & & & & & & & & & \\
\hline Gal'Perina, 1981 & $<1300$ & & 14.2 & 12.0 & & & 5.6 & & & & & & 5.7 & 1.4 & & 2.9 & 0.4 & & 3.1 & & & & & & 48.1 & & & & & 0.4 & & & 6.2 & & \\
\hline Gal'Perina, 1981 & $<1300$ & & 12.8 & 18.3 & & & 8.9 & & & & & & 5.3 & 1.3 & & 2.8 & 0.3 & & 3.1 & & & & & & 45.8 & & & & & 0.4 & & & & & \\
\hline Galushko, 1971 & $\sim 1350$ & & 18.7 & & & & 7.2 & & & & & & 13.1 & & & 6.7 & & & 3.9 & & & & & & 49.4 & & & & & 1.2 & & & & & \\
\hline Galushko, 1971 & $\sim 1350$ & & 18.5 & & & & 7.1 & & & 1.1 & & & 12.8 & & & 6.9 & & & 4.0 & & & & & & 48.6 & & & & & 1.2 & & & & & \\
\hline Galushko, 1971 & $\sim 1350$ & & 17.5 & & & & 6.5 & & & & & & 11.5 & & & 6.0 & & & 3.7 & & & & & & 53.8 & & & & & 1.0 & & & & & \\
\hline Galushko, 1971 & $\sim 1350$ & & 16.3 & & & & 5.8 & & & & & & 10.1 & & & 5.2 & & & 3.3 & & & & & & 58.2 & & & & & 1.0 & & & & & \\
\hline Galushko, 1971 & $\sim 1350$ & & 17.7 & & & & 9.4 & & & 1.1 & & & 11.8 & & & 8.7 & & & 3.8 & & & & & & 45.8 & & & & & 1.1 & & & & & \\
\hline Galushko, 1971 & $\sim 1350$ & & 20.2 & & & & 6.6 & & & 1.1 & & & 11.8 & & & 6.5 & & & 3.7 & & & & & & 48.9 & & & & & 1.2 & & & & & \\
\hline Galushko, 1974 & & & 18.7 & & & & 7.2 & & & & & & 13.1 & & & 6.7 & & & 3.9 & & & & & & 49.4 & & & & & 1.2 & & & & 1300 & \\
\hline Galushko, 1974 & & & 18.5 & & & & 7.1 & & & 1.1 & & & 12.8 & & & 6.9 & & & 4.0 & & & & & & 48.6 & & & & & 1.2 & & & & $>1350$ & \\
\hline Galushko, 1974 & & & 17.5 & & & & 6.5 & & & & & & 11.5 & & & 6.0 & & & 3.7 & & & & & & 53.8 & & & & & 1.0 & & & & $>1200$ & \\
\hline Galushko, 1974 & & & 16.3 & & & & 5.8 & & & & & & 10.1 & & & 5.2 & & & 3.3 & & & & & & 58.2 & & & & & 1.0 & & & & $>1200$ & \\
\hline Galushko, 1974 & & & 17.7 & & & & 9.4 & & & 1.1 & & & 11.8 & & & 8.7 & & & 3.8 & & & & & & 45.8 & & & & & 1.1 & & & & $>1350$ & \\
\hline Galushko, 1974 & & & 20.2 & & & & 6.6 & & & 1.1 & & & 11.8 & & & 6.5 & & & 3.7 & & & & & & 48.9 & & & & & 1.2 & & & & $>1350$ & \\
\hline Gerasimov, 2004 & 1300 & & 5.7 & 19.7 & & & & & & & & & & 2.1 & & & & & 7.9 & & & & & & 59.9 & & & & & 4.7 & & & & & \\
\hline Gerasimov, 2004 & 1250 & & 10.6 & 23.6 & & & 10.4 & & & & & & & & & & & & 5.4 & & & & & & 44.4 & & & & & & & & 5.4 & & \\
\hline Gerasimov, 2004 & & & & 8.0 & 5.5 & 42.5 & & & & & 8.0 & & & & & & & & & & & & & & 30.0 & & & & & 2.0 & & 5.5 & & & \\
\hline Gerasimov, 2004 & 1450 & & 7.8 & 16.0 & 2.0 & & & & & & & & & & & 2.3 & & & 8.0 & & & & & & 61.5 & & & & & & & & 2.5 & & \\
\hline Gerasimov, 2004 & 1250 & & 10.9 & 18.1 & & & 6.2 & & & & & & & 3.8 & & 5.3 & & & 5.4 & & & & & & 50.4 & & & & & & & & & & \\
\hline Gerasimov, 2004 & 1250 & & 9.6 & 16.3 & & & 13.2 & & & & & & & 10.3 & & & & & & & & & & & 48.6 & & 1.1 & & & & & & & & \\
\hline Gerasimov, 2004 & & & 25.5 & 5.2 & 3.0 & & 9.0 & & & & & & & & & 7.0 & & & 1.5 & & & & & & 50.0 & & & & & & & & & & \\
\hline Gerasimov, 2004 & 1400 & & 7.5 & 36.0 & 6.0 & & 4.3 & & & & & & & 3.3 & & & & & 3.5 & & & & & & 37.0 & & & & & & & & & & \\
\hline Gerasimov, 2004 & & & 13.5 & 5.7 & & & 0.7 & & & & & 8.5 & 0.6 & & & 0.3 & & & 18.6 & & & & & & 52.7 & & & & & & & & & & \\
\hline Gerasimov, 2004 & 1250 & & 7.4 & & & & 4.3 & & & & & & 13.1 & 3.3 & & 0.7 & & & 1.5 & & & 3.6 & & & 59.7 & & & & & & & 4.3 & & & \\
\hline Gerasimov, 2004 & & & 12.0 & 10.1 & 0.5 & & & 2.8 & & & 4.0 & & 0.3 & 2.5 & & & & & 12.4 & & & & & & 54.8 & & & & & & & & & & \\
\hline Gerasimov, 2004 & & & 9.0 & 13.5 & & & 5.5 & & & & & & 0.3 & 2.0 & & 0.3 & & & 8.0 & & & & & & 46.0 & & & & & & & 5.0 & 9.5 & & \\
\hline Gerasimov, 2004a & 1325 & & 11.6 & 13.2 & 5.1 & & 4.0 & & & & & & & & & & & & 4.8 & & & & & & 57.7 & & 3.6 & & & & & & & & \\
\hline Gerasimov, 2004a & 1290 & & 11.9 & 24.2 & & & 2.9 & & & & & & 3.1 & 2.8 & & 1.8 & 1.1 & & 2.8 & & & & & & 42.5 & & & & & 7.5 & & & & & \\
\hline Gerasimov, 2004a & 1300 & & 11.4 & 15.3 & & & 4.6 & & & & & & 0.3 & 1.3 & & 0.3 & & & 1.4 & & & & & & 44.4 & & & & & & & 8.2 & 12.7 & & \\
\hline Gerasimov, 2004a & 1275 & & 9.0 & 27.0 & & & & & & & & & & 5.5 & & & & & 5.5 & & & & & & 36.0 & & & & & & & 5.0 & 9.0 & & \\
\hline Gerasimov, 2004a & 1325 & & 10.0 & 10.5 & & & 9.0 & & & & & & & 0.8 & & 8.5 & & & 0.8 & & & & & & 60.5 & & & & & & & & & & \\
\hline Gerasimov, 2004a & 1300 & & 13.0 & 15.3 & & & 7.9 & & & & & & 0.6 & 1.8 & & 0.6 & & & 1.8 & & & & & & 56.6 & & & & & & & & 2.5 & & \\
\hline Gerasimov, 2004a & 1150 & & 2.5 & 17.5 & & 1.5 & & & & & & & 0.2 & 13.5 & & & & & & & & & & & 42.5 & 1.8 & & & & 22.5 & & & & & \\
\hline Gerasimov, 2004a & 1400 & & 4.6 & 11.4 & & & 14.8 & & & & & & & 6.5 & & 2.0 & & & 0.7 & & & & & & 53.0 & 3.0 & & & & 5.0 & & & & & \\
\hline Gerasimov, 2004a & 950 & & 7.4 & 36.2 & & & & & & & & & 0.2 & 0.8 & & & & & 17.1 & & & & & 11.1 & 21.9 & & & & & & & 3.6 & & & \\
\hline Gerasimov, 2004a & 1315 & & 5.8 & 17.8 & 6.5 & & & & & & & & & & & & & & 7.8 & & & & & & 40.4 & & 3.8 & & & & & 7.8 & 6.4 & & \\
\hline Giordano, 2002 & & 1968 & 18.0 & & & & 2.9 & & & & & & 3.4 & 7.9 & & 0.7 & 0.1 & & 4.5 & & & & 0.2 & & 60.1 & & & & & 0.4 & & & & & \\
\hline Giordano, 2002 & & 1951 & 18.0 & & & & 2.9 & & & & & & 3.8 & 8.4 & & 0.9 & 0.1 & & 4.1 & & & & 0.2 & & 60.0 & & & & & 0.4 & & & & & \\
\hline Giordano, 2002 & & 1792 & 18.5 & & & & 2.8 & & & & & & 3.6 & 8.5 & & 0.7 & 0.2 & & 3.9 & & & & 0.2 & & 59.7 & & & & & 0.5 & & & & & \\
\hline Giordano, 2002 & & 1211 & 12.1 & & & & 15.7 & & & & & & 10.1 & 3.0 & & 11.2 & & & 2.8 & & & & 1.0 & & 41.2 & & & & & 2.7 & & & & & \\
\hline
\end{tabular}


Table A1. Glass Compositions from Literature Review (continued)

\begin{tabular}{|c|c|c|c|c|c|c|c|c|c|c|c|c|c|c|c|c|c|c|c|c|c|c|c|c|c|c|c|c|c|c|c|c|c|c|c|}
\hline Ref & 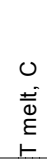 & $\begin{array}{l}0 \\
\stackrel{0}{0} \\
\stackrel{0}{0} \\
\stackrel{5}{1}\end{array}$ & $\begin{array}{l}00 \\
\stackrel{N}{\alpha}\end{array}$ & $\underset{\infty}{\widetilde{\infty}}$ & $\begin{array}{l}\text { \& } \\
\infty\end{array}$ & $\begin{array}{c}\text { Oొ } \\
\stackrel{N}{\infty}\end{array}$ & $\begin{array}{l}0 \\
\pi \\
0\end{array}$ & 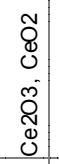 & $\begin{array}{l}m \\
0 \\
0 \\
0 \\
0 \\
0 \\
0\end{array}$ & $\begin{array}{l}\text { Õ } \\
\text { Ũ }\end{array}$ & $\begin{array}{l}0 \\
3 \\
0\end{array}$ & $\leftarrow$ & 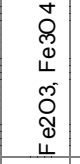 & $\begin{array}{l}\underset{\mathcal{X}}{x} \\
\end{array}$ & 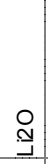 & $\begin{array}{l}0 \\
\stackrel{O}{\Sigma}\end{array}$ & $\begin{array}{l}\tilde{O} \\
\stackrel{\Sigma}{\Sigma}\end{array}$ & $\begin{array}{l}0 \\
0 \\
\Sigma \\
\Sigma\end{array}$ & $\begin{array}{l}\text { Q } \\
\text { Z } \\
Z\end{array}$ & $\frac{0}{z}$ & $\begin{array}{l}\mathcal{N} \\
\tilde{0}\end{array}$ & $\overline{\bar{I}}$ & $\begin{array}{l}\text { ฉి } \\
\vdots \\
a\end{array}$ & $\frac{8}{2}$ & $\frac{\widetilde{O}}{\omega}$ & Oొ & $\begin{array}{l}\text { Q } \\
\text { is }\end{array}$ & 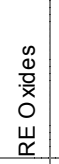 & 杄 & $\begin{array}{l}\tilde{O} \\
\stackrel{2}{F}\end{array}$ & 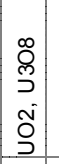 & $\begin{array}{l}\text { Q } \\
N\end{array}$ & $\begin{array}{c}\tilde{N} \\
\text { N }\end{array}$ & $\begin{array}{l}\hat{0} \\
\underline{1} \\
\hat{1}\end{array}$ & $\begin{array}{l}\bar{\Xi} \\
\text { क् } \\
\underline{\underline{0}} \\
\dot{z}\end{array}$ \\
\hline Giordano, 2002 & & 1360 & 16.3 & & & & 10.5 & & & & & & 10.1 & 1.9 & & 5.2 & 0.2 & & 3.8 & & & & 0.6 & & 47.0 & & & & & 1.6 & & & & & \\
\hline Giordano, 2002 & & 2122 & 12.5 & & & & & & & & & & & 4.2 & & & & & 4.6 & & & & & & 78.6 & & & & & & & & & & \\
\hline Giordano, 2002 & & 1782 & 19.2 & & & & 2.1 & & & & & & 3.4 & 6.3 & & 0.3 & 0.2 & & 5.3 & & & & 0.1 & & 60.7 & & & & & 0.3 & & & & & \\
\hline Giordano, 2002 & & 1829 & 17.1 & & & & 1.8 & & & & & & 2.9 & 6.8 & & 0.2 & 0.1 & & 5.7 & & & & 0.1 & & 63.9 & & & & & 0.3 & & & & & \\
\hline Giordano, 2002 & & 2045 & 17.3 & & & & 5.8 & & & & & & 5.4 & 1.4 & & 3.4 & & & 3.9 & & & & & & 61.2 & & & & & 0.8 & & & & & \\
\hline Giordano, 2002 & & 1205 & 10.2 & & & & 26.1 & & & & & & & 1.0 & & 9.2 & & & 7.6 & & & & & & 43.6 & & & & & 3.0 & & & & & \\
\hline Giordano, 2002 & & 1879 & 17.0 & & & & 0.8 & & & & & & 2.6 & 6.4 & & 0.3 & 0.1 & & 6.3 & & & & 0.1 & & 64.0 & & & & & 0.5 & & & & & \\
\hline Giordano, 2002 & & 1811 & 18.8 & & & & 0.7 & & & & & & 3.3 & 5.5 & & 0.4 & 0.2 & & 9.8 & & & & 0.1 & & 60.5 & & & & & 0.6 & & & & & \\
\hline Giordano, 2002 & & 1758 & 15.2 & & & & 5.0 & & & & & & 4.1 & 2.2 & & 2.2 & 0.1 & & 3.8 & & & & 0.1 & & 66.0 & & & & & 0.4 & & & & & \\
\hline Giordano, 2002 & & 1606 & 19.1 & & & & 6.5 & & & & & & 4.6 & 8.0 & & 1.7 & 0.1 & & 4.6 & & & & 0.7 & & 51.2 & & & & & 0.6 & & & & & \\
\hline Giordano, 2002 & & 1427 & 16.4 & & & & 10.2 & & & & & & 7.2 & 6.5 & & 5.1 & 0.1 & & 2.7 & & & & 0.7 & & 49.2 & & & & & 0.8 & & & & & \\
\hline Giordano, 2002 & & 1563 & 19.3 & & & & 6.6 & & & & & & 4.7 & 7.7 & & 1.7 & 0.1 & & 4.5 & & & & 0.7 & & 52.0 & & & & & 0.6 & & & & & \\
\hline Giordano, 2002 & & 1528 & 18.6 & & & & 7.3 & & & & & & 6.1 & 7.9 & & 2.5 & 0.1 & & 3.8 & & & & 0.4 & & 51.2 & & & & & 0.7 & & & & & \\
\hline Giordano, 2002 & & 1846 & 19.4 & & & & 2.4 & & & & & & & 7.4 & & 1.9 & & & 9.3 & & & & & & 58.8 & & & & & 0.8 & & & & & \\
\hline Giordano, 2002 & & 1799 & 16.7 & & & & 5.4 & & & & & & & 3.4 & & 2.9 & & & 6.7 & & & & & & 64.5 & & & & & 0.5 & & & & & \\
\hline Giordano, 2002 & & 1313 & 14.0 & & & & 15.0 & & & & & & & 3.0 & & 8.8 & & & 7.0 & & & & & & 50.6 & & & & & 2.4 & & & & & \\
\hline Giordano, 2003 & & 1212 & 16.3 & & & & 10.5 & & & & & & 10.9 & 1.9 & & 5.2 & 0.2 & 0.0 & 3.8 & & & & 0.6 & & 47.0 & & & & & 1.6 & & & & & \\
\hline Giordano, 2006 & & & 18.0 & & & & 2.9 & & & & & & 3.4 & 7.9 & & 0.7 & 0.1 & & 4.5 & & & & 0.2 & & 60.1 & & & & & 0.4 & & & & & \\
\hline Giordano, 2006 & & & 18.0 & & & & 2.9 & & & & & & 3.8 & 8.4 & & 0.9 & 0.1 & & 4.1 & & & & 0.2 & & 60.0 & & & & & 0.4 & & & & & \\
\hline Giordano, 2006 & & & 18.8 & & & & 2.9 & & & & & & 3.7 & 8.6 & & 0.7 & 0.2 & & 4.0 & & & & 0.2 & & 60.7 & & & & & 0.5 & & & & & \\
\hline Giordano, 2006 & & 1833 & 12.6 & & & & 3.4 & & & & & & 3.2 & 6.2 & & 1.2 & 0.1 & & 4.0 & & & & 0.0 & & 68.8 & & & & & 0.2 & & & & & \\
\hline Giordano, 2006 & & & 12.1 & & & & 15.7 & & & & & & 10.1 & 3.0 & & 11.2 & 0.2 & & 2.8 & & & & 1.0 & & 41.1 & & & & & 2.7 & & & & & \\
\hline Giordano, 2006 & & & 16.3 & & & & 10.5 & & & & & & 10.1 & 1.9 & & 5.2 & 0.2 & & 3.8 & & & & 0.6 & & 47.0 & & & & & 1.6 & & & & & \\
\hline Giordano, 2006 & & 1600 & 18.4 & & & & 5.8 & & & & & & 7.3 & 4.6 & & 2.4 & 0.2 & & 4.2 & & & & & & 55.4 & & & & & 0.7 & & & & & \\
\hline Giordano, 2006 & & & 12.5 & & & & & & & & & & & 4.2 & & & & & 4.6 & & & & & & 78.6 & & & & & & & & & & \\
\hline Giordano, 2006 & & & 15.6 & & & & 2.1 & & & & & & 1.0 & 3.8 & & & & & 4.4 & & & & & & 73.6 & & & & & & & & & & \\
\hline Giordano, 2006 & & & 17.3 & & & & 4.0 & & & & & & & 3.5 & & & & & 4.2 & & & & & & 71.5 & & & & & & & & & & \\
\hline Giordano, 2006 & & & 23.1 & & & & 8.7 & & & & & & & 1.9 & & & & & 2.6 & & & & & & 64.0 & & & & & & & & & & \\
\hline Giordano, 2006 & & & 27.2 & & & & 13.3 & & & & & & & 1.5 & & & & & 1.6 & & & & & & 56.2 & & & & & & & & & & \\
\hline Giordano, 2006 & & & 19.2 & & & & 2.1 & & & & & & 3.4 & 6.3 & & 0.3 & 0.2 & & 5.3 & & & & 0.1 & & 60.7 & & & & & 0.3 & & & & & \\
\hline Giordano, 2006 & & 2065 & 9.9 & & & & 2.4 & & & & & & 1.9 & 3.4 & & 1.6 & 0.0 & & 0.5 & & & & & & 79.4 & & & & & 0.2 & & & & & \\
\hline Giordano, 2006 & & & 20.0 & & & & 9.1 & & & & & & 0.0 & 0.9 & & 3.2 & 0.0 & & 3.5 & & & & 0.1 & & 62.5 & & & & & 0.6 & & & & & \\
\hline Giordano, 2006 & & & 16.1 & & & & 9.9 & & & & & & 7.5 & 3.7 & & 5.6 & 0.1 & & 2.3 & & & & & & 52.3 & & & & & 0.8 & & & & & \\
\hline Giordano, 2006 & & & 17.5 & & & & 8.1 & & & & & & 7.2 & 3.5 & & 3.8 & 0.2 & & 3.6 & & & & & & 53.7 & & & & & 0.6 & & & & & \\
\hline Giordano, 2006 & & & 17.1 & & & & 1.8 & & & & & & 2.9 & 6.8 & & 0.2 & 0.1 & & 5.7 & & & & 0.1 & & 63.9 & & & & & 0.3 & & & & & \\
\hline Giordano, 2006 & & 1493 & 19.0 & & & & 9.2 & & & & & & 9.0 & 1.6 & & 3.4 & 0.2 & & 3.5 & & & & & & 53.5 & & & & & 0.8 & & & & & \\
\hline Giordano, 2006 & & & 17.1 & & & & 4.7 & & & & & & 5.0 & 1.3 & & 1.8 & 0.1 & & 4.3 & & & & 0.1 & & 65.3 & & & & & 0.6 & & & & & \\
\hline Giordano, 2006 & & 1622 & 18.3 & & & & 7.1 & & & & & & 6.4 & 0.9 & & 2.6 & 0.2 & & 3.6 & & & & & & 60.7 & & & & & 0.6 & & & & & \\
\hline Giordano, 2006 & & & 20.0 & & & & 9.1 & & & & & & 0.0 & 0.9 & & 3.2 & 0.0 & & 3.5 & & & & 0.1 & & 62.4 & & & & & 0.6 & & & & & \\
\hline Giordano, 2006 & & & 10.2 & & & & 26.1 & & & & & & & 1.0 & & 9.2 & & & 7.6 & & & & & & 43.6 & & & & & 3.0 & & & & & \\
\hline Giordano, 2006 & & & 15.0 & & & & 10.4 & & & & & & 12.0 & 5.6 & & 3.7 & 0.3 & & 6.9 & & & & 1.2 & & 41.1 & & & & & 2.8 & & & & & \\
\hline Giordano, 2006 & & & 18.4 & & & & 4.0 & & & & & & 5.0 & 7.7 & & 1.4 & 0.1 & & 3.4 & & & & & & 58.8 & & & & & 0.5 & & & & & \\
\hline Giordano, 2006 & & & 17.3 & & & & 0.9 & & & & & & 2.6 & 6.5 & & 0.3 & 0.1 & & 6.5 & & & & 0.1 & & 65.3 & & & & & 0.5 & & & & & \\
\hline Giordano, 2006 & & & 13.3 & & & & 10.0 & & & & & & 11.2 & 1.4 & & 9.2 & & & 5.6 & & & & & & 46.6 & & & & & 2.5 & & & & & \\
\hline Giordano, 2006 & & & 12.8 & & & & 9.6 & & & & & & 9.9 & 1.5 & & 8.1 & & & 6.1 & & & & & & 49.3 & & & & & 2.1 & & & & & \\
\hline Giordano, 2006 & & & 12.1 & & & & 9.2 & & & & & & 8.9 & 1.8 & & 7.2 & & & 6.5 & & & & & & 51.6 & & & & & 1.5 & & & & & \\
\hline Giordano, 2006 & & & 9.9 & & & & 5.3 & & & & & & 7.2 & 2.3 & & 4.6 & & & 9.0 & & & & & & 59.0 & & & & & 1.6 & & & & & \\
\hline Giordano, 2006 & & & 13.1 & & & & 10.0 & & & & & & 10.2 & 1.5 & & 8.9 & & & 5.6 & & & & & & 48.2 & & & & & 2.3 & & & & & \\
\hline Giordano, 2006 & & & 4.2 & & & & 2.0 & & & & & & 1.2 & 3.4 & & 2.0 & & & 11.3 & & & & & & 74.8 & & & & & 0.5 & & & & & \\
\hline Giordano, 2006 & & 1204 & 12.5 & & & & 11.5 & & & & & & 11.3 & 1.1 & & 11.4 & 0.3 & & 2.7 & & & & 0.9 & & 45.8 & & & & & 2.3 & & & & & \\
\hline
\end{tabular}


Table A1. Glass Compositions from Literature Review (continued)

\begin{tabular}{|c|c|c|c|c|c|c|c|c|c|c|c|c|c|c|c|c|c|c|c|c|c|c|c|c|c|c|c|c|c|c|c|c|c|c|c|}
\hline Ref & 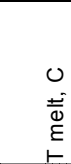 & $\begin{array}{l}0 \\
\stackrel{0}{0} \\
\stackrel{0}{0} \\
\vdash\end{array}$ & 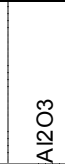 & ర్లి & $\begin{array}{l}\mathscr{D} \\
\infty\end{array}$ & @્̊ & $\begin{array}{l}0 \\
\mathbb{0} \\
0\end{array}$ & $\begin{array}{l}\tilde{D} \\
0 \\
0 \\
\tilde{N} \\
0 \\
0\end{array}$ & $\begin{array}{l}\text { Oొ } \\
\text { రิ } \\
0 \\
0 \\
0 \\
0\end{array}$ & 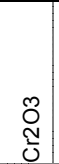 & $\stackrel{0}{3}$ & 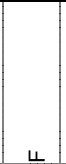 & 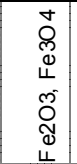 & $\underset{\tilde{\pi}}{\underline{x}}$ & $\stackrel{\stackrel{\Upsilon}{\beth}}{ }$ & $\begin{array}{l}\circ \\
\sum \\
\sum\end{array}$ & $\begin{array}{l}\tilde{O} \\
\sum \\
\Sigma\end{array}$ & $\begin{array}{l}0 \\
\stackrel{0}{\Sigma}\end{array}$ & $\begin{array}{l}\underset{\pi}{\pi} \\
\underset{z}{Z}\end{array}$ & $\frac{0}{z}$ & 正 & $\overline{\bar{\Xi}}$ & 足 & $\frac{8}{0}$ & $\stackrel{\widetilde{O}}{\omega}$ & $\begin{array}{l}0 \\
0 \\
\infty\end{array}$ & 옹 & 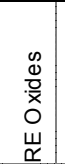 & 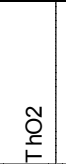 & $\stackrel{\widetilde{N}}{\stackrel{\tilde{O}}{1}}$ & 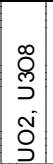 & $\stackrel{q}{N}$ & $\begin{array}{l}\text { ญे } \\
\text { N }\end{array}$ & $\underset{1}{0}$ & $\begin{array}{l}\bar{\partial} \\
\text { 을 } \\
\bar{m} \\
z\end{array}$ \\
\hline Giordano, 2006 & & 1386 & 16.9 & & & & 10.9 & & & & & & 8.4 & 2.2 & & 5.7 & 0.2 & & 2.6 & & & & & & 49.1 & & & & & 1.0 & & & & & \\
\hline Giordano, 2006 & & & 18.8 & & & & 0.7 & & & & & & 3.3 & 5.5 & & 0.4 & 0.2 & & 9.8 & & & & 0.1 & & 60.5 & & & & & 0.6 & & & & & \\
\hline Giordano, 2006 & & & 15.2 & & & & 5.0 & & & & & & 4.1 & 2.2 & & 2.2 & 0.1 & & 3.8 & & & & 0.1 & & 66.0 & & & & & 0.4 & & & & & \\
\hline Giordano, 2006 & & & 19.1 & & & & 6.5 & & & & & & 4.6 & 8.0 & & 1.7 & 0.1 & & 4.6 & & & & 0.7 & & 51.2 & & & & & 0.6 & & & & & \\
\hline Giordano, 2006 & & & 16.6 & & & & 10.3 & & & & & & 7.3 & 6.6 & & 5.2 & 0.1 & & 2.7 & & & & 0.7 & & 49.7 & & & & & 0.8 & & & & & \\
\hline Giordano, 2006 & & & 19.3 & & & & 6.6 & & & & & & 4.7 & 7.7 & & 1.7 & 0.1 & & 4.5 & & & & 0.7 & & 52.0 & & & & & 0.6 & & & & & \\
\hline Giordano, 2006 & & & 18.9 & & & & 7.4 & & & & & & 6.2 & 8.0 & & 2.5 & 0.1 & & 3.8 & & & & 0.4 & & 51.9 & & & & & 0.7 & & & & & \\
\hline Giordano, 2006 & & & 19.4 & & & & 2.4 & & & & & & & 7.4 & & 1.9 & & & 9.3 & & & & & & 58.8 & & & & & 0.8 & & & & & \\
\hline Giordano, 2006 & & & 16.7 & & & & 5.4 & & & & & & & 3.4 & & 2.9 & & & 6.7 & & & & & & 64.5 & & & & & 0.5 & & & & & \\
\hline Giordano, 2006 & & & 14.0 & & & & 15.0 & & & & & & & 3.0 & & 8.8 & & & 7.0 & & & & & & 50.6 & & & & & 2.4 & & & & & \\
\hline Grandy, 1993 & & 973 & 7.4 & & & & 8.3 & & & & & & 34.5 & 2.4 & & 4.6 & & & 4.8 & & & & & & 38.0 & & & & & & & & & 1450 & \\
\hline Grandy, 1993 & & & 13.0 & & & & 10.2 & & & & & & 4.5 & 2.9 & & 2.4 & & & 1.7 & & & & & & 65.4 & & & & 0.6 & & & & & & \\
\hline Grandy, 1993 & & 1187 & 9.0 & & & & 9.1 & & & & & & 26.4 & 2.5 & & 4.0 & & & 3.9 & & & & & & 45.1 & & & & & & & & & 1350 & \\
\hline Grandy, 1993 & & 1251 & 10.3 & & & & 9.7 & & & & & & 19.6 & 2.6 & & 3.5 & & & 3.2 & & & & & & 51.0 & & & & & & & & & 1350 & \\
\hline Grandy, 1993 & & 1408 & 11.4 & & & & 10.3 & & & & & & 13.9 & 2.7 & & 3.1 & & & 2.6 & & & & & & 56.0 & & & & & & & & & 1275 & \\
\hline Grandy, 1993 & & 1593 & 12.1 & & & & 10.0 & & & & & & 9.5 & 2.8 & & 2.8 & & & 2.2 & & & & & & 60.6 & & & & & & & & & & \\
\hline Grum-Grzhimailo, & & & 2.5 & 27.4 & 1.9 & & 7.6 & & & & & 0.9 & & & & & & & 6.1 & & & & & & 45.8 & & & & & & & & 7.9 & 925 & \\
\hline Grum-Grzhimailo, & & & 2.5 & 27.7 & & & 8.4 & & & & & 1.0 & & & & & & & 6.2 & & & & & & 46.3 & & & & & & & & 7.9 & 850 & \\
\hline Grum-Grzhimailo, & & & 2.6 & 28.4 & & & & & & & & 1.0 & & & & 6.2 & & & 6.3 & & & & & & 47.5 & & & & & & & & 8.1 & 725 & \\
\hline Grum-Grzhimailo, & & & 2.5 & 27.4 & & & 6.9 & & & & & 0.9 & & & & & & & 6.1 & & & & & & 45.8 & & 2.6 & & & & & & 7.9 & 895 & \\
\hline Grum-Grzhimailo, & & & 2.4 & 25.9 & & & & & & & & 0.9 & & & & & & & 5.8 & & & & & & 43.3 & & 14.4 & & & & & & 7.4 & 875 & \\
\hline Grum-Grzhimailo, & & & 2.4 & 26.7 & & & & & & & & 0.9 & & & & & & & 5.9 & & & & & & 44.7 & & & & & & & 11.7 & 7.7 & 720 & \\
\hline Grum-Grzhimailo, & & & 12.9 & 14.8 & 1.9 & & 6.1 & & & & & & 0.5 & 0.3 & & 4.2 & & & 5.2 & & & & & & 44.7 & & & & & & & 2.5 & 5.2 & & \\
\hline Grum-Grzhimailo, & & & 12.4 & 16.1 & 1.9 & & 6.2 & & & & & 0.6 & 0.4 & 0.3 & & 4.0 & & & 5.2 & & & & & & 44.1 & & & & & & & 2.7 & 5.2 & & \\
\hline Grum-Grzhimailo, & & & 12.7 & 15.6 & 1.9 & & 5.8 & & & & & 1.4 & 0.5 & 0.4 & & 3.9 & & & 5.3 & & & & & & 44.8 & & & & & & & 2.4 & 4.8 & & \\
\hline Grum-Grzhimailo, & & & 12.9 & 15.4 & 1.9 & & 5.6 & & & & & 2.2 & 0.6 & 0.4 & & 3.8 & & & 5.6 & & & & & & 44.3 & & & & & & & 2.4 & 4.7 & & \\
\hline Grum-Grzhimailo, & & & 12.9 & 14.8 & 1.8 & & 5.8 & & & & & 2.8 & 0.5 & 0.5 & & 3.4 & & & 5.6 & & & & & & 44.8 & & & & & & & 2.3 & 4.5 & & \\
\hline Hrma, 1994 & 1546 & 1407 & 12.0 & 8.5 & 0.2 & & & 0.3 & & 0.2 & 0.3 & 0.5 & 2.0 & & 1.0 & & 0.2 & 0.5 & 9.5 & 1.0 & & 2.5 & 0.2 & & 57.0 & 0.5 & 0.2 & 3.3 & & & & & & & \\
\hline Hrma, 1994 & 1266 & 1203 & 14.0 & 15.5 & 0.1 & & 10.0 & 0.2 & & 0.2 & 0.2 & 0.4 & 2.0 & & 1.0 & & 0.2 & 0.4 & 7.5 & 0.8 & & 2.0 & 0.1 & & 42.0 & 0.4 & 0.1 & 2.7 & & & & & & & \\
\hline Hrma, 1994 & 1070 & 1203 & 14.4 & 5.0 & 0.0 & & & 0.0 & & 0.0 & 0.0 & 0.1 & 8.6 & & 1.0 & 8.0 & 0.0 & 0.1 & 18.7 & 0.1 & & 0.3 & 0.0 & & 43.3 & 0.0 & 0.0 & 0.3 & & & & & & & \\
\hline Hrma, 1994 & 1266 & 1182 & 15.0 & 5.0 & 0.0 & & 10.0 & 0.0 & & 0.0 & 0.0 & 0.1 & 2.0 & & 7.0 & & 0.0 & \begin{tabular}{|l|} 
\\
\end{tabular} & 5.0 & 0.1 & & 0.3 & 0.0 & & 55.0 & 0.0 & 0.0 & \begin{tabular}{|l|}
0.3 \\
\end{tabular} & & & & & & & \\
\hline Hrma, 1994 & 1072 & 1011 & 14.0 & 20.0 & 0.0 & & & 0.0 & & 0.0 & 0.0 & 0.1 & 2.0 & & 7.0 & 8.0 & 0.0 & 0.1 & 5.0 & 0.1 & & 0.3 & 0.0 & & 42.0 & 0.0 & 0.0 & 0.3 & & & & & 1.0 & & \\
\hline Hrma, 1994 & 1277 & 1183 & 8.0 & 5.0 & 0.0 & & & 0.0 & & 0.0 & 0.0 & 0.1 & 15.0 & & 7.0 & & 0.0 & 0.1 & 7.0 & 0.1 & & 0.3 & 0.0 & & 57.0 & 0.0 & 0.0 & 0.3 & & & & & & & \\
\hline Hrma, 1994 & 1224 & 1137 & 10.4 & 17.0 & 0.1 & & & 0.1 & & 0.1 & 0.1 & 0.2 & 6.7 & & 2.0 & & 0.1 & 0.2 & 13.1 & 0.3 & & 0.7 & 0.1 & & 47.0 & 0.1 & 0.1 & 1.0 & & & & & 1.0 & & \\
\hline Hrma, 1994 & & & 10.0 & 9.7 & & & 0.8 & & & & & & 6.6 & & 3.5 & 0.8 & & & 10.4 & & 5.5 & & & & 49.3 & & & & & & & & 3.6 & & \\
\hline Hrma, 1994 & & & 15.0 & 9.2 & & & 0.7 & & & & & & 6.3 & & 3.3 & 0.7 & & & 9.8 & & 5.2 & & & & 46.6 & & & & & & & & 3.4 & & \\
\hline Hrma, 1994 & 1107 & 1037 & 9.0 & 17.0 & 0.0 & & 7.0 & 0.1 & & 0.1 & 0.1 & 0.1 & 4.0 & & 4.7 & 1.0 & 0.1 & 0.1 & 7.0 & 0.3 & & 0.6 & \begin{tabular}{|l|}
0.0 \\
\end{tabular} & & 46.8 & 0.1 & 0.0 & 0.8 & & & & & 1.0 & & \\
\hline Hrma, 1994 & 1204 & 1122 & 8.0 & 8.7 & 0.1 & & & 0.2 & & 0.2 & 0.2 & 0.4 & 4.0 & & 6.0 & 5.0 & 0.2 & 0.4 & 7.0 & 0.8 & & 2.0 & 0.1 & & 52.3 & 0.4 & 0.1 & 2.7 & & & & & 1.0 & & \\
\hline Hrma, 1994 & 1194 & 1116 & 9.0 & 7.0 & 0.0 & & 3.0 & 0.1 & & 0.1 & 0.1 & 0.1 & 4.0 & & 2.3 & 5.0 & 0.1 & 0.1 & 16.9 & 0.3 & 2.5 & 0.6 & \begin{tabular}{|l|}
5 \\
\end{tabular} & & 49.4 & 0.1 & 0.0 & 0.8 & & & & & 1.0 & & \\
\hline Hrma, 1994 & 1138 & 1065 & 7.6 & 7.0 & 0.1 & & 6.0 & 0.2 & & 0.2 & 0.2 & 0.4 & 4.0 & & 2.1 & & 0.2 & 0.4 & 17.0 & 0.8 & & 2.0 & 0.1 & & 47.3 & 0.4 & 0.1 & 2.7 & & & & & 1.0 & & \\
\hline Hrma, 1994 & 1177 & 1099 & 11.0 & 7.0 & 0.0 & & 7.0 & 0.1 & & 0.1 & 0.1 & 0.1 & 4.5 & & 6.0 & & 0.1 & 0.1 & 8.8 & 0.3 & & 0.6 & 0.0 & & 50.2 & 0.1 & 0.0 & 0.8 & & & & & 3.0 & & \\
\hline Hrma, 1994 & 1037 & 970 & 9.6 & 20.0 & 0.2 & & & 0.3 & & 0.2 & 0.3 & 0.5 & 2.0 & & 7.0 & & 0.2 & 0.5 & 7.4 & 1.0 & & 2.4 & \begin{tabular}{|l|} 
\\
\end{tabular} & & 44.7 & 0.5 & 0.2 & 3.1 & & & & & & & \\
\hline Hrma, 1994 & 1279 & 1191 & 7.8 & 5.0 & 0.0 & & & 0.0 & & 0.0 & 0.0 & 0.1 & 2.0 & & 1.6 & 8.0 & 0.0 & 0.1 & 20.0 & 0.1 & & 0.3 & \begin{tabular}{|l|l|}
3 & 0.0 \\
\end{tabular} & & 54.6 & 0.0 & 0.0 & 0.3 & & & & & & & \\
\hline Hrma, 1994 & 1162 & 1090 & 10.3 & 13.2 & 0.0 & & 7.0 & 0.1 & & 0.1 & 0.1 & 0.1 & 4.5 & & 4.4 & 1.0 & 0.1 & 0.1 & 7.0 & 0.3 & & 0.6 & \begin{tabular}{|l|}
5 \\
\end{tabular} & & 46.5 & 0.1 & 0.0 & 0.8 & & & & & 3.7 & & \\
\hline Hrma, 1994 & 1051 & 986 & 8.9 & 5.4 & 0.2 & & & 0.3 & & 0.2 & 0.3 & 0.5 & 2.0 & & 3.6 & 8.0 & 0.2 & 0.5 & 20.0 & 1.0 & & 2.5 & \begin{tabular}{|l|l|}
5 & 0.2 \\
\end{tabular} & & 42.0 & 0.5 & 0.2 & 3.3 & & & & & & & \\
\hline Hrma, 1994 & 1012 & 953 & 13.9 & 17.4 & 0.0 & & & 0.0 & & 0.0 & 0.0 & 0.1 & 2.0 & & \begin{tabular}{|l|}
3.7 \\
\end{tabular} & & 0.0 & 0.1 & 20.0 & 0.1 & & 0.3 & \begin{tabular}{|l|l|}
3 & 0.0 \\
\end{tabular} & & 42.0 & 0.0 & 0.0 & 0.3 & & & & & & & \\
\hline Hrma, 1994 & 1080 & 1009 & 13.4 & 5.0 & 0.0 & & 8.0 & 0.0 & & 0.0 & 0.0 & 0.1 & 6.3 & & 4.3 & & 0.0 & 0.1 & 20.0 & 0.1 & & 0.3 & \begin{tabular}{|l|l|}
3 & 0.0 \\
\end{tabular} & & 42.0 & 0.0 & 0.0 & 0.3 & & & & & & & \\
\hline Hrma, 1994 & 1278 & 1177 & 14.0 & 8.4 & 0.0 & & & 0.0 & & 0.0 & 0.0 & 0.1 & 2.0 & & 7.0 & & 0.0 & 0.1 & 10.6 & 0.1 & & 0.3 & \begin{tabular}{|l|l|}
3 & 0.0 \\
\end{tabular} & & 57.0 & 0.0 & 0.0 & 0.3 & & & & & & & \\
\hline Hrma, 1994 & 1187 & 1109 & 10.0 & \begin{tabular}{|l|l|}
6.4 \\
\end{tabular} & 0.1 & & 2.0 & 0.1 & & 0.1 & 0.1 & 0.2 & 2.0 & & 4.2 & 5.0 & 0.1 & 0.2 & 15.0 & 0.3 & & \begin{tabular}{|l|}
0.8 \\
\end{tabular} & \begin{tabular}{|l|l|}
3 & 0.1 \\
\end{tabular} & & 50.4 & 0.1 & 0.1 & 1.0 & & & & & 2.0 & & \\
\hline Hrma, 1994 & 1175 & 1098 & 10.0 & 6.9 & 0.1 & & 5.0 & 0.1 & & 0.1 & 0.1 & 0.2 & 3.0 & & 7.0 & 2.0 & 0.1 & 0.2 & 7.8 & 0.3 & & 0.8 & \begin{tabular}{|l|l|}
3 & 0.1 \\
\end{tabular} & & 53.3 & 0.1 & 0.1 & 1.0 & & & & & 2.0 & & \\
\hline
\end{tabular}


Table A1. Glass Compositions from Literature Review (continued)

\begin{tabular}{|c|c|c|c|c|c|c|c|c|c|c|c|c|c|c|c|c|c|c|c|c|c|c|c|c|c|c|c|c|c|c|c|c|c|c|c|}
\hline Ref & 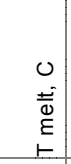 & $\begin{array}{l}0 \\
0 \\
0 \\
0 \\
\stackrel{0}{E} \\
⺊\end{array}$ & 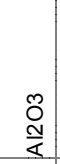 & 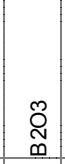 & $\begin{array}{l}\text { O } \\
\infty\end{array}$ & $\begin{array}{l}\text { Õ } \\
\text { N }\end{array}$ & $\begin{array}{l}0 \\
\pi \\
0\end{array}$ & 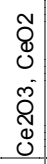 & $\begin{array}{l}0 \\
0 \\
0 \\
0 \\
0 \\
0 \\
0\end{array}$ & ญ̃ & O̊ & 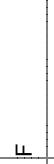 & 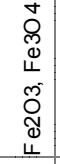 & $\begin{array}{l}\stackrel{\mathbb{N}}{\underline{y}} \\
\end{array}$ & 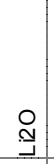 & $\begin{array}{l}0 \\
\text { On } \\
\Sigma\end{array}$ & $\begin{array}{l}\mathcal{O} \\
\stackrel{\Sigma}{\Sigma}\end{array}$ & $\begin{array}{l}0 \\
0 \\
\Sigma\end{array}$ & $\begin{array}{l}\text { D } \\
\underset{\pi}{Z}\end{array}$ & $\stackrel{\circ}{z}$ & $\begin{array}{l}\mathcal{N} \\
\text { ثี }\end{array}$ & $\overline{\bar{c}}$ & 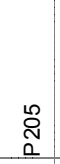 & $\frac{8}{a}$ & $\frac{\tilde{O}}{n}$ & $\begin{array}{l}\text { Oొ } \\
\text { c) }\end{array}$ & $\begin{array}{l}\text { Q } \\
\text { is }\end{array}$ & 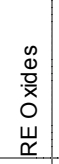 & $\begin{array}{c}\tilde{Q} \\
\stackrel{\varepsilon}{1}\end{array}$ & $\stackrel{\widetilde{N}}{\stackrel{N}{F}}$ & 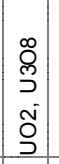 & $\frac{Q}{N}$ & $\begin{array}{l}\tilde{D} \\
N\end{array}$ & 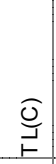 & $\begin{array}{l}\bar{\Xi} \\
\frac{0}{0} \\
\vec{z}\end{array}$ \\
\hline Hrma, 1994 & 1142 & 1068 & 7.5 & 12.6 & 0.1 & & 2.0 & 0.2 & & 0.1 & 0.2 & 0.4 & 2.0 & & 7.0 & 2.0 & 0.1 & 0.4 & 5.8 & 0.7 & & 1.6 & 0.1 & & 52.7 & 0.3 & 0.1 & 2.2 & & & & & 2.0 & & \\
\hline Hrma, 1994 & 1123 & 1054 & 8.5 & 14.4 & 0.1 & & 5.0 & 0.1 & & 0.1 & 0.1 & 0.3 & 2.0 & & 3.9 & 2.0 & 0.1 & 0.3 & 9.7 & 0.6 & & 1.4 & 0.1 & & 47.0 & 0.3 & 0.1 & 1.8 & & & & & 2.0 & & \\
\hline Hrma, 1994 & 1180 & 1102 & 7.9 & 13.6 & 0.1 & & 2.0 & 0.1 & & 0.1 & 0.1 & 0.2 & 5.2 & & 4.1 & 2.0 & 0.1 & 0.2 & 9.6 & 0.3 & & 0.8 & 0.1 & & 50.7 & 0.1 & 0.1 & 1.0 & & & & & 2.0 & & \\
\hline Hrma, 1994 & 1207 & 1129 & 8.0 & 15.9 & 0.1 & & 3.5 & 0.2 & & 0.2 & 0.2 & 0.4 & 4.0 & & 2.0 & & 0.2 & 0.4 & 10.1 & 0.8 & & 2.0 & 0.1 & & 47.5 & 0.4 & 0.1 & 2.7 & & & & & 1.0 & & \\
\hline Hrma, 1994 & & & 8.0 & 8.7 & 0.1 & & & 0.2 & & 0.2 & 0.2 & 0.4 & 4.0 & & 6.0 & 5.0 & 0.2 & 0.4 & 7.0 & 0.8 & & 2.0 & 0.1 & & 52.3 & 0.4 & 0.1 & 0.9 & & & 1.8 & & 1.0 & & \\
\hline Hrma, 1994 & 1038 & 979 & 15.0 & 20.0 & 0.0 & & 2.0 & 0.0 & & 0.0 & 0.0 & 0.1 & 2.0 & & 7.0 & 8.0 & 0.0 & 0.1 & 5.0 & 0.1 & & 0.3 & 0.0 & & 39.0 & 0.0 & 0.0 & 0.3 & & & & & 1.0 & & \\
\hline Hrma, 1994 & 973 & 913 & 11.5 & 17.2 & 0.0 & & 3.8 & 0.0 & & 0.0 & 0.0 & 0.1 & 2.0 & & 7.3 & 0.1 & 0.0 & 0.1 & 12.7 & 0.1 & & 0.3 & 0.0 & & 43.8 & 0.1 & 0.0 & 0.3 & & & & & 0.8 & & \\
\hline Hrma, 1994 & 1063 & 988 & 9.3 & 8.8 & 0.0 & & 0.6 & 0.0 & & 0.0 & 0.0 & 0.1 & 2.0 & & 7.4 & 0.1 & 0.0 & 0.1 & 17.3 & 0.1 & & 0.3 & 0.0 & & 52.8 & 0.1 & 0.0 & 0.4 & & & & & 0.8 & & \\
\hline Hrma, 1994 & 1279 & 1182 & 16.3 & 6.6 & 0.0 & & & 0.0 & & 0.0 & 0.0 & 0.1 & 2.0 & & 7.3 & & 0.0 & 0.1 & 12.0 & 0.1 & & 0.3 & 0.0 & & 52.8 & 0.1 & 0.0 & 0.4 & & & & & 1.8 & & \\
\hline Hrma, 1994 & 1068 & 1003 & 18.0 & 17.2 & 0.0 & & 10.0 & 0.0 & & 0.0 & 0.0 & 0.1 & 2.0 & & 0.5 & & 0.0 & 0.1 & 19.0 & 0.1 & & 0.3 & 0.0 & & 32.3 & 0.0 & 0.0 & 0.3 & & & & & & & \\
\hline Hrma, 1994 & 1215 & 1128 & 11.8 & 9.2 & 0.1 & & 1.0 & & & 1.1 & & 0.1 & 3.9 & & 5.2 & 0.6 & 1.2 & 0.6 & 12.1 & 0.5 & & & & & 51.9 & & & 0.4 & & & & & 0.3 & & \\
\hline Hrma, 1994 & 1231 & 1137 & 20.4 & 15.9 & & & 0.2 & & & 0.1 & & 0.1 & 0.0 & & 5.8 & 0.0 & & & 10.9 & & & & 0.5 & & 46.0 & 0.0 & & & & & & & & & \\
\hline Hrma, 1994 & 1350 & 1223 & 16.4 & 13.6 & & & 0.1 & & & 3.0 & & 0.6 & 0.5 & 0.2 & 7.0 & 0.0 & 0.2 & 0.1 & 8.0 & & & & & & 50.4 & 0.0 & & & & & & 0.1 & 0.0 & & \\
\hline Hrma, 1994 & 1336 & 1236 & 8.2 & 7.8 & & & 0.8 & & & 2.4 & 0.2 & 0.1 & 3.3 & & 7.2 & 0.3 & 1.2 & & 6.7 & & & 0.7 & 3.3 & 0.1 & 56.8 & \begin{tabular}{|l|}
0.7 \\
\end{tabular} & & 0.2 & & & & & 0.1 & & \\
\hline Hrma, 1994 & 1257 & 1162 & 18.2 & 14.2 & & & 0.1 & 0.1 & & 1.2 & 0.2 & & 0.8 & & 6.9 & 0.1 & 0.3 & & 8.1 & 0.1 & & 0.8 & 0.1 & & 48.6 & 0.0 & & 0.1 & & & & & 0.1 & & \\
\hline Hrma, 1994 & 1208 & 1121 & 11.8 & 9.2 & 0.1 & & 1.0 & 0.1 & & 0.1 & 0.1 & 0.2 & 3.9 & & 5.2 & 0.6 & 0.1 & 0.2 & 12.1 & 0.4 & & 1.1 & 0.1 & & 51.8 & 0.2 & 0.1 & 1.4 & & & & & 0.3 & & \\
\hline Hrma, 1994 & 1131 & 1054 & 9.5 & 17.0 & 0.1 & & 0.9 & 0.2 & & 0.1 & 0.2 & 0.3 & 4.0 & & 5.9 & & 0.1 & 0.3 & 7.0 & 0.7 & & 1.6 & 0.1 & & 48.2 & 0.3 & 0.1 & 2.2 & & & & & 1.0 & & \\
\hline Hrma, 1994 & 1223 & 1133 & 16.4 & 13.6 & 0.1 & & 0.1 & 0.1 & & 0.1 & 0.1 & 0.2 & 0.5 & & 7.0 & 0.0 & 0.1 & 0.2 & 8.0 & 0.4 & & 1.0 & 0.1 & & 50.4 & 0.2 & 0.1 & 1.4 & & & & & 0.0 & & \\
\hline Hrma, 1994 & 1234 & 1144 & 8.2 & 7.8 & 0.2 & & 0.8 & 0.2 & & 0.2 & 0.2 & 0.5 & 3.3 & & 7.1 & 0.3 & 0.2 & 0.5 & 6.6 & 0.9 & & 2.3 & 0.2 & & 56.6 & 0.5 & 0.2 & 3.0 & & & & & 0.1 & & \\
\hline Hrma, 1994 & 1223 & 1132 & 18.2 & 14.2 & 0.1 & & 0.1 & 0.1 & & 0.1 & 0.1 & 0.2 & 0.8 & & 6.9 & 0.1 & 0.1 & 0.2 & 8.1 & 0.3 & & 0.8 & 0.1 & & 48.6 & 0.2 & 0.1 & 1.0 & & & & & 0.1 & & \\
\hline Hrma, 1994 & 1122 & 1047 & 17.0 & 6.0 & 0.1 & & 0.5 & 0.1 & & 0.1 & 0.1 & 0.2 & 0.5 & & 7.0 & 2.0 & 0.1 & 0.2 & 18.0 & 0.5 & & 1.1 & 0.1 & & 44.0 & 0.2 & 0.1 & 1.5 & & & & & 0.5 & & \\
\hline Hrma, 1994 & 1373 & 1275 & 17.0 & 6.0 & 0.1 & & 4.0 & 0.1 & & 0.1 & 0.1 & 0.2 & 0.5 & & 1.4 & 0.5 & 0.1 & 0.2 & 18.0 & 0.5 & & 1.1 & 0.1 & & 47.7 & 0.2 & 0.1 & 1.5 & & & & & 0.5 & & \\
\hline Hrma, 1994 & 1295 & 1207 & 9.9 & 8.0 & 0.0 & & 1.4 & 0.1 & & 0.0 & 0.1 & 0.1 & 2.5 & & 1.8 & 0.5 & 0.0 & 0.1 & 18.0 & 0.2 & & 0.5 & 0.0 & & 49.8 & 0.1 & 0.0 & 0.7 & & & & & 6.1 & & \\
\hline Hrma, 1994 & 1115 & 1050 & 10.5 & 6.0 & 0.0 & & 4.0 & 0.1 & & 0.0 & 0.1 & 0.1 & 2.5 & & 7.0 & 0.5 & 0.0 & 0.1 & 14.0 & 0.2 & & 0.5 & 0.0 & & 46.0 & 0.1 & 0.0 & 0.7 & & & & & 7.5 & & \\
\hline Hrma, 1994 & 934 & 884 & 7.0 & 16.0 & 0.0 & & 4.0 & 0.1 & & 0.0 & 0.1 & 0.1 & 2.7 & & 5.3 & 0.5 & 0.0 & 0.1 & 18.0 & 0.2 & & 0.5 & 0.0 & & 44.0 & 0.1 & 0.0 & 0.7 & & & & & 0.5 & & \\
\hline Hrma, 1994 & 1026 & 957 & 13.9 & 17.4 & 0.0 & & & 0.0 & & 0.0 & 0.0 & 0.1 & 2.0 & & 3.7 & & 0.0 & 0.1 & 20.0 & 0.1 & & 0.3 & 0.0 & & 42.0 & \begin{tabular}{|l|} 
\\
\end{tabular} & 0.0 & 0.3 & & & & & & & \\
\hline Hrma, 1999 & & & 8.0 & 7.0 & & & & & & 0.7 & & & 13.0 & & 4.5 & 0.6 & 0.5 & & 15.7 & 2.0 & & & & & 45.0 & & & & & & & & 3.0 & & \\
\hline Hrma, 1999 & & & 8.0 & 7.0 & & & & & & 0.7 & & & 13.0 & & 4.5 & 0.6 & 0.5 & & 15.7 & 2.0 & & & & & 45.0 & & & & & & & & 3.0 & & \\
\hline Hrma, 1999 & & & 8.0 & 7.0 & & & & & & 0.5 & & & 14.5 & & 4.0 & 0.6 & 0.5 & & 15.3 & 1.2 & & & & & 42.4 & & & & & & & & 6.0 & & \\
\hline Hrma, 1999 & & & 8.0 & 7.0 & & & & & & 0.5 & & & 14.5 & & 4.0 & 0.6 & 0.5 & & 15.3 & 1.2 & & & & & 42.4 & & & & & & & & 6.0 & & \\
\hline Hrma, 1999 & & & 8.0 & 7.0 & & & & & & 0.5 & & & 11.5 & & 4.0 & 0.6 & 0.5 & & 15.3 & 1.2 & & & & & 45.4 & & & & & & & & 6.0 & & \\
\hline Hrma, 1999 & & & 8.0 & 7.0 & & & & & & 0.5 & & & 11.5 & & 4.0 & 0.6 & 0.5 & & 15.3 & 1.2 & & & & & 45.4 & & & & & & & & 6.0 & & \\
\hline Hrma, 1999 & & & 8.0 & 7.0 & & & & & & 0.5 & & & 8.9 & & 4.5 & 0.6 & 0.5 & & 15.3 & 1.2 & & & & & 47.5 & & & & & & & & 6.0 & & \\
\hline Hrma, 1999 & & & 8.0 & 7.0 & & & & & & 0.5 & & & 8.9 & & 4.5 & 0.6 & 0.5 & & 15.3 & 1.2 & & & & & 47.5 & & & & & & & & 6.0 & & \\
\hline Hrma, 1999 & & & 8.0 & 7.0 & & & & & & 0.5 & & & 11.5 & & 4.1 & 0.6 & 0.5 & & 15.3 & 0.7 & & & & & 45.8 & & & & & & & & 6.0 & & \\
\hline Hrma, 1999 & & & 8.0 & 7.0 & & & & & & 0.4 & & & 12.5 & & 3.0 & 0.6 & 0.4 & & 15.7 & 1.4 & & & & & 46.0 & & & & & & & & 5.1 & & \\
\hline Hrma, 1999 & & & 8.0 & 7.0 & & & & & & 0.3 & & & 11.5 & & 4.5 & 0.6 & 0.5 & & 15.3 & 1.0 & & & & & 45.3 & & & & & & & & 6.0 & & \\
\hline Hrma, 1999 & & & 8.0 & 7.0 & & & & & & 0.4 & & & 12.5 & & 3.0 & 0.6 & 0.4 & & 15.7 & 0.8 & & & & & 46.0 & & & & & & & & 5.7 & & \\
\hline Hrma, 1999 & & & 8.0 & 7.0 & & & & & & 1.0 & & & 11.0 & & 4.0 & 0.6 & 0.4 & & 15.7 & 1.0 & & & & & 45.3 & & & & & & & & 6.0 & & \\
\hline Hrma, 1999 & 1250 & & 8.0 & 5.0 & & & 0.3 & & & 0.1 & & & 15.0 & 1.5 & 6.0 & 2.5 & 1.0 & & 6.0 & 2.0 & & 0.1 & & & 52.4 & & & & & 0.1 & & & & & \\
\hline Hrma, 1999 & 1322 & & 8.0 & 5.0 & & & 2.0 & & & 0.1 & & & 15.0 & 3.8 & 3.0 & 0.5 & 1.0 & & 11.0 & 0.0 & & 0.1 & & & 49.9 & & & & & 0.6 & & & & & \\
\hline Hrma, 1999 & 1294 & & 8.0 & 10.0 & & & 0.3 & & & 0.3 & & & 6.0 & 3.8 & 6.0 & 2.5 & 3.0 & & 6.0 & 0.0 & & 0.1 & & & 53.9 & & & & & 0.1 & & & & & \\
\hline Hrma, 1999 & 1200 & & 8.0 & 10.0 & & & 2.0 & & & 0.3 & & & 15.0 & 1.5 & 6.0 & 0.5 & 1.0 & & 6.0 & 0.0 & & 0.1 & & & 44.0 & & & & & 0.1 & 5.5 & & & & \\
\hline Hrma, 1999 & 1140 & & 8.0 & 10.0 & & & 0.3 & & & 0.3 & & & 6.0 & 3.8 & 6.0 & 0.5 & 1.0 & & 11.0 & 2.0 & & 0.1 & & & 45.4 & & & & & 0.1 & 5.5 & & & & \\
\hline Hrma, 1999 & 1240 & & 8.0 & 5.0 & & & 2.0 & & & 0.1 & & & 6.0 & 1.5 & 6.0 & 2.5 & 1.0 & & 11.0 & 0.0 & & 0.1 & & & 50.7 & & & & & 0.6 & 5.5 & & & & \\
\hline Hrma, 1999 & 1333 & & 8.0 & 10.0 & & & 0.3 & & & 0.1 & & & 15.0 & 3.8 & 3.0 & 2.5 & 1.0 & & 6.0 & 2.0 & & 0.1 & & & 48.1 & & & & & 0.1 & & & & & \\
\hline Hrma, 1999 & 1280 & & 8.0 & 5.0 & & & 0.3 & & & 0.1 & & & 6.0 & 1.5 & 6.0 & 0.5 & 3.0 & & 11.0 & 0.0 & & 0.1 & & & 52.4 & & & & & 0.6 & 5.5 & & & & \\
\hline Hrma, 1999 & 1157 & & 8.0 & 5.0 & & & 2.0 & & & 0.1 & & & 6.0 & 3.8 & 6.0 & 2.5 & 3.0 & & 11.0 & 2.0 & & 0.1 & & & 44.9 & & & & & 0.1 & 5.5 & & & & \\
\hline Hrma, 1999 & 1149 & & 8.0 & 10.0 & & & 2.0 & & & 0.1 & & & 14.9 & 3.8 & 6.0 & 2.5 & 3.0 & & 6.0 & 0.0 & & 0.1 & & & 43.0 & & & & & 0.6 & & & & & \\
\hline Hrma, 1999 & 1250 & & 8.0 & 10.0 & & & 0.3 & & & 0.1 & & & 15.0 & 1.5 & 6.0 & 0.5 & 1.0 & & 11.0 & 2.0 & & 0.1 & & & 44.0 & & & & & 0.6 & & & & & \\
\hline
\end{tabular}


Table A1. Glass Compositions from Literature Review (continued)

\begin{tabular}{|c|c|c|c|c|c|c|c|c|c|c|c|c|c|c|c|c|c|c|c|c|c|c|c|c|c|c|c|c|c|c|c|c|c|c|c|}
\hline Ref & 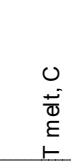 & $\begin{array}{l}0 \\
0 \\
8 \\
0 \\
⺊ \\
-\end{array}$ & 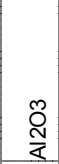 & 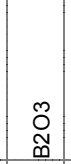 & $\begin{array}{l}0 \\
\mathbb{\mathbb { N }} \\
\boldsymbol{\infty}\end{array}$ & $\begin{array}{l}\text { Õ } \\
\overline{\tilde{\omega}}\end{array}$ & $\begin{array}{l}0 \\
0 \\
0\end{array}$ & 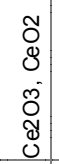 & $\begin{array}{l}\tilde{D} \\
0 \\
0 \\
0 \\
0 \\
0\end{array}$ & $\begin{array}{l}\text { Oొ } \\
\text { ָ̦ }\end{array}$ & $\stackrel{O}{\mathcal{O}}$ & 4 & 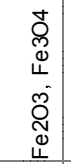 & $\stackrel{\stackrel{\sim}{\tilde{y}}}{ }$ & 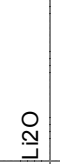 & $\frac{8}{\Sigma}$ & $\begin{array}{l}\tilde{Q} \\
\text { है }\end{array}$ & $\frac{\tilde{8}}{2}$ & $\begin{array}{l}\text { શิ } \\
\stackrel{\pi}{z}\end{array}$ & 을 & $\begin{array}{l}\text { N } \\
\text { ț }\end{array}$ & $\overline{\bar{c}}$ & 足 & $\begin{array}{l}\circ \\
\stackrel{0}{Q} \\
\end{array}$ & ก̃ & ల్ & 음 & 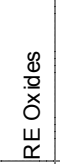 & $\begin{array}{l}\tilde{O} \\
\stackrel{F}{F}\end{array}$ & 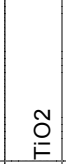 & $\begin{array}{l}\infty \\
0 \\
\text { ग } \\
د \\
\text { Ñ } \\
\text { ว }\end{array}$ & $\begin{array}{l}0 \\
\stackrel{5}{N}\end{array}$ & ัั & 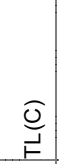 & 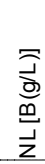 \\
\hline Hrma, 1999 & 1145 & & 8.0 & 10.0 & & & 2.0 & & & 0.3 & & & 6.0 & 3.8 & 6.0 & 0.5 & 3.0 & & 11.0 & 2.0 & & 0.1 & & & 46.8 & & & & & 0.6 & & & & & \\
\hline Hrma, 1999 & 1285 & & 8.0 & 10.0 & & & 2.0 & & & 0.3 & & & 14.5 & 1.5 & 3.0 & 2.5 & 3.0 & & 6.0 & 0.0 & & 0.1 & & & 43.0 & & & & & 0.6 & 5.5 & & & & \\
\hline Hrma, 1999 & 1134 & & 8.0 & 5.0 & & & 0.3 & & & 0.3 & & & 14.5 & 3.8 & 6.0 & 2.5 & 3.0 & & 11.0 & 2.0 & & 0.1 & & & 43.0 & & & & & 0.6 & & & & & \\
\hline Hrma, 1999 & 1300 & & 8.0 & 10.0 & & & 0.3 & & & 0.3 & & & 6.0 & 1.5 & 3.0 & 2.5 & 1.0 & & 11.0 & 2.0 & & 0.1 & & & 48.3 & & & & & 0.6 & 5.5 & & & & \\
\hline Hrma, 1999 & 1300 & & 8.0 & 10.0 & & & 2.0 & & & 0.1 & & & 15.0 & 1.5 & 3.0 & 0.5 & 3.0 & & 6.0 & 2.0 & & 0.1 & & & 43.2 & & & & & 0.1 & 5.5 & & & & \\
\hline Hrma, 1999 & 1326 & & 8.0 & 5.0 & & & 2.0 & & & 0.3 & & & 15.0 & 3.8 & 3.0 & 0.5 & 1.0 & & 11.0 & 0.0 & & 0.1 & & & 50.2 & & & & & 0.1 & & & & & \\
\hline Hrma, 2006 & 1150 & & 8.0 & 12.0 & & 1.5 & 1.5 & 0.5 & & 1.0 & & 0.5 & 1.5 & 3.0 & 4.0 & & 0.5 & & 16.0 & 0.2 & & 0.3 & 0.8 & 0.1 & 44.2 & & 0.5 & & & 1.3 & & 0.3 & 2.5 & & \\
\hline Huang, 2004 & & & 13.9 & & & & 11.3 & & & 0.5 & & & 21.1 & & & 9.4 & & & 3.3 & & & & & & 40.4 & & & & & & & & & & \\
\hline Ioffe, 1987 & & & 7.8 & 15.3 & & & 2.0 & & 0.9 & & 0.4 & & 0.5 & 2.6 & & 1.4 & & & 16.5 & & & & 6.3 & & 39.3 & & & & & 5.4 & & & 1.6 & & \\
\hline Ioffe, 1987 & & & 7.5 & 15.0 & & & 2.0 & & 0.4 & & 1.0 & & & 3.0 & & 0.8 & & & 16.7 & & & & 7.0 & & 40.0 & & & & & 6.2 & & & 0.5 & & \\
\hline Ioffe, 1987 & & & 7.6 & 15.1 & & & 2.0 & & 0.5 & & 0.5 & & 0.5 & 2.6 & & 1.4 & & & 16.0 & & & & 7.1 & & 39.5 & & & & & 6.0 & & & 1.2 & & \\
\hline Ioffe, 1987 & & & 8.6 & 15.0 & & & 2.2 & & 0.6 & & 0.2 & & 1.7 & 2.7 & & 1.8 & & & 14.0 & & & & 6.8 & & 42.0 & & & & & 4.0 & & & 0.4 & & \\
\hline Ioffe, 1987 & & & 9.0 & 15.5 & & & 2.5 & & 0.1 & & 0.9 & & 1.5 & 2.8 & & 2.0 & & & 14.7 & & & & 4.0 & & 35.0 & & & & & 11.0 & & & 1.0 & & \\
\hline \begin{tabular}{|l|l|} 
Ioffe, 1987 \\
\end{tabular} & & & 7.5 & 15.5 & & & 2.5 & & 0.6 & & 0.3 & & 0.5 & & 0.8 & 1.5 & & & 17.0 & & & & 6.3 & & 41.1 & & & & & 5.0 & & & 1.3 & & \\
\hline Jain, 1990 & & 1100 & 7.3 & 11.0 & 0.1 & & 0.7 & 0.1 & 0.1 & 0.1 & & & 12.2 & 3.5 & 3.1 & 0.8 & 0.9 & & 11.6 & 0.2 & 0.0 & 0.0 & 2.6 & & 41.7 & 0.3 & 0.1 & 0.1 & & 0.8 & & 0.5 & 2.2 & & \\
\hline \begin{tabular}{|l|} 
Jain, 1990 \\
\end{tabular} & & 1073 & 7.3 & 11.0 & 0.1 & & 0.7 & 0.1 & 0.1 & 0.1 & & & 12.2 & 3.5 & 3.1 & 0.8 & 0.9 & & 11.6 & 0.2 & 0.0 & 0.0 & 2.6 & & 41.7 & 0.3 & 0.1 & 0.1 & & 0.8 & & 0.5 & 2.2 & & \\
\hline Jantzen, 1995 & & & 13.5 & 10.8 & & & 0.4 & & & & & & 4.6 & & 4.1 & 1.4 & 2.5 & & 14.1 & 0.6 & 0.4 & & & & 46.5 & & & & & 0.7 & & & 0.3 & & \\
\hline Jantzen, 1995 & & & 7.1 & 8.3 & & & 1.3 & & & & & & 14.0 & 3.1 & 4.0 & 0.5 & 3.4 & & 9.9 & 1.1 & & 0.1 & & & 45.2 & & 0.1 & & & 1.3 & & & 0.6 & & \\
\hline Jantzen, 1995 & & & 13.5 & 7.4 & & & 0.5 & & & & & & 4.6 & & 4.2 & 0.7 & 2.6 & & 10.7 & 0.7 & & & & & 54.2 & & & & & & & & 0.8 & & \\
\hline Jantzen, 1995 & & & 13.5 & 10.3 & & & 0.5 & & & & & & 4.4 & 3.1 & 2.7 & 1.3 & 2.5 & & 10.7 & 0.6 & & & & & 48.7 & & & & & 1.7 & & & 0.0 & & \\
\hline Jantzen, 1995 & & & 14.0 & 7.5 & & & 0.4 & & & & & & 4.3 & 3.3 & 4.2 & 1.3 & 2.5 & & 7.4 & 0.6 & & & & & 52.7 & & & & & 1.7 & & & 0.0 & & \\
\hline Jantzen, 1995 & & & 7.1 & 7.0 & 0.1 & & 1.0 & & & 0.1 & 0.2 & & 7.8 & 2.2 & 4.6 & 1.5 & 1.8 & 0.2 & 8.6 & 0.4 & & 0.0 & & & 55.8 & & & 0.5 & & 0.6 & & & 0.3 & & \\
\hline Jantzen, 1995 & & & 7.1 & 7.0 & 0.1 & & 1.0 & & & 0.1 & 0.2 & & 7.8 & 2.2 & 4.6 & 1.5 & 1.8 & 0.2 & 8.6 & 0.4 & & 0.0 & & & 55.8 & & & 0.5 & & 0.6 & & & 0.3 & & \\
\hline Jantzen, 1995 & & & 7.1 & 7.0 & 0.1 & & 1.0 & & & 0.1 & 0.2 & & 7.8 & 2.2 & 4.6 & 1.5 & 1.8 & 0.2 & 8.6 & 0.4 & & 0.0 & & & 55.8 & & & 0.5 & & 0.6 & & & 0.3 & & \\
\hline Jantzen, 1995 & & & 7.5 & 6.6 & 0.1 & & 0.7 & & & 0.5 & 0.1 & & 9.9 & 1.6 & 4.4 & 1.5 & 2.7 & & 10.2 & 0.7 & & & & & 52.9 & & 0.1 & & & 0.1 & & 0.1 & 0.1 & & \\
\hline Jantzen, 1995 & & & 7.7 & 6.7 & 0.1 & & 0.7 & & & 0.5 & 0.1 & & 9.9 & 1.8 & 4.4 & 1.5 & 2.8 & & 10.4 & 0.7 & & & & & 52.4 & & 0.2 & & & 0.1 & & 0.1 & 0.1 & & \\
\hline Jantzen, 1995 & & & 7.5 & 6.7 & 0.1 & & 0.7 & & & 0.4 & 0.1 & & 9.4 & 1.9 & 4.6 & 1.5 & 2.7 & & 10.5 & 0.6 & & & & & 52.7 & & 0.1 & & & 0.1 & & 0.1 & 0.1 & & \\
\hline Jantzen, 1995 & & & 7.7 & 6.5 & 0.1 & & 0.7 & & & 0.4 & 0.1 & & 9.5 & 2.2 & 4.2 & 1.5 & 2.6 & & 10.8 & 0.6 & & & & & 52.8 & & 0.1 & & & 0.1 & & 0.1 & 0.1 & & \\
\hline Jantzen, 1995 & & & 8.6 & 6.7 & 0.1 & & 0.7 & & & 0.4 & 0.2 & & 10.0 & 2.2 & 4.3 & 1.5 & 2.8 & & 10.6 & 0.6 & & & & & 51.0 & & 0.1 & & & 0.1 & & 0.1 & 0.1 & & \\
\hline Jantzen, 1995 & & & 9.1 & 6.7 & 0.1 & & 0.8 & & & 0.4 & 0.2 & & 10.4 & 2.3 & 4.2 & 1.6 & 2.9 & & 10.1 & 0.6 & & & & & 50.3 & & 0.2 & & & 0.1 & & 0.1 & 0.1 & & \\
\hline Jantzen, 1995 & & & 13.1 & 13.9 & & & 3.0 & & & & & & 8.3 & & & & & & 15.7 & & & & & & 46.0 & & & & & & & & & & \\
\hline Jantzen, 1995 & & & 15.0 & 9.4 & & & & & & & & & 11.4 & & & & & & 17.2 & & & & & & 46.9 & & & & & & & & & & \\
\hline Jantzen, 1995 & & & 8.5 & 6.8 & 0.1 & & 0.8 & & & 0.4 & 0.2 & & 9.5 & 2.1 & 4.3 & 1.6 & 2.8 & & 10.4 & 0.6 & & & & & 51.5 & & 0.1 & & & 0.1 & & 0.1 & 0.1 & & \\
\hline Jensen. C., 1999 & & 1242 & 1.5 & & & & 29.5 & & & & & & 0.5 & 0.8 & & \begin{tabular}{|c|}
8.7 \\
\end{tabular} & & & 4.8 & & & & & & 54.2 & & & & & & & & & & \\
\hline Jensen. C., 1999 & & 1191 & 2.0 & & & & 30.5 & & & & & & 0.5 & 0.7 & & 9.9 & & & 4.6 & & & & & & 50.8 & & & & & & & & & & \\
\hline Jensen. C., 1999 & & 1216 & 0.8 & & & & 29.9 & & & & & & 0.6 & 0.2 & & 16.4 & & & 1.0 & & & & & & 49.1 & & & & & 0.1 & & & & & \\
\hline Jensen. C., 1999 & & 1140 & 2.1 & & & & 22.5 & & & & & & 4.5 & 0.8 & & 12.5 & & & 3.5 & & & & & & 53.2 & & & & & 0.2 & & & & & \\
\hline Jensen. C., 1999 & & 1163 & 2.0 & & & & 23.2 & & & & & & 6.0 & 0.8 & & 11.0 & & & 2.0 & & & & & & 53.5 & & & & & 0.1 & & & & & \\
\hline Jensen. C., 1999 & & 1257 & 12.2 & & & & 14.3 & & & & & & 6.9 & 1.1 & & 11.7 & & & 2.4 & & & & & & 47.6 & & & & & 1.8 & & & & & \\
\hline Jensen. C., 1999 & & 1263 & 12.8 & & & & 13.1 & & & & & & 6.6 & 1.1 & & 11.3 & & & 2.4 & & & & & & 48.5 & & & & & 1.9 & & & & & \\
\hline Jiricka, 2000 & & & 4.5 & 14.4 & & & & & & 2.9 & & & 4.5 & 3.8 & 3.2 & 0.4 & & & 8.0 & & & & & & 53.2 & & & & & 5.3 & & & & & \\
\hline Jiricka, 2000 & & & 1.0 & & & & 8.1 & & & & & & 0.1 & 0.3 & & 3.9 & & & 13.7 & & & & & & 72.5 & 0.3 & & & & & & & & & \\
\hline Johnston, 1990 & & & 7.9 & 9.5 & 0.6 & & 0.6 & 0.2 & & 0.2 & & & 11.3 & 3.3 & 3.0 & 1.2 & 1.3 & & 10.5 & 0.7 & & 0.1 & 2.4 & & 43.2 & 0.1 & & & & 1.0 & & & 3.0 & & \\
\hline Johnston, 1990 & & & 8.8 & 9.4 & 0.6 & & 0.6 & 0.2 & & 0.2 & & & 11.2 & 3.3 & 2.9 & 1.2 & 1.3 & & 10.4 & 0.7 & & 0.1 & 2.4 & & 42.8 & 0.1 & & & & 0.9 & & & 2.9 & & \\
\hline Johnston, 1990 & & & 8.0 & 7.4 & 0.2 & & 0.8 & & & & & & 12.3 & 1.6 & 3.0 & 1.3 & 1.3 & & 10.2 & & 2.7 & & 2.5 & & 44.7 & & & & 3.6 & & 0.6 & & & & \\
\hline Johnston, 1990 & & & 8.0 & 9.9 & 0.2 & & 0.7 & & & & & & 12.1 & 1.6 & 2.0 & 1.3 & 1.3 & & 8.9 & & 2.6 & & 2.5 & & 44.7 & & & & 3.6 & & 0.6 & & & & \\
\hline Johnston, 1990 & & & 8.0 & 12.4 & 0.1 & & 0.6 & & & & & & 12.1 & 1.3 & 2.0 & 1.3 & 1.3 & & 9.2 & & 2.6 & & 2.5 & & 42.3 & & & & 3.6 & & 0.6 & & & & \\
\hline Johnston, 1990 & & & 8.6 & 9.6 & 0.2 & & 1.0 & & & & & & 13.1 & 2.5 & 2.9 & 0.9 & 1.4 & & 10.7 & & 2.9 & & 0.6 & & 41.0 & & & & 3.9 & & 0.6 & & & & \\
\hline Johnston, 1990 & & & 9.9 & 12.3 & 0.2 & & 0.8 & & & & & & 11.9 & 1.6 & 2.2 & 0.8 & 1.2 & & 9.8 & & 3.1 & & 2.5 & & 39.6 & & & & 3.5 & & 0.6 & & & & \\
\hline
\end{tabular}


ORP-56297 Rev. 0

The Catholic University of America

High Level Waste Vitrification System Improvements Vitreous State Laboratory

Table A1. Glass Compositions from Literature Review (continued)

\begin{tabular}{|c|c|c|c|c|c|c|c|c|c|c|c|c|c|c|c|c|c|c|c|c|c|c|c|c|c|c|c|c|c|c|c|c|c|c|c|}
\hline Ref & 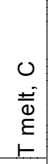 & 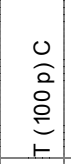 & $\begin{array}{l}00 \\
\stackrel{N}{<}\end{array}$ & 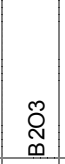 & $\begin{array}{l}\text { O } \\
\infty\end{array}$ & $\begin{array}{l}\text { Oొ } \\
\text { Non }\end{array}$ & $\begin{array}{l}0 \\
\mathbb{N} \\
0\end{array}$ & $\begin{array}{l}\tilde{0} \\
0 \\
0 \\
\tilde{ల} \\
0 \\
0 \\
0\end{array}$ & $\begin{array}{l}0 \\
\text { Oิ } \\
0 \\
0 \\
0 \\
0 \\
0\end{array}$ & $\begin{array}{l}\text { Oొ } \\
\text { Un }\end{array}$ & $\stackrel{O}{3}$ & 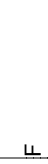 & 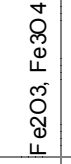 & $\underset{\underset{\mathbb{X}}{x}}{ }$ & $\stackrel{\stackrel{ }{J}}{\beth}$ & $\begin{array}{l}\circ \\
\text { O } \\
\Sigma\end{array}$ & $\begin{array}{l}\tilde{O} \\
\stackrel{C}{\Sigma} \\
\Sigma\end{array}$ & $\begin{array}{l}00 \\
\text { Oొ } \\
\Sigma\end{array}$ & $\begin{array}{l}\text { N } \\
\underset{z}{z}\end{array}$ & $\frac{0}{z}$ & $\stackrel{\mathcal{N}}{\stackrel{ \pm}{0}}$ & $\begin{array}{l}\overline{\underline{I}} \\
\text { Ot }\end{array}$ & $\begin{array}{l}\text { ฉి } \\
\text { an }\end{array}$ & $\frac{8}{a}$ & $\frac{\tilde{O}}{\mathrm{~s}}$ & $\begin{array}{l}\text { on } \\
\text { is }\end{array}$ & $\begin{array}{l}\text { o } \\
\text { in }\end{array}$ & 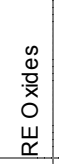 & $\begin{array}{c}\tilde{N} \\
\stackrel{1}{F}\end{array}$ & $\stackrel{\widetilde{N}}{\stackrel{N}{\vdash}}$ & 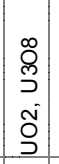 & $\stackrel{q}{N}$ & $\begin{array}{l}\text { ญ̃ } \\
\text { N }\end{array}$ & 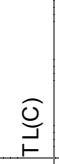 & $\begin{array}{l}\bar{\Xi} \\
\frac{0}{0} \\
\bar{\Xi} \\
z\end{array}$ \\
\hline \begin{tabular}{|l|l} 
Johnston, 1990 \\
\end{tabular} & & & 10.2 & 12.6 & & & 0.2 & & & & & & 12.2 & 1.0 & 2.0 & & 1.0 & & 10.9 & & 1.9 & & 2.6 & & 41.1 & & & & 3.6 & & 0.6 & & & & \\
\hline Johnston, 1990 & & & 10.4 & 13.4 & 0.2 & & 0.7 & & & & & & 12.6 & 1.4 & 4.6 & 0.7 & 1.0 & & 8.6 & & 1.9 & & 2.6 & & 42.0 & & & & 0.0 & & 0.0 & & & & \\
\hline Johnston, 1990 & & & 8.8 & 14.0 & 0.2 & & 0.8 & & & & & & 10.9 & 1.6 & 2.4 & 0.9 & 1.2 & & 8.9 & & 3.2 & & 0.6 & & 42.0 & & & & 3.9 & & 0.7 & & & & \\
\hline Johnston, 1990 & & & 10.0 & 12.3 & 0.2 & & 0.9 & & & & & & 12.0 & 2.5 & 2.7 & 1.2 & 1.3 & & 9.8 & & 2.6 & & 0.6 & & 39.8 & & & & 3.5 & & 0.6 & & & & \\
\hline Johnston, 1990 & & & 11.9 & 12.3 & 0.2 & & 0.9 & & & & & & 12.0 & 2.5 & 2.7 & 1.3 & 1.3 & & 9.8 & & 2.6 & & 0.6 & & 37.8 & & & & 3.5 & & 0.6 & & & & \\
\hline Johnston, 1990 & & & 10.0 & 12.4 & 0.2 & & 0.9 & & & & & & 12.0 & 2.5 & 2.7 & 1.3 & 1.3 & & 9.8 & & 2.6 & & 2.4 & & 37.9 & & & & 3.5 & & 0.6 & & & & \\
\hline Johnston, 1990 & & & 10.0 & 10.4 & 0.2 & & 0.9 & & & & & & 12.0 & 2.5 & 2.7 & 1.3 & 1.3 & & 9.8 & & 2.6 & & 2.5 & & 39.9 & & & & 3.5 & & 0.6 & & & & \\
\hline Johnston, 1990 & & & 9.9 & 12.3 & 0.2 & & 0.8 & & & & & & 11.9 & 1.6 & 2.2 & 0.8 & 1.2 & & 9.8 & & 3.1 & & 2.5 & & 39.6 & & & & 3.5 & & 0.6 & & & & \\
\hline \begin{tabular}{|l|} 
Johnston, 1990 \\
\end{tabular} & & & 9.7 & 12.1 & 0.2 & & 0.8 & & & & & & 11.7 & 1.6 & 2.2 & 0.8 & 1.2 & & 9.6 & & 3.0 & & 2.4 & & 38.9 & & & & 5.2 & & 0.6 & & & & \\
\hline Johnston, 1990 & & & 9.7 & 12.0 & 0.2 & & 0.8 & & & & & & 11.7 & 1.6 & 2.2 & 0.8 & 1.2 & & 11.6 & & 3.0 & & 2.4 & & 38.8 & & & & 3.5 & & 0.6 & & & & \\
\hline Johnston, 1990 & & & 10.1 & 12.5 & 0.2 & & 0.8 & & & & & & 12.2 & 1.6 & 2.3 & 0.8 & 1.2 & & 10.0 & & 3.0 & & 2.5 & & 38.5 & & & & 3.6 & & 0.6 & & & & \\
\hline Johnston, 1990 & & & 9.7 & 14.1 & 0.2 & & 0.8 & & & & & & 11.7 & 1.6 & 2.2 & 0.8 & 1.2 & & 9.6 & & 3.0 & & 2.4 & & 38.8 & & & & 3.5 & & 0.6 & & & & \\
\hline Johnston, 1990 & & & 9.5 & 11.8 & 1.8 & & 0.8 & & & & & & 15.3 & 1.5 & 2.1 & 0.8 & 1.2 & & 9.4 & & 3.0 & & 2.4 & & 38.1 & & & & 3.4 & & 0.6 & & & & \\
\hline \begin{tabular}{|l|} 
Johnston, 1990 \\
\end{tabular} & & & 9.9 & 10.3 & 0.2 & & 0.7 & & & & & & 11.9 & 1.9 & 2.0 & 1.3 & 1.3 & & 11.8 & & 2.7 & & 2.5 & & 39.6 & & & & 3.5 & & 0.6 & & & & \\
\hline \begin{tabular}{|l|} 
Johnston, 1990 \\
\end{tabular} & & & 9.8 & 12.2 & 0.2 & & 0.8 & & & & & & 11.9 & 1.6 & 2.2 & 0.8 & 1.2 & & 9.7 & & 3.1 & & 2.5 & & 39.4 & & & & 3.5 & & 1.2 & & & & \\
\hline Johnston, 1990 & & & 9.8 & 12.2 & 0.2 & & 0.7 & & & & & & 11.9 & 1.9 & 2.1 & 1.3 & 1.3 & & 8.9 & & 2.7 & & 3.5 & & 39.5 & & & & 3.5 & & 0.6 & & & & \\
\hline Johnston, 1990 & & & 10.1 & 12.5 & 0.2 & & 0.8 & & & & & & 12.2 & 1.6 & 2.3 & 0.8 & 1.2 & & 10.0 & & 3.0 & & 2.5 & & 38.5 & & & & 3.6 & & 0.6 & & & & \\
\hline \begin{tabular}{|l|} 
Johnston, 1990 \\
\end{tabular} & & & 10.0 & 12.4 & & & 0.8 & & & & & & 12.0 & 0.9 & 2.1 & 0.8 & 1.0 & & 10.7 & & 2.1 & & 2.6 & & 40.4 & & & & 3.6 & & 0.6 & & & & \\
\hline \begin{tabular}{|l|} 
Johnston, 1990 \\
\end{tabular} & & & 10.0 & 12.4 & & & 0.8 & & & & & & 12.0 & 0.9 & 2.1 & & 1.0 & & 10.7 & & 2.9 & & 2.6 & & 40.4 & & & & 3.6 & & 0.6 & & & & \\
\hline Johnston, 1990 & & & 9.9 & 12.6 & 0.2 & & 0.8 & & & & & & 12.0 & 1.3 & 4.3 & 0.9 & 1.2 & & 8.1 & & 3.0 & & 2.5 & & 39.7 & & & & 3.5 & & 0.0 & & & & \\
\hline \begin{tabular}{|l|} 
Johnston, 1990 \\
\end{tabular} & & & 10.1 & 12.9 & 0.2 & & 0.8 & & & & & & 12.2 & 1.3 & 4.4 & 0.9 & 1.2 & & 8.3 & & 3.1 & & 2.5 & & 40.4 & & & & 1.8 & & 0.0 & & & & \\
\hline \begin{tabular}{|l|} 
Johnston, 1990 \\
\end{tabular} & & & 9.9 & 12.6 & 0.2 & & 0.8 & & & & & & 12.0 & 1.3 & 4.3 & 0.9 & 1.2 & & 8.1 & & 6.5 & & 2.5 & & 39.7 & & & & 0.0 & & 0.0 & & & & \\
\hline \begin{tabular}{|l|} 
Johnston, 1990 \\
\end{tabular} & & & 10.1 & 12.9 & 0.2 & & 0.8 & & & & & & 12.2 & 1.3 & 4.4 & 0.9 & 1.2 & & 8.3 & & 4.8 & & 2.5 & & 40.5 & & & & 0.0 & & 0.0 & & & & \\
\hline Johnston, 1990 & & & 13.8 & 12.2 & 0.2 & & 0.8 & & & & & & 11.9 & 1.6 & 2.3 & 0.8 & 1.2 & & 9.8 & & 2.9 & & 2.5 & & 35.9 & & & & 3.5 & & 0.6 & & & & \\
\hline \begin{tabular}{|l|} 
Johnston, 1990 \\
\end{tabular} & & & 9.9 & 12.2 & 0.2 & & 0.8 & & & & & & 15.9 & 1.6 & 2.3 & 0.8 & 1.2 & & 9.8 & & 2.9 & & 2.5 & & 35.9 & & & & 3.5 & & 0.6 & & & & \\
\hline \begin{tabular}{|l|} 
Johnston, 1990 \\
\end{tabular} & & & 9.9 & 12.2 & & & 0.8 & & & & & & 11.8 & 0.9 & 2.0 & 1.6 & 1.0 & & 10.5 & & 2.8 & & 2.5 & & 39.8 & & & & 3.5 & & 0.6 & & & & \\
\hline \begin{tabular}{|l|} 
Johnston, 1990 \\
\end{tabular} & & & 9.9 & 12.2 & 0.2 & & 0.8 & & & & & & 11.9 & 1.6 & 2.3 & 0.8 & 1.2 & & 9.8 & & 2.9 & & 2.5 & & 39.8 & & & & 3.5 & & 0.6 & & & & \\
\hline 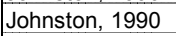 & & & 9.9 & 12.2 & 0.2 & & 0.8 & & & & & & 11.9 & 1.6 & 2.3 & 0.8 & 1.2 & & \begin{tabular}{|c|}
9.8 \\
\end{tabular} & & 6.9 & & 2.5 & & 35.9 & & & & 3.5 & & 0.6 & & & & \\
\hline Johnston, 1990 & & & 10.0 & 12.3 & & & 0.8 & & & & & & 11.9 & 0.9 & 2.1 & 0.8 & 1.0 & & 10.6 & & 2.9 & & 2.6 & & 40.1 & & & & 3.5 & & 0.6 & & & & \\
\hline \begin{tabular}{|l|} 
Johnston, 1990 \\
\end{tabular} & & & 10.0 & 12.3 & & & 0.2 & & & & & & 11.9 & 0.9 & 2.1 & 1.4 & 1.0 & & 10.6 & & 2.9 & & 2.6 & & 40.1 & & & & 3.5 & & 0.6 & & & & \\
\hline \begin{tabular}{|l|} 
Johnston, 1990 \\
\end{tabular} & & & 9.9 & 12.7 & 0.2 & & 0.8 & & & & & & 12.0 & 1.3 & 4.4 & 0.9 & 1.0 & & 8.2 & & 2.1 & & 2.5 & & 40.0 & & & & 3.5 & & 0.6 & & & & \\
\hline \begin{tabular}{|l|} 
Johnston, 1990 \\
\end{tabular} & & & 10.0 & 12.9 & 0.2 & & 0.8 & & & & & & 12.1 & 1.4 & 2.2 & 0.8 & 1.2 & & 8.5 & & 2.9 & & 2.5 & & 40.3 & & & & 3.5 & & 0.6 & & & & \\
\hline \begin{tabular}{|l|} 
Johnston, 1990 \\
\end{tabular} & & & 8.1 & 12.8 & 0.2 & & 0.8 & & & & & & 12.1 & 1.6 & 2.3 & 0.8 & 1.2 & & 10.2 & & 2.9 & & 2.5 & & 40.2 & & & & 3.6 & & 0.6 & & & & \\
\hline Johnston, 1990 & & & 9.9 & 12.7 & 0.2 & & 0.8 & & & & & & 12.0 & 1.6 & 2.2 & 0.8 & 1.2 & & 10.0 & & 2.9 & & 1.5 & & 39.9 & & & & 3.6 & & 0.6 & & & & \\
\hline Johnston, 1990 & & & 9.8 & 12.5 & 0.2 & & 0.9 & & & & & & 11.9 & 3.4 & 2.9 & 1.0 & 1.2 & & 10.5 & & 2.9 & & 2.5 & & 37.5 & & & & 3.5 & & 0.6 & & & & \\
\hline Johnston, 1990 & & & 9.8 & 12.2 & 0.2 & & 0.8 & & & & & & 11.9 & 1.6 & 2.2 & 0.8 & 1.2 & & 9.8 & & 3.3 & & 2.5 & & 39.5 & & & & 3.5 & & 0.6 & & & & \\
\hline \begin{tabular}{|l|} 
Johnston, 1990 \\
\end{tabular} & & & 9.7 & 12.0 & 0.2 & & 0.8 & & & & & & 13.7 & 1.6 & 2.2 & 0.8 & 1.2 & & 9.6 & & 3.0 & & 2.4 & & 38.8 & & & & 3.5 & & 0.6 & & & & \\
\hline \begin{tabular}{|l|} 
Johnston, 1990 \\
\end{tabular} & & & 9.6 & 12.0 & 0.2 & & 0.8 & & & & & & 11.7 & 1.6 & 2.2 & 0.8 & 1.2 & & 9.6 & & 3.0 & & 2.4 & & 41.0 & & & & 3.4 & & 0.6 & & & & \\
\hline Johnston, 1990 & & & 7.4 & 10.6 & 0.2 & & 0.8 & & & & & & 11.9 & 3.6 & 3.1 & 0.9 & 1.2 & & 11.3 & & 3.0 & & 2.3 & & 39.5 & & & & 3.5 & & 0.6 & & & & \\
\hline Johnston, 1990 & & & 8.4 & 10.1 & 0.2 & & 0.8 & & & & & & 11.9 & 3.5 & 3.0 & 0.9 & 1.2 & & 10.9 & & 3.0 & & 2.3 & & 39.5 & & & & 3.5 & & 0.6 & & & & \\
\hline Johnston, 1990 & & & 8.4 & 9.2 & 0.2 & & 0.8 & & & & & & 12.0 & 3.3 & 2.8 & 0.9 & 1.2 & & 10.1 & & 3.0 & & 2.4 & & 41.6 & & & & 3.5 & & 0.6 & & & & \\
\hline Johnston, 1990 & & & 9.9 & 9.5 & 0.2 & & 0.8 & & & & & & 12.0 & 3.3 & 2.9 & 0.9 & 1.2 & & 10.3 & & 3.0 & & 2.4 & & 39.6 & & & & 3.5 & & 0.6 & & & & \\
\hline Johnston, 1990 & & & 8.2 & 9.3 & 0.2 & & 0.9 & & & & & & 11.6 & 3.3 & 2.8 & 1.2 & 1.4 & & 10.2 & & 2.6 & & 2.3 & & 41.9 & & & & 3.4 & & 0.6 & & & & \\
\hline Karell, 2006 & & 1337 & & & & & 9.3 & & & & & & & & & & & & 15.4 & & & & & & 73.8 & & & & & & & & 1.5 & & \\
\hline Karell, 2006 & & 1338 & & & & & 9.2 & & & & & & & & & & & & 15.3 & & & & & & 71.0 & & & & & & & & 4.5 & & \\
\hline Karell, 2006 & & 1331 & & & & & 9.1 & & & & & & & & & & & & 15.1 & & & & & & 68.4 & & & & & & & & 7.4 & & \\
\hline Karell, 2006 & & & & & & & 9.0 & & & & & & & & & & & & 15.0 & & & & & & 65.7 & & & & & & & & 10.3 & & \\
\hline Karell, 2006 & & & & & & & 9.4 & & & & & & & & & & & & 15.5 & & & & & & 75.1 & & & & & & & & & & \\
\hline Karell, 2006 & & 1278 & & & & & 9.3 & & & & & & & & & & & & 15.4 & & & & & & 73.8 & & & & & & & & 1.5 & & \\
\hline Karell, 2006 & & 1316 & & & & & 9.2 & & & & & & & & & & & & 15.3 & & & & & & 71.0 & & & & & & & & 4.5 & & \\
\hline
\end{tabular}


Table A1. Glass Compositions from Literature Review (continued)

\begin{tabular}{|c|c|c|c|c|c|c|c|c|c|c|c|c|c|c|c|c|c|c|c|c|c|c|c|c|c|c|c|c|c|c|c|c|c|c|c|}
\hline Ref & 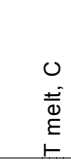 & $\begin{array}{l}0 \\
0 \\
\circ \\
\stackrel{0}{E} \\
\vdash\end{array}$ & $\begin{array}{l}\substack{N \\
\mathbb{N}} \\
\end{array}$ & 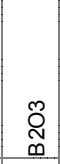 & $\begin{array}{l}\text { Oి } \\
\infty\end{array}$ & $\begin{array}{c}\text { Oొ } \\
\stackrel{N}{\infty}\end{array}$ & $\begin{array}{l}0 \\
\pi \\
0\end{array}$ & 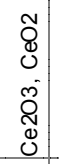 & $\begin{array}{l}m \\
0 \\
0 \\
0 \\
0 \\
0 \\
0\end{array}$ & $\begin{array}{l}\text { Oొ } \\
\text { บิ }\end{array}$ & $\stackrel{0}{3}$ & 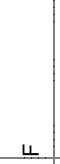 & 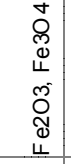 & $\begin{array}{l}\underset{\mathcal{X}}{x} \\
\end{array}$ & 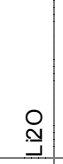 & $\begin{array}{l}\circ \\
\sum \\
\Sigma\end{array}$ & $\begin{array}{l}\tilde{O} \\
\text { C } \\
\Sigma\end{array}$ & $\begin{array}{l}0 \\
0 \\
\Sigma\end{array}$ & $\begin{array}{l}\text { N } \\
\underset{z}{z}\end{array}$ & $\frac{0}{z}$ & $\begin{array}{l}\text { N } \\
\text { tै }\end{array}$ & 吾 & 足 & $\frac{8}{a}$ & $\frac{\widetilde{O}}{\infty}$ & O) & $\begin{array}{l}\text { Q } \\
\text { in }\end{array}$ & 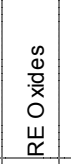 & 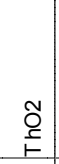 & 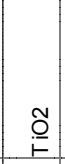 & 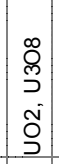 & $\stackrel{q}{N}$ & $\begin{array}{l}\text { ò } \\
\text { N }\end{array}$ & $\underset{1}{\underline{0}}$ & $\begin{array}{l}\bar{\Xi} \\
\frac{0}{0} \\
\frac{1}{z}\end{array}$ \\
\hline Karell, 2006 & & 1340 & & & & & 9.1 & & & & & & & & & & & & 15.1 & & & & & & 68.4 & & & & & & & & 7.4 & & \\
\hline Karell, 2006 & & 1370 & & & & & 9.0 & & & & & & & & & & & & 15.0 & & & & & & 65.7 & & & & & & & & 10.3 & & \\
\hline Khizanishvili, 1968 & 1300 & & 19.9 & 5.3 & & & 3.2 & & & & & & 4.3 & 5.5 & & 1.3 & & & 8.6 & & & & & & 51.3 & & & & & 0.3 & & & & & \\
\hline Khizanishvili, 1970 & 1130 & & 12.9 & 6.7 & & & 0.7 & & & & & & 3.9 & & & 0.1 & 3.4 & & 6.4 & & & & & & 65.6 & & 0.3 & & & & & & & & \\
\hline Khizanishvili, 1970 & 1100 & & 14.3 & & & & 0.8 & & & & & & 0.5 & 7.5 & & 0.2 & & & 3.7 & & & & & & 72.7 & & 0.3 & & & & & & & & \\
\hline Khizanishvili, 1970 & 1200 & & 14.9 & & & & 0.8 & & & & & & 0.5 & 3.7 & & 0.2 & & & 3.9 & & & & & & 75.7 & & 0.3 & & & & & & & & \\
\hline Khizanishvili, 1970 & 1130 & & 12.7 & 6.7 & & & 0.7 & & & & & & 8.5 & & & 0.1 & & & 6.3 & & & & & & 64.7 & & 0.3 & & & & & & & & \\
\hline Khizanishvili, 1970 & 1335 & & 13.8 & & & & 0.7 & & & & & & 0.5 & & & 0.2 & & & 3.6 & & 10.6 & & & & 70.3 & & 0.3 & & & & & & & & \\
\hline Khizanishvili, 1973 & 1275 & & 9.3 & 15.5 & & & 3.2 & & & & & & 0.4 & 1.5 & & 0.3 & & & 1.6 & & & & & & 46.9 & & 6.6 & & & & & 9.4 & 5.6 & & \\
\hline Kim, 1996 & & 1409 & 5.6 & 0.1 & & 1.6 & 1.4 & 1.9 & & 0.6 & & 0.4 & 7.8 & & & 0.1 & 1.3 & 0.1 & 14.1 & 1.6 & 0.3 & 0.1 & 0.9 & & 50.1 & 0.2 & & 6.9 & & & & & 5.0 & & \\
\hline Kim, 1996 & & 1344 & 6.0 & 0.1 & & 1.7 & 1.6 & 2.1 & & 0.7 & & 0.4 & 8.4 & & & 0.1 & 1.4 & 0.1 & 15.3 & 1.7 & 0.3 & 0.1 & 1.0 & & 45.7 & 0.3 & & 7.5 & & & & & 5.4 & & \\
\hline Kim, 1996 & & 1282 & 6.5 & 0.1 & & 1.8 & 1.7 & 2.3 & & 0.7 & & 0.5 & 9.1 & & & 0.1 & 1.5 & 0.1 & 16.5 & 1.9 & 0.4 & 0.1 & 1.1 & & 41.3 & 0.3 & & 8.1 & & & & & 5.9 & & \\
\hline Kim, 1996 & & 1229 & 6.9 & 0.1 & & 2.0 & 1.8 & 2.4 & & 0.8 & & 0.5 & 9.7 & & & 0.1 & 1.6 & 0.1 & 17.5 & 2.0 & 0.4 & 0.1 & 1.1 & & 37.8 & 0.3 & & 8.5 & & & & & 6.2 & & \\
\hline Kim, 1996 & & & 4.5 & 0.0 & 0.2 & & 0.4 & 0.3 & & 0.2 & 0.3 & 0.6 & 14.1 & & & 0.1 & 0.3 & 0.6 & 10.7 & 1.1 & 1.2 & 2.7 & 0.2 & & 52.0 & 0.5 & 0.2 & 2.4 & & & & & 7.5 & & \\
\hline Kim, 1996 & & & 4.5 & 4.0 & 0.2 & & 0.4 & 0.3 & & 0.2 & 0.3 & 0.6 & 14.1 & & & 0.1 & 0.3 & 0.6 & 10.7 & 1.1 & 1.2 & 2.7 & 0.2 & & 48.0 & 0.5 & 0.2 & 2.4 & & & & & 7.5 & & \\
\hline Kim, 1996 & & & 4.5 & 15.0 & 0.2 & & 0.4 & 0.3 & & 0.2 & 0.3 & 0.6 & 14.1 & & & 0.1 & 0.3 & 0.6 & 10.7 & 1.1 & 1.2 & 2.7 & 0.2 & & 37.0 & 0.5 & 0.2 & 2.4 & & & & & 7.5 & & \\
\hline Kim, 1996 & & 1381 & 4.5 & 0.0 & 0.2 & & 0.4 & 0.3 & & 0.2 & 0.3 & 0.6 & 14.1 & & & 0.1 & 0.3 & 0.6 & 14.4 & 1.1 & 1.2 & 2.7 & 0.2 & & 48.3 & 0.5 & 0.2 & 2.4 & & & & & 7.5 & & \\
\hline Kim, 1996 & & 1351 & 4.5 & 0.0 & 0.2 & & 0.4 & 0.3 & & 0.2 & 0.3 & 0.6 & 14.1 & & 1.7 & 0.1 & 0.3 & 0.6 & 10.7 & 1.1 & 1.2 & 2.7 & 0.2 & & 50.3 & 0.5 & 0.2 & 2.4 & & & & & 7.5 & & \\
\hline Kim, 1996 & & 1344 & 4.5 & 0.0 & 0.2 & & 0.4 & 0.3 & & 0.2 & 0.3 & 0.6 & 14.1 & & & 0.1 & 0.3 & 0.6 & 16.7 & 1.1 & 1.2 & 2.7 & 0.2 & & 46.0 & 0.5 & 0.2 & 2.4 & & & & & 7.5 & & \\
\hline Kim, 1996 & & 1297 & 4.5 & 0.0 & 0.2 & & 0.4 & 0.3 & & 0.2 & 0.3 & 0.6 & 14.1 & & 2.7 & 0.1 & 0.3 & 0.6 & 10.7 & 1.1 & 1.2 & 2.7 & 0.2 & & 49.3 & 0.5 & 0.2 & 2.4 & & & & & 7.5 & & \\
\hline Kim, 2001 & 1177 & & 9.0 & 13.3 & & & 13.6 & & & 0.3 & & & 2.0 & & & 1.5 & & & 14.0 & & & & & & 45.1 & & & & & & & & & & \\
\hline Kim, 2001 & 1177 & & 8.7 & 12.8 & & & 13.1 & & & 0.4 & & & 1.9 & & & 1.5 & & & 17.2 & & & & & & 43.5 & & & & & & & & & & \\
\hline Kukolev, 1969 & & & 6.5 & 18.7 & 4.6 & & 6.5 & & & & & & & 1.1 & & 0.5 & & & 4.5 & & & & & & 45.8 & & & & & & & 2.8 & 8.8 & & \\
\hline Kukolev, 1969 & & & 11.9 & & & & 10.0 & & & & & & & & & 4.3 & & & 4.7 & & & & & & 57.6 & & 1.5 & & & & & 3.6 & 7.0 & & \\
\hline Kukolev, 1969 & & & 17.7 & & 0.1 & & 6.8 & & & & & & & 0.2 & 1.6 & 0.4 & & & 5.4 & & & & 10.5 & & 44.4 & & 0.5 & & & & & 4.6 & 7.8 & & \\
\hline Kukolev, 1969 & & & 14.0 & & & & 7.2 & & & & & 2.4 & & & 0.7 & 2.0 & & & 10.4 & & & & 6.0 & & 47.3 & & 2.5 & & & & & 2.1 & 5.4 & & \\
\hline Kukolev, 1969 & & & 7.8 & & 0.1 & & 5.3 & & & & & & & 0.3 & 0.4 & & & & 10.8 & & & & 7.2 & & 54.9 & & 8.1 & & & & & 2.4 & 2.7 & & \\
\hline Kukolev, 1969 & & & 5.6 & 19.6 & 4.7 & & 5.5 & & & & & & & & & 1.8 & & & 3.0 & & & & & & 40.0 & & 2.3 & & & & & 9.4 & 8.1 & & \\
\hline Kukolev, 1969 & & & 3.8 & 14.2 & 11.3 & & 8.6 & & & & & & & 0.2 & 0.2 & 1.0 & & & 2.0 & & & & & & 42.6 & & 2.8 & & & & & 5.1 & 8.1 & & \\
\hline Li, 1997 & & & 7.8 & 8.6 & & & 1.2 & & & & & & 10.6 & 0.1 & 4.8 & 0.7 & & & 21.7 & & 3.1 & & & & 41.1 & & & & & & & & 0.3 & & \\
\hline Li, 1997 & & & 17.7 & 7.6 & & & 1.1 & & & & & & 9.5 & 0.1 & 4.3 & 0.7 & & & 19.4 & & 2.7 & & & & 36.6 & & & & & & & & 0.3 & & \\
\hline Li, 1997 & & & 19.3 & 7.5 & & & 1.1 & & & & & & 9.3 & 0.1 & 4.2 & 0.6 & & & 19.0 & & 2.7 & & & & 35.9 & & & & & & & & 0.3 & & \\
\hline Li, 1997 & & & 15.0 & & & & 1.2 & & & & & & 10.8 & 0.1 & 4.9 & 0.7 & & & 22.0 & & 3.1 & & & & 41.7 & & & & & & & & 0.3 & & \\
\hline Li, 1997 & & & 12.0 & 20.0 & & & 1.0 & & & & & & 8.6 & 0.1 & 3.9 & 0.6 & & & 17.6 & & 2.5 & & & & 33.4 & & & & & & & & 0.3 & & \\
\hline Li, 1997 & & & 13.8 & 8.0 & & & 1.1 & & & & & & 9.9 & 0.1 & 4.5 & 0.7 & & & 20.3 & & 2.9 & & & & 38.4 & & & & & & & & 0.3 & & \\
\hline Li, 1997 & & & 13.9 & 8.1 & & & & & & & & & 10.1 & 0.1 & 4.6 & 0.7 & & & 20.5 & & 2.9 & & & & 38.8 & & & & & & & & 0.3 & & \\
\hline Li, 1997 & & & 12.5 & 7.3 & & & 10.0 & & & & & & 9.1 & 0.1 & 4.1 & 0.6 & & & 18.5 & & 2.6 & & & & 34.9 & & & & & & & & 0.3 & & \\
\hline Li, 1997 & & & 14.4 & 8.4 & & & 1.2 & & & & & & 6.0 & 0.1 & 4.7 & 0.7 & & & 21.2 & & 3.0 & & & & 40.1 & & & & & & & & 0.3 & & \\
\hline Li, 1997 & & & 14.1 & 8.2 & & & 1.2 & & & & & & 8.0 & 0.1 & 4.6 & 0.7 & & & 20.8 & & 2.9 & & & & 39.3 & & & & & & & & 0.3 & & \\
\hline Li, 1997 & & & 13.3 & 7.7 & & & 1.1 & & & & & & 13.0 & 0.1 & 4.4 & 0.7 & & & 19.6 & & 2.8 & & & & 37.1 & & & & & & & & 0.3 & & \\
\hline Li, 1997 & & & 13.4 & 7.8 & & & 1.1 & & & & & & 9.7 & 3.0 & 4.4 & 0.7 & & & 19.7 & & 2.8 & & & & 37.3 & & & & & & & & 0.3 & & \\
\hline Li, 1997 & & & 13.0 & 7.5 & & & 1.1 & & & & & & 9.4 & 6.0 & 4.2 & 0.6 & & & 19.1 & & 2.7 & & & & 36.1 & & & & & & & & 0.3 & & \\
\hline Li, 1997 & & & 14.4 & 8.4 & & & 1.2 & & & & & & 10.4 & 0.1 & & 0.7 & & & 21.2 & & 3.0 & & & & 40.2 & & & & & & & & 0.3 & & \\
\hline Li, 1997 & & & 13.3 & 7.7 & & & 1.1 & & & & & & 9.6 & 0.1 & 8.0 & 0.7 & & & 19.5 & & 2.8 & & & & 37.0 & & & & & & & & 0.3 & & \\
\hline Li, 1997 & & & 15.0 & 8.7 & & & 1.2 & & & & & & 10.8 & 0.1 & 4.9 & 0.7 & & & 13.3 & & 3.1 & & & & 41.7 & & & & & & & & 0.3 & & \\
\hline Li, 1997 & & & 14.7 & 8.6 & & & 1.2 & & & & & & 10.6 & 0.1 & 4.8 & 0.7 & & & 14.8 & & 3.1 & & & & 41.0 & & & & & & & & 0.3 & & \\
\hline Li, 1997 & & & 12.2 & 7.1 & & & 1.0 & & & & & & 8.8 & 0.1 & 4.0 & 0.6 & & & 18.0 & & 2.5 & & & & 45.3 & & & & & & & & 0.3 & & \\
\hline Li, 1997 & & & 11.8 & 6.8 & & & 1.0 & & & & & & 8.5 & 0.1 & 3.8 & 0.6 & & & 17.3 & & 2.4 & & & & 47.4 & & & & & & & & 0.3 & & \\
\hline Mahoney, 2005 & & & 12.0 & & & & 4.6 & & & 0.1 & & 0.1 & 7.8 & 2.1 & & 1.2 & & & 17.0 & & & & 0.6 & & 52.5 & 0.7 & & & & 1.2 & & & & & v \\
\hline Mahoney, 2005 & & & 11.6 & & & & 4.4 & & & 0.1 & & 0.1 & 7.5 & 2.1 & & 1.2 & & & 20.0 & & & & 0.6 & & 50.3 & 0.8 & & & & 1.2 & & & & & v \\
\hline
\end{tabular}


Table A1. Glass Compositions from Literature Review (continued)

\begin{tabular}{|c|c|c|c|c|c|c|c|c|c|c|c|c|c|c|c|c|c|c|c|c|c|c|c|c|c|c|c|c|c|c|c|c|c|c|c|}
\hline Ref & 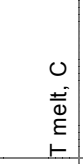 & $\begin{array}{l}0 \\
0 \\
\circ \\
0 \\
1\end{array}$ & $\begin{array}{l}\text { Õ } \\
\stackrel{N}{<}\end{array}$ & 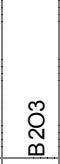 & $\begin{array}{l}\text { ષ } \\
\infty\end{array}$ & 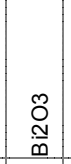 & $\begin{array}{l}0 \\
\mathbb{\pi} \\
0\end{array}$ & 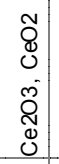 & $\begin{array}{l}0 \\
0 \\
0 \\
0 \\
0 \\
0 \\
0\end{array}$ & $\begin{array}{l}\text { Oొ } \\
\text { บิ }\end{array}$ & O̊ & 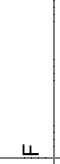 & 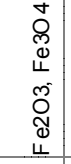 & $\begin{array}{l}\underset{\mathcal{X}}{x} \\
\end{array}$ & 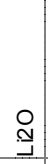 & $\stackrel{\circ}{\circ}$ & $\begin{array}{l}\text { Õ } \\
\text { C. }\end{array}$ & $\begin{array}{l}0 \\
0 \\
\Sigma\end{array}$ & $\begin{array}{l}\text { Q } \\
\text { Z } \\
Z\end{array}$ & $\frac{\circ}{z}$ & $\begin{array}{l}\mathcal{N} \\
\tilde{0}\end{array}$ & $\overline{\bar{I}}$ & ڤ̊ & $\frac{8}{2}$ & $\frac{\widetilde{O}}{\omega}$ & $\begin{array}{l}\text { Oొ } \\
\text { in }\end{array}$ & $\begin{array}{l}\text { Q } \\
\text { is }\end{array}$ & 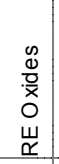 & $\begin{array}{l}\text { Õ } \\
\text { 尺े } \\
\end{array}$ & $\stackrel{\widetilde{N}}{\stackrel{N}{⺊}}$ & 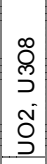 & $\frac{Q}{N}$ & $\begin{array}{l}\text { Õ } \\
\text { N }\end{array}$ & $\begin{array}{l}\overline{0} \\
\overrightarrow{1}\end{array}$ & $\begin{array}{l}\bar{\Xi} \\
0 \\
\underline{0} \\
z\end{array}$ \\
\hline Mahoney, 2005 & & & 11.2 & & & & 4.2 & & & 0.1 & & 0.1 & 7.2 & 2.0 & & 1.1 & & & 23.0 & & & & 0.7 & & 48.2 & 1.0 & & & & 1.1 & & & & & $\mathrm{v}$ \\
\hline Mahoney, 2005 & & & 10.8 & & & & 4.1 & & & 0.1 & & 0.1 & 6.8 & 1.9 & & 1.1 & & & 26.0 & & & & 0.8 & & 46.0 & 1.1 & & & & 1.1 & & & & & $v, p$ \\
\hline Mahoney, 2005 & & & 10.9 & & & & 4.2 & & & 0.1 & & 0.1 & 7.0 & 1.9 & & 1.1 & & & 20.0 & & & & 0.6 & & 47.1 & 0.8 & & & & 1.1 & & & 5.0 & & $v$ \\
\hline Mahoney, 2005 & & & 10.9 & 2.5 & & & 4.2 & & & 0.1 & & 0.1 & 7.0 & 1.9 & & 1.1 & & & 20.0 & & & & 0.6 & & 47.1 & 0.8 & & & & 1.1 & & & 2.5 & & $v$ \\
\hline Mahoney, 2005 & & & 10.5 & & & & 4.0 & & & 0.1 & & 0.1 & 6.7 & 1.9 & & 1.0 & & & 23.0 & & & & 0.7 & & 44.9 & 1.0 & & & & 1.0 & & & 5.0 & & $\mathrm{v}$ \\
\hline Mahoney, 2005 & & & 10.5 & 2.5 & & & 4.0 & & & 0.1 & & 0.1 & 6.7 & 1.9 & & 1.0 & & & 23.0 & & & & 0.7 & & 44.9 & 1.0 & & & & 1.0 & & & 2.5 & & $v$ \\
\hline Mahoney, 2005 & & & 8.5 & & & & 4.1 & & & 0.1 & & 0.1 & 6.9 & 1.9 & & 1.1 & & & 23.0 & & & & 0.7 & & 46.4 & 1.0 & & & & 1.1 & & & 5.0 & & $v$ \\
\hline Mahoney, 2005 & & & 12.5 & & & & 3.8 & & & 0.1 & & 0.1 & 6.5 & 1.8 & & 1.0 & & & 23.0 & & & & 0.7 & & 43.4 & 1.0 & & & & 1.0 & & & 5.0 & & $v, c$ \\
\hline Mahoney, 2005 & & & 10.8 & & & & 4.1 & & & 0.1 & & 0.1 & 4.7 & 1.9 & & 1.1 & & & 23.0 & & & & 0.7 & & 46.4 & 1.0 & & & & 1.1 & & & 5.0 & & $v$ \\
\hline Mahoney, 2005 & & & 10.2 & & & & 3.8 & & & 0.1 & & 0.1 & 8.7 & 1.8 & & 1.0 & & & 23.0 & & & & 0.7 & & 43.5 & 1.0 & & & & 1.0 & & & 5.0 & & v \\
\hline Mahoney, 2005 & & & 9.9 & 5.0 & & & 3.8 & & & 0.1 & & 0.1 & 6.3 & 1.8 & & 1.0 & & & 20.0 & & & & 0.6 & & 42.6 & 0.8 & & & & 1.0 & & & 7.0 & & \\
\hline Mahoney, 2005 & & & 9.5 & 5.0 & & & 3.6 & & & 0.1 & & 0.1 & 6.0 & 1.7 & & 0.9 & & & 20.0 & & & & 0.6 & & 40.6 & 0.8 & & & & 0.9 & & & 10.0 & & c \\
\hline Mahoney, 2005 & & & 9.5 & 5.0 & & & 3.6 & & & 0.1 & & 0.1 & 6.0 & 1.7 & & 0.9 & & & 20.0 & & & & 1.6 & & 40.6 & 0.8 & & & & 1.9 & & & 6.0 & & v \\
\hline Mahoney, 2005 & & & 9.9 & 5.0 & & & 3.8 & & & 0.1 & & 0.1 & 6.4 & 1.8 & & 1.0 & & & 17.0 & & & & 0.5 & & 45.8 & 0.7 & & & & 1.0 & & & 7.0 & & \\
\hline Mahoney, 2005 & & & 9.1 & 5.0 & & & 2.8 & & & 0.1 & & 0.0 & 4.3 & 1.5 & & 1.4 & & & 20.0 & & & & 0.6 & & 46.8 & 0.4 & & & & 0.8 & & & 7.0 & & \\
\hline Mahoney, 2005 & & & 9.8 & 5.0 & & & 5.1 & & & 0.1 & & 0.0 & 6.3 & 1.0 & & 2.8 & & & 20.0 & & & & 0.3 & & 40.7 & 0.8 & & & & 1.0 & & & 7.0 & & \\
\hline Mahoney, 2005 & & & 9.7 & 5.0 & & & 5.1 & & & 0.6 & & 0.0 & 6.5 & 1.0 & & 2.8 & & & 20.0 & & & & 0.2 & & 40.8 & 0.2 & & & & 1.0 & & & 7.0 & & \\
\hline Mahoney, 2005 & & & 9.4 & 4.2 & & & 2.9 & & & 0.1 & & 0.0 & 4.5 & 1.6 & & 1.4 & & & 20.0 & & & & 0.6 & & 48.2 & 0.4 & & & & 0.8 & & & 5.8 & & \\
\hline Mahoney, 2005 & & & 9.7 & 3.3 & & & 2.9 & & & 0.1 & & 0.0 & 4.6 & 1.6 & & 1.4 & & & 20.0 & & & & 0.6 & & 49.6 & 0.4 & & & & 0.8 & & & 4.7 & & v \\
\hline Mahoney, 2005 & & & 9.9 & 2.5 & & & 3.0 & & & 0.1 & & 0.0 & 4.7 & 1.6 & & 1.5 & & & 20.0 & & & & 0.6 & & 51.0 & 0.4 & & & & 0.9 & & & 3.5 & & v \\
\hline Mahoney, 2005 & & & 10.2 & 1.7 & & & 3.1 & & & 0.1 & & 0.0 & 4.9 & 1.7 & & 1.5 & & & 20.0 & & & & 0.6 & & 52.4 & 0.4 & & & & 0.9 & & & 2.3 & & v \\
\hline Mahoney, 2005 & & & 8.9 & 5.0 & & & 2.7 & & & 0.2 & & 0.0 & 4.2 & 1.5 & & 1.3 & & & 22.0 & & & & 0.7 & & 45.3 & 0.4 & & & & 0.8 & & & 7.0 & & \\
\hline Mahoney, 2005 & & & 8.6 & 5.0 & & & 2.6 & & & 0.2 & & 0.0 & 4.1 & 1.4 & & 1.3 & & & 24.0 & & & & 0.7 & & 43.8 & 0.5 & & & & 0.7 & & & 7.0 & & v \\
\hline Mahoney, 2005 & & & 11.7 & 4.9 & & & 2.7 & & & 0.1 & & 0.0 & 4.2 & 1.5 & & 1.4 & & & 19.4 & & & & 0.6 & & 45.4 & 0.4 & & & & 0.8 & & & 6.8 & & \\
\hline Mahoney, 2005 & & & 14.2 & 4.7 & & & 2.7 & & & 0.1 & & 0.0 & 4.1 & 1.4 & & 1.5 & & & 18.8 & & & & 0.6 & & 44.0 & 0.3 & & & & 0.8 & & & 6.6 & & \\
\hline Mahoney, 2005 & & & 16.8 & 4.6 & & & 2.7 & & & 0.1 & & 0.0 & 4.0 & 1.4 & & 1.6 & & & 18.2 & & & & 0.6 & & 42.6 & 0.3 & & & & 0.7 & & & 6.4 & & \\
\hline Mahoney, 2005 & & & 16.0 & 2.0 & & & 2.5 & & & 0.4 & & 0.1 & 4.0 & 2.5 & & 3.0 & & & 18.0 & & & & 1.6 & & 39.0 & 1.0 & & & & 2.0 & & & 7.8 & & $t, p, c$ \\
\hline Mahoney, 2005 & & & 8.0 & 6.0 & & & 2.5 & & & 0.4 & & 0.1 & 11.0 & 2.5 & & 3.0 & & & 18.0 & & & & 0.2 & & 44.0 & 0.1 & & & & 2.0 & & & 2.0 & & \\
\hline Mahoney, 2005 & & & 8.0 & 6.0 & & & 2.5 & & & 0.4 & & 0.1 & 11.0 & 2.5 & & 0.9 & & & 18.0 & & & & 1.6 & & 43.9 & 1.0 & & & & 2.0 & & & 2.0 & & $t, p$ \\
\hline Mahoney, 2005 & & & 8.0 & 2.0 & & & 5.5 & & & 0.4 & & 0.1 & 11.0 & 2.5 & & 0.9 & & & 20.1 & & & & 0.2 & & 39.0 & 0.1 & & & & 2.0 & & & 8.0 & & $t, p, c$ \\
\hline Mahoney, 2005 & & & 12.5 & 2.0 & & & 2.5 & & & 0.4 & & 0.1 & 4.0 & 2.5 & & 3.0 & & & 23.5 & & & & 0.2 & & 39.0 & 0.1 & & & & 2.0 & & & 8.0 & & \\
\hline Mahoney, 2005 & & & 8.0 & 3.0 & & & 5.5 & & & 0.4 & & 0.1 & 11.0 & 0.9 & & 3.0 & & & 18.0 & & & & 0.2 & & 46.1 & 1.0 & & & & 0.7 & & & 2.0 & & \\
\hline Mahoney, 2005 & & & 8.0 & 2.0 & & & 2.5 & & & 0.4 & & 0.1 & 4.0 & 0.9 & & 3.0 & & & 18.0 & & & & 0.2 & & 54.8 & 1.0 & & & & 2.0 & & & 3.0 & & \\
\hline Mahoney, 2005 & & & 8.0 & 2.0 & & & 5.5 & & & 0.4 & & 0.1 & 4.0 & 0.9 & & 0.9 & & & 19.3 & & & & 1.6 & & 47.1 & 0.1 & & & & 2.0 & & & 8.0 & & c \\
\hline Mahoney, 2005 & & & 11.3 & 2.0 & & & 2.5 & & & 0.4 & & 0.1 & 11.0 & 0.9 & & 3.0 & & & 18.0 & & & & 1.6 & & 39.0 & 1.0 & & & & 1.1 & & & 8.0 & & c \\
\hline Mahoney, 2005 & & & 14.7 & 6.0 & & & 5.5 & & & 0.4 & & 0.1 & 4.0 & 2.5 & & 3.0 & & & 18.0 & & & & 1.6 & & 39.0 & 0.1 & & & & 0.7 & & & 4.2 & & c \\
\hline Mahoney, 2005 & & & 16.0 & 6.0 & & & 2.5 & & & 0.4 & & 0.1 & 11.0 & 0.9 & & 0.9 & & & 18.0 & & & & 0.2 & & 39.0 & 0.1 & & & & 2.0 & & & 2.8 & & c \\
\hline Mahoney, 2005 & & & 16.0 & 2.0 & & & 5.5 & & & 0.4 & & 0.1 & 4.0 & 0.9 & & 0.9 & & & 21.5 & & & & 0.2 & & 39.0 & 1.0 & & & & 2.0 & & & 6.4 & & $t, p, c$ \\
\hline Mahoney, 2005 & & & 14.1 & 2.0 & & & 5.5 & & & 0.4 & & 0.1 & 11.0 & 2.5 & & 0.9 & & & 18.0 & & & & 1.6 & & 39.0 & 0.1 & & & & 0.7 & & & 4.0 & & $t, p, c$ \\
\hline Mahoney, 2005 & & & 9.9 & 5.0 & & & 3.8 & & & 0.1 & & 0.1 & 6.3 & 1.8 & & 1.0 & & & 20.0 & & & & 0.6 & & 42.6 & 0.8 & & & & 1.0 & & & 7.0 & & \\
\hline Mahoney, 2005 & & & 9.9 & 5.0 & & & 3.8 & & & 0.1 & & 0.1 & 6.4 & 1.8 & & 1.0 & & & 17.0 & & & & 0.5 & & 45.8 & 0.7 & & & & 1.0 & & & 7.0 & & \\
\hline Mahoney, 2005 & & & 10.3 & 5.0 & & & 3.9 & & & 0.1 & & 0.1 & 6.7 & 1.8 & & 1.0 & & & 17.0 & & & & 0.5 & & 44.7 & 0.7 & & & & 1.0 & & & 7.0 & & \\
\hline Mahoney, 2005 & & & 9.6 & 5.0 & & & 3.6 & & & 0.1 & & 0.1 & 6.1 & 1.7 & & 0.9 & & & 22.0 & & & & 0.6 & & 41.1 & 0.9 & & & & 0.9 & & & 7.0 & & \\
\hline Mahoney, 2005 & & & 9.3 & 5.0 & & & 3.5 & & & 0.1 & & 0.1 & 5.9 & 1.7 & & 0.9 & & & 24.0 & & & & 0.7 & & 39.7 & 1.0 & & & & 0.9 & & & 7.0 & & v \\
\hline Mangat, 1997 & $\sim 1400$ & & 7.4 & 16.7 & & 42.0 & & & & & & & & & & & & & & & & & & & 33.9 & & & & & & & & & & \\
\hline Mangat, 1997 & $\sim 1400$ & & 3.4 & 18.9 & & 31.1 & & & & & & & & & & 1.7 & & & & & & & & & 41.0 & & & & & & & & 4.0 & & \\
\hline Mangat, 1997 & $\sim 1400$ & & 4.9 & 19.1 & & 47.6 & & & & & & & & & & & & & & & & & & & 28.5 & & & & & & & & & & \\
\hline Mangat, 1997 & $\sim 1400$ & & 4.9 & 14.3 & & 47.6 & & & & & & & & & & & & & & & & & & & 33.3 & & & & & & & & & & \\
\hline Mangat, 1997 & $\sim 1400$ & & 3.4 & 18.8 & & 30.9 & & & & & & & & & & 2.4 & & & 1.5 & & & & & & 40.7 & & & & & & & & 2.3 & & \\
\hline Mangat, 1997 & $\sim 1400$ & & 3.4 & 19.0 & & 31.1 & & & & & & & & & & 1.7 & & & & & & & & & 40.8 & & & & & & & & 4.0 & & \\
\hline
\end{tabular}




\begin{tabular}{|c|c|c|c|c|c|c|c|c|c|c|c|c|c|c|c|c|c|c|c|c|c|c|c|c|c|c|c|c|c|c|c|c|c|c|c|}
\hline Ref & 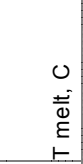 & 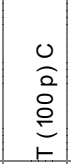 & $\begin{array}{l}\text { Oొ } \\
\stackrel{N}{<}\end{array}$ & 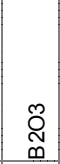 & $\begin{array}{l}\text { D } \\
\infty\end{array}$ & $\begin{array}{l}\text { Oొ } \\
\text { N }\end{array}$ & $\begin{array}{l}0 \\
\pi \\
0\end{array}$ & $\begin{array}{l}\widetilde{N} \\
\delta \\
0 \\
\tilde{N} \\
0 \\
0 \\
0\end{array}$ & $\begin{array}{l}\tilde{0} \\
0 \\
0 \\
0 \\
0 \\
0 \\
0\end{array}$ & $\begin{array}{l}\text { Oొ } \\
\text { ்ָ }\end{array}$ & O & 4 & 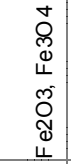 & 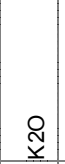 & 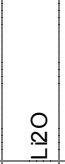 & $\stackrel{\circ}{\sum}$ & $\begin{array}{l}\tilde{O} \\
\text { ธิ }\end{array}$ & $\begin{array}{l}0 \\
0 \\
\Sigma\end{array}$ & $\begin{array}{l}\text { Q } \\
\stackrel{\text { Z }}{Z}\end{array}$ & $\frac{0}{z}$ & $\begin{array}{l}\tilde{N} \\
\tilde{\Xi}\end{array}$ & 吾 & مٌ & $\frac{8}{0}$ & $\stackrel{\text { ô }}{\infty}$ & $\begin{array}{l}\text { Oొ } \\
\text { ஸ) }\end{array}$ & 옹 & 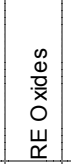 & 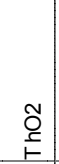 & $\underset{\mathscr{N}}{\stackrel{\sim}{\vdash}}$ & 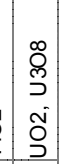 & $\stackrel{q}{N}$ & กั & $\underset{1}{0}$ & 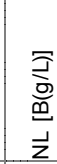 \\
\hline Mangat, 1997 & $\sim 1400$ & & 6.4 & 15.8 & & 30.9 & & & & & & & & & & 1.7 & & & 1.5 & & & & & & 40.7 & & & & & & & & 3.0 & & \\
\hline Mangat, 1997 & $\sim 1400$ & & 3.4 & 18.6 & & 30.5 & & & & & & & & & & 1.7 & & & & & & & & & 40.0 & & & 2.0 & & & & & 3.9 & & \\
\hline Mangat, 1997 & $\sim 1400$ & & 3.4 & 18.7 & & 30.7 & & & & & & & & & & 1.7 & & & 1.5 & & & & & & 40.3 & & & & & 1.0 & & & 2.7 & & \\
\hline Mangat, 1997 & $\sim 1400$ & & 5.0 & 20.0 & & 31.4 & & & & & & & & & 1.5 & & & & & & & & & & 34.1 & & & & & 1.0 & & 4.0 & \begin{tabular}{|l|l}
0 & 3.0 \\
\end{tabular} & & \\
\hline Mangat, 1997 & $\sim 1400$ & & 3.0 & 18.2 & & 25.3 & & & & & & & & & 2.0 & & & & & & & & & & 48.2 & & & & & & & 1.0 & \begin{tabular}{|l|l|}
0 & 2.3 \\
\end{tabular} & & \\
\hline Mangat, 1997 & $\sim 1400$ & & 3.0 & 18.1 & & 24.9 & & & & & & & & & 3.0 & & & & & & & & & & 47.8 & & & & & & & 1.0 & \begin{tabular}{|l|l|}
0 & 2.3 \\
\end{tabular} & & \\
\hline Mangat, 1997 & $\sim 1400$ & & 5.0 & 16.0 & & 24.9 & & & & & & & & & 3.7 & & & & & & & & & & 45.4 & & & & & & & & 5.0 & & \\
\hline Mangat, 1997 & $\sim 1400$ & & 3.6 & 19.6 & & 32.2 & & & & & & & & & 0.8 & 1.7 & & & & & & & & & 42.2 & & & & & & & & & & \\
\hline Mangat, 1997 & $\sim 1400$ & & 3.4 & 13.9 & & 35.8 & & & & & & & & & & 1.7 & & & 1.5 & & & & & & 40.7 & & & & & & & & 3.0 & & \\
\hline Mangat, 1997 & $\sim 1400$ & & 3.4 & 15.8 & & 33.8 & & & & & & & & & & 1.7 & & & 1.5 & & & & & & 40.7 & & & & & & & & 3.0 & & \\
\hline Mangat, 1997 & $\sim 1400$ & & 6.5 & 16.2 & & 31.5 & & & & & & & & & & & & & 1.6 & & & & & & 41.3 & & & & & & & & 3.0 & & \\
\hline Mangat, 1997 & $\sim 1400$ & & 3.1 & 16.4 & & 32.2 & & & & & & & & & 1.6 & & & & & & & & & & 42.5 & & & & & 1.0 & & & 3.1 & & \\
\hline Mangat, 1997 & $\sim 1400$ & & 3.6 & 19.6 & & 32.2 & 2.1 & & & & & & & & \begin{tabular}{|l|}
0.8 \\
\end{tabular} & \begin{tabular}{|l|}
1.7 \\
\end{tabular} & & & & & & & & & 40.1 & & & & & & & & & & \\
\hline Mangat, 1997 & $\sim 1400$ & & 7.4 & 20.5 & & 41.6 & & & & & & & & & & & & & & & & & & & 30.6 & & & & & & & & & & \\
\hline Mangat, 1997 & $\sim 1400$ & & 7.3 & 16.6 & & 41.7 & & & & & & & & & 0.5 & & & & & & & & & & 33.7 & & & & & & & & & & \\
\hline Mangat, 1997 & $\sim 1400$ & & 7.2 & 16.4 & & 41.2 & & & & & & & & & 2.0 & & & & & & & & & & 33.2 & & & & & & & & & & \\
\hline Mangat, 1997 & $\sim 1400$ & & 7.2 & 16.5 & & 41.5 & & & & & & & & & 1.0 & & & & & & & 0.5 & & & 33.4 & & & & & & & & & & \\
\hline Mangat, 1997 & $\sim 1400$ & & 7.3 & 16.6 & & 41.6 & & & & & & & & 1.0 & & & & & & & & & & & 33.6 & & & & & & & & & & \\
\hline Mangat, 1997 & $\sim 1400$ & & 7.2 & 16.3 & & 41.0 & 2.4 & & & & & & & & & & & & & & & & & & 33.1 & & & & & & & & & & \\
\hline Mangat, 1997 & $\sim 1400$ & & 7.2 & 16.4 & & 41.2 & & & & & & & & & 1.0 & & & & & & & & & & 33.2 & & & & & & & & 1.0 & & \\
\hline Mangat, 1997 & $\sim 1400$ & & 9.3 & 16.5 & & 41.6 & & & & & & & & & 1.0 & & & & & & & & & & 31.6 & & & & & & & & & & \\
\hline Mangat, 1997 & $\sim 1400$ & & 11.2 & 16.5 & & 41.6 & & & & & & & & & 1.0 & & & & & & & & & & 29.6 & & & & & & & & & & \\
\hline Mangat, 1997 & $\sim 1400$ & & 5.3 & 14.6 & & 27.8 & & & & & & & & & 0.1 & & & & 1.4 & & & 2.9 & & & 43.1 & & & & & & & 2.8 & \begin{tabular}{|l|l|}
3 & 2.0 \\
\end{tabular} & & \\
\hline Mangat, 1997 & $\sim 1400$ & & 7.0 & 17.0 & & 24.9 & & & & & & & & & & & & & 2.0 & & & 2.0 & & & 39.9 & & & & & & & 5.0 & $\begin{array}{l}2.2 \\
\end{array}$ & & \\
\hline Mangat, 1997 & $\sim 1400$ & & 5.4 & 15.2 & & 27.3 & & & & & & & & & 1.3 & & & 1.6 & & & & & & & 42.2 & & & & & & & 3.9 & 3.2 & & \\
\hline Mangat, 1997 & $\sim 1400$ & & 6.1 & 14.9 & & 27.6 & & & & & & & & & 1.6 & & & & & & & & 1.5 & & 42.1 & & & & & & & 3.9 & $\begin{array}{l}2.3 \\
\end{array}$ & & \\
\hline Matlack, 1997 & & & 7.9 & 8.1 & & & 4.5 & & & 0.4 & & & 6.3 & 2.0 & 4.1 & 2.3 & & & 8.7 & 0.1 & 0.1 & 0.2 & 2.2 & 0.0 & 43.2 & 1.3 & & & & & & 4.1 & \begin{tabular}{|l|} 
\\
\end{tabular} & & \\
\hline Matlack, 1997 & & & 7.9 & 8.1 & & & 4.5 & & & 0.4 & & & 6.3 & 2.0 & 4.1 & 2.3 & & & 8.7 & 0.1 & 0.1 & 0.2 & 2.2 & 0.0 & 43.2 & 1.3 & & & & & & 4.1 & 3.0 & & \\
\hline Matlack, 1997 & & & 7.9 & 8.1 & & & 4.5 & & & 0.4 & & & 6.3 & 2.0 & 4.1 & 2.3 & & & 8.7 & 0.1 & 0.1 & 0.2 & 2.2 & 0.0 & 43.2 & 1.3 & & & & & & 4.1 & $\begin{array}{l}3.0 \\
3.0\end{array}$ & & \\
\hline Matlack, 1997 & & & 7.9 & 8.1 & & & 4.5 & & & 0.4 & & & 6.3 & 2.0 & 4.1 & 2.3 & & & 8.7 & 0.1 & 0.1 & 0.2 & 2.2 & 0.0 & 43.2 & 1.3 & & & & & & 4.1 & 3.0 & & \\
\hline Matlack, 1997 & & & 7.9 & 8.1 & & & 4.5 & & & 0.4 & & & 6.3 & 2.0 & 4.1 & 2.3 & & & 8.7 & 0.1 & 0.1 & 0.2 & 2.2 & 0.0 & 43.2 & 1.3 & & & & & & 4.1 & $\begin{array}{l}3.0 \\
3.0\end{array}$ & & \\
\hline Matlack, 1997 & & & 7.9 & 8.1 & & & 4.5 & & & 0.4 & & & 6.3 & 2.0 & 4.1 & 2.3 & & & 8.7 & 0.1 & 0.1 & 0.2 & 2.2 & 0.0 & 43.2 & 1.3 & & & & & & 4.1 & 3.0 & & \\
\hline Matlack, 1997 & & & 7.9 & 8.1 & & & 4.5 & & & 0.4 & & & 6.3 & 2.0 & 4.1 & 2.3 & & & 8.7 & 0.1 & 0.1 & 0.2 & 2.2 & 0.0 & 43.2 & \begin{tabular}{|l|}
1.3 \\
\end{tabular} & & & & & & $\begin{array}{l}4.1 \\
4.1\end{array}$ & $\begin{array}{l}3.0 \\
3.0\end{array}$ & & \\
\hline Matlack, 1997 & & & 7.9 & 8.1 & & & 4.5 & & & 0.4 & & & 6.3 & 2.0 & 4.1 & 2.3 & & & 8.7 & 0.1 & 0.1 & 0.2 & 2.2 & 0.0 & 43.2 & 1.3 & & & & & & 4.1 & 3.0 & & \\
\hline Matlack, 1997 & & & 7.9 & 8.1 & & & 4.5 & & & 0.4 & & & 6.3 & 2.0 & 4.1 & 2.3 & & & 8.7 & 0.1 & 0.1 & 0.2 & 2.2 & 0.0 & 43.2 & 1.3 & & & & & & 4.1 & 3.0 & & \\
\hline Matlack, 1997 & & & 8.0 & 8.1 & & & 4.6 & & & 0.4 & & & 6.4 & 2.0 & 4.1 & 2.3 & & & 8.8 & 0.1 & 0.1 & 0.2 & 2.2 & 0.0 & 43.5 & 0.7 & & & & & & 4.1 & 3.0 & & \\
\hline Matlack, 1997 & & & 8.0 & 8.1 & & & 4.6 & & & 0.4 & & & 6.4 & 2.0 & 4.1 & 2.3 & & & 8.8 & 0.1 & 0.1 & 0.2 & 2.2 & 0.0 & 43.5 & \begin{tabular}{|l|}
0.7 \\
\end{tabular} & & & & & & 4.1 & \begin{tabular}{|l|} 
\\
\end{tabular} & & \\
\hline Matlack, 1997 & & & 8.0 & 8.1 & & & 4.6 & & & 0.4 & & & 6.4 & 2.0 & 4.1 & 2.3 & & & 8.8 & 0.1 & 0.1 & 0.2 & 2.2 & 0.0 & 43.5 & 0.7 & & & & & & 4.1 & 3.0 & & \\
\hline Matlack, 1997 & & 1195 & 9.9 & 4.0 & 0.0 & & 2.7 & & & 0.3 & & 0.8 & 6.3 & 4.0 & & 2.0 & & & 20.0 & 0.1 & 0.1 & 0.3 & 1.3 & 0.1 & 40.9 & 0.4 & & 0.0 & & & & 3.3 & 3.0 & & \\
\hline Matlack, 1997 & & & 9.9 & 4.0 & 0.0 & & 2.7 & & & 0.3 & & 0.8 & 6.3 & 4.0 & & 2.0 & & & 20.0 & 0.1 & 0.1 & 0.3 & 1.3 & 0.1 & 40.9 & \begin{tabular}{|l|l|}
0.4 \\
\end{tabular} & & 0.0 & & & & 3.3 & 3.0 & & \\
\hline Matlack, 1997 & & & 9.9 & 4.0 & 0.0 & & 2.7 & & & 0.3 & & 0.8 & 6.3 & 4.0 & & 2.0 & & & 20.0 & 0.1 & 0.1 & 0.3 & 1.3 & 0.1 & 40.9 & 0.4 & & 0.0 & & & & 3.3 & 3.0 & & \\
\hline Matlack, 1997 & & 1209 & 9.9 & 4.0 & 0.0 & & 2.7 & & & 0.3 & & 0.8 & 6.3 & 4.0 & & 2.0 & & & 20.0 & 0.1 & 0.1 & 0.3 & 1.3 & 0.1 & 40.9 & 0.4 & & 0.0 & & & & 3.3 & 3.0 & & \\
\hline Matlack, 1997 & & & 9.9 & 4.0 & 0.0 & & 2.7 & & & 0.3 & & 0.8 & 6.3 & 4.0 & & 2.0 & & & 20.0 & 0.1 & 0.1 & 0.3 & 1.3 & 0.1 & 40.9 & 0.4 & & 0.0 & & & & 3.3 & 3.0 & & \\
\hline Matlack, 1997 & & 1200 & 9.9 & 4.0 & 0.0 & & 2.7 & & & 0.3 & & 0.8 & 6.3 & 4.0 & & 2.0 & & & 20.0 & 0.1 & 0.1 & 0.3 & 1.3 & 0.1 & 40.9 & 0.4 & & 0.0 & & & & 3.3 & 3.0 & & \\
\hline Matlack, 1997 & & & 9.9 & 4.0 & 0.0 & & 2.7 & & & 0.3 & & 0.8 & 6.3 & 4.0 & & 2.0 & & & 20.0 & 0.1 & 0.1 & 0.3 & 1.3 & 0.1 & 40.9 & 0.4 & & 0.0 & & & & 3.3 & 3.0 & & \\
\hline Matlack, 1997 & & & 9.9 & 4.0 & 0.0 & & 2.7 & & & 0.3 & & 0.8 & 6.3 & 4.0 & & 2.0 & & & 20.0 & 0.1 & 0.1 & 0.3 & 1.3 & 0.1 & 40.9 & 0.4 & & 0.0 & & & & 3.3 & 3.0 & & \\
\hline Matlack, 1997 & & & 9.9 & 4.0 & 0.0 & & 2.7 & & & 0.3 & & 0.8 & 6.3 & 4.0 & & 2.0 & & & 20.0 & 0.1 & 0.1 & 0.3 & 1.3 & 0.1 & 40.9 & 0.4 & & 0.0 & & & & 3.3 & 3.0 & & \\
\hline Mcgetchin, 1978 & & 1060 & 8.8 & & & & 7.5 & & & & & & 7.3 & 0.3 & & 24.6 & & & 1.4 & & & & & & 51.4 & & & & & & & & & & \\
\hline Mcgetchin, 1978 & & 967 & 11.3 & & & & 9.5 & & & & & & 14.4 & 0.4 & & 17.3 & & & 1.8 & & & & & & 44.5 & & & & & & & & & & \\
\hline Merrill, 1993 & & & 20.0 & 5.0 & & & 4.7 & & & & & & & 1.8 & 0.5 & 10.2 & & & 6.0 & & 8.1 & 4.1 & 9.4 & 0.2 & 30.0 & & & & & & & & & & \\
\hline
\end{tabular}


ORP-56297 Rev. 0

Table A1. Glass Compositions from Literature Review (continued)

\begin{tabular}{|c|c|c|c|c|c|c|c|c|c|c|c|c|c|c|c|c|c|c|c|c|c|c|c|c|c|c|c|c|c|c|c|c|c|c|c|}
\hline Ref & 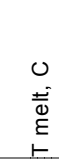 & $\begin{array}{l}0 \\
0 \\
\vdots \\
\stackrel{0}{0} \\
上\end{array}$ & 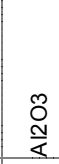 & 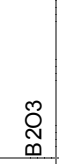 & $\begin{array}{l}\text { O } \\
\infty\end{array}$ & 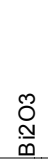 & $\begin{array}{l}0 \\
\mathbb{J} \\
0\end{array}$ & $\begin{array}{l}\tilde{O} \\
0 \\
0 \\
\tilde{ల} \\
0 \\
0 \\
0\end{array}$ & $\begin{array}{l}m \\
0 \\
0 \\
0 \\
0 \\
0 \\
0\end{array}$ & ญ̃ & $\stackrel{0}{3}$ & \llcorner & 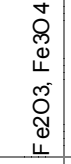 & $\begin{array}{l}\text { Dे } \\
\check{x}\end{array}$ & $\stackrel{ }{\stackrel{ }{J}}$ & $\begin{array}{l}0 \\
\stackrel{0}{m} \\
\Sigma\end{array}$ & $\begin{array}{l}\tilde{O} \\
\stackrel{\complement}{\Sigma}\end{array}$ & $\begin{array}{l}0 \\
0 \\
\Sigma\end{array}$ & $\begin{array}{l}\mathbb{Z} \\
\mathbb{N} \\
Z\end{array}$ & $\stackrel{\circ}{\bar{z}}$ & $\begin{array}{l}\text { N } \\
\text { Õ } \\
\end{array}$ & $\overline{\bar{z}}$ & 足 & $\frac{8}{2}$ & $\frac{\tilde{N}}{\infty}$ & $\begin{array}{l}\text { Oొ } \\
\text { in }\end{array}$ & $\begin{array}{l}\text { Q } \\
\text { is }\end{array}$ & 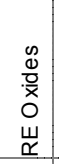 & $\begin{array}{l}\text { ô } \\
\text { \& }\end{array}$ & $\begin{array}{l}\widetilde{N} \\
\stackrel{\underline{F}}{F}\end{array}$ & 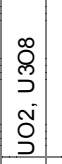 & $\stackrel{g}{N}$ & $\begin{array}{l}\tilde{D} \\
\end{array}$ & 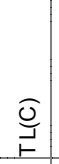 & $\begin{array}{l}\bar{\Xi} \\
\text { O্ } \\
\underline{\underline{z}} \\
\bar{z}\end{array}$ \\
\hline Merrill, 1995 & 1300 & & 33.6 & 4.9 & & & & & 0.0 & 0.0 & & 0.2 & 0.0 & 1.0 & & 0.0 & 0.0 & 0.1 & 18.0 & & & 0.1 & 41.6 & & & \begin{tabular}{|l|} 
\\
\end{tabular} & & & & & & & & & \\
\hline Merrill, 1995 & 1400 & & 35.5 & 2.7 & & & & & 0.4 & & & & & 0.7 & & 0.0 & & & 18.3 & & & & 41.9 & & & 0.2 & & & & & & & & & \\
\hline Merrill, 1995 & 1400 & & 35.7 & 2.7 & & & & & & & & & & 0.2 & & & & & 18.8 & & & & 42.0 & & & 0.3 & & & & & & & & & \\
\hline Merrill, 1995 & 1300 & & 34.7 & 4.7 & & & & & 0.0 & 0.0 & & 0.1 & 0.0 & 0.0 & & 0.0 & 0.0 & 0.1 & 18.2 & & & 0.1 & 41.9 & & & & & & & & & & & & \\
\hline Mika, 1997 & & & 8.0 & 7.0 & 0.3 & & 1.0 & 0.1 & 0.1 & 0.2 & 0.0 & 0.1 & 12.5 & 0.3 & 3.0 & 0.6 & 0.4 & 0.0 & 15.7 & 0.5 & 2.9 & 0.9 & 0.5 & 0.2 & 46.0 & 0.2 & & 0.5 & & 0.0 & & 0.0 & 1.8 & & \\
\hline Mika, 1997 & & & 8.0 & 7.0 & 0.3 & & 1.0 & 0.1 & 0.1 & 0.2 & 0.0 & 0.1 & 12.5 & 0.3 & 3.0 & 0.6 & 0.4 & 0.0 & 15.7 & 0.5 & 2.9 & 0.9 & 0.5 & 0.2 & 46.0 & 0.2 & & 0.5 & & 0.0 & & 0.0 & 1.8 & & \\
\hline Mika, 1997 & & & 8.0 & 7.0 & 0.3 & & 1.0 & 0.1 & 0.1 & 0.2 & 0.0 & 0.1 & 12.5 & 0.3 & 3.0 & 0.6 & 0.4 & 0.0 & 15.7 & 0.5 & 2.9 & 0.9 & 0.5 & 0.2 & 46.0 & 0.2 & & 0.5 & & 0.0 & & 0.0 & 1.8 & & \\
\hline Mika, 1997 & & & 8.0 & 7.0 & 0.3 & & 1.0 & 0.1 & 0.1 & 0.2 & 0.0 & 0.1 & 12.5 & 0.3 & 3.0 & 0.6 & 0.4 & 0.0 & 15.7 & 0.5 & 2.9 & 0.9 & 0.5 & 0.2 & 46.0 & 0.2 & & 0.5 & & 0.0 & & 0.0 & 1.8 & & \\
\hline Mika, 1997 & & & 8.0 & 7.0 & 0.3 & & 1.0 & 0.1 & 0.1 & 0.2 & 0.0 & 0.1 & 12.5 & 0.3 & 3.0 & 0.6 & 0.4 & 0.0 & 15.7 & 0.5 & 2.9 & 0.9 & 0.5 & 0.2 & 46.0 & 0.2 & & 0.5 & & 0.0 & & 0.0 & 1.8 & & \\
\hline Mika, 1997 & & & 8.0 & 7.0 & 0.3 & & 1.0 & 0.1 & 0.1 & 0.2 & 0.0 & 0.1 & 12.5 & 0.3 & 3.0 & 0.6 & 0.4 & 0.0 & 15.7 & 0.5 & 2.9 & 0.9 & 0.5 & 0.2 & 46.0 & 0.2 & & 0.5 & & 0.0 & & 0.0 & 1.8 & & \\
\hline Mika, 1997 & & & 8.0 & 7.0 & 0.3 & & 1.0 & 0.1 & 0.1 & 0.2 & 0.0 & 0.1 & 12.5 & 0.3 & 3.0 & 0.6 & 0.4 & 0.0 & 15.7 & 0.5 & 2.9 & 0.9 & 0.5 & 0.2 & 46.0 & 0.2 & & 0.5 & & 0.0 & & 0.0 & 1.8 & & \\
\hline Mika, 1997 & & & 8.0 & 7.0 & 0.3 & & 1.0 & 0.1 & 0.1 & 0.2 & 0.0 & 0.1 & 12.5 & 0.3 & 3.0 & 0.6 & 0.4 & 0.0 & 15.7 & 0.5 & 2.9 & 0.9 & 0.5 & 0.2 & 46.0 & 0.2 & & 0.5 & & 0.0 & & 0.0 & 1.8 & & \\
\hline Mika, 1997 & & & 8.0 & 7.0 & 0.3 & & 1.0 & 0.1 & 0.1 & 0.2 & 0.0 & 0.1 & 12.5 & 0.3 & 3.0 & 0.6 & 0.4 & 0.0 & 15.7 & 0.5 & 2.9 & 0.9 & 0.5 & 0.2 & 46.0 & 0.2 & & 0.5 & & 0.0 & & 0.0 & 1.8 & & \\
\hline Mika, 1997 & & & 12.0 & 6.7 & 0.3 & & 1.0 & 0.1 & 0.1 & 0.2 & 0.0 & 0.1 & 12.0 & 0.3 & 2.9 & 0.6 & 0.3 & 0.0 & 15.0 & 0.5 & 2.8 & 0.9 & 0.4 & 0.2 & 44.0 & 0.2 & & 0.4 & & 0.0 & & 0.0 & 1.8 & & \\
\hline Mika, 1997 & & & 16.0 & 6.4 & 0.3 & & 0.9 & 0.1 & 0.1 & 0.2 & 0.0 & 0.1 & 11.4 & 0.3 & 2.7 & 0.5 & 0.3 & 0.0 & 14.4 & 0.5 & 2.6 & 0.8 & 0.4 & 0.2 & 42.0 & 0.2 & & 0.4 & & 0.0 & & 0.0 & 1.7 & & \\
\hline Mika, 1997 & & & 8.6 & & 0.3 & & 1.1 & 0.1 & 0.1 & 0.2 & 0.0 & 0.1 & 13.4 & 0.3 & 3.2 & 0.6 & 0.4 & 0.0 & 16.9 & 0.6 & 3.1 & 1.0 & 0.5 & 0.2 & 49.5 & 0.2 & & 0.5 & & 0.0 & & 0.0 & 2.0 & & \\
\hline Mika, 1997 & & & 8.5 & 1.0 & 0.3 & & 1.1 & 0.1 & 0.1 & 0.2 & 0.0 & 0.1 & 13.3 & 0.3 & 3.2 & 0.6 & 0.4 & 0.0 & 16.7 & 0.6 & 3.1 & 1.0 & 0.5 & 0.2 & 49.0 & 0.2 & & 0.5 & & 0.0 & & 0.0 & 2.0 & & \\
\hline Mika, 1997 & & & 8.3 & 4.0 & 0.3 & & 1.0 & 0.1 & 0.1 & 0.2 & 0.0 & 0.1 & 12.9 & 0.3 & 3.1 & 0.6 & 0.4 & 0.0 & 16.2 & 0.5 & 3.0 & 0.9 & 0.5 & 0.2 & 47.5 & 0.2 & & 0.5 & & 0.0 & & 0.0 & 1.9 & & \\
\hline Mika, 1997 & & & 7.6 & 12.0 & 0.3 & & 0.9 & 0.1 & 0.1 & 0.2 & 0.0 & 0.1 & 11.8 & 0.3 & 2.8 & 0.6 & 0.3 & 0.0 & 14.9 & 0.5 & 2.7 & 0.9 & 0.4 & 0.2 & 43.5 & 0.2 & & 0.4 & & 0.0 & & 0.0 & 1.7 & & \\
\hline Mika, 1997 & & & 7.8 & 6.9 & 0.3 & & 3.0 & 0.1 & 0.1 & 0.2 & 0.0 & 0.1 & 12.2 & 0.3 & 2.9 & 0.6 & 0.4 & 0.0 & 15.4 & 0.5 & 2.8 & 0.9 & 0.5 & 0.2 & 45.1 & 0.2 & & 0.4 & & 0.0 & & 0.0 & 1.8 & & \\
\hline Mika, 1997 & & & 7.7 & 6.7 & 0.3 & & 5.0 & 0.1 & 0.1 & 0.2 & 0.0 & 0.1 & 12.0 & 0.3 & 2.9 & 0.6 & 0.3 & 0.0 & 15.1 & 0.5 & 2.8 & 0.9 & 0.4 & 0.2 & 44.1 & 0.2 & & 0.4 & & 0.0 & & 0.0 & 1.8 & & \\
\hline Mika, 1997 & & & 8.0 & 7.0 & 0.3 & & 1.0 & 0.1 & 0.1 & & 0.0 & 0.1 & 12.5 & 0.3 & 3.0 & 0.6 & 0.4 & 0.0 & 15.8 & 0.5 & 2.9 & 0.9 & 0.5 & 0.2 & 46.1 & 0.2 & & 0.5 & & 0.0 & & 0.0 & 1.9 & & \\
\hline Mika, 1997 & & & 8.0 & 7.0 & 0.3 & & 1.0 & 0.1 & 0.1 & 0.5 & 0.0 & 0.1 & 12.5 & 0.3 & 3.0 & 0.6 & 0.4 & 0.0 & 15.7 & 0.5 & 2.9 & 0.9 & 0.5 & 0.2 & 45.9 & 0.2 & & 0.5 & & 0.0 & & 0.0 & 1.8 & & \\
\hline Mika, 1997 & & & 8.0 & 7.0 & 0.3 & & 1.0 & 0.1 & 0.1 & 0.8 & 0.0 & 0.1 & 12.4 & 0.3 & 3.0 & 0.6 & 0.4 & 0.0 & 15.6 & 0.5 & 2.9 & 0.9 & 0.5 & 0.2 & 45.7 & 0.2 & & 0.5 & & 0.0 & & 0.0 & 1.8 & & \\
\hline Mika, 1997 & & & 7.9 & 6.9 & 0.3 & & 1.0 & 0.1 & 0.1 & 1.2 & 0.0 & 0.1 & 12.4 & 0.3 & 3.0 & 0.6 & 0.4 & 0.0 & 15.6 & 0.5 & 2.9 & 0.9 & 0.5 & 0.2 & 45.5 & 0.2 & & 0.4 & & 0.0 & & 0.0 & 1.8 & & \\
\hline Mika, 1997 & & & 8.6 & 7.5 & 0.3 & & 1.1 & 0.1 & 0.1 & 0.2 & 0.0 & 0.1 & 6.0 & 0.3 & 3.2 & 0.6 & 0.4 & 0.0 & 16.9 & 0.6 & 3.1 & 1.0 & 0.5 & 0.2 & 49.4 & 0.2 & & 0.5 & & 0.0 & & 0.0 & 2.0 & & \\
\hline Mika, 1997 & & & 8.3 & 7.3 & 0.3 & & 1.0 & 0.1 & 0.1 & 0.2 & 0.0 & 0.1 & 9.0 & 0.3 & 3.1 & 0.6 & 0.4 & 0.0 & 16.4 & 0.5 & 3.0 & 1.0 & 0.5 & 0.2 & 47.8 & 0.2 & & 0.5 & & 0.0 & & 0.0 & 1.9 & & \\
\hline Mika, 1997 & & & 7.8 & 6.8 & 0.3 & & 1.0 & 0.1 & 0.1 & 0.2 & 0.0 & 0.1 & 15.0 & 0.3 & 2.9 & 0.6 & 0.3 & 0.0 & 15.3 & 0.5 & 2.8 & 0.9 & 0.5 & 0.2 & 44.7 & 0.2 & & 0.4 & & 0.0 & & 0.0 & 1.8 & & \\
\hline Mika, 1997 & & & 7.0 & 6.2 & 0.3 & & 0.9 & 0.1 & 0.1 & 0.2 & 0.0 & 0.1 & 23.0 & 0.2 & 2.6 & 0.5 & 0.3 & 0.0 & 13.8 & 0.5 & 2.5 & 0.8 & 0.4 & 0.2 & 40.5 & 0.2 & & 0.4 & & 0.0 & & 0.0 & 1.6 & & \\
\hline Mika, 1997 & & & 7.9 & 6.9 & 0.3 & & 1.0 & 0.1 & 0.1 & 0.2 & 0.0 & 0.1 & 12.3 & 2.0 & 2.9 & 0.6 & 0.4 & 0.0 & 15.5 & 0.5 & 2.8 & 0.9 & 0.5 & 0.2 & 45.2 & 0.2 & & 0.4 & & 0.0 & & 0.0 & 1.8 & & \\
\hline Mika, 1997 & & & 7.7 & 6.7 & 0.3 & & 1.0 & 0.1 & 0.1 & 0.2 & 0.0 & 0.1 & 12.0 & 4.0 & 2.9 & 0.6 & 0.3 & 0.0 & 15.1 & 0.5 & 2.8 & 0.9 & 0.4 & 0.2 & 44.3 & 0.2 & & 0.4 & & 0.0 & & 0.0 & 1.8 & & \\
\hline Mika, 1997 & & & 8.2 & 7.2 & 0.3 & & 1.0 & 0.1 & 0.1 & 0.2 & 0.0 & 0.1 & 12.9 & 0.3 & & 0.6 & 0.4 & 0.0 & 16.2 & 0.5 & 3.0 & 0.9 & 0.5 & 0.2 & 47.4 & 0.2 & & 0.5 & & 0.0 & & 0.0 & 1.9 & & \\
\hline Mika, 1997 & & & 8.2 & 7.1 & 0.3 & & 1.0 & 0.1 & 0.1 & 0.2 & 0.0 & 0.1 & 12.8 & 0.3 & 1.0 & 0.6 & 0.4 & 0.0 & 16.1 & 0.5 & 2.9 & 0.9 & 0.5 & 0.2 & 46.9 & 0.2 & & 0.5 & & 0.0 & & 0.0 & 1.9 & & \\
\hline Mika, 1997 & & & 8.0 & 7.0 & 0.3 & & 1.0 & 0.1 & 0.1 & 0.2 & 0.0 & 0.1 & 12.5 & 0.3 & 3.0 & 0.6 & 0.4 & 0.0 & 15.7 & 0.5 & 2.9 & 0.9 & 0.5 & 0.2 & 46.0 & 0.2 & & 0.5 & & 0.0 & & 0.0 & 1.8 & & \\
\hline Mika, 1997 & & & 7.9 & 6.9 & 0.3 & & 1.0 & 0.1 & 0.1 & 0.2 & 0.0 & 0.1 & 12.3 & 0.3 & 4.5 & 0.6 & 0.4 & 0.0 & 15.5 & 0.5 & 2.8 & 0.9 & 0.5 & 0.2 & 45.3 & 0.2 & & 0.4 & & 0.0 & & 0.0 & 1.8 & & \\
\hline Mika, 1997 & & & 7.6 & 6.7 & 0.3 & & 1.0 & 0.1 & 0.1 & 0.2 & 0.0 & 0.1 & 11.9 & 0.3 & 7.5 & 0.6 & 0.3 & 0.0 & 15.0 & 0.5 & 2.7 & 0.9 & 0.4 & 0.2 & 43.9 & 0.2 & & 0.4 & & 0.0 & & 0.0 & 1.8 & & \\
\hline Mika, 1997 & & & 8.0 & 7.0 & 0.3 & & 1.0 & 0.1 & 0.1 & 0.2 & 0.0 & 0.1 & 12.4 & 0.3 & 3.0 & 1.0 & 0.4 & 0.0 & 15.7 & 0.5 & 2.9 & 0.9 & 0.5 & 0.2 & 45.8 & 0.2 & & 0.5 & & 0.0 & & 0.0 & 1.8 & & \\
\hline Mika, 1997 & & & 7.9 & 6.9 & 0.3 & & 1.0 & 0.1 & 0.1 & 0.2 & 0.0 & 0.1 & 12.3 & 0.3 & 3.0 & 2.0 & 0.4 & 0.0 & 15.5 & 0.5 & 2.8 & 0.9 & 0.5 & 0.2 & 45.4 & 0.2 & & 0.4 & & 0.0 & & 0.0 & 1.8 & & \\
\hline Mika, 1997 & & & 7.6 & 6.6 & 0.3 & & 0.9 & 0.1 & 0.1 & 0.2 & 0.0 & 0.1 & 11.8 & 0.3 & 2.8 & 6.0 & 0.3 & 0.0 & 14.9 & 0.5 & 2.7 & 0.9 & 0.4 & 0.2 & 43.5 & 0.2 & & 0.4 & & 0.0 & & 0.0 & 1.7 & & \\
\hline Mika, 1997 & & & 8.0 & 7.0 & 0.3 & & 1.0 & 0.1 & 0.1 & 0.2 & 0.0 & 0.1 & 12.5 & 0.3 & 3.0 & 0.6 & & 0.0 & 15.8 & 0.5 & 2.9 & 0.9 & 0.5 & 0.2 & 46.2 & 0.2 & & 0.5 & & 0.0 & & 0.0 & 1.9 & & \\
\hline Mika, 1997 & & 1056 & 7.9 & 7.0 & 0.3 & & 1.0 & 0.1 & 0.1 & 0.2 & 0.0 & 0.1 & 12.4 & 0.3 & 3.0 & 0.6 & 1.0 & 0.0 & 15.6 & 0.5 & 2.9 & 0.9 & 0.5 & 0.2 & 45.7 & 0.2 & & 0.5 & & 0.0 & & 0.0 & 1.8 & & \\
\hline Mika, 1997 & & & 7.7 & 6.7 & 0.3 & & 1.0 & 0.1 & 0.1 & 0.2 & 0.0 & 0.1 & 12.0 & 0.3 & 2.9 & 0.6 & 4.0 & 0.0 & 15.2 & 0.5 & 2.8 & 0.9 & 0.4 & 0.2 & 44.3 & 0.2 & & 0.4 & & 0.0 & & 0.0 & 1.8 & & \\
\hline Mika, 1997 & & & 8.7 & 7.6 & 0.3 & & 1.1 & 0.1 & 0.1 & 0.2 & 0.0 & 0.1 & 13.6 & 0.3 & 3.3 & 0.7 & 0.4 & 0.0 & 8.0 & 0.6 & 3.1 & 1.0 & 0.5 & 0.2 & 50.2 & 0.2 & & 0.5 & & 0.0 & & 0.0 & 2.0 & & \\
\hline Mika, 1997 & & & 8.4 & 7.3 & 0.3 & & 1.0 & 0.1 & 0.1 & 0.2 & 0.0 & 0.1 & 13.1 & 0.3 & 3.1 & 0.6 & 0.4 & 0.0 & 12.0 & 0.5 & 3.0 & 1.0 & 0.5 & 0.2 & 48.0 & 0.2 & & 0.5 & & 0.0 & & 0.0 & 1.9 & & \\
\hline Mika, 1997 & & & 7.6 & 6.6 & 0.3 & & 0.9 & 0.1 & 0.1 & 0.2 & 0.0 & 0.1 & 11.9 & 0.3 & 2.8 & 0.6 & 0.3 & 0.0 & 20.0 & 0.5 & 2.7 & 0.9 & 0.4 & 0.2 & 43.7 & 0.2 & & 0.4 & & 0.0 & & 0.0 & 1.8 & & \\
\hline Mika, 1997 & & & 8.0 & 7.0 & 0.3 & & 1.0 & 0.1 & 0.1 & 0.2 & 0.0 & 0.1 & 12.6 & 0.3 & 3.0 & 0.6 & 0.4 & 0.0 & 15.8 & & 2.9 & 0.9 & 0.5 & 0.2 & 46.2 & 0.2 & & 0.5 & & 0.0 & & 0.0 & 1.9 & & \\
\hline Mika, 1997 & & & 8.0 & 7.0 & 0.3 & & 1.0 & 0.1 & 0.1 & 0.2 & 0.0 & 0.1 & 12.4 & 0.3 & 3.0 & 0.6 & 0.4 & 0.0 & 15.7 & 1.0 & 2.9 & 0.9 & 0.5 & 0.2 & 45.8 & 0.2 & & 0.5 & & 0.0 & & 0.0 & 1.8 & & \\
\hline Mika, 1997 & & & 7.8 & 6.8 & 0.3 & & 1.0 & 0.1 & 0.1 & 0.2 & 0.0 & 0.1 & 12.2 & 0.3 & 2.9 & 0.6 & 0.4 & 0.0 & 15.3 & 3.0 & 2.8 & 0.9 & 0.5 & 0.2 & 44.9 & 0.2 & & 0.4 & & 0.0 & & 0.0 & 1.8 & & \\
\hline Mika, 1997 & & & 9.2 & 8.0 & 0.3 & & 1.1 & 0.1 & 0.1 & 0.3 & 0.0 & 0.1 & \begin{tabular}{|l|l|}
14.4 \\
\end{tabular} & 0.3 & 3.4 & 0.7 & 0.4 & 0.0 & 18.1 & 0.6 & 3.3 & 1.1 & 0.5 & 0.2 & 38.0 & 0.2 & & 0.5 & & 0.0 & & 0.0 & 2.1 & & \\
\hline Mika, 1997 & & & 7.1 & 6.2 & 0.3 & & 0.9 & 0.1 & 0.1 & 0.2 & 0.0 & 0.1 & 11.1 & 0.2 & 2.7 & 0.5 & 0.3 & 0.0 & 14.0 & 0.5 & 2.6 & 0.8 & 0.4 & 0.2 & 52.0 & 0.2 & & 0.4 & & 0.0 & & 0.0 & 1.6 & & \\
\hline
\end{tabular}


Table A1. Glass Compositions from Literature Review (continued)

\begin{tabular}{|c|c|c|c|c|c|c|c|c|c|c|c|c|c|c|c|c|c|c|c|c|c|c|c|c|c|c|c|c|c|c|c|c|c|c|c|}
\hline Ref & 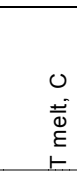 & $\begin{array}{l}0 \\
0 \\
\circ \\
0 \\
1\end{array}$ & 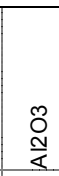 & 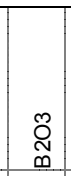 & $\begin{array}{l}\text { \& } \\
\infty\end{array}$ & $\begin{array}{l}\text { Oొ } \\
\stackrel{N}{\infty}\end{array}$ & $\begin{array}{l}0 \\
\mathbb{\sigma} \\
0\end{array}$ & 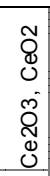 & $\begin{array}{l}m \\
0 \\
0 \\
0 \\
0 \\
0 \\
0\end{array}$ & $\begin{array}{l}\text { Õ } \\
\text { บे }\end{array}$ & $\stackrel{\circ}{3}$ & 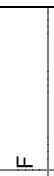 & 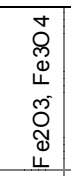 & $\underset{\mathbb{N}}{\stackrel{Q}{n}}$ & $\stackrel{\stackrel{ }{J}}{]}$ & $\stackrel{\circ}{\circ}$ & $\begin{array}{l}\tilde{O} \\
\text { C } \\
\Sigma\end{array}$ & $\begin{array}{l}0 \\
\text { Oे } \\
\Sigma\end{array}$ & $\begin{array}{l}\text { Q } \\
\text { Z } \\
Z\end{array}$ & $\stackrel{\circ}{z}$ & $\begin{array}{l}\mathcal{N} \\
\tilde{0}\end{array}$ & 豆 & $\begin{array}{l}\text { ฉి } \\
\Sigma\end{array}$ & $\frac{8}{0}$ & $\frac{\mathrm{O}}{\mathrm{n}}$ & $\begin{array}{l}0 \\
0 \\
\infty\end{array}$ & $\begin{array}{l}\text { Q } \\
\text { in }\end{array}$ & 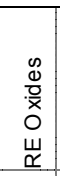 & $\begin{array}{l}\text { Qิ } \\
\text { \& }\end{array}$ & 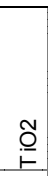 & 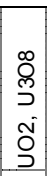 & $\stackrel{q}{N}$ & $\begin{array}{l}\text { ญ̃ } \\
\stackrel{N}{N}\end{array}$ & 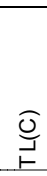 & 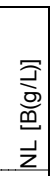 \\
\hline Mohammad, 2003 & 1300 & & 8.4 & 13.7 & 0.1 & & 0.6 & 0.0 & & 0.1 & & 0.0 & 9.2 & 0.0 & 0.7 & 0.5 & 3.5 & & 8.1 & 0.1 & & 5.8 & 0.1 & 0.2 & 48.3 & 0.0 & & 0.1 & & 0.0 & & & 0.1 & & 0.3 \\
\hline Mohammad, 2003 & 1300 & & 11.4 & 8.0 & 0.1 & & 0.8 & 0.0 & & 0.2 & & 0.0 & 12.5 & 0.0 & 0.6 & 0.5 & 4.7 & & 10.2 & 0.2 & & 7.8 & 0.2 & 0.2 & 42.0 & 0.0 & & 0.1 & & 0.0 & & & 0.1 & & 0.2 \\
\hline Mohammad, 2003 & 1300 & & 12.9 & 6.1 & 0.1 & & 0.9 & 0.0 & & 0.2 & & 0.0 & 14.0 & 0.0 & 0.5 & 0.5 & 5.3 & & 11.1 & 0.2 & & 8.8 & 0.2 & 0.3 & 38.1 & 0.0 & & 0.1 & & 0.0 & & & 0.1 & & 0.2 \\
\hline Mohammad, 2003 & 1300 & & 14.3 & 4.6 & 0.1 & & 0.9 & 0.0 & & 0.2 & & 0.0 & 15.5 & 0.0 & 0.4 & 0.5 & 5.9 & & 12.0 & 0.2 & & 9.7 & 0.2 & 0.3 & 34.0 & 0.0 & & 0.1 & & 0.1 & & & 0.2 & & 0.2 \\
\hline Mohammad, 2003 & 1300 & & 16.9 & 2.4 & 0.1 & & 1.1 & 0.0 & & 0.3 & & 0.0 & 18.4 & 0.0 & 0.3 & 0.5 & 7.0 & & 13.7 & 0.2 & & 11.6 & 0.2 & 0.3 & 25.7 & 0.0 & & 0.2 & & 0.1 & & & 0.2 & & 0.2 \\
\hline Mohammad, 2003 & 1300 & & 6.9 & 21.0 & 0.0 & & 0.5 & 0.0 & & 0.1 & & 0.0 & 7.5 & 0.0 & 0.7 & 0.4 & 2.8 & & 6.9 & 0.1 & & 4.7 & 0.1 & 0.1 & 47.6 & 0.0 & & 0.1 & & 0.0 & & & 0.1 & & 3.5 \\
\hline Mukhamedzhanov: & $1250 ?$ & & 4.3 & 3.3 & 6.0 & & & & & & & & & 1.6 & & & & & 5.5 & & & 4.0 & & & 66.1 & & & & & 7.0 & & 1.3 & & & \\
\hline Muller, 1998 & & 1045 & 9.3 & 6.1 & 0.0 & & 7.0 & & & 0.3 & & 1.1 & 4.8 & 5.5 & & & & & 20.0 & 0.1 & & 0.3 & 1.7 & 0.1 & 37.9 & 0.5 & & 0.0 & & & & & 4.3 & & \\
\hline Muller, 1998 & & 1139 & 14.8 & 6.1 & 0.0 & & 2.1 & & & 0.3 & & 1.1 & 4.8 & 5.5 & & & & & 20.0 & 0.1 & & 0.3 & 1.7 & 0.1 & 35.4 & 0.5 & & 0.0 & & & & 3.1 & 3.1 & & \\
\hline Muller, 1998 & & & 12.4 & 6.1 & 0.0 & & 2.1 & & 2.4 & 0.3 & & 1.1 & 4.8 & 5.5 & & & & & 20.0 & 0.1 & & 0.3 & 1.7 & 0.1 & 35.4 & 0.5 & & 0.0 & & & & 3.1 & 3.1 & & \\
\hline Muller, 1998 & & & 12.4 & 6.1 & 0.0 & & 2.1 & & & 0.3 & 2.4 & 1.1 & 4.8 & 5.5 & & & & & 20.0 & 0.1 & & 0.3 & 1.7 & 0.1 & 35.4 & 0.5 & & 0.0 & & & & 3.1 & 3.1 & & \\
\hline Muller, 1998 & & 1124 & 14.8 & 4.6 & 0.0 & & 2.1 & & & 0.3 & & 1.1 & 4.8 & 5.5 & 1.5 & & & & 20.0 & 0.1 & & 0.3 & 1.7 & 0.1 & 35.4 & 0.5 & & 0.0 & & & & 3.1 & 3.1 & & \\
\hline Muller, 1998 & & 1088 & 9.2 & 2.9 & 0.0 & & 9.7 & & & 0.3 & & 1.1 & 6.0 & 5.5 & & & & & 20.0 & 0.1 & & 0.3 & 1.7 & 0.1 & 38.7 & 0.5 & & 0.0 & & & & & 2.8 & & \\
\hline Muller, 1998 & & 1122 & 9.9 & 2.9 & & & 4.4 & & & 0.0 & & 0.1 & 7.3 & 3.1 & 2.0 & 2.0 & & & 20.0 & & & & 0.1 & & 41.5 & 0.0 & & & & & & 3.3 & 3.0 & & \\
\hline Muller, 1998 & & 1080 & 9.9 & 4.2 & & & 4.4 & & & 0.0 & & 0.1 & 7.3 & 3.1 & 2.0 & 2.0 & & & 20.0 & & & & 0.1 & & 40.2 & 0.0 & & & & & & 3.3 & 3.0 & & \\
\hline Muller, 1998 & & & 12.4 & 6.1 & 0.0 & & 3.3 & & & 0.3 & & 1.1 & 6.0 & 5.5 & & & & & 20.0 & 0.1 & & 0.3 & 1.7 & 0.1 & 35.4 & 0.5 & & 0.0 & & & & 3.1 & 3.1 & & \\
\hline Muller, 1998 & & 1133 & 10.7 & 1.8 & 0.0 & & 1.7 & & & 0.4 & & 1.3 & 5.7 & 4.1 & & 1.0 & & & 25.5 & 0.2 & & 0.4 & 1.0 & 0.1 & 39.7 & 0.3 & & 0.0 & & & & 2.8 & 2.8 & & \\
\hline Muller, 1998 & & & 10.5 & 1.8 & 0.0 & & 1.7 & & & 0.4 & & 1.3 & 5.6 & 4.0 & & 1.0 & & & 25.1 & 0.2 & & 0.4 & 2.0 & 0.1 & 39.0 & 0.6 & & 0.0 & & & & 2.8 & 2.8 & & \\
\hline Muller, 1998 & & 1145 & 10.7 & & 0.0 & & 1.7 & & & 0.4 & & 1.3 & 4.0 & 4.1 & 1.1 & & & & 25.5 & 0.2 & & 0.4 & 1.0 & 0.1 & 43.1 & 0.3 & & 0.0 & & & & 2.8 & 2.8 & & \\
\hline Muller, 1998 & & & 10.5 & & 0.0 & & 1.7 & & & 0.4 & & 1.3 & 3.9 & 4.0 & 1.1 & & & & 25.1 & 0.2 & & 0.4 & 2.0 & 0.1 & 42.3 & 0.6 & & 0.0 & & & & 2.8 & 2.8 & & \\
\hline Muller, 1998 & & 1156 & 9.9 & 6.0 & 0.0 & & 3.6 & & & 0.4 & & 0.9 & 8.3 & 2.1 & 2.0 & 2.0 & & & 12.0 & 0.1 & & 0.1 & 2.2 & 0.0 & 41.9 & 1.4 & & & & & & 3.3 & 3.0 & & \\
\hline Muller, 1998 & & 1113 & 8.9 & 6.0 & 0.0 & & 3.6 & & & 0.4 & & 0.9 & 8.3 & 2.1 & 3.0 & 2.0 & & & 13.0 & 0.1 & & 0.1 & 2.2 & 0.0 & 40.9 & 1.4 & & & & & & 3.3 & 3.0 & & \\
\hline Muller, 1998 & & 1067 & 8.9 & 6.0 & 0.0 & & 3.6 & & & 0.4 & & 0.9 & 6.3 & 2.1 & 3.0 & 2.0 & & & 15.0 & 0.1 & & 0.1 & 2.2 & 0.0 & 40.9 & 1.4 & & & & & & 3.3 & 3.0 & & \\
\hline Muller, 1998 & & 1156 & 8.9 & 6.0 & 0.0 & & 3.6 & & & 0.4 & & 0.9 & 6.3 & 2.1 & & 2.0 & & & 18.1 & 0.1 & & 0.1 & 2.2 & 0.0 & 40.9 & 1.4 & & & & & & 3.3 & 3.0 & & \\
\hline Muller, 1998 & & & 9.0 & 6.1 & 0.0 & & 3.7 & & & 0.4 & & 0.9 & 6.4 & 2.1 & 3.1 & 2.1 & & & 15.3 & 0.1 & & 0.1 & 2.3 & 0.0 & 41.6 & 0.4 & & & & & & 3.3 & 3.1 & & \\
\hline Muller, 1998 & & & 9.0 & 6.1 & 0.0 & & 3.7 & & & 0.4 & & 0.9 & 6.4 & 2.1 & 3.1 & 2.1 & & & 15.2 & 0.1 & & 0.1 & 2.3 & 0.0 & 41.4 & 0.7 & & & & & & 3.3 & 3.1 & & \\
\hline Muller, 1998 & & & 8.9 & 6.0 & 0.0 & & 3.7 & & & 0.4 & & 0.9 & 6.3 & 2.1 & 3.0 & 2.1 & & & 15.1 & 0.1 & & 0.1 & 2.3 & 0.0 & 41.1 & 1.0 & & & & & & 3.3 & 3.0 & & \\
\hline Muller, 1998 & & 1090 & 8.0 & 8.1 & 0.0 & & 4.5 & & & 0.4 & & 0.9 & 6.4 & 2.1 & 4.1 & 2.3 & & & 8.5 & 0.1 & & 0.1 & 2.2 & 0.0 & 43.2 & 1.4 & & & & & & 4.1 & 3.0 & & \\
\hline Muller, 1998 & & 1111 & 8.0 & 8.1 & 0.0 & & 4.5 & & & 0.4 & & 0.9 & 6.4 & 2.1 & 3.0 & 2.3 & & & 10.5 & 0.1 & & 0.1 & 2.2 & 0.0 & 42.3 & 1.4 & & & & & & 4.1 & 3.0 & & \\
\hline Muller, 1998 & & 1087 & 8.0 & 8.1 & 0.0 & & 4.5 & & & 0.4 & & 0.9 & 6.4 & 2.1 & 2.0 & 2.3 & & & 12.5 & 0.1 & & 0.1 & 2.2 & 0.0 & 41.3 & 1.4 & & & & & & 4.1 & 3.0 & & \\
\hline Muller, 1998 & & 1070 & 8.0 & 8.1 & & & 7.0 & & & 0.1 & & & 8.0 & 0.4 & 4.1 & 3.0 & & & 10.0 & & & & 0.0 & & 43.9 & 0.0 & & & & & & 4.0 & 3.0 & & \\
\hline Muller, 1998 & & 1050 & 9.9 & 6.1 & 0.0 & & 2.1 & & & 0.3 & & 1.1 & 9.7 & 5.5 & & & & & 20.0 & 0.1 & & 0.3 & 1.7 & 0.1 & 35.4 & 0.5 & 0.1 & 0.0 & & & & 3.1 & 3.1 & & \\
\hline Muller, 1998 & & 1045 & 9.9 & 6.1 & 0.0 & & 2.1 & & & 0.3 & & 1.1 & 4.8 & 5.5 & & & & & 20.0 & 0.1 & & 0.3 & 1.7 & 0.1 & 35.4 & 0.5 & 0.1 & 4.9 & & & & 3.1 & 3.1 & & \\
\hline Muller, 1998 & & 1097 & 9.9 & 6.1 & 0.0 & & 2.1 & & & 0.3 & & 1.1 & 4.8 & 5.5 & & & & & 20.0 & 0.1 & 4.9 & 0.3 & 1.7 & 0.1 & 35.4 & 0.5 & 0.1 & 0.0 & & & & 3.1 & 3.1 & & \\
\hline Muller, 1998 & & & 9.6 & 3.0 & 0.0 & & 10.3 & & & 0.3 & & 1.0 & 6.5 & 5.1 & & & & & 18.5 & 0.1 & & 0.3 & 0.8 & 0.1 & 40.0 & 0.5 & 0.1 & 0.0 & & & & & 3.0 & & \\
\hline Muller, 1998 & & 1079 & 9.2 & 2.9 & 0.0 & & 6.8 & & & 0.3 & & 1.1 & 6.0 & 5.5 & & & & & 20.0 & 0.1 & & 0.3 & 1.7 & 0.1 & 38.7 & 0.5 & 0.1 & 0.0 & & & & 2.9 & 2.8 & & \\
\hline Muller, 1998 & & 1018 & 9.9 & 3.0 & & & 4.8 & & & 0.0 & & 0.0 & 6.3 & 0.1 & 4.0 & 3.0 & & & 20.0 & 0.0 & & & 0.1 & 0.0 & 41.9 & 0.4 & & & & & & 3.3 & 3.0 & & \\
\hline Muller, 1998 & & 1150 & 9.9 & 4.0 & 0.0 & & 2.7 & & & 0.3 & & 0.8 & 6.3 & 4.0 & & 2.0 & & & 20.0 & 0.1 & & 0.3 & 1.3 & 0.1 & 40.9 & 0.4 & 0.1 & & & & & 3.3 & 3.0 & & \\
\hline Muller, 1998 & & 1105 & 9.9 & 4.0 & 0.0 & & 2.7 & & & 0.3 & 3.0 & 0.8 & 6.3 & 4.0 & & 2.0 & & & 20.0 & 0.1 & & 0.3 & 1.3 & 0.1 & 38.0 & 0.4 & 0.1 & & & & & 3.3 & 3.0 & & \\
\hline Muller, 1998 & & 1114 & 9.9 & 4.0 & 0.0 & & 2.7 & & & 0.3 & & 0.8 & 6.3 & 4.0 & & 2.0 & & & 20.0 & 0.1 & & 3.3 & 1.3 & 0.1 & 38.0 & 0.4 & 0.1 & & & & & 3.3 & 3.0 & & \\
\hline Muller, 1998 & & 1168 & 9.9 & 1.0 & 0.0 & & 2.7 & & & 0.3 & 2.0 & 0.8 & 6.3 & 4.0 & & 2.0 & & & 20.0 & 0.1 & & 2.3 & 1.3 & 0.1 & 40.0 & 0.4 & 0.1 & & & & & 3.3 & 3.0 & & \\
\hline Muller, 1998 & & 1092 & 9.9 & 2.0 & 0.0 & & 2.7 & & & 0.3 & & 0.8 & 6.3 & 4.0 & 2.0 & 2.0 & & & 20.0 & 0.1 & & 0.3 & 1.3 & 0.1 & 40.9 & 0.4 & 0.1 & & & & & 3.3 & 3.0 & & \\
\hline Muller, 1998 & & 1210 & 9.9 & 3.0 & & & 4.8 & & & 0.0 & & 0.0 & 6.3 & 0.1 & 4.0 & 3.0 & & & 20.0 & 0.0 & & & 0.1 & 0.0 & 41.9 & 0.4 & & & & & & 3.3 & 3.0 & & \\
\hline Muller, 1998 & & 1130 & 9.9 & 3.0 & & & 4.8 & & & 0.0 & & 0.0 & 6.3 & 0.1 & 4.0 & 3.0 & & & 20.0 & 0.0 & & & 0.1 & 0.0 & 41.9 & 0.4 & & & & & & 3.3 & 3.0 & & \\
\hline Muller, 1998 & & 1122 & 9.8 & 3.9 & 0.0 & & 4.7 & & & 0.3 & & 0.8 & 6.3 & 4.0 & & 2.0 & & & 20.0 & 0.1 & & 0.3 & 1.3 & 0.1 & 38.7 & 0.8 & 0.1 & & & & & 3.3 & 3.0 & & \\
\hline Musick, 2000 & & & 9.3 & 7.2 & & & 12.3 & & & 0.1 & & 5.1 & 2.8 & 0.2 & 6.2 & & 0.1 & & 10.7 & 0.0 & & & 1.3 & 0.0 & 37.1 & 1.1 & 0.3 & & & & & & 6.1 & & \\
\hline Musick, 2000 & & & 9.8 & 7.1 & & & 12.9 & & & 0.2 & & 5.4 & 2.7 & 0.2 & 6.0 & & 0.1 & & 10.5 & 0.0 & & & 1.3 & 0.0 & 35.9 & 1.2 & 0.3 & & & & & & 6.3 & & \\
\hline Musick, 2000 & & & 9.3 & 6.3 & & & 12.3 & & & 0.1 & & 5.1 & 3.1 & 0.2 & 6.1 & & 0.1 & & 11.0 & 0.0 & & & 1.3 & 0.0 & 38.6 & 1.1 & 0.3 & & & & & & 5.1 & & \\
\hline Musick, 2000 & & & 9.6 & 6.5 & & & 12.6 & & & 0.2 & & 2.6 & 3.2 & 0.2 & 6.2 & & 0.1 & & 11.3 & 0.0 & & & 1.3 & 0.0 & 39.6 & 1.2 & 0.3 & & & & & & 5.3 & & \\
\hline Musick, 2000 & & & 9.3 & 4.5 & & & 12.3 & & & 0.1 & & 5.1 & 4.6 & 0.2 & 5.6 & & 0.1 & & 12.3 & 0.0 & & & 1.3 & 0.0 & 38.1 & 1.1 & 0.3 & & & & & & 5.1 & & \\
\hline
\end{tabular}


ORP-56297 Rev. 0

The Catholic University of America

High Level Waste Vitrification System Improvements Vitreous State Laboratory

Table A1. Glass Compositions from Literature Review (continued)

\begin{tabular}{|c|c|c|c|c|c|c|c|c|c|c|c|c|c|c|c|c|c|c|c|c|c|c|c|c|c|c|c|c|c|c|c|c|c|c|c|}
\hline Ref & 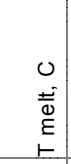 & $\begin{array}{l}0 \\
0 \\
\circ \\
\stackrel{0}{0} \\
上\end{array}$ & $\begin{array}{l}\stackrel{0}{N} \\
\underset{N}{<}\end{array}$ & 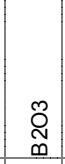 & $\begin{array}{l}\text { D } \\
\infty\end{array}$ & $\begin{array}{l}\text { Oొ } \\
\stackrel{N}{m}\end{array}$ & $\begin{array}{l}0 \\
\mathbb{J} \\
0\end{array}$ & $\begin{array}{l}\tilde{O} \\
0 \\
0 \\
\tilde{ల} \\
0 \\
0 \\
0\end{array}$ & $\begin{array}{l}0 \\
0 \\
0 \\
0 \\
0 \\
0 \\
0\end{array}$ & Oొ & $\begin{array}{l}0 \\
3 \\
0\end{array}$ & 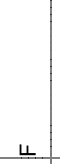 & 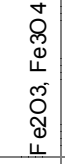 & 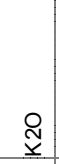 & $\stackrel{?}{\stackrel{ }{J}}$ & $\begin{array}{l}\circ \\
\text { O } \\
\Sigma\end{array}$ & $\begin{array}{l}\tilde{O} \\
\stackrel{\complement}{\Sigma}\end{array}$ & $\begin{array}{l}0 \\
0 \\
\Sigma \\
\Sigma\end{array}$ & $\begin{array}{l}\text { Q } \\
\text { Z }\end{array}$ & $\stackrel{\circ}{z}$ & $\begin{array}{l}\mathfrak{N} \\
\tilde{0} \\
\end{array}$ & $\overline{\bar{I}}$ & ฉ̃ & $\frac{8}{a}$ & $\stackrel{\widetilde{O}}{\omega}$ & O̊ & $\begin{array}{l}\text { Q } \\
\text { is }\end{array}$ & 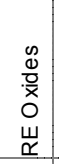 & $\begin{array}{l}\text { 实 } \\
\text { - }\end{array}$ & 尽 & 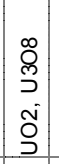 & $\stackrel{\text { Q }}{N}$ & $\stackrel{\text { Õ }}{N}$ & $\underset{\hat{O}}{\vec{O}}$ & $\begin{array}{l}\vec{\Xi} \\
\underline{0} \\
\underline{\underline{\omega}} \\
\vec{z}\end{array}$ \\
\hline Musick, 2000 & & & 9.6 & 4.6 & & & 12.6 & & & 0.2 & & 2.6 & 4.8 & 0.2 & 5.7 & & 0.1 & & 12.6 & 0.0 & & & 1.3 & 0.0 & 39.1 & 1.2 & 0.3 & & & & & & 5.3 & & \\
\hline Musick, 2000 & & & 9.3 & 4.5 & & & 12.3 & & & 0.1 & & 5.1 & 2.8 & 0.2 & 7.4 & & 0.1 & & 10.3 & 0.0 & & & 1.3 & 0.0 & 40.0 & 1.1 & 0.3 & & & & & & 5.1 & & \\
\hline Musick, 2000 & & & 9.6 & 4.6 & & & 12.6 & & & 0.2 & & 2.6 & 2.8 & 0.2 & 7.6 & & 0.1 & & 10.6 & 0.0 & & & 1.3 & 0.0 & 41.1 & 1.2 & 0.3 & & & & & & 5.3 & & \\
\hline Musick, 2000 & & & 9.3 & 5.7 & & & 12.3 & & & 0.1 & & 5.1 & 5.3 & 0.2 & 5.6 & & 0.1 & & 12.2 & 0.0 & & & 1.3 & 0.0 & 36.3 & 1.1 & 0.3 & & & & & & 5.1 & & \\
\hline Musick, 2000 & & & 9.6 & 5.8 & & & 12.6 & & & 0.2 & & 2.6 & 5.4 & 0.2 & 5.7 & & 0.1 & & 12.5 & 0.0 & & & 1.3 & 0.0 & 37.2 & 1.2 & 0.3 & & & & & & 5.3 & & \\
\hline Musick, 2000 & & & 9.3 & 6.3 & & & 12.3 & & & 0.1 & & 5.1 & 0.3 & 0.2 & 6.1 & & 0.1 & & 11.0 & 0.0 & & & 1.3 & 0.0 & 38.6 & 1.1 & 0.3 & 2.8 & & & & & 5.1 & & \\
\hline Musick, 2000 & & & 9.6 & 6.5 & & & 12.6 & & & 0.2 & & 2.6 & 0.3 & 0.2 & 6.2 & & 0.1 & & 11.3 & 0.0 & & & 1.3 & 0.0 & 39.6 & 1.2 & 0.3 & 2.9 & & & & & 5.3 & & \\
\hline Musick, 2000 & & & 9.3 & 5.7 & & & 12.3 & & & 0.1 & & 5.1 & 2.2 & 0.2 & 5.6 & & 0.1 & & 12.2 & 0.0 & & & 1.3 & 0.0 & 36.3 & 1.1 & 0.3 & 3.1 & & & & & 5.1 & & \\
\hline Musick, 2000 & & & 9.6 & 5.8 & & & 12.6 & & & 0.2 & & 2.6 & 2.2 & 0.2 & 5.7 & & 0.1 & & 12.5 & 0.0 & & & 1.3 & 0.0 & 37.2 & 1.2 & 0.3 & 3.2 & & & & & 5.3 & & \\
\hline Musick, 2000 & & & 9.3 & 7.2 & & & 12.3 & & & 0.1 & & 5.1 & 0.3 & 0.2 & 6.2 & & 0.1 & & 10.7 & 0.0 & & & 1.3 & 0.0 & 37.1 & 1.1 & 0.3 & 2.5 & & & & & 6.1 & & \\
\hline Musick, 2000 & & & 9.8 & 7.1 & & & 12.9 & & & 0.2 & & 5.4 & 0.3 & 0.2 & 6.0 & & 0.1 & & 10.5 & 0.0 & & & 1.3 & 0.0 & 35.9 & 1.2 & 0.3 & 2.4 & & & & & 6.3 & & \\
\hline Musick, 2000 & & & 7.3 & 5.3 & & & 13.3 & & & 0.1 & & 6.3 & 2.9 & 0.3 & 5.8 & & 0.1 & & 11.1 & 0.0 & & & 0.1 & 0.0 & 40.7 & 1.3 & & & & & & & 5.3 & & \\
\hline Musick, 2000 & & & 7.9 & 5.1 & & & 14.4 & & & 0.2 & & 6.8 & 2.8 & 0.3 & 5.5 & & 0.1 & & 10.8 & 0.0 & & & 0.1 & 0.0 & 38.8 & 1.4 & & & & & & & 5.8 & & \\
\hline Musick, 2000 & & & 8.6 & 5.1 & & & 11.3 & & & 0.1 & & 4.7 & 3.0 & 0.1 & 6.0 & & 0.1 & & 11.4 & 0.0 & & & 1.2 & 0.0 & 42.3 & 1.0 & 0.2 & & & & & & 4.7 & & \\
\hline Musick, 2000 & & & 8.8 & 5.2 & & & 11.6 & & & 0.1 & & 2.4 & 3.1 & 0.1 & 6.1 & & 0.1 & & 11.7 & 0.0 & & & 1.2 & 0.0 & 43.3 & 1.1 & 0.2 & & & & & & 4.8 & & \\
\hline Musick, 2000 & 1102 & & 10.3 & 8.1 & & & 7.0 & & & 0.1 & & 2.9 & 1.7 & 0.3 & 6.0 & & 0.1 & & 11.8 & 0.0 & & & 3.8 & & 40.5 & 0.3 & & 0.4 & & & & & 6.8 & & \\
\hline Musick, 2000 & 1102 & & 9.3 & 9.2 & & & 6.9 & & & 0.1 & & 2.5 & 1.8 & 0.3 & 5.5 & & 0.1 & & 12.1 & 0.0 & & & 3.6 & & 41.1 & 0.2 & & 0.3 & & & & & 7.0 & & \\
\hline Musick, 2000 & 1102 & & 8.7 & 8.5 & & & 6.7 & & & 0.1 & & 2.7 & 1.7 & 0.3 & 6.0 & & 0.1 & & 12.3 & 0.0 & & & 3.9 & & 41.2 & 0.3 & & 0.3 & & & & & 7.1 & & \\
\hline Musick, 2000 & 1028 & & 8.2 & 7.2 & & & 11.5 & & & 0.1 & & 6.0 & 0.8 & 0.1 & 6.0 & & 0.1 & & 11.2 & 0.0 & & & 3.1 & & 37.0 & 0.6 & & 2.5 & & & & & 5.7 & & \\
\hline Musick, 2000 & 1028 & & 7.7 & 9.9 & & & 11.1 & & & 0.1 & & 6.5 & 0.3 & 0.1 & 5.7 & & 0.1 & & 10.6 & 0.0 & & & 2.9 & & 36.3 & 0.5 & & 2.8 & & & & & 5.5 & & \\
\hline Oksoy, 1994 & & & 16.8 & 16.3 & & & 8.8 & & & & & & & & & & & & 14.3 & & & & & & 43.9 & & & & & & & & & & \\
\hline Oksoy, 1994 & & & 18.3 & 14.5 & & & & & & & & & & & & & & & 22.4 & & & & & & 44.9 & & & & & & & & & & \\
\hline Oksoy, 1994 & & & 17.5 & 4.8 & & & 8.4 & & & & & & & & & & & & 22.9 & & & & & & 46.4 & & & & & & & & & & \\
\hline Oksoy, 1994 & & & 14.8 & 4.1 & & & & & & & & & 20.9 & & & & & & 19.4 & & & & & & 40.7 & & & & & & & & & & \\
\hline Oksoy, 1994 & & & 11.5 & 13.0 & & & 7.0 & & & & & & 19.2 & & & & & & 11.5 & & & & & & 37.9 & & & & & & & & & & \\
\hline Oksoy, 1994 & & & 16.1 & 14.8 & & & & & & & & & & & & & & & 14.1 & & & & & & 55.0 & & & & & & & & & & \\
\hline Oksoy, 1994 & & & 11.3 & 8.8 & & & 3.2 & & & & & & 9.6 & & & & & & 17.0 & & & & & & 50.2 & & & & & & & & & & \\
\hline Oksoy, 1994 & & & 18.4 & 9.5 & & & 4.2 & & & & & & 12.6 & & & & & & 16.0 & & & & & & 39.4 & & & & & & & & & & \\
\hline Oksoy, 1994 & & & 22.6 & 7.6 & & & 2.8 & & & & & & 8.3 & & & & & & 15.8 & & & & & & 42.9 & & & & & & & & & & \\
\hline Oksoy, 1994 & & & 7.4 & 10.5 & & & 4.6 & & & & & & 14.0 & & & & & & 17.2 & & & & & & 46.3 & & & & & & & & & & \\
\hline Oksoy, 1994 & & & 13.0 & 13.7 & & & 3.0 & & & & & & 9.4 & & & & & & 15.5 & & & & & & 45.5 & & & & & & & & & & \\
\hline Oksoy, 1994 & & & 11.2 & 4.4 & & & 4.6 & & & & & & 14.0 & & & & & & 17.6 & & & & & & \begin{tabular}{|l|l|}
48.4 \\
\end{tabular} & & & & & & & & & & \\
\hline Oksoy, 1994 & & & 14.5 & 8.1 & & & 7.7 & & & & & & 9.1 & & & & & & 15.7 & & & & & & 45.0 & & & & & & & & & & \\
\hline Oksoy, 1994 & & & 14.6 & 9.2 & & & & & & & & & 13.3 & & & & & & 16.9 & & & & & & 45.9 & & & & & & & & & & \\
\hline Oksoy, 1994 & & & 11.5 & 7.4 & & & 2.7 & & & & & & 20.2 & & & & & & 14.0 & & & & & & 44.3 & & & & & & & & & & \\
\hline Oksoy, 1994 & & & 16.3 & 5.3 & & & 9.0 & & & & & & & & & & & & 14.6 & & & & & & 54.8 & & & & & & & & & & \\
\hline Oksoy, 1994 & & & 10.7 & 12.3 & & & 5.5 & & & & & & & & & & & & 19.6 & & & & & & 51.9 & & & & & & & & & & \\
\hline Oksoy, 1994 & & & 14.8 & 7.8 & & & 3.0 & & & & & & 9.2 & & & & & & 20.7 & & & & & & 44.5 & & & & & & & & & & \\
\hline Oksoy, 1994 & & & 16.3 & 9.3 & & & 4.3 & & & & & & 12.9 & & & & & & 12.3 & & & & & & 44.9 & & & & & & & & & & \\
\hline Oksoy, 1994 & & & 17.9 & 8.7 & & & 3.4 & & & & & & 10.3 & & & & & & 15.8 & & & & & & 43.8 & & & & & & & & & & \\
\hline Oksoy, 1994 & & & 17.2 & 4.6 & & & & & & & & & & & & & & & 23.2 & & & & & & 55.0 & & & & & & & & & & \\
\hline Olson, 1988 & & & 8.2 & 9.0 & 0.1 & & 0.4 & 0.6 & & 0.1 & 0.0 & & 11.5 & 3.4 & 2.9 & 1.0 & 1.0 & 0.0 & 10.5 & 0.3 & 0.0 & 0.2 & 2.5 & & 42.5 & 0.2 & 0.0 & 0.1 & 3.4 & 0.8 & 0.5 & 0.0 & 0.4 & & \\
\hline \begin{tabular}{|l} 
Olson, 1988 \\
\end{tabular} & & & 10.0 & 7.2 & 0.1 & & 0.7 & 0.3 & & 0.1 & 0.0 & & 15.0 & 3.7 & 3.1 & 1.7 & 1.6 & 0.0 & 11.3 & 0.1 & 0.0 & 0.1 & 0.1 & & 38.0 & 0.1 & 0.0 & 0.1 & 2.8 & 0.4 & 2.5 & 0.0 & 0.2 & & \\
\hline \begin{tabular}{|l|} 
Olson, 1988 \\
\end{tabular} & & & 10.0 & 7.8 & 0.0 & & 0.1 & 1.8 & & 0.4 & 0.1 & & 9.8 & 3.9 & 3.3 & 0.4 & 0.1 & 0.1 & 11.9 & 0.8 & 0.1 & 0.4 & 0.5 & & 42.0 & 0.6 & 0.1 & 0.4 & 2.1 & 2.2 & 0.2 & 0.1 & 1.0 & & \\
\hline \begin{tabular}{|l} 
Olson, 1988 \\
\end{tabular} & & & 10.2 & 7.6 & 0.0 & & 0.2 & 1.8 & & 0.4 & 0.1 & & 9.7 & 3.3 & 2.8 & 0.4 & 1.6 & 0.1 & 10.0 & 0.8 & 0.1 & 0.4 & 0.6 & & 40.9 & 0.6 & 0.1 & 0.4 & 4.1 & 2.3 & 0.1 & 0.1 & 1.1 & & \\
\hline Olson, 1988 & & & 10.1 & 11.2 & 0.1 & & 0.7 & 0.5 & & 0.1 & 0.0 & & 9.3 & 3.6 & 3.1 & 1.6 & 0.1 & 0.0 & 11.0 & 0.2 & 0.0 & 0.1 & 3.8 & & 38.0 & 0.2 & 0.0 & 0.1 & 5.1 & 0.6 & 0.1 & 0.0 & 0.3 & & \\
\hline Olson, 1988 & & & 10.0 & 12.4 & 0.0 & & 0.2 & 0.4 & & 0.1 & 0.0 & & 9.5 & 2.7 & 2.3 & 0.5 & 1.6 & 0.0 & 8.2 & 0.2 & 0.0 & 0.1 & 2.4 & & 42.0 & 0.1 & 0.0 & 0.1 & 4.1 & 0.5 & 2.0 & 0.0 & 0.2 & & \\
\hline Olson, 1988 & & & 8.3 & 11.5 & 0.0 & & 0.2 & 0.4 & & 0.1 & 0.0 & & 15.8 & 3.5 & 3.0 & 0.5 & 0.1 & 0.0 & 10.8 & 0.2 & 0.0 & 0.1 & 0.6 & & 38.0 & 0.1 & 0.0 & 0.1 & 3.7 & 0.5 & 2.2 & 0.0 & 0.2 & & \\
\hline Olson, 1988 & & & 10.2 & 7.1 & 0.0 & & 0.2 & 0.4 & & 0.1 & 0.0 & & 15.5 & 3.8 & 3.3 & 0.4 & 1.6 & 0.0 & 11.7 & 0.2 & 0.0 & 0.1 & 3.9 & & 38.0 & 0.1 & 0.0 & 0.1 & 1.8 & 0.5 & 0.1 & 0.0 & 0.2 & & \\
\hline \begin{tabular}{|l} 
Olson, 1988 \\
\end{tabular} & & & 9.4 & 10.6 & 0.1 & & 0.7 & 2.0 & & 0.4 & 0.1 & & 9.2 & 2.7 & 2.3 & 1.7 & 0.1 & 0.1 & 8.2 & 0.9 & 0.1 & 0.4 & 4.3 & & 38.0 & 0.7 & 0.1 & 0.4 & 2.1 & 2.4 & 2.0 & 0.1 & 1.1 & & \\
\hline
\end{tabular}


ORP-56297 Rev. 0

The Catholic University of America

High Level Waste Vitrification System Improvements Vitreous State Laboratory

Table A1. Glass Compositions from Literature Review (continued)

\begin{tabular}{|c|c|c|c|c|c|c|c|c|c|c|c|c|c|c|c|c|c|c|c|c|c|c|c|c|c|c|c|c|c|c|c|c|c|c|c|}
\hline Ref & 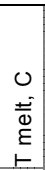 & $\begin{array}{l}0 \\
0 \\
0 \\
0 \\
上\end{array}$ & 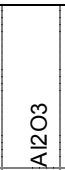 & 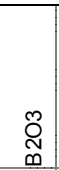 & $\begin{array}{l}\mathbb{D} \\
\infty\end{array}$ & 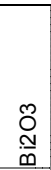 & $\begin{array}{l}0 \\
\mathbb{\pi} \\
0\end{array}$ & $\begin{array}{l} \\
0 \\
0 \\
0 \\
\tilde{ల} \\
0 \\
0 \\
0\end{array}$ & 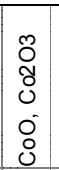 & $\begin{array}{c}\text { Oొ } \\
\text { N }\end{array}$ & O & 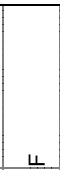 & 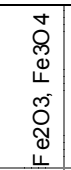 & 尺্்ָ & $\stackrel{\stackrel{ }{ }}{]}$ & $\begin{array}{l}0 \\
\sum\end{array}$ & $\begin{array}{l}\text { Õ } \\
\text { E }\end{array}$ & $\begin{array}{l}00 \\
\text { O) }\end{array}$ & $\begin{array}{l}\underset{N}{ } \\
\underset{Z}{Z}\end{array}$ & $\frac{0}{z}$ & \begin{tabular}{l}
$\mathcal{N}$ \\
\multirow{0}{0}{}
\end{tabular} & 突 & $\begin{array}{l}\text { مి } \\
\text { م. }\end{array}$ & $\frac{8}{0}$ & $\stackrel{\widetilde{N}}{\omega}$ & Õ & 옴 & 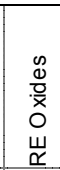 & $\begin{array}{l}\text { 足 } \\
\text { - }\end{array}$ & $\stackrel{\widetilde{N}}{\stackrel{N}{\vdash}}$ & 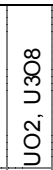 & $\stackrel{q}{N}$ & Oั & 产 & $\begin{array}{l}\bar{\partial} \\
\frac{0}{0} \\
\frac{0}{z}\end{array}$ \\
\hline Olson, 1993 & & 1103 & 7.7 & 16.6 & 0.3 & & 0.5 & 0.3 & & 0.1 & & & 15.5 & 3.1 & 2.3 & 0.9 & 0.8 & & 5.7 & 0.3 & & & 1.2 & & 39.5 & \begin{tabular}{|l|} 
\\
\end{tabular} & 0.3 & & 1.8 & 0.8 & 0.6 & 0.3 & 1.3 & & \\
\hline Olson, 1993 & & 880 & 7.7 & 16.6 & 0.3 & & 0.5 & 0.3 & & 0.1 & & & 8.6 & 6.9 & 5.1 & 0.9 & 0.8 & & 10.3 & 0.3 & & & 1.2 & & 35.3 & 0.2 & 0.3 & & 1.8 & 0.8 & 0.6 & 0.3 & 1.3 & & \\
\hline Olson, 1994 & & 1088 & 7.7 & 9.2 & 0.3 & & 0.5 & 0.3 & & 0.1 & & & 8.6 & 3.1 & 4.3 & 0.9 & 0.8 & & 10.3 & 0.3 & & & 1.2 & & 47.1 & 0.2 & 0.3 & & 1.8 & 0.8 & 0.6 & 0.3 & 1.3 & & \\
\hline Olson, 1994 & & 1249 & 7.7 & 9.2 & 0.3 & & 0.5 & 0.3 & & 0.1 & & & 8.6 & 6.9 & 2.3 & 0.9 & 0.8 & & 5.7 & 0.3 & & & 1.2 & & 46.4 & 0.2 & 0.3 & & 5.3 & 0.8 & 0.6 & 0.3 & 1.3 & & \\
\hline Olson, 1994 & & 1028 & 7.7 & 9.2 & 0.3 & & 0.5 & 0.3 & & 0.1 & & & 15.5 & 6.9 & 2.5 & 0.9 & 0.8 & & 10.3 & 0.3 & & & 1.2 & & 34.8 & 0.2 & 0.3 & & 5.3 & 0.8 & 0.6 & 0.3 & 1.3 & & \\
\hline Peeler, 1999 & & & 11.9 & 6.5 & & & & 8.4 & & & & & & & & & & & & & & 23.0 & & & 21.9 & & 1.4 & 26.9 & & & & & & 1308 & \\
\hline Peeler, 1999 & & & 17.0 & 9.3 & & & & 8.4 & & & & & & & & & & & & & & 23.0 & & & 16.1 & & 1.4 & 24.8 & & & & & & 1257 & \\
\hline Peeler, 1999 & & & 11.9 & 9.3 & & & & 8.4 & & & & & & & & & & & & & & 23.0 & & & 16.1 & & 2.0 & 29.3 & & & & & & 1313 & \\
\hline Peeler, 1999 & & & 11.9 & 6.5 & & & & 3.4 & & & & & & & & & & & & & & 23.0 & & & 16.1 & & 2.0 & 37.1 & & & & & & 1405 & \\
\hline Peeler, 1999 & & & 17.0 & 6.5 & & & & 3.4 & & & & & & & & & & & & & & 23.0 & & & 22.9 & & 2.0 & 25.2 & & & & & & 1241 & \\
\hline Peeler, 1999 & & & 11.9 & 9.3 & & & & 3.4 & & & & & & & & & & & & & & 23.0 & & & 16.1 & & 1.4 & 34.9 & & & & & & 1309 & \\
\hline Peeler, 1999 & & & 17.0 & 6.5 & & & & 3.4 & & & & & & & & & & & & & & 23.0 & & & 22.9 & & 1.4 & 25.7 & & & & & & 1209 & \\
\hline Peeler, 1999 & & & 17.0 & 6.5 & & & & 8.4 & & & & & & & & & & & & & & 18.4 & & & 21.6 & & 2.0 & 26.0 & & & & & & 1242 & \\
\hline Peeler, 1999 & & & 11.9 & 6.5 & & & & 8.4 & & & & & & & & & & & & & & 18.4 & & & 16.1 & & 1.4 & 37.3 & & & & & & 1323 & \\
\hline Peeler, 1999 & & & 11.9 & 9.3 & & & & 8.4 & & & & & & & & & & & & & & 18.4 & & & 22.8 & & 2.0 & 27.2 & & & & & & 1255 & \\
\hline Peeler, 1999 & & & 17.0 & 6.5 & & & & 8.4 & & & & & & & & & & & & & & 18.4 & & & 16.1 & & 1.4 & 32.2 & & & & & & 1292 & \\
\hline Peeler, 1999 & & & 11.9 & 6.5 & & & & 3.4 & & & & & & & & & & & & & & 18.4 & & & 22.9 & & 2.0 & 34.9 & & & & & & 1333 & \\
\hline Peeler, 1999 & & & 17.0 & 9.3 & & & & 3.4 & & & & & & & & & & & & & & 18.4 & & & 22.9 & & 1.4 & 27.6 & & & & & & 1181 & \\
\hline Peeler, 1999 & & & 17.0 & 9.3 & & & & 3.4 & & & & & & & & & & & & & & 18.4 & & & 16.1 & & 2.0 & 33.8 & & & & & & 1250 & \\
\hline Peeler, 1999 & & & 11.9 & 9.3 & & & & 3.4 & & & & & & & & & & & & & & 18.4 & & & 22.9 & & 1.4 & 32.7 & & & & & & 1279 & \\
\hline Peeler, 1999 & & & 17.0 & 6.5 & & & & 3.5 & & & & & & & & & & & & & & 18.4 & & & 16.1 & & 2.0 & 36.5 & & & & & & 1298 & \\
\hline Peeler, 1999 & & & 14.4 & 7.9 & & & & 5.8 & & & & & & & & & & & & & & 20.6 & & & 19.4 & & 1.7 & 30.2 & & & & & & 1273 & \\
\hline Peeler, 1999 & & & 11.9 & 6.5 & & & & 9.7 & & & & & & & & & & & & & & 17.0 & & & 16.1 & & 2.0 & 36.8 & & & & & & 1366 & \\
\hline Peeler, 1999 & & & 11.9 & 9.3 & & & & 9.7 & & & & & & & & & & & & & & 24.0 & & & 22.9 & & 2.0 & 20.2 & & & & & & 1249 & \\
\hline \begin{tabular}{|l} 
Peeler, 1999 \\
\end{tabular} & & & 11.9 & 6.5 & & & & 9.7 & & & & & & & & & & & & & & 24.0 & & & 16.1 & & 1.4 & 30.4 & & & & & & 1343 & \\
\hline Peeler, 1999 & & & 17.0 & 9.3 & & & & 9.7 & & & & & & & & & & & & & & 24.0 & & & 16.1 & & 2.0 & 21.9 & & & & & & 1242 & \\
\hline Peeler, 1999 & & & 11.9 & 9.3 & & & & 2.5 & & & & & & & & & & & & & & 24.0 & & & 22.9 & & 2.0 & 27.4 & & & & & & 1284 & \\
\hline Peeler, 1999 & & & 17.0 & 6.5 & & & & 2.5 & & & & & & & & & & & & & & 24.0 & & & 16.1 & & 2.0 & 31.9 & & & & & & 1282 & \\
\hline Peeler, 1999 & & & 11.9 & 9.3 & & & & 2.5 & & & & & & & & & & & & & & 24.0 & & & 16.1 & & 1.4 & 34.8 & & & & & & 1319 & \\
\hline Peeler, 1999 & & & 17.0 & 6.5 & & & & 2.5 & & & & & & & & & & & & & & 24.0 & & & 16.1 & & 2.0 & 31.9 & & & & & & 1305 & \\
\hline Peeler, 1999 & & & 17.0 & 6.5 & & & & 2.8 & & & & & & & & & & & & & & 24.0 & & & 22.9 & & 1.4 & 25.4 & & & & & & 1208 & \\
\hline Peeler, 1999 & & & 16.3 & 6.5 & & & & 9.7 & & & & & & & & & & & & & & 16.8 & & & 22.9 & & 1.4 & 26.4 & & & & & & 1242 & \\
\hline Peeler, 1999 & & & 17.0 & 9.3 & & & & 9.7 & & & & & & & & & & & & & & 16.8 & & & 16.1 & & 2.0 & 29.1 & & & & & & 1260 & \\
\hline Peeler, 1999 & & & 11.9 & 9.3 & & & & 2.5 & & & & & & & & & & & & & & 16.8 & & & 16.1 & & 1.4 & 42.0 & & & & & & 1300 & \\
\hline Peeler, 1999 & & & 17.0 & 9.3 & & & & 2.5 & & & & & & & & & & & & & & 16.8 & & & 22.9 & & 1.4 & 30.1 & & & & & & 1192 & \\
\hline Peeler, 1999 & & & 11.9 & 6.5 & & & & 2.5 & & & & & & & & & & & & & & 16.8 & & & 22.9 & & 2.0 & 37.4 & & & & & & 1305 & \\
\hline Peeler, 1999 & & & 14.4 & 7.9 & & & & 6.0 & & & & & & & & & & & & & & 20.3 & & & 19.4 & & 1.7 & 30.2 & & & & & & 1264 & \\
\hline Peeler, 1999 & & & 14.4 & 7.9 & & & & 5.6 & & & & & & & & & & & & & & 21.0 & & & 19.5 & & 1.7 & 29.9 & & & & & & 1271 & \\
\hline Peeler, 1999 & & & 14.2 & 7.7 & & & & 5.7 & & & & & & & & & & & & & & 20.9 & & & 19.2 & & 1.7 & 30.6 & & & & & & 1232 & \\
\hline Peeler, 1999 & & & 13.9 & 7.6 & & & & 5.9 & & & & & & & & & & & & & & 20.8 & & & 18.9 & & 1.6 & 31.3 & & & & & & 1289 & \\
\hline Peeler, 1999 & & & 13.7 & 7.5 & & & & 6.0 & & & & & & & & & & & & & & 20.7 & & & 18.5 & & 1.6 & 32.0 & & & & & & 1294 & \\
\hline Peeler, 1999 & & & 13.4 & 7.3 & & & & 6.1 & & & & & & & & & & & & & & 20.6 & & & 18.2 & & 1.6 & 32.7 & & & & & & 1305 & \\
\hline Peeler, 1999 & & & 13.2 & 7.2 & & & & 6.3 & & & & & & & & & & & & & & 20.5 & & & 17.9 & & 1.9 & 33.4 & & & & & & 1308 & \\
\hline Peeler, 1999 & & & 12.9 & 7.0 & & & & 6.4 & & & & & & & & & & & & & & 20.4 & & & 17.5 & & 1.5 & 34.1 & & & & & & 1318 & \\
\hline Peeler, 1999 & & & 12.7 & 6.9 & & & & 6.5 & & & & & & & & & & & & & & 20.3 & & & 17.2 & & 1.5 & 34.8 & & & & & & 1325 & \\
\hline Peeler, 1999 & & & 12.4 & 6.8 & & & & 6.7 & & & & & & & & & & & & & & 20.2 & & & 16.8 & & 1.5 & 35.5 & & & & & & 1324 & \\
\hline Peeler, 1999 & & & 16.9 & 9.2 & & & & 4.3 & & & & & & & & & & & & & & 21.9 & & & 22.9 & & 2.0 & 22.8 & & & & & & 1180 & \\
\hline Peeler, 1999 & & & 15.7 & 8.5 & & & & 4.9 & & & & & & & & & & & & & & 21.5 & & & 21.2 & & 1.8 & 26.3 & & & & & & 1227 & \\
\hline Peeler, 1999 & & & 11.9 & 6.5 & & & & 6.9 & & & & & & & & & & & & & & 20.0 & & & 16.2 & & 1.4 & 37.0 & & & & & & 1305 & \\
\hline Peeler, 1999 & & & 14.4 & 7.9 & & & & 10.9 & & & & & & & & & & & & & & 19.9 & & & 19.5 & & 1.7 & 25.7 & & & & & & 1259 & \\
\hline
\end{tabular}


ORP-56297 Rev. 0

The Catholic University of America

Table A1. Glass Compositions from Literature Review (continued)

\begin{tabular}{|c|c|c|c|c|c|c|c|c|c|c|c|c|c|c|c|c|c|c|c|c|c|c|c|c|c|c|c|c|c|c|c|c|c|c|c|}
\hline Ref & 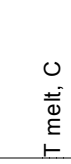 & $\begin{array}{l}0 \\
0 \\
\circ \\
0 \\
1\end{array}$ & $\begin{array}{l}\stackrel{0}{N} \\
\stackrel{N}{<}\end{array}$ & 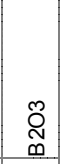 & $\begin{array}{l}\text { O } \\
\infty\end{array}$ & $\begin{array}{c}\text { Oొ } \\
\stackrel{N}{\infty}\end{array}$ & $\begin{array}{l}0 \\
\underset{\pi}{0}\end{array}$ & $\begin{array}{l}\tilde{O} \\
0 \\
0 \\
\tilde{\delta} \\
0 \\
0 \\
0\end{array}$ & $\begin{array}{l}m \\
0 \\
0 \\
0 \\
0 \\
0 \\
0\end{array}$ & ญ̃ & $\stackrel{\circ}{3}$ & \llcorner & 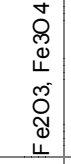 & $\begin{array}{l}\underset{\mathcal{X}}{x} \\
\end{array}$ & 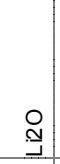 & $\begin{array}{l}0 \\
\text { On } \\
\sum\end{array}$ & $\begin{array}{l}\text { Õ } \\
\text { ¿ } \\
\Sigma\end{array}$ & $\begin{array}{l}0 \\
0 \\
\Sigma\end{array}$ & $\begin{array}{l}\mathbb{Z} \\
\mathbb{N} \\
Z\end{array}$ & $\frac{0}{z}$ & \begin{tabular}{l} 
N \\
\multirow{0}{0}{}
\end{tabular} & 王 & 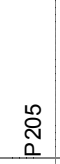 & $\frac{8}{2}$ & $\frac{\widetilde{O}}{\infty}$ & O) & $\begin{array}{l}\text { Q } \\
\text { c) }\end{array}$ & 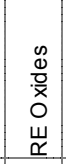 & 尽 & 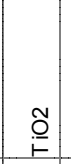 & 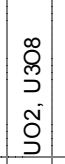 & $\stackrel{o}{N}$ & $\begin{array}{c}\tilde{N} \\
\text { N }\end{array}$ & 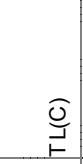 & $\begin{array}{l}\bar{\Xi} \\
\frac{⿹}{0} \\
\frac{1}{z}\end{array}$ \\
\hline Peeler, 1999 & & & 13.2 & 7.2 & & & & 6.3 & & & & & & & & & & & & & & 20.5 & & & 17.9 & & 1.5 & 33.4 & & & & & & 1333 & \\
\hline Peeler, 1999 & & & 14.4 & 7.9 & & & & 11.2 & & & & & & & & & & & & & & 21.0 & & & 19.6 & & 1.7 & 24.3 & & & & & & 1255 & \\
\hline Peeler, 1999 & & & 7.7 & 11.0 & & & & & & 0.1 & & & 2.4 & 0.2 & 3.7 & 0.1 & & & 6.8 & 0.0 & & 0.1 & 0.0 & & 62.4 & 0.0 & 0.1 & 0.0 & & & & & 5.4 & & \\
\hline Peeler, 1999 & & & 7.7 & 11.0 & & & & & & 0.1 & & & 2.4 & 0.2 & 3.7 & 0.1 & & & 6.8 & 0.0 & & 0.1 & 0.0 & & 62.4 & 0.0 & 0.1 & 0.0 & & & & & 5.4 & & \\
\hline Peeler, 1999 & & & 7.5 & 10.6 & & & 3.0 & & & 0.1 & & & 2.4 & 0.1 & 3.5 & 0.1 & & & 6.6 & 0.0 & & 0.1 & 0.0 & & 60.5 & 0.0 & 0.1 & 0.0 & & & & & 5.2 & & \\
\hline Peeler, 1999 & & & 7.5 & 10.6 & & & 3.0 & & & 0.1 & & & 2.4 & 0.1 & 3.5 & 0.1 & & & 6.6 & 0.0 & & 0.1 & 0.0 & & 60.5 & 0.0 & 0.1 & 0.0 & & & & & 5.2 & & \\
\hline Peeler, 2005 & & & 8.0 & 5.2 & 0.1 & & 0.8 & 0.1 & & 0.1 & 0.0 & & 9.2 & 0.4 & 5.2 & 0.7 & 2.1 & & 15.6 & 1.3 & & & & 0.1 & 47.8 & & & 0.0 & 0.0 & 0.0 & 3.3 & 0.0 & 0.1 & & 1.0 \\
\hline Peeler, 2005 & & & 8.0 & 5.2 & 0.1 & & 0.8 & 0.1 & & 0.1 & 0.0 & & 9.2 & 0.4 & & 0.7 & 2.1 & & 22.1 & 1.3 & & & & 0.1 & 46.5 & & & 0.0 & 0.0 & 0.0 & 3.3 & 0.0 & 0.1 & & 0.9 \\
\hline Peeler, 2005 & & & 8.0 & 5.2 & 0.1 & & 0.8 & 0.1 & & 0.1 & 0.0 & & 9.2 & 0.4 & 3.9 & 0.7 & 2.1 & & 17.5 & 1.3 & & & & 0.1 & 47.1 & & & 0.0 & 0.0 & 0.0 & 3.3 & 0.0 & 0.1 & & 1.0 \\
\hline Peeler, 2005 & & & 8.0 & 5.2 & 0.1 & & 0.8 & 0.1 & & 0.1 & 0.0 & & 9.2 & 0.4 & 2.6 & 0.7 & 2.1 & & 18.8 & 1.3 & & & & 0.1 & 47.1 & & & 0.0 & 0.0 & 0.0 & 3.3 & 0.0 & 0.1 & & 0.9 \\
\hline Peeler, 2005 & & & 8.0 & 5.2 & 0.1 & & 0.8 & 0.1 & & 0.1 & 0.0 & & 9.2 & 0.4 & 1.3 & 0.7 & 2.1 & & 20.1 & 1.3 & & & & 0.1 & 47.1 & & & 0.0 & 0.0 & 0.0 & 3.3 & 0.0 & 0.1 & & 0.9 \\
\hline Peeler, 2005 & & & 8.0 & 5.2 & 0.1 & & 0.8 & 0.1 & & 0.1 & 0.0 & & 9.2 & 0.4 & & 0.7 & 2.1 & & 21.4 & 1.3 & & & & 0.1 & 47.1 & & & 0.0 & 0.0 & 0.0 & 3.3 & 0.0 & 0.1 & & 0.9 \\
\hline Peeler, 2005 & & & 8.0 & 5.2 & 0.1 & & 0.8 & 0.1 & & 0.1 & 0.0 & & 9.2 & 0.4 & 3.9 & 0.7 & 2.1 & & 16.9 & 1.3 & & & & 0.1 & 47.8 & & & 0.0 & 0.0 & 0.0 & 3.3 & 0.0 & 0.1 & & 0.8 \\
\hline Peeler, 2005 & & & 8.0 & 5.2 & 0.1 & & 0.8 & 0.1 & & 0.1 & 0.0 & & 9.2 & 0.4 & 2.6 & 0.7 & 2.1 & & 18.2 & 1.3 & & & & 0.1 & 47.8 & & & 0.0 & 0.0 & 0.0 & 3.3 & 0.0 & 0.1 & & 0.8 \\
\hline Peeler, 2005 & & & 8.0 & 5.2 & 0.1 & & 0.8 & 0.1 & & 0.1 & 0.0 & & 9.2 & 0.4 & 1.3 & 0.7 & 2.1 & & 19.5 & 1.3 & & & & 0.1 & 47.8 & & & 0.0 & 0.0 & 0.0 & 3.3 & 0.0 & 0.1 & & 0.7 \\
\hline Peeler, 2005 & & & 8.0 & 5.2 & 0.1 & & 0.8 & 0.1 & & 0.1 & 0.0 & & 9.2 & 0.4 & & 0.7 & 2.1 & & 20.8 & 1.3 & & & & 0.1 & 47.8 & & & 0.0 & 0.0 & 0.0 & 3.3 & 0.0 & 0.1 & & 0.8 \\
\hline Peeler, 2005 & & & 8.0 & 5.2 & 0.1 & & 0.8 & 0.1 & & 0.1 & 0.0 & & 9.2 & 0.4 & 3.3 & 0.7 & 2.1 & & 17.5 & 1.3 & & & & 0.1 & 47.8 & & & 0.0 & 0.0 & 0.0 & 3.3 & 0.0 & 0.1 & & 0.8 \\
\hline Peeler, 2005 & & & 8.0 & 5.2 & 0.1 & & 0.8 & 0.1 & & 0.1 & 0.0 & & 9.2 & 0.4 & 3.9 & 0.7 & 2.1 & & 18.2 & 1.3 & & & & 0.1 & 46.5 & & & 0.0 & 0.0 & 0.0 & 3.3 & 0.0 & 0.1 & & 1.1 \\
\hline Peeler, 2005 & & & 8.0 & 5.2 & 0.1 & & 0.8 & 0.1 & & 0.1 & 0.0 & & 9.2 & 0.4 & 2.6 & 0.7 & 2.1 & & 19.5 & 1.3 & & & & 0.1 & 46.5 & & & 0.0 & 0.0 & 0.0 & 3.3 & 0.0 & 0.1 & & 1.0 \\
\hline Peeler, 2005 & & & 8.0 & 5.2 & 0.1 & & 0.8 & 0.1 & & 0.1 & 0.0 & & 9.2 & 0.4 & 1.3 & 0.7 & 2.1 & & 22.1 & 1.3 & & & & 0.1 & 45.2 & & & 0.0 & 0.0 & 0.0 & 3.3 & 0.0 & 0.1 & & 1.0 \\
\hline Peeler, 2005a & 1150 & & 12.6 & 4.7 & 0.0 & & 0.9 & 0.1 & & 0.1 & 0.0 & & 8.4 & 0.7 & 4.6 & 0.7 & 1.8 & & 17.1 & 0.5 & & & & 0.1 & 43.1 & 0.4 & & 0.0 & 0.1 & 0.0 & 2.0 & 0.0 & 0.1 & & 0.8 \\
\hline Peeler, 2005a & 1150 & & 11.9 & 4.6 & 0.0 & & 0.8 & 0.1 & & 0.1 & 0.0 & & 7.9 & 0.7 & 4.7 & 0.6 & 1.7 & & 17.5 & 0.5 & & & & 0.1 & 42.9 & 0.4 & & 0.0 & 0.1 & 1.0 & 2.0 & 0.0 & 0.1 & & 0.8 \\
\hline Peeler, 2005a & 1150 & & 8.6 & 4.7 & 0.1 & & 0.9 & 0.1 & & 0.1 & 0.0 & & 9.2 & 0.4 & 4.7 & 0.7 & 2.2 & & 17.1 & 1.2 & & & & 0.1 & 43.3 & 0.5 & & 0.0 & 0.1 & 0.9 & 3.4 & 0.1 & 0.1 & & 1.2 \\
\hline Peeler, 2005a & 1150 & & 12.8 & 4.7 & 0.0 & & 0.9 & 0.1 & & 0.1 & 0.0 & & 8.4 & 0.8 & 4.7 & 0.7 & 1.8 & & 14.4 & 0.5 & & & & 0.1 & 46.1 & 0.4 & & 0.0 & 0.1 & 0.0 & 2.0 & 0.0 & 0.1 & & 0.6 \\
\hline Peeler, 2005a & 1150 & & 9.2 & 4.7 & 0.1 & & 0.9 & 0.1 & & 0.1 & 0.0 & & 9.7 & 0.5 & 4.7 & 0.7 & 2.2 & & 16.6 & 1.3 & & & & 0.1 & 43.4 & 0.4 & & 0.0 & 0.1 & 0.0 & 3.5 & 0.1 & 0.1 & & 1.1 \\
\hline Peeler, 2005a & 1150 & & 8.6 & 4.6 & 0.1 & & 0.7 & 0.1 & & 0.1 & 0.0 & & 8.4 & 0.5 & 4.7 & 0.4 & 2.2 & & 17.0 & 1.9 & & & & 0.1 & 43.3 & 0.4 & & 0.0 & 0.1 & 0.9 & 3.7 & 0.1 & 0.1 & & 1.2 \\
\hline Peeler, 2005a & 1150 & & 9.3 & 4.7 & 0.1 & & 0.7 & 0.1 & & 0.1 & 0.0 & & 8.9 & 0.5 & 4.8 & 0.4 & 2.3 & & 16.3 & 2.1 & & & & 0.1 & 43.6 & 0.4 & & 0.0 & 0.2 & 0.0 & 4.2 & 0.1 & 0.1 & & 1.1 \\
\hline Peeler, 2005a & 1150 & & 12.0 & 4.7 & 0.0 & & 0.8 & 0.1 & & 0.1 & 0.0 & & 7.9 & 0.7 & \begin{tabular}{|l|}
4.7 \\
\end{tabular} & 0.6 & 1.7 & & 15.2 & 0.5 & & & & 0.1 & 45.9 & 0.4 & & 0.0 & 0.1 & 0.9 & 2.0 & 0.0 & 0.1 & & 0.7 \\
\hline Peeler, 2005a & 1150 & & 11.2 & 5.1 & 0.0 & & 0.8 & 0.1 & & 0.1 & 0.0 & & 7.6 & 0.7 & 5.1 & 0.6 & 1.6 & & 16.2 & 0.4 & & & & 0.1 & 47.1 & 0.3 & & 0.0 & 0.1 & 0.0 & 1.8 & 0.0 & 0.1 & & 0.7 \\
\hline Peeler, 2005a & 1150 & & 8.3 & 4.7 & 0.1 & & 0.9 & 0.1 & & 0.1 & 0.0 & & 9.4 & 0.4 & 4.7 & 0.7 & 2.3 & & 17.2 & 1.3 & & & & 0.1 & 43.3 & 0.5 & & 0.0 & 0.1 & 0.9 & 3.4 & 0.1 & 0.1 & & 1.2 \\
\hline Peeler, 2005a & 1150 & & 8.9 & 4.7 & 0.1 & & 0.9 & 0.1 & & 0.1 & 0.0 & & 9.7 & 0.4 & 4.7 & 0.8 & 2.3 & & 16.3 & 1.3 & & & & 0.1 & 43.6 & \begin{tabular}{|l|}
0.4 \\
\end{tabular} & & 0.0 & 0.2 & 0.0 & 3.7 & 0.1 & 0.1 & & 1.1 \\
\hline Peeler, 2005a & 1150 & & 10.4 & 5.0 & 0.0 & & 0.7 & 0.1 & & 0.1 & 0.0 & & 6.9 & 0.6 & 5.1 & 0.6 & 1.5 & & 16.8 & 0.4 & & & & 0.1 & 46.3 & 0.4 & & 0.0 & 0.1 & 0.8 & 1.7 & 0.0 & 0.1 & & 0.8 \\
\hline Peters, 1993 & & & 9.1 & & & 1.4 & 1.4 & & & 0.6 & & & 7.7 & & & & & & 17.7 & & 11.8 & & 3.3 & & 42.1 & & & & & & & & 4.9 & & \\
\hline Peters, 1993 & & & 9.1 & & & 1.4 & 1.4 & & & 0.8 & & & 7.7 & & & & & & 17.6 & & 11.8 & & 3.3 & & 42.1 & & & & & & & & 4.9 & & \\
\hline Peters, 1993 & & & 8.9 & & & 1.3 & 1.4 & & & 0.3 & & & 7.6 & & & & & & 17.4 & & 11.7 & & 5.0 & & 41.5 & & & & & & & & 4.9 & & \\
\hline Peters, 1993 & & & 8.7 & & & 1.3 & 1.4 & & & 0.3 & & & 7.4 & & & & & & 17.0 & & 11.4 & & 7.0 & & 40.7 & & & & & & & & 4.8 & & \\
\hline Peters, 1993 & & & 8.6 & & & 1.3 & 1.4 & & & 0.3 & & & 7.2 & & & & & & 16.7 & & 11.2 & & 9.0 & & 39.8 & & & & & & & & 4.7 & & \\
\hline Peters, 1993 & & & 9.0 & & & 1.4 & 1.4 & & & 0.3 & & & 7.6 & & & & & & 17.5 & & 11.7 & & 3.3 & & 41.8 & & & & & 1.0 & & & 4.9 & & \\
\hline Peters, 1993 & & & 8.8 & & & 1.3 & 1.4 & & & 0.3 & & & 7.5 & & & & & & 17.2 & & 11.5 & & 3.2 & & 41.0 & & & & & 3.0 & & & 4.8 & & \\
\hline Peters, 1993 & & & 8.6 & & & 1.3 & 1.4 & & & 0.3 & & & 7.3 & & & & & & 16.8 & & 11.3 & & 3.1 & & 40.2 & & & & & 5.0 & & & 4.7 & & \\
\hline Peters, 1993 & & & 9.1 & & & 1.4 & 1.4 & & & 0.3 & & & 7.7 & & & & & & 17.7 & & 11.9 & & 3.3 & & 42.3 & & & & & & & & 5.0 & & \\
\hline Peters, 1993 & 1250 & 1141 & 9.7 & 0.1 & & 1.5 & 1.5 & 2.0 & & 0.3 & & 0.4 & 8.2 & 0.2 & & 0.1 & 1.1 & 0.1 & 18.9 & 1.7 & 0.1 & 0.1 & 3.5 & 0.3 & 37.5 & 0.3 & 0.3 & 6.9 & & & & & 5.3 & & \\
\hline Peters, 1993 & 1350 & 1115 & 10.1 & 0.1 & & 1.5 & 1.6 & 2.1 & & 0.4 & & 0.4 & 8.5 & 0.2 & & 0.1 & 1.2 & 0.1 & 19.6 & 1.8 & 0.1 & 0.1 & 3.7 & 0.3 & 35.2 & 0.3 & 0.3 & 7.1 & & & & & 5.5 & & \\
\hline Peters, 1993 & 1215 & 1090 & 10.4 & 0.1 & & 1.6 & 1.7 & 2.2 & & 0.4 & & 0.4 & 8.8 & 0.2 & & 0.1 & 1.2 & 0.1 & 120.3 & 1.8 & 0.1 & 0.1 & 3.8 & 0.3 & 33.0 & 0.3 & 0.3 & 7.3 & & & & & 5.7 & & \\
\hline Peters, 1993 & 1350 & 1065 & 10.8 & 0.1 & & 1.6 & 1.7 & 2.3 & & 0.4 & & 0.5 & 9.1 & 0.2 & & 0.1 & 1.2 & 0.1 & 120.9 & 1.9 & 0.1 & 0.1 & 3.9 & 0.3 & 30.8 & 0.3 & 0.3 & 7.6 & & & & & 5.9 & & \\
\hline Peters, 1993 & 1250 & 1041 & 11.1 & 0.1 & & 1.7 & 1.8 & 2.3 & & 0.4 & & 0.5 & 9.4 & 0.2 & & 0.1 & 1.3 & 0.1 & 121.6 & 1.9 & 0.2 & 0.1 & 4.0 & 0.3 & 28.5 & 0.3 & 0.4 & 7.8 & & & & & 6.1 & & \\
\hline Peters, 1993 & 1350 & 1017 & 11.5 & 0.1 & & 1.7 & 1.8 & 2.4 & & 0.4 & & 0.5 & 9.7 & 0.2 & & 0.1 & 1.3 & 0.1 & 122.3 & 2.0 & 0.2 & 0.1 & 4.2 & 0.3 & 26.3 & 0.3 & 0.4 & 8.1 & & & & & 6.2 & & \\
\hline Peters, 1993 & 1300 & 993 & 11.8 & 0.1 & & 1.8 & 1.9 & 2.5 & & 0.4 & & 0.5 & 10.0 & 0.2 & & 0.1 & 1.4 & 0.1 & 23.0 & 2.1 & 0.2 & 0.1 & 4.3 & 0.3 & 24.1 & 0.3 & 0.4 & 8.3 & & & & & 6.4 & & \\
\hline Peters, 1993 & 1150 & & 8.7 & 13.8 & & & 0.8 & 0.1 & & 2.4 & 0.2 & 0.1 & 3.6 & & 3.0 & 0.3 & 1.2 & 0.0 & 17.8 & 0.1 & & 0.7 & 3.3 & 0.1 & 42.6 & 0.7 & & 0.2 & & 0.0 & & & 0.1 & & \\
\hline Peters, 1993 & 1144 & & 8.9 & 14.0 & & & 0.9 & 0.1 & & & 0.2 & 0.1 & 3.7 & & 7.4 & 0.3 & 1.3 & 0.0 & \begin{tabular}{|l|} 
\\
\end{tabular} & 0.1 & & 0.8 & 3.4 & 0.1 & 53.5 & 0.7 & & 0.3 & & 0.0 & & & 0.1 & & \\
\hline
\end{tabular}


ORP-56297 Rev. 0

The Catholic University of America

Table A1. Glass Compositions from Literature Review (continued)

\begin{tabular}{|c|c|c|c|c|c|c|c|c|c|c|c|c|c|c|c|c|c|c|c|c|c|c|c|c|c|c|c|c|c|c|c|c|c|c|c|}
\hline Ref & 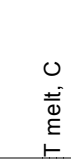 & $\begin{array}{l}0 \\
0 \\
0 \\
\stackrel{0}{0} \\
1\end{array}$ & $\begin{array}{l}\stackrel{0}{N} \\
\underset{N}{<}\end{array}$ & 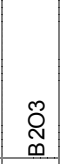 & $\begin{array}{l}\text { O } \\
\infty\end{array}$ & 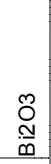 & $\begin{array}{l}0 \\
\mathbb{J} \\
0\end{array}$ & 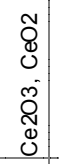 & $\begin{array}{l}m \\
0 \\
0 \\
0 \\
0 \\
0 \\
0\end{array}$ & $\begin{array}{l}\text { Oొ } \\
\text { บิ }\end{array}$ & $\stackrel{O}{3}$ & \llcorner & 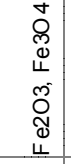 & $\begin{array}{l}\stackrel{2}{\mathbb{N}} \\
\underline{x}\end{array}$ & $\stackrel{ }{\stackrel{ }{J}}$ & $\begin{array}{l}\circ \\
\Sigma \\
\Sigma\end{array}$ & $\begin{array}{l}\tilde{N} \\
\text { C } \\
\Sigma\end{array}$ & $\begin{array}{l}0 \\
0 \\
\Sigma \\
\Sigma\end{array}$ & $\begin{array}{l}\mathbb{Z} \\
\mathbb{N} \\
Z\end{array}$ & $\stackrel{\circ}{z}$ & \begin{tabular}{l}
$\mathcal{N}$ \\
\multirow{5}{0}{}
\end{tabular} & $\overline{\bar{I}}$ & $\begin{array}{l}\text { م) } \\
\vdots \\
0\end{array}$ & $\frac{8}{2}$ & 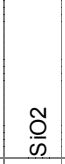 & $\begin{array}{l}\text { Oొ } \\
\text { in }\end{array}$ & $\begin{array}{l}\text { Q } \\
\text { is }\end{array}$ & 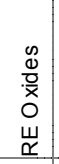 & $\begin{array}{l}\tilde{Q} \\
\stackrel{\varepsilon}{F}\end{array}$ & $\begin{array}{l}\tilde{O} \\
\stackrel{2}{F}\end{array}$ & 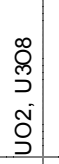 & $\stackrel{O}{N}$ & $\begin{array}{l}\text { ญ̃ } \\
\stackrel{N}{N}\end{array}$ & $\begin{array}{l}\hat{\underline{O}} \\
\overrightarrow{1}\end{array}$ & $\begin{array}{l}\bar{\Xi} \\
\text { ô } \\
\underline{\underline{\omega}} \\
\bar{z}\end{array}$ \\
\hline Peters, 1993 & 1130 & & 8.7 & 13.8 & & & 0.8 & 0.1 & & 2.4 & 0.2 & 0.1 & 3.6 & & & 0.3 & 1.2 & 0.0 & 20.8 & 0.1 & & 0.7 & 3.3 & 0.1 & 42.6 & \begin{tabular}{|l|l|} 
& \\
\end{tabular} & & 0.2 & & 0.0 & & & 0.1 & & \\
\hline Peters, 1993 & 1150 & & 8.7 & 13.7 & & & 0.9 & 0.1 & & & 0.2 & 0.1 & 3.6 & & 7.2 & 0.3 & 1.3 & 0.0 & 4.2 & 0.1 & & 0.7 & 5.5 & 0.1 & 52.3 & 0.7 & & 0.2 & & 0.0 & & & 0.1 & & \\
\hline Peters, 1993 & 1150 & & 9.0 & 14.1 & & & 0.9 & 0.1 & & 2.4 & 0.2 & 0.1 & 3.7 & & 7.4 & 0.3 & 1.2 & 0.0 & 4.4 & 0.1 & & 0.7 & 3.3 & 0.1 & 54.0 & 0.7 & & 0.2 & & 0.0 & & & 0.1 & & \\
\hline Peters, 1993 & 1150 & & 8.7 & 13.6 & & & 0.9 & 0.1 & & 2.4 & 0.2 & 0.1 & 3.6 & & 7.2 & 0.3 & 1.2 & 0.0 & 4.2 & 0.1 & & 0.7 & 3.3 & 0.1 & 52.2 & 0.7 & & 0.2 & & 0.0 & & & 0.1 & & \\
\hline Peters, 1993 & 1250 & & 8.5 & 13.3 & & & 0.8 & 0.1 & & 2.4 & 0.2 & 0.1 & 3.5 & & 7.0 & 0.3 & 1.2 & 0.0 & 4.1 & 0.1 & & 0.7 & 5.3 & 0.1 & 51.1 & 0.7 & & 0.2 & & 0.0 & & & 0.1 & & \\
\hline Peters, 1993 & 1250 & & 8.3 & 13.1 & & & 0.8 & 0.1 & & 2.3 & 0.2 & 0.1 & 3.4 & & 6.9 & 0.3 & 1.2 & 0.0 & 4.0 & 0.1 & & 0.7 & 7.3 & 0.1 & 50.0 & 0.7 & & 0.2 & & 0.0 & & & 0.1 & & \\
\hline Peters, 1993 & & & 9.9 & 12.3 & 0.2 & & 0.8 & 0.7 & 0.0 & 0.1 & 0.0 & & 11.9 & 1.6 & 2.2 & 0.8 & 1.2 & 0.0 & 9.8 & 0.3 & & 0.2 & 2.5 & & 39.6 & 0.2 & 0.0 & 0.2 & 3.5 & 0.8 & 0.6 & 0.0 & 0.4 & & \\
\hline Peters, 1993 & & & 10.3 & 12.7 & 0.2 & & 0.8 & 0.7 & 0.0 & 0.2 & 0.0 & & 12.4 & 1.6 & 2.4 & 0.8 & 1.2 & 0.0 & 10.2 & 0.3 & & 0.1 & 2.6 & & 37.4 & 0.2 & 0.0 & 0.1 & 3.7 & 0.8 & 0.6 & 0.0 & 0.4 & & \\
\hline Peters, 1993 & & & 10.1 & 12.5 & & & 0.5 & 0.7 & & 0.1 & & & 12.9 & 0.2 & 1.8 & 0.2 & 1.0 & 0.0 & 11.4 & 0.3 & & 0.1 & 2.6 & & 40.8 & 0.2 & & 0.3 & 3.6 & & 0.6 & & 0.2 & & \\
\hline Peters, 1993 & & & 9.3 & 11.5 & 0.2 & & 0.8 & 0.7 & 0.0 & 0.1 & 0.0 & & 17.2 & 1.5 & 2.1 & 0.8 & 1.1 & 0.0 & 9.2 & 0.3 & & 0.2 & 2.3 & & 37.2 & 0.2 & 0.0 & 0.2 & 3.3 & 0.8 & 0.6 & 0.0 & 0.4 & & \\
\hline Peters, 1993 & & & 8.3 & 10.1 & 0.2 & & 0.7 & 0.7 & 0.0 & 0.1 & 0.0 & & 12.0 & 3.6 & 3.1 & 0.9 & 1.0 & 0.0 & 11.1 & 0.3 & & 0.3 & 2.4 & & 39.6 & 0.2 & 0.0 & 0.2 & 3.6 & 0.8 & 0.6 & 0.0 & 0.3 & & \\
\hline Peters, 1993 & & & 8.3 & 9.4 & 0.2 & & 0.7 & 0.7 & 0.0 & 0.1 & 0.0 & & 12.0 & 3.4 & 2.9 & 0.9 & 1.0 & 0.0 & 10.3 & 0.3 & & 0.3 & 2.4 & & 41.6 & 0.2 & 0.0 & 0.2 & 3.6 & 0.8 & 0.6 & 0.0 & 0.3 & & \\
\hline Peters, 1993 & & 1048 & 8.3 & 12.6 & 0.2 & & 0.7 & 0.2 & 0.0 & 0.1 & 0.0 & & 12.0 & 3.1 & 2.7 & 0.9 & 1.0 & 0.0 & 9.6 & 0.3 & & 0.3 & 2.4 & & 39.8 & 0.2 & 0.0 & 0.2 & 3.6 & 0.8 & 0.6 & 0.0 & 0.3 & & \\
\hline Peters, 1993 & & 1113 & 8.3 & 11.7 & 0.2 & & 0.7 & 0.2 & 0.0 & 0.1 & 0.0 & & 12.0 & 2.9 & 2.5 & 0.9 & 1.0 & 0.0 & 9.0 & 0.3 & & 0.3 & 2.4 & & 41.8 & 0.2 & 0.0 & 0.2 & 3.6 & 0.8 & 0.6 & 0.0 & 0.3 & & \\
\hline Peters, 1993 & & & 8.3 & 10.1 & 0.2 & & 0.7 & 0.7 & 0.0 & 0.1 & 0.0 & & 12.0 & 3.6 & 3.1 & 0.9 & 1.0 & 0.0 & 11.1 & 0.3 & & 0.3 & 2.4 & & 39.6 & 0.2 & 0.0 & 0.2 & 3.6 & 0.8 & 0.6 & 0.0 & 0.3 & & \\
\hline Peters, 1993 & & & 8.3 & 12.6 & 0.2 & & 0.7 & 0.2 & 0.0 & 0.1 & 0.0 & & 12.0 & 3.1 & 2.7 & 0.9 & 1.0 & 0.0 & 9.6 & 0.3 & & 0.3 & 2.4 & & 39.8 & 0.2 & 0.0 & 0.2 & 3.6 & 0.8 & 0.6 & 0.0 & 0.3 & & \\
\hline Peters, 1993 & & & 10.0 & 12.4 & 0.2 & & 0.7 & 0.5 & 0.0 & 0.3 & 0.0 & & 12.3 & 2.0 & 2.0 & 1.8 & 1.3 & & 9.1 & 0.4 & & 0.1 & 2.6 & & 38.8 & & & 0.2 & 3.6 & 0.7 & 0.6 & 0.0 & 0.4 & & \\
\hline Peters, 1993 & & & 10.0 & 12.5 & 0.2 & & 0.8 & 0.7 & 0.0 & 0.3 & 0.0 & & 12.2 & 1.9 & 2.0 & 1.3 & 1.3 & & 9.9 & 0.3 & & 0.1 & 2.5 & & 38.8 & & & 0.2 & 3.6 & 0.7 & 0.6 & 0.0 & 0.5 & & \\
\hline Peters, 1993 & & & 9.8 & 11.2 & 0.2 & & 0.7 & 0.8 & 0.0 & 0.3 & 0.0 & & 13.6 & 1.9 & 2.2 & 1.3 & 1.3 & & 9.6 & 0.3 & & 0.1 & 2.5 & & 38.1 & & & 0.2 & 4.0 & 0.7 & 0.7 & 0.0 & 0.4 & & \\
\hline Peters, 1993 & & & 11.6 & 12.0 & 0.2 & & 0.8 & 0.7 & 0.0 & 0.1 & 0.0 & & 11.7 & 1.6 & 2.2 & 0.8 & 1.2 & 0.0 & 9.6 & 0.3 & & 0.2 & 2.4 & & 38.8 & 0.2 & 0.0 & 0.2 & 3.5 & 0.8 & 0.6 & 0.0 & 0.4 & & \\
\hline Peters, 1993 & & & 9.9 & 12.2 & 0.2 & & 0.8 & 0.7 & 0.0 & 0.1 & 0.0 & & 11.9 & 1.6 & 2.3 & 0.8 & 1.2 & 0.0 & 9.8 & 0.3 & & 0.1 & 2.5 & & 35.9 & 0.2 & 0.0 & 0.1 & 3.5 & 4.8 & 0.6 & 0.0 & 0.4 & & \\
\hline Peters, 1995 & 1150 & & 20.0 & 5.1 & & & 5.4 & & & & & & 0.5 & 4.5 & & 0.3 & & & 11.6 & & & & 38.5 & & 10.2 & 1.1 & & & & & & & & & 0.2 \\
\hline Peters, 1995 & 1350 & & 8.1 & & & & 11.5 & & & & & & 1.1 & 7.4 & & 0.6 & & & 12.0 & & & & 0.3 & & 52.0 & 2.1 & & & & & & & & & 0.3 \\
\hline Peters, 1995 & 1150 & & 11.9 & 11.3 & & & 10.2 & & & & & & 1.0 & 6.6 & & 0.6 & & & 10.6 & & & & 0.2 & & 41.4 & 1.8 & & & & & & & & & 0.3 \\
\hline Peters, 1995 & 1100 & & 20.4 & & & & 10.0 & & & & & & 1.0 & 6.4 & & 0.5 & & & 10.4 & & & & 37.7 & & 7.6 & 1.8 & & & & & & & & & 0.3 \\
\hline Peters, 1995 & 1150 & & 15.4 & & & & 10.7 & & & & & & 1.1 & 6.9 & & 0.6 & & & 11.2 & & & & 39.5 & & 8.2 & 1.9 & & & & & & & & & 0.5 \\
\hline Peters, 1995 & 1150 & & 20.7 & 5.2 & & & 7.1 & & & & & & 0.7 & 4.5 & & 0.4 & & & 12.6 & & & & 39.1 & & 5.4 & 1.3 & & & & & & & & & 0.2 \\
\hline Peters, 1995 & 1200 & & 25.0 & 6.3 & & & 8.5 & & & & & & 0.8 & 5.5 & & 0.5 & & & 8.9 & & & & 32.8 & & 6.5 & 1.5 & & & & & & & & & 0.2 \\
\hline Peters, 1995 & 1450 & & 3.2 & & & & 10.2 & & & & & & 1.0 & 6.6 & & 0.6 & & & 10.6 & & & & 0.2 & & 61.5 & 1.8 & & & & & & & & & 0.2 \\
\hline Peters, 1995 & 1200 & & 19.8 & 3.3 & & & 8.9 & & & & & & 0.9 & 5.7 & & 0.5 & & & 9.2 & & & & 39.6 & & 6.8 & 1.6 & & & & & & & & & 0.2 \\
\hline Peters, 1995 & 1200 & & 25.7 & & & & 6.9 & & & & & & 0.7 & 5.7 & & 0.4 & & & 8.3 & & & & 34.3 & & 13.1 & 1.4 & & & & & & & & & 0.1 \\
\hline Pittman, 2001 & & & 12.5 & 6.0 & & & 15.0 & & & & & 4.0 & & & 7.0 & 8.0 & & & & & & & & & 36.5 & 0.5 & & 2.5 & & & & & 8.0 & & \\
\hline Pittman, 2001 & & & 7.0 & 15.0 & & & 10.0 & & & & & 6.5 & & & 7.0 & 8.0 & & & & & & & & & 36.0 & 1.5 & & 5.0 & & & & & 4.0 & & \\
\hline Pittman, 2001 & & & 7.0 & 15.0 & & & 10.0 & 5.0 & & & & 4.0 & & & 7.0 & 8.0 & & & & & & & & & 36.0 & 1.5 & & 2.5 & & & & & 4.0 & & \\
\hline Pittman, 2001 & & & 7.0 & 6.0 & & & 15.0 & 5.0 & & & & 6.5 & & & 4.5 & 8.0 & & & 2.5 & & & & & & 36.0 & 0.5 & & 5.0 & & & & & 4.0 & & \\
\hline Pittman, 2001 & & & 7.0 & 6.0 & & & 15.0 & & & & & 6.5 & & & 7.0 & 8.0 & & & 2.5 & & & & & & 36.0 & 1.5 & & 2.5 & & & & & 8.0 & & \\
\hline Pittman, 2001 & & & 7.0 & 6.0 & & & 15.0 & 5.0 & & & & 4.0 & & & 7.0 & 8.0 & & & 2.5 & & & & & & 36.0 & 0.5 & & 5.0 & & & & & 4.0 & & \\
\hline Pittman, 2001 & & & 7.0 & 6.0 & & & 15.0 & & & & & 4.0 & & & 4.5 & 8.0 & & & & & & & & & 45.0 & 1.5 & & 5.0 & & & & & 4.0 & & \\
\hline Pittman, 2001 & & & 7.0 & 6.0 & & & 9.5 & 5.0 & & & & 6.5 & & & 4.5 & 8.0 & & & & & & & & & 46.5 & 0.5 & & 2.5 & & & & & 4.0 & & \\
\hline Pittman, 2001 & & & 7.0 & 6.0 & & & 9.5 & & & & & 6.5 & & & 7.0 & 13.0 & & & 2.5 & & & & & & 41.5 & 0.5 & & 2.5 & & & & & 4.0 & & \\
\hline Pittman, 2001 & & & 7.0 & 6.0 & & & 9.5 & 5.0 & & & & 4.0 & & & 4.5 & 13.0 & & & & & & & & & 36.5 & 1.5 & & 5.0 & & & & & 8.0 & & \\
\hline Pittman, 2001 & & & 7.0 & 6.0 & & & 9.5 & 5.0 & & & & 4.0 & & & 7.0 & 13.0 & & & & & & & & & 37.5 & 0.5 & & 2.5 & & & & & 8.0 & & \\
\hline Pittman, 2001 & & & 12.5 & 6.0 & & & 15.0 & 1.0 & & & & 4.0 & & & 4.5 & 13.0 & & & & & & & & & 36.0 & 1.5 & & 2.5 & & & & & 4.0 & & \\
\hline Pittman, 2001 & & & 7.0 & 6.0 & & & 10.0 & & & & & 4.0 & & & 4.5 & 8.0 & & & & & & & & & 47.0 & 0.5 & & 5.0 & & & & & 8.0 & & \\
\hline Pittman, 2001 & & & 7.0 & 14.5 & & & 15.0 & & & & & 4.0 & & & 4.5 & 8.0 & & & & & & & & & 36.0 & 0.5 & & 2.5 & & & & & 8.0 & & \\
\hline Pittman, 2001 & & & 7.0 & 6.5 & & & 9.5 & & & & & 6.5 & & & 7.0 & 13.0 & & & & & & & & & 36.0 & 1.5 & & 5.0 & & & & & 8.0 & & \\
\hline Pittman, 2001 & & & 12.0 & 6.0 & & & 15.0 & & & & & 6.5 & & & 4.5 & 13.0 & & & & & & & & & 36.0 & 0.5 & & 2.5 & & & & & 4.0 & & \\
\hline Pittman, 2001 & & & 8.0 & 6.0 & & & 9.5 & & & & & 4.0 & & & 7.0 & 8.0 & & & 2.5 & & & & & & 47.0 & 1.5 & & 2.5 & & & & & 4.0 & & \\
\hline Pittman, 2001 & & & 12.5 & 6.0 & & & 9.5 & 5.0 & & & & 6.5 & & & 7.0 & 8.0 & & & & & & & & & 37.5 & 1.5 & & 2.5 & & & & & 4.0 & & \\
\hline Pittman, 2001 & & & 12.5 & 6.0 & & & 9.5 & & & & & 6.5 & & & 4.5 & 8.0 & & & 2.5 & & & & & & 36.0 & 1.5 & & 5.0 & & & & & 8.0 & & \\
\hline
\end{tabular}


Table A1. Glass Compositions from Literature Review (continued)

\begin{tabular}{|c|c|c|c|c|c|c|c|c|c|c|c|c|c|c|c|c|c|c|c|c|c|c|c|c|c|c|c|c|c|c|c|c|c|c|c|}
\hline Ref & 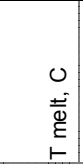 & $\begin{array}{l}0 \\
\text { a } \\
\stackrel{0}{0} \\
\stackrel{0}{=} \\
\vdash\end{array}$ & $\begin{array}{l}\text { Oొ } \\
\frac{\mathbb{1}}{\alpha}\end{array}$ & ్ֻలె & $\begin{array}{c}\stackrel{\bigcirc}{\mathbb{D}} \\
\stackrel{\infty}{2}\end{array}$ & 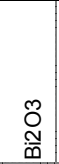 & $\stackrel{0}{0}$ & $\begin{array}{l}\text { N } \\
\mathcal{D} \\
0 \\
\tilde{N} \\
\mathcal{N} \\
\mathcal{S}\end{array}$ & $\begin{array}{l}\text { Oొ } \\
\text { రิ } \\
0 \\
0 \\
8\end{array}$ & 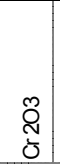 & $\stackrel{\circ}{3}$ & 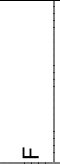 & 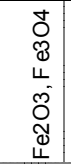 & 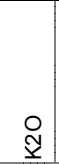 & ণ্ & $\begin{array}{l}\circ \\
\sum \\
\Sigma\end{array}$ & $\begin{array}{l}\tilde{O} \\
\sum \\
\Sigma\end{array}$ & $\begin{array}{l}0 \\
\stackrel{0}{2} \\
\stackrel{2}{2}\end{array}$ & $\begin{array}{l}\stackrel{O}{\mathbb{n}} \\
\mathbb{\mathbb { N }}\end{array}$ & $\stackrel{\circ}{z}$ & ָ̃ & 吾 & $\begin{array}{l}\text { 怘 } \\
\text { న }\end{array}$ & $\begin{array}{l}\circ \\
\frac{0}{0}\end{array}$ & ก๊ & ర్ల & 임 & $\begin{array}{l}\infty \\
\mathbb{8} \\
. \bar{x} \\
0 \\
\text { 山్ }\end{array}$ & 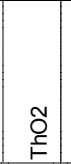 & $\stackrel{\widetilde{N}}{\stackrel{N}{L}}$ & $\begin{array}{l}\infty \\
\text { ల } \\
\text { ગ } \\
\text { N } \\
\text { อ }\end{array}$ & ํ & ั๊ & 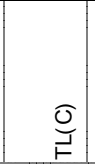 & $\begin{array}{l}\bar{\partial} \\
\frac{0}{0} \\
\frac{1}{z}\end{array}$ \\
\hline Pittman, 2001 & & & 12.5 & 6.0 & & & 9.5 & 5.0 & & & & 4.0 & & & 4.5 & 8.0 & & & 2.5 & & & & & & 36.0 & 1.5 & & 2.5 & & & & & 8.0 & & \\
\hline Pittman, 2001 & & & 12.5 & 6.0 & & & 9.5 & & & & & 4.0 & & & 7.0 & 13.0 & & & 2.5 & & & & & & 36.0 & 0.5 & & 5.0 & & & & & 4.0 & & \\
\hline Pittman, 2001 & & & 12.5 & 13.5 & & & 9.5 & & & & & 4.0 & & & 7.0 & 8.0 & & & & & & & & & 36.0 & 0.5 & & 5.0 & & & & & 4.0 & & \\
\hline Pittman, 2001 & & & 7.0 & 15.0 & & & 9.5 & & & & & 6.5 & & & 4.5 & 8.0 & & & 2.5 & & & & & & 36.0 & 0.5 & & 2.5 & & & & & 8.0 & & \\
\hline Pittman, 2001 & & & 7.0 & 15.0 & & & 9.5 & & & & & 4.0 & & & 4.5 & 13.0 & & & 2.5 & & & & & & 36.5 & 1.5 & & 2.5 & & & & & 4.0 & & \\
\hline Pittman, 2001 & & & 9.0 & 9.0 & & & 12.0 & 2.5 & & & & 5.0 & & & 5.0 & 10.5 & & & 1.5 & & & & & & 38.8 & 1.0 & & & & & & & 5.0 & & \\
\hline Pittman, 2001 & & & 8.6 & 5.1 & & & 11.3 & 3.0 & & & & 4.7 & & & 6.0 & 11.4 & & & 1.2 & & & & & & 42.3 & 1.1 & & & & & & & 4.7 & & \\
\hline Pittman, 2001 & & & 8.9 & 8.2 & & & 11.4 & 1.8 & & & & 5.1 & & & 5.6 & 9.8 & & & 1.1 & & & & & & 38.0 & 1.0 & & 3.6 & & & & & 5.6 & & \\
\hline Plodinec, 1978 & & 1280 & 21.6 & 7.5 & & & 3.9 & & & & & & 1.5 & & & & 1.2 & & 16.9 & 0.2 & & & & & 39.4 & & & & & 7.5 & 0.4 & & & & \\
\hline Plodinec, 1978 & & 1150 & 11.6 & 7.5 & & & 4.6 & & & & & & 7.9 & & & & 2.6 & & 16.9 & 0.6 & & & & & 39.4 & & & & & 7.5 & 1.5 & & & & \\
\hline Plodinec, 1978 & & 1094 & 9.0 & 6.5 & & & 4.3 & & & & & & 15.2 & & & & 4.1 & & 14.6 & 1.8 & & & & & 34.1 & & & & & 6.5 & 3.9 & & & & \\
\hline Plodinec, 1978 & & 1020 & 8.6 & 9.0 & & & 4.5 & & & & & & 0.6 & & 3.6 & & 0.5 & & 16.7 & 0.1 & & & & & 47.3 & & & & & 9.0 & 0.2 & & & & \\
\hline Plodinec, 1978 & & 1104 & 12.9 & 8.5 & & & 4.3 & & & & & & 0.9 & & 3.4 & & 0.7 & & 15.7 & 0.1 & & & & & 44.6 & & & & & 8.5 & 0.2 & & & & \\
\hline Plodinec, 1978 & & 1134 & 17.3 & 8.0 & & & 4.1 & & & & & & 1.2 & & 3.2 & & 1.0 & & 14.8 & 0.2 & & & & & 42.0 & & & & & 8.0 & 0.3 & & & & \\
\hline Plodinec, 1978 & & 1027 & 9.3 & 8.0 & & & 4.7 & & & & & & 6.3 & & 3.2 & & 2.1 & & 14.8 & 0.5 & & & & & 42.0 & & & & & 8.0 & 1.2 & & & & \\
\hline Plodinec, 1978 & & 1157 & 21.6 & 7.5 & & & 3.9 & & & & & & 1.5 & & 3.0 & & 1.2 & & 13.9 & 0.2 & & & & & 39.4 & & & & & 7.5 & 0.4 & & & & \\
\hline Plodinec, 1978 & & 1036 & 11.6 & 7.5 & & & 4.6 & & & & & & 7.9 & & 3.0 & & 2.6 & & 13.9 & 0.6 & & & & & 39.4 & & & & & 7.5 & 1.5 & & & & \\
\hline Plodinec, 1978 & & 1004 & 7.7 & 7.0 & & & 4.4 & & & & & & 13.1 & & 2.8 & & 3.5 & & 13.0 & 1.5 & & & & & 36.8 & & & & & 7.0 & 3.3 & & & & \\
\hline Plodinec, 1978 & & 1180 & 13.9 & 7.0 & & & 4.5 & & & & & & 9.5 & & 2.8 & & 3.1 & & 13.0 & 0.7 & & & & & 36.8 & & & & & 7.0 & 1.8 & & & & \\
\hline Plodinec, 1978 & & & 30.2 & 6.5 & & & 3.4 & & & & & & 2.1 & & 2.6 & & 1.7 & & 12.0 & 0.3 & & & & & 34.1 & & & & & 6.5 & 0.5 & & & & \\
\hline Prunchak, 1997 & & & & 7.8 & & 63.4 & & & & & & & & 0.9 & & & & & 1.2 & & & & & & 19.8 & & & & & & & 5.0 & 2.0 & & \\
\hline Prunchak, 1997 & & & & 6.8 & & 61.0 & & & & & & & & 0.9 & & & & & 0.9 & & & & & & 20.9 & & & & & & & 7.6 & 1.9 & & \\
\hline Prunchak, 1997 & & & & 8.6 & & 63.1 & & & & & & 1.2 & & 0.9 & & & & & 1.3 & & & & & & 18.6 & & & & & & & 4.9 & 1.3 & & \\
\hline Pye, 1985 & & 1055 & 9.3 & 7.4 & & & 1.1 & & & & & & 6.4 & & 5.1 & 0.8 & 2.2 & & 11.8 & 0.7 & & & & & 54.3 & & & & & & & & 1.0 & & \\
\hline Quang, 2003 & 1200 & & 6.2 & 13.0 & & & 5.7 & & & & & & & & & & & & 8.8 & & 12.0 & 2.0 & 3.7 & & 36.0 & & & & & & & 5.6 & 7.1 & & \\
\hline Radchenko, 2001 & $<1250$ & & 8.2 & 32.5 & & & 3.9 & & & & & & 5.0 & 0.8 & & 1.9 & 0.1 & & 11.6 & & & 0.5 & & & 35.5 & & & & & & & & & & \\
\hline Radchenko, 2001 & $<1250$ & & 12.2 & 5.0 & & & 10.8 & & & & & & 7.5 & 1.2 & & 2.9 & 0.1 & & 12.3 & & & 0.8 & & & 47.2 & & & & & & & & & & \\
\hline Rapp, 1985 & & 1434 & 12.4 & & & & 16.6 & & & & & 0.1 & 11.7 & 0.9 & & 4.5 & 0.2 & & 2.6 & & & & 0.3 & & 48.4 & 0.1 & & & & 1.9 & & & & 1216 & \\
\hline Rapp, 1985 & & 1337 & 11.2 & & & & 24.8 & & & & & 0.1 & 10.5 & 0.8 & & 4.0 & 0.2 & & 2.4 & & & & 0.3 & & 43.6 & 0.1 & & & & 1.7 & & & & 1251 & \\
\hline Rapp, 1985 & & 1413 & 18.6 & & & & 19.6 & & & & & 0.1 & 10.2 & 0.8 & & 3.9 & 0.2 & & 2.3 & & & & 0.3 & & 42.1 & 0.1 & & & & 1.6 & & & & 1309 & \\
\hline Rapp, 1985 & & & 11.5 & & & & 16.3 & & & & & 0.1 & 10.8 & 0.8 & & 10.7 & 0.2 & & 2.4 & & & & 0.3 & & 44.7 & 0.1 & & & & 1.7 & & & & 1253 & \\
\hline Rapp, 1985 & & 1259 & 9.9 & & & & 33.2 & & & & & 0.1 & 9.4 & 0.7 & & 3.6 & 0.2 & & 2.1 & & & & 0.3 & & 38.7 & 0.1 & & & & 1.5 & & & & 1288 & \\
\hline Rapp, 1985 & & & 11.2 & & & & 21.9 & & & & & 0.1 & 10.5 & 0.8 & & 6.2 & 0.2 & & 2.4 & & & & 0.3 & & 44.4 & 0.1 & & & & 1.7 & & & & 1254 & \\
\hline Rapp, 1985 & & & 11.5 & & & & 16.6 & & & & & 0.1 & 10.8 & 0.8 & & 10.4 & 0.2 & & 2.4 & & & & 0.3 & & 44.7 & 0.1 & & & & 1.7 & & & & 1285 & \\
\hline Rapp, 1985 & & & 12.0 & & & & 16.3 & & & & & 0.1 & 11.3 & 0.9 & & 4.3 & 0.2 & & 2.5 & & & & 0.3 & & 46.8 & 0.1 & & & & 4.9 & & & & 1243 & \\
\hline Rapp, 1985 & & 1455 & 25.4 & & & & 14.9 & & & & & 0.1 & 9.8 & 0.7 & & 3.8 & 0.2 & & 2.2 & & & & 0.3 & & 40.7 & 0.1 & & & & 1.6 & & & & 1380 & \\
\hline Rapp, 1985 & & 1560 & 13.6 & & & & 8.6 & & & & & 0.1 & 12.8 & 1.0 & & 4.9 & 0.2 & & 2.9 & & & & 0.3 & & 53.0 & 0.1 & & & & 2.1 & & & & 1271 & \\
\hline Reimus, 1988 & & & 10.0 & 10.7 & 0.1 & & 0.6 & 0.1 & & 0.2 & & & 12.2 & 2.4 & 2.6 & 1.3 & 1.3 & 0.0 & 9.6 & 0.3 & 0.0 & 0.3 & 2.5 & & 40.0 & 0.3 & 0.0 & 0.1 & 3.6 & 1.0 & 0.6 & & 0.3 & & \\
\hline Reimus, 1988 & & & 10.0 & 10.7 & 0.1 & & 0.6 & 0.1 & & 0.2 & & & 12.2 & 2.4 & 2.6 & 1.3 & 1.3 & 0.0 & 9.6 & 0.3 & 0.0 & 0.3 & 2.5 & & 40.0 & 0.3 & 0.0 & 0.1 & 3.6 & 1.0 & 0.6 & & 0.3 & & \\
\hline Reimus, 1988 & & & 10.0 & 10.7 & 0.1 & & 0.6 & 0.1 & & 0.2 & & & 12.2 & 2.4 & 2.6 & 1.3 & 1.3 & 0.0 & 9.6 & 0.3 & 0.0 & 0.3 & 2.5 & & 40.0 & 0.3 & 0.0 & 0.1 & 3.6 & 1.0 & 0.6 & & 0.3 & & \\
\hline Reimus, 1988 & & & 10.0 & 10.7 & 0.1 & & 0.6 & 0.1 & & 0.2 & & & 12.2 & 2.4 & 2.6 & 1.3 & 1.3 & 0.0 & 9.6 & 0.3 & 0.0 & 0.3 & 2.5 & & 40.0 & 0.3 & 0.0 & 0.1 & 3.6 & 1.0 & 0.6 & & 0.3 & & \\
\hline Reimus, 1988 & & & 10.0 & 10.7 & 0.1 & & 0.6 & 0.1 & & 0.2 & & & 12.2 & 2.4 & 2.6 & 1.3 & 1.3 & 0.0 & 9.6 & 0.3 & 0.0 & 0.3 & 2.5 & & 40.0 & 0.3 & 0.0 & 0.1 & 3.6 & 1.0 & 0.6 & & 0.3 & & \\
\hline Reimus, 1988 & & & 10.0 & 10.7 & 0.1 & & 0.6 & 0.1 & & 0.2 & & & 12.2 & 2.4 & 2.6 & 1.3 & 1.3 & 0.0 & 9.6 & 0.3 & 0.0 & 0.3 & 2.5 & & 40.0 & 0.3 & 0.0 & 0.1 & 3.6 & 1.0 & 0.6 & & 0.3 & & \\
\hline Reimus, 1988 & & & 10.0 & 10.7 & 0.1 & & 0.6 & 0.1 & & 0.2 & & & 12.2 & 2.4 & 2.6 & 1.3 & 1.3 & 0.0 & 9.6 & 0.3 & 0.0 & 0.3 & 2.5 & & 40.0 & 0.3 & 0.0 & 0.1 & 3.6 & 1.0 & 0.6 & & 0.3 & & \\
\hline Reimus, 1988 & & & 10.0 & 10.7 & 0.1 & & 0.6 & 0.1 & & 0.2 & & & 12.2 & 2.4 & 2.6 & 1.3 & 1.3 & 0.0 & 9.6 & 0.3 & 0.0 & 0.3 & 2.5 & & 40.0 & 0.3 & 0.0 & 0.1 & 3.6 & 1.0 & 0.6 & & 0.3 & & \\
\hline Reimus, 1988 & & & 10.0 & 10.7 & 0.1 & & 0.6 & 0.1 & & 0.2 & & & 12.2 & 2.4 & 2.6 & 1.3 & 1.3 & 0.0 & 9.6 & 0.3 & 0.0 & 0.3 & 2.5 & & 40.0 & 0.3 & 0.0 & 0.1 & 3.6 & 1.0 & 0.6 & & 0.3 & & \\
\hline Reimus, 1988 & & & 10.0 & 10.7 & 0.1 & & 0.6 & 0.1 & & 0.2 & & & 12.2 & 2.4 & 2.6 & 1.3 & 1.3 & 0.0 & 9.6 & 0.3 & 0.0 & 0.3 & 2.5 & & 40.0 & 0.3 & 0.0 & 0.1 & 3.6 & 1.0 & 0.6 & & 0.3 & & \\
\hline Reimus, 1988 & & & 10.0 & 10.7 & 0.1 & & 0.6 & 0.1 & & 0.2 & & & 12.2 & 2.4 & 2.6 & 1.3 & 1.3 & 0.0 & 9.6 & 0.3 & 0.0 & 0.3 & 2.5 & & 40.0 & 0.3 & 0.0 & 0.1 & 3.6 & 1.0 & 0.6 & & 0.3 & & \\
\hline Reimus, 1988 & & & 10.6 & 10.8 & 0.0 & & 0.6 & 0.1 & & 0.2 & & & 11.9 & 2.3 & 2.6 & 1.3 & 1.3 & 0.0 & 9.1 & 0.3 & 0.0 & 0.3 & 2.5 & & 41.2 & 0.3 & 0.0 & 0.1 & 2.5 & 0.9 & 0.5 & & 0.5 & & \\
\hline Reimus, 1988 & & & 10.0 & 10.7 & 0.1 & & 0.6 & 0.1 & & 0.2 & & & 12.2 & 2.4 & 2.6 & 1.3 & 1.3 & 0.0 & 9.6 & 0.3 & 0.0 & 0.3 & 2.5 & & 40.0 & 0.3 & 0.0 & 0.1 & 3.6 & 1.0 & 0.6 & & 0.3 & & \\
\hline
\end{tabular}


ORP-56297 Rev. 0

The Catholic University of America

High Level Waste Vitrification System Improvements Vitreous State Laboratory

Table A1. Glass Compositions from Literature Review (continued)

\begin{tabular}{|c|c|c|c|c|c|c|c|c|c|c|c|c|c|c|c|c|c|c|c|c|c|c|c|c|c|c|c|c|c|c|c|c|c|c|c|}
\hline Ref & 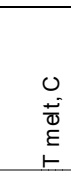 & $\begin{array}{l}0 \\
0 \\
8 \\
5 \\
⺊ \\
上\end{array}$ & $\frac{\text { NO }}{<}$ & ్ֻల్ల & $\begin{array}{l}\stackrel{0}{\mathbb{\Xi}} \\
\stackrel{\infty}{ }\end{array}$ & 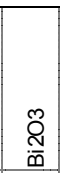 & $\begin{array}{l}8 \\
0 \\
0\end{array}$ & 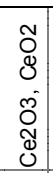 & $\begin{array}{l}\text { ల్ల } \\
0 \\
0 \\
8 \\
0 \\
0\end{array}$ & ญ్రి & $\begin{array}{l}9 \\
0\end{array}$ & 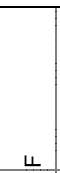 & 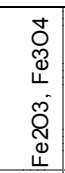 & $\begin{array}{l}\stackrel{\Upsilon}{Y} \\
\check{Y}\end{array}$ & $\stackrel{\stackrel{ }{J}}{ }$ & $\stackrel{8}{\Sigma}$ & $\begin{array}{l}\tilde{O} \\
\text { E } \\
\Sigma\end{array}$ & $\begin{array}{l}0 \\
\text { O̊ } \\
\Sigma\end{array}$ & $\begin{array}{l}\text { ㅇ } \\
\underset{\pi}{Z}\end{array}$ & $\frac{0}{z}$ & 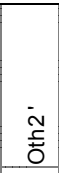 & 究 & 足 & $\begin{array}{l}\circ \\
\stackrel{0}{2}\end{array}$ & $\begin{array}{l}\text { N } \\
\text { के }\end{array}$ & రొ & $\begin{array}{l}\text { O } \\
\text { क் }\end{array}$ & 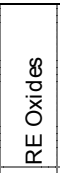 & $\begin{array}{l}\tilde{N} \\
\stackrel{\Gamma}{F}\end{array}$ & $\stackrel{N}{\text { Oิ }}$ & $\begin{array}{l}\infty \\
0 \\
\text { ల } \\
د \\
\tilde{~} \\
\text { ป }\end{array}$ & $\begin{array}{l}0 \\
\tilde{N}\end{array}$ & กั & $\underset{\vec{J}}{\widehat{O}}$ & $\begin{array}{l}\overline{\vec{J}} \\
\frac{\partial}{\underline{m}} \\
\frac{\vec{z}}{z}\end{array}$ \\
\hline Reimus, 1988 & & & 10.0 & 10.7 & 0.1 & & 0.6 & 0.1 & & 0.2 & & & 12.2 & 2.4 & 2.6 & 1.3 & 1.3 & 0.0 & 9.6 & 0.3 & 0.0 & 0.3 & 2.5 & & 40.0 & 0.3 & 0.0 & 0.1 & 3.6 & 1.0 & 0.6 & & 0.3 & & \\
\hline Reimus, 1988 & & & 10.0 & 10.7 & 0.1 & & 0.6 & 0.1 & & 0.2 & & & 12.2 & 2.4 & 2.6 & 1.3 & 1.3 & 0.0 & 9.6 & 0.3 & 0.0 & 0.3 & 2.5 & & 40.0 & 0.3 & 0.0 & 0.1 & 3.6 & 1.0 & 0.6 & & 0.3 & & \\
\hline Reimus, 1988 & & & 10.0 & 10.7 & 0.1 & & 0.6 & 0.1 & & 0.2 & & & 12.2 & 2.4 & 2.6 & 1.3 & 1.3 & 0.0 & 9.6 & 0.3 & 0.0 & 0.3 & 2.5 & & 40.0 & 0.3 & 0.0 & 0.1 & 3.6 & 1.0 & 0.6 & & 0.3 & & \\
\hline Reimus, 1988 & & & 10.3 & 10.9 & 0.1 & & 0.6 & 0.1 & & 0.2 & & & 11.7 & 2.3 & 2.6 & 1.3 & 1.3 & 0.0 & 9.2 & 0.3 & 0.0 & 0.3 & 2.5 & & 41.4 & 0.3 & 0.0 & 0.1 & 2.5 & 0.9 & 0.5 & & 0.5 & & \\
\hline Reimus, 1988 & & & 10.0 & 10.7 & 0.1 & & 0.6 & 0.1 & & 0.2 & & & 12.2 & 2.4 & 2.6 & 1.3 & 1.3 & 0.0 & 9.6 & 0.3 & 0.0 & 0.3 & 2.5 & & 40.0 & 0.3 & 0.0 & 0.1 & 3.6 & 1.0 & 0.6 & & 0.3 & & \\
\hline Reimus, 1988 & & & 10.4 & 10.8 & 0.0 & & 0.7 & 0.1 & & 0.2 & & & 11.8 & 2.4 & 2.6 & 1.3 & 1.4 & 0.0 & 9.2 & 0.3 & 0.0 & 0.3 & 2.5 & & 41.3 & 0.3 & 0.0 & 0.1 & 2.5 & 0.9 & 0.5 & & 0.5 & & \\
\hline Reimus, 1988 & & & 10.0 & 10.7 & 0.1 & & 0.6 & 0.1 & & 0.2 & & & 12.2 & 2.4 & 2.6 & 1.3 & 1.3 & 0.0 & 9.6 & 0.3 & 0.0 & 0.3 & 2.5 & & 40.0 & 0.3 & 0.0 & 0.1 & 3.6 & 1.0 & 0.6 & & 0.3 & & \\
\hline Reimus, 1988 & & & 10.4 & 10.9 & 0.1 & & 0.6 & 0.1 & & 0.2 & & & 11.8 & 2.2 & 2.5 & 1.3 & 1.4 & 0.0 & 9.3 & 0.3 & 0.0 & 0.3 & 2.5 & & 41.4 & 0.3 & 0.0 & 0.1 & 2.5 & 0.9 & 0.5 & & 0.4 & & \\
\hline Reimus, 1988 & & & 10.0 & 10.7 & 0.1 & & 0.6 & 0.1 & & 0.2 & & & 12.2 & 2.4 & 2.6 & 1.3 & 1.3 & 0.0 & 9.6 & 0.3 & 0.0 & 0.3 & 2.5 & & 40.0 & 0.3 & 0.0 & 0.1 & 3.6 & 1.0 & 0.6 & & 0.3 & & \\
\hline Reimus, 1988 & & & 10.3 & 11.0 & 0.1 & & 0.6 & 0.1 & & 0.2 & & & 11.6 & 2.4 & 2.6 & 1.3 & 1.3 & 0.0 & 9.2 & 0.3 & 0.0 & 0.3 & 2.5 & & 41.2 & 0.3 & 0.0 & 0.1 & 2.6 & 0.9 & 0.5 & & 0.5 & & \\
\hline Reimus, 1988 & & & 10.0 & 10.7 & 0.1 & & 0.6 & 0.1 & & 0.2 & & & 12.2 & 2.4 & 2.6 & 1.3 & 1.3 & 0.0 & 9.6 & 0.3 & 0.0 & 0.3 & 2.5 & & 40.0 & 0.3 & 0.0 & 0.1 & 3.6 & 1.0 & 0.6 & & 0.3 & & \\
\hline Reimus, 1988 & & & 10.4 & 10.9 & 0.1 & & 0.6 & 0.1 & & 0.2 & & & 11.7 & 2.2 & 2.6 & 1.3 & 1.4 & 0.0 & 9.1 & 0.3 & 0.0 & 0.3 & 2.6 & & 41.4 & 0.3 & 0.0 & 0.1 & 2.6 & 0.9 & 0.5 & & 0.5 & & \\
\hline Reimus, 1988 & & & 10.0 & 10.7 & 0.1 & & 0.6 & 0.1 & & 0.2 & & & 12.2 & 2.4 & 2.6 & 1.3 & 1.3 & 0.0 & 9.6 & 0.3 & 0.0 & 0.3 & 2.5 & & 40.0 & 0.3 & 0.0 & 0.1 & 3.6 & 1.0 & 0.6 & & 0.3 & & \\
\hline Reimus, 1988 & & & 10.0 & 10.7 & 0.1 & & 0.6 & 0.1 & & 0.2 & & & 12.2 & 2.4 & 2.6 & 1.3 & 1.3 & 0.0 & 9.6 & 0.3 & 0.0 & 0.3 & 2.5 & & 40.0 & 0.3 & 0.0 & 0.1 & 3.6 & 1.0 & 0.6 & & 0.3 & & \\
\hline Reimus, 1988 & & & 10.0 & 10.7 & 0.1 & & 0.6 & 0.1 & & 0.2 & & & 12.2 & 2.4 & 2.6 & 1.3 & 1.3 & 0.0 & 9.6 & 0.3 & 0.0 & 0.3 & 2.5 & & 40.0 & 0.3 & 0.0 & 0.1 & 3.6 & 1.0 & 0.6 & & 0.3 & & \\
\hline Reimus, 1988 & & & 11.3 & 10.7 & 0.1 & & 0.6 & 0.1 & & & 0.0 & 0.5 & 11.5 & 2.8 & 2.6 & 1.3 & 1.2 & 0.0 & 9.0 & & 0.0 & 0.3 & 2.6 & & 40.6 & 0.3 & & 0.1 & & 3.0 & 1.0 & 0.0 & & & \\
\hline \begin{tabular}{|l|} 
Reinherz, 1990 \\
\end{tabular} & 1205 & 2023 & & 13.7 & & 41.9 & & & & & & & & & & & & & 6.4 & & & & & & 30.6 & & & & & & & 3.0 & 1.4 & & \\
\hline \begin{tabular}{|l|} 
Reinherz, 1990 \\
\end{tabular} & 1205 & & & 13.7 & & 41.9 & & & & & & & & & & & & & 6.4 & & & 3.0 & & & 30.6 & & & & & & & 3.0 & 1.4 & & \\
\hline \begin{tabular}{|l|} 
Reinherz, 1990 \\
\end{tabular} & 1205 & & & 13.4 & & 41.0 & & & & & & & & & & & & & 6.2 & & & & & & 29.8 & & & & & 2.6 & & 2.9 & 1.0 & & \\
\hline \begin{tabular}{|l|} 
Reinherz, 1990 \\
\end{tabular} & 1205 & & & 13.4 & & 41.0 & & & & & & & & & & & & & 6.2 & & & 3.0 & & & 29.8 & & & & & 2.6 & & 2.9 & 1.0 & & \\
\hline \begin{tabular}{|l|} 
Reinherz, 1990 \\
\end{tabular} & 1260 & & & 13.0 & & 37.5 & & & & & & & & & & & & & 7.6 & & & & & & 32.0 & & & & & 2.2 & & 3.1 & 1.5 & & \\
\hline \begin{tabular}{|l|} 
Reinherz, 1990 \\
\end{tabular} & 1260 & & & 13.0 & & 37.5 & & & & & & & & & & & & & 7.6 & & & 3.2 & & & 32.0 & & & & & 2.2 & & 3.1 & 1.5 & & \\
\hline \begin{tabular}{|l|} 
Reinherz, 1990 \\
\end{tabular} & 1205 & & & 18.7 & & 35.0 & & & & & & & & & & & & & 7.1 & & & & & & 29.9 & & & & & 2.0 & & 2.9 & 1.4 & & \\
\hline Reinherz, 1990 & 1205 & & & 18.7 & & 35.0 & & & & & & & & & & & & & 7.1 & & & 3.0 & & & 29.9 & & & & & 2.0 & & 2.9 & 1.4 & & \\
\hline Reinherz, 1990 & 1205 & & & 17.7 & & 38.6 & & & & & & & & & & & & & 6.7 & & & & & & 28.1 & & & & & 2.2 & & 2.8 & 1.1 & & \\
\hline Reinherz, 1990 & 1205 & & & 17.7 & & 38.6 & & & & & & & & & & & & & 6.7 & & & 2.8 & & & 28.1 & & & & & 2.2 & & 2.8 & 1.1 & & \\
\hline Reinherz, 1990 & 1175 & & & 15.7 & & 41.8 & & & & & & & & & & & & & 5.5 & & & & & & 30.3 & & & & & 2.7 & & 3.0 & 1.0 & & \\
\hline \begin{tabular}{|l|} 
Reinherz, 1990 \\
\end{tabular} & 1175 & & & 15.7 & & 41.8 & & & & & & & & & & & & & 5.5 & & & & & & 30.3 & & & & & 2.7 & & 3.0 & 1.0 & & \\
\hline \begin{tabular}{|l|} 
Reinherz, 1990 \\
\end{tabular} & 1175 & & & 15.5 & & 41.0 & & & & & & & & & & & & & 7.1 & & & & & & 29.8 & & & & & 2.6 & & & 1.0 & & \\
\hline \begin{tabular}{|l|} 
Reinherz, 1990 \\
\end{tabular} & 1175 & & & 15.5 & & 41.0 & & & & & & & & & & & & & 7.1 & & & 3.0 & & & 29.8 & & & & & 2.6 & & & 1.0 & & \\
\hline Reinherz, 1990 & 1260 & & & 15.5 & & 35.2 & & & & & & & & & & & & & 7.1 & & & & & & 30.0 & & & & & 2.1 & & 5.9 & 1.4 & & \\
\hline Reinherz, 1990 & 1260 & & & 15.5 & & 35.2 & & & & & & & & & & & & & 7.1 & & & 3.0 & & & 30.0 & & & & & 2.1 & & 5.9 & 1.4 & & \\
\hline \begin{tabular}{|l|} 
Reinherz, 1990 \\
\end{tabular} & 1205 & & & 17.2 & & 37.6 & & & & & & & & & & & & & 6.5 & & & & & & 27.5 & & & & & 1.9 & & 5.4 & 1.3 & & \\
\hline \begin{tabular}{|l|} 
Reinherz, 1990 \\
\end{tabular} & 1205 & & & 17.2 & & 37.6 & & & & & & & & & & & & & 6.5 & & & 2.7 & & & 27.5 & & & & & 1.9 & & 5.4 & 1.3 & & \\
\hline \begin{tabular}{|l|} 
Reinherz, 1990 \\
\end{tabular} & 1205 & & & 17.9 & & 33.5 & & & & & & & & & & & & & 6.8 & & & & & & 33.9 & & & & & 1.4 & & 2.8 & 0.9 & & \\
\hline Reinherz, 1990 & 1205 & & & 17.9 & & 33.5 & & & & & & & & & & & & & 6.8 & & & 2.8 & & & 33.9 & & & & & 1.4 & & 2.8 & 0.9 & & \\
\hline Reinherz, 1990 & 1205 & & & 16.4 & & 37.5 & & & & & & & & & & & & & 6.8 & & & & & & 27.4 & & & & & 1.9 & & 2.7 & 1.3 & & \\
\hline Reinherz, 1990 & 1205 & & & 16.4 & & 37.5 & & & & & & & & & & & & & 6.8 & & & 6.1 & & & 27.4 & & & & & 1.9 & & 2.7 & 1.3 & & \\
\hline Reinherz, 1990 & 1175 & & & 15.2 & & 40.4 & & & & & & & & & & & & & 7.0 & & & & & & 28.9 & & & & & 2.6 & & 2.9 & & & \\
\hline Reinherz, 1990 & 1175 & & & 15.2 & & 40.4 & & & & & & & & & & & & & 7.0 & & & 2.9 & & & 28.9 & & & & & 2.6 & & 2.9 & & & \\
\hline Reinherz, 1990 & 1205 & & & 19.8 & & 35.7 & & & & & & & & & & & & & 7.8 & & & & & & 26.1 & & & & & 1.8 & & 5.1 & 1.2 & & \\
\hline Reinherz, 1990 & 1205 & & & 19.8 & & 35.7 & & & & & & & & & & & & & 7.8 & & & 2.6 & & & 26.1 & & & & & 1.8 & & 5.1 & 1.2 & & \\
\hline \begin{tabular}{|l|} 
Reinherz, 1990 \\
\end{tabular} & 1205 & & & 18.9 & & 39.3 & & & & & & & & & & & & & 6.8 & & & & & & 28.6 & & & & & 2.5 & & & 0.9 & & \\
\hline Reinherz, 1990 & 1205 & & & 18.9 & & 39.3 & & & & & & & & & & & & & 6.8 & & & 2.9 & & & 28.6 & & & & & 2.5 & & & 0.9 & & \\
\hline \begin{tabular}{|l|} 
Reinherz, 1990 \\
\end{tabular} & 1205 & & & 20.8 & & 38.7 & & 1.1 & & & & & & & & & & & 5.1 & & & & & & 28.1 & & & & & 2.5 & & 2.8 & 0.9 & & \\
\hline Reinherz, 1990 & 1205 & & & 20.8 & & 38.7 & & 1.1 & & & & & & & & & & & 5.1 & & & & & & 28.1 & & & & & 2.5 & & 2.8 & 0.9 & & \\
\hline \begin{tabular}{|l|} 
Reinherz, 1990 \\
\end{tabular} & 1175 & & & 15.4 & & 40.9 & & & & & & & & & & & & & 7.1 & & & & & & 29.7 & & & & & & & 2.9 & 1.0 & & \\
\hline Reinherz, 1990 & 1175 & & & 15.4 & & 40.9 & & & & & & & & & & & & & 7.1 & & & 3.0 & & & 29.7 & & & & & & & 2.9 & 1.0 & & \\
\hline Reinherz, 1990 & 1260 & & & 19.5 & & 29.3 & & & & & & & & & & & & & 8.9 & & & & & & 30.0 & & & & & 2.1 & & 5.9 & 1.4 & & \\
\hline
\end{tabular}


Table A1. Glass Compositions from Literature Review (continued)

\begin{tabular}{|c|c|c|c|c|c|c|c|c|c|c|c|c|c|c|c|c|c|c|c|c|c|c|c|c|c|c|c|c|c|c|c|c|c|c|c|}
\hline Ref & 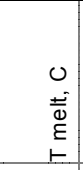 & $\begin{array}{l}0 \\
\stackrel{0}{\circ} \\
\stackrel{\circ}{\circ} \\
上\end{array}$ & 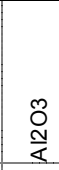 & 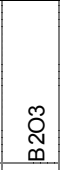 & $\begin{array}{l}\text { Q } \\
\infty\end{array}$ & 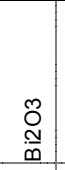 & $\begin{array}{l}0 \\
\pi \\
0\end{array}$ & 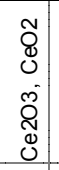 & $\begin{array}{l}0 \\
\text { Nิ } \\
0 \\
0 \\
0 \\
0 \\
0\end{array}$ & Oొ & Oे & 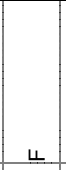 & 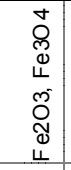 & $\begin{array}{l}\stackrel{\mathbb{N}}{x} \\
\end{array}$ & $\stackrel{\stackrel{ }{]}}{]}$ & $\begin{array}{l}0 \\
\text { D } \\
\Sigma\end{array}$ & $\begin{array}{l}\text { Õ } \\
\stackrel{\complement}{\Sigma}\end{array}$ & $\begin{array}{l}0 \\
0 \\
\Sigma\end{array}$ & $\begin{array}{l}2 \\
\text { J } \\
z\end{array}$ & $\frac{O}{z}$ & $\begin{array}{l}\text { N } \\
\text { ָै }\end{array}$ & $\begin{array}{l}\overline{\underline{z}} \\
\text { t }\end{array}$ & ฉి & $\frac{8}{2}$ & $\frac{\widetilde{O}}{\mathrm{O}}$ & $\begin{array}{l}0 \\
\text { on } \\
\end{array}$ & 高 & 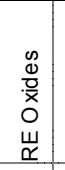 & $\begin{array}{l}\text { 实 } \\
\text { ⺊ }\end{array}$ & 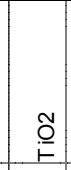 & 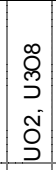 & $\stackrel{Q}{N}$ & $\stackrel{\frac{N}{N}}{N}$ & $\begin{array}{l}\frac{0}{3} \\
1\end{array}$ & $\begin{array}{l}\bar{\Xi} \\
\text { Ð్ } \\
\underline{\underline{m}} \\
\dot{z}\end{array}$ \\
\hline Reinherz, 1990 & 1260 & & & 19.5 & & 29.3 & & & & & & & & & & & & & 8.9 & & & 3.0 & & & 30.0 & & & & & 2.1 & & 5.9 & 1.4 & & \\
\hline Resce, 1995 & $<1350$ & & 14.4 & 5.9 & 4.3 & & 13.5 & & & & & & 4.5 & & & & & & 29.9 & 2.1 & & & & 3.22 & 22.1 & & & & & & & & & & \\
\hline Resce, 1995 & $<1350$ & & & 6.3 & 4.6 & & 14.4 & & & & & & 4.8 & & & & & & 31.7 & 2.2 & & & & 3.43 & 32.6 & & & & & & & & & & \\
\hline Resce, 1995 & $<1350$ & & 14.6 & & 4.4 & & 13.6 & & & & & & 4.6 & & & & & & 30.1 & 2.1 & & & & 3.22 & 27.5 & & & & & & & & & & \\
\hline Resce, 1995 & $<1350$ & & & & 4.7 & & 14.5 & & & & & & 4.9 & & & & & & 32.0 & 2.3 & & & & 3.43 & 38.3 & & & & & & & & & & \\
\hline Resce, 1995 & $<1350$ & & 14.7 & 6.0 & 4.4 & & 27.4 & & & & & & 4.6 & & & & & & 15.1 & 2.1 & & & & 3.22 & 22.5 & & & & & & & & & & \\
\hline Resce, 1995 & $<1350$ & & & 6.4 & 4.7 & & 29.2 & & & & & & 4.9 & & & & & & 16.1 & 2.3 & & & & 3.43 & 33.1 & & & & & & & & & & \\
\hline Resce, 1995 & $<1350$ & & 14.8 & & 4.4 & & 27.6 & & & & & & 4.6 & & & & & & 15.3 & 2.2 & & & & 3.22 & 27.9 & & & & & & & & & & \\
\hline Resce, 1995 & $<1350$ & & & & 4.7 & & 29.4 & & & & & & 4.9 & & & & & & 16.3 & 2.3 & & & & 3.43 & 38.9 & & & & & & & & & & \\
\hline Resce, 1995 & $<1350$ & & 14.4 & 8.8 & 4.3 & & 7.9 & & & & & & 4.5 & & & & & & 17.5 & 2.1 & & & & 3.1 & 37.3 & & & & & & & & & & \\
\hline Resce, 1995 & $<1350$ & & & 9.4 & 4.6 & & 8.4 & & & & & & 4.8 & & & & & & 18.6 & 2.2 & & & & 3.3 & 48.6 & & & & & & & & & & \\
\hline Resce, 1995 & $<1350$ & & 14.6 & & 4.4 & & 8.0 & & & & & & 4.6 & & & & & & 17.7 & 2.1 & & & & 3.2 & 45.5 & & & & & & & & & & \\
\hline Resce, 1995 & $<1350$ & & & & 4.7 & & 8.5 & & & & & & 4.9 & & & & & & 18.8 & 2.3 & & & & 3.4 & 57.5 & & & & & & & & & & \\
\hline Resce, 1995 & $<1350$ & & 14.5 & 8.9 & 4.4 & & 16.0 & & & & & & 4.5 & & & & & & 8.8 & 2.1 & & & & 3.2 & 37.6 & & & & & & & & & & \\
\hline Resce, 1995 & $<1350$ & & & 9.5 & 4.6 & & 17.0 & & & & & & 4.8 & & & & & & 9.4 & 2.3 & & & & 3.4 & 49.1 & & & & & & & & & & \\
\hline Resce, 1995 & $<1350$ & & 14.7 & & 4.4 & & 16.2 & & & & & & 4.6 & & & & & & 8.9 & 2.2 & & & & 3.2 & 45.9 & & & & & & & & & & \\
\hline Resce, 1995 & $<1350$ & & & & 4.7 & & 17.2 & & & & & & 4.9 & & & & & & 9.5 & 2.3 & & & & 3.4 & 58.0 & & & & & & & & & & \\
\hline Resce, 1995 & $<1350$ & & 11.8 & 4.0 & 3.5 & & 9.1 & & & & & & 33.3 & & & & & & 20.1 & 1.7 & & & & 2.6 & 13.9 & & & & & & & & & & \\
\hline Resce, 1995 & $<1350$ & & & 4.2 & 3.7 & & 9.5 & & & & & & 34.9 & & & & & & 21.1 & 1.8 & & & & 2.7 & 21.9 & & & & & & & & & & \\
\hline Resce, 1995 & $<1350$ & & 11.9 & & 3.6 & & 9.1 & & & & & & 33.4 & & & & & & 20.2 & 1.7 & & & & 2.6 & 17.5 & & & & & & & & & & \\
\hline Resce, 1995 & $<1350$ & & & & 3.8 & & 9.6 & & & & & & 35.2 & & & & & & 21.2 & 1.8 & & & & 2.7 & 25.7 & & & & & & & & & & \\
\hline Resce, 1995 & $<1350$ & & 11.9 & 4.1 & 3.6 & & 18.3 & & & & & & 33.6 & & & & & & 10.1 & 1.7 & & & & 2.6 & 14.0 & & & & & & & & & & \\
\hline Resce, 1995 & $<1350$ & & & 4.3 & 3.8 & & 19.3 & & & & & & 35.3 & & & & & & \begin{tabular}{|l|}
10.7 \\
\end{tabular} & 1.8 & & & & 2.7 & 22.1 & & & & & & & & & & \\
\hline Resce, 1995 & $<1350$ & & 12.0 & & 3.6 & & 18.4 & & & & & & 33.8 & & & & & & 10.2 & 1.8 & & & & 2.6 & 17.6 & & & & & & & & & & \\
\hline Resce, 1995 & $<1350$ & & & & 3.8 & & 19.4 & & & & & & 35.5 & & & & & & 10.7 & 1.8 & & & & 2.8 & 26.0 & & & & & & & & & & \\
\hline Resce, 1995 & $<1350$ & & 12.3 & 6.9 & 3.7 & & 5.3 & & & & & & 27.0 & & & & & & 11.6 & 1.8 & & & & 2.7 & 28.7 & & & & & & & & & & \\
\hline Resce, 1995 & $<1350$ & & & 6.7 & 3.7 & & 4.8 & & & & & & 34.8 & & & & & & 10.5 & 1.8 & & & & 2.7 & 34.9 & & & & & & & & & & \\
\hline Resce, 1995 & $<1350$ & & 13.1 & & 3.9 & & 6.1 & & & & & & 20.5 & & & & & & 13.5 & 1.9 & & & & 2.9 & 38.1 & & & & & & & & & & \\
\hline Resce, 1995 & $<1350$ & & & & 3.7 & & \begin{tabular}{|l|l|}
4.8 \\
\end{tabular} & & & & & & 35.1 & & & & & & 10.6 & 1.8 & & & & 2.7 & 41.1 & & & & & & & & & & \\
\hline Resce, 1995 & $<1350$ & & 12.5 & 7.1 & 3.8 & & 10.9 & & & & & & 25.9 & & & & & & 6.0 & 1.8 & & & & 2.7 & 29.3 & & & & & & & & & & \\
\hline Resce, 1995 & $<1350$ & & & 6.8 & 3.7 & & 9.6 & & & & & & 35.0 & & & & & & 5.3 & 1.8 & & & & 2.7 & 35.1 & & & & & & & & & & \\
\hline Resce, 1995 & $<1350$ & & 12.9 & & 3.9 & & 11.6 & & & & & & 23.4 & & & & & & 6.4 & 1.9 & & & & 2.8 & 37.1 & & & & & & & & & & \\
\hline Resce, 1995 & $<1350$ & & & & 3.8 & & 9.6 & & & & & & 35.3 & & & & & & 5.3 & 1.8 & & & & 2.7 & 41.3 & & & & & & & & & & \\
\hline Reynolds, 1997 & 1150 & & 8.3 & 5.0 & & 1.3 & 1.3 & 1.8 & & 0.3 & & 0.4 & 7.1 & 0.1 & 1.9 & 0.1 & 1.0 & 0.1 & 16.2 & 1.5 & 0.1 & 0.1 & 3.0 & 0.2 & 39.6 & 0.2 & 0.3 & 5.9 & & & & & 4.5 & & \\
\hline Riley, 2001 & & & 7.0 & 5.2 & & & 11.5 & 3.1 & & & & & & 0.1 & 6.1 & & & & 11.6 & 0.0 & & & 1.2 & & 43.0 & 1.1 & 0.2 & & & & & & 4.8 & & \\
\hline Riley, 2001 & & & 12.5 & 4.9 & & & 10.8 & 2.9 & & & & & & 0.1 & 5.7 & & & & 10.9 & 0.0 & & & 1.1 & & 40.5 & 1.0 & 0.2 & & & & & & 4.5 & & \\
\hline Riley, 2001 & & & 8.5 & 6.0 & & & 11.2 & 3.0 & & & & & & 0.1 & 5.9 & & & & 11.3 & 0.0 & & & 1.2 & & 41.9 & 1.0 & 0.2 & & & & & & 4.7 & & \\
\hline Riley, 2001 & & & 7.7 & 15.0 & & & 10.1 & 2.7 & & & & & & 0.1 & 5.4 & & & & 10.2 & 0.0 & & & 1.1 & & 37.9 & 0.9 & 0.2 & & & & & & 4.2 & & \\
\hline Roa, 1997 & & & & 20.0 & & & & 0.3 & & & & & 2.0 & & 7.0 & & & & 5.0 & & & 9.6 & 0.2 & & 44.0 & & & & & & & & 12.0 & $>1118$ & \\
\hline Roa, 1997 & & & & 5.0 & & & 10.0 & 0.0 & & & & & 3.4 & & 1.0 & & & & 9.6 & & & 1.0 & 0.0 & & 57.0 & & & & & & & & 13.0 & 1187 & \\
\hline Roa, 1997 & & & & 5.0 & & & & 0.0 & & & & & 15.0 & & 1.0 & 8.0 & & & 8.4 & & & 1.0 & 0.0 & & 53.6 & & & & & & & & 8.0 & $>1118$ & \\
\hline Roa, 1997 & & & 10.3 & 13.2 & & & 7.0 & 0.1 & & & & & 4.5 & & 4.4 & 1.0 & & & 7.0 & & & 2.4 & 0.1 & & 46.5 & & & & & & & & 3.7 & 931 & \\
\hline Roa, 1997 & & & 1.4 & 9.4 & & & & 0.1 & & & & & 7.1 & & 6.0 & & & & 9.2 & & & 2.4 & 0.1 & & 54.3 & & & & & & & & 10.0 & 1018 & \\
\hline Roa, 1997 & & & & 5.0 & & & 2.0 & 0.2 & & & & & 2.0 & & 7.0 & 8.0 & & & 11.9 & & & 8.6 & 0.2 & & 42.1 & & & & & & & & 13.0 & $>1118$ & \\
\hline Roa, 1997 & & & 6.4 & 11.4 & & & 2.8 & 0.1 & & & & & 5.7 & & 3.8 & 3.6 & & & 10.0 & & & 3.9 & 0.1 & & 48.0 & & & & & & & & 4.3 & 916 & \\
\hline Roa, 1997 & & & 2.4 & 13.1 & & & 5.0 & 0.1 & & & & & 4.0 & & 4.9 & 2.0 & & & 8.0 & & & 4.4 & 0.1 & & 46.0 & & & & & & & & 10.0 & 1090 & \\
\hline Roa, 1997 & & & 2.4 & 10.5 & & & 0.8 & 0.0 & & & & & 7.3 & & 3.7 & 0.8 & & & 11.3 & & & 5.8 & 0.1 & & 53.3 & & & & & & & & 3.9 & 862 & \\
\hline Roa, 1997 & & & 1.0 & 5.0 & & & & 0.0 & & & & & 6.0 & & 6.7 & & & & 10.3 & & & 1.0 & 0.0 & & 57.0 & & & & & & & & 13.0 & 1081 & \\
\hline Roa, 1997 & & & 2.6 & 20.0 & & & 8.0 & 0.0 & & & & & 2.0 & & 7.0 & & & & 5.1 & & & 1.0 & 0.0 & & 44.3 & & & & & & & & 10.0 & 1049 & \\
\hline Roa, 1997 & & & & 20.0 & & & 8.0 & 0.3 & & & & & 2.0 & & 1.0 & & & & 6.8 & & & 9.6 & 0.2 & & 43.9 & & & & & & & & 8.3 & 1108 & \\
\hline
\end{tabular}


ORP-56297 Rev. 0

The Catholic University of America

High Level Waste Vitrification System Improvements Vitreous State Laboratory

Table A1. Glass Compositions from Literature Review (continued)

\begin{tabular}{|c|c|c|c|c|c|c|c|c|c|c|c|c|c|c|c|c|c|c|c|c|c|c|c|c|c|c|c|c|c|c|c|c|c|c|c|}
\hline Ref & 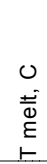 & $\begin{array}{l}0 \\
\stackrel{0}{\circ} \\
\stackrel{0}{0} \\
\vdash\end{array}$ & $\begin{array}{l}\text { Oొ } \\
\stackrel{N}{<}\end{array}$ & ల్లి & రి & 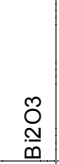 & $\begin{array}{l}0 \\
\mathbb{\pi} \\
0\end{array}$ & $\begin{array}{l}\widetilde{̃} \\
0 \\
0 \\
\tilde{ల} \\
0 \\
0 \\
0\end{array}$ & $\begin{array}{l}0 \\
0 \\
0 \\
0 \\
0 \\
0 \\
0\end{array}$ & $\begin{array}{l}\text { Õ } \\
\text { Uू }\end{array}$ & $\begin{array}{l}0 \\
3\end{array}$ & 4 & 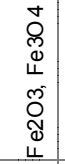 & ָָ & 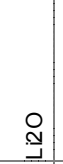 & $\stackrel{\circ}{\sum}$ & $\begin{array}{l}\text { Õ } \\
\text { ¿ } \\
\Sigma\end{array}$ & $\begin{array}{l}\text { Oొ } \\
\text { ¿ }\end{array}$ & $\begin{array}{l}\text { Q } \\
\underset{z}{z}\end{array}$ & $\frac{\mathrm{O}}{\mathrm{z}}$ & $\begin{array}{l}\mathcal{N} \\
\tilde{\Xi}\end{array}$ & $\overline{\bar{f}}$ & 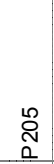 & $\frac{8}{0}$ & $\frac{\widetilde{N}}{(\infty)}$ & $\begin{array}{l}\text { OO } \\
\text { CD }\end{array}$ & o & 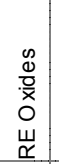 & 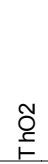 & $\begin{array}{l}\mathbb{N} \\
\stackrel{N}{F}\end{array}$ & 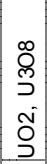 & $\stackrel{Q}{N}$ & $\begin{array}{l}\tilde{D} \\
\stackrel{N}{N}\end{array}$ & $\begin{array}{l}\hat{0} \\
\text {, }\end{array}$ & $\begin{array}{l}\bar{\Xi} \\
\text { 음 } \\
\underline{\underline{m}}\end{array}$ \\
\hline Roa, 1997 & & & 0.3 & 5.0 & & & & 0.0 & & & & & 2.0 & & 4.3 & & & & 20.0 & & & 1.0 & 0.0 & & 54.5 & & & & & & & & 13.0 & 1054 & \\
\hline Roa, 1997 & & & 2.6 & 5.0 & & & & 0.0 & & & & & 7.4 & & 7.0 & 8.0 & & & 13.6 & & & 1.0 & 0.0 & & 48.4 & & & & & & & & 7.0 & 1029 & \\
\hline Roa, 1997 & & & 5.0 & 14.8 & & & 2.0 & 0.1 & & & & & 3.0 & & 6.5 & 3.0 & & & 5.0 & & & 2.9 & 0.1 & & 50.7 & & & & & & & & 7.0 & 1090 & \\
\hline Roa, 1997 & & & 2.0 & 11.3 & & & 0.1 & & & & & & 0.1 & & 7.0 & 0.0 & & & 8.6 & & & 2.0 & & & 53.4 & & & & & & & & 15.5 & 1168 & \\
\hline Roa, 1997 & & & 2.0 & 6.0 & & & 0.5 & & & & & & 0.5 & & 7.0 & 0.5 & & & 18.0 & & & 9.0 & & & 45.5 & & & & & & & & 11.0 & 1027 & \\
\hline Roa, 1997 & & & 2.0 & 16.0 & & & 0.5 & 0.0 & & & & & 7.0 & & 2.5 & 4.0 & & & 5.0 & & & 2.0 & & & 56.0 & & & & & & & & 5.0 & 1129 & \\
\hline Roa, 1997 & & & 2.0 & 16.0 & & & 0.5 & & & & & & 10.5 & & 1.8 & 4.0 & & & 5.0 & & & 2.0 & & & 50.7 & & & & & & & & 7.5 & $>1454$ & \\
\hline Roa, 1997 & & & 2.0 & 6.0 & & & 0.5 & & & & & & 10.5 & & 7.0 & 0.5 & & & 18.0 & & & 2.0 & & & 45.5 & & & & & & & & 8.0 & 966 & \\
\hline Roa, 1997 & & & 10.5 & 6.0 & & & 4.0 & & & & & & 2.5 & & 7.0 & 0.5 & & & 14.0 & & & 2.0 & & & 46.0 & & & & & & & & 7.5 & 929 & \\
\hline Roa, 1997 & & & 2.0 & 16.0 & & & 0.5 & 0.2 & & & & & 0.5 & & 7.0 & 4.0 & & & 10.0 & & & 8.6 & 0.2 & & 44.0 & & & & & & & & 7.0 & 897 & \\
\hline Roa, 1997 & & & 1.0 & 7.5 & & & 7.0 & 0.2 & & & & & 4.0 & & 6.0 & 1.0 & & & 8.3 & & & 6.4 & 0.1 & & 49.2 & & & & & & & & 9.4 & 1035 & \\
\hline Roa, 1997 & & & 7.5 & 7.7 & & 5.1 & 0.9 & & & & & & 4.1 & & 4.3 & 0.3 & & & 10.8 & & & 4.0 & 0.8 & & 44.3 & & & & & & & & 10.1 & 1308 & \\
\hline Roa, 1997 & & & 4.0 & 8.0 & & 5.3 & 1.0 & & & & & & 4.3 & & 4.5 & 0.3 & & & 11.2 & & & 4.2 & 0.9 & & 46.0 & & & & & & & & 10.5 & 1069 & \\
\hline Roa, 1997 & & & 6.0 & 7.8 & & 5.2 & 0.9 & & & & & & 4.2 & & 4.4 & 0.3 & & & 11.0 & & & 4.1 & 0.8 & & 45.0 & & & & & & & & 10.3 & 1196 & \\
\hline Roa, 1997 & & & 2.5 & 2.0 & & 5.8 & 1.0 & & & & & & 4.7 & & 4.9 & 0.3 & & & 12.2 & & & 4.5 & 0.9 & & 49.9 & & & & & & & & 11.4 & 1031 & \\
\hline Roa, 1997 & & & 2.4 & 5.0 & & 5.6 & 1.0 & & & & & & 4.5 & & 4.7 & 0.3 & & & 11.8 & & & 4.4 & 0.9 & & 48.4 & & & & & & & & 11.1 & 1030 & \\
\hline Roa, 1997 & & & 2.2 & 12.0 & & 5.2 & 0.9 & & & & & & 4.2 & & 4.4 & 0.3 & & & 10.9 & & & 4.1 & 0.8 & & 44.8 & & & & & & & & 10.3 & 992 & \\
\hline Roa, 1997 & & & 2.4 & 8.4 & & 2.5 & 1.0 & & & & & & 4.5 & & 4.7 & 0.3 & & & 11.7 & & & 4.4 & 0.9 & & 48.2 & & & & & & & & 11.0 & 1017 & \\
\hline Roa, 1997 & & & 2.4 & 8.6 & & & 1.0 & & & & & & 4.6 & & 4.9 & 0.3 & & & 12.0 & & & 4.5 & 0.9 & & 49.4 & & & & & & & & 11.3 & 1015 & \\
\hline Roa, 1997 & & & 2.3 & 8.0 & & 7.5 & 1.0 & & & & & & 4.3 & & 4.5 & 0.3 & & & 11.1 & & & 4.2 & 0.9 & & 45.7 & & & & & & & & 10.5 & 1009 & \\
\hline Roa, 1997 & & & 2.2 & 7.8 & & 10.0 & 0.9 & & & & & & 4.1 & & 4.4 & 0.3 & & & 10.8 & & & 4.0 & 0.8 & & 44.5 & & & & & & & & 10.2 & 1023 & \\
\hline Roa, 1997 & & & 2.3 & 8.2 & & 5.4 & 1.0 & & & & & & 4.4 & & 4.6 & 0.3 & & & 11.4 & & & 4.2 & 0.9 & & 46.8 & & & & & & & & 10.7 & 1012 & \\
\hline Roa, 1997 & & & 2.3 & 8.2 & & 5.4 & 1.0 & & & & & & 4.4 & & 4.6 & 0.3 & & & 11.4 & & & 4.2 & 0.9 & & 46.8 & & & & & & & & 10.7 & 1012 & \\
\hline Roa, 1997 & & & 2.3 & 8.0 & & 5.3 & 1.0 & 1.5 & & & & & 4.3 & & 4.5 & 0.3 & & & 11.2 & & & 4.2 & 0.9 & & 46.1 & & & & & & & & 10.5 & 1019 & \\
\hline \begin{tabular}{|l|} 
Roa, 1997 \\
\end{tabular} & & & 2.2 & 7.9 & & 5.3 & 1.0 & 3.0 & & & & & 4.2 & & 4.5 & 0.3 & & & 11.1 & & & 4.1 & 0.9 & & 45.4 & & & & & & & & 10.4 & 1002 & \\
\hline Roa, 1997 & & & 2.4 & 8.5 & & 5.7 & 1.0 & & & & & & 4.6 & & & 0.3 & & & 11.9 & & & 4.5 & 0.9 & & 49.0 & & & & & & & & 11.2 & 1271 & \\
\hline Roa, 1997 & & & 2.4 & 8.4 & & 5.6 & 1.0 & & & & & & 4.5 & & 1.5 & 0.3 & & & 11.8 & & & 4.4 & 0.9 & & 48.3 & & & & & & & & 11.0 & 1155 & \\
\hline Roa, 1997 & & & 2.3 & 8.3 & & 5.5 & 1.0 & & & & & & 4.4 & & 3.0 & 0.3 & & & 11.6 & & & 4.3 & 0.9 & & 47.5 & & & & & & & & 10.9 & 1074 & \\
\hline Roa, 1997 & & & 2.3 & 8.0 & & 5.3 & 1.0 & & & & & & 4.3 & & 6.0 & 0.3 & & & 11.2 & & & 4.2 & 0.9 & & 46.1 & & & & & & & & 10.5 & 982 & \\
\hline Roa, 1997 & & & 2.4 & 8.6 & & 5.7 & 1.0 & & & & & & 4.6 & & 4.8 & 0.3 & & & 7.0 & & & 4.5 & 0.9 & & 49.1 & & & & & & & & 11.2 & 1223 & \\
\hline $\begin{array}{l}\text { Roa, } 1997 \\
\end{array}$ & & & 2.4 & 8.3 & & 5.5 & 1.0 & & & & & & 4.5 & & 4.7 & 0.3 & & & 9.5 & & & 4.3 & 0.9 & & 47.8 & & & & & & & & 10.9 & 1110 & \\
\hline Roa, 1997 & & & 2.3 & 8.0 & & 5.3 & 1.0 & & & & & & 4.3 & & 4.5 & 0.3 & & & 13.0 & & & 4.2 & 0.9 & & 45.9 & & & & & & & & 10.5 & 930 & \\
\hline Roa, 1997 & & & 2.5 & 8.8 & & 5.8 & 1.1 & & & & & & 4.7 & & 4.9 & 0.3 & & & 4.5 & & & 4.6 & 0.9 & & 50.4 & & & & & & & & 11.5 & 1350 & \\
\hline Roa, 1997 & & & 2.3 & 8.1 & & 5.4 & 1.0 & & & & & & 4.3 & & 4.5 & 0.3 & & & 11.3 & & & 4.2 & 2.0 & & 46.2 & & & & & & & & 10.6 & 1040 & \\
\hline Roa, 1997 & & & 2.3 & 8.0 & & 5.3 & 1.0 & & & & & & 4.3 & & 4.5 & 0.3 & & & 11.2 & & & 4.2 & 3.0 & & 45.8 & & & & & & & & 10.5 & 1052 & \\
\hline Roa, 1997 & & & 2.2 & 7.9 & & 5.2 & 1.0 & & & & & & 4.2 & & 4.4 & 0.3 & & & 11.0 & & & 4.1 & 4.0 & & 45.3 & & & & & & & & 10.4 & 1057 & \\
\hline Roa, 1997 & & & 2.2 & 7.8 & & 5.2 & 0.9 & & & & & & 4.2 & & 4.4 & 0.3 & & & 10.9 & & & 4.1 & 5.0 & & 44.8 & & & & & & & & 10.3 & 1065 & \\
\hline Roa, 1997 & & & 2.6 & 9.0 & & 6.0 & 1.1 & & & & & & 4.8 & & 5.1 & 0.3 & & & 12.6 & & & 4.7 & 1.0 & & 41.0 & & & & & & & & 11.9 & 1012 & \\
\hline Roa, 1997 & & & 2.1 & 7.4 & & 4.9 & 0.9 & & & & & & 3.9 & & 4.1 & 0.2 & & & 10.3 & & & 3.8 & 0.8 & & 52.0 & & & & & & & & 9.6 & 1022 & \\
\hline Roa, 1997 & & & 1.9 & 6.6 & & 4.4 & 0.8 & & & & & & 3.5 & & 3.7 & 0.2 & & & 9.2 & & & 3.4 & 0.7 & & 57.0 & & & & & & & & 8.6 & 1022 & \\
\hline Roa, 1997 & & & 2.4 & 8.4 & & 5.6 & 1.0 & & & & & & 4.5 & & 4.7 & 0.3 & & & 11.7 & & & 4.4 & 0.9 & & 48.2 & & & & & & & & 8.0 & 937 & \\
\hline Roa, 1997 & & & 2.3 & 8.0 & & 5.3 & 1.0 & & & & & & 4.3 & & 4.5 & 0.3 & & & 11.2 & & & 4.2 & 0.9 & & 46.1 & & & & & & & & 12.0 & 1074 & \\
\hline Roa, 1997 & & & 2.2 & 7.9 & & 5.2 & 0.9 & & & & & & 4.2 & & 4.4 & 0.3 & & & 11.0 & & & 4.1 & 0.8 & & 45.0 & & & & & & & & 14.0 & 1182 & \\
\hline Rocabois, 2001 & & & 24.5 & & & & 33.7 & & & & & & & & & 0.3 & & & & & & & & & 41.3 & & & & & & & & & 1360 & \\
\hline Rocabois, 2001 & & & 20.0 & & & & 38.2 & & & & & & & & & & & & & & & & & & 41.3 & & & & & & & & & 1347 & \\
\hline Rocabois, 2001 & & & 21.2 & & & & 35.7 & & & & & & & & & 0.4 & & & & & & & & & 42.5 & & & & & & & & & 1305 & \\
\hline Rocabois, 2001 & & & 20.3 & & & & 34.0 & & & & & & & & & 5.1 & & & & & & & & & 40.6 & & & & & & & & & 1387 & \\
\hline Rocabois, 2001 & & & 20.0 & & & & 33.5 & & & & & & & & & 6.1 & & & & & & & & & 39.9 & & & & & & & & & 1396 & \\
\hline Rocabois, 2001 & & & 19.5 & & & & 33.4 & & & & & & & & & 7.3 & & & & & & & & & 39.5 & & & & & & & & & 1408 & \\
\hline Rocabois, 2001 & & & 20.6 & & & & 34.4 & & & & & & & & & 2.8 & & & & & & & & & 42.2 & & & & & & & & & 1343 & \\
\hline
\end{tabular}


Table A1. Glass Compositions from Literature Review (continued)

\begin{tabular}{|c|c|c|c|c|c|c|c|c|c|c|c|c|c|c|c|c|c|c|c|c|c|c|c|c|c|c|c|c|c|c|c|c|c|c|c|}
\hline Ref & 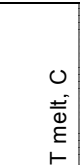 & $\begin{array}{l}0 \\
\frac{0}{2} \\
\stackrel{0}{0} \\
\text { - }\end{array}$ & 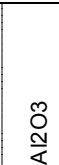 & ్ֻల్ల & $\begin{array}{l}\mathcal{D} \\
\infty\end{array}$ & Oొָ & 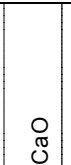 & $\begin{array}{l}\tilde{O} \\
\mathbb{d} \\
0 \\
\tilde{N} \\
0 \\
0 \\
0\end{array}$ & $\begin{array}{l}\text { Oొ } \\
\text { Oे } \\
0 \\
0 \\
0 \\
0\end{array}$ & $\begin{array}{l}\text { Oొ } \\
\text { Ũ }\end{array}$ & O̊ & $\longleftarrow$ & 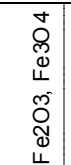 & 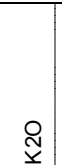 & 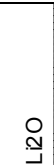 & $\begin{array}{l}\circ \\
\sum \\
\sum\end{array}$ & $\begin{array}{l}\tilde{O} \\
\stackrel{\Gamma}{\Sigma}\end{array}$ & $\begin{array}{l}0 \\
0 \\
\Sigma\end{array}$ & $\begin{array}{l}\stackrel{\mathbb{N}}{\pi} \\
\underset{Z}{Z}\end{array}$ & $\frac{}{z}$ & $\begin{array}{l}\tilde{N} \\
\tilde{0}\end{array}$ & $\begin{array}{l}\overline{\bar{c}} \\
\text { Oे }\end{array}$ & $\begin{array}{l}\text { مి } \\
\text { م) }\end{array}$ & $\frac{8}{0}$ & $\frac{\tilde{O}}{\infty}$ & Oొ & $\begin{array}{l}\frac{9}{i n} \\
\text { in }\end{array}$ & $\begin{array}{l}\infty \\
\stackrel{0}{0} \\
\cdot \frac{0}{x} \\
0 \\
\underset{\sim}{山}\end{array}$ & 正 & $\stackrel{\widetilde{N}}{\underline{\delta}}$ & $\begin{array}{l}\text { o̊ } \\
\text { ગ } \\
\text { دิ } \\
\text { రิ }\end{array}$ & 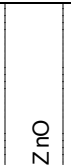 & $\frac{\tilde{D}}{N}$ & 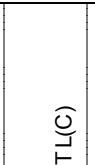 & 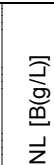 \\
\hline Rocabois, 2001 & & & 17.0 & & & & 21.7 & & & & & & & & & & & & & & & & & & 60.9 & & & & & & & & & 1219 & \\
\hline Rocabois, 2001 & & & 24.2 & & & & 31.9 & & & & & & & & & 5.0 & & & & & & & & & 39.0 & & & & & & & & & 1380 & \\
\hline Rocabois, 2001 & & & 17.2 & & & & 21.1 & & & & & & & & & 5.1 & & & & & & & & & 56.7 & & & & & & & & & 1196 & \\
\hline Rocabois, 2001 & & & 13.7 & & & & 35.5 & & & & & & & & & & & & & & & & & & 50.5 & & & & & & & & & 1376 & \\
\hline Rocabois, 2001 & & & 10.3 & & & & 27.8 & & & & & & & & & & & & & & & & & & 61.6 & & & & & & & & & 1323 & \\
\hline Rocabois, 2001 & & & 22.9 & & & & 30.6 & & & & & & & & & 9.0 & & & & & & & & & 37.4 & & & & & & & & & 1403 & \\
\hline Rocabois, 2001 & & & 16.2 & & & & 19.9 & & & & & & & & & 9.9 & & & & & & & & & 54.0 & & & & & & & & & 1244 & \\
\hline Rudkovskaya, 200 & $\sim 1300$ & & 8.2 & 27.2 & & & 0.4 & & & & & & 0.4 & 2.7 & & & & & 2.6 & & & & & & 46.3 & & & & & & & 12.1 & & & \\
\hline Rudkovskaya, 200 & $\sim 1330$ & & 8.0 & & 0.1 & & 4.1 & & & & & & 0.3 & 1.3 & & 1.9 & 3.1 & & 7.5 & & & & 0.0 & & 49.4 & & & & & & & 25.6 & & & \\
\hline Rudkovskaya, 200 & $\sim 1350$ & & 8.8 & & & & 4.0 & & & & & & 0.3 & 1.8 & & 2.6 & & & 7.4 & 1.0 & & & & & 55.2 & & & & & 0.0 & & 24.9 & & & \\
\hline Rudkovskaya, 200 & $\sim 1250$ & & 9.2 & & 8.3 & & 3.5 & & & & & & & 5.6 & & & & & 1.9 & & & & & & 49.1 & & & & & 2.6 & & 19.8 & & & \\
\hline Rudkovskaya, 200 & $\sim 1350$ & & 9.1 & & 4.0 & & 1.3 & & & & & & & 6.5 & & & & & 2.0 & & & & & & 44.3 & & & & & 0.5 & & 32.3 & & & \\
\hline Rudkovskaya, 200 & $\sim 1350$ & & 9.6 & & 9.0 & & 3.3 & & & & & & & 6.2 & & & & & 1.9 & & & & & & 49.2 & & & & & & & 20.9 & & & \\
\hline Russell, 2005 & & 2050 & 19.4 & & & & & & & & & & & & & & & & 111.8 & & & & & & 68.7 & & & & & & & & & & \\
\hline Russell, 2005 & & 1585 & 32.5 & & & & 15.3 & & & & & & & & & & & & 2.8 & & & & & & 49.3 & & & & & & & & & & \\
\hline Russell, 2005 & & 1675 & 28.3 & & & & 10.4 & & & & & & & & & & & & 5.7 & & & & & & 55.6 & & & & & & & & & & \\
\hline Russell, 2005 & & 1821 & 23.0 & & & & 4.2 & & & & & & & & & & & & 9.3 & & & & & & 63.4 & & & & & & & & & & \\
\hline Russell, 2005 & & 1499 & 36.6 & & & & 20.2 & & & & & & & & & & & & & & & & & & 43.2 & & & & & & & & & & \\
\hline Russell, 2005 & & & 24.8 & & & & 6.3 & & & & & & & & & & & & 8.1 & & & & & & 60.8 & & & & & & & & & & \\
\hline Russell, 2005 & & & 25.8 & & & & 7.4 & & & & & & & & & & & & 7.5 & & & & & & 59.3 & & & & & & & & & & \\
\hline Russell, 2005 & & & 26.9 & & & & 8.7 & & & & & & & & & & & & 6.7 & & & & & & 57.7 & & & & & & & & & & \\
\hline Russell, 2005 & & 1510 & 29.2 & & & & 11.4 & & & & & & & & & & & & 5.1 & & & & & & 54.3 & & & & & & & & & & \\
\hline Russell, 2005 & & 1792 & 30.4 & & & & 12.8 & & & & & & & & & & & & 4.3 & & & & & & 52.5 & & & & & & & & & & \\
\hline Russell, 2005 & & 1589 & 33.7 & & & & 16.7 & & & & & & & & & & & & 2.0 & & & & & & 47.6 & & & & & & & & & & \\
\hline Russell, 2005 & & 1563 & 35.1 & & & & 18.4 & & & & & & & & & & & & 1.0 & & & & & & 45.5 & & & & & & & & & & \\
\hline Russell, 2005 & & 1261 & & & & & 35.8 & & & & & & & & & 25.8 & & & & & & & & & 38.4 & & & & & & & & & & \\
\hline Russell, 2005 & & & 34.5 & & & & 21.1 & & & & & & & & & 1.5 & & & & & & & & & 42.9 & & & & & & & & & & \\
\hline Russell, 2005 & & & 26.7 & & & & 15.3 & & & & & & & & & 3.0 & & & 3.9 & & & & & & 51.1 & & & & & & & & & & \\
\hline Russell, 2005 & & & 16.9 & & & & 4.7 & & & & & & & & & 3.4 & & & 10.3 & & & & & & 64.8 & & & & & & & & & & \\
\hline Russell, 2005 & & 1433 & 32.1 & & & & 22.1 & & & & & & & & & 3.2 & & & & & & & & & 42.6 & & & & & & & & & & \\
\hline Russell, 2005 & & & 18.5 & & & & 7.0 & & & & & & & & & 3.5 & & & 9.0 & & & & & & 62.0 & & & & & & & & & & \\
\hline Russell, 2005 & & 1727 & 16.2 & & & & 5.9 & & & & & & & & & 4.3 & & & 9.9 & & & & & & 63.7 & & & & & & & & & & \\
\hline Russell, 2005 & & & 18.9 & & & & 10.4 & & & & & & & & & 4.8 & & & 7.4 & & & & & & 58.4 & & & & & & & & & & \\
\hline Russell, 2005 & & & 15.5 & & & & 7.3 & & & & & & & & & 5.2 & & & 9.4 & & & & & & 62.6 & & & & & & & & & & \\
\hline Russell, 2005 & & 1413 & 29.5 & & & & 23.2 & & & & & & & & & 5.0 & & & & & & & & & 42.3 & & & & & & & & & & \\
\hline Russell, 2005 & & & 19.2 & & & & 14.2 & & & & & & & & & 6.4 & & & 5.8 & & & & & & 54.5 & & & & & & & & & & \\
\hline Russell, 2005 & & & 15.5 & & & & 11.1 & & & & & & & & & 6.9 & & & 7.8 & & & & & & 58.7 & & & & & & & & & & \\
\hline Russell, 2005 & & 1378 & 26.7 & & & & 24.4 & & & & & & & & & 7.0 & & & & & & & & & 41.9 & & & & & & & & & & \\
\hline Russell, 2005 & & & 19.4 & & & & 18.2 & & & & & & & & & 8.0 & & & 4.0 & & & & & & 50.4 & & & & & & & & & & \\
\hline Russell, 2005 & & 1454 & 12.2 & & & & 13.4 & & & & & & & & & 9.6 & & & 7.4 & & & & & & 57.4 & & & & & & & & & & \\
\hline Russell, 2005 & & 1323 & 23.5 & & & & 25.8 & & & & & & & & & 9.3 & & & & & & & & & 41.5 & & & & & & & & & & \\
\hline Russell, 2005 & & 1378 & 19.2 & & & & 22.1 & & & & & & & & & 9.8 & & & 2.4 & & & & & & 46.5 & & & & & & & & & & \\
\hline Russell, 2005 & & & 14.7 & & & & 18.9 & & & & & & & & & 10.8 & & & 4.6 & & & & & & 51.1 & & & & & & & & & & \\
\hline Russell, 2005 & & 1299 & 20.6 & & & & 27.0 & & & & & & & & & 11.3 & & & & & & & & & 41.1 & & & & & & & & & & \\
\hline Russell, 2005 & & & 10.3 & & & & 16.9 & & & & & & & & & 12.2 & & & 6.2 & & & & & & 54.4 & & & & & & & & & & \\
\hline Russell, 2005 & & 1311 & 18.3 & & & & 28.0 & & & & & & & & & 12.9 & & & & & & & & & 40.8 & & & & & & & & & & \\
\hline Russell, 2005 & & & 8.3 & & & & 20.6 & & & & & & & & & 14.8 & & & 5.0 & & & & & & 51.3 & & & & & & & & & & \\
\hline Russell, 2005 & & 1302 & 7.0 & & & & 23.0 & & & & & & & & & 16.5 & & & 4.2 & & & & & & 49.3 & & & & & & & & & & \\
\hline Russell, 2005 & & 1280 & 11.3 & & & & 31.0 & & & & & & & & & $\mid 17.8$ & & & & & & & & & 39.9 & & & & & & & & & & \\
\hline Russell, 2005 & & & 4.7 & & & & 27.3 & & & & & & & & & 19.6 & & & 2.8 & & & & & & 45.7 & & & & & & & & & & \\
\hline
\end{tabular}


Table A1. Glass Compositions from Literature Review (continued)

\begin{tabular}{|c|c|c|c|c|c|c|c|c|c|c|c|c|c|c|c|c|c|c|c|c|c|c|c|c|c|c|c|c|c|c|c|c|c|c|c|}
\hline Ref & 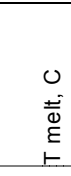 & 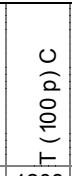 & 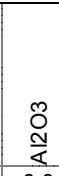 & 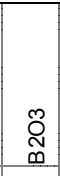 & $\begin{array}{l}\mathscr{D} \\
\infty\end{array}$ & $\begin{array}{l}\stackrel{0}{0} \\
\stackrel{0}{\infty}\end{array}$ & $\begin{array}{l}0 \\
0 \\
0\end{array}$ & $\begin{array}{l}\widetilde{̃} \\
0 \\
0 \\
\tilde{\delta}^{-} \\
0 \\
0 \\
0\end{array}$ & $\begin{array}{l}0 \\
0 \\
0 \\
0 \\
0 \\
0 \\
0\end{array}$ & ญొ & Oे & 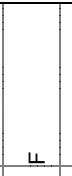 & 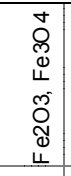 & $\underset{\mathbb{X}}{\underline{Z}}$ & $\stackrel{\stackrel{ }{ב}}{=}$ & $\begin{array}{l}0 \\
\vdots \\
\Sigma\end{array}$ & $\begin{array}{l}\tilde{O} \\
\stackrel{\complement}{\Sigma}\end{array}$ & $\begin{array}{l}0 \\
0 \\
\Sigma \\
\Sigma\end{array}$ & $\begin{array}{l}0 \\
\pi \\
z\end{array}$ & $\frac{0}{z}$ & 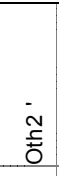 & $\begin{array}{l}\overline{\bar{c}} \\
\overline{0}\end{array}$ & $\begin{array}{l}\text { مٌ } \\
a \\
a\end{array}$ & $\frac{8}{2}$ & $\frac{\tilde{O}}{\omega}$ & $\begin{array}{l}0 \\
0 \\
\text { os }\end{array}$ & 옹 & 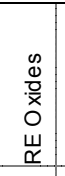 & 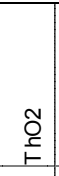 & 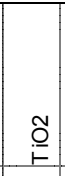 & 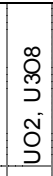 & $\stackrel{q}{N}$ & ญั & 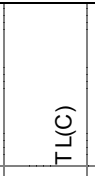 & 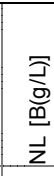 \\
\hline Russell, 2005 & & 1208 & 6.0 & & & & 33.3 & & & & & & & & & 21.5 & & & & & & & & & 39.2 & & & & & & & & & & \\
\hline Russell, 2005 & & 1213 & 1.6 & & & & 32.9 & & & & & & & & & 23.7 & & & 1.0 & & & & & & 40.9 & & & & & & & & & & \\
\hline Russell, 2005 & & 1783 & 18.4 & & & & 2.0 & & & & & & & & & 1.4 & & & 11.2 & & & & & & 67.0 & & & & & & & & & & \\
\hline Sato, 2005 & & & 16.8 & & & & 9.7 & & & & & & 10.1 & 1.0 & & 5.2 & 0.2 & & 2.6 & & & & 0.1 & & 52.5 & & & & & 1.4 & & & & 1230 & \\
\hline Sato, 2005 & & & 16.7 & & & & 9.6 & & & & & & 10.3 & 1.1 & & 5.3 & 0.2 & & 2.6 & & & & 0.1 & & 52.4 & & & & & 1.4 & & & & 1210 & \\
\hline Sato, 2005 & & & 16.1 & & & & 9.5 & & & & & & 10.3 & 1.0 & & 5.3 & 0.2 & & 2.7 & & & & 0.1 & & 52.2 & & & & & 1.4 & & & & 1190 & \\
\hline Sato, 2005 & & & 15.0 & & & & 9.1 & & & & & & 11.1 & 1.0 & & 5.9 & 0.2 & & 2.6 & & & & 0.1 & & 52.0 & & & & & 1.5 & & & & 1170 & \\
\hline Sato, 2005 & & & 14.2 & & & & 8.8 & & & & & & 12.3 & 1.1 & & 6.3 & 0.2 & & 2.5 & & & & 0.2 & & 52.4 & & & & & 1.7 & & & & 1150 & \\
\hline Sato, 2005 & & & 13.5 & & & & 8.7 & & & & & & 12.5 & 1.2 & & 5.8 & 0.2 & & 2.4 & & & & 0.2 & & 52.6 & & & & & 1.8 & & & & 1130 & \\
\hline Scarfe, 1983 & & 1444 & 29.3 & & & & 21.3 & & & & & & & & & 3.7 & & & & & & & & & 45.7 & & & & & & & & & 1488 & \\
\hline Scarfe, 1983 & & 1411 & 25.7 & & & & 21.9 & & & & & & & & & 5.6 & & & & & & & & & 46.9 & & & & & & & & & 1438 & \\
\hline Scarfe, 1983 & & 1353 & 22.0 & & & & 22.5 & & & & & & & & & 7.4 & & & & & & & & & 48.1 & & & & & & & & & 1388 & \\
\hline Scarfe, 1983 & & 1356 & 18.3 & & & & 23.0 & & & & & & & & & 9.3 & & & & & & & & & 49.3 & & & & & & & & & 1323 & \\
\hline Scarfe, 1983 & & 1331 & 15.4 & & & & 23.5 & & & & & & & & & 10.8 & & & & & & & & & 50.3 & & & & & & & & & 1274 & \\
\hline Scarfe, 1983 & & 1230 & 7.3 & & & & 24.7 & & & & & & & & & 14.9 & & & & & & & & & 53.0 & & & & & & & & & 1343 & \\
\hline Scarfe, 1983 & & 1196 & 3.7 & & & & 25.3 & & & & & & & & & 16.8 & & & & & & & & & 54.3 & & & & & & & & & 1368 & \\
\hline Scarfe, 1983 & & 1481 & 36.6 & & & & 20.2 & & & & & & & & & & & & & & & & & & 43.2 & & & & & & & & & 1553 & \\
\hline Scarfe, 1983 & & 1127 & & & & & 25.9 & & & & & & & & & 18.6 & & & & & & & & & 55.5 & & & & & & & & & 1392 & \\
\hline Scarfe, 1986 & & 1141 & & & & & 25.9 & & & & & & & & & 18.6 & & & & & & & & & 55.5 & & & & & & & & & 1392 & \\
\hline Scarfe, 1986 & & 1199 & 2.3 & & & & 25.5 & & & & & & & & & 17.4 & & & & & & & & & 54.7 & & & & & & & & & 1378 & \\
\hline Scarfe, 1986 & & 1280 & 11.0 & & & & 24.2 & & & & & & & & & 13.0 & & & & & & & & & 51.8 & & & & & & & & & 1324 & \\
\hline Scarfe, 1986 & & 1480 & 20.6 & & & & 22.7 & & & & & & & & & 8.1 & & & & & & & & & 48.6 & & & & & & & & & 1272 & \\
\hline Scarfe, 1986 & & 1709 & 29.1 & & & & 21.3 & & & & & & & & & 3.0 & & & & & & & & & 45.7 & & & & & & & & & 1217 & \\
\hline Scarfe, 1986 & & 2051 & 36.6 & & & & 20.2 & & & & & & & & & & & & & & & & & & 43.2 & & & & & & & & & 1118 & \\
\hline Scholes, 2000 & 1178 & & 9.0 & 3.0 & & 1.4 & 1.4 & 1.9 & & 0.3 & & 0.4 & 7.6 & 0.2 & 2.0 & 0.1 & 1.0 & 0.1 & 17.6 & 1.6 & 0.1 & 0.1 & 3.3 & 0.2 & 37.0 & 0.2 & 0.3 & 6.4 & & & & & 4.9 & & \\
\hline \begin{tabular}{|l} 
\\
Scholes, 2000
\end{tabular} & 1118 & & 8.3 & 3.0 & & 1.2 & 1.3 & 1.7 & & 0.3 & & 0.4 & 7.0 & 0.1 & 2.9 & 0.0 & 1.0 & 0.0 & 19.8 & 1.5 & 0.1 & 0.1 & 3.0 & 0.2 & 37.0 & 0.2 & 0.3 & 5.9 & & & & & 4.5 & & \\
\hline Scholes, 2000 & 1155 & & 8.3 & 8.0 & & 1.2 & 1.3 & 1.7 & & 0.3 & & 0.4 & 7.0 & 0.1 & 1.2 & 0.0 & 1.0 & 0.0 & 16.4 & 1.5 & 0.1 & 0.1 & 3.0 & 0.2 & 37.0 & 0.2 & 0.3 & 5.9 & & & & & 4.5 & & \\
\hline Scholes, 2000 & 1102 & & 7.6 & 5.0 & & 1.1 & 1.2 & 1.6 & & 0.3 & & 0.3 & 6.5 & 0.1 & 2.2 & 0.0 & 0.9 & 0.0 & 20.8 & 1.3 & 0.1 & 0.1 & 2.8 & 0.2 & 37.8 & 0.2 & 0.2 & 5.4 & & & & & 4.2 & & \\
\hline Scholes, 2000 & 1155 & & 7.6 & 10.0 & & 1.1 & 1.2 & 1.6 & & 0.3 & & 0.3 & 6.5 & 0.1 & & 0.0 & 0.9 & 0.0 & 18.6 & 1.3 & 0.1 & 0.1 & 2.8 & 0.2 & 37.1 & 0.2 & 0.2 & 5.4 & & & & & 4.2 & & \\
\hline Scholes, 2000 & & & 8.5 & 9.3 & & & 4.9 & 0.0 & & 0.1 & & 2.2 & 2.3 & 0.9 & 4.0 & 0.2 & 0.0 & 0.7 & 12.5 & 0.7 & 0.0 & 0.0 & 1.1 & & 42.1 & 0.2 & 1.8 & 2.3 & & & & & 6.2 & & \\
\hline Scholes, 2000 & & & 4.5 & 16.3 & & & 10.6 & 0.0 & & 0.1 & & 0.4 & 0.5 & 0.2 & 0.8 & 0.2 & 0.0 & 1.4 & 6.5 & 0.2 & 0.0 & 0.0 & 0.2 & & 51.6 & 0.4 & 0.4 & 4.5 & & & & & 1.2 & & \\
\hline Scholes, 2000 & & & 4.0 & 5.0 & & & & 0.0 & & 0.1 & & & & 2.0 & 0.8 & 0.2 & 0.0 & 1.5 & 20.0 & 1.5 & 0.0 & 0.0 & 2.5 & & 42.7 & 0.5 & & 5.0 & & & & & 14.0 & & \\
\hline Scholes, 2000 & & & 3.5 & 18.0 & & & & 0.0 & & 0.1 & & & 3.0 & & 2.7 & 0.2 & 0.0 & & 5.0 & 1.5 & 0.0 & 0.0 & 2.5 & & 54.3 & & 4.0 & 5.0 & & & & & & & \\
\hline Scholes, 2000 & 1150 & & 3.5 & 5.0 & & & 12.0 & 0.0 & & 0.1 & & 4.7 & & 2.0 & & 0.2 & 0.0 & 1.5 & 12.0 & 1.5 & 0.0 & 0.0 & & & 53.3 & & 4.0 & 0.0 & & & & & & & \\
\hline Scholes, 2000 & 1350 & & 4.0 & 5.0 & & & 12.0 & 0.0 & & 0.1 & & 3.3 & & 2.0 & 3.0 & 0.2 & 0.0 & & 5.0 & 1.5 & 0.0 & 0.0 & 2.5 & & 42.1 & & & 5.0 & & & & & 14.0 & $>1500$ & \\
\hline Scholes, 2000 & 1250 & & 16.9 & 18.0 & & & & 0.0 & & 0.1 & & & 8.0 & & & 0.2 & 0.0 & 1.5 & 18.6 & 1.5 & 0.0 & 0.0 & & & 35.0 & & & 0.0 & & & & & & & \\
\hline Scholes, 2000 & 1275 & & 5.4 & 15.4 & & & 1.5 & 0.0 & & 0.1 & & 0.7 & 6.3 & 0.3 & 1.2 & 0.2 & 0.0 & 1.3 & 11.9 & 1.3 & 0.0 & 0.0 & 0.3 & & 40.9 & 0.4 & 0.6 & 0.7 & & & & & 11.7 & $>1350$ & \\
\hline Scholes, 2000 & 1150 & & 3.5 & 5.0 & & & & 0.0 & & 0.1 & & 5.6 & 8.0 & 2.0 & 9.0 & 0.2 & 0.0 & 1.5 & 5.7 & & 0.0 & 0.0 & 2.5 & & 56.3 & 0.5 & & 0.0 & & & & & & & \\
\hline Scholes, 2000 & 1250 & & 4.9 & 16.3 & & & 1.0 & 0.0 & & 0.1 & & 3.3 & 4.6 & 1.8 & 3.9 & 0.2 & 0.0 & 0.2 & 6.5 & 1.4 & 0.0 & 0.0 & 0.2 & & 36.4 & \begin{tabular}{|l|}
0.4 \\
\end{tabular} & 3.6 & 2.8 & & & & & 12.4 & $>1500$ & \\
\hline Scholes, 2000 & 1150 & & 12.1 & 5.0 & & & 12.0 & 0.0 & & 0.1 & & & 8.0 & & 1.5 & 0.2 & 0.0 & & 20.0 & & 0.0 & 0.0 & & & 36.5 & 0.5 & 4.0 & 0.0 & & & & & & 1013 & \\
\hline Scholes, 2000 & 1150 & & 3.5 & 18.0 & & & 9.0 & 0.0 & & 0.1 & & & 3.0 & & & 0.2 & 0.0 & & 11.5 & & 0.0 & 0.0 & & & 35.0 & 0.5 & 4.0 & 5.0 & & & & & 10.0 & 973 & \\
\hline Scholes, 2000 & 1150 & & 4.0 & 7.5 & & & & 0.0 & & 0.1 & & 6.0 & 8.0 & & 4.7 & 0.2 & 0.0 & & 13.4 & 1.5 & 0.0 & 0.0 & 1.4 & & 35.0 & & 4.0 & 0.0 & & & & & 14.0 & & \\
\hline Scholes, 2000 & 1150 & & 8.0 & 6.0 & & & 4.0 & 0.0 & & 0.1 & & 2.5 & 3.0 & 1.0 & 6.0 & 0.2 & 0.0 & 1.0 & 10.0 & 0.5 & 0.0 & 0.0 & 1.5 & & 49.3 & 0.3 & 1.0 & 1.5 & & & & & 4.0 & 943 & \\
\hline Scholes, 2000 & 1200 & & 10.0 & 6.0 & & & 8.0 & 0.0 & & 0.1 & & 2.5 & 0.5 & 1.0 & 4.3 & 0.2 & 0.0 & 1.0 & 10.0 & 1.0 & 0.0 & 0.0 & 0.5 & & 40.0 & 0.3 & 3.0 & 3.5 & & & & & 8.0 & 1203 & \\
\hline Scholes, 2000 & 1150 & & 9.9 & 7.5 & & & 8.0 & 0.0 & & 0.1 & & 1.0 & 0.5 & 1.0 & 6.0 & 0.2 & 0.0 & 1.0 & 10.0 & 0.5 & 0.0 & 0.0 & 1.5 & & 40.0 & 0.1 & 3.0 & 1.5 & & & & & 8.0 & 1133 & \\
\hline Scholes, 2000 & 1150 & & 8.9 & 6.0 & & & 8.0 & 0.0 & & 0.1 & & 1.0 & 3.0 & 1.0 & 6.0 & \begin{tabular}{|l|}
0.2 \\
\end{tabular} & 0.0 & 1.0 & 10.0 & 0.5 & 0.0 & 0.0 & 1.5 & & 40.0 & 0.1 & 3.0 & 1.5 & & & & & 8.0 & 1173 & \\
\hline Scholes, 2000 & 1150 & & 12.0 & 6.0 & & & 4.0 & 0.0 & & 0.1 & & 1.0 & 0.5 & 1.0 & 5.8 & 0.2 & 0.0 & 1.0 & 15.0 & 1.0 & 0.0 & 0.0 & 1.5 & & 40.0 & 0.3 & 3.0 & 1.5 & & & & & 6.0 & 1023 & \\
\hline Scholes, 2000 & 1150 & & 12.0 & 6.0 & & & 8.0 & 0.0 & & 0.1 & & 2.5 & 1.5 & 1.0 & 6.0 & 0.2 & 0.0 & 1.0 & 10.5 & 0.5 & 0.0 & 0.0 & 1.5 & & 40.0 & 0.1 & 1.0 & 3.5 & & & & & 4.4 & 893 & \\
\hline Scholes, 2000 & 1150 & & 8.0 & 12.0 & & & 8.0 & 0.0 & & 0.1 & & 2.5 & 3.0 & 0.5 & 3.1 & 0.2 & 0.0 & 1.0 & 10.0 & 0.5 & 0.0 & 0.0 & 0.5 & & 41.2 & 0.3 & 3.0 & 2.0 & & & & & 4.0 & 893 & \\
\hline Scholes, 2000 & 1150 & & 10.0 & 11.2 & & & 4.0 & 0.0 & & 0.1 & & 1.0 & 1.5 & 0.5 & 6.0 & $\begin{array}{l}0.2 \\
\end{array}$ & 0.0 & 1.0 & 10.0 & 0.5 & 0.0 & 0.0 & 0.5 & & 40.8 & 0.1 & 1.0 & 3.5 & & & & & 8.0 & 1013 & \\
\hline
\end{tabular}




\begin{tabular}{|c|c|c|c|c|c|c|c|c|c|c|c|c|c|c|c|c|c|c|c|c|c|c|c|c|c|c|c|c|c|c|c|c|c|c|c|}
\hline Ref & 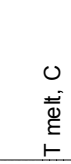 & $\begin{array}{l}0 \\
\frac{0}{2} \\
8 \\
= \\
⺊\end{array}$ & 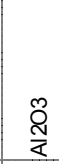 & 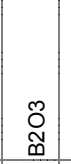 & 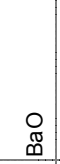 & 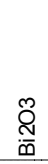 & $\begin{array}{l}0 \\
0 \\
0\end{array}$ & 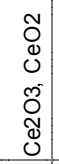 & $\begin{array}{l}0 \\
0 \\
0 \\
0 \\
8 \\
0\end{array}$ & ญ్ & O̧ & 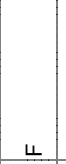 & 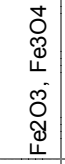 & $\begin{array}{l}\stackrel{\Xi}{\widetilde{Y}} \\
\end{array}$ & 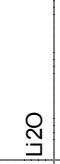 & $\begin{array}{l}0 \\
\text { OD }\end{array}$ & $\begin{array}{l}\tilde{O} \\
\text { I } \\
\Sigma\end{array}$ & $\begin{array}{l}0 \\
0 \\
\Sigma\end{array}$ & 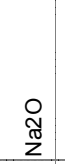 & $\frac{0}{z}$ & ָָ & 곰 & $\begin{array}{l}\text { ¿ } \\
\text { N }\end{array}$ & $\begin{array}{l}O \\
0 \\
Q\end{array}$ & 斻 & ס & 임 & 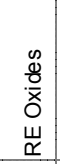 & $\begin{array}{l}\text { N } \\
\text { Oे } \\
F\end{array}$ & $\underset{\mathcal{O}}{\stackrel{N}{*}}$ & 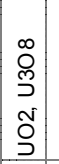 & $\begin{array}{l}O \\
\text { N }\end{array}$ & ั๊ & 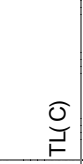 & $\begin{array}{l}\vec{\partial} \\
\text { क् } \\
\underline{\underline{m}} \\
\vec{z}\end{array}$ \\
\hline Scholes, 2000 & 1200 & & 10.0 & 6.0 & & & 4.0 & 0.0 & & 0.1 & & 1.0 & 3.0 & 0.5 & 5.4 & 0.2 & 0.0 & 0.5 & 15.0 & 0.5 & 0.0 & 0.0 & 0.5 & & 40.0 & 0.3 & 2.9 & 2.0 & & & & & 8.0 & 1163 & \\
\hline Scholes, 2000 & 1150 & & 12.0 & 6.0 & & & 4.0 & 0.0 & & 0.1 & & 2.5 & 0.5 & 0.5 & 3.0 & 0.2 & 0.0 & 1.0 & 15.0 & 1.0 & 0.0 & 3.3 & 0.5 & & 44.8 & & & 1.5 & & & & & 4.0 & 903 & \\
\hline Scholes, 2000 & 1150 & & 12.0 & 6.0 & & & 4.0 & 0.0 & & 0.1 & & 2.5 & 0.5 & 0.5 & 3.2 & 0.2 & 0.0 & 0.5 & 15.0 & 1.0 & 0.0 & 1.3 & 0.5 & & 45.1 & & & 3.5 & & & & & 4.0 & 883 & \\
\hline Scholes, 2000 & 1150 & & 12.0 & 6.0 & & & 4.0 & 0.0 & & 0.1 & & 2.5 & 0.5 & 0.5 & 3.0 & 0.2 & 0.0 & 0.5 & 14.4 & 0.5 & 0.0 & 3.3 & 0.5 & & 44.3 & & & 3.5 & & & & & 4.0 & 903 & \\
\hline Scholes, 2000 & 1150 & & 12.0 & 8.4 & & & 8.0 & 0.0 & & 0.1 & & 1.0 & 1.5 & 0.5 & 3.0 & 0.2 & 0.0 & 1.0 & 10.0 & 0.5 & 0.0 & 1.2 & 1.5 & & 41.5 & & & 3.5 & & & & & 6.0 & 1063 & \\
\hline Scholes, 2000 & 1150 & & 8.4 & 9.2 & & & 4.8 & 0.0 & & 0.1 & & 2.2 & 2.3 & 0.9 & 3.9 & 0.2 & 0.0 & 0.7 & 12.4 & 0.7 & 0.0 & 2.9 & 1.1 & & 41.7 & & & 2.3 & & & & & 6.2 & 953 & \\
\hline Scholes, 2000 & 1150 & & 8.5 & 9.3 & & & 4.9 & 0.0 & & 0.1 & & 2.2 & 2.3 & 0.9 & 4.0 & 0.2 & 0.0 & 0.7 & 12.5 & 0.7 & 0.0 & 2.1 & 1.1 & & 42.1 & & & 2.3 & & & & & 6.2 & 873 & \\
\hline Scholes, 2000 & 1150 & & 15.0 & 15.0 & & & 0.1 & & & 0.0 & & & 0.1 & & 8.5 & 0.1 & 0.0 & 0.0 & 5.0 & & 0.0 & 0.0 & & & 53.1 & & & 0.0 & & & & & 3.0 & 873 & \\
\hline Scholes, 2000 & 1150 & & 3.8 & 12.5 & & & 0.1 & & & 0.1 & & & 0.1 & 2.5 & 6.3 & 0.2 & 0.0 & & 8.8 & & 0.0 & 0.1 & 1.3 & & 54.0 & & & 0.0 & & & & & 10.5 & 883 & \\
\hline Scholes, 2002 & & & 10.0 & 9.0 & 2.0 & & 2.2 & & & 0.1 & & 0.6 & 4.1 & 2.8 & 2.0 & & 0.5 & 0.0 & 17.7 & 0.1 & & & 1.2 & & 45.5 & & & 0.0 & & & & & 1.2 & & \\
\hline Scholes, 2002 & & & 9.8 & 9.0 & 2.0 & & 2.2 & & & 0.1 & & 0.6 & 6.1 & 2.8 & 3.0 & & 0.7 & 0.0 & 14.1 & 0.1 & & & 0.8 & & 46.5 & & & 0.0 & & & & & 1.2 & & \\
\hline Scholes, 2002 & & & 9.8 & 9.0 & 2.0 & & 2.2 & & & 0.1 & & 0.4 & 4.1 & 2.8 & 2.0 & & 0.5 & 0.0 & 14.1 & 0.1 & & & 0.8 & & 50.3 & & & 0.0 & & & & & 0.8 & & \\
\hline Scholes, 2002 & & & 9.8 & 11.0 & 2.0 & & 2.2 & & & 0.1 & & 0.4 & 4.1 & 3.6 & 3.0 & & 0.7 & 0.0 & 14.5 & 0.1 & & & 1.2 & & 45.5 & & & 0.0 & & & & & 0.8 & & \\
\hline Seetharaman, 2000 & & 1355 & & & & & 21.6 & & & & & & 10.0 & & & 9.0 & & & & & & & & & 59.4 & & & & & & & & & & \\
\hline Seetharaman, 2000 & & 1097 & & & & & 22.5 & & & & & & 10.0 & & & 22.5 & & & & & & & & & 45.0 & & & & & & & & & & \\
\hline Seetharaman, 2000 & & 1327 & & & & & 18.8 & & & & & & 25.0 & & & 18.8 & & & & & & & & & 37.5 & & & & & & & & & & \\
\hline Sheng, 2001 & & & 24.4 & 4.2 & & & 2.6 & & & & & & 6.4 & 1.2 & & 1.2 & & & 6.1 & & & & & & 45.5 & 0.3 & & & & & & & & & \\
\hline Sheng, 2001 & & & 23.0 & 6.4 & & & 2.4 & & & & & & 6.0 & 1.1 & & 1.1 & & & 8.9 & & & & & & 43.0 & 0.3 & & & & & & & & & \\
\hline Sheng, 2001 & & & 21.7 & 8.5 & & & 2.3 & & & & & & 5.7 & 1.0 & & 1.1 & & & 11.7 & & & & & & 40.5 & 0.2 & & & & & & & & & \\
\hline Sheng, 2001 & & & 20.3 & 10.6 & & & 2.2 & & & & & & 5.3 & 1.0 & & 1.1 & & & 14.5 & & & & & & 38.0 & 0.2 & & & & & & & & & \\
\hline Sheng, 2001 & & & 19.0 & 12.7 & & & 2.1 & & & & & & 5.0 & 0.9 & & 1.1 & & & 17.2 & & & & & & 35.4 & 0.2 & & & & & & & & & \\
\hline Sheng, 2001 & & & 17.6 & 14.9 & & & 2.0 & & & & & & 4.6 & 0.8 & & 1.1 & & & 20.0 & & & & & & 32.9 & 0.2 & & & & & & & & & \\
\hline Sheng, 2001 & & & 16.3 & 17.0 & & & 1.8 & & & & & & 4.3 & 0.8 & & 1.0 & & & 22.8 & & & & & & 30.4 & 0.2 & & & & & & & & & \\
\hline Sheng, 2001 & & & 13.6 & 21.2 & & & 1.6 & & & & & & 3.6 & 0.7 & & 1.0 & & & 28.4 & & & & & & 25.3 & 0.2 & & & & & & & & & \\
\hline \begin{tabular}{|l|} 
Sipp, 2002 \\
\end{tabular} & & 2050 & 19.4 & & & & & & & & & & & & & & & & 11.8 & & & & & & 68.7 & & & & & & & & & & \\
\hline Sipp, 2002 & & 1318 & 3.2 & 4.5 & & & 7.2 & & & & & & 0.2 & 1.6 & & 3.1 & & & 15.8 & & & & & & 64.3 & & & & & 0.1 & & & & & \\
\hline \begin{tabular}{|l|} 
Sipp, 2002 \\
\end{tabular} & & 1349 & 74.0 & & & & 26.0 & & & & & & & & & & & & & & & & & & & & & & & & & & & & \\
\hline Sipp, 2002 & & 1369 & 58.6 & & & & 32.2 & & & & & & & & & & & & & & & & & & 9.2 & & & & & & & & & & \\
\hline Sipp, 2002 & & 1513 & 22.7 & & & & 37.3 & & & & & & & & & & & & & & & & & & 40.0 & & & & & & & & & & \\
\hline Sipp, 2002 & & 1521 & 36.6 & & & & 20.2 & & & & & & & & & & & & & & & & & & 43.2 & & & & & & & & & & \\
\hline Sipp, 2002 & & 1292 & & & & & 25.9 & & & & & & & & & 18.6 & & & & & & & & & 55.5 & & & & & & & & & & \\
\hline Sipp, 2002 & & 1414 & 14.3 & 6.4 & & & 18.4 & & & & & & 0.5 & 0.3 & & 2.7 & & & 0.5 & & & & & & 56.7 & & & & & 0.1 & & & & & \\
\hline Sipp, 2002 & & 1127 & & & & & & & & & & & & 32.0 & & & & & & & & & & & 40.8 & & & & & 27.2 & & & & & \\
\hline Sipp, 2002 & & 1415 & 0.6 & & & & 8.6 & & & & & & 0.8 & 0.2 & & 3.6 & & & 14.2 & & & & & & 72.0 & & & & & & & & & & \\
\hline Sipp, 2002 & & 1447 & & & & & & & & & & & & & & & & & 20.5 & & & & & & 79.5 & & & & & & & & & & \\
\hline Sipp, 2002 & & 1262 & & & & & & & & & & & & & & & & & 16.2 & & & & & & 62.9 & & & & & 20.9 & & & & & \\
\hline \begin{tabular}{|l|} 
Solvang, 2002 \\
\end{tabular} & & 1432 & 36.1 & & & & 20.5 & & & & & & & & & & & & & & & & & & 42.6 & & & & & & & & & & \\
\hline Solvang, 2002 & & 1257 & 13.5 & & & & 38.1 & & & & & & & & & & & & & & & & & & 47.7 & & & & & & & & & & \\
\hline \begin{tabular}{|l|} 
Solvang, 2002 \\
\end{tabular} & & 1255 & 36.4 & & & & 37.2 & & & & & & & & & & & & & & & & & & 25.5 & & & & & & & & & & \\
\hline Solvang, 2002 & & 1251 & 16.9 & & & & 39.0 & & & & & & & & & & & & & & & & & & 43.4 & & & & & & & & & & \\
\hline Solvang, 2002 & & 1242 & 20.2 & & & & 40.4 & & & & & & & & & & & & & & & & & & 38.8 & & & & & & & & & & \\
\hline Solvang, 2002 & & 1237 & 23.2 & & & & 41.5 & & & & & & & & & & & & & & & & & & 34.6 & & & & & & & & & & \\
\hline Solvang, 2002 & & 1155 & 25.6 & & & & 43.3 & & & & & & & & & & & & & & & & & & 30.3 & & & & & & & & & & \\
\hline Solvang, 2002 & & 1332 & 23.6 & & & & 30.1 & & & & & & & & & & & & & & & & & & 45.8 & & & & & & & & & & \\
\hline \begin{tabular}{|l|} 
Solvang, 2002 \\
\end{tabular} & & 1305 & 26.8 & & & & 32.6 & & & & & & & & & & & & & & & & & & 40.0 & & & & & & & & & & \\
\hline Solvang, 2002 & & 1287 & 30.3 & & & & 33.9 & & & & & & & & & & & & & & & & & & 35.4 & & & & & & & & & & \\
\hline Solvang, 2002 & & 1256 & 33.6 & & & & 35.0 & & & & & & & & & & & & & & & & & & 30.6 & & & & & & & & & & \\
\hline Solvang, 2002 & & 1208 & 37.1 & & & & 41.1 & & & & & & & & & & & & & & & & & & 21.3 & & & & & & & & & & \\
\hline Solvang, 2002 & & 1168 & 0.2 & & & & 48.1 & & & & & & & & & & & & & & & & & & 50.9 & & & & & & & & & & \\
\hline
\end{tabular}


Table A1. Glass Compositions from Literature Review (continued)

\begin{tabular}{|c|c|c|c|c|c|c|c|c|c|c|c|c|c|c|c|c|c|c|c|c|c|c|c|c|c|c|c|c|c|c|c|c|c|c|c|}
\hline Ref & 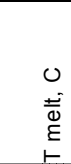 & $\begin{array}{l}0 \\
\stackrel{0}{2} \\
\stackrel{0}{0} \\
上\end{array}$ & 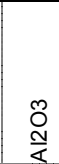 & 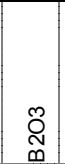 & $\begin{array}{l}\mathscr{D} \\
\infty\end{array}$ & 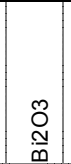 & $\begin{array}{l}0 \\
\mathbb{\sigma} \\
0\end{array}$ & 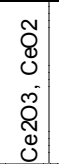 & $\begin{array}{l}\text { Oొ } \\
\text { N } \\
0 \\
0 \\
0 \\
0\end{array}$ & 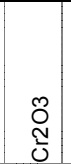 & $\stackrel{0}{3}$ & L & 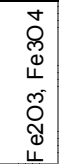 & ָิ & $\stackrel{\cap}{\beth}$ & $\begin{array}{l}\circ \\
\sum\end{array}$ & $\begin{array}{l}\tilde{O} \\
\sum \\
\sum\end{array}$ & $\begin{array}{l}00 \\
0 \\
\Sigma\end{array}$ & $\begin{array}{l}\stackrel{O}{N} \\
\underset{Z}{Z}\end{array}$ & $\frac{O}{z}$ & $\begin{array}{l}\mathcal{N} \\
\tilde{0}\end{array}$ & $\overline{\bar{I}}$ & مి & $\frac{8}{0}$ & $\frac{\tilde{O}}{\infty}$ & $\begin{array}{l}\text { Oొ } \\
\text { C) }\end{array}$ & o & 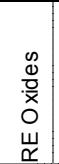 & 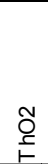 & $\stackrel{\widetilde{o}}{\stackrel{\mathrm{o}}{1}}$ & 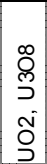 & $\stackrel{q}{N}$ & ָั & 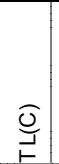 & $\begin{array}{l}\vec{\Xi} \\
\stackrel{0}{\underline{\underline{U}}} \\
\bar{z}\end{array}$ \\
\hline Soper, 1982 & & & 14.7 & 10.5 & & & 0.4 & & & & & & 4.0 & 0.3 & 4.1 & 1.4 & 3.2 & & 14.7 & 0.6 & & & & & 44.7 & & & 0.4 & & 0.7 & & & 0.4 & & \\
\hline Soper, 1982 & & 1143 & 14.7 & 10.5 & & & 0.4 & & & & & & 4.0 & 0.3 & 2.8 & 1.4 & 3.2 & & 14.6 & 0.6 & & & & & 45.4 & & & 0.7 & & 1.4 & & & & & \\
\hline Soper, 1982 & & & 14.7 & 10.1 & & & 0.4 & & & & & & 4.0 & 0.3 & 2.7 & 0.6 & 3.2 & & 10.8 & 0.6 & & & & & 50.5 & & & & & 1.4 & & & 0.6 & & \\
\hline Soper, 1982 & & & 14.7 & 9.9 & & & 0.4 & & & & & & 4.0 & 0.3 & 4.0 & 1.4 & 3.2 & & 10.6 & 0.6 & & & & & 49.7 & & & & & 1.4 & & & & & \\
\hline Soper, 1982 & & 1130 & 14.7 & 10.6 & & & 0.4 & & & & & & 4.0 & 0.3 & 4.2 & 0.7 & 3.2 & & 14.7 & 0.6 & & & & & 45.8 & & & & & & & & 0.7 & & \\
\hline Soper, 1982 & & & 14.7 & 7.9 & & & 0.4 & & & & & & 4.0 & 0.3 & 4.0 & 1.4 & 3.2 & & 13.9 & 0.6 & & & & & 49.7 & & & & & & & & & & \\
\hline Soper, 1982 & & 1150 & 14.7 & 7.9 & & & 0.4 & & & & & & 4.0 & 0.3 & 4.0 & 1.4 & 3.2 & & 13.9 & 0.6 & & & & & 48.4 & & & & & & & & & & \\
\hline Soper, 1982 & & & 14.7 & 9.5 & & & 0.4 & & & & & & 4.0 & 0.3 & 3.1 & 0.8 & 3.2 & & 12.3 & 0.6 & & & & & 51.0 & & & & & & & & & & \\
\hline Soper, 1982 & & 1148 & 14.7 & 10.9 & & & 0.4 & & & & & & 4.0 & 0.3 & 4.4 & 1.4 & 3.2 & & 11.5 & 0.6 & & & & & 47.1 & & & 0.7 & & & & & 0.7 & & \\
\hline Soper, 1982 & & & 14.7 & 8.3 & & & 0.4 & & & & & & 4.0 & 0.3 & 2.8 & 1.4 & 3.2 & & 11.0 & 0.6 & & & & & 51.9 & & & 0.7 & & & & & 0.7 & & \\
\hline Soper, 1982 & & 1152 & 14.7 & 8.6 & & & 0.4 & & & & & & 4.0 & 0.3 & 2.9 & 1.4 & 3.2 & & 15.0 & 0.6 & & & & & 46.7 & & & & & 1.4 & & & 0.7 & & \\
\hline Soper, 1982 & & 1148 & 14.7 & 9.0 & & & 0.4 & & & & & & 4.0 & 0.3 & 4.5 & 0.8 & 3.2 & & 11.8 & 0.6 & & & & & 48.5 & & & 0.8 & & 1.5 & & & & & \\
\hline Soper, 1982 & & 1177 & 14.7 & 9.8 & & & 0.4 & & & & & & 4.0 & 0.3 & 2.6 & 0.6 & 3.2 & & 13.8 & 0.6 & & & & & 49.2 & & & 0.6 & & & & & & & \\
\hline Soper, 1982 & & & 14.7 & 7.8 & & & 0.4 & & & & & & 4.0 & 0.3 & 4.5 & 0.5 & 3.2 & & 11.9 & 0.6 & & & & & 51.6 & & & & & & & & & & \\
\hline Soper, 1982 & & & 14.7 & 8.1 & & & 0.4 & & & & & & 4.0 & 0.3 & 4.6 & 1.3 & 3.2 & & 15.8 & 0.6 & & & & & 46.4 & & & 0.6 & & & & & & & \\
\hline Soper, 1982 & & & 14.7 & 7.4 & & & 0.4 & & & & & & 4.0 & 0.3 & 3.0 & 0.5 & 3.2 & & 14.8 & 0.6 & & & & & 49.8 & & & & & 1.4 & & & & & \\
\hline Soper, 1982 & & & 14.7 & 9.9 & & & 0.4 & & & & & & 4.0 & 0.3 & 4.5 & 0.5 & 3.2 & & 15.4 & 0.6 & & & & & 45.1 & & & & & 1.4 & & & & & \\
\hline Soper, 1982 & & & 14.7 & 9.3 & & & 0.4 & & & & & & 4.0 & 0.3 & 2.5 & 0.6 & 3.2 & & 10.4 & 0.6 & & & & & 52.3 & & & & & 1.4 & & & 0.3 & & \\
\hline Soper, 1982 & & & 14.7 & 6.9 & & & 0.4 & & & & & & 4.0 & 0.3 & 2.9 & 1.1 & 3.2 & & 11.3 & 0.6 & & & & & 52.9 & & & 0.4 & & 1.4 & & & & & \\
\hline Soper, 1982 & & & 14.7 & 9.8 & & & 0.4 & & & & & & 4.0 & 0.3 & 3.2 & 1.2 & 3.2 & & 12.2 & 0.6 & & & & & 50.3 & & & & & & & & & & \\
\hline Soper, 1982 & & & 14.7 & 7.6 & & & 0.4 & & & & & & 4.0 & 0.3 & 2.6 & 1.3 & 3.2 & & 10.7 & 0.6 & & & & & 54.0 & & & 0.4 & & & & & 0.3 & & \\
\hline Soper, 1982 & & & 14.7 & 9.3 & & & 0.4 & & & & & & 4.0 & 0.3 & 2.9 & 0.7 & 3.2 & & 11.3 & 0.6 & & & & & 51.6 & & & & & 0.7 & & & 0.4 & & \\
\hline Soper, 1982 & & & 14.7 & 9.3 & & & 0.4 & & & & & & 4.0 & 0.3 & 3.2 & 0.7 & 3.2 & & 10.6 & 0.6 & & & & & 51.9 & & & & & 0.7 & & & 0.4 & & \\
\hline Soper, 1982 & & & 14.7 & 8.6 & & & 0.4 & & & & & & 4.0 & 0.3 & 3.9 & 0.7 & 3.2 & & 12.0 & 0.6 & & & & & 51.2 & & & & & & & & 0.4 & & \\
\hline Soper, 1982 & & & 14.7 & 7.1 & & & 0.4 & & & & & & 4.0 & 0.3 & 5.0 & 0.7 & 3.2 & & 11.3 & 0.6 & & & & & 51.9 & & & & & & & & 0.7 & & \\
\hline Soper, 1982 & & & 14.7 & 8.6 & & & 0.4 & & & & & & 4.0 & 0.3 & 3.9 & 0.7 & 3.2 & & 12.0 & 0.6 & & & & & 51.2 & & & & & & & & 0.4 & & \\
\hline Sridharam, 2005 & & & & & 1.4 & 70.3 & & & & & & & & 1.0 & 0.5 & & & & & & & & & & 21.3 & & & 3.1 & & & & 2.4 & & & \\
\hline Sridharam, 2005 & & & & & 2.2 & 54.1 & & & & 4.5 & & & & & 1.8 & & 13.5 & & & & & & & & 19.0 & & & 2.8 & & & & 2.1 & & & \\
\hline Sridharam, 2005 & & & & & 1.4 & 70.8 & & & & & & & & 1.0 & 0.5 & & & & & & & & & & 21.5 & & & & & 2.4 & & 2.4 & & & \\
\hline Sridharam, 2005 & & & & & 1.4 & 69.2 & & & & & & & & 1.0 & 0.5 & & & & & & & & & & 21.0 & & & & & 4.6 & & 2.3 & & & \\
\hline Sridharam, 2005 & & & & 4.8 & & 48.4 & & & & & & & & 4.9 & 1.6 & & & & & & & & & & 28.2 & & & & & 12.1 & & & & & \\
\hline Sridharam, 2005 & & & & 4.8 & & 48.4 & & & & & & & & 4.9 & 1.6 & & & & & & & & & & 20.2 & & & & & 20.1 & & & & & \\
\hline Sridharam, 2005 & & & & 3.3 & & 62.8 & & & & & & & & 3.4 & 1.1 & & & & & & & & & & 15.9 & & & & & 8.4 & & 5.1 & & & \\
\hline Sridharam, 2005 & & & & 3.6 & & 66.5 & & & & & & & & 3.6 & 0.6 & & & & & & & & & & 16.8 & & & & & 8.9 & & & & & \\
\hline Sridharam, 2005 & & & & 5.8 & & 8.8 & & & & & & & & 4.4 & 1.4 & & & & & & & & & & 32.1 & & & & & 23.3 & & 24.2 & & & \\
\hline Sridharam, 2005 & & & & 5.2 & & 23.1 & & & & & & & & 4.2 & 1.2 & & & & & & & & & & 28.3 & & & & & 19.8 & & 18.2 & & & \\
\hline Sridharam, 2005 & & & & 3.0 & & 57.5 & & & & 13.5 & & & & 3.1 & 0.6 & & & & & & & & & & 14.6 & & & & & 7.7 & & & & & \\
\hline Sridharam, 2005 & & & & 3.4 & & 63.3 & & & & & 4.8 & & & 3.4 & 0.6 & & & & & & & & & & 16.0 & & & & & 8.5 & & & & & \\
\hline Staples, 1999 & 1150 & 1143 & 15.0 & 15.0 & 0.0 & & 0.1 & 0.1 & & & 0.1 & & 0.1 & & 8.5 & & & 0.0 & 5.0 & & & 0.1 & & & 53.1 & & 0.0 & & & & & & 3.0 & & \\
\hline Staples, 1999 & 1150 & 1183 & 13.1 & 4.4 & 0.0 & & 0.1 & 0.1 & & & 0.1 & & 0.0 & 8.7 & 7.2 & & & 0.0 & \begin{tabular}{|l|}
8.2 \\
\end{tabular} & & & 0.1 & 13.1 & & 42.2 & & 0.0 & & & & & & 2.6 & & \\
\hline Staples, 1999 & 1150 & 1172 & 14.6 & 5.0 & 0.0 & & 0.1 & 0.1 & & & 0.1 & & 0.1 & & 5.1 & & & 0.0 & 20.0 & & & 0.1 & & & 54.8 & & 0.0 & & & & & & & & \\
\hline Staples, 1999 & 1150 & 977 & 7.6 & 5.0 & 0.0 & & 0.1 & 0.1 & & & 0.1 & & 0.1 & 10.0 & 7.1 & & & 0.0 & 17.2 & & & 0.1 & & & 52.7 & & 0.0 & & & & & & & & \\
\hline Staples, 1999 & 1150 & 1119 & 10.9 & 7.2 & 0.0 & & 0.1 & 0.1 & & & 0.1 & & 0.1 & 2.4 & 5.1 & & & 0.0 & 16.7 & & & 0.1 & 3.6 & & 50.2 & & 0.0 & & & & & & 3.4 & & \\
\hline Staples, 1999 & 1150 & 1125 & 7.2 & 7.2 & 0.0 & & 0.1 & 0.1 & & & 0.1 & & 0.1 & 2.4 & 4.6 & & & 0.0 & 16.7 & & & 0.1 & 3.6 & & 47.5 & & 0.0 & & & & & & 10.1 & & \\
\hline Staples, 1999 & 1150 & 1041 & 8.9 & 12.1 & 0.0 & & 0.1 & 0.1 & & & 0.1 & & 0.1 & 7.2 & 6.5 & & & 0.0 & 9.5 & & & 0.1 & 3.6 & & 43.2 & & 0.0 & & & & & & 8.5 & & \\
\hline Staples, 1999 & 1150 & & 10.1 & 11.2 & 0.0 & & 0.1 & 0.1 & & & 0.1 & & 0.1 & 2.3 & 6.1 & & & 0.0 & 13.2 & & & 0.1 & 10.2 & & 40.4 & & 0.0 & & & & & & 6.1 & & \\
\hline Staples, 1999 & 1150 & 1121 & 9.8 & 6.8 & 0.0 & & 0.1 & 0.1 & & & 0.1 & & 0.1 & 6.6 & 6.1 & & & 0.0 & 10.9 & & & 0.1 & 10.2 & & 42.8 & & 0.0 & & & & & & 6.5 & & \\
\hline Staples, 1999 & 1150 & 1037 & 8.6 & 12.1 & 0.0 & & 0.1 & 0.1 & & & 0.1 & & 0.1 & 7.2 & 6.5 & & & 0.0 & 9.7 & & & 0.1 & 3.6 & & 43.0 & & 0.0 & & & & & & 8.8 & & \\
\hline Staples, 1999 & 1150 & 1106 & 7.2 & 7.2 & 0.0 & & 0.1 & 0.1 & & & 0.1 & & 0.1 & 7.2 & 4.9 & & & 0.0 & 13.3 & & & 0.1 & 3.6 & & 45.8 & & 0.0 & & & & & & 10.1 & & \\
\hline Staples, 1999 & 1150 & 1178 & 13.1 & 4.4 & 0.0 & & 0.1 & 0.1 & & & 0.1 & & 0.0 & 8.7 & 7.2 & & & 0.0 & 8.2 & & & 0.1 & 13.1 & & 42.2 & & 0.0 & & & & & & 2.6 & & \\
\hline
\end{tabular}


Table A1. Glass Compositions from Literature Review (continued)

\begin{tabular}{|c|c|c|c|c|c|c|c|c|c|c|c|c|c|c|c|c|c|c|c|c|c|c|c|c|c|c|c|c|c|c|c|c|c|c|c|}
\hline Ref & 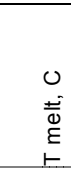 & $\begin{array}{l}0 \\
0 \\
0 \\
0 \\
0 \\
⺊\end{array}$ & $\begin{array}{l}\text { Oొ } \\
\text { N̦ }\end{array}$ & ల్లి & $\begin{array}{l}\mathbb{D} \\
\infty\end{array}$ & $\begin{array}{l}\text { Oొ } \\
\stackrel{N}{\infty}\end{array}$ & $\begin{array}{l}0 \\
\mathbb{\pi} \\
0\end{array}$ & 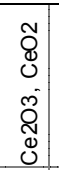 & $\begin{array}{l}\text { Oొ } \\
\text { Oे } \\
0 \\
0 \\
0 \\
0\end{array}$ & Õ & $\begin{array}{l}0 \\
3 \\
0\end{array}$ & 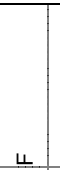 & 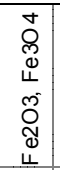 & 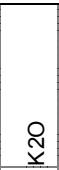 & $\stackrel{\stackrel{ }{J}}{ }$ & $\stackrel{\circ}{D^{\circ}}$ & $\begin{array}{l}\text { Õ } \\
\text { L } \\
\Sigma\end{array}$ & $\begin{array}{l}0 \\
\text { O̊ } \\
\Sigma\end{array}$ & $\begin{array}{l}\underset{\sim}{0} \\
\underset{Z}{Z}\end{array}$ & $\stackrel{\circ}{\bar{z}}$ & $\begin{array}{l}\tilde{N} \\
\tilde{0} \\
0\end{array}$ & $\overline{\bar{c}}$ & $\begin{array}{l}\text { ¿ి } \\
0 \\
0\end{array}$ & $\frac{8}{0}$ & $\stackrel{\widetilde{O}}{\infty}$ & $\begin{array}{l}\text { Oొ } \\
\text { ஸ) }\end{array}$ & o & 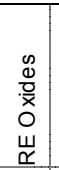 & ઠิ & $\stackrel{\widetilde{N}}{\stackrel{\sigma}{F}}$ & 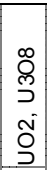 & $\stackrel{Q}{N}$ & $\begin{array}{l}\tilde{D} \\
\text { N }\end{array}$ & $\underset{1}{0}$ & $\begin{array}{l}\bar{\Xi} \\
\text { J్ } \\
\underline{\underline{v}} \\
\dot{z}\end{array}$ \\
\hline Staples, 1999 & 1150 & 1176 & 13.1 & 4.4 & 0.0 & & 0.1 & 0.1 & & & 0.1 & & 0.0 & 8.7 & 7.2 & & & 0.0 & 8.2 & & & 0.1 & 13.1 & & 42.2 & & 0.0 & & & & & & 2.6 & & \\
\hline Staples, 1999 & 1150 & 1185 & 14.0 & 8.4 & 0.1 & & & 0.0 & & 0.0 & 0.0 & & 2.0 & & 7.0 & & 0.0 & 0.1 & 10.6 & 0.1 & & 0.1 & 0.1 & & 57.0 & & 0.1 & 0.2 & & & & & & & \\
\hline Staples, 1999 & 1150 & 963 & 15.0 & 15.0 & 0.0 & & 0.1 & 0.1 & & & 0.1 & & 0.1 & & 9.0 & & & & 11.9 & & & 0.2 & & 0.0 & 45.7 & & 0.0 & & & & & & 3.0 & & \\
\hline Staples, 1999 & 1150 & 1195 & 15.0 & 5.0 & 0.0 & & 0.1 & 0.1 & & & 0.1 & & 0.1 & 10.0 & 8.4 & & & & 5.0 & & & 0.2 & 5.0 & 0.0 & 51.2 & & 0.0 & & & & & & & & \\
\hline Staples, 1999 & 1150 & 1139 & 7.5 & 15.0 & 0.0 & & 0.1 & 0.1 & & & 0.1 & & 0.1 & & 0.2 & & & & 20.0 & & & 0.2 & 5.0 & 0.0 & 51.9 & & 0.0 & & & & & & & & \\
\hline Staples, 1999 & 1150 & 1015 & 7.5 & 5.0 & 0.0 & & 0.1 & 0.1 & & & 0.1 & & 0.1 & 10.0 & 9.0 & & & & 11.7 & & & 0.2 & & 0.0 & 56.3 & & 0.0 & & & & & & & & \\
\hline Staples, 1999 & 1150 & 1093 & 7.6 & 10.1 & 0.0 & & 0.1 & 0.1 & & & 0.1 & & 0.1 & 5.0 & 5.7 & & & & 11.9 & & & 0.2 & 2.6 & 0.0 & 49.0 & & 0.0 & & & & & & 7.6 & & \\
\hline Staples, 1999 & 1150 & 1024 & 7.5 & 12.5 & 0.0 & & 0.1 & 0.1 & & & 0.1 & & 0.1 & 7.5 & 6.8 & & & & 8.8 & & & 0.2 & 1.3 & 0.0 & 44.7 & & 0.0 & & & & & & 10.5 & & \\
\hline Staples, 1999 & 1150 & 1129 & 10.8 & 7.5 & 0.0 & & 0.1 & 0.1 & & & 0.1 & & 0.1 & 7.3 & 6.8 & & & & 8.8 & & & 0.2 & 3.8 & 0.0 & 47.4 & & 0.0 & & & & & & 7.2 & & \\
\hline Staples, 1999 & 1150 & 1123 & 7.0 & 12.5 & 0.0 & & 0.1 & 0.1 & & & 0.1 & & 0.1 & 2.5 & 2.5 & & & & 16.3 & & & 0.2 & 3.8 & 0.0 & 44.5 & & 0.0 & & & & & & 10.5 & & \\
\hline Staples, 1999 & 1250 & 1325 & 19.9 & 5.0 & 0.0 & & & 0.0 & & 0.1 & & 6.0 & & & & 0.2 & 0.0 & 0.0 & 19.9 & 1.5 & 0.0 & 0.1 & & 0.0 & 47.2 & 0.2 & 0.1 & 0.0 & & & & & & & \\
\hline Staples, 1999 & 1250 & 1347 & 19.9 & 17.9 & 0.0 & & 11.9 & 0.0 & & 0.1 & & & & & & 0.2 & 0.0 & 0.0 & 5.0 & & 0.0 & 0.1 & 3.0 & 0.0 & 41.7 & 0.2 & 0.1 & 0.0 & & & & & & & \\
\hline Staples, 1999 & 1150 & 944 & 19.9 & 17.9 & 0.0 & & & 0.0 & & 0.1 & & & & 9.9 & 8.9 & 0.2 & 0.0 & 0.0 & 5.0 & 1.5 & 0.0 & 0.1 & & 0.0 & 34.7 & 0.2 & 0.1 & 0.0 & & & & & 1.5 & & \\
\hline Staples, 1999 & 1250 & 1254 & 7.9 & 5.0 & 0.0 & & 11.9 & 0.0 & & 0.1 & & 6.0 & & 9.9 & & 0.2 & 0.0 & 0.0 & 5.0 & 1.5 & 0.0 & 0.1 & 3.0 & 0.0 & 35.2 & 0.2 & 0.1 & 0.0 & & & & & 13.9 & & \\
\hline Staples, 1999 & 1150 & 975 & 19.9 & 5.0 & 0.0 & & 11.9 & 0.0 & & 0.1 & & 6.0 & 7.9 & & 7.4 & 0.2 & 0.0 & 0.0 & 5.0 & & 0.0 & 0.1 & & 0.0 & 36.3 & 0.2 & 0.1 & 0.0 & & & & & & & \\
\hline Staples, 1999 & 1250 & 1400 & 19.9 & 6.1 & 0.0 & & & 0.0 & & 0.1 & & & 7.9 & 9.9 & & 0.2 & 0.0 & 0.0 & 14.9 & & 0.0 & 0.1 & 3.0 & 0.0 & 35.6 & 0.2 & 0.1 & 0.0 & & & & & 2.0 & & \\
\hline Staples, 1999 & 1150 & 1050 & 7.9 & 6.0 & 0.0 & & 4.0 & 0.0 & & 0.1 & & 1.0 & 0.5 & 3.0 & 6.0 & 0.2 & 0.0 & 0.0 & 14.9 & 0.5 & 0.0 & 0.1 & 2.0 & 0.0 & 49.6 & 0.2 & 0.1 & 0.0 & & & & & 4.0 & & \\
\hline Staples, 1999 & 1150 & 1077 & 10.9 & 11.9 & 0.0 & & 4.0 & 0.0 & & 0.1 & & 1.0 & 0.5 & 3.0 & 3.0 & 0.2 & 0.0 & 0.0 & 14.9 & 0.5 & 0.0 & 0.1 & 2.0 & 0.0 & 39.7 & 0.2 & 0.1 & 0.0 & & & & & 7.9 & & \\
\hline Staples, 1999 & 1250 & 1175 & 7.9 & 6.0 & 0.0 & & 7.9 & 0.0 & & 0.1 & & 2.5 & 0.5 & 3.0 & 3.0 & 0.2 & 0.0 & 0.0 & 9.9 & 0.5 & 0.0 & 0.1 & 2.0 & 0.0 & 48.1 & 0.2 & 0.1 & 0.0 & & & & & 7.9 & & \\
\hline Staples, 1999 & 1250 & 1125 & 11.9 & 6.0 & 0.0 & & 4.0 & 0.0 & & 0.1 & & 1.0 & 3.0 & 6.0 & 3.0 & 0.2 & 0.0 & 0.0 & 14.9 & 0.5 & 0.0 & 0.1 & 1.0 & 0.0 & 40.2 & 0.2 & 0.1 & 0.0 & & & & & 7.9 & & \\
\hline Staples, 1999 & 1150 & 1043 & 7.9 & 6.0 & 0.0 & & 4.0 & 0.0 & & 0.1 & & 1.0 & 0.5 & 3.0 & 6.0 & 0.2 & 0.0 & 0.0 & 14.9 & 1.0 & 0.0 & 0.1 & 2.0 & 0.0 & 49.1 & 0.2 & 0.1 & 0.0 & & & & & 4.0 & & \\
\hline Staples, 1999 & 1150 & 1115 & 7.9 & 6.0 & 0.0 & & 4.0 & 0.0 & & 0.1 & & 1.0 & 0.5 & 6.0 & 3.0 & 0.2 & 0.0 & 0.0 & 14.9 & 1.0 & 0.0 & 0.1 & 2.0 & 0.0 & 49.1 & 0.2 & 0.1 & 0.0 & & & & & 4.0 & & \\
\hline Staples, 1999 & 1150 & 1167 & 7.9 & 6.0 & 0.0 & & 7.9 & 0.0 & & 0.1 & & 1.0 & 2.0 & 6.0 & 3.0 & 0.2 & 0.0 & 0.0 & 9.9 & 1.0 & 0.0 & 0.1 & 1.0 & 0.0 & 49.6 & 0.2 & 0.1 & 0.0 & & & & & 4.0 & & \\
\hline Staples, 1999 & 1250 & 1115 & 11.3 & 6.5 & 0.0 & & 3.8 & 0.0 & & 0.1 & & 0.9 & 0.5 & 21.0 & 6.1 & 0.2 & 0.0 & & 1.4 & 0.4 & 0.0 & 0.1 & 1.7 & 0.0 & 38.2 & 0.2 & 0.0 & 0.0 & & & & & 7.5 & & \\
\hline Staples, 1999 & 1150 & 1058 & 10.5 & 11.9 & 0.0 & & 4.0 & 0.0 & & 0.1 & & 1.4 & 0.5 & 3.0 & 3.0 & 0.2 & 0.0 & 0.0 & 14.9 & 0.5 & 0.0 & 0.1 & 2.0 & 0.0 & 39.7 & 0.2 & 0.1 & 0.0 & & & & & 7.9 & & \\
\hline Staples, 1999 & 1250 & 1059 & 11.9 & 6.0 & 0.0 & & 7.9 & 0.0 & & 0.1 & & 1.0 & 0.5 & 6.0 & 5.6 & 0.2 & 0.0 & 0.0 & 9.9 & 1.0 & 0.0 & 0.1 & 1.0 & 0.0 & 40.5 & 0.2 & 0.1 & 0.0 & & & & & 7.9 & & \\
\hline Staples, 1999 & 1250 & 1062 & 11.9 & 6.0 & 0.0 & & 7.9 & 0.0 & & 0.1 & & 1.0 & 0.5 & 6.0 & 5.8 & 0.2 & 0.0 & 0.0 & 9.9 & 0.5 & 0.0 & 0.1 & 1.0 & 0.0 & 40.9 & 0.2 & 0.1 & 0.0 & & & & & 7.9 & & \\
\hline Staples, 1999 & 1150 & 1167 & 8.2 & 6.1 & 0.0 & & 2.6 & 0.0 & & 0.1 & & & 0.5 & 6.1 & 5.1 & 0.2 & 0.0 & 0.0 & 10.2 & 0.5 & 0.0 & 0.1 & 1.0 & & 50.9 & \begin{tabular}{|l|}
0.2 \\
\end{tabular} & 0.1 & 0.0 & & & & & 8.1 & & \\
\hline Staples, 1999 & 1250 & 1185 & 9.8 & 6.0 & 0.0 & & 7.9 & 0.0 & & 0.1 & & 2.5 & 0.5 & 3.0 & 3.1 & 0.2 & 0.0 & 0.0 & 9.9 & 1.0 & 0.0 & 0.1 & 2.0 & 0.0 & 49.6 & 0.2 & 0.1 & 0.0 & & & & & 4.0 & & \\
\hline Staples, 1999 & 1150 & 1124 & 7.9 & 6.0 & 0.0 & & 7.4 & 0.0 & & 0.1 & & 1.0 & 3.0 & 6.0 & 3.0 & 0.2 & 0.0 & 0.0 & 9.9 & 1.0 & 0.0 & 0.1 & 1.0 & 0.0 & 49.2 & 0.2 & 0.1 & 0.0 & & & & & 4.0 & & \\
\hline Staples, 1999 & 1150 & 1068 & 9.9 & 9.1 & & & 4.9 & & & & & 2.1 & 2.6 & 4.4 & 3.9 & & & & 11.5 & 0.7 & & & 1.4 & & 43.6 & & & & & & & & 5.8 & & \\
\hline Staples, 1999 & 1150 & 1160 & 15.0 & 15.0 & 0.0 & & 0.1 & 0.1 & & & & & 0.0 & & 8.5 & & & 0.0 & 5.0 & & & 0.1 & & & 53.1 & & 0.0 & & & & & & 3.0 & & \\
\hline Staples, 1999 & 1150 & 1081 & 9.8 & 9.1 & 0.0 & & 4.9 & 0.0 & & 0.1 & & 2.1 & 2.5 & 4.4 & 3.9 & 0.2 & 0.0 & 0.0 & 11.5 & 0.7 & 0.0 & 0.1 & 1.4 & 0.0 & 43.3 & 0.2 & 0.1 & 0.0 & & & & & 5.8 & & \\
\hline Staples, 1999 & 1150 & 1055 & 9.8 & 9.1 & 0.0 & & 4.9 & 0.0 & & 0.1 & & 2.1 & 2.5 & 4.4 & 3.9 & 0.2 & 0.0 & 0.0 & 11.5 & 0.7 & 0.0 & 0.1 & 1.4 & 0.0 & 43.3 & 0.2 & 0.1 & 0.0 & & & & & 5.7 & & \\
\hline Staples, 1999 & 1250 & 1076 & 9.6 & 8.9 & 0.0 & & 4.8 & 0.0 & & 0.1 & & 2.0 & 2.5 & 4.3 & 3.8 & 0.2 & 0.0 & 2.0 & 11.2 & 0.7 & 0.0 & 0.1 & 1.4 & 0.0 & 42.4 & 0.2 & 0.1 & 0.0 & & & & & 5.6 & & \\
\hline Staples, 1999 & 1150 & 1068 & 9.8 & 9.0 & 0.0 & & 4.8 & 0.0 & & 0.1 & & 2.1 & 2.5 & 4.3 & 3.9 & 0.2 & 0.0 & 0.5 & 11.4 & 0.7 & 0.0 & 0.1 & 1.4 & 0.0 & 43.0 & 0.2 & 0.1 & 0.0 & & & & & 5.7 & & \\
\hline Staples, 1999 & 1150 & 1064 & 9.6 & 8.9 & 0.0 & & 4.8 & 0.0 & & 0.1 & & 2.0 & 2.5 & 4.3 & 3.8 & 0.2 & 0.0 & 0.0 & 11.2 & 0.7 & 0.0 & 0.1 & 1.4 & 0.0 & 42.4 & 0.2 & 2.1 & 0.0 & & & & & 5.6 & & \\
\hline Staples, 1999 & 1150 & 1067 & 9.4 & 8.7 & 0.0 & & 4.7 & 0.0 & & 0.1 & & 2.0 & 2.4 & 4.2 & 3.7 & 0.2 & 0.0 & 0.0 & 11.0 & 0.7 & 0.0 & 0.1 & 1.4 & 0.0 & 41.5 & 0.2 & 4.1 & 0.0 & & & & & 5.5 & & \\
\hline Staples, 2000 & & 1317 & 19.9 & 5.0 & & & & & & & & 6.0 & & & & & & & 19.9 & 1.5 & & 0.7 & & & 47.2 & & & & & & & & & & 0.5 \\
\hline Staples, 2000 & & 1341 & 19.9 & 17.9 & & & 11.9 & & & & & & & & & & & & 5.0 & & & 0.7 & 3.0 & & 41.7 & & & & & & & & & & 0.2 \\
\hline Staples, 2000 & & NM & 3.5 & 17.9 & & & & & & & & 4.0 & 7.9 & & & & & & 5.0 & 1.5 & & 0.7 & & & 59.6 & & & & & & & & & & 24.8 \\
\hline Staples, 2000 & & 938 & 19.9 & 17.9 & & & & & & & & & & 9.9 & 8.9 & & & & 5.0 & 1.5 & & 0.7 & & & 34.7 & & & & & & & & 1.5 & & 5.8 \\
\hline Staples, 2000 & & 1065 & 3.5 & 5.0 & & & 11.9 & & & & & & 7.9 & 1.0 & & & & & 19.9 & 1.5 & & 0.7 & & & 34.8 & & & & & & & & 13.9 & & 1.0 \\
\hline Staples, 2000 & & 913 & 6.5 & 17.9 & & & & & & & & & 7.9 & & 8.9 & & & & 5.0 & 1.5 & & 0.7 & 3.0 & & 34.8 & & & & & & & & 13.9 & & 4.1 \\
\hline Staples, 2000 & & 1253 & 7.9 & 5.0 & & & 11.9 & & & & & 6.0 & & 9.9 & & & & & 5.0 & 1.5 & & 0.7 & 3.0 & & 35.3 & & & & & & & & 13.9 & & 0.6 \\
\hline Staples, 2000 & & 966 & 3.5 & 17.9 & & & 11.9 & & & & & & & 9.9 & & & & & 12.7 & & & 0.7 & & & 43.4 & & & & & & & & & & 0.9 \\
\hline Staples, 2000 & & 970 & 19.9 & 5.0 & & & 11.9 & & & & & 6.0 & 7.9 & & 7.4 & & & & 5.0 & & & 0.7 & & & 36.3 & & & & & & & & & & 0.2 \\
\hline Staples, 2000 & & 1028 & 3.5 & 5.0 & & & & & & & & 6.0 & 7.9 & 9.9 & 8.3 & & & & 5.0 & & & 0.7 & 3.0 & & 50.7 & & & & & & & & & & 2.8 \\
\hline Staples, 2000 & & 939 & 3.5 & 5.0 & & & 7.5 & & & & & & & & 8.9 & & & & 15.9 & 1.5 & & 0.7 & 3.0 & & 54.1 & & & & & & & & & & 4.0 \\
\hline Staples, 2000 & & 1010 & 3.5 & 17.9 & & & & & & & & 6.0 & & & 0.5 & & & & 19.9 & & & 0.7 & & & 40.4 & & & & & & & & 11.2 & & 23.4 \\
\hline
\end{tabular}


Table A1. Glass Compositions from Literature Review (continued)

\begin{tabular}{|c|c|c|c|c|c|c|c|c|c|c|c|c|c|c|c|c|c|c|c|c|c|c|c|c|c|c|c|c|c|c|c|c|c|c|c|}
\hline Ref & 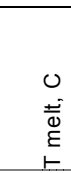 & $\begin{array}{l}0 \\
\frac{0}{2} \\
\stackrel{0}{0} \\
\stackrel{0}{1}\end{array}$ & $\begin{array}{l}\text { Oొ } \\
\stackrel{N}{<}\end{array}$ & ల్లి & $\begin{array}{l}\mathscr{D} \\
\infty \\
\infty\end{array}$ & $\begin{array}{l}\text { Oొ } \\
\stackrel{N}{\infty}\end{array}$ & $\begin{array}{l}0 \\
\mathbb{0} \\
0\end{array}$ & $\begin{array}{l}\widetilde{N} \\
\delta \\
0 \\
\tilde{N} \\
0 \\
0 \\
0\end{array}$ & $\begin{array}{l}\text { Õ } \\
\text { Nิ } \\
0 \\
0 \\
0 \\
0\end{array}$ & 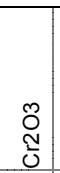 & $\stackrel{O}{3}$ & 4 & 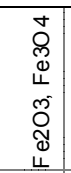 & $\begin{array}{l}\text { 尺े } \\
\text { x }\end{array}$ & $\stackrel{\stackrel{ }{J}}{ }$ & $\begin{array}{l}\circ \\
\sum \\
\Sigma\end{array}$ & $\begin{array}{l}\tilde{O} \\
\text { C } \\
\Sigma\end{array}$ & $\begin{array}{l}0 \\
0 \\
\Sigma\end{array}$ & $\begin{array}{l}\text { Q } \\
\text { Z } \\
Z\end{array}$ & 을 & $\begin{array}{l}\text { N } \\
\text { tँ }\end{array}$ & 㝙 & 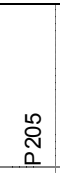 & $\frac{8}{0}$ & $\frac{\text { O }}{\infty}$ & Oo & 옹 & 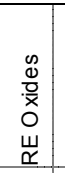 & $\begin{array}{l}\text { 足 } \\
\stackrel{1}{⺊}\end{array}$ & 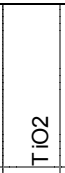 & 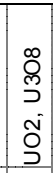 & $\frac{Q}{N}$ & $\begin{array}{l}\text { Qิ } \\
\text { N }\end{array}$ & 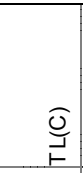 & 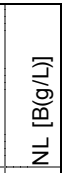 \\
\hline Staples, 2000 & & 1228 & 4.9 & 7.1 & & & & & & & & & & & 8.9 & & & & 5.0 & & & 0.7 & & & 59.6 & & & & & & & & 13.9 & & 0.6 \\
\hline Staples, 2000 & & 1400 & 19.9 & 6.1 & & & & & & & & & 7.9 & 9.9 & & & & & 14.9 & & & 0.7 & 3.0 & & 35.6 & & & & & & & & 2.0 & & 0.9 \\
\hline Staples, 2000 & & 1053 & 7.9 & 6.0 & & & 4.0 & & & & & 1.0 & 0.5 & 3.0 & 6.0 & & & & 14.9 & 0.5 & & 0.7 & 2.0 & & 49.6 & & & & & & & & 4.0 & & 1.5 \\
\hline Staples, 2000 & & 1077 & 10.9 & 11.9 & & & 4.0 & & & & & 1.0 & 0.5 & 3.0 & 3.0 & & & & 14.9 & 0.5 & & 0.7 & 2.0 & & 39.7 & & & & & & & & 7.9 & & 1.5 \\
\hline Staples, 2000 & & 1178 & 7.9 & 6.0 & & & 7.9 & & & & & 2.5 & 0.6 & 3.0 & 3.0 & & & & 9.9 & 0.5 & & 0.7 & 2.0 & & 48.1 & & & & & & & & 7.9 & & 0.4 \\
\hline Staples, 2000 & & 1125 & 11.9 & 6.0 & & & 4.0 & & & & & 1.0 & 3.0 & 6.0 & 3.0 & & & & 14.9 & 0.5 & & 0.7 & 1.0 & & 40.2 & & & & & & & & 7.9 & & 0.8 \\
\hline Staples, 2000 & & 1046 & 7.9 & 6.0 & & & 4.0 & & & & & 1.0 & 0.5 & 3.0 & 6.0 & & & & 14.9 & 1.0 & & 0.7 & 2.0 & & 49.1 & & & & & & & & 4.0 & & 1.5 \\
\hline Staples, 2000 & & 1120 & 7.9 & 6.0 & & & 4.0 & & & & & 1.0 & 0.5 & 6.0 & 3.0 & & & & 14.9 & 1.0 & & 0.7 & 2.0 & & 49.1 & & & & & & & & 4.0 & & 1.2 \\
\hline Staples, 2000 & & 1169 & 7.9 & 6.0 & & & 7.9 & & & & & 1.0 & 2.0 & 6.0 & 3.0 & & & & 9.9 & 1.0 & & 0.7 & 1.0 & & 49.6 & & & & & & & & 4.0 & & 0.5 \\
\hline Staples, 2000 & & 1115 & 11.9 & 6.0 & & & 4.0 & & & & & 1.1 & 0.5 & 3.0 & 6.0 & & & & 14.9 & 0.5 & & 0.7 & 2.0 & & 41.6 & & & & & & & & 7.9 & & 1.2 \\
\hline Staples, 2000 & & 1058 & 10.5 & 11.9 & & & 4.0 & & & & & 1.4 & 0.5 & 3.0 & 3.0 & & & & 14.9 & 0.5 & & 0.7 & 2.0 & & 39.7 & & & & & & & & 7.9 & & 1.8 \\
\hline Staples, 2000 & & 1059 & 11.9 & 6.0 & & & 7.9 & & & & & 1.0 & 0.5 & 6.0 & 5.6 & & & & 9.9 & 1.0 & & 0.7 & 1.0 & & 40.5 & & & & & & & & 7.9 & & 0.8 \\
\hline Staples, 2000 & & 1062 & 11.9 & 6.0 & & & 7.9 & & & & & 1.0 & 0.5 & 6.0 & 5.8 & & & & 9.9 & 0.5 & & 0.5 & 1.0 & & 40.9 & & & & & & & & 7.9 & & 0.9 \\
\hline Staples, 2000 & & 1169 & 8.0 & 6.0 & & & 4.0 & & & & & 1.0 & 0.5 & 6.0 & 4.9 & & & & 9.9 & 0.5 & & 0.7 & 1.0 & & 49.6 & & & & & & & & 7.9 & & 0.6 \\
\hline Staples, 2000 & & 1186 & 9.8 & 6.0 & & & 7.9 & & & & & 2.5 & 0.5 & 3.0 & 3.1 & & & & 9.9 & 1.0 & & 0.7 & 2.0 & & 49.6 & & & & & & & & 4.0 & & 0.6 \\
\hline Staples, 2000 & & 1128 & 7.9 & 6.0 & & & 7.4 & & & & & 1.0 & 3.0 & 6.0 & 3.0 & & & & 9.9 & 1.0 & & 0.7 & 1.0 & & 49.2 & & & & & & & & 4.0 & & 0.8 \\
\hline Staples, 2000 & & 1068 & 9.8 & 9.2 & & & 4.9 & & & & & 2.1 & 2.5 & 4.4 & 3.9 & & & & 11.5 & 0.7 & & & 1.4 & & 43.6 & & & & & & & & 5.8 & & 1.0 \\
\hline Staples, 2000 & & 1162 & 15.0 & 15.0 & & & & & & & & & & & 8.5 & & & & 5.0 & & & 0.5 & & & 53.1 & & & & & & & & 3.0 & & 1.1 \\
\hline Staples, 2000 & & 1136 & 3.8 & 12.5 & & & & & & & & & & 2.5 & 6.3 & & & & 8.8 & & & 0.5 & 1.3 & & 54.0 & & & & & & & & 10.5 & & 3.2 \\
\hline Staples, 2000 & & 1081 & 9.8 & 9.1 & & & 4.9 & & & & & 2.1 & 2.5 & 4.4 & 3.9 & & & & 11.5 & 0.7 & & 0.7 & 1.4 & & 43.3 & & & & & & & & 5.8 & & 1.1 \\
\hline Staples, 2000 & & 1055 & 9.8 & 9.1 & & & 4.9 & & & & & 2.1 & 2.5 & 4.4 & 3.9 & & & & 11.5 & 0.7 & & 0.7 & 1.4 & & 43.3 & & & & & & & & 5.8 & & 0.9 \\
\hline Staples, 2000 & & 1081 & 9.6 & 8.9 & & & 4.8 & & & & & 2.0 & 2.5 & 4.3 & 3.8 & & & & 11.2 & 0.7 & & 2.7 & 1.4 & & 42.4 & & & & & & & & 5.6 & & 1.0 \\
\hline Staples, 2000 & & 1068 & 9.3 & 8.6 & & & 4.6 & & & & & 2.0 & 2.4 & 4.2 & 3.7 & & & & 10.9 & 0.7 & & 0.8 & 1.4 & & 41.1 & & & & & & & & 5.5 & & 0.8 \\
\hline Staples, 2000 & & 1065 & 9.6 & 8.9 & & & 4.8 & & & & & 2.0 & 2.5 & 4.3 & 3.8 & & & & 11.2 & 0.7 & & 2.7 & 1.4 & & 42.4 & & & & & & & & 5.6 & & 0.7 \\
\hline Staples, 2000 & & 1071 & 9.4 & 8.7 & & & 4.7 & & & & & 2.0 & 2.4 & 4.2 & 3.7 & & & & 11.0 & 7.1 & & 0.7 & 1.4 & & 41.6 & & & & & & & & 5.5 & & 0.8 \\
\hline Stempin, 1985 & 1450 & & 17.7 & & 13.3 & & & & & & & 4.4 & & & & 22.2 & & & & & & & & & 32.6 & & 9.8 & & & & & & & & \\
\hline Stempin, 1985 & 1450 & & 16.8 & & 12.6 & & & & & 4.2 & 4.6 & 4.2 & & & & 21.0 & & & & & & & & & 24.1 & & 9.2 & & & & & & & & \\
\hline Stempin, 1985 & 1450 & & 12.9 & & 12.9 & & & & & 3.9 & & 6.4 & & & & 17.6 & & & & & & & & & 39.7 & & 9.7 & & & & & & & & \\
\hline Stempin, 1985 & 1450 & & 15.8 & & 11.9 & & & & & 2.0 & & 7.8 & & & & 12.5 & & & & 0.9 & & & & & 37.5 & & 8.7 & & & & & & & & \\
\hline Stempin, 1985 & 1450 & & 17.5 & & 13.2 & & & & & 0.6 & 0.3 & 4.4 & & & & 21.9 & & & & 0.6 & & & & & 31.4 & & 9.6 & & & & & & & & \\
\hline Stempin, 1985 & 1450 & & 17.4 & & 13.1 & & & & & 1.1 & & 4.3 & & & & 21.8 & & & & & & & & & 31.2 & & 9.6 & & & & & & & & \\
\hline Stempin, 1985 & 1450 & & 17.2 & & 13.0 & & & & & 4.3 & & 4.3 & & & & 21.6 & & & & & & & & & 30.0 & & 9.5 & & & & & & & & \\
\hline Stempin, 1985 & 1450 & & 17.4 & & 13.1 & & & & & 2.2 & & 4.3 & & & & 21.8 & & & & 1.1 & & & & & 30.4 & & 9.6 & & & & & & & & \\
\hline Stempin, 1985 & 1450 & & 17.3 & & 13.0 & & & & & 2.2 & 0.1 & 4.3 & & & & 21.7 & & & & 0.1 & & & & & 31.5 & & 9.5 & & & & & & & & \\
\hline Stempin, 1985 & 1450 & & 17.6 & & 13.2 & & & & & 1.1 & & 4.4 & & & & 22.0 & & & & 0.5 & & & & & 32.0 & & 9.7 & & & & & & & & \\
\hline Stempin, 1985 & 1450 & & 17.5 & & 13.1 & & & & & 2.2 & & 4.4 & & & & 21.9 & & & & & & & & & 31.3 & & 9.6 & & & & & & & & \\
\hline Stempin, 1985 & 1450 & & 17.1 & & 12.8 & & & & & 2.1 & & 4.2 & & & & 21.4 & & & & & & & & & 29.8 & & 9.4 & & & & & & & & \\
\hline Taniguchi, 1993 & & 1306 & 16.9 & & & & 8.5 & & & & & & 9.4 & 1.8 & & 8.8 & 0.1 & & 3.5 & & & 2.5 & 0.6 & & 44.8 & & & & & & & & & & \\
\hline Toplis, 1994 & & 1341 & 10.5 & & & & 9.4 & & & & & & 13.4 & 0.4 & & 4.0 & & & 2.4 & & & & 9.5 & & 47.4 & & & & & 3.8 & & & & & \\
\hline Toplis, 1994 & & 1294 & 11.4 & & & & 9.6 & & & & & & 14.2 & 0.5 & & 4.2 & & & 3.1 & & & & 0.1 & & 50.9 & & & & & 4.1 & & & & & \\
\hline Toplis, 1994 & & 1315 & 11.1 & & & & 8.5 & & & & & & 12.6 & 0.5 & & 3.9 & & & 2.9 & & & & 5.6 & & 50.5 & & & & & 3.4 & & & & & \\
\hline Toplis, 1994 & & 1282 & 11.4 & & & & 9.6 & & & & & & 13.9 & 0.5 & & 3.9 & & & 3.0 & & & & 2.4 & & 50.7 & & & & & 4.0 & & & & & \\
\hline Vienna, 1996 & 1516 & 1456 & 25.0 & 0.0 & & 1.1 & 1.0 & 1.3 & & 0.4 & & 0.3 & 5.4 & 0.1 & 2.3 & 0.0 & 0.7 & 0.0 & 9.9 & 1.1 & 0.1 & 0.0 & 0.6 & & 42.0 & 0.1 & & 5.0 & & & & & 3.5 & & \\
\hline Vienna, 1996 & 1506 & 1416 & 18.0 & 0.0 & & 1.2 & 1.1 & 1.5 & & 0.5 & & 0.3 & 5.9 & 0.1 & 2.5 & 0.0 & 0.8 & 0.0 & 10.9 & 1.2 & 0.1 & 0.0 & 0.7 & & 46.0 & 0.1 & & 5.4 & & & & & 3.8 & & \\
\hline Vienna, 1996 & 1457 & 1363 & 13.0 & 0.0 & & 1.3 & 1.2 & 1.5 & & 0.5 & & 0.3 & 6.2 & 0.1 & 2.6 & 0.0 & 0.8 & 0.0 & 11.5 & 1.3 & 0.1 & 0.1 & 0.7 & & 48.8 & 0.2 & & 5.7 & & & & & 4.0 & & \\
\hline Vienna, 1999 & & 1122 & 9.6 & 9.5 & & & 0.8 & & & 0.1 & & 0.3 & 7.9 & 2.8 & 1.7 & 0.0 & 0.3 & 0.1 & 17.5 & 0.2 & 0.0 & 0.0 & 0.4 & 0.1 & 44.7 & 1.3 & & & & 2.0 & & & 0.4 & & \\
\hline Vienna, 1999a & & & 4.5 & 5.0 & & & 0.1 & 0.0 & & & & & & 0.4 & 7.5 & & & & 14.8 & & & 0.9 & 0.2 & & 52.3 & & & & & & & & 15.0 & 1005 & 0.3 \\
\hline Vienna, 1999a & & & 4.5 & 10.0 & & & 0.1 & 0.0 & & & & & & 0.4 & 7.5 & & & & 11.0 & & & 0.9 & 0.2 & & 51.1 & & & & & & & & 15.0 & 1064 & 0.2 \\
\hline Vienna, 1999a & & & 4.5 & 10.0 & & & 0.1 & 0.0 & & & & & & 0.4 & 7.5 & & & & 11.0 & & & 0.9 & 0.2 & & 51.1 & & & & & & & & 15.0 & 1064 & 0.3 \\
\hline Vienna, 1999a & & & 4.5 & 10.0 & & & 0.1 & 0.0 & & & & & & 0.4 & 9.0 & & & & 7.8 & & & 0.9 & 0.2 & & 52.9 & & & & & & & & 15.0 & 1140 & 0.2 \\
\hline
\end{tabular}


ORP-56297 Rev. 0

The Catholic University of America

High Level Waste Vitrification System Improvements Vitreous State Laboratory

Table A1. Glass Compositions from Literature Review (continued)

\begin{tabular}{|c|c|c|c|c|c|c|c|c|c|c|c|c|c|c|c|c|c|c|c|c|c|c|c|c|c|c|c|c|c|c|c|c|c|c|c|}
\hline Ref & 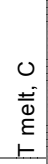 & $\begin{array}{l}0 \\
\stackrel{0}{0} \\
\stackrel{0}{0} \\
\stackrel{5}{匕}\end{array}$ & $\begin{array}{l}00 \\
\stackrel{N}{<}\end{array}$ & 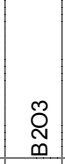 & $\begin{array}{l}\text { Q } \\
\infty\end{array}$ & $\begin{array}{l}\text { Oొ } \\
\stackrel{N}{m}\end{array}$ & $\begin{array}{l}0 \\
\mathbb{\pi} \\
0\end{array}$ & $\begin{array}{l}\tilde{O} \\
0 \\
0 \\
\tilde{ల} \\
0 \\
0 \\
0\end{array}$ & $\begin{array}{l}m \\
0 \\
0 \\
0 \\
0 \\
0 \\
0\end{array}$ & $\begin{array}{l}\text { Oొ } \\
\text { ป̂ }\end{array}$ & $\stackrel{0}{3}$ & 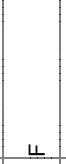 & 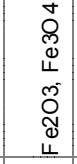 & $\begin{array}{l}\stackrel{\mathcal{N}}{\underline{x}} \\
\text { }\end{array}$ & $\stackrel{?}{\stackrel{ }{J}}$ & $\begin{array}{l}\circ \\
\text { O } \\
\Sigma\end{array}$ & $\begin{array}{l}\tilde{D} \\
\stackrel{\Sigma}{\Sigma}\end{array}$ & $\begin{array}{l}0_{0}^{0} \\
\text { ¿ }\end{array}$ & $\begin{array}{l}\text { N } \\
\underset{\pi}{2}\end{array}$ & $\frac{0}{z}$ & $\begin{array}{l}\text { N } \\
\text { בे }\end{array}$ & 吾 & $\begin{array}{l}\text { ¿ } \\
\Sigma \\
\Sigma\end{array}$ & $\frac{8}{2}$ & $\stackrel{\widetilde{O}}{\omega}$ & $\begin{array}{l}\text { Oొ } \\
\text { c) }\end{array}$ & 高 & 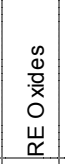 & $\begin{array}{l}\tilde{Q} \\
\stackrel{\varepsilon}{F}\end{array}$ & 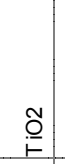 & 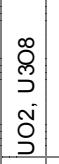 & $\stackrel{q}{N}$ & $\begin{array}{l}\text { ò } \\
\text { N }\end{array}$ & $\underset{\hat{O}}{\vec{O}}$ & 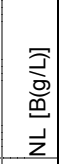 \\
\hline Vienna, 1999a & & & 6.0 & 15.0 & & & 0.1 & 0.0 & & & & & & 0.5 & 9.0 & & & & 4.0 & & & 0.9 & 0.2 & & 48.6 & & & & & & & & 16.5 & 1250 & 0.2 \\
\hline Vienna, 1999a & & & 6.0 & 15.0 & & & 0.1 & 0.0 & & & & & & 0.5 & 6.0 & & & & 10.5 & & & 1.0 & 0.2 & & 45.1 & & & & & & & & 16.5 & 1189 & 0.4 \\
\hline Vienna, 1999a & & & 6.0 & 5.0 & & & 0.1 & 0.0 & & & & & & 0.5 & 9.0 & & & & 11.6 & & & 1.0 & 0.2 & & 51.0 & & & & & & & & 16.5 & 1314 & $\mathrm{~nm}$ \\
\hline Vienna, 1999a & & & 6.0 & 5.0 & & & 0.1 & 0.0 & & & & & & 0.5 & 6.0 & & & & 18.1 & & & 1.0 & 0.2 & & 47.5 & & & & & & & & 16.5 & 1336 & 0.2 \\
\hline Vienna, 1999a & & & 4.5 & 10.0 & & & 0.1 & 0.0 & & & & & & 0.4 & 7.5 & & & & 11.0 & & & 0.9 & 0.2 & & 51.1 & & & & & & & & 15.0 & 1067 & 0.2 \\
\hline Vienna, 1999a & & & 3.0 & 15.0 & & & 0.1 & 0.0 & & & & & & 0.5 & 9.0 & & & & 3.6 & & & 1.0 & 0.2 & & 51.9 & & & & & & & & 16.5 & 1228 & 0.3 \\
\hline Vienna, 1999a & & & 3.0 & 15.0 & & & 0.1 & 0.0 & & & & & & 0.5 & 6.0 & & & & 10.1 & & & 1.0 & 0.2 & & 48.4 & & & & & & & & 16.5 & 1125 & 0.7 \\
\hline Vienna, 1999a & & & 3.0 & 5.0 & & & 0.1 & 0.0 & & & & & & 0.5 & 9.0 & & & & 11.2 & & & 0.9 & 0.2 & & 54.3 & & & & & & & & 16.5 & 1034 & 0.3 \\
\hline Vienna, 1999a & & & 4.5 & 10.0 & & & 0.1 & 0.0 & & & & & & 0.4 & 7.5 & & & & 11.2 & & & 0.8 & 0.2 & & 52.6 & & & & & & & & 13.5 & 964 & 0.3 \\
\hline Vienna, 1999a & & & 3.0 & 5.0 & & & 0.1 & 0.0 & & & & & & 0.5 & 6.0 & & & & 17.7 & & & 1.0 & 0.2 & & 50.9 & & & & & & & & 16.5 & 1113 & 0.5 \\
\hline Vienna, 1999a & & & 6.0 & 15.0 & & & 0.1 & 0.0 & & & & & & 0.4 & 9.0 & & & & 4.3 & & & 0.8 & 0.2 & & 51.4 & & & & & & & & 13.5 & 1180 & 0.3 \\
\hline Vienna, 1999a & & & 6.0 & 15.0 & & & 0.1 & 0.0 & & & & & & 0.4 & 6.0 & & & & 10.8 & & & 0.8 & 0.2 & & 47.9 & & & & & & & & 13.5 & 1087 & 1.0 \\
\hline Vienna, 1999a & & & 6.0 & 5.0 & & & 0.1 & 0.0 & & & & & & 0.4 & 9.0 & & & & 11.9 & & & 0.8 & 0.2 & & 53.8 & & & & & & & & 13.5 & 1003 & 0.3 \\
\hline Vienna, 1999a & & & 6.0 & 5.0 & & & 0.1 & 0.0 & & & & & & 0.4 & 6.0 & & & & 18.4 & & & 0.8 & 0.2 & & 50.4 & & & & & & & & 13.5 & 1105 & 0.3 \\
\hline Vienna, 1999a & & & 3.0 & 15.0 & & & 0.1 & 0.0 & & & & & & 0.4 & 9.0 & & & & 4.0 & & & 0.8 & 0.2 & & 54.8 & & & & & & & & 13.5 & 1137 & 0.4 \\
\hline Vienna, 1999a & & & 3.0 & 15.0 & & & 0.1 & 0.0 & & & & & & 0.4 & 6.0 & & & & 10.4 & & & 0.8 & 0.2 & & 51.3 & & & & & & & & 13.5 & 1070 & 0.6 \\
\hline Vienna, 1999a & & & 3.0 & 5.0 & & & 0.1 & 0.0 & & & & & & 0.4 & 9.0 & & & & 11.5 & & & 0.8 & \begin{tabular}{|l|}
0.2 \\
\end{tabular} & & 57.2 & & & & & & & & 13.5 & 1081 & 0.6 \\
\hline Vienna, 1999a & & & 4.5 & 10.0 & & & 0.1 & 0.0 & & & & & & 0.4 & 7.5 & & & & 11.0 & & & 0.9 & 0.2 & & 51.1 & & & & & & & & 15.0 & 1064 & 0.2 \\
\hline Vienna, 1999a & & & 3.0 & 5.0 & & & 0.1 & 0.0 & & & & & & 0.4 & 6.0 & & & & 18.0 & & & 0.8 & \begin{tabular}{|l|}
0.2 \\
\end{tabular} & & 53.7 & & & & & & & & 13.5 & 1059 & 3.9 \\
\hline Vienna, 1999a & & & 4.5 & 10.0 & & & 0.1 & 0.0 & & & & & & 0.5 & 7.5 & & & & 10.8 & & & 1.0 & 0.2 & & 49.7 & & & & & & & & 16.5 & 1110 & 0.2 \\
\hline Vienna, 1999a & & & 3.0 & 10.0 & & & 0.1 & 0.0 & & & & & & 0.4 & 7.5 & & & & 10.8 & & & 0.9 & 0.2 & & 52.8 & & & & & & & & 15.0 & 1038 & 0.3 \\
\hline Vienna, 1999a & & & 6.0 & 10.0 & & & 0.1 & 0.0 & & & & & & 0.4 & 7.5 & & & & 11.2 & & & 0.9 & 0.2 & & 49.5 & & & & & & & & 15.0 & 1085 & 0.2 \\
\hline Vienna, 1999a & & & 4.5 & 10.0 & & & 0.1 & 0.0 & & & & & & 0.4 & 7.5 & & & & 11.0 & & & 0.9 & 0.2 & & 51.1 & & & & & & & & 15.0 & 1064 & 0.3 \\
\hline Vienna, 1999a & & & 4.5 & 15.0 & & & 0.1 & 0.0 & & & & & & 0.4 & 7.5 & & & & 7.2 & & & 0.9 & 0.2 & & 49.9 & & & & & & & & 15.0 & 1177 & 0.4 \\
\hline Vienna, 1999a & & & 4.5 & 10.0 & & & 0.1 & 0.0 & & & & & & 0.4 & 6.0 & & & & 14.3 & & & 0.9 & 0.2 & & 49.4 & & & & & & & & 15.0 & 913 & 0.3 \\
\hline Vienna, 2000 & & & 12.9 & 5.0 & 0.9 & & 2.8 & & 0.0 & 0.2 & 0.0 & & 12.5 & 0.3 & & 1.8 & 0.4 & & 22.1 & 0.2 & & 0.1 & 0.9 & & 38.5 & & 0.0 & & & & & 0.0 & 0.5 & & \\
\hline Vienna, 2000 & & & 12.1 & 5.0 & 0.9 & & 2.6 & & & 0.2 & 0.0 & & 11.7 & 0.3 & 2.5 & 1.7 & 0.4 & & 18.9 & 0.2 & & 0.1 & 0.9 & & 41.3 & & 0.0 & & & & & 0.0 & 0.4 & & \\
\hline Vienna, 2000 & & & 9.4 & 8.3 & 0.7 & & 2.0 & & 0.0 & 0.2 & 0.0 & & 9.1 & 0.2 & 4.0 & 1.3 & 0.3 & & 14.6 & 0.1 & & 0.0 & \begin{tabular}{|l|}
0.7 \\
\end{tabular} & & 48.1 & & 0.0 & & & & & 0.0 & 0.3 & & \\
\hline Vienna, 2000 & & & 16.0 & 18.5 & 0.0 & & 0.2 & & & 0.2 & 0.0 & & 8.7 & & 3.5 & 0.1 & 0.4 & & 13.2 & & & 0.4 & 1.1 & & 37.0 & & 0.0 & & & 0.0 & & 0.0 & 0.1 & & \\
\hline Vienna, 2000 & & & 14.4 & 10.9 & 0.0 & & 0.2 & & & 0.2 & 0.0 & & 7.8 & & 6.0 & 0.1 & 0.4 & & 13.1 & & & 0.4 & 0.9 & & 45.0 & & 0.0 & & & 0.0 & & 0.0 & 0.1 & & \\
\hline Vienna, 2000 & & & 8.1 & 10.9 & 0.3 & & 1.0 & 0.1 & 0.1 & 0.2 & 0.0 & 0.1 & 12.8 & 0.3 & 1.0 & 0.6 & 0.3 & 0.0 & 17.3 & 0.5 & 0.3 & 1.0 & 0.5 & & 41.1 & 0.2 & 0.0 & \begin{tabular}{|l|}
0.2 \\
\end{tabular} & & 0.0 & & 0.0 & 1.8 & & \\
\hline Vienna, 2000 & & & 10.1 & 7.0 & 0.5 & & 4.0 & 0.1 & 0.2 & 0.0 & 0.1 & 0.1 & 23.0 & 0.9 & 3.0 & 0.1 & 0.6 & 0.0 & 15.8 & 0.1 & 0.5 & 1.6 & 0.8 & 0.3 & 30.2 & 0.3 & 0.1 & 0.3 & & 0.2 & & 0.1 & & & \\
\hline Vienna, 2000 & & & 11.0 & 6.8 & 0.4 & & & 0.1 & 0.1 & 0.6 & 0.0 & 0.1 & 12.2 & & 2.9 & 0.6 & 0.3 & 0.0 & 12.7 & 2.0 & 0.3 & 1.1 & 0.6 & 0.2 & 44.9 & 0.2 & 0.0 & 0.2 & & 0.0 & & 0.1 & 2.3 & & \\
\hline Vienna, 2000 & & & 11.0 & 7.0 & 0.4 & & & 0.1 & 0.1 & 0.6 & 0.0 & 0.1 & 12.5 & & 3.0 & 0.6 & 0.4 & 0.0 & 12.7 & 0.1 & 0.3 & 1.2 & 0.6 & 0.2 & 46.0 & 0.2 & 0.0 & 0.2 & & 0.0 & & 0.1 & 2.3 & & \\
\hline Vienna, 2000 & & & 11.0 & 6.9 & 0.4 & & & 0.1 & 0.1 & 0.1 & 0.0 & 0.1 & 12.3 & & 3.0 & 0.6 & 0.4 & 0.0 & 12.7 & 2.0 & 0.3 & 1.1 & 0.6 & 0.2 & 45.2 & 0.2 & 0.0 & 0.2 & & 0.0 & & 0.1 & 2.3 & & \\
\hline Vienna, 2000 & & & 11.0 & 7.1 & 0.4 & & & 0.1 & 0.1 & 0.0 & 0.0 & 0.1 & 12.6 & & 3.0 & 0.6 & 0.4 & 0.0 & 12.7 & 0.1 & 0.3 & 1.2 & 0.6 & 0.2 & 46.4 & 0.2 & 0.0 & 0.2 & & 0.0 & & 0.1 & 2.4 & & \\
\hline Vienna, 2000 & & & 11.0 & 7.1 & 0.4 & & & 0.1 & 0.1 & 0.0 & 0.0 & 0.1 & 12.6 & & 3.0 & 0.6 & 0.4 & 0.0 & 12.7 & 0.1 & 0.3 & 1.2 & 0.6 & 0.2 & 46.4 & 0.2 & 0.0 & 0.2 & & 0.0 & & 0.1 & 2.4 & & \\
\hline Vienna, 2000 & & & 11.0 & 7.0 & 0.4 & & & 0.1 & 0.1 & 0.2 & 0.0 & 0.1 & 12.5 & & 3.0 & 0.6 & 0.4 & 0.0 & 12.7 & 0.5 & 0.3 & 1.2 & 0.6 & 0.2 & 46.0 & 0.2 & 0.0 & 0.2 & & 0.0 & & 0.1 & 2.3 & & \\
\hline Vienna, 2000 & & & 7.8 & 6.8 & 0.3 & & 1.0 & 0.1 & 0.1 & 0.2 & 0.0 & 0.1 & 12.2 & 0.3 & 2.9 & 0.6 & 0.4 & 0.0 & 15.3 & 0.5 & 0.3 & 0.9 & 0.5 & 0.2 & 44.9 & 0.2 & 0.0 & 0.2 & & 2.5 & & 0.0 & 1.8 & & \\
\hline Vienna, 2000 & & & 7.6 & 6.7 & 0.3 & & 1.0 & 0.1 & 0.1 & 0.2 & 0.0 & 0.1 & 11.9 & 0.3 & 2.9 & 0.6 & 0.3 & 0.0 & 14.9 & 0.5 & 0.3 & 0.9 & 0.4 & 0.2 & 43.7 & 0.2 & 0.0 & 0.2 & & 5.0 & & 0.0 & 1.8 & & \\
\hline Vienna, 2000 & & & 16.0 & 15.0 & & & & & & & & & 5.0 & 4.0 & 6.0 & & & & 10.0 & 3.0 & & & & & 36.0 & & & & & 5.0 & & & & & \\
\hline Vienna, 2000 & & & 8.0 & 6.8 & 0.4 & & & 0.1 & 0.1 & 0.6 & 0.0 & 0.1 & 12.2 & & 2.9 & 0.6 & 0.3 & 0.0 & 15.7 & 2.0 & 0.3 & 1.1 & 0.6 & 0.2 & 44.9 & 0.2 & 0.0 & 0.2 & & 0.0 & & 0.1 & 2.3 & & \\
\hline Vienna, 2000 & & & 8.0 & 6.8 & 0.4 & & & 0.1 & 0.1 & 0.6 & 0.0 & 0.1 & 12.2 & & 2.9 & 0.6 & 0.3 & 0.0 & 15.7 & 2.0 & 0.3 & 1.1 & 0.6 & 0.2 & 44.9 & 0.2 & 0.0 & 0.2 & & 0.0 & & 0.1 & 2.3 & & \\
\hline Vienna, 2000 & & & 8.0 & 7.0 & 0.4 & & & 0.1 & 0.1 & 0.6 & 0.0 & 0.1 & 12.5 & & 3.0 & 0.6 & 0.4 & 0.0 & 15.7 & 0.1 & 0.3 & 1.2 & 0.6 & 0.2 & 46.0 & 0.2 & 0.0 & 0.2 & & 0.0 & & 0.1 & 2.3 & & \\
\hline Vienna, 2000 & & & 8.0 & 6.9 & 0.4 & & & 0.1 & 0.1 & 0.1 & 0.0 & 0.1 & 12.3 & & 3.0 & 0.6 & 0.4 & 0.0 & 15.7 & 2.0 & 0.3 & 1.1 & 0.6 & 0.2 & 45.2 & 0.2 & 0.0 & 0.2 & & 0.0 & & 0.1 & 2.3 & & \\
\hline Vienna, 2000 & & & 8.0 & 7.1 & 0.4 & & & 0.1 & 0.1 & 0.0 & 0.0 & 0.1 & 12.6 & & 3.0 & 0.6 & 0.4 & 0.0 & 15.7 & 0.1 & 0.3 & 1.2 & 0.6 & 0.2 & 46.4 & 0.2 & 0.0 & 0.2 & & 0.0 & & 0.1 & 2.4 & & \\
\hline Vienna, 2000 & & & 8.0 & 7.1 & 0.4 & & & 0.1 & 0.1 & 0.0 & 0.0 & 0.1 & 12.6 & & 3.0 & 0.6 & 0.4 & 0.0 & 15.7 & 0.1 & 0.3 & 1.2 & 0.6 & 0.2 & 46.4 & 0.2 & 0.0 & 0.2 & & 0.0 & & 0.1 & 2.4 & & \\
\hline Vienna, 2000 & & & 8.0 & 7.0 & 0.4 & & & 0.1 & 0.1 & 0.2 & 0.0 & 0.1 & 12.5 & & 3.0 & 0.6 & 0.4 & 0.0 & 15.7 & 0.5 & 0.3 & 1.2 & 0.6 & 0.2 & 46.0 & 0.2 & 0.0 & 0.2 & & 0.0 & & 0.1 & 2.3 & & \\
\hline Vienna, 2000 & & & 7.8 & 6.8 & 0.6 & & 1.0 & 0.1 & 0.2 & 0.2 & 0.1 & 0.1 & 12.1 & 0.3 & 2.9 & 0.6 & 0.7 & 0.0 & 15.3 & 0.5 & 0.5 & 1.7 & 0.8 & 0.3 & 44.7 & 0.4 & 0.1 & 0.3 & & 0.0 & & 0.1 & 1.8 & & \\
\hline Vienna, 2000 & & & 8.0 & 7.0 & 0.4 & & & 0.1 & 0.1 & 0.2 & 0.0 & 0.1 & 12.5 & & 3.0 & 0.6 & 0.4 & 0.0 & 15.7 & 0.5 & 0.3 & 1.2 & 0.6 & 0.2 & 46.0 & 0.2 & 0.0 & 0.2 & & 0.0 & & 0.1 & 2.3 & & \\
\hline
\end{tabular}


Table A1. Glass Compositions from Literature Review (continued)

\begin{tabular}{|c|c|c|c|c|c|c|c|c|c|c|c|c|c|c|c|c|c|c|c|c|c|c|c|c|c|c|c|c|c|c|c|c|c|c|c|}
\hline Ref & 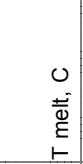 & $\begin{array}{l}0 \\
0 \\
8 \\
0 \\
上 \\
1\end{array}$ & $\frac{\substack{0 \\
\frac{N}{\alpha}}}{4}$ & 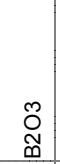 & 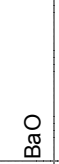 & 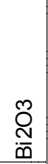 & $\begin{array}{l}0 \\
0 \\
0\end{array}$ & $\begin{array}{l}\widetilde{O} \\
\text { d } \\
0 \\
\tilde{O} \\
\widetilde{J} \\
0 \\
0\end{array}$ & $\begin{array}{l}0 \\
0 \\
0 \\
0 \\
0 \\
0 \\
0\end{array}$ & ָֻ & $\begin{array}{l}0 \\
\mathcal{O} \\
0\end{array}$ & 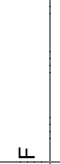 & 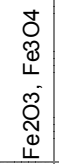 & 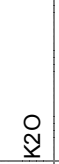 & 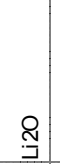 & $\begin{array}{l}\circ \\
\text { I } \\
\Sigma\end{array}$ & $\begin{array}{l}\tilde{O} \\
\text { E } \\
\text { L }\end{array}$ & $\begin{array}{l}0 \\
\text { On } \\
\stackrel{0}{2}\end{array}$ & $\begin{array}{l}\text { 尺్ } \\
\text { Z }\end{array}$ & $\frac{0}{z}$ & 䇣 & 吾 & 岕 & $\begin{array}{l}\circ \\
\frac{0}{0}\end{array}$ & ণ̃ & סొ & 옳 & 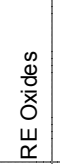 & $\begin{array}{l}\text { Õ } \\
\risingdotseq\end{array}$ & $\stackrel{\tilde{o}}{\stackrel{\rho}{F}}$ & 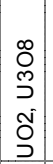 & $\begin{array}{l}O \\
\stackrel{N}{N}\end{array}$ & ญั & 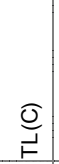 & $\begin{array}{l}\bar{\Xi} \\
\frac{\sigma}{0} \\
\underline{\underline{z}} \\
\bar{z}\end{array}$ \\
\hline Vienna, 2000 & & & 8.0 & 7.0 & 0.4 & & & 0.1 & 0.1 & 0.2 & 0.0 & 0.1 & 12.5 & & 3.0 & 0.6 & 0.4 & 0.0 & 15.7 & 0.5 & 0.3 & 1.2 & 0.6 & 0.2 & 46.0 & 0.2 & 0.0 & 0.2 & & 0.0 & & 0.1 & 2.3 & & \\
\hline Vienna, 2000 & & & 10.4 & 9.1 & 0.4 & & 1.3 & 0.1 & 0.1 & 0.3 & 0.0 & 0.1 & 16.2 & 0.4 & 3.9 & 0.8 & 0.5 & 0.0 & 20.4 & 0.7 & 0.4 & 1.2 & 0.6 & 0.2 & 30.0 & 0.2 & 0.0 & 0.2 & & 0.0 & & 0.1 & 2.4 & & \\
\hline Vienna, 2000 & & & 7.8 & 6.8 & 0.3 & & 1.0 & 0.1 & 0.1 & 0.2 & 0.0 & 0.1 & 12.2 & 0.3 & 2.9 & 0.6 & 0.4 & 0.0 & 15.4 & 0.5 & 0.3 & 0.9 & 0.5 & 0.2 & 45.0 & 0.2 & 0.0 & 0.2 & & 0.0 & & 0.0 & 4.0 & & \\
\hline Vojtech, 1996 & $\sim 1200$ & & 16.2 & 17.1 & & & 0.2 & & & 4.1 & & 0.0 & 1.4 & 0.1 & 6.7 & 0.0 & 0.4 & 0.1 & 0.3 & 0.1 & 0.1 & & 0.1 & & 48.8 & 0.1 & & 0.0 & & & & 0.2 & 0.0 & & \\
\hline Vojtech, 1996 & 1300 & & 16.2 & 17.1 & & & 0.2 & & & 4.1 & & 0.0 & 1.4 & 0.1 & & 0.0 & 0.4 & 0.1 & 12.9 & 0.1 & \begin{tabular}{|l|}
0.1 \\
\end{tabular} & & 0.1 & & 46.8 & 0.1 & & 0.0 & & & & 0.2 & 0.0 & & \\
\hline Vojtech, 1996 & $\sim 1250$ & & 13.1 & 17.1 & & & 0.2 & & & 4.1 & & 0.0 & 1.4 & 0.1 & & 0.0 & 0.4 & 0.1 & 16.1 & 0.1 & 0.1 & & 0.1 & & 46.8 & 0.1 & & 0.0 & & & & 0.2 & 0.0 & & \\
\hline Vojtech, 1996 & 1150 & & 13.1 & 17.1 & & & 0.2 & & & 4.1 & & 0.0 & 1.4 & 0.1 & 6.7 & 0.0 & 0.4 & 0.1 & 9.4 & 0.1 & 0.1 & & 0.1 & & 46.8 & 0.1 & & 0.0 & & & & 0.2 & 0.0 & & \\
\hline Vojtech, 1996 & 1150 & & 10.0 & 17.1 & & & 0.2 & & & 4.1 & & 0.0 & 1.4 & 0.1 & & 0.0 & 0.4 & 0.1 & 19.2 & 0.1 & \begin{tabular}{|l|}
0.1 \\
\end{tabular} & & 0.1 & & 46.8 & 0.1 & & 0.0 & & & & 0.2 & 0.0 & & \\
\hline Vojtech, 1996 & $\sim 1100$ & & 10.0 & 17.1 & & & 0.2 & & & 4.1 & & 0.0 & 1.4 & 0.1 & 6.7 & 0.0 & 0.4 & 0.1 & 12.5 & 0.1 & 0.1 & & 0.1 & & 46.8 & 0.1 & & 0.0 & & & & 0.2 & 0.0 & & \\
\hline Wiese HEWC Pame & & & 20.7 & 21.7 & & & 3.9 & & & & & & & & 3.1 & & & & 8.6 & & & 0.8 & & & 38.8 & 0.9 & & & & 1.6 & & & & & \\
\hline Wroniciewicz, 199 & 1300 & & 3.3 & & & & 12.9 & 2.2 & & 1.5 & & & 16.2 & 0.7 & & 0.6 & & & 11.2 & 0.6 & & & & & 27.8 & & & & & 12.9 & 2.2 & & 7.9 & & \\
\hline Wroniciewicz, 199 & 1400 & & 3.2 & & & & 6.3 & 2.2 & & 2.2 & & & 39.9 & 0.7 & & 0.6 & & & 0.3 & 1.1 & & & & 2.2 & 16.6 & & & & & 0.4 & 2.2 & 22.0 & & & \\
\hline Wroniciewicz, 199 & 1500 & & 6.8 & & & & 4.0 & & & 0.0 & & & 2.8 & 1.4 & & 1.3 & & & 0.6 & & & 20.3 & & & 34.8 & & & & & 27.9 & & 0.0 & 0.0 & & \\
\hline Wroniciewicz, 199: & 1500 & & 27.5 & & & & 4.1 & & & 0.0 & & & 2.8 & 1.5 & & 1.3 & & & 0.7 & & & 25.2 & & & 35.8 & & & & & 0.9 & & 0.0 & 0.0 & & \\
\hline Wroniciewicz, 199 & 1500 & & 7.0 & & & & 14.6 & 2.3 & & 0.0 & & & 2.9 & 1.5 & & 1.3 & & & 0.7 & & & & & 2.3 & 36.2 & & & & & 24.1 & 2.3 & 0.0 & 0.0 & & \\
\hline Wroniciewicz, 199: & 1350 & & 8.1 & & & & 14.8 & 2.3 & & 0.0 & & & 6.9 & 1.3 & & 1.1 & & & 15.3 & & & & 16.9 & & 30.3 & & & & & 0.7 & 2.2 & 0.0 & 0.0 & & \\
\hline Wroniciewicz, 199 & 1500 & & 3.2 & & & & 1.9 & 2.2 & & 16.0 & & & 55.7 & 0.7 & & 0.6 & & & 0.3 & & & & & & 16.7 & & & & & 0.4 & 2.2 & & & & \\
\hline Wroniciewicz, 199 & 1500 & & 3.2 & & & & 1.9 & 2.1 & & & & & 55.8 & 0.7 & & 0.6 & & & 0.3 & 16.0 & & & & & 16.7 & & & & & 0.4 & 2.1 & & & & \\
\hline Wroniciewicz, 199: & 1500 & & 7.7 & & & & 14.6 & 1.8 & & 1.5 & & & 38.0 & 0.7 & & 0.7 & & & 0.3 & 0.6 & & & & 1.8 & 21.8 & & & & & 5.1 & 1.8 & 0.0 & 3.7 & & \\
\hline Wroniciewicz, 199 & 1500 & & 8.7 & & & & 15.9 & 2.0 & & 1.7 & & & 41.2 & 0.8 & & 0.7 & & & 0.4 & 0.8 & & & & 2.0 & 23.4 & & & & & 0.5 & 1.9 & & & & \\
\hline Wroniciewicz, 199: & 1500 & & 7.5 & & & & 14.2 & 1.7 & & 1.4 & & & 37.0 & 0.7 & & 0.6 & & & 0.3 & 0.6 & & & & 1.7 & 21.2 & & & & & 11.1 & 1.7 & 0.0 & & & \\
\hline Wroniciewicz, 199 & 1500 & & 7.5 & & & & 14.2 & 1.7 & & 1.4 & & & 37.0 & 0.7 & & 0.6 & & & 0.3 & 0.6 & & & & 1.7 & 21.2 & & & & & 11.1 & 1.7 & 0.0 & & & \\
\hline Wroniciewicz, 199 & 1500 & & 4.0 & & & & 12.6 & 1.8 & & 1.4 & & & 36.0 & 0.8 & & 0.8 & & & 0.4 & 0.6 & & & & 1.8 & 20.5 & & & & & 10.2 & 1.8 & & 7.4 & & \\
\hline Wroniciewicz, 199: & 1500 & & 4.0 & & & & 12.6 & 1.8 & & 1.4 & & & 36.0 & 0.8 & & 0.8 & & & 0.4 & 0.6 & & & & 1.8 & 20.5 & & & & & 10.2 & 1.8 & & 7.4 & & \\
\hline WVNS, 1995 & & & 7.1 & 15.3 & 0.3 & & 0.5 & 0.3 & & 0.1 & & & 9.7 & 6.9 & 2.3 & 0.9 & 0.8 & & 5.7 & 0.3 & & & 1.2 & & 40.6 & 0.2 & 0.3 & & 4.5 & 0.8 & 1.2 & 0.3 & 1.3 & & \\
\hline WVNS, 1995 & & & 7.1 & 10.4 & 0.3 & & 0.5 & 0.3 & & 0.1 & & & 14.3 & 6.9 & 5.1 & 0.9 & 0.8 & & 8.8 & 0.3 & & & 1.2 & & 36.9 & 0.2 & 0.3 & & 2.7 & 0.8 & 1.2 & 0.3 & 1.3 & & \\
\hline WVNS, 1995 & & & 7.7 & 16.6 & 0.3 & & 0.5 & 0.3 & & 0.1 & & & 15.5 & 3.1 & 2.3 & 0.9 & 0.8 & & 5.7 & 0.3 & & & 1.2 & & 39.5 & 0.2 & 0.3 & & 1.8 & 0.8 & 1.2 & 0.3 & 1.3 & & \\
\hline WVNS, 1995 & & & 7.7 & 16.6 & 0.3 & & 0.5 & 0.3 & & 0.1 & & & 8.6 & 6.9 & 5.1 & 0.9 & 0.8 & & 10.3 & 0.3 & & & 1.2 & & 35.3 & 0.2 & 0.3 & & 1.8 & 0.8 & 1.2 & 0.3 & 1.3 & & \\
\hline WVNS, 1995 & & & 7.7 & 9.2 & 0.3 & & 0.5 & 0.3 & & 0.1 & & & 8.6 & 3.1 & 4.3 & 0.9 & 0.8 & & 10.3 & 0.3 & & & 1.2 & & 47.1 & 0.2 & 0.3 & & 1.8 & 0.8 & 1.2 & 0.3 & 1.3 & & \\
\hline WVNS, 1995 & & & 7.7 & 9.2 & 0.3 & & 0.5 & 0.3 & & 0.1 & & & 8.6 & 6.9 & 2.3 & 0.9 & 0.8 & & 5.7 & 0.3 & & & 1.2 & & 46.4 & 0.2 & 0.3 & & 5.3 & 0.8 & 1.2 & 0.3 & 1.3 & & \\
\hline WVNS, 1995 & & & 7.7 & 9.2 & 0.3 & & 0.5 & 0.3 & & 0.1 & & & 15.5 & 6.9 & 2.5 & 0.9 & 0.8 & & 10.3 & 0.3 & & & 1.2 & & 34.8 & 0.2 & 0.3 & & 5.3 & 0.8 & 1.2 & 0.3 & 1.3 & & \\
\hline WVNS, 1995 & & & 7.4 & 9.0 & 0.3 & & 0.5 & 0.3 & & 0.1 & & & 6.6 & 6.0 & 2.8 & 0.9 & 0.8 & & 8.4 & 0.3 & & & 1.2 & & 47.7 & 0.2 & 0.3 & & 4.8 & 0.8 & 1.2 & 0.3 & 0.7 & & \\
\hline WVNS, 1995 & & & 7.4 & 7.1 & 0.3 & & 0.5 & 0.3 & & 0.1 & & & 17.4 & 5.9 & 4.0 & 0.9 & 0.8 & & 5.2 & 0.3 & & & 1.2 & & 43.0 & 0.2 & 0.3 & & 2.7 & 0.8 & 1.2 & 0.3 & 0.7 & & \\
\hline WVNS, 1995 & & & 7.7 & 7.1 & 0.3 & & 0.5 & 0.3 & & 0.1 & & & 17.4 & 4.2 & 4.7 & 0.9 & 0.8 & & 6.0 & 0.3 & & & 1.2 & & 39.3 & 0.2 & 0.3 & & 5.2 & 0.8 & 1.2 & 0.3 & 1.9 & & \\
\hline Yamagishi, 1985 & 1350 & & & & & & & & & & & & & 5.8 & & & & & & & 26.3 & & & 16.1 & 46.6 & & & & & & & 4.2 & 0.9 & & \\
\hline Yamagishi, 1985 & 1250 & & & 10.6 & & & & & & & & & & & & & & & 6.7 & & 41.5 & & & & 38.5 & & & & & & & 2.7 & & & \\
\hline Yamagishi, 1985 & 1300 & & & & & & & & & & & & & 11.1 & & & & & & & 34.8 & & & & 36.9 & & & & & & & 16.7 & 0.5 & & \\
\hline Yamagishi, 1985 & 1250 & & & 15.0 & & & & & & & & & & & & & & & 14.9 & & 21.5 & & & & 45.6 & & & & & & & 3.1 & & & \\
\hline Yamagishi, 1985 & 1400 & & & & & & & & & & & & & 17.7 & & 6.7 & & & & & 35.2 & & & & 39.9 & & & & & & & & 0.5 & & \\
\hline Yamagishi, 1985 & 1250 & & & & & & & & & & & & & & & & & & 9.3 & & 42.4 & & & & 44.2 & & & & & & & 4.1 & & & \\
\hline Yamagishi, 1985 & 1400 & & & & & & & & & & & & & & & & & & & & 41.2 & & & & 35.0 & & & & & & & 23.3 & 0.4 & & \\
\hline Yamagishi, 1985 & 1300 & & & 0.7 & & & & & & & & & & 11.3 & & & & & & & 30.9 & & & & 31.2 & & & & & & & 25.4 & 0.5 & & \\
\hline Yamagishi, 1985 & 1250 & & & & & & & & & & & & & & & & & & 5.8 & & 39.72 & 20.7 & & & 22.4 & & & & & & & 11.0 & 0.4 & & \\
\hline Yamagishi, 1985 & 1300 & & & & & & & & & & & & & 2.7 & & & & & & & 54.8 & & & & 29.1 & & & & & & & 13.1 & 0.4 & & \\
\hline Yamagishi, 1985 & 1300 & & & & & & & & & & & & & 6.0 & & & & & & & 46.4 & & & & 35.6 & & & & & 10.9 & & & 0.4 & & \\
\hline Yamagishi, 1985 & 1400 & & 1.0 & 1.4 & & & & & & & & & & 9.4 & & & & & & & 40.1 & & & & 41.2 & & & & & & & 6.5 & 0.5 & & \\
\hline Yamagishi, 1985 & 1250 & & & & & & & & & & & & & & & & & & & & 74.8 & & & & 25.0 & & & & & & & & 0.2 & & \\
\hline Yamagishi, 1985 & 1350 & & 4.7 & & & & & & & & & & & 8.8 & & & & & & & 39.6 & & & & 28.0 & & & & & & & 18.9 & & & \\
\hline Yamagishi, 1985 & 1250 & & & 4.8 & 26.6 & & & & & & & & & 8.2 & & & & & & & 33.2 & & & & 26.1 & & & & & & & & 1.2 & & \\
\hline
\end{tabular}


Table A1. Glass Compositions from Literature Review (continued)

\begin{tabular}{|c|c|c|c|c|c|c|c|c|c|c|c|c|c|c|c|c|c|c|c|c|c|c|c|c|c|c|c|c|c|c|c|c|c|c|c|}
\hline Ref & $\begin{array}{l}0 \\
0 \\
\stackrel{ \pm}{0} \\
E \\
⺊\end{array}$ & $\begin{array}{l}0 \\
0 \\
8 \\
\frac{8}{5} \\
1\end{array}$ & $\begin{array}{c}\text { ֻొ } \\
\frac{\pi}{4}\end{array}$ & 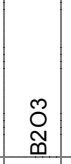 & $\begin{array}{l}\text { O } \\
\underset{\mathbb{D}}{ } \\
\end{array}$ & 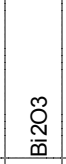 & $\begin{array}{l}0 \\
\mathbb{0} \\
0\end{array}$ & 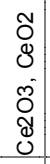 & $\begin{array}{l}\text { ON } \\
0 \\
0 \\
0 \\
0 \\
0\end{array}$ & $\begin{array}{l}\text { ర్ల } \\
\text { ปn }\end{array}$ & $\stackrel{9}{0}$ & \llcorner & 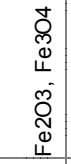 & $\begin{array}{l}\stackrel{\mathcal{\Upsilon}}{\underline{y}} \\
\end{array}$ & 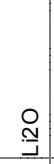 & $\frac{8}{2}$ & $\begin{array}{l}\tilde{Q} \\
\text { है }\end{array}$ & $\frac{\pi}{2}$ & $\underset{\underset{\mathbb{T}}{Z}}{\stackrel{O}{2}}$ & $\stackrel{0}{z}$ & $\begin{array}{l}\text { N } \\
\text { ठ̃ }\end{array}$ & $\begin{array}{l}\overline{\underline{\Xi}} \\
\overline{0}\end{array}$ & 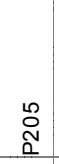 & $\begin{array}{l}0 \\
\frac{2}{2}\end{array}$ & $\begin{array}{l}\text { Õ } \\
\text { in }\end{array}$ & 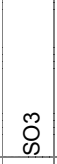 & $\begin{array}{l}\text { 잉 } \\
\text { (n) }\end{array}$ & 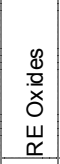 & $\begin{array}{l}\tilde{O} \\
\stackrel{5}{F}\end{array}$ & $\stackrel{\widetilde{N}}{\mathrm{I}}$ & 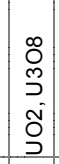 & $\begin{array}{l}O \\
\stackrel{N}{N}\end{array}$ & ญั & 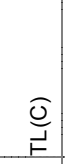 & $\begin{array}{l}\bar{J} \\
\frac{\vec{b}}{\tilde{\omega}} \\
\frac{1}{z}\end{array}$ \\
\hline Yamagishi, 1985 & 1350 & & & & & & & & & & & & & 11.2 & & & & & & & 30.9 & & & & 36.2 & & 8.6 & & & & & 12.7 & 0.5 & & \\
\hline Yamagishi, 1985 & 1300 & & & & & & & & & & & & & 10.7 & & & & & & & 35.8 & 4.0 & & & 35.8 & & & & & 4.8 & & 8.9 & & & \\
\hline Yamagishi, 1985 & 1250 & & & & & & 3.8 & & & & & & & 9.6 & & & & & 4.2 & & 41.1 & & & & 29.1 & & & & & & & 11.8 & 0.4 & & \\
\hline Yamagishi, 1985 & 1300 & & & & & & & & & & & & & 16.8 & & 6.3 & & & & & 33.1 & & & & 37.5 & & & & & 5.8 & & & 0.5 & & \\
\hline Yamagishi, 1985 & 1250 & & & 3.0 & & & & & & & & & & 2.4 & 1.8 & 1.7 & & & & & 47.4 & 2.3 & & & 29.4 & & & 8.4 & & & & 3.5 & & & \\
\hline Yamagishi, 1985 & 1200 & & 2.2 & 11.2 & & 19.8 & & & & & & & & 24.0 & & & & & & & 10.8 & & & & 26.0 & & & & & & & 5.5 & 0.4 & & \\
\hline Yan, 2001 & & 1277 & 15.2 & & & & 9.4 & & & & & & 17.1 & 1.4 & & 7.8 & & & 2.8 & & & 0.9 & & & 45.5 & & & & & & & & & & \\
\hline Yan, 2001 & & 1282 & 15.7 & & & & 8.5 & & & & & & 17.0 & 0.6 & & 7.1 & & & 1.6 & & & 1.1 & & & 48.0 & & & & & 0.4 & & & & & \\
\hline Yan, 2001 & & 1230 & 15.7 & & & & 12.5 & & & & & & 17.0 & 0.7 & & 8.0 & & & 1.6 & & & 0.6 & & & 44.0 & & & & & & & & & & \\
\hline Yee, 1955 & & 1112 & 6.6 & 23.5 & & & & & 0.6 & & & & & & & & 1.7 & & 21.7 & & & & & & 46.0 & & & & & & & & & & \\
\hline Yee, 1955 & & 1004 & 6.0 & 23.6 & & & & & 0.5 & & & 7.2 & & & & & 1.5 & & 20.8 & & & & & & 38.9 & & & & & & & & & & \\
\hline Yee, 1955 & & 1065 & 4.2 & 24.9 & & & & & 0.5 & & & 5.1 & & & & & 1.5 & & 18.7 & 0.5 & & & & & 43.5 & & & & & & & & & & \\
\hline Yee, 1955 & & 957 & 4.2 & 24.8 & & & & & 0.5 & & & 10.2 & & & & & 1.5 & & 19.8 & 0.5 & & & & & 36.3 & & & & & & & & & & \\
\hline Yee, 1955 & & 1032 & 4.4 & 25.5 & & & & & 0.5 & & & 6.1 & & & & & 1.5 & & 19.1 & 0.5 & & & & & 41.1 & & & & & & & & & & \\
\hline Yee, 1955 & & 965 & 5.4 & 25.5 & 8.2 & & & & 0.5 & & & 5.1 & & & & & 1.5 & & 19.0 & 0.5 & & & & & 33.3 & & & & & & & & & & \\
\hline Yee, 1955 & & 1089 & 6.5 & 23.2 & & & & & 0.6 & & & 1.0 & & & & & 1.7 & & 21.5 & & & & & & 45.5 & & & & & & & & & & \\
\hline Yee, 1955 & & 1057 & 6.4 & 22.8 & & & & & 0.5 & & & 3.0 & & & & & 1.6 & & 21.0 & & & & & & 44.6 & & & & & & & & & & \\
\hline Yee, 1955 & & 1031 & 6.3 & 22.3 & & & & & 0.5 & & & 4.9 & & & & & 1.6 & & 20.7 & & & & & & 43.7 & & & & & & & & & & \\
\hline Yee, 1955 & & 1022 & 6.2 & 22.1 & & & & & 0.5 & & & 5.7 & & & & & 1.6 & & 20.5 & & & & & & 43.3 & & & & & & & & & & \\
\hline Yee, 1955 & & 1007 & 6.1 & 21.9 & & & & & 0.5 & & & 6.9 & & & & & 1.6 & & 20.2 & & & & & & 42.8 & & & & & & & & & & \\
\hline Yee, 1955 & & 994 & 6.1 & 21.6 & & & & & 0.5 & & & 8.1 & & & & & 1.6 & & 19.9 & & & & & & 42.2 & & & & & & & & & & \\
\hline Yee, 1955 & & 972 & 5.9 & 21.1 & & & & & 0.5 & & & 10.3 & & & & & 1.5 & & 19.5 & & & & & & 41.2 & & & & & & & & & & \\
\hline Yee, 1955 & & 995 & 5.7 & 22.5 & & & & & 0.5 & & & 6.9 & 4.5 & & & & 1.5 & & 19.8 & & & & & & 37.1 & & & & & & & & & & \\
\hline Yee, 1955 & & 1001 & 5.5 & 21.6 & & & & & 0.5 & & & 6.6 & 8.3 & & & & 1.4 & & 19.0 & & & & & & 35.6 & & & & & & & & & & \\
\hline Yee, 1955 & & 997 & 5.3 & 20.9 & & & & & 0.5 & & & 6.4 & 11.5 & & & & 1.4 & & 18.4 & & & & & & 34.4 & & & & & & & & & & \\
\hline Yee, 1955 & & 995 & 5.2 & 20.2 & & & & & 0.4 & & & 6.2 & 14.3 & & & & 1.3 & & 17.8 & & & & & & 33.3 & & & & & & & & & & \\
\hline Yee, 1955 & & 1010 & 5.5 & 21.7 & & & & & 0.5 & & & 5.6 & & & & & 1.4 & & 19.1 & & & & & & 35.7 & & & & & & & & & & \\
\hline Yee, 1955 & & 1046 & 5.4 & 21.0 & & & & & 0.5 & & & 6.2 & & & & & 1.4 & & 18.5 & & & & & & 34.7 & & & & & & & & & & \\
\hline Yee, 1955 & & 1001 & 3.7 & 22.5 & 2.5 & & & & 0.2 & & & 5.6 & & & & & 0.7 & & 10.3 & & & & & & 41.8 & & & & & & & & & & \\
\hline Yoshida, 1993 & $\sim 1300$ & & 2.0 & 24.0 & 5.0 & & 5.0 & & & & & & & 6.0 & & & & & 2.0 & & & & & & 49.0 & & & 2.0 & & & & & 5.0 & & \\
\hline Yoshida, 1993 & $\sim 1300$ & & 1.5 & 22.0 & 5.0 & & & & & & & & & 6.0 & & & & & 4.0 & & & & & & 45.5 & & & 5.0 & & & & 7.0 & 4.0 & & \\
\hline Yoshida, 1993 & $\sim 1300$ & & 2.0 & 21.0 & 4.5 & & & & & & & & & 5.0 & & & & & 3.0 & & & & & & 50.0 & & & 5.0 & & & & 4.5 & 5.0 & & \\
\hline Yoshida, 1993 & $\sim 1300$ & & 3.0 & 29.0 & 7.0 & & & & & & & & & 5.0 & & & & & 2.0 & & & & & & 40.0 & & & 2.0 & & & & 7.0 & 5.0 & & \\
\hline Yoshida, 1993 & $\sim 1300$ & & 6.0 & 38.0 & & & & & & & & & & 2.0 & 2.0 & & & & 2.0 & & & & & & 37.0 & & & 5.0 & & & & 8.0 & & & \\
\hline Yoshida, 1993 & $\sim 1300$ & & 2.0 & 24.0 & 5.0 & & & & & & & & & 6.0 & & & & & 2.0 & & & & & & 49.0 & & 5.0 & 2.0 & & & & & 5.0 & & \\
\hline Yoshida, 1993 & $\sim 1300$ & & 2.5 & 22.5 & 4.5 & 13.0 & & & & & & & & 5.0 & & & & & 2.5 & & & & & & 39.0 & & & 2.0 & & & & 4.5 & 4.5 & & \\
\hline Yoshida, 1993 & $\sim 1300$ & & 3.0 & 26.0 & 5.0 & & & & & & & & & 6.0 & & & & & 3.0 & & & & & & 45.0 & & & 2.0 & & & & 5.0 & 5.0 & & \\
\hline Zubekhin, 1993 & $\leq 1260$ & & 20.2 & & & & 18.2 & & & & & & 4.0 & 6.4 & & 3.2 & 2.0 & & 5.6 & & & & & & 40.3 & & & & & & & & & & \\
\hline Zubekhin, 1993 & $\leq 1260$ & & 20.2 & & & & 22.2 & & & & & & 4.0 & 6.4 & & 3.2 & 2.0 & & 5.6 & & & & & & 36.3 & & & & & & & & & & \\
\hline Zubekhin, 1993 & $\leq 1260$ & & 24.2 & & & & 18.2 & & & & & & 4.0 & 6.4 & & 3.2 & 2.0 & & 5.6 & & & & & & 36.3 & & & & & & & & & & \\
\hline Zubekhin, 1993 & $>1260$ & & 20.2 & & & & 30.2 & & & & & & 4.0 & 6.4 & & 3.2 & 2.0 & & 5.6 & & & & & & 28.2 & & & & & & & & & & \\
\hline Zubekhin, 1993 & $\leq 1260$ & & 28.2 & & & & 22.2 & & & & & & 4.0 & 6.4 & & 3.2 & 2.0 & & 5.6 & & & & & & 28.2 & & & & & & & & & & \\
\hline Zubekhin, 1993 & $\leq 1260$ & & 36.3 & & & & 14.1 & & & & & & 4.0 & 6.4 & & 3.2 & 2.0 & & 5.6 & & & & & & 28.2 & & & & & & & & & & \\
\hline Zubekhin, 1993 & $\gg>1260$ & & 20.2 & & & & 38.3 & & & & & & 4.0 & 6.4 & & 3.2 & 2.0 & & 5.6 & & & & & & 20.2 & & & & & & & & & & \\
\hline Zubekhin, 1993 & $>1260$ & & 28.2 & & & & 30.2 & & & & & & 4.0 & 6.4 & & 3.2 & 2.0 & & 5.6 & & & & & & 20.2 & & & & & & & & & & \\
\hline Zubekhin, 1993 & $\leq 1260$ & & 36.3 & & & & 22.2 & & & & & & 4.0 & 6.4 & & 3.2 & 2.0 & & 5.6 & & & & & & 20.2 & & & & & & & & & & \\
\hline Zubekhin, 1993 & $>1260$ & & 44.3 & & & & 14.1 & & & & & & 4.0 & 6.4 & & 3.2 & 2.0 & & 5.6 & & & & & & 20.2 & & & & & & & & & & \\
\hline Zubekhin, 1993 & $>>1260$ & & 20.2 & & & & 46.4 & & & & & & 4.0 & 6.4 & & 3.2 & 2.0 & & 5.6 & & & & & & 12.1 & & & & & & & & & & \\
\hline Zubekhin, 1993 & $\gg>1260$ & & 28.2 & & & & 38.3 & & & & & & 4.0 & 6.4 & & 3.2 & 2.0 & & 5.6 & & & & & & 12.1 & & & & & & & & & & \\
\hline
\end{tabular}




\begin{tabular}{|c|c|c|c|c|c|c|c|c|c|c|c|c|c|c|c|c|c|c|c|c|c|c|c|c|c|c|c|c|c|c|c|c|c|c|c|}
\hline Ref & 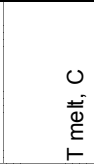 & 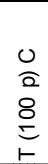 & 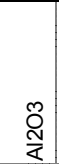 & ָొ & 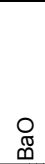 & $\stackrel{\widetilde{n}}{\frac{\tilde{\omega}}{\omega}}$ & O్ర & 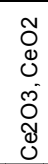 & $\begin{array}{l}\widetilde{N} \\
\text { Oे } \\
0 \\
0 \\
0\end{array}$ & O̊ & @̧ & 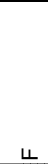 & 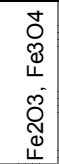 & 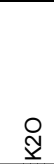 & 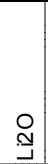 & $\begin{array}{l}0 \\
\stackrel{D}{\Sigma}\end{array}$ & $\begin{array}{l}\tilde{N} \\
\tilde{\Sigma} \\
\Sigma\end{array}$ & $\begin{array}{l}0 \\
\stackrel{0}{2} \\
\stackrel{0}{\Sigma}\end{array}$ & $\begin{array}{l}\stackrel{\sim}{\mathbb{Z}} \\
\mathbb{Z}\end{array}$ & 을 & 胥 & 疋 & 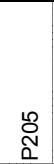 & $\begin{array}{l}\circ \\
\frac{2}{\alpha}\end{array}$ & ก̃ & ర్రి & 오ํ & 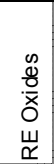 & $\begin{array}{l}\widetilde{O} \\
\stackrel{5}{F}\end{array}$ & $\stackrel{\widetilde{N}}{\stackrel{\rho}{\circ}}$ & $\begin{array}{l}\text { o } \\
\text { O } \\
\text { S } \\
\text { Ñ } \\
\text { O }\end{array}$ & $\stackrel{0}{\grave{N}}$ & ญั & $\underset{\mapsto}{\stackrel{0}{J}}$ & $\begin{array}{l}\bar{\partial} \\
\text { ప্ర } \\
\bar{z}\end{array}$ \\
\hline Zubekhin, 1993 & $>>1260$ & & 36.3 & & & & 30.2 & & & & & & 4.0 & 6.4 & & 3.2 & 2.0 & & 5.6 & & & & & & 12.1 & & & & & & & & & & \\
\hline Zubekhin, 1993 & $>>1260$ & & 44.3 & & & & 22.2 & & & & & & 4.0 & 6.4 & & 3.2 & 2.0 & & 5.6 & & & & & & 12.1 & & & & & & & & & & \\
\hline Zubekhin, 1993 & $>>1260$ & & 52.4 & & & & 14.1 & & & & & & 4.0 & 6.4 & & 3.2 & 2.0 & & 5.6 & & & & & & 12.1 & & & & & & & & & & \\
\hline Zubekhin, 1993 & $>1260$ & & 12.6 & & & & 10.9 & & & & & & 3.4 & 5.4 & & 2.7 & 1.7 & & 4.7 & & & & & & 58.7 & & & & & & & & & & \\
\hline Zubekhin, 1993 & $>1260$ & & 21.0 & & & & 10.9 & & & & & & 3.4 & 5.4 & & 2.7 & 1.7 & & 4.7 & & & & & & 50.3 & & & & & & & & & & \\
\hline Zubekhin, 1993 & $>1260$ & & 12.6 & & & & 27.7 & & & & & & 3.4 & 5.4 & & 2.7 & 1.7 & & 4.7 & & & & & & 41.9 & & & & & & & & & & \\
\hline Zubekhin, 1993 & $\leq 1260$ & & 29.4 & & & & 10.9 & & & & & & 3.4 & 5.4 & & 2.7 & 1.7 & & 4.7 & & & & & & 41.9 & & & & & & & & & & \\
\hline Zubekhin, 1993 & $\leq 1260$ & & 12.6 & & & & 31.9 & & & & & & 3.4 & 5.4 & & 2.7 & 1.7 & & 4.7 & & & & & & 37.8 & & & & & & & & & & \\
\hline Zubekhin, 1993 & $\leq 1260$ & & 33.6 & & & & 10.9 & & & & & & 3.4 & 5.4 & & 2.7 & 1.7 & & 4.7 & & & & & & 37.8 & & & & & & & & & & \\
\hline Zubekhin, 1993 & $>1260$ & & 13.5 & & & & 36.0 & & & & & & 3.6 & 5.8 & & 2.9 & 1.8 & & 5.0 & & & & & & 31.5 & & & & & & & & & & \\
\hline Zubekhin, 1993 & $>>1260$ & & 41.9 & & & & 10.9 & & & & & & 3.4 & 5.4 & & 2.7 & 1.7 & & 4.7 & & & & & & 29.4 & & & & & & & & & & \\
\hline Zubekhin, 1993 & $>1260$ & & 12.6 & & & & 19.3 & & & & & & 3.4 & 5.4 & & 2.7 & 1.7 & & 4.7 & & & & & & 50.3 & & & & & & & & & & \\
\hline
\end{tabular}

